\title{
Maternal, Fetal, and Child Outcomes of Mental Health Treatments in Women: A Systematic Review of Perinatal Pharmacologic Interventions
}


Number 236

\title{
Maternal, Fetal, and Child Outcomes of Mental Health Treatments in Women: A Systematic Review of Perinatal Pharmacologic Interventions
}

\author{
Prepared for: \\ Agency for Healthcare Research and Quality \\ U.S. Department of Health and Human Services \\ 5600 Fishers Lane \\ Rockville, MD 20857 \\ www.ahrq.gov
}

Contract No. 290-2015-00011-I

Prepared by:

RTI International-University of North Carolina at Chapel Hill Evidence-based Practice Center

Research Triangle Park, NC

\section{Investigators:}

Meera Viswanathan, Ph.D.

Jennifer Cook Middleton, Ph.D.

Alison Stuebe, M.D., M.Sc.

Nancy Berkman, Ph.D.

Alison N. Goulding, M.D., M.S.C.R.

Skyler McLaurin-Jiang, M.D., M.P.H.

Andrea B. Dotson, M.D., M.S.P.H.

Manny Coker-Schwimmer, M.P.H.

Claire Baker

Christiane Voisin, M.S.L.S.

Carla Bann, Ph.D.

Bradley N. Gaynes, M.D., M.P.H.

AHRQ Publication No. 21-EHC001

April 2021 
This report is based on research conducted by the RTI International-University of North Carolina at Chapel Hill Evidence-based Practice Center (EPC) under contract to the Agency for Healthcare Research and Quality (AHRQ), Rockville, MD (Contract No. 290-2015-00011-I). The findings and conclusions in this document are those of the authors, who are responsible for its contents; the findings and conclusions do not necessarily represent the views of AHRQ. Therefore, no statement in this report should be construed as an official position of AHRQ or of the U.S. Department of Health and Human Services.

\section{None of the investigators have any affiliations or financial involvement that conflicts with the material presented in this report.}

The information in this report is intended to help healthcare decision makers - patients and clinicians, health system leaders, and policymakers, among others-make well-informed decisions and thereby improve the quality of healthcare services. This report is not intended to be a substitute for the application of clinical judgment. Anyone who makes decisions concerning the provision of clinical care should consider this report in the same way as any medical reference and in conjunction with all other pertinent information, i.e., in the context of available resources and circumstances presented by individual patients.

This report is made available to the public under the terms of a licensing agreement between the author and the Agency for Healthcare Research and Quality. This report may be used and reprinted without permission except those copyrighted materials that are clearly noted in the report. Further reproduction of those copyrighted materials is prohibited without the express permission of copyright holders.

AHRQ or U.S. Department of Health and Human Services endorsement of any derivative products that may be developed from this report, such as clinical practice guidelines, other quality enhancement tools, or reimbursement or coverage policies, may not be stated or implied.

AHRQ appreciates appropriate acknowledgment and citation of its work. Suggested language for acknowledgment: This work was based on an evidence report, Maternal, Fetal, and Child Outcomes of Mental Health Treatments in Women: A Systematic Review of Perinatal Pharmacologic Interventions, by the Evidence-based Practice Center Program at the Agency for Healthcare Research and Quality (AHRQ).

Suggested citation: Viswanathan M, Middleton JC, Stuebe A., Berkman N., Goulding AN, McLaurin-Jiang S, Dotson AB, Coker-Schwimmer M, Baker C, Voisin C, Bann C, Gaynes BN. Maternal, Fetal, and Child Outcomes of Mental Health Treatments in Women: A Systematic Review of Perinatal Pharmacologic Interventions. Comparative Effectiveness Review No. 236. (Prepared by the RTI International-University of North Carolina at Chapel Hill Evidence-based Practice Center under Contract No. 290-2015-00011-I.) AHRQ Publication No. 21-EHC001. Rockville, MD: Agency for Healthcare Research and Quality; April 2021. DOI: https://doi.org/10.23970/AHRQEPCCER236. Posted final reports are located on the Effective Health Care Program search page. 


\section{Preface}

The Agency for Healthcare Research and Quality (AHRQ), through its Evidence-based Practice Centers (EPCs), sponsors the development of systematic reviews to assist public- and private-sector organizations in their efforts to improve the quality of healthcare in the United States. These reviews provide comprehensive, science-based information on common, costly medical conditions, and new healthcare technologies and strategies.

Systematic reviews are the building blocks underlying evidence-based practice; they focus attention on the strength and limits of evidence from research studies about the effectiveness and safety of a clinical intervention. In the context of developing recommendations for practice, systematic reviews can help clarify whether assertions about the value of the intervention are based on strong evidence from clinical studies. For more information about AHRQ EPC systematic reviews, see https://effectivehealthcare.ahrq.gov/about/epc/evidence-synthesis.

AHRQ expects that these systematic reviews will be helpful to health plans, providers, purchasers, government programs, and the healthcare system as a whole. Transparency and stakeholder input are essential to the Effective Health Care Program. Please visit the website (www.effectivehealthcare.ahrq.gov) to see draft research questions and reports or to join an email list to learn about new program products and opportunities for input.

If you have comments on this systematic review, they may be sent by mail to the Task Order Officer named below at: Agency for Healthcare Research and Quality, 5600 Fishers Lane, Rockville, MD 20857, or by email to epc@ahrq.hhs.gov.

David Meyers, M.D. Acting Director Agency for Healthcare Research and Quality

Christine Chang, M.D., M.P.H.

Acting Director

Evidence-based Practice Center Program

Center for Evidence and Practice Improvement Agency for Healthcare Research and Quality
Arlene S. Bierman, M.D., M.S.

Director

Center for Evidence and Practice Improvement

Agency for Healthcare Research and Quality

Jill Huppert, M.D.

Task Order Officer

Center for Evidence and Practice Improvement

Agency for Healthcare Research and Quality 


\section{Acknowledgments}

The authors gratefully acknowledge the following individuals for their contributions to this project and deeply appreciate their considerable support, commitment, and contributions: Christine Chang, M.D., our medical officer; Jill Huppert, M.D., M.P.H., our AHRQ Task Order Officer (TOO); Aysegul Gozu, M.D., M.P.H., our previous TOO; Ian Saldanha, M.B.S, M.P.H., Ph.D., our EPC Associate Editor; RTI International-University of North Carolina at Chapel Hill EPC staff: Rania Ali, M.P.H.; Sharon Barrell, M.A.; Rachel Clark, B.A.; Kayla Giger, B.S.; Valerie Forman Hoffman, Ph.D., M.P.H; Linda Lux, M.P.A.; Kathleen Lohr, Ph.D.; Loraine Monroe; Kara Suvada, B.S.; Rachel Palmieri Weber, Ph.D.; and Carol Woodell, B.S.P.H. We also thank representatives from the American Psychiatric Association (Jennifer Medicus, M.B.A.; Laura Fochtmann, M.D., M.B.I.) and American College of Obstetricians and Gynecologists (Jessica Butler, M.P.H).

\section{Key Informants}

In designing the study questions, the EPC consulted several Key Informants who represent the end-users of research. The EPC sought the Key Informant input on the priority areas for research and synthesis. Key Informants are not involved in the analysis of the evidence or the writing of the report. Therefore, in the end, study questions, design, methodological approaches, and/or conclusions do not necessarily represent the views of individual Key Informants.

Key Informants must disclose any financial conflicts of interest greater than $\$ 5,000$ and any other relevant business or professional conflicts of interest. Because of their role as end-users, individuals with potential conflicts may be retained. The TOO and the EPC work to balance, manage, or mitigate any conflicts of interest.

The list of Key Informants who provided input to this report follows:

Madeleine Becker, M.D., FACLP*

Sidney Kimmel Medical College

Thomas Jefferson University and Jefferson

Health

Philadelphia, PA

Beth Collins Sharp, Ph.D., R.N., FAAN

Office of Women's Health, HHS

Washington, DC

*Provided input on Draft Report.
Tiffany A. Moore Simas, M.D., M.P.H., M.Ed.*

University of Massachusetts Medical School UMass Memorial Health Care

Worcester, MA

Michael O'Hara, Ph.D.

University of Iowa

Iowa City, IA

\section{Technical Expert Panel}

In designing the study questions and methodology at the outset of this report, the EPC consulted several technical and content experts. Broad expertise and perspectives were sought. Divergent and conflicted opinions are common and perceived as healthy scientific discourse that results in a thoughtful, relevant systematic review. Therefore, in the end, study questions, design, methodologic approaches, and/or conclusions do not necessarily represent the views of individual technical and content experts. 
Technical Experts must disclose any financial conflicts of interest greater than $\$ 5,000$ and any other relevant business or professional conflicts of interest. Because of their unique clinical or content expertise, individuals with potential conflicts may be retained. The TOO and the EPC work to balance, manage, or mitigate any potential conflicts of interest identified.

The list of Technical Experts who provided input to this report follows:

Madeleine Becker, M.D., FACLP*

Sidney Kimmel Medical College

Thomas Jefferson University and Jefferson

Health

Philadelphia, PA

Lee S. Cohen, M.D.

Massachusetts General Hospital

Harvard Medical School

Boston, MA

Beth Collins Sharp, Ph.D., R.N., FAAN

Office of Women's Health, HHS

Washington, DC
Deidre Gifford, M.D., M.P.H.

Connecticut Department of Public Health

Hartford, CT

Samantha Meltzer-Brody, M.D., M.P.H.

University of North Carolina at Chapel Hill

Chapel Hill, NC

Tiffany A. Moore Simas, M.D., M.P.H., M.Ed.*

University of Massachusetts Medical School

UMass Memorial Health Care

Worcester, MA

Michael O’Hara, Ph.D.

University of Iowa

Iowa City, IA

*Provided input on Draft Report.

\section{Peer Reviewers}

Prior to publication of the final evidence report, EPCs sought input from independent Peer Reviewers without financial conflicts of interest. However, the conclusions and synthesis of the scientific literature presented in this report do not necessarily represent the views of individual reviewers.

Peer Reviewers must disclose any financial conflicts of interest greater than $\$ 5,000$ and any other relevant business or professional conflicts of interest. Because of their unique clinical or content expertise, individuals with potential nonfinancial conflicts may be retained. The TOO and the EPC work to balance, manage, or mitigate any potential nonfinancial conflicts of interest identified. 
The list of Peer Reviewers follows:

Amritha Bhat, M.B.B.S., M.D., M.P.H.

University of Washington

Seattle, WA

Krista F. Huybrechts, M.S., Ph.D.

Harvard Medical School

Brigham and Women's Hospital

Boston, MA
Pedro Martinez, M.D.

National Institute of Mental Health

Bethesda, MD 


\section{Maternal, Fetal, and Child Outcomes of Mental Health Treatments in Women: A Systematic Review of Perinatal Pharmacologic Interventions}

Background. Untreated maternal mental health disorders can have devastating sequelae for the mother and child. For women who are currently or planning to become pregnant or are breastfeeding, a critical question is whether the benefits of treating psychiatric illness with pharmacologic interventions outweigh the harms for mother and child.

Methods. We conducted a systematic review to assess the benefits and harms of pharmacologic interventions compared with placebo, no treatment, or other pharmacologic interventions for pregnant and postpartum women with mental health disorders. We searched four databases and other sources for evidence available from inception through June 5, 2020 and surveilled the literature through March 2, 2021; dually screened the results; and analyzed eligible studies. We included studies of pregnant, postpartum, or reproductive-age women with a new or preexisting diagnosis of a mental health disorder treated with pharmacotherapy; we excluded psychotherapy. Eligible comparators included women with the disorder but no pharmacotherapy or women who discontinued the pharmacotherapy before pregnancy.

Results. A total of 164 studies (168 articles) met eligibility criteria. Brexanolone for depression onset in the third trimester or in the postpartum period probably improves depressive symptoms at 30 days (least square mean difference in the Hamilton Rating Scale for Depression, -2.6; $\mathrm{p}=0.02 ; \mathrm{N}=209$ ) when compared with placebo. Sertraline for postpartum depression may improve response (calculated relative risk [RR], 2.24; 95\% confidence interval [CI], 0.95 to 5.24; $\mathrm{N}=36$ ), remission (calculated $\mathrm{RR}, 2.51 ; 95 \% \mathrm{CI}, 0.94$ to $6.70 ; \mathrm{N}=36$ ), and depressive symptoms (p-values ranging from 0.01 to 0.05 ) when compared with placebo. Discontinuing use of mood stabilizers during pregnancy may increase recurrence (adjusted hazard ratio [AHR], 2.2; 95\% CI, 1.2 to $4.2 ; \mathrm{N}=89$ ) and reduce time to recurrence of mood disorders ( 2 vs. 28 weeks, AHR, 12.1; $95 \% \mathrm{CI}, 1.6$ to $91 ; \mathrm{N}=26$ ) for bipolar disorder when compared with continued use. Brexanolone for depression onset in the third trimester or in the postpartum period may increase the risk of sedation or somnolence, leading to dose interruption or reduction when compared with placebo (5\% vs. $0 \%$ ). More than 95 percent of studies reporting on harms were observational in design and unable to fully account for confounding. These studies suggested some associations between benzodiazepine exposure before conception and ectopic pregnancy; between specific antidepressants during pregnancy and adverse maternal outcomes such as postpartum hemorrhage, preeclampsia, and spontaneous abortion, and child outcomes such as respiratory issues, low Apgar scores, persistent pulmonary hypertension of the newborn, depression in children, and autism spectrum disorder; between quetiapine or olanzapine and gestational diabetes; and between benzodiazepine and neonatal intensive care admissions. Causality cannot be inferred from these studies. We found insufficient evidence on benefits and harms from comparative effectiveness studies, with one exception: one study suggested a higher risk of overall congenital anomalies (adjusted RR [ARR], 1.85; 95\% CI, 1.23 to 2.78; $\mathrm{N}=2,608$ ) and cardiac anomalies (ARR, 2.25; 95\% CI, 1.17 to $4.34 ; \mathrm{N}=2,608$ ) for lithium compared with lamotrigine during first- 
trimester exposure.

Conclusions. Few studies have been conducted in pregnant and postpartum women on the benefits of pharmacotherapy; many studies report on harms but are of low quality. The limited evidence available is consistent with some benefit, and some studies suggested increased adverse events. However, because these studies could not rule out underlying disease severity as the cause of the association, the causal link between the exposure and adverse events is unclear. Patients and clinicians need to make an informed, collaborative decision on treatment choices. 


\section{Contents}

Evidence Summary ............................................................................................................................. ES-1

Introduction ................................................................................................................................ 1

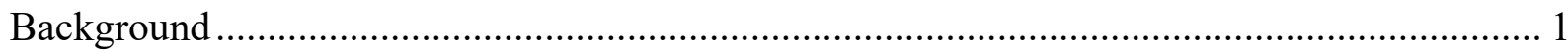

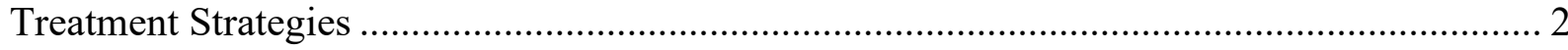

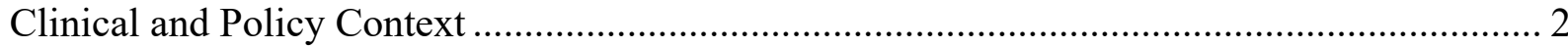

Purpose and Scope of the Systematic Review .................................................................... 3

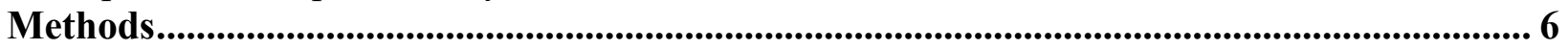

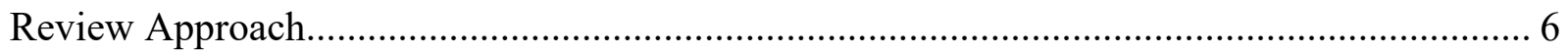

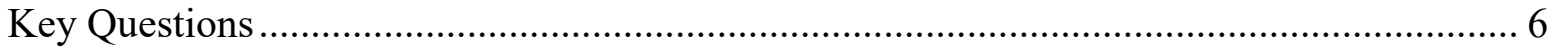

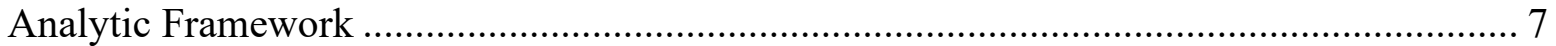

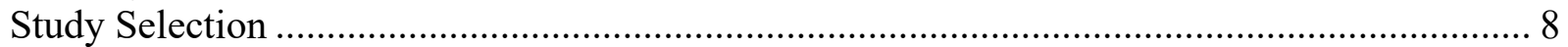

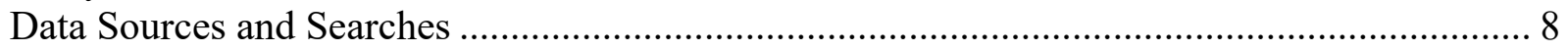

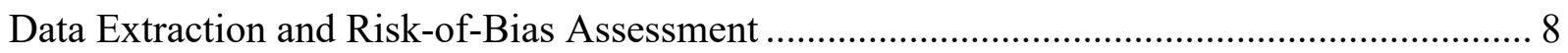

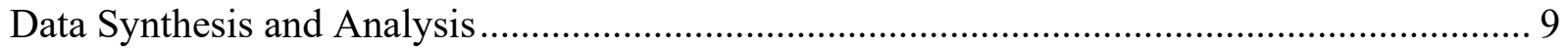

Grading the Strength of the Body of Evidence ................................................................... 9

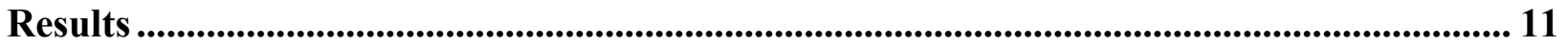

Literature Searches and Study Characteristics..................................................................... 11

Description of Included Evidence ......................................................................................... 11

KQ 1: Benefits of Pharmacologic Treatments Versus No Treatment or Placebo for Pregnant and Postpartum Women With Anxiety, Depression, Bipolar Disorder, or Schizophrenia ....... 12

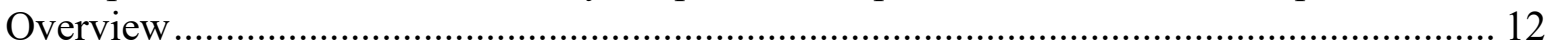

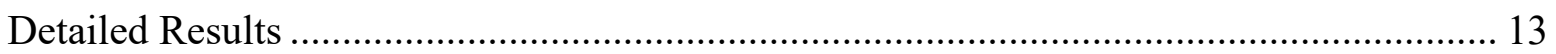

KQ 2: Comparative Benefits of Pharmacologic Treatments for Pregnant and Postpartum

Women With Anxiety, Depression, Bipolar Disorder, or Schizophrenia................................. 19

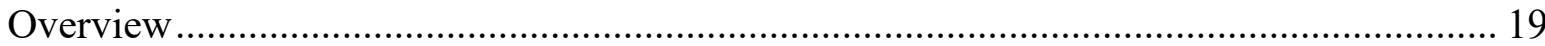

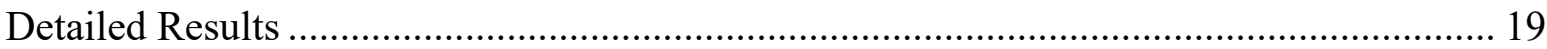

KQ 3: Harms of Pharmacologic Treatments Versus No Treatment or Placebo for Pregnant and

Postpartum Women With Mental Health Disorders ……………......................................... 20

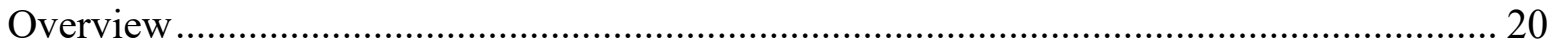

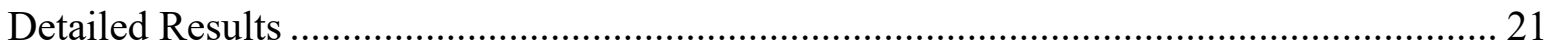

KQ 4: Comparative Harms of Pharmacologic Treatments for Pregnant and Postpartum

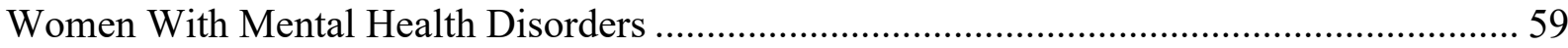

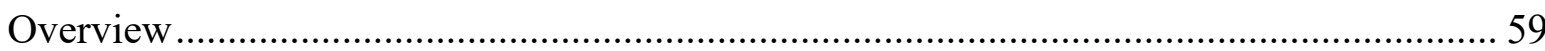

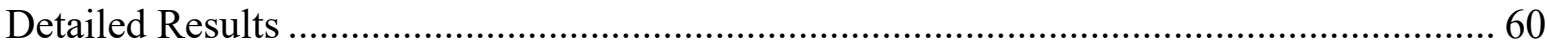

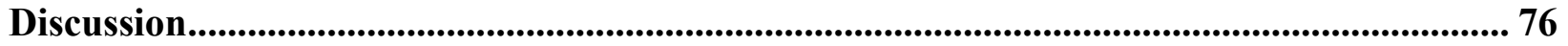

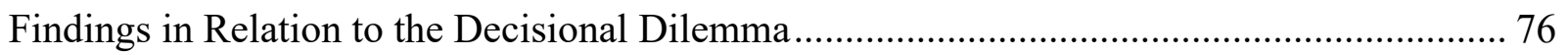

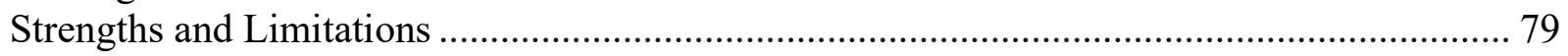

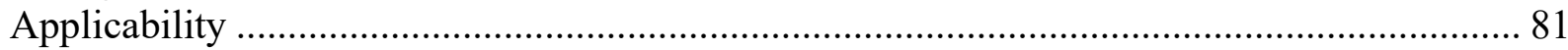

Implications for Clinical Practice, Education, Research, or Health Policy ............................. 81

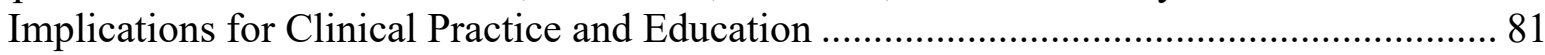

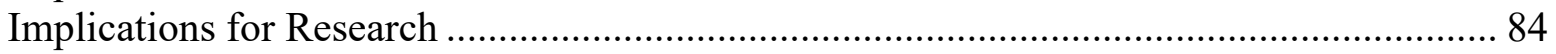

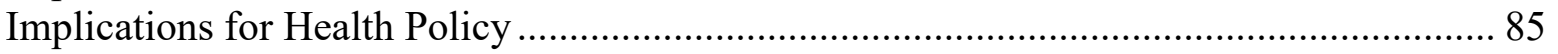

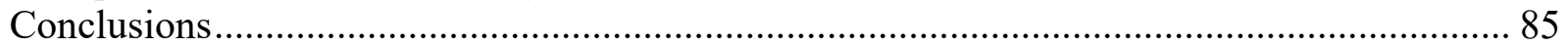

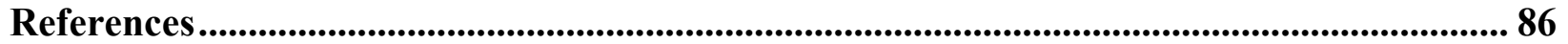

Abbreviations and Acronyms .............................................................................................................. 96 


\section{Tables}

Table 1. Concordance and uncertainty in clinical practice guidelines........................................ 4

Table 2. Key characteristics for included studies ........................................................... 11

Table 3. Summary of evidence for maternal benefit for treatment versus no treatment for mental health disorders in pregnancy and postpartum................................................. 13

Table 4. Strength of evidence for maternal benefit for brexanolone versus placebo for depression

Table 5. Strength of evidence for maternal benefit of sertraline versus no treatment for depression

Table 6. Strength of evidence for maternal benefit of mood stabilizers versus no treatment for bipolar disorder

Table 7. Summary of evidence from comparative effectiveness studies for maternal benefits of pharmacologic treatments for mental health disorders in pregnancy or postpartum.... 19

Table 8. Summary of evidence for harms from pharmacologic treatments versus no treatment for mental health disorders in pregnancy or postpartum

Table 9. Strength of evidence from effectiveness studies for harm to the mother (intervention versus placebo or no treatment)

Table 10. Strength of evidence from effectiveness studies for harm to the fetus, infant, or child (intervention versus placebo or no treatment)

Table 11. Insufficient strength of evidence from effectiveness studies for harm to the mother

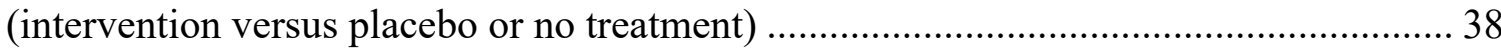

Table 12. Insufficient strength of evidence from effectiveness studies for harm to the fetus, infant, or child (intervention versus placebo or no treatment).

Table 13. Summary of evidence from comparative effectiveness studies for harm of pharmacologic treatments for mental health disorders in pregnancy or postpartum

Table 14. Strength of evidence from effectiveness studies for harm to the fetus, infant, or child (intervention versus placebo or no treatment)

Table 15. Insufficient strength of evidence from comparative effectiveness studies for harm to the mother 70

Table 16. Strength of evidence for harms outcomes: SSRIs versus SNRIs............................... 71

Table 17. Absolute risk differences for key outcomes .......................................................... 82

\section{Figures}

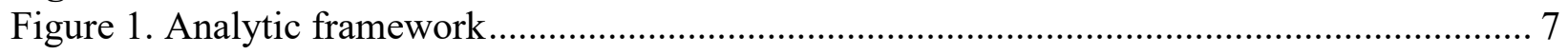

Figure 2. Exposure to psychotropic medications and major congenital anomalies.

\section{Appendixes}

Appendix A. Detailed Methods

Appendix B. Results

Appendix C. References for Appendixes 


\section{Evidence Summary}

\section{Main Points}

- Few studies have been conducted in pregnant and postpartum women on the benefits of pharmacotherapy; many studies report on harms but are of low quality.

- Brexanolone probably improves depressive symptoms; it may increase the risk of sedation or somnolence, leading to dose interruption or reduction.

- Sertraline may improve response, remission, and depression and anxiety symptoms.

- Mood stabilizers may reduce recurrence and increase time to recurrence.

- Although associations may exist between psychotropic medications and adverse events, causality cannot be inferred.

- First-trimester exposure to lithium is more likely to be associated with overall congenital and cardiac anomalies than first trimester exposure to lamotrigine, which can inform the decision to switch a medication in a successfully treated individual.

- We did not find eligible evidence on congenital anomalies for triazolam, alprazolam, valproate, carbamazepine, clonazepam, and topiramate, although evidence is available from studies of other populations ineligible for this review.

- The paucity of evidence does not mean that pharmacotherapy is not beneficial, nor that harms do not exist; rather, it underscores the absence of high-quality research.

\section{Background and Purpose}

Untreated mental health disorders in perinatal (pregnant and postpartum, including breastfeeding) women can have devasting sequelae. Pregnancy-associated suicide kills more women than hemorrhage or preeclampsia. Depressive symptoms are associated with reduced safety for the child, increased harsh punishment, impaired development of infant emotional regulation and attachment, and greater risk of psychiatric disease in the child. Treatment choices for mental health disorders include pharmacotherapy, psychotherapy, and other approaches (e.g., yoga, mindfulness, self-care, nutritional or herbal supplements). For women who are currently or planning to become pregnant, a critical question is whether the benefits for mother and fetus of treating psychiatric illness with pharmacologic interventions outweigh the harms; a systematic review will help clarify the balance of benefits and harms.

\section{Methods}

We employed methods consistent with those outlined in the Agency for Healthcare Quality and Research Evidence-based Practice Center Program Methods Guidance (https://effectivehealthcare.ahrq.gov/topics/cer-methods-guide/overview), and we describe these in the full report. Our searches covered publication dates in PubMed ${ }^{\circledR}$, the Cochrane Library, Embase $^{\circledR}$, and PsycINFO ${ }^{\circledR}$ from inception through June 5, 2020. We surveilled key journals and PubMed through March 2, 2021.

\section{Results}

A total of 164 studies (168 articles) met eligibility criteria. Most studies were observational in design and had high risk of bias; they cannot fully address confounding. The associations 
between psychotropic medications and outcomes reported in observational studies cannot be inferred to be causal and varied by exposure and outcome.

Key Question (KQ) 1: Benefits of Pharmacologic Treatments Versus No Treatment or Placebo for Pregnant and Postpartum Women With Anxiety, Depression, Bipolar Disorder, or Schizophrenia. Substantial evidence exists on the effectiveness of medications in the general population, but evidence in pregnant and postpartum women is sparse ( 9 randomized controlled trials [RCTs] and 10 observational studies). When evidence was available, we found low to moderate strength of evidence of benefit. Specifically, for depression, three RCTs offered evidence that brexanolone for depression onset in the third trimester or postpartum is associated with improved depressive symptoms shortly after infusion (60 hours) and at 30 days after treatment (moderate strength of evidence); two RCTs reported that sertraline in the postpartum period achieves response, remission, and improvements in depressive symptoms (low strength of evidence). For bipolar disorders, two cohort studies found that discontinuing mood stabilizers during pregnancy may increase recurrence and reduce time to recurrence (low strength of evidence).

KQ 2: Comparative Benefits of Pharmacologic Treatments for Pregnant and Postpartum Women With Anxiety, Depression, Bipolar Disorder, or Schizophrenia. For depression and bipolar disorder, we found insufficient evidence to judge the comparative effectiveness of a very limited number of outcomes and interventions from one RCT and four observational studies of exposure during pregnancy. For anxiety and schizophrenia, we found no evidence on comparative effectiveness.

KQ 3: Harms of Pharmacologic Treatments Versus No Treatment or Placebo for Pregnant and Postpartum Women With Mental Health Disorders. We found 5 RCTs and 70 observational studies. As a result, most studies could not assert a causal relationship between exposure and resultant harms. We found low strength of evidence for several outcomes. Regarding maternal harms, benzodiazepine before conception may be associated with an increased risk of ectopic pregnancy. During pregnancy, exposure to several antidepressants may be associated with a higher risk of postpartum hemorrhage; serotonin-norepinephrine reuptake inhibitor (SNRIs) and tricyclic antidepressants may be associated with an increased risk of preeclampsia; SNRIs may be associated with spontaneous abortion; and quetiapine and olanzapine may be associated with an increased risk of gestational diabetes. For depression onset in the third trimester or within 4 weeks of birth, brexanolone may be associated with the risk of sedation or somnolence leading to dose interruption or reduction. For child adverse outcomes, we found an association between benzodiazepine and neonatal intensive care unit admissions; between selective serotonin reuptake inhibitors and respiratory issues, low Apgar scores, persistent pulmonary hypertension of the newborn, and depression in children; and between citalopram and autism spectrum disorder. Signals of harms that we identified above may be partially or wholly attributable to residual confounding. Importantly, we found insufficient evidence on congenital anomalies and cardiac defects from studies included our review. We note, however, that we did not find eligible evidence on congenital anomalies for triazolam, alprazolam, valproate, carbamazepine, clonazepam, and topiramate, although evidence is available from studies of other populations ineligible for this review.

KQ 4: Comparative Harms of Pharmacologic Treatments for Pregnant and Postpartum Women With Mental Health Disorders. We found 1 RCT and 55 observational studies; limiting causal inference regarding exposures and resultant harms. Evidence from one study suggested that the association between first trimester exposure to lithium and overall congenital 
anomalies and cardiac anomalies may be greater than the association between first trimester exposure to lamotrigine and the same outcomes (low strength of evidence) during pregnancy, which can inform the decision to switch a medication in a successfully treated individual. The evidence is insufficient for all other comparisons and outcomes.

\section{Limitations}

We identified few randomized controlled RCTs of pharmacotherapy for mental health disorders during pregnancy or lactation; therefore we relied on observational studies for the bulk of this review. A significant constraint to interpreting the evidence is the widespread risk of confounding. Underlying mental health disorders result in the use of psychotropic medications. Underlying mental health disorders may also result in some of the harms investigated in this review regardless of exposure to medications. Studies varied greatly in the extent to which they were able to address underlying severity of mental health disorders. The majority were unable to address confounding, often because of a lack of the necessary variables in registry datasets. A small subset of studies attempted various approaches to address confounding (e.g., propensity score adjustment, stratification by number of disorders). In many instances, controls for confounding reduced the effect size and, in some instances, reversed the direction of effect. Most studies were unable to identify dose and duration of exposure. For the benefits question (KQ 1), eligible studies had comparator arms of women with the same disorder as in the treatment arm. For the harms question (KQ 3), however, we were more inclusive and included studies with comparator arms comprising women with prior exposure to the drug, even if the disorder status was not specified. As a result, our KQ 1 evidence base controls for underlying severity as a confounder better than the KQ 3 evidence base.

We elected to restrict the evidence to women with mental health disorders as a means of reducing the potential for confounding in the evidence base. However, this criterion excluded studies of well-conducted negative controls that might bolster the evidence on the association between the exposure and the outcome. Also, this criterion resulted in the exclusion of studies reporting on relevant outcomes for exposures to the intervention for other clinical conditions. Studies of multiple drug exposures presented results for each exposure but did not always present results separately for the women with multiple drug exposures. In these studies with overlapping arms, we were not able to attribute the effect of the intervention to a single drug. As a result, we excluded these studies. The exclusion of studies with overlapping arms also restricted the comprehensiveness of our review. These limitations of the evidence and of our review criteria mean that the signals of harms that we identified above may be partially or wholly attributable to residual confounding. We may also have missed eligible studies because of our restriction to English language studies.

\section{Implications and Conclusions}

The central decisional dilemma facing pregnant and postpartum women with mental health disorders and their healthcare providers is how to balance benefits and harms of psychotropic drugs for both themselves and their children. One such critical trade-off is whether improved symptoms in the mother outweigh the harms from potential congenital anomalies in the fetus. Given long-standing restrictions on including pregnant and lactating women in clinical trials, few clinical trials have evaluated the effectiveness of pharmacotherapy. By contrast, evidence is voluminous but of low quality on the harms of pharmacotherapy. Our findings indicate the need for clear communication to patients on four primary points: (1) evidence exists that medication 
works in the general populations; (2) few studies have measured effectiveness in pregnant women; (3) the limited evidence available is consistent with some benefit; and (4) some studies suggested some increased adverse events, many of which are rare or transient. However, because these studies could not rule out the severity of the underlying mental health disorder as the cause of the association, the causal link between the exposure and adverse events is unclear. The patient and her provider are uniquely qualified to determine whether the mother's medical need for treatment exceeds any potential harms. 


\section{Introduction}

\section{Background}

Untreated mental health disorders among perinatal women (i.e., women who are pregnant or postpartum through 1 year, including those who are breastfeeding) can have devasting sequelae. Pregnancy-associated suicide kills more women than hemorrhage or preeclampsia. ${ }^{1}$ In addition to the negative effects on women's health and well-being, depressive symptoms are associated with adverse parenting practices such as reduced use of safety (such as always using car seats) and child development practices (such as limiting television or video watching) and increased use of harsh punishment. ${ }^{2}$ In addition, postpartum depression is associated with reduced maternal sensitivity, ${ }^{3}$ which may adversely affect development of infant emotional regulation and attachment. ${ }^{4,5}$ Insecure attachment, in turn, increases the risk of psychiatric disease in the child. ${ }^{3,6}$

According to Census Bureau data, 86 percent of women in the United States will give birth at least once by age $44 .^{7}$ The risk of a psychiatric illness during the period immediately before pregnancy, during pregnancy, and through 12 months postpartum is substantial. A nationally representative survey of 14,019 women in the 2001-2002 National Epidemiologic Survey on Alcohol and Related Conditions compared the prevalence of mental health disorders in past-year pregnant women (pregnant at the time of the survey or pregnant in the prior 12 months) and nonpregnant women of childbearing age. The survey found that 25.3 percent of women pregnant in the past year reported any mental health or substance use disorder or combination of disorders. $^{8}$

However, pregnancy does not appear to be associated with a higher or lower prevalence of mental health disorders in general. Differences in the prevalence for specific mental health disorders between nonpregnant women of childbearing age and past-year pregnant women all lie within the margin of error; major depressive disorder: 8.1 percent versus 8.4 percent, adjusted odds ratio (AOR), 1.24, (95\% confidence interval [CI], 0.94 to 1.64), bipolar disorder: 2.3 percent versus 2.8 percent, AOR, 1.09, (95\% CI, 0.70 to 1.70$)$, anxiety disorders: 14.9 percent versus 13.0 percent, AOR, 0.99, (95\% CI, 0.68 to 1.43$)$ and any psychotic disorder (including schizophrenia): 0.3 percent versus 0.4 percent, AOR, 1.50 , (95\% CI, 0.54 to 4.18$).{ }^{8}$

Among mental health disorders in the perinatal period and the first year postpartum, depression (major depressive disorder and other depressive disorders) is the most common form, although depression and anxiety often coexist. In women with postpartum depression, for example, 66 percent have been reported to have a comorbid anxiety disorder diagnosis. ${ }^{9}$

In addition to understanding how to manage prevalent psychiatric illness, healthcare providers also need to consider how best to manage new-onset mental health disorders during the perinatal period. The incidence of these disorders may be difficult to quantify accurately because most women with a psychiatric condition do not receive mental healthcare, regardless of pregnancy status: many women have few encounters with the healthcare system prior to their pregnancy.

The National Epidemiologic Survey did find significantly higher odds of depression among postpartum women when compared with nonpregnant women. However, some uncertainty persists as to whether higher rates of postpartum depression reflect poor identification during pregnancy rather than new onset of the disorder in the postpartum period ${ }^{10}$ and whether the differences in prevalence of depression between past-year pregnant women and nonpregnant women might be overstated. ${ }^{11}$ 
A recent Danish study of perinatal women found no differences between pregnancy and postpartum in first-time psychiatric episodes treated at outpatient facilities. It did, however, report a significant increase in first-time psychiatric episodes treated at inpatient facilities shortly following childbirth when compared with during pregnancy, suggesting a potentially different etiology for severe mental health disorders. The authors suggested that childbirth could serve as a "potent and highly significant trigger" of severe psychiatric episodes. ${ }^{12}$

\section{Treatment Strategies}

Treatment choices for mental health disorders include pharmacotherapy, psychotherapy, and other approaches (e.g., yoga, mindfulness, self-care, nutritional or herbal supplements). The likelihood of perinatal exposure to psychotropic drugs for mental health disorders is increasing, but no clear and comprehensive summary of their effects exists. Among women of childbearing age, use of prescription antidepressants in the 30 days before the National Health and Nutrition Examination Survey has increased markedly over the past 30 years, from 2.3 percent (1988 through 1994) to 14.8 percent (2007 through 2010). ${ }^{13}$ Regarding exposure during pregnancy specifically, more recent data (2006 through 2011) on women with private insurance indicate a rate of exposure of 10.1 percent to psychotropic medications during pregnancy. ${ }^{14}$ Rates of exposure during pregnancy vary by drug: Medicaid claims data from 2000 through 2007 show that 8.1 percent of women were exposed to an antidepressant during pregnancy. ${ }^{15}$ From 2001 through 2007, use of second-generation antipsychotics by pregnant women more than doubled, from 0.3 percent to 0.8 percent; ${ }^{16}$ use of antiepileptic drugs increased from 1.57 percent of deliveries to 2.19 percent. ${ }^{17}$ Rates of exposure to psychotropic drugs may continue to rise as newer, more targeted therapies are approved: one recently approved drug, brexanolone, targets postpartum depression specifically. ${ }^{18,19}$

Treatment strategies need to account for the balance between benefits and harms. Food and Drug Administration (FDA) offers information on potential harms through warning language in prescription labeling and boxed warnings. FDA offers cautionary language in prescription labeling sections that are specific to pregnancy, lactation, and males and females with reproductive potential. ${ }^{20}$ Additionally, FDA may offer its most prominent warning in the form of a boxed warning. Boxed warnings are issued when the risk of adverse reactions is "so serious in proportion to the potential benefit" 21, p. 11 that the risks must be considered when deciding to use the drug. Through 2014, FDA also used to apply letter categories to drugs to indicate toxicity but has discontinued their use because of concerns about misinterpretation and oversimplification. ${ }^{22}$

\section{Clinical and Policy Context}

For women who are currently or planning to be pregnant, a critical question is whether the benefits for mother and fetus of treating psychiatric illness with pharmacologic interventions outweigh the harms. Similarly, a key decision for breastfeeding women is whether the harms of infants' medication exposure via breastmilk outweighs either harms to women of not being treated with pharmacotherapy $4,5,23,24$ or harms to mother and infant of not breastfeeding. ${ }^{25}$

In addressing these decisional dilemmas, clinical practice guidelines on the use of psychotropic medications during three stages of the perinatal period (before pregnancy, during pregnancy, and during lactation) must address multiple clinical scenarios. Specifically, guidance is needed at each stage on treatments for new diagnoses and whether to continue, add to, or switch psychotropic medications for preexisting diagnoses. ${ }^{26,27}$ These guidelines need to address 
the high rate of unplanned pregnancies ${ }^{28}$ and the consequent need for a change in medication management or reassurance to women in early stages of pregnancy.

The evidence base has two important constraints in providing support for clinical practice guidelines: (1) lack of trial data, which leads to reliance on observational data, and (2) heterogeneity of the included conditions, medications, and comparators.

Trial evidence on psychotropic medications (for any indication) in pregnant or breastfeeding women is "historically lacking." ${ }^{29}$ Concerns about maternal and fetal safety, ethics, and the logistics of trial design serve as constraints, ${ }^{30}$ leaving patients and clinicians with a dearth of information to make therapeutic decisions. To address this gap, a recent report to Congress advocates for inclusion of pregnant and lactating women in clinical trials. ${ }^{31}$ At the same time, high-quality safety data are also limited. ${ }^{32}$ Absent high-quality evidence, clinical

recommendations may vary on key decisions - such as whether to continue antidepressants in the preconception, pregnancy, and breastfeeding phases and what agent to use ${ }^{33}$ - or fall back on expert opinion. ${ }^{34,35}$

The heterogeneity of included populations and pharmacologic interventions further complicates interpretation of the evidence. Some medications are prescribed across numerous conditions; coexisting mental health conditions are common. Complexity and severity of mental health conditions, baseline symptom control, and health status also require consideration. For example, dosage for a second-generation antipsychotic in hard-to-treat major depression is much lower than dosage used to treat a primary bipolar disorder; the balance of benefits and harms would differ as a result. Underlying variations in population characteristics and medication dosing might explain important differences in harms. The number and diversity of interventions also introduce complexity. A woman's decision to pursue pharmacologic or psychological therapy may turn on socioeconomic factors such as locally available psychotherapists, insurance coverage, transportation, paid time off from work to attend therapy, and access to childcare. These social determinants of health may confound observed associations between use of pharmacologic therapy and clinical outcomes.

Three recent reviews have reported on guidelines for treating depression, bipolar disorder, and schizophrenia in perinatal women; ${ }^{26,33,36}$ there is no clear consensus on what best practices are. Table 1 summarizes areas where multiple guidelines agree, disagree, or indicate uncertainty. Areas of concordance are not always based on evidence: they may be based on clinical judgment. They do not necessarily represent consensus in the field because not all guidelines may comment on a topic. Notably, recommendations regarding the same drug class (e.g., antipsychotics or antidepressants) varied by mental health condition. Guidelines also varied in whether the recommendation was issued for a specific drug or drug class. Other areas of uncertainty include treatment options (starting, continuing, switching, or stopping medications) for breastfeeding women or symptomatic women.

\section{Purpose and Scope of the Systematic Review}

Inconsistencies and uncertainties in the management of maternal mental health disorders point to the need for a systematic review to help clarify the balance of benefits and harms from psychotropic drugs for these disorders. Guideline developers will benefit from a systematic review that addresses both benefits and harms of psychotropic drugs so that they can address net benefit. A systematic review of the harms of psychotropic drugs that accounts for underlying severity and disorders but also addresses drug class effects can help resolve inconsistencies in 
recommendations. Finally, guideline developers will benefit from a synthesis of the evidence on the consequences of stopping or switching drugs.

The Agency for Healthcare Research and Quality and its partners, the American College of Obstetricians and Gynecologists and the American Psychiatric Association, will use this review to inform vital questions of safe management of psychotropic drugs in the perinatal period, considering both benefits (Key Questions [KQs] 1 and 2) and harms (KQs 3 and 4). Specifically, in pregnant or breastfeeding women with an anxiety, depressive, or bipolar disorder or schizophrenia, KQ 1 will consider the benefits of pharmacotherapy compared with placebo or no treatment, while KQ 2 will evaluate the comparative benefits of pharmacologic interventions. In women with any mental health condition during the preconception, pregnancy, or postpartum phase, KQ 3 will focus on maternal and fetal/child harms of pharmacotherapy compared with placebo or no treatment, while KQ 4 will evaluate the comparative harms of pharmacologic interventions. A Contextual Question asks about the harms of not treating a disorder or stopping or switching medications.

This review, as framed, does not span all eligible interventions. Notably, the review does not address the efficacy of nonpharmacologic interventions (e.g., psychological interventions vs. usual care, wait-list, or no treatment). Although this exclusion limits the scope of the review, it reduces the heterogeneity of the included populations: the efficacy population for psychotherapyonly interventions may have lower severity of psychiatric illness. The review also specifies eligible populations as those with a mental health condition; as a result, it excludes studies of negative controls.

Table 1. Concordance ${ }^{*}$ and uncertainty in clinical practice guidelines

\begin{tabular}{|c|c|c|c|}
\hline Perinatal Period & Depression $^{33}$ & Bipolar Disorder $^{37}$ & Schizophrenia ${ }^{26}$ \\
\hline Preconception & $\begin{array}{l}\text { Concordance } \\
\text { - Psychotherapy as initial } \\
\text { treatment, } \\
\text { antidepressants as } \\
\text { initial therapy in severe } \\
\text { cases }\end{array}$ & $\begin{array}{l}\text { Concordance } \\
\text { - Potential teratogenic } \\
\text { effects for lithium, } \\
\text { sodium valproate, and } \\
\text { carbamazepine }\end{array}$ & $\begin{array}{l}\text { Concordance } \\
\text { - Inform patients and } \\
\text { partners of the risk and } \\
\text { benefits of antipsychotic } \\
\text { treatment in pregnancy, } \\
\text { especially risks for } \\
\text { teratogenicity and } \\
\text { malformations in } \\
\text { pregnancy } \\
\text { - Prescribe } 5 \text { mg folate } \\
\text { per day } 3 \text { months } \\
\text { before conception and } \\
\text { throughout pregnancy }\end{array}$ \\
\hline
\end{tabular}




\begin{tabular}{|c|c|c|c|}
\hline Perinatal Period & Depression $^{33}$ & Bipolar Disorder ${ }^{37}$ & Schizophrenia ${ }^{26}$ \\
\hline During pregnancy & $\begin{array}{l}\text { Concordance } \\
\text { - Four guidelines advise } \\
\text { continuation of } \\
\text { antidepressants, five do } \\
\text { not make a } \\
\text { recommendation } \\
\text { - Avoid paroxetine because } \\
\text { of the risk of congenital } \\
\text { cardiac malformations in } \\
\text { the newborn } \\
\text { Uncertainty } \\
\text { - Switching: three } \\
\text { guidelines discourage } \\
\text { switching, and one } \\
\text { promotes switching away } \\
\text { from unfavorable } \\
\text { medications (paroxetine } \\
\text { and fluoxetine) } \\
\text { Optimal treatment for } \\
\text { patients with current } \\
\text { symptoms despite } \\
\text { antidepressant use }\end{array}$ & $\begin{array}{l}\text { Concordance } \\
\text { - Consider the balance } \\
\text { between risk and benefits in } \\
\text { deciding whether to } \\
\text { continue treatment } \\
\text { - } \text { Medication is warranted for } \\
\text { symptoms or when relapse } \\
\text { is of concern } \\
\text { - Consider lithium for women } \\
\text { with severe bipolar disorder } \\
\text { - Antipsychotics are safer } \\
\text { than mood stabilizers } \\
\text { - Antidepressants are } \\
\text { relatively safe but carry } \\
\text { potential neonatal side } \\
\text { effects } \\
\text { Uncertainty } \\
\text { - Discontinuing or reducing } \\
\text { lithium dose just before } \\
\text { delivery to avoid lithium } \\
\text { toxicity in the infant (one } \\
\text { guideline only) } \\
\text { - Safety of typical vs. atypical } \\
\text { antipsychotics } \\
\text { - Safety of lamotrigine vs. } \\
\text { other drugs } \\
\text { Specific antidepressant } \\
\text { recommendations within and } \\
\text { across drug classes }\end{array}$ & $\begin{array}{l}\text { Concordance } \\
\text { - Use antipsychotics that } \\
\text { allow control of } \\
\text { schizophrenia symptoms } \\
\text { and are not contraindicated } \\
\text { - If clinically tolerable, avoid } \\
\text { introducing an antipsychotic } \\
\text { drug until the second or } \\
\text { third trimester due to } \\
\text { teratogenic risk } \\
\text { - Consider the lowest } \\
\text { necessary dosage of } \\
\text { haloperidol for treatment } \\
\text { - Other first-generation } \\
\text { antipsychotic should not be } \\
\text { initiated in pregnancy } \\
\text { - Among second-generation } \\
\text { antipsychotics, olanzapine, } \\
\text { risperidone, and quetiapine } \\
\text { may be considered as } \\
\text { treatment, but they all carry } \\
\text { risks } \\
\text { - Clozapine should not be } \\
\text { used in pregnancy } \\
\text { - Other second-generation } \\
\text { antipsychotic should not be } \\
\text { initiated in pregnancy } \\
\text { Do not stop medications } \\
\text { without clear safety reasons }\end{array}$ \\
\hline During breastfeeding & $\begin{array}{l}\text { Concordance } \\
\text { - For new episodes, start } \\
\text { with psychotherapy as } \\
\text { initial treatment and } \\
\text { consider antidepressants } \\
\text { for severe cases } \\
\text { - Continue antidepressants } \\
\text { to avoid relapse } \\
\text { - Sertraline preferred due } \\
\text { to its favorable profile } \\
\text { during lactation } \\
\text { - Two guidelines also } \\
\text { recommend citalopram } \\
\text { - Avoid fluoxetine due to its } \\
\text { long half-life and } \\
\text { presence in breastmilk }\end{array}$ & $\begin{array}{l}\text { Concordance } \\
\text { - Reintroduce lithium after } \\
\text { delivery } \\
\text { - Breastfeeding is not } \\
\text { recommended with lithium } \\
\text { due to a risk of toxicity for } \\
\text { the baby } \\
\text { Uncertainty } \\
\text { - Lithium dose at } \\
\text { reintroduction } \\
\text { - Safety of breastfeeding } \\
\text { with anticonvulsant mood } \\
\text { stabilizers, including } \\
\text { sodium valproate, } \\
\text { carbamazepine, and } \\
\text { lamotrigine, and second- } \\
\text { generation antipsychotics } \\
\text { - Safety of breastfeeding } \\
\text { with antidepressants }\end{array}$ & $\begin{array}{l}\text { Uncertainty } \\
\text { - Benefits and harms of } \\
\text { specific antipsychotics } \\
\text { during breastfeeding }\end{array}$ \\
\hline
\end{tabular}

*Concordance indicates that at least two guidelines made similar recommendations and no guidelines disagreed. 


\section{Methods}

Below we describe the approach used in this systematic review, list the Key Questions (KQs), and describe the analytic framework. We also outline briefly the study selection criteria, data sources and searches, data extraction and risk of bias, data synthesis and analysis, and grading of the strength of the body of evidence. Additional details can be found in Appendix A.

\section{Review Approach}

The methods for this systematic review followed the Agency for Healthcare Research and Quality's (AHRQ's) Methods Guide for Effectiveness and Comparative Effectiveness Reviews (available at https://effectivehealthcare.ahrq.gov/topics/cer-methods-guide/overview). This systematic review also reports in accordance with the Preferred Items for Reporting in Systematic Reviews and Meta-Analyses. ${ }^{38}$

The topic of this report was developed by AHRQ in consultation with the American College of Obstetricians and Gynecologists and the American Psychiatric Association. A panel of Key Informants gave feedback on the initial proposed KQs; these KQs were posted on AHRQ's Effective Health Care (EHC) website for public comment in July 2018 for 3 weeks and revised in response to comments. We then drafted a protocol for the systematic review and recruited a panel of technical experts to provide high-level content and methodological expertise throughout the development of the review. The final protocol is posted on the EHC website at https://effectivehealthcare.ahrq.gov/products/mental-health-pregnancy/protocol. The PROSPERO registration is CRD42019124057.

\section{Key Questions}

The following KQs and Contextual Question (CQ) guided our systematic review.

KQ 1. Among pregnant and postpartum women, what is the effectiveness of pharmacologic interventions on maternal outcomes

a. Among those with a new or preexisting anxiety disorder?

b. Among those with a new or preexisting depressive disorder?

c. Among those with a new or preexisting bipolar disorder?

d. Among those with new or preexisting schizophrenia?

KQ 2. Among pregnant and postpartum women, what is the comparative effectiveness of pharmacologic interventions on maternal outcomes

a. Among those with a new or preexisting anxiety disorder?

b. Among those with a new or preexisting depressive disorder?

c. Among those with a new or preexisting bipolar disorder?

d. Among those with new or preexisting schizophrenia?

KQ 3. Among reproductive-age women with any mental health disorder, what are the maternal and fetal harms associated with pharmacologic interventions for a mental health disorder during preconception, pregnancy, and postpartum? 
KQ 4. Among reproductive-age women with any mental health disorder, what are the comparative maternal and fetal harms of pharmacologic interventions for a mental health disorder during preconception, pregnancy, and postpartum?

CQ 1. Among women who are preconceptional, pregnant, or postpartum, within a given disorder, what are the harms of not treating the disorder, stopping a pharmacologic treatment, or switching medications?

\section{Analytic Framework}

The analytic framework in Figure 1 illustrates the KQs.

Figure 1. Analytic framework

KQs 1-2:

Pregnant or postpartum women with new or preexisting anxiety, depression, bipolar disorder, or schizophrenia

KQs 3-4:

Reproductive-age women with any mental health disorder (new or preexisting)

\section{Pharmacologic intervention}

(e.g., antianxiety agents, antidepressants, antipsychotics, mood stabilizers)

Final health outcomes

- Symptoms (including suicidal ideation)

- Functional capacity

- Quality of life

(KQs 1, 2)

- Peripartum events

- Adherence to treatment/care

- Suicidal events

$\mathrm{KQ}=$ Key Question.

Maternal: Miscarriage, danger to self or infant, misuse of prescription medication, treatmentrelated harms, death

Fetus/Infant/Child: Death, preterm birth/small or large for gestational age, congenital anomalies, perinatal complications, poor infant attachment/bonding, delayed development 

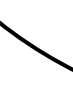

(KQs 3, 4)

\section{Harms}

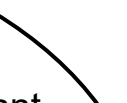




\section{Study Selection}

Eligible studies met the following criteria: (1) included pregnant, postpartum, or reproductive-age women receiving a pharmacologic intervention for a mental health disorder; (2) included an eligible study design (i.e., randomized controlled trials [RCTs], controlled clinical trials (assignment without randomization), case-control studies, and cohort studies with comparison arms); and (3) were published in English. For KQ 1 and KQ 2, studies met the following additional criteria: (1) included pregnant or postpartum women with a new or preexisting diagnosis of anxiety, depression, bipolar disorder, or schizophrenia; (2) examined an outcome that measured maternal benefit; and (3) had a followup period that ranged from conception up to 1 year postpartum. For KQ 2 and KQ 4, studies also had to meet the following criteria: (1) included women who were of reproductive age (15-44 years old during preconception $[\leq 12$ weeks before pregnancy], pregnant, or postpartum [through 1 year]) with any mental health disorder; (2) included an eligible comparison arm; and (3) examined an eligible outcome of maternal or fetal/infant/child harm. For KQ 1 and KQ 3, eligible comparators included placebo or no treatment. For KQ 2 and KQ 4, eligible comparators included active pharmacotherapy.

In applying the criterion of "new or preexisting diagnosis" we did not restrict eligibility to studies with a clinical diagnosis. Many studies reported outcomes based on prescription refills, which used clinician judgment that the underlying disorder would benefit from a pharmacologic intervention as a proxy for diagnosis. We excluded analysis of negative controls (women without a mental health disorder). We also excluded comparisons with nonpharmacologic interventions.

Appendix A lists detailed inclusion and exclusion criteria, organized by population, intervention, comparator, outcome, timing, setting, and study design. In brief, eligible interventions include antipsychotics, selective serotonin reuptake inhibitors (SSRIs), serotoninnorepinephrine reuptake inhibitors, tricyclic antidepressants, other antidepressants, mood stabilizers, antianxiety agents, and other medications for mental health disorders. We excluded psychotherapy.

\section{Data Sources and Searches}

We conducted focused searches of PubMed, the Cochrane Library, Embase, and PsycINFO from inception to December 11, 2018, and updated searches on June 4 and 5, 2020. We also searched relevant systematic reviews and gray literature. We surveilled key journals and PubMed through March 2, 2021.

\section{Data Extraction and Risk-of-Bias Assessment}

For each included study, one investigator extracted information about design, population, intervention, and outcomes, and a second investigator reviewed the information for completeness and accuracy. The criteria set forth by AHRQ's Methods Guide for Comparative Effectiveness Reviews guided our assessment of methodological risk of bias. Two independent investigators assessed the risk of bias of each study, using predefined criteria established in the Risk Of Bias In Non-Randomized Studies - of Interventions (ROBINS-I) ${ }^{39}$ tool for observational studies and the Cochrane $\mathrm{RCT}^{40}$ tool for RCTs. We rated outcomes as low, moderate, or high risk of bias for each study. 


\section{Data Synthesis and Analysis}

For KQs 2 and 4, we grouped studies by exposure rather than underlying disorder for two reasons: first, many studies did not describe the specific underlying disorder, and second, some outcomes may be relevant across disorder categories. We synthesized all data qualitatively. When studies provided adjusted effect sizes, we used these data to interpret the evidence. When studies did not provide adjusted effect sizes, we calculated relative risks, odds ratios, and risk differences. Additionally, when at least three independent and similar studies were available, random-effects models using the inverse-variance weighted method of DerSimonian and Laird were used to estimate pooled effects for relative risks and risk differences for categorical outcomes and standardized and weighted mean differences for continuous outcomes. All quantitative analyses were conducted using Comprehensive Meta-Analysis (Version 3.3) software. ${ }^{41}$ We did not exclude high risk-of-bias studies. We planned to conduct quantitative sensitivity analyses with and without high risk-of-bias studies when the volume of studies on a given outcome permitted such analyses.

\section{Grading the Strength of the Body of Evidence}

We graded the strength of evidence based on the Grading of Recommendations Assessment, Development and Evaluation (GRADE) working group guidance ${ }^{42}$ and guidance established for the Evidence-based Practice Center Program. ${ }^{43}$ Developed to grade the overall strength of a body of evidence, this approach incorporates five key domains: risk of bias (includes study design and aggregate quality), consistency, directness, precision of the evidence, and reporting bias. This approach requires looking beyond statistical significance alone. It requires considering whether studies are consistent and of high quality and outcomes are direct and clinically relevant. It emphasizes the adequacy of the sample size to rule out spurious associations and results that are not clinically relevant.

The domains listed above are reflected in an overall rating regarding the strength of the evidence of high, moderate, low, or insufficient.

- A high strength of evidence reflects high confidence that the evidence reflects the true effect. Further research is very unlikely to change our confidence in the estimate of effect.

- A moderate rating implies moderate confidence that the evidence reflects the true effect. Further research may change our confidence in the estimate of the effect and may change the estimate.

- A low rating implies low confidence that the evidence reflects the true effect. Further research is likely to change our confidence in the estimate of the effect and is likely to change the estimate.

- An insufficient rating indicates that the evidence does not permit estimation of an effect because multiple domain ratings indicate weakness in the evidence base (i.e., the evidence base may comprise studies with limitations; be inconsistent, indirect, or imprecise; or be biased in reporting). When high risk-of-bias studies are likely to alter the judgment, we offer a strength-of-evidence grade that relies on the better quality evidence. When the signals from the evidence base are conflicting and we cannot attribute the differences to risk of bias alone, we assigned the grade as insufficient. 
- We also note evidence bases where we found no eligible evidence based on our inclusion and exclusion criteria; evidence may be available from studies of other populations ineligible for this review.

Evidence bases consisting of RCTs begin with an overall rating of high; downgrading any domain (study limitations, precision, consistency, directness, and reporting bias) results in lower ratings. Evidence bases consisting of observational studies begin with a rating of low. They may be downgraded for the domains listed above. They may also be upgraded on three domains: dose-response association, plausible confounding that would decrease the observed effect, and strength of association (magnitude of effect).

In Chapter 3, we focus on evidence with high, moderate, or low strength of evidence and summarize insufficient evidence only for bodies of evidence for which we were unable to arrive at a conclusion despite multiple studies on a topic. Insufficient ratings from single studies with imprecise results (i.e., small sample sizes, few events, and very wide confidence intervals; adequately sized samples with wide confidence intervals that do not permit an inference of benefit, harm, or absence of benefit or harm) are described in detail in Appendix B. 


\section{Results}

In this chapter, we present the yield from literature searches first, followed by a brief description of the characteristics of included studies. The remainder of the chapter presents results organized by Key Question (KQ) and then by evidence grade. Within each KQ section, we first present an overview followed by detailed results. The detailed results for KQ 1 and KQ 3 present evidence separately for disorder first (anxiety, depression, bipolar disorder, and lastly schizophrenia) and then by intervention. The detailed results for KQ 2 and KQ 4 are not specific to disorder groups but also present results for interventions in the same order as in KQs 1 and 3 for consistency; that is, we present results for drugs used for anxiety, depression, bipolar disorder, and then schizophrenia.

For each KQ, first we present results for outcomes graded as moderate or low. Second, we discuss outcomes rated as insufficient for which two or more studies provided evidence. Outcomes for which we found a single study with imprecise results are not discussed in detail. Third, we summarize outcomes and interventions for which we found no eligible evidence based on our review parameters. Summary tables in this chapter list populations; interventions; comparators; outcomes; study details; references; and factors that affect the strength of evidence, the strength of evidence grade, and the direction of effect. We do not provide ratings for directness: we selected outcomes a priori that are directly relevant to the KQ.

Details on results of literature searches, included studies, detailed results for all outcomes, meta-analyses, and excluded studies can be found in Appendix B. Appendix C lists references used in the appendixes.

\section{Literature Searches and Study Characteristics}

The electronic search, gray literature, and reference mining identified 31,846 citations. After title and abstract screening, 1,812 studies were retrieved for full-text review. A total of 164 studies (168 articles) met eligibility criteria. Surveillance through March 2, 2021 did not identify additional eligible studies. Thirty-three studies were not included in the data synthesis because they reported only unadjusted effectiveness data. ${ }^{44-76}$

A total of 131 studies (135 articles) were included in the analyses (see Appendix B). The evidence base predominantly comprises observational studies with high risk of bias, focusing on pregnant women (rather than postpartum women). About a third were set in the United States; another third were set in Canada and Denmark. More than three-quarters do not appear to be funded by industry (Table 2).

\section{Description of Included Evidence}

Table 2. Key characteristics for included studies

\begin{tabular}{llll}
\hline Study Characteristics & Subcharacteristics & $\begin{array}{l}\text { Number of } \\
\text { Studies } \\
\text { (Articles) }\end{array}$ & Percent \\
\hline Study quality & Low risk-of-bias studies & 4 & 3.0 \\
\cline { 2 - 4 } & Some concerns for risk-of-bias studies & 44 & 33.6 \\
\cline { 2 - 4 } & Some concerns/high risk-of-bias studies* & 6 & 4.6 \\
\cline { 2 - 4 } & High risk-of-bias studies & $77(81)$ & 58.8 \\
\hline Population characteristics: & Pregnant & $119(122)$ & 90.8 \\
\cline { 2 - 4 } Pregnant or postpartum & Postpartum & $11(12)$ & 8.4 \\
\cline { 2 - 4 } & Pregnant and postpartum & 1 & 0.8 \\
\hline
\end{tabular}




\begin{tabular}{|c|c|c|c|}
\hline Study Characteristics & Subcharacteristics & $\begin{array}{l}\text { Number of } \\
\text { Studies } \\
\text { (Articles) }\end{array}$ & Percent \\
\hline Population characteristics: & Majority white (>50\%) & $42(44)$ & 32.1 \\
\hline \multirow[t]{2}{*}{ Race } & Majority nonwhite (>50\%) & 7 & 5.3 \\
\hline & Not reported & $82(84)$ & 62.6 \\
\hline \multirow[t]{2}{*}{ Design } & RCTs & $10(11)$ & 7.6 \\
\hline & Observational & $121(124)$ & 92.4 \\
\hline \multirow[t]{2}{*}{ Comparator $^{\star *}$} & Active comparator & $60(62)$ & 45.8 \\
\hline & Placebo or no treatment comparator & $86(88)$ & 65.6 \\
\hline \multirow[t]{18}{*}{ Geographic setting } & United States & $48(50)$ & 36.6 \\
\hline & United Kingdom & 6 & 4.6 \\
\hline & Canada & $26(27)$ & 19.8 \\
\hline & China & 1 & 0.7 \\
\hline & Denmark & $11(12)$ & 8.4 \\
\hline & Finland & 3 & 2.3 \\
\hline & Australia & 11 & 8.4 \\
\hline & Multiple countries & 6 & 4.6 \\
\hline & Netherlands & 1 & 0.7 \\
\hline & India & 1 & 0.7 \\
\hline & Israel & 1 & 0.7 \\
\hline & Italy & 2 & 2.3 \\
\hline & Japan & 1 & 0.7 \\
\hline & Norway & 3 & 2.3 \\
\hline & Spain & 1 & 0.7 \\
\hline & Sweden & 7 & 5.3 \\
\hline & Taiwan & 1 & 0.7 \\
\hline & Turkey & 1 & 0.7 \\
\hline \multirow[t]{4}{*}{ Funding } & Industry & $14(16)$ & 10.7 \\
\hline & No industry & $93(95)$ & 71.0 \\
\hline & No funding source can be ascertained & 22 & 16.8 \\
\hline & Unfunded & 2 & 1.5 \\
\hline
\end{tabular}

*Some concerns for risk of bias for KQs 1/3, high risk of bias for KQs 2/4.

**Some studies had multiple comparator arms; Ns sum to more than 100 percent.

$\mathrm{KQ}=$ Key Question; RCT $=$ randomized controlled trial.

\section{KQ 1: Benefits of Pharmacologic Treatments Versus No Treatment or Placebo for Pregnant and Postpartum Women With Anxiety, Depression, Bipolar Disorder, or Schizophrenia}

\section{Overview}

- Nineteen studies reported on maternal benefits.

- For depression and bipolar disorder, the evidence was sparse, whereas for anxiety and schizophrenia, evidence was insufficient or unavailable.

- For depression, the strongest evidence is for a drug targeting postpartum depression, brexanolone. Three randomized controlled trials (RCTs) suggested moderate strength of evidence that brexanolone is associated with improved depressive symptoms shortly after infusion (60 hours) and at 30 days after treatment. 
- For depression also, low strength of evidence from two RCTs supports the use of sertraline for postpartum depression to achieve response, remission, and improvements in symptoms of depression and anxiety.

- For depression, single small studies with few events results provided insufficient evidence on benefits for fluoxetine or paroxetine.

- For bipolar disorders, low strength of evidence from two cohort studies supports the continuation of mood stabilizers during pregnancy to prevent recurrence.

- For anxiety, we found one study reporting one outcome (response) on hydroxyzine only; the evidence was rated as insufficient.

- For schizophrenia, we found no eligible studies. Evidence may be available from studies of other populations ineligible for this review

\section{Detailed Results}

Table 3 provides an overview of the findings on benefits of pharmacotherapy in the perinatal period. Appendix B includes detailed tables on all outcomes. Nineteen studies reported on maternal benefit. We found very limited evidence supporting benefits among pregnant women for depression, anxiety, and bipolar disorder and none at all for schizophrenia.

Table 3. Summary of evidence for maternal benefit for treatment versus no treatment for mental health disorders in pregnancy and postpartum

\begin{tabular}{|c|c|c|c|c|c|c|c|c|c|c|c|c|}
\hline Disorder & Exposure & 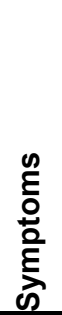 & 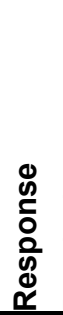 & 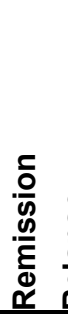 & 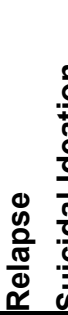 & 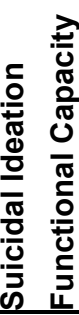 & $\begin{array}{l}0 \\
\stackrel{0}{4} \\
4 \\
0 \\
\stackrel{ \pm}{0} \\
\frac{\pi}{0} \\
0\end{array}$ & $\begin{array}{l}\frac{0}{0} \\
\frac{0}{\Sigma} \\
\geq \\
\searrow \\
\frac{\lambda}{0}\end{array}$ & 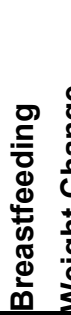 & 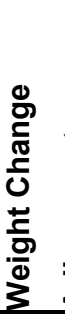 & 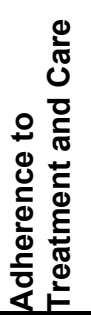 & 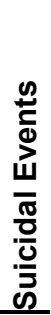 \\
\hline \multirow[t]{4}{*}{ Anxiety } & Benzodiazepine & - & - & - & - & $-\quad-$ & - & - & - & - & - & - \\
\hline & Hydroxyzine & - & I & - & - & - & - & - & - & - & - & - \\
\hline & All other anxiolytics & - & - & - & - & - & - & - & - & - & - & - \\
\hline & Sedatives $^{*}$ & - & - & - & - & - & - & - & - & - & - & - \\
\hline \multirow[t]{5}{*}{ Depression } & SSRIs (unspecified) & - & - & - & - & - & - & I & - & - & - & - \\
\hline & Fluoxetine & I & - & - & - & - & - & - & - & - & - & - \\
\hline & Paroxetine & I & $\mathrm{I}$ & I & - & - & - & - & - & - & - & - \\
\hline & Sertraline & $\mathrm{L}$ & $\mathrm{L}$ & $\mathrm{L}$ & - & $\mathrm{I}$ & - & - & - & - & - & - \\
\hline & Brexanolone & $\mathrm{M}$ & - & - & - & I & - & - & - & - & - & - \\
\hline \multirow[t]{2}{*}{ Bipolar disorder } & Mood stabilizers (unspecified) & - & - & - & $\mathrm{L}$ & - & - & - & - & - & - & - \\
\hline & Lamotrigine & - & - & - & $\mathrm{L}$ & - & - & - & - & - & - & - \\
\hline Schizophrenia & All antipsychotics & - & - & - & - & - & - & - & - & - & - & - \\
\hline
\end{tabular}

The remainder of this section describes the evidence of benefit, insufficient evidence, and no evidence in greater detail. 


\section{Evidence of Benefit: Brexanolone for Postpartum Depression}

\section{Overview}

- Three small RCTs, enrolling women with depression onset in the third trimester or within 4 weeks of birth, demonstrated benefit for brexanolone to reduce depressive symptoms compared with placebo at 60 hours and 30 days after infusion, providing moderate evidence of benefit that brexanolone improves symptoms.

\section{Detailed Synthesis}

Three RCTs (rated low risk of bias) reported on the efficacy for brexanolone versus placebo for depression symptoms with onset in the third trimester of pregnancy or within 4 weeks of birth. ${ }^{18,77}$ The prespecified primary outcome of the RCTs was change from baseline to 60 hours in the 17-Item Hamilton Rating Scale for Depression (HAM-D) score. Brexanolone was administered as a 60-hour continuous infusion with a peak dose of either $60 \mu \mathrm{g} / \mathrm{kg}$ per hour or 90 $\mu \mathrm{g} / \mathrm{kg}$ per $\mathrm{h}$. The BRX60 schedule was administered at $30 \mu \mathrm{g} / \mathrm{kg}$ per hour $(0-4$ hours $), 60 \mu \mathrm{g} / \mathrm{kg}$ per hour (4-56 hours), and $30 \mu \mathrm{g} / \mathrm{kg}$ per hour (56-60 hours). The BRX90 schedule was administered at $30 \mu \mathrm{g} / \mathrm{kg}$ per hour (0-4 hours), $60 \mu \mathrm{g} / \mathrm{kg}$ per hour (4-24 hours), $90 \mu \mathrm{g} / \mathrm{kg}$ per hour (24-52 hours), $60 \mu \mathrm{g} / \mathrm{kg}$ per hour (52-56 hours), and $30 \mu \mathrm{g} / \mathrm{kg}$ per hour (56-60 hours).

The first study ${ }^{18}$ was a phase 2 clinical trial enrolling a total of 21 women from four hospitals in the United States, randomized to placebo $(\mathrm{N}=11)$ versus BRX90 $(\mathrm{N}=10)$. The second and third trials were reported together in a single publication. ${ }^{77}$ Women were recruited at 30 centers in the United States. Among the latter two trials, one was a three-arm trial of placebo, BRX60, or BRX90 ( $\mathrm{N}=138$ randomized, 122 treated), and the second was a two-arm trial comparing BRX90 with placebo ( $\mathrm{N}=108$ randomized, 104 treated). The second publication included an integrated analysis that pooled participants treated with BRX $90(\mathrm{~N}=102)$ versus placebo $(\mathrm{N}=107)$.

In the integrated analysis, BRX90 infusion reduced HAM-D scores more than placebo at 60 hours (least square [LS] mean difference, standard error [SE] -4.1, 0.9, $\mathrm{p}<0.001$ ) and at 30 days (LS mean difference, SE -2.6, 1.1, $\mathrm{p}=0.02$ ). Whereas BRX significantly reduced HAM-D scores, results were mixed for other depression measures. BRX90 reduced Montgomery-Åsberg Depression Rating Scale (MADRS) and Edinburgh Postnatal Depression Scale (EPDS) scores at 30 days in the phase 2 clinical trial, but in the phase 3 trials, only BRX60 differed from placebo. At 30 days post-treatment, there was no difference in Patient Health Questionnaire, 9 item (PHQ9) or Generalized Anxiety Disorder 7-item (GAD-7) scores in any of the treatment groups. The phase 2 trial evaluated maternal function and found no difference at day 30 . The authors note that the trials were powered to observe differences in HAM-D scores; the results were underpowered for other outcomes. Whether the specific focus of the instruments (depression only vs. depression and anxiety) and mode of data collection (clinical interview vs. self-report) may have influenced the magnitude of outcomes is unclear.

We rated the strength of evidence as moderate across all depression symptom outcomes (Table 4). We based this judgment on findings from three small RCTs with statistically significant results and consistent findings for HAM-D and statistically nonsignificant but largely consistent findings for other measures of depression. We also note that the magnitude of benefit declined between the 60-hour measurement and the 30-day measurement of HAM-D; the mean effect for brexanolone was stable, but the placebo group improved from 60 hours to 30 days. 
Table 4. Strength of evidence for maternal benefit for brexanolone versus placebo for depression

\begin{tabular}{|c|c|c|c|c|c|c|c|}
\hline Population & $\begin{array}{l}\text { Intervention } \\
\text { and } \\
\text { Comparator }\end{array}$ & Outcome & $\begin{array}{l}\text { Incidence } \\
\text { or Mean } \\
\text { Effect by } \\
\text { Arm }\end{array}$ & Results & $\begin{array}{l}\text { Study } \\
\text { Design and } \\
\text { Sample Size }\end{array}$ & $\begin{array}{l}\text { Factors That } \\
\text { Affect the } \\
\text { Strength of } \\
\text { Evidence }\end{array}$ & $\begin{array}{l}\text { Overall } \\
\text { Evidence } \\
\text { Strength } \\
\text { (Direction of } \\
\text { Effect) }\end{array}$ \\
\hline $\begin{array}{l}\text { Women with } \\
\text { postpartum } \\
\text { depression }\end{array}$ & $\begin{array}{l}\text { Brexanolone } \\
90 \text { vs. placebo, } \\
\text { integrated } \\
\text { analysis }\end{array}$ & $\begin{array}{l}\text { Symptom } \\
\text { response: } \\
\text { Change in } \\
\text { HAM-D score } \\
\text { from baseline } \\
\text { to } 60 \text { hours }\end{array}$ & $\begin{array}{l}\text { Placebo: } \\
-12.8 \\
\text { BRX90: } \\
-17.0\end{array}$ & $\begin{array}{l}\text { SD not reported } \\
\text { by RCT, results } \\
\text { cannot be } \\
\text { pooled } \\
\text { LS mean } \\
\text { difference (SE) } \\
\text { BRX } 90 \text { vs. } \\
\text { placebo: } \\
-4.1(0.9), \\
\text { p<0.001 }\end{array}$ & $\begin{array}{l}3 \text { RCTs, } \\
\mathrm{N}=209^{18,77}\end{array}$ & $\begin{array}{l}\text { Low study } \\
\text { limitations, } \\
\text { precise } \\
\text { (statistically } \\
\text { significant } \\
\text { results } \\
\text { suggestive of } \\
\text { benefit), } \\
\text { consistent }\end{array}$ & $\begin{array}{l}\text { Moderate } \\
\text { across all } \\
\text { symptom } \\
\text { outcome } \\
\text { measures }\end{array}$ \\
\hline $\begin{array}{l}\text { Women with } \\
\text { postpartum } \\
\text { depression }\end{array}$ & $\begin{array}{l}\text { Brexanolone } \\
90 \text { vs. placebo, } \\
\text { integrated } \\
\text { analysis }\end{array}$ & $\begin{array}{l}\text { Symptom } \\
\text { response: } \\
\text { Change in } \\
\text { HAM-D score } \\
\text { from baseline } \\
\text { to } 30 \text { days }\end{array}$ & $\begin{array}{l}\text { Placebo: } \\
-14.3 \\
\text { BRX90: } \\
-16.9\end{array}$ & $\begin{array}{l}\text { SD not reported } \\
\text { by RCT, results } \\
\text { cannot be } \\
\text { pooled } \\
\text { LS mean } \\
\text { difference (SE) } \\
\text { BRX } 90 \text { vs. } \\
\text { placebo: } \\
-2.6(1.1) \text {, } \\
p=0.02\end{array}$ & $\begin{array}{l}\text { RCTs, } \\
\mathrm{N}=209^{18,77}\end{array}$ & $\begin{array}{l}\text { Low study } \\
\text { limitations, } \\
\text { consistent, } \\
\text { precise } \\
\text { (statistically } \\
\text { significant } \\
\text { results } \\
\text { suggestive of } \\
\text { benefit) }\end{array}$ & $\begin{array}{l}\text { Moderate } \\
\text { across all } \\
\text { symptom } \\
\text { outcome } \\
\text { measures }\end{array}$ \\
\hline
\end{tabular}

$\mathrm{BRX}=$ brexanolone for postpartum depression; HAM-D = Hamilton Depression Rating; LS = least square; $\mathrm{N}=$ number; RCT = randomized controlled trial; $\mathrm{SD}=$ standard deviation; $\mathrm{SE}=$ standard error; vs. $=$ versus.

\section{Evidence of Benefit: Sertraline for Postpartum Depression}

\section{Overview}

- Two RCTs compared sertraline with placebo for postpartum depression.

- For response, two RCTs comparing sertraline with placebo support a low strength of evidence that sertraline improves response rate.

- For remission, similarly, two RCTs comparing sertraline with placebo support a low strength of evidence that sertraline improves remission rates relative to placebo; the evidence for sertraline plus psychotherapy versus psychotherapy alone is insufficient.

- For depression onset between 0 and 1 month postpartum, the evidence from one RCT suggested greater improvement in depression severity with sertraline compared with placebo (low strength of evidence).

- For reduction in anxiety associated with perinatal depression, one low risk-of-bias study provided a low strength of evidence that sertraline produces a greater benefit severity than placebo.

\section{Detailed Results}

For response, two RCTs provided evidence of response for sertraline versus placebo (Table 5); ${ }^{78,79}$ one low risk-of-bias study with a small sample and a mean dose of $100 \mathrm{mg}$ reported a statistically significant benefit for depression that onsets within 1 to 3 months postpartum. ${ }^{78} \mathrm{~A}$ more restrictive definition of postpartum depression with onset up to 1 month postpartum from the same study also reported statistically significant differences favoring sertraline. ${ }^{78}$ Calculated 
relative risks suggest imprecise results with wide confidence intervals (CIs), few events, and small numbers of participants, but a large effect size. A second study, a high risk-of-bias study (because of high attrition) with a median dose of $150 \mathrm{mg}$, found no difference in response rates. ${ }^{79}$

Table 5. Strength of evidence for maternal benefit of sertraline versus no treatment for depression

\begin{tabular}{|c|c|c|c|c|c|c|c|}
\hline Population & $\begin{array}{l}\text { Intervention } \\
\text { and } \\
\text { Comparator }\end{array}$ & Outcome & $\begin{array}{l}\text { Incidence or } \\
\text { Mean Effect } \\
\text { by Arm }\end{array}$ & Results & $\begin{array}{l}\text { Study } \\
\text { Design } \\
\text { and } \\
\text { Sample } \\
\text { Size }\end{array}$ & $\begin{array}{l}\text { Factors That Affect } \\
\text { the Strength of } \\
\text { Evidence }\end{array}$ & $\begin{array}{l}\text { Overall } \\
\text { Evidence } \\
\text { Strength } \\
\text { (Direction of } \\
\text { Effect) }\end{array}$ \\
\hline $\begin{array}{l}\text { Women with } \\
\text { depression } \\
\text { onset } 0-3 \\
\text { months } \mathrm{pp}^{78} \\
\text { or women } \\
\text { with } \\
\text { depression } \\
\text { onset } 0-12 \\
\text { months } \mathrm{pp}^{79}\end{array}$ & $\begin{array}{l}\text { Sertraline vs. } \\
\text { placebo }\end{array}$ & $\begin{array}{l}\text { Response at } 6 \\
\text { weeks in ITT } \\
\text { group ( } \geq 50 \% \\
\text { reduction in } \\
\text { HAM-D-19 } \\
\text { symptoms and } \\
\text { CGI-I score of } \\
\text { "much } \\
\text { improved" or } \\
\text { "very much } \\
\text { improved") } \\
\text { response at } 12 \\
\text { weeks ( } \geq 50 \% \\
\text { reduction in } \\
\text { HAM-D-17 } \\
\text { symptoms) }\end{array}$ & $\begin{array}{l}10 / 17(59 \%) \\
\text { vs. } 5 / 19 \\
(26 \%),{ }^{78} \mathrm{NR}^{79}\end{array}$ & $\begin{array}{l}\text { AOR=NR, } \\
p=0.05 ; \\
\text { calculated RR: } \\
2.24(95 \% \mathrm{Cl}, \\
0.95 \text { to } 5.24) ; \\
\text { ARD: } \\
326 / 1,000(11 \\
\text { to } 1116 \\
\text { more) }{ }^{78} \text { no } \\
\text { difference } \\
\text { between two } \\
\text { arms, } \\
p=0.054^{79}\end{array}$ & $\begin{array}{l}2 \text { RCTs, } \\
\mathrm{N}=145^{78,} \\
79\end{array}$ & $\begin{array}{l}\text { High study } \\
\text { limitations (one high } \\
\text { risk-of-bias study }{ }^{79} \\
\text { imprecise (few } \\
\text { events, small N, } \\
\text { wide Cls), } \\
\text { inconsistent; large } \\
\text { effect for low risk-of- } \\
\text { bias study }\end{array}$ & $\begin{array}{l}\text { Low that } \\
\text { response rate } \\
\text { at } 6 \text { weeks is } \\
\text { greater with } \\
\text { sertraline }\end{array}$ \\
\hline $\begin{array}{l}\text { Women with } \\
\text { depression } \\
\text { onset } 0-3 \\
\text { months } \mathrm{pp}^{78} \\
\text { or women } \\
\text { with } \\
\text { depression } \\
\text { onset } 0-12 \\
\text { months } \text { pp }^{79}\end{array}$ & $\begin{array}{l}\text { Sertraline vs. } \\
\text { placebo }\end{array}$ & $\begin{array}{l}\text { Remission at } 6 \\
\text { weeks in ITT } \\
\text { group (meeting } \\
\text { response } \\
\text { above and with } \\
\text { HAM-D-19 } \\
\text { score } \leq 7)^{78} \text { or } \\
\text { remission at } 12 \\
\text { weeks (HAM- } \\
\text { D-17 } \leq 7 \text { ) }\end{array}$ & $\begin{array}{l}\text { 9/17 (53\%) vs. } \\
4 / 19(23 \%) ; 78 \\
\text { NR }^{79}\end{array}$ & $\begin{array}{l}\text { AOR=NR, } \\
p=0.05 \\
\text { calculated RR: } \\
2.51(95 \% \mathrm{Cl}, \\
0.94 \text { to } 6.70), \\
\text { ARD: } \\
320 / 1,000 \text { (4 to } \\
619 \text { more) } ;^{78} \\
\text { no difference } \\
\text { between two } \\
\text { arms, } \\
p=0.372^{79}\end{array}$ & $\begin{array}{l}2 \text { RCTs, } \\
\mathrm{N}=145^{78,} \\
79\end{array}$ & $\begin{array}{l}\text { High study } \\
\text { limitations (one high } \\
\text { risk-of-bias study } \\
\text { imprecise (few } \\
\text { events, small N, } \\
\text { wide Cls), } \\
\text { inconsistent; large } \\
\text { effect for low risk-of- } \\
\text { bias study }\end{array}$ & $\begin{array}{l}\text { Low that } \\
\text { remission rate } \\
\text { at } 6 \text { weeks is } \\
\text { greater with } \\
\text { sertraline }\end{array}$ \\
\hline $\begin{array}{l}\text { Women with } \\
\text { depression } \\
\text { onset } 0-1 \\
\text { month pp }\end{array}$ & $\begin{array}{l}\text { Sertraline vs. } \\
\text { placebo }\end{array}$ & $\begin{array}{l}\text { Decrease in } \\
\text { HAM-D-19 } \\
\text { scores at } 6 \\
\text { weeks }\end{array}$ & $\begin{array}{l}\text { Sertraline: } \\
\text { baseline HAM- } \\
\text { D-19 NR, final } \\
\text { NR } \\
\text { Placebo: } \\
\text { baseline HAM- } \\
\text { D NR, final NR }\end{array}$ & $\begin{array}{l}\text { Regression } \\
\text { coefficient } 1.18 \\
\text { favoring } \\
\text { sertraline } \\
\text { group, } p=0.01^{78}\end{array}$ & $\begin{array}{l}1 \mathrm{RCT}, \\
\mathrm{N}=27^{78}\end{array}$ & $\begin{array}{l}\text { Low study } \\
\text { limitations, statistical } \\
\text { significance with } \\
\text { small sample size, } \\
\text { consistency } \\
\text { unknown }\end{array}$ & $\begin{array}{l}\text { Low that } \\
\text { reduction in } \\
\text { depressive } \\
\text { severity is } \\
\text { greater with } \\
\text { sertraline }\end{array}$ \\
\hline $\begin{array}{l}\text { Women with } \\
\text { depression } \\
\text { onset } 0-1 \\
\text { month pp }\end{array}$ & $\begin{array}{l}\text { Sertraline vs. } \\
\text { placebo }\end{array}$ & $\begin{array}{l}\text { Decrease in } \\
\text { EPDS scores } \\
\text { at } 6 \text { weeks }\end{array}$ & $\begin{array}{l}\text { Sertraline: } \\
\text { baseline EPDS } \\
\text { NR, final NR } \\
\text { Placebo: } \\
\text { baseline EPDS } \\
\text { NR, final NR } \\
\end{array}$ & $\begin{array}{l}\text { Regression } \\
\text { coefficient } 0.91 \\
\text { favoring } \\
\text { sertraline } \\
\text { group, } p=0.04^{78} \\
\end{array}$ & $\begin{array}{l}1 \mathrm{RCT}, \\
\mathrm{N}=27^{78}\end{array}$ & $\begin{array}{l}\text { Low study } \\
\text { limitations, statistical } \\
\text { significance with } \\
\text { small sample size, } \\
\text { consistency } \\
\text { unknown }\end{array}$ & $\begin{array}{l}\text { Low that } \\
\text { reduction in } \\
\text { depressive } \\
\text { severity is } \\
\text { greater with } \\
\text { sertraline } \\
\end{array}$ \\
\hline $\begin{array}{l}\text { Women with } \\
\text { depression } \\
\text { onset } 0-1 \\
\text { month pp }\end{array}$ & $\begin{array}{l}\text { Sertraline vs. } \\
\text { placebo }\end{array}$ & $\begin{array}{l}\text { Decrease in } \\
\text { HAM-A at } 6 \\
\text { weeks }\end{array}$ & $\begin{array}{l}\text { Sertraline: } \\
\text { baseline HAM- } \\
\text { A NR, final NR } \\
\text { Placebo: } \\
\text { baseline HAM- } \\
\text { A NR, final NR }\end{array}$ & $\begin{array}{l}\text { Regression } \\
\text { coefficient } 1.19 \\
\text { favoring } \\
\text { sertraline } \\
\text { group, } p=0.03^{78}\end{array}$ & $\begin{array}{l}1 \mathrm{RCT}, \\
\mathrm{N}=27^{78}\end{array}$ & $\begin{array}{l}\text { Low study } \\
\text { limitations, } \\
\text { imprecise (small N), } \\
\text { consistency } \\
\text { unknown }\end{array}$ & $\begin{array}{l}\text { Low that } \\
\text { reduction in } \\
\text { anxiety severity } \\
\text { is greater with } \\
\text { sertraline }\end{array}$ \\
\hline
\end{tabular}

$\mathrm{ARD}=$ absolute risk difference; $\mathrm{AOR}=$ adjusted odds ratio; CGI-I = Clinical Global Impression-Improvement; $\mathrm{CI}=$ confidence interval; EPDS = Edinburgh Postnatal Depression Scale; HAM-A = Hamilton Anxiety Rating Scale; HAM-D-17 = Hamilton 
Depression Rating scale, 17-item version; HAM-D-19 = Hamilton Depression Rating scale, 19-item version; ITT = intention to treat; $\mathrm{N}=$ number; $\mathrm{NR}=$ not reported; $\mathrm{pp}=$ postpartum; $\mathrm{RCT}=$ randomized controlled trial; $\mathrm{RR}=$ relative risk; vs. $=$ versus.

For remission, which was measured in the same two RCTs, evidence indicated that sertraline improves remission rates in one low risk-of-bias study whether onset is defined as 0 to 3 months postpartum or 0 to 1 month postpartum. ${ }^{78}$ As with the results on response, calculated relative risks suggest imprecise results with wide CIs, few events, and small numbers of participants, but a large effect size. A high risk-of-bias study did not find any statistically significant improvement. ${ }^{79}$ As with the results on response, we graded the strength of evidence as low for benefit for sertraline compared with placebo.

One low risk-of-bias RCT provided low strength of evidence of benefit that sertraline produces a greater reduction in depressive severity (rated separately for the EPDS and the HAMD) when onset is between birth and 1 month when compared with placebo. ${ }^{78}$ For reduction in anxiety associated with perinatal depression, one low risk-of-bias RCT provided a low grade of evidence that sertraline produces a greater benefit than placebo for those with onset within 1 month of delivery. ${ }^{78}$

\section{Evidence of Benefit: Antipsychotics for Bipolar Disorder}

\section{Overview}

- Evidence from two single small cohorts of women with bipolar disorder on recurrence and time to recurrence suggested benefit for treatment with mood stabilizers compared with discontinuation of treatment (low for benefit).

\section{Detailed Synthesis}

Two publications reported on mood stabilizers (Table 6); ${ }^{80,81}$ of these, one reported on mood stabilizers as a class, ${ }^{80}$ and one focused on lamotrigine. ${ }^{81}$ One was rated high risk of bias, ${ }^{81}$ and the other as having some risk-of-bias concerns ${ }^{80}$ Both were nonrandomized observational cohort studies. These publications drew from two cohorts from the United States (one from Massachusetts $^{80}$ and one from Georgia ${ }^{81}$ ). Publications compared pregnant women who were exposed to mood stabilizers with women with mood disorders who discontinued the use of mood stabilizers. ${ }^{80,81}$ Although the studies are small (and one is high risk of bias ${ }^{81}$ ), they reported substantially higher risks of recurrence or shorter time to recurrence for discontinuation of medications, suggesting low strength of evidence of benefit with mood stabilizers. We graded the evidence as low for benefit, after upgrading the evidence for time to recurrence, based on a large effect size.

\section{Insufficient Evidence}

- We assigned insufficient grades to functional capacity (measured by the Postpartum Adjustment Questionnaire for sertraline and the Barkin Index of Maternal Function for brexanolone) and delivery mode for all reported interventions.

- We found no evidence of benefit for fluoxetine and paroxetine for any reported outcome.

- Insufficient grades were assigned based on study limitations, bias, consistency, and precision. The evidence for these outcomes comprised single studies for which we could not infer consistency of the evidence base. Additionally, some effects were imprecise (i.e., the results relied on small sample sizes, few events, or had wide CIs suggestive of 
both benefits and harms) and had high study limitations (see Appendix B for further details).

Table 6. Strength of evidence for maternal benefit of mood stabilizers versus no treatment for bipolar disorder

\begin{tabular}{|c|c|c|c|c|c|c|c|}
\hline Population & $\begin{array}{l}\text { Intervention } \\
\text { and } \\
\text { Comparator }\end{array}$ & Outcome & $\begin{array}{l}\text { Incidence } \\
\text { by Arm }\end{array}$ & Results & $\begin{array}{l}\text { Study } \\
\text { Design } \\
\text { and } \\
\text { Sample } \\
\text { Size }\end{array}$ & $\begin{array}{l}\text { Factors That } \\
\text { Affect the } \\
\text { Strength of } \\
\text { Evidence }\end{array}$ & $\begin{array}{l}\text { Overall } \\
\text { Evidence } \\
\text { Strength } \\
\text { (Direction } \\
\text { of Effect) }\end{array}$ \\
\hline $\begin{array}{l}\text { Bipolar } \\
\text { disorder }\end{array}$ & $\begin{array}{l}\text { Women with } \\
\text { bipolar } \\
\text { disorder who } \\
\text { discontinued } \\
\text { mood } \\
\text { stabilizers vs. } \\
\text { women } \\
\text { exposed to } \\
\text { mood } \\
\text { stabilizers in } \\
\text { pregnancy }\end{array}$ & $\begin{array}{l}\text { Recurrence } \\
\text { of bipolar } \\
\text { disorder }\end{array}$ & $\begin{array}{l}53 / 62 \\
(85.5 \%) \\
\text { vs. } \\
10 / 27 \\
(37 \%)^{80}\end{array}$ & $\begin{array}{l}\text { AHR for treatment } \\
\text { discontinuation vs. } \\
\text { exposure to mood } \\
\text { stabilizers: } 2.2(95 \% \mathrm{Cl} \text {, } \\
1.2 \text { to } 4.2)^{80} \mathrm{ARD} \text { : } \\
268 / 1,000(95 \% \mathrm{Cl}, 56 \\
\text { more to } 486 \text { more) }\end{array}$ & $\begin{array}{l}1 \text { cohort, } \\
n=89^{80}\end{array}$ & $\begin{array}{l}\text { Moderate } \\
\text { study } \\
\text { limitations, } \\
\text { imprecise (few } \\
\text { events); } \\
\text { consistency } \\
\text { unknown }\end{array}$ & $\begin{array}{l}\text { Low } \\
\text { (favoring } \\
\text { treatment } \\
\text { with mood } \\
\text { stabilizers) }\end{array}$ \\
\hline $\begin{array}{l}\text { Bipolar } \\
\text { disorder }\end{array}$ & $\begin{array}{l}\text { Women with } \\
\text { bipolar } \\
\text { disorder who } \\
\text { discontinued } \\
\text { mood } \\
\text { stabilizers vs. } \\
\text { women } \\
\text { exposed to } \\
\text { lamotrigine in } \\
\text { pregnancy }\end{array}$ & $\begin{array}{l}\text { Time-to- } \\
25 \%- \\
\text { recurrence of } \\
\text { bipolar } \\
\text { disorder }\end{array}$ & $\begin{array}{l}16 / 16 \\
(100 \%) \\
\text { vs. } \\
3 / 10 \\
(30 \%)^{81}\end{array}$ & $\begin{array}{l}2 \text { vs, } 28 \text { weeks, AHR for } \\
\text { discontinuation vs. } \\
\text { exposure to lamotrigine: } \\
12.1(95 \% \mathrm{Cl}, 1.6 \text { to } \\
91.7)^{81} ; \mathrm{ARD}: 687 / 1,000 \\
(95 \% \mathrm{Cl}, 135 \text { more to } 700 \\
\text { more) }\end{array}$ & $\begin{array}{l}1 \text { cohort, } \\
n=26^{81}\end{array}$ & $\begin{array}{l}\text { High study } \\
\text { limitations } \\
\text { (high risk of } \\
\text { bias }^{81} \text { ) } \\
\text { imprecise (few } \\
\text { events, small } \\
\mathrm{N} \text {, wide Cls); } \\
\text { consistency } \\
\text { unknown; very } \\
\text { large effect } \\
\text { size }\end{array}$ & $\begin{array}{l}\text { Low } \\
\text { (favoring } \\
\text { treatment } \\
\text { with } \\
\text { lamotrigine) } \\
\end{array}$ \\
\hline
\end{tabular}

$\overline{\mathrm{ARD}}=$ absolute risk difference; $\mathrm{AHR}=$ adjusted hazard ratio; $\mathrm{CI}=$ confidence interval; $\mathrm{n}=$ number; vs. $=$ versus.

\section{No Evidence in Populations of Interest (Pregnant Women With Mental Health Disorders)}

- For schizophrenia treatments, we found no evidence on benefits for any outcomes.

- For anxiety treatments, we found no evidence on symptoms, remission, relapse, functional outcomes, quality of life, delivery mode, breastfeeding, weight change, adherence to treatment, or suicidal ideation or events.

- For depression treatment, we found no evidence on relapse, functional outcomes, quality of life, breastfeeding, weight change, adherence to treatment, or suicidal ideation or events.

- For bipolar disorder. we found no evidence on symptoms, response, remission, functional capacity, quality of life, delivery mode, breastfeeding, weight change, adherence to treatment, or suicidal ideation or events. 


\section{KQ 2: Comparative Benefits of Pharmacologic Treatments for Pregnant and Postpartum Women With Anxiety, Depression, Bipolar Disorder, or Schizophrenia}

\section{Overview}

- Five studies (7 articles) reported on comparative benefits.

- For depression and bipolar disorder, we found insufficient evidence to judge the comparative effectiveness of a very limited number of outcomes and interventions.

- For anxiety and schizophrenia, we found no evidence on comparative effectiveness for anxiety or schizophrenia.

\section{Detailed Results}

Table 7 provides an overview of the findings on comparative benefits of pharmacotherapy in the perinatal period. Appendix B includes detailed tables on all outcomes. The evidence on comparative benefits was sparse. Five studies ( 7 articles) reported on comparative benefits. The limited evidence that we identified was rated as insufficient.

Table 7. Summary of evidence from comparative effectiveness studies for maternal benefits of pharmacologic treatments for mental health disorders in pregnancy or postpartum

\begin{tabular}{|c|c|c|c|c|c|c|c|c|c|c|c|c|c|c|c|c|}
\hline Disorder & Intervention & Comparator & $\begin{array}{l}\stackrel{\infty}{\varepsilon} \\
\text { के } \\
\text { के }\end{array}$ & 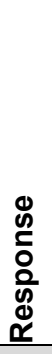 & 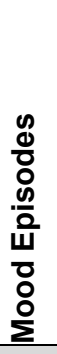 & 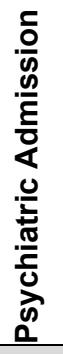 & 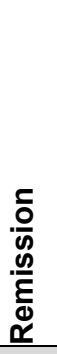 & 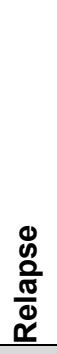 & $\begin{array}{l}\frac{c}{0} \\
\stackrel{0}{\pi} \\
\frac{\pi}{0} \\
\frac{0}{0} \\
\bar{\pi} \\
\frac{\pi}{0} \\
\frac{0}{0} \\
\frac{0}{3} \\
\text { 心 }\end{array}$ & 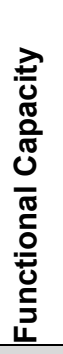 & 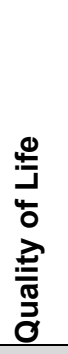 & 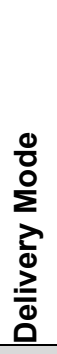 & 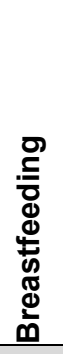 & 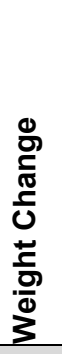 & 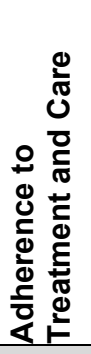 & 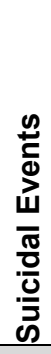 \\
\hline \multirow[t]{2}{*}{ Anxiety } & All anxiolytics & NA & - & - & - & - & - & - & - & - & - & - & - & - & - & - \\
\hline & Sedatives* & NA & - & - & - & - & - & - & - & - & - & - & - & - & - & - \\
\hline \multirow[t]{2}{*}{ Depression } & Fluoxetine & TCA & - & - & - & - & - & - & - & - & - & I & - & - & - & - \\
\hline & Sertraline & Nortriptyline & - & I & I & - & I & - & - & I & - & - & - & - & - & - \\
\hline \multirow[t]{5}{*}{$\begin{array}{l}\text { Bipolar } \\
\text { disorder }\end{array}$} & Lamotrigine & Lithium & - & - & - & I & - & - & - & - & - & - & - & - & - & - \\
\hline & Olanzapine & Lithium & - & - & I & - & - & - & - & - & - & - & - & - & - & - \\
\hline & $\begin{array}{l}\text { Sodium } \\
\text { valproate }\end{array}$ & $\begin{array}{l}\text { Lithium plus } \\
\text { sodium } \\
\text { valproate }\end{array}$ & - & - & - & - & - & 1 & - & - & - & - & - & - & - & - \\
\hline & Lithium & $\begin{array}{l}\text { Lithium plus } \\
\text { sodium } \\
\text { valproate }\end{array}$ & - & - & - & - & - & I & - & - & - & - & - & - & - & - \\
\hline & Lithium & Paroxetine & - & - & I & - & - & - & - & - & - & - & - & - & - & - \\
\hline Schizophrenia & $\begin{array}{l}\text { All } \\
\text { antipsychotics }\end{array}$ & NA & - & - & - & - & - & - & - & - & - & - & - & - & - & - \\
\hline
\end{tabular}




\section{Evidence of Benefit}

For interventions for which evidence was available (fluoxetine vs. tricyclic antidepressants [TCAs], sertraline vs. nortriptyline, lamotrigine vs. lithium, olanzapine vs. lithium, and lithium vs. paroxetine, Table 7), no drug was judged as having greater benefit than a comparison drug for any evaluated disorder.

\section{Insufficient Evidence}

\section{Overview}

- We assigned insufficient grades to several outcomes (response, mood disorders, psychiatric admissions, remission, functional status, and mode of delivery) listed in Table 7. Insufficient grades were assigned based on study limitations, bias, consistency, and precision. The evidence for these outcomes comprised single studies for which we could not infer consistency of the evidence base. Additionally, some were imprecise (i.e., the results relied on small sample sizes, few events, or had wide CIs suggestive of both benefits and harms) and had high study limitations.

\section{No Evidence in Populations of Interest (Pregnant Women With Mental Health Disorders)}

\section{Overview}

- For anxiety and schizophrenia treatments, we found no evidence on comparative benefits for any outcomes in pregnant and postpartum women.

- For depression treatment, we found no evidence on symptoms, psychiatric admission, relapse, suicidal ideation, quality of life, breastfeeding, weight change, adherence to treatment and care, or suicidal events.

- For bipolar disorder treatment, we also found no evidence on symptoms, response, remission, relapse, suicidal ideation, functional capacity, delivery mode, quality of life, breastfeeding, weight change, adherence to treatment and care, or suicidal events.

\section{KQ 3: Harms of Pharmacologic Treatments Versus No Treatment or Placebo for Pregnant and Postpartum Women With Mental Health Disorders}

\section{Overview}

- Seventy-five studies (77 articles) reported on harms; 70 studies were observational and could not assert a causal relationship between exposure and the outcome. Harms in the results below include any eligible adverse event; the events may not be a direct result of the exposure.

- Most studies reporting on adverse outcomes characterized the population by exposure to the drug (rather than by the presence of a disorder). These studies generally included women with current or past depression, anxiety, or any mental health disorder as untreated controls. The findings on adverse outcomes are therefore not specific to a disorder. 
- Interventions for which we found evidence of an association between exposure during pregnancy and adverse events for one or more outcome included benzodiazepines, SSRIs (unspecified), fluoxetine, citalopram, escitalopram, paroxetine, sertraline, SNRIs (unspecified), venlafaxine, bupropion, brexanolone, quetiapine, and olanzapine. In all instances, the strength of evidence on the harms from these drugs was rated low. The magnitude of the association varied by outcome.

- The evidence on harms was not consistent within or across interventions. For example, the association between exposure to the drug and postpartum hemorrhage varied by intervention and by timing of exposure within each intervention.

- Interventions for which we found insufficient evidence to judge the strength of association between exposure during pregnancy and adverse events included the sedativehypnotics temazepam, zopiclone, and zolpidem; the antidepressants fluvoxamine, trazodone, duloxetine, clomipramine, amitriptyline/nortriptyline, and mirtazapine; mood stabilizers as a class, lamotrigine, valproate, topiramate, carbamazepine, oxcarbazepine, lithium; second-generation antipsychotics and first-generation antipsychotics, respectively, as a class; and the second-generation antipsychotics aripiprazole, ziprasidone, and risperidone.

- Maternal outcomes for which we found low strength of evidence of harms for one or more medications for exposure during pregnancy included ectopic pregnancy, preeclampsia, spontaneous abortion, postpartum hemorrhage, and gestational diabetes. The evidence on harms was not consistent within or across outcomes. The most consistent evidence of harm is for postpartum hemorrhage. For other outcomes, harms were detected for a subset of evaluated exposures.

- Child outcomes for which we found low strength of evidence of harms for one or more medications for exposure during pregnancy included neonatal intensive care unit (NICU) admission, respiratory issues, low Apgar scores, primary persistent pulmonary hypertension of the newborn, and depression in the child. These outcomes vary in severity. More serious outcomes such as persistent pulmonary hypertension of the newborn are very rare.

- Outcomes for which we found insufficient evidence included abruption, gestational hypertension; perinatal death, preterm birth; major congenital anomalies; cardiac defects; small for gestational age; large for gestational age; birthweight; delayed social, emotional, and cognitive development; attention-deficit/hyperactivity disorder (ADHD); and anxiety in children for all reported exposures.

- Outcomes for which we found no eligible evidence, based on our review parameters, for any interventions included infertility, glucose intolerance, reduced milk production or unwanted weaning, and withdrawal symptoms in the newborn. Evidence may be available from studies of other populations ineligible for this review.

- We did not find eligible evidence on congenital anomalies for triazolam, alprazolam, valproate, carbamazepine, clonazepam, and topiramate compared with no treatment or placebo, although evidence is available from studies of other populations ineligible for this review.

\section{Detailed Results}

Table 8 provides an overview of the findings on harms of pharmacotherapy in the perinatal period. Appendix B includes detailed tables on all outcomes. Seventy-five studies (77 articles) 
reported on harms. The remainder of this section describes the evidence of maternal harm; evidence of fetal, neonatal, infant, or child harm; insufficient evidence of maternal harm; insufficient evidence of fetal, neonatal, infant, or child harm; and no evidence in greater detail.

\section{Evidence of Maternal Harm}

\section{Overview}

- The evidence on maternal harms from treatments for mental health disorders generally comes from single-study bodies of evidence with moderate limitations and precise results (i.e., sample size, event rate, and CIs permit inference of benefit, harm, or absence of benefit or harm).

- When compared with unexposed women with at least one anxiety diagnosis in the year before conception, evidence from one study suggested benzodiazepine exposure 90 days before conception may be associated with an increased risk of ectopic pregnancy (low strength of evidence of harms).

- The evidence suggested potential associations between exposures to specific psychotropic medications (detailed below) during pregnancy and postpartum hemorrhage, preeclampsia, spontaneous abortion, and gestational diabetes.

- When compared with unexposed women with depression or anxiety, evidence from one study suggests that exposure during pregnancy to selective serotonin reuptake inhibitors (SSRIs) as a class, citalopram, escitalopram, fluoxetine, paroxetine, sertraline, serotonin-norepinephrine reuptake inhibitors (SNRIs) as a class, venlafaxine, and bupropion may be associated with an increased risk of postpartum hemorrhage (low strength of evidence of harms). The association may vary by timing of exposure. Studies report associations between current (SSRIs and SNRIs), recent exposure (SSRIs), and past exposure (bupropion) and postpartum hemorrhage.

- When compared with unexposed women with depression, evidence from two studies each suggested that SNRIs and TCAs exposure during pregnancy may be associated with an increased risk of preeclampsia (low strength of evidence).

$\circ$ When compared with women who discontinued quetiapine and olanzapine during pregnancy, evidence from one study suggested that continued use of quetiapine or olanzapine may be associated with an increased risk of gestational diabetes (low strength of evidence).

- When compared with women with a depression diagnosis in the 4 years preceding pregnancy but not exposed to any antidepressant in the first trimester, evidence from one cohort suggested that SNRI exposure in the first trimester was associated with a higher risk of spontaneous abortion (low strength of evidence). When compared with unexposed women with a history of mood or anxiety disorders, evidence from two studies suggested that benzodiazepine exposure early during pregnancy may be associated with an increased risk of spontaneous abortion when compared with no exposure to benzodiazepines (low strength of evidence of harms).

- When compared with unexposed women with depression, evidence from the Food and Drug Administration (FDA) label for brexanolone exposure for depression onset in the third trimester or within 4 weeks of birth compared with placebo suggested sedation or loss of consciousness led to dose interruption or reduction in some patients during the infusion (low strength of evidence of harms). 
Table 8. Summary of evidence for harms from pharmacologic treatments versus no treatment for mental health disorders in pregnancy or postpartum

\begin{tabular}{|c|c|c|c|c|c|c|c|c|c|c|c|c|c|c|c|c|c|c|c|c|c|c|c|c|c|}
\hline Condition & Exposure & 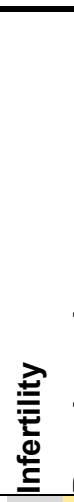 & 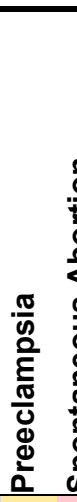 & 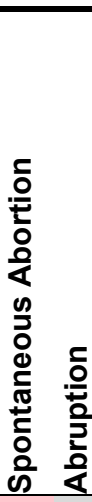 & 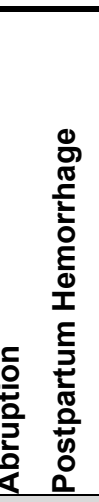 & 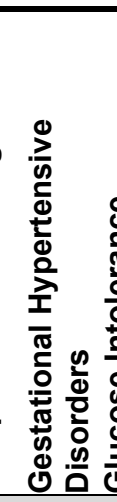 & 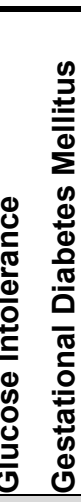 & 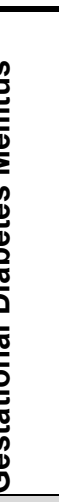 & 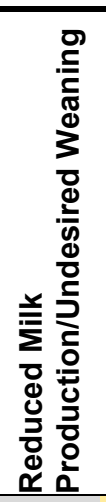 & 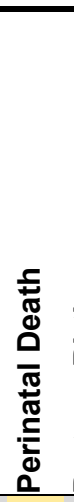 & 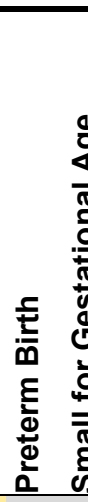 & 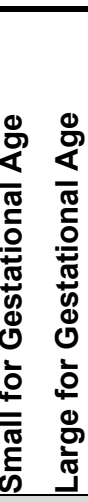 & 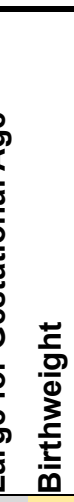 & 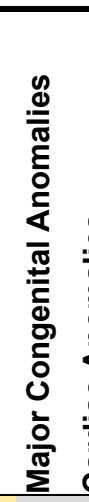 & 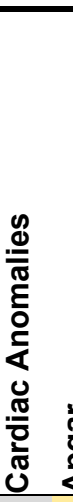 & $\begin{array}{r}\frac{\pi}{3} \\
\frac{\pi}{0} \\
\frac{\pi}{0} \\
\frac{0}{2} \\
\frac{10}{3}\end{array}$ & 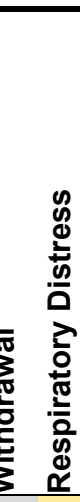 & 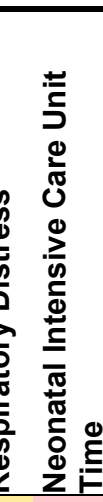 & 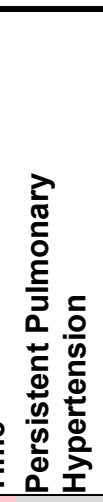 & 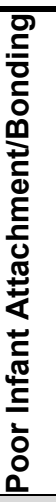 & 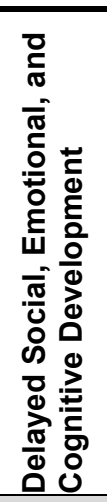 & 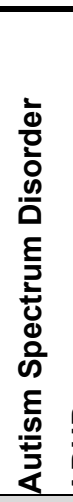 & 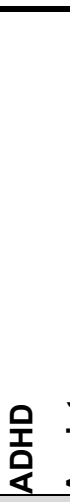 & & \\
\hline Depression or anxiety & Benzodiazepine & - & 1 & L $\quad-$ & $-\quad-$ & - & - & - & - & I & - & $-\quad-$ & -1 & - & - & $1-$ & -1 & I L & - & - & - & - & - & - & \\
\hline Depression or anxiety & Diazepam* & - & - & - & - & - & - & - & - & - & - & - & - & 1 & - & - & - & - & - & - & - & - & - & - & - \\
\hline Depression or anxiety & Temazepam* & - & - & - & - & - & - & - & - & - & - & - & - & 1 & - & - & - & - & - & - & - & - & - & - & - \\
\hline Depression or anxiety & Zopiclone* & - & - & - & - & - & - & - & - & - & - & - & - & 1 & - & - & - & - & - & - & - & - & - & - & - \\
\hline Mental health disorders & Zolpidem* $^{*}$ & - & 1 & - & - & I & - & - & - & - & I & - & - & - & - & 1 & I & I & - & - & - & - & - & - & - \\
\hline Mental health disorders & Hydroxyzine & - & - & - & - & - & - & - & - & - & - & - & - & - & - & - & - & - & - & - & - & - & - & - & - \\
\hline Anxiety & Other anxiolytics & - & - & - & - & - & - & - & - & - & - & - & - & - & - & - & - & - & - & - & - & - & - & - & - \\
\hline $\begin{array}{l}\text { Depression and/or } \\
\text { anxiety and/or other } \\
\text { mental health disorders }\end{array}$ & SSRIs (unspecified) & - & 1 & 1 & L & 1 & - & 1 & - & I & 1 & 1 & 1 & I & I & L & L & 1 & L & - & 1 & I & I & I & L \\
\hline $\begin{array}{l}\text { Depression and/or } \\
\text { anxiety and/or other } \\
\text { mental health disorders }\end{array}$ & Citalopram & - & 1 & - & L & - & - & 1 & - & - & - & - & - & 1 & I & - & - & - & - & - & - & L & - & - & \\
\hline $\begin{array}{l}\text { Depression, mood, or } \\
\text { anxiety disorder }\end{array}$ & Escitalopram & - & 1 & - & L & - & - & - & - & - & - & - & - & 1 & I & - & - & - & - & - & - & - & - & - & - \\
\hline $\begin{array}{l}\text { Depression, mood, or } \\
\text { anxiety disorder }\end{array}$ & Fluoxetine & - & 1 & - & L & - & - & 1 & - & - & - & - & - & 1 & 1 & - & - & - & - & - & - & I & - & - & - \\
\hline $\begin{array}{l}\text { Depression, mood, or } \\
\text { anxiety disorder }\end{array}$ & Fluvoxamine & - & - & - & - & - & - & - & - & - & - & - & - & I & - & - & - & - & - & - & - & - & - & - & - \\
\hline $\begin{array}{l}\text { Depression, anxiety, or } \\
\text { other mental health } \\
\text { disorders }\end{array}$ & Paroxetine & - & 1 & - & $\mathrm{L}$ & - & - & 1 & - & - & - & - & - & 1 & 1 & - & - & - & - & - & - & 1 & - & - & \\
\hline
\end{tabular}




\begin{tabular}{|c|c|c|c|c|c|c|c|c|c|c|c|c|c|c|c|c|c|c|c|c|c|c|c|c|c|c|c|c|c|}
\hline Condition & Intervention & 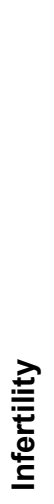 & 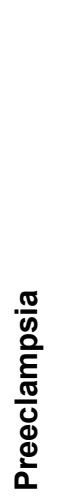 & 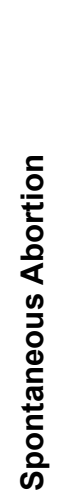 & $\frac{5}{ㅇ ㅡ ㄹ ~}$ & 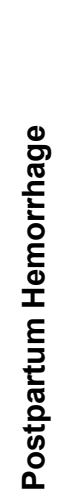 & 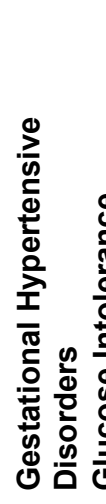 & 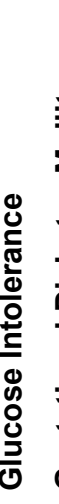 & 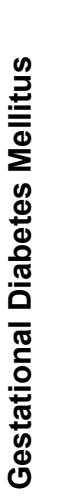 & 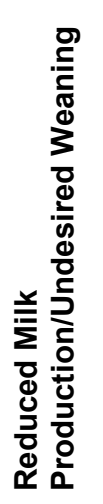 & $\begin{array}{l}0 \\
\frac{5}{0} \\
0 \\
\frac{1}{\pi} \\
\frac{\pi}{\pi} \\
. \frac{1}{0} \\
0 \\
0\end{array}$ & 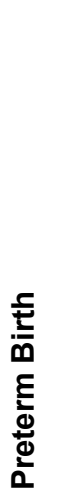 & 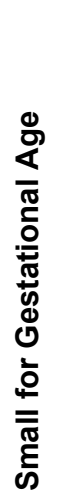 & 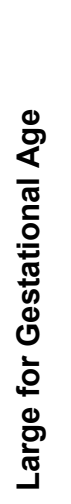 & 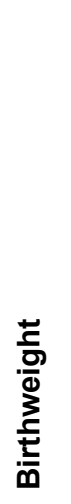 & 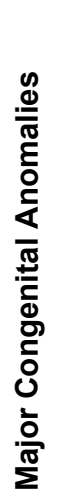 & 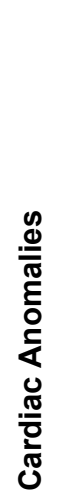 & $\frac{1}{\frac{\pi}{0}}$ & $\begin{array}{l}\frac{\pi}{0} \\
\frac{\pi}{0} \\
\frac{10}{0} \\
\frac{5}{5} \\
3\end{array}$ & 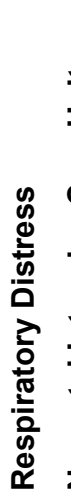 & 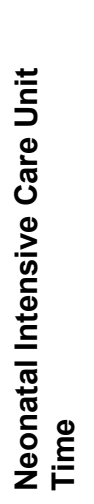 & 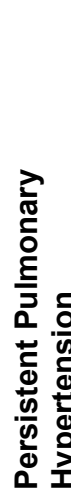 & 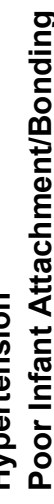 & 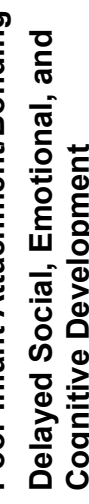 & & 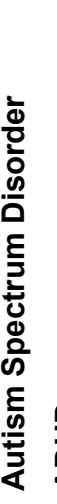 & $\begin{array}{l}0 \\
\text { 문 } \\
\text { \& }\end{array}$ & 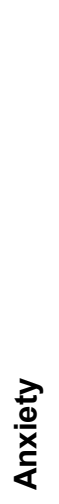 & 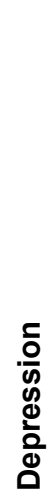 \\
\hline $\begin{array}{l}\text { Depression and/or } \\
\text { anxiety and/or other } \\
\text { mental health disorders }\end{array}$ & Sertraline & - & I & - & - & $\mathrm{L}$ & - & - & 1 & - & - & - & - & - & - & 1 & 1 & - & - & - & - & - & - & - & & I & - & - & - \\
\hline $\begin{array}{l}\text { Mood or anxiety } \\
\text { disorders or prescribed } \\
\text { medication }^{* * *}\end{array}$ & Trazodone & - & 1 & - & - & I & - & - & - & - & - & - & - & - & - & - & - & - & - & - & - & - & - & - & & - & - & - & - \\
\hline $\begin{array}{l}\text { Depression, mood, or } \\
\text { anxiety disorder }\end{array}$ & SNRIs (unspecified) & - & L & $L$ & - & $\mathrm{L}$ & - & - & - & - & - & - & - & - & - & 1 & I & - & - & - & - & - & - & - & & - & - & - & - \\
\hline Prescribed medication*** & Duloxetine & - & - & - & - & - & - & - & - & - & - & - & - & - & - & - & 1 & - & - & - & - & - & - & - & & I & - & - & - \\
\hline $\begin{array}{l}\text { Depression, mood, or } \\
\text { anxiety disorder }\end{array}$ & Venlafaxine & - & $\mathrm{L}$ & - & - & $\mathrm{L}$ & 1 & - & 1 & - & - & - & 1 & - & - & - & 1 & - & - & - & - & - & - & - & & I & - & - & - \\
\hline $\begin{array}{l}\text { Depression and/or } \\
\text { anxiety and/or other } \\
\text { mental health disorders }\end{array}$ & TCAs (unspecified) & - & $\mathrm{L}$ & I & - & - & - & - & - & - & I & - & 1 & - & - & I & 1 & - & - & - & - & - & - & - & & - & - & - & - \\
\hline Mental health disorders & Clomipramine & - & - & - & - & - & - & - & - & - & - & - & - & - & - & - & - & - & - & - & - & - & - & - & & । & - & - & - \\
\hline Mental health disorders & Amitriptyline & - & - & - & - & I & - & - & - & - & - & - & - & - & - & - & - & - & - & - & - & - & - & - & & - & - & - & - \\
\hline Mental health disorders & $\begin{array}{l}\text { Amitriptyline or } \\
\text { nortriptyline }\end{array}$ & - & - & - & - & - & - & - & - & - & - & - & - & - & - & - & - & - & - & - & - & - & - & - & & 1 & - & - & - \\
\hline Postpartum depression & Brexanolone $^{* *}$ & - & - & - & - & - & - & - & - & - & - & - & - & - & - & - & - & - & - & - & - & - & - & - & & - & - & - & - \\
\hline $\begin{array}{l}\text { Depression, mood, or } \\
\text { anxiety disorder }\end{array}$ & Bupropion & - & 1 & - & - & $\mathrm{L}$ & - & - & 1 & - & - & - & - & - & - & - & 1 & - & - & - & - & - & - & - & & - & - & - & - \\
\hline $\begin{array}{l}\text { Depression, mood, or } \\
\text { anxiety disorder }\end{array}$ & Mirtazapine & - & - & - & - & I & - & - & - & - & - & I & - & - & I & - & - & - & - & - & I & - & - & - & & 1 & - & - & - \\
\hline Bipolar disorder & Mood stabilizers & - & 1 & - & I & - & - & - & - & - & - & I & 1 & - & - & - & - & - & - & - & - & - & - & - & & - & - & - & - \\
\hline Bipolar disorder & Lamotrigine & - & 1 & - & I & - & - & - & 1 & - & - & I & 1 & - & - & - & - & - & - & - & - & - & - & - & & - & - & - & - \\
\hline Bipolar disorder & Valproate & - & 1 & - & 1 & - & - & - & - & - & - & 1 & I & - & - & - & - & - & - & - & - & - & - & - & & - & - & - & - \\
\hline
\end{tabular}




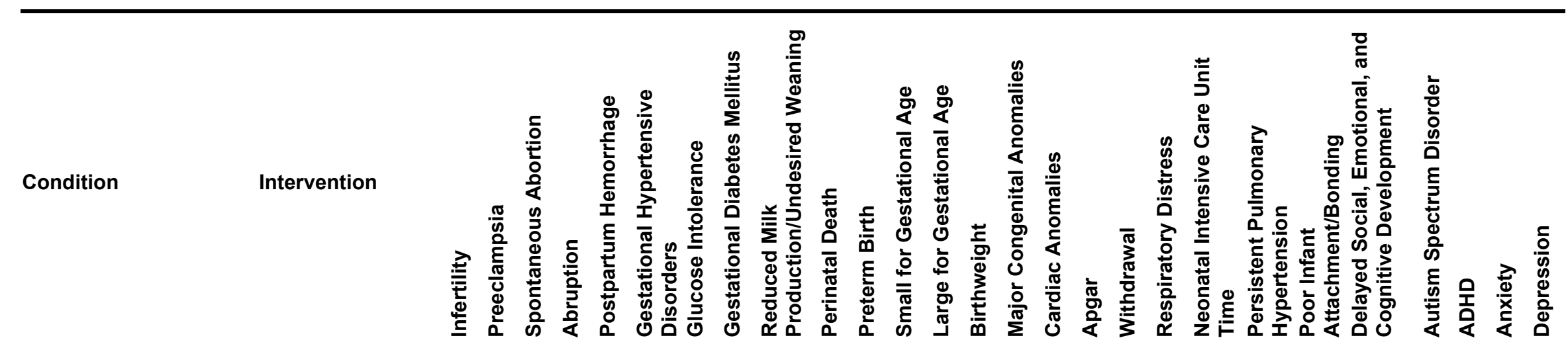

\begin{tabular}{|c|c|c|c|c|c|c|c|c|c|c|c|c|c|c|c|c|c|c|c|c|c|c|c|c|c|c|c|c|}
\hline Bipolar disorder & Topiramate & - & I & - & I & - & - & - & - & - & - & I & 1 & - & - & - & - & - & - & - & - & - & - & - & - & - & - & - \\
\hline Bipolar disorder & Carbamazepine & - & I & - & I & - & - & - & - & - & - & I & I & - & - & - & - & - & - & - & - & - & - & - & - & - & - & - \\
\hline Bipolar disorder & Oxcarbazepine & - & I & - & 1 & - & - & - & - & - & - & 1 & 1 & - & - & - & - & - & - & - & - & - & - & - & - & - & - & - \\
\hline Bipolar or mood disorder & Lithium & - & I & - & 1 & - & - & - & I & - & - & I & 1 & - & - & - & - & - & - & - & - & - & - & I & - & - & - & - \\
\hline $\begin{array}{l}\text { Mental health disorders } \\
\text { or schizophrenia, bipolar } \\
\text { disorder, or psychosis }\end{array}$ & $\begin{array}{l}\text { Second-generation } \\
\text { antipsychotics } \\
\text { (unspecified) }\end{array}$ & - & - & - & - & - & - & - & I & - & - & I & 1 & I & I & I & 1 & - & - & - & - & - & - & - & - & - & - & - \\
\hline $\begin{array}{l}\text { Schizophrenia, bipolar } \\
\text { disorder, or psychosis or } \\
\text { prescribed medication }\end{array}$ & $\begin{array}{l}\text { First-generation } \\
\text { antipsychotics } \\
\text { (unspecified) }\end{array}$ & - & - & - & - & - & - & - & - & - & - & 1 & 1 & 1 & 1 & 1 & 1 & - & - & - & - & - & - & - & - & - & - & - \\
\hline Prescribed medication ${ }^{* * *}$ & Aripiprazole & - & - & - & - & - & - & - & I & - & - & - & - & - & - & - & - & - & - & - & - & - & - & - & - & - & - & - \\
\hline $\begin{array}{l}\text { Mental health } \\
\text { disorders }{ }^{\star \star \star} \text { or } \\
\text { prescribed medication }\end{array}$ & Ziprasidone & - & - & - & - & - & - & - & I & - & - & - & - & - & - & - & - & - & - & - & - & - & - & - & - & - & - & - \\
\hline Prescribed medication ${ }^{* * *}$ & Quetiapine & - & - & - & - & - & - & - & L & - & - & - & - & - & - & I & - & - & - & - & - & - & - & - & - & - & - & - \\
\hline Prescribed medication ${ }^{* * *}$ & Risperidone & - & - & - & - & - & - & - & I & - & - & - & - & - & - & I & 1 & - & - & - & - & - & - & - & - & - & - & - \\
\hline Prescribed medication ${ }^{* * *}$ & Olanzapine & - & - & - & - & - & - & - & $L$ & - & - & - & - & - & - & - & - & - & - & - & - & - & - & - & - & - & - & - \\
\hline
\end{tabular}

*: Used as a sedative-hypnotic to address sleep difficulty, not targeted at underlying mental health disorder.

**: Although we found no evidence of harms for brexanolone for a priori outcomes, we rated the evidence on dose interruption or reduction due to somnolence or sedation as low.

***: Underlying mental health indication and exposure to medications are generally not specified; study populations are based on cohorts with prescriptions.

I: Insufficient for all measures for the outcome domain; L: Low evidence of harm for at least one measure for the outcome domain; -: No eligible evidence.

$\mathrm{ADHD}=$ attention-deficit/hyperactivity disorder; SNRI $=$ serotonin-norepinephrine reuptake inhibitor; SSRI $=$ selective serotonin reuptake inhibitor; TCA $=$ tricyclic

antidepressant. 


\section{Detailed Synthesis}

Table 9 provides an overview of the findings on maternal harms of pharmacotherapy in the perinatal period. Appendix B includes detailed tables on all outcomes. We describe results for six publications (7 studies) for harms below (Table 9).

The evidence for brexanolone came from three RCTs and is rated as low for harms. The evidence on other harms from treatments for mental health disorders, with one exception (two SNRI studies reported on preeclampsia), derives from single-study bodies of evidence with moderate limitations and precise results (studies large enough to detect a difference [or no difference] in effect estimates). The relative paucity of evidence for these harms led us to rate the strength of evidence as low. Although the potential for maternal harm may exist, we have low confidence that future studies will support this association.

Specifically, the identified harms include ectopic pregnancy for benzodiazepine exposure before conception; postpartum hemorrhage for SSRIs overall, citalopram, escitalopram, paroxetine, sertraline, SNRIs overall, venlafaxine, and bupropion; preeclampsia for SNRIs overall and TCAs overall; gestational diabetes for quetiapine olanzapine; and spontaneous abortion for SNRIs and benzodiazepine for exposure during pregnancy; and somnolence or sedation leading to dose interruption or reduction for brexanolone for late-pregnancy or postpartum depression.

\section{Ectopic Pregnancy for Benzodiazepines}

Evidence from one study ${ }^{82}$ suggested an increased risk of ectopic pregnancy with benzodiazepine exposure 90 days before conception when compared with no exposure before conception, among pregnant women with at least one anxiety disorder diagnosis in the year before conception (graded low strength of evidence of harms). Risk factors for ectopic pregnancy include previous ectopic pregnancy, history of pelvic infection, infertility, cigarette smoking and age older than 35 years. ${ }^{83}$ To the extent that such risk factors are associated with an indication for treatment with benzodiazepines, confounding by indication may explain these results; however, one suggested mechanism of action could be through the central relaxation of smooth muscle and the direct effect on gamma-aminobutyric acid receptors in the fallopian tube, potentially resulting in a higher incidence of ectopic pregnancy as a result. ${ }^{82}$ The absolute risk difference is 7 more cases per $1,000(95 \% \mathrm{CI}, 4$ to 11$)$.

\section{Postpartum Hemorrhage for SSRIs, Citalopram, Escitalopram, Sertraline, SNRIs, and Venlafaxine}

Regarding postpartum hemorrhage, results from one propensity-score adjusted study suggested that an association between exposure to several antidepressants and postpartum hemorrhage that may vary by timing. ${ }^{84}$ Current exposure (at the time of delivery) is associated with postpartum hemorrhage for SSRIs as a class, citalopram, escitalopram, sertraline, SNRIs as a class, and venlafaxine. Recent exposure (up to 1 month before delivery) is also associated with an increased risk of postpartum hemorrhage for SSRIs as a class, paroxetine, and sertraline (low strength of evidence of harms). For bupropion, however, the association is with past exposure to bupropion (supply of drug 1 to 5 months before delivery). Previous studies suggested that SSRIs in particular can reduce platelet function and result in bleeding because the drugs inhibit serotonin reuptake into platelets. ${ }^{85}$ In a sample with a prevalence of 2.75 percent in the unexposed arm, the absolute risk difference for SSRIs is 13 more cases per 1,000 (95\% CI, 9 more to 16 more); for SNRIs, the risk is 25 more per 1,000 (95\% CI, 10 more to 45 more). The 
mechanism of action for other antidepressants is not clear, and associations could potentially be attributable to chance; the authors suggested more research to confirm the findings. ${ }^{84}$ The risk of residual confounding also remains: the study was not able to control for confounding factors of inadequate diet, the use of tobacco, and severity of disorder.

Table 9. Strength of evidence from effectiveness studies for harm to the mother (intervention versus placebo or no treatment)

\begin{tabular}{|c|c|c|c|c|c|c|c|}
\hline Population & $\begin{array}{l}\text { Intervention } \\
\text { and } \\
\text { Comparator }\end{array}$ & Outcome & $\begin{array}{l}\text { Incidence or } \\
\text { Mean Effect } \\
\text { by Arm }\end{array}$ & Results & $\begin{array}{l}\text { Study } \\
\text { Design and } \\
\text { Sample Size }\end{array}$ & $\begin{array}{l}\text { Factors That } \\
\text { Affect the } \\
\text { Strength of } \\
\text { Evidence }\end{array}$ & $\begin{array}{l}\text { Overall } \\
\text { Evidence } \\
\text { Strength } \\
\text { (Direction of } \\
\text { Effect) }\end{array}$ \\
\hline $\begin{array}{l}\text { Pregnant } \\
\text { women with } \\
\text { at least one } \\
\text { anxiety } \\
\text { diagnosis in } \\
\text { the year } \\
\text { before } \\
\text { conception }\end{array}$ & $\begin{array}{l}\text { Benzodiaz- } \\
\text { epine } \\
\text { exposure } 90 \\
\text { days before } \\
\text { conception } \\
\text { vs. no } \\
\text { benzodiaz- } \\
\text { epine } \\
\text { exposure } \\
\text { before } \\
\text { conception }\end{array}$ & $\begin{array}{l}\text { Ectopic } \\
\text { pregnancy }\end{array}$ & $\begin{array}{l}249 / 9,188 \\
(2.71 \%) \text { vs. } \\
1,730 / 81,291 \\
(2.13 \%)^{82}\end{array}$ & $\begin{array}{l}\text { ARR: } 1.33 \\
(95 \% \mathrm{Cl}, 1.17 \\
\text { to } 1.51)^{82}\end{array}$ & $\begin{array}{l}1 \text { cohort, } \\
\mathrm{N}=90,479^{82}\end{array}$ & $\begin{array}{l}\text { Moderate } \\
\text { study } \\
\text { limitations, } \\
\text { precise, } \\
\text { consistency } \\
\text { unknown }\end{array}$ & $\begin{array}{l}\text { Low for } \\
\text { harms with } \\
\text { benzodiaz- } \\
\text { epine }\end{array}$ \\
\hline $\begin{array}{l}\text { Mood or } \\
\text { anxiety } \\
\text { disorder }\end{array}$ & $\begin{array}{l}\text { Current SSRI } \\
\text { exposure } \\
\text { (exposure } \\
\text { during } \\
\text { delivery) vs. } \\
\text { unexposed } \\
\text { with mood or } \\
\text { anxiety } \\
\text { disorder }\end{array}$ & $\begin{array}{l}\text { Postpartum } \\
\text { hemorrhage }\end{array}$ & $\begin{array}{l}503 / 12,710 \\
\text { (3.96\%) vs. } \\
1,896 / 69,044 \\
(2.75 \%)^{84}\end{array}$ & $\begin{array}{l}\text { ARR, } 1.47 \\
(95 \% \mathrm{Cl}, 1.33 \\
\text { to } 1.62)^{84}\end{array}$ & $\begin{array}{l}1 \text { cohort, } \\
n=81,754^{84}\end{array}$ & $\begin{array}{l}\text { Moderate } \\
\text { study } \\
\text { limitations, } \\
\text { precise, } \\
\text { consistency } \\
\text { unknown }\end{array}$ & $\begin{array}{l}\text { Low for } \\
\text { harms for } \\
\text { current } \\
\text { exposure with } \\
\text { SSRIs }\end{array}$ \\
\hline $\begin{array}{l}\text { Mood or } \\
\text { anxiety } \\
\text { disorder }\end{array}$ & $\begin{array}{l}\text { Recent SSRI } \\
\text { exposure } \\
\text { (within } 1 \\
\text { month before } \\
\text { delivery) vs. } \\
\text { unexposed } \\
\text { with mood or } \\
\text { anxiety } \\
\text { disorder }\end{array}$ & $\begin{array}{l}\text { Postpartum } \\
\text { hemorrhage }\end{array}$ & $\begin{array}{l}196 / 6,096 \\
(3.2 \%) \text { vs. } \\
1,896 / 69,044 \\
(2.75 \%)^{84}\end{array}$ & $\begin{array}{l}\text { ARR, } 1.19 \\
(95 \% \mathrm{Cl}, 1.03 \\
\text { to } 1.38)^{84}\end{array}$ & $\begin{array}{l}1 \text { cohort, } n= \\
75,140^{84}\end{array}$ & $\begin{array}{l}\text { Moderate } \\
\text { study } \\
\text { limitations, } \\
\text { precise, } \\
\text { consistency } \\
\text { unknown }\end{array}$ & $\begin{array}{l}\text { Low for } \\
\text { harms for } \\
\text { recent } \\
\text { exposure with } \\
\text { SSRIs }\end{array}$ \\
\hline $\begin{array}{l}\text { Pregnant } \\
\text { women with } \\
\text { mood or } \\
\text { anxiety } \\
\text { disorder } \\
\text { d4 }\end{array}$ & $\begin{array}{l}\text { Current } \\
\text { citalopram } \\
\text { use vs. no } \\
\text { treatment }\end{array}$ & $\begin{array}{l}\text { Postpartum } \\
\text { hemorrhage }\end{array}$ & $\begin{array}{l}36 / 891(4 \%) \\
\text { vs. } \\
1,896 / 69,044 \\
(2.75 \%)\end{array}$ & $\begin{array}{l}\text { ARR, } 1.48 \\
(95 \% \mathrm{Cl}, 1.07 \\
\text { to } 2.04)\end{array}$ & $\begin{array}{l}1 \text { cohort, } \\
\mathrm{N}=69,935^{84}\end{array}$ & $\begin{array}{l}\text { Moderate } \\
\text { study } \\
\text { limitations, } \\
\text { precise, } \\
\text { consistency } \\
\text { unknown }\end{array}$ & $\begin{array}{l}\text { Low for } \\
\text { harms with } \\
\text { citalopram }\end{array}$ \\
\hline
\end{tabular}




\begin{tabular}{|c|c|c|c|c|c|c|c|}
\hline Population & $\begin{array}{l}\text { Intervention } \\
\text { and } \\
\text { Comparator }\end{array}$ & Outcome & $\begin{array}{l}\text { Incidence or } \\
\text { Mean Effect } \\
\text { by Arm }\end{array}$ & Results & $\begin{array}{l}\text { Study } \\
\text { Design and } \\
\text { Sample Size }\end{array}$ & $\begin{array}{l}\text { Factors That } \\
\text { Affect the } \\
\text { Strength of } \\
\text { Evidence }\end{array}$ & $\begin{array}{l}\text { Overall } \\
\text { Evidence } \\
\text { Strength } \\
\text { (Direction of } \\
\text { Effect) }\end{array}$ \\
\hline $\begin{array}{l}\text { Mood or } \\
\text { anxiety } \\
\text { disorder }\end{array}$ & $\begin{array}{l}\text { Current } \\
\text { escitalopram } \\
\text { exposure vs. } \\
\text { unexposed } \\
\text { with mood or } \\
\text { anxiety } \\
\text { disorder }\end{array}$ & $\begin{array}{l}\text { Postpartum } \\
\text { hemorrhage }\end{array}$ & $\begin{array}{l}43 / 1,022 \\
(4.21 \%) \text { vs. } \\
1,896 / 69,044 \\
(2.75 \%)^{84}\end{array}$ & $\begin{array}{l}\text { ARR, } 1.56 \\
(95 \% \mathrm{Cl}, 1.16 \\
\text { to } 2.09)^{84}\end{array}$ & $\begin{array}{l}1 \text { cohort, } \\
n=70,006^{84}\end{array}$ & $\begin{array}{l}\text { Moderate } \\
\text { study } \\
\text { limitations, } \\
\text { precise, } \\
\text { consistency } \\
\text { unknown }\end{array}$ & $\begin{array}{l}\text { Low for } \\
\text { harms with } \\
\text { escitalopram }\end{array}$ \\
\hline $\begin{array}{l}\text { Women with } \\
\text { diagnosis } \\
\text { code for } \\
\text { mood or } \\
\text { anxiety } \\
\text { disorder } 1-5 \\
\text { months } \\
\text { before } \\
\text { delivery }\end{array}$ & $\begin{array}{l}\text { Paroxetine } \\
\text { current (at } \\
\text { delivery) or } \\
\text { unexposed }\end{array}$ & $\begin{array}{l}\text { Postpartum } \\
\text { hemorrhage } \\
\text { billing } \\
\text { diagnosis }\end{array}$ & $\begin{array}{l}77 / 2,055 \\
(3.75 \%) \\
\text { vs. } \\
1,896 / 69,044 \\
(2.75 \%)\end{array}$ & $\begin{array}{l}\text { ARR, } 1.39 \\
(95 \% \mathrm{Cl}, 1.09 \\
\text { to } 1.71)\end{array}$ & $\begin{array}{l}1 \text { cohort, } N= \\
71,099^{84}\end{array}$ & $\begin{array}{l}\text { Moderate } \\
\text { study } \\
\text { limitations, } \\
\text { precise, } \\
\text { consistency } \\
\text { unknown }\end{array}$ & $\begin{array}{l}\text { Low for } \\
\text { harms with } \\
\text { paroxetine }\end{array}$ \\
\hline $\begin{array}{l}\text { Women with } \\
\text { diagnosis } \\
\text { code for } \\
\text { mood or } \\
\text { anxiety } \\
\text { disorder 1-5 } \\
\text { months } \\
\text { before } \\
\text { delivery }\end{array}$ & $\begin{array}{l}\text { Sertraline } \\
\text { current (at } \\
\text { delivery) or } \\
\text { unexposed }\end{array}$ & $\begin{array}{l}\text { Postpartum } \\
\text { hemorrhage } \\
\text { billing } \\
\text { diagnosis }\end{array}$ & $\begin{array}{l}162 / 4,526 \\
(3.58 \%) \text { vs. } \\
1,896 / 69,044 \\
(2.75 \%)\end{array}$ & $\begin{array}{l}\text { ARR, 1.31 } \\
(95 \% \mathrm{Cl}, 1.12 \\
\text { to } 1.54)\end{array}$ & $\begin{array}{l}1 \text { cohort, } N= \\
73,570^{84}\end{array}$ & $\begin{array}{l}\text { Moderate } \\
\text { study } \\
\text { limitations, } \\
\text { precise, } \\
\text { consistency } \\
\text { unknown }\end{array}$ & $\begin{array}{l}\text { Low for } \\
\text { harms with } \\
\text { sertraline }\end{array}$ \\
\hline $\begin{array}{l}\text { Women with } \\
\text { diagnosis } \\
\text { code for } \\
\text { mood or } \\
\text { anxiety } \\
\text { disorder } 1-5 \\
\text { months } \\
\text { before } \\
\text { delivery }\end{array}$ & $\begin{array}{l}\text { Sertraline } \\
\text { recent }(<1 \\
\text { month before } \\
\text { delivery) or } \\
\text { unexposed }\end{array}$ & $\begin{array}{l}\text { Postpartum } \\
\text { hemorrhage } \\
\text { billing } \\
\text { diagnosis }\end{array}$ & $\begin{array}{l}78 / 2,226 \\
(3.50 \%) \text { vs. } \\
1,896 / 69,044 \\
(2.75 \%)\end{array}$ & $\begin{array}{l}\text { ARR, 1.27 } \\
(95 \% \mathrm{Cl}, 1.01 \\
\text { to } 1.59)\end{array}$ & $\begin{array}{l}1 \text { cohort, } N= \\
71,270^{84}\end{array}$ & $\begin{array}{l}\text { Moderate } \\
\text { study } \\
\text { limitations, } \\
\text { precise, } \\
\text { consistency } \\
\text { unknown }\end{array}$ & $\begin{array}{l}\text { Low for } \\
\text { harms with } \\
\text { sertraline }\end{array}$ \\
\hline $\begin{array}{l}\text { Pregnant } \\
\text { women with } \\
\text { mood or } \\
\text { anxiety } \\
\text { disorders }\end{array}$ & $\begin{array}{l}\text { SNRI } \\
\text { exposure at } \\
\text { time of } \\
\text { delivery vs. } \\
\text { unexposed }\end{array}$ & $\begin{array}{l}\text { Postpartum } \\
\text { hemorrhage }\end{array}$ & $\begin{array}{l}35 / 702 \\
(5.0 \%) \text { vs. } \\
1,896 / 69,044 \\
(2.75 \%)^{84}\end{array}$ & $\begin{array}{l}\text { ARR, } 1.90 \\
(1.37 \text { to } 2.63)\end{array}$ & $\begin{array}{l}1 \text { cohort, } \\
\mathrm{N}=69,746^{84}\end{array}$ & $\begin{array}{l}\text { Moderate } \\
\text { study } \\
\text { limitations, } \\
\text { precise; } \\
\text { consistency } \\
\text { unknown }\end{array}$ & $\begin{array}{l}\text { Low for } \\
\text { harms with } \\
\text { SNRIs }\end{array}$ \\
\hline $\begin{array}{l}\text { Pregnant } \\
\text { women with } \\
\text { mood or } \\
\text { anxiety } \\
\text { disorders }\end{array}$ & $\begin{array}{l}\text { Venlafaxine } \\
\text { exposure at } \\
\text { time of } \\
\text { delivery vs. } \\
\text { unexposed }\end{array}$ & $\begin{array}{l}\text { Postpartum } \\
\text { hemorrhage }\end{array}$ & $\begin{array}{l}46 / 763 \\
(6.0 \%) \text { vs. } \\
1,896 / 69,044 \\
(2.75 \%)^{84}\end{array}$ & $\begin{array}{l}\text { ARR, } 2.24 \\
\text { (1.69 to } 2.97)\end{array}$ & $\begin{array}{l}1 \text { cohort, } \\
\mathrm{N}=69,807^{84}\end{array}$ & $\begin{array}{l}\text { Moderate } \\
\text { study } \\
\text { limitations, } \\
\text { precise; } \\
\text { consistency } \\
\text { unknown }\end{array}$ & $\begin{array}{l}\text { Low for } \\
\text { harms with } \\
\text { venlafaxine }\end{array}$ \\
\hline $\begin{array}{l}\text { Mood } \\
\text { disorder or } \\
\text { anxiety or } \\
\text { bupropion- } \\
\text { exposed } \\
\text { women }\end{array}$ & $\begin{array}{l}\text { Past } \\
\text { bupropion } \\
\text { exposure in } \\
\text { pregnancy } \\
\text { vs. bupropion } \\
\text { unexposed } \\
\text { women with } \\
\text { mood } \\
\text { disorder or } \\
\text { anxiety }\end{array}$ & $\begin{array}{l}\text { Postpartum } \\
\text { hemorrhage }\end{array}$ & $\begin{array}{l}61 / 1712 \\
(3.6 \%) \text { vs. } \\
1,896 / 69,044 \\
(2.75 \%)^{84}\end{array}$ & $\begin{array}{l}\text { ARR, } 1.32 \\
(95 \% \mathrm{Cl}, 1.02 \\
\text { to } 1.69)^{84}\end{array}$ & $\begin{array}{l}1 \text { cohort, } \\
n=70,206^{84}\end{array}$ & $\begin{array}{l}\text { Moderate } \\
\text { study } \\
\text { limitations, } \\
\text { precise, } \\
\text { consistency } \\
\text { unknown }\end{array}$ & $\begin{array}{l}\text { Low for } \\
\text { harms of } \\
\text { bupropion }\end{array}$ \\
\hline
\end{tabular}




\begin{tabular}{|c|c|c|c|c|c|c|c|}
\hline Population & $\begin{array}{l}\text { Intervention } \\
\text { and } \\
\text { Comparator }\end{array}$ & Outcome & $\begin{array}{l}\text { Incidence or } \\
\text { Mean Effect } \\
\text { by Arm }\end{array}$ & Results & $\begin{array}{l}\text { Study } \\
\text { Design and } \\
\text { Sample Size }\end{array}$ & $\begin{array}{l}\text { Factors That } \\
\text { Affect the } \\
\text { Strength of } \\
\text { Evidence }\end{array}$ & $\begin{array}{l}\text { Overall } \\
\text { Evidence } \\
\text { Strength } \\
\text { (Direction of } \\
\text { Effect) }\end{array}$ \\
\hline $\begin{array}{l}\text { Pregnant } \\
\text { women: SNRI } \\
\text { exposure or } \\
\text { depression } \\
\text { diagnosis, } \\
\text { through } \\
\text { second } \\
\text { trimester }\end{array}$ & $\begin{array}{l}\text { SNRI } \\
\text { exposure } \\
\text { through } \\
\text { second } \\
\text { trimester vs. } \\
\text { unexposed }\end{array}$ & Preeclampsia & $\begin{array}{l}107 / 1,216 \\
(9 \%) \text { vs. } \\
3,215 / 59,219 \\
(5 \%)^{86} ; \\
23 / 408 \\
(5.6 \%) \text { vs. } \\
1,569 / 65,392 \\
(2.4 \%)^{87}\end{array}$ & $\begin{array}{l}\text { ARR, 1.52 } \\
(95 \% \mathrm{Cl}, 1.25 \\
\text { to } 1.83)^{86} ; \\
\text { ARR, } 1.59 \\
(95 \% \mathrm{Cl}, 1.26 \\
\text { to } 3.03)^{87}\end{array}$ & $\begin{array}{l}2 \text { cohorts, } \\
\mathrm{N}=126,235^{86} \\
87\end{array}$ & $\begin{array}{l}\text { Moderate } \\
\text { study } \\
\text { limitations, } \\
\text { precise; } \\
\text { consistent }\end{array}$ & $\begin{array}{l}\text { Low for } \\
\text { harms with } \\
\text { SNRIs }\end{array}$ \\
\hline $\begin{array}{l}\text { Depressed } \\
\text { women }\end{array}$ & $\begin{array}{l}\text { TCA } \\
\text { exposure in } \\
\text { pregnancy } \\
\text { vs. } \\
\text { unexposed } \\
\text { women with } \\
\text { depression in } \\
\text { pregnancy }\end{array}$ & Preeclampsia & $\begin{array}{l}47 / 441 \\
(10.7 \%) \text { vs, } \\
3,215 / / 59,219 \\
(5.4 \%)^{86} ; \\
14 / 146 \\
(9.59 \%) \text { vs. } \\
1,569 / 65,392 \\
(2.40 \%)^{87} \\
\end{array}$ & $\begin{array}{l}\text { ARR, 1.62 } \\
(95 \% \mathrm{Cl}, 1.23 \\
\text { to } 2.12)^{86} ; \\
\text { ARR, 3.23 } \\
(95 \% \mathrm{Cl}, 1.87 \\
\text { to } 5.59)^{87}\end{array}$ & $\begin{array}{l}2 \text { cohorts, } \\
\mathrm{n}=125,198^{86} \\
87\end{array}$ & $\begin{array}{l}\text { Moderate } \\
\text { study } \\
\text { limitations, } \\
\text { precise, } \\
\text { consistent }\end{array}$ & $\begin{array}{l}\text { Low for } \\
\text { harms of TCA }\end{array}$ \\
\hline $\begin{array}{l}\text { Women } \\
\text { prescribed a } \\
\text { second- } \\
\text { generation } \\
\text { antipsychotic }\end{array}$ & $\begin{array}{l}\text { Quetiapine } \\
\text { continued vs. } \\
\text { quetiapine } \\
\text { discontinued }\end{array}$ & $\begin{array}{l}\text { Gestational } \\
\text { diabetes }\end{array}$ & $\begin{array}{l}110 / 1,543 \\
(7.1 \%) \text { vs. } \\
122 / 2,990 \\
(4.1 \%)^{88}\end{array}$ & $\begin{array}{l}\text { ARR, } 1.28 \\
(95 \% \mathrm{Cl}, 1.01 \\
\text { to } 1.62)^{88}\end{array}$ & $\begin{array}{l}1 \text { cohort, } \\
n=10,379^{88}\end{array}$ & $\begin{array}{l}\text { Moderate } \\
\text { study } \\
\text { limitations, } \\
\text { precise, } \\
\text { consistency } \\
\text { unknown }\end{array}$ & $\begin{array}{l}\text { Low for } \\
\text { harms of } \\
\text { continued } \\
\text { quetiapine }\end{array}$ \\
\hline $\begin{array}{l}\text { Women } \\
\text { prescribed an } \\
\text { second- } \\
\text { generation } \\
\text { antipsychotic }\end{array}$ & $\begin{array}{l}\text { Olanzapine } \\
\text { continued vs. } \\
\text { olanzapine } \\
\text { discontinued }\end{array}$ & $\begin{array}{l}\text { Gestational } \\
\text { diabetes }\end{array}$ & $\begin{array}{l}46 / 384 \\
(12.0 \%) \text { vs. } \\
49 / 1,041 \\
(4.7 \%)^{88}\end{array}$ & $\begin{array}{l}\text { ARR, } 1.61 \\
(95 \% \mathrm{Cl}, 1.13 \\
\text { to } 2.29)^{88}\end{array}$ & $\begin{array}{l}1 \text { cohort, } \\
n=10,379^{88}\end{array}$ & $\begin{array}{l}\text { Moderate } \\
\text { study } \\
\text { limitations, } \\
\text { precise, } \\
\text { consistency } \\
\text { unknown }\end{array}$ & $\begin{array}{l}\text { Low for } \\
\text { harms of } \\
\text { continued } \\
\text { quetiapine }\end{array}$ \\
\hline $\begin{array}{l}\text { Pregnant } \\
\text { women: SNRI } \\
\text { exposure or } \\
\text { depression } \\
\text { diagnosis in } \\
\text { past } 4 \text { years }\end{array}$ & $\begin{array}{l}\text { SNRI } \\
\text { exposure in } \\
1^{\text {st }} \text { trimester } \\
\text { vs. } \\
\text { unexposed to } \\
\text { any } \\
\text { antidepressa } \\
\text { nt }\end{array}$ & $\begin{array}{l}\text { Spontaneous } \\
\text { abortion }\end{array}$ & $\begin{array}{l}20 / 90(22 \%) \\
\text { vs. } 720 / 7,034 \\
(10 \%) ; 89 \\
\text { results } \\
\text { corrected for } \\
\text { induced } \\
\text { abortions: } \\
20 / 137(15 \%) \\
\text { vs. } 720 / 8,877 \\
(8.1 \%)\end{array}$ & $\begin{array}{l}\text { ARR, } 2.1 \\
(95 \% \mathrm{Cl}, 1.4 \\
\text { to } 3.0) ; \\
\text { corrected for } \\
\text { induced } \\
\text { abortions } \\
\text { ARR, } 1.7 \\
(95 \% \mathrm{Cl}, 1.2 \\
\text { to } 2.6)^{89}\end{array}$ & $\begin{array}{l}1 \text { cohort, } \\
n=7,134, \\
\text { corrected for } \\
\text { induced } \\
\text { abortion, } \\
n=9,014^{89}\end{array}$ & $\begin{array}{l}\text { Moderate } \\
\text { study } \\
\text { limitations, } \\
\text { precise; } \\
\text { consistency } \\
\text { unknown }\end{array}$ & $\begin{array}{l}\text { Low for } \\
\text { harms with } \\
\text { SNRIs }\end{array}$ \\
\hline $\begin{array}{l}\text { Pregnant } \\
\text { women with } \\
\text { depression or } \\
\text { anxiety }\end{array}$ & $\begin{array}{l}\text { Benzodiaz- } \\
\text { epine } \\
\text { exposure in } \\
\text { first } \\
\text { trimester }{ }^{90} \text { or } \\
\text { within the first } \\
19 \text { weeks }^{91} \\
\text { vs. untreated } \\
\text { or a history of } \\
\text { mood } \\
\text { disorders or } \\
\text { anxiety } \\
\text { during } \\
\text { pregnancy }\end{array}$ & $\begin{array}{l}\text { Spontaneous } \\
\text { abortion }\end{array}$ & $\begin{array}{l}386 / 2,384 \\
(16 \%) \text { vs. } \\
442 / 3,647 \\
(12 \%)^{90} \\
198 \\
\text { cases/570 } \\
\text { controls vs. } \\
3,221 \\
\text { cases } / 15,382_{\text {controls }^{91}}\end{array}$ & $\begin{array}{l}\text { ARR, } 1.6 \\
(95 \% \mathrm{Cl}, 1.3 \\
\text { to } 1.9)^{90} \\
\text { AOR: } 2.85 \\
(95 \% \mathrm{Cl}, 1.72 \\
\text { to } 4.72)^{91}\end{array}$ & $\begin{array}{l}1 \text { cohort, } 1 \\
\text { case-control } \\
\text { study, } \\
\mathrm{N}=21,983^{90,91}\end{array}$ & $\begin{array}{l}\text { Moderate } \\
\text { study } \\
\text { limitations } \\
\text { (high risk of } \\
\text { bias }^{90} \text { ) } \\
\text { precise, } \\
\text { consistent }\end{array}$ & $\begin{array}{l}\text { Low for } \\
\text { harms with } \\
\text { benzodiaz- } \\
\text { epine }\end{array}$ \\
\hline
\end{tabular}




\begin{tabular}{|c|c|c|c|c|c|c|c|}
\hline Population & $\begin{array}{l}\text { Intervention } \\
\text { and } \\
\text { Comparator }\end{array}$ & Outcome & $\begin{array}{l}\text { Incidence or } \\
\text { Mean Effect } \\
\text { by Arm }\end{array}$ & Results & $\begin{array}{l}\text { Study } \\
\text { Design and } \\
\text { Sample Size }\end{array}$ & $\begin{array}{l}\text { Factors That } \\
\text { Affect the } \\
\text { Strength of } \\
\text { Evidence }\end{array}$ & $\begin{array}{l}\text { Overall } \\
\text { Evidence } \\
\text { Strength } \\
\text { (Direction of } \\
\text { Effect) }\end{array}$ \\
\hline \multirow[t]{3}{*}{$\begin{array}{l}\text { Women with } \\
\text { postpartum } \\
\text { depression }\end{array}$} & $\begin{array}{l}\text { Brexanolone } \\
\text { vs. placebo }\end{array}$ & $\begin{array}{l}\text { Dose } \\
\text { interruption or } \\
\text { reduction in } \\
\text { some } \\
\text { patients } \\
\text { during the } \\
\text { infusion due } \\
\text { to sedation or } \\
\text { somnolence }\end{array}$ & $\begin{array}{l}\text { Treatment } \\
\text { arm: } 5 \% \\
\text { Placebo: } 0 \\
\text { (from FDA } \\
\text { prescribing } \\
\text { information }^{92} \text { ) }\end{array}$ & $\begin{array}{l}\text { Data not } \\
\text { reported by } \\
\text { study, pooled } \\
\text { RR not } \\
\text { calculable }\end{array}$ & $\begin{array}{l}3 \text { RCTs, } \\
\mathrm{N}=209^{18,77}\end{array}$ & $\begin{array}{l}\text { Low study } \\
\text { limitations, } \\
\text { precision and } \\
\text { consistency } \\
\text { unknown }\end{array}$ & $\begin{array}{l}\text { Low for } \\
\text { harms with } \\
\text { exposure to } \\
\text { brexanolone }\end{array}$ \\
\hline & $\begin{array}{l}\text { Brexanolone } \\
\text { vs. placebo }\end{array}$ & $\begin{array}{l}\text { Loss of con- } \\
\text { sciousness }\end{array}$ & $\begin{array}{l}\text { BRX60: } 5 \% \text {; } \\
\text { BRX90: } 3 \% ; \\
\text { Placebo: } 0 \% \\
\text { (from FDA } \\
\text { prescribing } \\
\text { information }{ }^{92} \text { ) }\end{array}$ & $\begin{array}{l}\text { Data not } \\
\text { reported by } \\
\text { study, cannot } \\
\text { be pooled }\end{array}$ & $\begin{array}{l}3 \text { RCTs, } \\
\mathrm{N}=209^{18,77}\end{array}$ & $\begin{array}{l}\text { Low study } \\
\text { limitations, } \\
\text { precision and } \\
\text { consistency } \\
\text { unknown }\end{array}$ & \\
\hline & $\begin{array}{l}\text { Brexanolone } \\
\text { vs. placebo }\end{array}$ & Somnolence & $\begin{array}{l}\text { BRX60: } 7 / 38 \\
(18.4 \%) \\
\text { BRX90: } \\
\text { 8/102 (7.8\%) } \\
\text { Placebo: } \\
\text { 5/107 }(4.7 \%)\end{array}$ & $\begin{array}{l}\text { Pooled RR, } \\
2.00,95 \% \mathrm{Cl} \text {, } \\
0.78 \text { to } 5.16 \text {, } \\
\mathrm{I}^{2}=0 \%\end{array}$ & $\begin{array}{l}3 \text { RCTs, } \\
\mathrm{N}=209^{18,77}\end{array}$ & $\begin{array}{l}\text { Low study } \\
\text { limitations, } \\
\text { inconsistent, } \\
\text { imprecise } \\
\text { (wide Cls, } \\
\text { few events) }\end{array}$ & \\
\hline
\end{tabular}

$\mathrm{ARR}=$ adjusted risk ratio; $\mathrm{BRX}=$ brexanolone; $\mathrm{CI}=$ confidence interval; FDA $=$ Food and Drug Administration; $\mathrm{N}=$ number; $\mathrm{RCT}=$ randomized controlled trial; $\mathrm{RR}=$ relative risk; $\mathrm{SNRI}=$ serotonin-norepinephrine reuptake inhibitor; $\mathrm{SSRI}=$ selective serotonin reuptake inhibitor; TCA = tricyclic antidepressant; vs. = versus.

\section{Preeclampsia for SNRIs and TCAs}

Two studies ${ }^{86,87}$ offered low strength of evidence, suggesting increased risk of preeclampsia for women exposed to non-SSRI drugs, specifically SNRIs (adjusted risk ratios [ARRs] range from 1.52 to 1.59 ) and TCAs (ARRs range from 1.62 to 3.23); absolute risk differences range from 14 more cases per 1,000 to 54 cases per 1,000. Risk factors for preeclampsia include maternal antiphospholipid antibody syndrome, family history, nulliparity, donor egg pregnancy, diabetes, obesity, and preexisting hypertension. These factors may cause placental hypoxia and ischemia. However, it is also possible that antidepressants increase serotonin and norepinephrine levels and therefore lead to preeclampsia. ${ }^{87}$ Because both serotonin and norepinephrine are vasoconstrictors, to the extent that placental ischemia ${ }^{93}$ contributes to the pathophysiology of preeclampsia, SNRIs could affect preeclampsia risk. It is unclear why the results are not consistent across exposures: one possible explanation is residual confounding due to inadequately controlled depression severity or other comorbid conditions. It is also plausible that underlying characteristics associated with therapeutic response to SNRIs or TCAs are risk factors for preeclampsia.

\section{Gestational Diabetes for Quetiapine or Olanzapine}

For gestational diabetes mellitus (GDM), low strength of evidence from one cohort study ${ }^{88}$ suggested that continuing use (as reflected in two or more prescription dispensings) during the first half of pregnancy of quetiapine (absolute risk difference: 11 per 1,000, 95\% CI, 0 to 25) or olanzapine (absolute risk difference: 29 per 1,000, 95\% CI, 6 to 61) may be associated with an increased risk of developing GDM compared with women discontinuing these respective medications before the start of pregnancy. Antipsychotics may result in changes in appetite and 
diet because of interactions with serotonergic, histaminergic, and dopaminergic neurotransmitter systems ${ }^{94}$ FDA notes the risk of metabolic side effects from second-generation antipsychotics in prescription labels for these drugs. ${ }^{95,96}$ The other second-generation antipsychotics analyzed that showed no clear difference in risk were aripiprazole, ziprasidone, and risperidone. Women who continued antipsychotic treatment during pregnancy generally had higher comorbidity and longer baseline antipsychotic use. Of note, these analyses involved a generalized linear model and propensity-score stratification to obtain risks of developing GDM with adjustment for confounders.

\section{Spontaneous Abortion and SNRIs}

Associations between pharmacologic treatment and spontaneous abortion may be confounded by the severity of the underlying mental health disorder. In a systematic review, exposure to psychological stress was associated with an increased risk of miscarriage (odds ratio [OR], $1.42 ; 95 \%$ CI, 1.19 to 1.70$).{ }^{97}$ Proposed mechanisms include dysregulation of the hypothalamic pituitary adrenal axis and early pregnancy immune function..$^{88}$ To the extent that women experiencing greater psychological stress would be more likely to be treated pharmacologically, confounding by indication by underlying observed associations.

One study suggested that SNRI exposure in the first trimester may be associated with a higher rate of spontaneous abortion when compared with no exposure (low strength of evidence).$^{89}$ Analyses controlling for induced abortion showed a slightly attenuated but still statistically significant difference (absolute risk difference: 62 per 1,000, 95\% CI, 16 to 130 ). Although we rated this outcome as low strength of evidence for potential harms, we note that the comparison group includes women with a depression diagnosis in the 4 years preceding pregnancy. Although the authors adjusted for use of teratogenic medication in the first trimester, number of prescription medication in 3 months before pregnancy, and number of mental health visits in 3 months before pregnancy, the two comparison groups likely had different baseline severity that is unaccounted for in the analysis.

\section{Spontaneous Abortion and Benzodiazepines}

Evidence from two studies ${ }^{90,91}$ suggested an increased risk of spontaneous abortion with benzodiazepine exposure 90 days before conception when compared with untreated or a history of mood disorders or anxiety (graded low strength of evidence of harms). Although residual confounding may explain these results, as with the results for ectopic pregnancy, the authors note that benzodiazepines cross the placental barrier easily and may accumulate in fetal issues. ${ }^{82}$ The absolute risk difference is 73 per $1,000(95 \%$ CI, 36 to 109).

\section{Dose Interruption or Reduction Due to Somnolence or Sedation for Brexanolone}

FDA includes a boxed warning on the prescribing information of excessive sedation or loss of consciousness in the active arm for brexanolone leading to dose interruption or reduction. In the prescribing instructions, the manufacturer reports higher rates of dizziness, loss of consciousness, and somnolence with brexanolone compared with placebo. Results could not be pooled for loss of consciousness from the individual studies. Rates of loss of consciousness did not appear to increase with dose intensity: 5 percent of women randomized to BRX60 versus 3 percent for BRX90 experienced loss of consciousness. 


\section{Evidence of Fetal, Infant, or Child Harm}

\section{Overview}

- The evidence on fetal, infant, or child harms from treatments for mental health disorders comes from bodies of evidence with moderate or high study limitations and precise results. Low strength-of-evidence grades from bodies of evidence with high limitations had precise results but also other factors beyond statistical significance alone (such as large effects or plausible confounding [i.e., one could infer that in the absence of confounding, the effect would be larger]) that contributed to moving the grade from insufficient to low.

- The evidence on fetal, infant, or child harms from treatments for mental health disorders comes from bodies of evidence with moderate or high study limitations but effects that did not cross the null. However, beyond statistical significance alone, other factors (such as large sample size or plausible confounding [i.e., the effect is larger when confounding is addressed]) contributed to moving the grade from insufficient to low.

- Among women with a history of mental health disorders, evidence from one study suggested that benzodiazepine exposure during pregnancy may be associated with an increased risk of NICU admission when compared with no exposure; hospital protocols may explain this association (low strength of evidence of harms).

- Among women with a history of mental health disorders or among women previously exposed to SSRIs, evidence from three studies suggested that exposure to SSRIs during pregnancy may be associated with increased risk of respiratory issues in the newborn (low strength of evidence); evidence from two studies suggested that exposure to SSRIs in pregnancy may be associated with an increased risk of low Apgar scores when compared with no exposure (low strength of evidence).

- When compared with women with untreated depression during pregnancy, evidence from one cohort suggested that exposure to SSRIs in pregnancy may be associated with an increased risk of primary persistent pulmonary hypertension of the newborn (without cardiac malformation or lung hypoplasia in full-term deliveries) (low strength of evidence); the absolute risk increase is small (33 more cases per 100,000 persons).

- Among women with a psychiatric diagnosis, evidence from one cohort indicated that an increased risk of childhood depression was observed among women exposed to SSRIs when compared women with no treatment; the study did not control for depression severity, and the direction of effect is unclear (low strength of evidence).

- When compared with unexposed women with a known mental health disorder, evidence from one cohort suggested that citalopram exposure in pregnant women may be associated with a higher risk of autism spectrum disorder in their children; residual confounding could potentially explain this effect (low strength of evidence).

\section{Detailed Synthesis}

Table 10 provides an overview of the findings on harms to the fetus, infant, or child of pharmacotherapy in the perinatal period. Appendix B includes detailed tables on all outcomes. We describe results from five publications on harms below (Table 10). 
Table 10. Strength of evidence from effectiveness studies for harm to the fetus, infant, or child (intervention versus placebo or no treatment)

\begin{tabular}{|c|c|c|c|c|c|c|c|}
\hline Population & $\begin{array}{l}\text { Intervention } \\
\text { and } \\
\text { Comparator }\end{array}$ & Outcome & $\begin{array}{l}\text { Incidence or } \\
\text { Mean Effect } \\
\text { by Arm }\end{array}$ & Results & $\begin{array}{l}\text { Study } \\
\text { Design and } \\
\text { Sample Size }\end{array}$ & $\begin{array}{l}\text { Factors That } \\
\text { Affect the } \\
\text { Strength of } \\
\text { Evidence }\end{array}$ & $\begin{array}{l}\text { Overall } \\
\text { Evidence } \\
\text { Strength } \\
\text { (Direction of } \\
\text { Effect) }\end{array}$ \\
\hline $\begin{array}{l}\text { Pregnant } \\
\text { women with a } \\
\text { mental health } \\
\text { disorder }^{99}\end{array}$ & $\begin{array}{l}\text { Benzodiaze- } \\
\text { pine exposure } \\
\text { during } \\
\text { pregnancy vs. } \\
\text { unexposed to } \\
\text { benzodiaze- } \\
\text { pine use } \\
\text { during } \\
\text { pregnancy }\end{array}$ & $\begin{array}{l}\text { NICU } \\
\text { admission }\end{array}$ & $\begin{array}{l}32 / 144 \\
(22.2 \%) \text { vs. } \\
125 / 649 \\
(19.3 \%)\end{array}$ & $\begin{array}{l}\text { AOR, } 2.02 \\
(95 \% \mathrm{Cl}, 1.11 \\
\text { to } 3.66)\end{array}$ & $\begin{array}{l}1 \text { cohort, } \\
\mathrm{N}=793^{99}\end{array}$ & $\begin{array}{l}\text { Moderate } \\
\text { study } \\
\text { limitations, } \\
\text { precise, } \\
\text { consistency } \\
\text { unknown }\end{array}$ & $\begin{array}{l}\text { Low for } \\
\text { harms of } \\
\text { benzodiaze- } \\
\text { pine }\end{array}$ \\
\hline \multirow[t]{2}{*}{$\begin{array}{l}\text { History of } \\
\text { SSRI } \\
\text { exposure }\end{array}$} & \multirow[t]{2}{*}{$\begin{array}{l}\text { SSRI } \\
\text { exposure in } \\
\text { pregnancy vs. } \\
\text { women who } \\
\text { discontinued } \\
\text { SSRIs }\end{array}$} & \multirow[t]{2}{*}{$\begin{array}{l}\text { Intrauterine } \\
\text { hypoxia and } \\
\text { birth } \\
\text { asphyxia }\end{array}$} & \multirow[t]{2}{*}{$\begin{array}{l}94 / 2,664 \\
(3.5 \%) \text { vs. } \\
124 / 5,141 \\
(2.4 \%)^{100}\end{array}$} & $\begin{array}{l}\text { Adjusted } \\
\text { prevalence } \\
\text { ratio in all } \\
\text { women: } \\
1.39(95 \% \mathrm{Cl} \text {, } \\
1.07 \text { to } 1.81)\end{array}$ & \multirow[t]{2}{*}{$\begin{array}{l}1 \text { cohort, } n= \\
7,805^{100}\end{array}$} & \multirow[t]{2}{*}{$\begin{array}{l}\text { High study } \\
\text { limitations } \\
\text { (high risk-of- } \\
\text { bias study) } \\
\text { precise, } \\
\text { consistency } \\
\text { unknown }\end{array}$} & \multirow[t]{2}{*}{$\begin{array}{l}\text { Low for } \\
\text { harms with } \\
\text { SSRIs }\end{array}$} \\
\hline & & & & $\begin{array}{l}\text { Adjusted } \\
\text { prevalence } \\
\text { ratio in women } \\
\text { with vaginal } \\
\text { deliveries in } \\
\text { the third } \\
\text { trimester: } \\
1.70(95 \% \mathrm{Cl} \text {, } \\
1.23 \text { to } 2.33)\end{array}$ & & & \\
\hline $\begin{array}{l}\text { History of } \\
\text { mental health } \\
\text { disorder or } \\
\text { depression or } \\
\text { SSRI- } \\
\text { exposed } \\
\text { women }\end{array}$ & $\begin{array}{l}\text { SSRI } \\
\text { exposure in } \\
\text { pregnancy vs. } \\
\text { unexposed } \\
\text { women with } \\
\text { depression or } \\
\text { mental health } \\
\text { disorder }\end{array}$ & $\begin{array}{l}\text { Respiratory } \\
\text { conditions } \\
\text { (respiratory } \\
\text { conditions of } \\
\text { newborns } \\
\text { other than } \\
\text { intrauterine } \\
\text { hypoxia and } \\
\text { birth } \\
\text { asphyxia, }{ }^{100} \\
\text { undefined } \\
\text { breathing } \\
\text { problems, }{ }^{101} \\
\text { respiratory }^{1} \\
\text { distress }^{102} \text { ) } \\
\end{array}$ & $\begin{array}{l}\text { Ranges from } \\
4.3 \% \text { to } 4.9 \% \\
\text { in the } \\
\text { treatment } \\
\text { arm and } \\
3.1 \% \text { to } 3.2 \text { in } \\
\text { the control } \\
\text { arm }{ }^{100,101 ;} \text { NR } \\
\text { in one } \\
\text { study }{ }^{102}\end{array}$ & $\begin{array}{l}\text { All three } \\
\text { studies } \\
\text { reported } \\
\text { increased risk. } \\
\text { Adjusted } \\
\text { prevalence } \\
\text { ratios and } \\
\text { AOR, range } \\
\text { from } 1.37 \text { to } \\
1.4^{100,101}\end{array}$ & $\begin{array}{l}\text { cohort } \\
\text { studies, } 100-102 \\
n>33,186 \\
(N=N R \text { in one } \\
\left.\text { study }^{102}\right)\end{array}$ & $\begin{array}{l}\text { High study } \\
\text { limitations ( } 2 \\
\text { of } 3 \text { are high } \\
\text { risk-of-bias } \\
\text { studies, }{ }^{100,101} \\
\text { precise, wide } \\
\text { Cls), } \\
\text { consistent }\end{array}$ & $\begin{array}{l}\text { Low for } \\
\text { harms with } \\
\text { SSRls }\end{array}$ \\
\hline $\begin{array}{l}\text { Exposed to } \\
\text { SSRIs during } \\
\text { pregnancy, } \\
\text { before } \\
\text { pregnancy, or } \\
\text { unexposed } \\
\text { with a } \\
\text { psychiatric } \\
\text { diagnosis }\end{array}$ & $\begin{array}{l}\text { Exposed to } \\
\text { SSRIs during } \\
\text { pregnancy vs. } \\
\text { before } \\
\text { pregnancy or } \\
\text { unexposed } \\
\text { with a } \\
\text { psychiatric } \\
\text { diagnosis }\end{array}$ & $\begin{array}{l}\text { Low Apgar } \\
\text { score (<7 at } \\
5 \mathrm{~min})\end{array}$ & $\begin{array}{l}376 / 15,729 \\
(2.4 \%) \text { vs. } \\
113 / 9,652 \\
(1.2 \%)^{101}\end{array}$ & $\begin{array}{l}\text { Adjusted } \\
\text { prevalence } \\
\text { ratio: } 1.69 \\
(95 \% \mathrm{Cl}, 1.02 \\
\text { to } 2.79)^{100} \\
\\
\text { AOR, } 1.68 \\
(95 \% \mathrm{Cl}, 1.34 \\
\text { to } 2.12)^{101}\end{array}$ & $\begin{array}{l}2 \text { cohorts, } \\
n=33,186^{100} \\
101\end{array}$ & $\begin{array}{l}\text { High study } \\
\text { limitations (all } \\
\text { risk-of-bias } \\
\text { studies }^{100,101} \text { ), } \\
\text { precise, } \\
\text { consistent }\end{array}$ & $\begin{array}{l}\text { Low for } \\
\text { harms with } \\
\text { SSRIs }\end{array}$ \\
\hline
\end{tabular}




\begin{tabular}{|c|c|c|c|c|c|c|c|}
\hline Population & $\begin{array}{l}\text { Intervention } \\
\text { and } \\
\text { Comparator }\end{array}$ & Outcome & $\begin{array}{l}\text { Incidence or } \\
\text { Mean Effect } \\
\text { by Arm }\end{array}$ & Results & $\begin{array}{l}\text { Study } \\
\text { Design and } \\
\text { Sample Size }\end{array}$ & $\begin{array}{l}\text { Factors That } \\
\text { Affect the } \\
\text { Strength of } \\
\text { Evidence }\end{array}$ & $\begin{array}{l}\text { Overall } \\
\text { Evidence } \\
\text { Strength } \\
\text { (Direction of } \\
\text { Effect) }\end{array}$ \\
\hline Depressed & $\begin{array}{l}\text { Exposed to } \\
\text { SSRIs during } \\
\text { pregnancy vs. } \\
\text { unexposed } \\
\text { during } \\
\text { pregnancy }\end{array}$ & $\begin{array}{l}\text { Primary } \\
\text { persistent } \\
\text { pulmonary } \\
\text { hyperten- } \\
\text { sion (without } \\
\text { cardiac } \\
\text { malforma- } \\
\text { tion or } \\
\text { hypoplasia } \\
\text { in full-term } \\
\text { deliveries) }\end{array}$ & $\begin{array}{l}94 / 54,281 \\
(0.2 \%) \text { vs. } \\
669 / 567,118 \\
(0.1 \%)^{103} \\
\end{array}$ & $\begin{array}{l}\text { Adjusted OR, } \\
1.28(95 \% \mathrm{Cl}, \\
1.01 \text { to } 1.70)^{103} \\
\text { AOR, when not } \\
\text { restricted to full- } \\
\text { term or by } \\
\text { outcome } \\
\text { (persistent } \\
\text { pulmonary } \\
\text { hypertension } \\
\text { rather than } \\
\text { primary } \\
\text { persistent } \\
\text { pulmonary } \\
\text { hypertension): } \\
1.08 \text { (95\% CI, } \\
0.92 \text { to } 1.27)^{103}\end{array}$ & $\begin{array}{l}1 \text { cohort, } \\
n=621,399^{103}\end{array}$ & $\begin{array}{l}\text { Moderate } \\
\text { study } \\
\text { limitations } \\
\text { precise, } \\
\text { consistency } \\
\text { unknown, } \\
\text { adjusting for } \\
\text { confounding } \\
\text { increased the } \\
\text { odds }\end{array}$ & $\begin{array}{l}\text { Low for } \\
\text { harms with } \\
\text { SSRIs }\end{array}$ \\
\hline $\begin{array}{l}\text { Exposed to } \\
\text { SSRIs during } \\
\text { pregnancy or } \\
\text { unexposed } \\
\text { with a } \\
\text { psychiatric } \\
\text { diagnosis }\end{array}$ & $\begin{array}{l}\text { Exposed to } \\
\text { SSRIs during } \\
\text { pregnancy vs. } \\
\text { unexposed } \\
\text { with a } \\
\text { psychiatric } \\
\text { diagnosis }\end{array}$ & Depression & $\begin{array}{l}60 / 15,729 \\
(0.4 \%) \text { vs. } \\
30 / 9,651 \\
(0.3 \%)\end{array}$ & $\begin{array}{l}\text { AHR, } 1.78(95 \% \\
\mathrm{Cl}, 1.12 \text { to } 2.82) \\
p=0.015\end{array}$ & $\begin{array}{l}1 \text { cohort, } \\
n=25,380^{104}\end{array}$ & $\begin{array}{l}\text { Moderate } \\
\text { study } \\
\text { limitations, } \\
\text { precise, } \\
\text { consistency } \\
\text { unknown }\end{array}$ & $\begin{array}{l}\text { Low for } \\
\text { harms with } \\
\text { SSRIs }\end{array}$ \\
\hline $\begin{array}{l}\text { Pregnant with } \\
\text { or without a } \\
\text { known } \\
\text { psychiatric } \\
\text { condition }\end{array}$ & $\begin{array}{l}\text { Maternal } \\
\text { exposure to } \\
\text { citalopram vs. } \\
\text { no maternal } \\
\text { exposure to } \\
\text { any } \\
\text { antidepressant } \\
\text { but with a } \\
\text { known } \\
\text { psychiatric } \\
\text { condition }\end{array}$ & $\begin{array}{l}\text { Autism } \\
\text { spectrum } \\
\text { disorder } \\
\text { without } \\
\text { intellectual } \\
\text { disabilities } \\
\text { among } \\
\text { offspring }\end{array}$ & $\begin{array}{l}46 / 1064 \\
(4.3 \%) \text { vs. } \\
291 / 12325 \\
(2.4 \%)\end{array}$ & $\begin{array}{l}\text { AOR, } 1.75(95 \% \\
\mathrm{Cl}, 1.25 \text { to } 2.45)\end{array}$ & $\begin{array}{l}1 \text { cohort, } \\
n=13,389^{105}\end{array}$ & $\begin{array}{l}\text { Moderate } \\
\text { study } \\
\text { limitations, } \\
\text { precise, } \\
\text { consistency } \\
\text { unknown }\end{array}$ & $\begin{array}{l}\text { Low for } \\
\text { harms with } \\
\text { citalopram }\end{array}$ \\
\hline
\end{tabular}

$\overline{\mathrm{AHR}}=$ adjusted hazard ratio; $\mathrm{AOR}=$ adjusted odds ratio; $\mathrm{CI}=$ confidence interval; $\mathrm{N} / \mathrm{n}=$ number; NICU $=$ neonatal intensive care unit; $\mathrm{NR}=$ not reported; $\mathrm{OR}=$ odds ratio; $\mathrm{SSRI}=$ selective serotonin reuptake inhibitor; vs. = versus.

The identified harms include NICU admissions for benzodiazepine; respiratory issues, low Apgar scores, persistent pulmonary hypertension of the newborn, and depression in children for SSRIs overall; and autism spectrum disorder for citalopram. The relative paucity of evidence for these harms led us to rate the strength of evidence as low. Although the potential for harm to the child may exist, we have low confidence that future studies will support this association.

The evidence on fetal, infant, or child harms from treatments for mental health disorders comes from bodies of evidence with moderate or high limitations and precise results. Bodies of evidence with high limitations and precise results were not automatically upgraded from insufficient to low strength of evidence: they had factors beyond adequate sample size/events and narrow CIs alone (such as large effect size) that resulted in the low strength-of-evidence grade. 


\section{NICU Admission and Benzodiazapines}

Regarding fetal harms, the evidence suggested that benzodiazepine exposure during pregnancy may be associated with an increased risk of NICU admission when compared with no exposure to benzodiazepines among women with a history of psychiatric disorders (absolute risk difference: $133 / 1,000,95 \%$ CI, 17 to 274 ) (low strength of evidence). ${ }^{99}$ The authors noted several differences between the comparison arms and the results adjusted for these confounders, but residual confounding cannot be ruled out. Also the study did not comment on dose or frequency.

NICU admission may be confounded by provider knowledge of antenatal exposure to psychotropic medications and hospital protocols. Until recently, neonatal abstinence syndrome due to prenatal exposure to opiates or benzodiazepines was typically treated in the NICU.

\section{Respiratory Issues and SSRIs}

Three studies reporting on respiratory distress and SSRIs used different measures; their results could not be pooled. One reported on respiratory conditions of newborns other than intrauterine hypoxia and birth asphyxia (International Statistical Classification of Diseases and Related Health Problems [ICD-9] code of other respiratory conditions), ${ }^{100}$ undefined breathing problems, ${ }^{101}$ and respiratory distress (ICD-9 codes of 769, 770.6, and $770.8^{102}$ ). The two studies that did specify that the ICD-9 codes included very heterogeneous outcomes, ranging from benign self-limiting conditions such as transient tachypnea of the newborn to more serious outcomes such as respiratory arrest of the newborn. Although we rated the outcome as low strength of evidence for harm, in the absence of more details on the proportion and differences between study arms for more serious outcomes, the clinical implications of this finding are unclear.

One study reported higher rates of intrauterine hypoxia and birth asphyxia. ${ }^{100}$ The study used the ICD-9 code of 768. One concern is whether the higher rate in the exposed arm may be attributable to the greater number of Caesarean sections in the exposed arm and the higher use of the ICD-9 code of 768.3 for fetal distress during labor for nonreassuring fetal testing. Analyses from the same study in a population of women with vaginal deliveries found a statistically significant difference in the third trimester only. Notably, the incidence of intrauterine hypoxia and birth asphyxia in the exposed arm (3.5\%) is much higher than the incidence of low Apgar scores ( $<7$ at 5 minutes) in the same study $(1.1 \%)$. It is unclear under what circumstances an infant would meet criteria for intrauterine hypoxia and birth asphyxia with a 5-minute Apgar $\geq 7$, so the clinical significance of this finding is unclear.

\section{Low Apgar Score and SSRIs}

Although both studies reporting on low Apgar scores and exposure to SSRIs are rated as high risk of bias, the direction is consistent. ${ }^{100,101}$ The inter-rater reliability and consistency of scoring within individuals can vary; of note, the prevalence of 5-minute Apgar $<7$ in the control group was 1.2 percent in one study and 0.6 percent in the other, suggesting differences in the study population or the local standards for assignment of scores. The absolute risk difference from the study with the larger effect size is 8 per 1,000 (95\% CI, 4 more to 13 more). The Apgar score does not predict long-term neurological outcomes ${ }^{106}$ and was developed to determine the immediate need for resuscitation. Although we have rated the strength of evidence as low rather than insufficient, based on consistency, the clinical implications are unclear. Also, given the high risk of bias, it is possible that both studies are consistently biased. 


\section{Persistent Pulmonary Hypertension of the Newborn and SSRIs}

For the study reporting an association between persistent pulmonary hypertension of the newborn and SSRIs, as with other observational cohorts, residual confounding and the potential for misclassification may exist. Risk factors, such as smoking, obesity, and Caesarean section, are all more prevalent in populations of psychiatric patients. ${ }^{107}$ However, adjustments for potential sources of confounding (restricting the sample to full-term births ${ }^{107}$ and restricting the outcome to those without cardiac anomalies or hypoplasia) resulted in higher odds than unrestricted results when compared with no exposure. ${ }^{103}$ Notably, the baseline risk $(0.1 \%$ in the unexposed arm) and absolute risk increase are very low (33 more cases per 100,000 persons, $95 \% \mathrm{CI}, 1$ to 83 more cases); in the analyses adjusting for potential sources of confounding, the risks are lower still at 9 more cases per 100,000 (95\% CI ranges from 9 fewer cases to 32 more cases). These results suggest that although the exposure may be associated with a higher risk of a potentially serious complication, the absolute risk of harm is very low (low strength of evidence).

\section{Depression in Children and SSRls}

A single large national Finnish registry cohort study provides low strength of evidence that offspring of mothers with psychiatric illness exposed to SSRIs during pregnancy were more likely to have had a diagnosis of depression by age 15 than mothers with psychiatric illness (nearly all of which were affective disorders) who were not exposed to SSRIs during pregnancy. ${ }^{104,108}$ Of note, the absolute incidence of a depression diagnosis was less than 0.4 percent in each group (absolute risk difference: $2 / 1,000,95 \%$ CI, 0 to 6 more). Further, there was no control for depressive severity (although the study controlled for previous diagnoses related to suicidal behavior). If depressed mothers receiving SSRIs were more severely depressed than depressed mothers not receiving SSRIs, the increased risk of depression in the offspring of those who received SSRIs may reflect a greater congenital risk of developing depression independent of any exposure.

\section{Autism Spectrum Disorder and Citalopram}

Results from one study suggested an increased risk of autism spectrum disorder in pregnant women exposed to citalopram (absolute risk difference: 17 per 1,000, 95\% CI, 6 to 32) (low strength of evidence). Residual confounding could explain these results: the study found a stronger effect for autism spectrum disorder without intellectual disabilities (adjusted odds ratio [AOR], $1.75 ; 95 \% \mathrm{CI}, 1.25$ to 2.45 ), which the study notes is more likely to be heritable. ${ }^{105}$ The study also notes that 95 percent of women who took antidepressants during pregnancy did not have a child with autism, and if no pregnant women took antidepressants, only 2 percent of autism cases in this population would be prevented. ${ }^{105}$

\section{Insufficient Evidence of Maternal Harms}

\section{Overview}

- Insufficient grades were assigned based on study limitations, bias, consistency, and precision. Some bodies of evidence comprised single studies for which we could not infer consistency of the evidence base. Additionally, the reported associations were imprecise (i.e., the results relied on small sample sizes, few events, or had wide CIs suggestive of both benefits and harms), were inconsistent, or had high study limitations.

- We found insufficient evidence to judge the risk of abruption and gestational hypertension in pregnancy for all reported exposures. 
- We found insufficient evidence to judge the risk of preeclampsia for all reported exposures other than SNRIs and TCAs during pregnancy. Exposures during pregnancy with insufficient evidence include benzodiazepine, zolpidem, SSRIs (unspecified), citalopram, escitalopram, fluoxetine, paroxetine, sertraline, trazodone, bupropion, and mood stabilizers (as a class and separately for lamotrigine, valproate, topiramate, carbamazepine, oxcarbazepine, and lithium).

- We found insufficient evidence to judge overall adverse events or dizziness from using brexanolone for depression onset in the third trimester or within 4 weeks of delivery but note that FDA requires a boxed warning for excessive sedation or sudden loss of consciousness during infusion.

- We found insufficient evidence to judge the risk of spontaneous abortion for SSRIs and benzodiazepine.

- We found insufficient evidence to judge the risk of postpartum hemorrhage for trazodone, TCAs, and mirtazapine.

\section{Detailed Synthesis}

We found single studies with imprecise results, often from high risk-of-bias studies for most outcomes, including evidence from single studies of different exposures on gestational hypertension (see Appendix B for further details). For a subset of outcomes and interventions, we identified multiple studies but graded the evidence as insufficient. We describe these results in greater detail below for preeclampsia for SSRIs, spontaneous abortion for SSRIs and TCAs, and brexanolone for any adverse events including dizziness (Table 11).

\section{Preeclampsia and SSRIs}

Two studies ${ }^{86,87}$ with some risk-of-bias concerns reported inconsistent and imprecise results (CIs for the estimate of effect span both appreciable benefit and appreciable harm) and inclusive of both appreciable benefits and appreciable harms, resulting in the evidence being graded as insufficient.

\section{Spontaneous Abortion and SSRIs or TCAs}

Two publications reported on spontaneous abortion when comparing SSRI or TCA exposure in pregnancy with no exposure. One, rated as having a high risk of bias, suggested an association between SSRI or TCA exposure and spontaneous abortion..$^{90}$ The second, rated as having some risk-of-bias concerns, reported imprecise results with wide CIs suggestive of both benefits and harms for both exposures. In accounting for study limitations, we rated the evidence base as insufficient. $^{89}$

\section{Adverse Events and Brexanolone}

Three RCTs (rated low risk of bias) reported on the harms of brexanolone versus placebo for depression symptoms with onset in the third trimester of pregnancy or within 4 weeks of birth. ${ }^{18,77}$ Brexanolone was administered as a 60 -hour continuous infusion with a peak dose of either $60 \mu \mathrm{g} / \mathrm{kg}$ per hour or $90 \mu \mathrm{g} / \mathrm{kg}$ per hour. The study reported similar rates of any adverse events during infusion in the brexanolone and placebo groups and a higher rate of dizziness in the brexanolone arm versus the placebo arm. The pooled results for any adverse events and dizziness are imprecise and inconsistent and were graded as insufficient. 
Table 11. Insufficient strength of evidence from effectiveness studies for harm to the mother (intervention versus placebo or no treatment)

\begin{tabular}{|c|c|c|c|c|c|c|c|}
\hline Population & $\begin{array}{l}\text { Intervention } \\
\text { and } \\
\text { Comparator }\end{array}$ & Outcome & $\begin{array}{l}\text { Incidence or } \\
\text { Mean Effect } \\
\text { by Arm }\end{array}$ & Results & $\begin{array}{l}\text { Study } \\
\text { Design and } \\
\text { Sample } \\
\text { Size }\end{array}$ & $\begin{array}{l}\text { Factors That } \\
\text { Affect the } \\
\text { Strength of } \\
\text { Evidence }\end{array}$ & $\begin{array}{l}\text { Overall } \\
\text { Evidence } \\
\text { Strength } \\
\text { (Direction of } \\
\text { Effect) }\end{array}$ \\
\hline $\begin{array}{l}\text { Depressed } \\
\text { women }\end{array}$ & $\begin{array}{l}\text { SSRI } \\
\text { exposure } \\
\text { during } \\
\text { pregnancy } \\
\text { vs. } \\
\text { unexposed } \\
\text { women with } \\
\text { depression }\end{array}$ & Preeclampsia & $\begin{array}{l}303 / 5215 \\
(5.8 \%) \text { vs. } \\
222 / 4,661 \\
(4.8 \%)^{86} ; \\
105 / 3,169 \\
(3.3 \%) \text { vs. } \\
1,569 / 65,392 \\
(2.4 \%)^{87}\end{array}$ & $\begin{array}{l}\text { ARR, } 1.21(95 \% \\
\mathrm{Cl}, 1.02 \text { to } 1.45)^{86} \\
\text { but bias corrected } \\
\mathrm{RR}=0.9 ; \mathrm{ARR}, \\
1.22(95 \% \mathrm{Cl}, \\
\left.0.97 \text { to } 1.54^{87}\right) ; \\
\text { severe } \\
\text { preeclampsia: } \\
\text { ARR, } 1.03(95 \% \\
\mathrm{Cl}, 0.81 \text { to } 1.18)\end{array}$ & $\begin{array}{l}2 \text { cohorts, } \\
n=78,437^{86,} \\
87\end{array}$ & $\begin{array}{l}\text { Moderate } \\
\text { study } \\
\text { limitations } \\
\text { (both have } \\
\text { some risk-of- } \\
\text { bias } \\
\text { concerns), } \\
\text { imprecise } \\
\text { (wide Cls), } \\
\text { inconsistent }\end{array}$ & Insufficient \\
\hline $\begin{array}{l}\text { Depressed or } \\
\text { TCA-exposed } \\
\text { women }\end{array}$ & $\begin{array}{l}\text { TCA } \\
\text { exposure in } \\
\text { pregnancy } \\
\text { vs. } \\
\text { unexposed } \\
\text { women with } \\
\text { depression }\end{array}$ & $\begin{array}{l}\text { Spontaneous } \\
\text { abortion }\end{array}$ & $\begin{array}{l}20 / 112 \\
(17.9 \%) \text { vs. } \\
720 / 7,034 \\
(10.2 \%)^{89} \\
\text { NR in } \\
\text { second }^{90}\end{array}$ & $\begin{array}{l}\text { ARR, } 1.5,(95 \% \\
\mathrm{Cl}, 0.96 \text { to } 2.2)^{89} \\
\text { and } \\
1.3(99 \% \mathrm{Cl}, 1.1 \\
\text { to } 1.5)^{90}\end{array}$ & $\begin{array}{l}2 \text { cohorts, } \\
n=7,146 \text { in } \\
\text { one cohort, } \\
\text { NR in } \\
\text { second }^{90}\end{array}$ & $\begin{array}{l}\text { Moderate } \\
\text { study } \\
\text { limitations } \\
\text { (one high } \\
\text { risk-of-bias } \\
\text { study }^{90} \text { ), } \\
\text { imprecise, } \\
\text { consistent }\end{array}$ & Insufficient \\
\hline $\begin{array}{l}\text { Depressed } \\
\text { women }\end{array}$ & $\begin{array}{l}\text { SSRI } \\
\text { exposure in } \\
\text { pregnancy } \\
\text { vs. } \\
\text { unexposed } \\
\text { women with } \\
\text { depression }\end{array}$ & $\begin{array}{l}\text { Spontaneous } \\
\text { abortion }\end{array}$ & $\begin{array}{l}93 / 938 \\
(9.9 \%) \text { vs. } \\
720 / 8,877 \\
(8.1 \%)^{89} \\
1,539 / 10,312 \\
(14.9) \text { vs. } \\
442 / 3647 \\
(12.1 \%)^{90}\end{array}$ & $\begin{array}{l}\text { ARR, } 1.2,(95 \% \\
\mathrm{Cl}, 0.94 \text { to } 1.5)^{89} \\
\text { and } 1.4(99 \% \mathrm{Cl} \text {, } \\
1.2 \text { to } 1.7)^{90}\end{array}$ & $\begin{array}{l}2 \text { cohorts, } \\
n=23,774^{89}, \\
90\end{array}$ & $\begin{array}{l}\text { Moderate } \\
\text { study } \\
\text { limitations } \\
\text { (one high } \\
\text { risk-of-bias } \\
\text { study, }{ }^{90} \text { ) } \\
\text { imprecise, } \\
\text { consistent }\end{array}$ & Insufficient \\
\hline $\begin{array}{l}\text { Women with } \\
\text { postpartum } \\
\text { depression }\end{array}$ & $\begin{array}{l}\text { Brexanolone } \\
\text { vs. placebo }\end{array}$ & $\begin{array}{l}\text { Any adverse } \\
\text { event }\end{array}$ & $\begin{array}{l}\text { Placebo: } \\
54 / 107 \\
\text { BRX60: } \\
\text { 19/38 } \\
\text { BRX90: } \\
51 / 102 \\
\end{array}$ & $\begin{array}{l}\text { Pooled RR, } 0.93 \\
(95 \% \mathrm{Cl}, 0.72 \text { to } \\
1.21), \mathrm{I}^{2}=1.5 \% \\
\text { Placebo: } 50.5 \% \\
\text { BRX60: } 50.0 \% \\
\text { BRX90: } 50.0 \% \\
\end{array}$ & $\begin{array}{l}3 \text { RCTs, } \\
\mathrm{N}=209^{18,77}\end{array}$ & $\begin{array}{l}\text { Low study } \\
\text { limitations, } \\
\text { inconsistent, } \\
\text { imprecise } \\
\text { (wide Cls) }\end{array}$ & Insufficient \\
\hline $\begin{array}{l}\text { Women with } \\
\text { postpartum } \\
\text { depression }\end{array}$ & $\begin{array}{l}\text { Brexanolone } \\
\text { vs. placebo }\end{array}$ & Dizziness & $\begin{array}{l}\text { Placebo: } \\
\text { 8/107 } \\
\text { BRX60: 6/38 } \\
\text { BRX90: } \\
\text { 13/102 }\end{array}$ & $\begin{array}{l}\text { Pooled RR, } 1.56 \\
(95 \% \mathrm{Cl}, 0.52 \text { to } \\
4.66), \mathrm{I}^{2}=31.3 \% \\
\text { Placebo: } 7.5 \% \\
\text { BRX60: } 15.8 \\
\text { BRX90: } 12.7 \%\end{array}$ & $\begin{array}{l}3 \text { RCTs, } \\
\mathrm{N}=209^{18,77}\end{array}$ & $\begin{array}{l}\text { Low study } \\
\text { limitations, } \\
\text { inconsistent, } \\
\text { imprecise } \\
\text { (wide Cls) }\end{array}$ & Insufficient \\
\hline
\end{tabular}

$\mathrm{ARR}=$ adjusted risk ratio; $\mathrm{BRX}=$ brexanolone for postpartum depression; $\mathrm{CI}=$ confidence interval; $\mathrm{n}=$ number; $\mathrm{NR}=$ not reported; = RR = relative risk; $\mathrm{SSRI}=$ selective serotonin reuptake inhibitor; $\mathrm{TCA}=$ tricyclic antidepressant; vs. $=$ versus.

\section{Insufficient Evidence of Fetal, Infant, or Child Harms}

\section{Overview}

- Insufficient grades were assigned based on study limitations, bias, consistency, and precision. Some bodies of evidence comprised single studies for which we could not infer consistency of the evidence base. Additionally, the reported associations were imprecise (i.e., the results relied on small sample sizes, few events, or had CIs suggestive of both benefits and harms), were inconsistent, or had high study limitations. 
- We found insufficient evidence for perinatal death, preterm birth; major congenital anomalies; cardiac defects; small for gestational age; large for gestational age; birthweight; neonatal convulsions; extended hospital stay; delayed social, emotional, and cognitive development; ADHD; and anxiety in children for all reported exposures.

- We found insufficient evidence to judge the risk of autism spectrum disorder for all reported exposures other than citalopram. Exposures during pregnancy with insufficient evidence include SSRIs (unspecified), fluoxetine, paroxetine, sertraline, venlafaxine, duloxetine, clomipramine, amitriptyline/nortriptyline, and mirtazapine.

Although diazepam, temazepam, and paroxetine include language suggesting risks of major congenital anomalies, we found insufficient evidence from eligible studies for our population of interest; evidence is available from studies of other populations ineligible for this review.

\section{Detailed Synthesis}

We found single studies with imprecise results, often from high risk-of-bias studies for most outcomes, including evidence from single studies of different exposures on perinatal death, large for gestational age, ADHD, and anxiety (see Appendix B for further details). For a subset of outcomes and interventions, we identified multiple studies but graded the evidence as insufficient. We describe these results in greater detail below. Specifically, we found multiple publications providing evidence for one or more intervention for preterm birth, neonatal convulsions, small for gestational age, birthweight, major congenital anomalies, cardiac defects, NICU admissions, extended hospital stay, infant and child development and behavior, and autism spectrum disorder (Table 12).

\section{Preterm Birth and SSRIs}

Six studies reported inconsistent results on the associations between preterm birth (all defined as $<37$ weeks) and SSRIs. ${ }^{101,102,109-112}$ The results could not be pooled because of variations in reported outcomes. One study reported statistically significant benefit; ${ }^{101}$ a second reported statistically significant harms. ${ }^{111}$ The other studies reported relative risks, odds, or incidences, with wide CIs suggestive of both benefits and harms. Four of the six studies did not account for severity of psychiatric illness. ${ }^{102,109,110}$ The studies that accounted for this variable in found no statistically significant differences between the groups. ${ }^{102,112}$ Inconsistency, imprecision, and high study limitations for most studies led to a rating of insufficient.

\section{Neonatal Convulsions and SSRIs}

Regarding neonatal convulsions, two studies ${ }^{100,102}$ provided consistent but imprecise evidence on the risks of neonatal convulsions associated with exposure to SSRIs. Only one study provided rates, but the numbers suggest a very small baseline risk $(0.1 \%$ in the unexposed arm) and a small absolute risk difference (2 in 1,000). 
Table 12. Insufficient strength of evidence from effectiveness studies for harm to the fetus, infant, or child (intervention versus placebo or no treatment)

\begin{tabular}{|c|c|c|c|c|c|c|c|}
\hline Population & $\begin{array}{l}\text { Intervention and } \\
\text { Comparator }\end{array}$ & Outcome & $\begin{array}{l}\text { Incidence or } \\
\text { Mean Effect by } \\
\text { Arm }\end{array}$ & Results & $\begin{array}{l}\text { Study Design and } \\
\text { Sample Size }\end{array}$ & $\begin{array}{l}\text { Factors That Affect the } \\
\text { Strength of Evidence }\end{array}$ & $\begin{array}{l}\text { Overall Evidence } \\
\text { Strength } \\
\text { (Direction of } \\
\text { Effect) }\end{array}$ \\
\hline $\begin{array}{l}\text { Depressed, } \\
\text { psychiatric } \\
\text { disorders, or } \\
\text { discontinued } \\
\text { SSRIs during } \\
\text { pregnancy } \\
\text { exposed to } \\
\text { SSRIs }\end{array}$ & $\begin{array}{l}\text { SSRI exposure } \\
\text { during pregnancy } \\
\text { vs. no exposure } \\
\text { (exposure prior to } \\
\text { pregnancy or } \\
\text { depressed or with } \\
\text { psychiatric } \\
\text { disorder) }\end{array}$ & Preterm birth & $\begin{array}{l}741 / 15,729 \\
(4.7 \%) \text { vs. } \\
515 / 9,652 ; \\
(5.3 \%)^{101} \\
17 / 192(8.8 \%) \text { vs. } \\
415 / 5,710 \\
(7.3 \%)^{: 110} 55 / 221 \\
(24.9 \%) \text { vs. } \\
185 / 1,566 \\
(11.8 \%)^{: 11} 3 / 37 \\
(8.11 \%) \text { vs. } 3 / 19 \\
(15.79 \%)^{112} \\
\\
\text { N=NR for two } \\
\text { publications }\end{array}$ & $\begin{array}{l}\text { Overall } 5 \text { of } 6 \\
\text { studies do not report } \\
\text { increased risks with } \\
\text { SSRIs. } \\
\text { Prevalence, AOR, } \\
\text { ARRs range from } \\
0.84^{101} \text { to } 2.68^{111} \\
\text { with Cls spanning } \\
\text { the null in } 2 \text { of } 4 \\
\text { studies null (one } \\
\text { study reported } \\
\text { higher odds in the } \\
\text { SSRI group, } 111 \text { the } \\
\text { other reported lower } \\
\text { odds in the SSRI } \\
\text { group }{ }^{101} ; \text { difference } \\
\text { in incidence: } 0.007 \\
\text { (95\% CI, -0.018 to } \\
0.034 \text { ); } 102 \mathrm{NR}, \mathrm{p}= \\
0.948^{112}\end{array}$ & $\begin{array}{l}6 \text { cohorts; } \mathrm{N}>33,666 \\
\mathrm{~N}=\mathrm{NR} \text { in one } \\
\left.\text { study }^{102}\right)^{101,109-112}\end{array}$ & $\begin{array}{l}\text { High study limitations (5 } \\
\text { high risk-of-bias } \\
\text { studies }^{101,109-112} \text { ), mostly } \\
\text { consistent, imprecise } \\
\text { (wide Cls in some } \\
\text { studies) }\end{array}$ & Insufficient \\
\hline $\begin{array}{l}\text { Exposed to } \\
\text { SSRIs during } \\
\text { pregnancy or } \\
\text { unexposed with } \\
\text { previous } \\
\text { exposure or } \\
\text { depressed }\end{array}$ & $\begin{array}{l}\text { Exposed to SSRIs } \\
\text { during pregnancy } \\
\text { vs. unexposed } \\
\text { depressed or } \\
\text { exposed before } \\
\text { but not during } \\
\text { pregnancy }\end{array}$ & $\begin{array}{l}\text { Neonatal } \\
\text { convulsions }\end{array}$ & $\begin{array}{l}9 / 2,664(0.3 \%) \text { vs. } \\
7 / 5,141(0.1 \%) ; 100 \\
\text { NR in one study }{ }^{102}\end{array}$ & $\begin{array}{l}\text { Adjusted prevalence } \\
\text { ratio: } 2.28(95 \% \mathrm{Cl} \text {, } \\
0.87 \text { to } 5.97)^{100} \\
\text { Difference in } \\
\text { incidence: } \\
0.00077(95 \% \mathrm{Cl} \text {, } \\
-0.001 \text { to } 0.0036) \\
\text { p-value } 0.3^{102}\end{array}$ & $\begin{array}{l}2 \text { cohort studies, } \\
\mathrm{n}>7,805(\mathrm{~N} N \mathrm{NR} \text { in } \\
\left.\text { one study }{ }^{102}\right)^{100,102}\end{array}$ & $\begin{array}{l}\text { High study limitations (1 } \\
\text { of } 2 \text { studies are high risk } \\
\left.\text { of bias }{ }^{100}\right) \text { imprecise, } \\
\text { consistent }\end{array}$ & Insufficient \\
\hline
\end{tabular}




\begin{tabular}{|c|c|c|c|c|c|c|c|}
\hline Population & $\begin{array}{l}\text { Intervention } \\
\text { and } \\
\text { Comparator }\end{array}$ & Outcome & $\begin{array}{l}\text { Incidence or } \\
\text { Mean Effect by } \\
\text { Arm }\end{array}$ & Results & $\begin{array}{l}\text { Study Design and } \\
\text { Sample Size }\end{array}$ & $\begin{array}{l}\text { Factors That Affect the } \\
\text { Strength of Evidence }\end{array}$ & $\begin{array}{l}\text { Overall Evidence } \\
\text { Strength } \\
\text { (Direction of } \\
\text { Effect) }\end{array}$ \\
\hline $\begin{array}{l}\text { History of mental } \\
\text { health disorder or } \\
\text { depression or } \\
\text { SSRI-exposed } \\
\text { women }\end{array}$ & $\begin{array}{l}\text { SSRI exposure } \\
\text { in pregnancy vs. } \\
\text { unexposed } \\
\text { women with } \\
\text { depression or } \\
\text { mental health } \\
\text { disorder }\end{array}$ & $\begin{array}{l}\text { Small for } \\
\text { gestational age }\end{array}$ & $\begin{array}{l}\text { Varies across } \\
\text { studies from } 2.5 \% \\
\text { to } 17.4 \% \text { in the } \\
\text { treatment arm, } \\
\text { and } 2.5 \% \text { to } \\
14.7 \% \text { in the } \\
\text { control arm }{ }^{100-102,} \\
110,113\end{array}$ & $\begin{array}{l}\text { Five }^{100-102,111,113} \text { of } 6 \\
\text { studies report } \\
\text { nonsignificant } \\
\text { results (adjusted } \\
\text { prevalence ratios, } \\
\text { ARR, AOR, } \\
\text { difference in } \\
\text { incidence) with Cls } \\
\text { spanning the null; } \\
\text { one study reported } \\
\text { AOR of } 1.68 \text { (95\% } \\
\text { Cl, } 1.03 \text { to } 2.74) ; 110 \\
\text { ARR varies by } \\
\text { trimester of } \\
\text { exposure from } 0.7 \text { to } \\
1.4,95 \% \text { Cl spans } \\
\text { the null }\end{array}$ & $\begin{array}{l}5 \text { cohort studies, } 1 \\
\text { case-control, } \mathrm{n} \\
\text { varies by trimester, } \\
\mathrm{n}=43,185^{100-102,110,111,} \\
113\end{array}$ & $\begin{array}{l}\text { High study limitations ( } 4 \\
\text { of } 6 \text { high risk-of-bias } \\
\text { studies }^{100,101,110,111} \text { ), } \\
\text { imprecise (wide Cls), } \\
\text { mostly consistent }\end{array}$ & Insufficient \\
\hline $\begin{array}{l}\text { Use of } \\
\text { antidepressants } \\
\text { before or during } \\
\text { pregnancy or } \\
\text { psychiatric illness }\end{array}$ & $\begin{array}{l}\text { SSRI exposure } \\
\text { during } \\
\text { pregnancy vs. } \\
\text { SSRI exposure } \\
\text { just before but } \\
\text { not during } \\
\text { pregnancy or } \\
\text { psychiatric } \\
\text { illness with no } \\
\text { exposure }\end{array}$ & $\begin{array}{l}\text { Low birth } \\
\text { weight }\end{array}$ & $\begin{array}{l}42 / 221(19.0 \%) \\
\text { vs. } 150 / 1,566 \\
(9.6 \%){ }^{111} \\
\text { NR in one } \\
\text { study; } 1094 / 36 \\
(11.11 \%) \text { vs. } 3 / 19 \\
(15.79 \%)^{112}\end{array}$ & $\begin{array}{l}\text { Adjusted prevalence } \\
\text { ratio: } 1.1(95 \% \mathrm{Cl} \text {, } \\
0.9 \text { to } 1.3)^{109} \\
\mathrm{AOR}, 2.26(95 \% \mathrm{Cl} \text {, } \\
1.31 \text { to } 3.91) \\
\mathrm{NR}, \mathrm{p}=0.613^{112}\end{array}$ & $\begin{array}{l}3 \text { cohorts, } \\
\mathrm{N}>1,842,111,112 \\
\mathrm{~N}=\mathrm{NR} \text { in one } \\
\text { study } 109\end{array}$ & $\begin{array}{l}\text { High study limitations } \\
\text { (high risk of bias }{ }^{109,111,} \\
112 \text { ), imprecise (wide } \\
\text { Cls), inconsistent }\end{array}$ & Insufficient \\
\hline $\begin{array}{l}\text { History of } \\
\text { depression or } \\
\text { anxiety or current } \\
\text { or past SSRI- } \\
\text { exposed women }\end{array}$ & $\begin{array}{l}\text { Exposed to } \\
\text { SSRIs in } \\
\text { pregnancy vs. } \\
\text { unexposed } \\
\text { women with } \\
\text { history of } \\
\text { depression, } \\
\text { anxiety or prior } \\
\text { exposure }\end{array}$ & $\begin{array}{l}\text { Major } \\
\text { congenital } \\
\text { anomalies }\end{array}$ & $\begin{array}{l}279 / 2,327 \\
(12.0 \%) \text { vs. } \\
1,650 / 14,847 \\
(11.1 \%)^{114} \\
204 / 7,683(2.7 \%) \\
\text { vs, } 380 / 13,432 \\
(2.8 \%)^{115} \\
208 / 4,183(5.0 \%) \\
\text { vs. } 36 / 806 \\
(4.5 \%)^{116}\end{array}$ & $\begin{array}{l}\text { Adjusted prevalence } \\
\text { ratio: } 1.07(95 \% \mathrm{Cl} \text {, } \\
0.93 \text { to } 1.22):{ }^{114} \\
\text { AOR, } 0.93(95 \% \mathrm{Cl} \text {, } \\
0.78 \text { to } 1.11) ; 115 \\
\text { adjusted effect NR } \\
\text { in one study, } \\
p=0.9^{116}\end{array}$ & $\begin{array}{l}3 \text { cohorts, } \\
n=43,299^{114-116}\end{array}$ & $\begin{array}{l}\text { High study limitations (all } \\
\text { risk-of-bias studies), }{ }^{114-} \\
116 \text { imprecise (wide Cls), } \\
\text { consistent }\end{array}$ & Insufficient \\
\hline
\end{tabular}




\begin{tabular}{|c|c|c|c|c|c|c|c|}
\hline Population & $\begin{array}{l}\text { Intervention and } \\
\text { Comparator }\end{array}$ & Outcome & $\begin{array}{l}\text { Incidence or } \\
\text { Mean Effect by } \\
\text { Arm }\end{array}$ & Results & $\begin{array}{l}\text { Study Design and } \\
\text { Sample Size }\end{array}$ & $\begin{array}{l}\text { Factors That Affect the } \\
\text { Strength of Evidence }\end{array}$ & $\begin{array}{l}\text { Overall Evidence } \\
\text { Strength } \\
\text { (Direction of } \\
\text { Effect) }\end{array}$ \\
\hline $\begin{array}{l}\text { Pregnant women } \\
\text { with diagnosis of } \\
\text { depression only }^{115} \\
\text { or depression and } \\
\text { or anxiety, or } \\
\text { exposed to } \\
\text { antidepressants }^{114}\end{array}$ & $\begin{array}{l}\text { Maternal exposure } \\
\text { to citalopram vs. } \\
\text { no exposure } \\
\text { anxiety and/or } \\
\text { depression }\end{array}$ & $\begin{array}{l}\text { Major } \\
\text { congenital } \\
\text { anomalies }\end{array}$ & $\begin{array}{l}\text { NR/1946 vs. } \\
666 / 23,833 \\
(2.8 \%) \text { in one } \\
\text { study, }{ }^{115} \text { NR in } \\
\text { second }{ }^{114}\end{array}$ & $\begin{array}{l}\text { AOR, } 1.36(95 \% \mathrm{Cl}, \\
1.08 \text { to } 1.73)^{114} ; \mathrm{OR} \text {, } \\
0.97(95 \% \mathrm{Cl}, 0.71 \\
\text { to } 1.31)^{115}\end{array}$ & $\begin{array}{l}2 \text { cohort studies; } \\
\mathrm{n}>25,779^{115}, 116\end{array}$ & $\begin{array}{l}\text { High study limitations } \\
\text { (high risk of bias }{ }^{114,115} \text { ), } \\
\text { imprecise (Cls } \\
\text { suggestive of both } \\
\text { benefits and harms in } \\
\text { one study }{ }^{115} \text { ), } \\
\text { inconsistent }\end{array}$ & Insufficient \\
\hline $\begin{array}{l}\text { Pregnant women: } \\
\text { Cohort 1: } \\
\text { depression and/or } \\
\text { anxiety diagnosis } \\
\text { and exposure to } \\
\text { antidepressants in } \\
\text { the year before } \\
\text { pregnancy; } \\
\text { Cohort 2: } \\
\text { depression } \\
\text { diagnosis from } \\
\text { the year before } \\
\text { conception } \\
\text { through the first } \\
\text { trimester }\end{array}$ & $\begin{array}{l}\text { Fluoxetine } \\
\text { exposure in the } \\
\text { first trimester vs. } \\
\text { unexposed }\end{array}$ & $\begin{array}{l}\text { Major } \\
\text { congenital } \\
\text { anomalies }\end{array}$ & $\begin{array}{l}\text { NR/191 vs. } \\
1,650 / 14,847 \\
(11.1 \%)^{114} ; \\
241 / 3,189(7.6 \%) \\
\text { vs. } 380 / 13,432 \\
(2.8 \%)^{115}\end{array}$ & $\begin{array}{l}\text { AOR, } 0.80(95 \% \mathrm{Cl} \text {, } \\
0.49 \text { to } 1.31) ; 114 \\
\text { AOR, } 0.85(95 \% \mathrm{Cl} \text {, } \\
0.66 \text { to } 1.09)^{115}\end{array}$ & $\begin{array}{l}2 \text { cohorts: } N= \\
15,038 ; 114 \\
N=27,022^{115}\end{array}$ & $\begin{array}{l}\text { High study limitations } \\
\text { (both high risk of bias), } \\
\text { imprecise (wide Cls); } \\
\text { consistent }\end{array}$ & Insufficient \\
\hline $\begin{array}{l}\text { Women with } \\
\text { depression or } \\
\text { anxiety in the year } \\
\text { before pregnancy }\end{array}$ & $\begin{array}{l}\text { Paroxetine in } 1^{\text {st }} \\
\text { trimester vs. not } \\
\text { exposed in } 1^{\text {st }} \\
\text { trimester }\end{array}$ & $\begin{array}{l}\text { Major } \\
\text { congenital } \\
\text { anomalies }\end{array}$ & $\begin{array}{l}168 / 1,132 \\
(14.8 \%) \text { vs. } \\
1,650 / 14,847 \\
(11.1 \%)^{114} \\
\text { Paroxetine } \\
\text { exposed: } 37 \\
\text { cases, } 375 \\
\text { controls } \\
\text { No } \\
\text { antidepressant: } \\
94 \text { cases, } 1134 \\
\text { controls }{ }^{117} \\
\\
36 / 1,200(3.0 \%) \\
\text { vs. } 380 / 13,432 \\
(2.8 \%)^{115}\end{array}$ & $\begin{array}{l}\text { Results not pooled } \\
\text { because two } \\
\text { publications }{ }^{114,117} \\
\text { potentially draw } \\
\text { from the same } \\
\text { population } \\
\text { AOR, } 1.24(95 \% \mathrm{Cl} \text {, } \\
0.99 \text { to } 1.55 ; 99 \% \\
\mathrm{Cl}, 0.79 \text { to } 1.66)^{114} \\
\text { AOR, } 1.27(95 \% \mathrm{Cl} \text {, } \\
0.78 \text { to } 2.06)^{117} \\
\text { AOR } 1.01(95 \% \mathrm{Cl} \text {, } \\
0.71 \text { to } 1.44)^{115}\end{array}$ & $\begin{array}{l}2 \text { cohorts, }{ }^{114,115} 1 \\
\text { case-control, } 117 \\
\mathrm{~N} \geq 33,119 \text { ( } \mathrm{N} \text { from } \\
\text { two studies; }{ }^{114,115} \\
\text { third study may be a } \\
\text { subset of one } \\
\text { study }^{117}\end{array}$ & $\begin{array}{l}\text { High study limitations } \\
\text { (two high risk-of-bias } \\
\text { studies } \text { sit, } 115^{115} \text { ), imprecise, } \\
\text { consistent }\end{array}$ & Insufficient \\
\hline
\end{tabular}




\begin{tabular}{|c|c|c|c|c|c|c|c|}
\hline Population & $\begin{array}{l}\text { Intervention and } \\
\text { Comparator }\end{array}$ & Outcome & $\begin{array}{l}\text { Incidence or } \\
\text { Mean Effect by } \\
\text { Arm }\end{array}$ & Results & $\begin{array}{l}\text { Study Design and } \\
\text { Sample Size }\end{array}$ & $\begin{array}{l}\text { Factors That Affect the } \\
\text { Strength of Evidence }\end{array}$ & $\begin{array}{l}\text { Overall Evidence } \\
\text { Strength } \\
\text { (Direction of } \\
\text { Effect) }\end{array}$ \\
\hline $\begin{array}{l}\text { Women with } \\
\text { depression or } \\
\text { anxiety in the year } \\
\text { before pregnancy }\end{array}$ & $\begin{array}{l}\text { Sertraline in } 1^{\text {st }} \\
\text { trimester vs. not } \\
\text { exposed in } 1^{\text {st }} \\
\text { trimester }\end{array}$ & $\begin{array}{l}\text { Major } \\
\text { congenital } \\
\text { anomalies }\end{array}$ & $\begin{array}{l}\text { NR/365 vs. } \\
1650 / 14,847 \\
(11.1 \%)^{114} 45 / 366 \\
(12.31099 \%) \\
\text { vs.1,651/14,868 } \\
(11.1 \%) \text { from one } \\
\text { study with } \\
\text { potentially } \\
\left.\text { overlapping }_{\text {participants }}^{118}\right) \\
\\
25 / 757(3.3 \%) \text { vs. } \\
380 / 13,432 \\
(2.8 \%)^{115}\end{array}$ & $\begin{array}{l}\text { Results not pooled } \\
\text { because two } \\
\text { publications }{ }^{114,118} \\
\text { potentially draw } \\
\text { from the same } \\
\text { population } \\
\text { AOR, } 1.09(95 \% \mathrm{Cl} \text {, } \\
0.80 \text { to } 1.50)^{114} \\
(\text { ARR, } 1.11(95 \% \mathrm{Cl} \\
0.81 \text { to } 1.52) \text { from } \\
\text { potentially } \\
\text { overlapping } \\
\text { citation } 118 \\
\\
\text { AOR, } 1.17(95 \% \mathrm{Cl} \text {, } \\
0.78 \text { to } 1.77)^{115}\end{array}$ & $\begin{array}{l}2 \text { cohorts, } \\
\mathrm{N}>32,676^{114} \\
\text { (potential overlap of } \\
\text { participants in two } \\
\text { publications }^{114,118} \text { ) }\end{array}$ & $\begin{array}{l}\text { High study limitations } \\
\text { (two high risk-of- bias } \\
\text { studies }^{114,115} \text { ), consistent }\end{array}$ & Insufficient \\
\hline $\begin{array}{l}\text { History of } \\
\text { depression, } \\
\text { depression or } \\
\text { anxiety or mental } \\
\text { health disorder or } \\
\text { TCA-exposed } \\
\text { women }\end{array}$ & $\begin{array}{l}\text { TCA exposure } \\
\text { during the first } \\
\text { trimester) vs. } \\
\text { unexposed with } \\
\text { history of } \\
\text { depression, } \\
\text { anxiety, or mental } \\
\text { health disorder }\end{array}$ & $\begin{array}{l}\text { Major } \\
\text { congenital } \\
\text { anomalies }\end{array}$ & $\begin{array}{l}51 / 382(13.4 \%) \\
\text { vs. } 1,650 / 14,847 \\
(11.1 \%)^{114 ;} ; \\
74 / 2,428(3.0 \%) \\
\text { vs. } 380 / 13,432 \\
(2.8 \%)^{115} ; \text { NR in } \\
\text { other study }{ }^{117}\end{array}$ & $\begin{array}{l}\text { Results not pooled } \\
\text { because two } \\
\text { publications }{ }^{114,117} \\
\text { potentially draw } \\
\text { from the same } \\
\text { population } \\
\text { AOR, } 1.16(95 \% \mathrm{Cl} \text {, } \\
0.86 \text { to } 1.56)^{114} \\
\text { AOR, } 1.02(95 \% \mathrm{Cl} \text {, } \\
0.79 \text { to } 1.32)^{115} \\
\text { AOR, } 0.78(95 \% \mathrm{Cl} \text {, } \\
0.30 \text { to } 2.02)^{117}\end{array}$ & $\begin{array}{l}1 \text { case-control, }{ }^{117} 2 \\
\text { cohorts, } \mathrm{n} \geq 31,089^{115}, \\
{ }^{119} \mathrm{~N} \text { from two } \\
\text { studies; }{ }^{114,115} \\
\text { (potential overlap of } \\
\text { participants in the } \\
\text { publications) }\end{array}$ & $\begin{array}{l}\text { High study limitations } \\
\text { (two high risk-of-bias } \\
\text { studies), }{ }^{114,} 117 \text { imprecise } \\
\text { (wide Cls), consistent }\end{array}$ & Insufficient \\
\hline $\begin{array}{l}\text { Women with a } \\
\text { psychiatric } \\
\text { condition or } \\
\text { women with } \\
\text { schizophrenia, } \\
\text { bipolar disorder, } \\
\text { or psychosis }\end{array}$ & $\begin{array}{l}\text { Second- } \\
\text { generation } \\
\text { antipsychotic } \\
\text { exposure in first } \\
\text { trimester vs. } \\
\text { unexposed to } \\
\text { second-generation } \\
\text { antipsychotic }\end{array}$ & $\begin{array}{l}\text { Major } \\
\text { congenital } \\
\text { anomalies }\end{array}$ & $\begin{array}{l}209 / 3,995(5.2 \%) \\
\text { vs, } 471 / 11,606 \\
(4.1 \%)^{120} \\
3 / 214(1.4 \%) \text { vs. } \\
1 / 89(1.1 \%)^{121}\end{array}$ & $\begin{array}{l}\text { AOR, } 1.16(95 \% \mathrm{Cl} \\
0.99 \text { to } 1.35)^{120} \\
\text { AOR, } 0.69(95 \% \mathrm{Cl} \text {, } \\
0.06 \text { to } 8.09)^{121}\end{array}$ & $\begin{array}{l}2 \text { cohorts, } \\
n=15,904^{120,121}\end{array}$ & $\begin{array}{l}\text { Moderate study } \\
\text { limitations (one high risk- } \\
\text { of-bias study }{ }^{121} \text { precise, } \\
\text { inconsistent }\end{array}$ & Insufficient \\
\hline
\end{tabular}




\begin{tabular}{|c|c|c|c|c|c|c|c|}
\hline Population & $\begin{array}{l}\text { Intervention and } \\
\text { Comparator }\end{array}$ & Outcome & $\begin{array}{l}\text { Incidence or } \\
\text { Mean Effect by } \\
\text { Arm }\end{array}$ & Results & $\begin{array}{l}\text { Study Design and } \\
\text { Sample Size }\end{array}$ & $\begin{array}{l}\text { Factors That Affect the } \\
\text { Strength of Evidence }\end{array}$ & $\begin{array}{l}\text { Overall Evidence } \\
\text { Strength } \\
\text { (Direction of } \\
\text { Effect) }\end{array}$ \\
\hline $\begin{array}{l}\text { Pregnant women } \\
\text { with diagnosis of } \text { depression only }^{115} \\
\text { or depression and } \\
\text { or anxiety, or } \\
\text { exposed to } \\
\text { antidepressants }^{114} \text {, } \\
{ }_{122}\end{array}$ & $\begin{array}{l}\text { Maternal exposure } \\
\text { to citalopram vs. } \\
\text { no exposure or } \\
\text { unexposed to } \\
\text { SSRIs in early } \\
\text { pregnancy }\end{array}$ & $\begin{array}{l}\text { Cardiac } \\
\text { congenital } \\
\text { anomalies }\end{array}$ & $\begin{array}{l}\text { NR in two } \\
\text { studies; }{ }^{114,115} 50 \\
\text { cases/39 controls } \\
\text { vs. } 149 \text { cases/125 } \\
\text { controls }^{122} \\
\end{array}$ & $\begin{array}{l}\text { Pooled OR, } 1.09 \\
(95 \% \mathrm{Cl}, 0.82 \text { to } \\
1.46), \mathrm{I}^{2}: 0 \% \\
\text { AOR, } 1.15(95 \% \mathrm{Cl} \text {, } \\
0.69 \text { to } 1.92)^{114} \\
\text { AOR, } 1.02(95 \% \mathrm{Cl} \text {, } \\
0.61 \text { to } 1.70)^{115} \\
\text { AOR, } 1.11(95 \% \mathrm{Cl} \\
0.68 \text { to } 1.83)^{122}\end{array}$ & $\begin{array}{l}2 \text { cohort studies, } 1 \\
\text { case control, } \\
N>363^{122} N \text { NR in } \\
\text { two studies }\end{array}$ & $\begin{array}{l}\text { High study limitations } \\
\text { (high risk of bias }{ }^{114,115} \text { ), } \\
\text { imprecision (wide Cls), } \\
\text { consistent }\end{array}$ & Insufficient \\
\hline $\begin{array}{l}\text { Depression or } \\
\text { antidepressant } \\
\text { exposed women }\end{array}$ & $\begin{array}{l}\text { Escitalopram } \\
\text { exposure during } \\
\text { early pregnancy } \\
\text { vs. unexposed } \\
\text { women with } \\
\text { depression or } \\
\text { unexposed to } \\
\text { SSRIs in early } \\
\text { pregnancy }\end{array}$ & $\begin{array}{l}\text { Cardiac } \\
\text { anomalies }\end{array}$ & $\begin{array}{l}3 / 333(0.9 \%) \text { vs. } \\
112 / 13,432 \\
(0.83 \%) ; 11543 \\
\text { cases/35 controls } \\
\text { vs. } 149 \text { cases } / 125 \\
\text { controls }{ }^{122}\end{array}$ & $\begin{array}{l}\text { AOR, } 1.09(95 \% \mathrm{Cl} \\
(0.34 \text { to } 3.50)^{115} \\
\text { AOR, } 1.16(0.69 \text { to } \\
1.97)^{122}\end{array}$ & $\begin{array}{l}1 \text { cohort, } \\
\mathrm{n}=13,765,{ }^{115} 1 \text { case- } \\
\text { control, } n=352^{122}\end{array}$ & $\begin{array}{l}\text { Serious study limitations } \\
\text { (high risk of bias }^{115} \text { ), } \\
\text { imprecision (wide Cls } \\
\text { spanning the null), } \\
\text { consistency unknown }\end{array}$ & Insufficient \\
\hline
\end{tabular}




\begin{tabular}{|c|c|c|c|c|c|c|c|}
\hline Population & $\begin{array}{l}\text { Intervention and } \\
\text { Comparator }\end{array}$ & Outcome & $\begin{array}{l}\text { Incidence or } \\
\text { Mean Effect by } \\
\text { Arm }\end{array}$ & Results & $\begin{array}{l}\text { Study Design and } \\
\text { Sample Size }\end{array}$ & $\begin{array}{l}\text { Factors That Affect the } \\
\text { Strength of Evidence }\end{array}$ & $\begin{array}{l}\text { Overall Evidence } \\
\text { Strength } \\
\text { (Direction of } \\
\text { Effect) }\end{array}$ \\
\hline $\begin{array}{l}\text { Women with } \\
\text { depression or } \\
\text { anxiety in the year } \\
\text { prior to pregnancy }\end{array}$ & $\begin{array}{l}\text { Sertraline in } 1^{\text {st }} \\
\text { trimester vs. } \\
\text { unexposed }\end{array}$ & $\begin{array}{l}\text { Cardiac } \\
\text { anomaly }\end{array}$ & $\begin{array}{l}\text { NR in one study: } \\
\text { NR/365 vs. } \\
\text { NR/14,847 } \\
\text { (results from } \\
\text { publication with } \\
\text { overlapping data: } \\
\text { 10/366) }(2.7 \%) \\
\text { vs. } 344 / 14,868 \\
\left.(2.3 \%)^{118}\right) ; \\
9 / 757(1.0 \%) \text { vs. } \\
\text { NR/13,432; } 115 \\
93 / 11,126(0.8 \%) \\
\text { vs. } 1,479 / 180,564 \\
(0.8 \%)^{123} \\
156 \text { cases/129 } \\
\text { controls vs. } 149 \\
\text { cases } / 125^{\text {controls }}{ }^{122}\end{array}$ & $\begin{array}{l}\text { Pooled AOR, } 1.08 \\
(95 \% \mathrm{Cl}, 0.91 \text { to } \\
\text { 1.28), } \text { I }^{2}: 0 \% \\
\text { AOR, } 1.14(95 \% \mathrm{Cl} \text {, } \\
0.60 \text { to } 2.15)^{114} \\
\text { (Results from one } \\
\text { publication } \\
\text { potentially } \\
\text { overlapping data } \\
\text { with study } 114 \\
\text { included in meta- } \\
\text { analysis is also } \\
\text { consistent, with } \\
\text { ARR, } 1.16(95 \% \mathrm{Cl}, \\
0.62 \text { to } 2.19)^{118} \\
\text { AOR, } 1.39(95 \% \mathrm{Cl}, \\
0.70 \text { to } 2.74)^{115} \\
\text { Propensity score } \\
\text { AOR, } 1.09(95 \% \mathrm{Cl}, \\
0.88 \text { to } 1.34, \\
\text { p=0.051) } \\
\text { AOR, } 0.97(95 \% \mathrm{Cl} \text {, } \\
0.69 \text { to } 1.37)\end{array}$ & $\begin{array}{l}3 \text { cohorts, } 1 \text { case- } \\
\text { control, } 5 \\
\text { publications, }{ }^{114,115,} \\
118,122,123 \mathrm{~N}>250,577 \\
\text { (potential overlap in } \\
\text { two publications }{ }^{114} \text {, } \\
{ }^{118} \text { ) }\end{array}$ & 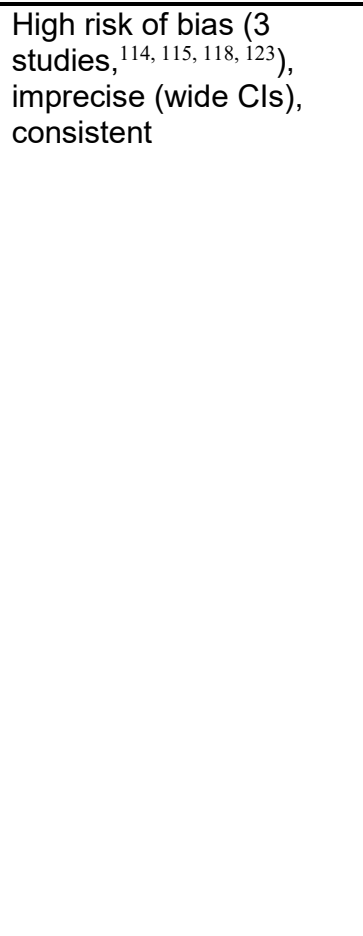 & Insufficient \\
\hline
\end{tabular}




\begin{tabular}{|c|c|c|c|c|c|c|c|}
\hline Population & $\begin{array}{l}\text { Intervention and } \\
\text { Comparator }\end{array}$ & Outcome & $\begin{array}{l}\text { Incidence or } \\
\text { Mean Effect by } \\
\text { Arm }\end{array}$ & Results & $\begin{array}{l}\text { Study Design and } \\
\text { Sample Size }\end{array}$ & $\begin{array}{l}\text { Factors That Affect the } \\
\text { Strength of Evidence }\end{array}$ & $\begin{array}{l}\text { Overall Evidence } \\
\text { Strength } \\
\text { (Direction of } \\
\text { Effect) }\end{array}$ \\
\hline $\begin{array}{l}\text { Women with } \\
\text { depression or } \\
\text { anxiety in the year } \\
\text { prior to pregnancy } \\
\text { or exposure to } \\
\text { antidepressants } \\
\text { outside of early } \\
\text { pregnancy }\end{array}$ & $\begin{array}{l}\text { Paroxetine in first } \\
\text { trimester vs. } \\
\text { unexposed }\end{array}$ & $\begin{array}{l}\text { Cardiac } \\
\text { anomalies }\end{array}$ & 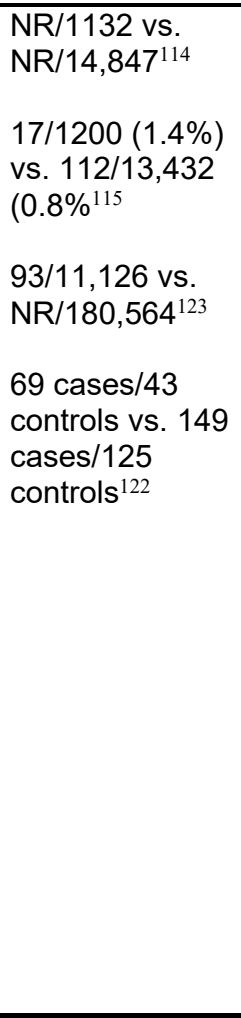 & $\begin{array}{l}\text { Pooled AOR, } 1.26 \text {, } \\
95 \% \mathrm{Cl}, 0.96 \text { to } \\
1.65, \mathrm{I}^{2}: 58 \% \text {; high } \\
\text { heterogeneity } \\
\text { potentially explained } \\
\text { by clinical } \\
\text { (differences in the } \\
\text { definition of cardiac } \\
\text { anomaly) and } \\
\text { statistical } \\
\text { heterogeneity } \\
\text { (differences in } \\
\text { direction of effect) } \\
\text { AOR, } 1.45(95 \% \mathrm{Cl} \text {, } \\
1.12-1.88 ; 99 \% \mathrm{Cl}, \\
0.87 \text { to } 2.03)^{114} \\
\text { AOR, } 1.67(95 \% \mathrm{Cl} \text {, } \\
1.00 \text { to } 2.80, \\
\text { p=0.051) } \\
\text { Propensity score } \\
\text { AOR, } 0.94(95 \% \mathrm{Cl} \text {, } \\
0.73 \text { to } 1.21)^{123} \\
\text { AOR, } 1.27(95 \% \mathrm{Cl} \text {, } \\
0.8 \text { to } 2)^{122}\end{array}$ & $\begin{array}{l}3 \text { cohorts, } 1 \text { case- } \\
\text { control, N=222,505 }\end{array}$ & $\begin{array}{l}\text { High study limitations (3 } \\
\text { studies }^{114,115,123} \text { ), } \\
\text { imprecision (wide Cls), } \\
\text { inconsistent }\end{array}$ & Insufficient \\
\hline
\end{tabular}




\begin{tabular}{|c|c|c|c|c|c|c|c|}
\hline Population & $\begin{array}{l}\text { Intervention and } \\
\text { Comparator }\end{array}$ & Outcome & $\begin{array}{l}\text { Incidence or } \\
\text { Mean Effect by } \\
\text { Arm }\end{array}$ & Results & $\begin{array}{l}\text { Study Design and } \\
\text { Sample Size }\end{array}$ & $\begin{array}{l}\text { Factors That Affect the } \\
\text { Strength of Evidence }\end{array}$ & $\begin{array}{l}\text { Overall Evidence } \\
\text { Strength } \\
\text { (Direction of } \\
\text { Effect) }\end{array}$ \\
\hline $\begin{array}{l}\text { Women with } \\
\text { depression or } \\
\text { anxiety in the year } \\
\text { before pregnancy }\end{array}$ & $\begin{array}{l}\text { Sertraline in } 1^{\text {st }} \\
\text { trimester vs. } \\
\text { unexposed }\end{array}$ & $\begin{array}{l}\text { Cardiac } \\
\text { anomalies }\end{array}$ & $\begin{array}{l}\text { NR in one study: } \\
\text { NR/365 vs. } \\
\text { NR/14,847 } \\
\text { (results from } \\
\text { publication with } \\
\text { overlapping data: } \\
10 / 366(2.7 \%) \text { vs. } \\
344 / 14,868 \\
\left.(2.3 \%)^{118}\right) ; \\
9 / 757(1.0 \%) \text { vs. } \\
\text { NR/13,432;115 } \\
93 / 11,126(0.8 \%) \\
\text { vs. } 1,479 / 180,564 \\
(0.8 \%)^{123} \\
\end{array}$ & $\begin{array}{l}\text { Pooled AOR, } 1.12 \\
(95 \% \mathrm{Cl}, 0.92 \text { to } \\
1.35), \mathrm{I}^{2}: 0 \%, \\
\text { AOR, } 1.14(95 \% \mathrm{Cl} \text {, } \\
0.60 \text { to } 2.15)^{114} \\
\text { Results from one } \\
\text { publication } \\
\text { potentially } \\
\text { overlapping data } \\
\text { with study }{ }^{114} \\
\text { included in meta- } \\
\text { analysis is also } \\
\text { consistent, with } \\
\text { ARR, } 1.16(95 \% \mathrm{Cl} \text {, } \\
0.62 \text { to } 2.19)^{118}\end{array}$ & $\begin{array}{l}3 \text { cohorts, } 4 \\
\text { publications, }{ }^{114,115,} \\
118,123 \mathrm{~N}>250,018 \\
\text { (potential overlap in } \\
\text { two publications }{ }^{114,} \\
118 \text { ) }\end{array}$ & $\begin{array}{l}\text { High risk of bias (all } \\
\text { studies), imprecise (wide } \\
\text { Cls), consistent }\end{array}$ & Insufficient \\
\hline $\begin{array}{l}\text { Depressed or } \\
\text { exposed to SSRIs } \\
\text { in pregnancy }\end{array}$ & $\begin{array}{l}\text { Exposed to SSRIs } \\
\text { vs. unexposed to } \\
\text { SSRIs during } \\
\text { pregnancy with } \\
\text { depression or } \\
\text { unexposed to } \\
\text { SSRIs in early } \\
\text { pregnancy }\end{array}$ & $\begin{array}{l}\text { Cardiac } \\
\text { anomalies }\end{array}$ & $\begin{array}{l}\text { 68/7683 }(0.9 \%) \\
\text { vs. } 112 / 13,432 \\
(0.8 \%) ; 115 \mathrm{NR} \text { in } \\
\text { second study; } 123 \\
466 \text { cases/341 } \\
\text { controls vs. } 149 \\
\text { cases/125 } \\
\text { controls }^{122}\end{array}$ & $\begin{array}{l}\text { Pooled OR, } 1.07 \\
(95 \% \mathrm{Cl}, 0.97 \text { to } \\
1.20), \mathrm{I}^{2}: 0 \% \\
\text { AOR, } 1.06(95 \% \mathrm{Cl} \text {, } \\
0.93 \text { to } 1.22)^{123} \\
\text { AOR, } 1.04(95 \% \mathrm{Cl} \text {, } \\
0.76 \text { to } 1.41)^{115} \\
\text { AOR, } 1.14(95 \% \mathrm{Cl} \text {, } \\
0.87 \text { to } 1.51)^{122}\end{array}$ & $\begin{array}{l}2 \text { cohorts } 1 \text { case } \\
\text { control, } 115,122,123 \\
\mathrm{~N}>22,196(\mathrm{~N}=\mathrm{NR} \text { in } \\
\left.\text { one study }^{123}\right)\end{array}$ & $\begin{array}{l}\text { High study limitations } \\
\text { (2 high risk-of-bias } \\
\text { studies }^{115,123} \text { ) imprecise } \\
\text { (wide Cls), consistent }\end{array}$ & Insufficient \\
\hline
\end{tabular}




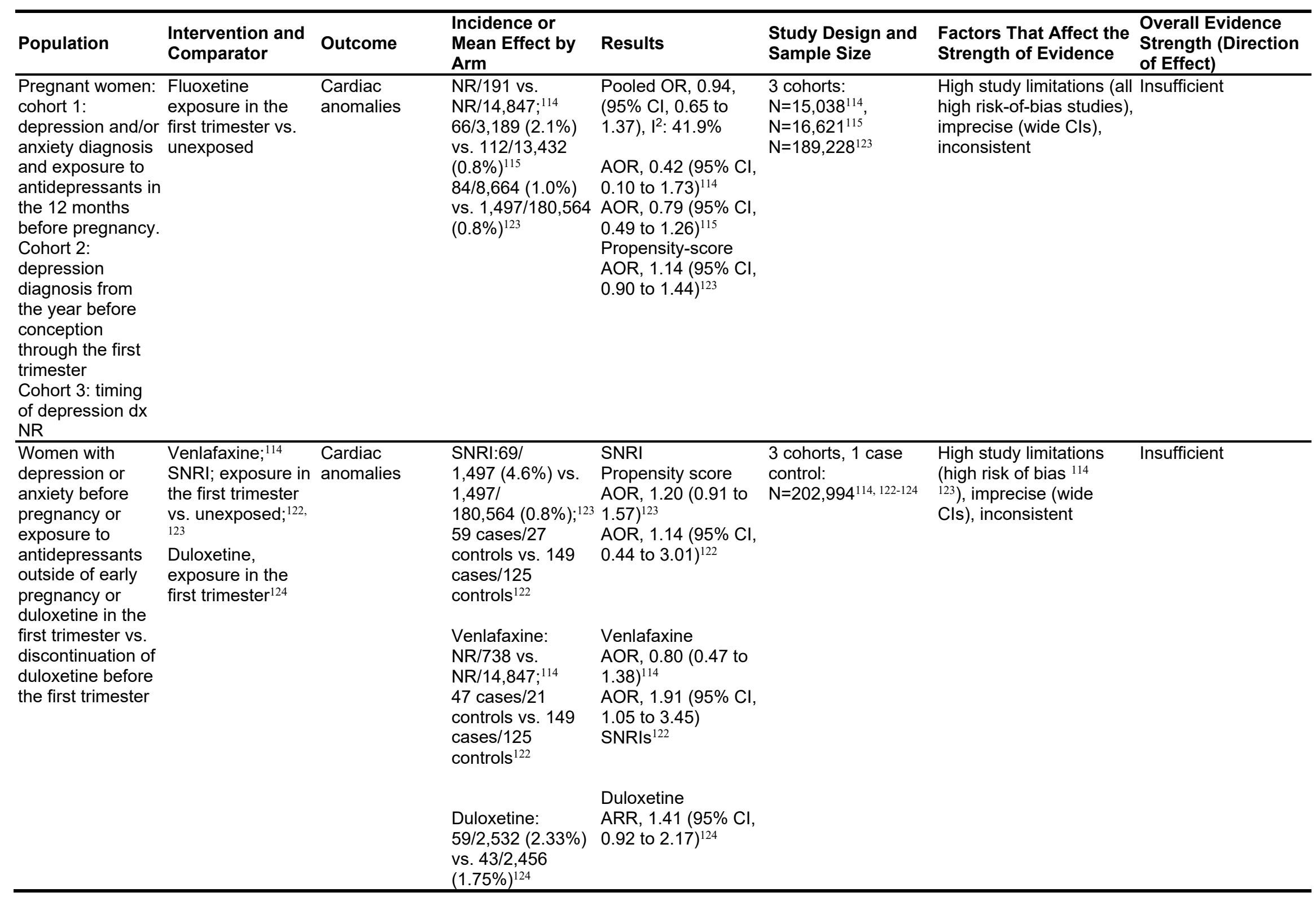




\begin{tabular}{|c|c|c|c|c|c|c|c|}
\hline Population & $\begin{array}{l}\text { Intervention and } \\
\text { Comparator }\end{array}$ & Outcome & $\begin{array}{l}\text { Incidence or } \\
\text { Mean Effect by } \\
\text { Arm }\end{array}$ & Results & $\begin{array}{l}\text { Study Design and } \\
\text { Sample Size }\end{array}$ & $\begin{array}{l}\text { Factors That Affect the } \\
\text { Strength of Evidence }\end{array}$ & $\begin{array}{l}\text { Overall Evidence } \\
\text { Strength } \\
\text { (Direction of } \\
\text { Effect) }\end{array}$ \\
\hline $\begin{array}{l}\text { History of } \\
\text { depression or } \\
\text { anxiety or TCA- } \\
\text { exposed women }\end{array}$ & $\begin{array}{l}\text { TCA in first } \\
\text { trimester vs. } \\
\text { unexposed } \\
\text { women with } \\
\text { history of } \\
\text { depression } \\
\end{array}$ & $\begin{array}{l}\text { Cardiac } \\
\text { anomalies }\end{array}$ & $\begin{array}{l}20 / 2,428(0.82 \%) \\
\text { vs. } 112 / 13,432 \\
(0.83 \%) ; 115 \text { NR in } \\
\text { other studies }^{114,123}\end{array}$ & $\begin{array}{l}\text { Pooled AOR, } 0.86 \\
(95 \% \text { Cl, } 0.65 \text { to } \\
1.13), 1^{2}: 0 \%{ }^{114,115,123}\end{array}$ & $\begin{array}{l}3 \text { cohorts, } \mathrm{n}>15,860 \\
(\mathrm{~N}=\mathrm{NR} \text { in two } \\
\text { studies) })^{114,115,123}\end{array}$ & $\begin{array}{l}\text { High study limitations (all } \\
\text { risk-of-bias studies), }{ }^{114,115} \text {, } \\
123 \text { imprecise (wide Cls), } \\
\text { consistent }\end{array}$ & Insufficient \\
\hline $\begin{array}{l}\text { Depressed or } \\
\text { bupropion- } \\
\text { exposed women }\end{array}$ & $\begin{array}{l}\text { Bupropion } \\
\text { exposure in } \\
\text { pregnancy vs. } \\
\text { unexposed } \\
\text { women with } \\
\text { depression or } \\
\text { unexposed in } \\
\text { early pregnancy }\end{array}$ & $\begin{array}{l}\text { Cardiac } \\
\text { anomalies }\end{array}$ & $\begin{array}{l}\text { NR; } 123 \\
57 \text { cases/45 } \\
\text { controls vs. } 149 \\
\text { cases } / 125 \\
\text { controls }^{122}\end{array}$ & $\begin{array}{l}\text { AOR, } 0.92(95 \% \mathrm{Cl}, \\
0.69 \text { to } 1.22) ; 123 \\
\text { AOR, } 1.06(0.66 \text { to } \\
1.71)^{122}\end{array}$ & $\begin{array}{l}1 \text { cohort, } 1 \text { case- } \\
\text { control, } \mathrm{n} \text { NR in } \\
\text { cohort }^{123} \mathrm{~N} \text { in case- } \\
\text { control }=376^{122}\end{array}$ & $\begin{array}{l}\text { Serious study limitations } \\
\text { (high risk of bias }^{123} \text { ) } \\
\text { imprecise (wide Cls } \\
\text { spanning the null), } \\
\text { consistency unknown }\end{array}$ & Insufficient \\
\hline $\begin{array}{l}\text { Exposed to SSRIs } \\
\text { during pregnancy } \\
\text { or unexposed with } \\
\text { a psychiatric } \\
\text { diagnosis }\end{array}$ & $\begin{array}{l}\text { Exposed to SSRIs } \\
\text { during pregnancy } \\
\text { vs. unexposed } \\
\text { with a psychiatric } \\
\text { diagnosis }\end{array}$ & $\mathrm{NICU}$ & $\begin{array}{l}2,405 / \\
15,729(15.3 \%) \text { vs. } \\
1,160 / 9,652 \\
(12.0 \%)^{: 101} 7 / 33 \\
(21.21 \%) \text { vs. } 5 / 19 \\
(26.32 \%)^{112}\end{array}$ & $\begin{array}{l}\text { Adjusted OR, } 1.24 \\
(95 \% \mathrm{Cl}, 1.14 \text { to } \\
1.35) ; 101 \mathrm{NR}, \\
\mathrm{p}=0.816^{112}\end{array}$ & $\begin{array}{l}2 \text { cohorts, } \\
n=25,433^{101,112}\end{array}$ & $\begin{array}{l}\text { High study limitations (high } \\
\text { risk of bias }{ }^{101,112} \text { ) precise, } \\
\text { inconsistent }\end{array}$ & Insufficient \\
\hline $\begin{array}{l}\text { Exposed to SSRIs } \\
\text { during pregnancy } \\
\text { or unexposed } \\
\text { depressed or with } \\
\text { a psychiatric } \\
\text { illness }\end{array}$ & $\begin{array}{l}\text { Exposed to SSRIs } \\
\text { during pregnancy } \\
\text { vs. unexposed } \\
\text { depressed during } \\
\text { pregnancy or with } \\
\text { a psychiatric } \\
\text { illness }\end{array}$ & 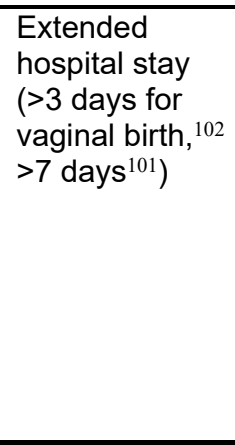 & $\begin{array}{l}1315 / 15,729(8.4 \%) \\
\text { vs. } 821 / 9,652 \\
(8.5 \%) ; 101 \\
(9.0 \%) \text { vs. } 75 / 1,566 \\
(4.8 \%) ; 11 \\
\text { NR in one study }{ }^{102} \\
\end{array}$ & $\begin{array}{l}\text { Inconsistent results } \\
\text { spanning benefits, } \\
\text { harms, and no effect } \\
\text { AOR, } 0.89(95 \% \mathrm{Cl} \text {, } \\
0.8 \text { to } 0.99) ; 101 \mathrm{AOR} \text {, } \\
1.93(95 \% \mathrm{Cl}, 1.11 \\
\text { to } 3.36)^{111} \\
\text { Difference in } \\
\text { incidence: } 0.035 \\
(95 \% \mathrm{Cl},-0.005 \text { to } \\
0.072), \mathrm{p} \text {-value } \\
0.08^{102}\end{array}$ & $\begin{array}{l}3 \text { cohort studies, } \\
\mathrm{n}>27,168 \mathrm{~N}=\mathrm{NR} \text { in } \\
\text { one study }{ }^{101,102,111}\end{array}$ & $\begin{array}{l}\text { High study limitations ( } 2 \text { of } \\
3 \text { studies are high risk of } \\
\text { bias }^{101,111} \text { ) imprecise, } \\
\text { inconsistent }\end{array}$ & Insufficient \\
\hline
\end{tabular}




\begin{tabular}{|c|c|c|c|c|c|c|c|}
\hline Population & $\begin{array}{l}\text { Intervention and } \\
\text { Comparator }\end{array}$ & Outcome & $\begin{array}{l}\text { Incidence or } \\
\text { Mean Effect by } \\
\text { Arm }\end{array}$ & Results & $\begin{array}{l}\text { Study Design and } \\
\text { Sample Size }\end{array}$ & $\begin{array}{l}\text { Factors That Affect the } \\
\text { Strength of Evidence }\end{array}$ & $\begin{array}{l}\text { Overall Evidence } \\
\text { Strength } \\
\text { (Direction of } \\
\text { Effect) }\end{array}$ \\
\hline $\begin{array}{l}\text { Depressed or } \\
\text { anxious or } \\
\text { exposed to SSRIs } \\
\text { in pregnancy }\end{array}$ & $\begin{array}{l}\text { Exposed to SSRIs } \\
\text { vs. unexposed } \\
\text { during pregnancy } \\
\text { with depression or } \\
\text { anxiety }\end{array}$ & $\begin{array}{l}\text { Infant and child } \\
\text { behavior and } \\
\text { development }\end{array}$ & $\begin{array}{l}\text { Varies by } \\
\text { measure } \\
\text { (measures } \\
\text { include CBCL } \\
\text { [including } \\
\text { subscales], } \\
\text { Behaviour Rating } \\
\text { Inventory of } \\
\text { Executive } \\
\text { Function- } \\
\text { Preschool version } \\
\text { (BRIEF-P), } \\
\text { Snijders-Oomen } \\
\text { Niet-verbale } \\
\text { intelligentie Test- } \\
\text { Revisie (SON-R 2 } \\
\text { 1/2-7), NEPSY-II, } \\
\text { NICU network } \\
\text { neurobehavioral } \\
\text { (NNNS [including } \\
\text { subscales])- } \\
\text { Attention scores, } \\
\text { MDI, PDI, BRS } \\
\end{array}$ & $\begin{array}{l}\text { Results vary by } \\
\text { specific outcome, } \\
\text { but the majority of } \\
\text { outcomes are not } \\
\text { statistically } \\
\text { significant; } \\
\text { exceptions include } 1 \\
\text { subscale measure } \\
\text { for CBCL and } \\
\text { NEPSY-II, and } 3 \text { of } \\
13 \text { NNNS subscale } \\
\text { measures; studies } \\
\text { with significant } \\
\text { findings did not } \\
\text { adjust for multiple } \\
\text { comparisons }\end{array}$ & $\begin{array}{l}4 \text { cohorts, } \\
\mathrm{N}=4,410^{125-128}\end{array}$ & $\begin{array}{l}\text { High study limitations ( } 3 \\
\text { of } 4 \text { are high risk of } \\
\text { bias, }{ }^{125-127} \text { imprecise, } \\
\text { consistency unknown } \\
\text { (single measures of } \\
\text { outcomes not repeated } \\
\text { in multiple studies) }\end{array}$ & Insufficient \\
\hline $\begin{array}{l}\text { Pregnant women: } \\
\text { Any lifetime } \\
\text { depression or } \\
\text { anxiety diagnosis }\end{array}$ & $\begin{array}{l}\text { Fluoxetine during } \\
\text { pregnancy vs. } \\
\text { unexposed } \\
\text { women }\end{array}$ & $\begin{array}{l}\text { Autism } \\
\text { spectrum } \\
\text { disorder }\end{array}$ & $\begin{array}{l}8 / 327(2.1 \%) \text { vs. } \\
282 / 14,805 \\
(1.9 \%) ; 129 \\
16 / 453(3.5 \%) \text { vs. } \\
353 / 12,325 \\
(2.9 \%)^{105}\end{array}$ & $\begin{array}{l}\text { By 7- or 8-year } \\
\text { followup ARR, } 1.08 \\
(095 \% \mathrm{Cl}, .53 \text { to } \\
2.21) ; 129 \\
\text { 4-year or more } \\
\text { followup: AOR, } 1.42 \\
(95 \% \mathrm{Cl}, 0.84 \text { to } \\
2.39)^{105}\end{array}$ & $\begin{array}{l}2 \text { cohorts: } \\
\mathrm{N}=15,132 ;{ }^{129} \\
\mathrm{~N}=12,778^{105} \\
\text { (potential overlap of } \\
\text { participants in the } \\
\text { publications) }\end{array}$ & $\begin{array}{l}\text { Moderate study } \\
\text { limitations, imprecise } \\
\text { (wide Cls); consistent }\end{array}$ & Insufficient \\
\hline
\end{tabular}




\begin{tabular}{|c|c|c|c|c|c|c|c|}
\hline Population & $\begin{array}{l}\text { Intervention and } \\
\text { Comparator }\end{array}$ & Outcome & $\begin{array}{l}\text { Incidence or } \\
\text { Mean Effect by } \\
\text { Arm }\end{array}$ & Results & $\begin{array}{l}\text { Study Design and } \\
\text { Sample Size }\end{array}$ & $\begin{array}{l}\text { Factors That Affect the } \\
\text { Strength of Evidence }\end{array}$ & $\begin{array}{l}\text { Overall Evidence } \\
\text { Strength } \\
\text { (Direction of } \\
\text { Effect) }\end{array}$ \\
\hline $\begin{array}{l}\text { Women with } \\
\text { current or past } \\
\text { mental health } \\
\text { disorder }\end{array}$ & $\begin{array}{l}\text { Paroxetine vs. } \\
\text { unexposed to } \\
\text { antidepressants } \\
\text { during pregnancy }\end{array}$ & $\begin{array}{l}\text { Autism } \\
\text { spectrum } \\
\text { disorder }\end{array}$ & $\begin{array}{l}5 / 264(1.9 \%) \text { vs. } \\
353 / 12,325 \\
(2.9 \%)^{105} \\
3 / 108(2.8 \%) \text { vs. } \\
282 / 14,805 \\
(1.9 \%)^{129}\end{array}$ & $\begin{array}{l}\text { ARR, } 0.61(95 \% \mathrm{Cl} \text {, } \\
0.25 \text { to } 1.49)^{105} \\
\text { ARR, } 1.21(95 \% \mathrm{Cl} \text {, } \\
0.38 \text { to } 3.8)^{129} \\
\text { Results adjusting for } \\
\text { the number of } \\
\text { mental health } \\
\text { disorders show } \\
\text { attenuating risks of } \\
\text { autism spectrum } \\
\text { disorder } \\
\geq 1 \text { mental health } \\
\text { disorder: ARR, } 1.36 \\
\text { (95\% Cl, } 0.51 \text { to } \\
3.64)^{129} \\
\geq 2 \text { mental health } \\
\text { disorders: ARR, } \\
1.02(95 \% \mathrm{Cl}, 0.38 \\
\text { to } 2.78)^{129} \\
\geq 3 \text { mental health } \\
\text { disorders: ARR, } \\
0.74(95 \% \text { Cl, } 0.27 \\
\text { to } 2.04)^{129}\end{array}$ & $\begin{array}{l}2 \text { cohorts, } \\
\text { N>35,218 } 105,129 \\
\text { (potential overlap of } \\
\text { participants in the } \\
\text { publications) }\end{array}$ & $\begin{array}{l}\text { Moderate study } \\
\text { limitations, imprecise } \\
\text { (wide Cls), consistency } \\
\text { unknown (potential } \\
\text { overlap of participants) }\end{array}$ & Insufficient \\
\hline
\end{tabular}




\begin{tabular}{|c|c|c|c|c|c|c|c|}
\hline Population & $\begin{array}{l}\text { Intervention and } \\
\text { Comparator }\end{array}$ & Outcome & $\begin{array}{l}\text { Incidence or } \\
\text { Mean Effect by } \\
\text { Arm }\end{array}$ & Results & $\begin{array}{l}\text { Study Design and } \\
\text { Sample Size }\end{array}$ & $\begin{array}{l}\text { Factors That Affect the } \\
\text { Strength of Evidence }\end{array}$ & $\begin{array}{l}\text { Overall Evidence } \\
\text { Strength (Direction } \\
\text { of Effect) }\end{array}$ \\
\hline $\begin{array}{l}\text { Women with } \\
\text { current or past } \\
\text { mental health } \\
\text { disorder }\end{array}$ & $\begin{array}{l}\text { Sertraline vs. } \\
\text { unexposed to } \\
\text { antidepressants } \\
\text { during pregnancy }\end{array}$ & $\begin{array}{l}\text { Autism } \\
\text { spectrum } \\
\text { disorder }\end{array}$ & $\begin{array}{l}31 / 912(3.4 \%) \text { vs. } \\
353 / 12,325 \\
(2.9 \%)^{105} \\
\\
16 / 672(2.4 \%) \text { vs. } \\
282 / 14,805 \\
(1.9 \%)^{129}\end{array}$ & $\begin{array}{l}\text { AOR, } 1.45(95 \% \mathrm{Cl}, \\
0.98 \text { to } 2.16)^{105} \\
\text { ARR, } 1.17,(95 \% \mathrm{Cl} \text {, } \\
0.99 \text { to } 2.32)^{129} \\
\text { Results adjusting for } \\
\text { the number of } \\
\text { mental health } \\
\text { disorders show } \\
\text { attenuating risks of } \\
\text { autism spectrum } \\
\text { disorder } \\
\geq 1 \text { mental health } \\
\text { disorder: ARR, } 1.32 \\
\text { (95\% Cl, } 0.86 \text { to } \\
2.24)^{129} \\
\geq 2 \text { mental health } \\
\text { disorders: ARR, } \\
0.99(95 \% \text { Cl, } 0.63 \\
\text { to } 1.55)^{129} \\
\geq 3 \text { mental health } \\
\text { disorders: ARR, } \\
0.71(95 \% \text { Cl, } 0.43 \\
\text { to } 1.17)^{129}\end{array}$ & $\begin{array}{l}2 \text { cohorts, } \\
\mathrm{N}>15,47728,714^{105} \\
129 \text { (potential overlap } \\
\text { of participants in the } \\
\text { publications) }\end{array}$ & $\begin{array}{l}\text { Moderate study } \\
\text { limitations, imprecise } \\
\text { (wide Cls), some } \\
\text { consistency }\end{array}$ & Insufficient \\
\hline
\end{tabular}




\begin{tabular}{|c|c|c|c|c|c|c|c|}
\hline Population & $\begin{array}{l}\text { Intervention and } \\
\text { Comparator }\end{array}$ & Outcome & $\begin{array}{l}\text { Incidence or } \\
\text { Mean Effect by } \\
\text { Arm }\end{array}$ & Results & $\begin{array}{l}\text { Study Design and } \\
\text { Sample Size }\end{array}$ & $\begin{array}{l}\text { Factors That Affect the } \\
\text { Strength of Evidence }\end{array}$ & $\begin{array}{l}\text { Overall Evidence } \\
\text { Strength } \\
\text { (Direction of } \\
\text { Effect) }\end{array}$ \\
\hline $\begin{array}{l}\text { Pregnant women: } \\
\text { Any lifetime } \\
\text { depression or } \\
\text { anxiety diagnosis }\end{array}$ & $\begin{array}{l}\text { Venlafaxine during } \\
\text { pregnancy vs. } \\
\text { unexposed women }\end{array}$ & $\begin{array}{l}\text { Autism } \\
\text { spectrum } \\
\text { disorder }\end{array}$ & $\begin{array}{l}4 / 195(2.1 \%) \text { vs. } \\
282 / 14,805 \\
(1.9 \%) ; 129 \\
11 / 213(5.1 \%) \text { vs. } \\
353 / 12,325 \\
(2.9 \%)^{105}\end{array}$ & $\begin{array}{l}\text { By 7- or 8-year } \\
\text { followup ARR, } 0.74 \\
\text { (0.32 to } 1.72) ; 129 \\
\text { 4-year or more } \\
\text { followup: AOR, } 1.81 \\
(0.89 \text { to } 3.71)^{105} \\
\geq 1 \text { mental health } \\
\text { disorder: ARR, } 1.36 \\
(95 \% \mathrm{Cl}, 0.61 \text { to } \\
3.04)^{129} \\
\geq 2 \text { mental health } \\
\text { disorders: ARR, } \\
1.01(95 \% \mathrm{Cl}, 0.44 \\
\text { to } 2.29)^{129} \\
\geq 3 \text { mental health } \\
\text { disorders: ARR, } \\
0.74(95 \% \mathrm{Cl}, 0.32 \\
\text { to } 1.72)^{129}\end{array}$ & $\begin{array}{l}2 \text { cohorts: } \mathrm{N}=27,538 \\
105,129\end{array}$ & $\begin{array}{l}\text { Moderate study } \\
\text { limitations, imprecise } \\
\text { (wide Cls); inconsistent }\end{array}$ & Insufficient \\
\hline
\end{tabular}




\begin{tabular}{|c|c|c|c|c|c|c|c|}
\hline Population & $\begin{array}{l}\text { Intervention and } \\
\text { Comparator }\end{array}$ & Outcome & $\begin{array}{l}\text { Incidence or } \\
\text { Mean Effect by } \\
\text { Arm }\end{array}$ & Results & $\begin{array}{l}\text { Study Design and } \\
\text { Sample Size }\end{array}$ & $\begin{array}{l}\text { Factors That Affect the } \\
\text { Strength of Evidence }\end{array}$ & $\begin{array}{l}\text { Overall Evidence } \\
\text { Strength } \\
\text { (Direction of } \\
\text { Effect) }\end{array}$ \\
\hline $\begin{array}{l}\text { Mental health } \\
\text { disorder or } \\
\text { clomipramine- } \\
\text { exposed women }\end{array}$ & $\begin{array}{l}\text { Clomipramine vs. } \\
\text { unexposed } \\
\text { women with } \\
\text { history of } \\
\text { psychiatric } \\
\text { disorder }\end{array}$ & $\begin{array}{l}\text { Autism } \\
\text { spectrum } \\
\text { disorder }\end{array}$ & $\begin{array}{l}16 / 235(6.8 \%) \text { vs. } \\
353 / 12,325 \\
(2.9 \%) ;{ }^{105} \text { NR in } \\
\text { second study }{ }^{129}\end{array}$ & $\begin{array}{l}\text { AOR, } 1.76(95 \% \mathrm{Cl} \text {, } \\
1.01 \text { to } 3.05)^{105} ; \\
\text { ARR, } 3.36,95 \% \mathrm{Cl} \text {, } \\
1.39 \text { to } 8.13 \text {, results } \\
\text { not statistically } \\
\text { significant when } \\
\text { corrected for } \\
\text { multiple testing } \\
\\
\geq 1 \text { mental health } \\
\text { disorder: ARR, } 3.36 \\
\text { (95\% Cl, } 1.39 \text { to } \\
8.13)^{129} \\
\geq 2 \text { mental health } \\
\text { disorders: ARR, } \\
2.53(95 \% \text { Cl, } 1.02 \\
\text { to } 6.22)^{129} \\
\geq 3 \text { mental health } \\
\text { disorders: ARR, } \\
1.88 \text { (95\% Cl, } 0.7 \text { to } \\
4.73)^{129}\end{array}$ & $\begin{array}{l}2 \text { cohorts, } \\
\mathrm{n}=36,936^{105,129} \\
\text { (potential overlap of } \\
\text { participants in the } \\
\text { publications) }\end{array}$ & $\begin{array}{l}\text { Moderate study } \\
\text { limitations, imprecise, } \\
\text { consistent }\end{array}$ & Insufficient \\
\hline
\end{tabular}

AOR = adjusted odds ratio; ARR = adjusted risk ratio; BRIEF-P = Behaviour Rating Inventory of Executive Function—Preschool version; BRS = Behavioral Rating Scale; CBCL $=$ childhood behavior checklist; $\mathrm{CI}=$ confidence interval; $\mathrm{dx}=$ diagnosis; $\mathrm{MDI}=$ Mental Development Index; $\mathrm{N}=$ number; NEPSY-II $=$ Developmental NEuroPSYchological Assessment-II; NICU = neonatal intensive care unit; NNNS = NICU Network Neurobehavioral Scale subscale measures; NR = not reported; OR = odds ratio; PDI = Provisional

Diagnostic Instrument; SON-R = Snijders-Oomen Niet-verbale intelligentie Test-Revisie; SNRI = serotonin-norepinephrine reuptake inhibitor; SSRI = selective serotonin reuptake inhibitor; TCA = tricyclic antidepressant; vs. = versus. 


\section{Small for Gestational Age and SSRIs}

Six studies reported on the association between small for gestational age and SSRI exposure. ${ }^{100-102,110,111,113}$ The results could not be pooled because of differences in reported outcomes. Five of six studies reported results with wide CIs suggestive of both benefits and harms. The only study that controlled for severity of psychiatric illness reported no statistically significant difference between the groups. Wide CIs indicated the potential for both appreciable benefit and appreciable harm. This imprecision, coupled with high study limitations for most studies, led to a rating of insufficient.

\section{Low Birthweight and SSRIs}

Although two studies ${ }^{109,111}$ provided consistent evidence of an increased association between low birth weight and SSRI exposure, both had limitations. Neither controlled for severity of maternal illness. A third study reported no statistically significant differences. ${ }^{112}$

\section{Congenital Anomalies and Pharmacologic Interventions}

Prescription labeling from FDA suggests potential fetal concerns for several psychotropic drugs. These include paroxetine, temazepam, triazolam, alprazolam, diazepam, valproate, carbamazepine, and topiramate for congenital anomalies and clonazepam for increased risk of hypothermia, hypotonia, respiratory depression, difficulty feeding, and withdrawal. We did not find eligible evidence on congenital anomalies for triazolam, alprazolam, valproate, carbamazepine, and topiramate, although evidence is available from studies of other populations ineligible for this review. This broader evidence formed the basis of the FDA warning language. For paroxetine, the concern was based on increased reports of cardiac malformation at the time of the labeling. For temazepam and diazepam, the concerns were based on increased reports of congenital anomalies.

Regarding major congenital anomalies, eight studies evaluated associations between various exposures (diazepam, temazepam, zopiclone, SSRIs as a class, citalopram, escitalopram, fluoxetine, fluvoxamine, paroxetine, sertraline, SNRIs as a class, TCAs as a class, quetiapine, risperidone, second-generation antipsychotics, and first-generation antipsychotics) and major congenital anomalies (any type). ${ }^{14-118,121,130,131}$ Figure 2 displays results for all reported exposures in a forest plot (the results are not pooled because of potential overlap of participants across studies for some interventions). The results almost always span the null with CIs suggesting benefits and harms (with the exception of one study each on citalopram ${ }^{114}$ and second-generation antipsychotics ${ }^{120}$ ), with adjusted odds ratios on both sides on the null. For both citalopram and second-generation antipsychotics, the evidence base comprised two studies with conflicting results. Inconsistency was one factor in downgrading the evidence base overall; another was the potential study limitations of the evidence base.

Regarding findings specific to temazepam and diazepam, our review identified a single eligible study with serious limitations arising from residual confounding and potential selection bias. The study reported AORs of 0.99 (95\% CI, 0.61 to 1.61) for diazepam and 1.04 (95\% CI, 0.47 to 2.32 ) for temazepam. ${ }^{131}$ FDA found that "an increased risk of congenital anomalies associated with the use of diazepam and chlordiazepoxide during the first trimester of pregnancy has been suggested in several studies,"132 but the studies are not cited.

Regarding cardiac anomalies, eight studies evaluated associations with cardiac anomalies ${ }^{114}$, $115,118,122,123,133-135$ and used different criteria to identify affected infants. Studies using the Medicaid Analytic eXtract cohort ${ }^{123,133}$ required either (1) ICD-9 diagnosis for a specific 
malformation on more than one date, (2) a diagnosis on one date and a relevant procedure code, or (3) a diagnosis on only one date with an infant death before 90 days. For infants born preterm, these studies excluded isolated cardiac anomalies associated with prematurity $(745.5$, atrial septal defect, 747.0, patent ductus arteriosus, 746.02, pulmonary-valve stenosis, and 747.3 anomalies of the pulmonary artery). A single U.K. study ${ }^{115}$ used European Surveillance of Congenital Anomalies (EUROCAT) criteria, which similarly exclude Q21.11, patent, or persistent foramen ovale, if gestational age at birth is $<37$ weeks. In contrast, studies from the Quebec Pregnancy Cohort ${ }^{114}, 118,134$ used diagnosis codes from hospitalizations in Quebec to identify cardiac anomalies using ICD-9 codes 745-746 or ICD-10 codes Q20-Q22, with no exclusion for atrial septal defect (ICD-9: 745.5, ICD-10: Q21.1) for preterm infants. The National Birth Defects Prevention Study ${ }^{122}$ relied on a classification of heart defects developed for the study. ${ }^{136}$ Lastly, a 1983 study ${ }^{135}$ used information from the Swedish Registry of Congenital Malformations and from special child cardiology clinics to identify six infants with heart defects; no further details were provided. Given that atrial septal defects are part of preterm infant physiology, the clinical validity of studies that do not exclude these cases is unclear.

Regarding findings specific to paroxetine (previously labeled Category X drug for cardiac defects), although we found no evidence supporting an association between exposure to psychotropic agents and cardiac defects, one study reported an association between paroxetine exposure and atrial or ventricular septal defects. ${ }^{114}$ A second study, focusing on ventricular septal defects, found no association with paroxetine exposure, raising the possibility that differences in findings could be attributable to how outcomes were defined. ${ }^{123}$ The clinical validity of an association with atrial septal defects is unclear. Beyond differences in outcome measurement, differences between studies in design and controls for confounding could potentially explain variations in results. The study that adjusted for numerous potential confounders through propensity score adjustment found no differences in ventricular septal defects. ${ }^{123}$

\section{NICU Admission and SSRIs}

Regarding fetal harms, evidence from two studies provided inconsistent evidence on SSRI exposure during pregnancy and NICU admissions. ${ }^{101,112}$ One study-adjusting for sex, birth period, maternal age at delivery, place of residence, marital status, parity, smoking, socioeconomic status, purchase of anxiolytics, sedative-hypnotics, or antiepileptic drugs, prepregnancy diabetes, and other chronic diseases, but not severity of mental health disorderreported significantly increased risk. ${ }^{101}$ The second study accounted for severity of the disorder and for smoking and found no difference. ${ }^{112}$ In addition to inconsistent adjustment for severity, as noted previously, NICU admission may be confounded by provider knowledge of antenatal exposure to psychotropic medications and hospital protocols. 
Figure 2. Exposure to psychotropic medications and major congenital anomalies

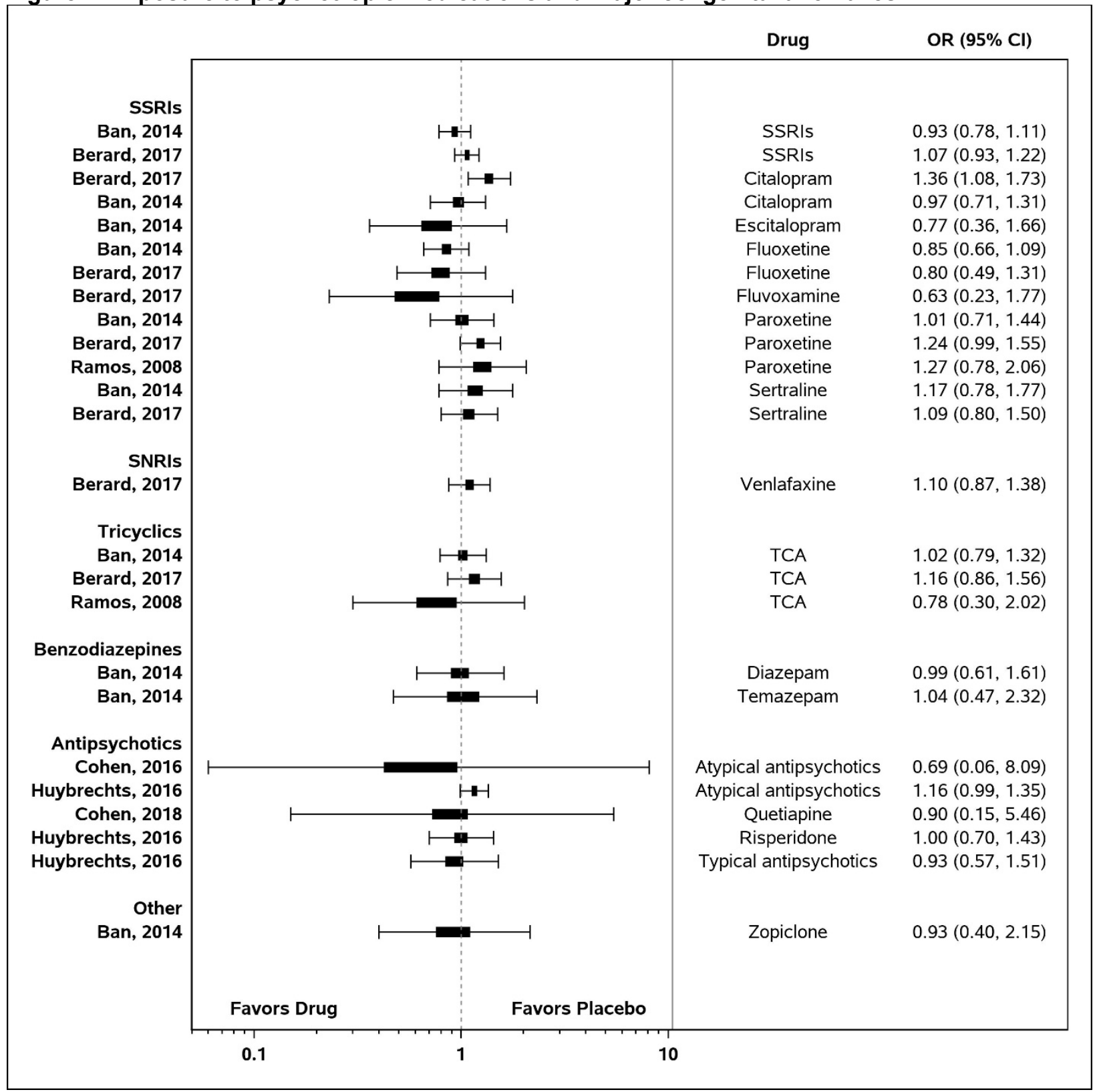

$\mathrm{CI}=$ confidence interval; $\mathrm{OR}=$ odds ratio; $\mathrm{SNRI}=$ serotonin-norepinephrine reuptake inhibitor; SSRI = selective serotonin reuptake inhibitor; $\mathrm{TCA}=$ tricyclic antidepressant.

\section{Extended Hospital Stay and SSRIs}

Three studies reported inconsistent results (one suggested benefit, ${ }^{101}$ the second no statistically significant difference, ${ }^{102}$ and the third suggested harm ${ }^{111}$ ) from exposure to SSRIs. The study reporting no statistically significant differences controlled for maternal illness severity; the other two did not. The inconsistencies, coupled with high study limitations, led to a strength-of-evidence grade of insufficient. 


\section{Infant and Child Behavior and Development and SSRIs}

Four studies ${ }^{125-128}$ reported on numerous scales and subscale measures of infant and child development and behavior associated with exposure to SSRIs. These results could not be pooled because of heterogeneity, but the majority of outcomes reported results that were not statistically significant.

\section{Autism Spectrum Disorder and Fluoxetine, Paroxetine, Sertraline, Venlafaxine, or Clomipramine}

Two studies, ${ }^{129} \mathrm{~N}=12,778^{105}$ with a potential overlap in participants, did not consistently find associations between exposure to fluoxetine, paroxetine, sertraline, venlafaxine, or clomipramine and autism spectrum disorder. When one study controlled for number of mental health disorders, the effect always attenuated, suggesting that severity of psychiatric illness was a strong confounder of the observed effect.

\section{No Evidence in Populations of Interest (Pregnant Women With Mental Health Disorders)}

\section{Overview}

- For outcomes prioritized for this review, we found no eligible studies that provided evidence about_infertility, abruption, glucose intolerance, reduced milk production or unwanted weaning, and withdrawal symptoms in the newborn.

- For interventions in our populations of interest, we found no eligible studies that provided evidence of fetal harms on several drugs with FDA pregnancy-related boxed warnings (valproate) or language suggesting fetal harm (alprazolam, valproate, carbamazepine, topiramate, clonazepam , triazolam, asenapine, brexpiprazole, chlordiazepoxide, clorazepate, fluphenazine, hydroxyzine, imipramine, and lorazepam). Evidence may be available from studies of other populations ineligible for this review (such as among pregnant or women without mental health disorders who were exposed to these drugs).

- We did not find eligible studies that provided evidence of the harms reported in warnings for amitriptyline and lithium, although we found insufficient evidence on other reported harms (autism spectrum disorder for amitriptyline; preeclampsia, placental abruption, preterm birth, and small-for-gestational-age for valproate, carbamazepine, topiramate; and preeclampsia, placental abruption, preterm birth, small-for-gestational-age, and child IQ for lithium).

\section{Detailed Synthesis}

We found no evidence of fetal harms for several drugs that had FDA pregnancy-related boxed warnings (valproate [for women without epilepsy]) or language suggesting fetal or neonatal harm in the past (alprazolam, valproate [for women with epilepsy], carbamazepine , topiramate, clonazepam, triazolam, amitriptyline, asenapine, brexpiprazole, chlordiazepoxide, clorazepate, fluphenazine, hydroxyzine, imipramine, lithium, and lorazepam).

Valproate has an FDA boxed warning related to pregnancy in women without epilepsy. This drug can cause serious birth defects, including neural tube defects, decreased IQ, and neurodevelopmental disorders. Triazolam is contraindicated in pregnant women because of the risk of congenital anomalies. Hydroxyzine does not have a boxed warning, but the prescription language notes that it is contraindicated for early pregnancy. Risks from other drugs (alprazolam, 
carbamazepine, clonazepam, diazepam, and topiramate) per FDA include congenital anomalies (alprazolam, carbamazepine), oral clefts (topiramate), small for gestational age (topiramate), and perinatal complications (clonazepam).

Per FDA prescribing information, other fetal risks include extrapyramidal and/or withdrawal symptoms following delivery (asenapine, brexpiprazole, and fluphenazine) and congenital anomalies (chlordiazepoxide, clorazepate, imipramine, and lorazepam).

The warning label for lithium notes that although early voluntary reporting suggested an increase in cardiovascular anomalies, subsequent studies suggested a small increased risk.

\section{KQ 4: Comparative Harms of Pharmacologic Treatments for Pregnant and Postpartum Women With Mental Health Disorders}

\section{Overview}

- Fifty-six studies (57 articles) reported on comparative adverse outcomes; 55 studies were observational and could not assert a causal relationship between exposure and resultant harms.

- As with the evidence on adverse outcomes of exposure versus no exposure, the studies reporting on comparative harms characterized the population by exposure to the drug (rather than by the presence of a disorder). The findings on adverse events are therefore not specific to a disorder.

- The strength of evidence on the adverse outcomes from these drugs was rated low primarily due to study limitations.

- We found evidence of two comparative adverse outcomes for lithium versus lamotrigine during pregnancy. Results from one study suggested that first trimester exposure to lithium may be more likely to be associated with overall congenital anomalies and cardiac anomalies than first trimester exposure to lamotrigine (low strength of evidence), a finding that can inform the decision to switch a medication in a successfully treated individual.

- We found evidence on one or more outcome for 108 comparisons; we found insufficient evidence on 107 of these comparisons, primarily due to study limitations arising from the lack of adjustment for confounding. Most comparisons had single-study bodies of evidence; consistency could not be ascertained.

- Interventions for which we found no eligible studies in our population of interest providing comparative evidence of adverse outcomes include anxiolytics, hypnotic sedatives, and brexanolone; evidence may be available from studies of other populations ineligible for this review.

- Maternal outcomes for which we found insufficient evidence include preeclampsia, spontaneous abortion, postpartum hemorrhage, gestational hypertensive disorders, and GDM.

- Child outcomes for which we found insufficient evidence include perinatal death; preterm birth; small for gestational age; large for gestational age; birthweight; low Apgar; withdrawal symptoms; respiratory distress; NICU time; delayed social, emotional, and cognitive development; autism spectrum disorder; and ADHD. 
- Outcomes for which we found no evidence include infertility, abruption, glucose intolerance, and reduced milk production/undesired weaning for women; we also found no evidence for persistent pulmonary hypertension of the newborn, poor infant attachment/bonding, anxiety in children, and depression in children.

\section{Detailed Results}

Table 13 provides an overview of the findings on harms of pharmacotherapy in the perinatal period. Appendix B includes detailed tables on all outcomes. Fifty-six studies (57 articles) reported on comparative harms. Although several studies drew from large databases, the vast majority of graded evidence was insufficient owing to failure to control for confounding. The remainder of this section describes in greater detail the evidence for comparative maternal harm; comparative fetal, neonatal, infant, or child harm; insufficient evidence of comparative maternal harm; insufficient evidence of fetal, neonatal, infant, or child harm; and no evidence.

\section{Evidence of Maternal Harm}

We did not find sufficient evidence to support a judgment of maternal harms from studies comparing pharmacotherapy in pregnancy or postpartum.

\section{Evidence of Fetal, Infant, or Child Harms}

\section{Overview}

- The evidence on fetal, infant, or child harms from treatments for mental health disorders comes from bodies of evidence with high limitations and precise results (i.e., sample size, event rate, and confidence intervals permit inference of benefit, harm, or absence of benefit or harm). Precise results came from bodies of evidence with high limitations but effects that did not cross the null. However, beyond statistical significance alone, other factors contributed to moving the grade from insufficient to low.

- Evidence from one cohort study suggested that exposure to lithium during pregnancy may be more likely to be associated with overall congenital anomalies and cardiac anomalies than exposure to lamotrigine, which can inform the decision to switch a medication in a successfully treated individual. 
Table 13. Summary of evidence from comparative effectiveness studies for harm of pharmacologic treatments for mental health disorders in pregnancy or postpartum

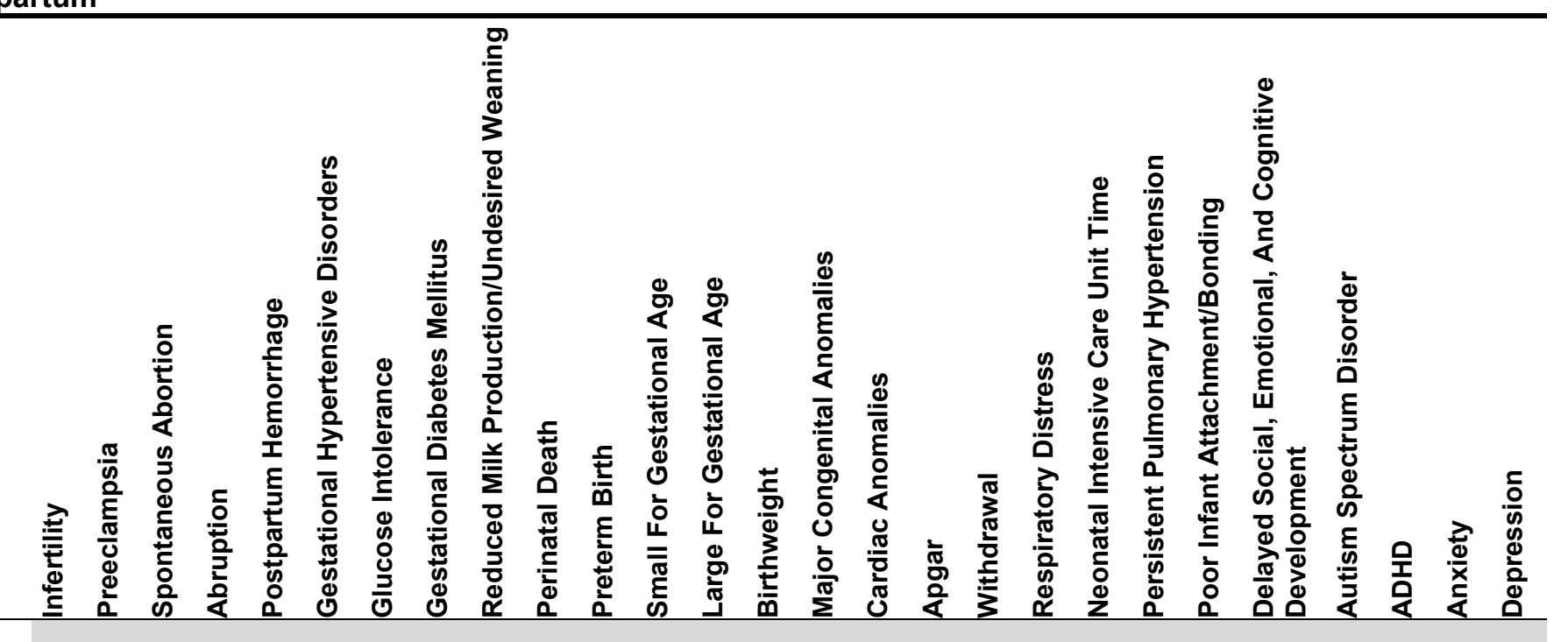

\begin{tabular}{|c|c|c|c|c|c|c|c|c|c|c|c|c|c|c|c|c|c|c|c|c|c|c|c|c|c|c|c|c|}
\hline Anxiolytics & Any other comparator & - & - & - & - & - & - & - & - & - & - & - & - & - & - & - & - & - & - & - & - & - & - & - & - & - & - & - \\
\hline $\begin{array}{l}\text { Sedative } \\
\text { hypnotics }\end{array}$ & Any other comparator & - & - & - & - & - & - & - & - & - & - & - & - & - & - & - & - & - & - & - & - & - & - & - & - & - & - & - \\
\hline SSRIs & $\begin{array}{l}\text { SSRIs plus } \\
\text { mirtazapine }\end{array}$ & - & - & - & - & - & - & - & - & - & - & I & - & - & I & - & - & - & - & - & I & - & - & - & - & - & - & - \\
\hline SSRIs & TCAs & - & I & I & - & - & - & - & - & - & - & I & I & - & 1 & I & - & I & - & - & I & - & - & - & 1 & I & - & - \\
\hline SSRIs & MAOIs & - & - & - & - & - & - & - & - & - & - & - & - & - & - & - & - & - & - & - & - & - & - & - & I & 1 & - & - \\
\hline Citalopram & Escitalopram & - & - & - & - & - & - & - & - & - & - & - & - & - & - & - & I & - & - & - & - & - & - & - & - & - & - & - \\
\hline Citalopram & Fluvoxamine & - & - & - & - & - & - & - & - & - & - & - & - & - & - & - & - & - & - & - & - & - & - & - & I & - & - & - \\
\hline Citalopram & Fluoxetine & - & - & - & - & - & - & - & - & - & - & - & - & - & - & - & I & - & - & - & - & - & - & - & I & - & - & - \\
\hline Citalopram & Paroxetine & - & - & - & - & - & - & - & - & - & - & - & - & - & - & - & I & - & - & - & - & - & - & - & I & - & - & - \\
\hline Citalopram & Sertraline & - & - & - & - & - & - & - & - & - & - & - & - & - & - & - & 1 & - & - & - & - & - & - & - & 1 & - & - & - \\
\hline
\end{tabular}




\begin{tabular}{|c|c|c|c|c|c|c|c|c|c|c|c|c|c|c|c|c|c|c|c|c|c|c|c|c|c|c|c|c|}
\hline Exposure & Comparator & 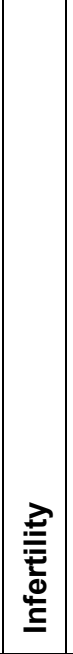 & 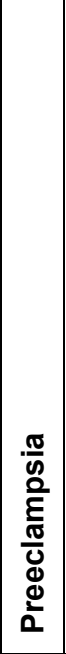 & 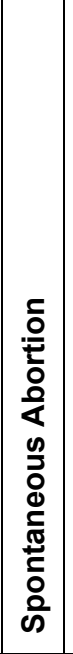 & 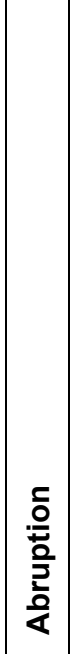 & 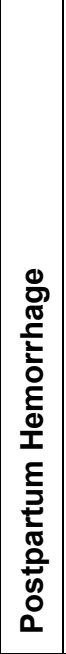 & 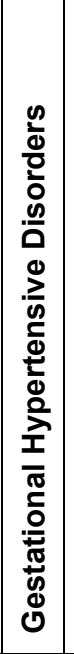 & 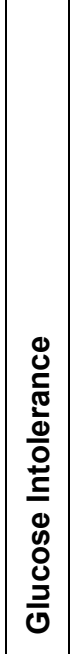 & 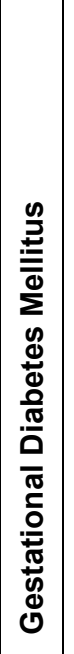 & 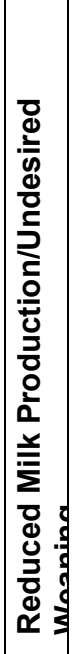 & 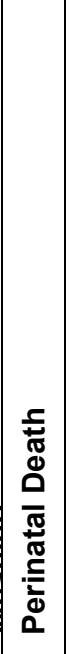 & 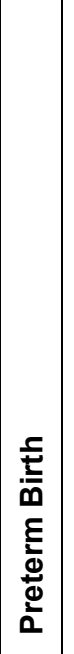 & 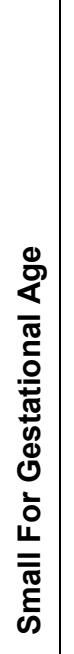 & 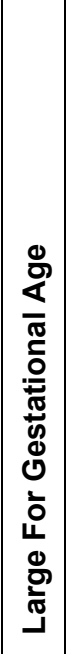 & 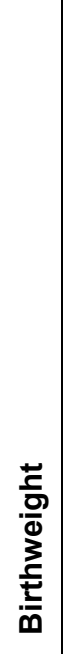 & 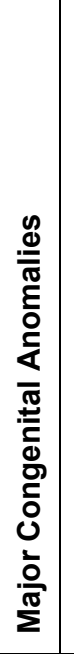 & 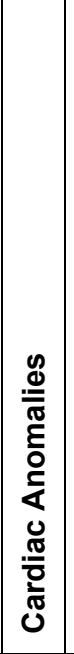 & $\begin{array}{l} \\
\frac{\grave{\sigma}}{\mathrm{g}} \\
\frac{0}{\mathbf{2}}\end{array}$ & 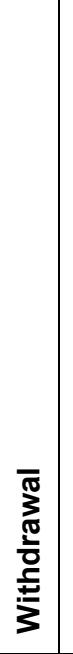 & 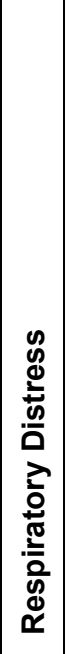 & 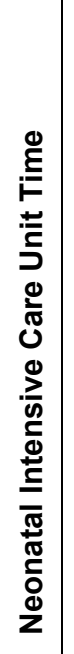 & 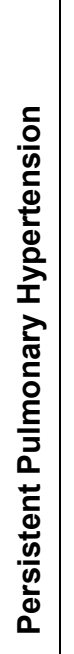 & 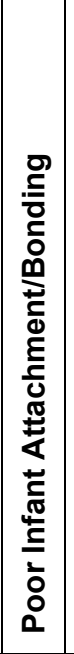 & 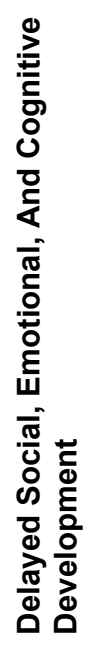 & 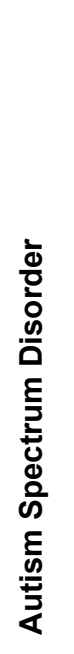 & $\begin{array}{l}\text { 오 } \\
\text { 虽 }\end{array}$ & 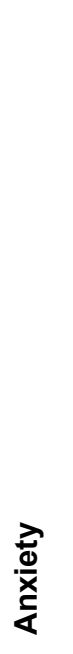 & 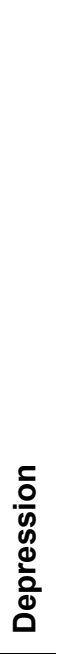 \\
\hline Citalopram & Venlafaxine & - & - & - & - & - & - & - & - & - & - & - & - & - & - & - & I & - & - & - & - & - & - & - & - & - & - & - \\
\hline Escitalopram & Bupropion & - & - & - & - & - & - & - & - & - & - & - & - & - & - & - & 1 & - & - & - & - & - & - & - & - & - & - & - \\
\hline Escitalopram & Duloxetine & - & - & - & - & - & - & - & - & - & - & - & - & - & - & - & I & - & - & - & - & - & - & - & - & - & - & - \\
\hline Escitalopram & Paroxetine & - & - & - & - & - & - & - & - & - & - & - & - & - & - & - & 1 & - & - & - & - & - & - & - & - & - & - & - \\
\hline Escitalopram & Sertraline & - & - & - & - & - & - & - & - & - & - & - & - & - & - & - & I & - & - & - & - & - & - & - & - & - & - & - \\
\hline Fluoxetine & Bupropion & - & - & - & - & - & - & - & - & - & - & - & - & - & - & - & 1 & - & - & - & - & - & - & - & - & - & - & - \\
\hline Fluoxetine & Duloxetine & - & - & - & - & - & - & - & - & - & - & - & - & - & - & - & 1 & - & - & - & - & - & - & - & - & - & - & - \\
\hline Fluoxetine & Escitalopram & - & - & - & - & - & - & - & - & - & - & - & - & - & - & - & I & - & - & - & - & - & - & - & - & - & - & - \\
\hline Fluoxetine & Sertraline & - & - & - & - & - & - & - & - & - & - & - & - & - & - & - & I & - & - & - & - & - & - & - & - & - & - & - \\
\hline Fluoxetine & Venlafaxine & - & - & - & - & - & - & - & - & - & - & - & - & - & - & - & I & - & - & - & - & - & - & - & - & - & - & - \\
\hline Fluoxetine & $\begin{array}{l}\text { Citalopram or } \\
\text { escitalopram }\end{array}$ & - & - & 1 & - & - & - & - & - & - & 1 & - & - & - & 1 & 1 & 1 & - & - & - & - & - & - & - & - & - & - & - \\
\hline Fluoxetine & $\begin{array}{l}\text { Escitalopram or } \\
\text { fluvoxamine }\end{array}$ & - & - & I & - & - & - & - & - & - & I & - & - & - & I & I & - & - & - & - & - & - & - & - & I & - & - & - \\
\hline
\end{tabular}




\begin{tabular}{|c|c|c|c|c|c|c|c|c|c|c|c|c|c|c|c|c|c|c|c|c|c|c|c|c|c|c|c|c|}
\hline Exposure & Comparator & 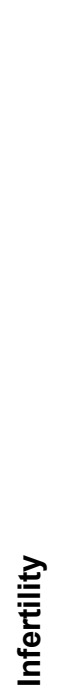 & 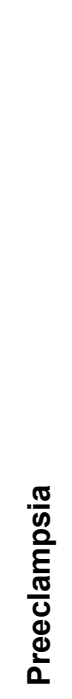 & 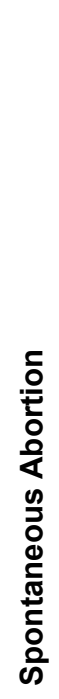 & $\begin{array}{l}\text { 을 } \\
\text { 을 } \\
\text { 눙 }\end{array}$ & 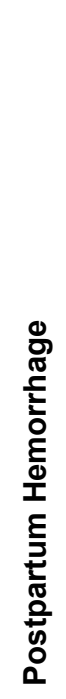 & 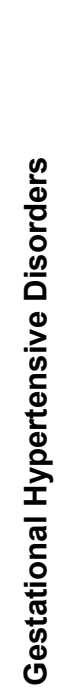 & 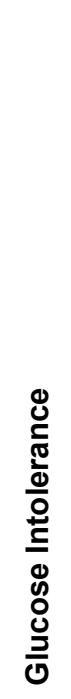 & 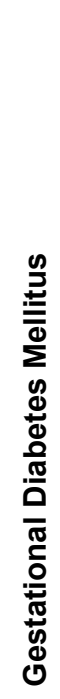 & 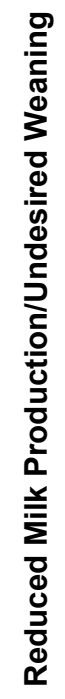 & 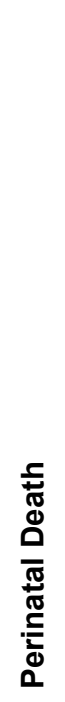 & 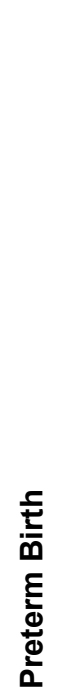 & 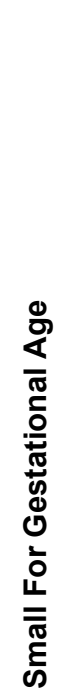 & 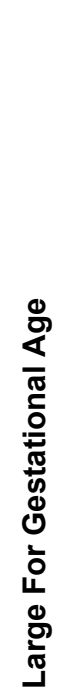 & & 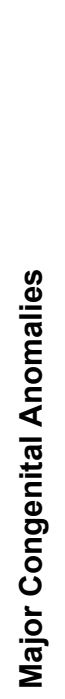 & 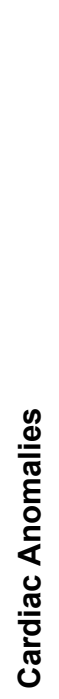 & $\frac{1}{\frac{\pi}{0}}$ & $\begin{array}{l}\bar{\pi} \\
\frac{3}{\pi} \\
\frac{1}{0} \\
\frac{5}{5} \\
3\end{array}$ & 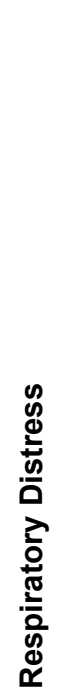 & 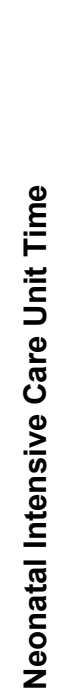 & 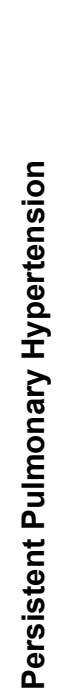 & 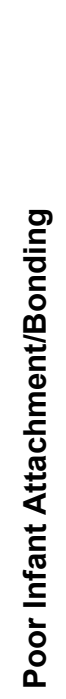 & 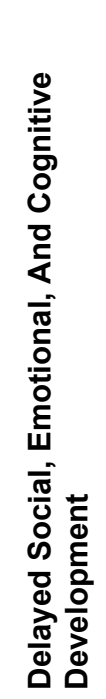 & 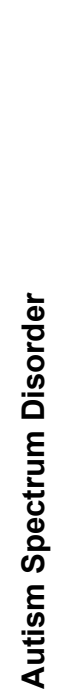 & $\begin{array}{l}\text { 오 } \\
\text { 足 }\end{array}$ & 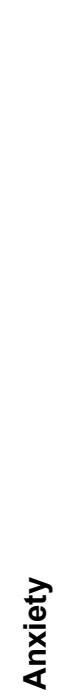 & $\begin{array}{l}\text { 음 } \\
\text { d } \\
\frac{0}{0}\end{array}$ \\
\hline Paroxetine & Duloxetine & - & - & - & - & - & - & - & - & - & - & - & - & - & - & - & I & - & - & - & - & - & - & - & - & - & - & - \\
\hline Paroxetine & Fluoxetine & - & I & I & - & - & - & - & - & - & I & - & - & - & I & I & I & - & - & - & - & - & - & - & - & - & - & - \\
\hline Paroxetine & Venlafaxine & - & - & I & - & - & - & - & - & - & - & - & - & - & - & - & I & - & - & - & - & - & - & - & - & - & - & - \\
\hline Paroxetine & $\begin{array}{l}\text { Citalopram or } \\
\text { escitalopram }\end{array}$ & - & - & I & - & - & - & - & - & - & - & - & - & - & I & I & - & - & - & - & - & - & - & - & - & - & - & - \\
\hline Paroxetine & Sertraline & - & - & I & - & - & - & - & - & - & - & - & - & - & I & I & I & - & - & - & - & - & - & - & I & - & - & - \\
\hline Paroxetine & SNRIs & - & - & - & - & - & - & - & - & - & I & - & - & - & I & I & - & - & - & - & - & - & - & - & - & - & - & - \\
\hline Paroxetine & $\begin{array}{l}\text { Other SSRI } \\
\text { comparators }\end{array}$ & - & - & - & - & - & - & - & - & - & - & - & I & I & I & I & I & - & I & - & - & - & - & - & - & - & - & - \\
\hline Paroxetine & TCAs & - & - & - & - & - & - & - & - & - & - & - & - & - & - & I & - & - & - & - & - & - & - & - & - & - & - & - \\
\hline Fluoxetine & SNRIs & - & - & I & - & - & - & - & - & - & I & - & - & - & I & I & - & - & - & - & - & - & - & - & - & - & - & - \\
\hline Fluoxetine & TCAs & - & - & - & - & - & - & - & - & - & - & - & - & - & I & I & - & - & - & - & - & - & - & I & - & - & - & - \\
\hline Fluvoxamine & $\begin{array}{l}\text { Citalopram or } \\
\text { escitalopram }\end{array}$ & - & - & I & - & - & - & - & - & - & I & - & - & - & I & I & - & - & - & - & - & - & - & - & I & - & - & - \\
\hline Fluvoxamine & Fluoxetine & - & - & I & - & - & - & - & - & - & I & - & - & - & I & I & - & - & - & - & - & - & - & - & I & - & - & - \\
\hline Fluvoxamine & Paroxetine & - & - & I & - & - & - & - & - & - & I & - & - & - & I & I & - & - & - & - & - & - & - & - & I & - & - & - \\
\hline Fluvoxamine & Sertraline & - & - & I & - & - & - & - & - & - & I & - & - & - & I & I & - & - & - & - & - & - & - & - & I & - & - & - \\
\hline
\end{tabular}




\begin{tabular}{|c|c|c|c|c|c|c|c|c|c|c|c|c|c|c|c|c|c|c|c|c|c|c|c|c|c|c|c|c|}
\hline Exposure & Comparator & & $\begin{array}{l}\frac{\pi}{\omega} \\
\frac{0}{\varepsilon} \\
\frac{\pi}{0} \\
\frac{\pi}{0} \\
\frac{0}{0}\end{array}$ & 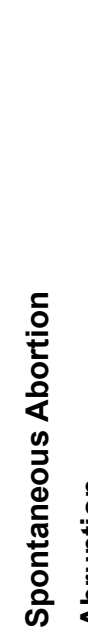 & 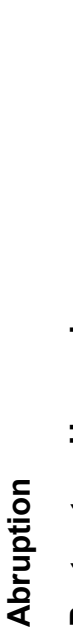 & 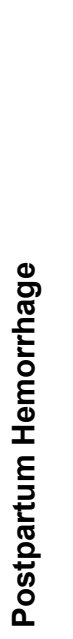 & 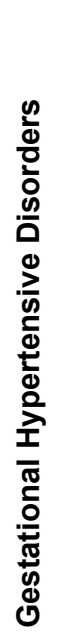 & 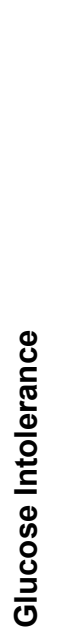 & 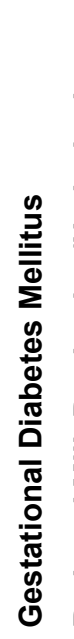 & 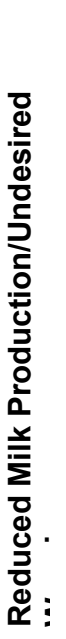 & 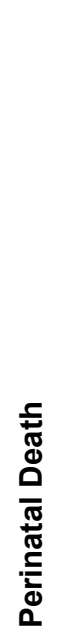 & 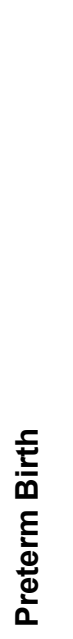 & 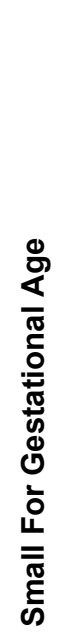 & 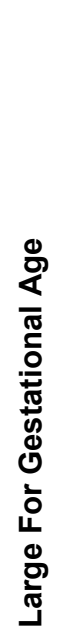 & 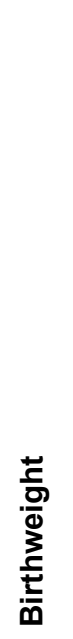 & 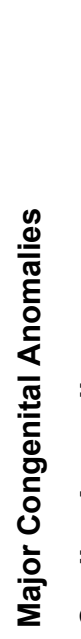 & 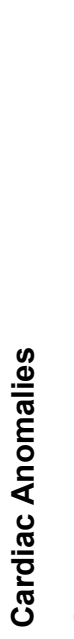 & & 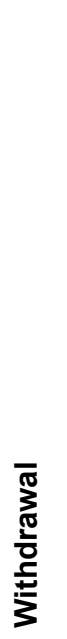 & 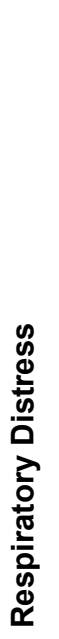 & 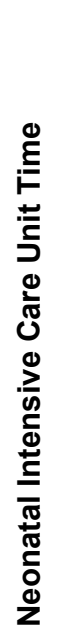 & 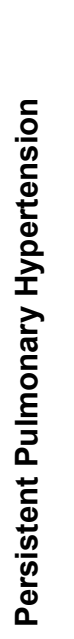 & 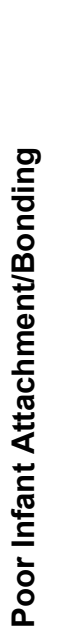 & 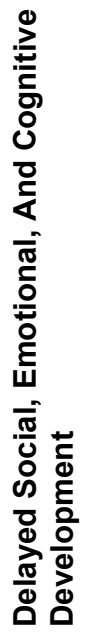 & 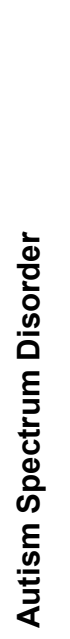 & $\begin{array}{l}\text { 옴 } \\
\text { 高 }\end{array}$ & 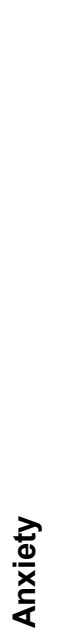 & 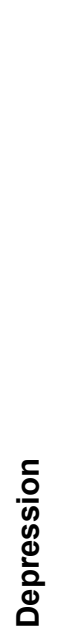 \\
\hline Fluvoxamine & $\begin{array}{l}\text { Venlafaxine or } \\
\text { desvenlafaxine }\end{array}$ & - & - & 1 & - & - & - & - & - & - & I & - & - & - & 1 & I & - & - & - & - & - & - & - & - & - & - & - & - \\
\hline Sertraline & $\begin{array}{l}\text { Citalopram or } \\
\text { escitalopram }\end{array}$ & - & - & 1 & - & - & - & - & - & - & 1 & - & - & - & 1 & 1 & 1 & - & - & - & - & - & - & - & - & - & - & - \\
\hline Sertraline & Duloxetine & - & - & - & - & - & - & - & - & - & - & - & - & - & - & - & I & - & - & - & - & - & - & - & - & - & - & - \\
\hline Sertraline & $\begin{array}{l}\text { Escitalopram or } \\
\text { fluvoxamine }\end{array}$ & - & - & - & - & - & - & - & - & - & - & - & - & - & - & - & - & - & - & - & - & - & - & - & 1 & - & - & - \\
\hline Sertraline & Fluoxetine & - & - & I & - & - & - & - & - & - & 1 & - & - & - & I & 1 & - & - & - & - & - & - & - & - & I & - & - & - \\
\hline Sertraline & Nonsertraline SSRIs & - & - & - & - & - & - & - & - & - & - & - & - & - & - & I & - & - & - & - & - & - & - & - & - & - & - & - \\
\hline Sertraline & $\begin{array}{l}\text { Venlafaxine or } \\
\text { desvenlafaxine }\end{array}$ & - & - & - & - & - & - & - & - & - & 1 & - & - & - & 1 & 1 & - & - & - & - & - & - & - & - & - & - & - & - \\
\hline Sertraline & Bupropion & - & - & - & - & - & - & - & - & - & - & - & - & - & - & - & 1 & - & - & - & - & - & - & - & - & - & - & - \\
\hline Sertraline & Nortriptyline & - & - & - & - & - & - & - & - & - & - & - & - & - & - & - & - & - & - & - & - & - & - & - & - & - & - & - \\
\hline Duloxetine & SSRIs & - & 1 & - & - & 1 & - & - & - & - & - & 1 & 1 & - & - & - & 1 & - & - & - & - & - & - & - & - & - & - & - \\
\hline Duloxetine & Venlafaxine & - & 1 & - & - & 1 & - & - & - & - & - & 1 & 1 & - & - & - & 1 & - & - & - & - & - & - & - & - & - & - & - \\
\hline Venlafaxine & SSRIs & - & - & 1 & - & - & - & - & - & - & - & I & I & - & I & 1 & - & - & - & - & - & - & - & - & - & - & - & - \\
\hline Venlafaxine & Bupropion & - & - & - & - & - & - & - & - & - & - & - & - & - & - & - & 1 & - & - & - & - & - & - & - & - & - & - & - \\
\hline Venlafaxine & Escitalopram & - & - & - & - & - & - & - & - & - & - & - & - & - & - & - & 1 & - & - & - & - & - & - & - & - & - & - & \\
\hline
\end{tabular}




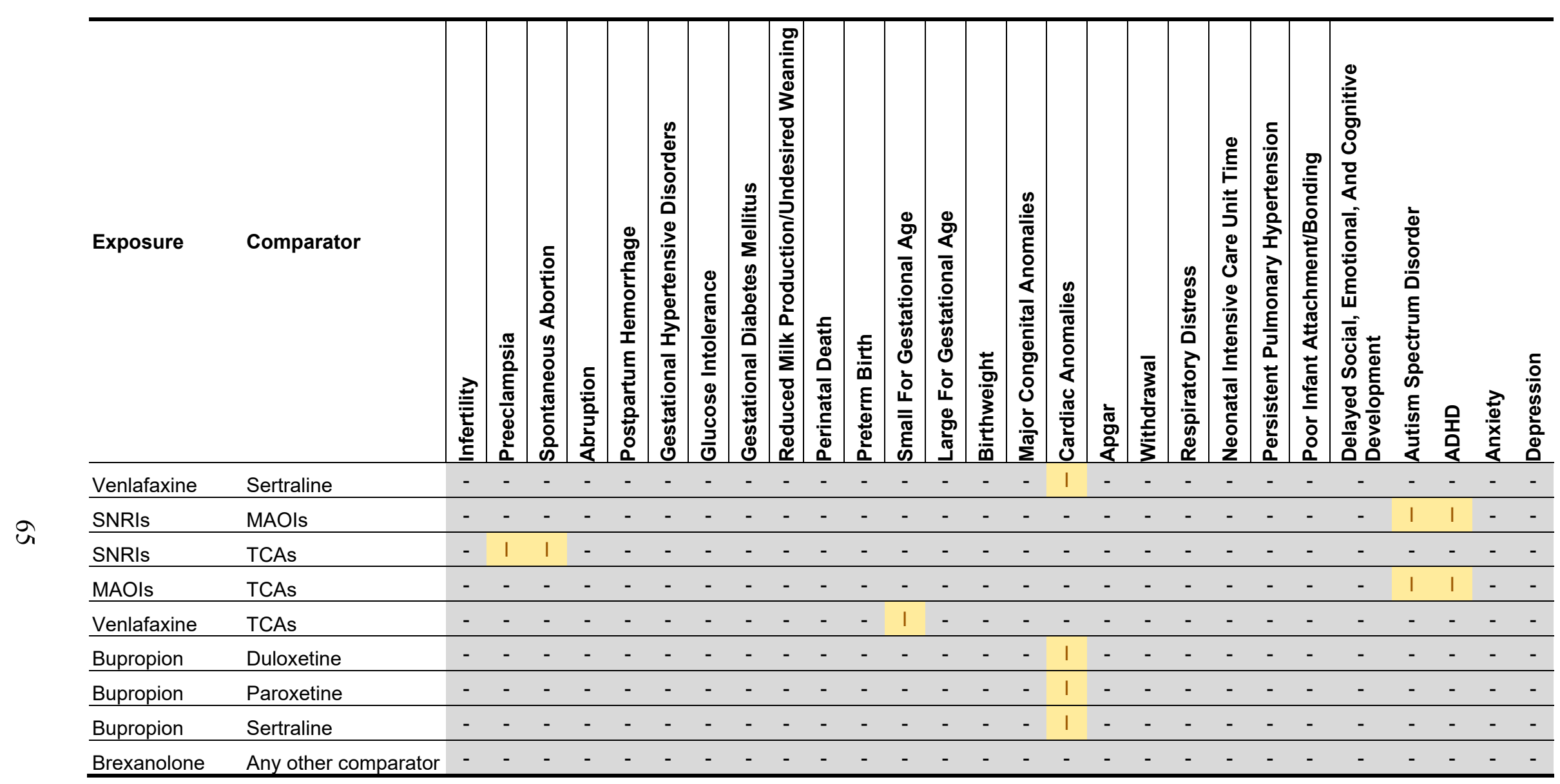




\begin{tabular}{|c|c|c|c|c|c|c|c|c|c|c|c|c|c|c|c|c|c|c|c|c|c|c|c|c|c|c|c|c|}
\hline Exposure & Comparator & 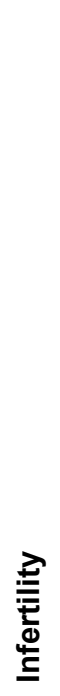 & 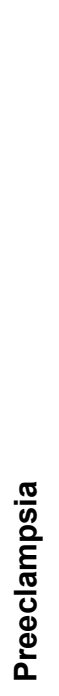 & 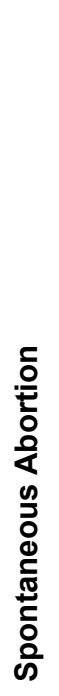 & $\frac{\text { 은 }}{\frac{1}{2}}$ & 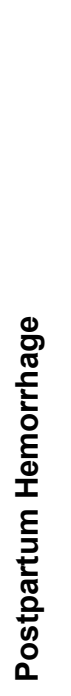 & 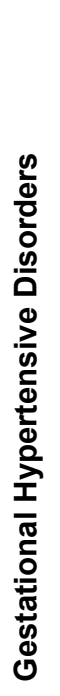 & 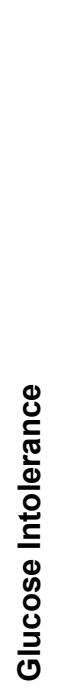 & 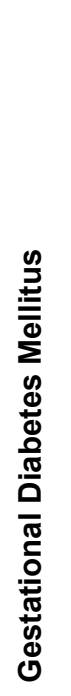 & 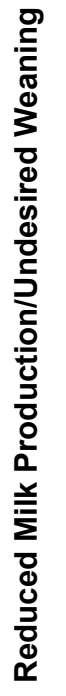 & 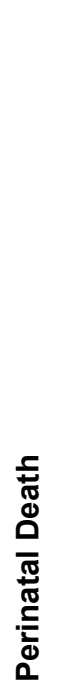 & 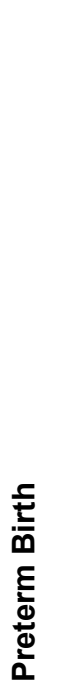 & 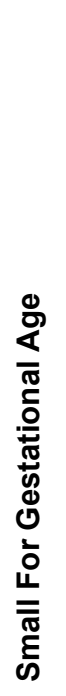 & 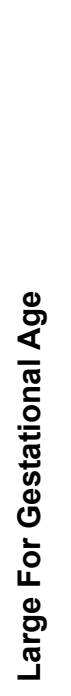 & 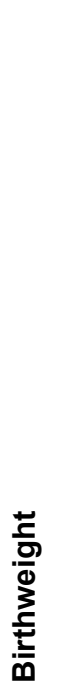 & 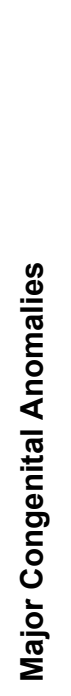 & 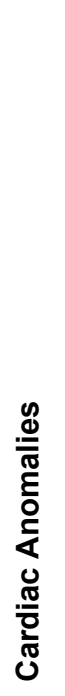 & $\begin{array}{l}\frac{1}{\bar{J}} \\
\frac{0}{0}\end{array}$ & $\begin{array}{l}\bar{\pi} \\
\frac{\pi}{\sqrt[T]{5}} \\
\frac{5}{5} \\
\stackrel{5}{3}\end{array}$ & 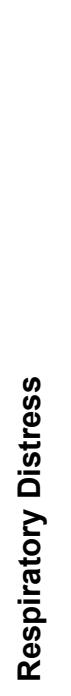 & 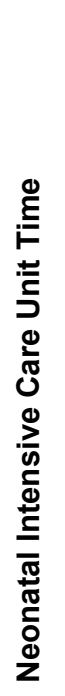 & 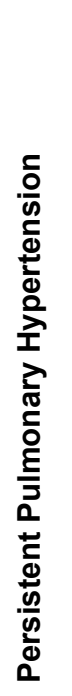 & 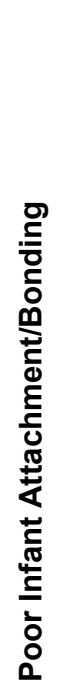 & 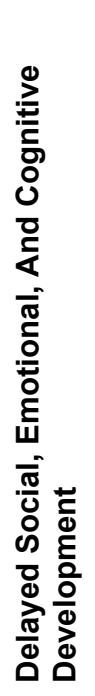 & 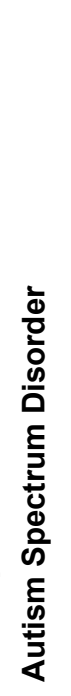 & $\begin{array}{l}\text { 욷 } \\
\text { 운 }\end{array}$ & 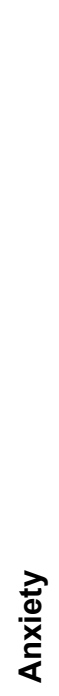 & 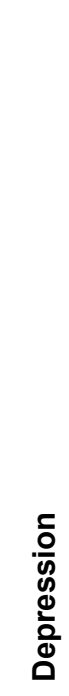 \\
\hline Mirtazapine & SSRIs & - & - & । & - & - & - & - & - & - & I & I & - & - & I & I & - & - & - & - & I & - & - & - & - & - & - & - \\
\hline Mirtazapine & $\begin{array}{l}\text { SSRIs plus } \\
\text { mirtazapine }\end{array}$ & - & - & - & - & - & - & - & - & - & - & I & - & - & I & - & - & - & - & - & I & - & - & - & - & - & - & - \\
\hline Lithium & Lamotrigine & - & - & - & - & - & - & - & I & - & - & - & - & - & - & $L$ & $L$ & - & - & - & - & - & - & - & - & - & - & - \\
\hline Haloperidol & Olanzapine & - & - & - & - & - & - & - & - & - & - & I & - & - & I & - & - & 1 & - & I & I & - & - & - & - & - & - & - \\
\hline Haloperidol & Quetiapine & - & - & - & - & - & - & - & - & - & - & I & - & - & I & - & - & I & - & I & I & - & - & - & - & - & - & - \\
\hline Haloperidol & Risperidone & - & - & - & - & - & - & - & - & - & - & I & - & - & I & - & - & । & - & I & I & - & - & - & - & - & - & - \\
\hline Aripiprazole & Risperidone & - & - & - & - & - & - & - & I & - & - & - & - & - & - & - & - & - & - & - & - & - & - & - & - & - & - & - \\
\hline Clozapine & Aripiprazole & - & - & - & - & - & - & - & I & - & - & - & - & - & - & - & - & - & - & - & - & - & - & - & - & - & - & - \\
\hline Clozapine & Risperidone & - & - & - & - & - & - & - & I & - & - & - & - & - & - & - & - & - & - & - & - & - & - & - & - & - & - & - \\
\hline Olanzapine & Aripiprazole & - & - & - & - & - & - & - & I & - & - & - & - & - & - & - & - & - & - & - & - & - & - & - & - & - & - & - \\
\hline Olanzapine & Clozapine & - & - & - & - & - & - & - & I & - & - & - & - & - & - & - & - & - & - & - & - & - & - & - & - & - & - & - \\
\hline Olanzapine & Quetiapine & - & - & - & - & - & - & - & I & - & - & I & - & - & I & - & - & - & - & 1 & I & - & - & - & - & - & - & - \\
\hline Olanzapine & Risperidone & - & - & - & - & - & - & - & I & - & - & I & - & - & I & - & - & - & - & 1 & I & - & - & - & - & - & - & - \\
\hline Quetiapine & Clozapine & - & - & - & - & - & - & - & I & - & - & - & - & - & - & - & - & - & - & - & - & - & - & - & - & - & - & - \\
\hline Quetiapine & Aripiprazole & - & - & - & - & - & - & - & I & - & - & - & - & - & - & - & - & - & - & - & - & - & - & - & - & - & - & - \\
\hline
\end{tabular}




\begin{tabular}{|c|c|c|c|c|c|c|c|c|c|c|c|c|c|c|c|c|c|c|c|c|c|c|c|c|c|c|c|c|}
\hline Exposure & Comparator & 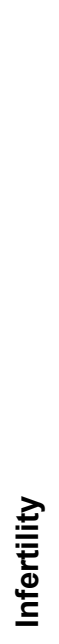 & 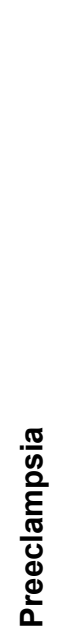 & 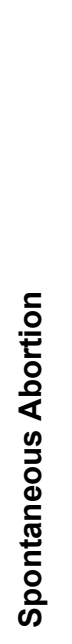 & 들 & 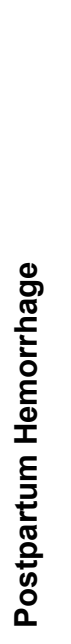 & 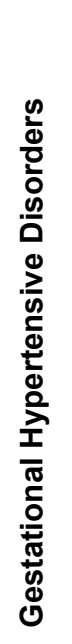 & 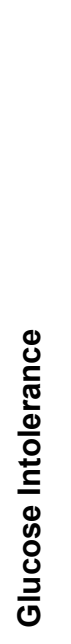 & 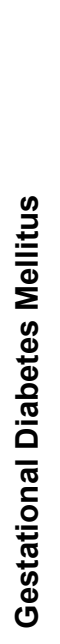 & 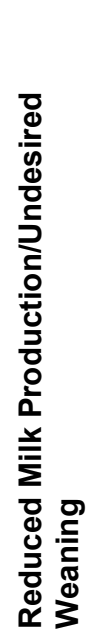 & 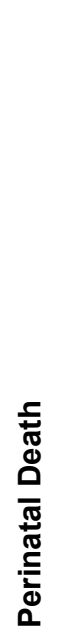 & 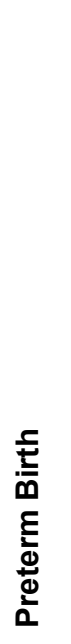 & 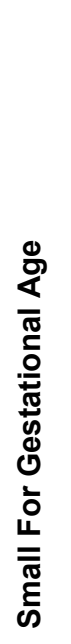 & 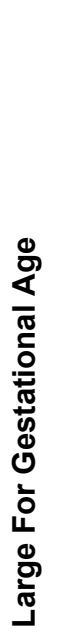 & 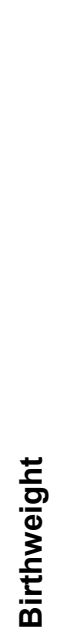 & 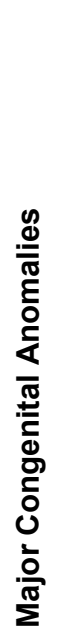 & 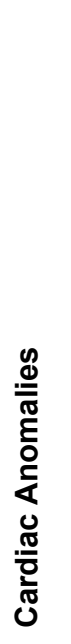 & $\begin{array}{l}\frac{1}{\pi} \\
\frac{0}{8} \\
\frac{0}{4}\end{array}$ & 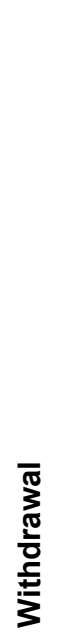 & 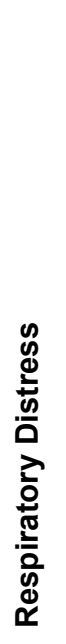 & 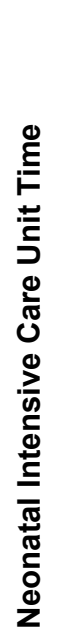 & 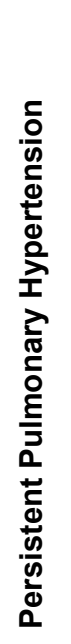 & 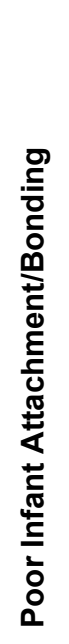 & 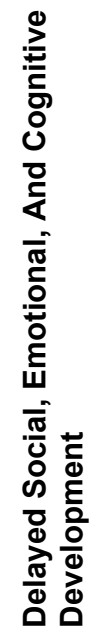 & 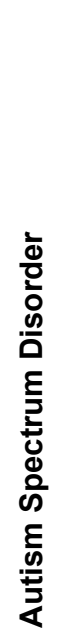 & $\begin{array}{l}\text { 오 } \\
\text { 足 }\end{array}$ & 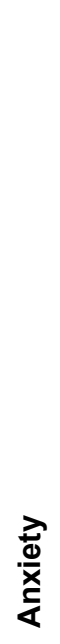 & 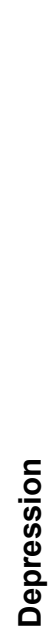 \\
\hline Quetiapine & Risperidone & - & - & - & - & - & - & - & I & - & - & I & - & - & I & - & - & - & - & I & I & - & - & - & - & - & - & - \\
\hline $\begin{array}{l}\text { First generation } \\
\text { antipsychotics }\end{array}$ & $\begin{array}{l}\text { Second generation } \\
\text { antipsychotics }\end{array}$ & - & - & - & - & - & - & - & - & - & - & - & 1 & I & I & - & - & - & - & - & - & - & - & I & - & - & - & - \\
\hline Olanzapine & Lithium & - & - & - & - & - & - & - & I & - & - & - & - & - & - & - & - & - & - & - & - & - & - & - & - & - & - & - \\
\hline Olanzapine & Lamotrigine & - & - & - & - & - & - & - & I & - & - & - & - & - & - & - & - & - & - & - & - & - & - & - & - & - & - & - \\
\hline Quetiapine & Lithium & - & - & - & - & - & - & - & I & - & - & - & - & - & - & - & - & - & - & - & - & - & - & - & - & - & - & - \\
\hline Quetiapine & Lamotrigine & - & - & - & - & - & - & - & I & - & - & - & - & - & - & - & - & - & - & - & - & - & - & - & - & - & - & - \\
\hline Aripiprazole & Lithium & - & - & - & - & - & - & - & I & - & - & - & - & - & - & - & - & - & - & - & - & - & - & - & - & - & - & - \\
\hline Aripiprazole & Lamotrigine & - & - & - & - & - & - & - & I & - & - & - & - & - & - & - & - & - & - & - & - & - & - & - & - & - & - & - \\
\hline Clozapine & Lithium & - & - & - & - & - & - & - & I & - & - & - & - & - & - & - & - & - & - & - & - & - & - & - & - & - & - & - \\
\hline Clozapine & Lamotrigine & - & - & - & - & - & - & - & I & - & - & - & - & - & - & - & - & - & - & - & - & - & - & - & - & - & - & - \\
\hline Risperidone & Lithium & - & - & - & - & - & - & - & I & - & - & - & - & - & - & - & - & - & - & - & - & - & - & - & - & - & - & - \\
\hline Risperidone & Lamotrigine & - & - & - & - & - & - & - & 1 & - & - & - & - & - & - & - & - & - & - & - & - & - & - & - & - & - & - & - \\
\hline
\end{tabular}

I: Insufficient for all measures for the outcome domain; L: Low evidence of harm for at least one measure for the outcome domain; -: No eligible evidence.

$\mathrm{ADHD}=$ attention-deficit/hyperactivity disorder; MAOI $=$ monoamine oxidase inhibitor; SNRI = serotonin-norepinephrine reuptake inhibitor; SSRI $=$ selective serotonin reuptake

inhibitor; TCA $=$ tricyclic antidepressant. 


\section{Detailed Synthesis}

Table 14 provides an overview of the findings on harms to the fetus, infant, or child of pharmacotherapy in the perinatal period. Appendix B includes detailed tables on all outcomes. We describe results from one high risk-of-bias publication that yielded evidence of harm below.

Table 14. Strength of evidence from effectiveness studies for harm to the fetus, infant, or child (intervention versus placebo or no treatment)

\begin{tabular}{|c|c|c|c|c|c|c|c|}
\hline Population & $\begin{array}{l}\text { Intervention } \\
\text { and } \\
\text { Comparator }\end{array}$ & Outcome & $\begin{array}{l}\text { Incidence } \\
\text { or Mean } \\
\text { Effect by } \\
\text { Arm }\end{array}$ & Results & $\begin{array}{l}\text { Study } \\
\text { Design and } \\
\text { Sample } \\
\text { Size }\end{array}$ & $\begin{array}{l}\text { Factors That } \\
\text { Affect the } \\
\text { Strength of } \\
\text { Evidence }\end{array}$ & $\begin{array}{l}\text { Overall } \\
\text { Evidence } \\
\text { Strength } \\
\text { (Direction } \\
\text { of Effect) }\end{array}$ \\
\hline $\begin{array}{l}\text { Lithium- or } \\
\text { lamotrigine- } \\
\text { exposed } \\
\text { women }\end{array}$ & $\begin{array}{l}\text { Lithium vs. } \\
\text { lamotrigine } \\
\text { exposure in } \\
\text { first- } \\
\text { trimester } \\
\text { pregnancy }\end{array}$ & $\begin{array}{l}\text { Fetal cardiac } \\
\text { anomalies }\end{array}$ & $\begin{array}{l}16 / 663 \\
(2.4 \%) \text { vs. } \\
27 / 1,945 \\
(1.4 \%)^{133}\end{array}$ & $\begin{array}{l}\text { ARR, } 2.25 \\
(95 \% \mathrm{Cl}, \\
1.17 \text { to } \\
4.34)\end{array}$ & $\begin{array}{l}1 \text { cohort; } \\
n=2,608^{133}\end{array}$ & $\begin{array}{l}\text { High study } \\
\text { limitations } \\
\text { (high risk of } \\
\text { bias), }{ }^{133} \\
\text { precise, large } \\
\text { effect size, } \\
\text { consistency } \\
\text { unknown }\end{array}$ & $\begin{array}{l}\text { Low for } \\
\text { greater } \\
\text { harms with } \\
\text { lithium than } \\
\text { lamotrigine }\end{array}$ \\
\hline $\begin{array}{l}\text { Lithium- or } \\
\text { lamotrigine- } \\
\text { exposed } \\
\text { women }\end{array}$ & $\begin{array}{l}\text { Lithium vs. } \\
\text { lamotrigine } \\
\text { exposure in } \\
\text { first- } \\
\text { trimester } \\
\text { pregnancy }\end{array}$ & $\begin{array}{l}\text { Overall } \\
\text { congenital } \\
\text { anomalies }\end{array}$ & $\begin{array}{l}38 / 663 \\
(5.7 \%) \text { vs. } \\
76 / 1,945 \\
(3.9 \%)^{133}\end{array}$ & $\begin{array}{l}\text { ARR, } 1.85 \\
(95 \% \mathrm{Cl}, \\
1.23 \text { to } \\
2.78)\end{array}$ & $\begin{array}{l}1 \text { cohort; } \\
n=2,608^{133}\end{array}$ & $\begin{array}{l}\text { High study } \\
\text { limitations } \\
\text { (high risk of } \\
\text { bias), }{ }^{133} \\
\text { precise, large } \\
\text { effect size, } \\
\text { consistency } \\
\text { unknown }\end{array}$ & $\begin{array}{l}\text { Low for } \\
\text { greater } \\
\text { harms with } \\
\text { lithium than } \\
\text { lamotrigine }\end{array}$ \\
\hline
\end{tabular}

$\overline{\mathrm{ARR}}=$ adjusted risk ratio; $\mathrm{CI}=$ confidence interval; $\mathrm{n}=$ number; vs. $=$ versus.

The evidence on fetal, infant, or child harms from treatments for mental health disorders comes from bodies of evidence with high limitations and precise results. Precise results from bodies of evidence with high limitations had factors beyond statistical significance alone that resulted in the low strength-of-evidence grade. Specifically, the identified harms include congenital anomalies and cardiac anomalies favoring lithium over lamotrigine, which can inform the decision to switch a medication in a successfully treated individual. ${ }^{133}$ Although the study was rated high risk of bias because of the restriction to live births and the resulting potential for selection bias, we upgraded the evidence from insufficient to low for lithium versus lamotrigine because of the large effect size and note the relatively small number of events in the lithium arm.

\section{Insufficient Evidence of Maternal Harms}

\section{Overview}

- Insufficient grades were assigned based study limitations, bias, consistency, and precision. Some bodies of evidence comprised single studies for which we could not infer consistency of the evidence base. Additionally, the reported associations were imprecise (i.e., the results relied on small sample sizes, few events, or had wide CIs suggestive of both benefits and harms) and had high study limitations. 
- The evidence is insufficient for comparative evidence on maternal harms for mental health disorders; the evidence comes from bodies of evidence with high limitations and imprecise results.

- Evidence from two cohort studies was insufficient to judge the strength of association between preeclampsia and exposure to SSRIs versus SNRIs during pregnancy.

- Evidence from two cohort studies was insufficient to judge the strength of association between gestational hypertension and exposure to SSRIs versus SNRIs during pregnancy.

- Evidence from two cohort studies was insufficient to judge the strength of association between spontaneous abortion and exposure to SSRIs versus venlafaxine during pregnancy.

\section{Detailed Synthesis}

Table 15 provides an overview of the findings on maternal harms from use of pharmacotherapy in the perinatal period. Appendix B includes detailed tables on all outcomes. We describe results from two publications on harms below.

We found single studies with imprecise results, often from high risk-of-bias studies for most outcomes (see Appendix B for further details). For three outcomes, we identified multiple studies but graded the evidence as insufficient. We describe these results in greater detail below.

\section{Preeclampsia for SSRIs Versus SNRIs}

Two high risk-of-bias studies ${ }^{87,137}$ reported results that suggest lower relative risks for preeclampsia with SSRIs compared with SNRIs (one was imprecise with a very small number of events). Neither study presented adjusted results for this comparison. With no controls for confounders, we rated the strength-of-evidence grade as insufficient.

\section{Gestational Hypertension for SSRIs Versus SNRIs}

Two high risk-of-bias studies ${ }^{137,138}$ reported results that suggest lower relative risks for gestational hypertension with SSRIs compared with SNRIs. Both were imprecise with a very small number of events. Neither study presented adjusted results for this comparison. With no controls for confounders, we rated the strength-of-evidence grade as insufficient.

\section{Spontaneous Abortion for SSRIs Versus Venlafaxine}

Two studies ${ }^{139,140}$ (one high risk-of-bias study ${ }^{139}$ ) reported no significant differences in spontaneous abortion associated with SSRIs compared with venlafaxine. One attempted to control for potential confounding. ${ }^{140}$ Both were imprecise with wide CIs spanning both appreciable benefit and appreciable harm; as a result, we could not conclude equivalence and graded the evidence as insufficient. 
Table 15. Insufficient strength of evidence from comparative effectiveness studies for harm to the mother

\begin{tabular}{|c|c|c|c|c|c|c|c|}
\hline Population & $\begin{array}{l}\text { Intervention } \\
\text { and } \\
\text { Comparator }\end{array}$ & Outcome & $\begin{array}{l}\text { Incidence or } \\
\text { Mean Effect } \\
\text { by Arm }\end{array}$ & Results & $\begin{array}{l}\text { Study } \\
\text { Design and } \\
\text { Sample } \\
\text { Size }\end{array}$ & $\begin{array}{l}\text { Factors That } \\
\text { Affect the } \\
\text { Strength of } \\
\text { Evidence }\end{array}$ & $\begin{array}{l}\text { Overall } \\
\text { Evidence } \\
\text { Strength } \\
\text { (Direction } \\
\text { of Effect) } \\
\end{array}$ \\
\hline $\begin{array}{l}\text { Pregnant } \\
\text { women who } \\
\text { used SSRIs } \\
\text { or SNRIs } \\
\text { during } \\
\text { pregnancy or } \\
\text { who had } \\
\text { depression }\end{array}$ & $\begin{array}{l}\text { SSRI vS. } \\
\text { SNRI }\end{array}$ & Preeclampsia & $\begin{array}{l}23 / 408(5.6 \%) \\
\text { vs. } 105 / 3,169 \\
(3.3 \%)^{87} \\
\text { Continued AD } \\
\text { use } \geq 20 \\
\text { weeks of } \\
\text { gestation: } \\
4 / 157(2.5 \%) \\
\text { vs. } 2 / 21 \\
(9.5 \%)^{137}\end{array}$ & $\begin{array}{l}\mathrm{RR}, 0.59 \\
(95 \% \mathrm{Cl}, \\
0.38 \text { to } \\
0.91)^{87} \\
\\
\mathrm{RR}, 0.27 \\
(95 \% \mathrm{Cl} \\
0.052 \text { to } \\
1.37)^{137}\end{array}$ & $\begin{array}{l}2 \text { cohorts; } \\
n=3,577 \text { in } \\
\text { one cohort, } \\
n=252 \text { in } \\
\text { second }^{137}\end{array}$ & $\begin{array}{l}\text { High study } \\
\text { limitations } \\
\text { (high risk of } \text { bias }^{87,137} \text { ), } \\
\text { imprecise (few } \\
\text { events), } \\
\text { consistent }\end{array}$ & Insufficient \\
\hline $\begin{array}{l}\text { Pregnant } \\
\text { women who } \\
\text { used SSRIs } \\
\text { or SNRIs } \\
\text { during } \\
\text { pregnancy }\end{array}$ & $\begin{array}{l}\text { SSRI vS. } \\
\text { SNRI }\end{array}$ & $\begin{array}{l}\text { Gestational } \\
\text { hypertension }\end{array}$ & $\begin{array}{l}\text { Continued AD } \\
\text { use } \geq 20 \text { weeks } \\
\text { of gestation: } \\
11 / 157(7.0 \%) \\
\text { vs } 3 / 21 \\
(14.6 \%)^{137} \\
2 / 23(8.3 \%) \\
\text { vs. } 23 / 60 \\
(39.1 \%)^{138} \\
\end{array}$ & $\begin{array}{l}\mathrm{RR}, 0.49 \\
(95 \% \mathrm{Cl}, \\
0.15 \text { to } \\
1.62)^{137} \\
\\
\mathrm{RR}, 0.24 \text {. } \\
(95 \% \mathrm{Cl} \\
0.06 \text { to } \\
0.89)^{138} \\
\end{array}$ & $\begin{array}{l}2 \text { cohorts, } \\
n=335^{137,138}\end{array}$ & $\begin{array}{l}\text { High study } \\
\text { limitations } \\
\text { (high risk of } \\
\text { bias }^{137,138} \text { ), } \\
\text { imprecise } \\
\text { (wide Cls), } \\
\text { consistent }\end{array}$ & Insufficient \\
\hline $\begin{array}{l}\text { Pregnant } \\
\text { women with } \\
\text { depression, } \\
\text { pregnant } \\
\text { women who } \\
\text { used SSRIs } \\
\text { or venlafaxine } \\
\text { during } \\
\text { pregnancy }\end{array}$ & $\begin{array}{l}\text { Venlafaxine } \\
\text { vs. SSRI, } \\
\text { first trimester } \\
\text { exposure }^{139} \\
\end{array}$ & $\begin{array}{l}\text { Spontaneous } \\
\text { abortion }\end{array}$ & $\begin{array}{l}18 / 150(12 \%) \\
\text { vs. } 16 / 150 \\
(10.7 \%)^{139} \\
46 / 281 \\
(16.37 \%) \text { vs. } \\
144 / 843 \\
(17.1 \%)^{140}\end{array}$ & $\begin{array}{l}\mathrm{RR}, 1.1 \\
(95 \% \mathrm{Cl}, \\
0.60 \text { to } \\
2.12) 139 \\
\mathrm{AOR}, 1.0 \\
(95 \% \mathrm{Cl}, \\
0.67 \text { to } \\
1.53)\end{array}$ & $\begin{array}{l}2 \text { cohorts, } \\
n=1424^{139,140}\end{array}$ & $\begin{array}{l}\text { Moderate study } \\
\text { limitations } \\
\text { (high risk of } \\
\text { bias }^{139} \text { ) } \\
\text { imprecise } \\
\text { (wide Cls), } \\
\text { consistent }\end{array}$ & Insufficient \\
\hline
\end{tabular}

$\mathrm{AD}=$ antidepressant; $\mathrm{AOR}=$ adjusted odds ratio; $\mathrm{CI}=$ confidence interval; $\mathrm{RR}=$ relative risk; SNRI $=$ serotonin-norepinephrine reuptake inhibitor; SSRI $=$ selective serotonin reuptake inhibitor; $\mathrm{n}=$ number; vs. $=$ versus.

\section{Insufficient Evidence of Fetal, Infant, or Child Harms}

\section{Overview}

- Insufficient grades were assigned based on study limitations, bias, consistency, and precision. Some bodies of evidence comprised single studies for which we could not infer consistency of the evidence base. Additionally, the reported associations were imprecise (i.e., the results relied on small sample sizes, few events, or had wide CIs suggestive of both benefits and harms) and had high study limitations.

- We found insufficient evidence to judge the comparative fetal, infant, or child harms of psychiatric medications during pregnancy or the postpartum period given evidence with high limitations and imprecise results.

- Evidence from three cohort studies provided insufficient evidence to judge the strength of association between anomalies and exposure to SSRIs versus TCAs during pregnancy. 
- Evidence from two cohort studies provided insufficient evidence to judge the strength of association between preterm births, and birthweight and exposure to SSRIs versus TCAs during pregnancy.

- Evidence from two cohort studies provided insufficient evidence to judge the strength of association between autism spectrum disorder and different SSRIs during pregnancy.

- Evidence from two cohort studies provided insufficient evidence to judge the strength of association between preterm births and birthweight and exposure to mirtazapine versus SSRIs during pregnancy.

- Evidence from two cohort studies provided insufficient evidence to judge the strength of association between small for gestational age and birthweight and exposure to venlafaxine versus SSRIs during pregnancy.

\section{Detailed Synthesis}

We found single studies with imprecise results, often from high risk-of-bias studies for most outcomes (see Appendix B for further details). For some outcomes, we identified multiple studies but graded the evidence as insufficient. Specifically, we found insufficient evidence on congenital anomalies, preterm births, small for gestational age, birthweight, and autism spectrum disorder (Table 16).

Table 16. Strength of evidence for harms outcomes: SSRIs versus SNRIs

\begin{tabular}{|c|c|c|c|c|c|c|c|}
\hline Population & $\begin{array}{l}\text { Intervention } \\
\text { and } \\
\text { Comparator }\end{array}$ & Outcome & $\begin{array}{l}\text { Incidence or } \\
\text { Mean Effect } \\
\text { by Arm }\end{array}$ & Results & $\begin{array}{l}\text { Study } \\
\text { Design and } \\
\text { Sample Size }\end{array}$ & $\begin{array}{l}\text { Factors } \\
\text { That Affect } \\
\text { the } \\
\text { Strength of } \\
\text { Evidence }\end{array}$ & $\begin{array}{l}\text { Overall Evidence } \\
\text { Strength } \\
\text { (Direction of } \\
\text { Effect) }\end{array}$ \\
\hline $\begin{array}{l}\text { Exposed to } \\
\text { SSRIs in the first } \\
\text { trimester vs. } \\
\text { exposed to TCAs } \\
\text { in the first } \\
\text { trimester (full- } \\
\text { term infants) }\end{array}$ & $\begin{array}{l}\text { SSRIs vs. } \\
\text { TCAs }\end{array}$ & $\begin{array}{l}\text { Preterm } \\
\text { birth }\end{array}$ & $\begin{array}{l}166 / 1,768 \\
(9.4 \%) \text { vs. } \\
42 / 381 \\
(11.0 \%)^{141} \\
3 / 42(7.1 \%) \text { vs. } \\
6 / 37 \\
(16.2 \%)^{142}\end{array}$ & $\begin{array}{l}\mathrm{RR}, 0.85 \\
(95 \% \mathrm{Cl}, \\
0.62 \text { to } \\
1.17)^{141} \\
\text { RR: } 2.27 \\
(95 \% \mathrm{Cl}, \\
0.61 \text { to } \\
8.44)^{142} \\
\end{array}$ & $\begin{array}{l}2 \text { cohort } \\
\text { study, } \\
\mathrm{N}=2,228^{141}, \\
142\end{array}$ & $\begin{array}{l}\text { High study } \\
\text { limitations } \\
\text { (high risk of } \\
\text { bias }^{141,142} \text { ), } \\
\text { imprecise } \\
\text { (wide Cls), } \\
\text { inconsistent }\end{array}$ & Insufficient \\
\hline $\begin{array}{l}\text { Women exposed } \\
\text { to therapeutic } \\
\text { agents during } \\
\text { pregnancy }\end{array}$ & $\begin{array}{l}\text { Mirtazapine } \\
\text { exposure } \\
\text { during } \\
\text { pregnancy vs. } \\
\text { SSRI exposure } \\
\text { during } \\
\text { pregnancy }\end{array}$ & $\begin{array}{l}\text { Preterm } \\
\text { delivery }\end{array}$ & $\begin{array}{l}31 / 279(11.1 \%) \\
\text { vs. } 32 / 302 \\
(10.6 \%)^{143} \\
1 / 16(6.25 \%) \\
\text { vs. } 3 / 40 \\
(7.50 \%)^{112}\end{array}$ & $\begin{array}{l}\text { OR, } 1.06 \\
(95 \% \mathrm{Cl} \\
(0.63 \text { to } \\
1.78)^{143} \text { the } \\
\text { OR did not } \\
\text { change after } \\
\text { adjusting for } \\
\text { concomitant } \\
\text { drug } \\
\text { treatment } \\
\text { RR, } 0.83 \\
(95 \% \mathrm{Cl}, \\
0.09 \text { to } \\
7.43)^{112}\end{array}$ & $\begin{array}{l}2 \text { cohorts, } \\
n=637^{112,143}\end{array}$ & $\begin{array}{l}\text { High study } \\
\text { limitations } \\
\text { (high risk of } \\
\text { bias }^{112,143} \text { ), } \\
\text { imprecise, } \\
\text { consistent }\end{array}$ & Insufficient \\
\hline
\end{tabular}




\begin{tabular}{|c|c|c|c|c|c|c|c|}
\hline Population & $\begin{array}{l}\text { Intervention } \\
\text { and } \\
\text { Comparator }\end{array}$ & Outcome & $\begin{array}{l}\text { Incidence or } \\
\text { Mean Effect } \\
\text { by Arm }\end{array}$ & Results & $\begin{array}{l}\text { Study } \\
\text { Design and } \\
\text { Sample Size }\end{array}$ & $\begin{array}{l}\text { Factors That } \\
\text { Affect the } \\
\text { Strength of } \\
\text { Evidence }\end{array}$ & $\begin{array}{l}\text { Overall } \\
\text { Evidence } \\
\text { Strength } \\
\text { (Direction of } \\
\text { Effect) }\end{array}$ \\
\hline $\begin{array}{l}\text { Exposed to } \\
\text { SSRIs in the } \\
\text { first } \\
\text { trimester vs. } \\
\text { exposed to } \\
\text { TCAs in the } \\
\text { first } \\
\text { trimester } \\
\text { (full-term } \\
\text { infants) } \\
\end{array}$ & $\begin{array}{l}\text { SSRIs vs. } \\
\text { TCAs }\end{array}$ & $\begin{array}{l}\text { Malforma- } \\
\text { tions (one or } \\
\text { more } \\
\text { malforma- } \\
\text { tions, major } \\
\text { congenital } \\
\text { malforma- } \\
\text { tions, minor } \\
\text { malforma- } \\
\text { tions) } \\
\end{array}$ & $\begin{array}{l}\text { Ranges from } \\
3.7 \% \text { to } 13.4 \% \\
\text { in the SSRI arm } \\
\text { vs. } 3.4 \% \text { to } \\
12.0 \% \text { in the } \\
\text { TCA arm }{ }^{113}\end{array}$ & $\begin{array}{l}\text { RRs range } \\
\text { from } 1.08 \text { to } \\
1.45, \text { all Cls } \\
\text { cross the } \\
\text { null }^{141,144,145}\end{array}$ & $\begin{array}{l}3 \text { cohort } \\
\text { studies, } \\
N=5,651^{141}, \\
144,145\end{array}$ & $\begin{array}{l}\text { High study } \\
\text { limitations } \\
\text { (high risk of } \\
\text { bias }^{141,144,145} \text { ), } \\
\text { imprecise } \\
\text { (wide Cls), } \\
\text { consistent }\end{array}$ & Insufficient \\
\hline $\begin{array}{l}\text { Pregnant } \\
\text { women with } \\
\text { a psychiatric } \\
\text { disorder } \\
\text { (second } \\
\text { trimester) or } \\
\text { exposed to } \\
\text { venlafaxine } \\
\text { or SSRls } \\
\text { (during } \\
\text { pregnancy) }\end{array}$ & $\begin{array}{l}\text { Venlafaxine vs. } \\
\text { SSRI during } \\
\text { first or second } \\
\text { trimester }\end{array}$ & $\begin{array}{l}\text { SGA }\left(<10^{\text {th }}\right. \\
\text { percentile })\end{array}$ & $\begin{array}{l}5 / 17(29.4 \%) \\
\text { vs. } 24 / 155 \\
(15.5 \%)^{113} \\
12 / 133(9.02 \%) \\
\text { vs. } 55 / 438 \\
(12.6 \%)^{140}\end{array}$ & $\begin{array}{l}\mathrm{RR}, 1.90,95 \% \\
\mathrm{Cl}, 0.83 \text { to } \\
4.33^{113} \mathrm{AOR}, \\
0.714(95 \% \\
\mathrm{Cl}, 0.353 \text { to } \\
1.35)\end{array}$ & $\begin{array}{l}2 \text { cohorts, } \\
\mathrm{n}=772^{113,140}\end{array}$ & $\begin{array}{l}\text { Moderate } \\
\text { study } \\
\text { limitations } \\
\text { (high risk of } \\
\text { bias, }{ }^{113} \text { ) } \\
\text { imprecise } \\
\text { (wide Cls), } \\
\text { inconsistent }\end{array}$ & Insufficient \\
\hline $\begin{array}{l}\text { Exposed to } \\
\text { SSRIs vs. } \\
\text { exposed to } \\
\text { TCAs }\end{array}$ & $\begin{array}{l}\text { SSRIs vs. } \\
\text { TCAs }\end{array}$ & $\begin{array}{l}\text { Birthweight } \\
\text { (birthweight } \\
\leq 2,500 \mathrm{~g} \text { or } \\
\text { total } \\
\text { birthweight }^{144} \\
\text { or } \\
\text { undefined }^{142} \text { ) }\end{array}$ & $\begin{array}{l}\text { 19/185 (10.3\%) } \\
\text { vs. } 21 / 209 \\
\text { (10.0\%), mean } \\
\text { difference: }-80 \\
\text { g }^{144 ;} ; 1 / 42 \\
(2.4 \%) \text { vs. } 2 / 37 \\
(5.4 \%)\end{array}$ & $\begin{array}{l}\text { RR, } 1.02(95 \% \\
\mathrm{Cl}, 0.57 \text { to } \\
1.84) ; \mathrm{Cls} \text { for } \\
\text { mean } \\
\text { difference } \\
\text { span the } \\
\text { null }^{144} ; \mathrm{RR}, \\
2.27(95 \% \mathrm{Cl} \text {, } \\
0.21 \text { to } \\
2.40)^{142}\end{array}$ & $\begin{array}{l}2 \text { cohort } \\
\text { study, } \\
\mathrm{N}=473^{142,144}\end{array}$ & $\begin{array}{l}\text { High study } \\
\text { limitations } \\
\text { (high risk of } \\
\left.\text { bias }^{142,144}\right) \text {, } \\
\text { imprecise, } \\
\text { consistent }\end{array}$ & Insufficient \\
\hline $\begin{array}{l}\text { Women } \\
\text { exposed to } \\
\text { therapeutic } \\
\text { agents } \\
\text { during } \\
\text { pregnancy }\end{array}$ & $\begin{array}{l}\text { Mirtazapine } \\
\text { exposure } \\
\text { during } \\
\text { pregnancy vs. } \\
\text { SSRI exposure } \\
\text { during } \\
\text { pregnancy }\end{array}$ & $\begin{array}{l}\text { Birth weight } \\
\text { (grams) }\end{array}$ & $\begin{array}{l}39 \text { (IQR 38-40) } \\
\text { vs. } 39 \text { (IQR 38- } \\
40)^{143} \\
3251.25 \mathrm{gm} \\
\text { (SD: } 502.27 \\
\mathrm{n}=16 \mathrm{vs} . \\
3,153.17 \mathrm{gm} \\
(\mathrm{SD}: 435.17) \\
\mathrm{n}=40^{112}\end{array}$ & $\begin{array}{l}\text { No difference } \\
\text { between } \\
\text { groups; }{ }^{143} \\
\text { mean } \\
\text { difference, } \\
98.08(95 \% \mathrm{Cl} \text {, } \\
-165.60 \text { to } \\
361.76)^{112} \\
\text { The OR did } \\
\text { not change } \\
\text { after adjusting } \\
\text { for } \\
\text { concomitant } \\
\text { drug } \\
\text { treatment }{ }^{143}\end{array}$ & $\begin{array}{l}2 \text { cohorts, } \\
\mathrm{n}=652^{112,143}\end{array}$ & $\begin{array}{l}\text { High study } \\
\text { limitations } \\
\text { (high risk of } \\
\text { bias }^{112,143} \text { ), } \\
\text { imprecise } \\
\text { (wide Cls), } \\
\text { consistent }\end{array}$ & Insufficient \\
\hline
\end{tabular}




\begin{tabular}{|c|c|c|c|c|c|c|c|}
\hline Population & $\begin{array}{l}\text { Intervention } \\
\text { and } \\
\text { Comparator }\end{array}$ & Outcome & $\begin{array}{l}\text { Incidence or } \\
\text { Mean Effect } \\
\text { by Arm }\end{array}$ & Results & $\begin{array}{l}\text { Study } \\
\text { Design and } \\
\text { Sample Size }\end{array}$ & $\begin{array}{l}\text { Factors That } \\
\text { Affect the } \\
\text { Strength of } \\
\text { Evidence }\end{array}$ & $\begin{array}{l}\text { Overall } \\
\text { Evidence } \\
\text { Strength } \\
\text { (Direction of } \\
\text { Effect) }\end{array}$ \\
\hline $\begin{array}{l}\text { Women } \\
\text { exposed to } \\
\text { antidepressant } \\
\mathrm{s} \text { in } \\
\text { second/third } \\
\text { trimester of } \\
\text { pregnancy }\end{array}$ & $\begin{array}{l}\text { Paroxetine vs. } \\
\text { sertraline }\end{array}$ & $\begin{array}{l}\text { Autism } \\
\text { spectrum } \\
\text { disorder }\end{array}$ & $\begin{array}{l}\text { Paroxetine: } \\
\text { 11/744 }(1.5 \%) \\
\text { vs. sertraline: } \\
1 / 292 \\
(0.34 \%)^{146} \\
\\
\text { Paroxetine: } \\
7 / 871(0.8 \%) \\
\text { vs. sertraline: } \\
9 / 1576 \\
(0.57 \%)^{146} \\
\end{array}$ & $\begin{array}{l}\text { RR, } 4.3(95 \% \\
\text { Cl, } 0.56 \text { to } \\
33.3)^{146} \\
\text { RR, } 1.41 \\
(95 \% \mathrm{Cl}, \\
0.53 \text { to } \\
3.77)^{147}\end{array}$ & $\begin{array}{l}2 \text { cohorts, N= } \\
3,483^{146,147}\end{array}$ & $\begin{array}{l}\text { High study } \\
\text { limitations } \\
\text { (high risk of } \\
\text { bias }^{146,147} \text { ), } \\
\text { imprecise } \\
\text { (wide Cls), } \\
\text { consistent }\end{array}$ & Insufficient \\
\hline $\begin{array}{l}\text { Pregnant } \\
\text { women taking } \\
\text { fluoxetine or } \\
\text { sertraline }\end{array}$ & $\begin{array}{l}\text { Sertraline } \\
\text { monotherapy vs. } \\
\text { fluoxetine } \\
\text { monotherapy }\end{array}$ & $\begin{array}{l}\text { Autism } \\
\text { spectrum } \\
\text { disorder }\end{array}$ & $\begin{array}{l}9 / 1,576(0.6 \%) \\
\text { vs. } 18 / 160 \\
(11 \%)^{147} \\
1 / 292(0.3 \%) \\
\text { vs. } 5 / 171 \\
(2.9 \%)^{146}\end{array}$ & $\begin{array}{l}\mathrm{RR}, 0.05 \\
(95 \% \mathrm{Cl}, \\
0.02 \text { to } \\
0.11)^{147} \\
\mathrm{RR}, 0.12 \\
(95 \% \mathrm{Cl}, \\
0.01 \text { to } \\
0.99)^{146}\end{array}$ & $\begin{array}{l}2 \text { cohorts; } \\
n=1,736 \text { in } \\
\text { one cohort, }{ }^{147} \\
n=463 \text { in one } \\
\text { cohort }^{146}\end{array}$ & $\begin{array}{l}\text { High study } \\
\text { limitations } \\
\text { (high risk of } \\
\text { bias) }{ }^{147} \\
\text { precise, } \\
\text { consistent }\end{array}$ & Insufficient \\
\hline $\begin{array}{l}\text { Pregnant } \\
\text { women taking } \\
\text { fluoxetine, } \\
\text { fluvoxamine or } \\
\text { escitalopram }\end{array}$ & $\begin{array}{l}\text { Fluoxetine } \\
\text { monotherapy vs. } \\
\text { fluvoxamine or } \\
\text { escitalopram } \\
\text { monotherapy: }{ }^{147} \\
\text { fluoxetine } \\
\text { monotherapy vs. } \\
\text { fluvoxamine }^{146}\end{array}$ & $\begin{array}{l}\text { Autism } \\
\text { spectrum } \\
\text { disorder }\end{array}$ & $\begin{array}{l}18 / 160(11 \%) \\
\text { vs. } 1 / 1047 \\
(0.09 \%)^{147} \\
\\
5 / 171(2.9 \%) \\
\text { vs. } 1 / 35 \\
(2.9 \%)^{146}\end{array}$ & $\begin{array}{l}\text { RR, } 117.79, \\
(95 \% \mathrm{Cl}, \\
15.83 \text { to } \\
876.27) \\
\mathrm{RR}, 1.02 \\
(95 \% \mathrm{Cl}, \\
0.12 \text { to } 8.49)\end{array}$ & $\begin{array}{l}2 \text { cohorts; } \\
n=1.207 \text { in } \\
\text { one cohort, } 147 \\
n=206 \text { in one } \\
\text { cohort }^{146}\end{array}$ & $\begin{array}{l}\text { High study } \\
\text { limitations } \\
\text { (high risk of } \\
\text { bias }^{146,147} \text { ), } \\
\text { imprecise } \\
\text { (wide Cls), } \\
\text { consistent }\end{array}$ & Insufficient \\
\hline $\begin{array}{l}\text { Pregnant } \\
\text { women taking } \\
\text { paroxetine or } \\
\text { fluoxetine }\end{array}$ & $\begin{array}{l}\text { Paroxetine } \\
\text { monotherapy vs. } \\
\text { fluoxetine } \\
\text { monotherapy }\end{array}$ & $\begin{array}{l}\text { Autism } \\
\text { spectrum } \\
\text { disorder }\end{array}$ & $\begin{array}{l}11 / 744(1.5 \%) \\
\text { vs. } 5 / 171 \\
(2.9 \%)^{146} \\
\\
7 / 871(0.6 \%) \\
\text { vs. } 18 / 160 \\
(11 \%)^{147}\end{array}$ & $\begin{array}{l}\text { RR, } 0.51 \\
(95 \% \mathrm{Cl}, \\
0.18 \text { to } \\
1.44)^{146} \\
\\
\mathrm{RR}, 0.07 \\
(95 \% \mathrm{Cl}, \\
0.03 \text { to } \\
0.17)^{147} \\
\end{array}$ & $\begin{array}{l}2 \text { cohorts, } \\
n=915 \text { in one } \\
\text { cohort }{ }^{146}, \\
n=1,031 \text { in } \\
\text { one } \text { cohort }^{147}\end{array}$ & $\begin{array}{l}\text { High study } \\
\text { limitations } \\
\text { (high risk of } \\
\text { bias }^{148} \text { ), } \\
\text { imprecise } \\
\text { (few events), } \\
\text { consistent }\end{array}$ & Insufficient \\
\hline $\begin{array}{l}\text { Pregnant } \\
\text { women taking } \\
\text { fluoxetine or } \\
\text { sertraline }\end{array}$ & $\begin{array}{l}\text { Sertraline } \\
\text { monotherapy vs. } \\
\text { fluoxetine } \\
\text { monotherapy }\end{array}$ & $\begin{array}{l}\text { Autism } \\
\text { spectrum } \\
\text { disorder }\end{array}$ & $\begin{array}{l}\text { 9/1576 (0.6\%) } \\
\text { vs. } 18 / 160 \\
(11 \%)^{147} \\
1 / 292(0.3 \%) \\
\text { vs. } 5 / 171 \\
(2.9 \%)^{146}\end{array}$ & $\begin{array}{l}\mathrm{RR}, 0.05 \\
(95 \% \mathrm{Cl}, \\
0.02 \text { to } \\
0.11)^{147} \\
\mathrm{RR}, 0.12 \\
(95 \% \mathrm{Cl} \\
0.01 \text { to } \\
0.99)^{146} \\
\end{array}$ & $\begin{array}{l}2 \text { cohorts; } \\
n=1,736 \text { in } \\
\text { one cohort, }{ }^{147} \\
n=463 \text { in one } \\
\text { cohort }^{146}\end{array}$ & $\begin{array}{l}\text { High study } \\
\text { limitations } \\
\text { (high risk of } \\
\text { bias, } \\
\text { potential } \\
\text { inaccuracy in } \\
\text { data147,148 } \\
\text { precise, } \\
\text { consistent } \\
\end{array}$ & Insufficient \\
\hline $\begin{array}{l}\text { Pregnant } \\
\text { women in } \\
\text { second or third } \\
\text { trimester }{ }^{146} \text { or } \\
\text { during } \\
\text { pregnancy }{ }^{147} \\
\text { exposed to } \\
\text { citalopram or } \\
\text { fluoxetine }\end{array}$ & $\begin{array}{l}\text { Maternal } \\
\text { exposure to } \\
\text { citalopram vs. } \\
\text { maternal } \\
\text { exposure to } \\
\text { fluoxetine }\end{array}$ & $\begin{array}{l}\text { Autism } \\
\text { spectrum } \\
\text { disorder }\end{array}$ & $\begin{array}{l}5 / 421(1.2 \%) \\
\text { vs. } 5 / 171 \\
(2.9 \%) ; 146 \\
14 / 1751 \\
(0.8 \%) \text { vs. } \\
18 / 160 \\
(11.3 \%)^{147}\end{array}$ & $\begin{array}{l}\text { RR, } 0.41 \\
(95 \% \mathrm{Cl}, \\
0.11 \text { to } \\
1.4))^{: 146} \mathrm{RR}, \\
7.1(95 \% \mathrm{Cl}, \\
0.036 \text { to } \\
0.14)^{147}\end{array}$ & $\begin{array}{l}2 \text { cohorts, } \\
N=2,503^{146}, \\
147\end{array}$ & $\begin{array}{l}\text { High study } \\
\text { limitations } \\
\text { (high risk of } \text { bias }^{146,147} \text { ), } \\
\text { imprecise, } \\
\text { inconsistent }\end{array}$ & Insufficient \\
\hline
\end{tabular}




\begin{tabular}{|c|c|c|c|c|c|c|c|}
\hline Population & $\begin{array}{l}\text { Intervention } \\
\text { and } \\
\text { Comparator }\end{array}$ & Outcome & $\begin{array}{l}\text { Incidence or } \\
\text { Mean Effect } \\
\text { by Arm }\end{array}$ & Results & $\begin{array}{l}\text { Study Design } \\
\text { and Sample } \\
\text { Size }\end{array}$ & $\begin{array}{l}\text { Factors That } \\
\text { Affect the } \\
\text { Strength of } \\
\text { Evidence }\end{array}$ & $\begin{array}{l}\text { Overall } \\
\text { Evidence } \\
\text { Strength } \\
\text { (Direction of } \\
\text { Effect) } \\
\end{array}$ \\
\hline $\begin{array}{l}\text { Pregnant } \\
\text { women in } \\
\text { second or } \\
\text { third } \\
\text { trimester }{ }^{146} \text { or } \\
\text { during } \\
\text { pregnancy }{ }^{147} \\
\text { exposed to } \\
\text { citalopram or } \\
\text { paroxetine }\end{array}$ & $\begin{array}{l}\text { Maternal } \\
\text { exposure to } \\
\text { citalopram vs. } \\
\text { maternal } \\
\text { exposure to } \\
\text { paroxetine }\end{array}$ & $\begin{array}{l}\text { Autism } \\
\text { spectrum } \\
\text { disorder }\end{array}$ & $\begin{array}{l}5 / 421(1.2 \%) \\
\text { vs. } 11 / 744 \\
(1.5 \%) ; 146 \\
14 / 1,751 \\
(0.8 \%) \text { vs. } \\
7 / 871 \\
(0.8 \%)^{147}\end{array}$ & $\begin{array}{l}\text { RR, } 0.80 \\
\text { (95\% Cl, } \\
0.28 \text { to } \\
2.3) ; 146 \mathrm{RR}, \\
0.99(95 \% \\
\mathrm{Cl}, 0.4 \text { to } \\
2.5)^{147}\end{array}$ & $\begin{array}{l}2 \text { cohorts, } \\
\mathrm{N}=3,787^{146,147}\end{array}$ & $\begin{array}{l}\text { High study } \\
\text { limitations } \\
\text { (high risk of } \\
\text { bias }^{147} \text { ), } \\
\text { imprecise, } \\
\text { consistent }\end{array}$ & Insufficient \\
\hline $\begin{array}{l}\text { Pregnant } \\
\text { women in } \\
\text { second or } \\
\text { third } \\
\text { trimester }{ }^{146} \text { or } \\
\text { during } \\
\text { pregnancy }{ }^{147} \\
\text { exposed to } \\
\text { citalopram or } \\
\text { sertraline }\end{array}$ & $\begin{array}{l}\text { Maternal } \\
\text { exposure to } \\
\text { citalopram vs. } \\
\text { maternal } \\
\text { exposure to } \\
\text { sertraline }\end{array}$ & $\begin{array}{l}\text { Autism } \\
\text { spectrum } \\
\text { disorder }\end{array}$ & $\begin{array}{l}5 / 421(1.2 \%) \\
\text { vs. } 1 / 292 \\
(0.34 \%) ; 146 \\
14 / 1,751 \\
(0.8 \%) \text { vs. } \\
9 / 1,576 \\
(0.57 \%)^{147}\end{array}$ & $\begin{array}{l}\text { RR, } 3.5(95 \% \\
\mathrm{Cl}, 0.41 \text { to } \\
29.5){ }^{146} \\
\mathrm{RR}, 1.4(95 \% \\
\mathrm{Cl}, 0.61 \text { to } \\
3.2)^{147}\end{array}$ & $\begin{array}{l}2 \text { cohorts, } \\
\mathrm{N}=4,040^{146,147}\end{array}$ & $\begin{array}{l}\text { High study } \\
\text { limitations } \\
\text { (high risk of } \\
\text { bias }^{146,147} \text { ), } \\
\text { imprecise, } \\
\text { consistent }\end{array}$ & Insufficient \\
\hline
\end{tabular}

\section{Congenital Anomalies for SSRIs Versus TCAs}

Three studies ${ }^{141,144,145}$ reported results that suggest higher relative risks for anomalies with SSRIs compared with TCAs, with wide CIs suggestive of both benefits and harms. No study presented adjusted relative risks, leading to high study limitations and an insufficient grade.

\section{Preterm Births for SSRIs Versus TCAs}

Two studies ${ }^{141,142}$ reported inconsistent results for preterm births for SSRIs compared with TCAs, with wide CIs suggestive of both benefits and harms. No study presented adjusted relative risks, leading to high study limitations and an insufficient grade.

\section{Preterm Births for Mirtazapine Versus SSRIs}

Two studies ${ }^{141,142}$ reported inconsistent results for preterm births for mirtazapine compared with SSRIs, with wide CIs suggestive of both benefits and harms. No study presented adjusted relative risks, leading to high study limitations and an insufficient grade.

\section{Small for Gestational Age for Venlafaxine Versus SSRIs}

Two studies ${ }^{113,140}$ reported inconsistent results for the risks of small for gestational age with venlafaxine versus SSRIs, with wide CIs suggestive of both benefits and harms. One study reported higher but nonsignificant risks for unadjusted results for venlafaxine, ${ }^{113}$ and a second reported lower but adjusted odds risks for venlafaxine. Inconsistency and imprecision from wide CIs in studies with small samples and events resulted in an insufficient grade. 


\section{Birthweight for SSRIs Versus TCAs}

Two studies ${ }^{142,144}$ reported results that suggest higher relative risks for low birthweight with SSRIs compared with TCAs, with wide CIs suggestive of both benefits and harms. No study presented adjusted relative risks, leading to high study limitations and an insufficient grade.

\section{Birthweight for Mirtazapine Versus SSRIs}

Two studies ${ }^{112,143}$ reported no significant differences in birthweight with mirtazapine versus SSRIs, with wide CIs suggestive of both benefits and harms. No study presented adjusted relative risks, leading to high study limitations and an insufficient grade.

\section{Autism Spectrum Disorder for Head-to-Head SSRI Comparisons}

Two studies ${ }^{146,147}$ reported results on autism spectrum disorder for paroxetine versus sertraline; sertraline versus fluoxetine; fluoxetine monotherapy versus fluvoxamine or escitalopram; paroxetine versus fluoxetine, sertraline versus fluoxetine, citalopram versus fluoxetine, citalopram versus paroxetine, and citalopram versus sertraline. No study presented adjusted relative risks, leading to high study limitations and an insufficient grade.

\section{No Evidence in Populations of Interest (Pregnant Women With Mental Health Disorders)}

\section{Overview}

- We found no evidence on active treatment in comparison with anxiolytics, hypnotic sedatives, and brexanolone.

- We found no evidence on maternal harms of infertility, abruption, glucose intolerance, and reduced milk production or undesired weaning.

- We also found no evidence on child harms of persistent pulmonary hypertension, poor infant attachment/bonding, anxiety in children, and depression. 


\section{Discussion}

\section{Findings in Relation to the Decisional Dilemma}

The central decisional dilemma facing pregnant and postpartum women with mental health disorders and their healthcare providers is how to balance benefits and harms of psychotropic drugs for themselves and their children. One such critical trade-off is whether improved symptoms in the mother outweigh the harms from potential congenital anomalies in the fetus. The available evidence from studies of pregnant or postpartum women with mental health disorders does little to clarify these issues. Despite the high reported prevalence for any mental health disorders among pregnant women $\left(25.3 \%\right.$ in the United States in 2001 to $\left.2002^{8}\right)$, the evidence is very sparse on the benefits of treatment, mostly reflecting how little controlled, highquality research this particular area has received. Indeed, only 10 randomized controlled trials involving psychotropic medication for any mental health disorder were eligible for this review. This limited evidence base reflects decades of exclusion of pregnant and lactating women from clinical trials. ${ }^{29}$ In contrast, evidence on harms of treatment is voluminous but of low quality primarily because of the inability to control for confounding.

Regarding benefits of treatment (Key Question [KQ] 1), the absence of evidence on the efficacy of many psychotropic drugs in pregnancy or in the postpartum period should not be interpreted as an absence of their benefit. For symptom response, for example, we found signals of benefits for symptom response for sertraline and brexanolone: for both drugs, we found evidence bases with two or more randomized controlled trials (RCTs). For fluoxetine and paroxetine, one small RCT apiece with imprecise results was not sufficient to arrive at a conclusion. For all other drugs, we found no eligible studies; evidence may be available from studies of other populations ineligible for this review.

Substantial evidence exists on the efficacy of psychotropic medications across a broad spectrum of persons with mental health disorders. Systematic reviews in the general population have found evidence of benefit for several pharmacologic agents for anxiety, depression, bipolar disorder, and schizophrenia. For example, an evidence-based treatment review on pharmacotherapy for generalized anxiety disorder, published in 2018, summarized findings from RCTs with adults across classes of treatment options. ${ }^{149}$ The authors concluded that evidence of efficacious treatment options include selective serotonin reuptake inhibitors (SSRIs) (paroxetine and escitalopram), serotonin-norepinephrine reuptake inhibitors (SNRIs) (venlafaxine and duloxetine), benzodiazepines, and tricyclic antidepressants (TCAs).

In adults with major depressive disorder, multiple systematic evidence reviews have demonstrated the efficacy of all classes of antidepressants, including second-generation antidepressants (SSRIs, serotonin modulators, SNRIs, bupropion, and mirtazapine) and TCAs compared with placebo. ${ }^{150-152}$ There is not a clear consensus on whether there are sex differences in antidepressant efficacy, likely due to variability in methodology and few specific investigations addressing this question. ${ }^{153}$ Some studies have shown that men experience a better therapeutic response to TCAs than women, while others show that women respond better to SSRI treatment than men.

A recent systematic review on treatments for bipolar disorder, published in 2018, found modest evidence of benefit for several drugs. ${ }^{154}$ This review synthesized evidence from 108 studies for 28 drugs. The review found that lithium, asenapine, cariprazine, olanzapine, quetiapine, risperidone, and ziprasidone may modestly improve acute mania symptoms in adults with bipolar I disorder when compared with placebo. Although eligible studies included men and 
women, the review did not find sufficient evidence to be able to ascertain effects specific to women but noted that the prevalence is similar in men and women.

Regarding schizophrenia, numerous guidelines consistently recommend antipsychotic medication for maintenance of remission following a first episode of schizophrenia and clozapine for treatment resistance. ${ }^{155}$ Some evidence suggested better response among women than men. ${ }^{156}$

In the absence of evidence comparing benefits from pharmacotherapy in pregnant or postpartum women with general populations, the results from general populations may be interpreted as applicable to pregnant and postpartum women. However, doses of medication may need to be adjusted to maintain comparable blood levels of medication given the physiological changes in blood volume and other pharmacokinetic considerations during pregnancy. ${ }^{157,158}$

When evidence from studies of pregnant and postpartum women was available, we found low to moderate strength of evidence of benefit. Specifically, for depression, we found moderate strength of evidence that brexanolone is associated with improved depressive symptoms shortly after infusion (60 hours) and at 30 days after treatment. Moreover, the substantial reduction in symptoms in the placebo group illustrates the importance of restorative sleep and material support for management of postpartum depression symptoms. However, the mode of delivery of the drug (infusion), requirements for healthcare personnel to be present for the duration of the infusion, and cost may serve as barriers to widespread use of brexanolone. We found low strength of evidence from two RCTs that sertraline achieves response, remission, and improvements in symptoms of depression and anxiety. For bipolar disorders, we found low strength of evidence from two cohort studies that discontinuing mood stabilizers increases recurrence and reduces time to recurrence. Other than one small study for hydroxyzine, we found no information on the efficacy of anxiolytics or sedative-hypnotics during the perinatal period and nothing on the efficacy of antipsychotics.

Regarding harms of treatment (KQ 3), we restricted the review to studies comparing women receiving psychotropic drugs with women with a mental health disorder who were not receiving psychotropic drugs. This requirement narrowed the pool of eligible evidence to studies that made some attempt to control for this possible source of underlying confounding, but the risk of residual confounding in this literature is substantial: no observational study could completely control for severity of psychiatric illness that could have predicted both exposure and outcome.

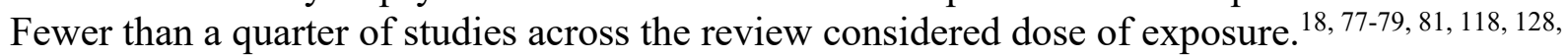
133, 143, 159-177 This limited information on dose of exposure further complicates the extent to which inferences can be drawn between the stated exposure and the outcome. Nonetheless, within this pool of evidence, we found some signals of harms for a limited set of exposures; all were rated as low strength of evidence. Our low confidence that future results will confirm these findings stems from multiple considerations: limited precision for rare outcomes, high study limitations primarily from an inability to address confounding fully, and concerns about exposure and outcome measurement. We note that our findings pertain to the population eligible for our review; evidence on the harms of these drugs in broader populations (such as nonpregnant populations and populations without mental health disorders) will also be relevant for clinical practice. These evidence bases may form the basis, for example, for the Food and Drug Administration (FDA) warnings on some psychotropic drugs.

Specifically, for maternal harms, we found evidence that several antidepressants are associated with increased risk of postpartum hemorrhage; SNRIs and TCAs may be associated with an increased risk of preeclampsia; and quetiapine or olanzapine may be associated with an 
increased risk of gestational diabetes. For child harms, we found an association between benzodiazepine and neonatal intensive care unit admissions; between SSRIs and respiratory issues, low Apgar scores, persistent pulmonary hypertension of the newborn, and depression in children; and between citalopram and autism spectrum disorder, although the studies were not able to fully control for confounding. Importantly, we found insufficient evidence on congenital anomalies and cardiac defects for most comparisons, although we did find some evidence of increased cardiac defects for paroxetine.

These findings need to be interpreted in the context of low absolute risks for several outcomes. For example, recent systematic reviews also found an increased risk of persistent pulmonary hypertension with SSRIs and SNRIs but noted the very high numbers of patients needed to harm, ranging from 286 to $1,000 .{ }^{178,179}$ Similarly, the study reporting higher rates of postpartum hemorrhage notes low absolute risk differences, with a number needed to harm ranging from 80 to $100 .^{84}$

The findings are consistent with other prior work on this topic. A 2009 report came to the same conclusions; ${ }^{180}$ unfortunately, our review indicates that the state of the science has not advanced substantially. This narrow evidence base is not limited to mental health disorders; a 2018 report to Congress detailed deficiencies in the evidence base and outlined strategies to improve the quantity, quality, and timeliness of research on safety and efficacy of therapeutic products used by pregnant women and lactating women. ${ }^{31}$

The findings on comparative benefits (KQ 2) and harms (KQ 4) were very sparse. Results on numerous comparisons yielded insufficient evidence to judge comparative benefits or harms, with one exception: results from one study suggested that exposure to lithium may be more likely to be associated with overall congenital anomalies and cardiac anomalies than exposure to lamotrigine. As with the results on comparative efficacy, the paucity or absence of evidence in this population cannot be interpreted as lack of benefit. Systematic reviews in nonpregnant populations suggested that different classes of antidepressants may be similar in benefits. ${ }^{151}$ Systematic reviews on benefits of medications for these disorders in the general population offer some insights on comparative effectiveness. Regarding anxiety, one review ${ }^{149}$ reported studies evaluating the benefits of paroxetine and other SSRIs. In one study, no difference in response or remission was found in relation to sertraline, ${ }^{181}$ and in a second, no difference was found in relation to escitalopram. ${ }^{182}$ Based on a recent meta-analysis, benzodiazepines were found to be more effective than SSRIs and SNRIs, ${ }^{183}$ and a Cochrane review found that benzodiazepines were more effective than azapirones. ${ }^{184}$

Regarding comparative effectiveness for depression, multiple comparative reviews have shown no clear benefit of one antidepressant versus any other for pharmacologic treatment of depression, although medications do differ by measures such as adherence and tolerability. ${ }^{150,151 \text {, }}$ 185 The types of antidepressants involved both direct and indirect comparisons of secondgeneration antidepressants (SSRIs, serotonin modulators, SNRIs, bupropion, and mirtazapine) and TCAs compared with each other in adults with major depressive disorder.

Regarding comparative effectiveness of treatments for bipolar disorder, one review found low strength of evidence that olanzapine and divalproex/valproate are not different in their effect on acute mania. ${ }^{154}$ The review authors also found that lithium improved acute mania better than topiramate (low strength of evidence). The review found insufficient evidence for all other drug comparisons with active controls.

Reviews have found that antipsychotics are similar in function, quality of life, and mortality. ${ }^{186,187}$ One report also noted that core illness symptoms improved more with 
olanzapine and risperidone than asenapine, quetiapine, and ziprasidone and with paliperidone than lurasidone. ${ }^{186}$ The review generally found insufficient evidence of differences in benefits by sex.

Our review findings do support some currently existing clinical guidelines. For example, our findings support clinical guidelines ${ }^{26,33,36,188,189}$ suggesting the need to consider the balance between risk and benefits in deciding whether to continue treatment and the use of medications for symptoms or when relapse is of concern. Our review is also consistent with current guidelines suggesting that antidepressants are relatively safe but may carry potential neonatal side effects. Guidelines have not yet offered recommendations for brexanolone and express uncertainty regarding lamotrigine: our findings may contribute to filling these gaps.

Some guidelines are silent on whether to continue exposure to medications during pregnancy. ${ }^{33}$ Our review of the contextual evidence on this question (harms of not treating the disorder, stopping a pharmacologic treatment, or switching medications) offers some clarity for the use of mood stabilizers for bipolar disorder and continued uncertainty for the use of antidepressants for depression. Two studies of women with bipolar disorder supported continued use of mood stabilizers to reduce recurrence and increase time to recurrence. ${ }^{80,81}$ A third study noted a higher rate of relapse among postpartum women who switched from lithium to sodium valproate to breastfeed (4/7) than among women who stayed on lithium (1/9); however, small numbers limit our ability to make conclusions. ${ }^{190}$ The evidence for depression is mixed. Three studies compared evaluated outcomes for those discontinuing medications with those continuing medications through pregnancy. One study of Medicaid claims data from 28,493 women found higher odds of relapse with continuation (odds ratio, 2.0; 95\% confidence interval [CI], 1.8 to 2.2) (authors note that this result could be explained by systematic biases but could not rule out the possibility that antidepressant medications are less effective in pregnancy). ${ }^{191}$ A second study of 778 women from 137 obstetrical practices and hospital-based clinics found an adjusted hazard ratio of 0.88 (95\% CI, 0.51 to 1.5$)$ of onset of a major depressive episode in pregnancy and after delivery. ${ }^{188}$ A third study of 201 women, drawing from three centers with specific expertise in treating psychiatric illness, found an increased risk of relapse with discontinuation (hazard ratio, 5.0; 95\% CI, 2.8 to 9.1). ${ }^{169}$ Potential explanation for these differences may lie in the severity of disorders in each population, difference in the type of care offered to the patient populations, or the limitations of claims data to fully capture the context around the decision to discontinue medications. ${ }^{188,191,192}$ One study of duloxetine, prescribed primarily for depression and anxiety, did not report a difference between duloxetine continuers and duloxetine discontinuers for the risk of any congenital malformations (adjusted relative risk, 1.09; 95\% CI. 0.84 to 1.42 ) or cardiovascular anomalies (adjusted relative risk, $1.41 ; 95 \% \mathrm{CI}, 0.92$ to 2.17 ). ${ }^{124}$

Some guidelines note uncertainty about how to treat new-onset psychiatric illness during the perinatal period and the implications of treatment during lactation. ${ }^{26,36}$ We did not identify any studies of treatment for new-onset disorders or for lactating women.

\section{Strengths and Limitations}

As noted above, a significant constraint to interpreting the evidence is the lack of data from RCTs and the widespread risk of confounding in observational studies. Underlying mental health disorders result in the use of psychotropic medications. They may also result in some of the harms investigated in this review regardless of exposure to medications. One strength of our review is that we required, for the effectiveness questions (KQs 1 and 3), that studies include comparison groups defined by exposure to a mental health disorder; however, it is likely that 
illness severity is correlated with pharmacologic treatment, confounding observed associations. We also required that studies made some attempt to adjust for confounding; this helped manage the scope and heterogeneity of the review but resulted in some limitations. Studies varied greatly in the extent to which they were able to address underlying severity of psychiatric illness. The majority were unable to address confounding adequately because they could not control for severity of psychiatric illness. A subset of studies attempted measures at addressing confounding such as multivariate regression with propensity score adjustment and stratification by number of disorders. In most instances, controls for confounding reduced the effect size and, in some instances, reversed the direction of effect.

For the benefits question (KQ 1), eligible studies had comparator arms of women with the same disorder as in the treatment arm. For the harms question (KQ 3), however, we were more inclusive and included studies with comparator arms comprising women with prior exposure to the drug, even if the disorder status was not specified. As a result, our KQ 1 evidence base controls for underlying severity as a confounder better than the KQ 3 evidence base.

We elected to restrict the evidence to women with mental health disorders as a means of reducing the potential for confounding in the evidence base. However, this criterion excluded studies of well-conducted negative controls that might bolster the evidence on the association between the exposure and the outcome. Also, this criterion resulted in the exclusion of studies reporting on relevant outcomes for exposures to the intervention for other clinical conditions. Studies of multiple drug exposures presented results for each exposure but did not always present results separately for the women with multiple drug exposures. In these studies with overlapping arms, we were not able to attribute the effect of the intervention to a single drug. As a result, we excluded these studies. The exclusion of studies with overlapping arms also restricted the comprehensiveness of our review. These limitations of the evidence and of our review mean that the signals of harms that we identified above may be partially or wholly attributable to residual confounding. A further consequence of our inclusion criteria is that we did not find eligible evidence on harms of some psychotropic drugs compared with no treatment for which FDA has issued warnings, such valproate, alprazolam, carbamazepine, topiramate, clonazepam, and triazolam. These evidence bases may draw on literature outside the parameters of our review, such as single-arm studies or cohorts of women exposed to psychotropic drugs, but either not pregnant or not with a mental health disorder.

Another limitation of the evidence pertains to common data sources and resultant issues with measurement of exposures and comparators. Rare outcomes and harms are difficult to identify prospectively; the use of registries for prospectively collected data obviates that problem but creates several others. Healthcare utilization databases studies often defined exposure as having a prescription. Some studies conducted sensitivity analyses with more than one prescription. Although this approach offers a proxy for adherence, it cannot fully address the concern that prescriptions, particularly during pregnancy, may not wholly represent exposure to the drug. Few studies reported dose or duration of exposure. Registry studies that attempted to address confounding generally defined the comparison arm as unexposed to the drug in pregnancy but with a mental health disorder. In some cases, the mental health disorder was further defined as depression or anxiety; in other cases, any history of mental health disorders allowed women to qualify for the comparison arm. Such broad inclusions for psychiatric history and duration of the lookback period allow for greater precision from amassing large sample sizes in the comparison arm but also increase the risk of unmeasured confounding. Another concern relates to the absence of information on participation in psychotherapy or other psychological interventions in 
the unexposed arm. The likely impact of poorly defined exposures and comparators further serves to weaken our confidence in the findings.

We may also have missed eligible studies because of our restriction to English language studies.

\section{Applicability}

The results from this review are generally applicable to populations of pregnant women with the same mental health condition as women included in the individual studies that comprise the body of evidence for each drug-treatment outcome. However, the criteria used to identify specific mental health conditions differed across studies and few studies controlled for baseline clinical severity. Therefore, we cannot comment on the additional effect, if any, of particular symptoms of a condition and whether the severity of the condition in the study populations would be similar to that in other similarly diagnosed patient populations. Criteria used to identify specific conditions differed across studies, and few studies controlled for baseline clinical severity.

Often studies determined receipt of pharmacotherapy through pharmacy and medical records of prescriptions filled. Some of these studies included women as receiving treatment with as few as one filled prescription, resulting in findings being limited to any possible exposure versus none. Other studies included a larger number of prescriptions to be filled, resulting in greater confidence that exposure occurred and continued over time. However, determining a specific level of exposure is further limited by adherence not being measured in studies. Also, some drugs are reported by class and the results may not extrapolate to all of the individual drugs in the class.

Most studies were conducted in majority-white populations and may not generalize to other races or ethnicities. Similarly, access to care may differ in U.S. versus non-U.S. cohorts, resulting in differences in outcomes between the populations that cannot be measured. Few studies reported on concurrent or recent psychological or behavioral treatment so that benefits of treatment cannot differentiate between efficacy and augmentation.

\section{Implications for Clinical Practice, Education, Research, or Health Policy}

\section{Implications for Clinical Practice and Education}

Pregnant women often seek information outside of the clinical context on psychotropic medications; information shared on popular internet message boards may be inaccurate, contradictory, or judgmental. ${ }^{193}$ Clinicians and health communicators can use the findings from our review as an evidence-based source to inform and educate patients.

Although our results do not substantially add to the evidence base on benefits, we did not find any evidence that benefit would be different (or similar) in pregnant populations. We found some evidence of associations with maternal and child harms but note that these may be explained by residual confounding, rather than by either exposure to the drug. Some harms are self-limiting (as in the case of Apgar scores, which assess immediate need for resuscitation and do not predict individual neonatal mortality or neurologic outcome ${ }^{106}$ ) or very rare (as in the case of persistent pulmonary hypertension or postpartum hemorrhage). The absolute risks of these harms should be included when considering the balance of benefits and harms. Table 17 
summarizes the absolute risks for some of the outcomes evaluated in this report. Additionally, the risks of proceeding without treatment should be compared with the specific pharmacologic treatment being considered. Given the well-documented maternal and fetal risks of untreated maternal mental health disorders, clinicians should consider the possible adverse effects of pharmacologic treatments in contrast with the known adverse effects of untreated psychiatric illness (rather than when the patient is without any mental health disorders and not in need of treatment). The severity of the patient's mental health disorder should be carefully considered. Depending on the severity of the mental health disorder, maternal and fetal risks of foregoing treatment may change significantly.

Given these complexities, a shared decision-making framework is essential for clinical decision making. Shared decision making includes at least two experts: the patient, who is an expert on her life experience and preferences, and the provider, who is an expert in clinical care. ${ }^{194,195}$ The provider's role is to elicit the patient's personal values and preferences and risk tolerance and share relevant clinical knowledge to inform the decision-making process. During patient counseling, the clinician should inform the patient about the limitations of current research on the topic of pharmacologic treatments for pregnant or postpartum women with mental health disorders. To provide informed consent to initiate or to forgo treatment, patients need to understand that the evidence base is limited and insufficient to provide a strong recommendation. Under these circumstances, a patient's values and preferences and her unique clinical circumstances become even more important in the decision-making process.

Warfarin provides an example of how important it is to consider the severity of maternal disease when making decisions about pharmacologic treatments during pregnancy. Warfarin is a well-known teratogen, and use is generally avoided during pregnancy. However, in those women at exceptionally high risk of thromboembolic events, such as those with mechanical heart valves, warfarin is the most effective anticoagulant, and this maternal risk reduction may outweigh the fetal risks in certain patients. As is the case with psychiatric illness, the balance of benefits and risks shifts significantly depending on the severity of maternal disease, and patients' individual values and preferences must inform such complex decision making.

These complex decisions require shared decision-making tools that clinicians and patients can use to jointly make informed decisions.

Table 17. Absolute risk differences for key outcomes

\begin{tabular}{|c|c|c|c|c|c|c|c|c|}
\hline Population & Outcome* & Exposure & Comparator & $\mathbf{N}$ & $\begin{array}{l}\text { ARD } \\
\text { per } \\
1000 \\
\text { Women }\end{array}$ & $\begin{array}{l}95 \% \\
\text { Lower } \\
\text { CI }\end{array}$ & $\begin{array}{l}95 \% \\
\text { Upper } \\
\text { Cl }\end{array}$ & $\begin{array}{l}\text { Key } \\
\text { Considerations }\end{array}$ \\
\hline $\begin{array}{l}\text { Women with } \\
\text { postpartum } \\
\text { depression }\end{array}$ & Response & Sertraline & Placebo & 36 & 326 & 11 & 1116 & $\begin{array}{l}\text { RCT evidence, } \\
\text { small sample, } \\
\text { imprecise results }\end{array}$ \\
\hline $\begin{array}{l}\text { Women with } \\
\text { postpartum } \\
\text { depression }\end{array}$ & Remission & Sertraline & Placebo & 36 & 320 & 4 & 619 & $\begin{array}{l}\text { RCT evidence, } \\
\text { small sample, } \\
\text { imprecise results }\end{array}$ \\
\hline $\begin{array}{l}\text { Women with bipolar } \\
\text { disorder }\end{array}$ & $\begin{array}{l}\text { Recurrence } \\
\text { from } \\
\text { discontinuation }\end{array}$ & $\begin{array}{l}\text { Discontinued } \\
\text { mood } \\
\text { stabilizers }\end{array}$ & $\begin{array}{l}\text { Continued } \\
\text { mood } \\
\text { stabilizers }\end{array}$ & 89 & 268 & 56 & 486 & $\begin{array}{l}\text { Observational } \\
\text { evidence, small } \\
\text { sample, imprecise } \\
\text { results }\end{array}$ \\
\hline $\begin{array}{l}\text { Women with bipolar } \\
\text { disorder }\end{array}$ & $\begin{array}{l}\text { Time-to-25\%- } \\
\text { recurrence } \\
\text { from } \\
\text { discontinuation }\end{array}$ & $\begin{array}{l}\text { Discontinued } \\
\text { mood } \\
\text { stabilizers }\end{array}$ & $\begin{array}{l}\text { Continued } \\
\text { lamotrigine }\end{array}$ & 26 & 687 & 135 & 700 & $\begin{array}{l}\text { Observational } \\
\text { evidence, study } \\
\text { has limitations, } \\
\text { small sample, } \\
\text { imprecise results }\end{array}$ \\
\hline
\end{tabular}




\begin{tabular}{|c|c|c|c|c|c|c|c|c|}
\hline Population & Outcome* & Exposure & Comparator & $\mathbf{N}$ & $\begin{array}{l}\text { ARD } \\
\text { per } 100 \\
\text { Women }\end{array}$ & $\begin{array}{l}95 \% \\
\text { Lower } \\
\text { Cl }\end{array}$ & $\begin{array}{l}95 \% \\
\text { Upper } \\
\text { Cl }\end{array}$ & $\begin{array}{l}\text { Key } \\
\text { Considerations }\end{array}$ \\
\hline $\begin{array}{l}\text { Women with at least } \\
\text { one anxiety diagnosis } \\
\text { in the year before } \\
\text { conception }\end{array}$ & $\begin{array}{l}\text { Ectopic } \\
\text { pregnancy }\end{array}$ & $\begin{array}{l}\text { Benzodiaz- } \\
\text { epine } \\
\text { exposure } 90 \\
\text { days before } \\
\text { conception }\end{array}$ & $\begin{array}{l}\text { No } \\
\text { benzodiaz- } \\
\text { epine } \\
\text { exposure } \\
\text { before } \\
\text { conception }\end{array}$ & 90479 & 7 & 4 & 11 & $\begin{array}{l}\text { Observational } \\
\text { evidence, potential } \\
\text { for residual } \\
\text { confounding }\end{array}$ \\
\hline $\begin{array}{l}\text { Women with mood or } \\
\text { anxiety disorder }\end{array}$ & $\begin{array}{l}\text { Postpartum } \\
\text { hemorrhage }\end{array}$ & $\begin{array}{l}\text { Exposed to } \\
\text { SSRIs during } \\
\text { delivery }\end{array}$ & $\begin{array}{l}\text { Unexposed to } \\
\text { SSRIs during } \\
\text { delivery }\end{array}$ & 81754 & 13 & 9 & 17 & $\begin{array}{l}\text { Observational } \\
\text { evidence, potential } \\
\text { for residual } \\
\text { confounding }\end{array}$ \\
\hline $\begin{array}{l}\text { Women with mood or } \\
\text { anxiety disorder }\end{array}$ & $\begin{array}{l}\text { Postpartum } \\
\text { hemorrhage }\end{array}$ & $\begin{array}{l}\text { Exposed to } \\
\text { SNRIs during } \\
\text { delivery }\end{array}$ & $\begin{array}{l}\text { Unexposed to } \\
\text { SNRIs during } \\
\text { delivery }\end{array}$ & 69746 & 25 & 10 & 45 & $\begin{array}{l}\text { Observational } \\
\text { evidence, potential } \\
\text { for residual } \\
\text { confounding }\end{array}$ \\
\hline $\begin{array}{l}\text { SNRI exposure or } \\
\text { depression diagnosis, } \\
\text { through second } \\
\text { trimester }\end{array}$ & Preeclampsia & $\begin{array}{l}\text { SNRIs } \\
\text { exposure } \\
\text { through } \\
\text { second } \\
\text { trimester }\end{array}$ & $\begin{array}{l}\text { Unexposed } \\
\text { depressed }\end{array}$ & 65800 & 14 & 6 & 49 & $\begin{array}{l}\text { Observational } \\
\text { evidence, potential } \\
\text { for residual } \\
\text { confounding }\end{array}$ \\
\hline $\begin{array}{l}\text { Women with } \\
\text { depression }\end{array}$ & Preeclampsia & $\begin{array}{l}\text { Exposed to } \\
\text { TCAs in } \\
\text { pregnancy }\end{array}$ & $\begin{array}{l}\text { Unexposed to } \\
\text { TCAs in } \\
\text { pregnancy }\end{array}$ & 65538 & 54 & 21 & 110 & $\begin{array}{l}\text { Observational } \\
\text { evidence, potential } \\
\text { for residual } \\
\text { confounding }\end{array}$ \\
\hline $\begin{array}{l}\text { Women prescribed } \\
\text { second-generation } \\
\text { antipsychotic }\end{array}$ & $\begin{array}{l}\text { Gestational } \\
\text { diabetes }\end{array}$ & $\begin{array}{l}\text { Quetiapine } \\
\text { continued in } \\
\text { pregnancy }\end{array}$ & $\begin{array}{l}\text { Quetiapine } \\
\text { discontinued } \\
\text { in pregnancy }\end{array}$ & 4533 & 11 & $<1$ & 25 & $\begin{array}{l}\text { Observational } \\
\text { evidence, potential } \\
\text { for residual } \\
\text { confounding } \\
\end{array}$ \\
\hline $\begin{array}{l}\text { Women prescribed } \\
\text { second-generation } \\
\text { antipsychotic }\end{array}$ & $\begin{array}{l}\text { Gestational } \\
\text { diabetes }\end{array}$ & $\begin{array}{l}\text { Olanzapine } \\
\text { continued in } \\
\text { pregnancy }\end{array}$ & $\begin{array}{l}\text { Olanzapine } \\
\text { discontinued } \\
\text { in pregnancy }\end{array}$ & 1425 & 29 & 6 & 61 & $\begin{array}{l}\text { Observational } \\
\text { evidence, potential } \\
\text { for residual } \\
\text { confounding }\end{array}$ \\
\hline $\begin{array}{l}\text { Women with } \\
\text { depression or anxiety }\end{array}$ & $\begin{array}{l}\text { Spontaneous } \\
\text { abortion }\end{array}$ & $\begin{array}{l}\text { Benzodiaz- } \\
\text { epine } \\
\text { exposure in } \\
\text { 1st trimester }\end{array}$ & $\begin{array}{l}\text { Unmedicated } \\
\text { mental illness }\end{array}$ & 6031 & 73 & 36 & 109 & $\begin{array}{l}\text { Observational } \\
\text { evidence, potential } \\
\text { for residual } \\
\text { confounding } \\
\end{array}$ \\
\hline $\begin{array}{l}\text { Women with SNRI } \\
\text { exposure or } \\
\text { depression diagnosis } \\
\text { in past } 4 \text { years }\end{array}$ & $\begin{array}{l}\text { Spontaneous } \\
\text { abortion }\end{array}$ & $\begin{array}{l}\text { SNRI } \\
\text { exposure in } \\
\text { 1st trimester }\end{array}$ & $\begin{array}{l}\text { Unexposed } \\
\text { with } \\
\text { depression } \\
\text { diagnosis in } \\
\text { past } 4 \text { years }\end{array}$ & 9014 & 62 & 16 & 130 & $\begin{array}{l}\text { Observational } \\
\text { evidence, potential } \\
\text { for residual } \\
\text { confounding }\end{array}$ \\
\hline $\begin{array}{l}\text { Women with a mental } \\
\text { health disorder }\end{array}$ & $\begin{array}{l}\text { NICU } \\
\text { admission }\end{array}$ & $\begin{array}{l}\text { Benzodiaz- } \\
\text { epine } \\
\text { exposure } \\
\text { during } \\
\text { pregnancy }\end{array}$ & $\begin{array}{l}\text { Unexposed to } \\
\text { benzodiaz- } \\
\text { epine during } \\
\text { pregnancy }\end{array}$ & 793 & 133 & 17 & 274 & $\begin{array}{l}\text { Observational } \\
\text { evidence, potential } \\
\text { for residual } \\
\text { confounding, } \\
\text { benzodiazepine } \\
\text { exposure may } \\
\text { result in NICU } \\
\text { admission by } \\
\text { policy }\end{array}$ \\
\hline
\end{tabular}




\begin{tabular}{|c|c|c|c|c|c|c|c|c|}
\hline Population & Outcome* & Exposure & Comparator & $\mathbf{N}$ & $\begin{array}{l}\text { ARD } \\
\text { per } 100 \\
\text { Women }\end{array}$ & $\begin{array}{l}95 \% \\
\text { Lower } \\
\text { Cl }\end{array}$ & $\begin{array}{l}95 \% \\
\text { Upper } \\
\text { Cl }\end{array}$ & $\begin{array}{l}\text { Key } \\
\text { Considerations }\end{array}$ \\
\hline $\begin{array}{l}\text { Women exposed to } \\
\text { SSRIs or unexposed } \\
\text { with a psychiatric } \\
\text { diagnosis }\end{array}$ & Apgar score & $\begin{array}{l}\text { Exposed to } \\
\text { SSRIs during } \\
\text { pregnancy }\end{array}$ & $\begin{array}{l}\text { Exposed to } \\
\text { SSRIs before } \\
\text { pregnancy or } \\
\text { unexposed with } \\
\text { a psychiatric } \\
\text { diagnosis }\end{array}$ & 25,381 & 8 & 4 & 13 & $\begin{array}{l}\text { Observational } \\
\text { evidence, } \\
\text { potential for } \\
\text { residual } \\
\text { confounding, } \\
\text { transient outcome }\end{array}$ \\
\hline $\begin{array}{l}\text { Women with } \\
\text { depression }\end{array}$ & $\begin{array}{l}\text { Persistent } \\
\text { pulmonary } \\
\text { hypertension } \\
\text { of the newborn }\end{array}$ & $\begin{array}{l}\text { Exposed to } \\
\text { SSRIs during } \\
\text { pregnancy }\end{array}$ & $\begin{array}{l}\text { Unexposed to } \\
\text { SSRIs during } \\
\text { pregnancy }\end{array}$ & $\begin{array}{l}621 \\
399\end{array}$ & $<1$ & $<1$ & $<1$ & $\begin{array}{l}\text { Observational } \\
\text { evidence, } \\
\text { potential for } \\
\text { residual } \\
\text { confounding } \\
\end{array}$ \\
\hline $\begin{array}{l}\text { Women with a mental } \\
\text { health disorder }\end{array}$ & $\begin{array}{l}\text { Childhood } \\
\text { depression }\end{array}$ & $\begin{array}{l}\text { Exposed to } \\
\text { SSRIs during } \\
\text { pregnancy }\end{array}$ & $\begin{array}{l}\text { Unexposed to } \\
\text { SSRIs during } \\
\text { pregnancy }\end{array}$ & 25,380 & 2 & $<1$ & 6 & $\begin{array}{l}\text { Observational } \\
\text { evidence, } \\
\text { potential for } \\
\text { residual } \\
\text { confounding } \\
\end{array}$ \\
\hline $\begin{array}{l}\text { Women with a mental } \\
\text { health disorder }\end{array}$ & $\begin{array}{l}\text { Autism } \\
\text { spectrum } \\
\text { disorder } \\
\text { without } \\
\text { intellectual } \\
\text { disabilities }\end{array}$ & $\begin{array}{l}\text { Exposed to } \\
\text { citalopram } \\
\text { during } \\
\text { pregnancy }\end{array}$ & $\begin{array}{l}\text { Unexposed to } \\
\text { any } \\
\text { antidepressants } \\
\text { during } \\
\text { pregnancy }\end{array}$ & 13,389 & 17 & 6 & 32 & $\begin{array}{l}\text { Observational } \\
\text { evidence, } \\
\text { potential for } \\
\text { residual } \\
\text { confounding }\end{array}$ \\
\hline
\end{tabular}

$\overline{\mathrm{ARD}}=$ absolute risk difference $\mathrm{CI}=$ confidence interval; $\mathrm{NICU}=$ neonatal intensive care unit; $\mathrm{RCT}=$ randomized controlled trial; SNRI = serotonin-norepinephrine reuptake inhibitor; SSRI $=$ selective serotonin reuptake inhibitor; TCA $=$ tricyclic antidepressant.

* Outcomes selected from evidence bases rated as low or moderate strength of evidence The ARD was calculated based on adjusted effect sizes when available. When multiple studies were available on harms, we selected cohort studies with the highest effect size to present the outer bound for harms.

\section{Implications for Research}

The paucity of definitive evidence on this topic offers a vast expanse of new opportunities. Clinical trials offer the greatest rigor, but feasibility and ethics have constrained their use. There is an emerging consensus that routine exclusion of pregnant and lactating women from clinical trials has harmful consequences, forcing patients and providers to make clinical decisions in the absence of evidence. Even when providers infer efficacy for pregnant women from studies of nonpregnant populations, little information is available on dose adjustment that may be needed given the physiological changes during pregnancy. ${ }^{196}$ In 2018, the Task Force on Research Specific to Pregnant Women and Lactating Women issued recommendations to include pregnant and lactating women in scientific studies and remove regulatory barriers to participation in research. ${ }^{31}$ It will be essential to leverage this consensus to conduct clinical trials evaluating the efficacy and effectiveness of pharmacologic ${ }^{196}$ mental health treatments in perinatal women. In addition, future observational studies should address confounding adequately. Pragmatic trial designs and collaborative care models that allow ongoing data collection may permit greater rigor while addressing confounding.

A large proportion of studies included in our review are observational and often draw from registries and prescription databases. The limitations posed by these data sources, particularly with regard to data collection on symptoms, can skew the overall evidence base to focus on harms rather than benefits. Additionally, clinical practice and protocols for women with known 
exposure to psychotropic medication may result in harms being more likely to be detected or suspected than among women without known psychotropic exposure.

New efforts, such as the Outcome Measures Framework, supported by the Agency for Healthcare Research and Quality, can help categorize outcomes and harmonize data collection. A recent publication suggested a minimum set of outcome measures for depression in patient registries and clinical practice and provides guidance for implementation and data collection. ${ }^{197}$ Claims data will also continue to be important in identifying harms but will need to offer better evidence of severity of disorders, dosing, and duration of exposure to adequately control for confounding. Linked databases of maternal and child outcomes can also help to control for selection bias and confounding.

\section{Implications for Health Policy}

The complexity of the clinical considerations calls for greater involvement of mental health professionals in treatment decisions (rather than primary care or obstetrics alone). Adequate specialist care may be lacking in rural and other underserved areas. Telemedicine may help provide access to care where it might otherwise be inaccessible. At the same time, coverage of mental health services, including both pharmacologic and nonpharmacologic treatment, is essential, with particular attention to ongoing coverage in the postpartum period. Medicaid provides insurance for nearly 50 percent of pregnancies in the United States, but coverage ends at 60 days postpartum, and in many states, a large proportion of women lose coverage at that time. ${ }^{198}$ Continued coverage is necessary to treat maternal mental health problems beyond the first 60 days after delivery.

\section{Conclusions}

The central decisional dilemma facing pregnant and postpartum women with mental health disorders and their provider is how to balance benefits and harms of psychotropic drugs for both themselves and their children. One such critical trade-off is whether improved symptoms in the mother outweigh the harms from potential congenital anomalies in the fetus. The evidence is very sparse on the benefits of pharmacotherapy. By contrast, evidence on harms of pharmacotherapy is voluminous but of low quality. Our findings indicate the need for clear communication to patients on four primary points: evidence exists that medications work in general populations; few studies have measured effectiveness in pregnant women; the limited evidence available is consistent with some benefit; and some studies suggested some potential risks, many of which are transient or rare. The patient and her provider are uniquely qualified to determine whether the mother's medical need for treatment exceeds any potential harms. 


\section{References}

1. Palladino CL, Singh V, Campbell J, et al. Homicide and suicide during the perinatal period: findings from the National Violent Death Reporting System. Obstet Gynecol. 2011 Nov;118(5):1056-63. doi: 10.1097/AOG.0b013e31823294da. PMID: 22015873.

2. McLearn KT, Minkovitz CS, Strobino DM, et al. The timing of maternal depressive symptoms and mothers' parenting practices with young children: implications for pediatric practice. Pediatrics. $2006 \mathrm{Jul} ; 118(1): \mathrm{e} 174-82$. doi: 10.1542/peds.2005-1551. PMID: 16818531.

3. Burke L. The impact of maternal depression on familial relationships. Int Rev Psychiatry. 2003 Aug;15(3):243-55. doi: 10.1080/0954026031000136866. PMID: 15276963.

4. Ashman SB, Dawson G. Maternal depression, infant psychobiological development, and risk for depression. Children of depressed parents: Mechanisms of risk and implications for treatment. Washington, DC, US: American Psychological Association; 2002:37-58

5. Field T, Sandberg D, Garcia R, et al. Pregnancy problems, postpartum depression, and early mother-infant interactions. Dev Psychol. 1985;21(6):1152-6. doi: 10.1037/00121649.21.6.1152.

6. Beck CT. The effects of postpartum depression on maternal-infant interaction: a meta-analysis. Nurs Res. 1995 Sep-Oct;44(5):298-304. PMID: 7567486.

7. Pew Research Center. They're waiting longer, but U.S. women today more likely to have children than a decade ago. 2018. http://assets.pewresearch.org/wpcontent/uploads/sites/3/2018/01/18092036/PewMotherhood-report-FINAL.pdf.

8. Vesga-Lopez O, Blanco C, Keyes K, et al. Psychiatric disorders in pregnant and postpartum women in the United States. Arch Gen Psychiatry. 2008 Jul;65(7):805-15. doi: 10.1001/archpsyc.65.7.805. PMID: 18606953.

9. Wisner KL, Sit DK, McShea MC, et al. Onset timing, thoughts of self-harm, and diagnoses in postpartum women with screen-positive depression findings. JAMA Psychiatry. 2013 May;70(5):490-8. doi:

10.1001/jamapsychiatry.2013.87. PMID: 23487258.

10. Howard LM, Molyneaux E, Dennis CL, et al. Non-psychotic mental disorders in the perinatal period. Lancet. 2014 Nov 15;384(9956):1775-88. doi: 10.1016/S0140-6736(14)61276-9. PMID: 25455248.

11. O'Hara MW, Wisner KL. Perinatal mental illness: definition, description and aetiology. Best Pract Res Clin Obstet Gynaecol. 2014 Jan;28(1):3-12. doi: 10.1016/j.bpobgyn.2013.09.002. PMID: 24140480.

12. Munk-Olsen T, Maegbaek ML, Johannsen BM, et al. Perinatal psychiatric episodes: a populationbased study on treatment incidence and prevalence. Transl Psychiatry. 2016 Oct 18;6(10):e919. doi: 10.1038/tp.2016.190. PMID: 27754485.

13. National Center for Health Statistics. Health, United States, 2013: In Brief. Hyattsville, MD: U.S. Department of Health and Human Services, Centers for Disease Control and Prevention, National Center for Health Statistics; 2014. https://www.cdc.gov/nchs/data/hus/hus13 InBrie f.pdf.

14. Hanley GE, Mintzes B. Patterns of psychotropic medicine use in pregnancy in the United States from 2006 to 2011 among women with private insurance. BMC Pregnancy Childbirth. $2014 \mathrm{Jul}$ 22;14:242. doi: 10.1186/1471-2393-14-242. PMID: 25048574.

15. Huybrechts KF, Palmsten K, Mogun H, et al. National trends in antidepressant medication treatment among publicly insured pregnant women. Gen Hosp Psychiatry. 2013 MayJun;35(3):265-71. doi: 10.1016/j.genhosppsych.2012.12.010. PMID: 23374897.

16. Toh S, Li Q, Cheetham TC, et al. Prevalence and trends in the use of antipsychotic medications during pregnancy in the U.S., 2001-2007: a population-based study of 585,615 deliveries. Arch Womens Ment Health. 2013 Apr;16(2):14957. doi: 10.1007/s00737-013-0330-6. PMID: 23389622.

17. Bobo WV, Davis RL, Toh S, et al. Trends in the use of antiepileptic drugs among pregnant women in the US, 2001-2007: a medication exposure in pregnancy risk evaluation program study. Paediatr Perinat Epidemiol. 2012 Nov;26(6):57888. doi: 10.1111/ppe.12004. PMID: 23061694.

18. Kanes S, Colquhoun H, Gunduz-Bruce H, et al. Brexanolone (SAGE-547 injection) in postpartum depression: a randomised controlled trial. Lancet. 2017 Jul 29;390(10093):480-9. doi: 10.1016/S0140-6736(17)31264-3. PMID: 28619476.

19. Kanes SJ, Colquhoun H, Doherty J, et al. Openlabel, proof-of-concept study of brexanolone in the treatment of severe postpartum depression. Hum Psychopharmacol. 2017 Mar;32(2). doi: 10.1002/hup.2576. PMID: 28370307.

20. Roca C. An evolution of labeling information for pregnant women: PLLR history and background. Silver Spring, MD: U.S. Food \& Drug Administration; 2018.

https://www.fda.gov/files/advisory $\% 20$ committee s/published/An-Evolution-of-LabelingInformation-for-Pregnant-Women--PLLRHistory.pdf. Accessed on February 18, 2020. 
21. U. S. Food and Drug Administration. Guidance for industry warnings and precautions, contraindications, and boxed warning sections for labeling for Human Prescription Drug and biological products--content and Format. Silver Spring, MD: US Department of Health and Human Services, Food and Drug Administration; 2011. https://www.fda.gov/media/71866/download. Accessed on February 6, 2020.

22. Dinatale M. The pregnancy and lactation labeling rule (PLLR). Silver Spring, MD: U.S. Food and Drug Administration; 2016.

https://www.fda.gov/media/100406/download.

23. Brand SR, Brennan PA. Impact of antenatal and postpartum maternal mental illness: how are the children? Clin Obstet Gynecol. 2009

Sep;52(3):441-55. doi:

10.1097/GRF.0b013e3181b52930. PMID: 19661760.

24. Grace SL, Evindar A, Stewart DE. The effect of postpartum depression on child cognitive development and behavior: a review and critical analysis of the literature. Arch Womens Ment Health. 2003 Nov;6(4):263-74. doi: 10.1007/s00737-003-0024-6. PMID: 14628179.

25. Bartick MC, Schwarz EB, Green BD, et al. Suboptimal breastfeeding in the United States: Maternal and pediatric health outcomes and costs. Matern Child Nutr. 2017 Jan;13(1). doi: 10.1111/mcn.12366. PMID: 27647492.

26. Hasan A, Falkai P, Wobrock T, et al. World Federation of Societies of Biological Psychiatry (WFSBP) guidelines for biological treatment of schizophrenia. Part 3: update 2015 management of special circumstances: depression, suicidality, substance use disorders and pregnancy and lactation. World J Biol Psychiatry. 2015 Apr;16(3):142-70. doi:

10.3109/15622975.2015.1009163. PMID: 25822804.

27. Gentile S. Antipsychotic therapy during early and late pregnancy. A systematic review. Schizophr Bull. 2010 May;36(3):518-44. doi: 10.1093/schbul/sbn107. PMID: 18787227.

28. Finer LB, Zolna MR. Declines in unintended pregnancy in the United States, 2008-2011. N Engl J Med. 2016 Mar 3;374(9):843-52. doi: 10.1056/NEJMsa1506575. PMID: 26962904.

29. Gee RE, Wood SF, Schubert KG. Women's health, pregnancy, and the U.S. Food and Drug Administration. Obstet Gynecol. 2014 Jan;123(1):161-5. doi: 10.1097/AOG.0000000000000063. PMID: 24463677.

30. Sheffield JS, Siegel D, Mirochnick M, et al. Designing drug trials: considerations for pregnant women. Clin Infect Dis. 2014 Dec 15;59 Suppl 7:S437-44. doi: 10.1093/cid/ciu709. PMID: 25425722

31. Task Force on Research Specific to Pregnant Women and Lactating Women. Report to
Secretary, Health and Human Services, Congress. Rockville, MD: National Institutes of Health; 2018. https://www.nichd.nih.gov/sites/default/files/201 8-09/PRGLAC Report.pdf. Accessed on May 20, 2020.

32. Thorpe PG, Gilboa SM, Hernandez-Diaz S, et al. Medications in the first trimester of pregnancy: most common exposures and critical gaps in understanding fetal risk. Pharmacoepidemiol Drug Saf. 2013 Sep;22(9):1013-8. doi: 10.1002/pds.3495. PMID: 23893932.

33. Molenaar NM, Kamperman AM, Boyce P, et al. Guidelines on treatment of perinatal depression with antidepressants: an international review. Aust N Z J Psychiatry. 2018 Apr;52(4):320-7. doi: $10.1177 / 0004867418762057$. PMID: 29506399.

34. Koren G, Nordeng H. Antidepressant use during pregnancy: the benefit-risk ratio. Am J Obstet Gynecol. 2012 Sep;207(3):157-63. doi: 10.1016/j.ajog.2012.02.009. PMID: 22425404.

35. Muzik M, Hamilton SE. Use of antidepressants during pregnancy?: What to consider when weighing treatment with antidepressants against untreated depression. Matern Child Health J. 2016 Nov;20(11):2268-79. doi: 10.1007/s10995016-2038-5. PMID: 27461022.

36. Parker GB, Graham RK, Tavella G. Is there consensus across international evidence-based guidelines for the management of bipolar disorder? Acta Psychiatr Scand. 2017 Jun;135(6):515-26. doi: 10.1111/acps.12717. PMID: 28260229.

37. Graham RK, Tavella G, Parker GB. Is there consensus across international evidence-based guidelines for the psychotropic drug management of bipolar disorder during the perinatal period? J Affect Disord. 2018 Mar 1;228:216-21. doi: 10.1016/j.jad.2017.12.022. PMID: 29274567.

38. Moher D, Liberati A, Tetzlaff J, et al. Preferred reporting items for systematic reviews and metaanalyses: the PRISMA statement. Ann Intern Med. 2009 Aug 18;151(4):264-9, W64. doi: 10.7326/0003-4819-151-4-200908180-00135. PMID: 19622511.

39. Sterne JA, Hernan MA, Reeves BC, et al. ROBINS-I: a tool for assessing risk of bias in non-randomised studies of interventions. BMJ. 2016 Oct 12;355:i4919. doi: 10.1136/bmj.i4919. PMID: 27733354.

40. Higgins JPT, Green S. Cochrane handbook for systematic reviews of interventions: John Wiley \& Sons, Ltd; 2011.

41. Borenstein M, Hedges L, Higgins J, et al. Comprehensive meta-analysis version 3 . Englewood, NJ: Biostat; 2013.

42. Akl E, Mustafa R, Wiercioch N, et al. 1. Overview of the GRADE approach. In: Schunemann H, Brozek J, Guyatt G, Oxman A, eds. GRADE handbook. Geneva, Switzerland: World Health Organization; 2013. 
43. Berkman ND, Lohr KN, Ansari MT, et al. Grading the strength of a body of evidence when assessing health care interventions: an EPC update. J Clin Epidemiol. 2015 Nov;68(11):131224. doi: 10.1016/j.jclinepi.2014.11.023. PMID: 25721570 .

44. Uguz F, Yuksel G, Onur OS, et al. Neonatal outcomes in pregnant women with untreated and treated panic disorder. Compr Psychiatry. 2018 Nov;87:107-11. doi:

10.1016/j.comppsych.2018.10.001. PMID: 30326358.

45. Rotem-Kohavi N, Williams LJ, Virji-Babul N, et al. Alterations in Resting-State Networks Following In Utero Selective Serotonin Reuptake Inhibitor Exposure in the Neonatal Brain. Biol Psychiatry Cogn Neurosci Neuroimaging. 2018 Aug 25. doi: 10.1016/j.bpsc.2018.08.004. PMID: 30292808.

46. Giardinelli L, Lelli L, Ugolini V, et al. Comparison of two different treatments in depressed pregnant women: fetal growth characteristics and neonatal outcomes. J Perinat Med. 2018 Jul 19. doi: 10.1515/jpm-2017-0367. PMID: 30024856.

47. Galbally M, Frayne J, Watson SJ, et al. Aripiprazole and pregnancy: A retrospective, multicentre study. J Affect Disord. 2018 Oct 1;238:593-6. doi: 10.1016/j.jad.2018.06.004. PMID: 29957476.

48. Pope CJ, Sharma V, Sommerdyk C, et al. Antidepressants and recurrence of depression in the postpartum period. Arch Womens Ment Health. 2018 Dec;21(6):821-8. doi: 10.1007/s00737-018-0877-3. PMID: 29943237.

49. Freeman MP, Sosinsky AZ, Goez-Mogollon L, et al. Gestational Weight Gain and Pre-pregnancy Body Mass Index Associated With SecondGeneration Antipsychotic Drug Use During Pregnancy. Psychosomatics. 2018 Mar Apr;59(2):125-34. doi: 10.1016/j.psym.2017.09.002. PMID: 29078988.

50. Lupattelli A, Wood M, Lapane K, et al. Risk of preeclampsia after gestational exposure to selective serotonin reuptake inhibitors and other antidepressants: A study from The Norwegian Mother and Child Cohort Study.

Pharmacoepidemiol Drug Saf. 2017

Oct;26(10):1266-76. doi: 10.1002/pds.4286. PMID: 28815791.

51. Driscoll KE, Sit DKY, Moses-Kolko EL, et al. Mood symptoms in pregnant and postpartum women with bipolar disorder: a naturalistic study. Bipolar Disord. 2017 Jun;19(4):295-304. doi: 10.1111/bdi.12500. PMID: 28665044.

52. Yang A, Ciolino JD, Pinheiro E, et al. Neonatal Discontinuation Syndrome in Serotonergic Antidepressant-Exposed Neonates. J Clin Psychiatry. 2017 May;78(5):605-11. doi: 10.4088/JCP.16m11044. PMID: 28570796.

53. Stone KC, Salisbury AL, Miller-Loncar CL, et al. Pregnancy and postpartum antidepressant use moderates the effects of sleep on depression. Arch Womens Ment Health. 2017 Oct;20(5):62132. doi: 10.1007/s00737-017-0726-9. PMID: 28488099.

54. Johnson KC, Smith AK, Stowe ZN, et al. Preschool outcomes following prenatal serotonin reuptake inhibitor exposure: differences in language and behavior, but not cognitive function. J Clin Psychiatry. 2016 Feb;77(2):e17682. doi: 10.4088/JCP.14m09348. PMID: 26930533.

55. Hermansen TK, Roysamb E, Augusti EM, et al. Behavior and inhibitory control in children with prenatal exposure to antidepressants and medically untreated depression.

Psychopharmacology (Berl). 2016 Apr;233(8):1523-35. doi: 10.1007/s00213-0164248-3. PMID: 26924747.

56. Johansen RL, Mortensen LH, Andersen AM, et al. Maternal use of selective serotonin reuptake inhibitors and risk of miscarriage - assessing potential biases. Paediatr Perinat Epidemiol. 2015 Jan;29(1):72-81. doi: 10.1111/ppe.12160. PMID: 25382157.

57. Santucci AK, Singer LT, Wisniewski SR, et al. Impact of prenatal exposure to serotonin reuptake inhibitors or maternal major depressive disorder on infant developmental outcomes. J Clin Psychiatry. 2014 Oct;75(10):1088-95. doi: 10.4088/JCP.13m08902. PMID: 25373117.

58. Diav-Citrin O, Shechtman S, Tahover E, et al. Pregnancy outcome following in utero exposure to lithium: a prospective, comparative, observational study. Am J Psychiatry. 2014 Jul;171(7):785-94. doi: 10.1176/appi.ajp.2014.12111402. PMID: 24781368.

59. Sahingoz M, Yuksel G, Karsidag C, et al. Birth weight and preterm birth in babies of pregnant women with major depression in relation to treatment with antidepressants. J Clin Psychopharmacol. 2014 Apr;34(2):226-9. doi: 10.1097/jcp.0000000000000077. PMID: 24525643.

60. Michielsen LA, van der Heijden FM, Janssen PK, et al. Effects of maternal psychotropic drug dosage on birth outcomes. Neuropsychiatr Dis Treat. 2014;10:13-8. doi: 10.2147/ndt.s53430. PMID: 24376355.

61. Engelstad HJ, Roghair RD, Calarge CA, et al. Perinatal outcomes of pregnancies complicated by maternal depression with or without selective serotonin reuptake inhibitor therapy. Neonatology. 2014;105(2):149-54. doi: 10.1159/000356774. PMID: 24356332.

62. Weikum WM, Oberlander TF, Hensch TK, et al. Prenatal exposure to antidepressants and depressed maternal mood alter trajectory of infant speech perception. Proc Natl Acad Sci U S A. 2012 Oct 16;109 Suppl 2:17221-7. doi: 10.1073/pnas.1121263109. PMID: 23045665. 
63. Hayes RM, Wu P, Shelton RC, et al. Maternal antidepressant use and adverse outcomes: a cohort study of 228,876 pregnancies. Am J Obstet Gynecol. 2012 Jul;207(1):49.e1-9. doi: 10.1016/j.ajog.2012.04.028. PMID: 22727349.

64. Suri R, Hellemann G, Stowe ZN, et al. A prospective, naturalistic, blinded study of early neurobehavioral outcomes for infants following prenatal antidepressant exposure. J Clin Psychiatry. $2011 \mathrm{Jul} ; 72(7): 1002-7$. doi: 10.4088/JCP.10m06135. PMID: 21672498.

65. Wisner KL, Sit DK, Hanusa BH, et al. Major depression and antidepressant treatment: impact on pregnancy and neonatal outcomes. Am J Psychiatry. 2009 May;166(5):557-66. doi: 10.1176/appi.ajp.2008.08081170. PMID: 19289451.

66. Jordan AE, Jackson GL, Deardorff D, et al. Serotonin reuptake inhibitor use in pregnancy and the neonatal behavioral syndrome. J Matern Fetal Neonatal Med. 2008 Oct;21(10):745-51. doi: 10.1080/14767050802255488. PMID: 19012191.

67. Suri R, Altshuler L, Hellemann G, et al. Effects of antenatal depression and antidepressant treatment on gestational age at birth and risk of preterm birth. Am J Psychiatry. 2007

Aug;164(8):1206-13. doi:

10.1176/appi.ajp.2007.06071172. PMID: 17671283.

68. Pearlstein TB, Zlotnick C, Battle CL, et al. Patient choice of treatment for postpartum depression: a pilot study. Arch Womens Ment Health. 2006 Nov;9(6):303-8. doi: 10.1007/s00737-006-0145-9. PMID: 16932988.

69. Sivojelezova A, Shuhaiber S, Sarkissian L, et al. Citalopram use in pregnancy: prospective comparative evaluation of pregnancy and fetal outcome. Am J Obstet Gynecol. 2005

Dec;193(6):2004-9. doi: 10.1016/j.ajog.2005.05.012. PMID: 16325604.

70. Wisner KL, Hanusa BH, Peindl KS, et al. Prevention of postpartum episodes in women with bipolar disorder. Biol Psychiatry. 2004 Oct 15;56(8):592-6. doi:

10.1016/j.biopsych.2004.07.022. PMID: 15476689 .

71. Suri R, Altshuler L, Hendrick V, et al. The impact of depression and fluoxetine treatment on obstetrical outcome. Arch Womens Ment Health. 2004 Jul;7(3):193-200. doi: 10.1007/s00737-0040057-5. PMID: 15241665.

72. Lee A, Woo J, Ito S. Frequency of infant adverse events that are associated with citalopram use during breast-feeding. Am J Obstet Gynecol. 2004 Jan;190(1):218-21. PMID: 14749663.

73. Troyer WA, Pereira GR, Lannon RA, et al. Association of maternal lithium exposure and premature delivery. J Perinatol. 1993 MarApr;13(2):123-7. PMID: 8515304.

74. Petersen I, Sammon CJ, McCrea RL, et al. Risks associated with antipsychotic treatment in pregnancy: Comparative cohort studies based on electronic health records. Schizophr Res. 2016;176(2-3):349-56. doi: 10.1016/j.schres.2016.07.023. PMID: 201637815-001.

75. Edvinsson Å, Hellgren C, Kunovac Kallak T, et al. The effect of antenatal depression and antidepressant treatment on placental tissue: a protein-validated gene expression study. BMC Pregnancy Childbirth. 2019 Dec 5;19(1):479. doi: 10.1186/s12884-019-2586-y. PMID: 31805950.

76. McLean K, Murphy KE, Dalfen A, et al. The effect of maternal antidepressants on third trimester uteroplacental hemodynamics and the neonatal abstinence syndrome: a retrospective cohort study. Arch Womens Ment Health. 2019 Dec;22(6):791-7. doi: 10.1007/s00737-01900954-8. PMID: 30798375.

77. Meltzer-Brody S, Colquhoun H, Riesenberg R, et al. Brexanolone injection in post-partum depression: two multicentre, double-blind, randomised, placebo-controlled, phase 3 trials. Lancet. 2018 Sep 22;392(10152):1058-70. doi: 10.1016/s0140-6736(18)31551-4. PMID: 30177236.

78. Hantsoo L, Ward-O'Brien D, Czarkowski KA, et al. A randomized, placebo-controlled, doubleblind trial of sertraline for postpartum depression. Psychopharmacology (Berl). 2014

Mar;231(5):939-48. doi: 10.1007/s00213-0133316-1. PMID: 24173623.

79. O'Hara MW, Pearlstein T, Stuart S, et al. A placebo controlled treatment trial of sertraline and interpersonal psychotherapy for postpartum depression. J Affect Disord. 2018 Oct 31;245:524-32. doi: 10.1016/j.jad.2018.10.361. PMID: 30447565.

80. Viguera AC, Whitfield T, Baldessarini RJ, et al. Risk of recurrence in women with bipolar disorder during pregnancy: prospective study of mood stabilizer discontinuation. Am J Psychiatry. 2007 Dec;164(12):1817-24; quiz 923. doi: 10.1176/appi.ajp.2007.06101639. PMID: 18056236.

81. Newport DJ, Stowe ZN, Viguera AC, et al. Lamotrigine in bipolar disorder: efficacy during pregnancy. Bipolar Disord. 2008 May;10(3):4326. doi: 10.1111/j.1399-5618.2007.00565.x. PMID: 18402631 .

82. Wall-Wieler E, Robakis TK, Lyell DJ, et al Benzodiazepine use before conception and risk of ectopic pregnancy. Hum Reprod. 2020 Jun 3. doi: 10.1093/humrep/deaa082. PMID: 32485732.

83. American College of Obstetricians, Gynecologists' Committee on Practice B-G. ACOG Practice Bulletin No. 193: Tubal Ectopic Pregnancy. Obstet Gynecol. 2018 Mar;131(3):e91-e103. doi: 10.1097/AOG.0000000000002560. PMID: 29470343.

84. Palmsten K, Hernandez-Diaz S, Huybrechts KF, et al. Use of antidepressants near delivery and risk of postpartum hemorrhage: cohort study of 
low income women in the United States. BMJ. 2013 Aug 21;347:f4877. doi: 10.1136/bmj.f4877. PMID: 23965506.

85. McCloskey DJ, Postolache TT, Vittone BJ, et al. Selective serotonin reuptake inhibitors: measurement of effect on platelet function. Transl Res. 2008 Mar;151(3):168-72. doi: 10.1016/j.trsl.2007.10.004. PMID: 18279816.

86. Palmsten K, Huybrechts KF, Michels KB, et al. Antidepressant use and risk for preeclampsia. Epidemiology. 2013 Sep;24(5):682-91. doi: 10.1097/EDE.0b013e31829e0aaa. PMID: 23873072.

87. Palmsten K, Setoguchi S, Margulis AV, et al. Elevated risk of preeclampsia in pregnant women with depression: depression or antidepressants? Am J Epidemiol. 2012 May 15;175(10):988-97. doi: 10.1093/aje/kwr394. PMID: 22442287.

88. Park Y, Hernandez-Diaz S, Bateman BT, et al. Continuation of atypical antipsychotic medication during early pregnancy and the risk of gestational diabetes. Am J Psychiatry. 2018;175(6):564-74. doi: 10.1176/appi.ajp.2018.17040393.

89. Almeida ND, Basso O, Abrahamowicz M, et al. Risk of miscarriage in women receiving antidepressants in early pregnancy, correcting for induced abortions. Epidemiology. 2016 Jul;27(4):538-46. doi:

10.1097/ede.0000000000000484. PMID: 27031036.

90. Ban L, Tata LJ, West J, et al. Live and non-live pregnancy outcomes among women with depression and anxiety: a population-based study. PLoS One. 2012;7(8):e43462. doi:

10.1371/journal.pone.0043462. PMID: 22937052.

91. Sheehy O, Zhao JP, Bérard A. Association between incident exposure to benzodiazepines in early pregnancy and risk of spontaneous abortion. JAMA Psychiatry. 2019 May 15;76(9):948-57. doi: 10.1001/jamapsychiatry.2019.0963. PMID: 31090881 .

92. U.S. Food and Drug Administration. Highlights of prescribing information. Reference ID: 4405779. Silver Spring, MD: U.S. Food and Drug Administration; 2019. https://www.accessdata.fda.gov/drugsatfda docs/ label/2019/211371lbl.pdf. Accessed on February 17, 2020.

93. Qu H, Khalil RA. Vascular mechanisms and molecular targets in hypertensive pregnancy and preeclampsia. Am J Physiol Heart Circ Physiol. 2020 Aug 7. doi: 10.1152/ajpheart.00202.2020. PMID: 32762557.

94. Bak M, Fransen A, Janssen J, et al. Almost all antipsychotics result in weight gain: a metaanalysis. PLoS One. 2014;9(4):e94112. doi: 10.1371/journal.pone.0094112. PMID: 24763306.

95. Lilly USA. Highlights of prescribing information - Zyprexa. Reference ID: 3675779. Indianapolis, IN: Eli Lilly and Company; 2009. https://www.accessdata.fda.gov/drugsatfda docs/ label/2014/020592s062021086s040021253s0481b 1.pdf. Accessed on May 20, 2020.

96. AstraZeneca Pharmaceuticals LP. Seroquel medicaiton guide. Reference ID: 3397413. Wilmington, DE: AstraZeneca Pharmaceuticals LP; 2013.

https://www.fda.gov/files/drugs/published/Seroqu el-Medication-Guide.pdf. Accessed on May 20, 2020.

97. Qu F, Wu Y, Zhu YH, et al. The association between psychological stress and miscarriage: A systematic review and meta-analysis. Sci Rep. 2017 May 11;7(1):1731. doi: 10.1038/s41598017-01792-3. PMID: 28496110.

98. Frazier T, Hogue CJR, Bonney EA, et al. Weathering the storm; a review of pre-pregnancy stress and risk of spontaneous abortion. Psychoneuroendocrinology. 2018;92:142-54. doi: 10.1016/j.psyneuen.2018.03.001. PMID: 29628283.

99. Freeman MP, Goez-Mogollon L, McInerney KA, et al. Obstetrical and neonatal outcomes after benzodiazepine exposure during pregnancy: results from a prospective registry of women with psychiatric disorders. Gen Hosp Psychiatry. 2018 Jul - Aug;53:73-9. doi: 10.1016/j.genhosppsych.2018.05.010. PMID: 29958100.

100. Cantarutti A, Merlino L, Giaquinto C, et al. Use of antidepressant medication in pregnancy and adverse neonatal outcomes: a population-based investigation. Pharmacoepidemiol Drug Saf. 2017 Sep;26(9):1100-8. doi: 10.1002/pds.4242. PMID: 28594145.

101. Malm H, Sourander A, Gissler M, et al. Pregnancy complications following prenatal exposure to SSRIs or maternal psychiatric disorders: results from population-based national register data. Am J Psychiatry. 2015 Dec;172(12):1224-32. doi: 10.1176/appi.ajp.2015.14121575. PMID: 26238606.

102. Oberlander TF, Warburton W, Misri S, et al. Neonatal outcomes after prenatal exposure to selective serotonin reuptake inhibitor antidepressants and maternal depression using population-based linked health data. Arch Gen Psychiatry. 2006 Aug;63(8):898-906. doi: 10.1001/archpsyc.63.8.898. PMID: 16894066.

103. Huybrechts KF, Bateman BT, Palmsten K, et al. Antidepressant use late in pregnancy and risk of persistent pulmonary hypertension of the newborn. JAMA. 2015 Jun 2;313(21):2142-51. doi: 10.1001/jama.2015.5605. PMID: 26034955.

104. Malm H, Brown AS, Gissler M, et al. Gestational exposure to selective serotonin reuptake inhibitors and offspring psychiatric disorders: a national register-based study. J Am Acad Child Adolesc Psychiatry. 2016 May;55(5):359-66. doi: 10.1016/j.jaac.2016.02.013. PMID: 27126849. 
105. Rai D, Lee BK, Dalman C, et al. Antidepressants during pregnancy and autism in offspring: population based cohort study. BMJ. 2017 Jul 19;358:j2811. doi: 10.1136/bmj.j2811. PMID: 28724519.

106. American Academy of Pediatrics Committee on Fetus and Newborn, American College of Obstetricians and Gynecologists Committee on Obstetric Practice. The Apgar score. Pediatrics. 2015 October;136. PMID: 10.1542/peds.20152651.

107. Steurer MA, Baer RJ, Oltman S, et al. Morbidity of persistent pulmonary hypertension of the newborn in the first year of life. J Pediatr. 2019 Oct;213:58-65 e4. doi: 10.1016/j.jpeds.2019.06.053. PMID: 31399244.

108. Venkatesh KK, Riley L, Castro VM, et al. Association of antenatal depression symptoms and antidepressant treatment with preterm birth. Obstet Gynecol. 2016 May;127(5):926-33. doi: 10.1097/aog.0000000000001397. PMID: 27054941.

109. Cantarutti A, Merlino L, Monzani E, et al. Is the risk of preterm birth and low birth weight affected by the use of antidepressant agents during pregnancy? A population-based investigation. PLoS One. 2016;11(12):e0168115. doi: 10.1371/journal.pone.0168115. PMID: 27977749.

110. Toh S, Mitchell AA, Louik C, et al. Antidepressant use during pregnancy and the risk of preterm delivery and fetal growth restriction. J Clin Psychopharmacol. 2009 Dec;29(6):555-60. doi: 10.1097/JCP.0b013e3181bf344c. PMID: 19910720.

111. Grzeskowiak LE, Gilbert AL, Morrison JL. Neonatal outcomes after late-gestation exposure to selective serotonin reuptake inhibitors. J Clin Psychopharmacol. 2012 Oct;32(5):615-21. doi: 10.1097/JCP.0b013e31826686bc. PMID: 22926594.

112. Güngör BB, Öztürk N, Atar AÖ, et al. Comparison of the groups treated with mirtazapine and selective serotonine reuptake inhibitors with respect to birth outcomes and severity of psychiatric disorder. Psychiatry and Clinical Psychopharmacology. 2019;29(4):82231. doi: 10.1080/24750573.2019.1673936.

113. Ramos E, St-Andre M, Berard A. Association between antidepressant use during pregnancy and infants born small for gestational age. Can J Psychiatry. 2010 Oct;55(10):643-52. PMID: 20964943.

114. Berard A, Zhao JP, Sheehy O. Antidepressant use during pregnancy and the risk of major congenital malformations in a cohort of depressed pregnant women: an updated analysis of the Quebec Pregnancy Cohort. BMJ Open. 2017 Jan 12;7(1):e013372. doi:

10.1136/bmjopen-2016-013372. PMID: 28082367.
115. Ban L, Gibson JE, West J, et al. Maternal depression, antidepressant prescriptions, and congenital anomaly risk in offspring: a population-based cohort study. BJOG. 2014 Nov;121(12):1471-81. doi: 10.1111/14710528.12682. PMID: 24612301.

116. Jimenez-Solem E, Andersen JT, Petersen M, et al. Exposure to selective serotonin reuptake inhibitors and the risk of congenital malformations: a nationwide cohort study. BMJ Open. 2012;2(3). doi: 10.1136/bmjopen-2012001148.

117. Ramos É, St-André M, Rey É, et al. Duration of antidepressant use during pregnancy and risk of major congenital malformations. Br J Psychiatry. 2008;192(5):344-50. doi:

10.1192/bjp.bp.107.042523. PMID: 2008-07004007.

118. Berard A, Zhao JP, Sheehy O. Sertraline use during pregnancy and the risk of major malformations. Am J Obstet Gynecol. 2015 Jun;212(6):795.e1-.e12. doi: 10.1016/j.ajog.2015.01.034. PMID: 25637841.

119. Lopez-Gimenez JF, de la Fuente Revenga M, Ruso-Julve F, et al. Validation of schizophrenia gene expression profile in a preclinical model of maternal infection during pregnancy. Schizophr Res. 2017;189:217-8. doi:

10.1016/j.schres.2017.02.005. PMID: 201754219-015.

120. Huybrechts KF, Hernandez-Diaz S, Patorno E, et al. Antipsychotic use in pregnancy and the risk for congenital malformations. JAMA Psychiatry. 2016 Sep 1;73(9):938-46. doi:

10.1001/jamapsychiatry.2016.1520. PMID: 27540849.

121. Cohen LS, Viguera AC, McInerney KA, et al. Reproductive safety of second-generation antipsychotics: Current data from the Massachusetts General Hospital National Pregnancy Registry for atypical antipsychotics. Am J Psychiatry. 2016 Mar 1;173(3):263-70. doi: 10.1176/appi.ajp.2015.15040506. PMID: 26441156.

122. Anderson KN, Lind JN, Simeone RM, et al. Maternal use of specific antidepressant medications during early pregnancy and the risk of selected birth defects. JAMA Psychiatry. 2020 Aug 5. doi: 10.1001/jamapsychiatry.2020.2453. PMID: 32777011.

123. Huybrechts KF, Palmsten K, Avorn J, et al. Antidepressant use in pregnancy and the risk of cardiac defects. N Engl J Med. 2014 Jun 19;370(25):2397-407. doi: 10.1056/NEJMoa1312828. PMID: 24941178.

124. Huybrechts KF, Bateman BT, Pawar A, et al. Maternal and fetal outcomes following exposure to duloxetine in pregnancy: cohort study. BMJ. 2020 Feb 19;368:m237. doi: 10.1136/bmj.m237. PMID: 32075794.

125. Lupattelli A, Wood M, Ystrom E, et al. Effect of time-dependent selective serotonin reuptake 
inhibitor antidepressants during pregnancy on behavioral, emotional, and social development in preschool-aged children. J Am Acad Child Adolesc Psychiatry. 2018 Mar;57(3):200-8. doi: 10.1016/j.jaac.2017.12.010. PMID: 29496129.

126. El Marroun H, White TJ, Fernandez G, et al. Prenatal exposure to selective serotonin reuptake inhibitors and non-verbal cognitive functioning in childhood. J Psychopharmacol. 2017

Mar;31(3):346-55. doi:

10.1177/0269881116665335. PMID: 27624153.

127. Salisbury AL, Wisner KL, Pearlstein T, et al. Newborn neurobehavioral patterns are differentially related to prenatal maternal major depressive disorder and serotonin reuptake inhibitor treatment. Depress Anxiety. 2011 Nov;28(11):1008-19. doi: 10.1002/da.20883. PMID: 21898709.

128. Casper RC, Fleisher BE, Lee-Ancajas JC, et al. Follow-up of children of depressed mothers exposed or not exposed to antidepressant drugs during pregnancy. J Pediatr. 2003

Apr;142(4):402-8. doi: 10.1067/mpd.2003.139. PMID: 12712058.

129. Viktorin A, Uher R, Reichenberg A, et al. Autism risk following antidepressant medication during pregnancy. Psychol Med. 2017 Dec;47(16):278796. doi: 10.1017/s0033291717001301. PMID: 28528584.

130. Cohen LS, Goez-Mogollon L, Sosinsky AZ, et al. Risk of major malformations in infants following first-trimester exposure to quetiapine. Am J Psychiatry. 2018 Aug 16:appiajp201818010098. doi: 10.1176/appi.ajp.2018.18010098. PMID: 30111186.

131. Ban L, West J, Gibson JE, et al. First trimester exposure to anxiolytic and hypnotic drugs and the risks of major congenital anomalies: a United Kingdom population-based cohort study. PLoS One. 2014;9(6):e100996. doi:

10.1371/journal.pone.0100996. PMID: 24963627.

132. Mallinckrodt, Inc. Restoril ${ }^{\mathrm{TM}}$ (temazepam) Capsules USP. FDA approved labeling text 2.25.08. Hazelwood, MO: Mallinckrodt Inc.; 2008.

https://www.accessdata.fda.gov/drugsatfda_docs/ label/2008/018163s058s059lbl.pdf. Accessed on February 18, 2020.

133. Patorno E, Huybrechts KF, Bateman BT, et al. Lithium use in pregnancy and the risk of cardiac malformations. N Engl J Med. 2017 Jun 8;376(23):2245-54. doi: 10.1056/NEJMoa1612222. PMID: 28591541.

134. Berard A, Ramos E, Rey E, et al. First trimester exposure to paroxetine and risk of cardiac malformations in infants: the importance of dosage. Birth Defects Res B Dev Reprod Toxicol. 2007 Feb;80(1):18-27. doi: 10.1002/bdrb.20099. PMID: 17187388.

135. Kallen B, Tandberg A. Lithium and pregnancy. A cohort study on manic-depressive women. Acta
Psychiatr Scand. 1983 Aug;68(2):134-9. PMID: 6624510.

136. Botto LD, Lin AE, Riehle-Colarusso T, et al. Seeking causes: classifying and evaluating congenital heart defects in etiologic studies. Birth Defects Res A Clin Mol Teratol. 2007 Oct;79(10):714-27. doi: 10.1002/bdra.20403. PMID: 17729292.

137. De Ocampo MPG, Araneta MRG, Macera CA, et al. Risk of gestational hypertension and preeclampsia in women who discontinued or continued antidepressant medication use during pregnancy. Arch Womens Ment Health. 2016 Dec;19(6):1051-61. doi: 10.1007/s00737-0160655-z. PMID: 27558246.

138. Galbally M, Watson SJ, Boyce P, et al. The mother, the infant and the mother-infant relationship: What is the impact of antidepressant medication in pregnancy. J Affect Disord. 2020;272:363-70. doi: 10.1016/j.jad.2020.03.116.

139. Einarson A, Fatoye B, Sarkar M, et al. Pregnancy outcome following gestational exposure to venlafaxine: a multicenter prospective controlled study. Am J Psychiatry. 2001 Oct;158(10):172830. doi: 10.1176/appi.ajp.158.10.1728. PMID: 11579012.

140. Richardson JL, Martin F, Dunstan H, et al. Pregnancy outcomes following maternal venlafaxine use: A prospective observational comparative cohort study. Reprod Toxicol. 2019 Mar;84:108-13. doi:

10.1016/j.reprotox.2019.01.003. PMID: 30639403.

141. Davis RL, Rubanowice D, McPhillips H, et al. Risks of congenital malformations and perinatal events among infants exposed to antidepressant medications during pregnancy.

Pharmacoepidemiol Drug Saf. 2007

Oct;16(10):1086-94. doi: 10.1002/pds. 1462. PMID: 17729378.

142. Pearson KH, Nonacs RM, Viguera AC, et al. Birth outcomes following prenatal exposure to antidepressants. J Clin Psychiatry. 2007

Aug;68(8):1284-9. PMID: 17854255.

143. Winterfeld U, Klinger G, Panchaud A, et al. Pregnancy outcome following maternal exposure to mirtazapine: a multicenter, prospective study. J Clin Psychopharmacol. 2015 Jun;35(3):250-9. doi: $10.1097 /$ jcp.0000000000000309. PMID: 25830592.

144. Simon GE, Cunningham ML, Davis RL. Outcomes of prenatal antidepressant exposure. Am J Psychiatry. 2002 Dec;159(12):2055-61. doi: 10.1176/appi.ajp.159.12.2055. PMID: 12450956.

145. Pedersen LH, Henriksen TB, Vestergaard M, et al. Selective serotonin reuptake inhibitors in pregnancy and congenital malformations: population based cohort study. BMJ. 2009 Sep 23;339:b3569. doi: 10.1136/bmj.b3569. PMID: 19776103. 
146. Berard A, Boukhris T, Sheehy O. Selective serotonin reuptake inhibitors and autism: additional data on the Quebec Pregnancy/Birth Cohort. Am J Obstet Gynecol. 2016

Dec;215(6):803-5. doi: 10.1016/j.ajog.2016.08.021. PMID: 27589900.

147. Hviid A, Melbye M, Pasternak B. Use of selective serotonin reuptake inhibitors during pregnancy and risk of autism. N Engl J Med. 2013 Dec 19;369(25):2406-15. doi: 10.1056/NEJMoa1301449. PMID: 24350950.

148. Sivathamboo N, Hitchcock A, Graham J, et al. The use of antidepressant drugs in pregnant women with epilepsy: a study from the Australian Pregnancy Register. Epilepsia. 2018 Sep;59(9):1696-704. doi: 10.1111/epi.14539. PMID: 30142256.

149. Strawn JR, Geracioti L, Rajdev N, et al. Pharmacotherapy for generalized anxiety disorder in adult and pediatric patients: an evidence-based treatment review. Expert Opin Pharmacother. 2018 Jul;19(10):1057-70. doi: 10.1080/14656566.2018.1491966. PMID: 30056792.

150. Cipriani A, Furukawa TA, Salanti G, et al. Comparative efficacy and acceptability of 21 antidepressant drugs for the acute treatment of adults with major depressive disorder: a systematic review and network meta-analysis. Lancet. 2018 Apr 7;391(10128):1357-66. doi: 10.1016/S0140-6736(17)32802-7. PMID: 29477251.

151. Gartlehner G, Hansen RA, Morgan LC, et al. Comparative benefits and harms of secondgeneration antidepressants for treating major depressive disorder: an updated meta-analysis. Ann Intern Med. 2011 Dec 6;155(11):772-85. doi: 10.7326/0003-4819-155-11-20111206000009. PMID: 22147715.

152. Gartlehner G, Wagner G, Matyas N, et al. Pharmacological and non-pharmacological treatments for major depressive disorder: review of systematic reviews. BMJ Open. 2017 Jun 14;7(6):e014912. doi: 10.1136/bmjopen-2016014912. PMID: 28615268.

153. LeGates TA, Kvarta MD, Thompson SM. Sex differences in antidepressant efficacy. Neuropsychopharmacology. 2019 Jan;44(1):14054. doi: 10.1038/s41386-018-0156-z. PMID: 30082889.

154. Butler M, Urosevic S, Desai P, et al. Treatment for bipolar disorder in adults: a systematic review. Comparative Effectiveness Review No. 208. (Prepared by the Minnesota Evidence-based Practice Center under Contract No. 290-201200016-I.) AHRQ Publication No. 18-EHC012EF. Rockville, MD: Quality AfHRa; August 2018. Posted final reports are located on the Effective Health Care Program search page. doi: https://doi.org/10.23970/AHRQEPCCER208.

155. Keating D, McWilliams S, Schneider I, et al. Pharmacological guidelines for schizophrenia: a systematic review and comparison of recommendations for the first episode. BMJ Open. 2017 Jan 6;7(1):e013881. doi: 10.1136/bmjopen-2016-013881. PMID: 28062471.

156. Wyatt RJ, Alexander RC, Egan MF, et al. Schizophrenia, just the facts. What do we know, how well do we know it? Schizophr Res. 1988 Jan-Feb;1(1):3-18. doi: 10.1016/09209964(88)90034-5. PMID: 3154503.

157. Seeman MV. Gender differences in the prescribing of antipsychotic drugs. Am J Psychiatry. 2004 Aug;161(8):1324-33. doi: 10.1176/appi.ajp.161.8.1324. PMID: 15285956.

158. Westin AA, Brekke M, Molden E, et al. Treatment With Antipsychotics in Pregnancy: Changes in Drug Disposition. Clin Pharmacol Ther. 2018 Mar;103(3):477-84. doi: 10.1002/cpt.770. PMID: 28643331.

159. Forsberg L, Adler M, Romer Ek I, et al. Maternal mood disorders and lithium exposure in utero were not associated with poor cognitive development during childhood. Acta Paediatr. 2018 Aug;107(8):1379-88. doi: 10.1111/apa.14152. PMID: 29150869.

160. Wesseloo R, Liu X, Clark CT, et al. Risk of postpartum episodes in women with bipolar disorder after lamotrigine or lithium use during pregnancy: a population-based cohort study. J Affect Disord. 2017 Aug 15;218:394-7. doi: 10.1016/j.jad.2017.04.070. PMID: 28501739.

161. Milgrom J, Gemmill AW, Ericksen J, et al. Treatment of postnatal depression with cognitive behavioural therapy, sertraline and combination therapy: a randomised controlled trial. Aust N Z J Psychiatry. 2015 Mar;49(3):236-45. doi: 10.1177/0004867414565474. PMID: 25586754.

162. Nulman I, Koren G, Rovet J, et al. Neurodevelopment of children following prenatal exposure to venlafaxine, selective serotonin reuptake inhibitors, or untreated maternal depression. Am J Psychiatry. 2012

Nov;169(11):1165-74. doi: 10.1176/appi.ajp.2012.11111721. PMID: 23128923.

163. Bloch M, Meiboom H, Lorberblatt M, et al. The effect of sertraline add-on to brief dynamic psychotherapy for the treatment of postpartum depression: a randomized, double-blind, placebocontrolled study. J Clin Psychiatry. 2012 Feb;73(2):235-41. doi: 10.4088/JCP.11m07117. PMID: 22401479.

164. Lanza di Scalea T, Hanusa BH, Wisner KL. Sexual function in postpartum women treated for depression: results from a randomized trial of nortriptyline versus sertraline. J Clin Psychiatry. 2009 Mar;70(3):423-8. PMID: 19284932.

165. Wisner KL, Hanusa BH, Perel JM, et al. Postpartum depression: a randomized trial of sertraline versus nortriptyline. J Clin Psychopharmacol. 2006 Aug;26(4):353-60. doi: 
10.1097/01.jcp.0000227706.56870.dd. PMID: 16855451.

166. Yonkers KA, Lin H, Howell HB, et al. Pharmacologic treatment of postpartum women with new-onset major depressive disorder: a randomized controlled trial with paroxetine. J Clin Psychiatry. 2008 Apr;69(4):659-65. PMID: 18363420.

167. Djulus J, Koren G, Einarson TR, et al. Exposure to mirtazapine during pregnancy: a prospective, comparative study of birth outcomes. J Clin Psychiatry. 2006 Aug;67(8):1280-4. PMID: 16965209.

168. Sharma V, Smith A, Mazmanian D. Olanzapine in the prevention of postpartum psychosis and mood episodes in bipolar disorder. Bipolar Disord. 2006 Aug;8(4):400-4. doi: 10.1111/j.1399-5618.2006.00335.x. PMID: 16879140.

169. Cohen LS, Altshuler LL, Harlow BL, et al. Relapse of major depression during pregnancy in women who maintain or discontinue antidepressant treatment. JAMA. $2006 \mathrm{Feb}$ 1;295(5):499-507. doi: 10.1001/jama.295.5.499. PMID: 16449615.

170. Oberlander TF, Misri S, Fitzgerald CE, et al. Pharmacologic factors associated with transient neonatal symptoms following prenatal psychotropic medication exposure. J Clin Psychiatry. 2004 Feb;65(2):230-7. PMID: 15003078.

171. Nulman I, Rovet J, Stewart DE, et al. Child development following exposure to tricyclic antidepressants or fluoxetine throughout fetal life: a prospective, controlled study. Am J Psychiatry. 2002 Nov;159(11):1889-95. doi: 10.1176/appi.ajp.159.11.1889. PMID: 12411224.

172. Reisner JG. Hydroxyzine for controlling postpartum anxiety: a double-blind study. Nebr State Med J. 1967 Nov;52(11):498-9. PMID: 4230037.

173. Babu GN, Desai G, Tippeswamy H, et al. Birth weight and use of olanzapine in pregnancy: a prospective comparative study. J Clin Psychopharmacol. 2010;30(3):331-2. doi: 10.1097/JCP.0b013e3181db8734. PMID: 201010616-020.

174. Juric S, Newport DJ, Ritchie JC, et al. Zolpidem

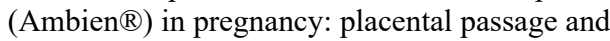
outcome. Arch Womens Ment Health. 2009; 12(6):441-6. doi: 10.1007/s00737-0090100-7. PMID: 2009-21413-008.

175. Rampono J, Simmer K, Ilett KF, et al. Placental transfer of SSRI and SNRI antidepressants and effects on the neonate. Pharmacopsychiatry. 2009 May;42(3):95-100. doi: 10.1055/s-00281103296. PMID: 19452377.

176. Newport DJ, Calamaras MR, DeVane CL, et al. Atypical antipsychotic administration during late pregnancy: placental passage and obstetrical outcomes. Am J Psychiatry. 2007

Aug;164(8):1214-20. doi: 10.1176/appi.ajp.2007.06111886. PMID: 17671284.

177. Misri S, Reebye P, Kendrick K, et al. Internalizing behaviors in 4-year-old children exposed in utero to psychotropic medications. Am J Psychiatry. 2006 Jun;163(6):1026-32. doi: 10.1176/ajp.2006.163.6.1026. PMID: 16741203.

178. Masarwa R, Bar-Oz B, Gorelik E, et al. Prenatal exposure to SSRIs and SNRIs and risk for persistent pulmonary hypertension of the newborn: a systematic review, meta-analysis and network meta-analysis. Am J Obstet Gynecol. 2018 Aug 28. doi: 10.1016/j.ajog.2018.08.030. PMID: 30170040.

179. Grigoriadis S, Vonderporten EH, Mamisashvili $\mathrm{L}$, et al. Prenatal exposure to antidepressants and persistent pulmonary hypertension of the newborn: systematic review and meta-analysis. BMJ. 2014 Jan 14;348:f6932. doi:

10.1136/bmj.f6932. PMID: 24429387.

180. Yonkers KA, Wisner KL, Stewart DE, et al. The management of depression during pregnancy: a report from the American Psychiatric Association and the American College of Obstetricians and Gynecologists. Obstet Gynecol. 2009

Sep;114(3):703-13. doi: 10.1097/AOG.0b013e3181ba0632. PMID: 19701065.

181. Ball SG, Kuhn A, Wall D, et al. Selective serotonin reuptake inhibitor treatment for generalized anxiety disorder: a double-blind, prospective comparison between paroxetine and sertraline. J Clin Psychiatry. 2005 Jan;66(1):94-9. doi: 10.4088/jcp.v66n0113. PMID: 15669894.

182. Bielski RJ, Bose A, Chang CC. A double-blind comparison of escitalopram and paroxetine in the long-term treatment of generalized anxiety disorder. Ann Clin Psychiatry. 2005 AprJun;17(2):65-9. doi:

10.1080/10401230590932326. PMID: 16075658.

183. Gomez AF, Barthel AL, Hofmann SG. Comparing the efficacy of benzodiazepines and serotonergic anti-depressants for adults with generalized anxiety disorder: a meta-analytic review. Expert Opin Pharmacother. 2018 Jun;19(8):883-94. doi: 10.1080/14656566.2018.1472767. PMID: 29806492.

184. Chessick CA, Allen MH, Thase M, et al. Azapirones for generalized anxiety disorder. Cochrane Database Syst Rev. 2006 Jul 19(3):CD006115. doi: 10.1002/14651858.CD006115. PMID: 16856115.

185. Gartlehner G, Gaynes BN, Amick HR, et al. Comparative benefits and harms of antidepressant, psychological, complementary, and exercise treatments for major depression: an evidence report for a clinical practice guideline from the American College of Physicians. Ann Intern Med. 2016 Mar 1;164(5):331-41. doi: 10.7326/M15-1813. PMID: 26857743. 
186. McDonagh MS, Dana T, Selph S, et al.

Treatments for schizophrenia in adults: a systematic review. Comparative Effectiveness Review No. 198. (Prepared by the Pacific Northwest Evidence-based Practice Center under Contract No. 290-2015-00009-I.) AHRQ Publication No. 17(18)-EHC031-EF. Rockville, MD: Agency for Healthcare Research and Quality; October 2017.

www.effectivehealthcare.ahrq.gov/reports/final.cf m. doi:

https://doi.org/10.23970/AHRQEPCCER198.

187. Stroup TS, Marder S. Pharmacotherapy for schizophrenia: acute and maintenance phase treatment. Waltham, MA: UpToDate; 2019. Accessed on February 6, 2020.

188. Yonkers KA, Gotman N, Smith MV, et al. Does antidepressant use attenuate the risk of a major depressive episode in pregnancy? Epidemiology. 2011 Nov;22(6):848-54. doi: 10.1097/EDE.0b013e3182306847. PMID: 21900825.

189. American Psychiatric Association. Clinical practice guidelines. Washington, DC: American Psychiatric Association; 2020.

https://www.psychiatry.org/psychiatrists/practice/ clinical-practice-guidelines. Accessed on September 3, 2020.

190. Lebedevs T, Gan M, Teoh SWK, et al. Analysis of perinatal women attending a mother and baby unit taking sodium valproate or lithium with a diagnosis of bipolar affective disorder. Psychiatr Q. 2020 Mar 10. doi: 10.1007/s11126-02009729-2. PMID: 32157548.

191. Swanson SA, Hernandez-Diaz S, Palmsten K, et al. Methodological considerations in assessing the effectiveness of antidepressant medication continuation during pregnancy using administrative data. Pharmacoepidemiol Drug Saf. 2015 Sep;24(9):934-42. doi: 10.1002/pds.3798. PMID: 26045370.
192. de Bruin NM, Kruse CG. 5-HT6 receptor antagonists: potential efficacy for the treatment of cognitive impairment in schizophrenia. Curr Pharm Des. 2015;21(26):3739-59. PMID: 26044973.

193. Denton LK, Creeley CE, Stavola B, et al. An analysis of online pregnancy message boards: Mother-to-mother advice on medication use. Women Birth. 2020 Feb;33(1):e48-e58. doi: 10.1016/j.wombi.2018.12.003. PMID: 30545755

194. Hargraves I, LeBlanc A, Shah ND, et al. Shared decision making: the need for patient-clinician conversation, not just information. Health Aff (Millwood). 2016 Apr;35(4):627-9. doi: 10.1377/hlthaff.2015.1354. PMID: 27044962.

195. Brito JP, Kunneman M, Montori VM. Shared decision making. EBM Toolkit home. Rochester, MN: Mayo Clinic; 2020.

https://bestpractice.bmj.com/info/us/toolkit/practi se-ebm/shared-decision-making/. Accessed on May 21, 2020.

196. Schoretsanitis G, Spigset O, Stingl JC, et al. The impact of pregnancy on the pharmacokinetics of antidepressants: a systematic critical review and meta-analysis. Expert Opin Drug Metab Toxicol. 2020 May;16(5):431-40. doi: 10.1080/17425255.2020.1750598. PMID: 32238008 .

197. Gliklich RE, Leavy MB, Cosgrove L, et al. Harmonized Outcome Measures for Use in Depression Patient Registries and Clinical Practice. Ann Intern Med. 2020 May 12. doi: 10.7326/M19-3818. PMID: 32422056.

198. Ranji U, Gomez I, Salganicoff A. Expanding postpartum Medicaid coverage. Washington, DC: Kaiser Family Foundation; 2019. Accessed on May 20, 2020. 


\section{Abbreviations and Acronyms}

$\mathrm{AD}=$ antidepressants

$\mathrm{ADD}=$ attention deficit disorder

ADHD $=$ attention-deficit/hyperactivity disorder

AEDs $=$ anti-epileptic drugs

$\mathrm{AHR}=$ adjusted hazard ratio

AHRQ = Agency for Healthcare Research and Quality

$\mathrm{AOR}=$ adjusted odds ratio

$\mathrm{ARR}=$ adjusted risk ratio

$\mathrm{ASD}=$ autism spectrum disorder

ASEX $=$ Arizona Sexual Experience Scale

$\mathrm{BDI}=$ Beck Depression Inventory

BDI-II = Beck Depression Inventory-II;

BRIEF-P = Behaviour Rating Inventory of Executive Function - Preschool version

$\mathrm{BRS}=$ Behavioral Rating Scale

$\mathrm{BRX}=$ brexanolone for postpartum depression

$\mathrm{CBCL}=$ childhood behavior checklist

$\mathrm{CBT}=$ cognitive behavioral therapy

CGI $=$ Clinical Global Impression

CGI-I = Clinical Global Impression- Improvement

CGI-S = Clinical Global Impression-Severity of Illness

$\mathrm{CI}=$ confidence interval

$\mathrm{dx}=$ diagnosis

$\mathrm{EHC}=$ Effective Health Care

EPDS $=$ Edinburgh Postnatal Depression Scale

FDA $=$ Food and Drug Administration

GAD-7 = Generalized Anxiety Disorder 7-item

GAS $=$ general adaptation syndrome

GRADE $=$ Grading of Recommendations Assessment, Development and Evaluation

HAM-A = Hamilton Anxiety Rating Scale

HAM-D = Hamilton Depression Rating

HAM-D-17= Hamilton Depression Rating scale, 17-item version

HAM-D-19= Hamilton Depression Rating scale, 19-item version

HRSD = Hamilton Rating Scale for Depression

IDAS-GD = Inventory of Depression and Anxiety Symptoms, General Depression scale

IDS-SR = Inventory of Depression Symptoms - SR

INFANIB = Infant Neurological International Battery

$\mathrm{IQR}=$ interquartile ratio 
ITT $=$ intention to treat

$\mathrm{KQ}=$ Key Question

LGA = large for gestational age

LMP = last menstrual period

$\mathrm{LS}=$ least square

MADRS = Montgomery-Åsberg Depression Rating Scale

MAOI = Monoamine oxidase inhibitors

MDI $=$ Mental Development Index

$\mathrm{N} / \mathrm{n}=$ number

$\mathrm{NA}=$ not applicable

NEPSY-II = Developmental NEuroPSYchological Assessment-II

$\mathrm{NICU}=$ neonatal intensive care unit

NNNS = NICU Network Neurobehavioral Scale

$\mathrm{NR}=$ not reported

$\mathrm{NS}=$ not significant

$\mathrm{OR}=$ odds ratio

$\mathrm{PDI}=$ Provisional Diagnostic Instrument

PHQ9 = Patient Health Questionnaire, 9 item

$\mathrm{pp}=$ postpartum

$\mathrm{PPH}=$ persistent pulmonary hypertension

PPAQ $=$ Postpartum Adjustment Questionnaire

$\mathrm{QPC}=$ Quebec Pregnancy/Children Cohort

$\mathrm{RCT}=$ randomized controlled trial

$\mathrm{RD}=$ risk difference

$\mathrm{ROB}=$ risk of bias

$\mathrm{RR}=$ relative risk

$\mathrm{RRR}=$ relative risk ratio

$\mathrm{SD}=$ standard deviation

$\mathrm{SE}=$ standard error

SON-R 2 1/2-7 = Snijders-Oomen Niet-verbale intelligentie Test-Revisie

$\mathrm{SNRI}=$ serotonin-norepinephrine reuptake inhibitor

$\mathrm{SPQ}=$ Social Problems Questionnaire

SSRI $=$ selective serotonin reuptake inhibitor

$\mathrm{TCA}=$ tricyclic antidepressant

vs. $=$ versus 


\section{Appendix A. Detailed Methods}

\section{Details of Data Sources and Searches}

We conducted focused searches of PubMed, the Cochrane Library, Embase, and PsycINFO from inception to June 5, 2020. The inception date varied by database. PubMed has some citations from the 1940s, but indexing starts reliably in 1950. Cochrane Database of Systematic Reviews records start from 1995. Cochrane Central Register of Controlled Trials is not a standard bibliographic database, rather a collection of records harvested primarily from other databases; therefore, it has no inception (start) date. For Embase, the inception date is 1947. For PsycINFO, the inception date is 1887 . Medical subject headings were used as search terms when available and keywords when appropriate, focusing on terms to describe relevant populations, interventions, outcomes, and study designs. Targeted searches for unpublished literature were conducted by searching ClinicalTrials.gov and the World Health Organization International Clinical Trials Registry Platform. The search strategy was developed by an experienced librarian with inputs from the study investigators. The search strategy was also peer reviewed by an independent experienced librarian. To supplement electronic searches, we reviewed the reference lists of pertinent systematic reviews published in 2013 or later to ensure the search strategy captured all relevant studies. A Federal Register notice (FRN) was posted between December 10, 2019 and January 9, 2020, as well as a request for supplemental evidence and data between December 16, 2019 and January 10, 2020. We updated the literature search during the public posting period of the draft report on June 4 to 5, 2020.

\section{Study Selection}

Table A-1 lists inclusion and exclusion criteria.

Table A-1. Inclusion/exclusion criteria

\begin{tabular}{|c|c|c|}
\hline \multirow{2}{*}{$\frac{\text { PICOTS }}{\text { Population }}$} & Inclusion & Exclusion \\
\hline & $\begin{array}{l}\text { KQs 1, 2: Women who are pregnant or postpartum with new or } \\
\text { preexisting diagnosis of anxiety, depression, bipolar disorder, } \\
\text { or schizophrenia } \\
\text { - Anxiety disorders include Diagnostic and Statistical } \\
\text { Manual (DSM) } 5 \text { and DSM-IV diagnoses (including } \\
\text { generalized anxiety disorder, panic disorder, social } \\
\text { anxiety disorder [social phobia], obsessive compulsive } \\
\text { disorder [OCD], and posttraumatic stress disorder } \\
\text { [PTSD]) } \\
\text { Depressive disorders include major depressive } \\
\text { disorder }\end{array}$ & $\begin{array}{l}\text { KQs 1, 2: Studies of women with } \\
\text { disorders other than anxiety } \\
\text { (including PTSD and OCD), } \\
\text { depression, bipolar disorder, and } \\
\text { schizophrenia } \\
\text { KQs } 3,4 \text { : <90\% of sample are } \\
\text { women reproductive age (15-44) } \\
\text { KQs 1-4: Studies with } 100 \% \\
\text { substance use disorders }\end{array}$ \\
\hline & $\begin{array}{l}\text { KQs } 3 \text {, } 4 \text { : Reproductive-aged women (15- } 44 \text { years old during } \\
\text { preconception }[\leq 12 \text { weeks before pregnancy], pregnancy, and } \\
\text { postpartum [through } 1 \text { year]) with any mental health disorder } \\
\text { (new or preexisting) }\end{array}$ & \\
\hline
\end{tabular}




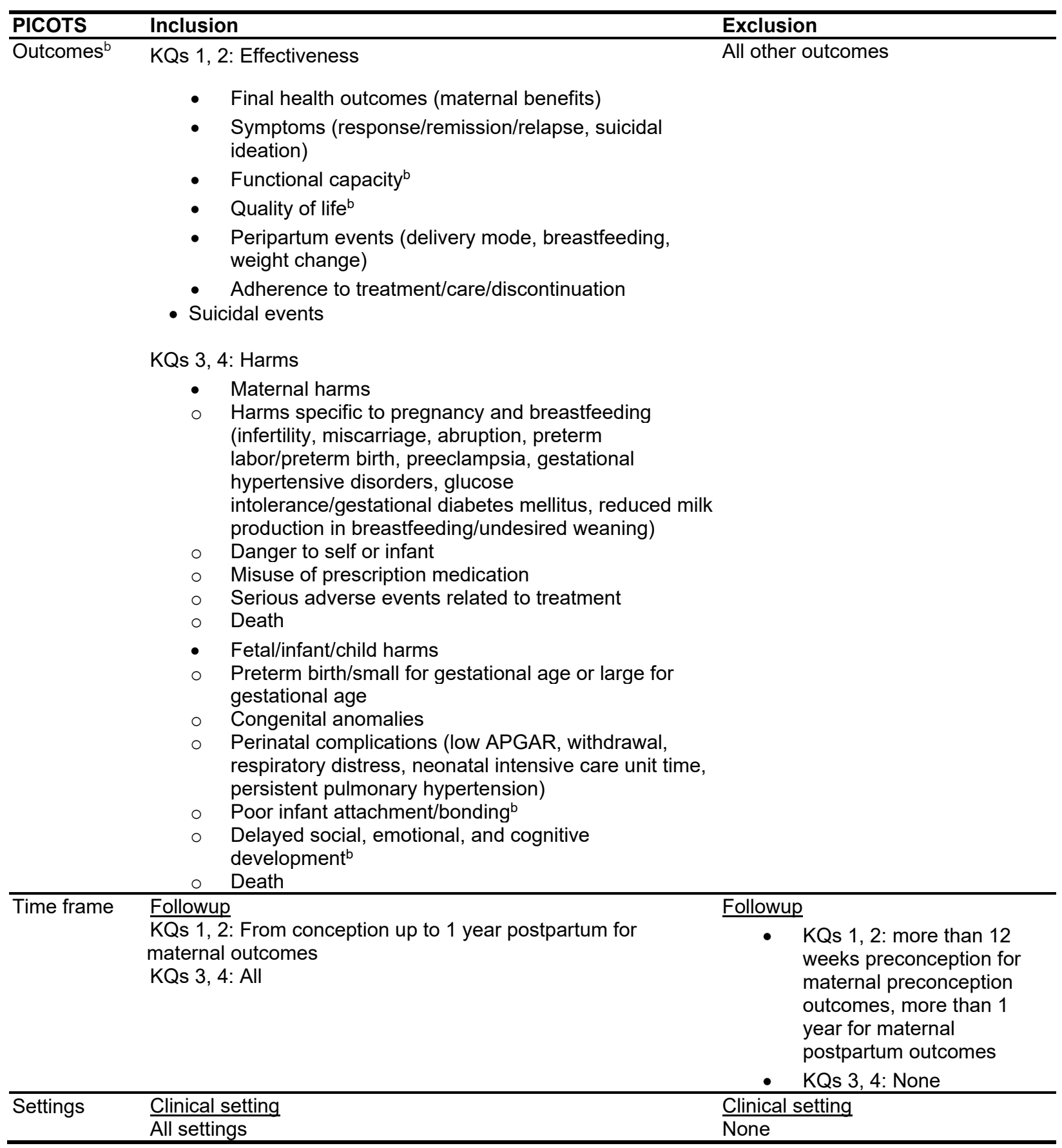




\begin{tabular}{|c|c|c|}
\hline PICOTS & Inclusion & Exclusion \\
\hline Study design ${ }^{c}$ & $\begin{array}{l}\text { Randomized controlled trials (RCTs), controlled clinical trials } \\
\text { (CCTs), case-control studies, cohort studies with comparison } \\
\text { arms } \\
\text { Reference lists of relevant systematic reviews published in } \\
2013 \text { or later are used to ensure our search strategies captured } \\
\text { all relevant studies. }\end{array}$ & $\begin{array}{l}\text { All other designs and studies using } \\
\text { included designs that do not meet } \\
\text { the sample size criterion }\end{array}$ \\
\hline Language & Studies published in English & $\begin{array}{l}\text { Studies published in languages other } \\
\text { than English }\end{array}$ \\
\hline $\begin{array}{l}{ }^{a} \text { Strength of evi } \\
\text { b Outcomes were }\end{array}$ & $\begin{array}{l}\text { dence (SOE) grades were focused on outcomes above prioritized by t } \\
\text { e limited to validated measures. }\end{array}$ & Technical Expert Panel (TEP). \\
\hline${ }^{\mathrm{c}} \mathrm{KQs} 1$ and 3 an & alyses were limited to studies that control for confounding. & \\
\hline $\begin{array}{l}\mathrm{CCT}=\text { case-con } \\
\text { disorder; PICOT } \\
\mathrm{RCT}=\text { randomiz } \\
\text { inhibitor; } \mathrm{SOE}=\end{array}$ & $\begin{array}{l}\text { olled trial; DSM = Diagnostic and Statistical Manual; KQ = Key Que } \\
=\text { population, interventions, comparisons, outcomes, timing, and setti } \\
\text { d controlled trial; SNRI = serotonin and norepinephrine reuptake inhil } \\
\text { trength of evidence; TEP = Technical Expert Panel. }\end{array}$ & $\begin{array}{l}\text { stion; } \mathrm{OCD}=\text { obsessive compulsive } \\
\text { ing; PTSD = posttraumatic stress disorder; } \\
\text { bitor; SSRI = selective serotonin reuptake }\end{array}$ \\
\hline
\end{tabular}

We imported all citations identified through searches and other sources into EndNote v.X9. Independent reviewers screened the titles and abstracts of all citations using the inclusion and exclusion criteria using Abstrackr. Studies included by either reviewer were retrieved for fulltext screening. Independent reviewers then screened the full-text version of eligible references. Discrepancies between the reviewers were resolved through discussions and consensus or consultation with a third reviewer. Excluded studies are listed in the Results Appendix.

\section{Data Abstraction}

We developed and pilot tested a standardized data extraction form to extract study characteristics (author, study design, inclusion and exclusion criteria, patient characteristics, interventions, comparisons, outcomes, settings, and related items for assessing study quality and applicability). Trained reviewers abstracted the relevant data from each included article into the evidence tables; a second member of the team reviewed all data abstractions for completeness and accuracy.

\section{Assessment of Methodological Risk of Bias of Individual Studies}

The criteria set forth by the Agency for Healthcare Research and Quality's Methods Guide for Comparative Effectiveness Reviews guided our assessment of methodological risk of bias. To assess the risk of bias (i.e., internal validity), we used the ROBINS-I ${ }^{1}$ tool for observational studies and the Cochrane RCT ${ }^{2}$ tool for RCTs. For both observational studies and RCTs, risk-ofbias assessment included questions to assess selection bias, confounding, performance bias, detection bias, and attrition bias; concepts covered include those about adequacy of randomization (for RCTs only), similarity of groups at baseline, masking, attrition, whether intention-to-treat analysis was used, method of handling dropouts and missing data, validity and reliability of outcome measures, and treatment fidelity. ${ }^{3}$

Two independent reviewers assigned risk-of-bias ratings for each study with disagreements resolved by discussion and consensus. Reviewers assigned a rating of low risk of bias (study met all criteria), some concerns (study met some criteria), high risk of bias (methodological 
shortcomings leading to high risk of bias in one or more categories), or unclear risk of bias (methods not reported clearly).

We did not assess the risk of bias or strength of evidence for studies eligible for the contextual question.

\section{Data Synthesis}

\section{Planned Analyses}

We summarized all included studies in narrative form and in summary tables that tabulate the important features of the study populations, design, intervention, comparison, outcomes, setting (including geographic location), and results.

If we found three or more studies with low levels of heterogeneity (similar populations, interventions, comparators, outcomes), we considered meta-analysis. To determine whether quantitative analyses were appropriate for bodies of evidence that contained three or more similar studies, we assessed the clinical and methodological heterogeneity of the studies under consideration following established guidance. ${ }^{3,4}$ For all analyses, we used random effects models to estimate pooled or comparative effects; unlike a fixed-effects model, this approach allowed for the likelihood that the true population effect may vary from study to study.

We calculated standardized differences (relative risks or standardized mean differences) for outcomes; when we graded the strength-of-evidence (SOE) grade as higher than insufficient, we also presented absolute differences in effect, when possible, in the detailed results to aid with interpretation of results.

We assessed statistical heterogeneity in effects between studies included in meta-analyses by calculating the chi-square statistic and the $\mathrm{I}^{2}$ statistic (the proportion of variation in study estimates due to heterogeneity). The importance of the observed value of $\mathrm{I}^{2}$ depends on the magnitude and direction of effects and on the SOE for heterogeneity (e.g., p-value from the chisquare test or a confidence interval for $\mathrm{I}^{2}$ ).

When possible, for each intervention/comparator grouping, we present findings clustered by perinatal status and disorder. We also note special characteristics of the sample required for study inclusions.

\section{Addressing Confounding}

As noted in the main report, one significant methodological limitation of the nonrandomized controlled trial (non-RCT) evidence base is confounding. All observational studies are subject to confounding by indication - that is, the extent to which exposure to maternal mental health morbidity influences both exposure to the medication and outcomes. To address this limitation, some studies have compared women receiving pharmacotherapy with women who have the same mental health disorder but who are not receiving treatment. ${ }^{5}$ However, this approach does not address confounding arising from disease severity, in that women who are receiving pharmacologic treatment may be likely to have more severe underlying disease than women who forgo pharmacologic therapy. Others trying to address residual confounding with highdimensional propensity scores have found that full adjustment leads to many associations being no longer statistically significant. ${ }^{6,7}$ In analyzing the evidence, we accounted for the manner of adjustment for confounding. 
Study designs most likely to report on harms associated with maternal use of psychotropic medications include case-control studies, pregnancy registry studies, observational cohort studies, and secondary analyses of administrative databases. Each of these study designs has strengths and limitations. Case-control studies allow assessment of associations with rare outcomes, such as congenital anomalies; however, both recall bias and selection of an appropriate control population are concerns. Pregnancy registry studies facilitate postmarketing surveillance of new medications but may be limited by selection bias. Observational cohort studies, particularly large birth cohorts such as the Norwegian Mother and Child Cohort Study ${ }^{8}$ and the Danish National Birth Cohort, ${ }^{9}$ capture sufficiently large samples to assess relatively rare outcomes with prospective assessment of exposure, but they cannot fully address confounding. Large administrative databases ${ }^{10,11}$ allow assessment of rare outcomes such as congenital anomalies but have problems in identifying pregnancies, misclassifying exposures, specifying outcomes and covariates, and addressing confounding. ${ }^{11}$

To address these limitations, we limited analysis of results of KQs 1 and 3 (benefits and harms of interventions compared with no treatment, usual care, or placebo) to studies that adjusted for confounding through matching, regression, or propensity score adjustments and provided these adjusted results for the comparison of interest (e.g., active intervention vs. no treatment for women with a diagnosis). We did not calculate indirect comparisons from studies that present adjusted results for comparisons outside the remit of this review. For example, several studies presented adjusted results for women with a drug exposure versus women with no drug exposure and no disorders and, separately, adjusted results comparing women with a disorder but no drug exposure versus women with no drug exposure and no disorder. Calculated indirect comparisons for women with a drug exposure versus women with a disorder but no drug exposure would be at risk of violating assumptions about transitivity and would likely have residual confounding. ${ }^{12}$

For KQs 2 and 4 (benefits and harms from head-to-head comparisons of interventions), we included studies that did not provide adjusted results that addressed confounding, but we marked down the evidence base for high potential for risk of bias. We excluded studies that did not present mutually exclusive arms: in studies with overlapping cases in exposure arms, the association between the intervention and the outcome cannot be established. We also required clarity on the interventions for both arms. We did not synthesize results from studies comparing an active intervention with "other antidepressants," polytherapy, or co-exposure to other drugs with no further elaboration. In these instances, because we could not discern the nature of the exposure, we could not interpret the clinical significance of differences in outcomes between the arms.

\section{Sensitivity Analyses}

When relevant (the evidence included both studies with high risk of bias and without high risk of bias), we conducted qualitative or quantitative sensitivity analyses to gauge the difference in conclusions upon including and excluding high risk-of-bias studies. For bodies of evidence with meta-analyses, we reported effect sizes with and without high risk-of-bias studies.

\section{Grading the Strength of Evidence}

We graded the SOE based on the Grading of Recommendations Assessment, Development and Evaluation (short GRADE) working group guidance ${ }^{13}$ and guidance established for the Evidence-based Practice Center Program. ${ }^{14}$ Developed to grade the overall strength of a body of 
evidence, this approach incorporates five key domains: risk of bias (includes study design and aggregate quality), consistency, directness, precision of the evidence, and reporting bias. This approach requires looking beyond statistical significance alone, even when studies are consistent and of high quality and outcomes are direct and clinically relevant. It emphasizes the adequacy of the sample size to rule out spurious associations and results that are not clinically relevant. It also considers other optional domains that may be relevant to increasing the SOE for some scenarios, such as a dose-response association, plausible confounding that would decrease the observed effect, and strength of association (magnitude of effect). We depart from GRADE guidance and follow EPC-specific guidance on the starting grade for observational studies and the rating of the consistency domain for single-study bodies of evidence. We rate bodies of evidence from observational studies as moderate if no other reasons arise to downgrade the evidence. We downgrade single-study bodies of evidence for unknown consistency.

Table 2 describes the grades of evidence that could be assigned. Grades reflect the strength of the body of evidence to answer KQs on the comparative effectiveness, efficacy, and harms of the interventions included in this review. Two reviewers assessed each domain for each key outcome with differences resolved by consensus. Because these are direct outcomes, the evidence was not downgraded for indirectness; the SOE tables do not explicitly grade for directness as a result.

Table A-2. Definitions of the grades of overall SOE ${ }^{14}$

\begin{tabular}{ll}
\hline Grade & Definition \\
\hline High & $\begin{array}{l}\text { High confidence that the evidence reflects the true effect. Further research is very unlikely to } \\
\text { change our confidence in the estimate of effect. }\end{array}$ \\
\hline Moderate & $\begin{array}{l}\text { Moderate confidence that the evidence reflects the true effect. Further research may change } \\
\text { our confidence in the estimate of the effect and may change the estimate. }\end{array}$ \\
\hline Low & $\begin{array}{l}\text { Low confidence that the evidence reflects the true effect. Further research is likely to change } \\
\text { our confidence in the estimate of the effect and is likely to change the estimate. }\end{array}$ \\
\hline Insufficient & Evidence either is unavailable or does not permit estimation of an effect. \\
\hline SOE = strength of evidence.
\end{tabular}

For bodies of evidence for which we could conduct sensitivity analyses, we based the final SOE grade on the evidence base without high risk-of-bias studies. We appended a footnote to SOE tables to indicate when sensitivity analyses changed the SOE grade.

\section{Assessing Applicability}

We assessed the applicability of individual studies and the applicability of a body of evidence following guidance from the Methods Guide for Effectiveness and Comparative Effectiveness Reviews. ${ }^{15}$ For individual studies, we examined conditions that may limit applicability based on the PICOTS (population, interventions, comparisons, outcomes, timing, and setting) structure. Some factors identified that may limit the applicability of evidence include the following: perinatal status, severity and type of disorder, comorbid conditions, history of previous depressive episodes or depression treatment, or setting (primary care vs. specialty care). We indicated perinatal status and type of disorder in the analysis and otherwise call out characteristics of the study populations that might limit applicability.

\section{Peer Review and Public Commentary}

Experts in the treatment of mental health conditions in perinatal populations were invited to provide external peer review of the draft systematic review that was entitled "Maternal and Fetal Effects of Mental Health Treatments in Pregnant and Breastfeeding Women: A Systematic 
Review of Pharmacological Interventions." AHRQ staff and an Associate Editor reviewed the draft systematic review before it went out for peer review. The EPC Associate Editors are leaders in their respective fields and are actively involved as directors or leaders at their EPCs. Their role is to assess adherence to established methodology and guidelines for EPC-based research. We revised the report in response to AHRQ staff, the Associate Editor, peer reviewer, and public comments.

\section{Search Strategy}

\section{Mental Health Treatment in Pregnant Women Published Literature Searches}

\section{PubMed}

12-9-2018

\begin{tabular}{|c|c|c|c|}
\hline Search & Query & Items Found & Notes \\
\hline \#1 & $\begin{array}{l}\text { Search ("Anxiety Disorders"[Mesh] OR anxiety[tiab] OR "Bipolar } \\
\text { Disorder"[Mesh] OR bipolar[tiab] OR "Depressive Disorder"[MeSH] OR } \\
\text { "Depressive Disorder, Major"[Mesh] OR Depression[Mesh] OR } \\
\text { depress*[tiab] OR depression[tiab] OR depressive[tiab] OR } \\
\text { depressed[tiab] OR "Dysthymic Disorder"[Mesh] OR dysthymia[tiab] OR } \\
\text { dysthymic[tiab] OR "Feeding and Eating Disorders"[Mesh] OR } \\
\text { anorexia[tiab] OR anorexic[tiab] OR "binge eating"[tiab] OR bulimic[tiab] } \\
\text { OR bulimia[tiab] OR GAD OR "Psychotic Disorders"[Mesh] OR "psychotic } \\
\text { disorder"[tiab] OR "psychotic disorders"[tiab] OR psychosis[tiab] OR } \\
\text { psychoses[tiab] OR (mental[Tiab] AND (health[Tiab] OR illness[Tiab] OR } \\
\text { disorders[Tiab])) OR "Mental Health"[Mesh] OR "Mental Disorders"[Mesh] } \\
\text { OR "Obsessive-Compulsive Disorder"[Mesh] OR "Obsessive-Compulsive } \\
\text { Disorder"[tiab] OR OCD[tiab] OR "panic disorder" OR "Persistent } \\
\text { Depressive Disorder"[tiab] OR phobia*[tiab] OR phobic[tiab] OR } \\
\text { psychotic*[tiab] OR "Stress Disorders, Post-Traumatic"[Mesh] OR "post- } \\
\text { traumatic stress disorder"[All Fields] OR "post-traumatic stress } \\
\text { disorders"[All Fields] OR "posttraumatic stress disorder"[All Fields] OR } \\
\text { "posttraumatic stress disorders"[All Fields] OR (disorder* AND "post- } \\
\text { traumatic"[tiab]) OR "Stress Disorders, Traumatic"[Mesh:NOEXP] OR } \\
\text { PTSD[tiab] OR "Schizophrenia"[Mesh] OR schizophren*[tiab] OR "stress } \\
\text { disorder"[All Fields]) }\end{array}$ & 1656505 & \\
\hline \#2 & $\begin{array}{l}\text { Search (alprazolam OR "Anti-Anxiety Agents"[Mesh] OR "Anti-Anxiety } \\
\text { Agents"[Pharmacological Action] OR anti-anxiety OR antianxiety OR } \\
\text { "Amitriptyline"[Mesh] OR amitriptyline OR "Amoxapine"[Mesh] OR } \\
\text { amoxapine OR Anticonvulsants[Mesh] OR } \\
\text { Anticonvulsants[Pharmacological Action] OR anticonvulsant OR } \\
\text { anticonvulsants OR "Antidepressive Agents"[MeSH] OR "Antidepressive } \\
\text { Agents, Second-Generation"[MeSH] OR anti-depress* OR } \\
\text { antidepressant* OR antidepressive* OR "antidepressive agent" OR } \\
\text { "antidepressive agents" OR "antidepressive drug" OR "antidepressive } \\
\text { drugs" OR "Antipsychotic Agents"[Mesh] OR antipsychotic* OR } \\
\text { anxiolytic* OR "Aripiprazole"[Mesh] OR aripiprazole OR } \\
\text { "Asenapine"[Supplementary Concept] OR Asenapine OR } \\
\text { benzodiazepine* OR brexanolone OR brexpiprazole OR } \\
\text { Bupropion[MeSH] OR Bupropion OR "Buspirone"[Mesh] OR Buspirone } \\
\text { OR "Carbamazepine"[Mesh] OR Carbamazepine OR Cariprazine OR } \\
\text { "Chlorpromazine"[Mesh] OR Chlorpromazine OR Citalopram[MeSH] OR } \\
\text { citalopram OR "clobazam" [Supplementary Concept] OR clobazam OR } \\
\text { "Clomipramine"[Mesh] OR Clomipramine OR "Clonazepam"[Mesh] OR } \\
\text { Clonazepam OR "Clonidine"[Mesh] OR Clonidine OR "Clorazepate } \\
\text { Dipotassium"[Mesh] OR Clorazepate OR "Chlordiazepoxide"[Mesh] OR } \\
\text { Chlordiazepoxide OR "Clozapine"[Mesh] OR clozapine) }\end{array}$ & 447815 & \\
\hline
\end{tabular}




\begin{tabular}{|c|c|c|c|}
\hline Search & Query & Items Found & Notes \\
\hline \#3 & $\begin{array}{l}\text { Search ("Desipramine"[Mesh] OR Desipramine OR "Desvenlafaxine } \\
\text { Succinate"[Mesh] OR Desvenlafaxine OR "Diazepam"[Mesh] OR } \\
\text { Diazepam OR "Diphenhydramine"[Mesh] OR Diphenhydramine OR } \\
\text { "Doxepin"[Mesh] OR doxepin OR "Duloxetine Hydrochloride"[Mesh] OR } \\
\text { duloxetine OR escitalopram OR "Eszopiclone"[Mesh] OR eszopiclone OR } \\
\text { Fluoxetine[MeSH] OR fluoxetine OR "Fluphenazine"[Mesh] OR } \\
\text { fluphenazine OR Fluvoxamine[MeSH] OR fluvoxamine OR } \\
\text { "gabapentin"[Supplementary Concept] OR gabapentin OR } \\
\text { "Haloperidol"[Mesh] OR Haloperidol OR "Hydroxyzine"[Mesh] OR } \\
\text { Hydroxyzine OR "iloperidone"[Supplementary Concept] OR iloperidone } \\
\text { OR "Imipramine"[Mesh] OR imipramine OR "lamotrigine"[Supplementary } \\
\text { Concept] OR lamotrigine OR "Levomilnacipran"[Mesh] OR } \\
\text { Levomilnacipran OR "Lisdexamfetamine Dimesylate "[Mesh] OR "Lithium } \\
\text { Carbonate"[Mesh] OR lithium OR "Lorazepam"[Mesh] OR lorazepam OR } \\
\text { "Lurasidone Hydrochloride"[Mesh] OR lurasidone OR } \\
\text { "milnacipran"[Supplementary Concept]) }\end{array}$ & 160740 & \\
\hline \#4 & $\begin{array}{l}\text { Search (milnacipran OR "Maprotiline"[Mesh] OR Maprotiline OR } \\
\text { mirtazapine[Supplementary Concept] OR mirtazapine OR } \\
\text { "nefazodone"[Supplementary Concept] OR nefazodone OR } \\
\text { "norepinephrine reuptake inhibitor" OR "norepinephrine reuptake } \\
\text { inhibitors" OR "Nortriptyline"[Mesh] OR nortriptyline OR } \\
\text { "olanzapine"[Supplementary Concept] OR olanzapine OR } \\
\text { "oxcarbazepine"[Supplementary Concept] OR oxcarbazepine OR } \\
\text { "Paliperidone Palmitate"[Mesh] OR Paliperidone OR Paroxetine[MeSH] } \\
\text { OR paroxetine OR "Perphenazine"[Mesh] OR perphenazine OR } \\
\text { "Protriptyline"[Mesh] OR protriptyline OR "Quetiapine Fumarate"[Mesh] } \\
\text { OR quetiapine OR "ramelteon" [Supplementary Concept] OR ramelteon } \\
\text { OR "Risperidone"[Mesh] OR risperidone OR "selective serotonin } \\
\text { reuptake inhibitor" OR "selective serotonin reuptake inhibitors" OR } \\
\text { "Serotonin Uptake Inhibitors"[MeSH] OR "serotonin norepinephrine } \\
\text { reuptake inhibitor"[All Fields] OR "serotonin norepinephrine reuptake } \\
\text { inhibitors"[All Fields] OR Sertraline[MeSH] OR sertraline OR SNRI* OR } \\
\text { SSRI OR SSRIs) }\end{array}$ & 70738 & \\
\hline \#5 & $\begin{array}{l}\text { Search ("Temazepam"[Mesh] OR Temazepam "Thioridazine"[Mesh] OR } \\
\text { Thioridazine OR "Thiothixene"[Mesh] OR Thiothixene OR } \\
\text { "topiramate"[Supplementary Concept] OR topiramate OR } \\
\text { Trazodone[Mesh] OR trazodone OR "Triazolam"[Mesh] OR triazolam OR } \\
\text { "Trifluoperazine"[Mesh] OR Trifluoperazine OR "Trimipramine"[Mesh] OR } \\
\text { Trimipramine OR "Valproic Acid"[Mesh] OR valproate OR "Venlafaxine } \\
\text { Hydrochloride"[Mesh] OR venlafaxine OR "Vilazodone } \\
\text { Hydrochloride"[Mesh] OR vilazodone OR "vortioxetine"[Supplementary } \\
\text { Concept] OR vortioxetine OR "zaleplon"[Supplementary Concept] OR } \\
\text { zaleplon OR "ziprasidone"[Supplementary Concept] OR ziprasidone OR } \\
\text { "zolpidem"[Supplementary Concept] OR zolpidem) }\end{array}$ & 42558 & \\
\hline$\# 6$ & Search (\#2 or \#3 or \#4 or \#5) & 534672 & \\
\hline$\# 7$ & Search (\#1 and \#6) & 184651 & \\
\hline \#8 & $\begin{array}{l}\text { Search ("Pregnant Women"[Mesh] OR Pregnancy[Mesh] OR } \\
\text { preconception[tiab] OR pregnant[tiab] OR pregnancy[tiab] OR } \\
\text { prenatal[tiab] OR "post-partum"[tiab] OR postpartum[tiab] OR } \\
\text { postnatal[tiab] OR perinatal[tiab] OR antenatal[tiab] OR "Maternal Health } \\
\text { Services"[Mesh] OR "maternal health"[tiab] OR "Infant Nutritional } \\
\text { Physiological Phenomena"[Mesh] OR "Breast Feeding"[Mesh] OR "breast } \\
\text { feeding"[tiab] OR breastfeed*[tiab] OR (breast[tiab] AND fed[tiab]) OR } \\
\text { breastfed[tiab] OR "Pregnancy Complications"[All Fields] OR "Maternal } \\
\text { Welfare"[All Fields] OR gestation*[tiab] OR maternal*[tiab] OR } \\
\text { "Pregnancy Outcome"[Mesh]) }\end{array}$ & 1220300 & \\
\hline$\# 9$ & Search (\#7 and \#8) & 6083 & \\
\hline \#10 & Search (\#7 and \#8) Filters: English & 5540 & \\
\hline \#11 & $\begin{array}{l}\text { Search ((\#10 AND Humans[Mesh:noexp]) OR (\#10 NOT } \\
\text { Animals[Mesh:noexp])) }\end{array}$ & 4340 & \\
\hline
\end{tabular}




\begin{tabular}{|c|c|c|c|}
\hline Search & Query & Items Found & Notes \\
\hline$\# 12$ & $\begin{array}{l}\text { Search (accident* OR "Adverse Effects" OR "adverse effect" or "adverse } \\
\text { event" OR "adverse events" OR "adverse outcome" OR "adverse } \\
\text { outcomes" OR "adverse reaction" OR "adverse reactions" OR "chemically } \\
\text { induced" OR complication* OR death* OR "Drug Allergies" OR "Drug } \\
\text { Dependency" OR "drug effects" OR "Drug Sensitivity" OR harm* OR } \\
\text { harms OR "Long Term Adverse Effects"[Mesh] OR "manic episode" OR } \\
\text { overdos* OR "Patient Safety" OR poisoning OR self damage* OR self } \\
\text { injur* OR self inflict* OR "Self-Injurious Behavior"[Mesh] OR "Side Effect" } \\
\text { OR "side effects" OR Suicide OR suicidal* OR toxicity) }\end{array}$ & 7964680 & \\
\hline$\# 13$ & $\begin{array}{l}\text { Search ("Abortion, Spontaneous"[Mesh] OR "Abruptio Placentae"[Mesh] } \\
\text { OR abruption*[tiab] OR "Apgar Score"[Mesh] OR "Birth } \\
\text { Weight/chemically induced"[Mesh] OR "Birth Weight/drug effects"[Mesh] } \\
\text { OR "Child Development Disorders, Pervasive/chemically induced"[Mesh] } \\
\text { OR "Child Development/drug effects"[Mesh] OR "Craniofacial } \\
\text { Abnormalities/chemically induced"[Mesh] OR "Congenital } \\
\text { Abnormalities"[Mesh] OR "congenital abnormality"[tiab] OR "congenital } \\
\text { abnormalities"[tiab] OR death[tiab] OR "delayed development"[ALL } \\
\text { FIELDS] OR "Glucose Intolerance"[Mesh] OR "glucose intolerance"[tiab] } \\
\text { OR "Infant, Extremely Premature/growth and development"[Mesh] OR } \\
\text { (infant* AND (attachment* OR bonding)) OR "Infertility, Female"[Mesh] } \\
\text { OR “Infantile Respiratory Distress Syndrome"[tiab] OR infertility[tiab] OR } \\
\text { ("Intellectual Disability"[Mesh] AND child*[tw]) OR "Low APGAR"[tiab] OR } \\
\text { miscarry[tiab] OR miscarriage*[tiab] OR Mortality[Mesh] OR mortality[tiab] } \\
\text { OR "Neonatal Abstinence Syndrome"[Mesh] OR "Neonatal Respiratory } \\
\text { Distress Syndrome"[tiab] OR "Obstetric Labor, Premature"[Mesh] OR } \\
\text { "Persistent Fetal Circulation Syndrome"[Mesh] OR "Persistent Pulmonary } \\
\text { Hypertension of Newborn"[tiab] OR "Pre-Eclampsia"[Mesh] OR } \\
\text { preeclampsia[tiab] OR pre-eclampsia[tiab] OR "Premature Birth"[Mesh] } \\
\text { OR "premature birth"[tiab] OR "preterm birth" OR "pre-term birth"[tiab] OR } \\
\text { "Prenatal Exposure Delayed Effects"[Mesh] OR "Prescription Drug } \\
\text { Misuse"[Mesh] OR ("Prescription Drug"[tiab] AND misuse[tiab]) OR } \\
\text { "preterm labor"[tiab] OR "pre-term labor"[tiab] OR "Postpartum } \\
\text { Hemorrhage"[Mesh] OR "Respiratory Distress Syndrome, } \\
\text { Newborn"[Mesh] OR "Uterine Inertia/chemically induced"[Mesh]) }\end{array}$ & 2154910 & $\begin{array}{l}\text { Now with all } \\
\text { harms } \\
\text { terms } \\
\text { mentioned } \\
\text { in PICOs } \\
\text { table }\end{array}$ \\
\hline$\# 14$ & Search $(\# 12$ or \#13) & 8795543 & \\
\hline$\# 15$ & 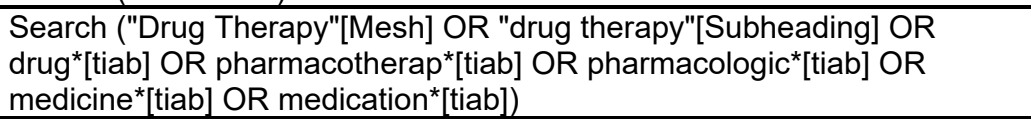 & 4349451 & \\
\hline \#16 & Search (\#14 and \#15 and \#8 and \#1) & 11864 & \\
\hline$\# 17$ & Search (\#14 and \#15 and \#8 and \#1) Filters: English & 10679 & \\
\hline$\# 18$ & $\begin{array}{l}\text { Search ((\#17 AND Humans[Mesh:noexp]) OR (\#17 NOT } \\
\text { Animals[Mesh:noexp])) }\end{array}$ & 8952 & \\
\hline \#19 & $\begin{array}{l}\text { Search ((\#10 AND Humans[Mesh:noexp]) OR (\#10 NOT } \\
\text { Animals[Mesh:noexp])) Filters: Case Reports }\end{array}$ & 550 & \\
\hline \#20 & $\begin{array}{l}\text { Search ((\#10 AND Humans[Mesh:noexp]) OR (\#10 NOT } \\
\text { Animals[Mesh:noexp])) Filters: Case Reports; Editorial }\end{array}$ & 615 & \\
\hline \#21 & Search (\#11 NOT (\#19 or \#20)) & 3725 & $\begin{array}{l}\text { Benefits } \\
\text { results }\end{array}$ \\
\hline \#22 & $\begin{array}{l}\text { Search ((\#17 AND Humans[Mesh:noexp]) OR (\#17 NOT } \\
\text { Animals[Mesh:noexp])) Filters: Case Reports }\end{array}$ & 902 & \\
\hline \#23 & $\begin{array}{l}\text { Search ((\#17 AND Humans[Mesh:noexp]) OR (\#17 NOT } \\
\text { Animals[Mesh:noexp])) Filters: Case Reports; Editorial }\end{array}$ & 1005 & \\
\hline \#24 & Search (\#18 NOT (\#22 OR \#23)) & 7947 & $\begin{array}{l}\text { Harms } \\
\text { results }\end{array}$ \\
\hline$\# 25$ & Search (\#21 and \#24) & 2384 & $\begin{array}{l}\text { Overlap of } \\
\text { benefits and } \\
\text { harms } \\
\text { results }\end{array}$ \\
\hline
\end{tabular}




\begin{tabular}{|c|c|c|c|}
\hline Search & Query & Items Found & Notes \\
\hline \#26 & Search (\#21 not \#25) & 1341 & $\begin{array}{l}\text { Unique } \\
\text { benefits } \\
\text { results not } \\
\text { in harms } \\
\text { results }\end{array}$ \\
\hline \#27 & Search (\#24 not \#25) & 5563 & $\begin{array}{l}\text { Unique } \\
\text { harms } \\
\text { results not } \\
\text { in benefits } \\
\text { results }\end{array}$ \\
\hline \#28 & Search ( $\# 25$ or $\# 26$ or $\# 27)$ & 9288 & $\begin{array}{l}\text { Total } \\
\text { number for } \\
\text { PubMed }\end{array}$ \\
\hline
\end{tabular}




\section{PubMed}

6-4-2020

\begin{tabular}{|c|c|c|}
\hline $\begin{array}{l}\text { Search } \\
\text { Number }\end{array}$ & Filters & Results \\
\hline 1 & $\begin{array}{l}\text { "Anxiety Disorders"[Mesh] OR anxiety[tiab] OR "Bipolar Disorder"[Mesh] } \\
\text { OR bipolar[tiab] OR "Depressive Disorder"[MeSH] OR "Depressive } \\
\text { Disorder, Major"[Mesh] OR Depression[Mesh] OR depress*[tiab] OR } \\
\text { depression[tiab] OR depressive[tiab] OR depressed[tiab] OR "Dysthymic } \\
\text { Disorder"[Mesh] OR dysthymia[tiab] OR dysthymic[tiab] OR "Feeding } \\
\text { AND Eating Disorders"[Mesh] OR anorexia[tiab] OR anorexic[tiab] OR } \\
\text { "binge eating"[tiab] OR bulimic[tiab] OR bulimia[tiab] OR GAD OR } \\
\text { "Psychotic Disorders"[Mesh] OR "psychotic disorder"[tiab] OR "psychotic } \\
\text { disorders"[tiab] OR psychosis[tiab] OR psychoses[tiab] OR (mental[Tiab] } \\
\text { AND (health[Tiab] OR illness[Tiab] OR disorders[Tiab])) OR "Mental } \\
\text { Health"[Mesh] OR "Mental Disorders"[Mesh] OR "Obsessive-Compulsive } \\
\text { Disorder"[Mesh] OR "Obsessive-Compulsive Disorder"[tiab] OR OCD[tiab] } \\
\text { OR "panic disorder" OR "Persistent Depressive Disorder"[tiab] OR } \\
\text { phobia*[tiab] OR phobic[tiab] OR psychotic*[tiab] OR "Stress Disorders, } \\
\text { Post-Traumatic"[Mesh] OR "post-traumatic stress disorder"[All Fields] OR } \\
\text { "post-traumatic stress disorders"[All Fields] OR "posttraumatic stress } \\
\text { disorder"[All Fields] OR "posttraumatic stress disorders"[All Fields] OR } \\
\text { (disorder* AND "post-traumatic"[tiab]) OR "Stress Disorders, } \\
\text { Traumatic"[Mesh:NOEXP] OR PTSD[tiab] OR "Schizophrenia"[Mesh] OR } \\
\text { schizophren*[tiab] OR "stress disorder"[All Fields] }\end{array}$ & $1,791,649$ \\
\hline 2 & $\begin{array}{l}\text { alprazolam OR "Anti-Anxiety Agents"[Mesh] OR "Anti-Anxiety } \\
\text { Agents"[Pharmacological Action] OR anti-anxiety OR antianxiety OR } \\
\text { "Amitriptyline"[Mesh] OR amitriptyline OR "Amoxapine"[Mesh] OR } \\
\text { amoxapine OR Anticonvulsants[Mesh] OR } \\
\text { Anticonvulsants[Pharmacological Action] OR anticonvulsant OR } \\
\text { anticonvulsants OR "Antidepressive Agents"[MeSH] OR "Antidepressive } \\
\text { Agents, Second-Generation"[MeSH] OR anti-depress* OR } \\
\text { antidepressant* OR antidepressive* OR "antidepressive agent" OR } \\
\text { "antidepressive agents" OR "antidepressive drug" OR "antidepressive } \\
\text { drugs" OR "Antipsychotic Agents"[Mesh] OR antipsychotic* OR anxiolytic* } \\
\text { OR "Aripiprazole"[Mesh] OR aripiprazole OR "Asenapine"[Supplementary } \\
\text { Concept] OR Asenapine OR benzodiazepine* OR brexanolone OR } \\
\text { brexpiprazole OR Bupropion[MeSH] OR Bupropion OR "Buspirone"[Mesh] } \\
\text { OR Buspirone OR "Carbamazepine"[Mesh] OR Carbamazepine OR } \\
\text { Cariprazine OR "Chlorpromazine"[Mesh] OR Chlorpromazine OR } \\
\text { Citalopram[MeSH] OR citalopram OR "clobazam" [Supplementary } \\
\text { Concept] OR clobazam OR "Clomipramine"[Mesh] OR Clomipramine OR } \\
\text { "Clonazepam"[Mesh] OR Clonazepam OR "Clonidine"[Mesh] OR } \\
\text { Clonidine OR "Clorazepate Dipotassium"[Mesh] OR Clorazepate OR } \\
\text { "Chlordiazepoxide"[Mesh] OR Chlordiazepoxide OR "Clozapine"[Mesh] } \\
\text { OR clozapine }\end{array}$ & 505,728 \\
\hline 3 & $\begin{array}{l}\text { "Desipramine"[Mesh] OR Desipramine OR "Desvenlafaxine } \\
\text { Succinate"[Mesh] OR Desvenlafaxine OR "Diazepam"[Mesh] OR } \\
\text { Diazepam OR "Diphenhydramine"[Mesh] OR Diphenhydramine OR } \\
\text { "Doxepin"[Mesh] OR doxepin OR "Duloxetine Hydrochloride"[Mesh] OR } \\
\text { duloxetine OR escitalopram OR "Eszopiclone"[Mesh] OR eszopiclone OR } \\
\text { Fluoxetine[MeSH] OR fluoxetine OR "Fluphenazine"[Mesh] OR } \\
\text { fluphenazine OR Fluvoxamine[MeSH] OR fluvoxamine OR } \\
\text { "gabapentin"[Supplementary Concept] OR gabapentin OR } \\
\text { "Haloperidol"[Mesh] OR Haloperidol OR "Hydroxyzine"[Mesh] OR } \\
\text { Hydroxyzine OR "iloperidone"[Supplementary Concept] OR iloperidone } \\
\text { OR "Imipramine"[Mesh] OR imipramine OR "lamotrigine"[Supplementary } \\
\text { Concept] OR lamotrigine OR "Levomilnacipran"[Mesh] OR } \\
\text { Levomilnacipran OR "Lisdexamfetamine Dimesylate "[Mesh] OR "Lithium } \\
\text { Carbonate"[Mesh] OR lithium OR "Lorazepam"[Mesh] OR lorazepam OR } \\
\text { "Lurasidone Hydrochloride"[Mesh] OR lurasidone OR } \\
\text { "milnacipran"[Supplementary Concept] }\end{array}$ & 170,275 \\
\hline
\end{tabular}




\begin{tabular}{|c|c|c|}
\hline $\begin{array}{l}\text { Search } \\
\text { Number }\end{array}$ & Filters & Results \\
\hline 4 & $\begin{array}{l}\text { milnacipran OR "Maprotiline"[Mesh] OR Maprotiline OR } \\
\text { mirtazapine[Supplementary Concept] OR mirtazapine OR } \\
\text { "nefazodone"[Supplementary Concept] OR nefazodone OR } \\
\text { "norepinephrine reuptake inhibitor" OR "norepinephrine reuptake } \\
\text { inhibitors" OR "Nortriptyline"[Mesh] OR nortriptyline OR } \\
\text { "olanzapine"[Supplementary Concept] OR olanzapine OR } \\
\text { "Oxcarbazepine"[Supplementary Concept] OR oxcarbazepine OR } \\
\text { "Paliperidone Palmitate"[Mesh] OR Paliperidone OR Paroxetine[MeSH] } \\
\text { OR paroxetine OR "Perphenazine"[Mesh] OR perphenazine OR } \\
\text { "Protriptyline"[Mesh] OR protriptyline OR "Quetiapine Fumarate"[Mesh] } \\
\text { OR quetiapine OR "ramelteon" [Supplementary Concept] OR ramelteon } \\
\text { OR "Risperidone"[Mesh] OR risperidone OR "selective serotonin reuptake } \\
\text { inhibitor" OR "selective serotonin reuptake inhibitors" OR "Serotonin } \\
\text { Uptake Inhibitors"[MeSH] OR "serotonin norepinephrine reuptake } \\
\text { inhibitor"[All Fields] OR "serotonin norepinephrine reuptake inhibitors"[All } \\
\text { Fields] OR Sertraline[MeSH] OR sertraline OR SNRI* OR SSRI OR } \\
\text { SSRIs }\end{array}$ & 74,815 \\
\hline$\overline{5}$ & $\begin{array}{l}\text { "Temazepam"[Mesh] OR Temazepam "Thioridazine"[Mesh] OR } \\
\text { Thioridazine OR "Thiothixene"[Mesh] OR Thiothixene OR } \\
\text { "topiramate"[Supplementary Concept] OR topiramate OR } \\
\text { Trazodone[Mesh] OR trazodone OR "Triazolam"[Mesh] OR triazolam OR } \\
\text { "Trifluoperazine"[Mesh] OR Trifluoperazine OR "Trimipramine"[Mesh] OR } \\
\text { Trimipramine OR "Valproic Acid"[Mesh] OR valproate OR "Venlafaxine } \\
\text { Hydrochloride"[Mesh] OR venlafaxine OR "Vilazodone } \\
\text { Hydrochloride"[Mesh] OR vilazodone OR "vortioxetine"[Supplementary } \\
\text { Concept] OR vortioxetine OR "zaleplon"[Supplementary Concept] OR } \\
\text { zaleplon OR "ziprasidone"[Supplementary Concept] OR ziprasidone OR } \\
\text { "zolpidem"[Supplementary Concept] OR zolpidem }\end{array}$ & 44,808 \\
\hline 6 & \#2 OR \#3 OR \#4 OR \#5 & 585,792 \\
\hline 7 & \#1 AND \#6 & 199,331 \\
\hline 8 & $\begin{array}{l}\text { "Pregnant Women"[Mesh] OR Pregnancy[Mesh] OR preconception[tiab] } \\
\text { OR pregnant[tiab] OR pregnancy[tiab] OR prenatal[tiab] OR "post- } \\
\text { partum"[tiab] OR postpartum[tiab] OR postnatal[tiab] OR perinatal[tiab] } \\
\text { OR antenatal[tiab] OR "Maternal Health Services"[Mesh] OR "maternal } \\
\text { health"[tiab] OR "Infant Nutritional Physiological Phenomena"[Mesh] OR } \\
\text { "Breast Feeding"[Mesh] OR "breast feeding"[tiab] OR breastfeed*[tiab] OR } \\
\text { (breast[tiab] AND fed[tiab]) OR breastfed[tiab] OR "Pregnancy } \\
\text { Complications"[All Fields] OR "Maternal Welfare"[All Fields] OR } \\
\text { gestation*[tiab] OR maternal*[tiab] OR "Pregnancy Outcome"[Mesh] }\end{array}$ & $1,296,988$ \\
\hline 9 & \#7 AND \#8 & 6,782 \\
\hline 10 & \#7 AND \#8 & 6,208 \\
\hline 11 & (\#10 AND Humans[Mesh:noexp]) OR (\#10 NOT Animals[Mesh:noexp]) & 4,843 \\
\hline 12 & $\begin{array}{l}\text { accident* OR "Adverse Effects" OR "adverse effect" OR "adverse event" } \\
\text { OR "adverse events" OR "adverse outcome" OR "adverse outcomes" OR } \\
\text { "adverse reaction" OR "adverse reactions" OR "chemically induced" OR } \\
\text { complication* OR death* OR "Drug Allergies" OR "Drug Dependency" OR } \\
\text { "drug effects" OR "Drug Sensitivity" OR harm* OR harms OR "Long Term } \\
\text { Adverse Effects"[Mesh] OR "manic episode" OR overdos* OR "Patient } \\
\text { Safety" OR poisoning OR self damage* OR self injur* OR self inflict* OR } \\
\text { "Self-Injurious Behavior"[Mesh] OR "Side Effect" OR "side effects" OR } \\
\text { Suicide OR suicidal* OR toxicity }\end{array}$ & $8,665,883$ \\
\hline
\end{tabular}




\begin{tabular}{|c|c|c|c|}
\hline $\begin{array}{l}\text { Search } \\
\text { Number }\end{array}$ & Query & Filters & Results \\
\hline 13 & $\begin{array}{l}\text { "Abortion, Spontaneous"[Mesh] OR "Abruptio Placentae"[Mesh] OR } \\
\text { abruption*tiab] OR "Apgar Score"[Mesh] OR "Birth Weight/chemically } \\
\text { induced"[Mesh] OR "Birth Weight/drug effects"[Mesh] OR "Child } \\
\text { Development Disorders, Pervasive/chemically induced"[Mesh] OR "Child } \\
\text { Development/drug effects"[Mesh] OR "Craniofacial } \\
\text { Abnormalities/chemically induced"[Mesh] OR "Congenital } \\
\text { Abnormalities"[Mesh] OR "congenital abnormality"[tiab] OR "congenital } \\
\text { abnormalities"[tiab] OR death[tiab] OR "delayed development"[ALL } \\
\text { FIELDS] OR "Glucose Intolerance"[Mesh] OR "glucose intolerance"[tiab] } \\
\text { OR "Infant, Extremely Premature/growth AND development"[Mesh] OR } \\
\text { (infant* AND (attachment* OR bonding)) OR "Infertility, Female"[Mesh] } \\
\text { OR “Infantile Respiratory Distress Syndrome"[tiab] OR infertility[tiab] OR } \\
\text { ("Intellectual Disability"[Mesh] AND child*[tw]) OR "Low APGAR"[tiab] OR } \\
\text { miscarry[tiab] OR miscarriage*[tiab] OR Mortality[Mesh] OR mortality[tiab] } \\
\text { OR "Neonatal Abstinence Syndrome"[Mesh] OR "Neonatal Respiratory } \\
\text { Distress Syndrome"[tiab] OR "Obstetric Labor, Premature"[Mesh] OR } \\
\text { "Persistent Fetal Circulation Syndrome"[Mesh] OR "Persistent Pulmonary } \\
\text { Hypertension of Newborn"[tiab] OR "Pre-Eclampsia"[Mesh] OR } \\
\text { preeclampsia[tiab] OR pre-eclampsia[tiab] OR "Premature Birth"[Mesh] } \\
\text { OR "premature birth"[tiab] OR "preterm birth" OR "pre-term birth"[tiab] OR } \\
\text { "Prenatal Exposure Delayed Effects"[Mesh] OR "Prescription Drug } \\
\text { Misuse"[Mesh] OR ("Prescription Drug"[tiab] AND misuse[tiab]) OR } \\
\text { "preterm labor"[tiab] OR "pre-term labor"[tiab] OR "Postpartum } \\
\text { Hemorrhage"[Mesh] OR "Respiratory Distress Syndrome, } \\
\text { Newborn"[Mesh] OR "Uterine Inertia/chemically induced"[Mesh] }\end{array}$ & & $2,345,644$ \\
\hline 14 & $\# 12$ OR \#13 & & $9,559,975$ \\
\hline 15 & $\begin{array}{l}\text { "Drug Therapy"[Mesh] OR "drug therapy"[Subheading] OR drug*[tiab] OR } \\
\text { pharmacotherap*[tiab] OR pharmacologic*[tiab] OR medicine*[tiab] OR } \\
\text { medication*[tiab] }\end{array}$ & & $4,692,865$ \\
\hline 16 & \#14 AND \#15 AND \#8 AND \#1 & & 13,011 \\
\hline 17 & \#14 AND \#15 AND \#8 AND \#1 & English & 11,788 \\
\hline 18 & (\#17 AND Humans[Mesh:noexp]) OR (\#17 NOT Animals[Mesh:noexp]) & & 9,848 \\
\hline 19 & (\#10 AND Humans[Mesh:noexp]) OR (\#10 NOT Animals[Mesh:noexp]) & Case Reports & 612 \\
\hline 20 & (\#10 AND Humans[Mesh:noexp]) OR (\#10 NOT Animals[Mesh:noexp]) & $\begin{array}{l}\text { Case Reports, } \\
\text { Editorial }\end{array}$ & 684 \\
\hline 21 & \#11 NOT (\#19 OR \#20) & & \\
\hline 22 & (\#17 AND Humans[Mesh:noexp]) OR (\#17 NOT Animals[Mesh:noexp]) & Case Reports & 967 \\
\hline 23 & (\#17 AND Humans[Mesh:noexp]) OR (\#17 NOT Animals[Mesh:noexp]) & $\begin{array}{l}\text { Case Reports, } \\
\text { Editorial }\end{array}$ & 1,077 \\
\hline 24 & \#18 NOT (\#22 OR \#23) & & 8,771 \\
\hline 25 & \#21 AND ("2018/06/09"[Date - Publication] : "3000"[Date - Publication]) & & 546 \\
\hline 26 & \#24 AND ("2018/06/09"[Date - Publication] : "3000"[Date - Publication]) & & 811 \\
\hline 27 & $\begin{array}{l}\text { retraction[All Fields] OR “Retracted Publication"[pt] OR Duplicate } \\
\text { Publication [pt] OR Erratum[All Fields] }\end{array}$ & & 116,824 \\
\hline 28 & \#27 AND (\#21 OR \#24) & & 6 \\
\hline
\end{tabular}




\section{Cochrane Library}

12-10-2018

\begin{tabular}{|c|c|c|}
\hline Search & Query & Results \\
\hline \#1 & $\begin{array}{l}\text { [mh "Anxiety Disorders"] OR anxiety:ti,ab OR [mh "Bipolar Disorder"] OR bipolar:ti,ab OR [mh } \\
\text { "Depressive Disorder"] OR [mh "Depressive Disorder, Major"] OR [mh Depression] OR } \\
\text { depress*:ti,ab OR depression:ti,ab OR depressive:ti,ab OR depressed:ti,ab OR [mh } \\
\text { "Dysthymic Disorder"] OR dysthymia:ti,ab OR dysthymic:ti,ab OR [mh "Feeding and Eating } \\
\text { Disorders"] OR anorexia:ti,ab OR anorexic:ti,ab OR "binge eating":ti,ab OR bulimic:ti,ab OR } \\
\text { bulimia:ti,ab OR GAD OR [mh "Psychotic Disorders"] OR "psychotic disorder":ti,ab OR } \\
\text { "psychotic disorders":ti,ab OR psychosis:ti,ab OR psychoses:ti,ab OR (mental:ti,ab AND } \\
\text { (health:ti,ab OR illness:ti,ab OR disorders:ti,ab)) OR [mh "Mental Health"] OR [mh "Mental } \\
\text { Disorders"] OR [mh "Obsessive-Compulsive Disorder"] OR "Obsessive-Compulsive } \\
\text { Disorder":ti,ab OR OCD:ti,ab OR "panic disorder" OR "Persistent Depressive Disorder":ti,ab } \\
\text { OR phobia*:ti,ab OR phobic:ti,ab OR psychotic*:ti,ab OR [mh "Stress Disorders, Post- } \\
\text { Traumatic"] OR "post-traumatic stress disorder" OR "post-traumatic stress disorders" OR } \\
\text { "posttraumatic stress disorder" OR "posttraumatic stress disorders" OR (disorder* AND "post- } \\
\text { traumatic":ti,ab) OR [mh ^"Stress Disorders, Traumatic"] OR PTSD:ti,ab OR [mh } \\
\text { "Schizophrenia"] OR schizophren*:ti,ab OR "stress disorder" }\end{array}$ & 135850 \\
\hline \#2 & $\begin{array}{l}\text { alprazolam OR [mh "Anti-Anxiety Agents"] OR anti-anxiety OR antianxiety OR [mh } \\
\text { "Amitriptyline"] OR amitriptyline OR [mh "Amoxapine"] OR amoxapine OR [mh } \\
\text { Anticonvulsants] OR anticonvulsant OR anticonvulsants OR [mh "Antidepressive Agents"] OR } \\
\text { [mh "Antidepressive Agents, Second-Generation"] OR anti-depress* OR antidepressant* OR } \\
\text { antidepressive* OR [mh "Antipsychotic Agents"] OR antipsychotic* OR anxiolytic* OR [mh } \\
\text { "Aripiprazole"] OR aripiprazole OR Asenapine OR benzodiazepine* OR brexanolone OR } \\
\text { brexpiprazole OR [mh Bupropion] OR Bupropion OR [mh "Buspirone"] OR Buspirone OR [mh } \\
\text { "Carbamazepine"] OR Carbamazepine OR Cariprazine OR [mh "Chlorpromazine"] OR } \\
\text { Chlorpromazine OR [mh Citalopram] OR citalopram OR clobazam OR [mh Clomipramine] OR } \\
\text { Clomipramine OR [mh "Clonazepam"] OR Clonazepam OR [mh "Clonidine"] OR Clonidine OR } \\
\text { [mh "Clorazepate Dipotassium"] OR Clorazepate OR [mh "Chlordiazepoxide"] OR } \\
\text { Chlordiazepoxide OR [mh "Clozapine"] OR clozapine }\end{array}$ & 41190 \\
\hline \#3 & $\begin{array}{l}\text { [mh "Desipramine"] OR Desipramine OR [mh "Desvenlafaxine Succinate"] OR Desvenlafaxine } \\
\text { OR [mh "Diazepam"] OR Diazepam OR [mh "Diphenhydramine"] OR Diphenhydramine OR } \\
\text { [mh "Doxepin"] OR doxepin OR [mh "Duloxetine Hydrochloride"] OR duloxetine OR } \\
\text { escitalopram OR [mh "Eszopiclone"] OR eszopiclone OR [mh Fluoxetine] OR fluoxetine OR } \\
\text { [mh "Fluphenazine"] OR fluphenazine OR [mh Fluvoxamine] OR fluvoxamine OR gabapentin } \\
\text { OR [mh "Haloperidol"] OR Haloperidol OR [mh "Hydroxyzine"] OR Hydroxyzine OR } \\
\text { iloperidone OR [mh "Imipramine"] OR imipramine OR lamotrigine OR Levomilnacipran [mh } \\
\text { "Lisdexamfetamine Dimesylate"] OR [mh "Lithium Carbonate"] OR lithium OR [mh } \\
\text { "Lorazepam"] OR lorazepam OR [mh "Lurasidone Hydrochloride"] OR lurasidone OR } \\
\text { Maprotiline }\end{array}$ & \\
\hline$\overline{\# 4}$ & $\begin{array}{l}\text { milnacipran OR mirtazapine OR nefazodone OR "norepinephrine reuptake inhibitor" OR } \\
\text { "norepinephrine reuptake inhibitors" OR [mh "Nortriptyline"] OR nortriptyline OR olanzapine } \\
\text { OR oxcarbazepine OR [mh "Paliperidone Palmitate"] OR Paliperidone OR [mh Paroxetine] } \\
\text { OR paroxetine OR [mh "Perphenazine"] OR perphenazine OR [mh "Protriptyline"] OR } \\
\text { protriptyline OR [mh "Quetiapine Fumarate"] OR quetiapine OR ramelteon OR [mh } \\
\text { "Risperidone"] OR risperidone OR "selective serotonin reuptake inhibitor" OR "selective } \\
\text { serotonin reuptake inhibitors" OR [mh "Serotonin Uptake Inhibitors"] OR "serotonin } \\
\text { norepinephrine reuptake inhibitor":ti,ab,kw OR "serotonin norepinephrine reuptake } \\
\text { inhibitors":ti,ab,kw OR [mh Sertraline] OR sertraline OR SNRI* OR SSRI* }\end{array}$ & 15699 \\
\hline \#5 & $\begin{array}{l}\text { [mh "Temazepam"] OR Temazepam OR Thioridazine OR Thiothixine OR topiramate OR [mh } \\
\text { Trazodone] OR trazodone OR [mh "Triazolam"] OR triazolam OR Trifluoperazine OR [mh } \\
\text { "Trimipramine"] OR Trimipramine OR [mh "Valproic Acid"] OR valproate OR [mh "Venlafaxine } \\
\text { Hydrochloride"] OR venlafaxine OR [mh "Vilazodone Hydrochloride"] OR vilazodone OR } \\
\text { Vistaril OR vortioxetine OR Vyvanse OR zaleplon OR ziprasidone OR zolpidem }\end{array}$ & 8257 \\
\hline \#6 & $(\# 2$ or \#3 or \#4 or \#5) & 61747 \\
\hline \#7 & (\#1 and \#6) & 35441 \\
\hline
\end{tabular}




\begin{tabular}{|c|c|c|}
\hline Search & Query & Results \\
\hline \#8 & $\begin{array}{l}\text { [mh "Pregnant Women"] OR [mh Pregnancy] OR preconception:ti,ab OR pregnant:ti,ab OR } \\
\text { pregnancy:ti,ab OR prenatal:ti,ab OR "post-partum":ti,ab OR postpartum:ti,ab OR } \\
\text { postnatal:ti,ab OR perinatal:ti,ab OR antenatal:ti,ab OR [mh "Maternal Health Services"] OR } \\
\text { "maternal health":ti,ab OR [mh "Infant Nutritional Physiological Phenomena"] OR [mh "Breast } \\
\text { Feeding"] OR "breast feeding":ti,ab OR breastfeed*:ti,ab OR (breast:ti,ab AND fed:ti,ab) OR } \\
\text { breastfed:ti,ab OR "Pregnancy Complications":ti,ab,kw OR "Maternal Welfare":ti,ab,kw OR } \\
\text { gestation*:ti,ab OR maternal*:ti,ab OR [mh "Pregnancy Outcome"] }\end{array}$ & 51540 \\
\hline \#9 & $(\# 7$ and \#8) & 443 \\
\hline$\# 10$ & (\#9 AND [mh ^Humans]) OR (\#9 NOT [mh ^^Animals]) & 443 \\
\hline \#11 & $\begin{array}{l}\text { [mh "Drug Therapy"] OR drug*:ti,ab OR pharmacotherap*:ti,ab OR pharmacologic*:ti,ab OR } \\
\text { medicine }^{*}: t i, a b \text { OR medication*:ti,ab }\end{array}$ & 321669 \\
\hline$\# 12$ & $\begin{array}{l}\text { accident* OR "Adverse Effects" OR "adverse effect" or "adverse event" OR "adverse events" } \\
\text { OR "adverse outcome" OR "adverse outcomes" OR "adverse reaction" OR "adverse } \\
\text { reactions" OR "chemically induced" OR complication* OR death* OR "Drug Allergies" OR } \\
\text { "Drug Dependency" OR "drug effects" OR "Drug Sensitivity" OR harm* OR harms OR [mh } \\
\text { "Long Term Adverse Effects"] OR "manic episode" OR overdos* OR "Patient Safety" OR } \\
\text { poisoning OR self damage* OR self injur* OR self inflict* OR [mh "Self-Injurious Behavior"] } \\
\text { OR "Side Effect" OR "side effects" OR Suicide OR suicidal* OR toxicity }\end{array}$ & 477736 \\
\hline$\overline{\# 13}$ & $\begin{array}{l}\text { [mh "Abortion, Spontaneous"] OR [mh "Abruptio Placentae"] OR abruption*:ti,ab OR [mh } \\
\text { "Apgar Score"] OR [mh "Birth Weight"/CI] OR [mh "Birth Weight"/DE] OR [mh "Child } \\
\text { Development Disorders, Pervasive"/Cl] OR [mh "Child Development"/DE] OR [mh } \\
\text { "Craniofacial Abnormalities"/CI] OR [mh "Congenital Abnormalities"] OR "congenital } \\
\text { abnormality":ti,ab OR "congenital abnormalities":ti,ab OR death:ti,ab OR "delayed } \\
\text { development":ti,ab,kw OR [mh "Glucose Intolerance"] OR "glucose intolerance":ti,ab OR [mh } \\
\text { "Infant, Extremely Premature"/GD] OR (infant* AND (attachment* OR bonding)) OR [mh } \\
\text { "Infertility, Female"] OR "Infantile Respiratory Distress Syndrome":ti,ab OR infertility:ti,ab OR } \\
\text { ([mh "Intellectual Disability"] AND child*:ti,ab,kw) OR "Low APGAR":ti,ab OR miscarry:ti,ab } \\
\text { OR miscarriage*:ti,ab OR [mh Mortality] OR mortality:ti,ab OR [mh "Neonatal Abstinence } \\
\text { Syndrome"] OR “Neonatal Respiratory Distress Syndrome":ti,ab OR [mh "Obstetric Labor, } \\
\text { Premature"] OR [mh "Persistent Fetal Circulation Syndrome"] OR "Persistent Pulmonary } \\
\text { Hypertension of Newborn":ti,ab OR [mh "Pre-Eclampsia"] OR preeclampsia:ti,ab OR "pre- } \\
\text { eclampsia":ti,ab OR [mh "Premature Birth"] OR "premature birth":ti,ab OR "preterm birth":ti,ab } \\
\text { OR "pre-term birth":ti,ab OR [mh "Prenatal Exposure Delayed Effects"] OR [mh "Prescription } \\
\text { Drug Misuse"] OR ("Prescription Drug":ti,ab AND misuse:ti,ab) OR "preterm labor":ti,ab OR } \\
\text { "pre-term labor":ti,ab OR [mh "Postpartum Hemorrhage"] OR [mh "Respiratory Distress } \\
\text { Syndrome, Newborn"] OR [mh "Uterine Inertia"/Cl] }\end{array}$ & 102127 \\
\hline \#14 & $\# 12$ OR \#13 & 507255 \\
\hline \#15 & $\# 1$ and \#8 and \#11 and \#14 & 785 \\
\hline$\overline{\# 16}$ & (\#15 AND [mh ^Humans]) OR (\#15 NOT [mh ^Animals]) & 785 \\
\hline \#17 & editorial:pt OR case study:pt OR [mh "case reports"] & 8739 \\
\hline \#18 & $\# 10$ not \#17 & 441 \\
\hline$\# 19$ & $\# 16$ not \#17 & 779 \\
\hline$\# 20$ & $\# 19$ not \#18 & 548 \\
\hline
\end{tabular}




\section{Cochrane Library}

6-5-2020

\begin{tabular}{|c|c|c|}
\hline Search & Query & Results \\
\hline \#1 & $\begin{array}{l}\text { [mh "Anxiety Disorders"] OR anxiety:ti,ab OR [mh "Bipolar Disorder"] OR bipolar:ti,ab } \\
\text { OR [mh "Depressive Disorder"] OR [mh "Depressive Disorder, Major"] OR [mh } \\
\text { Depression] OR depress*:ti,ab OR depression:ti,ab OR depressive:ti,ab OR } \\
\text { depressed:ti,ab OR [mh "Dysthymic Disorder"] OR dysthymia:ti,ab OR dysthymic:ti,ab } \\
\text { OR [mh "Feeding AND Eating Disorders"] OR anorexia:ti,ab OR anorexic:ti,ab OR } \\
\text { "binge eating":ti,ab OR bulimic:ti,ab OR bulimia:ti,ab OR GAD OR [mh "Psychotic } \\
\text { Disorders"] OR "psychotic disorder":ti,ab OR "psychotic disorders":ti,ab OR } \\
\text { psychosis:ti,ab OR psychoses:ti,ab OR (mental:ti,ab AND (health:ti,ab OR illness:ti,ab } \\
\text { OR disorders:ti,ab)) OR [mh "Mental Health"] OR [mh "Mental Disorders"] OR [mh } \\
\text { "Obsessive-Compulsive Disorder"] OR "Obsessive-Compulsive Disorder":ti,ab OR } \\
\text { OCD:ti,ab OR "panic disorder" OR "Persistent Depressive Disorder":ti,ab OR } \\
\text { phobia*:ti,ab OR phobic:ti,ab OR psychotic*:ti,ab OR [mh "Stress Disorders, Post- } \\
\text { Traumatic"] OR "post-traumatic stress disorder" OR "post-traumatic stress disorders" } \\
\text { OR "posttraumatic stress disorder" OR "posttraumatic stress disorders" OR (disorder* } \\
\text { AND "post-traumatic":ti,ab) OR [mh ^"Stress Disorders, Traumatic"] OR PTSD:ti,ab } \\
\text { OR [mh "Schizophrenia"] OR schizophren*:ti,ab OR "stress disorder" }\end{array}$ & 175238 \\
\hline \#2 & $\begin{array}{l}\text { alprazolam OR [mh "Anti-Anxiety Agents"] OR anti-anxiety OR antianxiety OR [mh } \\
\text { "Amitriptyline"] OR amitriptyline OR [mh "Amoxapine"] OR amoxapine OR [mh } \\
\text { Anticonvulsants] OR anticonvulsant OR anticonvulsants OR [mh "Antidepressive } \\
\text { Agents"] OR [mh "Antidepressive Agents, Second-Generation"] OR anti-depress* OR } \\
\text { antidepressant* OR antidepressive* OR [mh "Antipsychotic Agents"] OR } \\
\text { antipsychotic* OR anxiolytic* OR [mh "Aripiprazole"] OR aripiprazole OR Asenapine } \\
\text { OR benzodiazepine* OR brexanolone OR brexpiprazole OR [mh Bupropion] OR } \\
\text { Bupropion OR [mh "Buspirone"] OR Buspirone OR [mh "Carbamazepine"] OR } \\
\text { Carbamazepine OR Cariprazine OR [mh "Chlorpromazine"] OR Chlorpromazine OR } \\
\text { [mh Citalopram] OR citalopram OR clobazam OR [mh Clomipramine] OR } \\
\text { Clomipramine OR [mh "Clonazepam"] OR Clonazepam OR [mh "Clonidine"] OR } \\
\text { Clonidine OR [mh "Clorazepate Dipotassium"] OR Clorazepate OR [mh } \\
\text { "Chlordiazepoxide"] OR Chlordiazepoxide OR [mh "Clozapine"] OR clozapine }\end{array}$ & 48106 \\
\hline \#3 & $\begin{array}{l}\text { [mh "Desipramine"] OR Desipramine OR [mh "Desvenlafaxine Succinate"] OR } \\
\text { Desvenlafaxine OR [mh "Diazepam"] OR Diazepam OR [mh "Diphenhydramine"] OR } \\
\text { Diphenhydramine OR [mh "Doxepin"] OR doxepin OR [mh "Duloxetine Hydrochloride"] } \\
\text { OR duloxetine OR escitalopram OR [mh "Eszopiclone"] OR eszopiclone OR [mh } \\
\text { Fluoxetine] OR fluoxetine OR [mh "Fluphenazine"] OR fluphenazine OR [mh } \\
\text { Fluvoxamine] OR fluvoxamine OR gabapentin OR [mh "Haloperidol"] OR Haloperidol } \\
\text { OR [mh "Hydroxyzine"] OR Hydroxyzine OR iloperidone OR [mh "Imipramine"] OR } \\
\text { imipramine OR lamotrigine OR Levomilnacipran [mh "Lisdexamfetamine Dimesylate"] } \\
\text { OR [mh "Lithium Carbonate"] OR lithium OR [mh "Lorazepam"] OR lorazepam OR } \\
\text { [mh "Lurasidone Hydrochloride"] OR lurasidone OR Maprotiline }\end{array}$ & 27651 \\
\hline \#4 & $\begin{array}{l}\text { milnacipran OR mirtazapine OR nefazodone OR "norepinephrine reuptake inhibitor" } \\
\text { OR "norepinephrine reuptake inhibitors" OR [mh "Nortriptyline"] OR nortriptyline OR } \\
\text { olanzapine OR oxcarbazepine OR [mh "Paliperidone Palmitate"] OR Paliperidone OR } \\
\text { [mh Paroxetine] OR paroxetine OR [mh "Perphenazine"] OR perphenazine OR [mh } \\
\text { "Protriptyline"] OR protriptyline OR [mh "Quetiapine Fumarate"] OR quetiapine OR } \\
\text { ramelteon OR [mh "Risperidone"] OR risperidone OR "selective serotonin reuptake } \\
\text { inhibitor" OR "selective serotonin reuptake inhibitors" OR [mh "Serotonin Uptake } \\
\text { Inhibitors"] OR "serotonin norepinephrine reuptake inhibitor":ti,ab,kw OR "serotonin } \\
\text { norepinephrine reuptake inhibitors":ti,ab,kw OR [mh Sertraline] OR sertraline OR } \\
\text { SNRI* OR SSRI* }\end{array}$ & 18539 \\
\hline \#5 & $\begin{array}{l}\text { [mh "Temazepam"] OR Temazepam OR Thioridazine OR Thiothixine OR topiramate } \\
\text { OR [mh Trazodone] OR trazodone OR [mh "Triazolam"] OR triazolam OR } \\
\text { Trifluoperazine OR [mh "Trimipramine"] OR Trimipramine OR [mh "Valproic Acid"] OR } \\
\text { valproate OR [mh "Venlafaxine Hydrochloride"] OR venlafaxine OR [mh "Vilazodone } \\
\text { Hydrochloride"] OR vilazodone OR Vistaril OR vortioxetine OR Vyvanse OR zaleplon } \\
\text { OR ziprasidone OR zolpidem }\end{array}$ & 9442 \\
\hline$\overline{\# 6}$ & (\#2 OR \#3 OR \#4 OR \#5) & 72329 \\
\hline
\end{tabular}




\begin{tabular}{|c|c|c|}
\hline Search & Query & Results \\
\hline$\overline{\# 7}$ & $(\# 1$ AND \#6) & 42201 \\
\hline \#8 & $\begin{array}{l}\text { [mh "Pregnant Women"] OR [mh Pregnancy] OR preconception:ti,ab OR } \\
\text { pregnant:ti,ab OR pregnancy:ti,ab OR prenatal:ti,ab OR "post-partum":ti,ab OR } \\
\text { postpartum:ti,ab OR postnatal:ti,ab OR perinatal:ti,ab OR antenatal:ti,ab OR [mh } \\
\text { "Maternal Health Services"] OR "maternal health":ti,ab OR [mh "Infant Nutritional } \\
\text { Physiological Phenomena"] OR [mh "Breast Feeding"] OR "breast feeding":ti,ab OR } \\
\text { breastfeed*:ti,ab OR (breast:ti,ab AND fed:ti,ab) OR breastfed:ti,ab OR "Pregnancy } \\
\text { Complications":ti,ab,kw OR "Maternal Welfare":ti,ab,kw OR gestation*:ti,ab OR } \\
\text { maternal*:ti,ab OR [mh "Pregnancy Outcome"] }\end{array}$ & 78260 \\
\hline$\# 9$ & $(\# 7$ AND \#8) & 1127 \\
\hline$\# 10$ & (\#9 AND [mh ^Humans]) OR (\#9 NOT [mh ^Animals]) & 1127 \\
\hline \#11 & $\begin{array}{l}\text { [mh "Drug Therapy"] OR drug*:ti,ab OR pharmacotherap*:ti,ab OR } \\
\text { pharmacologic*:ti,ab OR medicine*:ti,ab OR medication*:ti,ab }\end{array}$ & 405459 \\
\hline \#12 & $\begin{array}{l}\text { accident* OR "Adverse Effects" OR "adverse effect" OR "adverse event" OR "adverse } \\
\text { events" OR "adverse outcome" OR "adverse outcomes" OR "adverse reaction" OR } \\
\text { "adverse reactions" OR "chemically induced" OR complication* OR death* OR "Drug } \\
\text { Allergies" OR "Drug Dependency" OR "drug effects" OR "Drug Sensitivity" OR harm } \\
\text { OR harms OR [mh "Long Term Adverse Effects"] OR "manic episode" OR overdos* } \\
\text { OR "Patient Safety" OR poisoning OR self damage* OR self injur* OR self inflict* OR } \\
\text { [mh "Self-Injurious Behavior"] OR "Side Effect" OR "side effects" OR Suicide OR } \\
\text { suicidal* OR toxicity }\end{array}$ & 590895 \\
\hline$\# 13$ & $\begin{array}{l}\text { [mh "Abortion, Spontaneous"] OR [mh "Abruptio Placentae"] OR abruption*:ti,ab OR } \\
\text { [mh "Apgar Score"] OR [mh "Birth Weight"/CI] OR [mh "Birth Weight"/DE] OR [mh } \\
\text { "Child Development Disorders, Pervasive"/CI] OR [mh "Child Development"/DE] OR } \\
\text { [mh "Craniofacial Abnormalities"/CI] OR [mh "Congenital Abnormalities"] OR } \\
\text { "congenital abnormality":ti,ab OR "congenital abnormalities":ti,ab OR death:ti,ab OR } \\
\text { "delayed development":ti,ab,kw OR [mh "Glucose Intolerance"] OR "glucose } \\
\text { intolerance":ti,ab OR [mh "Infant, Extremely Premature"/GD] OR (infant* AND } \\
\text { (attachment* OR bonding)) OR [mh "Infertility, Female"] OR “Infantile Respiratory } \\
\text { Distress Syndrome":ti,ab OR infertility:ti,ab OR ([mh "Intellectual Disability"] AND } \\
\text { child*:ti,ab,kw) OR "Low APGAR":ti,ab OR miscarry:ti,ab OR miscarriage*:ti,ab OR } \\
\text { [mh Mortality] OR mortality:ti,ab OR [mh "Neonatal Abstinence Syndrome"] OR } \\
\text { "Neonatal Respiratory Distress Syndrome":ti,ab OR [mh "Obstetric Labor, Premature"] } \\
\text { OR [mh "Persistent Fetal Circulation Syndrome"] OR "Persistent Pulmonary } \\
\text { Hypertension of Newborn":ti,ab OR [mh "Pre-Eclampsia"] OR preeclampsia:ti,ab OR } \\
\text { "pre-eclampsia":ti,ab OR [mh "Premature Birth"] OR "premature birth":ti,ab OR } \\
\text { "preterm birth":ti,ab OR "pre-term birth":ti,ab OR [mh "Prenatal Exposure Delayed } \\
\text { Effects"] OR [mh "Prescription Drug Misuse"] OR ("Prescription Drug":ti,ab AND } \\
\text { misuse:ti,ab) OR "preterm labor":ti,ab OR "pre-term labor":ti,ab OR [mh "Postpartum } \\
\text { Hemorrhage"] OR [mh "Respiratory Distress Syndrome, Newborn"] OR [mh "Uterine } \\
\text { Inertia"/CI] }\end{array}$ & 134167 \\
\hline$\overline{\# 14}$ & $\# 12$ OR \#13 & 630035 \\
\hline$\# 15$ & \#1 AND \#8 AND \#11 AND \#14 & 1673 \\
\hline \#16 & (\#15 AND [mh ^Humans]) OR (\#15 NOT [mh ^Animals]) & 1673 \\
\hline \#17 & editorial:pt OR case study:pt OR [mh "case reports"] & 10833 \\
\hline$\# 18$ & $\# 10$ not \#17 & 1124 \\
\hline$\# 19$ & $\# 16$ not \#17 & 1666 \\
\hline$\# 20$ & $\# 19$ not \#18 & 1130 \\
\hline
\end{tabular}




\section{Embase}

12-10-2018

\begin{tabular}{ll}
\hline No. Query & Results \\
\hline$\# 1$ 'anxiety disorder'/exp/mj OR 'anxiety disorder' OR 'anxiety'/exp OR anxiety OR 'bipolar & $2,834,461$ \\
disorder'/exp/mj OR 'bipolar disorder' OR bipolar OR 'depression'/exp OR 'depression' OR depress* & \\
OR 'dysthymia' OR 'dysthymia'/exp OR dysthymia OR dysthymic OR 'eating disorder'/exp OR \\
'eating disorder' OR 'anorexia'/exp OR 'anorexia' OR 'anorexic'/exp OR anorexic OR 'binge \\
eating'/exp OR 'binge eating' OR bulimic OR 'bulimia'/exp OR bulimia OR 'gad'/exp OR gad OR \\
'psychosis' OR 'psychotic disorder'/exp OR 'psychotic disorder' OR 'psychotic disorders'/exp OR \\
'psychotic disorders' OR 'psychosis'/exp OR psychosis OR 'psychoses'/exp OR psychoses OR \\
(mental AND ('health'/exp OR health OR 'illness'/exp OR illness OR 'disorders'/exp OR disorders)) \\
OR 'mental health'/exp OR 'mental health' OR 'mental disease'/exp OR 'mental disease' OR 'mental \\
disorder'/exp OR 'mental disorder' OR 'mental disorders'/exp OR 'mental disorders' OR 'obsessive \\
compulsive disorder'/exp OR 'obsessive compulsive disorder' OR 'obsessive-compulsive \\
disorder'/exp OR 'obsessive-compulsive disorder' OR ocd OR 'panic disorder'/exp OR 'panic \\
disorder' OR 'panic'/exp OR 'panic' OR 'persistent depressive disorder'/exp OR 'persistent \\
depressive disorder' OR 'phobia'/exp OR 'phobia' OR phobia* OR phobic OR psychotic* OR 'post- \\
traumatic stress disorder'/exp OR 'post-traumatic stress disorder' OR 'post-traumatic stress \\
disorders' OR 'posttraumatic stress disorder'/exp OR 'posttraumatic stress disorder' OR \\
'posttraumatic stress disorders' OR (disorder* AND 'post-traumatic') OR 'acute stress disorder'/exp \\
OR 'acute stress disorder' OR 'schizophrenia'/exp OR 'schizophrenia' OR schizophren* OR 'stress \\
disorder' OR 'ptsd'/exp OR pts \\
\hline
\end{tabular}

\#2 ('anxiolytic agent'/exp OR 'anti anxiety' OR antianxiety OR 'amitriptyline'/exp OR amitriptyline OR 560,87 'amoxapine'/exp OR amoxapine OR 'anticonvulsive agent'/exp OR anticonvulsant OR anticonvulsants OR 'antidepressant agent'/exp) AND antidepressant* OR antidepressive* OR 'antidepressive agent' OR 'antidepressive agents' OR 'antidepressive drug' OR 'antidepressive drugs' OR 'neuroleptic agent'/exp OR antipsychotic* OR anxiolytic* OR 'aripiprazole'/exp OR aripiprazole OR 'asenapine'/exp OR asenapine OR benzodiazepine* OR 'brexanolone'/exp OR brexanolone OR 'brexpiprazole'/exp OR brexpiprazole OR 'amfebutamone'/exp OR bupropion OR 'buspirone'/exp OR buspirone OR 'carbamazepine'/exp OR carbamazepine OR 'cariprazine'/exp OR cariprazine OR 'chlorpromazine'/exp OR chlorpromazine OR 'citalopram'/exp OR citalopram OR 'clobazam'/exp OR clobazam OR clomipramine OR 'clonazepam'/exp OR clonazepam OR 'clonidine'/exp OR clonidine OR 'clorazepate'/exp OR clorazepate OR 'chlordiazepoxide'/exp OR chlordiazepoxide OR 'clozapine'/exp OR clozapine

\#3 'desipramine'/exp OR desipramine OR 'desvenlafaxine'/exp OR desvenlafaxine OR 'diazepam'/exp 361,628 OR diazepam OR 'diphenhydramine'/exp OR diphenhydramine OR 'doxepin'/exp OR doxepin OR 'duloxetine'/exp OR duloxetine OR 'escitalopram'/exp OR escitalopram OR 'eszopiclone'/exp OR eszopiclone OR 'fluoxetine'/exp OR fluoxetine OR 'fluphenazine'/exp OR fluphenazine OR 'fluvoxamine'/exp OR fluvoxamine OR 'gabapentin'/exp OR gabapentin OR 'haloperidol'/exp OR haloperidol OR 'hydroxyzine'/exp OR hydroxyzine OR 'iloperidone'/exp OR iloperidone OR 'imipramine'/exp OR imipramine OR 'lamotrigine'/exp OR lamotrigine OR levomilnacipran OR 'lisdexamfetamine'/exp OR 'lithium carbonate'/exp OR lithium OR 'lorazepam'/exp OR lorazepam OR 'lurasidone'/exp OR lurasidone OR maprotiline OR 'milnacipran'/exp OR milnacipran

\#4 'mirtazapine'/exp OR mirtazapine OR 'nefazodone'/exp OR nefazodone OR 'noradrenalin uptake inhibitor'/exp OR 'norepinephrine reuptake inhibitors' OR 'nortriptyline'/exp OR nortriptyline OR 'olanzapine'/exp OR olanzapine OR 'oxcarbazepine'/exp OR oxcarbazepine OR 'paliperidone'/exp OR paliperidone OR 'paroxetine'/exp OR paroxetine OR 'perphenazine'/exp OR perphenazine OR 'protriptyline'/exp OR protriptyline OR 'quetiapine'/exp OR quetiapine OR 'ramelteon'/exp OR ramelteon OR 'risperidone'/exp OR risperidone OR 'serotonin uptake inhibitor'/exp OR 'selective serotonin reuptake inhibitor' OR 'selective serotonin reuptake inhibitors' OR 'serotonin noradrenalin reuptake inhibitor'/exp OR 'serotonin norepinephrine reuptake inhibitor' OR 'serotonin norepinephrine reuptake inhibitors' OR 'sertraline'/exp OR sertraline OR snri* OR ssri OR ssris

\#5 'temazepam'/exp OR temazepam OR thioridazine OR thiothixene OR 'topiramate'/exp OR topiramate OR 'trazodone'/exp OR trazodone OR 'triazolam'/exp OR triazolam OR trifluoperazine OR 'trimipramine'/exp OR trimipramine OR 'valproic acid'/exp OR valproate OR 'venlafaxine'/exp OR venlafaxine OR 'vilazodone'/exp OR vilazodone OR vistaril OR 'vortioxetine'/exp OR vortioxetine OR 'zaleplon'/exp OR zaleplon OR 'ziprasidone'/exp OR ziprasidone OR 'zolpidem'/exp OR zolpidem 


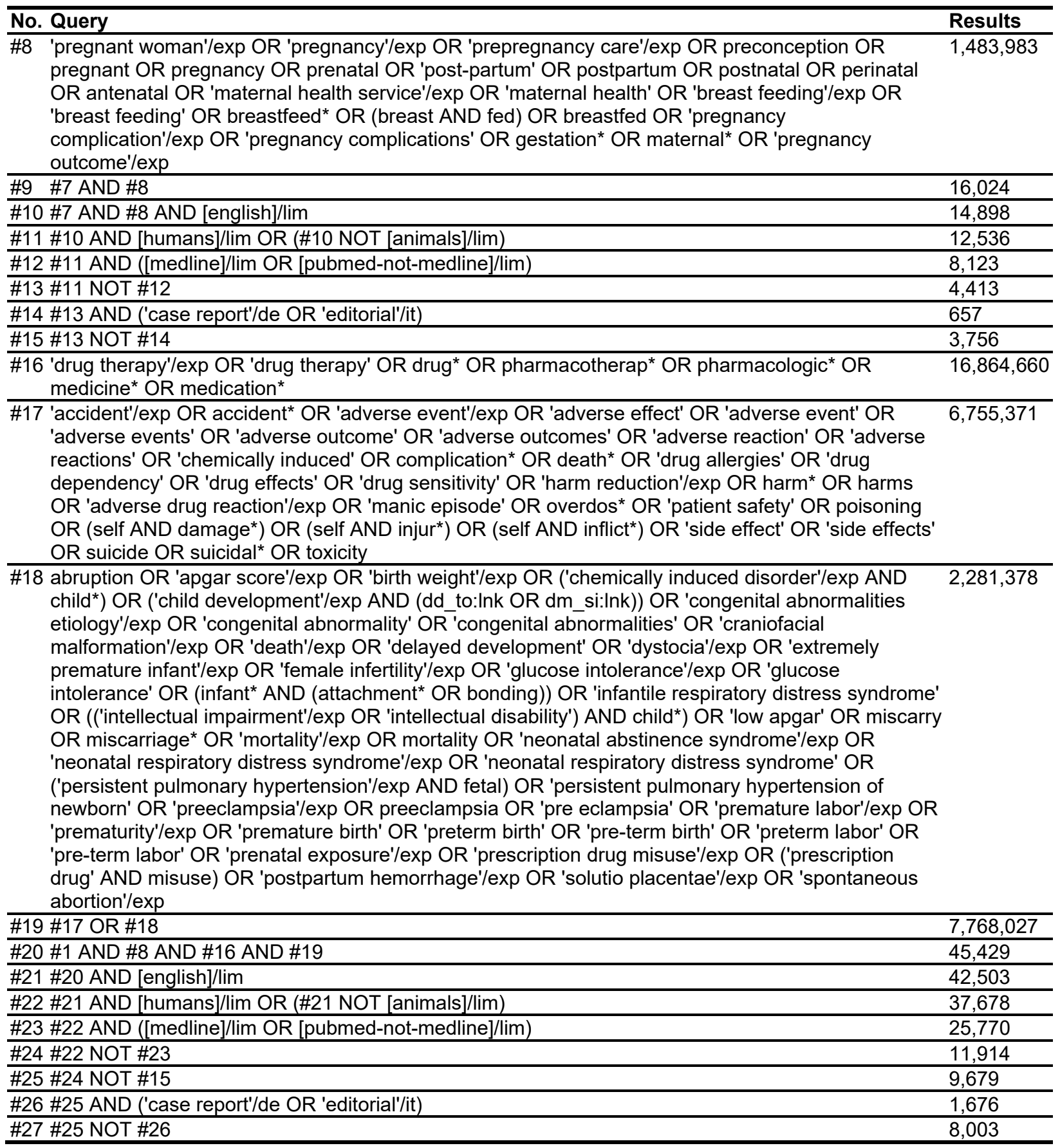


Embase

6-4-2020

\begin{tabular}{ll}
\hline No. Query & Results \\
\hline \#1 'anxiety disorder'/exp/mj OR 'anxiety disorder' OR 'anxiety'/exp OR anxiety OR 'bipolar & $3,112,575$ \\
disorder'/exp/mj OR 'bipolar disorder' OR bipolar OR 'depression'/exp OR 'depression' OR depress* & \\
OR 'dysthymia' OR 'dysthymia'/exp OR dysthymia OR dysthymic OR 'eating disorder'/exp OR \\
'eating disorder' OR 'anorexia'/exp OR 'anorexia' OR 'anorexic'/exp OR anorexic OR 'binge \\
eating'/exp OR 'binge eating' OR bulimic OR 'bulimia'/exp OR bulimia OR 'gad'/exp OR gad OR \\
'psychosis' OR 'psychotic disorder'/exp OR 'psychotic disorder' OR 'psychotic disorders'/exp OR \\
'psychotic disorders' OR 'psychosis'/exp OR psychosis OR 'psychoses'/exp OR psychoses OR \\
(mental AND ('health'/exp OR health OR 'illness'/exp OR illness OR 'disorders'/exp OR disorders)) \\
OR 'mental health'/exp OR 'mental health' OR 'mental disease'/exp OR 'mental disease' OR 'mental \\
disorder'/exp OR 'mental disorder' OR 'mental disorders'/exp OR 'mental disorders' OR 'obsessive \\
compulsive disorder'/exp OR 'obsessive compulsive disorder' OR 'obsessive-compulsive \\
disorder'/exp OR 'obsessive-compulsive disorder' OR ocd OR 'panic disorder'/exp OR 'panic \\
disorder' OR 'panic'/exp OR 'panic' OR 'persistent depressive disorder'/exp OR 'persistent \\
depressive disorder' OR 'phobia'/exp OR 'phobia' OR phobia* OR phobic OR psychotic* OR 'post- \\
traumatic stress disorder'/exp OR 'post-traumatic stress disorder' OR 'post-traumatic stress \\
disorders' OR 'posttraumatic stress disorder'/exp OR 'posttraumatic stress disorder' OR \\
'posttraumatic stress disorders' OR (disorder* AND 'post-traumatic') OR 'acute stress disorder'/exp \\
OR 'acute stress disorder' OR 'schizophrenia'/exp OR 'schizophrenia' OR schizophren* OR 'stress \\
disorder' OR 'ptsd'/exp OR ptsd \\
\hline ('anxiolytic agent'/exp OR 'anti anxiety' OR antianxiety OR 'amitriptyline'/exp OR amitriptyline OR
\end{tabular}

\#2 ('anxiolytic agent'/exp OR 'anti anxiety' OR antianxiety OR 'amitriptyline'/exp OR amitriptyline OR 590,700

'amoxapine'/exp OR amoxapine OR 'anticonvulsive agent'/exp OR anticonvulsant OR

anticonvulsants OR 'antidepressant agent'/exp) AND antidepressant* OR antidepressive* OR

'antidepressive agent' OR 'antidepressive agents' OR 'antidepressive drug' OR 'antidepressive drugs' OR 'neuroleptic agent'/exp OR antipsychotic* OR anxiolytic* OR 'aripiprazole'/exp OR aripiprazole OR 'asenapine'/exp OR asenapine OR benzodiazepine* OR 'brexanolone'/exp OR brexanolone OR 'brexpiprazole'/exp OR brexpiprazole OR 'amfebutamone'/exp OR bupropion OR 'buspirone'/exp OR buspirone OR 'carbamazepine'/exp OR carbamazepine OR 'cariprazine'/exp OR cariprazine OR 'chlorpromazine'/exp OR chlorpromazine OR 'citalopram'/exp OR citalopram OR 'clobazam'/exp OR clobazam OR clomipramine OR 'clonazepam'/exp OR clonazepam OR 'clonidine'/exp OR clonidine OR 'clorazepate'/exp OR clorazepate OR 'chlordiazepoxide'/exp OR chlordiazepoxide OR 'clozapine'/exp OR clozapine

\#3 'desipramine'/exp OR desipramine OR 'desvenlafaxine'/exp OR desvenlafaxine OR 'diazepam'/exp 384,886 OR diazepam OR 'diphenhydramine'/exp OR diphenhydramine OR 'doxepin'/exp OR doxepin OR 'duloxetine'/exp OR duloxetine OR 'escitalopram'/exp OR escitalopram OR 'eszopiclone'/exp OR eszopiclone OR 'fluoxetine'/exp OR fluoxetine OR 'fluphenazine'/exp OR fluphenazine OR 'fluvoxamine'/exp OR fluvoxamine OR 'gabapentin'/exp OR gabapentin OR 'haloperidol'/exp OR haloperidol OR 'hydroxyzine'/exp OR hydroxyzine OR 'iloperidone'/exp OR iloperidone OR 'imipramine'/exp OR imipramine OR 'lamotrigine'/exp OR lamotrigine OR levomilnacipran OR 'lisdexamfetamine'/exp OR 'lithium carbonate'/exp OR lithium OR 'lorazepam'/exp OR lorazepam OR 'lurasidone'/exp OR lurasidone OR maprotiline OR 'milnacipran'/exp OR milnacipran

\#4 'mirtazapine'/exp OR mirtazapine OR 'nefazodone'/exp OR nefazodone OR 'noradrenalin uptake inhibitor'/exp OR 'norepinephrine reuptake inhibitors' OR 'nortriptyline'/exp OR nortriptyline OR 'olanzapine'/exp OR olanzapine OR 'oxcarbazepine'/exp OR oxcarbazepine OR 'paliperidone'/exp OR paliperidone OR 'paroxetine'/exp OR paroxetine OR 'perphenazine'/exp OR perphenazine OR 'protriptyline'/exp OR protriptyline OR 'quetiapine'/exp OR quetiapine OR 'ramelteon'/exp OR ramelteon OR 'risperidone'/exp OR risperidone OR 'serotonin uptake inhibitor'/exp OR 'selective serotonin reuptake inhibitor' OR 'selective serotonin reuptake inhibitors' OR 'serotonin noradrenalin reuptake inhibitor'/exp OR 'serotonin norepinephrine reuptake inhibitor' OR 'serotonin norepinephrine reuptake inhibitors' OR 'sertraline'/exp OR sertraline OR snri* OR ssri OR ssris

\#5 'temazepam'/exp OR temazepam OR thioridazine OR thiothixene OR 'topiramate'/exp OR 149,136 topiramate OR 'trazodone'/exp OR trazodone OR 'triazolam'/exp OR triazolam OR trifluoperazine OR 'trimipramine'/exp OR trimipramine OR 'valproic acid'/exp OR valproate OR 'venlafaxine'/exp OR venlafaxine OR 'vilazodone'/exp OR vilazodone OR vistaril OR 'vortioxetine'/exp OR vortioxetine OR 'zaleplon'/exp OR zaleplon OR 'ziprasidone'/exp OR ziprasidone OR 'zolpidem'/exp OR zolpidem

916,325

417,309




\begin{tabular}{|c|c|}
\hline No. Query & Results \\
\hline $\begin{array}{l}\text { \#8 'pregnant woman'/exp OR 'pregnancy'/exp OR 'prepregnancy care'/exp OR preconception OR } \\
\text { pregnant OR pregnancy OR prenatal OR 'post-partum' OR postpartum OR postnatal OR perinatal } \\
\text { OR antenatal OR 'maternal health service'/exp OR 'maternal health' OR 'breast feeding'/exp OR } \\
\text { 'breast feeding' OR breastfeed* OR (breast AND fed) OR breastfed OR 'pregnancy } \\
\text { complication'/exp OR 'pregnancy complications' OR gestation* OR maternal* OR 'pregnancy } \\
\text { outcome'/exp }\end{array}$ & $1,602,653$ \\
\hline \#9 \#7 AND \#8 & 17,798 \\
\hline \#10 \#7 AND \#8 AND [english]/lim & 16,630 \\
\hline \#11 \#10 AND [humans]/lim OR (\#10 NOT [animals]/lim) & 13,950 \\
\hline \#12 \#11 AND ([medline]/lim OR [pubmed-not-medline]/lim) & 9,279 \\
\hline \#13 \#11 NOT \#12 & 4,671 \\
\hline \#14 \#13 AND ('case report'/de OR 'editorial'/it) & 711 \\
\hline \#15 \#13 NOT \#14 & 3,960 \\
\hline $\begin{array}{l}\text { \#16 'drug therapy'/exp OR 'drug therapy' OR drug* OR pharmacotherap* OR pharmacologic* OR } \\
\text { medicine* OR medication* }\end{array}$ & $18,908,546$ \\
\hline $\begin{array}{l}\text { \#17 'accident'/exp OR accident* OR 'adverse event'/exp OR 'adverse effect' OR 'adverse event' OR } \\
\text { 'adverse events' OR 'adverse outcome' OR 'adverse outcomes' OR 'adverse reaction' OR 'adverse } \\
\text { reactions' OR 'chemically induced' OR complication* OR death* OR 'drug allergies' OR 'drug } \\
\text { dependency' OR 'drug effects' OR 'drug sensitivity' OR 'harm reduction'/exp OR harm* OR harms } \\
\text { OR 'adverse drug reaction'/exp OR 'manic episode' OR overdos* OR 'patient safety' OR poisoning } \\
\text { OR (self AND damage* }) \text { OR (self AND injur*) OR (self AND inflict*) OR 'side effect' OR 'side effects' } \\
\text { OR suicide OR suicidal* OR toxicity }\end{array}$ & $7,370,502$ \\
\hline $\begin{array}{l}\text { \#18 abruption OR 'apgar score'/exp OR 'birth weight'/exp OR ('chemically induced disorder'/exp AND } \\
\text { child*) OR ('child development'/exp AND (dd_to:Ink OR dm_si:Ink)) OR 'congenital abnormalities } \\
\text { etiology'/exp OR 'congenital abnormality' OR 'congenital abnormalities' OR 'craniofacial } \\
\text { malformation'/exp OR 'death'/exp OR 'delayed development' OR 'dystocia'/exp OR 'extremely } \\
\text { premature infant'/exp OR 'female infertility'/exp OR 'glucose intolerance'/exp OR 'glucose } \\
\text { intolerance' OR (infant* AND (attachment* OR bonding)) OR 'infantile respiratory distress syndrome' } \\
\text { OR (('intellectual impairment'/exp OR 'intellectual disability') AND child*) OR 'low apgar' OR miscarry } \\
\text { OR miscarriage* OR 'mortality'/exp OR mortality OR 'neonatal abstinence syndrome'/exp OR } \\
\text { 'neonatal respiratory distress syndrome'/exp OR 'neonatal respiratory distress syndrome' OR } \\
\text { ('persistent pulmonary hypertension'/exp AND fetal) OR 'persistent pulmonary hypertension of } \\
\text { newborn' OR 'preeclampsia'/exp OR preeclampsia OR 'pre eclampsia' OR 'premature labor'/exp OR } \\
\text { 'prematurity'/exp OR 'premature birth' OR 'preterm birth' OR 'pre-term birth' OR 'preterm labor' OR } \\
\text { 'pre-term labor' OR 'prenatal exposure'/exp OR 'prescription drug misuse'/exp OR ('prescription } \\
\text { drug' AND misuse) OR 'postpartum hemorrhage'/exp OR 'solutio placentae'/exp OR 'spontaneous } \\
\text { abortion'/exp }\end{array}$ & $2,533,578$ \\
\hline \#19 \#17 OR \#18 & $8,500,781$ \\
\hline \#20 \#1 AND \#8 AND \#16 AND \#19 & 51,856 \\
\hline \#21 \#20 AND [english]/lim & 48,832 \\
\hline \#22 \#21 AND [humans]/lim OR (\#21 NOT [animals]/lim) & 43,352 \\
\hline \#23 \#22 AND ([medline]/lim OR [pubmed-not-medline]/lim) & 30,464 \\
\hline \#24 \#22 NOT \#23 & 12,888 \\
\hline \#25 \#24 NOT \#15 & 10,537 \\
\hline \#26 \#25 AND ('case report'/de OR 'editorial'/it) & 1,966 \\
\hline \#27 \#25 NOT \#26 & 8,573 \\
\hline \#28 (\#15 OR \#27) AND '(retraction of publication or retracted publication).pt' & 0 \\
\hline \#29 (\#15 OR \#27) AND erratum & 6 \\
\hline \#30 \#15 AND [2018-2020]/py & 544 \\
\hline \#31 \#27 AND [2018-2020]/py & 1,721 \\
\hline
\end{tabular}




\section{PsycINFO}

12-11-2018

\begin{tabular}{|c|c|c|c|}
\hline \# & Query & $\begin{array}{l}\text { Limiters/ } \\
\text { Expanders }\end{array}$ & Results \\
\hline 1 & 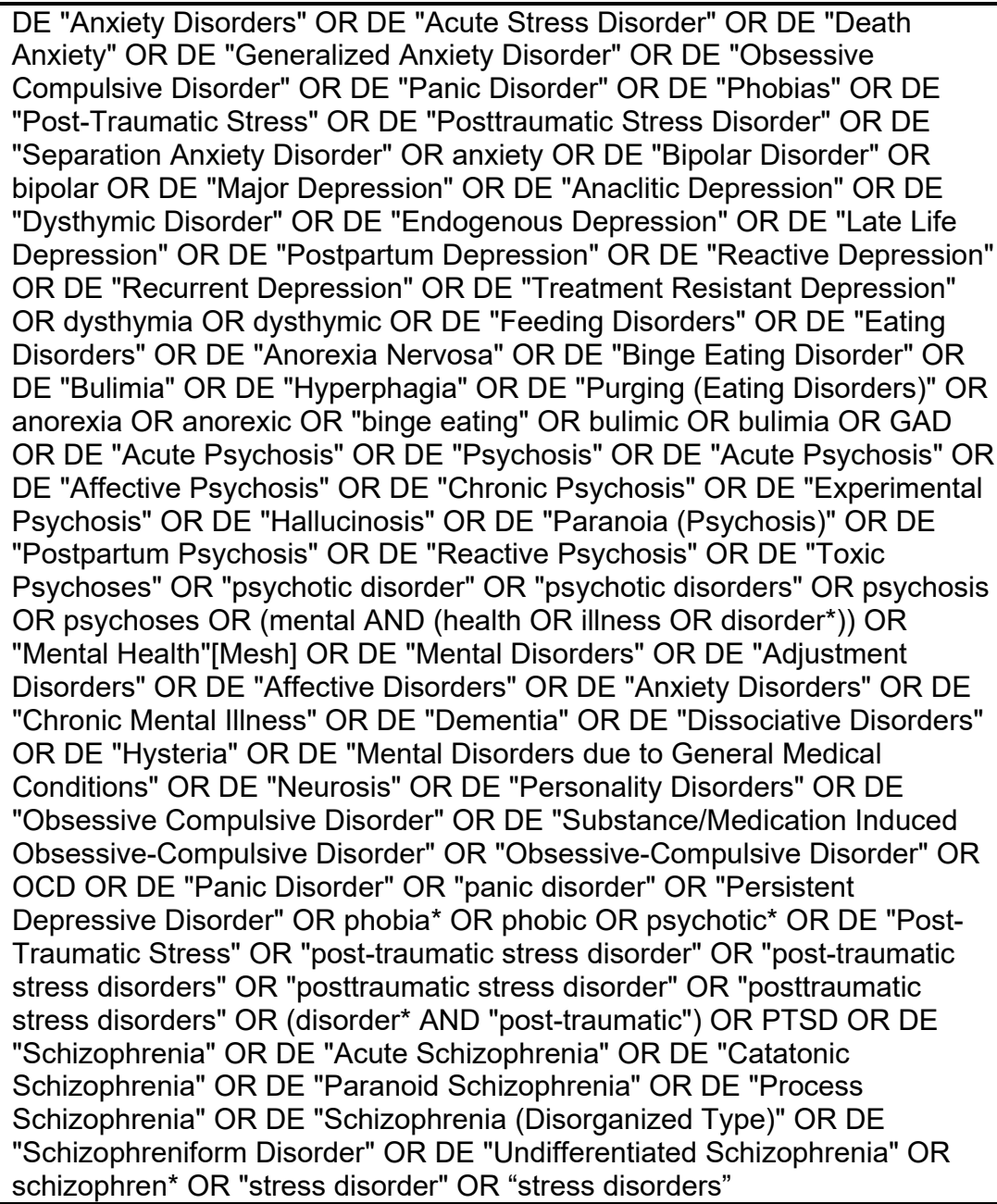 & & $1,188,879$ \\
\hline 2 & $\begin{array}{l}\text { (alprazolam OR "Anti-Anxiety Agents" OR anti-anxiety OR antianxiety OR } \\
\text { DE "Tranquilizing Drugs" OR DE "Amitriptyline" OR DE "Doxepin" OR DE } \\
\text { "Haloperidol" OR DE "Minor Tranquilizers" OR DE "Neuroleptic Drugs" OR } \\
\text { amitriptyline OR OR amoxapine OR DE "Anticonvulsive Drugs" OR } \\
\text { anticonvulsant OR anticonvulsants OR DE "Antidepressant Drugs" OR } \\
\text { "Antidepressive Agents, Second-Generation" OR anti-depress* OR } \\
\text { antidepressant* OR antidepressive* OR "antidepressive agent" OR } \\
\text { "antidepressive agents" OR "antidepressive drug" OR "antidepressive drugs" } \\
\text { OR antipsychotic* OR anxiolytic* OR DE "Aripiprazole" OR aripiprazole OR } \\
\text { Asenapine OR DE "Benzodiazepines" OR benzodiazepine* OR brexanolone } \\
\text { OR brexpiprazole OR DE "Bupropion" OR Bupropion OR DE "Buspirone" } \\
\text { OR Buspirone OR DE "Carbamazepine" OR Carbamazepine OR } \\
\text { Cariprazine OR DE "Chlorpromazine" OR Chlorpromazine OR DE } \\
\text { "Citalopram" OR citalopram OR clobazam OR clomipramine OR DE } \\
\text { "Clonazepam" OR Clonazepam OR DE "Clonidine" OR Clonidine OR } \\
\text { Clorazepate OR DE "Chlordiazepoxide" OR Chlordiazepoxide OR DE } \\
\text { "Clozapine" OR clozapine }\end{array}$ & $\begin{array}{l}\text { Search modes - } \\
\text { Boolean/Phrase }\end{array}$ & 123,726 \\
\hline
\end{tabular}




\begin{tabular}{|c|c|c|c|}
\hline \# & Query & $\begin{array}{l}\text { Limiters/ } \\
\text { Expanders }\end{array}$ & Results \\
\hline 3 & $\begin{array}{l}\text { DE "Desipramine" OR Desipramine OR Desvenlafaxine OR DE "Diazepam" } \\
\text { OR Diazepam OR DE "Diphenhydramine" OR Diphenhydramine OR DE } \\
\text { "Doxepin" OR doxepin OR duloxetine OR escitalopram OR eszopiclone OR } \\
\text { DE "Fluoxetine" OR fluoxetine OR DE "Fluphenazine" OR fluphenazine OR } \\
\text { DE "Fluvoxamine" OR fluvoxamine OR DE "Gabapentin" OR gabapentin OR } \\
\text { DE "Haloperidol" OR Haloperidol OR DE "Hydroxyzine" OR Hydroxyzine OR } \\
\text { iloperidone OR DE "Imipramine" OR imipramine OR lamotrigine OR } \\
\text { Levomilnacipran OR "Lisdexamfetamine Dimesylate" OR DE "Lithium" OR } \\
\text { lithium OR DE "Lorazepam" OR lorazepam OR lurasidone OR Maprotiline } \\
\text { OR milnacipran }\end{array}$ & $\begin{array}{l}\text { Search modes - } \\
\text { Boolean/Phrase }\end{array}$ & 46,720 \\
\hline 4 & $\begin{array}{l}\text { mirtazapine OR DE "Nefazodone" OR nefazodone OR DE "Neuroleptic } \\
\text { Drugs" OR "norepinephrine reuptake inhibitor" OR "norepinephrine reuptake } \\
\text { inhibitors" OR DE "Nortriptyline" OR nortriptyline OR DE "Olanzapine" OR } \\
\text { olanzapine OR oxcarbazepine OR Paliperidone OR DE "Paroxetine" OR } \\
\text { paroxetine OR DE "Perphenazine" OR perphenazine OR protriptyline OR } \\
\text { Prozac OR DE "Quetiapine" OR quetiapine OR ramelteon OR DE } \\
\text { "Risperidone" OR risperidone OR DE "Serotonin Reuptake Inhibitors" OR } \\
\text { "selective serotonin reuptake inhibitor" OR "selective serotonin reuptake } \\
\text { inhibitors" OR DE "Serotonin Norepinephrine Reuptake Inhibitors" OR } \\
\text { "serotonin norepinephrine reuptake inhibitor" OR "serotonin norepinephrine } \\
\text { reuptake inhibitors" OR DE "Sertraline" OR sertraline OR SNRI* OR SSRI } \\
\text { OR SSRIs }\end{array}$ & $\begin{array}{l}\text { Search modes - } \\
\text { Boolean/Phrase }\end{array}$ & 43,811 \\
\hline 5 & $\begin{array}{l}\text { Temazepam OR Thioridazine OR Thiothixene OR Topiramate OR DE } \\
\text { "Tranquilizing Drugs" OR DE "Trazodone" OR trazodone OR DE "Triazolam" } \\
\text { OR triazolam OR Trifluoperazine OR Trimipramine OR DE "Valproic Acid" } \\
\text { OR valproate OR DE "Venlafaxine" OR venlafaxine OR vilazodone OR } \\
\text { Vistaril OR vortioxetine OR zaleplon OR ziprasidone OR zolpidem }\end{array}$ & $\begin{array}{l}\text { Search modes - } \\
\text { Boolean/Phrase }\end{array}$ & 16,407 \\
\hline 6 & S2 or S3 or S4 or S5 & $\begin{array}{l}\text { Search modes - } \\
\text { Boolean/Phrase }\end{array}$ & 145,539 \\
\hline 7 & S1 and S6 & $\begin{array}{l}\text { Search modes - } \\
\text { Boolean/Phrase }\end{array}$ & 101,270 \\
\hline 8 & $\begin{array}{l}\text { DE "Pregnancy" OR preconception[tiab] OR pregnant" OR pregnancy OR } \\
\text { prenatal OR "post-partum" OR postpartum OR postnatal OR perinatal OR } \\
\text { antenatal OR "Maternal Health Services" OR "maternal health" OR "Infant } \\
\text { Nutritional Physiological Phenomena" OR "Breast Feeding"[Mesh] OR } \\
\text { "breast feeding" OR breastfeed* OR (breast AND fed) OR breastfed OR } \\
\text { "Maternal Welfare" OR gestation* OR maternal* }\end{array}$ & $\begin{array}{l}\text { Search modes - } \\
\text { Boolean/Phrase }\end{array}$ & 97,482 \\
\hline$\overline{9}$ & S7 and S8 & $\begin{array}{l}\text { Search modes - } \\
\text { Boolean/Phrase }\end{array}$ & 1,827 \\
\hline 10 & S7 and S8 & $\begin{array}{l}\text { Limiters - English } \\
\text { Search modes - } \\
\text { Boolean/Phrase }\end{array}$ & 1,773 \\
\hline 11 & S10 & $\begin{array}{l}\text { Limiters - } \\
\text { Population Group: } \\
\text { Human } \\
\text { Search modes - } \\
\text { Boolean/Phrase } \\
\end{array}$ & 1,328 \\
\hline 12 & S10 & $\begin{array}{l}\text { Limiters - } \\
\text { Population Group: } \\
\text { Animal } \\
\text { Search modes - } \\
\text { Boolean/Phrase }\end{array}$ & 435 \\
\hline 13 & S10 not S12 & $\begin{array}{l}\text { Limiters - } \\
\text { Population Group: } \\
\text { Animal } \\
\text { Search modes - } \\
\text { Boolean/Phrase } \\
\end{array}$ & 0 \\
\hline 14 & $\mathrm{~S} 11$ or $\mathrm{S} 13$ & $\begin{array}{l}\text { Search modes - } \\
\text { Boolean/Phrase }\end{array}$ & 1,328 \\
\hline
\end{tabular}




\begin{tabular}{|c|c|c|c|}
\hline \# & Query & $\begin{array}{l}\text { Limiters/ } \\
\text { Expanders }\end{array}$ & Results \\
\hline 15 & DE "Case Report" & $\begin{array}{l}\text { Search modes - } \\
\text { Boolean/Phrase }\end{array}$ & 22,842 \\
\hline 16 & S14 not S15 & $\begin{array}{l}\text { Search modes - } \\
\text { Boolean/Phrase }\end{array}$ & 1,284 \\
\hline 17 & PZ editorial & $\begin{array}{l}\text { Search modes - } \\
\text { Boolean/Phrase }\end{array}$ & 42,667 \\
\hline 18 & S16 NOT S17 & $\begin{array}{l}\text { Search modes - } \\
\text { Boolean/Phrase }\end{array}$ & 1,271 \\
\hline 19 & $\begin{array}{l}\text { DE "Drug Therapy" OR "drug therapy" OR drug*[tiab] OR pharmacotherap*: } \\
\text { ti,ab OR pharmacologic* OR medicine* OR medication* }\end{array}$ & $\begin{array}{l}\text { Search modes - } \\
\text { Boolean/Phrase }\end{array}$ & 700,317 \\
\hline 20 & $\begin{array}{l}\text { DE "Accidents" OR accident* OR "adverse effects" OR "adverse effect" or } \\
\text { "adverse event" OR "adverse events" OR "adverse outcome" OR "adverse } \\
\text { outcomes" OR "adverse reaction" OR "adverse reactions" OR "chemically } \\
\text { induced" OR complication* OR DE "Death and Dying" OR death* OR DE } \\
\text { "Drug Allergies" OR "Drug Allergies" OR DE "Drug Dependency" OR "Drug } \\
\text { Dependency" OR "drug effects" OR DE "Drug Sensitivity" OR "Drug } \\
\text { Sensitivity" OR DE "Harm Reduction" OR harm* OR harms OR "manic } \\
\text { episode" OR overdos* OR DE "Patient Safety" OR "Patient Safety" OR } \\
\text { poisoning OR DE "Self-Destructive Behavior" OR DE "Attempted Suicide" } \\
\text { OR DE "Head Banging" OR DE "Self-Inflicted Wounds" OR DE "Self- } \\
\text { Injurious Behavior" OR DE "Self-Mutilation" OR DE "Suicide" OR self } \\
\text { damage* OR self injur* OR self inflict* OR "Self-Injurious Behavior" OR DE } \\
\text { "Sequelae" OR DE "Side Effects (Drug)" OR "Side Effect" OR "side effects" } \\
\text { OR Suicide OR suicidal* OR DE "Toxic Disorders" OR toxicity }\end{array}$ & $\begin{array}{l}\text { Search modes - } \\
\text { Boolean/Phrase }\end{array}$ & 382,221 \\
\hline$\overline{21}$ & $\begin{array}{l}\text { Abruption* OR DE "Agenesis" OR "Apgar Score" OR DE "Anencephaly" OR } \\
\text { (DE "Birth Weight" AND chemical*) OR (DE "Birth Weight" AND chemical*) } \\
\text { OR DE "Childhood Development" OR DE "Cleft Palate" OR DE "Congenital } \\
\text { Disorders" OR "congenital abnormality" OR "congenital abnormalities" OR } \\
\text { DE "Conjoined Twins" OR ("Craniofacial Abnormalities" AND chemical*) OR } \\
\text { death OR DE "Death and Dying" OR DE "Delayed Development" OR DE } \\
\text { "Delayed Speech" OR DE "Developmental Disabilities" OR DE "Down's } \\
\text { Syndrome" OR "Drug Induced Congenital Disorders" OR DE "Failure to } \\
\text { Thrive" OR "Glucose Intolerance" OR DE "Hemorrhage" OR DE } \\
\text { "Hermaphroditism" OR (infant* AND (DE "Attachment Behavior" OR } \\
\text { attachment* OR bonding)) OR "Infantile Respiratory Distress Syndrome" OR } \\
\text { DE "Infertility" OR ("Intellectual Disability" AND child*) OR DE "Klinefelters } \\
\text { Syndrome" OR DE "Language Delay" OR ((DE "Learning Disabilities" OR } \\
\text { DE "Multiple Disabilities" OR DE "Reading Disabilities") AND child*) OR } \\
\text { "Low APGAR" OR DE "Microcephaly" OR miscarry OR miscarriage* OR } \\
\text { mortality OR "Neonatal Abstinence Syndrome" OR DE "Neonatal Disorders" } \\
\text { OR "Neonatal Respiratory Distress Syndrome" OR "Persistent Fetal } \\
\text { Circulation Syndrome" OR "Persistent Pulmonary Hypertension of Newborn" } \\
\text { OR DE "Phenylketonuria" OR "Postpartum Hemorrhage" OR DE "Prader } \\
\text { Willi Syndrome" OR DE "Preeclampsia" OR preeclampsia OR pre-eclampsia } \\
\text { OR DE "Premature Birth" OR "premature birth" OR "Premature labor" OR } \\
\text { "Prescription Drug Misuse" OR ("Prescription Drug" AND misuse) OR } \\
\text { "preterm birth" OR "pre-term birth" OR "preterm labor" OR "pre-term labor" } \\
\text { OR DE "Prenatal Exposure" OR ("Respiratory Distress Syndrome" AND } \\
\text { newborn) OR DE "Spina Bifida" OR DE "Spontaneous Abortion" OR DE "Tay } \\
\text { Sachs Disease" OR DE "Turners Syndrome" OR ("Uterine Inertia" AND } \\
\text { chemical*) }\end{array}$ & $\begin{array}{l}\text { Search modes - } \\
\text { Boolean/Phrase }\end{array}$ & 260,875 \\
\hline 22 & $\mathrm{~S} 20$ or S21 & $\begin{array}{l}\text { Search modes - } \\
\text { Boolean/Phrase }\end{array}$ & 529,322 \\
\hline 23 & S1 and S8 and S19 and S22 & $\begin{array}{l}\text { Search modes - } \\
\text { Boolean/Phrase }\end{array}$ & 4,407 \\
\hline$\overline{24}$ & $\mathrm{~S} 23$ & $\begin{array}{l}\text { Limiters - English } \\
\text { Search modes - } \\
\text { Boolean/Phrase }\end{array}$ & 4,364 \\
\hline
\end{tabular}




\begin{tabular}{|c|c|c|c|}
\hline \# & Query & $\begin{array}{l}\text { Limiters/ } \\
\text { Expanders }\end{array}$ & Results \\
\hline & S24 & $\begin{array}{l}\text { Limiters - } \\
\text { Population Group: } \\
\text { Human } \\
\text { Search modes - } \\
\text { Boolean/Phrase } \\
\end{array}$ & 3,999 \\
\hline 26 & S24 & $\begin{array}{l}\text { Limiters - } \\
\text { Population Group: } \\
\text { Animal } \\
\text { Search modes - } \\
\text { Boolean/Phrase } \\
\end{array}$ & 275 \\
\hline 27 & S24 not S26 & $\begin{array}{l}\text { Search modes - } \\
\text { Boolean/Phrase }\end{array}$ & 4,089 \\
\hline & $\mathrm{S} 25$ or $\mathrm{S} 27$ & $\begin{array}{l}\text { Search modes - } \\
\text { Boolean/Phrase }\end{array}$ & 4,139 \\
\hline 29 & S28 NOT (DE "Case Report" OR PZ Editorial) & $\begin{array}{l}\text { Search modes - } \\
\text { Boolean/Phrase }\end{array}$ & 4,067 \\
\hline & S29 NOT S18 & $\begin{array}{l}\text { Search modes - } \\
\text { Boolean/Phrase }\end{array}$ & 3,393 \\
\hline
\end{tabular}




\section{PsyciNfo}

6-4-2020

\begin{tabular}{|c|c|c|c|}
\hline$\#$ & Query & $\begin{array}{l}\text { Limiters/ } \\
\text { Expanders }\end{array}$ & Results \\
\hline S1 & 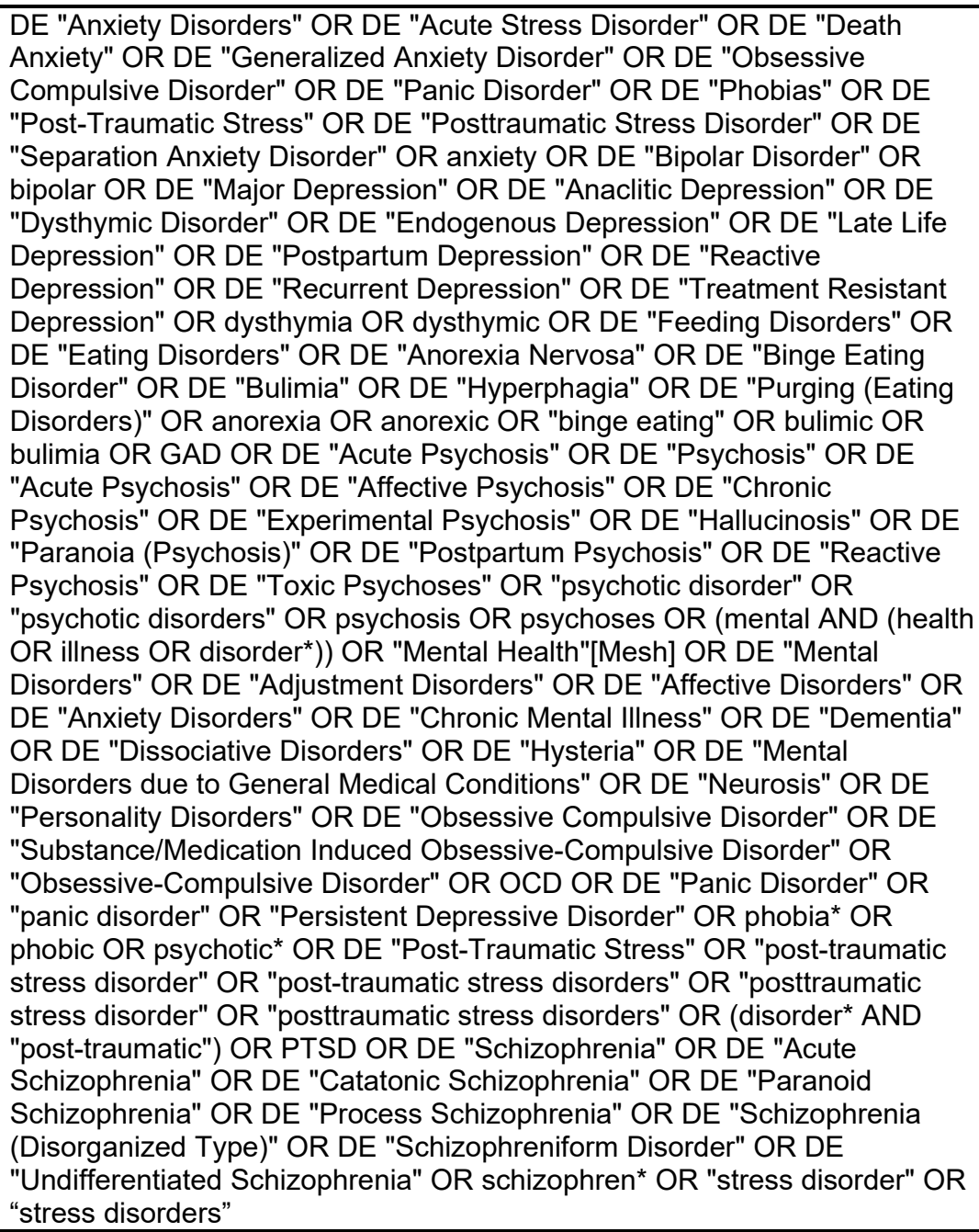 & & $1,266,162$ \\
\hline S2 & $\begin{array}{l}\text { (alprazolam OR "Anti-Anxiety Agents" OR anti-anxiety OR antianxiety OR } \\
\text { DE "Tranquilizing Drugs" OR DE "Amitriptyline" OR DE "Doxepin" OR DE } \\
\text { "Haloperidol" OR DE "Minor Tranquilizers" OR DE "Neuroleptic Drugs" OR } \\
\text { amitriptyline OR OR amoxapine OR DE "Anticonvulsive Drugs" OR } \\
\text { anticonvulsant OR anticonvulsants OR DE "Antidepressant Drugs" OR } \\
\text { "Antidepressive Agents, Second-Generation" OR anti-depress* OR } \\
\text { antidepressant* OR antidepressive* OR "antidepressive agent" OR } \\
\text { "antidepressive agents" OR "antidepressive drug" OR "antidepressive } \\
\text { drugs" OR antipsychotic* OR anxiolytic* OR DE "Aripiprazole" OR } \\
\text { aripiprazole OR Asenapine OR DE "Benzodiazepines" OR benzodiazepine* } \\
\text { OR brexanolone OR brexpiprazole OR DE "Bupropion" OR Bupropion OR } \\
\text { DE "Buspirone" OR Buspirone OR DE "Carbamazepine" OR } \\
\text { Carbamazepine OR Cariprazine OR DE "Chlorpromazine" OR } \\
\text { Chlorpromazine OR DE "Citalopram" OR citalopram OR clobazam OR } \\
\text { clomipramine OR DE "Clonazepam" OR Clonazepam OR DE "Clonidine" } \\
\text { OR Clonidine OR Clorazepate OR DE "Chlordiazepoxide" OR } \\
\text { Chlordiazepoxide OR DE "Clozapine" OR clozapine }\end{array}$ & $\begin{array}{l}\text { Search modes - } \\
\text { Boolean/Phrase }\end{array}$ & 129,042 \\
\hline
\end{tabular}




\begin{tabular}{|c|c|c|c|}
\hline \# & Query & $\begin{array}{l}\text { Limiters/ } \\
\text { Expanders }\end{array}$ & Results \\
\hline S3 & $\begin{array}{l}\text { DE "Desipramine" OR Desipramine OR Desvenlafaxine OR DE "Diazepam" } \\
\text { OR Diazepam OR DE "Diphenhydramine" OR Diphenhydramine OR DE } \\
\text { "Doxepin" OR doxepin OR duloxetine OR escitalopram OR eszopiclone OR } \\
\text { DE "Fluoxetine" OR fluoxetine OR DE "Fluphenazine" OR fluphenazine OR } \\
\text { DE "Fluvoxamine" OR fluvoxamine OR DE "Gabapentin" OR gabapentin } \\
\text { OR DE "Haloperidol" OR Haloperidol OR DE "Hydroxyzine" OR } \\
\text { Hydroxyzine OR iloperidone OR DE "Imipramine" OR imipramine OR } \\
\text { lamotrigine OR Levomilnacipran OR "Lisdexamfetamine Dimesylate" OR } \\
\text { DE "Lithium" OR lithium OR DE "Lorazepam" OR lorazepam OR lurasidone } \\
\text { OR Maprotiline OR milnacipran }\end{array}$ & $\begin{array}{l}\text { Search modes - } \\
\text { Boolean/Phrase }\end{array}$ & 49,631 \\
\hline S4 & $\begin{array}{l}\text { mirtazapine OR DE "Nefazodone" OR nefazodone OR DE "Neuroleptic } \\
\text { Drugs" OR "norepinephrine reuptake inhibitor" OR "norepinephrine reuptake } \\
\text { inhibitors" OR DE "Nortriptyline" OR nortriptyline OR DE "Olanzapine" OR } \\
\text { olanzapine OR oxcarbazepine OR Paliperidone OR DE "Paroxetine" OR } \\
\text { paroxetine OR DE "Perphenazine" OR perphenazine OR protriptyline OR } \\
\text { Prozac OR DE "Quetiapine" OR quetiapine OR ramelteon OR DE } \\
\text { "Risperidone" OR risperidone OR DE "Serotonin Reuptake Inhibitors" OR } \\
\text { "selective serotonin reuptake inhibitor" OR "selective serotonin reuptake } \\
\text { inhibitors" OR DE "Serotonin Norepinephrine Reuptake Inhibitors" OR } \\
\text { "serotonin norepinephrine reuptake inhibitor" OR "serotonin norepinephrine } \\
\text { reuptake inhibitors" OR DE "Sertraline" OR sertraline OR SNRI* OR SSRI } \\
\text { OR SSRIs }\end{array}$ & $\begin{array}{l}\text { Search modes - } \\
\text { Boolean/Phrase }\end{array}$ & 47,743 \\
\hline$\overline{\mathrm{S} 5}$ & $\begin{array}{l}\text { Temazepam OR Thioridazine OR Thiothixene OR Topiramate OR DE } \\
\text { "Tranquilizing Drugs" OR DE "Trazodone" OR trazodone OR DE } \\
\text { "Triazolam" OR triazolam OR Trifluoperazine OR Trimipramine OR DE } \\
\text { "Valproic Acid" OR valproate OR DE "Venlafaxine" OR venlafaxine OR } \\
\text { vilazodone OR Vistaril OR vortioxetine OR zaleplon OR ziprasidone OR } \\
\text { zolpidem }\end{array}$ & $\begin{array}{l}\text { Search modes - } \\
\text { Boolean/Phrase }\end{array}$ & 16,983 \\
\hline S6 & S2 OR S3 OR S4 OR S5 & $\begin{array}{l}\text { Search modes - } \\
\text { Boolean/Phrase }\end{array}$ & 152,221 \\
\hline$\overline{\mathrm{S} 7}$ & S1 AND S6 & $\begin{array}{l}\text { Search modes - } \\
\text { Boolean/Phrase }\end{array}$ & 106,198 \\
\hline$\overline{S 8}$ & $\begin{array}{l}\text { DE "Pregnancy" OR preconception[tiab] OR pregnant" OR pregnancy OR } \\
\text { prenatal OR "post-partum" OR postpartum OR postnatal OR perinatal OR } \\
\text { antenatal OR "Maternal Health Services" OR "maternal health" OR "Infant } \\
\text { Nutritional Physiological Phenomena" OR "Breast Feeding"[Mesh] OR } \\
\text { "breast feeding" OR breastfeed* OR (breast AND fed) OR breastfed OR } \\
\text { "Maternal Welfare" OR gestation* OR maternal* }\end{array}$ & $\begin{array}{l}\text { Search modes - } \\
\text { Boolean/Phrase }\end{array}$ & 103,648 \\
\hline$\overline{\mathrm{S} 9}$ & S7 AND S8 & $\begin{array}{l}\text { Search modes - } \\
\text { Boolean/Phrase }\end{array}$ & 1,981 \\
\hline$\overline{\mathrm{S} 10}$ & S7 AND S8 & $\begin{array}{l}\text { Limiters - English } \\
\text { Search modes - } \\
\text { Boolean/Phrase } \\
\end{array}$ & 1,925 \\
\hline$\overline{\mathrm{S} 11}$ & S10 & $\begin{array}{l}\text { Limiters - } \\
\text { Population Group: } \\
\text { Human } \\
\text { Search modes - } \\
\text { Boolean/Phrase } \\
\end{array}$ & 1,436 \\
\hline$\overline{\mathrm{S} 12}$ & S10 & $\begin{array}{l}\text { Limiters - } \\
\text { Population Group: } \\
\text { Animal } \\
\text { Search modes - } \\
\text { Boolean/Phrase } \\
\end{array}$ & 479 \\
\hline $\mathrm{S} 13$ & $\mathrm{~S} 10$ not $\mathrm{S} 12$ & $\begin{array}{l}\text { Limiters - } \\
\text { Population Group: } \\
\text { Animal } \\
\text { Search modes - } \\
\text { Boolean/Phrase }\end{array}$ & 0 \\
\hline
\end{tabular}




\begin{tabular}{|c|c|c|c|}
\hline \# & Query & $\begin{array}{l}\text { Limiters/ } \\
\text { Expanders }\end{array}$ & Results \\
\hline$\overline{S 14}$ & S11 OR S13 & $\begin{array}{l}\text { Search modes - } \\
\text { Boolean/Phrase }\end{array}$ & 1,436 \\
\hline$\overline{S 15}$ & DE "Case Report" & $\begin{array}{l}\text { Search modes - } \\
\text { Boolean/Phrase }\end{array}$ & 22,945 \\
\hline$\overline{\mathrm{S} 16}$ & S14 not S15 & $\begin{array}{l}\text { Search modes - } \\
\text { Boolean/Phrase }\end{array}$ & 1,392 \\
\hline$S 17$ & PZ editorial & $\begin{array}{l}\text { Search modes - } \\
\text { Boolean/Phrase }\end{array}$ & 44,005 \\
\hline$\overline{S 18}$ & S16 NOT S17 & $\begin{array}{l}\text { Search modes - } \\
\text { Boolean/Phrase }\end{array}$ & 1,379 \\
\hline$\overline{\mathrm{S} 19}$ & $\begin{array}{l}\text { DE "Drug Therapy" OR "drug therapy" OR drug*[tiab] OR pharmacotherap*: } \\
\text { ti,ab OR pharmacologic* OR medicine* OR medication* }\end{array}$ & $\begin{array}{l}\text { Search modes - } \\
\text { Boolean/Phrase }\end{array}$ & 766,776 \\
\hline$\overline{\mathrm{S} 20}$ & $\begin{array}{l}\text { DE "Accidents" OR accident* OR "adverse effects" OR "adverse effect" OR } \\
\text { "adverse event" OR "adverse events" OR "adverse outcome" OR "adverse } \\
\text { outcomes" OR "adverse reaction" OR "adverse reactions" OR "chemically } \\
\text { induced" OR complication* OR DE "Death AND Dying" OR death* OR DE } \\
\text { "Drug Allergies" OR "Drug Allergies" OR DE "Drug Dependency" OR "Drug } \\
\text { Dependency" OR "drug effects" OR DE "Drug Sensitivity" OR "Drug } \\
\text { Sensitivity" OR DE "Harm Reduction" OR harm OR harms OR "manic } \\
\text { episode" OR overdos* OR DE "Patient Safety" OR "Patient Safety" OR } \\
\text { poisoning OR DE "Self-Destructive Behavior" OR DE "Attempted Suicide" } \\
\text { OR DE "Head Banging" OR DE "Self-Inflicted Wounds" OR DE "Self- } \\
\text { Injurious Behavior" OR DE "Self-Mutilation" OR DE "Suicide" OR self } \\
\text { damage* OR self injur* OR self inflict* OR "Self-Injurious Behavior" OR DE } \\
\text { "Sequelae" OR DE "Side Effects (Drug)" OR "Side Effect" OR "side effects" } \\
\text { OR Suicide OR suicidal* OR DE "Toxic Disorders" OR toxicity }\end{array}$ & $\begin{array}{l}\text { Search modes - } \\
\text { Boolean/Phrase }\end{array}$ & 423,684 \\
\hline$\overline{\mathrm{S} 21}$ & $\begin{array}{l}\text { Abruption* OR DE "Agenesis" OR "Apgar Score" OR DE "Anencephaly" OR } \\
\text { (DE "Birth Weight" AND chemical") OR (DE "Birth Weight" AND chemical*) } \\
\text { OR DE "Childhood Development" OR DE "Cleft Palate" OR DE "Congenital } \\
\text { Disorders" OR "congenital abnormality" OR "congenital abnormalities" OR } \\
\text { DE "Conjoined Twins" OR ("Craniofacial Abnormalities" AND chemical*) OR } \\
\text { death OR DE "Death AND Dying" OR DE "Delayed Development" OR DE } \\
\text { "Delayed Speech" OR DE "Developmental Disabilities" OR DE "Down's } \\
\text { Syndrome" OR "Drug Induced Congenital Disorders" OR DE "Failure to } \\
\text { Thrive" OR "Glucose Intolerance" OR DE "Hemorrhage" OR DE } \\
\text { "Hermaphroditism" OR (infant* AND (DE "Attachment Behavior" OR } \\
\text { attachment* OR bonding)) OR "Infantile Respiratory Distress Syndrome" } \\
\text { OR DE "Infertility" OR ("Intellectual Disability" AND child") OR DE } \\
\text { "Klinefelters Syndrome" OR DE "Language Delay" OR ((DE "Learning } \\
\text { Disabilities" OR DE "Multiple Disabilities" OR DE "Reading Disabilities") } \\
\text { AND child*) OR "Low APGAR" OR DE "Microcephaly" OR miscarry OR } \\
\text { miscarriage* OR mortality OR "Neonatal Abstinence Syndrome" OR DE } \\
\text { "Neonatal Disorders" OR "Neonatal Respiratory Distress Syndrome" OR } \\
\text { "Persistent Fetal Circulation Syndrome" OR "Persistent Pulmonary } \\
\text { Hypertension of Newborn" OR DE "Phenylketonuria" OR "Postpartum } \\
\text { Hemorrhage" OR DE "Prader Willi Syndrome" OR DE "Preeclampsia" OR } \\
\text { preeclampsia OR pre-eclampsia OR DE "Premature Birth" OR "premature } \\
\text { birth" OR "Premature labor" OR "Prescription Drug Misuse" OR } \\
\text { ("Prescription Drug" AND misuse) OR "preterm birth" OR "pre-term birth" } \\
\text { OR "preterm labor" OR "pre-term labor" OR DE "Prenatal Exposure" OR } \\
\text { ("Respiratory Distress Syndrome" AND newborn) OR DE "Spina Bifida" OR } \\
\text { DE "Spontaneous Abortion" OR DE "Tay Sachs Disease" OR DE "Turners } \\
\text { Syndrome" OR ("Uterine Inertia" AND chemical*) }\end{array}$ & $\begin{array}{l}\text { Search modes - } \\
\text { Boolean/Phrase } \\
\text { Pan }\end{array}$ & 276,659 \\
\hline$\overline{\mathrm{S} 22}$ & S20 OR S21 & $\begin{array}{l}\text { Search modes - } \\
\text { Boolean/Phrase }\end{array}$ & 579,290 \\
\hline$\overline{S 23}$ & S1 AND S8 AND S19 AND S22 & $\begin{array}{l}\text { Search modes - } \\
\text { Boolean/Phrase }\end{array}$ & 4,955 \\
\hline
\end{tabular}




\begin{tabular}{|c|c|c|c|}
\hline$\#$ & Query & $\begin{array}{l}\text { Limiters/ } \\
\text { Expanders }\end{array}$ & Results \\
\hline$\overline{\mathrm{S} 24}$ & S23 & $\begin{array}{l}\text { Limiters - English } \\
\text { Search modes - } \\
\text { Boolean/Phrase }\end{array}$ & 4,903 \\
\hline$\overline{S 25}$ & S24 & $\begin{array}{l}\text { Limiters - } \\
\text { Population Group: } \\
\text { Human } \\
\text { Search modes - } \\
\text { Boolean/Phrase }\end{array}$ & :4,467 \\
\hline$\overline{S 26}$ & S24 & $\begin{array}{l}\text { Limiters - } \\
\text { Population Group: } \\
\text { Animal } \\
\text { Search modes - } \\
\text { Boolean/Phrase } \\
\end{array}$ & 319 \\
\hline S27 & S24 not S26 & $\begin{array}{l}\text { Search modes - } \\
\text { Boolean/Phrase }\end{array}$ & 4,584 \\
\hline$\overline{\mathrm{S} 28}$ & S25 OR S27 & $\begin{array}{l}\text { Search modes - } \\
\text { Boolean/Phrase }\end{array}$ & 4,639 \\
\hline $\mathrm{S} 29$ & S28 NOT (DE "Case Report" OR PZ Editorial) & $\begin{array}{l}\text { Search modes - } \\
\text { Boolean/Phrase }\end{array}$ & 4,563 \\
\hline$\overline{S 30}$ & S29 NOT S18 & $\begin{array}{l}\text { Search modes - } \\
\text { Boolean/Phrase } \\
\end{array}$ & 3,827 \\
\hline$\overline{\text { S31 }}$ & & $\begin{array}{l}\text { Limiters - } \\
\text { Published Date: } \\
\text { 20180601- } \\
\text { 20200631 } \\
\text { Expanders - Apply } \\
\text { equivalent } \\
\text { subjects } \\
\text { Search modes - } \\
\text { Boolean/Phrase } \\
\end{array}$ & 264,781 \\
\hline S32 & S18 AND S31 & $\begin{array}{l}\text { Expanders - Apply } \\
\text { equivalent } \\
\text { subjects } \\
\text { Search modes - } \\
\text { Boolean/Phrase }\end{array}$ & 101 \\
\hline S33 & S30 AND S31 & $\begin{array}{l}\text { Expanders - Apply } \\
\text { equivalent } \\
\text { subjects } \\
\text { Search modes - } \\
\text { Boolean/Phrase } \\
\end{array}$ & 416 \\
\hline$\overline{\text { S34 }}$ & S18 OR S30 & $\begin{array}{l}\text { Limiters - } \\
\text { Document Type: } \\
\text { Erratum/Correctio } \\
\text { n, Retraction } \\
\text { Expanders - Apply } \\
\text { equivalent } \\
\text { subjects } \\
\text { Search modes - } \\
\text { Boolean/Phrase }\end{array}$ & 35 \\
\hline
\end{tabular}




\title{
Gray Literature Searches for MHT in Pregnancy: ClinicalTrials.gov and WHO ICRTP Strategies
}

\author{
ClinicalTrials.gov results $=\mathbf{4 4 6}$ \\ WHO ICTRP results $=\mathbf{1 1 2}$ \\ ClinicalTrials.gov (12-14-2018) \\ BENEFITS
}

Condition box:

anxiety OR bipolar OR depress* OR dysthymi* OR "Eating Disorders" OR anorexi* OR "binge eating" OR bulimi* OR GAD OR "psychotic disorder" OR "psychotic disorders" OR psychosis OR psychoses OR "Mental Health" OR "Mental Disorders" OR "Obsessive-Compulsive Disorder" OR OCD OR "panic disorder" OR phobia* OR phobic OR psychotic* OR "posttraumatic stress disorder" OR "post-traumatic stress disorders" OR "posttraumatic stress disorder" OR "posttraumatic stress disorders" OR (disorder* AND "post-traumatic") OR PTSD OR schizophren* OR "stress disorder" OR "stress disorders"

\section{Intervention box:}

alprazolam OR anti-anxiety OR antianxiety OR amitriptyline OR amoxapine OR anticonvulsant* OR anti-depress* OR antidepressant* OR antidepressive* OR antipsychotic* OR anxiolytic* OR aripiprazole OR Asenapine OR benzodiazepine* OR brexanolone OR brexpiprazole OR Bupropion OR Buspirone OR Carbamazepine OR cariprazine OR Chlorpromazine OR citalopram OR clobazam OR clomipramine OR Clonazepam OR Clonidine OR Clorazepate OR Chlordiazepoxide OR clozapine OR Desipramine OR Desvenlafaxine OR Diazepam OR Diphenhydramine OR doxepin OR duloxetine OR escitalopram OR eszopiclone OR fluoxetine OR fluphenazine OR fluvoxamine OR gabapentin OR Haloperidol OR Hydroxyzine OR iloperidone OR imipramine OR lamotrigine OR levomilnacipran OR "lisdexamfetamine dimesylate" OR lithium OR lorazepam OR lurasidone OR Maprotiline OR milnacipran OR mirtazapine OR nefazodone OR "norepinephrine reuptake inhibitor" OR "norepinephrine reuptake inhibitors" OR nortriptyline OR olanzapine OR oxcarbazepine OR Paliperidone OR paroxetine OR perphenazine OR protriptyline OR quetiapine OR ramelteon OR risperidone OR "selective serotonin reuptake inhibitor" OR "selective serotonin reuptake inhibitors" OR "Serotonin Uptake Inhibitors" OR "serotonin norepinephrine reuptake inhibitor" OR "serotonin norepinephrine reuptake inhibitors" OR sertraline OR SNRI* OR SSRI OR SSRIs OR Temazepam OR thioridazine OR thiothixene OR topiramate OR trazodone OR triazolam OR trifluoperazine OR Trimipramine OR valproate OR venlafaxine OR vilazodone OR Vistaril OR vortioxetine OR zaleplon OR ziprasidone OR zolpidem

\section{Population (Other Terms box):}

Preconception OR pregnant OR pregnancy OR prenatal OR "post-partum" OR postpartum OR postnatal OR perinatal OR antenatal OR "maternal health" OR "Infant Nutritional Physiological Phenomena" OR "breast feeding" OR breastfeed* OR (breast AND fed) OR breastfed OR gestation* OR maternal*

Benefits/effectiveness KQs - Whole block: $=74$ results, all imported (Preconception OR pregnant OR pregnancy OR prenatal OR "post-partum" OR postpartum OR postnatal OR perinatal OR antenatal OR "maternal health" OR "Infant Nutritional Physiological 
Phenomena" OR "breast feeding" OR breastfeed* OR breastfed or breast-fed OR gestation* OR maternal*) AND (anxiety OR bipolar OR depress* OR dysthymi* OR "Eating Disorders" OR anorexi* OR "binge eating" OR bulimi* OR GAD OR "psychotic disorder" OR "psychotic disorders" OR psychosis OR psychoses OR "Mental Health" OR "Mental Disorders" OR "Obsessive-Compulsive Disorder" OR OCD OR "panic disorder" OR phobia* OR phobic OR psychotic* OR "post-traumatic stress disorder" OR "post-traumatic stress disorders" OR "posttraumatic stress disorder" OR "posttraumatic stress disorders" OR (disorder* AND "posttraumatic") OR PTSD OR schizophren* OR "stress disorder" OR "stress disorders") [DISEASE] AND ( alprazolam OR anti-anxiety OR antianxiety OR amitriptyline OR amoxapine OR anticonvulsant* OR anti-depress* OR antidepressant* OR antidepressive* OR antipsychotic* OR anxiolytic* OR aripiprazole OR Asenapine OR benzodiazepine* OR brexanolone OR brexpiprazole OR Bupropion OR Buspirone OR Carbamazepine OR cariprazine OR Chlorpromazine OR citalopram OR clobazam OR clomipramine OR Clonazepam OR Clonidine OR Clorazepate OR Chlordiazepoxide OR clozapine OR Desipramine OR Desvenlafaxine OR Diazepam OR Diphenhydramine OR doxepin OR duloxetine OR escitalopram OR eszopiclone OR fluoxetine OR fluphenazine OR fluvoxamine OR gabapentin OR Haloperidol OR Hydroxyzine OR iloperidone OR imipramine OR lamotrigine OR levomilnacipran OR "lisdexamfetamine dimesylate" OR lithium OR lorazepam OR lurasidone OR Maprotiline OR milnacipran OR mirtazapine OR nefazodone OR "norepinephrine reuptake inhibitor" OR "norepinephrine reuptake inhibitors" OR nortriptyline OR olanzapine OR oxcarbazepine OR Paliperidone OR paroxetine OR perphenazine OR protriptyline OR quetiapine OR ramelteon OR risperidone OR "selective serotonin reuptake inhibitor" OR "selective serotonin reuptake inhibitors" OR "Serotonin Uptake Inhibitors" OR "serotonin norepinephrine reuptake inhibitor" OR "serotonin norepinephrine reuptake inhibitors" OR sertraline OR SNRI* OR SSRI OR SSRIs OR Temazepam OR thioridazine OR thiothixene OR topiramate OR trazodone OR triazolam OR trifluoperazine OR Trimipramine OR valproate OR venlafaxine OR vilazodone OR Vistaril OR vortioxetine OR zaleplon OR ziprasidone OR zolpidem) [TREATMENT]

\section{HARMS}

445 found, 372 imported and the rest were duplicates with the benefits search above.

\section{Use condition terms from above}

\section{Guide to below search: Did not use treatment terms, as including them did not net any results}

Instead used population string AND concatenated harms string AND condition terms with [DISEASE] qualifier

(Preconception OR pregnant OR pregnancy OR prenatal OR "post-partum" OR postpartum OR postnatal OR perinatal OR antenatal OR "maternal health" OR "Infant Nutritional Physiological Phenomena" OR "breast feeding" OR breastfeed* OR breast AND fed OR breastfed OR gestation* OR maternal*) AND ( accident* OR "adverse effect" OR "Adverse Effects" OR "adverse event" OR "adverse events" OR "adverse outcome" OR "adverse outcomes" OR "adverse reaction" OR "adverse reactions" OR "chemically induced" OR complication* OR death* OR "Drug Allergies" OR "Drug Dependency" OR "drug effects" OR "Drug Sensitivity" OR harm* OR harms OR "manic episode" OR overdos* OR "Patient Safety" OR poisoning OR self damage* OR self injur* OR self inflict* OR "Self-Injurious Behavior" OR "Side Effect" OR "side effects" OR Suicide OR suicidal* OR toxicity OR "Abruptio Placentae" OR abruption* OR 
"Apgar Score" OR (("Birth Weight" OR "Craniofacial Abnormalities" OR "Extremely Premature" OR "Uterine Inertia") AND ("chemically induced" OR "drug effects" OR "growth and development")) OR "Child Development" OR "Congenital Abnormalities" OR "congenital abnormality" OR "congenital abnormalities" OR (infant* AND (attachment* OR bonding)) OR "Infantile Respiratory Distress Syndrome" OR "delayed development" OR "Glucose Intolerance" OR (Infertility AND female) OR ("Intellectual Disability" AND child*) OR "Low APGAR" OR miscarry OR miscarriage* OR Mortality OR "Neonatal Abstinence Syndrome" OR "Neonatal Respiratory Distress Syndrome" OR "Persistent Fetal Circulation Syndrome" OR "Persistent Pulmonary Hypertension of Newborn" OR "Postpartum Hemorrhage" OR "Pre-Eclampsia" OR preeclampsia OR pre-eclampsia OR "premature birth" OR "Prenatal Exposure Delayed Effects" OR "Prescription Drug Misuse" OR ("Prescription Drug" AND misuse) OR "preterm birth" OR "pre-term birth" OR "preterm labor" OR "pre-term labor" OR ("Respiratory Distress Syndrome" AND newborn ) OR "spontaneous abortion" ) AND ( anxiety OR bipolar OR depress* OR dysthymi* OR "Eating Disorders" OR anorexi* OR "binge eating" OR bulimi* OR GAD OR "psychotic disorder" OR "psychotic disorders" OR psychosis OR psychoses OR "Mental Health" OR "Mental Disorders" OR "Obsessive-Compulsive Disorder" OR OCD OR "panic disorder" OR phobia* OR phobic OR psychotic* OR "post-traumatic stress disorder" OR "post-traumatic stress disorders" OR "posttraumatic stress disorder" OR "posttraumatic stress disorders" OR disorder* AND "post-traumatic" OR PTSD OR schizophren* OR "stress disorder" OR "stress disorders" ) [DISEASE]

\section{WHO ICTRP searches (Advanced Search) 12-17-2018}

\section{Benefits}

256 maximum character limit per search box.

Recruitment status: ALL and no other limits selected.

(7 searches; same condition and Title (population) search terms as listed below for all searches, but split into 7 queries in order to capture all pharmacotherapy terms used in the ClinicalTrials.gov search)

Title box (used for population)

Preconception OR pregnant OR pregnancy OR prenatal OR "post-partum" OR postpartum OR postnatal OR perinatal OR antenatal OR "maternal health" OR "breast feeding" OR breastfeed* OR (breast AND fed) OR breastfed OR gestation* OR maternal*

\section{Condition box:}

Anxiety OR anxio* OR bipolar OR depress* OR anorexi* OR binge OR bulimi* OR psycho* OR "Mental Disorder" OR "Obsessive-Compulsive" OR OCD OR panic OR phobi* OR "posttraumatic" OR posttraumatic stress disorder" OR PTSD OR schizophren* OR stress

\section{Intervention boxes:}

Search 1 - 9 results, 9 imported alprazolam OR anti-anxiety OR antianxiety OR amitriptyline OR amoxapine OR anticonvulsant* OR anti-depress* OR antidepressant* OR antidepressive* OR antipsychotic* OR anxiolytic* OR aripiprazole OR Asenapine OR benzodiazepine* OR brexanolone Search $2-7$ results, 5 imported 
brexpiprazole OR Bupropion OR Buspirone OR Carbamazepine OR cariprazine OR

Chlorpromazine OR citalopram OR clobazam OR clomipramine OR Clonazepam OR Clonidine OR Clorazepate OR Chlordiazepoxide OR clozapine

Search 3 - 102 results, 92 imported

Desipramine OR Desvenlafaxine OR Diazepam OR Diphenhydramine OR doxepin OR

duloxetine OR escitalopram OR eszopiclone OR fluoxetine OR fluphenazine OR fluvoxamine OR gabapentin OR Haloperidol OR Hydroxyzine OR iloperidone

Search $4-5$ results, 2 imported

imipramine OR lamotrigine OR levomilnacipran OR "lisdexamfetamine dimesylate" OR lithium OR lorazepam OR lurasidone OR Maprotiline OR milnacipran OR mirtazapine OR nefazodone OR "norepinephrine reuptake inhibitor"

Search $5-6$ results, 1 imported

"norepinephrine reuptake inhibitors" OR nortriptyline OR olanzapine OR oxcarbazepine OR Paliperidone OR paroxetine OR perphenazine OR protriptyline OR quetiapine OR ramelteon OR risperidone

Search 6 - 11 results, 3 imported

"selective serotonin reuptake inhibitor" OR "selective serotonin reuptake inhibitors" OR

"Serotonin Uptake Inhibitors" OR "serotonin norepinephrine reuptake inhibitor" OR "serotonin norepinephrine reuptake inhibitors" OR sertraline OR SNRI* OR SSRI

Search 7 - 104 results, 0 imported

SSRIs OR Temazepam OR thioridazine OR thiothixene OR topiramate OR trazodone OR triazolam OR trifluoperazine OR Trimipramine OR valproate OR venlafaxine OR vilazodone OR Vistaril OR vortioxetine OR zaleplon OR ziprasidone OR zolpidem

\section{Harms searches (12-18-2018)}

\section{Advanced Search}

256 maximum character limit per search box.

Recruitment status: ALL and no other limits selected.

Various searches broken up into shorter chunks of harms terms to keep title box 256 char or less

Title box (used for population):

Format for this search box:

(Preconception OR pregnant OR pregnancy OR prenatal OR "post-partum" OR postpartum OR postnatal OR perinatal OR antenatal OR "maternal health") AND (partial harms terms from below)

Search $1-0$ results

(Preconception OR pregnant OR pregnancy OR prenatal OR "post-partum" OR postpartum OR postnatal OR perinatal OR antenatal OR "maternal health") AND (accident* OR "adverse effect" OR "Adverse Effects" OR "adverse event" OR "adverse events")

Search 2 - 0 results

(Preconception OR pregnant OR pregnancy OR prenatal OR "post-partum" OR postpartum OR postnatal OR perinatal OR antenatal OR "maternal health") AND ("adverse outcome" OR "adverse outcomes" OR "adverse reaction" OR "adverse reactions" OR "chemically induced") Search 3 - 0 results 
(Preconception OR pregnant OR pregnancy OR prenatal OR "post-partum" OR postpartum OR postnatal OR perinatal OR antenatal OR "maternal health") AND (complication* OR death* OR "Drug Allergies" OR "Drug Dependency" OR "drug effects" OR "Drug Sensitivity")

Search $4-10$ results, 0 imported (duplicates to other gray literature search results)

(Preconception OR pregnant OR pregnancy OR prenatal OR "post-partum" OR postpartum OR postnatal OR perinatal OR antenatal OR "maternal health") AND (harm* OR harms OR "manic episode" OR overdos* OR "Patient Safety" OR poisoning OR self damage*)

Search 5 - 0 results

(Preconception OR pregnant OR pregnancy OR prenatal OR "post-partum" OR postpartum OR postnatal OR perinatal OR antenatal OR "maternal health") AND (self injur* OR self inflict* OR "Self-Injurious Behavior" OR "Side Effect" OR "side effects" OR Suicide)

Search 6 - 0 results

(Preconception OR pregnant OR pregnancy OR prenatal OR "post-partum" OR postpartum OR postnatal OR perinatal OR antenatal OR "maternal health") AND (suicidal* OR toxicity OR

"Abruptio Placentae" OR abruption* OR "Apgar Score")

Search 7 - 0 results

(Preconception OR pregnant OR pregnancy OR prenatal OR "post-partum" OR postpartum OR postnatal OR perinatal OR antenatal OR "maternal health") AND (("Birth Weight" OR

"Craniofacial Abnormalities" OR "Extremely Premature" OR "Uterine Inertia") AND

("chemically induced" OR "drug effects" OR "growth and development"))

Search 8 - 0 results

(Preconception OR pregnant OR pregnancy OR prenatal OR "post-partum" OR postpartum OR postnatal OR perinatal OR antenatal OR "maternal health") AND ("Child Development" OR "Congenital Abnormalities" OR "congenital abnormality" OR "congenital abnormalities")

Search 9 - 0 results

(Preconception OR pregnant OR pregnancy OR prenatal OR "post-partum" OR postpartum OR postnatal OR perinatal OR antenatal OR "maternal health") AND ((infant* AND (attachment* OR bonding)) OR "Infantile Respiratory Distress Syndrome" OR "delayed development")

Search 10 - 0 results

(Preconception OR pregnant OR pregnancy OR prenatal OR "post-partum" OR postpartum OR postnatal OR perinatal OR antenatal OR "maternal health") AND ("Glucose Intolerance" OR (Infertility AND female) OR ("Intellectual Disability" AND child*) OR "Low APGAR")

Search $11-1$ result, 0 imported (duplicate to other gray literature search results)

(Preconception OR pregnant OR pregnancy OR prenatal OR "post-partum" OR postpartum OR postnatal OR perinatal OR antenatal OR "maternal health") AND (miscarry OR miscarriage* OR Mortality OR "Neonatal Abstinence Syndrome" OR "Neonatal Respiratory Distress Syndrome") Search 12 - 0 results

(Preconception OR pregnant OR pregnancy OR prenatal OR "post-partum" OR postpartum OR postnatal OR perinatal OR antenatal OR "maternal health") AND ("Persistent Fetal Circulation Syndrome" OR "Persistent Pulmonary Hypertension of Newborn")

Search $13-1$ result, 0 imported (duplicate to other gray literature search results)

(Preconception OR pregnant OR pregnancy OR prenatal OR "post-partum" OR postpartum OR postnatal OR perinatal OR antenatal OR "maternal health") AND ("Postpartum Hemorrhage" OR "Pre-Eclampsia" OR preeclampsia OR pre-eclampsia OR "premature birth")

Search 14 - 0 results 
(Preconception OR pregnant OR pregnancy OR prenatal OR "post-partum" OR postpartum OR postnatal OR perinatal OR antenatal OR "maternal health") AND ("Prenatal Exposure Delayed Effects" OR "Prescription Drug Misuse" OR ("Prescription Drug" AND misuse))

Search 15 - 0 results

(Preconception OR pregnant OR pregnancy OR prenatal OR "post-partum" OR postpartum OR postnatal OR perinatal OR antenatal OR "maternal health") AND ("preterm birth" OR "pre-term birth" OR "preterm labor" OR "pre-term labor")

Search $16-0$ results

(Preconception OR pregnant OR pregnancy OR prenatal OR "post-partum" OR postpartum OR postnatal OR perinatal OR antenatal OR "maternal health") AND (("Respiratory Distress Syndrome" AND newborn) OR "spontaneous abortion")

\section{Condition box:}

Anxiety OR anxio* OR bipolar OR depress* OR anorexi* OR binge OR bulimi* OR psycho* OR "Mental Disorder" OR "Obsessive-Compulsive" OR OCD OR panic OR phobi* OR "posttraumatic" OR posttraumatic stress disorder" OR PTSD OR schizophren* OR stress

Intervention box:

Generic drug therapy string:

drug* OR pharmacotherap* OR pharmacologic* OR medicine* OR medication*

\section{Gray Literature Searches for MHT in Pregnancy: ClinicalTrials.gov}

ClinicalTrials.gov results $=\mathbf{2 9 0}$

ClinicalTrials.gov (12-14-2018)

BENEFITS

\section{Condition box:}

anxiety OR bipolar OR depress* OR dysthymi* OR "Eating Disorders" OR anorexi* OR "binge eating" OR bulimi* OR GAD OR "psychotic disorder" OR "psychotic disorders" OR psychosis OR psychoses OR "Mental Health" OR "Mental Disorders" OR "Obsessive-Compulsive Disorder" OR OCD OR "panic disorder" OR phobia* OR phobic OR psychotic* OR "posttraumatic stress disorder" OR "post-traumatic stress disorders" OR "posttraumatic stress disorder" OR "posttraumatic stress disorders" OR (disorder* AND "post-traumatic") OR PTSD OR schizophren* OR "stress disorder" OR "stress disorders"

\section{Intervention box:}

alprazolam OR anti-anxiety OR antianxiety OR amitriptyline OR amoxapine OR anticonvulsant* OR anti-depress* OR antidepressant* OR antidepressive* OR antipsychotic* OR anxiolytic* OR aripiprazole OR Asenapine OR benzodiazepine* OR brexanolone OR brexpiprazole OR Bupropion OR Buspirone OR Carbamazepine OR cariprazine OR Chlorpromazine OR citalopram OR clobazam OR clomipramine OR Clonazepam OR Clonidine OR Clorazepate OR Chlordiazepoxide OR clozapine OR Desipramine OR Desvenlafaxine OR Diazepam OR Diphenhydramine OR doxepin OR duloxetine OR escitalopram OR eszopiclone OR fluoxetine OR fluphenazine OR fluvoxamine OR gabapentin OR Haloperidol OR Hydroxyzine OR iloperidone OR imipramine OR lamotrigine OR levomilnacipran OR "lisdexamfetamine dimesylate" OR lithium OR lorazepam OR lurasidone OR Maprotiline OR milnacipran OR mirtazapine OR nefazodone OR "norepinephrine reuptake inhibitor" OR "norepinephrine reuptake inhibitors" OR nortriptyline OR olanzapine OR oxcarbazepine OR Paliperidone OR 
paroxetine OR perphenazine OR protriptyline OR quetiapine OR ramelteon OR risperidone OR "selective serotonin reuptake inhibitor" OR "selective serotonin reuptake inhibitors" OR

"Serotonin Uptake Inhibitors" OR "serotonin norepinephrine reuptake inhibitor" OR "serotonin norepinephrine reuptake inhibitors" OR sertraline OR SNRI* OR SSRI OR SSRIs OR Temazepam OR thioridazine OR thiothixene OR topiramate OR trazodone OR triazolam OR trifluoperazine OR Trimipramine OR valproate OR venlafaxine OR vilazodone OR Vistaril OR vortioxetine OR zaleplon OR ziprasidone OR zolpidem

\section{Population (Other Terms box):}

Preconception OR pregnant OR pregnancy OR prenatal OR "post-partum" OR postpartum OR postnatal OR perinatal OR antenatal OR "maternal health" OR "Infant Nutritional Physiological Phenomena" OR "breast feeding" OR breastfeed* OR (breast AND fed) OR breastfed OR gestation* OR maternal*

\section{Benefits/effectiveness KQs - Whole block: $=26$ results, 26 imported}

(Preconception OR pregnant OR pregnancy OR prenatal OR "post-partum" OR postpartum OR postnatal OR perinatal OR antenatal OR "maternal health" OR "Infant Nutritional Physiological Phenomena" OR "breast feeding" OR breastfeed* OR (breast AND fed) OR breastfed OR gestation* OR maternal*) AND AREA[ConditionSearch] (anxiety OR bipolar OR depress* OR dysthymi* OR "Eating Disorders" OR anorexi* OR "binge eating" OR bulimi* OR GAD OR "psychotic disorder" OR "psychotic disorders" OR psychosis OR psychoses OR "Mental Health" OR "Mental Disorders" OR "Obsessive-Compulsive Disorder" OR OCD OR "panic disorder" OR phobia* OR phobic OR psychotic* OR "post-traumatic stress disorder" OR "post-traumatic stress disorders" OR "posttraumatic stress disorder" OR "posttraumatic stress disorders" OR (disorder* AND "post-traumatic") OR PTSD OR schizophren* OR "stress disorder" OR "stress disorders") AND AREA[InterventionSearch] (alprazolam OR anti-anxiety OR antianxiety OR amitriptyline OR amoxapine OR anticonvulsant* OR anti-depress* OR antidepressant* OR antidepressive* OR antipsychotic* OR anxiolytic* OR aripiprazole OR Asenapine OR benzodiazepine* OR brexanolone OR brexpiprazole OR Bupropion OR Buspirone OR Carbamazepine OR cariprazine OR Chlorpromazine OR citalopram OR clobazam OR clomipramine OR Clonazepam OR Clonidine OR Clorazepate OR Chlordiazepoxide OR clozapine OR Desipramine OR Desvenlafaxine OR Diazepam OR Diphenhydramine OR doxepin OR duloxetine OR escitalopram OR eszopiclone OR fluoxetine OR fluphenazine OR fluvoxamine OR gabapentin OR Haloperidol OR Hydroxyzine OR iloperidone OR imipramine OR lamotrigine OR levomilnacipran OR "lisdexamfetamine dimesylate" OR lithium OR lorazepam OR lurasidone OR Maprotiline OR milnacipran OR mirtazapine OR nefazodone OR "norepinephrine reuptake inhibitor" OR "norepinephrine reuptake inhibitors" OR nortriptyline OR olanzapine OR oxcarbazepine OR Paliperidone OR paroxetine OR perphenazine OR protriptyline OR quetiapine OR ramelteon OR risperidone OR "selective serotonin reuptake inhibitor" OR "selective serotonin reuptake inhibitors" OR "Serotonin Uptake Inhibitors" OR "serotonin norepinephrine reuptake inhibitor" OR "serotonin norepinephrine reuptake inhibitors" OR sertraline OR SNRI* OR SSRI OR SSRIs OR Temazepam OR thioridazine OR thiothixene OR topiramate OR trazodone OR triazolam OR trifluoperazine OR Trimipramine OR valproate OR venlafaxine OR vilazodone OR Vistaril OR vortioxetine OR zaleplon OR ziprasidone OR zolpidem) AND AREA[LastUpdatePostDate] EXPAND[Term] RANGE[06/14/2018, 06/05/2020] 


\section{HARMS}

290 found, 264 imported and the rest were duplicates with the benefits search above.

\section{Use condition terms from above}

Guide to below search: Did not use treatment terms, as including them did not net any results

Instead used population string AND concatenated harms string AND condition terms with [DISEASE] qualifier

(Preconception OR pregnant OR pregnancy OR prenatal OR "post-partum" OR postpartum OR postnatal OR perinatal OR antenatal OR "maternal health" OR "Infant Nutritional Physiological Phenomena" OR "breast feeding" OR breastfeed* OR breast AND fed OR breastfed OR gestation* OR maternal*) AND ( accident* OR "adverse effect" OR "Adverse Effects" OR "adverse event" OR "adverse events" OR "adverse outcome" OR "adverse outcomes" OR "adverse reaction" OR "adverse reactions" OR "chemically induced" OR complication* OR death* OR "Drug Allergies" OR "Drug Dependency" OR "drug effects" OR "Drug Sensitivity" OR harm* OR harms OR "manic episode" OR overdos* OR "Patient Safety" OR poisoning OR self damage* OR self injur* OR self inflict* OR "Self-Injurious Behavior" OR "Side Effect" OR "side effects" OR Suicide OR suicidal* OR toxicity OR "Abruptio Placentae" OR abruption* OR "Apgar Score" OR (("Birth Weight" OR "Craniofacial Abnormalities" OR "Extremely Premature" OR "Uterine Inertia") AND ("chemically induced" OR "drug effects" OR "growth and development")) OR "Child Development" OR "Congenital Abnormalities" OR "congenital abnormality" OR "congenital abnormalities" OR (infant* AND (attachment* OR bonding)) OR "Infantile Respiratory Distress Syndrome" OR "delayed development" OR "Glucose Intolerance" OR (Infertility AND female) OR ("Intellectual Disability" AND child*) OR "Low APGAR" OR miscarry OR miscarriage* OR Mortality OR "Neonatal Abstinence Syndrome" OR "Neonatal Respiratory Distress Syndrome" OR "Persistent Fetal Circulation Syndrome" OR "Persistent Pulmonary Hypertension of Newborn" OR "Postpartum Hemorrhage" OR "Pre-Eclampsia" OR preeclampsia OR pre-eclampsia OR "premature birth" OR "Prenatal Exposure Delayed Effects" OR "Prescription Drug Misuse" OR ("Prescription Drug" AND misuse) OR "preterm birth" OR "pre-term birth" OR "preterm labor" OR "pre-term labor" OR ("Respiratory Distress Syndrome" AND newborn ) OR "spontaneous abortion" ) AND ( anxiety OR bipolar OR depress* OR dysthymi* OR "Eating Disorders" OR anorexi* OR "binge eating" OR bulimi* OR GAD OR "psychotic disorder" OR "psychotic disorders" OR psychosis OR psychoses OR "Mental Health" OR "Mental Disorders" OR "Obsessive-Compulsive Disorder" OR OCD OR "panic disorder" OR phobia* OR phobic OR psychotic* OR "post-traumatic stress disorder" OR "post-traumatic stress disorders" OR "posttraumatic stress disorder" OR "posttraumatic stress disorders" OR disorder* AND "post-traumatic" OR PTSD OR schizophren* OR "stress disorder" OR "stress disorders" ) [DISEASE] 


\section{Appendix B. Results}

\section{Results of Literature Searches}

The electronic search, gray literature, and reference mining identified 31,846 citations. After title and abstract screening, 1,812 studies were retrieved for full-text review. A total of 164 studies (168 articles) met eligibility criteria. Thirty-three studies were not included in the data synthesis due to only reporting unadjusted effectiveness data. A total of 131 studies (135 articles) were included in the analyses (Figure B-1).

\section{Description of Included Studies}

For KQ 1, we identified 9 trials and 10 observational studies. Four studies were assessed as having low risk of bias, 8 studies were assessed as having some concerns for bias, and 7 studies were assessed as having high risk of bias. ${ }^{16-34}$

For KQ 2, we identified one trial ( 2 articles) and four observational studies ( 5 articles). One study was assessed as having some concerns for risk of bias and four studies ( 6 articles) were assessed as having high risk of bias. ${ }^{35-41}$

For KQ 3, we identified 5 trials and 70 observational studies (72 articles). Three studies were assessed as having low risk of bias, 32 studies were assessed as having some concerns for bias, 35 studies (37 articles) were assessed as having high risk of bias, and 5 studies were rated as having some concerns/high risk of bias. ${ }^{6-9}, 16,17,19,23,25,27,31,33,42-106$

For KQ 4, we identified one trial ( 2 articles) and 55 observational studies. Seven studies were assessed as having some concerns for bias, 44 studies (45 articles) were assessed as having high

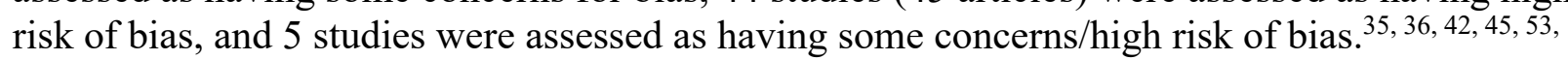
$55,64,70,81,83-85,87,95-98,107-146$

An overview of the pharmacologic interventions that were examined are provided in Table B-1. All of the included studies were published in English. Additional details of the risk of bias assessments are downloadable on SRDR. 
Figure B-1. Article flow diagram

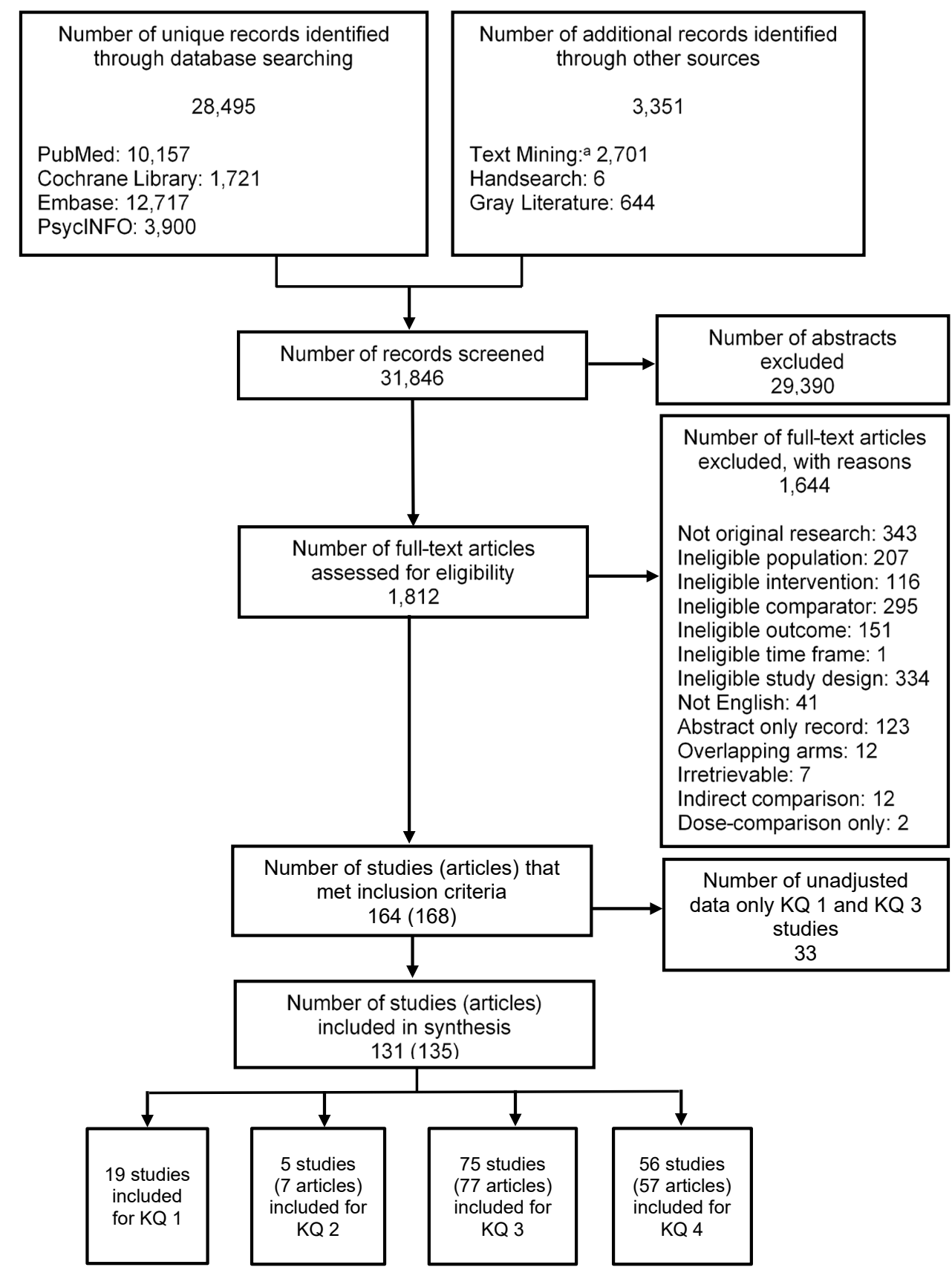

Note: The sum of the number of studies per KQ exceeds the total number of studies because some studies were applicable to multiple KQs.

${ }^{\text {a }}$ As part of a methods project, an independent search was undertaken, using text-mining software to identify additional relevant keywords and MeSH search terms. This search was also independently peer reviewed. Duplicate citations were removed prior to screening.

$\mathrm{KQ}=$ Key Question 
Table B-1. Pharmacologic interventions for included studies Pharmacologic Intervention Amitriptyline

Antipsychotics (Second-generation) Number of Studies

Antipsychotics (First-generation) $2^{56,74}$

Percent 1.5

Antipsychotics associated with increased risk of T2DM

$7^{44,50,62,85,97,119,125}$

(Quetiapine [high-dose], Olanzapine, Risperidone, or

Clozapine)

Anxiolytics

Aripiprazole

Benzodiazepine

Brexanolone

Bupropion

Carbamazepine

Citalopram

Citalopram or Escitalopram

Clomipramine

Clozapine

Duloxetine

Escitalopram

Escitalopram or Fluvoxamine

Fluoxetine

Fluvoxamine

$4^{30,46,71,79} \quad 3.1$

Haloperidol

Lamotrigine

$2^{90,97}$

2

$2^{17,19}$

6

$1^{96}$

$9^{51,57,72,74,75,104,111,117,141}$

3

$2^{51,}$

Lithium

Mirtazapine

Mood Stabilizers

MAOIs

$1^{97}$

$4^{56,75,98,141}$

$5^{72,74,75,106,141}$

$1^{117}$

$18^{7,29,39,40,51,56,57,72,74,75,81,104,106,107,111,117,134,136,141}$

$3^{57,107,111}$

3.1

1.5

1.5

Nortriptyline

Olanzapine

Oxcarbazepine

Paroxetine

$1^{127}$

$6^{24,37,38,96,97,108}$

$6^{37,38,41,47,97,108}$

$6^{56,74,75,95,116}$

1.5

Paroxeline 20

Quetiapine

Risperidone

$2^{26,96}$

4.6

Sertraline

SNRIs

Sodium Valproate

SSRIs

$2^{53,114}$

0.8

6.9

2.3

1.5

Nonsertraline SSRIs

TCA

$2^{35,36,56}$

3

$3^{9}$

196

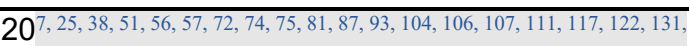
141

$4^{43,90,97,127}$

2.3

$4^{62,90}$

$4^{62,90,97,127}$

$19^{7}, 16,21-23,35,36,51,56,57,70,72,74,75,104,106,107,111,117,141$

$19^{7,51,53,56,57,64,74,75,81,83,98,104,106,107,110,112,114,124,140} 14.5$

$2^{41,96}$

1.5

$47^{6-9,27,53,54,57-61,63,64,66,69,70,72,74-76,79-84,87,88,91,93,95 \text {, }}$

$104,106,110,112,114,116,123,124,126,131,133,135$

Topiramate

Trazadone

$1^{70}$

Venlafaxine

$18^{7,39,40,53,57,64,72,75,79,81,83,93,110,114,123,126,133,134,136}$

196

$2^{74,75}$

Venlafaxine or desvenlafaxine

$6^{83,98,122,135,141,144}$

0.8

Ziprasidone

$1^{107}$

Zolpidem

$1^{90}$

$1^{92}$ 


\begin{tabular}{|c|c|c|}
\hline Pharmacologic Intervention & Number of Studies & Percent \\
\hline Zopiclone & $1^{71}$ & 0.8 \\
\hline Pharmacologic Combinations & $9^{41,45,72,95,115,120,129,132,141}$ & 6.9 \\
\hline Nonspecific/Undefined Pharmacologic Interventions & 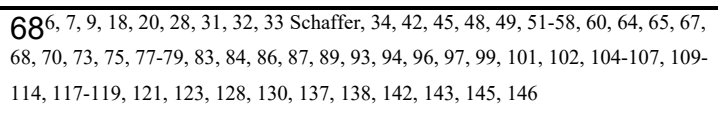 & 51.9 \\
\hline
\end{tabular}




\section{Detailed Results}

Key Question 1: Among pregnant and postpartum women, what is the effectiveness of pharmacologic interventions on maternal outcomes among those with a new or preexisting anxiety disorder, depressive disorder, bipolar disorder, or schizophrenia?

\section{Anxiety: Anti-Anxiety Medications}

\section{Overview}

- The evidence is insufficient to judge maternal benefits from anxiolytics following delivery.

\section{Detailed Results}

One randomized controlled trial (RCT), rated high risk of bias, reported on the maternal benefits of anxiolytics in $1967 .{ }^{30}$ The trial included 51 postpartum women with anxiety in one hospital site in the United States. Women were given either hydroxyzine or placebo for anxiety over a period of 3 days following delivery.

This trial used an unvalidated tool to measure "a series of behavioral responses" 30 and did not present data on variance by arm. We judged the evidence to be insufficient due to bias, potential imprecision, and lack of information on consistency (Table B-2).

Table B-2. Strength of evidence for effectiveness: Anxiolytics versus no treatment

\begin{tabular}{|c|c|c|c|c|c|c|c|}
\hline Population & $\begin{array}{l}\text { Intervention } \\
\text { and } \\
\text { Comparator }\end{array}$ & Outcome & $\begin{array}{l}\text { Incidence } \\
\text { or Mean } \\
\text { Effect by } \\
\text { Arm }\end{array}$ & Results & $\begin{array}{l}\text { Study Design } \\
\text { and Sample } \\
\text { Size }\end{array}$ & $\begin{array}{l}\text { Factors That } \\
\text { Affect the } \\
\text { Strength of } \\
\text { Evidence }\end{array}$ & $\begin{array}{l}\text { Overall } \\
\text { Evidence } \\
\text { Strength } \\
\text { (Direction } \\
\text { of Effect) }\end{array}$ \\
\hline $\begin{array}{l}\text { Postpartum } \\
\text { women with } \\
\text { anxiety }\end{array}$ & $\begin{array}{l}\text { Hydroxyzine } \\
\text { vs. placebo for } \\
3 \text { days } \\
\text { following } \\
\text { delivery }\end{array}$ & $\begin{array}{l}\text { Maternal } \\
\text { behavioral } \\
\text { response }\end{array}$ & $\begin{array}{l}\text { Average } \\
\text { "response } \\
\text { rating": } \\
7.24 \text { vs. } \\
3.25^{30}\end{array}$ & $\begin{array}{l}\text { Effect not } \\
\text { calculable } \\
\text { (variance NR) }\end{array}$ & $1 \mathrm{RCT}, \mathrm{N}=51^{30}$ & $\begin{array}{l}\text { High study } \\
\text { limitations } \\
\text { (high risk of } \text { bias }^{30} \text { ), likely } \\
\text { imprecise } \\
\text { (small Ns), } \\
\text { consistency } \\
\text { unknown }\end{array}$ & Insufficient \\
\hline
\end{tabular}

KQ = Key Question; $\mathrm{N}=$ number; $\mathrm{NR}=$ not reported; $\mathrm{RCT}=$ randomized controlled trial; vs. = versus.

\section{Depression: Selective Serotonin Reuptake Inhibitors (SSRIs)}

\section{Overview}

- The evidence is insufficient to judge the risk of benefits of SSRIs during pregnancy as a drug class for mode of delivery.

- Included studies did not report on other maternal benefits.

\section{Detailed Results}

One publication with some risk-of-bias concerns reported on mode of delivery, specifically, incidence of Cesarean section (C-section), among depressed women with and without SSRI 
exposure during pregnancy. ${ }^{27}$ The evidence is insufficient to judge the effect of SSRI exposure (Table B-3).

Table B-3. Strength of evidence for effectiveness: SSRIs versus no treatment

\begin{tabular}{|c|c|c|c|c|c|c|c|}
\hline Population & $\begin{array}{l}\text { Intervention } \\
\text { and } \\
\text { Comparator }\end{array}$ & Outcome & $\begin{array}{l}\text { Incidence } \\
\text { or Mean } \\
\text { Effect by } \\
\text { Arm }\end{array}$ & Results & $\begin{array}{l}\text { Study Design } \\
\text { and Sample Size }\end{array}$ & $\begin{array}{l}\text { Factors That } \\
\text { Affect the Strength } \\
\text { of Evidence }\end{array}$ & $\begin{array}{l}\text { Overall } \\
\text { Evidence } \\
\text { Strength } \\
\text { (Direction } \\
\text { of Effect) } \\
\end{array}$ \\
\hline $\begin{array}{l}\text { Depressed } \\
\text { women }\end{array}$ & $\begin{array}{l}\text { SSRI } \\
\text { exposure in } \\
\text { pregnancy } \\
\text { vs. } \\
\text { unexposed } \\
\text { women with } \\
\text { depression }\end{array}$ & $\begin{array}{l}\text { Cesarean } \\
\text { section }\end{array}$ & NR & $\begin{array}{l}\text { Difference in } \\
\text { incidence: } \\
-0.009(95 \% \mathrm{Cl} \text {, } \\
-0.05 \text { to } 0.036) \\
p \text {-value }=0.69^{27}\end{array}$ & 1 cohort, $n=N^{27}$ & $\begin{array}{l}\text { Moderate study } \\
\text { limitations }^{27} \\
\text { imprecise, } \\
\text { consistency } \\
\text { unknown }\end{array}$ & Insufficient \\
\hline
\end{tabular}

$\overline{\mathrm{CI}}=$ confidence interval; $\mathrm{N}=$ number; $\mathrm{NR}=$ not reported; SSRI $=$ selective serotonin reuptake inhibitor; $\mathrm{TCA}=$ tricyclic antidepressant; vs. $=$ versus.

\section{Depression: Fluoxetine}

\section{Overview}

- The evidence is insufficient to judge the risk of benefits of fluoxetine for postpartum depression.

- No other maternal benefits were evaluated.

\section{Detailed Results}

One RCT of women in an urban health district in the United Kingdom reported on a fluoxetine benefit outcome. ${ }^{29}$ The RCT examined the effect of fluoxetine versus placebo exposure on depression outcomes during the postpartum period. ${ }^{29}$ Both arms also received one session of CBT in one fluoxetine/placebo comparison and six sessions in a second; the dose of fluoxetine was not specified. ${ }^{29}$ The study risk of bias was rated as some concerns. Hamilton depression scores improved in all groups, but results were not superior in the fluoxetine arm in either the one-session or six-session CBT co-intervention comparisons (Table B-4).

Table B-4. Strength of evidence for effectiveness outcomes: Fluoxetine versus no treatment

\begin{tabular}{|c|c|c|c|c|c|c|}
\hline Population & $\begin{array}{l}\text { Intervention } \\
\text { and } \\
\text { Comparator }\end{array}$ & Outcome & $\begin{array}{l}\text { Incidence or Mean } \\
\text { Effect by Arm }\end{array}$ & $\begin{array}{l}\text { Study } \\
\text { Design } \\
\text { and } \\
\text { Sample } \\
\text { Size }\end{array}$ & $\begin{array}{l}\text { Factors That } \\
\text { Affect the } \\
\text { Strength of } \\
\text { Evidence }\end{array}$ & $\begin{array}{l}\text { Overall } \\
\text { Evidence } \\
\text { Strength } \\
\text { (Direction } \\
\text { of Effect) }\end{array}$ \\
\hline $\begin{array}{l}\text { Postpartum } \\
\text { women: } \\
\text { Fluoxetine } \\
\text { exposure or } \\
\text { depression } \\
\text { diagnosis } 12 \\
\text { weeks after } \\
\text { delivery }\end{array}$ & $\begin{array}{l}\text { Fluoxetine } \\
\text { exposure for } 3 \\
\text { months vs. } \\
\text { placebo. Both } \\
\text { arms also } \\
\text { received CBT (1 } \\
\text { or } 6 \text { sessions) }\end{array}$ & $\begin{array}{l}\text { Hamilton } \\
\text { Depression } \\
\text { score }\end{array}$ & $\begin{array}{l}\text { Intent-to-treat findings: } 1 \text { ARR } \\
\text { Session of CBT: } 4.4 \\
\text { (2.4 to } 7.4) \text { vs. } 8.1(6.1 \\
\text { to } 10.7) \text {; calculated } \\
\text { mean difference: } \\
-3.7(95 \% \mathrm{Cl},-8.8 \text { to } \\
1.4) \\
6 \text { sessions of CBT: } 5.1 \\
\text { (2.6 to 9.2) vs. } 4.9(3.0 \\
\text { to } 8.9) ; \text { calculated mean } \\
\text { difference: } 0.2(95 \% \mathrm{Cl} \text {, } \\
-10.0 \text { to } 10.4)^{29}\end{array}$ & $\begin{array}{l}1 \text { cohort: } \\
\mathrm{N}=87^{29}\end{array}$ & $\begin{array}{l}\text { Moderate study } \\
\text { limitations, } \\
\text { seriously } \\
\text { imprecise (small } \\
\text { sample size, Cls } \\
\text { spanning the } \\
\text { null), } \\
\text { consistency } \\
\text { unknown }\end{array}$ & Insufficient \\
\hline
\end{tabular}

$\mathrm{ARR}=$ adjusted risk ratio; $\mathrm{CBT}=$ cognitive behavioral therapy; $\mathrm{CI}=$ confidence interval; $\mathrm{N}=$ number; vs. $=$ versus. 


\section{Depression: Paroxetine}

\section{Overview}

- The evidence is insufficient to rate the strength of evidence for the likelihood of response, remission, or symptoms for women with postpartum onset of depression compared with women on placebo.

\section{Detailed Results}

A single publication reported on women with onset of depressive symptoms at 0 to 3 months postpartum, randomized to paroxetine versus placebo with the primary outcome of change in depressive symptoms indexed by HAM-D-17 at 8 weeks after treatment initiation. ${ }^{25}$ Enrollment of 120 women was planned, but only 70 women were recruited. Women in the active arm received one capsule (10 $\mathrm{mg}$ of immediate release paroxetine) daily for the first and second week with increases up to $40 \mathrm{mg}$ if participants did not experience sufficient improvement. There was no change in HAM-D-17 score between the two groups. More women allocated to paroxetine were more likely to achieve remission, indexed by HAM-D-17 $\leq 8$ (adjusted odds ratio [AOR], $3.5 ; 95 \% \mathrm{CI}, 1.1$ to 11.5 ); however, only 31 of 70 women completed the 8 -week followup (Table B-5).

\section{Depression: Sertraline}

\section{Overview}

- The evidence for sertraline for postpartum depression was mixed and varied by outcome.

- For response, two studies comparing sertraline with placebo only showed that sertraline improves response rate (low strength of evidence); the evidence for sertraline plus psychotherapy versus psychotherapy alone is insufficient.

- For remission, similarly, two studies comparing sertraline with placebo only showed that sertraline improves remission rates relative to placebo (low strength of evidence); the evidence for sertraline plus psychotherapy versus psychotherapy alone is insufficient.

- For reduction in depression severity, when onset of depression occurred after the first month postpartum, the evidence was insufficient for sertraline with placebo only and for sertraline plus psychotherapy versus psychotherapy alone.

- For reduction in depression severity, when onset of depression occurred, between 0 and 1 month postpartum, the evidence from one study suggested greater improvement with sertraline compared with placebo.

- For reduction in anxiety among women with depression, one low risk-of-bias study reported that sertraline produces a greater reduction in severity than placebo (low strength of evidence).

- Evidence addressing differences in postpartum adjustment was insufficient. 
Table B-5. Strength of evidence for effectiveness: Paroxetine versus no treatment

\begin{tabular}{|c|c|c|c|c|c|c|c|}
\hline Population & $\begin{array}{l}\text { Intervention } \\
\text { and } \\
\text { Comparator }\end{array}$ & Outcome & $\begin{array}{l}\text { Incidence } \\
\text { or Mean } \\
\text { Effect by } \\
\text { Arm }\end{array}$ & Results & $\begin{array}{l}\text { Study Design } \\
\text { and Sample } \\
\text { Size }\end{array}$ & $\begin{array}{l}\text { Factors That } \\
\text { Affect the } \\
\text { Strength of } \\
\text { Evidence }\end{array}$ & $\begin{array}{l}\text { Overall } \\
\text { Evidence } \\
\text { Strength } \\
\text { (Direction } \\
\text { of Effect) }\end{array}$ \\
\hline $\begin{array}{l}\text { Women with } \\
\text { depression } \\
\text { onset } 0-3 \\
\text { months } \\
\text { postpartum }\end{array}$ & $\begin{array}{l}\text { Paroxetine } \\
\text { vs. placebo }\end{array}$ & $\begin{array}{l}\text { Response: } \\
\text { CGI-I score } \\
=1 \text { or } 2\end{array}$ & $\begin{array}{l}\text { Paroxetine: } \\
\text { 15/35 } \\
\text { Placebo: } \\
\text { 11/35 }\end{array}$ & $\begin{array}{l}\text { AOR, 1.04 } \\
(0.33-3.26) \\
p=0.94\end{array}$ & $1 \mathrm{RCT}, \mathrm{N}=70^{25}$ & $\begin{array}{l}\text { High study } \\
\text { limitations (high } \\
\text { risk of bias }{ }^{25} \text { ), } \\
\text { serious } \\
\text { imprecision } \\
\text { (wide Cls, small } \\
\text { sample size), } \\
\text { consistency } \\
\text { unknown }\end{array}$ & Insufficient \\
\hline $\begin{array}{l}\text { Women with } \\
\text { depression } \\
\text { onset 0-3 } \\
\text { months } \\
\text { postpartum }\end{array}$ & $\begin{array}{l}\text { Paroxetine } \\
\text { vs. placebo }\end{array}$ & $\begin{array}{l}\text { Remission: } \\
\text { HAM-D-17 } \\
\text { total score } \\
\leq 8\end{array}$ & $\begin{array}{l}\text { Paroxetine: } \\
\text { 13/35 } \\
\text { Placebo: } \\
5 / 35\end{array}$ & $\begin{array}{l}\text { AOR, } 3.5(1.1- \\
11.5), p=0.04\end{array}$ & $1 \mathrm{RCT}, \mathrm{N}=70^{25}$ & $\begin{array}{l}\text { High study } \\
\text { limitations (high } \\
\text { risk of bias }{ }^{25} \text { ), } \\
\text { imprecision } \\
\text { (small sample } \\
\text { size), } \\
\text { consistency } \\
\text { unknown }\end{array}$ & Insufficient \\
\hline $\begin{array}{l}\text { Women with } \\
\text { depression } \\
\text { onset 0-3 } \\
\text { months } \\
\text { postpartum }\end{array}$ & $\begin{array}{l}\text { Paroxetine } \\
\text { vs. placebo }\end{array}$ & $\begin{array}{l}\text { Symptoms: } \\
\text { IDS-SR } \\
\text { symptom } \\
\text { score }\end{array}$ & $\begin{array}{l}\text { Paroxetine: } \\
\text { baseline } \\
38.6 \text {, final } \\
14.0 \\
\text { Placebo: } \\
\text { baseline } \\
42.8 \text {, final } \\
22.6\end{array}$ & $\begin{array}{l}\text { Group effect: } \\
-4.98 \\
(p=0.019) \text {, but } \\
\text { group } x \text { time } \\
\text { NS }\end{array}$ & $1 \mathrm{RCT}, \mathrm{N}=70^{25}$ & $\begin{array}{l}\text { High study } \\
\text { limitations (high } \\
\text { risk of bias }{ }^{25} \text { ), } \\
\text { imprecision } \\
\text { (small sample } \\
\text { size), } \\
\text { consistency } \\
\text { unknown }\end{array}$ & Insufficient \\
\hline $\begin{array}{l}\text { Women with } \\
\text { depression } \\
\text { onset } 0-3 \\
\text { months } \\
\text { postpartum }\end{array}$ & $\begin{array}{l}\text { Paroxetine } \\
\text { vs. placebo }\end{array}$ & $\begin{array}{l}\text { Symptoms: } \\
\text { HAM-D-17 } \\
\text { score }\end{array}$ & $\begin{array}{l}\text { Paroxetine: } \\
\text { baseline } \\
23.6 \text {, final } \\
8.6 \\
\text { Placebo: } \\
\text { baseline } \\
24.7 \text {, final } \\
13.3\end{array}$ & $\begin{array}{l}\text { Group effect: } \\
-1.62(p=0.22)\end{array}$ & $1 \mathrm{RCT}, \mathrm{N}=70^{25}$ & $\begin{array}{l}\text { High study } \\
\text { limitations (high } \\
\text { risk of bias }{ }^{25} \text { ), } \\
\text { serious } \\
\text { imprecision } \\
\text { (wide Cls, small } \\
\text { sample size), } \\
\text { consistency } \\
\text { unknown }\end{array}$ & Insufficient \\
\hline $\begin{array}{l}\text { Women with } \\
\text { depression } \\
\text { onset 0-3 } \\
\text { months } \\
\text { postpartum }\end{array}$ & $\begin{array}{l}\text { Paroxetine } \\
\text { vs. placebo }\end{array}$ & $\begin{array}{l}\text { Symptoms: } \\
\text { CGI-S } \\
\text { symptom } \\
\text { score }\end{array}$ & $\begin{array}{l}\text { Paroxetine: } \\
\text { baseline } \\
4.2 \text {, final } 1.8 \\
\text { Placebo: } \\
\text { baseline } \\
4.5 \text {, final } 3.1\end{array}$ & $\begin{array}{l}\text { Group effect: } \\
-0.48 \\
(p=0.047)\end{array}$ & $1 \mathrm{RCT}, \mathrm{N}=70^{25}$ & $\begin{array}{l}\text { High study } \\
\text { limitations (high } \\
\text { risk of bias }{ }^{25} \text { ), } \\
\text { imprecision } \\
\text { (small sample } \\
\text { size), } \\
\text { consistency } \\
\text { unknown }\end{array}$ & Insufficient \\
\hline
\end{tabular}

AOR = adjusted odds ratio; CGI-I = Clinical Global Impression-Improvement; CGI-S = Clinical Global Impression-Severity of Illness; CI = confidence interval; HAM-D-17= Hamilton Depression Rating scale, 17-item version; IDS-SR = Inventory of Depression Symptoms - SR; N = number; NS = not significant; RCT = randomized controlled trial; vs. = versus.

\section{Detailed Results}

Four publications compared the benefits of sertraline with no sertraline treatment; these study designs compared sertraline with placebo (Table B-6) ${ }^{16,22}$ or compared sertraline plus a psychotherapy (cognitive behavioral therapy ${ }^{21}$ or brief dynamic psychotherapy ${ }^{23}$ ) with that respective psychotherapy alone. All four studies were RCTs. ${ }^{16,21-23}$ Two studies were high risk of bias ${ }^{16,21}$ and two had low risk of bias. ${ }^{22,23}$ 
The four trials addressed postpartum depression (onset varied between 2 months and 12 months following delivery). ${ }^{16,21-23}$ One study looked at two intervals: onset within 3 months of delivery (the primary outcome, which is consistent with how DSM-5 defines the postpartum specifier), and onset within the more strict DSM-IV definition of 1 month postpartum. ${ }^{22}$ Three studies allowed doses ranging from $50 \mathrm{mg}$ to $200 \mathrm{mg},{ }^{16,21,22}$ while the other allowed doses ranging from $25 \mathrm{mg}$ to $100 \mathrm{mg} .^{23}$

Table B-6. Strength of evidence for effectiveness: Sertraline versus no treatment

\begin{tabular}{|c|c|c|c|c|c|c|c|}
\hline Population & $\begin{array}{l}\text { Intervention } \\
\text { and } \\
\text { Comparator }\end{array}$ & Outcome & $\begin{array}{l}\text { Incidence or } \\
\text { Mean Effect } \\
\text { by Arm }\end{array}$ & Results & $\begin{array}{l}\text { Study } \\
\text { Design and } \\
\text { Sample Size }\end{array}$ & $\begin{array}{l}\text { Factors That } \\
\text { Affect the } \\
\text { Strength of } \\
\text { Evidence }\end{array}$ & $\begin{array}{l}\text { Overall } \\
\text { Evidence } \\
\text { Strength } \\
\text { (Direction of } \\
\text { Effect) }\end{array}$ \\
\hline $\begin{array}{l}\text { Women with } \\
\text { depression } \\
\text { onset 0-3 } \\
\text { months } \\
\text { postpartum } 22 \\
\text { or women } \\
\text { with } \\
\text { depression } \\
\text { onset 0-12 } \\
\text { months } \\
\text { postpartum }\end{array}$ & $\begin{array}{l}\text { Sertraline vs. } \\
\text { placebo }\end{array}$ & $\begin{array}{l}\text { Response at } \\
6 \text { weeks in } \\
\text { ITT group } \\
\text { ( } \geq 50 \% \\
\text { reduction in } \\
\text { HAM-D-19 } \\
\text { symptoms } \\
\text { and CGI-I } \\
\text { score of } \\
\text { "much } \\
\text { improved" or } \\
\text { "very much } \\
\text { improved") } 22 \\
\text { or response } \\
\text { at } 12 \text { weeks } \\
\text { ( } \geq 50 \% \\
\text { reduction in } \\
\text { HAM-D-17 } \\
\text { symptoms) }\end{array}$ & $\begin{array}{l}10 / 17(59 \%) \\
\text { vs. } 5 / 19 \\
(26 \%),{ }^{22} \mathrm{NR}^{16}\end{array}$ & $\begin{array}{l}\text { AOR, = NR, } \\
p=0.05 \\
\text { calculated } \\
\text { RR: } 2.24 \\
(95 \% \mathrm{Cl}, 0.95 \\
\text { to } 5.24)^{: 22} \text { no } \\
\text { difference } \\
\text { between two } \\
\text { arms, } \\
p=0.054^{16}\end{array}$ & $\begin{array}{l}2 \text { RCTs, } \\
\mathrm{N}=145^{16,22}\end{array}$ & $\begin{array}{l}\text { Moderate } \\
\text { study } \\
\text { limitations } \\
\text { (one high } \\
\text { risk-of-bias } \\
\text { study) } \\
\text { imprecise } \\
\text { (few events, } \\
\text { small N, wide } \\
\text { Cls), } \\
\text { inconsistent; } \\
\text { large effect } \\
\text { for low risk of } \\
\text { bias study }\end{array}$ & $\begin{array}{l}\text { Low that } \\
\text { response at } 6 \\
\text { weeks is } \\
\text { greater with } \\
\text { sertraline }\end{array}$ \\
\hline $\begin{array}{l}\text { Women with } \\
\text { depression } \\
\text { onset } 0-2 \\
\text { months } \\
\text { postpartum }\end{array}$ & $\begin{array}{l}\text { Sertraline } \\
\text { plus brief } \\
\text { dynamic } \\
\text { psychotherap } \\
\text { y vs. placebo }\end{array}$ & $\begin{array}{l}\text { Response at } \\
8 \text { weeks } \\
(>50 \% \\
\text { reduction in } \\
\text { MADRS or } \\
\text { EPDS score) }\end{array}$ & $\begin{array}{l}14 / 20(70 \%) \\
\text { vs. } 11 / 20 \\
(55 \%)^{23}\end{array}$ & $\begin{array}{l}\text { RR = NR, } \\
\text { between } \\
\text { groups } p- \\
\text { value }=0.33^{23}\end{array}$ & $\begin{array}{l}1 \mathrm{RCT}, \\
\mathrm{N}=40^{23}\end{array}$ & $\begin{array}{l}\text { Low risk of } \\
\text { bias }^{23} \text { ), } \\
\text { seriously } \\
\text { imprecise } \\
\text { (few events, } \\
\text { likely Cls } \\
\text { span the null), } \\
\text { consistency } \\
\text { unknown }\end{array}$ & Insufficient \\
\hline $\begin{array}{l}\text { Women with } \\
\text { depression } \\
\text { onset 0-3 } \\
\text { months } \\
\text { postpartum } 22 \\
\text { or women } \\
\text { with } \\
\text { depression } \\
\text { onset 0-12 } \\
\text { months } \\
\text { postpartum }\end{array}$ & $\begin{array}{l}\text { Sertraline vs. } \\
\text { placebo }\end{array}$ & $\begin{array}{l}\text { Remission at } \\
6 \text { weeks in } \\
\text { ITT group } \\
\text { (meeting } \\
\text { response } \\
\text { above and } \\
\text { with HAM-D- } \\
19 \text { score } \leq 7 \text { ) } 22 \\
\text { or remission } \\
\text { at } 12 \text { weeks } \\
(\text { HAMD-17 } \\
\leq 7)^{16}\end{array}$ & $\begin{array}{l}\text { 9/17 (53\%) } \\
\text { vs. } 4 / 19 \\
(23 \%) ; 22 \\
N^{16}\end{array}$ & $\begin{array}{l}\text { AOR = NR, } \\
p=0.05 \\
\text { calculated } \\
\text { RR: } 2.51 \\
(95 \% \mathrm{Cl}, 0.94 \\
\text { to } 6.70)^{: 22} \\
\text { no difference } \\
\text { between two } \\
\text { arms, } \\
p=0.372^{16}\end{array}$ & $\begin{array}{l}2 \text { RCTs, } \\
\mathrm{N}=145^{16,22}\end{array}$ & $\begin{array}{l}\text { Moderate } \\
\text { study } \\
\text { limitations } \\
\text { (one high } \\
\text { risk-of-bias } \\
\text { study }^{16} \\
\text { imprecise } \\
\text { (few events), } \\
\text { inconsistent; } \\
\text { large effect } \\
\text { for low risk of } \\
\text { bias study }\end{array}$ & $\begin{array}{l}\text { Low that } \\
\text { remission at } 6 \\
\text { weeks is } \\
\text { greater with } \\
\text { sertraline }\end{array}$ \\
\hline
\end{tabular}




\begin{tabular}{|c|c|c|c|c|c|c|c|}
\hline Population & $\begin{array}{l}\text { Intervention } \\
\text { and } \\
\text { Comparator }\end{array}$ & Outcome & $\begin{array}{l}\text { Incidence or } \\
\text { Mean Effect } \\
\text { by Arm }\end{array}$ & Results & $\begin{array}{l}\text { Study } \\
\text { Design and } \\
\text { Sample Size }\end{array}$ & $\begin{array}{l}\text { Factors That } \\
\text { Affect the } \\
\text { Strength of } \\
\text { Evidence }\end{array}$ & $\begin{array}{l}\text { Overall } \\
\text { Evidence } \\
\text { Strength } \\
\text { (Direction of } \\
\text { Effect) }\end{array}$ \\
\hline $\begin{array}{l}\text { Women with } \\
\text { depression } \\
\text { onset 0-2 } \\
\text { months } \\
\text { postpartum }\end{array}$ & $\begin{array}{l}\text { Sertraline plus } \\
\text { brief dynamic } \\
\text { psychotherapy } \\
\text { vs. placebo }\end{array}$ & $\begin{array}{l}\text { Remission at } \\
8 \text { weeks (final } \\
\text { score of }<10 \\
\text { on MADRS or } \\
<7 \text { on EPDS) }\end{array}$ & $\begin{array}{l}13 / 20(65 \%) \\
\text { vs. } 10 / 20 \\
(50 \%)^{23}\end{array}$ & $\begin{array}{l}\text { RR = NR, } \\
\text { between } \\
\text { groups } p- \\
\text { value } \\
p=0.34^{23}\end{array}$ & $\begin{array}{l}1 \mathrm{RCT}, \\
\mathrm{N}=40^{23}\end{array}$ & $\begin{array}{l}\text { Low risk of } \\
\text { bias, }{ }^{23} \text { likely } \\
\text { imprecise } \\
\text { (few events, } \\
\text { Cls likely } \\
\text { span the null), } \\
\text { consistency } \\
\text { unknown }\end{array}$ & \\
\hline \multirow[t]{6}{*}{$\begin{array}{l}\text { Women with } \\
\text { depression } \\
\text { onset } 0-3 \\
\text { months } \\
\text { postpartum }{ }^{22} \\
\text { or women } \\
\text { with } \\
\text { depression } \\
\text { onset } 0-12 \\
\text { months } \\
\text { postpartum }{ }^{16} \\
\end{array}$} & $\begin{array}{l}\text { Sertraline vs. } \\
\text { placebo }\end{array}$ & $\begin{array}{l}\text { Decrease in } \\
\text { HAM-D-19 } \\
\text { scores at } 6 \\
\text { weeks }\end{array}$ & $\begin{array}{l}\text { Sertraline: } \\
\text { baseline } \\
\text { HAM-D-19 } \\
\text { 20.6, final NR } \\
\text { Placebo: } \\
\text { baseline } \\
\text { HAM-D 23.2, } \\
\text { final NR }\end{array}$ & $\begin{array}{l}\text { Regression } \\
\text { coefficient } 0.6 \\
\text { favoring } \\
\text { sertraline } \\
\text { group, } \\
p=0.15^{22}\end{array}$ & $\begin{array}{l}1 \mathrm{RCT}, \\
\mathrm{N}=36^{22}\end{array}$ & $\begin{array}{l}\text { High study } \\
\text { limitations } \\
\text { (one high } \\
\text { risk-of-bias } \\
\text { stud)y }^{16} \\
\text { imprecise } \\
\text { (few events), } \\
\text { consistency } \\
\text { unknown }\end{array}$ & $\begin{array}{l}\text { Insufficient } \\
\text { across all } \\
\text { measures of } \\
\text { change in } \\
\text { symptom } \\
\text { severity }\end{array}$ \\
\hline & & $\begin{array}{l}\text { Decrease in } \\
\text { EPDS scores } \\
\text { at } 6 \text { weeks }\end{array}$ & $\begin{array}{l}\text { Sertraline: } \\
\text { baseline } \\
\text { EPDS 18.8, } \\
\text { final NR } \\
\text { Placebo: } \\
\text { baseline } \\
\text { EPDS 20.8, } \\
\text { final NR }\end{array}$ & $\begin{array}{l}\text { Regression } \\
\text { coefficient } \\
0.25 \text { favoring } \\
\text { sertraline } \\
\text { group, } \\
p=0.51^{22}\end{array}$ & $\begin{array}{c}1 \mathrm{RCT}, \\
\mathrm{N}=36^{22}\end{array}$ & & \\
\hline & & $\begin{array}{l}\text { Decrease in } \\
\text { depressive } \\
\text { severity at } 12 \\
\text { weeks (HAM- } \\
\text { D-17) }\end{array}$ & $\mathrm{NR}^{16}$ & $\begin{array}{l}\text { No difference } \\
\text { between two } \\
\text { arms, } p=\mathrm{NR}^{16}\end{array}$ & $\begin{array}{l}1 \mathrm{RCT} \\
\mathrm{N}=109^{16}\end{array}$ & & \\
\hline & & $\begin{array}{l}\text { Decrease in } \\
\text { depressive } \\
\text { severity at } 12 \\
\text { weeks (BDI) }\end{array}$ & $\mathrm{NR}^{16}$ & $\begin{array}{l}\text { No difference } \\
\text { between two } \\
\text { arms, } p=N^{16}\end{array}$ & $\begin{array}{l}1 \mathrm{RCT} \\
\mathrm{N}=109^{16}\end{array}$ & & \\
\hline & & $\begin{array}{l}\text { Decrease in } \\
\text { depressive } \\
\text { severity at } 12 \\
\text { weeks (IDAS- } \\
\text { GD) }\end{array}$ & $\mathrm{NR}^{16}$ & $\begin{array}{l}\text { No difference } \\
\text { between two } \\
\text { arms, } p=N^{16}\end{array}$ & $\begin{array}{l}1 \mathrm{RCT} \\
\mathrm{N}=109^{16}\end{array}$ & & \\
\hline & & $\begin{array}{l}\text { CGI-S at } 12 \\
\text { weeks }\end{array}$ & $\mathrm{NR}^{16}$ & $\begin{array}{l}\text { No difference } \\
\text { between two } \\
\text { arms, } p=\mathrm{NR}^{16}\end{array}$ & $\begin{array}{l}1 \mathrm{RCT}, \\
\mathrm{N}=109^{16}\end{array}$ & & \\
\hline $\begin{array}{l}\text { Women with } \\
\text { depression } \\
\text { onset } 0-12 \\
\text { months } \\
\text { postpartum }\end{array}$ & $\begin{array}{l}\text { Sertraline vs. } \\
\text { placebo }\end{array}$ & $\begin{array}{l}\text { CGI-I at } 12 \\
\text { weeks }\end{array}$ & $\mathrm{NR}^{16}$ & $\begin{array}{l}\text { No difference } \\
\text { between two } \\
\text { arms, } p=\mathrm{NR}^{16}\end{array}$ & $\begin{array}{l}1 \mathrm{RCT} \\
\mathrm{N}=\text { 109 }^{16}\end{array}$ & $\begin{array}{l}\text { High study } \\
\text { limitations } \\
\text { (high risk of } \\
\text { bias }^{16} \text { ), likely } \\
\text { imprecise, } \\
\text { consistency } \\
\text { unknown }\end{array}$ & Insufficient \\
\hline
\end{tabular}




\begin{tabular}{|c|c|c|c|c|c|c|c|}
\hline Population & $\begin{array}{l}\text { Intervention } \\
\text { and } \\
\text { Comparator }\end{array}$ & Outcome & $\begin{array}{l}\text { Incidence or } \\
\text { Mean Effect } \\
\text { by Arm }\end{array}$ & Results & $\begin{array}{l}\text { Study } \\
\text { Design and } \\
\text { Sample Size }\end{array}$ & $\begin{array}{l}\text { Factors That } \\
\text { Affect the } \\
\text { Strength of } \\
\text { Evidence }\end{array}$ & $\begin{array}{l}\text { Overall } \\
\text { Evidence } \\
\text { Strength } \\
\text { (Direction of } \\
\text { Effect) }\end{array}$ \\
\hline
\end{tabular}

\begin{tabular}{|c|c|c|c|c|}
\hline $\begin{array}{l}\text { Women with } \\
\text { depression } \\
\text { onset } \\
\text { between } 2 \\
\text { and } 8 \text { months } \\
\text { postpartum }\end{array}$ & $\begin{array}{l}\text { Sertraline } \\
\text { plus CBT vs. } \\
\text { CBT }\end{array}$ & $\begin{array}{l}\text { Decrease in } \\
\text { BDI-Il at } 24 \\
\text { weeks }\end{array}$ & $\begin{array}{l}\text { Sertraline } \\
\text { plus CBT: - } \\
14.54 \\
\text { CBT: }-16.2^{21}\end{array}$ & $\begin{array}{l}\text { Between- } \\
\text { group mean } \\
\text { difference: } \\
1.6 \text { favoring } \\
\text { CBT group, } \\
\mathrm{p}=\mathrm{NS}^{21}\end{array}$ \\
\hline
\end{tabular}

High study Insufficient

\section{limitations}

(high risk of

bias $^{21}$ )

seriously

imprecise

(small sample

size, Cls

likely span

the null), consistency

unknown

\begin{tabular}{|c|c|c|c|c|c|c|c|}
\hline $\begin{array}{l}\text { Women with } \\
\text { depression } \\
\text { onset } 0-1 \\
\text { month } \\
\text { postpartum }\end{array}$ & $\begin{array}{l}\text { Sertraline vs. } \\
\text { placebo }\end{array}$ & $\begin{array}{l}\text { Decrease in } \\
\text { HAM-D-19 } \\
\text { scores at } 6 \\
\text { weeks }\end{array}$ & $\begin{array}{l}\text { Sertraline: } \\
\text { baseline } \\
\text { HAM-D-19 } \\
\text { NR, final NR } \\
\text { Placebo: } \\
\text { baseline } \\
\text { HAM-D NR, } \\
\text { final NR }\end{array}$ & $\begin{array}{l}\text { Regression } \\
\text { coefficient } \\
1.18 \text { favoring } \\
\text { sertraline } \\
\text { group, } \\
p=0.01^{22}\end{array}$ & $\begin{array}{l}1 \mathrm{RCT}, \\
\mathrm{N}=27^{22}\end{array}$ & $\begin{array}{l}\text { Low study } \\
\text { limitations, } \\
\text { imprecise } \\
\text { (small sample } \\
\text { size), } \\
\text { consistency } \\
\text { unknown }\end{array}$ & $\begin{array}{l}\text { Low that } \\
\text { reduction in } \\
\text { depressive } \\
\text { severity is } \\
\text { greater with } \\
\text { sertraline }\end{array}$ \\
\hline $\begin{array}{l}\text { Women with } \\
\text { depression } \\
\text { onset } 0-1 \\
\text { month } \\
\text { postpartum }\end{array}$ & $\begin{array}{l}\text { Sertraline vs. } \\
\text { placebo }\end{array}$ & $\begin{array}{l}\text { Decrease in } \\
\text { EPDS scores } \\
\text { at } 6 \text { weeks }\end{array}$ & $\begin{array}{l}\text { Sertraline: } \\
\text { baseline } \\
\text { EPDS NR, } \\
\text { final NR } \\
\text { Placebo: } \\
\text { baseline } \\
\text { EPDS NR, } \\
\text { final NR }\end{array}$ & $\begin{array}{l}\text { Regression } \\
\text { coefficient } \\
0.91 \text { favoring } \\
\text { sertraline } \\
\text { group, } \\
p=0.04^{22}\end{array}$ & $\begin{array}{l}\begin{array}{l}1 \mathrm{RCT}, \\
\mathrm{N}=27^{22}\end{array}\end{array}$ & $\begin{array}{l}\text { Low study } \\
\text { limitations, } \\
\text { imprecise } \\
\text { (small sample } \\
\text { size), } \\
\text { consistency } \\
\text { unknown }\end{array}$ & $\begin{array}{l}\text { Low that } \\
\text { reduction in } \\
\text { depressive } \\
\text { severity is } \\
\text { greater with } \\
\text { sertraline }\end{array}$ \\
\hline $\begin{array}{l}\text { Women with } \\
\text { depression } \\
\text { onset 0-3 } \\
\text { months } \\
\text { postpartum }\end{array}$ & $\begin{array}{l}\text { Sertraline vs. } \\
\text { placebo }\end{array}$ & $\begin{array}{l}\text { Decrease in } \\
\text { HAM-A at } 6 \\
\text { weeks }\end{array}$ & $\begin{array}{l}\text { Sertraline: } \\
\text { baseline } \\
\text { HAM-A 21.3, } \\
\text { final NR } \\
\text { Placebo: } \\
\text { baseline } \\
\text { HAM-A 24.5, } \\
\text { final NR }\end{array}$ & $\begin{array}{l}\text { Regression } \\
\text { coefficient } \\
0.92 \text { favoring } \\
\text { sertraline } \\
\text { group, } \\
p=0.08^{22}\end{array}$ & $\begin{array}{l}1 \mathrm{RCT}, \\
\mathrm{N}=36^{22}\end{array}$ & $\begin{array}{l}\text { Low study } \\
\text { limitations, } \\
\text { imprecise } \\
\text { (small sample } \\
\text { size), } \\
\text { consistency } \\
\text { unknown }\end{array}$ & Insufficient \\
\hline $\begin{array}{l}\text { Women with } \\
\text { depression } \\
\text { onset } 0-1 \\
\text { month } \\
\text { postpartum }\end{array}$ & $\begin{array}{l}\text { Sertraline vs. } \\
\text { placebo }\end{array}$ & $\begin{array}{l}\text { Decrease in } \\
\text { HAM-A at } 6 \\
\text { weeks }\end{array}$ & $\begin{array}{l}\text { Sertraline: } \\
\text { baseline } \\
\text { HAM-A NR, } \\
\text { final NR } \\
\text { Placebo: } \\
\text { baseline } \\
\text { HAM-A NR, } \\
\text { final NR }\end{array}$ & $\begin{array}{l}\text { Regression } \\
\text { coefficient } \\
1.19 \text { favoring } \\
\text { sertraline } \\
\text { group, } \\
p=0.03^{22}\end{array}$ & $\begin{array}{l}1 \mathrm{RCT}, \\
\mathrm{N}=27^{22}\end{array}$ & $\begin{array}{l}\text { Low study } \\
\text { limitations, } \\
\text { imprecise } \\
\text { (few } \\
\text { events),con- } \\
\text { sistency } \\
\text { unknown }\end{array}$ & $\begin{array}{l}\text { Low that } \\
\text { reduction in } \\
\text { anxiety } \\
\text { severity is } \\
\text { greater with } \\
\text { sertraline }\end{array}$ \\
\hline $\begin{array}{l}\text { Women with } \\
\text { depression } \\
\text { onset } 0-12 \\
\text { months } \\
\text { postpartum }\end{array}$ & $\begin{array}{l}\text { Sertraline vs. } \\
\text { placebo }\end{array}$ & $\begin{array}{l}\text { PPAQ at } 12 \\
\text { weeks }\end{array}$ & $\mathrm{NR}^{16}$ & $\begin{array}{l}\text { No difference } \\
\text { between two } \\
\text { arms, } p=N^{16}\end{array}$ & $\begin{array}{l}1 \mathrm{RCT} \\
\mathrm{N}=109^{16}\end{array}$ & $\begin{array}{l}\text { High study } \\
\text { limitations } \\
\text { (high risk of } \\
\text { bias }^{16} \text { ), likely } \\
\text { imprecise, } \\
\text { consistency } \\
\text { unknown }\end{array}$ & Insufficient \\
\hline
\end{tabular}

AOR = adjusted odds ratio; BDI = Beck Depression Inventory; BDI-II = Beck Depression Inventory-II; CBT = cognitive

behavioral therapy; CGI-S = Clinical Global Impression-Severity of Illness; CGI-I = Clinical Global Impression- Improvement; $\mathrm{CI}=$ confidence interval; EPDS = Edinburgh Postnatal Depression Scale; HAM-A = Hamilton Anxiety Rating Scale; HAM-D-

17= Hamilton Depression Rating scale, 17-item version; HAM-D-19= Hamilton Depression Rating scale, 19-item version; IDA- 
GD = Inventory of Depression and Anxiety Symptoms, General Depression scale; ITT = intention to treat; NR = not reported; NS = not significant; PPAQ = Postpartum Adjustment Questionnaire; RCT = randomized controlled trial; vs. = versus.

The evidence for sertraline was mixed and varied by outcome. For response, two studies provided evidence of response for sertraline versus placebo; ${ }^{16,22}$ a third study provided evidence from a comparison of sertraline plus psychotherapy versus psychotherapy alone. Because of the clinical heterogeneity in comparators, we did not pool results. The evidence from the two studies of sertraline versus placebo only offers low strength of evidence of benefit: one low risk-of-bias study with a small sample and a mean dose of $100 \mathrm{mg}$ reported a statistically significant benefit for depression with onset within 1 or 3 months postpartum, respectively. ${ }^{22}$ A more restrictive definition of postpartum depression with onset up to 1 month postpartum from the same study also reported statistically significant differences favoring sertraline. ${ }^{22}$ Calculated relative risks suggest imprecise results with wide CIs, few events, and small numbers of participants, but a large effect size. A second study, a high risk-of-bias study with a median dose of $150 \mathrm{mg}$, found no difference in response rates. ${ }^{16}$

A low risk-of-bias study comparing sertraline (mean dose between 65 and $70 \mathrm{mg}$ ) plus brief dynamic psychotherapy with brief dynamic psychotherapy alone showed a benefit in the same direction, which does not reach statistical significance. ${ }^{23}$ In this trial, the control group was an active treatment, brief dynamic psychotherapy, which would raise the response rate in the control group and make significance harder to demonstrate. ${ }^{23}$

For remission, which involves the same three studies, there is evidence that sertraline improves remission rates in one low risk-of-bias study whether onset is defined as 0 to 3 months postpartum or 0 to 1 month postpartum. ${ }^{22}$ A high risk-of-bias study did not report any statistically significant improvement. ${ }^{16}$ As with the results on remission, we graded the strength of evidence as low for benefit for sertraline compared with placebo. A low risk-of-bias study comparing sertraline plus brief dynamic psychotherapy with brief dynamic psychotherapy alone did not report any statistically significant differences. ${ }^{23}$

For reduction in depressive severity, two studies provided evidence on change in symptom response for sertraline versus placebo; ${ }^{16,22}$ a third study provided evidence from a comparison of sertraline plus CBT versus CBT alone. As with the results on response and remission, because of the clinical heterogeneity in comparators, we did not pool results.

Two trials with onset of depression between 0 to 3 and 0 to 12 months compared sertraline with placebo only. These trials (1 low risk of bias and 1 high risk of bias) did not demonstrate differences in any of a range of outcomes measuring differences in symptoms (HAM-D 19, EPDS, HAM-D 17, BDI, IDA-GD, CGI-S). ${ }^{16,22}$

One low risk-of-bias study provided low strength of evidence of benefit that sertraline produces a greater reduction in depressive severity when onset is between birth and 1 month when compared with placebo. ${ }^{22}$

One high risk-of-bias study provided insufficient data to judge the strength of evidence for this outcome when comparing sertraline plus CBT with placebo plus CBT. ${ }^{21}$ As with the trial on psychodynamic therapy, differences are likely to be harder to demonstrate.

For reduction in anxiety, one low risk-of-bias study reported that sertraline produces a greater reduction in anxiety severity than placebo (low strength of evidence). ${ }^{22}$ For those with onset of postpartum depression within 3 months of delivery, the reduction favors sertraline but not to a statistically significant degree; for those with onset within 1 month of delivery, the statistically significant reduction favors sertraline despite the small sample size. Evidence addressing differences in postpartum adjustment was insufficient. ${ }^{22}$ 


\section{Depression: Brexanolone}

\section{Overview}

- Three small randomized controlled trials demonstrated benefit for brexanolone to reduce depressive symptoms compared with placebo at 60 hours and 30 days after infusion for depression onset in the third trimester of pregnancy or within 4 weeks of birth. These trials provided moderate evidence of benefit that brexanolone improves symptoms.

- The evidence is insufficient to judge the benefits of brexanolone for functional outcomes.

\section{Detailed Results}

Three RCTs (rated low risk of bias) reported on the efficacy for brexanolone versus placebo for depression symptoms with onset in the third trimester of pregnancy or within 4 weeks of birth. ${ }^{17,19}$ The prespecified primary outcome of the trials was change from baseline to 60 hours in the 17-Item Hamilton Rating Scale for Depression (HAM-D) score. Brexanolone was administered as a 60-hour continuous infusion with a peak dose of either $60 \mu \mathrm{g} / \mathrm{kg}$ per hour or 90 $\mu \mathrm{g} / \mathrm{kg}$ per hour. The BRX60 schedule was administered at $30 \mu \mathrm{g} / \mathrm{kg}$ per hour ( $0-4$ hours $), 60$ $\mu \mathrm{g} / \mathrm{kg}$ per hour (4-56 hours), or $30 \mu \mathrm{g} / \mathrm{kg}$ per hour (56-60 hours). The BRX90 schedule was administered at $30 \mu \mathrm{g} / \mathrm{kg}$ per hour (0-4 hours), $60 \mu \mathrm{g} / \mathrm{kg}$ per hour (4-24 hours), $90 \mu \mathrm{g} / \mathrm{kg}$ per hour (24-52 hours), $60 \mu \mathrm{g} / \mathrm{kg}$ per hour (52-56 hours), or $30 \mu \mathrm{g} / \mathrm{kg}$ per hour (56-60 hours).

The first study ${ }^{19}$ was a phase 2 clinical trial enrolling a total of 21 women from four hospitals in the United States, randomized to placebo $(\mathrm{N}=11)$ versus BRX90 $(\mathrm{N}=10)$. The second and third trials were reported together in a single publication. ${ }^{17}$ Women were recruited at 30 centers in the United States. Two trials were conducted, including a three-arm trial of placebo, BRX60 or BRX90 ( $\mathrm{N}=138$ randomized, 122 treated), and a two-arm trial comparing BRX90 with placebo $(\mathrm{N}=108$ randomized, 104 treated). The second publication included an integrated analysis that pooled participants treated with $\mathrm{BRX} 90(\mathrm{~N}=102)$ versus placebo $(\mathrm{N}=107)$.

In the integrated analysis, BRX90 infusion reduced HAM-D scores more than placebo at 60 hours (least square [LS] mean difference, $\mathrm{SE}-4.1,0.9, \mathrm{p}<0.001$ ) and at 30 days (LS mean difference, $\mathrm{SE}-2.6,1.1, \mathrm{p}=0.02$ ). Whereas BRX reduced HAM-D scores, results were mixed for other depression measures. BRX90 reduced MADRS and EPDS scores at 30 days in the phase 2 clinical trial, but in the phase 3 trials, only BRX60 differed from placebo. At 30 days posttreatment, there was no difference in PHQ-9 or GAD-9 scores in any of the treatment groups. The phase 2 trial evaluated maternal function and found no difference at day 30 . The authors note that the trials were powered to observe differences in HAM-D scores; the trials were underpowered for other outcomes. Whether the specific focus of the instruments used (depression only vs. depression and anxiety) and mode of data collection (clinical interview vs. self-report) may have influenced the magnitude of outcomes is unclear.

We rated the strength of evidence as moderate across all depression symptom outcomes (Table B-7). We based this judgment on findings from three small trials with precise and consistent findings for HAM-D and imprecise but largely consistent for other measures of depression. We also note that the magnitude of benefit declined between the 60-hour measurement and the 30-day measurement of HAM-D. 
Table B-7. Strength of evidence for effectiveness outcomes for brexanolone versus placebo

\begin{tabular}{|c|c|c|c|c|c|c|c|}
\hline Population & $\begin{array}{l}\text { Intervention } \\
\text { and } \\
\text { Comparator }\end{array}$ & Outcome & $\begin{array}{l}\text { Incidence or } \\
\text { Mean Effect } \\
\text { by Arm }\end{array}$ & Results & $\begin{array}{l}\text { Study Design } \\
\text { and Sample } \\
\text { Size }\end{array}$ & $\begin{array}{l}\text { Factors That } \\
\text { Affect the } \\
\text { Strength of } \\
\text { Evidence }\end{array}$ & $\begin{array}{l}\text { Overall } \\
\text { Evidence } \\
\text { Strength } \\
\text { (Direction of } \\
\text { Effect) }\end{array}$ \\
\hline $\begin{array}{l}\text { Women with } \\
\text { postpartum } \\
\text { depression }\end{array}$ & $\begin{array}{l}\text { Brexanolone } \\
90 \text { vs. } \\
\text { placebo, } \\
\text { integrated } \\
\text { analysis }\end{array}$ & $\begin{array}{l}\text { Symptom } \\
\text { response: } \\
\text { Change in } \\
\text { HAM-D score } \\
\text { from baseline } \\
\text { to } 60 \text { hours }\end{array}$ & $\begin{array}{l}\text { Placebo: } \\
-12.8 \\
\text { BRX90: }-17.0\end{array}$ & $\begin{array}{l}\text { SD not reported } \\
\text { by RCT, results } \\
\text { cannot be pooled } \\
\text { LS mean } \\
\text { difference (SE) } \\
\text { BRX } 90 \text { vs. } \\
\text { placebo: }-4.1 \\
(0.9), p<.001\end{array}$ & $\begin{array}{l}\text { RCTs, } \\
\mathrm{N}=209, \\
\text { BRX90=102, } \\
\text { placebo- } 107^{17}\end{array}$ & $\begin{array}{l}\text { Low study } \\
\text { limitations, } \\
\text { precise } \\
\text { (statistically } \\
\text { significant } \\
\text { results } \\
\text { suggestive of } \\
\text { benefit), } \\
\text { consistent }\end{array}$ & $\begin{array}{l}\text { Moderate } \\
\text { across all } \\
\text { symptom } \\
\text { outcome } \\
\text { measures }\end{array}$ \\
\hline $\begin{array}{l}\text { Women with } \\
\text { postpartum } \\
\text { depression }\end{array}$ & $\begin{array}{l}\text { Brexanolone } \\
90 \text { vs. } \\
\text { placebo, } \\
\text { integrated } \\
\text { analysis }\end{array}$ & $\begin{array}{l}\text { Symptom } \\
\text { response: } \\
\text { Change in } \\
\text { HAM-D score } \\
\text { from baseline } \\
\text { to } 30 \text { days }\end{array}$ & $\begin{array}{l}\text { Placebo: } \\
-14.3 \\
\text { BRX90: }-16.9\end{array}$ & $\begin{array}{l}\text { SD not reported } \\
\text { by RCT, results } \\
\text { cannot be pooled } \\
\text { LS mean } \\
\text { difference (SE) } \\
\text { BRX } 90 \text { vs. } \\
\text { placebo: }-2.6 \\
(1.1), p=0.02\end{array}$ & $\begin{array}{l}\text { RCTs, } \\
\mathrm{N}=209, \\
\mathrm{BRX} 90=102, \\
\text { placebo- } 107^{17}\end{array}$ & $\begin{array}{l}\text { Low study } \\
\text { limitations, } \\
\text { precise } \\
\text { (statistically } \\
\text { significant } \\
\text { results } \\
\text { suggestive of } \\
\text { benefit), } \\
\text { consistent }\end{array}$ & \\
\hline $\begin{array}{l}\text { Women with } \\
\text { postpartum } \\
\text { depression }\end{array}$ & $\begin{array}{l}\text { Brexanolone } \\
60 \text { vs. } 90 \text { vs. } \\
\text { placebo }\end{array}$ & $\begin{array}{l}\text { Symptom } \\
\text { response: } \\
\text { change in } \\
\text { EPDS score } \\
\text { from baseline } \\
\text { to } 30 \text { days }\end{array}$ & $\begin{array}{l}\text { Study 1: } \\
\text { Placebo: }-5.3 \\
\text { BRX90: }-13.5 \\
\text { Study 2: } \\
\text { Placebo: }-9.2 \\
\text { BRX60: }-12.8 \\
\text { BRX90: }-11.9 \\
\text { Study 3: } \\
\text { Placebo: - } \\
\text { 11.2 } \\
\text { BRX90: }-10.8\end{array}$ & $\begin{array}{l}\text { SD not reported } \\
\text { by RCT, results } \\
\text { cannot be pooled } \\
\text { Study1: p using } \\
\text { mixed effects } \\
\text { model: } 0.024 \\
\text { LS mean } \\
\text { difference (SE) } \\
\text { Study } 2: \\
\text { BRX60 vs. } \\
\text { placebo, }-3.7 \\
(1.7), p=0.03 \\
\text { BRX90 vs. } \\
\text { placebo, }-1.8 \\
(1.6), p=0.55\end{array}$ & $\begin{array}{l}\text { RCT } \\
\text { Study 1: } \\
\text { N=21, } \\
\text { BRX90=10, } \\
\text { placebo-11 } \\
\text { Study 2, } \\
\mathrm{N}=122, \\
\text { placebo=43, } \\
\text { BRX60=38, } \\
\text { BRX90=41 } \\
\text { Study } 3, \\
\text { N=104, } \\
\text { placebo=53, } \\
\text { BRX90 }=51^{17}\end{array}$ & $\begin{array}{l}\text { Low study } \\
\text { limitations, } \\
\text { seriously } \\
\text { imprecise } \\
\text { (nonsignifican } \\
\text { t results, } \\
\text { underpowered } \\
\text { ), consistent }\end{array}$ & \\
\hline & & & & $\begin{array}{l}\text { Study } 3 \text { : BRX90 } \\
\text { vs. placebo, } 0.4 \\
\text { (1.2), } p=0.72\end{array}$ & & & \\
\hline
\end{tabular}




\begin{tabular}{|c|c|c|c|c|c|c|c|}
\hline Population & $\begin{array}{l}\text { Intervention } \\
\text { and } \\
\text { Comparator }\end{array}$ & Outcome & $\begin{array}{l}\text { Incidence } \\
\text { or Mean } \\
\text { Effect by } \\
\text { Arm }\end{array}$ & Results & $\begin{array}{l}\text { Study Design } \\
\text { and Sample } \\
\text { Size }\end{array}$ & $\begin{array}{l}\text { Factors That } \\
\text { Affect the } \\
\text { Strength of } \\
\text { Evidence }\end{array}$ & $\begin{array}{l}\text { Overall } \\
\text { Evidence } \\
\text { Strength } \\
\text { (Direction } \\
\text { of Effect) }\end{array}$ \\
\hline $\begin{array}{l}\text { Women with } \\
\text { postpartum } \\
\text { depression }\end{array}$ & $\begin{array}{l}\text { Brexanolone } \\
60 \text { vs. } 90 \text { vs. } \\
\text { placebo }\end{array}$ & $\begin{array}{l}\text { Symptom } \\
\text { response: } \\
\text { change in } \\
\text { MADRS } \\
\text { score from } \\
\text { baseline to } \\
30 \text { days }\end{array}$ & $\begin{array}{l}\text { Study 1: } \\
\text { Placebo: } \\
-11.3 \\
\text { BRX90: - } \\
26.2 \\
\text { Study 2: } \\
\text { Placebo: - } \\
\text { 19.4 } \\
\text { BRX60: - } \\
\text { 25.1 } \\
\text { BRX90: - } \\
23.0 \\
\\
\text { Study 3: } \\
\text { Placebo: - } \\
20.5 \\
\text { BRX90: - } \\
20.5\end{array}$ & $\begin{array}{l}\text { SD not } \\
\text { reported by } \\
\text { RCT, results } \\
\text { cannot be } \\
\text { pooled } \\
\text { Study 1: p } \\
\text { using mixed } \\
\text { effects } \\
\text { model:.01 } \\
\text { LS mean } \\
\text { difference (SE) } \\
\text { Study } 2: \\
\text { BRX60 vs. } \\
\text { placebo, }-5.6 \\
(2.8), p=.045 . \\
\text { BRX90 vs. } \\
\text { placebo, }-3.6 \\
(2.7), p=.19 \\
\text { Study } 3: \\
\text { BRX90 vs. } \\
\text { placebo, } 0 \\
(1.8), p=.98\end{array}$ & $\begin{array}{l}\text { RCT } \\
\text { Study } 1: \\
\text { N=21, } \\
\text { BRX90=10, } \\
\text { placebo-11 } \\
\text { Study 2, } \\
\text { N=122, } \\
\text { placebo=43, } \\
\text { BRX60=38, } \\
\text { BRX90=4117 } \\
\text { Study } 3, \\
\text { N=104, } \\
\text { placebo=53, } \\
\text { BRX90=51 }\end{array}$ & $\begin{array}{l}\text { Low study } \\
\text { limitations, } \\
\text { seriously imprecise } \\
\text { (nonsignificant } \\
\text { results, } \\
\text { underpowered), } \\
\text { consistent }\end{array}$ & \\
\hline $\begin{array}{l}\text { Women with } \\
\text { postpartum } \\
\text { depression }\end{array}$ & $\begin{array}{l}\text { Brexanolone } \\
60 \text { vs. } 90 \text { vs. } \\
\text { placebo }\end{array}$ & $\begin{array}{l}\text { Symptom } \\
\text { response: } \\
\text { change in } \\
\text { PHQ9 score } \\
\text { from } \\
\text { baseline to } \\
30 \text { days }\end{array}$ & $\begin{array}{l}\text { Study 1: } \\
\text { Placebo: - } \\
\text { 8.3 } \\
\text { BRX90: - } \\
\text { 11.0 } \\
\text { Study 2: } \\
\text { Placebo: - } \\
\text { 9.5 } \\
\text { BRX60: - } \\
\text { 12.0 } \\
\text { BRX90: - } \\
\text { 11.9 } \\
\text { Study 3: } \\
\text { Placebo: } \\
\text {-8.3 } \\
\text { BRX90: } \\
\text {-11.0 }\end{array}$ & $\begin{array}{l}\text { SD not } \\
\text { reported by } \\
\text { RCT, results } \\
\text { cannot be } \\
\text { pooled } \\
\text { Study 1: p } \\
\text { using mixed- } \\
\text { effects } \\
\text { model:.47 } \\
\text { LS mean } \\
\text { difference (SE) } \\
\text { Study } 2: \\
\text { BRX60 vs. } \\
\text { placebo, }-2.5 \\
(1.6), p=0.13 \\
\text { BRX90 vs. } \\
\text { placebo, }-2.4 \\
(1.6), p=0.13 \\
\text { Study } 3: \\
\text { BRX90 vs. } \\
\text { placebo, }-0.5 \\
(1.1), p=0.69\end{array}$ & $\begin{array}{l}\text { RCT } \\
\text { Study } 1: \\
\text { N=21, } \\
\text { BRX90=10, } \\
\text { placebo-11 } \\
\text { Study 2, } \\
\text { N=122, } \\
\text { placebo=43, } \\
\text { BRX60=38, } \\
\text { BRX90=4117 } \\
\text { Study 3, } \\
\text { N=104, } \\
\text { placebo=53, } \\
\text { BRX90=5117 }\end{array}$ & $\begin{array}{l}\text { Low study } \\
\text { limitations, } \\
\text { seriously imprecise } \\
\text { (nonsignificant } \\
\text { results, } \\
\text { underpowered), } \\
\text { consistent }\end{array}$ & \\
\hline
\end{tabular}




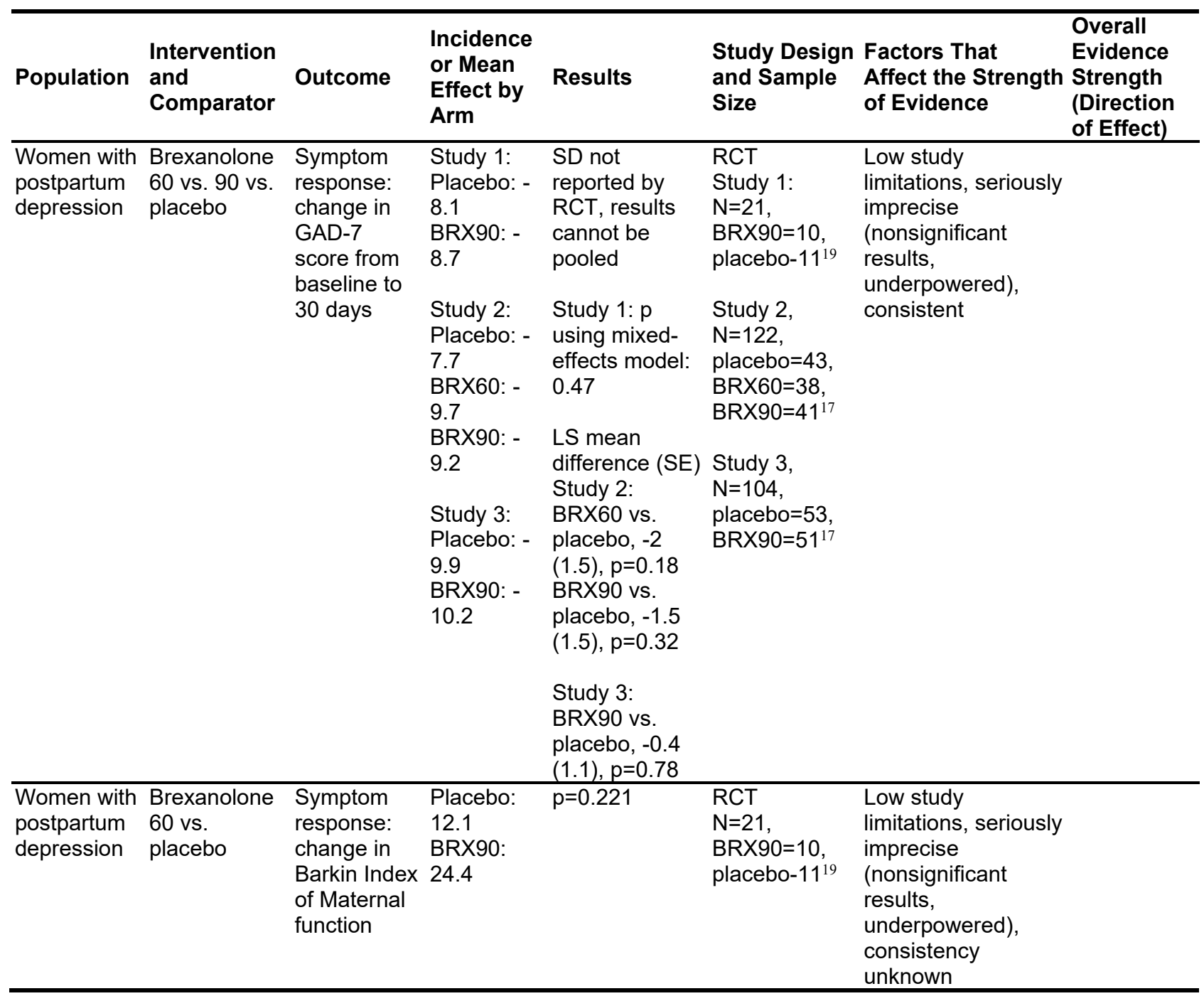

BRX90 = brexanolone for postpartum depression; EPDS = Edinburgh Postnatal Depression Scale; GAD-7 = Generalized Anxiety Disorder 7-item; HAM-D = Hamilton Depression Rating; LS = least square; MADRS = Montgomery-Åsberg Depression Rating Scale; $\mathrm{N}=$ number; PHQ9 = Patient Health Questionnaire, 9 item; RCT = randomized controlled trial; $\mathrm{SD}=$ standard deviation; $\mathrm{SE}=$ standard error; vs. $=$ versus.

\section{Depression: Other Drugs}

\section{Overview}

- No included publications reported on the benefits of citalopram, escitalopram, fluvoxamine, trazodone, serotonin-norepinephrine reuptake inhibitors (SNRIs), tricyclic antidepressants (TCAs), monoamine oxidase inhibitors (MAOIs), bupropion, or mirtazapine. 


\section{Bipolar Disorder: Mood Stabilizers}

\section{Overview}

- Evidence from single small trials on recurrence and time to recurrence suggest benefit for treatment with mood stabilizers compared with discontinuation of treatment (low for benefit) during pregnancy.

\section{Detailed Results}

Two publications reported on mood stabilizers; ${ }^{24,26}$ of these, one reported on mood stabilizers as a class, ${ }^{26}$ and one focused on lamotrigine. ${ }^{24}$ For the study evaluating mood stabilizers as a class, participants were exposed to lithium (61.8\%), anticonvulsants (36.0\%; valproic acid, lamotrigine, carbamazepine, and gabapentin), and atypical antipsychotics (2.3\%; olanzapine and quetiapine). ${ }^{26}$ One was rated high risk of bias ${ }^{24}$ and the other as having some risk-of-bias concerns. ${ }^{26}$ Both were nonrandomized observational cohort studies. These publications drew from two cohorts from the United States (one from Massachusetts ${ }^{26}$ and one from Georgia ${ }^{24}$ ). Publications compared pregnant women who were exposed to mood stabilizers with women with mood disorders who discontinued mood stabilizers. ${ }^{24,26}$

Although the studies are small (and one is high risk of bias ${ }^{24}$ ), they reported substantially higher risks of recurrence or time to recurrence (time-to-25\%-recurrence was 28.0 versus 2.0 weeks) for discontinuation of medications, suggesting low strength of evidence of benefit with mood stabilizers (Table B-8).

Table B-8. Strength of evidence for effectiveness: Mood stabilizers versus no treatment

\begin{tabular}{|c|c|c|c|c|c|c|c|}
\hline Population & $\begin{array}{l}\text { Intervention } \\
\text { and } \\
\text { Comparator }\end{array}$ & Outcome & $\begin{array}{l}\text { Incidence or } \\
\text { Mean Effect } \\
\text { by Arm }\end{array}$ & Results & $\begin{array}{l}\text { Study } \\
\text { Design } \\
\text { and } \\
\text { Sample } \\
\text { Size }\end{array}$ & $\begin{array}{l}\text { Factors That } \\
\text { Affect the } \\
\text { Strength of } \\
\text { Evidence }\end{array}$ & $\begin{array}{l}\text { Overall } \\
\text { Evidence } \\
\text { Strength } \\
\text { (Direction of } \\
\text { Effect) }\end{array}$ \\
\hline $\begin{array}{l}\text { Bipolar } \\
\text { disorder }\end{array}$ & $\begin{array}{l}\text { Women with } \\
\text { bipolar disorder } \\
\text { who } \\
\text { discontinued } \\
\text { mood stabilizers } \\
\text { vs. women } \\
\text { exposed to mood } \\
\text { stabilizers in } \\
\text { pregnancy }\end{array}$ & $\begin{array}{l}\text { Recurrence } \\
\text { of bipolar } \\
\text { disorder }\end{array}$ & $\begin{array}{l}53 / 62 \\
(85.5 \%) \\
\text { vs.10/27 } \\
(37 \%)^{26}\end{array}$ & $\begin{array}{l}\text { AHR for } \\
\text { treatment } \\
\text { discontinuation } \\
\text { vs. exposure } \\
\text { to mood } \\
\text { stabilizers: } 2.2 \\
(95 \% \mathrm{Cl}, 1.2 \text { to } \\
4.2)^{26}\end{array}$ & $\begin{array}{l}1 \text { cohort, } \\
N=89^{26}\end{array}$ & $\begin{array}{l}\text { Moderate study } \\
\text { limitations, } \\
\text { imprecise (few } \\
\text { events); } \\
\text { consistency } \\
\text { unknown }\end{array}$ & $\begin{array}{l}\text { Low (favoring } \\
\text { treatment with } \\
\text { mood } \\
\text { stabilizers) }\end{array}$ \\
\hline $\begin{array}{l}\text { Bipolar } \\
\text { disorder }\end{array}$ & $\begin{array}{l}\text { Women with } \\
\text { bipolar disorder } \\
\text { who } \\
\text { discontinued } \\
\text { mood stabilizers } \\
\text { vs. women } \\
\text { exposed to } \\
\text { lamotrigine in } \\
\text { pregnancy }\end{array}$ & $\begin{array}{l}\text { Recurrence } \\
\text { of bipolar } \\
\text { disorder }\end{array}$ & $\begin{array}{l}16 / 16(100 \%) \\
\text { vs } 3 / 10 \\
(30 \%) .{ }^{24}\end{array}$ & $\begin{array}{l}\text { AHR for } \\
\text { discontinuation } \\
\text { vs. exposure } \\
\text { to lamotrigine: } \\
12.1(95 \% \mathrm{Cl} \text {, } \\
1.6 \text { to } 91)^{24}\end{array}$ & $\begin{array}{l}1 \text { cohort, } \\
n=26^{24}\end{array}$ & $\begin{array}{l}\text { High study } \\
\text { limitations (high } \\
\text { risk of bias }{ }^{24} \text { ) } \\
\text { imprecise (few } \\
\text { events, small N, } \\
\text { wide Cls); } \\
\text { consistency } \\
\text { unknown; very } \\
\text { large effect size }\end{array}$ & $\begin{array}{l}\text { Low (favoring } \\
\text { treatment with } \\
\text { lamotrigine) }\end{array}$ \\
\hline
\end{tabular}

$\mathrm{AHR}=$ adjusted hazard ratio; $\mathrm{CI}=$ confidence interval; $\mathrm{N}=$ number; $\mathrm{vs.}=$ versus.

\section{Schizophrenia: Antipsychotics}

\section{Overview}

- No included publications reported on benefits of antipsychotic medications. 


\section{Pharmacologic Combinations}

\section{Overview}

- No included publications reported on the benefits of pharmacologic combinations.

\section{Nonspecific or Undefined Pharmacologic Interventions}

\section{Overview}

- Seven publications reported on nonspecific or undefined pharmacologic interventions on maternal outcomes (C-section, deliberate self-harm, inpatient hospital stays, postnatal depression symptom severity, or relapse of major depression). Because the clinical utility is limited, we did not judge the risk of harms for maternal or child outcomes.

\section{Detailed Results}

Seven publications reported on nonspecific/undefined pharmacologic interventions. ${ }^{18,20,28,31}$, 32, 34 These publications drew from seven cohorts (1 from the Multinational Medication Use in Pregnancy Study, ${ }^{18} 1$ from the U.S. Medicaid Analytic eXtract ${ }^{20} 1$ from MothertoBaby Antidepressants in Pregnancy Cohort,$^{32} 1$ from a perinatal mental health unit based in Spain, ${ }^{34}$ one based in Canada, ${ }^{31} 1$ based on a cohort in Australia, ${ }^{33}$ and 1 based in the United States). ${ }^{28}$ All were nonrandomized studies and were rated as having some risk of bias. Six of the publications focused on exposure during pregnancy, ${ }^{20,28,31-34}$ and one focused on exposure during pregnancy and the postpartum period.$^{18}$ Five publications compared any antidepressant use to not exposed to antidepressants. ${ }^{18,20,28,31,32}$ These five studies evaluated the outcomes of inpatient hospital stays, deliberate self-harm, postnatal depression symptom severity, relapse of major depression, and the likelihood of a C-section. Two studies evaluated the likelihood of a C-section: one study compared lithium plus antidepressants with not exposed to lithium plus antidepressants, ${ }^{34}$ and one study compared antipsychotics use with not exposed to antipsychotics. ${ }^{33}$ Because the interventions of these studies were not clearly defined, they are not evaluated any further given their limited clinical utility.

Key Question 2: Among pregnant and postpartum women, what is the comparative effectiveness of pharmacologic interventions on maternal outcomes among those with a new or preexisting anxiety disorder, depressive disorder, bipolar disorder, or schizophrenia?

\section{Anxiety: Anti-Anxiety Medications}

\section{Overview}

- No included publications reported on the comparative benefits of anxiolytics.

\section{Depression: Fluoxetine Versus TCAs}

\section{Overview}

- The evidence is insufficient to judge the comparative risk of harms from fluoxetine versus TCAs during pregnancy for any maternal outcomes (emergency C-section or 
repeated C-section) or child outcomes (gestational age, birthweight, major congenital anomalies, or infant mental development).

\section{Detailed Results}

One high risk-of-bias publication from the Canadian Motherisk cohort reported on the comparative harms of fluoxetine versus TCAs as a class. ${ }^{39}$ The publication reported on women who used one of the medications during the first trimester or longer. ${ }^{39}$

No significant differences were found in the risk of harms for maternal outcomes (emergency $\mathrm{C}$-section or repeated C-section). The strength of evidence was rated as insufficient because of imprecision and potential risk of bias (Table B-9).

Table B-9. Strength of evidence for comparative effectiveness outcomes: Fluoxetine versus TCAs

\begin{tabular}{|c|c|c|c|c|c|c|c|}
\hline Population & $\begin{array}{l}\text { Intervention } \\
\text { and } \\
\text { Comparator }\end{array}$ & Outcome & $\begin{array}{l}\text { Incidence } \\
\text { or Mean } \\
\text { Effect by } \\
\text { Arm }\end{array}$ & Results & $\begin{array}{l}\text { Study } \\
\text { Design } \\
\text { and } \\
\text { Sample } \\
\text { Size } \\
\end{array}$ & $\begin{array}{l}\text { Factors That Affect } \\
\text { the Strength of } \\
\text { Evidence }\end{array}$ & $\begin{array}{l}\text { Overall } \\
\text { Evidence } \\
\text { Strength } \\
\text { (Direction of } \\
\text { Effect) }\end{array}$ \\
\hline $\begin{array}{l}\text { Pregnant } \\
\text { women with } \\
\text { depression }\end{array}$ & $\begin{array}{l}\text { Fluoxetine vs. } \\
\text { TCAs }\end{array}$ & $\begin{array}{l}\text { Emergency } \\
\text { C-section }\end{array}$ & $\begin{array}{l}4 / 42(9.5 \%) \\
\text { vs. } 5 / 43 \\
(12 \%)^{39}\end{array}$ & $\begin{array}{l}\text { RR, } 0.82 \\
(95 \% \mathrm{Cl}, \\
0.24 \text { to } \\
2.84)^{39}\end{array}$ & $\begin{array}{l}1 \text { cohort, } \\
n=85^{39}\end{array}$ & $\begin{array}{l}\text { High study limitations } \\
\text { (high risk of bias }^{39} \text { ), } \\
\text { seriously imprecise } \\
\text { (few events, wide } \\
\text { Cls), inconsistency } \\
\text { unknown }\end{array}$ & Insufficient \\
\hline $\begin{array}{l}\text { Pregnant } \\
\text { women with } \\
\text { depression }\end{array}$ & $\begin{array}{l}\text { Fluoxetine vs. } \\
\text { TCAs }\end{array}$ & $\begin{array}{l}\text { Repeated } \\
\text { C-section }\end{array}$ & $\begin{array}{l}6 / 42(14 \%) \\
\text { Vs. } 6 / 43 \\
(14 \%)^{39}\end{array}$ & $\begin{array}{l}\text { RR, } 1.02 \\
(95 \% \mathrm{Cl} \\
0.36 \text { to } \\
2.92)^{39}\end{array}$ & $\begin{array}{l}1 \text { cohort, } \\
n=85^{39}\end{array}$ & $\begin{array}{l}\text { High study limitations } \\
\text { (high risk of bias }{ }^{39} \text { ), } \\
\text { seriously imprecise } \\
\text { (few events, wide } \\
\text { Cls), inconsistency } \\
\text { unknown }\end{array}$ & Insufficient \\
\hline
\end{tabular}

$\overline{\mathrm{CI}}=$ confidence interval; $\mathrm{n}=$ number; $\mathrm{RR}=$ relative risk; $\mathrm{TCA}=$ tricyclic antidepressant; $\mathrm{vs} .=$ versus.

\section{Depression: Sertraline Versus Nortriptyline}

\section{Overview}

- One high risk-of-bias trial did not report any differences between sertraline and nortriptyline for postpartum depression for response; remission; time to response and remission; and general, sexual, or psychosocial functioning, but we rated the evidence as insufficient because of imprecision and potential bias.

\section{Detailed Results}

One RCT (rated high risk of bias) reported on the comparative effectiveness of sertraline versus nortriptyline for women with postpartum depression. ${ }^{35,36}$ Some women may also have had chronic depression: the study began including women with chronic depression after the trial started. Women were treated with a fixed dose strategy of $25 \mathrm{mg} /$ day of sertraline or $10 \mathrm{mg} /$ day of nortriptyline initially. The dose was escalated to $50 \mathrm{mg} /$ day of sertraline or $25 \mathrm{mg} /$ day of nortriptyline after 2 days and then gradually increased up to a maximum of $200 \mathrm{mg} / \mathrm{day}$ of sertraline and $150 \mathrm{mg}$ /day nortriptyline if response or side effects did not prohibit further escalation. The study was rated high risk of bias because of high and differential attrition by 8 weeks ( $42 \%$ vs. $24 \%$ ). The study was powered to detect differences in proportion greater than 32 percent, differences in time to remission of 28 percent, and differences in HRSD scores of less than -1.7 to more than 3.7 points and in GAS scores of less than -5.72 to more than 4.9 points. 
The study was not powered to test for equivalence. The study did not report differences at 8 weeks or at other time points for response; remission; time to response and remission; and general, sexual, or psychosocial functioning. We graded the evidence as insufficient because of imprecision and potential for bias due to attrition (Table B-10).

Table B-10. Strength of evidence for comparative effectiveness outcomes: Sertraline versus nortriptyline

\begin{tabular}{|c|c|c|c|c|c|c|c|}
\hline Population & $\begin{array}{l}\text { Intervention } \\
\text { and } \\
\text { Comparator }\end{array}$ & Outcome & $\begin{array}{l}\text { Incidence } \\
\text { or Mean } \\
\text { Effect by } \\
\text { Arm }\end{array}$ & Results & $\begin{array}{l}\text { Study } \\
\text { Design } \\
\text { and } \\
\text { Sample } \\
\text { Size }\end{array}$ & $\begin{array}{l}\text { Factors That } \\
\text { Affect the } \\
\text { Strength of } \\
\text { Evidence }\end{array}$ & $\begin{array}{l}\text { Overall } \\
\text { Evidence } \\
\text { Strength } \\
\text { (Direction of } \\
\text { Effect) }\end{array}$ \\
\hline $\begin{array}{l}\text { Women with } \\
\text { postpartum } \\
\text { depression (with } \\
\text { or without } \\
\text { chronic } \\
\text { depression) } \\
\end{array}$ & $\begin{array}{l}\text { Sertraline vs. } \\
\text { nortriptyline }\end{array}$ & $\begin{array}{l}\text { Response at } \\
8 \text { weeks } \\
(50 \% \\
\text { reduction in } \\
\text { HRSD) }\end{array}$ & $\begin{array}{l}31 / 55 \\
(56.4 \%) \text { vs. } \\
37 / 54 \\
(68.5 \%)^{36}\end{array}$ & $\begin{array}{l}\text { RR, } 0.94 \\
(95 \% \mathrm{Cl} \text {, } \\
0.63 \text { to } \\
1.41)^{36}\end{array}$ & $\begin{array}{l}1 \text { trial, } \\
\mathrm{N}=108^{36}\end{array}$ & $\begin{array}{l}\text { High study } \\
\text { limitations (high } \\
\text { risk of bias, }{ }^{36} \text { ) } \\
\text { imprecise (wide } \\
\text { Cls), consistency } \\
\text { unknown }\end{array}$ & Insufficient \\
\hline $\begin{array}{l}\text { Women with } \\
\text { postpartum } \\
\text { depression (with } \\
\text { or without } \\
\text { chronic } \\
\text { depression) }\end{array}$ & $\begin{array}{l}\text { Sertraline vs. } \\
\text { nortriptyline }\end{array}$ & $\begin{array}{l}\text { Time to } \\
\text { response } \\
(50 \% \\
\text { reduction in } \\
\text { HRSD) }\end{array}$ & NR & $N R, p=.21$ & $\begin{array}{l}1 \text { trial, } \\
\mathrm{N}=95^{36}\end{array}$ & $\begin{array}{l}\text { High study } \\
\text { limitations (high } \\
\text { risk of bias }{ }^{36} \text { ) } \\
\text { likely imprecise, } \\
\text { consistency } \\
\text { unknown }\end{array}$ & Insufficient \\
\hline $\begin{array}{l}\text { Women with } \\
\text { postpartum } \\
\text { depression (with } \\
\text { or without } \\
\text { chronic } \\
\text { depression) } \\
\end{array}$ & $\begin{array}{l}\text { Sertraline vs. } \\
\text { nortriptyline }\end{array}$ & $\begin{array}{l}\text { Remission at } \\
8 \text { weeks } \\
(\text { HRSD }<7 \text { at } \\
\text { week } 8)\end{array}$ & $\begin{array}{l}25 / 55 \\
(45.5 \%) \text { vs. } \\
26 / 54 \\
(48.1 \%)^{36}\end{array}$ & $\begin{array}{l}\text { RR, } 0.82 \\
(95 \% \mathrm{Cl}, \\
0.61 \text { to } \\
1.10)^{36}\end{array}$ & $\begin{array}{l}1 \text { trial, } \\
\mathrm{N}=108^{36}\end{array}$ & $\begin{array}{l}\text { High study } \\
\text { limitations (high } \\
\text { risk of bias, }{ }^{36} \text { ) } \\
\text { imprecise (wide } \\
\text { Cls), consistency } \\
\text { unknown }\end{array}$ & Insufficient \\
\hline $\begin{array}{l}\text { Women with } \\
\text { postpartum } \\
\text { depression (with } \\
\text { or without } \\
\text { chronic } \\
\text { depression) }\end{array}$ & $\begin{array}{l}\text { Sertraline vs. } \\
\text { nortriptyline }\end{array}$ & $\begin{array}{l}\text { Time to } \\
\text { remission at } \\
8 \text { weeks } \\
\text { (HRSD }<7 \text { at } \\
\text { week } 8)\end{array}$ & $\begin{array}{l}\text { Median: } 5 \\
\text { weeks vs. } 5 \\
\text { weeks }^{36}\end{array}$ & $\begin{array}{l}N R, \\
p=0.82^{36}\end{array}$ & $\begin{array}{c}1 \text { trial, } \\
\mathrm{N}=95^{36}\end{array}$ & $\begin{array}{l}\text { High study } \\
\text { limitations (high } \\
\text { risk of bias, }{ }^{36} \text { ) } \\
\text { imprecise (likely } \\
\text { imprecise), } \\
\text { consistency } \\
\text { unknown }\end{array}$ & Insufficient \\
\hline $\begin{array}{l}\text { Women with } \\
\text { postpartum } \\
\text { depression (with } \\
\text { or without } \\
\text { chronic } \\
\text { depression) } \\
\end{array}$ & $\begin{array}{l}\text { Sertraline vs. } \\
\text { nortriptyline }\end{array}$ & $\begin{array}{l}\text { Symptom } \\
\text { scores } \\
\text { (HRSD) at } 8 \\
\text { weeks }\end{array}$ & NR & $\begin{array}{l}\text { Effect size: } \\
0.13(95 \% \\
\mathrm{Cl},-0.26 \text { to } \\
0.55)^{36}\end{array}$ & $\begin{array}{l}1 \text { trial, } \\
\mathrm{N}=95^{36}\end{array}$ & $\begin{array}{l}\text { High study } \\
\text { limitations (high } \\
\text { risk of bias }{ }^{36} \text { ) } \\
\text { imprecise (wide } \\
\text { Cls), consistency } \\
\text { unknown }\end{array}$ & Insufficient \\
\hline $\begin{array}{l}\text { Women with } \\
\text { postpartum } \\
\text { depression (with } \\
\text { or without } \\
\text { chronic } \\
\text { depression) }\end{array}$ & $\begin{array}{l}\text { Sertraline vs. } \\
\text { nortriptyline }\end{array}$ & $\begin{array}{l}\text { Symptom } \\
\text { scores (CGI } \\
\text { score of } 0 \text { or } \\
\text { 1) at } 8 \text { weeks }\end{array}$ & $\begin{array}{l}32 / 36 \\
(88.9 \%) \text { vs. } \\
44 / 47 \\
(93.6 \%)^{36}\end{array}$ & $\begin{array}{l}\mathrm{RR}, 0.95 \\
(95 \% \mathrm{Cl} \text {, } \\
0.83 \text { to } \\
1.09)^{36}\end{array}$ & $\begin{array}{l}1 \text { trial, } \\
\mathrm{N}=83^{36}\end{array}$ & $\begin{array}{l}\text { High study } \\
\text { limitations (high } \\
\text { risk of bias, }{ }^{36} \text { ) } \\
\text { imprecise (wide } \\
\text { Cls), consistency } \\
\text { unknown }\end{array}$ & Insufficient \\
\hline
\end{tabular}




\begin{tabular}{|c|c|c|c|c|c|c|c|}
\hline Population & $\begin{array}{l}\text { Intervention } \\
\text { and } \\
\text { Comparator }\end{array}$ & Outcome & $\begin{array}{l}\text { Incidence } \\
\text { or Mean } \\
\text { Effect by } \\
\text { Arm }\end{array}$ & Results & $\begin{array}{l}\text { Study } \\
\text { Design } \\
\text { and } \\
\text { Sample } \\
\text { Size }\end{array}$ & $\begin{array}{l}\text { Factors That } \\
\text { Affect the } \\
\text { Strength of } \\
\text { Evidence }\end{array}$ & $\begin{array}{l}\text { Overall } \\
\text { Evidence } \\
\text { Strength } \\
\text { (Direction of } \\
\text { Effect) }\end{array}$ \\
\hline $\begin{array}{l}\text { Women with } \\
\text { postpartum } \\
\text { depression (with } \\
\text { or without } \\
\text { chronic } \\
\text { depression) }\end{array}$ & $\begin{array}{l}\text { Sertraline vs. } \\
\text { nortriptyline }\end{array}$ & $\begin{array}{l}\text { Global } \\
\text { functioning } \\
\text { (GAS) at } 8 \\
\text { weeks }\end{array}$ & NR & $\begin{array}{l}\text { Effect size } \\
0.005 \\
(95 \% \\
\mathrm{Cl},-0.44 \text { to } \\
0.38)^{36}\end{array}$ & $\begin{array}{l}1 \text { trial, } \\
\mathrm{N}=95^{36}\end{array}$ & $\begin{array}{l}\text { High study } \\
\text { limitations (high } \\
\text { risk of bias }{ }^{36} \text { ) } \\
\text { imprecise (wide } \\
\text { Cls), consistency } \\
\text { unknown }\end{array}$ & Insufficient \\
\hline $\begin{array}{l}\text { Women with } \\
\text { postpartum } \\
\text { depression (with } \\
\text { or without } \\
\text { chronic } \\
\text { depression) } \\
\end{array}$ & $\begin{array}{l}\text { Sertraline vs. } \\
\text { nortriptyline }\end{array}$ & $\begin{array}{l}\text { Social } \\
\text { functioning } \\
\text { (SPQ) at } 8 \\
\text { weeks }\end{array}$ & NR & $\begin{array}{l}N R, \\
p=0.33^{36}\end{array}$ & $\begin{array}{c}1 \text { trial, } \\
\mathrm{N}=83^{36}\end{array}$ & $\begin{array}{l}\text { High study } \\
\text { limitations (high } \\
\text { risk of bias, }{ }^{36} \text { ) } \\
\text { imprecise (wide } \\
\text { Cls), consistency } \\
\text { unknown }\end{array}$ & Insufficient \\
\hline $\begin{array}{l}\text { Women with } \\
\text { postpartum } \\
\text { depression (with } \\
\text { or without } \\
\text { chronic } \\
\text { depression) }\end{array}$ & $\begin{array}{l}\text { Sertraline vs. } \\
\text { nortriptyline }\end{array}$ & $\begin{array}{l}\text { Arizona } \\
\text { Sexual } \\
\text { Experience } \\
\text { Scale (ASEX) }\end{array}$ & NR & $\begin{array}{l}\text { AOR, } 2.35 \\
\text { (95\% Cl, } \\
0.6 \text { to } \\
9.26)^{35}\end{array}$ & $\begin{array}{l}1 \text { trial, } \\
\mathrm{N}=70^{35}\end{array}$ & $\begin{array}{l}\text { High study } \\
\text { limitations (high } \\
\text { risk of bias, }{ }^{35} \text { ) } \\
\text { imprecise (wide } \\
\text { Cls), consistency } \\
\text { unknown }\end{array}$ & Insufficient \\
\hline
\end{tabular}

ASEX = Arizona Sexual Experience Scale; AOR = adjusted odds ratio; CGI = Clinical Global Impression; CI = confidence interval; GAS = general adaptation syndrome; HRSD = Hamilton Rating Scale for Depression; $N=$ number; $N R=$ not reported; $\mathrm{RR}=$ relative risk; SPQ $=$ Social Problems Questionnaire.

\section{Depression: Other Drugs}

\section{Overview}

- No included publications reported on the comparative benefits of other medications for depression, including brexanolone, bupropion, mirtazapine, MAOIs, SNRIs, or TCAs.

\section{Bipolar Disorder: Lamotrigine Versus Lithium}

\section{Overview}

- The evidence for lamotrigine versus lithium during pregnancy is insufficient to judge the comparative effectiveness of associated maternal benefits (specifically reduced postpartum psychiatric admissions).

\section{Detailed Results}

One publication, focusing on comparing lithium and lamotrigine ${ }^{37}$ drew from a cohort from Denmark. The study was rated as high risk of bias. The publication focused on any exposure from preconception through postpartum. ${ }^{37}$

The evidence is insufficient to judge the associated maternal benefits (reduced postpartum psychiatric admissions) (Table B-11). 
Table B-11. Strength of evidence for comparative effectiveness: Lamotrigine versus lithium

\begin{tabular}{|c|c|c|c|c|c|c|c|}
\hline Population & $\begin{array}{l}\text { Intervention } \\
\text { and } \\
\text { Comparator }\end{array}$ & Outcome & $\begin{array}{l}\text { Incidence or } \\
\text { Mean Effect by } \\
\text { Arm }\end{array}$ & Results & $\begin{array}{l}\text { Study Design } \\
\text { and Sample } \\
\text { Size }\end{array}$ & $\begin{array}{l}\text { Factors } \\
\text { That Affect } \\
\text { the Strength } \\
\text { of Evidence }\end{array}$ & $\begin{array}{l}\text { Overall } \\
\text { Evidence } \\
\text { Strength } \\
\text { (Direction } \\
\text { of Effect) }\end{array}$ \\
\hline $\begin{array}{l}\text { Women with } \\
\text { bipolar } \\
\text { disorder }\end{array}$ & $\begin{array}{l}\text { Lamotrigine } \\
\text { vs. lithium } \\
\text { exposure } \\
\text { during } \\
\text { pregnancy }\end{array}$ & $\begin{array}{l}\text { Postpartum } \\
\text { psychiatric } \\
\text { admission }\end{array}$ & $\begin{array}{l}\text { 4/55 (7.3\%) vs. } \\
9 / 59(15.2 \%)^{37}\end{array}$ & $\begin{array}{l}\text { OR, } 0.83(95 \% \\
\mathrm{Cl}, 0.22 \text { to } \\
3.14)^{37}\end{array}$ & $\begin{array}{l}1 \text { cohort, } \\
n=114^{37}\end{array}$ & $\begin{array}{l}\text { High study } \\
\text { limitations } \\
\text { high risk of } \\
\text { bias, }{ }^{37} \\
\text { imprecise } \\
\text { (wide Cls), } \\
\text { consistency } \\
\text { unknown }\end{array}$ & Insufficient \\
\hline
\end{tabular}

$\mathrm{CI}=$ confidence interval; $\mathrm{n}=$ number; $\mathrm{OR}=$ odds ratio; vs. $=$ versus.

\section{Bipolar Disorder: Olanzapine Versus Lithium}

\section{Overview}

- The evidence for olanzapine versus lithium during pregnancy is insufficient to judge the comparative effectiveness of associated maternal benefits (specifically mood episodes).

\section{Detailed Results}

One publication, focusing on comparing olanzapine and lamotrigine, ${ }^{38}$ drew from a cohort from Canada. The study was rated as high risk of bias. The publication focused on any exposure during pregnancy. ${ }^{38}$

The evidence is insufficient to judge the associated maternal benefits (postpartum mood episodes) (Table B-12).

Table B-12. Strength of evidence for comparative effectiveness: Olanzapine versus lithium

\begin{tabular}{|c|c|c|c|c|c|c|c|}
\hline Population & $\begin{array}{l}\text { Intervention and } \\
\text { Comparator }\end{array}$ & Outcome & $\begin{array}{l}\text { Incidence } \\
\text { or Mean } \\
\text { Effect by } \\
\text { Arm }\end{array}$ & Results & $\begin{array}{l}\text { Study } \\
\text { Design } \\
\text { and } \\
\text { Sample } \\
\text { Size }\end{array}$ & $\begin{array}{l}\text { Factors That Affect the } \\
\text { Strength of Evidence }\end{array}$ & $\begin{array}{l}\text { Overall } \\
\text { Evidence } \\
\text { Strength } \\
\text { (Direction } \\
\text { of Effect) }\end{array}$ \\
\hline $\begin{array}{l}\text { Women with } \\
\text { bipolar } \\
\text { disorder }\end{array}$ & $\begin{array}{l}\text { Olanzapine vs. } \\
\text { Lithium exposure } \\
\text { during pregnancy }\end{array}$ & $\begin{array}{l}\text { Postpartum } \\
\text { mood } \\
\text { episode }\end{array}$ & $\begin{array}{l}2 / 9 \\
(22.2 \%) \\
\text { Vs. } 2 / 2 \\
(100 \%)^{38}\end{array}$ & $\begin{array}{l}\text { RR, } 0.3 \\
(95 \% \\
\text { Cl, } 0.09 \\
\text { to } \\
0.98)^{38}\end{array}$ & $\begin{array}{l}1 \text { cohort, } \\
n=25^{38}\end{array}$ & $\begin{array}{l}\text { High study limitations, } \\
\text { high risk of bias, }{ }^{38} \\
\text { imprecise (few events, } \\
\text { small N, wide Cls), } \\
\text { consistency unknown }\end{array}$ & Insufficient \\
\hline
\end{tabular}

$\mathrm{CI}=$ confidence interval; $\mathrm{n}=$ number; $\mathrm{RR}=$ relative risk; $\mathrm{vs}=$ versus.

\section{Bipolar Disorder: Lithium Versus Sodium Valproate}

\section{Overview}

- The evidence for lithium versus sodium valproate postpartum is insufficient to judge the comparative effectiveness of associated maternal benefits (relapse).

\section{Detailed Results}

One high risk-of-bias publication, comparing lithium and sodium valproate, ${ }^{41}$ drew from a mother-baby-unit in a hospital-based cohort from Australia. The publication focused on exposure during the postpartum period, but the duration of the period was not defined. Women were on 
lithium, sodium valproate, or a combination on discharge. Relapse was defined as readmission to the mother-baby-unit or detailed as such in patient notes.

The evidence is insufficient to judge the associated maternal benefits (relapse) (Table B-13).

Table B-13. Strength of evidence for comparative effectiveness: Lithium versus sodium valproate

\begin{tabular}{|c|c|c|c|c|c|c|c|}
\hline Population & $\begin{array}{l}\text { Intervention and } \\
\text { Comparator }\end{array}$ & Outcome & $\begin{array}{l}\text { Incidence } \\
\text { or Mean } \\
\text { Effect by } \\
\text { Arm }\end{array}$ & Results & $\begin{array}{l}\text { Study } \\
\text { Design } \\
\text { and } \\
\text { Sample } \\
\text { Size }\end{array}$ & $\begin{array}{l}\text { Factors That Affect the } \\
\text { Strength of Evidence }\end{array}$ & $\begin{array}{l}\text { Overall } \\
\text { Evidence } \\
\text { Strength } \\
\text { (Direction } \\
\text { of Effect) }\end{array}$ \\
\hline $\begin{array}{l}\text { Women with } \\
\text { bipolar } \\
\text { disorder }\end{array}$ & $\begin{array}{l}\text { Lithium vs. sodium } \\
\text { valproate exposure } \\
\text { postpartum }\end{array}$ & Relapse & $\begin{array}{l}13 / 34 \\
(38.2 \%) \\
\text { vs. } 14 / 30 \\
(46.7 \%)^{41}\end{array}$ & $\begin{array}{l}\mathrm{RR}, \\
0.82 \\
(95 \% \\
\mathrm{Cl}, 0.46 \\
\text { to } \\
1.45)^{41}\end{array}$ & $\begin{array}{l}1 \text { cohort, } \\
n=64^{41}\end{array}$ & $\begin{array}{l}\text { High study limitations (high } \\
\text { risk of bias, }{ }^{41} \text { ) imprecise } \\
\text { (few events, small Ns, wide } \\
\text { Cls), consistency unknown }\end{array}$ & Insufficient \\
\hline
\end{tabular}

$\mathrm{CI}=$ confidence interval; $\mathrm{n}=$ number; $\mathrm{RR}=$ relative risk; $\mathrm{vs}=$ versus.

\section{Bipolar Disorder: Lithium Versus Lithium Plus Sodium Valproate}

\section{Overview}

- The evidence for lithium versus lithium plus sodium valproate postpartum is insufficient to judge the comparative effectiveness of associated maternal benefits (relapse).

\section{Detailed Results}

One high risk-of-bias publication, comparing lithium and lithium plus sodium valproate, ${ }^{41}$ drew from a mother-baby-unit in a hospital-based cohort from Australia. The publication focused on exposure during the postpartum period, but the duration of the period was not defined.

Women were on lithium, sodium valproate, or a combination on discharge. Relapse was defined as readmission to the mother-baby-unit or detailed as such in patient notes.

The evidence is insufficient to judge the associated maternal benefits (relapse) (Table B-14).

Table B-14. Strength of evidence for comparative effectiveness: Lithium versus lithium plus sodium valproate

\begin{tabular}{|c|c|c|c|c|c|c|c|}
\hline Population & $\begin{array}{l}\text { Intervention and } \\
\text { Comparator }\end{array}$ & Outcome & $\begin{array}{l}\text { Incidence } \\
\text { or Mean } \\
\text { Effect by } \\
\text { Arm }\end{array}$ & Results & $\begin{array}{l}\text { Study } \\
\text { Design } \\
\text { and } \\
\text { Sample } \\
\text { Size } \\
\end{array}$ & $\begin{array}{l}\text { Factors That Affect the } \\
\text { Strength of Evidence }\end{array}$ & $\begin{array}{l}\text { Overall } \\
\text { Evidence } \\
\text { Strength } \\
\text { (Direction } \\
\text { of Effect) }\end{array}$ \\
\hline $\begin{array}{l}\text { Women with } \\
\text { bipolar } \\
\text { disorder }\end{array}$ & $\begin{array}{l}\text { Lithium vs. lithium } \\
\text { plus sodium } \\
\text { valproate exposure } \\
\text { postpartum }\end{array}$ & Relapse & $\begin{array}{l}13 / 34 \\
(38.2 \%) \\
\text { vs. } 1 / 3 \\
(33.3 \%)^{41}\end{array}$ & $\begin{array}{l}\mathrm{RR}, \\
1.15 \\
(95 \% \\
\mathrm{Cl}, 0.22 \\
\text { to } \\
6.01)^{41}\end{array}$ & $\begin{array}{l}1 \text { cohort, } \\
n=38^{41}\end{array}$ & $\begin{array}{l}\text { High study limitations (high } \\
\text { risk of bias, }{ }^{41} \text { ) imprecise } \\
\text { (few events, small Ns, wide } \\
\text { Cls), consistency unknown }\end{array}$ & Insufficient \\
\hline
\end{tabular}

$\mathrm{CI}=$ confidence interval; $\mathrm{n}=$ number; $\mathrm{RR}=$ relative risk; $\mathrm{vs}=$ versus. 


\section{Bipolar Disorder: Sodium Valproate Versus Lithium Plus Sodium Valproate}

\section{Overview}

- The evidence for sodium valproate versus lithium plus sodium valproate postpartum is insufficient to judge the comparative effectiveness of associated maternal benefits (relapse).

\section{Detailed Results}

One high risk-of-bias publication, comparing sodium valproate versus lithium plus sodium valproate ${ }^{41}$ drew from a mother-baby-unit in a hospital-based cohort from Australia. The publication focused on exposure during the postpartum period, but the duration of the period was not defined. Women were on lithium, sodium valproate, or a combination on discharge. Relapse was defined as readmission to the mother-baby-unit or detailed as such in patient notes.

The evidence is insufficient to judge the associated maternal benefits (relapse) (Table B-15).

Table B-15. Strength of evidence for comparative effectiveness: Sodium valproate versus lithium plus sodium valproate

\begin{tabular}{|c|c|c|c|c|c|c|c|}
\hline Population & $\begin{array}{l}\text { Intervention and } \\
\text { Comparator }\end{array}$ & Outcome & $\begin{array}{l}\text { Incidence } \\
\text { or Mean } \\
\text { Effect by } \\
\text { Arm }\end{array}$ & Results & $\begin{array}{l}\text { Study } \\
\text { Design } \\
\text { and } \\
\text { Sample } \\
\text { Size }\end{array}$ & $\begin{array}{l}\text { Factors That Affect the } \\
\text { Strength of Evidence }\end{array}$ & $\begin{array}{l}\text { Overall } \\
\text { Evidence } \\
\text { Strength } \\
\text { (Direction } \\
\text { of Effect) }\end{array}$ \\
\hline $\begin{array}{l}\text { Women with } \\
\text { bipolar } \\
\text { disorder }\end{array}$ & $\begin{array}{l}\text { Sodium valproate vs. } \\
\text { lithium plus sodium } \\
\text { valproate exposure } \\
\text { postpartum }\end{array}$ & Relapse & $\begin{array}{l}14 / 30 \\
(46.7 \%) \\
\text { vs. } 1 / 3 \\
(33.3 \%)^{41}\end{array}$ & $\begin{array}{l}\text { RR, } \\
1.40 \\
(95 \% \\
\mathrm{Cl}, 0.27 \\
\text { to } \\
7.26)^{41}\end{array}$ & $\begin{array}{l}1 \text { cohort, } \\
\mathrm{n}=33^{41}\end{array}$ & $\begin{array}{l}\text { High study limitations } \\
\text { (high risk of bias }{ }^{41} \text { ) } \\
\text { imprecise (few events, } \\
\text { small Ns, wide Cls), } \\
\text { consistency unknown }\end{array}$ & Insufficient \\
\hline
\end{tabular}

$\mathrm{CI}=$ confidence interval; $\mathrm{n}=$ number; $\mathrm{RR}=$ relative risk; $\mathrm{vs}=$ versus.

\section{Bipolar Disorder: Lithium Versus Paroxetine}

\section{Overview}

- The evidence for lithium versus paroxetine during pregnancy is insufficient to judge the comparative effectiveness of associated maternal benefits (specifically a mood episode).

\section{Detailed Results}

One publication, focusing on comparing lithium versus paroxetine,${ }^{38}$ drew from a cohort from Canada. The study was rated as high risk of bias. The publication focused on any exposure during pregnancy. ${ }^{38}$

The evidence is insufficient to judge the associated maternal benefits (postpartum mood episodes) (Table B-16). 
Table B-16. Strength of evidence for comparative effectiveness: Lithium versus paroxetine

\begin{tabular}{|c|c|c|c|c|c|c|c|}
\hline Population & $\begin{array}{l}\text { Intervention and } \\
\text { Comparator }\end{array}$ & Outcome & $\begin{array}{l}\text { Incidence } \\
\text { or Mean } \\
\text { Effect by } \\
\text { Arm }\end{array}$ & Results & $\begin{array}{l}\text { Study } \\
\text { Design } \\
\text { and } \\
\text { Sample } \\
\text { Size }\end{array}$ & $\begin{array}{l}\text { Factors That Affect } \\
\text { the Strength of } \\
\text { Evidence }\end{array}$ & $\begin{array}{l}\text { Overall } \\
\text { Evidence } \\
\text { Strength } \\
\text { (Direction } \\
\text { of Effect) }\end{array}$ \\
\hline $\begin{array}{l}\text { Women with } \\
\text { bipolar } \\
\text { disorder }\end{array}$ & $\begin{array}{l}\text { Lithium vs. } \\
\text { Paroxetine } \\
\text { exposure during } \\
\text { pregnancy }\end{array}$ & $\begin{array}{l}\text { Postpartum } \\
\text { mood } \\
\text { episode }\end{array}$ & $\begin{array}{l}2 / 2 \\
(100 \%) \\
\text { vs. } 2 / 2 \\
(100 \%)^{38}\end{array}$ & $\begin{array}{l}\text { Not } \\
\text { calculatec }\end{array}$ & $\begin{array}{l}1 \text { cohort, } \\
n=25^{38}\end{array}$ & $\begin{array}{l}\text { High study limitations, } \\
\text { high risk of bias, }{ }^{38} \\
\text { imprecise, } \\
\text { consistency unknown }\end{array}$ & Insufficient \\
\hline
\end{tabular}

$\mathrm{n}=$ number; vs. $=$ versus.

\section{Schizophrenia: Antipsychotics Versus Specific Active Comparators}

\section{Overview}

- No included publications reported on the comparative benefits on antipsychotic medications.

\section{Pharmacologic Combinations Versus Specific Active Comparators}

\section{Overview}

- No included publications reported on the benefits of pharmacologic combinations.

\section{Nonspecific or Undefined Pharmacologic Interventions Versus Specific Active Comparators}

\section{Overview}

- No included publications reported on the benefits of nonspecific/undefined pharmacologic interventions.

Key Question 3: Among reproductive-aged women with any mental health disorder, what are the maternal and fetal harms associated with pharmacologic interventions for a mental health disorder during preconception, pregnancy, and postpartum?

\section{Anti-Anxiety Medications}

\section{Overview}

- Benzodiazepine exposure 90 days before conception may be associated with an increased risk of ectopic pregnancy when compared with no exposure to benzodiazepines among unexposed women having at least one anxiety diagnosis in the year before conception (low strength of evidence of harms).

- Benzodiazepine exposure early during pregnancy may be associated with an increased risk of spontaneous abortion when compared with no exposure to benzodiazepines among unexposed women with a history of mood or anxiety disorders (low strength of evidence of harms). 
- Benzodiazepine exposure during pregnancy may be associated with an increased risk of neonatal intensive care unit (NICU) admission when compared with no exposure to benzodiazepines among women with a history of psychiatric disorders (low strength of evidence of harms).

- The evidence is insufficient to judge the risk of harms for maternal (spontaneous abortion, preeclampsia) or child outcomes (perinatal mortality, prematurity, birth weight, breathing difficulty, feeding difficulty, Apgar score, or major congenital anomalies).

\section{Detailed Results}

Five publications reported on maternal or fetal harms of anxiolytics, specifically on benzodiazepines and sedative-hypnotics (Table B-17). ${ }^{46,71,79,100,103}$ Medications such as SSRIs, TCAs, and SNRIs that may also be prescribed for anxiety are described elsewhere in this review. All but one were cohort studies (1 was a case-control) and focused on exposure to anxiolytics immediately before or during pregnancy, with two studies exclusively focusing on first trimester exposure. ${ }^{71,79}$ The five studies drew from four cohorts of pregnant women (the Health improvement Network [THIN] based in the United Kingdom, ${ }^{71,79}$ the Quebec Pregnancy Cohort in Canada, ${ }^{100}$ the MarketScan Research Databases, ${ }^{103}$ and National Pregnancy Registry for Psychiatric Medications based in the United States ${ }^{46}$ ).

Every study reported on benzodiazepines as a class, although one trial specifically evaluated diazepam and temazepam. ${ }^{71}$ Two studies $^{71,79}$ compared pregnant women exposed to anxiolytics with untreated anxiety. One of those studies also compared women who continued benzodiazepine use during the first trimester to women who discontinue medication. ${ }^{79}$ Three studies compared women with benzodiazepine exposure to those without exposure to benzodiazepine during pregnancy but with a history of psychiatric morbidity. ${ }^{46,100,103}$

One study was rated as having some concerns ${ }^{46}$ and two were rated as high risk of bias. ${ }^{71,79}$

Evidence from one study ${ }^{103}$ suggests an increased risk of ectopic pregnancy with benzodiazepine exposure 90 days before conception when compared with no exposure before conception, among pregnant women with at least one anxiety disorder diagnosis in the year before conception (graded low strength of evidence of harms). Although residual confounding may explain these results, one suggested mechanism of action could be through the central relaxation of smooth muscle and the direct effect on gamma-aminobutyric acid receptors in the fallopian tube, potentially resulting in a higher incidence of ectopic pregnancy as a result. ${ }^{103}$ The absolute risk difference is 7 per 1,000 (95\% CI, 4 to 11$)$.

Evidence from two studies ${ }^{79,100}$ suggests an increased risk of spontaneous abortion with benzodiazepine exposure 90 days before conception when compared with untreated or a history of mood disorders or anxiety (graded low strength of evidence of harms). Although residual confounding may explain these results, as with the results for ectopic pregnancy, the authors note that benzodiazepines cross the placental barrier easily and may accumulate in fetal issues. ${ }^{103}$ The absolute risk difference is 73 per 1,000 (95\% CI, 36 to 109). 
Table B-17. Strength of evidence for harms outcomes: Anxiolytics versus no treatment

\begin{tabular}{|c|c|c|c|c|c|c|c|}
\hline Population & $\begin{array}{l}\text { Intervention } \\
\text { and } \\
\text { Comparator }\end{array}$ & Outcome & $\begin{array}{l}\text { Incidence or } \\
\text { Mean Effect } \\
\text { by Arm }\end{array}$ & Results & $\begin{array}{l}\text { Study } \\
\text { Design and } \\
\text { Sample } \\
\text { Size }\end{array}$ & $\begin{array}{l}\text { Factors That } \\
\text { Affect the } \\
\text { Strength of } \\
\text { Evidence }\end{array}$ & $\begin{array}{l}\text { Overall } \\
\text { Evidence } \\
\text { Strength } \\
\text { (Direction of } \\
\text { Effect) }\end{array}$ \\
\hline $\begin{array}{l}\text { Pregnant women } \\
\text { with least one } \\
\text { anxiety diagnosis } \\
\text { in the year } \\
\text { before } \\
\text { conception }\end{array}$ & $\begin{array}{l}\text { Benzodiazepine } \\
\text { exposure } 90 \\
\text { days before } \\
\text { conception vs. } \\
\text { no } \\
\text { benzodiazepine } \\
\text { exposure } \\
\text { before } \\
\text { conception }\end{array}$ & $\begin{array}{l}\text { Ectopic } \\
\text { pregnancy }\end{array}$ & $\begin{array}{l}249 / 9,188 \\
(2.71 \%) \text { vs. } \\
1,730 / 81,291 \\
(2.13 \%)^{103}\end{array}$ & $\begin{array}{l}\text { ARR: } \\
1.33 \\
(95 \% \\
\mathrm{Cl}, 1.17 \\
\text { to } \\
1.51)^{103}\end{array}$ & $\begin{array}{l}1 \text { cohort, } \\
N=90,479^{103}\end{array}$ & $\begin{array}{l}\text { Moderate study } \\
\text { limitations, precise, } \\
\text { consistency } \\
\text { unknown }\end{array}$ & $\begin{array}{l}\text { Low for harms } \\
\text { with } \\
\text { benzodiazepine }\end{array}$ \\
\hline $\begin{array}{l}\text { Pregnant women } \\
\text { with depression } \\
\text { or anxiety }\end{array}$ & $\begin{array}{l}\text { Benzodiazepine } \\
\text { exposure in first } \\
\text { trimester vs. } \\
\text { untreated } \\
\text { depression or } \\
\text { anxiety during } \\
\text { pregnancy }\end{array}$ & $\begin{array}{l}\text { Perinatal } \\
\text { death }\end{array}$ & $\begin{array}{l}16 / 2,384 \\
(0.7 \%) \text { vs. } \\
20 / 3,647 \\
(0.5 \%)^{79}\end{array}$ & $\begin{array}{l}\text { RRR, } \\
1.4 \\
(95 \% \\
\mathrm{Cl}, 0.6 \\
\text { to } 1.9)^{79}\end{array}$ & $\begin{array}{l}1 \text { cohort, } \\
\mathrm{N}=6,031^{79}\end{array}$ & $\begin{array}{l}\text { High study } \\
\text { limitations (high } \\
\text { risk of bias }{ }^{79} \text { ), } \\
\text { imprecision (wide } \\
\text { Cls spanning the } \\
\text { null, few events), } \\
\text { consistency } \\
\text { unknown }\end{array}$ & Insufficient \\
\hline $\begin{array}{l}\text { Pregnant women } \\
\text { with depression } \\
\text { or anxiety }\end{array}$ & $\begin{array}{l}\text { Benzodiazepine } \\
\text { exposure in first } \\
\text { trimester }^{79} \text { or } \\
\text { within the first } \\
19 \text { weeks }^{100} \mathrm{vs.} \\
\text { untreated or a } \\
\text { history of mood } \\
\text { disorders or } \\
\text { anxiety during } \\
\text { pregnancy }\end{array}$ & $\begin{array}{l}\text { Spontaneous } \\
\text { abortion }\end{array}$ & $\begin{array}{l}386 / 2,384 \\
(16 \%) \text { vs. } \\
442 / 3,647 \\
(12 \%)^{79} \\
198 \\
\text { cases/570 } \\
\text { controls vs. } \\
3,221 \\
\text { cases/15,382 } \\
\text { controls }^{100}\end{array}$ & $\begin{array}{l}\text { RRR, } \\
1.6 \\
(95 \% \\
\mathrm{Cl}, 1.3 \\
\text { to } 1.9)^{79} \\
\\
\text { AOR: } \\
2.85 \\
(95 \% \\
\mathrm{Cl}, 1.72 \\
\text { to } \\
4.72)^{100} \\
\end{array}$ & $\begin{array}{l}1 \text { cohort, } 1 \\
\text { case-control } \\
\text { study, } \\
\mathrm{N}=21,983^{79} \\
100\end{array}$ & $\begin{array}{l}\text { Moderate study } \\
\text { limitations (high } \\
\text { risk of bias }{ }^{79} \text { ) } \\
\text { precise, consistent }\end{array}$ & $\begin{array}{l}\text { Low for harms } \\
\text { with } \\
\text { benzodiazepine }\end{array}$ \\
\hline $\begin{array}{l}\text { Pregnant women } \\
\text { with depression } \\
\text { or anxiety }\end{array}$ & $\begin{array}{l}\text { Continuation of } \\
\text { benzodiazepine } \\
\text { through first } \\
\text { trimester vs. } \\
\text { discontinuation } \\
\text { of } \\
\text { benzodiazepine } \\
\text { during first } \\
\text { trimester }\end{array}$ & $\begin{array}{l}\text { Perinatal } \\
\text { death }\end{array}$ & $\begin{array}{l}6 / 611(1 \%) \\
\text { vs. } 19 / 2,717 \\
(0.7 \%)^{79}\end{array}$ & $\begin{array}{l}\text { RRR, } \\
1.7 \\
(95 \% \\
\mathrm{Cl}, 0.5 \\
\text { to } 6.0)^{79}\end{array}$ & $\begin{array}{l}1 \text { cohort, } \\
\mathrm{N}=3,328^{79}\end{array}$ & $\begin{array}{l}\text { High study } \\
\text { limitations (high } \\
\text { risk of bias }^{79} \text { ) } \\
\text { imprecision (wide } \\
\text { Cls spanning the } \\
\text { null, few events), } \\
\text { consistency } \\
\text { unknown }\end{array}$ & Insufficient \\
\hline $\begin{array}{l}\text { Pregnant women } \\
\text { with depression } \\
\text { or anxiety }\end{array}$ & $\begin{array}{l}\text { Continuation of } \\
\text { benzodiazepine } \\
\text { through first } \\
\text { trimester vs. } \\
\text { discontinuation } \\
\text { of } \\
\text { benzodiazepine } \\
\text { during first } \\
\text { trimester }\end{array}$ & $\begin{array}{l}\text { Spontaneous } \\
\text { abortion }\end{array}$ & $\begin{array}{l}105 / 611 \\
(17 \%) \text { vs. } \\
415 / 2,717 \\
(15 \%)^{79}\end{array}$ & $\begin{array}{l}\text { RRR, } \\
1.5 \\
(95 \% \\
\text { Cl, } 1.0 \\
\text { to } 2.1)^{79}\end{array}$ & $\begin{array}{l}1 \text { cohort, } \\
\mathrm{N}=3,328^{79}\end{array}$ & $\begin{array}{l}\text { High study } \\
\text { limitations (high } \\
\text { risk of bias }^{79} \text { ) } \\
\text { precise, } \\
\text { consistency } \\
\text { unknown }\end{array}$ & Insufficient \\
\hline $\begin{array}{l}\text { Pregnant women } \\
\text { with depression } \\
\text { or anxiety }\end{array}$ & $\begin{array}{l}\text { Diazepam use } \\
\text { in first trimester } \\
\text { vs. untreated } \\
\text { depression or } \\
\text { anxiety during } \\
\text { pregnancy }\end{array}$ & $\begin{array}{l}\text { Major } \\
\text { congenital } \\
\text { anomalies }\end{array}$ & $\begin{array}{l}31 / 1,159 \\
(2.7 \%) \text { vs. } \\
518 / 19,193 \\
(2.7 \%)^{71}\end{array}$ & $\begin{array}{l}\text { AOR, } \\
0.99 \\
(95 \% \\
\mathrm{Cl}, 0.61 \\
\text { to } \\
1.61)^{71}\end{array}$ & $\begin{array}{l}1 \text { cohort, } \\
\mathrm{N}=20,352^{71}\end{array}$ & $\begin{array}{l}\text { High study } \\
\text { limitations (high } \\
\text { risk of bias }{ }^{71} \text { ) } \\
\text { imprecision (wide } \\
\text { Cls spanning the } \\
\text { null), consistency } \\
\text { unknown }\end{array}$ & Insufficient \\
\hline
\end{tabular}




\begin{tabular}{|c|c|c|c|c|c|c|c|}
\hline Population & $\begin{array}{l}\text { Intervention } \\
\text { and } \\
\text { Comparator }\end{array}$ & Outcome & $\begin{array}{l}\text { Incidence or } \\
\text { Mean Effect } \\
\text { by Arm }\end{array}$ & Results & $\begin{array}{l}\text { Study } \\
\text { Design and } \\
\text { Sample } \\
\text { Size }\end{array}$ & $\begin{array}{l}\text { Factors That } \\
\text { Affect the } \\
\text { Strength of } \\
\text { Evidence }\end{array}$ & $\begin{array}{l}\text { Overall } \\
\text { Evidence } \\
\text { Strength } \\
\text { (Direction of } \\
\text { Effect) }\end{array}$ \\
\hline $\begin{array}{l}\text { Pregnant women } \\
\text { with depression } \\
\text { or anxiety }\end{array}$ & $\begin{array}{l}\text { Temazepam } \\
\text { use in first } \\
\text { trimester vs. } \\
\text { untreated } \\
\text { depression or } \\
\text { anxiety during } \\
\text { pregnancy } \\
\end{array}$ & $\begin{array}{l}\text { Major } \\
\text { congenital } \\
\text { anomalies }\end{array}$ & $\begin{array}{l}11 / 379 \\
(2.9 \%) \text { vs. } \\
518 / 19,193 \\
(2.7 \%)^{71}\end{array}$ & $\begin{array}{l}\text { AOR, } \\
1.04 \\
(95 \% \\
\mathrm{Cl}, 0.47 \\
\text { to } \\
2.32)^{71}\end{array}$ & $\begin{array}{l}1 \text { cohort, } \\
\mathrm{N}=19,572^{71}\end{array}$ & $\begin{array}{l}\text { High study } \\
\text { limitations (high } \\
\text { risk of bias }{ }^{71} \text { ) } \\
\text { imprecision (wide } \\
\text { Cls spanning the } \\
\text { null), consistency } \\
\text { unknown }\end{array}$ & Insufficient \\
\hline $\begin{array}{l}\text { Pregnant women } \\
\text { with a psychiatric } \\
\text { disorder }\end{array}$ & $\begin{array}{l}\text { Benzodiazepine } \\
\text { exposure } \\
\text { during } \\
\text { pregnancy vs. } \\
\text { unexposed to } \\
\text { benzodiazepine } \\
\text { use during } \\
\text { pregnancy }\end{array}$ & $\begin{array}{l}\text { Low birth } \\
\text { weight }\end{array}$ & $\begin{array}{l}7 / 112(6.3 \%) \\
\text { vs. } 16 / 398 \\
(4 \%)^{46}\end{array}$ & $\begin{array}{l}\text { AOR, } \\
2.33 \\
(95 \% \\
\mathrm{Cl}, 0.63 \\
\text { to } \\
8.59)^{46}\end{array}$ & $\begin{array}{l}1 \text { cohort, } \\
N=510^{46}\end{array}$ & $\begin{array}{l}\text { Moderate study } \\
\text { limitations, serious } \\
\text { imprecision (wide } \\
\text { Cls spanning the } \\
\text { null, few events), } \\
\text { consistency } \\
\text { unknown }\end{array}$ & Insufficient \\
\hline $\begin{array}{l}\text { Pregnant women } \\
\text { with a psychiatric } \\
\text { disorder }\end{array}$ & $\begin{array}{l}\text { Benzodiazepine } \\
\text { exposure } \\
\text { during } \\
\text { pregnancy vs. } \\
\text { unexposed to } \\
\text { benzodiazepine } \\
\text { use during } \\
\text { pregnancy }\end{array}$ & $\begin{array}{l}\text { High birth } \\
\text { weight }\end{array}$ & $\begin{array}{l}11 / 112 \\
(9.8 \%) \text { vs. } \\
47 / 398 \\
(11.8 \%)^{46}\end{array}$ & $\begin{array}{l}\text { AOR, } \\
0.94 \\
(95 \% \\
\mathrm{Cl}, 0.38 \\
\text { to } 2.3)^{46}\end{array}$ & $\begin{array}{l}1 \text { cohort, } \\
\mathrm{N}=510^{46}\end{array}$ & $\begin{array}{l}\text { Moderate study } \\
\text { limitations, serious } \\
\text { imprecision (wide } \\
\text { Cls spanning the } \\
\text { null, few events), } \\
\text { consistency } \\
\text { unknown }\end{array}$ & Insufficient \\
\hline $\begin{array}{l}\text { Pregnant women } \\
\text { with a psychiatric } \\
\text { disorder }\end{array}$ & $\begin{array}{l}\text { Benzodiazepine } \\
\text { exposure } \\
\text { during } \\
\text { pregnancy vs. } \\
\text { unexposed to } \\
\text { benzodiazepine } \\
\text { use during } \\
\text { pregnancy }\end{array}$ & $\begin{array}{l}\text { Breathing } \\
\text { difficulty in } \\
\text { neonate }\end{array}$ & $\begin{array}{l}20 / 96 \\
(20.8 \%) \text { vs. } \\
78 / 387 \\
(20.2 \%)^{46}\end{array}$ & $\begin{array}{l}\text { AOR, } \\
1.84 \\
(95 \% \\
\mathrm{Cl}, 0.87 \\
\text { to } \\
3.93)^{46}\end{array}$ & $\begin{array}{l}1 \text { cohort, } \\
\mathrm{N}=483^{46}\end{array}$ & $\begin{array}{l}\text { Moderate study } \\
\text { limitations, serious } \\
\text { imprecision (wide } \\
\text { Cls spanning the } \\
\text { null, few events), } \\
\text { consistency } \\
\text { unknown }\end{array}$ & Insufficient \\
\hline $\begin{array}{l}\text { Pregnant women } \\
\text { with a psychiatric } \\
\text { disorder } \\
\end{array}$ & $\begin{array}{l}\text { Benzodiazepine } \\
\text { exposure } \\
\text { during } \\
\text { pregnancy vs. } \\
\text { unexposed to } \\
\text { benzodiazepine } \\
\text { use during } \\
\text { pregnancy }\end{array}$ & $\begin{array}{l}\text { Feeding } \\
\text { difficulties in } \\
\text { neonate }\end{array}$ & $\begin{array}{l}21 / 96 \\
(21.9 \%) \text { vs. } \\
78 / 387 \\
(20.2 \%)^{46}\end{array}$ & $\begin{array}{l}\text { AOR, } \\
1.4 \\
(95 \% \\
\mathrm{Cl}, 0.65 \\
\text { to } \\
3.04)^{46}\end{array}$ & $\begin{array}{l}1 \text { cohort, } \\
\mathrm{N}=483^{46}\end{array}$ & $\begin{array}{l}\text { Moderate study } \\
\text { limitations, serious } \\
\text { imprecision (wide } \\
\text { Cls spanning the } \\
\text { null, few events), } \\
\text { consistency } \\
\text { unknown }\end{array}$ & Insufficient \\
\hline $\begin{array}{l}\text { Pregnant women } \\
\text { with a psychiatric } \\
\text { disorder }\end{array}$ & $\begin{array}{l}\text { Benzodiazepine } \\
\text { exposure } \\
\text { during } \\
\text { pregnancy vs. } \\
\text { unexposed to } \\
\text { benzodiazepine } \\
\text { use during } \\
\text { pregnancy }\end{array}$ & $\begin{array}{l}\text { Low 5-minute } \\
\text { Apgar } \\
\text { scores }^{46}\end{array}$ & $\begin{array}{l}6 / 108(5.6 \%) \\
\text { Vs. } 12 / 378 \\
(3.2 \%)^{46}\end{array}$ & $\begin{array}{l}\text { AOR, } \\
4.91 \\
(95 \% \\
\mathrm{Cl}, 0.69 \\
\text { to } \\
34.96)^{46}\end{array}$ & $\begin{array}{l}1 \text { cohort, } \\
\mathrm{N}=486^{46}\end{array}$ & $\begin{array}{l}\text { Moderate study } \\
\text { limitations, serious } \\
\text { imprecision (wide } \\
\text { Cls spanning the } \\
\text { null, few events), } \\
\text { consistency } \\
\text { unknown }\end{array}$ & Insufficient \\
\hline $\begin{array}{l}\text { Pregnant women } \\
\text { with a psychiatric } \\
\text { disorder }\end{array}$ & $\begin{array}{l}\text { Benzodiazepine } \\
\text { exposure } \\
\text { during } \\
\text { pregnancy vs. } \\
\text { unexposed to } \\
\text { benzodiazepine } \\
\text { use during } \\
\text { pregnancy }\end{array}$ & $\begin{array}{l}\text { NICU } \\
\text { admission }\end{array}$ & $\begin{array}{l}32 / 144 \\
(22.2 \%) \text { vs. } \\
125 / 649 \\
(19.3 \%)^{46}\end{array}$ & $\begin{array}{l}\text { AOR, } \\
2.02(95 \\
\% \mathrm{Cl}, \\
1.11 \text { to } \\
3.66)^{46}\end{array}$ & $\begin{array}{l}1 \text { cohort, } \\
\mathrm{N}=793^{46}\end{array}$ & $\begin{array}{l}\text { Moderate study } \\
\text { limitations, precise, } \\
\text { consistency } \\
\text { unknown }\end{array}$ & $\begin{array}{l}\text { Low for harms } \\
\text { of } \\
\text { benzodiazepine }\end{array}$ \\
\hline
\end{tabular}




\begin{tabular}{|c|c|c|c|c|c|c|}
\hline Population & $\begin{array}{l}\text { Intervention } \\
\text { and } \\
\text { Comparator }\end{array}$ & $\begin{array}{l}\text { Incidence } \\
\text { or Mean } \\
\text { Effect by } \\
\text { Arm }\end{array}$ & Results & $\begin{array}{l}\text { Study } \\
\text { Design and } \\
\text { Sample } \\
\text { Size }\end{array}$ & $\begin{array}{l}\text { Factors That } \\
\text { Affect the } \\
\text { Strength of } \\
\text { Evidence }\end{array}$ & $\begin{array}{l}\text { Overall } \\
\text { Evidence } \\
\text { Strength } \\
\text { (Direction of } \\
\text { Effect) }\end{array}$ \\
\hline $\begin{array}{l}\text { Pregnant women } \\
\text { with a psychiatric } \\
\text { disorder }\end{array}$ & $\begin{array}{l}\text { Benzodiazepine Prematurity } \\
\text { exposure } \\
\text { during } \\
\text { pregnancy vs. } \\
\text { unexposed to } \\
\text { benzodiazepine } \\
\text { use during } \\
\text { pregnancy }\end{array}$ & $\begin{array}{l}17 / 144 \\
(11.8 \%) \text { vs. } \\
87 / 650 \\
(13.4 \%)^{46}\end{array}$ & $\begin{array}{l}\text { AOR, } \\
1.31 \\
(95 \% \\
\mathrm{Cl}, 0.55 \\
\text { to } \\
2.32)^{46}\end{array}$ & $\begin{array}{l}1 \text { cohort, } \\
N=794^{46}\end{array}$ & $\begin{array}{l}\text { Moderate study } \\
\text { limitations, serious } \\
\text { imprecision (wide } \\
\text { Cls spanning the } \\
\text { null, few events), } \\
\text { consistency } \\
\text { unknown }\end{array}$ & Insufficient \\
\hline $\begin{array}{l}\text { Pregnant women } \\
\text { with a psychiatric } \\
\text { disorder }\end{array}$ & $\begin{array}{l}\text { Benzodiazepine Preeclampsia } \\
\text { exposure } \\
\text { during } \\
\text { pregnancy vs. } \\
\text { unexposed to } \\
\text { benzodiazepine } \\
\text { use during } \\
\text { pregnancy }\end{array}$ & $\begin{array}{l}30 / 144 \\
(20.8 \%) \text { vs. } \\
118 / 650 \\
(18.2 \%)^{46}\end{array}$ & $\begin{array}{l}\text { AOR, } \\
0.81 \\
(95 \% \\
\mathrm{Cl}, 0.43 \\
\text { to } \\
1.52)^{46}\end{array}$ & $\begin{array}{l}1 \text { cohort, } \\
\mathrm{N}=794^{46}\end{array}$ & $\begin{array}{l}\text { Moderate study } \\
\text { limitations, serious } \\
\text { imprecision (wide } \\
\text { Cls spanning the } \\
\text { null, few events), } \\
\text { consistency } \\
\text { unknown }\end{array}$ & Insufficient \\
\hline
\end{tabular}

$\mathrm{AOR}=$ adjusted odds ratio; $\mathrm{CI}=$ confidence interval; $\mathrm{KQ}=$ Key Question; $\mathrm{N}=$ number; $\mathrm{NICU}=$ neonatal intensive care unit; $\mathrm{RRR}=$ relative risk reduction; vs. $=$ versus.

The evidence for anxiolytics is insufficient to draw conclusions on preeclampsia. Regarding fetal harms, the evidence suggests that benzodiazepine exposure during pregnancy may be associated with an increased risk of NICU admission when compared with no exposure to benzodiazepines among women with a history of psychiatric disorders. ${ }^{46}$ The authors noted several differences between the comparison arms and the results adjusted for these confounders, but residual confounding cannot be ruled out. Additionally, NICU admission ${ }^{147}$ may be confounded by provider knowledge of antenatal exposure to psychotropic medications and hospital protocols. Until recently, neonatal abstinence syndrome due to prenatal exposure to opiates or benzodiazepines was typically treated in the neonatal intensive care unit. In an analysis of discharges from 23 tertiary care pediatric hospitals between January 2013 and March 2016, 93 percent of infants receiving pharmacologic treatment and 72 percent of infants receiving nonpharmacologic treatment were admitted to the NICU. ${ }^{147}$

\section{Hypnotic Sedatives}

\section{Overview}

- The evidence on hypnotic sedatives (zolpidem and zopiclone) during pregnancy is insufficient to judge the risk of harms for maternal (hypertension/preeclampsia) or child outcomes (preterm delivery, major congenital anomalies, NICU admission, respiratory difficulties, lethargy, hypotonia, Apgar scores)

\section{Detailed Results}

Two publications reported on other medications. ${ }^{71,92}$ These publications drew from two cohorts (one from THIN ${ }^{71}$ and one from a cohort based in the United States ${ }^{92}$ ). Both were nonrandomized studies and rated as high risk of bias. ${ }^{71,92}$

Both publications focused on exposure during pregnancy. One compared zopiclone exposure to untreated depression or anxiety, ${ }^{71}$ and one compared zolpidem exposure to untreated psychiatric illness. $^{92}$ 
The evidence for other medications, is insufficient to judge the risk of maternal harms (preterm delivery, hypertension/preeclampsia) or child outcomes (major congenital anomalies, NICU admission, respiratory difficulties, lethargy, hypotonia, Apgar score) (Table B-18).

Table B-18. Strength of evidence for harms outcomes: Other medications versus no treatment

\begin{tabular}{|c|c|c|c|c|c|c|c|}
\hline Population & $\begin{array}{l}\text { Intervention and } \\
\text { Comparator }\end{array}$ & Outcome & $\begin{array}{l}\text { Incidence or } \\
\text { Mean Effect } \\
\text { by Arm }\end{array}$ & Results & $\begin{array}{l}\text { Study } \\
\text { Design } \\
\text { and } \\
\text { Sample } \\
\text { Size } \\
\end{array}$ & $\begin{array}{l}\text { Factors That } \\
\text { Affect the } \\
\text { Strength of } \\
\text { Evidence }\end{array}$ & $\begin{array}{l}\text { Overall Evidence } \\
\text { Strength } \\
\text { (Direction of } \\
\text { Effect) }\end{array}$ \\
\hline $\begin{array}{l}\text { Women with } \\
\text { psychiatric } \\
\text { illness }\end{array}$ & $\begin{array}{l}\text { Zolpidem exposure } \\
\text { during pregnancy } \\
\text { vs. unexposed to } \\
\text { zolpidem during } \\
\text { pregnancy }\end{array}$ & $\begin{array}{l}\text { Hypertension/ } \\
\text { Preeclampsia }\end{array}$ & $\begin{array}{l}3 / 45(6.7 \%) \\
\text { vs. } 4 / 45 \\
(8.9 \%)^{92}\end{array}$ & $\begin{array}{l}\text { NR, NS based on } \\
\text { multivariate } \\
\text { conditional logistic } \\
\text { regression, } p= \\
1.00^{92}\end{array}$ & $\begin{array}{l}1 \text { cohort, } \\
\mathrm{n}=90^{92}\end{array}$ & $\begin{array}{l}\text { Imprecise, } \\
\text { consistent, } \\
\text { high risk of } \\
\text { bias }^{92}\end{array}$ & Insufficient \\
\hline $\begin{array}{l}\text { Women with } \\
\text { psychiatric } \\
\text { illness }\end{array}$ & $\begin{array}{l}\text { Zolpidem exposure } \\
\text { during pregnancy } \\
\text { vs. unexposed to } \\
\text { zolpidem during } \\
\text { pregnancy }\end{array}$ & $\begin{array}{l}\text { Preterm } \\
\text { delivery }\end{array}$ & $\begin{array}{l}12 / 45 \\
(26.7 \%) \text { vs. } \\
6 / 45 \\
(13.3 \%)^{92}\end{array}$ & $\begin{array}{l}\text { NR, NS based on } \\
\text { multivariate } \\
\text { conditional logistic } \\
\text { regression, } \mathrm{p} \\
<.18^{92} \\
\end{array}$ & $\begin{array}{l}1 \text { cohort, } \\
\mathrm{n}=90^{92}\end{array}$ & $\begin{array}{l}\text { Imprecise, } \\
\text { consistent, } \\
\text { high risk of } \\
\text { bias }^{92}\end{array}$ & Insufficient \\
\hline $\begin{array}{l}\text { Women with } \\
\text { psychiatric } \\
\text { illness }\end{array}$ & $\begin{array}{l}\text { Zolpidem exposure } \\
\text { during pregnancy } \\
\text { vs. unexposed to } \\
\text { zolpidem during } \\
\text { pregnancy }\end{array}$ & Hypotonia & $\begin{array}{l}0 / 45(0.0 \%) \\
\text { vs. } 4 / 45 \\
(8.9 \%)^{92}\end{array}$ & $\begin{array}{l}\mathrm{NR}, \mathrm{NS} \text { based on } \\
\text { multivariate } \\
\text { conditional logistic } \\
\text { regression, } \mathrm{p} \\
<.13^{92} \\
\end{array}$ & $\begin{array}{l}1 \text { cohort, } \\
\mathrm{n}=90^{92}\end{array}$ & $\begin{array}{l}\text { Imprecise, } \\
\text { consistent, } \\
\text { high risk of } \\
\text { bias }^{92}\end{array}$ & Insufficient \\
\hline $\begin{array}{l}\text { Women with } \\
\text { psychiatric } \\
\text { illness }\end{array}$ & $\begin{array}{l}\text { Zolpidem exposure } \\
\text { during pregnancy } \\
\text { vs. unexposed to } \\
\text { zolpidem during } \\
\text { pregnancy }\end{array}$ & Lethargy & $\begin{array}{l}1 / 45(2.2 \%) \\
\text { vs. } 4 / 45 \\
(8.9 \%)^{92}\end{array}$ & $\begin{array}{l}\text { NR, NS based on } \\
\text { multivariate } \\
\text { conditional logistic } \\
\text { regression, } p \\
<.38^{92} \\
\end{array}$ & $\begin{array}{l}1 \text { cohort, } \\
\mathrm{n}=90^{92}\end{array}$ & $\begin{array}{l}\text { Imprecise, } \\
\text { consistent, } \\
\text { high risk of } \\
\text { bias }^{92}\end{array}$ & Insufficient \\
\hline $\begin{array}{l}\text { Women with } \\
\text { psychiatric } \\
\text { illness }\end{array}$ & $\begin{array}{l}\text { Zolpidem exposure } \\
\text { during pregnancy } \\
\text { vs. unexposed to } \\
\text { zolpidem during } \\
\text { pregnancy }\end{array}$ & $\begin{array}{l}\text { NICU } \\
\text { admission }\end{array}$ & $\begin{array}{l}4 / 45(8.9 \%) \\
\text { vs. } 6 / 45 \\
(13.3 \%)^{92}\end{array}$ & $\begin{array}{l}\mathrm{NR}, \mathrm{NS} \text { based on } \\
\text { multivariate } \\
\text { conditional logistic } \\
\text { regression, } \mathrm{p} \\
<.76^{92}\end{array}$ & $\begin{array}{l}1 \text { cohort, } \\
\mathrm{n}=90^{92}\end{array}$ & $\begin{array}{l}\text { Imprecise, } \\
\text { consistent, } \\
\text { high risk of } \\
\text { bias }^{92}\end{array}$ & Insufficient \\
\hline $\begin{array}{l}\text { Women with } \\
\text { psychiatric } \\
\text { illness }\end{array}$ & $\begin{array}{l}\text { Zolpidem exposure } \\
\text { during pregnancy } \\
\text { vs. unexposed to } \\
\text { zolpidem during } \\
\text { pregnancy }\end{array}$ & $\begin{array}{l}\text { Respiratory } \\
\text { difficulty }\end{array}$ & $\begin{array}{l}10 / 45 \\
(22.2 \%) \text { vs. } \\
14 / 45 \\
(31.1 \%)^{92}\end{array}$ & $\begin{array}{l}\text { NR, NS based on } \\
\text { multivariate } \\
\text { conditional logistic } \\
\text { regression, } \mathrm{p} \\
<.49^{92} \\
\end{array}$ & $\begin{array}{l}1 \text { cohort, } \\
\mathrm{n}=90^{92}\end{array}$ & $\begin{array}{l}\text { Imprecise, } \\
\text { consistent, } \\
\text { high risk of } \\
\text { bias }^{92}\end{array}$ & Insufficient \\
\hline $\begin{array}{l}\text { Women with } \\
\text { psychiatric } \\
\text { illness }\end{array}$ & $\begin{array}{l}\text { Zolpidem exposure } \\
\text { during pregnancy } \\
\text { vs. unexposed to } \\
\text { zolpidem during } \\
\text { pregnancy }\end{array}$ & $\begin{array}{l}\text { Apgar score } \\
\text { at } 5 \text { minutes }\end{array}$ & $\mathrm{NR}^{92}$ & $\begin{array}{l}\mathrm{NR}, \mathrm{NS} \text { based on } \\
\text { multivariate } \\
\text { conditional logistic } \\
\text { regression, } \mathrm{p} \\
<.10^{92} \\
\end{array}$ & $\begin{array}{l}1 \text { cohort, } \\
\mathrm{n}=90^{92}\end{array}$ & $\begin{array}{l}\text { Imprecise, } \\
\text { consistent, } \\
\text { high risk of } \\
\text { bias }^{92}\end{array}$ & Insufficient \\
\hline $\begin{array}{l}\text { Women with } \\
\text { depression or } \\
\text { anxiety }\end{array}$ & $\begin{array}{l}\text { Zopiclone } \\
\text { exposure during } \\
\text { pregnancy vs. } \\
\text { unexposed women } \\
\text { with untreated } \\
\text { depression or } \\
\text { anxiety }\end{array}$ & $\begin{array}{l}\text { Major } \\
\text { congenital } \\
\text { anomalies }\end{array}$ & $\begin{array}{l}10 / 406 \\
(2.5 \%) \text { vs. } \\
518 / 19193 \\
(2.7 \%)^{71}\end{array}$ & $\begin{array}{l}\text { AOR: } 0.93(95 \% \\
\mathrm{Cl},(0.4 \text { to } 2.15)^{71}\end{array}$ & $\begin{array}{l}1 \text { cohort; } \\
n=19599^{71}\end{array}$ & $\begin{array}{l}\text { Imprecise, } \\
\text { consistent, } \\
\text { high risk of } \\
\text { bias }^{71}\end{array}$ & Insufficient \\
\hline
\end{tabular}

$\overline{\mathrm{AOR}}=$ adjusted odds ratio; $\mathrm{CI}=$ confidence interval; $\mathrm{N}=$ number; $\mathrm{NICU}=$ neonatal intensive care unit; $\mathrm{NR}=$ not reported; $\mathrm{NS}=$ not sufficient; vs. $=$ versus 


\section{SSRIs as a Drug Class}

\section{Overview}

- Exposure to SSRIs in pregnancy may be associated with an increased risk of low Apgar scores, respiratory distress, and primary persistent hypertension of the newborn (without cardiac anomaly or lung hypoplasia in full-term deliveries) compared with no treatment (low strength of evidence).

- Current or recent exposure (at or up to 1 month prior to delivery) may be associated with an increased risk of postpartum hemorrhage; the evidence for past exposure (1 to 5 months prior to delivery) or undefined exposure is insufficient.

- Exposure to SSRIs in pregnancy may be associated with an increased risk of depression in the child compared with no treatment (low strength of evidence).

- The evidence for SSRIs as a class during pregnancy is insufficient to judge the risk of harms for maternal (spontaneous abortion, gestational diabetes, hypertension, preeclampsia) or child outcomes (preterm birth, perinatal mortality, major congenital anomalies, cardiac anomalies, small for gestational age, low birth weight, birthweight, gestational age, neonatal convulsions, NICU admissions, extended hospital stay, feeding problems, jaundice, infant and child behavior and development, infant or child weight, autism spectrum disorder, attention-deficit/hyperactivity disorder, or anxiety).

\section{Detailed Results}

Thirty-two publications (31 studies) ${ }^{6-9,}, 27,54,57-61,63,64,66,69,70,72,74-76,79-84,88,91,93,95,104,106$ reported on harms associated with SSRI exposure in pregnancy compared with no exposure (Table B-19). Multiple publications reported on different outcomes from the same database (British Columba, ${ }^{27,81}$ Finland population register, ${ }^{59,63,66}$ Lombardy healthcare utilization database, ${ }^{54,58}$ THIN,,${ }^{72,79}$ and the U.S. Medicaid Analytic eXtract ${ }^{6,7,74,75}$ ) or potentially from the same population source (Danish Medical Birth Registry ${ }^{91}$ and Danish National Birth Cohort;, ${ }^{9,} 60$ one or more databases from Quebec including the Quebec Pregnancy/Children Cohort, the public health plan administrator database [Regie I'assurance maladie du Quebec or RAMQ], the hospitalization database [Med-Echo], and the Quebec birth and death registries ${ }^{57,64,70,83,93}$ ). Other cohorts represented by single publications include the Generation R study ${ }^{61}{ }^{1}$ the Lifestyle During Pregnancy Study ${ }^{69}$ the Norwegian Mother and Child Cohort Study (MoBa), ${ }^{8}$ the Slone Epidemiology Center Birth Defects Study, ${ }^{84}$ the Women's and Children's Health Network, ${ }^{80}$ a Kaiser Permanente Washington cohort, ${ }^{104}$ the National Birth Defects Prevention Study, ${ }^{106}$ and unnamed cohorts. ${ }^{76,82,88,95}$ 
Table B-19. Strength of evidence for harms: SSRIs versus no treatment

\begin{tabular}{|c|c|c|c|c|c|c|c|}
\hline Population & $\begin{array}{l}\text { Intervention } \\
\text { and } \\
\text { Comparator }\end{array}$ & Outcome & $\begin{array}{l}\text { Incidence or } \\
\text { Mean Effect } \\
\text { by Arm }\end{array}$ & Results & $\begin{array}{l}\text { Study } \\
\text { Design and } \\
\text { Sample Size }\end{array}$ & $\begin{array}{l}\text { Factors That } \\
\text { Affect the } \\
\text { Strength of } \\
\text { Evidence }\end{array}$ & $\begin{array}{l}\text { Overall } \\
\text { Evidence } \\
\text { Strength } \\
\text { (Direction of } \\
\text { Effect) } \\
\end{array}$ \\
\hline $\begin{array}{l}\text { Depressed } \\
\text { women }\end{array}$ & $\begin{array}{l}\text { SSRI } \\
\text { exposure in } \\
\text { pregnancy } \\
\text { vs. } \\
\text { unexposed } \\
\text { women with } \\
\text { depression }\end{array}$ & $\begin{array}{l}\text { Spontaneous } \\
\text { abortion }\end{array}$ & $\begin{array}{l}93 / 938(9.9 \%) \\
\text { vs. } 720 / 8,877 \\
(8.1 \%)^{64} \\
1,539 / \\
10,312(14.9) \\
\text { vs. } 442 / 3,647 \\
(12.1 \%)^{79}\end{array}$ & $\begin{array}{l}\text { ARR, } 1.2(95 \% \\
\mathrm{Cl}, 0.94 \text { to } \\
1.5)^{64} \text { and } \\
1.4(99 \% \mathrm{Cl}, \\
1.2 \text { to } 1.7)^{79}\end{array}$ & $\begin{array}{l}2 \text { cohorts; } \\
n=23,774^{64,79}\end{array}$ & $\begin{array}{l}\text { Moderate } \\
\text { study } \\
\text { limitations } \\
\text { (one high risk } \\
\text { of bias } \\
\text { study, }{ }^{79} \text { ) } \\
\text { imprecise } \\
\text { (wide Cls), } \\
\text { consistent }\end{array}$ & Insufficient \\
\hline $\begin{array}{l}\text { Psychiatric } \\
\text { disorder } \\
\text { during } \\
\text { pregnancy or } \\
\text { exposed to } \\
\text { SSRIs }\end{array}$ & $\begin{array}{l}\text { SSRI } \\
\text { exposure } \\
\text { during } \\
\text { pregnancy } \\
\text { vs. } \\
\text { unexposed } \\
\text { women with a } \\
\text { psychiatric } \\
\text { during } \\
\text { pregnancy }\end{array}$ & $\begin{array}{l}\text { Bleeding } \\
\text { during and } \\
\text { after delivery }\end{array}$ & $\begin{array}{l}520 / 15,729 \\
(3.3 \%) \text { vs. } \\
342 / 9,652 \\
(3.5 \%)^{66}\end{array}$ & $\begin{array}{l}\text { AOR, } 0.83 \\
(95 \% \mathrm{Cl}, 0.71 \\
\text { to } 0.96)^{66} \\
\text { AOR, for } \\
\text { exposure in the } \\
\text { first trimester: } \\
0.82(95 \% \mathrm{Cl} \text {, } \\
0.69 \text { to } 0.99) \\
\text { AOR, for } \\
\text { exposure in the } \\
\text { second and/or } \\
\text { third trimesters: } \\
0.84 \text { (95\% Cl, } \\
0.71 \text { to } 1.00)\end{array}$ & $\begin{array}{l}1 \text { cohort, } \\
n=104,841^{66}\end{array}$ & $\begin{array}{l}\text { High study } \\
\text { limitations } \\
\text { (high risk of } \\
\text { bias }^{66} \text { ), } \\
\text { precise, } \\
\text { consistency }\end{array}$ & Insufficient \\
\hline $\begin{array}{l}\text { Mood or } \\
\text { anxiety } \\
\text { disorder }\end{array}$ & $\begin{array}{l}\text { Current SSRI } \\
\text { exposure } \\
\text { (exposure } \\
\text { during } \\
\text { delivery) vs. } \\
\text { unexposed } \\
\text { with mood or } \\
\text { anxiety } \\
\text { disorder }\end{array}$ & $\begin{array}{l}\text { Postpartum } \\
\text { hemorrhage }\end{array}$ & $\begin{array}{l}503 / 12,710 \\
(3.96 \%) \text { vs. } \\
1,896 / 69,044 \\
(2.75 \%)^{74}\end{array}$ & $\begin{array}{l}\text { ARR, } 1.47 \\
(95 \% \mathrm{Cl}, 1.33 \\
\text { to } 1.62)^{74}\end{array}$ & $\begin{array}{l}1 \text { cohort, } \\
n=81,754^{74}\end{array}$ & $\begin{array}{l}\text { Moderate } \\
\text { study } \\
\text { limitations, } \\
\text { precise, } \\
\text { consistency } \\
\text { unknown }\end{array}$ & $\begin{array}{l}\text { Low for } \\
\text { harms for } \\
\text { current } \\
\text { exposure with } \\
\text { SSRls }\end{array}$ \\
\hline $\begin{array}{l}\text { Mood or } \\
\text { anxiety } \\
\text { disorder }\end{array}$ & $\begin{array}{l}\text { Recent SSRI } \\
\text { exposure } \\
\text { (within } 1 \\
\text { month before } \\
\text { delivery) vs. } \\
\text { unexposed } \\
\text { with mood or } \\
\text { anxiety } \\
\text { disorder }\end{array}$ & $\begin{array}{l}\text { Postpartum } \\
\text { hemorrhage }\end{array}$ & $\begin{array}{l}196 / 6,096 \\
(3.2 \%) \text { vs. } \\
1,896 / \\
69,044 \\
(2.75 \%)^{74}\end{array}$ & $\begin{array}{l}\text { ARR, } 1.19 \\
(95 \% \mathrm{Cl}, 1.03 \\
\text { to } 1.38)^{74}\end{array}$ & $\begin{array}{l}1 \text { cohort, } n= \\
75,140^{74}\end{array}$ & $\begin{array}{l}\text { Moderate } \\
\text { study } \\
\text { limitations, } \\
\text { precise, } \\
\text { consistency } \\
\text { unknown }\end{array}$ & $\begin{array}{l}\text { Low for } \\
\text { harms for } \\
\text { recent } \\
\text { exposure with } \\
\text { SSRIs }\end{array}$ \\
\hline $\begin{array}{l}\text { Mood or } \\
\text { anxiety } \\
\text { disorder }\end{array}$ & $\begin{array}{l}\text { Past SSRI } \\
\text { exposure (1 } \\
\text { to } 5 \text { months } \\
\text { before } \\
\text { delivery) vs. } \\
\text { unexposed } \\
\text { with mood or } \\
\text { anxiety } \\
\text { disorder }\end{array}$ & $\begin{array}{l}\text { Postpartum } \\
\text { hemorrhage }\end{array}$ & $\begin{array}{l}264 / 10,416 \\
(2.5 \%) \text { vs. } \\
1,896 / \\
69,044 \\
(2.75 \%)^{74}\end{array}$ & $\begin{array}{l}\text { ARR, } 0.93 \\
(95 \% \mathrm{Cl}, 0.82 \\
\text { to } 1.06)^{74}\end{array}$ & $\begin{array}{l}1 \text { cohort, } n= \\
69,984^{74}\end{array}$ & $\begin{array}{l}\text { Moderate } \\
\text { study } \\
\text { limitations, } \\
\text { imprecision } \\
\text { (wide Cls } \\
\text { spanning the } \\
\text { null), } \\
\text { consistency } \\
\text { unknown }\end{array}$ & Insufficient \\
\hline
\end{tabular}




\begin{tabular}{|c|c|c|c|c|c|c|c|}
\hline Population & $\begin{array}{l}\text { Intervention } \\
\text { and } \\
\text { Comparator }\end{array}$ & Outcome & $\begin{array}{l}\text { Incidence or } \\
\text { Mean Effect } \\
\text { by Arm }\end{array}$ & Results & $\begin{array}{l}\text { Study } \\
\text { Design and } \\
\text { Sample Size }\end{array}$ & $\begin{array}{l}\text { Factors That } \\
\text { Affect the } \\
\text { Strength of } \\
\text { Evidence }\end{array}$ & $\begin{array}{l}\text { Overall } \\
\text { Evidence } \\
\text { Strength } \\
\text { (Direction of } \\
\text { Effect) }\end{array}$ \\
\hline $\begin{array}{l}\text { Depressed, } \\
\text { psychiatric } \\
\text { disorders, or } \\
\text { discontinued } \\
\text { SSRIs during } \\
\text { pregnancy } \\
\text { exposed to } \\
\text { SSRIs }\end{array}$ & $\begin{array}{l}\text { SSRI } \\
\text { exposure } \\
\text { during } \\
\text { pregnancy } \\
\text { vs. no } \\
\text { exposure } \\
\text { (exposure } \\
\text { prior to } \\
\text { pregnancy or } \\
\text { depressed or } \\
\text { with } \\
\text { psychiatric } \\
\text { disorder) }\end{array}$ & Preterm birth & $\begin{array}{l}741 / 15,729 \\
(4.7 \%) \text { vs. } \\
515 / 9,652 ; \\
(5.3 \%)^{66} \\
17 / 192(8.8 \%) \\
\text { vs. } 415 / 5,710 \\
(7.3 \%) ; 4 \\
55 / 221(24.9 \%) \\
\text { vs. } 185 / 1,566 \\
(11.8 \%)^{80} 3 / 37 \\
(8.11 \%) \text { vs. } \\
3 / 19 \\
(15.79 \%)^{95} \\
\\
\text { N=NR for two } \\
\text { publications }{ }^{27}, \\
58\end{array}$ & $\begin{array}{l}\text { Overall } 5 \text { of } 6 \\
\text { studies do not } \\
\text { report } \\
\text { increased risks } \\
\text { with SSRIs. } \\
\text { Prevalence, } \\
\text { AOR, ARRs } \\
\text { range from } \\
0.84^{66} \text { to } 2.68^{80} \\
\text { with Cls } \\
\text { spanning the } \\
\text { null in } 2 \text { of } 4 \\
\text { studies null } \\
\text { (one study } \\
\text { reported } \\
\text { higher odds in } \\
\text { the SSRI } \\
\text { group, }{ }^{80} \text { the } \\
\text { other reported } \\
\text { lower odds in } \\
\text { the SSRI } \\
\text { group }{ }^{66} ; \\
\text { difference in } \\
\text { incidence: } \\
0.007 \text { ( } 95 \% \mathrm{Cl} \text {, } \\
-0.018 \text { to } \\
0.034) ; 27 \mathrm{NR} \text {, } \\
\text { p=0.948 }\end{array}$ & $\begin{array}{l}\text { 6 cohorts; } \\
\mathrm{N}>33,666 \\
\mathrm{~N}=\mathrm{NR} \text { in one } \\
\left.\text { study }{ }^{27}\right)^{58,66} \\
80,84,95\end{array}$ & $\begin{array}{l}\text { High study } \\
\text { limitations ( } 5 \\
\text { high risk-of- } \\
\text { bias studies }{ }^{58,} \\
66,80,84,95 \text { ), } \\
\text { mostly } \\
\text { consistent, } \\
\text { imprecise } \\
\text { (wide Cls in } \\
\text { some } \\
\text { studies) }\end{array}$ & Insufficient \\
\hline $\begin{array}{l}\text { Exposure to } \\
\text { anti- } \\
\text { depressants } \\
\text { before } \\
\text { pregnancy or } \\
\text { exposed to } \\
\text { SSRIs during } \\
\text { pregnancy }\end{array}$ & $\begin{array}{l}\text { SSRI } \\
\text { exposure } \\
\text { during } \\
\text { pregnancy } \\
\text { vs. exposure } \\
\text { before but not } \\
\text { during } \\
\text { pregnancy }\end{array}$ & $\begin{array}{l}\text { Gestational } \\
\text { diabetes }\end{array}$ & $\begin{array}{l}126 / 1,403 \\
(8.90 \%) \text { vs. } \\
90 / 1,211 \\
(7.43 \%)^{104}\end{array}$ & $\begin{array}{l}\text { RR (inverse } \\
\text { probability of } \\
\text { treatment } \\
\text { weighted): } \\
1.10(95 \% \mathrm{Cl}, \\
0.84 \text { to } 1.45)^{104}\end{array}$ & $\begin{array}{l}1 \text { cohort, } \\
n=2,614^{104}\end{array}$ & $\begin{array}{l}\text { Moderate } \\
\text { study } \\
\text { limitations, } \\
\text { imprecise } \\
\text { (wide Cls), } \\
\text { consistency } \\
\text { unknown }\end{array}$ & Insufficient \\
\hline $\begin{array}{l}\text { Depressed } \\
\text { women }\end{array}$ & $\begin{array}{l}\text { SSRI } \\
\text { exposure } \\
\text { during } \\
\text { pregnancy } \\
\text { vs. } \\
\text { unexposed } \\
\text { women with } \\
\text { depression }\end{array}$ & Preeclampsia & $\begin{array}{l}303 / 5215 \\
(5.8 \%) \text { vs. } \\
222 / 4,661 \\
(4.8 \%) ;^{75} \\
105 / 3,169 \\
(3.3 \%) \text { vs. } \\
1569 / 65,392 \\
(2.4 \%)^{81}\end{array}$ & $\begin{array}{l}\text { ARR, } 1.21 \\
(95 \% \mathrm{Cl}, 1.02 \\
\text { to } 1.45)^{75} \text { but } \\
\text { bias corrected } \\
\mathrm{RR}=0.9 ; \mathrm{ARR}, \\
1.22(95 \% \mathrm{Cl} \text {, } \\
0.97 \text { to } 1.54^{81} \text {; } \\
\text { severe } \\
\text { preeclampsia: } \\
\mathrm{ARR}, 1.03 \text {, } \\
95 \% \mathrm{Cl}, 0.81 \\
\text { to } 1.18\end{array}$ & $\begin{array}{l}2 \text { cohorts, } \\
\mathrm{n}=78,437^{75,81}\end{array}$ & $\begin{array}{l}\text { Moderate } \\
\text { study } \\
\text { limitations } \\
\text { (both have } \\
\text { some risk-of- } \\
\text { bias } \\
\text { concerns), } \\
\text { imprecise } \\
\text { (wide Cls), } \\
\text { inconsistent }\end{array}$ & Insufficient \\
\hline
\end{tabular}




\begin{tabular}{|c|c|c|c|c|c|c|c|}
\hline Population & $\begin{array}{l}\text { Intervention } \\
\text { and } \\
\text { Comparator }\end{array}$ & Outcome & $\begin{array}{l}\text { Incidence or } \\
\text { Mean Effect } \\
\text { by Arm }\end{array}$ & Results & $\begin{array}{l}\text { Study } \\
\text { Design and } \\
\text { Sample Size }\end{array}$ & $\begin{array}{l}\text { Factors That } \\
\text { Affect the } \\
\text { Strength of } \\
\text { Evidence }\end{array}$ & $\begin{array}{l}\text { Overall } \\
\text { Evidence } \\
\text { Strength } \\
\text { (Direction of } \\
\text { Effect) }\end{array}$ \\
\hline $\begin{array}{l}\text { Psychiatric } \\
\text { disorder or } \\
\text { exposed to } \\
\text { SSRIs }\end{array}$ & $\begin{array}{l}\text { SSRI } \\
\text { exposure } \\
\text { during } \\
\text { pregnancy } \\
\text { vs. } \\
\text { unexposed } \\
\text { with } \\
\text { psychiatric } \\
\text { disorders }\end{array}$ & Hypertension & $\begin{array}{l}813 / 15,729 \\
(5.2 \%) \text { vs. } \\
434 / 9,652 \\
(4.5 \%)^{66}\end{array}$ & $\begin{array}{l}\text { AOR, } 1.1 \\
(95 \% \mathrm{Cl}, 0.97 \\
\text { to } 1.26)^{66}\end{array}$ & $\begin{array}{l}1 \text { cohort, } \\
n=25,381^{66}\end{array}$ & $\begin{array}{l}\text { High study } \\
\text { limitations } \\
\text { (high risk of } \\
\text { bias }^{66} \text { ) } \\
\text { imprecise } \\
\text { (wide Cls } \\
\text { spanning the } \\
\text { null), } \\
\text { consistency } \\
\text { unknown }\end{array}$ & Insufficient \\
\hline $\begin{array}{l}\text { Depressed or } \\
\text { anxious } \\
\text { women }\end{array}$ & $\begin{array}{l}\text { SSRI } \\
\text { exposure in } \\
\text { pregnancy } \\
\text { vs. } \\
\text { unexposed } \\
\text { women with } \\
\text { depression }\end{array}$ & $\begin{array}{l}\text { Perinatal } \\
\text { mortality }\end{array}$ & $\begin{array}{l}57 / 10312 \\
(0.6 \%) \text { vs. } \\
20 / 3,647 \\
(0.6 \%)^{79}\end{array}$ & $\begin{array}{l}\text { ARR, } 1.2(99 \% \\
\text { CI, } 0.6 \text { to } \\
2.3)^{79}\end{array}$ & $\begin{array}{l}1 \text { cohort, } \\
n=13,959^{79}\end{array}$ & $\begin{array}{l}\text { High study } \\
\text { limitations } \\
\text { (high study }^{\text {limitations }}{ }^{79} \text { ), } \\
\text { imprecise } \\
\text { (wide Cls), } \\
\text { consistency } \\
\text { unknown }\end{array}$ & Insufficient \\
\hline $\begin{array}{l}\text { History of } \\
\text { psychiatric } \\
\text { disorder or } \\
\text { depression or } \\
\text { SSRI- } \\
\text { exposed } \\
\text { women }\end{array}$ & $\begin{array}{l}\text { SSRI } \\
\text { exposure in } \\
\text { pregnancy } \\
\text { vs. } \\
\text { unexposed } \\
\text { women with } \\
\text { depression or } \\
\text { psychiatric } \\
\text { disorder }\end{array}$ & $\begin{array}{l}\text { Small for } \\
\text { gestational } \\
\text { age }\end{array}$ & $\begin{array}{l}\text { Varies across } \\
\text { studies from } \\
2.5 \% \text { to } 17.4 \% \\
\text { in the } \\
\text { treatment arm, } \\
\text { and } 2.5 \% \text { to } \\
14.7 \% \text { in the } \\
\text { control arm }{ }^{27,54,} \\
66,83,84\end{array}$ & $\begin{array}{l}\text { Five }{ }^{27,54,66,80,83} \\
\text { of six studies } \\
\text { report } \\
\text { nonsignificant } \\
\text { results } \\
\text { (adjusted } \\
\text { prevalence } \\
\text { ratios, ARR, } \\
\text { AOR, } \\
\text { difference in } \\
\text { incidence) with } \\
\text { Cls spanning } \\
\text { the null; one } \\
\text { study reported } \\
\text { AOR, of } 1.68 \\
(95 \% \text { CI, } 1.03 \\
\text { to } 2.74)^{84} \\
\text { ARR, varies by } \\
\text { trimester of } \\
\text { exposure from } \\
0.7 \text { to } 1.4 \text {, } \\
95 \% \text { CI, spans } \\
\text { the null } 83\end{array}$ & $\begin{array}{l}\text { cohort } \\
\text { studies, } 1 \\
\text { case-control, } \\
\text { n varies by } \\
\text { trimester, } \\
\mathrm{n}=43,185^{27,54,} \\
66,80,83,84 \\
\end{array}$ & $\begin{array}{l}\text { High study } \\
\text { limitations ( } 4 \\
\text { of } 6 \text { high risk- } \\
\text { of-bias } \\
\text { studies }^{54,66,80,} \\
\text { 84), imprecise } \\
\text { (wide Cls), } \\
\text { mostly } \\
\text { consistent }\end{array}$ & Insufficient \\
\hline $\begin{array}{l}\text { Use of } \\
\text { antidepressa } \\
\text { nts before or } \\
\text { during } \\
\text { pregnancy or } \\
\text { psychiatric } \\
\text { illness }\end{array}$ & $\begin{array}{l}\text { SSRI } \\
\text { exposure } \\
\text { during } \\
\text { pregnancy } \\
\text { vs. SSRI } \\
\text { exposure just } \\
\text { before but not } \\
\text { during } \\
\text { pregnancy or } \\
\text { psychiatric } \\
\text { illness with } \\
\text { no exposure }\end{array}$ & $\begin{array}{l}\text { Low birth } \\
\text { weight }\end{array}$ & $\begin{array}{l}\text { 42/221 }(19.0 \%) \\
\text { vs. } 150 / 1,566 \\
(9.6 \%) ; \\
\text { NR in one } \\
\text { study; } 58^{58} 436 \\
(11.11 \%) \text { vs. } \\
3 / 19 \\
(15.79 \%)^{95}\end{array}$ & $\begin{array}{l}\text { Adjusted } \\
\text { prevalence } \\
\text { ratio: } 1.1(95 \% \\
\mathrm{Cl}, 0.9 \text { to } \\
1.3)^{58} \\
\mathrm{AOR}, 2.26 \\
(95 \% \mathrm{Cl}, 1.31 \\
\text { to } 3.91) \\
\mathrm{NR}, \mathrm{p}=0.613^{95}\end{array}$ & $\begin{array}{l}3 \text { cohorts, } \\
\mathrm{N}>1,842,80,95 \\
\mathrm{~N}=\mathrm{NR} \text { in one } \\
\text { study }^{58}\end{array}$ & $\begin{array}{l}\text { High study } \\
\text { limitations } \\
\text { (high risk of } \\
\text { bias }^{58,80,95} \text { ), } \\
\text { imprecise } \\
\text { (wide Cls), } \\
\text { inconsistent }\end{array}$ & Insufficient \\
\hline
\end{tabular}




\begin{tabular}{|c|c|c|c|c|c|c|c|}
\hline Population & $\begin{array}{l}\text { Intervention } \\
\text { and } \\
\text { Comparator }\end{array}$ & Outcome & $\begin{array}{l}\text { Incidence or } \\
\text { Mean Effect } \\
\text { by Arm }\end{array}$ & Results & $\begin{array}{l}\text { Study } \\
\text { Design and } \\
\text { Sample Size }\end{array}$ & $\begin{array}{l}\text { Factors That } \\
\text { Affect the } \\
\text { Strength of } \\
\text { Evidence }\end{array}$ & $\begin{array}{l}\text { Overall } \\
\text { Evidence } \\
\text { Strength } \\
\text { (Direction of } \\
\text { Effect) }\end{array}$ \\
\hline $\begin{array}{l}\text { Exposed to } \\
\text { SSRIs during } \\
\text { pregnancy or } \\
\text { depressed }\end{array}$ & $\begin{array}{l}\text { SSRI } \\
\text { exposure } \\
\text { during } \\
\text { pregnancy } \\
\text { vs. untreated } \\
\text { depressed }\end{array}$ & Birthweight & NR & $\begin{array}{l}\text { Adjusted } \\
\text { difference }(\mathrm{g}) \text { : } \\
10(95 \% \mathrm{Cl} \text {, } \\
-43 \text { to } 70) \mathrm{p}- \\
\text { value } 0.72^{27}\end{array}$ & $\begin{array}{l}1 \text { cohort, } \\
N=15,685^{27}\end{array}$ & $\begin{array}{l}\text { Moderate } \\
\text { study } \\
\text { limitations, } \\
\text { imprecise } \\
\text { (wide Cls), } \\
\text { consistency } \\
\text { unknown }\end{array}$ & Insufficient \\
\hline $\begin{array}{l}\text { Exposed to } \\
\text { SSRIs during } \\
\text { pregnancy or } \\
\text { depressed }\end{array}$ & $\begin{array}{l}\text { SSRI } \\
\text { exposure } \\
\text { during } \\
\text { pregnancy } \\
\text { vs. untreated } \\
\text { depressed }\end{array}$ & $\begin{array}{l}\text { Gestational } \\
\text { age }\end{array}$ & NR & $\begin{array}{l}\text { Adjusted } \\
\text { difference } \\
\text { (weeks): }-0.14 \\
\text { (95\% Cl, }-0.34 \\
\text { to } 0.06 \text { ) p- } \\
\text { value } 0.18^{27}\end{array}$ & $\begin{array}{l}1 \text { cohort, } \\
\mathrm{N}=15,685^{27}\end{array}$ & $\begin{array}{l}\text { Moderate } \\
\text { study } \\
\text { limitations, } \\
\text { imprecise } \\
\text { (wide Cls), } \\
\text { consistency } \\
\text { unknown }\end{array}$ & Insufficient \\
\hline $\begin{array}{l}\text { History of } \\
\text { depression or } \\
\text { anxiety or } \\
\text { current or } \\
\text { past SSRI- } \\
\text { exposed } \\
\text { women }\end{array}$ & $\begin{array}{l}\text { Exposed to } \\
\text { SSRIs in } \\
\text { pregnancy } \\
\text { vs. } \\
\text { unexposed } \\
\text { women with } \\
\text { history of } \\
\text { depression, } \\
\text { anxiety or } \\
\text { prior } \\
\text { exposure }\end{array}$ & $\begin{array}{l}\text { Major } \\
\text { congenital } \\
\text { anomalies }\end{array}$ & $\begin{array}{l}279 / 2,327 \\
(12.0 \%) \text { vs. } \\
1,650 / 14,847 \\
(11.1 \%)^{57} ; \\
204 / 7,683 \\
(2.7 \%) \text { vs. } \\
380 / 13,432 \\
(2.8 \%)^{72} \\
208 / 4183 \\
(5.0 \%) \text { vs. } \\
36 / 806 \\
(4.5 \%)^{91}\end{array}$ & $\begin{array}{l}\text { Adjusted } \\
\text { prevalence } \\
\text { ratio: } 1.07 \\
(95 \% \mathrm{Cl}, 0.93 \\
\text { to } 1.22) ; 57 \\
\text { AOR, } 0.93 \\
(95 \% \mathrm{Cl}, 0.78 \\
\text { to } 1.11) ;^{72} \\
\text { adjusted effect } \\
\text { NR in one } \\
\text { study, } \mathrm{p}=0.9^{91}\end{array}$ & $\begin{array}{l}3 \text { cohorts, } \\
\mathrm{n}=43,299^{57,}, 72, \\
91\end{array}$ & $\begin{array}{l}\text { High study } \\
\text { limitations (all } \\
\text { risk of bias } \\
\text { studies), }{ }^{57,72,} \\
91 \text { imprecise } \\
\text { (wide Cls), } \\
\text { consistent }\end{array}$ & $\begin{array}{l}\text { Low for no } \\
\text { harms with } \\
\text { SSRIs }\end{array}$ \\
\hline $\begin{array}{l}\text { Psychiatric } \\
\text { disorder with } \\
\text { prescription } \\
\text { prior to } \\
\text { pregnancy }\end{array}$ & $\begin{array}{l}\text { Exposed to } \\
\text { SSRIs other } \\
\text { than } \\
\text { sertraline vs. } \\
\text { unexposed to } \\
\text { SSRls during } \\
\text { pregnancy } \\
\text { with a } \\
\text { psychiatric } \\
\text { disorder }\end{array}$ & $\begin{array}{l}\text { Major } \\
\text { congenital } \\
\text { anomalies }\end{array}$ & $\begin{array}{l}236 / 1,936 \\
(12.0 \%) \text { vs. } \\
1,651 / 14,868 \\
(11.1 \%)^{70}\end{array}$ & $\begin{array}{l}\text { ARR, } 1.08 \\
(95 \% \mathrm{Cl}, 0.93 \\
\text { to } 1.25)^{70}\end{array}$ & $\begin{array}{l}1 \text { cohort, } \\
N=16,831^{70}\end{array}$ & $\begin{array}{l}\text { High study } \\
\text { limitations } \\
(\text { high risk of } \\
\left.\text { bias }^{70}\right) \text {, } \\
\text { imprecise } \\
\text { (wide Cls), } \\
\text { consistency } \\
\text { unknown }\end{array}$ & Insufficient \\
\hline $\begin{array}{l}\text { Psychiatric } \\
\text { disorder with } \\
\text { prescription } \\
\text { prior to } \\
\text { pregnancy }\end{array}$ & $\begin{array}{l}\text { Exposed to } \\
\text { SSRIs other } \\
\text { than } \\
\text { paroxetine } \\
\text { vs. } \\
\text { unexposed to } \\
\text { SSRIs during } \\
\text { pregnancy } \\
\text { with a } \\
\text { psychiatric } \\
\text { disorder }\end{array}$ & $\begin{array}{l}\text { Major } \\
\text { congenital } \\
\text { anomalies }\end{array}$ & $\begin{array}{l}\text { Not applicable } \\
\text { for case- } \\
\text { control }\end{array}$ & $\begin{array}{l}\text { AOR, } 1.19 \\
(95 \% \mathrm{Cl}, 0.71 \\
\text { to } 1.97)^{93}\end{array}$ & $\begin{array}{l}1 \text { case- } \\
\text { control, N } \\
\mathrm{NR}^{93}\end{array}$ & $\begin{array}{l}\text { Medium } \\
\text { study } \\
\text { limitations, } \\
\text { imprecise } \\
\text { (wide Cls), } \\
\text { consistency } \\
\text { unknown }\end{array}$ & Insufficient \\
\hline
\end{tabular}




\begin{tabular}{|c|c|c|c|c|c|c|c|}
\hline Population & $\begin{array}{l}\text { Intervention } \\
\text { and } \\
\text { Comparator }\end{array}$ & Outcome & $\begin{array}{l}\text { Incidence or } \\
\text { Mean Effect } \\
\text { by Arm }\end{array}$ & Results & $\begin{array}{l}\text { Study } \\
\text { Design and } \\
\text { Sample Size }\end{array}$ & $\begin{array}{l}\text { Factors That } \\
\text { Affect the } \\
\text { Strength of } \\
\text { Evidence }\end{array}$ & $\begin{array}{l}\text { Overall } \\
\text { Evidence } \\
\text { Strength } \\
\text { (Direction of } \\
\text { Effect) }\end{array}$ \\
\hline $\begin{array}{l}\text { Depressed or } \\
\text { exposed to } \\
\text { SSRIs in } \\
\text { pregnancy }\end{array}$ & $\begin{array}{l}\text { Exposed to } \\
\text { SSRIs vs. } \\
\text { unexposed to } \\
\text { SSRIs during } \\
\text { pregnancy } \\
\text { with } \\
\text { depression or } \\
\text { unexposed to } \\
\text { SSRIs in } \\
\text { early } \\
\text { pregnancy }\end{array}$ & $\begin{array}{l}\text { Cardiac } \\
\text { anomalies }\end{array}$ & $\begin{array}{l}68 / 7683 \\
(0.9 \%) \text { vs. } \\
112 / 13,432 \\
(0.8 \%)^{72} \mathrm{NR} \\
\text { in second } \\
\text { study; }{ }^{7} \\
466 \\
\text { cases/341 } \\
\text { controls vs. } \\
149 \\
\text { cases/125 }^{\text {controls }^{106}}\end{array}$ & $\begin{array}{l}\text { Pooled OR, } \\
1.07(95 \% \mathrm{Cl} \text {, } \\
0.96 \text { to } 1.20) \text {, } \\
\mathrm{I}^{2}: 0 \% \\
\text { AOR, } 1.06 \\
(95 \% \mathrm{Cl}, 0.93 \\
\text { to } 1.22)^{7} \\
\text { AOR, } 1.04 \\
(95 \% \mathrm{Cl}, 0.76 \\
\text { to } 1.41)^{72} \\
\text { AOR, } 1.14 \\
(95 \% \mathrm{Cl}, 0.87 \\
\text { to } 1.51)^{106}\end{array}$ & $\begin{array}{l}2 \text { cohorts } 1 \\
\text { case control, } \\
\mathrm{N}>22,196 \\
(\mathrm{~N}=\mathrm{NR} \text { in one } \\
\left.\text { study }^{7}\right)^{72,106}\end{array}$ & $\begin{array}{l}\text { High study } \\
\text { limitations } \\
\text { (two high risk } \\
\text { of bias } \\
\text { studies }^{7,72} \text { ) } \\
\text { imprecise } \\
\text { (wide Cls), } \\
\text { consistent }\end{array}$ & Insufficient \\
\hline $\begin{array}{l}\text { Psychiatric } \\
\text { disorder with } \\
\text { prescription } \\
\text { prior to } \\
\text { pregnancy }\end{array}$ & $\begin{array}{l}\text { Exposed to } \\
\text { SSRIs other } \\
\text { than } \\
\text { sertraline vs. } \\
\text { unexposed to } \\
\text { SSRIs during } \\
\text { pregnancy } \\
\text { with a } \\
\text { psychiatric } \\
\text { disorder }\end{array}$ & $\begin{array}{l}\text { Cardiac } \\
\text { anomalies }\end{array}$ & $\begin{array}{l}51 / 1936 \\
(2.6 \%) \text { vs. } \\
344 / 14,868 \\
(2.3 \%)^{70}\end{array}$ & $\begin{array}{l}\text { ARR, } 1.08 \\
(95 \% \mathrm{Cl}, 0.93 \\
\text { to } 1.25)^{70}\end{array}$ & $\begin{array}{l}1 \text { cohort, } \\
\mathrm{N}=16,831^{70}\end{array}$ & $\begin{array}{l}\text { High study } \\
\text { limitations } \\
\text { (high risk of } \\
\text { bias }^{70} \text { ) } \\
\text { imprecise } \\
\text { (wide Cls } \\
\text { spanning the } \\
\text { null), } \\
\text { consistency } \\
\text { unknown }\end{array}$ & Insufficient \\
\hline $\begin{array}{l}\text { Exposed to } \\
\text { SSRIs during } \\
\text { pregnancy, } \\
\text { before } \\
\text { pregnancy, or } \\
\text { unexposed } \\
\text { with a } \\
\text { psychiatric } \\
\text { diagnosis } \\
\end{array}$ & $\begin{array}{l}\text { Exposed to } \\
\text { SSRIs during } \\
\text { pregnancy } \\
\text { vs. before } \\
\text { pregnancy or } \\
\text { unexposed } \\
\text { with a } \\
\text { psychiatric } \\
\text { diagnosis } \\
\end{array}$ & $\begin{array}{l}\text { Low Apgar } \\
\text { score }(<7 \text { at } 5 \\
\text { min) }\end{array}$ & $\begin{array}{l}28 / 2,664 \\
(1.1 \%) \text { vs. } \\
31 / 5,141 \\
(0.6 \%)^{54} \\
\\
376 / 15,729 \\
(2.4 \%) \text { vs. } \\
113 / 9,652 \\
(1.2 \%)^{66} \\
\end{array}$ & $\begin{array}{l}\text { Adjusted } \\
\text { prevalence } \\
\text { ratio: } 1.69 \\
(95 \% \mathrm{Cl}, 1.02 \\
\text { to } 2.79)^{54} \\
\text { AOR, } 1.68 \\
(95 \% \mathrm{Cl}, 1.34 \\
\text { to } 2.12)^{66}\end{array}$ & $\begin{array}{l}2 \text { cohorts, } \\
\mathrm{n}=33,186^{54,66}\end{array}$ & $\begin{array}{l}\text { High study } \\
\text { limitations (all } \\
\text { risk-of-bias } \\
\text { studies }^{54,66} \text { ), } \\
\text { precise, } \\
\text { consistent }\end{array}$ & $\begin{array}{l}\text { Low for } \\
\text { harms with } \\
\text { SSRIs }\end{array}$ \\
\hline $\begin{array}{l}\text { History of } \\
\text { SSRI- } \\
\text { exposure }\end{array}$ & $\begin{array}{l}\text { SSRI } \\
\text { exposure in } \\
\text { pregnancy } \\
\text { vs. women } \\
\text { who } \\
\text { discontinued } \\
\text { SSRIs }\end{array}$ & $\begin{array}{l}\text { Intrauterine } \\
\text { hypoxia and } \\
\text { birth asphyxia }\end{array}$ & $\begin{array}{l}94 / 2664 \\
(3.5 \%) \text { vs. } \\
124 / 5141 \\
(2.4 \%)^{54}\end{array}$ & $\begin{array}{l}\text { Adjusted } \\
\text { prevalence } \\
\text { ratio in all } \\
\text { women: } \\
1.39(95 \% \mathrm{Cl} \text {, } \\
1.07 \text { to } 1.81) \\
\\
\text { Adjusted } \\
\text { prevalence } \\
\text { ratio in women } \\
\text { with vaginal } \\
\text { deliveries in } \\
\text { the third } \\
\text { trimester: } \\
1.70(95 \% \mathrm{Cl} \text {, } \\
1.23 \text { to } 2.33)^{54}\end{array}$ & $\begin{array}{l}1 \text { cohort, } n= \\
7805^{54}\end{array}$ & $\begin{array}{l}\text { High study } \\
\text { limitations } \\
\text { (high risk-of- } \\
\text { bias study }{ }^{54} \\
\text { precise, } \\
\text { consistency } \\
\text { unknown }\end{array}$ & $\begin{array}{l}\text { Low for } \\
\text { harms with } \\
\text { SSRIs }\end{array}$ \\
\hline
\end{tabular}




\begin{tabular}{|c|c|c|c|c|c|c|c|}
\hline Population & $\begin{array}{l}\text { Intervention } \\
\text { and } \\
\text { Comparator }\end{array}$ & Outcome & $\begin{array}{l}\text { Incidence } \\
\text { or Mean } \\
\text { Effect by } \\
\text { Arm }\end{array}$ & Results & $\begin{array}{l}\text { Study } \\
\text { Design and } \\
\text { Sample Size }\end{array}$ & $\begin{array}{l}\text { Factors That } \\
\text { Affect the } \\
\text { Strength of } \\
\text { Evidence }\end{array}$ & $\begin{array}{l}\text { Overall } \\
\text { Evidence } \\
\text { Strength } \\
\text { (Direction of } \\
\text { Effect) }\end{array}$ \\
\hline $\begin{array}{l}\text { History of } \\
\text { psychiatric } \\
\text { disorder or } \\
\text { depression or } \\
\text { SSRI- } \\
\text { exposed } \\
\text { women }\end{array}$ & $\begin{array}{l}\text { SSRI } \\
\text { exposure in } \\
\text { pregnancy } \\
\text { vs. } \\
\text { unexposed } \\
\text { women with } \\
\text { depression or } \\
\text { psychiatric } \\
\text { disorder }\end{array}$ & $\begin{array}{l}\text { Respiratory } \\
\text { conditions } \\
\text { (respiratory } \\
\text { conditions of } \\
\text { newborns } \\
\text { other than } \\
\text { intrauterine } \\
\text { hypoxia and } \\
\text { birth } \\
\text { asphyxia, }{ }^{54} \\
\text { undefined } \\
\text { breathing } \\
\text { problems, }{ }^{66} \\
\text { respiratory }^{\text {distress }}{ }^{27} \text { ) }\end{array}$ & $\begin{array}{l}\text { Ranges } \\
\text { from } 4.3 \% \text { to } \\
4.9 \% \text { in the } \\
\text { treatment } \\
\text { arm and } \\
3.1 \% \text { to } 3.2 \\
\text { in the } \\
\text { control } \\
\text { arm }{ }^{54,66 ;} \mathrm{NR} \\
\text { in one } \\
\text { study }^{27}\end{array}$ & $\begin{array}{l}\text { All three } 27,54,66 \\
\text { studies reported } \\
\text { increased risk. } \\
\text { Adjusted } \\
\text { prevalence } \\
\text { ratios and AOR, } \\
\text { range from } 1.37 \\
\text { to } 1.4^{54,66}\end{array}$ & $\begin{array}{l}3 \text { cohort } \\
\text { studies, } \\
n>33,186 \\
(N=N R \text { in one } \\
{\left.\text { study }{ }^{27}\right)^{27,54,66}}\end{array}$ & $\begin{array}{l}\text { High study } \\
\text { limitations ( } 2 \\
\text { of } 3 \text { are high } \\
\text { risk-of-bias } \\
\text { studies, }{ }^{54,66} \\
\text { precise, } \\
\text { consistent }\end{array}$ & $\begin{array}{l}\text { Low for } \\
\text { harms with } \\
\text { SSRIs }\end{array}$ \\
\hline $\begin{array}{l}\text { Exposed to } \\
\text { SSRIs during } \\
\text { pregnancy or } \\
\text { unexposed } \\
\text { with previous } \\
\text { exposure or } \\
\text { depressed }\end{array}$ & $\begin{array}{l}\text { Exposed to } \\
\text { SSRIs during } \\
\text { pregnancy } \\
\text { vs. } \\
\text { unexposed } \\
\text { depressed or } \\
\text { exposed prior } \\
\text { to but not } \\
\text { during } \\
\text { pregnancy }\end{array}$ & $\begin{array}{l}\text { Neonatal } \\
\text { convulsions }\end{array}$ & $\begin{array}{l}9 / 2,664 \\
(0.3 \%) \text { vs. } \\
7 / 5,141 \\
(0.1 \%) ; 54 \\
\text { NR in one } \\
\text { study }^{27}\end{array}$ & $\begin{array}{l}\text { Adjusted } \\
\text { prevalence } \\
\text { ratio: } 2.28 \text { (95\% } \\
\mathrm{Cl}, 0.87 \text { to } \\
5.97)^{54} \\
\text { Difference in } \\
\text { incidence: } \\
0.00077 \text { (95\% } \\
\mathrm{Cl},-0.001 \text { to } \\
0.0036) \text { p-value } \\
0.3^{27}\end{array}$ & $\begin{array}{l}2 \text { cohort } \\
\text { studies, } \\
n>7,805 \\
(\mathrm{~N}=\mathrm{NR} \text { in one } \\
\left.\text { study }^{27}\right)^{27,54} \\
\end{array}$ & $\begin{array}{l}\text { High study } \\
\text { limitations ( } 1 \\
\text { of } 2 \text { studies is } \\
\text { high risk of } \\
\text { bias }^{54} \text { ) } \\
\text { imprecise } \\
\text { (wide Cls } \\
\text { spanning the } \\
\text { null), } \\
\text { consistent }\end{array}$ & Insufficient \\
\hline $\begin{array}{l}\text { Exposed to } \\
\text { SSRIs during } \\
\text { pregnancy or } \\
\text { unexposed } \\
\text { with a } \\
\text { psychiatric } \\
\text { diagnosis }\end{array}$ & $\begin{array}{l}\text { Exposed to } \\
\text { SSRIs during } \\
\text { pregnancy } \\
\text { vs. } \\
\text { unexposed } \\
\text { with a } \\
\text { psychiatric } \\
\text { diagnosis }\end{array}$ & NICU & $\begin{array}{l}2,405 / \\
15,729 \\
(15.3 \%) \text { vs. } \\
1,160 / 9,652 \\
(12.0 \%)^{; 66} \\
7 / 33 \\
(21.21 \%) \text { vs. } \\
5 / 19 \\
(26.32 \%)^{95} \\
\end{array}$ & $\begin{array}{l}\text { Adjusted OR, } \\
1.24(95 \% \mathrm{Cl}, \\
1.14 \text { to } 1.35) ; 66 \\
\mathrm{NR}, \mathrm{p}=0.816^{95}\end{array}$ & $\begin{array}{l}2 \text { cohorts, } \\
\mathrm{n}=25,433^{66,95}\end{array}$ & $\begin{array}{l}\text { High study } \\
\text { limitations } \\
\text { (high risk of } \\
\text { bias }^{66,95} \text { ) } \\
\text { precise, } \\
\text { inconsistent }\end{array}$ & Insufficient \\
\hline $\begin{array}{l}\text { Exposed to } \\
\text { SSRIs during } \\
\text { pregnancy or } \\
\text { unexposed } \\
\text { depressed or } \\
\text { with a } \\
\text { psychiatric } \\
\text { illness }\end{array}$ & $\begin{array}{l}\text { Exposed to } \\
\text { SSRIs during } \\
\text { pregnancy } \\
\text { vs. } \\
\text { unexposed } \\
\text { depressed } \\
\text { during } \\
\text { pregnancy or } \\
\text { with a } \\
\text { psychiatric } \\
\text { illness }\end{array}$ & $\begin{array}{l}\text { Extended } \\
\text { hospital stay } \\
(>3 \text { days for } \\
\text { vaginal birth, }{ }^{27} \\
\left.>7 \text { days }^{66}\right)\end{array}$ & $\begin{array}{l}1,315 / 15,72 \\
9(8.4 \%) \text { vs. } \\
8,21 / 9,652 \\
(8.5 \%) ; 66 \\
20 / 221 \\
(9.0 \%) \text { vs. } \\
75 / 1,566 \\
(4.8 \%)^{; 80} \\
\text { NR in one } \\
\text { study }^{27}\end{array}$ & $\begin{array}{l}\text { Inconsistent } \\
\text { results } \\
\text { spanning } \\
\text { benefits, harms, } \\
\text { and no effect } \\
\text { AOR, } 0.89 \\
\text { (95\% } \mathrm{Cl}, 0.8 \text { to } \\
0.99) ; 6 \mathrm{AOR}, \\
1.93 \text { (95\% Cl, } \\
1.11 \text { to } 3.36) \\
\text { Difference in } \\
\text { incidence: } \\
0.035 \text { (95\% Cl, } \\
-0.005 \text { to } \\
0.072) \text {, p-value } \\
0.0827\end{array}$ & $\begin{array}{l}3 \text { cohort } \\
\text { studies, } \\
n>27,168(\mathrm{~N}= \\
\text { NR in one } \\
\left.\text { study }^{27}\right)^{66,80}\end{array}$ & $\begin{array}{l}\text { High study } \\
\text { limitations ( } 2 \\
\text { of } 3 \text { studies } \\
\text { are high risk } \\
\text { of bias }{ }^{66,80} \text { ) } \\
\text { imprecise, } \\
\text { inconsistent }\end{array}$ & Insufficient \\
\hline
\end{tabular}




\begin{tabular}{|c|c|c|c|c|c|c|c|}
\hline Population & $\begin{array}{l}\text { Intervention } \\
\text { and } \\
\text { Comparator }\end{array}$ & Outcome & $\begin{array}{l}\text { Incidence } \\
\text { or Mean } \\
\text { Effect by } \\
\text { Arm }\end{array}$ & Results & $\begin{array}{l}\text { Study } \\
\text { Design and } \\
\text { Sample Size }\end{array}$ & $\begin{array}{l}\text { Factors That } \\
\text { Affect the } \\
\text { Strength of } \\
\text { Evidence }\end{array}$ & $\begin{array}{l}\text { Overall } \\
\text { Evidence } \\
\text { Strength } \\
\text { (Direction of } \\
\text { Effect) }\end{array}$ \\
\hline $\begin{array}{l}\text { Exposed to } \\
\text { SSRIs during } \\
\text { pregnancy or } \\
\text { unexposed } \\
\text { depressed }\end{array}$ & $\begin{array}{l}\text { Exposed to } \\
\text { SSRIs during } \\
\text { pregnancy } \\
\text { vs. } \\
\text { unexposed } \\
\text { depressed } \\
\text { during } \\
\text { pregnancy }\end{array}$ & $\begin{array}{l}\text { Feeding } \\
\text { problems }\end{array}$ & NR & $\begin{array}{l}\text { Difference in } \\
\text { incidence: } \\
0.011(95 \% \mathrm{Cl} \text {, } \\
\text {-0.009 to } 0.03) \text {, } \\
\text { p-value } 0.28^{27}\end{array}$ & $\begin{array}{l}1 \text { cohort, n } \\
\text { NR }^{27}\end{array}$ & $\begin{array}{l}\text { Moderate } \\
\text { study } \\
\text { limitations, } \\
\text { imprecise } \\
\text { (Cls spanning } \\
\text { the null), } \\
\text { consistency } \\
\text { unknown }\end{array}$ & Insufficient \\
\hline $\begin{array}{l}\text { Exposed to } \\
\text { SSRIs during } \\
\text { pregnancy or } \\
\text { unexposed } \\
\text { depressed }\end{array}$ & $\begin{array}{l}\text { Exposed to } \\
\text { SSRIs during } \\
\text { pregnancy } \\
\text { vs. } \\
\text { unexposed } \\
\text { depressed } \\
\text { during } \\
\text { pregnancy }\end{array}$ & Jaundice & NR & $\begin{array}{l}\text { Difference } \\
\text { Incidence: } 0.01 \\
(95 \% \mathrm{Cl},-0.02 \\
\text { to } 0.04), \mathrm{p}- \\
\text { value } 0.45^{27}\end{array}$ & $\begin{array}{l}1 \text { cohort, n } \\
\text { NR }^{27}\end{array}$ & $\begin{array}{l}\text { Moderate } \\
\text { study } \\
\text { limitations, } \\
\text { imprecise } \\
\text { (Cls spanning } \\
\text { the null), } \\
\text { consistency } \\
\text { unknown }\end{array}$ & Insufficient \\
\hline Depressed & $\begin{array}{l}\text { Exposed to } \\
\text { SSRIs during } \\
\text { pregnancy } \\
\text { vs. } \\
\text { unexposed } \\
\text { during } \\
\text { pregnancy }\end{array}$ & $\begin{array}{l}\text { Primary } \\
\text { persistent } \\
\text { pulmonary } \\
\text { hypertension } \\
\text { (without } \\
\text { cardiac } \\
\text { anomaly or } \\
\text { hypoplasia in } \\
\text { full-term } \\
\text { deliveries) }\end{array}$ & $\begin{array}{l}94 / 54,281 \\
(0.2 \%) \text { vs. } \\
669 / 567,118 \\
(0.1 \%)^{6}\end{array}$ & $\begin{array}{l}\text { Adjusted } \mathrm{OR}, \\
1.28(95 \% \mathrm{Cl}, \\
1.01 \text { to } 1.70)^{6} \\
\text { AOR, when not } \\
\text { restricted to full- } \\
\text { term or by } \\
\text { outcome } \\
\text { (primary } \\
\text { persistent } \\
\text { pulmonary } \\
\text { hypertension } \\
\text { rather than } \\
\text { primary } \\
\text { persistent } \\
\text { pulmonary } \\
\text { hypertension): } \\
1.08(95 \% \mathrm{Cl}, \\
0.92 \text { to } 1.27)^{6}\end{array}$ & $\begin{array}{l}1 \text { cohort, } \\
n=621,399^{6}\end{array}$ & $\begin{array}{l}\text { Moderate } \\
\text { study } \\
\text { limitations, } \\
\text { precise, } \\
\text { consistency } \\
\text { unknown, } \\
\text { adjusting for } \\
\text { confounding } \\
\text { increased the } \\
\text { odds }\end{array}$ & $\begin{array}{l}\text { Low for } \\
\text { harms with } \\
\text { SSRIs }\end{array}$ \\
\hline
\end{tabular}




\begin{tabular}{|c|c|c|c|c|c|c|c|}
\hline Population & $\begin{array}{l}\text { Intervention } \\
\text { and } \\
\text { Comparator }\end{array}$ & Outcome & $\begin{array}{l}\text { Incidence } \\
\text { or Mean } \\
\text { Effect by } \\
\text { Arm }\end{array}$ & Results & $\begin{array}{l}\text { Study } \\
\text { Design and } \\
\text { Sample Size }\end{array}$ & $\begin{array}{l}\text { Factors That } \\
\text { Affect the } \\
\text { Strength of } \\
\text { Evidence }\end{array}$ & $\begin{array}{l}\text { Overall } \\
\text { Evidence } \\
\text { Strength } \\
\text { (Direction of } \\
\text { Effect) }\end{array}$ \\
\hline $\begin{array}{l}\text { Depressed or } \\
\text { anxious or } \\
\text { exposed to } \\
\text { SSRIs in } \\
\text { pregnancy }\end{array}$ & $\begin{array}{l}\text { Exposed to } \\
\text { SSRIs vs. } \\
\text { unexposed } \\
\text { during } \\
\text { pregnancy } \\
\text { with } \\
\text { depression or } \\
\text { anxiety }\end{array}$ & $\begin{array}{l}\text { Infant and } \\
\text { child behavior } \\
\text { and } \\
\text { development }\end{array}$ & $\begin{array}{l}\text { Varies by } \\
\text { measure } \\
\text { (measures } \\
\text { include } \\
\text { CBCL } \\
\text { [including } \\
\text { subscales], } \\
\text { Behaviour } \\
\text { Rating } \\
\text { Inventory of } \\
\text { Executive } \\
\text { Function - } \\
\text { Preschool } \\
\text { version } \\
\text { (BRIEF-P), } \\
\text { Snijders- } \\
\text { Oomen Niet- } \\
\text { verbale } \\
\text { intelligentie } \\
\text { Test-Revisie } \\
\text { (SON-R 2 } \\
\text { 1/2-7), } \\
\text { NEPSY-II, } \\
\text { NICU } \\
\text { network } \\
\text { neurobe- } \\
\text { havioral } \\
\text { (NNNS } \\
\text { [including } \\
\text { subscales]) - } \\
\text { Attention } \\
\text { scores, MDI, } \\
\text { PDI, BRS) }\end{array}$ & $\begin{array}{l}\text { Results vary by } \\
\text { specific } \\
\text { outcome, but } \\
\text { the majority of } \\
\text { outcomes are } \\
\text { not statistically } \\
\text { significant; } \\
\text { exceptions } \\
\text { include } 1 \\
\text { subscale } \\
\text { measure for } \\
\text { CBCL, NEPSY- } \\
\text { II, and } 3 \text { of } 13 \\
\text { NNNS subscale } \\
\text { measures; } \\
\text { studies with } \\
\text { significant } \\
\text { findings did not } \\
\text { adjust for } \\
\text { multiple } \\
\text { comparisons }\end{array}$ & $\begin{array}{l}4 \text { cohorts, } \\
\mathrm{N}=4,410^{8,61}, \\
82,88\end{array}$ & $\begin{array}{l}\text { High study } \\
\text { limitations ( } 3 \\
\text { of } 4 \text { are high } \\
\text { risk of bias } \\
61,82 \text { ) } \\
\text { imprecise, } \\
\text { consistency } \\
\text { unknown } \\
\text { (single } \\
\text { measures of } \\
\text { outcomes not } \\
\text { repeated in } \\
\text { multiple } \\
\text { studies) }\end{array}$ & Insufficient \\
\hline
\end{tabular}




\begin{tabular}{|c|c|c|c|c|c|c|c|}
\hline Population & $\begin{array}{l}\text { Intervention } \\
\text { and } \\
\text { Comparator }\end{array}$ & Outcome & $\begin{array}{l}\text { Incidence } \\
\text { or Mean } \\
\text { Effect by } \\
\text { Arm }\end{array}$ & Results & $\begin{array}{l}\text { Study } \\
\text { Design and } \\
\text { Sample Size }\end{array}$ & $\begin{array}{l}\text { Factors That } \\
\text { Affect the } \\
\text { Strength of } \\
\text { Evidence }\end{array}$ & $\begin{array}{l}\text { Overall } \\
\text { Evidence } \\
\text { Strength } \\
\text { (Direction of } \\
\text { Effect) }\end{array}$ \\
\hline $\begin{array}{l}\text { Depressed in } \\
\text { pregnancy }\end{array}$ & $\begin{array}{l}\text { Exposed to } \\
\text { SSRls vs. } \\
\text { unexposed } \\
\text { during } \\
\text { pregnancy } \\
\text { with } \\
\text { depression }\end{array}$ & $\begin{array}{l}\text { Infant weight } \\
\text { at } 52 \text { weeks }\end{array}$ & NR & $N R, p=0.6^{76}$ & $\begin{array}{l}1 \text { cohort, } \\
\mathrm{N}=73^{76}\end{array}$ & $\begin{array}{l}\text { High study } \\
\text { limitations } \\
\text { (high risk of } \\
\text { bias }^{76} \text { ) likely } \\
\text { imprecise, } \\
\text { consistency } \\
\text { unknown }\end{array}$ & Insufficient \\
\hline $\begin{array}{l}\text { Psychiatric } \\
\text { illness in } \\
\text { pregnancy or } \\
\text { exposed to } \\
\text { SSRIs }\end{array}$ & $\begin{array}{l}\text { Exposed to } \\
\text { SSRIs vs. } \\
\text { unexposed } \\
\text { during } \\
\text { pregnancy } \\
\text { with } \\
\text { psychiatric } \\
\text { illness and no } \\
\text { psychotropic } \\
\text { medication } \\
\text { use } \\
\end{array}$ & $\begin{array}{l}\text { Child weight at } \\
7 \text { years }\end{array}$ & NR & $\begin{array}{l}\text { Regression } \\
\text { coefficient: } 0.04 \text {, } \\
95 \% \mathrm{Cl} \text {, } \\
-0.19 \text { to } 0.26^{9,60}\end{array}$ & $\begin{array}{l}1 \text { cohort, } \\
\mathrm{N}=617^{9,60}\end{array}$ & $\begin{array}{l}\text { High study } \\
\text { limitations } \\
\text { (high risk of } \\
\text { bias }^{9,60}{ }^{60} \text { likely } \\
\text { precise, } \\
\text { consistency } \\
\text { unknown }\end{array}$ & Insufficient \\
\hline $\begin{array}{l}\text { Depression- } \\
\text { related } \\
\text { psychiatric } \\
\text { disorder }\end{array}$ & $\begin{array}{l}\text { Exposed to } \\
\text { SSRIs during } \\
\text { pregnancy } \\
\text { vs. } \\
\text { unexposed } \\
\text { with a } \\
\text { depression- } \\
\text { related } \\
\text { psychiatric } \\
\text { disorder }\end{array}$ & $\begin{array}{l}\text { Incidence of } \\
\text { developmental } \\
\text { disorders } \\
\text { (speech/ } \\
\text { language, } \\
\text { motor, } \\
\text { scholastic) }\end{array}$ & $\begin{array}{l}\text { Rates range } \\
0.4 \% \text { to } \\
1.6 \% \text { in the } \\
\text { treatment } \\
\text { arm and } \\
0.6 \% \text { to } \\
1.8 \% \text { in the } \\
\text { control arm }{ }^{59}\end{array}$ & $\begin{array}{l}\text { AHR ranges } \\
\text { from } 0.88 \text { to } \\
1.3 \text {, all Cls } \\
\text { cross the null; } \\
\text { adjustments } \\
\text { that resulted in } \\
\text { greater risk of } \\
\text { confounding by } \\
\text { restricting to } \\
\text { women with } \\
\text { more than one } \\
\text { prescription in } \\
\text { the control arm } \\
\text { increased the } \\
\text { likelihood of } \\
\text { disorders }\end{array}$ & $\begin{array}{l}1 \text { cohort, } \\
\mathrm{N}=25,133^{59}\end{array}$ & $\begin{array}{l}\text { Moderate } \\
\text { study } \\
\text { limitations, } \\
\text { imprecise } \\
\text { (wide Cls), } \\
\text { consistency } \\
\text { unknown }\end{array}$ & Insufficient \\
\hline $\begin{array}{l}\text { Exposed to } \\
\text { SSRIs during } \\
\text { pregnancy or } \\
\text { unexposed } \\
\text { with a } \\
\text { psychiatric } \\
\text { diagnosis }\end{array}$ & $\begin{array}{l}\text { Exposed to } \\
\text { SSRIs during } \\
\text { pregnancy } \\
\text { vs. } \\
\text { unexposed } \\
\text { with a } \\
\text { psychiatric } \\
\text { diagnosis } \\
\end{array}$ & $\begin{array}{l}\text { Autism } \\
\text { spectrum } \\
\text { disorder }\end{array}$ & $\begin{array}{l}88 / 15,729 \\
(0.6 \%) \text { vs. } \\
79 / 9,651 \\
(0.8 \%)^{63}\end{array}$ & $\begin{array}{l}\text { AHR: } 0.88 \\
(95 \% \mathrm{Cl}, 0.65 \\
\text { to } 1.2) \\
p=0.428^{63}\end{array}$ & $\begin{array}{l}1 \text { cohort, } \\
n=25,380^{63}\end{array}$ & $\begin{array}{l}\text { Moderate } \\
\text { study } \\
\text { limitations, } \\
\text { imprecise } \\
\text { (wide Cls), } \\
\text { consistency } \\
\text { unknown }\end{array}$ & Insufficient \\
\hline $\begin{array}{l}\text { Exposed to } \\
\text { SSRIs during } \\
\text { pregnancy or } \\
\text { unexposed } \\
\text { with a } \\
\text { psychiatric } \\
\text { diagnosis }\end{array}$ & $\begin{array}{l}\text { Exposed to } \\
\text { SSRIs during } \\
\text { pregnancy } \\
\text { vs. } \\
\text { unexposed } \\
\text { with a } \\
\text { psychiatric } \\
\text { diagnosis }\end{array}$ & ADHD & $\begin{array}{l}160 / 15,729 \\
(1.0 \%) \text { vs. } \\
137 / 9,651 \\
(1.4 \%)^{63}\end{array}$ & $\begin{array}{l}\text { AHR: } 0.98 \\
(95 \% \mathrm{Cl}, 0.77 \\
\text { to } 1.24) \\
p=0.847^{63}\end{array}$ & $\begin{array}{l}1 \text { cohort, } \\
n=25,380^{63}\end{array}$ & $\begin{array}{l}\text { Moderate } \\
\text { study } \\
\text { limitations, } \\
\text { imprecise } \\
\text { (wide Cls), } \\
\text { consistency } \\
\text { unknown }\end{array}$ & Insufficient \\
\hline
\end{tabular}




\begin{tabular}{|c|c|c|c|c|c|c|c|}
\hline Population & $\begin{array}{l}\text { Intervention } \\
\text { and } \\
\text { Comparator }\end{array}$ & Outcome & $\begin{array}{l}\text { Incidence } \\
\text { or Mean } \\
\text { Effect by } \\
\text { Arm }\end{array}$ & Results & $\begin{array}{l}\text { Study } \\
\text { Design and } \\
\text { Sample Size }\end{array}$ & $\begin{array}{l}\text { Factors That } \\
\text { Affect the } \\
\text { Strength of } \\
\text { Evidence }\end{array}$ & $\begin{array}{l}\text { Overall } \\
\text { Evidence } \\
\text { Strength } \\
\text { (Direction of } \\
\text { Effect) }\end{array}$ \\
\hline $\begin{array}{l}\text { Exposed to } \\
\text { SSRIs during } \\
\text { pregnancy or } \\
\text { unexposed } \\
\text { with a } \\
\text { psychiatric } \\
\text { diagnosis }\end{array}$ & $\begin{array}{l}\text { Exposed to } \\
\text { SSRIs during } \\
\text { pregnancy } \\
\text { vs. } \\
\text { unexposed } \\
\text { with a } \\
\text { psychiatric } \\
\text { diagnosis }\end{array}$ & Anxiety & $\begin{array}{l}65 / 15,729 \\
(0.4 \%) \text { vs. } \\
39 / 9,651 \\
(0.4 \%)^{63}\end{array}$ & $\begin{array}{l}\text { AHR: } 1.3(95 \% \\
C l, 0.84 \text { to } 2.01) \\
p=0.234^{63}\end{array}$ & $\begin{array}{l}1 \text { cohort, } \\
n=25,380^{63}\end{array}$ & $\begin{array}{l}\text { Moderate } \\
\text { study } \\
\text { limitations, } \\
\text { imprecise } \\
\text { (wide Cls), } \\
\text { consistency } \\
\text { unknown }\end{array}$ & Insufficient \\
\hline $\begin{array}{l}\text { Exposed to } \\
\text { SSRIs during } \\
\text { pregnancy or } \\
\text { unexposed } \\
\text { with a } \\
\text { psychiatric } \\
\text { diagnosis }\end{array}$ & $\begin{array}{l}\text { Exposed to } \\
\text { SSRIs during } \\
\text { pregnancy } \\
\text { vs. } \\
\text { unexposed } \\
\text { with a } \\
\text { psychiatric } \\
\text { diagnosis }\end{array}$ & Depression & $\begin{array}{l}60 / 15,729 \\
(0.4 \%) \text { vs. } \\
30 / 9,651 \\
(0.3 \%)^{63}\end{array}$ & $\begin{array}{l}\text { AHR: } 1.78 \\
(95 \% \mathrm{Cl}, 1.12 \\
\text { to } 2.82) \\
p=0.015^{63}\end{array}$ & $\begin{array}{l}1 \text { cohort, } \\
n=25,380^{63}\end{array}$ & $\begin{array}{l}\text { Moderate } \\
\text { study } \\
\text { limitations, } \\
\text { precise, } \\
\text { consistency } \\
\text { unknown }\end{array}$ & $\begin{array}{l}\text { Low for } \\
\text { harms with } \\
\text { SSRIs }\end{array}$ \\
\hline
\end{tabular}

$\mathrm{ADHD}=$ attention-deficit/hyperactivity disorder; $\mathrm{AHR}=$ adjusted hazard ratio; $\mathrm{AOR}=$ adjusted odds ratio; $\mathrm{ARR}=$ adjusted risk ratio; BRIEF-P = Behaviour Rating Inventory of Executive Function - Preschool version; BRS = Behavior Rating Scale; $\mathrm{CBCL}=$ childhood behavior checklist; $\mathrm{CI}=$ confidence interval; $\mathrm{MDI}=$ Mental Development Index; $\mathrm{n} / \mathrm{N}=$ number; NEPSY-II $=\mathrm{A}$ Developmental NEuroPSYchological Assessment-II; NICU = neonatal intensive care unit; NNNS = NICU Network Neurobehavioral Scale; NR = not reported; OR = odds ratio; PDI = Psychomotor Development Index; RR = relative risk; SON-R 2 1/2-7 = Snijders-Oomen Niet-verbale intelligentie Test-Revisie; SSRI = selective serotonin reuptake inhibitor; vs. = versus.

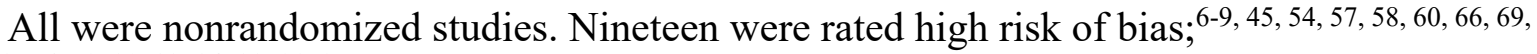
$70,72,76,79,80,82,84,88,91,95$ others were rated as having some risk-of-bias concerns.

All publications focused on exposure during pregnancy. Publications compared pregnant women exposed to SSRIs with women with untreated depression,, , 57, 64, 72, 75, 81 depression and anxiety, ${ }^{79}$ mood or anxiety disorders, ${ }^{74}$ history of a psychiatric disorder, ${ }^{51,83,93,95}$ or history of antidepressant exposure before the last menstrual period. ${ }^{104}$ Studies specifying a history of disorders varied significantly in their definition and included depression only, depression or anxiety, or a history of psychiatric illness. The most inclusive specified the control arm as having a lifetime history of psychiatric illness but no drug exposure during pregnancy. ${ }^{80,83,93}$ Among those specifying the control arm with respect to exposure during pregnancy, the allowable period of exposure also varied: no exposure in the past year to 20 weeks gestation, ${ }^{81}$ no exposure in the trimester of interest, ${ }^{79,83}$ no exposure in an unspecified period of "late gestation," after the last menstrual period, ${ }^{104}$ and a history of anxiety or depression but no supply of antidepressants in the 5 months before delivery in another. ${ }^{74}$ These sources of clinical heterogeneity, coupled with statistical heterogeneity, precluded meta-analyses.

Consistent and precise evidence from two or more of three studies indicated a risk of harm with SSRIs for some neonatal outcomes, specifically, low Apgar score, $, 4,66$ and respiratory distress. ${ }^{27,54,66}$ Two of these studies were rated as high risk of bias, ${ }^{54,66}$ leading to low confidence in the reported effects in these outcomes. Three studies reported on respiratory distress used different measures and could not be pooled. One reported on respiratory conditions of newborns other than intrauterine hypoxia and birth asphyxia (ICD-9 code of other respiratory conditions),${ }^{54}$ undefined breathing problems, ${ }^{66}$ and respiratory distress (ICD-9 codes of 769, 770.6 , and $770.8^{27}$ ). The two studies that do specify the ICD-9 codes include very heterogeneous outcomes, ranging from benign self-limiting conditions such as transient tachypnea of the newborn to more serious outcomes such as respiratory arrest of the newborn. Although we 
graded the outcome as low strength of evidence for harm, in the absence of more details on the proportion and differences between study arms for more serious outcomes, the clinical implications of this finding are unclear.

One study reported higher rates of intrauterine hypoxia and birth asphyxia. ${ }^{54}$ The study uses the ICD-9 code of 768. One concern is whether the higher rate in the exposed arm may be attributable to the greater number of c-sections in the exposed arm and the higher use of the ICD9 code of 768.3 for fetal distress during labor for nonreassuring fetal testing. Analyses from the same study in a population of women with vaginal deliveries found a statistically significant difference in the third trimester only. Notably, the incidence of this outcome in the exposed arm $(3.5 \%)$ is much lower than the incidence of low Apgar scores ( $<7$ at 5 minutes) in the same study $(1.1 \%)$, so the clinical significance of this finding is unclear.

Evidence from a single study suggests harms of postpartum hemorrhage with current or recent exposure to SSRIs (measured as having a prescription at or in the month prior to delivery) but not for past exposure (defined as having a prescription 1 to 5 months prior to delivery), when compared with no exposure to SSRIs among women with depression or anxiety. ${ }^{74}$ The association between SSRI exposure and postpartum hemorrhage persisted in multiple sensitivity analyses. A second high risk-of-bias study found a protective association between SSRIs and bleeding during and after delivery. ${ }^{66}$

Evidence from a single study suggested harms of primary persistent pulmonary hypertension. ${ }^{6}$ We graded the evidence as low for harms. The study reported higher odds (AOR, $1.28 ; 95 \%$ CI, 1.01 to 1.64 ) after restricting the cohort to term deliveries and redefining the outcome as primary persistent pulmonary hypertension (the absence of congenital cardiac anomalies and lung hypoplasia) than when the study included all deliveries and defined the outcome as all primary persistent pulmonary hypertension cases (AOR, 1.28; 95\% CI, 1.01 to 1.64). We note, however, that risk factors, such as smoking, obesity, and C-section, are all more prevalent in populations of psychiatric patients. ${ }^{148}$ Evidence from one study with high risk of bias suggested a greater risk of harms of depression in children who were exposed in utero to SSRIs. ${ }^{63}$ As with other results, the risk of residual confounding cannot be ruled out.

The evidence for SSRIs as a class is insufficient to judge the risk of harms for maternal (spontaneous abortion, gestational diabetes, hypertension) or child outcomes (perinatal mortality, small for gestational age, birthweight, gestational age, neonatal convulsions, NICU admissions, extended hospital stay, feeding problems, jaundice, infant and child behavior and development, infant or child weight, autism spectrum disorder, attention-deficit hyperactivity disorder, or anxiety) (Table B-19). These bodies of evidence generally comprised one or two studies with nonsignificant results. One exception was for NICU studies: one of two studies, with high riskof-bias reported higher rates of NICU admissions in the treatment group. ${ }^{66}$ The study found higher odds ratios for unadjusted analyses than for unadjusted analyses; because residual confounding could potentially explain the association between SSRI exposure and the outcome, we rated the evidence as insufficient.

Multiple SSRI studies reported on some maternal (preeclampsia) and child (preterm birth, low birth weight, small for gestational age, outcomes, major congenital anomalies) outcomes. We were unable to pool most of these results because of the variations in outcome measures (with the exception of cardiac anomalies). Although studies consistently (or nearly consistently) reported no statistically significant effects and, in some cases, drew from large samples, the span of the CIs in these studies included appreciable benefit and appreciable harm. As a result, we could not infer that the results were equivalent. Additionally, many of the evidence bases solely 
comprised or included high risk-of-bias studies, so we judge the strength of the evidence as insufficient for these outcomes. Three publications ${ }^{7,72,106}$ ( 2 high risk of bias ${ }^{7,72}$ ) reporting on cardiac anomalies were pooled, resulting in a finding of no increased risk from SSRIs as a drug class $\left(1.07 ; 95 \%\right.$ CI, 0.96 to $1.20 ; \mathrm{I}^{2}, 0 \%$; Figure B-2). The CIs were wide and spanned both appreciable benefit and appreciable harm; as a result, we could not conclude equivalence.

Three studies also reported numerous other birth defects. One found an increased risk of craniosynostosis (RR, 2.43; 95\% CI, 1.44 to 4.11 ; 19 exposed cases) and musculoskeletal defects (RR, 1.28; 95\% CI, 1.03 to $1.58 ; 104$ exposed cases) with nonsertraline SSRIs. ${ }^{70}$ A second found a decreased risk of genital system anomalies with SSRIs (AOR, 0.57; 95\% CI, 0.35 to 0.92 ). ${ }^{72}$ Neither study adjusted for multiple comparisons, and both were rated as high risk of bias. A third study with moderate study limitations that also did not adjust for multiple comparisons generally reported no difference in several other birth defects, including neural tube defects (AOR, 0.81; $95 \% \mathrm{CI}, 0.48$ to 1.36$).{ }^{106} \mathrm{We}$ also found insufficient evidence from a single study each on the harms of SSRIs other than sertraline ${ }^{70}$ (major congenital anomalies and cardiac anomalies) and SSRIs other than paroxetine (major congenital anomalies) ${ }^{93}$ when compared with no treatment.

\section{SSRIs: Citalopram}

\section{Overview}

- When compared with unexposed women with depression or anxiety, citalopram exposure at the time of delivery may be associated with a risk of postpartum hemorrhage (low strength of evidence of harms).

- When compared with unexposed women with a known psychiatric disorder, citalopram exposure in pregnant women may be associated with a higher risk of autism spectrum disorder in their children (low strength of evidence of harms).

- The evidence is insufficient to judge the risk of harms for maternal (gestational diabetes, preeclampsia, postpartum hemorrhage for recent or past exposure to citalopram during pregnancy) or child outcomes (congenital anomalies or cardiac anomalies).

\section{Detailed Results}

Six publications reported on either maternal or fetal harms of citalopram (Table B-20). ${ }^{51,57 \text {, }}$ 72, 74, 75, 104 All of these studies drew from large cohorts including THIN from the United Kingdom, ${ }^{72}$ the Quebec Pregnancy/Children Cohort (QPC) in Canada, ${ }^{57}$ the Stockholm Youth Cohort in Sweden, ${ }^{51}$ a U.S. Kaiser Permanente Washington cohort, ${ }^{104}$ the National Birth Defects Prevention Study, ${ }^{106}$ and the Medicaid Analytic eXtract in the United States. ${ }^{74,75}$ Two studies were rated as high risk of bias; ${ }^{57,72}$ the rest had some risk-of-bias concerns.

The studies looked at a variety of outcomes; three focused on maternal harms, including gestational diabetes, ${ }^{104}$ postpartum hemorrhage, and preeclampsia, ${ }^{74,75}$ and four evaluated fetal harms, specifically autism spectrum disorder and congenital anomalies. ${ }^{51,57,72,106}$ Regarding maternal harms, one study reported a higher risk of postpartum hemorrhage with current use (at delivery) of citalopram, but no difference with recent (up to 1 month before delivery) or past use (1 to 5 months before delivery) of citalopram when compared with pregnant women with a diagnosis of depression of anxiety. ${ }^{74}$ Other studies did not find a difference in risk of gestational diabetes ${ }^{104}$ or preeclampsia with citalopram. ${ }^{75}$ Study results for fetal harms were conflicting. Two studies evaluated risk of major congenital anomalies with one study finding increased risk of major congenital anomalies with citalopram, ${ }^{72}$ and another study found no change in risk with 
major congenital anomalies. ${ }^{72}$ Three studies found no increased risk of cardiac anomalies (pooled OR, 1.09; 95\% CI, 0.82 to $1.46 ; \mathrm{I}^{2}, 0 \%$; Figure B-3). ${ }^{57,72,106}$ The CIs were wide and spanned both appreciable benefit and appreciable harm; as a result, we could not conclude equivalence. One study also found no increased association with other anomalies, with the exception of a higher risk of craniosynostosis congenital anomalies, but the study had not conducted testing for multiple comparisons. ${ }^{57} \mathrm{~A}$ third study with moderate study limitations that also did not adjust for multiple comparisons generally reported no difference in several other birth defects, including neural tube defects (AOR, 0.56; 95\% CI, 0.18 to 1.76 ). ${ }^{106}$ One study evaluated risk of autism spectrum disorder both with and without intellectual disabilities and found an increased risk with citalopram use ${ }^{51}$ (Table B-20).

Table B-20. Strength of evidence for harms outcomes: citalopram versus no treatment

\begin{tabular}{|c|c|c|c|c|c|c|c|}
\hline Population & $\begin{array}{l}\text { Intervention } \\
\text { and } \\
\text { Comparator }\end{array}$ & Outcome & $\begin{array}{l}\text { Incidence or } \\
\text { Mean Effect } \\
\text { by Arm }\end{array}$ & Results & $\begin{array}{l}\text { Study } \\
\text { Design and } \\
\text { Sample Size }\end{array}$ & $\begin{array}{l}\text { Factors } \\
\text { That Affect } \\
\text { the } \\
\text { Strength } \\
\text { of } \\
\text { Evidence }\end{array}$ & $\begin{array}{l}\text { Overall } \\
\text { Evidence } \\
\text { Strength } \\
\text { (Direction of } \\
\text { Effect) }\end{array}$ \\
\hline $\begin{array}{l}\text { Exposure to } \\
\text { antidepressants } \\
\text { before pregnancy } \\
\text { or exposed to } \\
\text { citalopram }\end{array}$ & $\begin{array}{l}\text { Citalopram } \\
\text { exposure } \\
\text { during } \\
\text { pregnancy } \\
\text { vs. exposure } \\
\text { before but } \\
\text { not during } \\
\text { pregnancy }\end{array}$ & $\begin{array}{l}\text { Gestational } \\
\text { diabetes }\end{array}$ & $\begin{array}{l}28 / 343 \\
(8.16 \%) \text { vs. } \\
90 / 1211 \\
(7.43 \%)\end{array}$ & $\begin{array}{l}\text { RR } \\
\text { (inverse } \\
\text { probability } \\
\text { of } \\
\text { treatment } \\
\text { weighted): } \\
0.82(95 \% \\
\mathrm{Cl}, 0.51 \text { to } \\
1.28)^{104}\end{array}$ & $\begin{array}{l}1 \text { cohort, } \\
n=1,554^{104}\end{array}$ & $\begin{array}{l}\text { Moderate } \\
\text { study } \\
\text { limitations, } \\
\text { imprecise } \\
\text { (wide Cls), } \\
\text { consistency } \\
\text { unknown }\end{array}$ & Insufficient \\
\hline $\begin{array}{l}\text { Pregnant women } \\
\text { with depression }^{75}\end{array}$ & $\begin{array}{l}\text { Use of } \\
\text { citalopram } \\
\text { vs. no } \\
\text { treatment }\end{array}$ & Preeclampsia & $\begin{array}{l}91 / 1680 \\
(5.4 \%) \text { vs. } \\
3,215 / 59,219 \\
(5.4 \%)^{75}\end{array}$ & $\begin{array}{l}\mathrm{RR}, 1.01 \\
(95 \% \mathrm{Cl}, \\
0.82 \text { to } \\
1.23)^{75}\end{array}$ & $\begin{array}{l}1 \text { cohort, } \\
\mathrm{N}=60,899^{75}\end{array}$ & $\begin{array}{l}\text { Moderate } \\
\text { study } \\
\text { limitations, } \\
\text { imprecision } \\
\text { (wide Cls), } \\
\text { consistency } \\
\text { unknown }\end{array}$ & Insufficient \\
\hline $\begin{array}{l}\text { Pregnant women } \\
\text { with mood or } \\
\text { anxiety disorder }\end{array}$ & $\begin{array}{l}\text { Current } \\
\text { citalopram } \\
\text { use vs. no } \\
\text { treatment }\end{array}$ & $\begin{array}{l}\text { Postpartum } \\
\text { hemorrhage }\end{array}$ & $\begin{array}{l}36 / 891(4 \%) \\
\text { vs. } \\
1,896 / 69,044 \\
(2.7 \%)^{74}\end{array}$ & $\begin{array}{l}\text { RR, } 1.48 \\
(95 \% \mathrm{Cl}, \\
1.07 \text { to } \\
2.04)^{74}\end{array}$ & $\begin{array}{l}1 \text { cohort, } \\
\mathrm{N}=69,935^{74}\end{array}$ & $\begin{array}{l}\text { Moderate } \\
\text { study } \\
\text { limitations, } \\
\text { precise, } \\
\text { consistency } \\
\text { unknown } \\
\end{array}$ & $\begin{array}{l}\text { Low for } \\
\text { harms with } \\
\text { citalopram }\end{array}$ \\
\hline $\begin{array}{l}\text { Pregnant women } \\
\text { with mood or } \\
\text { anxiety disorder }\end{array}$ & $\begin{array}{l}\text { Recent } \\
\text { citalopram } \\
\text { use (1-30 } \\
\text { days before } \\
\text { delivery) vs. } \\
\text { no treatment }\end{array}$ & $\begin{array}{l}\text { Postpartum } \\
\text { hemorrhage }\end{array}$ & $\begin{array}{l}\text { NR/830 vs. } \\
1,896 / 69,044 \\
(2.7 \%)^{74}\end{array}$ & $\begin{array}{l}\text { RR, } 0.7 \\
(95 \% \mathrm{Cl}, \\
0.37 \text { to } \\
1.34)^{74}\end{array}$ & $\begin{array}{l}1 \text { cohort, } \\
N=69,874^{74}\end{array}$ & $\begin{array}{l}\text { Moderate } \\
\text { study } \\
\text { limitations, } \\
\text { imprecision } \\
\text { (wide Cls), } \\
\text { consistency } \\
\text { unknown }\end{array}$ & Insufficient \\
\hline $\begin{array}{l}\text { Pregnant women } \\
\text { with mood or } \\
\text { anxiety disorder }\end{array}$ & $\begin{array}{l}\text { Past } \\
\text { citalopram } \\
\text { use (1-5 } \\
\text { months } \\
\text { before } \\
\text { delivery) vs. } \\
\text { no treatment }\end{array}$ & $\begin{array}{l}\text { Postpartum } \\
\text { hemorrhage }\end{array}$ & $\begin{array}{l}17 / 830(2 \%) \\
\text { vs. } \\
1,896 / 69,044 \\
(2.7 \%)^{74}\end{array}$ & $\begin{array}{l}\text { RR, } 0.76 \\
(95 \% \mathrm{Cl}, \\
0.47 \text { to } \\
1.23)^{74}\end{array}$ & $\begin{array}{l}1 \text { cohort, } \\
\mathrm{N}=69,874^{74}\end{array}$ & $\begin{array}{l}\text { Moderate } \\
\text { study } \\
\text { limitations, } \\
\text { imprecision } \\
\text { (wide Cls), } \\
\text { consistency } \\
\text { unknown }\end{array}$ & Insufficient \\
\hline
\end{tabular}




\begin{tabular}{|c|c|c|c|c|c|c|c|}
\hline Population & $\begin{array}{l}\text { Intervention } \\
\text { and } \\
\text { Comparator }\end{array}$ & Outcome & $\begin{array}{l}\text { Incidence or } \\
\text { Mean Effect } \\
\text { by Arm }\end{array}$ & Results & $\begin{array}{l}\text { Study } \\
\text { Design and } \\
\text { Sample Size }\end{array}$ & $\begin{array}{l}\text { Factors } \\
\text { That Affect } \\
\text { the } \\
\text { Strength } \\
\text { of } \\
\text { Evidence }\end{array}$ & $\begin{array}{l}\text { Overall } \\
\text { Evidence } \\
\text { Strength } \\
\text { (Direction of } \\
\text { Effect) }\end{array}$ \\
\hline $\begin{array}{l}\text { Pregnant women } \\
\text { with diagnosis of } \\
\text { depression only }{ }^{72} \\
\text { or depression and } \\
\text { or anxiety, or } \\
\text { exposed to } \\
\text { antidepressants }^{57}\end{array}$ & $\begin{array}{l}\text { Maternal } \\
\text { exposure to } \\
\text { citalopram } \\
\text { vs. no } \\
\text { exposure } \\
\text { anxiety } \\
\text { and/or } \\
\text { depression }\end{array}$ & $\begin{array}{l}\text { Major } \\
\text { congenital } \\
\text { anomalies }\end{array}$ & $\begin{array}{l}52 / 1,946 \\
(2.7 \%) \text { vs. } \\
666 / 23,833 \\
(2.8 \%) \text { in one } \\
\text { study, }^{72} \text { NR in } \\
\text { second }^{57}\end{array}$ & $\begin{array}{l}\text { AOR, } 1.36 \\
(95 \% \mathrm{Cl}, \\
1.08 \text { to } \\
1.73)^{57} \\
\text { OR, } 0.97 \\
(95 \% \mathrm{Cl}, \\
0.71 \text { to } \\
1.31)^{72}\end{array}$ & $\begin{array}{l}2 \text { cohort } \\
\text { studies; } \\
n>25,779^{57,72}\end{array}$ & $\begin{array}{l}\text { High study } \\
\text { limitations } \\
\text { (high risk of } \text { bias }^{57,72} \text { ), } \\
\text { imprecision } \\
(\text { Cls span } \\
\text { null in one } \\
\text { study }^{72} \text { ), } \\
\text { inconsistent }\end{array}$ & Insufficient \\
\hline $\begin{array}{l}\text { Pregnant women } \\
\text { with diagnosis of } \\
\text { depression only }{ }^{72} \\
\text { or depression and } \\
\text { or anxiety, or } \\
\text { exposed to } \\
\text { antidepressants }{ }^{57} \text {, } \\
106\end{array}$ & $\begin{array}{l}\text { Maternal } \\
\text { exposure to } \\
\text { citalopram } \\
\text { vs. no } \\
\text { exposure or } \\
\text { unexposed to } \\
\text { SSRIs in } \\
\text { early } \\
\text { pregnancy }\end{array}$ & $\begin{array}{l}\text { Cardiac } \\
\text { congenital } \\
\text { anomalies }\end{array}$ & $\begin{array}{l}\text { NR in two } \\
\text { studies; } 57,72 \\
50 \text { cases/39 } \\
\text { controls vs. } \\
149 \\
\text { cases/125 } \\
\text { controls }^{106}\end{array}$ & $\begin{array}{l}\text { Pooled } \\
\text { OR, } 1.09 \\
(95 \% \mathrm{Cl}, \\
0.82 \text { to } \\
1.46), \mathrm{I}^{2} \text { : } \\
0 \% \\
\\
\text { AOR, } 1.15 \\
(95 \% \mathrm{Cl}, \\
0.69 \text { to } \\
1.92)^{57} \\
\mathrm{AOR}, 1.02 \\
(95 \% \mathrm{Cl}, \\
0.61 \text { to } \\
1.70)^{72} \\
\mathrm{AOR}, 1.11 \\
(95 \% \mathrm{Cl}, \\
0.68 \text { to } \\
1.83)^{106}\end{array}$ & $\begin{array}{l}2 \text { cohort } \\
\text { studies, } 1 \\
\text { case control, } \\
\mathrm{N}>363^{106} \mathrm{~N} \\
\mathrm{NR} \text { in two } \\
\text { studies }^{57,72}\end{array}$ & $\begin{array}{l}\text { High study } \\
\text { limitations } \\
\text { (high risk of } \\
\text { bias }^{57,72} \text { ), } \\
\text { imprecision } \\
\text { (wide Cls), } \\
\text { consistent }\end{array}$ & Insufficient \\
\hline $\begin{array}{l}\text { Pregnant with or } \\
\text { without a known } \\
\text { psychiatric } \\
\text { condition }\end{array}$ & $\begin{array}{l}\text { Maternal } \\
\text { exposure to } \\
\text { citalopram } \\
\text { vs. no } \\
\text { maternal } \\
\text { exposure to } \\
\text { any } \\
\text { antidepressa } \\
\text { nt but with a } \\
\text { known } \\
\text { psychiatric } \\
\text { condition }\end{array}$ & $\begin{array}{l}\text { Autism } \\
\text { spectrum } \\
\text { disorder } \\
\text { among } \\
\text { offspring }\end{array}$ & $\begin{array}{l}52 / 1,064 \\
(4.9 \%) \text { vs. } \\
353 / 12,325 \\
(2.9 \%)^{51}\end{array}$ & $\begin{array}{l}\text { AOR, } 1.65 \\
(95 \% \mathrm{Cl} \\
1.2 \text { to } \\
2.26)^{51}\end{array}$ & $\begin{array}{l}1 \text { cohort } \\
\text { study, } n= \\
13,389^{51}\end{array}$ & $\begin{array}{l}\text { Moderate } \\
\text { study } \\
\text { limitations, } \\
\text { precise, } \\
\text { consistency } \\
\text { unknown }\end{array}$ & $\begin{array}{l}\text { Low for } \\
\text { harms with } \\
\text { citalopram }\end{array}$ \\
\hline $\begin{array}{l}\text { Pregnant with or } \\
\text { without a known } \\
\text { psychiatric } \\
\text { condition }\end{array}$ & $\begin{array}{l}\text { Maternal } \\
\text { exposure to } \\
\text { citalopram } \\
\text { vs. no } \\
\text { maternal } \\
\text { exposure to } \\
\text { any } \\
\text { antidepressa } \\
\text { nt but with a } \\
\text { known } \\
\text { psychiatric } \\
\text { condition }\end{array}$ & $\begin{array}{l}\text { Autism } \\
\text { spectrum } \\
\text { disorder } \\
\text { without } \\
\text { intellectual } \\
\text { disabilities } \\
\text { among } \\
\text { offspring }\end{array}$ & $\begin{array}{l}46 / 1,064 \\
(4.3 \%) \text { vs. } \\
291 / 12,325 \\
(2.4 \%)\end{array}$ & $\begin{array}{l}\text { AOR, } 1.75 \\
\text { (95\% Cl, } \\
1.25 \text { to } \\
2.45)\end{array}$ & $\begin{array}{l}\text { Cohort study } \\
13,389^{51}\end{array}$ & $\begin{array}{l}\text { Moderate } \\
\text { study } \\
\text { limitations, } \\
\text { precise, } \\
\text { consistency } \\
\text { unknown }\end{array}$ & $\begin{array}{l}\text { Low for } \\
\text { harms with } \\
\text { citalopram }\end{array}$ \\
\hline
\end{tabular}

$\mathrm{AOR}=$ adjusted odds ratio; $\mathrm{CI}=$ confidence interval; $\mathrm{N}=$ number; $\mathrm{NR}=$ not reported; $\mathrm{RR}=$ relative risk; vs. $=$ versus. 


\section{SSRIs: Escitalopram}

\section{Overview}

- When compared with unexposed women with depression or anxiety, escitalopram exposure at the time of delivery may be associated with a risk of postpartum hemorrhage (low strength of evidence of harms).

- The evidence of postpartum hemorrhage was judged to be insufficient if escitalopram exposure was prior to delivery (either 1 to 30 days or 1 to 5 months prior to delivery).

- The evidence is also insufficient to judge the risk of harms for exposure to escitalopram during pregnancy for other maternal (preeclampsia) or child outcomes (major congenital or heart anomalies).

\section{Detailed Results}

Four publications reported on escitalopram. ${ }^{72,74,75,106}$ These publications drew from three cohorts: two from the U.S. Medicaid Analytic eXtract, ${ }^{74,75}$ one from the U.K. THIN, ${ }^{72}$ and one from the National Birth Defect Study. ${ }^{106}$ All were nonrandomized studies. One was rated as high risk of bias; ${ }^{72}$ the other two were rated as having some risk-of-bias concerns. ${ }^{74,75,106}$

All publications focused on exposure during pregnancy. Publications compared pregnant women exposed to escitalopram in the first trimester with women with untreated depression; ${ }^{72}$ pregnant women with current (on the date of delivery), recent (1 to 30 days before delivery), or past (1 to 5 months before delivery) exposure to escitalopram with women with untreated mood or anxiety disorders $;{ }^{74}$ pregnant women with escitalopram exposure in the second to third trimester with women with untreated depression; ${ }^{75}$ and women exposed to escitalopram during early pregnancy to women not exposed during early pregnancy. ${ }^{106}$

When compared with unexposed women with depression or anxiety, escitalopram exposure at the time of delivery may be associated with a risk of postpartum hemorrhage (low strength of evidence of harms). ${ }^{74}$ The results are insufficient to rate the harms of escitalopram on other maternal (recent exposure for postpartum hemorrhage or preeclampsia) or fetal outcomes (major congenital or heart anomalies) (Table B-21). One study with moderate study limitations that did not adjust for multiple comparisons reported no differences in several other birth defects, including neural tube defects (AOR, $1.14 ; 95 \% \mathrm{CI}, 0.44$ to 3.01 ). ${ }^{106}$ 
Table B-21. Strength of evidence for harms: Escitalopram versus no treatment

\begin{tabular}{|c|c|c|c|c|c|c|c|}
\hline Population & $\begin{array}{l}\text { Intervention } \\
\text { and } \\
\text { Comparator }\end{array}$ & Outcome & $\begin{array}{l}\text { Incidence or } \\
\text { Mean Effect } \\
\text { by Arm }\end{array}$ & Results & $\begin{array}{l}\text { Study Design } \\
\text { and Sample } \\
\text { Size }\end{array}$ & $\begin{array}{l}\text { Factors That } \\
\text { Affect the } \\
\text { Strength of } \\
\text { Evidence }\end{array}$ & $\begin{array}{l}\text { Overall } \\
\text { Evidence } \\
\text { Strength } \\
\text { (Direction of } \\
\text { Effect) }\end{array}$ \\
\hline $\begin{array}{l}\text { Mood or } \\
\text { anxiety } \\
\text { disorder }\end{array}$ & $\begin{array}{l}\text { Current } \\
\text { escitalopram } \\
\text { exposure vs. } \\
\text { unexposed with } \\
\text { mood or anxiety } \\
\text { disorder }\end{array}$ & $\begin{array}{l}\text { Postpartum } \\
\text { hemorrhage }\end{array}$ & $\begin{array}{l}43 / 1,022 \\
(4.21 \%) \text { vs. } \\
1,896 / 69,044 \\
(2.75 \%)^{74}\end{array}$ & $\begin{array}{l}\text { ARR, } 1.56 \\
(95 \% \mathrm{Cl}, 1.16 \\
\text { to } 2.09)^{74}\end{array}$ & $\begin{array}{l}1 \text { cohort, } \\
n=70,006^{74}\end{array}$ & $\begin{array}{l}\text { Moderate study } \\
\text { limitations, } \\
\text { precise, } \\
\text { consistency } \\
\text { unknown }\end{array}$ & $\begin{array}{l}\text { Low for harms } \\
\text { with } \\
\text { escitalopram }\end{array}$ \\
\hline $\begin{array}{l}\text { Mood or } \\
\text { anxiety } \\
\text { disorder }\end{array}$ & $\begin{array}{l}\text { Recent } \\
\text { escitalopram } \\
\text { exposure } \\
\text { (within } 1 \text { month } \\
\text { before delivery) } \\
\text { vs. unexposed } \\
\text { with mood or } \\
\text { anxiety disorder }\end{array}$ & $\begin{array}{l}\text { Postpartum } \\
\text { hemorrhage }\end{array}$ & $\begin{array}{l}14 / 520 \\
(2.69 \%) \text { vs. } \\
1,896 / 69,044 \\
(2.75 \%)^{74}\end{array}$ & $\begin{array}{l}\text { ARR, } 1.01 \\
(95 \% \mathrm{Cl}, 0.61 \\
\text { to } 1.7)^{74}\end{array}$ & $\begin{array}{l}1 \text { cohort, } n= \\
69,564^{74}\end{array}$ & $\begin{array}{l}\text { Moderate study } \\
\text { limitations, } \\
\text { imprecision } \\
\text { (wide Cls } \\
\text { spanning the } \\
\text { null), } \\
\text { consistency } \\
\text { unknown }\end{array}$ & Insufficient \\
\hline $\begin{array}{l}\text { Mood or } \\
\text { anxiety } \\
\text { disorder }\end{array}$ & $\begin{array}{l}\text { Past } \\
\text { escitalopram } \\
\text { exposure (1 to } \\
5 \text { months } \\
\text { before delivery) } \\
\text { vs. unexposed } \\
\text { with mood or } \\
\text { anxiety disorder }\end{array}$ & $\begin{array}{l}\text { Postpartum } \\
\text { hemorrhage }\end{array}$ & $\begin{array}{l}24 / 940 \\
(2.55 \%) \text { vs. } \\
1,896 / 69,044 \\
(2.75 \%)^{74}\end{array}$ & $\begin{array}{l}\text { ARR, } 0.96 \\
(95 \% \mathrm{Cl}, 0.64 \\
\text { to } 1.42)^{74}\end{array}$ & $\begin{array}{l}1 \text { cohort, } n= \\
69,984^{74}\end{array}$ & $\begin{array}{l}\text { Moderate study } \\
\text { limitations, } \\
\text { imprecision } \\
\text { (wide Cls } \\
\text { spanning the } \\
\text { null), } \\
\text { consistency } \\
\text { unknown }\end{array}$ & Insufficient \\
\hline Depression & $\begin{array}{l}\text { Escitalopram } \\
\text { exposure in } \\
\text { 2nd-3rd } \\
\text { trimester vs. } \\
\text { unexposed } \\
\text { women with } \\
\text { depression }\end{array}$ & Preeclampsia & $\begin{array}{l}125 / 1,936 \\
(6.46 \%) \text { vs. } \\
3,215 / 59,219 \\
(5.43 \%)^{75}\end{array}$ & $\begin{array}{l}\text { ARR, } 1.14 \\
(95 \% \mathrm{Cl}, 0.96 \\
\text { to } 1.36)^{75}\end{array}$ & $\begin{array}{l}1 \text { cohort, } \\
n=61,155^{75}\end{array}$ & $\begin{array}{l}\text { Moderate study } \\
\text { limitations, } \\
\text { imprecision } \\
\text { (wide Cls } \\
\text { spanning the } \\
\text { null), } \\
\text { consistency } \\
\text { unknown }\end{array}$ & Insufficient \\
\hline Depression & $\begin{array}{l}\text { Escitalopram } \\
\text { exposure in first } \\
\text { trimester vs. } \\
\text { unexposed } \\
\text { women with } \\
\text { depression }\end{array}$ & $\begin{array}{l}\text { Major } \\
\text { congenital } \\
\text { anomalies }\end{array}$ & $\begin{array}{l}7 / 333(2.1 \%) \\
\text { vs. } 380 / 13,432 \\
(2.83 \%)^{72}\end{array}$ & $\begin{array}{l}\text { AOR, } 0.77 \\
(95 \% \mathrm{Cl},(0.36 \\
\text { to } 1.66)^{72}\end{array}$ & $\begin{array}{l}1 \text { cohort, } \\
n=13,765^{72}\end{array}$ & $\begin{array}{l}\text { Serious study } \\
\text { limitations (high } \\
\text { risk of bias }{ }^{72} \text { ), } \\
\text { imprecision } \\
\text { (wide Cls } \\
\text { spanning the } \\
\text { null), } \\
\text { consistency } \\
\text { unknown }\end{array}$ & Insufficient \\
\hline $\begin{array}{l}\text { Depression } \\
\text { or anti- } \\
\text { depressant } \\
\text { exposed } \\
\text { women }\end{array}$ & $\begin{array}{l}\text { Escitalopram } \\
\text { exposure during } \\
\text { early pregnancy } \\
\text { vs. unexposed } \\
\text { women with } \\
\text { depression or } \\
\text { unexposed to } \\
\text { SSRls in early } \\
\text { pregnancy }\end{array}$ & $\begin{array}{l}\text { Cardiac } \\
\text { anomalies }\end{array}$ & $\begin{array}{l}3 / 333(0.9 \%) \\
\text { vs. } 112 / 13,432 \\
(0.83 \%)^{72} 43 \\
\text { cases/35 } \\
\text { controls vs. } \\
149 \text { cases/125 }^{\text {controls }}{ }^{106}\end{array}$ & $\begin{array}{l}\text { AOR, } 1.09 \\
(95 \% \mathrm{Cl},(0.34 \\
\text { to } 3.50)^{72} \\
\text { AOR, } 1.16 \\
(0.69 \text { to } 1.97)^{106}\end{array}$ & $\begin{array}{l}1 \text { cohort, } \\
n=13,765,{ }^{72} 1 \\
\text { case-control, } \\
n=352^{106}\end{array}$ & $\begin{array}{l}\text { Serious study } \\
\text { limitations (high } \\
\text { risk of bias }^{72} \text { ), } \\
\text { imprecision } \\
\text { (wide Cls } \\
\text { spanning the } \\
\text { null), } \\
\text { consistency } \\
\text { unknown }\end{array}$ & Insufficient \\
\hline
\end{tabular}

$\mathrm{AOR}=$ adjusted odds ratio; $\mathrm{ARR}=$ adjusted risk ratio; $\mathrm{CI}=$ confidence interval; $\mathrm{n}=$ number; vs. $=$ versus. 


\section{SSRIs: Fluoxetine}

\section{Overview}

- When compared with unexposed women with mood or anxiety disorders, fluoxetine exposure at the time of delivery may be associated with a risk of postpartum hemorrhage (low strength of evidence of harms). The evidence of postpartum hemorrhage was judged to be insufficient if fluoxetine exposure was prior to delivery (either 1 to 30 days or 1 to 5 months prior to delivery).

- The evidence was judged as insufficient for exposure to fluoxetine during pregnancy for all other examined maternal outcomes (gestational diabetes or preeclampsia) and child outcomes (major congenital anomalies, cardiac anomalies, or autism spectrum disorder).

\section{Detailed Results}

Nine publications reported on the evidence for harms from fluoxetine (Table B-22). $7,51,56,57$, 72, 74, 75, 104, 106 All publications were nonrandomized studies and drew data from seven cohorts (3 studies from the U.S. Medicaid Analytic eXtract, $, 74,75$ and 1 each from a U.S. Kaiser Permanente Washington cohort, ${ }^{104}$ the U.S. National Birth Defects Prevention Study, ${ }^{106}$ the Canadian QPC, ${ }^{57}$ the United Kingdom's THIN, ${ }^{72}$ the Swedish Stockholm youth cohort, ${ }^{51}$ and a Swedish birth cohort). ${ }^{56}$ Five studies' risk of bias was rated as some concerns; $;{ }^{51,56,75,104,106}$ the other three studies were rated as high.

Table B-22. Strength of evidence for harms: Fluoxetine versus no treatment

\begin{tabular}{|c|c|c|c|c|c|c|c|}
\hline Population & $\begin{array}{l}\text { Intervention } \\
\text { and } \\
\text { Comparator }\end{array}$ & Outcome & $\begin{array}{l}\text { Incidence or } \\
\text { Mean Effect } \\
\text { by Arm }\end{array}$ & Results & $\begin{array}{l}\text { Study Design } \\
\text { and Sample } \\
\text { Size }\end{array}$ & $\begin{array}{l}\text { Factors } \\
\text { That Affect } \\
\text { the } \\
\text { Strength } \\
\text { of } \\
\text { Evidence }\end{array}$ & $\begin{array}{l}\text { Overall } \\
\text { Evidence } \\
\text { Strength } \\
\text { (Direction } \\
\text { of Effect) }\end{array}$ \\
\hline $\begin{array}{l}\text { Women with an } \\
\text { antidepressant } \\
\text { prescription fill } \\
\text { during the } 6 \\
\text { months before } \\
\text { pregnancy }\end{array}$ & $\begin{array}{l}\text { Fluoxetine } \\
\text { exposure } \\
\text { before } \\
\text { gestational } \\
\text { diabetes } \\
\text { screening } \\
\text { (24-28 } \\
\text { weeks } \\
\text { gestation) or } \\
\text { unexposed } \\
\end{array}$ & $\begin{array}{l}\text { Gestational } \\
\text { diabetes }\end{array}$ & $\begin{array}{l}35 / 474 \\
(7.17 \%) \text { vs. } \\
90 / 1211 \\
(7.43 \%)^{104}\end{array}$ & $\begin{array}{l}\text { ARR: } 1.00 \\
(0.66-1.52)^{104}\end{array}$ & $\begin{array}{l}1 \text { cohort, } \\
N=1,685^{104}\end{array}$ & $\begin{array}{l}\text { Moderate } \\
\text { study } \\
\text { limitations, } \\
\text { imprecise } \\
\text { (Cls span } \\
\text { the null), } \\
\text { consistency } \\
\text { unknown }\end{array}$ & Insufficient \\
\hline $\begin{array}{l}\text { Pregnant } \\
\text { women: } \\
\text { Fluoxetine } \\
\text { exposure } \\
\text { gestational } \\
\text { days } 90 \text { to } 225 \\
\text { or depression } \\
\text { diagnosis, LMP } \\
\text { to gestational } \\
\text { day } 225 \\
\end{array}$ & $\begin{array}{l}\text { Fluoxetine } \\
\text { exposure, } \\
\text { 2nd to first } \\
\text { half of } 3 r d \\
\text { trimester vs. } \\
\text { unexposed }\end{array}$ & Preeclampsia & $\begin{array}{l}299 / 5,650 \\
\text { (5\%) vs. } \\
3,215 / 59,219 \\
(5 \%)^{75}\end{array}$ & $\begin{array}{l}\text { ARR, } 0.97 \\
(95 \% \mathrm{Cl}, 0.87 \\
\text { to } 1.09)^{75}\end{array}$ & $\begin{array}{l}1 \text { cohort: } \\
\mathrm{N}=64,869^{75}\end{array}$ & $\begin{array}{l}\text { Moderate } \\
\text { study } \\
\text { limitations, } \\
\text { imprecise } \\
\text { (Cls span } \\
\text { the null), } \\
\text { consistency } \\
\text { unknown }\end{array}$ & Insufficient \\
\hline $\begin{array}{l}\text { Women with } \\
\text { diagnosis code } \\
\text { for mood or } \\
\text { anxiety disorder } \\
1-5 \text { months } \\
\text { prior to delivery }\end{array}$ & $\begin{array}{l}\text { Fluoxetine } \\
\text { current (at } \\
\text { delivery) or } \\
\text { unexposed }\end{array}$ & $\begin{array}{l}\text { Postpartum } \\
\text { hemorrhage } \\
\text { billing } \\
\text { diagnosis }\end{array}$ & $\begin{array}{l}\text { Current: } \\
137 / 3,322 \\
(4.1 \%) \\
\text { Unexposed: } \\
1,896 / 69,044 \\
(2.8 \%)^{74} \\
\end{array}$ & $\begin{array}{l}\text { ARR, } \\
\text { current vs. } \\
\text { unexposed: } \\
1.51(95 \% \mathrm{Cl} \text {, } \\
1.27 \text { to } 1.79)^{74}\end{array}$ & $\begin{array}{l}1 \text { cohort, } \\
\mathrm{N}=72,366^{74}\end{array}$ & $\begin{array}{l}\text { Moderate } \\
\text { study } \\
\text { limitations, } \\
\text { precise, } \\
\text { consistency } \\
\text { unknown } \\
\end{array}$ & $\begin{array}{l}\text { Low for } \\
\text { harms } \\
\text { with } \\
\text { fluoxetine } \\
\end{array}$ \\
\hline
\end{tabular}




\begin{tabular}{|c|c|c|c|c|c|c|c|}
\hline Population & $\begin{array}{l}\text { Intervention } \\
\text { and } \\
\text { Comparator }\end{array}$ & Outcome & $\begin{array}{l}\text { Incidence or } \\
\text { Mean Effect } \\
\text { by Arm }\end{array}$ & Results & $\begin{array}{l}\text { Study Design } \\
\text { and Sample } \\
\text { Size }\end{array}$ & $\begin{array}{l}\text { Factors } \\
\text { That Affect } \\
\text { the } \\
\text { Strength of } \\
\text { Evidence }\end{array}$ & $\begin{array}{l}\text { Overall } \\
\text { Evidence } \\
\text { Strength } \\
\text { (Direction } \\
\text { of Effect) } \\
\end{array}$ \\
\hline $\begin{array}{l}\text { Women with } \\
\text { diagnosis code } \\
\text { for mood or } \\
\text { anxiety disorder } \\
1-5 \text { months } \\
\text { prior to delivery }\end{array}$ & $\begin{array}{l}\text { Fluoxetine } \\
\text { recent }(\leq 1 \\
\text { month } \\
\text { before } \\
\text { delivery), or } \\
\text { unexposed }\end{array}$ & $\begin{array}{l}\text { Postpartum } \\
\text { hemorrhage } \\
\text { billing } \\
\text { diagnosis }\end{array}$ & $\begin{array}{l}\text { Recent: } \\
50 / 1,628 \\
(3.1 \%) \\
\text { Unexposed: } \\
1,896 / 69,044 \\
(2.8 \%)^{74}\end{array}$ & $\begin{array}{l}\text { ARR, recent vs. } \\
\text { unxexp: } 1.14 \\
(95 \% \mathrm{Cl}, 0.86 \text { to } \\
1.50)^{74}\end{array}$ & $\begin{array}{l}1 \text { cohort, } \\
\mathrm{N}=70,672^{74}\end{array}$ & $\begin{array}{l}\text { Moderate } \\
\text { study } \\
\text { limitations, } \\
\text { imprecision } \\
\text { (wide Cls), } \\
\text { consistency } \\
\text { unknown } \\
\end{array}$ & Insufficient \\
\hline $\begin{array}{l}\text { Women with } \\
\text { diagnosis code } \\
\text { for mood or } \\
\text { anxiety disorder } \\
1-5 \text { months } \\
\text { prior to delivery }\end{array}$ & $\begin{array}{l}\text { Fluoxetine } \\
\text { past (>1 to } 5 \\
\text { months } \\
\text { before } \\
\text { delivery), or } \\
\text { unexposed }\end{array}$ & $\begin{array}{l}\text { Postpartum } \\
\text { hemorrhage } \\
\text { billing } \\
\text { diagnosis }\end{array}$ & $\begin{array}{l}\text { Past: } 78 / 3,075 \\
(2.5 \%) \\
\text { Unexposed: } \\
1,896 / 69,044 \\
(2.8 \%)^{74}\end{array}$ & $\begin{array}{l}\text { ARR, past vs. } \\
\text { unxexp: } \\
0.93(95 \% \mathrm{Cl} \text {, } \\
0.75 \text { to } 1.17)^{74}\end{array}$ & $\begin{array}{l}1 \text { cohort, } \\
\mathrm{N}=72,119^{74}\end{array}$ & $\begin{array}{l}\text { Moderate } \\
\text { study } \\
\text { limitations, } \\
\text { precision } \\
\text { (wide Cls), } \\
\text { consistency } \\
\text { unknown }\end{array}$ & Insufficient \\
\hline $\begin{array}{l}\text { Pregnant } \\
\text { women: Cohort } \\
\text { 1: depression } \\
\text { and/or anxiety } \\
\text { diagnosis and } \\
\text { exposure to } \\
\text { antidepressants } \\
\text { in the year } \\
\text { before } \\
\text { pregnancy; } \\
\text { Cohort 2: } \\
\text { depression } \\
\text { diagnosis from } \\
\text { the year before } \\
\text { conception } \\
\text { through the first } \\
\text { trimester }\end{array}$ & $\begin{array}{l}\text { Fluoxetine } \\
\text { exposure in } \\
\text { the first } \\
\text { trimester vs. } \\
\text { unexposed } \\
\end{array}$ & $\begin{array}{l}\text { Major } \\
\text { congenital } \\
\text { anomalies }\end{array}$ & $\begin{array}{l}\text { NR/191 vs. } \\
1,650 / 14,847 \\
(11.1 \%)^{57} ; \\
241 / 3,189 \\
(7.6 \%) \text { vs. } \\
380 / 13,432 \\
(2.8 \%)^{72}\end{array}$ & $\begin{array}{l}\text { AOR, } 0.80(95 \% \\
\mathrm{Cl}, 0.49 \text { to } 1.31) ; 57 \\
\text { AOR, } 0.85(95 \% \\
\mathrm{Cl}, 0.66 \text { to } 1.09)^{72}\end{array}$ & $\begin{array}{l}2 \text { cohorts: } \\
N=15,038 ; 57 \\
N=27,022^{72}\end{array}$ & $\begin{array}{l}\text { High study } \\
\text { limitations } \\
\text { (both high } \\
\text { risk of bias), } \\
\text { imprecise } \\
\text { (wide Cls); } \\
\text { consistent }\end{array}$ & Insufficient \\
\hline $\begin{array}{l}\text { Women with } \\
\text { depression or } \\
\text { anxiety before } \\
\text { pregnancy or } \\
\text { exposure to } \\
\text { antidepressants } \\
\text { outside of early } \\
\text { pregnancy }\end{array}$ & $\begin{array}{l}\text { Fluoxetine } \\
\text { exposure in } \\
\text { the first } \\
\text { trimester vs. } \\
\text { unexposed } \\
\end{array}$ & $\begin{array}{l}\text { Cardiac } \\
\text { anomalies }\end{array}$ & $\begin{array}{l}\text { NR/191 vs. } \\
\text { NR/14,847; } 57 \\
66 / 3,189 \\
(2.1 \%) \text { vs. } \\
112 / 13,432 \\
(0.8 \%)^{72} \\
84 / 8,664 \\
(1.0 \%) \text { vs. } \\
1,497 / 180,564 \\
(0.8 \%)^{7} 125 \\
\text { cases/81 } \\
\text { controls vs. } \\
149 \text { cases/125 }^{1} / 125 \\
\text { controls }\end{array}$ & $\begin{array}{l}\text { Pooled OR, } 1.06 \\
\text { (95\% Cl, } 0.82 \text { to } \\
\text { 1.39), I2: } 35.9 \% \\
\text { AOR, } 0.42(95 \% \\
\mathrm{Cl}, 0.10 \text { to } 1.73)^{57} \\
\text { AOR, } 0.79(95 \% \\
\mathrm{Cl}, 0.49 \text { to } 1.26)^{72} \\
\text { Propensity score } \\
\text { AOR, } 1.14(95 \% \\
\mathrm{Cl}, 0.90 \text { to } 1.44)^{7} \\
\text { AOR, } 1.33(95 \% \\
\mathrm{Cl}, 0.91 \text { to } 1.95)^{106}\end{array}$ & $\begin{array}{l}3 \text { cohorts, } 1 \\
\text { case-control: } \\
N=15,038,{ }^{57} \\
N=16,621,{ }^{72} \\
N=189,228,{ }^{7} \\
N=480^{106}\end{array}$ & $\begin{array}{l}\text { High study } \\
\text { limitations } \\
\text { (all high risk- } \\
\text { of-bias } \\
\text { studies), } \\
\text { imprecise } \\
\text { (wide Cls), } \\
\text { inconsistent }\end{array}$ & Insufficient \\
\hline $\begin{array}{l}\text { Pregnant } \\
\text { women: Any } \\
\text { lifetime } \\
\text { depression or } \\
\text { anxiety } \\
\text { diagnosis }\end{array}$ & $\begin{array}{l}\text { Fluoxetine } \\
\text { during } \\
\text { pregnancy } \\
\text { vs. } \\
\text { unexposed } \\
\text { women }\end{array}$ & $\begin{array}{l}\text { Autism } \\
\text { spectrum } \\
\text { disorder }\end{array}$ & $\begin{array}{l}8 / 327(2.1 \%) \\
\text { vs. } 282 / 14,805 \\
(1.9 \%) ; 56 \\
16 / 453(3.5 \%) \\
\text { vs. } 353 / 12,325 \\
(2.9 \%)^{51}\end{array}$ & $\begin{array}{l}\text { By } 7 \text { or 8-year } \\
\text { followup ARR, } \\
1.08(0.53 \text { to } \\
2.21)^{-56} \\
4 \text { year or more } \\
\text { followup: AOR, } \\
1.42(0.84 \text { to } \\
2.39)^{51}\end{array}$ & $\begin{array}{l}2 \text { cohorts: } \\
\mathrm{N}=15,132 ; 56 \\
\mathrm{~N}=12,778^{51} \\
\text { (potential } \\
\text { overlap of } \\
\text { participants in } \\
\text { the } \\
\text { publications) }\end{array}$ & $\begin{array}{l}\text { Moderate } \\
\text { study } \\
\text { limitations, } \\
\text { imprecise } \\
\text { (wide Cls); } \\
\text { consistent }\end{array}$ & Insufficient \\
\hline
\end{tabular}

$\mathrm{AOR}=$ adjusted odds ratio; $\mathrm{ARR}=$ adjusted risk ratio; $\mathrm{CI}=$ confidence interval; $\mathrm{dx}=$ diagnosis; $\mathrm{LMP}=$ last menstrual period; $\mathrm{N}$ $=$ number; $\mathrm{NR}=$ not reported; vs. $=$ versus. 
Study cohorts consisted of pregnant women with depression or depression/anxiety, comparing women exposed to fluoxetine with those not exposed. The time period used to determine depression or depression/anxiety in the not exposed group differed across studies, including a period during pregnancy that was comparable to the fluoxetine exposure ${ }^{72,74,75}$ the 6 months before pregnancy; ${ }^{104}$ exposed to antidepressants outside of early pregnancy; ${ }^{106}$ the year prior to pregnancy; ${ }^{57}$ any lifetime diagnosis, ${ }^{51,56}$ or, in one publication, the time period was not reported. $^{7}$

The evidence for harms from fluoxetine was graded as low strength of evidence for increased risk of postpartum hemorrhage among women exposed at the time of delivery $(R R=1.51)$, based on evidence from one large Medicaid cohort $(\mathrm{N}=72,366) .{ }^{74}$ In this study, the risk of postpartum hemorrhage was not significantly different between the two groups if fluoxetine exposure was prior to delivery (either $\leq 1$ month or $>1$ to 5 months) (insufficient strength of evidence).

The evidence was insufficient to judge any of the other studied outcomes. The outcomes include maternal outcomes (gestational diabetes or preeclampsia) and child adverse outcomes (major congenital anomalies, cardiac anomalies, or autism spectrum disorder) (Table B-19).

One publication reporting on gestational diabetes $(\mathrm{N}=1,685)$ did not find an increased risk (ARR, 1.00) and the CI surrounding the estimate is large (imprecise), precluding no difference. Similarly, preeclampsia, only reported in one publication, did not find an increased risk. ${ }^{75}$ Based on a large cohort $(\mathrm{N}=64,869)$, the $\mathrm{ARR}$ is close to 1 ( $\mathrm{ARR}=0.97)$, but the $\mathrm{CI}$ surrounding the estimate is large (imprecise), precluding concluding no difference. Two high risk-of-bias publications, reporting on the risk of major congenital anomalies related to exposure to fluoxetine during the first trimester, found no increased risk in either study, but similar to the preeclampsia finding, large confidence intervals preclude concluding no difference. ${ }^{57,72}$ Four high risk-of-bias publications reporting on cardiac anomalies were pooled, resulting in a finding of no increased risk from fluoxetine (OR, 1.06; 95\% CI, 0.82 to 1.39, Figure B-4). The CIs were wide and spanned both appreciable benefit and appreciable harm; as a result, we could not conclude equivalence. One study with moderate study limitations that also did not adjust for multiple comparisons generally reported no difference in several other birth defects, including

neural tube defects (AOR, 0.94; 95\% CI, 0.46 to 1.93). ${ }^{106}$ Lastly, two publications, with potential overlaps in the cohort, reporting on autism spectrum disorder results ${ }^{51,56}$ were consistent in reporting an increased association in the fluoxetine group, but the confidence intervals were large in both studies, resulting in the association not being significantly different from that of the not exposed group. When the referent group was restricted to women with more than one psychiatric diagnosis, the point estimate for relative risk moved from 1.18 ( $\geq 1$ disorder, with CIs spanning the null) to 0.66 ( $\geq 3$ disorders, also with CIs spanning the null). ${ }^{56}$ This adjustment for confounding further suggests that the incidence of autism cannot be attributed to exposure to fluoxetine.

\section{SSRIs: Fluvoxamine}

\section{Overview}

- The evidence for fluvoxamine during pregnancy, when analyzed individually, is insufficient to judge the risk of harms for the child outcome (major congenital anomalies). 


\section{Detailed Results}

One publication reported on fluvoxamine. ${ }^{57}$ This study drew from one cohort (the Canadian QPC), which was a nonrandomized observational study and was rated as high risk of bias. This publication focused on exposure 12 months preconception or during pregnancy. The publication compared pregnant women exposed to fluvoxamine with women with untreated depression or anxiety. ${ }^{57}$

The evidence for fluvoxamine, when analyzed individually, is insufficient to judge the risk of harms for the child outcome (major congenital anomalies) (Table B-23). This study found no association between fluvoxamine use in pregnancy or preconception and the prevalence odds of major congenital anomalies. There was no study to which to compare findings, so the consistency of results is not known.

Table B-23. Strength of evidence for harms: Fluvoxamine versus no treatment

\begin{tabular}{|c|c|c|c|c|c|c|c|}
\hline Population & $\begin{array}{l}\text { Intervention and } \\
\text { Comparator }\end{array}$ & Outcome & $\begin{array}{l}\text { Incidence } \\
\text { or Mean } \\
\text { Effect by } \\
\text { Arm }\end{array}$ & Results & $\begin{array}{l}\text { Study } \\
\text { Design and } \\
\text { Sample } \\
\text { Size }\end{array}$ & $\begin{array}{l}\text { Factors That } \\
\text { Affect the } \\
\text { Strength of } \\
\text { Evidence }\end{array}$ & $\begin{array}{l}\text { Overall } \\
\text { Evidence } \\
\text { Strength } \\
\text { (Direction of } \\
\text { Effect) }\end{array}$ \\
\hline $\begin{array}{l}\text { Depression/ } \\
\text { anxiety or } \\
\text { fluvoxamine- } \\
\text { exposed } \\
\text { women }\end{array}$ & $\begin{array}{l}\text { Fluvoxamine } \\
\text { exposure in } \\
\text { preconception or } \\
\text { pregnancy vs. } \\
\text { unexposed women } \\
\text { with depression or } \\
\text { anxiety }\end{array}$ & $\begin{array}{l}\text { Major congenital } \\
\text { anomalies }\end{array}$ & $\mathrm{NR}^{57}$ & $\begin{array}{l}\text { AOR, } 0.63, \\
95 \% \mathrm{Cl},(0.23 \\
\text { to } 1.77)^{57}\end{array}$ & $\begin{array}{l}1 \text { cohort, } \\
\text { NR }^{57}\end{array}$ & $\begin{array}{l}\text { High study } \\
\text { limitations (high } \\
\text { risk of bias }{ }^{57} \text { ), } \\
\text { likely imprecise, } \\
\text { consistency } \\
\text { unknown }\end{array}$ & Insufficient \\
\hline
\end{tabular}

$\overline{\mathrm{AOR}}=$ adjusted odds ratio; $\mathrm{CI}=$ confidence interval; $\mathrm{NR}=$ not reported.

\section{SSRIs: Paroxetine}

\section{Overview}

- When compared with unexposed women with depression or anxiety, paroxetine exposure within 1 month prior to delivery may be associated with a risk of postpartum hemorrhage (low strength of evidence of harms). The evidence of postpartum hemorrhage was judged to be insufficient if paroxetine exposure was at the time of delivery or 1 to 5 months prior to delivery.

- Paroxetine exposure in the first trimester is associated with a higher risk of atrial septal defect but not with other cardiac anomalies.

- The evidence is insufficient to judge the risk of harms for exposure to paroxetine for other maternal (discontinuation due to adverse events, preeclampsia, or gestational diabetes) or child outcomes ("harm to infant," major congenital anomalies, cardiac anomalies, or autism spectrum disorder).

\section{Detailed Results}

Eleven publications reported on harms of paroxetine (Table B-24). 7, 25, 51, 56, 57, 72, 74, 75, 93, 104, 106 Of these, four reported on maternal harms, $25,74,75,104$ and eight reported on fetal or infant harms..$^{7,25,51,56,57,72,93,106}$ Four were rated high risk of bias. ${ }^{7,25,57,72}$ The rest had some risk-ofbias concerns. 
Table B-24. Strength of evidence for harms outcomes: Paroxetine versus no treatment

\begin{tabular}{|c|c|c|c|c|c|c|c|}
\hline Population & $\begin{array}{l}\text { Intervention } \\
\text { and } \\
\text { Comparator }\end{array}$ & Outcome & $\begin{array}{l}\text { Incidence or } \\
\text { Mean Effect } \\
\text { by Arm }\end{array}$ & Results & $\begin{array}{l}\text { Study } \\
\text { Design and } \\
\text { Sample Size }\end{array}$ & $\begin{array}{l}\text { Factors That } \\
\text { Affect the } \\
\text { Strength of } \\
\text { Evidence }\end{array}$ & $\begin{array}{l}\text { Overall } \\
\text { Evidence } \\
\text { Strength } \\
\text { (Direction of } \\
\text { Effect) }\end{array}$ \\
\hline $\begin{array}{l}\text { Women with } \\
\text { depression } \\
\text { onset 0-3 } \\
\text { months pp }\end{array}$ & $\begin{array}{l}\text { Paroxetine } \\
\text { vs. placebo }\end{array}$ & $\begin{array}{l}\text { Discontin- } \\
\text { uation due to } \\
\text { adverse } \\
\text { events }\end{array}$ & $\begin{array}{l}1 / 35(2.86 \%) \\
\text { vs } 4 / 35 \\
(16 \%)^{25}\end{array}$ & No difference & $\mathrm{RCT}, \mathrm{N}=70^{25}$ & $\begin{array}{l}\text { High study } \\
\text { limitations } \\
\text { (high risk of } \\
\text { bias, }{ }^{25} \\
\text { serious } \\
\text { imprecision } \\
\text { (few events, } \\
\text { small sample } \\
\text { size), } \\
\text { consistency } \\
\text { unknown }\end{array}$ & Insufficient \\
\hline $\begin{array}{l}\text { Women with } \\
\text { history of } \\
\text { anti- } \\
\text { depressant } \\
\text { use at least } 6 \\
\text { months } \\
\text { before } \\
\text { pregnancy }\end{array}$ & $\begin{array}{l}\text { Paroxetine } \\
\text { vs. no anti- } \\
\text { depressant fill }\end{array}$ & $\begin{array}{l}\text { Gestational } \\
\text { Diabetes }\end{array}$ & $\begin{array}{l}12 / 147(8 \%) \\
\text { vs. } 90 / 1,211 \\
(7 \%)^{104}\end{array}$ & $\begin{array}{l}\text { Weighted RR, } \\
0.88(95 \% \\
\mathrm{Cl}, .49 \text { to } \\
1.60)^{104}\end{array}$ & $\begin{array}{l}1 \text { cohort, } \\
\mathrm{N}=1,358^{104}\end{array}$ & $\begin{array}{l}\text { Moderate } \\
\text { study } \\
\text { limitations, } \\
\text { imprecision } \\
\text { (small sample } \\
\text { size, wide } \\
\text { Cls), } \\
\text { consistency } \\
\text { unknown } \\
\end{array}$ & Insufficient \\
\hline $\begin{array}{l}\text { Women with } \\
\text { a diagnosis } \\
\text { code for } \\
\text { depression } \\
\text { between LMP } \\
\text { and } 225 \\
\text { gestational } \\
\text { days }\end{array}$ & $\begin{array}{l}\text { Paroxetine } \\
\text { dispensed } \\
\text { between } 90 \\
\text { and } 225 \text { days } \\
\text { of gestation } \\
\text { vs. no } \\
\text { antidepres- } \\
\text { sant, LMP to } \\
225 \text { days of } \\
\text { gestation }\end{array}$ & $\begin{array}{l}\text { Preeclampsia } \\
\text { billing code } \\
\text { between } 140 \\
\text { days of } \\
\text { gestation and } \\
30 \text { days } \\
\text { postpartum }\end{array}$ & $\begin{array}{l}183 / 3517 \\
(5 \%) \text { vs. } \\
3,215 / 59,219 \\
(5 \%)\end{array}$ & $\begin{array}{l}\text { ARR, } \\
\text { paroxetine vs. } \\
\text { unexposed: } \\
0.99(95 \% \mathrm{Cl} \text {, } \\
0.86 \text { to } 1.15)\end{array}$ & $\begin{array}{l}1 \text { cohort, } \mathrm{N}= \\
100,942^{75}\end{array}$ & $\begin{array}{l}\text { Moderate } \\
\text { study } \\
\text { limitations, } \\
\text { precision } \\
\text { (wide Cls), } \\
\text { consistency } \\
\text { unknown }\end{array}$ & Insufficient \\
\hline $\begin{array}{l}\text { Women with } \\
\text { diagnosis } \\
\text { code for } \\
\text { mood or } \\
\text { anxiety } \\
\text { disorder } 1-5 \\
\text { months prior } \\
\text { to delivery }\end{array}$ & $\begin{array}{l}\text { Paroxetine } \\
\text { current (at } \\
\text { delivery) or } \\
\text { unexposed }\end{array}$ & $\begin{array}{l}\text { Postpartum } \\
\text { hemorrhage } \\
\text { billing } \\
\text { diagnosis }\end{array}$ & $\begin{array}{l}77 / 2,055 \\
(3.75 \%) \text { vs. } \\
1,896 / 69,044 \\
(2.75 \%)^{74}\end{array}$ & $\begin{array}{l}\text { ARR, } \\
\text { Current vs. } \\
\text { unexposed: } \\
1.39(95 \% \mathrm{Cl} \text {, } \\
1.09 \text { to } 1.71)^{74}\end{array}$ & $\begin{array}{l}1 \text { cohort, } N= \\
71,099^{74}\end{array}$ & $\begin{array}{l}\text { Moderate } \\
\text { study } \\
\text { limitations, } \\
\text { precise, } \\
\text { consistency } \\
\text { unknown }\end{array}$ & $\begin{array}{l}\text { Low for } \\
\text { harms with } \\
\text { paroxetine }\end{array}$ \\
\hline $\begin{array}{l}\text { Women with } \\
\text { diagnosis } \\
\text { code for } \\
\text { mood or } \\
\text { anxiety } \\
\text { disorder } 1-5 \\
\text { months prior } \\
\text { to delivery }\end{array}$ & $\begin{array}{l}\text { Paroxetine } \\
\text { recent }(<1 \\
\text { month before } \\
\text { delivery), or } \\
\text { unexposed }\end{array}$ & $\begin{array}{l}\text { Postpartum } \\
\text { hemorrhage } \\
\text { billing } \\
\text { diagnosis }\end{array}$ & $\begin{array}{l}40 / 962 \\
(4.16 \%) \text { vs, } \\
1,896 / 69,044 \\
(2.75 \%)^{74}\end{array}$ & $\begin{array}{l}\text { ARR, recent vs. } \\
\text { unexposed: } \\
1.52(95 \% \mathrm{Cl} \text {, } \\
1.12-2.07) \\
\text { Past: } 1.13(95 \% \\
\mathrm{Cl}, 0.85 \text { to } \\
1.49)^{74}\end{array}$ & $\begin{array}{l}1 \text { cohort, } N= \\
70,006^{74}\end{array}$ & $\begin{array}{l}\text { Moderate } \\
\text { study } \\
\text { limitations, } \\
\text { imprecision } \\
\text { (wide Cls), } \\
\text { consistency } \\
\text { unknown }\end{array}$ & Insufficient \\
\hline $\begin{array}{l}\text { Women with } \\
\text { diagnosis } \\
\text { code for } \\
\text { mood or } \\
\text { anxiety } \\
\text { disorder } 1-5 \\
\text { months prior } \\
\text { to delivery }\end{array}$ & $\begin{array}{l}\text { Paroxetine } \\
\text { past (>1 to } 5 \\
\text { months } \\
\text { before } \\
\text { delivery), or } \\
\text { unexposed }\end{array}$ & $\begin{array}{l}\text { Postpartum } \\
\text { hemorrhage } \\
\text { billing } \\
\text { diagnosis }\end{array}$ & $\begin{array}{l}49 / 1617 \\
(3.03 \%) \text { vs. } \\
1,896 / 69,044 \\
(2.75 \%)^{74}\end{array}$ & $\begin{array}{l}\text { ARR, past vs. } \\
\text { unexposed: } \\
1.13(95 \% \mathrm{Cl} \text {, } \\
0.85 \text { to } 1.49)^{74}\end{array}$ & $\begin{array}{l}1 \text { cohort, } N= \\
70,661^{74}\end{array}$ & $\begin{array}{l}\text { Moderate } \\
\text { study } \\
\text { limitations, } \\
\text { precision } \\
\text { (wide Cls), } \\
\text { consistency } \\
\text { unknown }\end{array}$ & Insufficient \\
\hline
\end{tabular}




\begin{tabular}{|c|c|c|c|c|c|c|c|}
\hline Population & $\begin{array}{l}\text { Intervention } \\
\text { and } \\
\text { Comparator }\end{array}$ & Outcome & $\begin{array}{l}\text { Incidence or } \\
\text { Mean Effect } \\
\text { by Arm }\end{array}$ & Results & $\begin{array}{l}\text { Study } \\
\text { Design and } \\
\text { Sample Size }\end{array}$ & $\begin{array}{l}\text { Factors That } \\
\text { Affect the } \\
\text { Strength of } \\
\text { Evidence }\end{array}$ & $\begin{array}{l}\text { Overall } \\
\text { Evidence } \\
\text { Strength } \\
\text { (Direction of } \\
\text { Effect) }\end{array}$ \\
\hline $\begin{array}{l}\text { Women with } \\
\text { depression or } \\
\text { anxiety in the } \\
\text { year prior to } \\
\text { pregnancy }\end{array}$ & $\begin{array}{l}\text { Paroxetine in } \\
\text { 1st trimester } \\
\text { vs. not } \\
\text { exposed in } \\
\text { 1st trimester }\end{array}$ & $\begin{array}{l}\text { Major } \\
\text { congenital } \\
\text { anomalies }\end{array}$ & $\begin{array}{l}\text { 168/1,132 } \\
(14.8 \%) \text { vs. } \\
1,650 / 14,847 \\
(11.1 \%)^{57} \\
\text { Paroxetine- } \\
\text { exposed: } 37 \\
\text { cases, } 375 \\
\text { controls } \\
\text { No } \\
\text { antidepres- } \\
\text { sant: } 94 \\
\text { cases, } 1,134 \\
\text { controls } \\
\\
36 / 1,200 \\
(3.0 \%) \text { vs. } \\
380 / 13,432 \\
(2.8 \%)^{72}\end{array}$ & $\begin{array}{l}\text { Results not } \\
\text { pooled because } \\
\text { two } \\
\text { publications }{ }^{57,93} \\
\text { potentially drew } \\
\text { from the same } \\
\text { population } \\
\text { AOR, } 1.24 \\
(95 \% \mathrm{Cl}, 0.99 \\
\text { to } 1.55 ; 99 \% \mathrm{Cl} \text {, } \\
0.79 \text { to } 1.66)^{57} \\
\text { AOR, } 1.27 \\
(95 \% \mathrm{Cl}, 0.78 \\
\text { to } 2.06)^{93} \\
\text { AOR, } 1.01 \\
(95 \% \mathrm{Cl}, 0.71 \\
\text { to } 1.44)^{72}\end{array}$ & $\begin{array}{l}2 \text { cohorts, }{ }^{57,72} \\
1 \text { case- } \\
\text { control, }{ }^{93} \\
\mathrm{~N} \geq 33,119(\mathrm{~N} \\
\text { from two } \\
\text { studies) }{ }^{57,}, 72 \\
\text { (study } \\
\text { samples may } \\
\text { overlap) }\end{array}$ & $\begin{array}{l}\text { High study } \\
\text { limitations } \\
\text { (two high } \\
\text { risk-of-bias } \\
\text { studies }^{57,72} \text { ), } \\
\text { imprecise, } \\
\text { consistent }\end{array}$ & Insufficient \\
\hline $\begin{array}{l}\text { Women with } \\
\text { depression or } \\
\text { anxiety in the } \\
\text { year prior to } \\
\text { pregnancy or } \\
\text { exposure to } \\
\text { anti- } \\
\text { depressants } \\
\text { outside of } \\
\text { early } \\
\text { pregnancy }\end{array}$ & $\begin{array}{l}\text { Paroxetine in } \\
\text { first trimester } \\
\text { vs. } \\
\text { unexposed }\end{array}$ & $\begin{array}{l}\text { Cardiac } \\
\text { anomalies }\end{array}$ & $\begin{array}{l}\text { NR/1132 vs. } \\
\text { NR/14,84757 } \\
17 / 1200 \\
(1.4 \%) \text { vs. } \\
112 / 13,432 \\
\left(0.8 \%^{72}\right. \\
93 / 11,126 \text { vs. } \\
\text { NR/180,5647 } \\
69 \text { cases/43 } \\
\text { controls vs. } \\
149 \\
\text { cases/125 }^{7} \\
\text { controls }^{106}\end{array}$ & 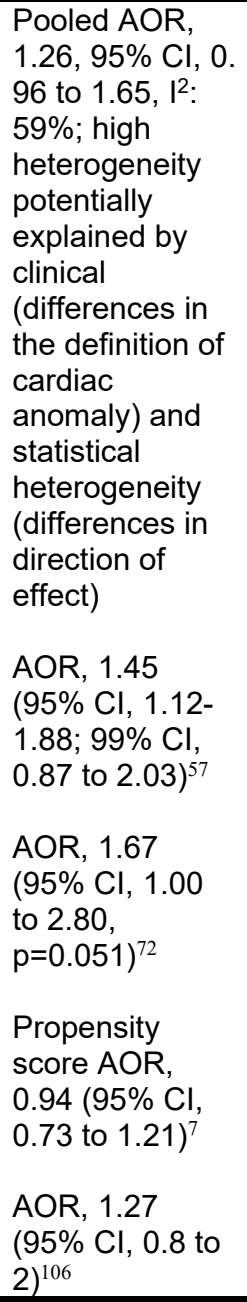 & $\begin{array}{l}3 \text { cohorts, } \\
721 \text {, } 1 \text { case- } \\
\text { control, }{ }^{106} \\
\mathrm{~N}=222,505\end{array}$ & $\begin{array}{l}\text { High study } \\
\text { limitations ( } 3 \\
\text { studies }^{7,57,72} \text { ), } \\
\text { imprecision } \\
\text { (wide Cls), } \\
\text { inconsistent }\end{array}$ & Insufficient \\
\hline
\end{tabular}




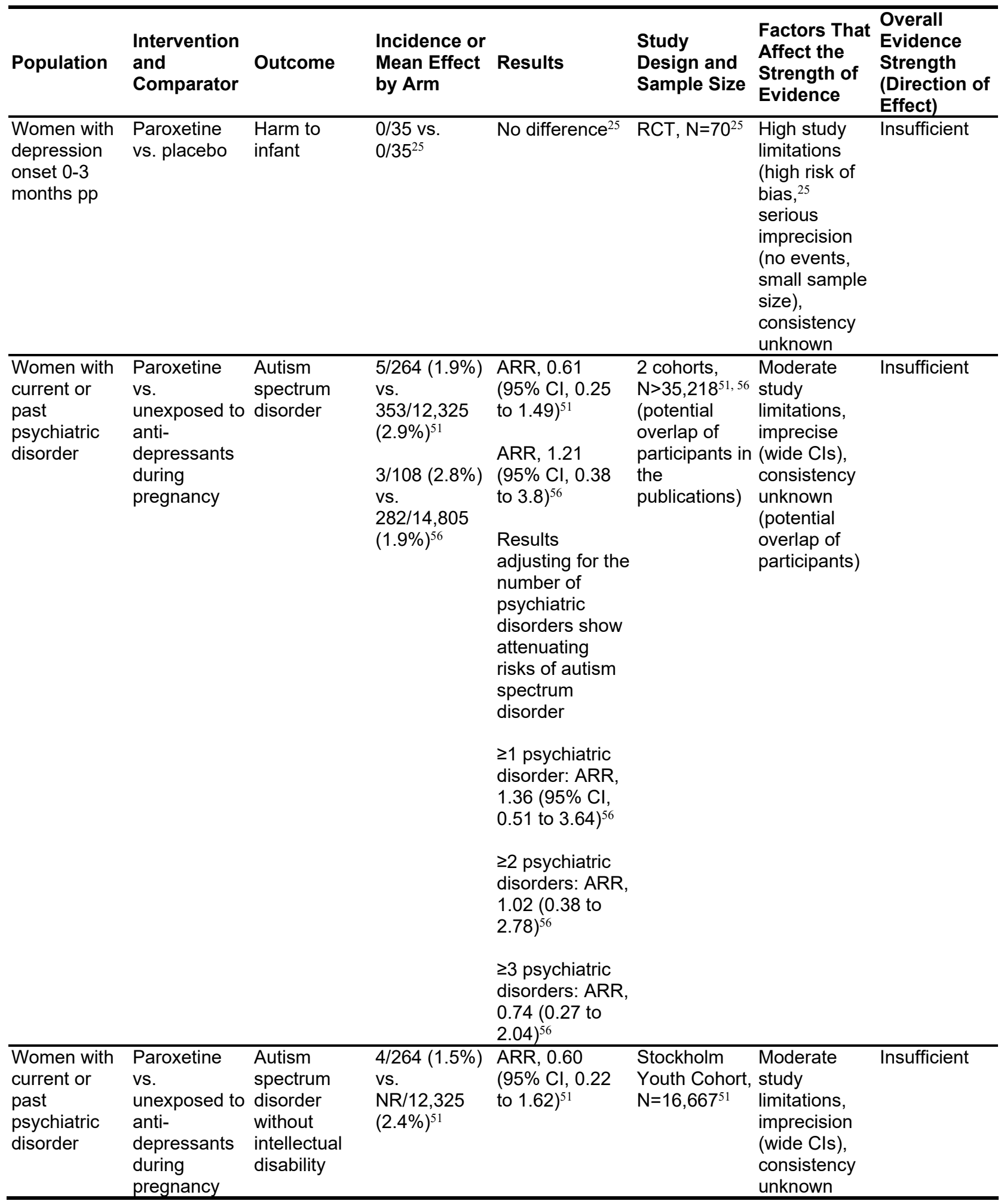

$\mathrm{AOR}=$ adjusted odds ratio; $\mathrm{ARR}=$ adjusted risk ratio; $\mathrm{CI}=$ confidence interval; $\mathrm{LMP}=$ last menstrual period; $\mathrm{N}=$ number; $\mathrm{NR}=$ not reported; $\mathrm{RCT}=$ randomized controlled trial; vs. = versus. 
Results were drawn from seven independent cohorts, including a small RCT; ${ }^{25}$ a British population sample; ${ }^{72}$ and cohorts from Sweden, ${ }^{51,56}$ Quebec, ${ }^{57,93}$ and the United States. ${ }^{7}$, 74, 75, 104, ${ }^{106}$ The two Swedish cohorts evaluated risk of autism spectrum disorder, and both included children residing in Stockholm County who were born in 2006 or 2007. ${ }^{51,56}$ The two Quebec studies evaluated congenital anomalies among births in Quebec from 1998 to $2002^{93}$ and from 1998 to $2008 .{ }^{57}$ The two publications from Quebec potentially drew participants from the same cohort, similarly, the Swedish publications may have drawn from the same cohort.

One randomized trial among postpartum women with onset of depression symptoms between 0 and 3 months postpartum compared paroxetine with placebo ${ }^{25}$ and found no differences in discontinuation for maternal side effects or harm to the infant. The remaining publications were nonrandomized studies. Two publications reported on maternal harms of hemorrhage or preeclampsia using data from the U.S. Medicaid Analytic eXtract. The hemorrhage analysis ${ }^{74}$ quantified the association between a diagnosis code for postpartum hemorrhage and dispensing of a supply of paroxetine that overlapped with the delivery date (current), within 1 month of delivery date (recent), of $>1$ to 5 months before delivery date (past), compared with a referent group of women with a depression or anxiety diagnosis within 1 to 5 months of delivery but no medical therapy. Current or recent, but not past, dispensing of paroxetine was associated with an increased risk of postpartum hemorrhage, with adjustment for multiple confounders. A second publication using the Medicaid Analytic eXtract sample among women with a depression diagnosis between the last menstrual period and 255 days of pregnancy found no association between paroxetine exposure and preeclampsia, adjusting for multiple confounders. ${ }^{75}$ One study, a Kaiser Permanente Washington cohort, ${ }^{104}$ reported no differences in the rate of gestational diabetes.

Three observational studies evaluated the association between paroxetine exposure in the first trimester compared with no antidepressant and the risk of major congenital anomalies ${ }^{57,72,93}$ among women with depression or anxiety in the year prior to pregnancy. Two publications, likely drawing from the same population source from Quebec, found modest associations, but confidence intervals were wide, ${ }^{57,93}$ a U.K. THIN publication found no association. ${ }^{72}$

Four studies specifically quantified associations between first trimester paroxetine exposure and cardiac anomalies. ${ }^{7,57,72,106}$ In the Quebec and THIN cohorts, exposure was associated with cardiac anomalies, but in the propensity-score-adjusted Medicaid eXtract study and a National Birth Defects Prevention case-control, no association was found. The pooled adjusted OR was 1.26 (95\% CI, 0.96 to $1.65, \mathrm{I}^{2}, 59 \%$; Figure B-5). These differences may reflect the diagnosis codes used to define cardiac anomalies: Whereas atrial septal defect/patent foramen ovale (ICD9 745.5, ICD10 Q21.1) was included in the definition of cardiac anomaly for the Quebec and U.K. cohorts, this code was excluded from the definition of cardiac anomalies in the Medicaid eXtract sample. The National Birth Defects Prevention Study relied on a classification of heart defects developed for the study. ${ }^{149}$ The publication from the Quebec cohort reported a higher risk of ASD/VSD $;{ }^{57}$ the Medicaid eXtract publication found no association with VSD. ${ }^{7}$ There was no association between paroxetine exposure and any other class of birth defects in adjusted analyses in two studies; ${ }^{57,72}$ a third generally reported no significant differences but did not control for multiple comparisons. ${ }^{106}$ Given differences in criteria used, it is unclear whether there is a clinically meaningful association between paroxetine exposure and cardiac anomalies.

Two publications from Swedish birth cohorts, with potential overlaps in the cohort, found no association between paroxetine exposure during pregnancy among women with a current or past psychiatric disorder and autism spectrum disorder in offspring. ${ }^{51,56}$ Moreover, when the referent 
group was restricted to women with more than one psychiatric diagnosis, the point estimate for relative risk moved from 1.36 ( $\geq 1$ disorder) to 0.74 ( $\geq 3$ disorders). ${ }^{56}$

\section{SSRIs: Sertraline}

\section{Overview}

- One moderate risk-of-bias cohort study reported that sertraline use during pregnancy increases the risk of postpartum hemorrhage for exposure at the time of delivery or exposure 1 to 30 days prior to delivery (low strength of evidence). The evidence of postpartum hemorrhage was judged to be insufficient if sertraline exposure was 1 to 5 months prior to delivery.

- The evidence for the remaining maternal outcome comparisons (side effects, attrition, preeclampsia, gestational diabetes), as well as all of the fetal outcomes (major congenital anomalies, cardiac anomalies, or autism spectrum disorder), is insufficient for the risks of harm with sertraline use during pregnancy.

\section{Detailed Results}

Twelve publications reported on either maternal or fetal harms of sertraline (Table B-25). ${ }^{7}$

16, $23,51,56,57,70,72,74,75,104,106$ Two studies were RCTs. ${ }^{16,23}$ Of the remaining ten studies, three drew from the United States Medicaid Analytic Extract; ${ }^{7,74,75}$ one from the United Kingdom's THI) ${ }^{72}$ two from Canada's QPC, likely drawing on the same population; ${ }^{57,70}$ two from Sweden, one from the Stockholm Youth Cohort ${ }^{51}$ and one from the Swedish National Registers; ${ }^{56}$ one from Kaiser Permanente Washington, ${ }^{104}$ and one from the U.S. National Birth Defects Prevention Study. ${ }^{106}$ Five were rated high risk of bias, ${ }^{7,} 16,57,70,72$ six were rated some risk-ofbias concerns, ${ }^{51,56,74,75,104,106}$ and one was rated low risk of bias. ${ }^{23}$

Table B-25. Strength of evidence for harms: Sertraline versus no treatment

\begin{tabular}{|c|c|c|c|c|c|c|c|}
\hline Population & $\begin{array}{l}\text { Intervention and } \\
\text { Comparator }\end{array}$ & Outcome & $\begin{array}{l}\text { Incidence or } \\
\text { Mean Effect } \\
\text { by Arm }\end{array}$ & Results & $\begin{array}{l}\text { Study Design } \\
\text { and Sample } \\
\text { Size }\end{array}$ & $\begin{array}{l}\text { Factors That } \\
\text { Affect the } \\
\text { Strength of } \\
\text { Evidence }\end{array}$ & $\begin{array}{l}\text { Overall } \\
\text { Evidence } \\
\text { Strength } \\
\text { (Direction } \\
\text { of Effect) }\end{array}$ \\
\hline $\begin{array}{l}\text { Women with } \\
\text { depression } \\
\text { onset } 0-12 \\
\text { months } \\
\text { postpartum }\end{array}$ & $\begin{array}{l}\text { Sertraline vs. } \\
\text { interpersonal } \\
\text { psychotherapy }\end{array}$ & $\begin{array}{l}\text { Overall } \\
\text { attrition }\end{array}$ & $\begin{array}{l}16 / 56(28.6 \%) \\
\text { vs. } 9 / 53 \\
(17.0 \%)^{16}\end{array}$ & $\begin{array}{l}\text { No difference } \\
\text { between two } \\
\text { arms } \\
(p=0.119)^{16}\end{array}$ & $\mathrm{RCT}, \mathrm{n}=109^{16}$ & $\begin{array}{l}\text { High study } \\
\text { limitations } \\
\text { (high risk of } \\
\text { bias }^{16} \text { ), likely } \\
\text { imprecise, } \\
\text { consistency } \\
\text { unknown }\end{array}$ & Insufficient \\
\hline $\begin{array}{l}\text { Women with } \\
\text { depression } \\
\text { onset } 0-2 \\
\text { month } \\
\text { postpartum }\end{array}$ & $\begin{array}{l}\text { Sertraline plus brief } \\
\text { dynamic } \\
\text { psychotherapy vs. } \\
\text { placebo }\end{array}$ & $\begin{array}{l}\text { UKU Side } \\
\text { Effects } \\
\text { Rating Scale } \\
\text { at } 8 \text { weeks } \\
\text { postpartum }\end{array}$ & $\begin{array}{l}\text { Mean change } \\
\text { in UKU with } \\
\text { sertraline = } \\
-8.4 ; \text { without } \\
\text { sertraline = } \\
-7.8\end{array}$ & $\begin{array}{l}\text { No difference } \\
\text { between two } \\
\text { arms } \\
(p=0.456)^{23}\end{array}$ & $\mathrm{RCT}, \mathrm{n}=40^{23}$ & $\begin{array}{l}\text { Low risk of } \\
\text { bias }^{23} \text { ) } \\
\text { seriously } \\
\text { imprecise (no } \\
\text { statistical } \\
\text { significance, } \\
\text { small sample } \\
\text { size), } \\
\text { consistency } \\
\text { unknown }\end{array}$ & Insufficient \\
\hline
\end{tabular}




\begin{tabular}{|c|c|c|c|c|c|c|c|}
\hline Population & $\begin{array}{l}\text { Intervention and } \\
\text { Comparator }\end{array}$ & Outcome & $\begin{array}{l}\text { Incidence or } \\
\text { Mean Effect } \\
\text { by Arm }\end{array}$ & Results & $\begin{array}{l}\text { Study Design } \\
\text { and Sample } \\
\text { Size }\end{array}$ & $\begin{array}{l}\text { Factors That } \\
\text { Affect the } \\
\text { Strength of } \\
\text { Evidence }\end{array}$ & $\begin{array}{l}\text { Overall } \\
\text { Evidence } \\
\text { Strength } \\
\text { (Direction } \\
\text { of Effect) }\end{array}$ \\
\hline $\begin{array}{l}\text { Women with } \\
\text { an } \\
\text { antidepressant } \\
\text { prescription } \\
\text { filled during } \\
\text { the } 6 \text { months } \\
\text { before } \\
\text { pregnancy }\end{array}$ & $\begin{array}{l}\text { Sertraline } \\
\text { continuation (1 or } \\
\text { more refills of } \\
\text { sertraline between } \\
\text { last menstrual } \\
\text { period and } 20-24 \\
\text { weeks gestation) } \\
\text { vs. sertraline } \\
\text { discontinuation (no } \\
\text { sertraline refill) }\end{array}$ & $\begin{array}{l}\text { Gestational } \\
\text { diabetes }\end{array}$ & $\begin{array}{l}52 / 462 \\
(11.23 \%) \text { vs. } \\
90 / 1211 \\
(7.43 \%)^{104}\end{array}$ & $\begin{array}{l}\text { IPTW RR, } \\
1.30(95 \% \mathrm{Cl}, \\
0.63 \text { to } \\
1.81)^{104}\end{array}$ & $\begin{array}{l}\text { Retrospective } \\
\text { study within } \\
\text { Kaiser } \\
\text { Permanente } \\
\text { Washington }^{104} \\
\mathrm{~N}=1,673\end{array}$ & $\begin{array}{l}\text { Moderate } \\
\text { study } \\
\text { limitations, } \\
\text { precise (wide } \\
\text { Cls), } \\
\text { consistency } \\
\text { unknown }\end{array}$ & Insufficient \\
\hline $\begin{array}{l}\text { Women with a } \\
\text { diagnosis code } \\
\text { for depression } \\
\text { between LMP } \\
\text { and } 225 \\
\text { gestational } \\
\text { days }\end{array}$ & $\begin{array}{l}\text { Sertraline } \\
\text { dispensed between } \\
90 \text { and } 225 \text { days of } \\
\text { gestation vs. no } \\
\text { antidepressant, } \\
\text { LMP to } 225 \text { days of } \\
\text { gestation }\end{array}$ & $\begin{array}{l}\text { Preeclampsia } \\
\text { billing code } \\
\text { between } 140 \\
\text { days of } \\
\text { gestation and } \\
30 \text { days } \\
\text { postpartum }\end{array}$ & $\begin{array}{l}3,98 / 7,143 \\
(5.6 \%) \text { vs. } \\
3,215 / 59,219 \\
(5 \%)^{75} \\
\\
\end{array}$ & $\begin{array}{l}\text { ARR, } 1.03 \\
(95 \% \mathrm{Cl}, 0.93 \\
\text { to } 1.14)^{75}\end{array}$ & $\begin{array}{l}1 \text { cohort, } N= \\
66,362^{75}\end{array}$ & $\begin{array}{l}\text { Moderate } \\
\text { study } \\
\text { limitations, } \\
\text { precise (wide } \\
\text { Cls), } \\
\text { consistency } \\
\text { unknown }\end{array}$ & Insufficient \\
\hline $\begin{array}{l}\text { Women with } \\
\text { diagnosis code } \\
\text { for mood or } \\
\text { anxiety } \\
\text { disorder } 1-5 \\
\text { months prior to } \\
\text { delivery }\end{array}$ & $\begin{array}{l}\text { Sertraline current } \\
\text { (at delivery), or } \\
\text { unexposed }\end{array}$ & $\begin{array}{l}\text { Postpartum } \\
\text { hemorrhage } \\
\text { billing } \\
\text { diagnosis }\end{array}$ & $\begin{array}{l}162 / 4526 \\
(3.6 \%) \\
\text { vs. } \\
1,896 / 69,044 \\
(2.7 \%)^{74}\end{array}$ & $\begin{array}{l}\text { ARR, } 1.31 \\
(95 \% \mathrm{Cl}, 1.12 \\
\text { to } 1.54)^{74}\end{array}$ & $\begin{array}{l}1 \text { cohort, } \\
N=73,570^{74}\end{array}$ & $\begin{array}{l}\text { Moderate } \\
\text { study } \\
\text { limitations, } \\
\text { precise, } \\
\text { consistency } \\
\text { unknown }\end{array}$ & $\begin{array}{l}\text { Low } \\
\text { strength of } \\
\text { evidence of } \\
\text { increased } \\
\text { risk of } \\
\text { postpartum } \\
\text { hemorrhag } \\
\text { e with } \\
\text { current } \\
\text { exposure to } \\
\text { sertraline }\end{array}$ \\
\hline $\begin{array}{l}\text { Women with } \\
\text { diagnosis code } \\
\text { for mood or } \\
\text { anxiety } \\
\text { disorder } 1-5 \\
\text { months prior to } \\
\text { delivery }\end{array}$ & $\begin{array}{l}\text { Sertraline recent } \\
(<1 \text { month before } \\
\text { delivery) or } \\
\text { unexposed }\end{array}$ & $\begin{array}{l}\text { Postpartum } \\
\text { hemorrhage } \\
\text { billing } \\
\text { diagnosis }\end{array}$ & $\begin{array}{l}78 / 2,226 \\
(3.5 \%) \\
\text { vs. } \\
1,896 / 69,044 \\
(2.7 \%)^{74}\end{array}$ & $\begin{array}{l}\text { ARR, } 1.27 \\
(95 \% \mathrm{Cl}, 1.01 \\
\text { to } 1.59)^{74}\end{array}$ & $\begin{array}{l}1 \text { cohort, } \\
N=71,270^{74}\end{array}$ & $\begin{array}{l}\text { Moderate } \\
\text { study } \\
\text { limitations, } \\
\text { imprecise } \\
\text { (wide Cls), } \\
\text { consistency } \\
\text { unknown }\end{array}$ & $\begin{array}{l}\text { Low } \\
\text { strength of } \\
\text { evidence of } \\
\text { increased } \\
\text { risk of } \\
\text { postpartum } \\
\text { hemorrhag } \\
\text { e with } \\
\text { recent } \\
\text { exposure to } \\
\text { sertraline }\end{array}$ \\
\hline $\begin{array}{l}\text { Women with } \\
\text { diagnosis code } \\
\text { for mood or } \\
\text { anxiety } \\
\text { disorder } 1-5 \\
\text { months prior to } \\
\text { delivery }\end{array}$ & $\begin{array}{l}\text { Sertraline past (>1 } \\
\text { to } 5 \text { months before } \\
\text { delivery) vs. } \\
\text { unexposed }\end{array}$ & $\begin{array}{l}\text { Postpartum } \\
\text { hemorrhage } \\
\text { billing } \\
\text { diagnosis }\end{array}$ & $\begin{array}{l}85 / 3,812 \\
(2.2 \%) \text { vs. } \\
1,896 / 69,044 \\
(2.7 \%)^{74}\end{array}$ & $\begin{array}{l}\text { ARR, } 0.82 \\
(95 \% \mathrm{Cl}, 0.66 \\
\text { to } 1.01)^{74}\end{array}$ & $\begin{array}{l}1 \text { cohort, } \\
\mathrm{N}=72,856^{74}\end{array}$ & $\begin{array}{l}\text { Moderate } \\
\text { study } \\
\text { limitations, } \\
\text { imprecise } \\
\text { (wide Cls), } \\
\text { consistency } \\
\text { unknown }\end{array}$ & Insufficient \\
\hline
\end{tabular}




\begin{tabular}{|c|c|c|c|c|c|c|c|}
\hline Population & $\begin{array}{l}\text { Intervention and } \\
\text { Comparator }\end{array}$ & Outcome & $\begin{array}{l}\text { Incidence or } \\
\text { Mean Effect } \\
\text { by Arm }\end{array}$ & Results & $\begin{array}{l}\text { Study Design } \\
\text { and Sample } \\
\text { Size }\end{array}$ & $\begin{array}{l}\text { Factors That } \\
\text { Affect the } \\
\text { Strength of } \\
\text { Evidence }\end{array}$ & $\begin{array}{l}\text { Overall } \\
\text { Evidence } \\
\text { Strength } \\
\text { (Direction } \\
\text { of Effect) }\end{array}$ \\
\hline $\begin{array}{l}\text { Women with } \\
\text { depression or } \\
\text { anxiety in the } \\
\text { year prior to } \\
\text { pregnancy }\end{array}$ & $\begin{array}{l}\text { Sertraline in 1st } \\
\text { trimester vs. not } \\
\text { exposed in 1st } \\
\text { trimester }\end{array}$ & $\begin{array}{l}\text { Major } \\
\text { congenital } \\
\text { anomaly }\end{array}$ & $\begin{array}{l}\text { NR/365 vs. } \\
1,650 / 14,847 \\
(11.1 \%)^{57} \\
45 / 366 \\
(12.30 \%) \\
\text { vs.1,651/ } \\
14,868 \\
(11.1 \%) \text { from } \\
\text { potentially } \\
\text { overlapping } \\
\left.\text { citation }{ }^{70}\right) \\
\\
25 / 757(3.3 \%) \\
\text { vs. } 380 / 13,432 \\
(2.8 \%)^{72}\end{array}$ & 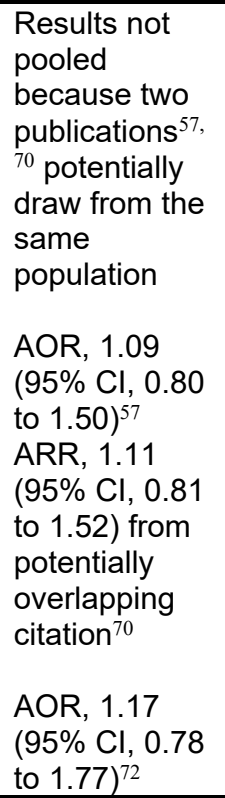 & $\begin{array}{l}2 \text { cohorts, } \\
\mathrm{N}>32,676^{57} \\
\text { (potential } \\
\text { overlap of } \\
\text { participants in } \\
\text { two } \\
\text { publications } \\
{ }^{70} \text { ) }\end{array}$ & $\begin{array}{l}\text { High study } \\
\text { limitations } \\
\text { (two high } \\
\text { risk-of-bias } \\
\text { studies }^{57,72} \text { ), } \\
\text { consistent }\end{array}$ & Insufficient \\
\hline $\begin{array}{l}\text { Women with } \\
\text { depression or } \\
\text { anxiety in the } \\
\text { year prior to } \\
\text { pregnancy }\end{array}$ & $\begin{array}{l}\text { Sertraline in } 1^{\text {st }} \\
\text { trimester vs. } \\
\text { unexposed }\end{array}$ & $\begin{array}{l}\text { Cardiac } \\
\text { anomaly }\end{array}$ & $\begin{array}{l}\text { NR in one } \\
\text { study: NR/365 } \\
\text { vs. } \\
\text { NR/14,847 }{ }^{57} \\
\text { (results from } \\
\text { publication } \\
\text { with } \\
\text { overlapping } \\
\text { data: } 10 / 366) \\
(2.7 \%) \text { vs. } \\
344 / 14,868 \\
\left.(2.3 \%)^{70}\right) ; \\
9 / 757(1.0 \%) \\
\text { vs. } \\
\text { NR/13,432; } 72 \\
93 / 11,126 \\
(0.8 \%) \text { vs. } \\
1,479 / 180,564 \\
(0.8 \%)^{7} \\
156 \text { cases/129 } \\
\text { controls vs. } \\
149 \text { cases } \\
/ 125 \\
\text { controls } 106 \\
\end{array}$ & $\begin{array}{l}\text { Pooled AOR, } \\
1.08(95 \% \mathrm{Cl} \text {, } \\
0.91 \text { to } 1.28) \text {, } \\
\mathrm{I}^{2}: 0 \% \\
\text { AOR, } 1.14 \\
(95 \% \mathrm{Cl}, 0.60 \\
\text { to } 2.15)^{57} \\
\text { (Results from } \\
\text { one } \\
\text { publication } \\
\text { potentially } \\
\text { overlapping } \\
\text { data with } \\
\text { study } \\
\text { included in } \\
\text { meta-analysis } \\
\text { is also } \\
\text { consistent, } \\
\text { with ARR, } 1.16 \\
\text { (95\% Cl, } 0.62 \\
\text { to } 2.19)^{70} \\
\text { AOR, } 1.39 \\
\text { (95\% Cl, } 0.70 \\
\text { to } 2.74)^{72} \\
\text { Propensity } \\
\text { score AOR, } \\
1.09(95 \% \mathrm{Cl}, \\
0.88 \text { to } 1.34, \\
\text { p=0.051) } \\
\text { AOR, } 0.97 \\
\text { (95\% Cl, } 0.69 \\
\text { to } 1.37) \\
\end{array}$ & $\begin{array}{l}3 \text { cohorts, } 1 \\
\text { case-control, } 5 \\
\text { publications } \\
57,70,72,106 \\
\mathrm{~N}>250,577 \\
\text { (potential } \\
\text { overlap in two }_{\text {publications }}^{57} \text {, } \\
{ }_{70} \text { ) }\end{array}$ & $\begin{array}{l}\text { High risk of } \\
\text { bias ( } 3 \\
\text { studies, } \\
72,57,70, \\
\text { (wide } \mathrm{Cls} \text { ), } \\
\text { consistent }\end{array}$ & Insufficient \\
\hline
\end{tabular}




\begin{tabular}{|c|c|c|c|c|c|c|c|}
\hline Population & $\begin{array}{l}\text { Intervention and } \\
\text { Comparator }\end{array}$ & Outcome & $\begin{array}{l}\text { Incidence or } \\
\text { Mean Effect } \\
\text { by Arm }\end{array}$ & Results & $\begin{array}{l}\text { Study Design } \\
\text { and Sample } \\
\text { Size }\end{array}$ & $\begin{array}{l}\text { Factors That } \\
\text { Affect the } \\
\text { Strength of } \\
\text { Evidence }\end{array}$ & $\begin{array}{l}\text { Overall } \\
\text { Evidence } \\
\text { Strength } \\
\text { (Direction } \\
\text { of Effect) }\end{array}$ \\
\hline $\begin{array}{l}\text { Women with } \\
\text { current or past } \\
\text { psychiatric } \\
\text { disorder }\end{array}$ & $\begin{array}{l}\text { Sertraline vs. } \\
\text { unexposed to } \\
\text { antidepressants } \\
\text { during pregnancy }\end{array}$ & $\begin{array}{l}\text { Autism } \\
\text { spectrum } \\
\text { disorder }\end{array}$ & 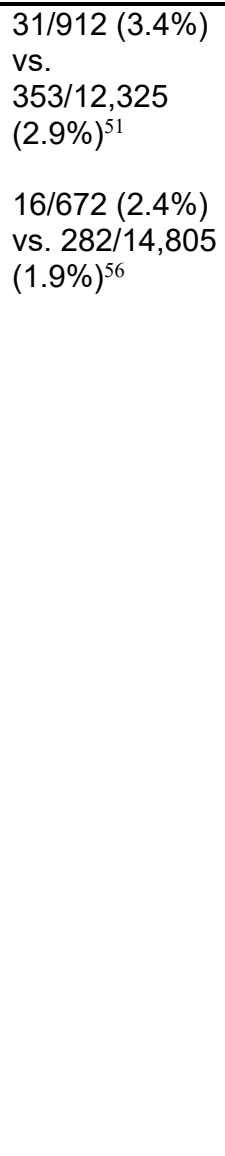 & $\begin{array}{l}\text { AOR, } 1.45 \\
(95 \% \mathrm{CI}, 0.98 \\
\text { to } 2.16)^{51} \\
\text { ARR, } 1.17 \\
(95 \% \mathrm{Cl}, 0.99 \\
\text { to } 2.32))^{56} \\
\text { results } \\
\text { adjusting for } \\
\text { the number of } \\
\text { psychiatric } \\
\text { disorders } \\
\text { show } \\
\text { attenuating } \\
\text { risks of autism } \\
\text { spectrum } \\
\text { disorder } \\
\geq 1 \text { psychiatric } \\
\text { disorder: ARR, } \\
1.32(95 \% \mathrm{Cl}, \\
0.86 \text { to } 2.24)^{56} \\
\geq 2 \text { psychiatric } \\
\text { disorders: } \\
\text { ARR, } 0.99 \\
(95 \% \mathrm{Cl}, 0.63 \\
\text { to } 1.55)^{56} \\
\geq 3 \text { psychiatric } \\
\text { disorders: } \\
\text { ARR, 0.71 } \\
(95 \% \mathrm{Cl}, 0.43 \\
\text { to } 1.17)^{56}\end{array}$ & $\begin{array}{l}2 \text { cohorts, } \\
\text { N>15,47751, } 56 \\
\text { (potential } \\
\text { overlap of } \\
\text { participants in } \\
\text { the } \\
\text { publications) }\end{array}$ & $\begin{array}{l}\text { Moderate } \\
\text { study } \\
\text { limitations, } \\
\text { imprecise } \\
\text { (wide Cls), } \\
\text { some } \\
\text { consistency }\end{array}$ & Insufficient \\
\hline $\begin{array}{l}\text { Women with } \\
\text { current or past } \\
\text { psychiatric } \\
\text { disorder }\end{array}$ & $\begin{array}{l}\text { Sertraline vs. } \\
\text { unexposed to } \\
\text { antidepressants } \\
\text { during pregnancy }\end{array}$ & $\begin{array}{l}\text { Autism } \\
\text { spectrum } \\
\text { disorder } \\
\text { without } \\
\text { intellectual } \\
\text { disability }\end{array}$ & $\begin{array}{l}27 / 912(3.0 \%) \\
\text { vs. } \\
\text { NR/12,325 }\end{array}$ & $\begin{array}{l}\text { AOR, } 1.52 \\
(95 \% \mathrm{Cl}, 0.22 \\
\text { to } 1.62)^{51}\end{array}$ & $\begin{array}{l}\text { Stockholm } \\
\text { Youth Cohort, } \\
N=16,667^{51}\end{array}$ & $\begin{array}{l}\text { Moderate } \\
\text { study } \\
\text { limitations, } \\
\text { imprecise } \\
\text { (wide Cls), } \\
\text { consistency } \\
\text { unknown }\end{array}$ & Insufficient \\
\hline
\end{tabular}

$\mathrm{AOR}=$ adjusted odds ratio; $\mathrm{ARR}=$ adjusted risk ratio; $\mathrm{CI}=$ confidence interval; IPTW =inverse probability of treatment weighted; LMP = last menstrual period; $\mathrm{n} / \mathrm{N}=$ number; $\mathrm{NR}=$ not reported; $\mathrm{RCT}=$ randomized controlled trials; $\mathrm{UKU}=\mathrm{Udvalg}$ for Kliniske Undersøgelser; vs. = versus.

The two RCTs focused on postpartum exposure, ${ }^{16,23}$ while the remaining nine focused on exposure at various times during pregnancy. Two focused on first trimester exposures, ${ }^{7,72}$ one focused on first and second trimester exposures, ${ }^{104}$ two focused on second and third trimester exposures $^{74,75}$ (with 1 limiting third trimester exposure to the first half ${ }^{75}$ ), and four looked at exposure at any time during pregnancy. ${ }^{51,56,57,70}$ The two RCTs involved comparison with psychotherapy: one compared sertraline versus interpersonal psychotherapy, ${ }^{16}$ while the other compared sertraline plus a brief dynamic psychotherapy with psychotherapy alone. ${ }^{23}$ The nine cohort studies compared sertraline exposure to no medication exposure, although one looked at discontinuation during the first two trimesters. ${ }^{104}$ Only the two RCTs reported doses, with one 
reporting a mean dose between 65 and $70 \mathrm{mg}^{23}$ and the other reporting a median dose of 150 mg. ${ }^{16}$

The evidence for sertraline varied by outcome. Overall, there is low strength of evidence that sertraline increases the risk of postpartum hemorrhage for current or recent use. ${ }^{74}$ Also, one low risk-of-bias RCT provided low strength of evidence of no increased side effects with sertraline compared with placebo. ${ }^{23}$ The evidence for the remaining maternal outcome comparisons (attrition, preeclampsia, and incident gestational diabetes), as well as all of the fetal outcomes (major congenital anomalies, cardiac anomalies, or autism spectrum disorder), is insufficient. Evidence from four cohorts, when pooled, spanned the null for cardiac anomalies, but with intervals that included appreciable benefit and appreciable harm (pooled AOR, 1.08; 95\% CI, 0.91 to $1.28 ; \mathrm{I}^{2}, 0 \%$; Figure $\left.\mathrm{B}-6\right) ;^{7,57,72,106}$ as a result, we rated the evidence as insufficient.

Notably, two publications, likely reporting on the same population, ${ }^{57,70}$ reported nonsignificant results for craniosynostosis and ventricular/atrial septal defects in one publication ${ }^{57}$ and statistically significant differences in the other. ${ }^{70}$ The authors attribute differences in findings to differences in inclusion criteria and exposure groups. ${ }^{57} \mathrm{~A}$ third study generally reported no significant differences for other birth defects but did not control for multiple comparisons. ${ }^{106}$

Two publications from Swedish birth cohorts, with potential overlaps in the cohort, found no association between paroxetine exposure during pregnancy among women with a current or past psychiatric disorder and autism spectrum disorder in offspring. ${ }^{51,56}$ Moreover, when the referent group was restricted to women with more than one psychiatric diagnosis, the point estimate for relative risk moved from 1.32 ( $\geq 1$ disorder) to 0.71 ( $\geq 3$ disorders). ${ }^{56}$

\section{SSRIs: Trazodone}

\section{Overview}

- The evidence for trazodone use during pregnancy, when analyzed individually, is insufficient to judge the risk of harms for maternal outcomes (postpartum hemorrhage or preeclampsia).

\section{Detailed Results}

Two publications reported on trazodone. ${ }^{74,75}$ These publications drew from one cohort (2000-2007 U.S. nationwide Medicaid Analytic eXtract [MAX] data). Both were nonrandomized observational studies and were rated as "some concerns" regarding risk of bias. ${ }^{74,75}$

Both publications focused on exposure during pregnancy. One publication ${ }^{74}$ also analyzed recent trazodone exposure (women with a supply of antidepressants on at least 1 day in the month before the delivery date but not on the delivery date) and past trazodone exposure (1 to 5 months prior to delivery). Publications compared pregnant women exposed to trazodone with women with nonpharmacologically treated depression ${ }^{75}$ or mood disorder and anxiety. ${ }^{74}$ One publication $^{75}$ compared pregnant women who continued versus discontinued trazodone during pregnancy.

The evidence for trazodone, when analyzed individually, is insufficient to judge the risk of harms for maternal outcomes (postpartum hemorrhage or preeclampsia) (Table B-26). The publications consistently found no association between trazodone exposure and maternal harms (postpartum hemorrhage, preeclampsia). Notably, there was no association detected with recent or current trazodone exposure in this publication. ${ }^{74}$ 
There was an insufficient number of studies to pool results.

Table B-26. Strength of evidence for harms: Trazodone versus no treatment

\begin{tabular}{|c|c|c|c|c|c|c|c|}
\hline Population & $\begin{array}{l}\text { Intervention } \\
\text { and } \\
\text { Comparator }\end{array}$ & Outcome & $\begin{array}{l}\text { Incidence } \\
\text { or Mean } \\
\text { Effect by } \\
\text { Arm }\end{array}$ & Results & $\begin{array}{l}\text { Study Design } \\
\text { and Sample } \\
\text { Size }\end{array}$ & $\begin{array}{l}\text { Factors That } \\
\text { Affect the } \\
\text { Strength of } \\
\text { Evidence }\end{array}$ & $\begin{array}{l}\text { Overall } \\
\text { Evidence } \\
\text { Strength } \\
\text { (Direction } \\
\text { of Effect) } \\
\end{array}$ \\
\hline $\begin{array}{l}\text { Mood } \\
\text { disorder or } \\
\text { anxiety or } \\
\text { bupropion- } \\
\text { exposed } \\
\text { women }\end{array}$ & $\begin{array}{l}\text { Current } \\
\text { trazodone } \\
\text { exposure in } \\
\text { pregnancy } \\
\text { vs. } \\
\text { unexposed } \\
\text { women with } \\
\text { mood } \\
\text { disorder or } \\
\text { anxiety } \\
\end{array}$ & $\begin{array}{l}\text { Postpartum } \\
\text { hemorrhage }\end{array}$ & NR & $\begin{array}{l}\text { ARR, } 1.85 \\
(95 \% \mathrm{Cl}, 0.90 \\
\text { to } 3.80)^{74}\end{array}$ & $\begin{array}{l}1 \text { cohort, } \\
n=69,183^{74}\end{array}$ & $\begin{array}{l}\text { Moderate study } \\
\text { limitations, } \\
\text { imprecise (wide } \\
\text { Cls), consistency } \\
\text { unknown }\end{array}$ & Insufficient \\
\hline $\begin{array}{l}\text { Mood } \\
\text { disorder or } \\
\text { anxiety or } \\
\text { bupropion- } \\
\text { exposed } \\
\text { women }\end{array}$ & $\begin{array}{l}\text { Recent } \\
\text { trazodone } \\
\text { exposure in } \\
\text { pregnancy } \\
\text { vs. } \\
\text { unexposed } \\
\text { women with } \\
\text { mood } \\
\text { disorder or } \\
\text { anxiety }\end{array}$ & $\begin{array}{l}\text { Postpartum } \\
\text { hemorrhage }\end{array}$ & NR & $\begin{array}{l}\text { ARR, } 2.01 \\
(95 \% \mathrm{Cl}, 0.77 \\
\text { to } 5.24)^{74}\end{array}$ & $\begin{array}{l}1 \text { cohort, } \\
n=69,117^{74}\end{array}$ & $\begin{array}{l}\text { Moderate study } \\
\text { limitations, } \\
\text { imprecise (wide } \\
\text { Cls), consistency } \\
\text { unknown }\end{array}$ & Insufficient \\
\hline $\begin{array}{l}\text { Mood } \\
\text { disorder or } \\
\text { anxiety or } \\
\text { bupropion- } \\
\text { exposed } \\
\text { women }\end{array}$ & $\begin{array}{l}\text { Past } \\
\text { trazodone } \\
\text { exposure in } \\
\text { pregnancy } \\
\text { vs. } \\
\text { unexposed } \\
\text { women with } \\
\text { mood } \\
\text { disorder or } \\
\text { anxiety }\end{array}$ & $\begin{array}{l}\text { Postpartum } \\
\text { hemorrhage }\end{array}$ & NR & $\begin{array}{l}\text { ARR, } 0.61 \\
(95 \% \mathrm{Cl}, 0.23 \\
\text { to } 1.67)^{74}\end{array}$ & $\begin{array}{l}1 \text { cohort, } \\
n=69,270^{74}\end{array}$ & $\begin{array}{l}\text { Moderate study } \\
\text { limitations, } \\
\text { imprecise (wide } \\
\text { Cls), consistency } \\
\text { unknown }\end{array}$ & Insufficient \\
\hline $\begin{array}{l}\text { Depressed } \\
\text { women }\end{array}$ & $\begin{array}{l}\text { Trazodone } \\
\text { exposure in } \\
\text { pregnancy } \\
\text { vs. } \\
\text { unexposed } \\
\text { women with } \\
\text { depression }\end{array}$ & Preeclampsia & $\begin{array}{l}14 / 339 \text { vs. } \\
3,215 / 5,91 \\
9^{75}\end{array}$ & $\begin{array}{l}\text { ARR, } 0.63 \\
(95 \% \mathrm{Cl}, 0.38 \\
\text { to } 1.05)^{75}\end{array}$ & $\begin{array}{l}1 \text { cohort, } \\
n=59,558^{75}\end{array}$ & $\begin{array}{l}\text { Moderate study } \\
\text { limitations, } \\
\text { imprecise (wide } \\
\text { Cls), consistency } \\
\text { unknown }\end{array}$ & Insufficient \\
\hline
\end{tabular}

$\overline{\mathrm{ARR}}=$ adjusted risk ratio; $\mathrm{CI}=$ confidence interval; $\mathrm{n}=$ number; $\mathrm{NR}=$ not reported; $\mathrm{vs} .=$ versus.

\section{SNRIs}

\section{Overview}

- For SNRIs overall, evidence suggests an increased association of postpartum hemorrhage with exposure to SNRIs at the time of delivery (low for harms). It also suggests an increased association of spontaneous abortion and preeclampsia for SNRIs (low strength of evidence for harms).

- For SNRIs overall, the evidence is insufficient for maternal postpartum hemorrhage with recent or past exposure or child outcomes (major congenital anomalies, cardiac anomalies). 
- For duloxetine exposure during pregnancy, the evidence is insufficient for duloxetine continuation versus discontinuation for child outcomes, specifically any congenital anomalies or cardiovascular anomalies.

- For venlafaxine, evidence suggests an increased association of postpartum hemorrhage with exposure to SNRIs at the time of delivery (low strength of evidence for harms). It also suggests an increased association with preeclampsia (low strength of evidence for harms). The evidence is insufficient to judge the risk of harms for gestational diabetes or for child outcomes (small for gestational age, cardiac anomalies, or autism spectrum disorder).

\section{Detailed Results}

Twelve publications reported on SNRIs. ${ }^{7,51,56,57,64,74,75,81,83,104,106}$ Of these, three analyzed only the effects of SNRIs as a class, ${ }^{7,64,81}$ three focused specifically on the effects of venlafaxine, ${ }^{51,83,104}$ one focused on duloxetine exposure versus discontinuation, ${ }^{98}$ three included analyses of both SNRIs as a class and venlafaxine more specifically, ${ }^{77,74,106}$ one included analyses of venlafaxine and duloxetine, ${ }^{56}$ and one included analyses of SNRIs as a class and both venlafaxine and duloxetine more specifically. ${ }^{75}$ These studies drew from five cohorts (three from the Canadian QPC, ${ }^{57,64,83}$ four from the U.S. Medicaid Analytic eXtract, ${ }^{7,74,75,98}$ and one each from a U.S. Kaiser Permanente Washington cohort, ${ }^{104}$ the U.S. National Birth Defects Prevention Study, ${ }^{106}$ a Swedish Stockholm youth cohort, ${ }^{51}$ a Swedish birth cohort, ${ }^{56}$ and a Canadian British Columbia birth cohort. ${ }^{81}$ All were nonrandomized studies. Two studies were rated high risk of bias. ${ }^{7,57}$

The evidence on the risk of maternal harms from SNRIs was reported in relation to spontaneous abortion, preeclampsia, gestational diabetes, and hemorrhage. One publication reported on the risk of spontaneous abortion during the first trimester for women exposed to SNRIs compared with women with a depression diagnosis during the 4 years before pregnancy and no prescription in the year before pregnancy. ${ }^{64}$ The difference in the adjusted risk of spontaneous abortion prior to 20 weeks was calculated both with and without a correction for induced abortions. The risk adjustment for induced abortions resulted in a lower estimate of risk. In both analyses, the risk of spontaneous abortion was significantly higher in the exposed group. We rated our confidence in the findings as low strength of evidence for harms (Table B-27). 
Table B-27. Strength of evidence for harms: SNRIs versus no treatment

\begin{tabular}{|c|c|c|c|c|c|c|c|}
\hline Population & $\begin{array}{l}\text { Intervention } \\
\text { and } \\
\text { Comparator }\end{array}$ & Outcome & $\begin{array}{l}\text { Incidence or } \\
\text { Mean Effect } \\
\text { by Arm }\end{array}$ & Results & $\begin{array}{l}\text { Study Design } \\
\text { and Sample } \\
\text { Size }\end{array}$ & $\begin{array}{l}\text { Factors That } \\
\text { Affect the } \\
\text { Strength of } \\
\text { Evidence }\end{array}$ & $\begin{array}{l}\text { Overall } \\
\text { Evidence } \\
\text { Strength } \\
\text { (Direction of } \\
\text { Effect) } \\
\end{array}$ \\
\hline $\begin{array}{l}\text { Pregnant } \\
\text { women: } \\
\text { SNRI } \\
\text { exposure or } \\
\text { depression } \\
\text { diagnosis in } \\
\text { past } 4 \text { years }\end{array}$ & $\begin{array}{l}\text { SNRI } \\
\text { exposure in } \\
\text { 1st trimester } \\
\text { vs. } \\
\text { unexposed }\end{array}$ & $\begin{array}{l}\text { Spontaneous } \\
\text { abortion }\end{array}$ & $\begin{array}{l}20 / 90(22 \%) \\
\text { vs. } 720 / 7,034 \\
(10 \%) ; 64 \\
\text { results } \\
\text { corrected for } \\
\text { induced } \\
\text { abortions: } \\
20 / 137(15 \%) \\
\text { vs. } 720 / 8,877 \\
(8.1 \%)^{64}\end{array}$ & $\begin{array}{l}\text { ARR, } 2.1 \\
\text { (95\% CI, } 1.4 \\
\text { to } 3.0) ; \\
\text { corrected for } \\
\text { induced } \\
\text { abortions } \\
\text { ARR, } 1.7 \\
(95 \% \mathrm{Cl}, 1.2 \\
\text { to } 2.6)^{64}\end{array}$ & $\begin{array}{l}1 \text { cohort, } \\
n=7,134 ; \\
\text { corrected for } \\
\text { inducted } \\
\text { abortion, } \\
n=9,014^{64}\end{array}$ & $\begin{array}{l}\text { Moderate study } \\
\text { limitations, } \\
\text { precise; } \\
\text { consistency } \\
\text { unknown }\end{array}$ & $\begin{array}{l}\text { Low for harms } \\
\text { with SNRIs }\end{array}$ \\
\hline $\begin{array}{l}\text { Pregnant } \\
\text { women: } \\
\text { SNRI } \\
\text { exposure or } \\
\text { depression } \\
\text { diagnosis, } \\
\text { through 2nd } \\
\text { trimester } \\
\end{array}$ & $\begin{array}{l}\text { SNRI } \\
\text { exposure } \\
\text { through 2nd } \\
\text { trimester vs. } \\
\text { unexposed }\end{array}$ & Preeclampsia & $\begin{array}{l}107 / 1,216 \\
(9 \%) \text { vs. } \\
3,215 / 59,219 \\
(5 \%)^{\cdot 75} \\
23 / 408(5.6 \%) \\
\text { vs. } \\
1,569 / 65,392 \\
(2.4 \%)^{81} \\
\end{array}$ & $\begin{array}{l}\text { ARR, 1.52 } \\
(95 \% \mathrm{Cl}, 1.26 \\
\text { to } 1.83)^{75} \text {; } \\
\text { ARR, } 1.59 \\
(95 \% \mathrm{Cl}, 1.26 \\
\text { to } 3.03)^{81}\end{array}$ & $\begin{array}{l}2 \text { cohorts: } \\
N=126,235^{75,81}\end{array}$ & $\begin{array}{l}\text { Moderate study } \\
\text { limitations, } \\
\text { precise; } \\
\text { consistent }\end{array}$ & $\begin{array}{l}\text { Low for harms } \\
\text { with SNRIs }\end{array}$ \\
\hline $\begin{array}{l}\text { Pregnant } \\
\text { women: } \\
\text { venlafaxine } \\
\text { exposure or } \\
\text { depression } \\
\text { diagnosis, } \\
\text { through 2nd } \\
\text { trimester }\end{array}$ & $\begin{array}{l}\text { Venlafaxine } \\
\text { exposure } \\
\text { through } 2 \mathrm{nd} \\
\text { trimester vs. } \\
\text { unexposed }\end{array}$ & Preeclampsia & $\begin{array}{l}100 / 1,113 \\
(9 \%) \text { vs. } \\
3,215 / 59,219 \\
(5 \%)^{75}\end{array}$ & $\begin{array}{l}\text { ARR, } 1.57 \\
(1.29 \text { to } \\
1.91)^{75}\end{array}$ & $\begin{array}{l}1 \text { cohort: } \\
N=60,332^{75}\end{array}$ & $\begin{array}{l}\text { Moderate study } \\
\text { limitations, } \\
\text { precise; } \\
\text { consistency } \\
\text { unknown }\end{array}$ & $\begin{array}{l}\text { Low for harms } \\
\text { with venlafaxine }\end{array}$ \\
\hline $\begin{array}{l}\text { Pregnant } \\
\text { women: } \\
\text { anti- } \\
\text { depressant } \\
\text { prescription } \\
\text { fill during the } \\
6 \text { months } \\
\text { before } \\
\text { pregnancy }\end{array}$ & $\begin{array}{l}\text { Venlafaxine } \\
\text { exposure } \\
\text { before } \\
\text { gestational } \\
\text { diabetes } \\
\text { screening } \\
\text { (24-28 weeks } \\
\text { gestations) } \\
\text { vs. } \\
\text { unexposed } \\
\end{array}$ & $\begin{array}{l}\text { Gestational } \\
\text { diabetes }\end{array}$ & $\begin{array}{l}15 / 104(14 \%) \\
\text { vs. 90/1,211 } \\
(7 \%)^{104}\end{array}$ & $\begin{array}{l}\text { ARR: } 1.52 \\
(0.87 \text { to } \\
2.68)^{104}\end{array}$ & $\begin{array}{l}1 \text { cohort, } \\
\mathrm{N}=1,315^{104}\end{array}$ & $\begin{array}{l}\text { Moderate study } \\
\text { limitations, } \\
\text { imprecise (Cls } \\
\text { span the null), } \\
\text { consistency } \\
\text { unknown }\end{array}$ & Insufficient \\
\hline $\begin{array}{l}\text { Pregnant } \\
\text { women with } \\
\text { mood or } \\
\text { anxiety } \\
\text { disorders }\end{array}$ & $\begin{array}{l}\text { SNRI recent } \\
\text { exposure } \\
\text { (<1 month } \\
\text { before } \\
\text { delivery) vs. } \\
\text { unexposed } \\
\end{array}$ & $\begin{array}{l}\text { Postpartum } \\
\text { hemorrhage } \\
\text { billing } \\
\text { diagnosis }\end{array}$ & $\begin{array}{l}\text { NR/217 } \\
\text { (NR\%) vs. } \\
1,898 / 69,044 \\
(2.8 \%)^{74}\end{array}$ & $\begin{array}{l}\text { ARR, } 1.21 \\
(0.58 \text { to } \\
2.54)^{74}\end{array}$ & $\begin{array}{l}1 \text { cohort: } \\
N=69,261^{74}\end{array}$ & $\begin{array}{l}\text { Moderate study } \\
\text { limitations, } \\
\text { imprecise, } \\
\text { consistency } \\
\text { unknown }\end{array}$ & Insufficient \\
\hline $\begin{array}{l}\text { Pregnant } \\
\text { women with } \\
\text { mood or } \\
\text { anxiety } \\
\text { disorders }\end{array}$ & $\begin{array}{l}\text { SNRI past } \\
\text { exposure (>1 } \\
\text { to } 5 \text { months } \\
\text { before } \\
\text { delivery) or } \\
\text { unexposed }\end{array}$ & $\begin{array}{l}\text { Postpartum } \\
\text { hemorrhage } \\
\text { billing } \\
\text { diagnosis }\end{array}$ & $\begin{array}{l}12 / 423(2.8 \%) \\
\text { vs. } \\
1,898 / 69,044 \\
(2.8 \%)^{74}\end{array}$ & $\begin{array}{l}\text { ARR, } 1.05 \\
(0.60 \text { to } \\
1.83)^{74}\end{array}$ & $\begin{array}{l}1 \text { cohort: } \\
\mathrm{N}=69,467^{74}\end{array}$ & $\begin{array}{l}\text { Moderate study } \\
\text { limitations, } \\
\text { imprecise, } \\
\text { consistency } \\
\text { unknown }\end{array}$ & Insufficient \\
\hline
\end{tabular}




\begin{tabular}{|c|c|c|c|c|c|c|c|}
\hline Population & $\begin{array}{l}\text { Intervention } \\
\text { and } \\
\text { Comparator }\end{array}$ & Outcome & $\begin{array}{l}\text { Incidence or } \\
\text { Mean Effect } \\
\text { by Arm }\end{array}$ & Results & $\begin{array}{l}\text { Study Design } \\
\text { and Sample } \\
\text { Size }\end{array}$ & $\begin{array}{l}\text { Factors That } \\
\text { Affect the } \\
\text { Strength of } \\
\text { Evidence }\end{array}$ & $\begin{array}{l}\text { Overall Evidence } \\
\text { Strength } \\
\text { (Direction of } \\
\text { Effect) }\end{array}$ \\
\hline $\begin{array}{l}\text { Pregnant } \\
\text { women with } \\
\text { mood or } \\
\text { anxiety } \\
\text { disorders }\end{array}$ & $\begin{array}{l}\text { Venlafaxine } \\
\text { recent } \\
\text { exposure } \\
\text { (<1 month } \\
\text { before } \\
\text { delivery) vs. } \\
\text { unexposed }\end{array}$ & $\begin{array}{l}\text { Postpartum } \\
\text { hemorrhage } \\
\text { billing } \\
\text { diagnosis }\end{array}$ & $\begin{array}{l}\text { NR/237 (6\%) } \\
\text { vs. } \\
1,898 / 69,044 \\
(2.8 \%)^{74}\end{array}$ & $\begin{array}{l}\text { ARR, } 1.10 \\
(95 \% \mathrm{Cl}, 0.53 \\
\text { to } 2.30)^{74}\end{array}$ & $\begin{array}{l}1 \text { cohort, } \\
\mathrm{N}=69,281^{74}\end{array}$ & $\begin{array}{l}\text { Moderate study } \\
\text { limitations, } \\
\text { imprecise, } \\
\text { consistency } \\
\text { unknown }\end{array}$ & Insufficient \\
\hline $\begin{array}{l}\text { Pregnant } \\
\text { women with } \\
\text { a psychiatric } \\
\text { disorder } \\
\text { diagnosis, } \\
\text { used AD } \\
\geq 30 \text { days in } \\
\text { prior year }\end{array}$ & $\begin{array}{l}\text { Venlafaxine } \\
\text { during 2nd } \\
\text { trimester vs. } \\
\text { unexposed }\end{array}$ & $\begin{array}{l}\text { Small for } \\
\text { gestational } \\
\text { age }\end{array}$ & $\begin{array}{l}\text { NA, case- } \\
\text { control }^{83}\end{array}$ & $\begin{array}{l}\text { ARR, } 2.55 \\
(1.04 \text { to } 6.27)^{83}\end{array}$ & $\begin{array}{l}1 \text { case-control: } \\
\mathrm{N}=755^{83}\end{array}$ & $\begin{array}{l}\text { Moderate study } \\
\text { limitations, } \\
\text { imprecise (few } \\
\text { events, wide } \\
\text { Cls); consistency } \\
\text { unknown }\end{array}$ & Insufficient \\
\hline $\begin{array}{l}\text { Duloxetine } \\
\text { in the first } \\
\text { trimester vs. } \\
\text { discontin- } \\
\text { uation of } \\
\text { duloxetine } \\
\text { before the } \\
\text { first } \\
\text { trimester }\end{array}$ & $\begin{array}{l}\text { Duloxetine, } \\
\text { exposure in } \\
\text { the first } \\
\text { trimester }\end{array}$ & $\begin{array}{l}\text { Any } \\
\text { congenital } \\
\text { malformation } \\
\text { s }\end{array}$ & $\begin{array}{l}\text { Duloxetine: } \\
128 / 2,532 \\
(5.06 \%) \text { vs. } \\
114 / 2456 \\
(4.64 \%)^{98}\end{array}$ & $\begin{array}{l}\text { ARR, } 1.09 \\
(95 \% \mathrm{Cl}, 0.84 \\
\text { to } 1.42)^{98}\end{array}$ & $\begin{array}{l}1 \text { cohorts: } \\
\mathrm{N}=4988^{98}\end{array}$ & $\begin{array}{l}\text { Moderate study } \\
\text { limitations, } \\
\text { imprecise (wide } \\
\text { Cls), consistency } \\
\text { unknown }\end{array}$ & Insufficient \\
\hline
\end{tabular}




\begin{tabular}{|c|c|c|c|c|c|c|c|}
\hline Population & $\begin{array}{l}\text { Intervention } \\
\text { and } \\
\text { Comparator }\end{array}$ & Outcome & $\begin{array}{l}\text { Incidence or } \\
\text { Mean Effect } \\
\text { by Arm }\end{array}$ & Results & $\begin{array}{l}\text { Study Design } \\
\text { and Sample } \\
\text { Size }\end{array}$ & $\begin{array}{l}\text { Factors That } \\
\text { Affect the } \\
\text { Strength of } \\
\text { Evidence }\end{array}$ & $\begin{array}{l}\text { Overall } \\
\text { Evidence } \\
\text { Strength } \\
\text { (Direction of } \\
\text { Effect) }\end{array}$ \\
\hline $\begin{array}{l}\text { Women with } \\
\text { depression } \\
\text { or anxiety } \\
\text { before } \\
\text { pregnancy } \\
\text { or exposure } \\
\text { to anti- } \\
\text { depressants } \\
\text { outside of } \\
\text { early } \\
\text { pregnancy } \\
\text { or } \\
\text { duloxetine in } \\
\text { the first } \\
\text { trimester vs. } \\
\text { discontinuati } \\
\text { on of } \\
\text { duloxetine } \\
\text { before the } \\
\text { first } \\
\text { trimester }\end{array}$ & $\begin{array}{l}\text { Venlafaxine; } \\
\text { SNRI; } \\
\text { exposure in } \\
\text { the first } \\
\text { trimester vs. } \\
\text { unexposed; } \\
106 \\
\text { Duloxetine, } \\
\text { exposure in } \\
\text { the first } \\
\text { trimester }^{98} \\
\end{array}$ & $\begin{array}{l}\text { Cardiac } \\
\text { anomalies }\end{array}$ & $\begin{array}{l}\text { SNRI:69/ } \\
1,497(4.6 \%) \\
\text { vs. } 1,497 / \\
180,564 \\
(0.8 \%)^{7} 59 \\
\text { cases/27 } \\
\text { controls vs. } \\
149 \\
\text { cases/125 } \\
\text { controls }^{106} \\
\\
\text { Venlafaxine: } \\
\text { NR/738 vs. } \\
\text { NR/14,847; } 57 \\
47 \text { cases/21 } \\
\text { controls vs. } \\
149 \\
\text { cases } / 125 \text { controls }{ }^{106} \\
\\
\text { Duloxetine: } \\
59 / 2,532 \\
(2.33 \%) \text { vs. } \\
43 / 2,456 \\
(1.75 \%)^{98}\end{array}$ & $\begin{array}{l}\text { SNRI } \\
\text { Propensity } \\
\text { score AOR, } \\
1.20(0.91 \text { to } \\
1.57)^{7} \\
\text { AOR, } 1.14 \\
(95 \% \mathrm{Cl}, 0.44 \\
\text { to } 3.01)^{106} \\
\\
\text { Venlafaxine } \\
\text { AOR, 0.80 } \\
(0.47 \text { to } \\
1.38)^{57} \\
\text { AOR, } 1.91 \\
(95 \% \text { CI, } 1.05 \\
\text { to } 3.45) \\
\text { SNRIs }{ }^{106} \\
\text { Duloxetine } \\
\text { ARR, } 1.41 \\
(95 \% \text { CI, } 0.92 \\
\text { to } 2.17)^{98}\end{array}$ & $\begin{array}{l}3 \text { cohorts, } 1 \text { case } \\
\text { control: } \\
N=202,994^{7,57,98,} \\
106\end{array}$ & $\begin{array}{l}\text { High study } \\
\text { limitations (high } \\
\text { risk of bias }{ }^{57} \\
{ }^{7} \text { ), imprecise } \\
\text { (wide Cls), } \\
\text { inconsistent }\end{array}$ & Insufficient \\
\hline $\begin{array}{l}\text { Pregnant } \\
\text { women: Any } \\
\text { lifetime } \\
\text { depression } \\
\text { or anxiety } \\
\text { diagnosis }\end{array}$ & $\begin{array}{l}\text { Venlafaxine } \\
\text { during } \\
\text { pregnancy } \\
\text { vs. } \\
\text { unexposed } \\
\text { women }\end{array}$ & $\begin{array}{l}\text { Autism } \\
\text { spectrum } \\
\text { disorder }\end{array}$ & $\begin{array}{l}4 / 195(2.1 \%) \\
\text { vs. } \\
282 / 14,805 \\
(1.9 \%) ; 5 \\
11 / 213(5.1 \%) \\
\text { vs. } \\
353 / 12,325 \\
(2.9 \%)^{51}\end{array}$ & $\begin{array}{l}\text { By } 7-\text { or } 8- \\
\text { year followup } \\
\text { ARR, } 0.74 \\
(0.32 \text { to } \\
1.72)^{: 56} \\
4 \text {-year or } \\
\text { more followup: } \\
\text { AOR, } 1.81 \\
(0.89 \text { to } \\
3.71)^{51} \\
\geq 1 \text { psychiatric } \\
\text { disorder: } \\
\text { ARR, } 1.36 \\
(95 \% \text { Cl, } 0.61 \\
\text { to } 3.04)^{56} \\
\geq 2 \text { psychiatric } \\
\text { disorders: } \\
\text { ARR, } 1.01 \\
(0.44 \text { to } \\
2.29)^{56} \\
\geq 3 \text { psychiatric } \\
\text { disorders: } \\
\text { ARR, } 0.74 \\
(0.32 \text { to } \\
1.72)^{56}\end{array}$ & $\begin{array}{l}2 \text { cohorts: } \\
\mathrm{N}=27,538^{51,56}\end{array}$ & $\begin{array}{l}\text { Moderate study } \\
\text { limitations, } \\
\text { imprecise (wide } \\
\text { Cls), inconsistent }\end{array}$ & Insufficient \\
\hline
\end{tabular}




\begin{tabular}{|c|c|c|c|c|c|c|c|}
\hline Population & $\begin{array}{l}\text { Intervention } \\
\text { and } \\
\text { Comparator }\end{array}$ & Outcome & $\begin{array}{l}\text { Incidence or } \\
\text { Mean Effect } \\
\text { by Arm }\end{array}$ & Results & $\begin{array}{l}\text { Study Design } \\
\text { and Sample } \\
\text { Size }\end{array}$ & $\begin{array}{l}\text { Factors That } \\
\text { Affect the } \\
\text { Strength of } \\
\text { Evidence }\end{array}$ & $\begin{array}{l}\text { Overall } \\
\text { Evidence } \\
\text { Strength } \\
\text { (Direction of } \\
\text { Effect) }\end{array}$ \\
\hline $\begin{array}{l}\text { Pregnant } \\
\text { women: Any } \\
\text { lifetime } \\
\text { psychiatric } \\
\text { disorders }\end{array}$ & $\begin{array}{l}\text { Duloxetine } \\
\text { during } \\
\text { pregnancy } \\
\text { vs. } \\
\text { unexposed } \\
\text { women }\end{array}$ & $\begin{array}{l}\text { Autism } \\
\text { spectrum } \\
\text { disorder }\end{array}$ & $\begin{array}{l}\text { NR/52 (NR\%) } \\
\text { vs. control } \geq 1 \\
\text { psychiatric } \\
\text { disorder: } \\
\text { NR/24,285 } \\
\text { (NR\%); } \\
\text { control } \geq 2 \\
\text { psychiatric } \\
\text { disorders } \\
\text { NR/5,839 } \\
\text { (NR\%); } \\
\text { control } \geq 3 \\
\text { psychiatric } \\
\text { disorders } \\
\text { NR/5,839 } \\
(\mathrm{NR} \%)^{56}\end{array}$ & $\begin{array}{l}\text { Results not } \\
\text { presented for } \\
\text { any } \\
\text { comparison }{ }^{56}\end{array}$ & $\begin{array}{l}\text { Duloxetine and } \\
\text { control } \geq 1 \\
\text { psychiatric } \\
\text { disorder: } \\
\mathrm{N}=24,337 \text {; } \\
\text { duloxetine and } \\
\text { control } \geq 2 \\
\text { psychiatric } \\
\text { disorders: } \\
\mathrm{N}=5,891 ; \\
\text { duloxetine and } \\
\text { control } \geq 3 \\
\text { psychiatric } \\
\text { disorders: } \\
\mathrm{N}=1946^{56}\end{array}$ & $\begin{array}{l}\text { Moderate study } \\
\text { limitations, no } \\
\text { result estimates }\end{array}$ & Insufficient \\
\hline
\end{tabular}

$\mathrm{AD}=$ antidepressants; $\mathrm{AOR}=$ adjusted odds ratio; $\mathrm{ARR}=$ adjusted risk ratio; $\mathrm{CI}=$ confidence interval; $\mathrm{dx}=$ diagnosis; $\mathrm{N}=$ number; NA = not applicable; NR = not reported; SNRI = serotonin-norepinephrine reuptake inhibitor; vs. $=$ versus.

Two publications, reporting on the risk of preeclampsia in independent cohorts, compared women with depression diagnoses while pregnant through the second trimester. ${ }^{75,81}$ Those exposed to SNRIs were found to be at significantly higher risk of preeclampsia in both analyses after controlling for potential confounders. We rated our confidence in the findings as low strength of evidence for harms. One of the publications also reported on risk of preeclampsia among the women exposed to venlafaxine more specifically. ${ }^{75}$ Similarly, those exposed to venlafaxine were found to be at significantly higher risk of preeclampsia, and we rated our confidence in the findings as low strength of evidence for harms.

One publication compared the risk of hemorrhage among women with mood or anxiety disorders exposed to SNRIs at the time of delivery at $\leq 1$ month prior to delivery and at 1 to 5 months prior to delivery compared with women with no exposure to antidepressants. ${ }^{74}$ In analyses controlling for potential confounders, the risk of hemorrhage was significantly higher among women exposed to SNRIs at the time of delivery (low strength of evidence for harms), but the evidence was insufficient for grading the outcome when exposure was prior to delivery. In this publication, we found a similar pattern of findings in relation to venlafaxine more specifically. $^{74}$

One publication reporting on gestational diabetes $(\mathrm{N}=1,315)$ found an increased risk from venlafaxine (ARR, 1.52), but the CI surrounding the estimate is large (imprecise). As a result, the evidence was graded as insufficient.

The evidence on the risk of child adverse outcomes from SNRIs reported on small for gestational age, major or any congenital anomalies, cardiac anomalies, and autism spectrum disorder. Each was graded as insufficient strength of evidence. Limited to one case-control study, women exposed to venlafaxine during the second trimester were found to be significantly more likely to give birth to children who were small for gestational age. ${ }^{83}$ However, when the sample population was divided into subgroups of women who used $\leq 150 \mathrm{mg} /$ day and those who used $>150 \mathrm{mg}$ /day during the second trimester, only women using the smaller dose were found to be at significantly increased risk of their child being small for gestational age. The authors speculated that the results could be the result of residual confounding by indication; women at 
lower dosages have poorer control of their psychiatric condition. Because of the potential for residual confounding, we rated this outcome as insufficient, despite the statistical significant result. Also limiting the strength of the findings was small sample sizes. Only five of the venlafaxine cases and 12 of the control subjects had a child small for gestational age.

One study of congenital anomalies ${ }^{57}$ also compared venlafaxine to controls in relation to organ specific anomalies: nervous system; eye, ear, face, and neck; circulatory system; respiratory system; digestive system; genital organs; urinary system; musculoskeletal system; craniosynostosis; ventricular/atrial septal defect; and cardiac anomalies discussed below. A significantly increased risk was found only in relation to respiratory anomalies (AOR, 2.17; 95\% CI, 2.07 to 4.38). One study reported some differences in birth defects for SNRIs and venlafaxine but had small numbers of cases and controls, had imprecise results, and did not control for multiple comparisons. ${ }^{106}$

One study evaluated the risk of any congenital malformations when comparing the risk for women on duloxetine in the first trimester when compared with women who discontinued duloxetine. Discontinuation was defined as having a duloxetine prescription dispensed between 6 months and 60 days before the last menstrual period but not during the first trimester. The study found no statistically significant results and wide CIs. Four studies reported on cardiac anomalies following exposure to SNRIs as a drug class, ${ }^{7,}{ }^{106}$ duloxetine, ${ }^{98}$ and venlafaxine. ${ }^{57,} 106$ Given the heterogeneity in exposure, we did not pool the results. The results were rated as insufficient as a result of inconsistent and imprecise results. Two publications with potential overlaps in the cohorts $^{51,56}$ reported inconsistent and imprecise results on autism spectrum disorder. These results were rated as insufficient as a result.

\section{TCAs}

\section{Overview}

- TCA exposure during pregnancy may be associated with an increased risk of preeclampsia compared with no treatment (low strength of evidence).

- For TCAs overall, the evidence is insufficient to judge the risk of harms for other maternal (spontaneous abortion, postpartum hemorrhage) or child outcomes (perinatal mortality, small for gestational age, major congenital anomalies, cardiac anomalies).

- For clomipramine and amitriptyline or nortriptyline exposure during pregnancy, the evidence is insufficient to judge the risk of harms for child outcomes (autism spectrum disorder).

\section{Detailed Results}

Twelve publications reported on TCAs; ${ }^{7,51,56,57,64,72,74,75,79,81,83,93}$ of these, none reported on TCAs as a class, and three focused on specific drugs (amitriptyline and nortriptyline, and clomipramine) (Table B-28). ${ }^{51,56,74}$ These publications drew from four cohorts (four from the Canadian QPC, ${ }^{57,64,83,93}$ three from the U.S. Medicaid Analytic eXtract, ${ }^{7,74,75}$ two each from the U.K. THIN ${ }^{72,79}$ and the Swedish Birth Cohort, ${ }^{51,56}$ and one from the Canadian British Columbia health utilization databases. ${ }^{81}$ All were nonrandomized studies. Four were rated high risk of bias; ${ }^{7,} 57,72,79$ others were rated as having some risk-of-bias concerns. 
Table B-28. Strength of evidence for harms: TCAs versus no treatment

\begin{tabular}{|c|c|c|c|c|c|c|c|}
\hline Population & $\begin{array}{l}\text { Intervention } \\
\text { and } \\
\text { Comparator }\end{array}$ & Outcome & $\begin{array}{l}\text { Incidence or } \\
\text { Mean Effect } \\
\text { by Arm }\end{array}$ & Results & $\begin{array}{l}\text { Study } \\
\text { Design and } \\
\text { Sample Size }\end{array}$ & $\begin{array}{l}\text { Factors That } \\
\text { Affect the } \\
\text { Strength of } \\
\text { Evidence }\end{array}$ & $\begin{array}{l}\text { Overall } \\
\text { Evidence } \\
\text { Strength } \\
\text { (Direction of } \\
\text { Effect) }\end{array}$ \\
\hline $\begin{array}{l}\text { Depressed or } \\
\text { TCA-exposed } \\
\text { women }\end{array}$ & $\begin{array}{l}\text { TCA } \\
\text { exposure in } \\
\text { pregnancy } \\
\text { vs. } \\
\text { unexposed } \\
\text { women with } \\
\text { depression }\end{array}$ & $\begin{array}{l}\text { Spontaneous } \\
\text { abortion }\end{array}$ & $\begin{array}{l}20 / 112 \\
(17.9 \%) \text { vs. } \\
720 / 7,034 \\
(10.2 \%)^{64} \\
\text { NR in } \\
\text { second }^{79}\end{array}$ & $\begin{array}{l}\text { ARR, } 1.5,95 \% \\
\mathrm{Cl}, 1.5(0.96 \text { to } \\
2.2)^{64} \text { and } 1.3 \\
(99 \% \mathrm{Cl}, 1.1 \text { to } \\
1.5)^{79}\end{array}$ & $\begin{array}{l}2 \text { cohorts; } \\
n=7,146 \text { in } \\
\text { one cohort, }{ }^{64} \\
\text { NR in } \\
\text { second }^{79}\end{array}$ & $\begin{array}{l}\text { Moderate } \\
\text { study } \\
\text { limitations } \\
\text { (one high } \\
\text { risk-of-bias } \\
\text { study }^{79} \text { ), } \\
\text { imprecise, } \\
\text { consistent }\end{array}$ & Insufficient \\
\hline $\begin{array}{l}\text { Mood or } \\
\text { anxiety } \\
\text { disorder } \\
\text { during } \\
\text { pregnancy or } \\
\text { exposed to } \\
\text { amitriptyline }\end{array}$ & $\begin{array}{l}\text { Current } \\
\text { amitriptyline } \\
\text { exposure (at } \\
\text { delivery), or } \\
\text { unexposed }\end{array}$ & $\begin{array}{l}\text { Postpartum } \\
\text { hemorrhage } \\
\text { billing } \\
\text { diagnosis }\end{array}$ & NR & $\begin{array}{l}\text { ARR, } 1.68(95 \% \\
\mathrm{Cl}, 0.89 \text { to } \\
3.16)^{74}\end{array}$ & $\begin{array}{l}1 \text { cohort, } \\
\mathrm{N}=69,220^{74}\end{array}$ & $\begin{array}{l}\text { Moderate } \\
\text { study } \\
\text { limitations, } \\
\text { precise, } \\
\text { consistency } \\
\text { unknown }\end{array}$ & Insufficient \\
\hline $\begin{array}{l}\text { Mood or } \\
\text { anxiety } \\
\text { disorder } \\
\text { during } \\
\text { pregnancy or } \\
\text { exposed to } \\
\text { amitriptyline }\end{array}$ & $\begin{array}{l}\text { Recent } \\
\text { amitriptyline } \\
\text { exposure (<1 } \\
\text { month before } \\
\text { delivery) or } \\
\text { unexposed }\end{array}$ & $\begin{array}{l}\text { Postpartum } \\
\text { hemorrhage } \\
\text { billing } \\
\text { diagnosis }\end{array}$ & NR & $\begin{array}{l}\text { ARR, } 1.13(95 \% \\
\mathrm{Cl}, 0.29 \text { to } \\
4.42)^{74}\end{array}$ & $\begin{array}{l}1 \text { cohort, } \\
N=69,113^{74}\end{array}$ & $\begin{array}{l}\text { Moderate } \\
\text { study } \\
\text { limitations, } \\
\text { imprecision } \\
\text { (wide Cls), } \\
\text { consistency } \\
\text { unknown }\end{array}$ & Insufficient \\
\hline $\begin{array}{l}\text { Mood or } \\
\text { anxiety } \\
\text { disorder } \\
\text { during } \\
\text { pregnancy or } \\
\text { exposed to } \\
\text { amitriptyline }\end{array}$ & $\begin{array}{l}\text { Past } \\
\text { amitriptyline } \\
\text { exposure (>1 } \\
\text { to } 5 \text { months } \\
\text { before } \\
\text { delivery) or } \\
\text { unexposed }\end{array}$ & $\begin{array}{l}\text { Postpartum } \\
\text { hemorrhage } \\
\text { billing } \\
\text { diagnosis }\end{array}$ & NR & $\begin{array}{l}\text { ARR, } 1.08(95 \% \\
\mathrm{Cl}, 0.48 \text { to } \\
2.42)^{74}\end{array}$ & $\begin{array}{l}1 \text { cohort, } \\
\mathrm{n}=69,250^{74}\end{array}$ & $\begin{array}{l}\text { Moderate } \\
\text { study } \\
\text { limitations, } \\
\text { precision } \\
\text { (wide Cls), } \\
\text { consistency } \\
\text { unknown }\end{array}$ & Insufficient \\
\hline $\begin{array}{l}\text { Depressed } \\
\text { women }\end{array}$ & $\begin{array}{l}\text { TCA } \\
\text { exposure in } \\
\text { pregnancy } \\
\text { vs. } \\
\text { unexposed } \\
\text { women with } \\
\text { depression in } \\
\text { pregnancy }\end{array}$ & Preeclampsia & $\begin{array}{l}47 / 441 \\
(10.7 \%) \text { vs. } \\
3,215 / / 59,219 \\
(5.4 \%) ; 75 \\
14 / 146 \\
(9.59 \%) \text { vs. } \\
1,569 / 65,392 \\
(2.40 \%)^{81}\end{array}$ & $\begin{array}{l}\text { ARR, } 1.62(95 \% \\
\mathrm{Cl}, 1.23 \text { to } \\
2.12)^{75} ; \mathrm{ARR}, \\
3.23(95 \% \mathrm{Cl} \\
1.87 \text { to } 5.59)^{81}\end{array}$ & $\begin{array}{l}2 \text { cohorts, } \\
n=125,198^{75}, \\
81\end{array}$ & $\begin{array}{l}\text { Moderate } \\
\text { study } \\
\text { limitations, } \\
\text { precise, } \\
\text { consistent }\end{array}$ & $\begin{array}{l}\text { Low for } \\
\text { harms of TCA }\end{array}$ \\
\hline $\begin{array}{l}\text { Depressed or } \\
\text { anxious or } \\
\text { TCA-exposed } \\
\text { women } \\
\end{array}$ & $\begin{array}{l}\text { TCA } \\
\text { exposure in } \\
\text { pregnancy } \\
\text { vs. } \\
\text { unexposed } \\
\text { women with } \\
\text { depression }\end{array}$ & $\begin{array}{l}\text { Perinatal } \\
\text { mortality }\end{array}$ & $\begin{array}{l}18 / 3,019 \\
(0.6 \%) \text { vs. } \\
20 / 3647 \\
(0.6 \%)^{79}\end{array}$ & $\begin{array}{l}\text { ARR, } 1.2(99 \% \\
\mathrm{Cl}, 0.5 \text { to } 2.7)\end{array}$ & $\begin{array}{l}1 \text { cohort, } \\
n=6,666^{79}\end{array}$ & $\begin{array}{l}\text { High study } \\
\text { limitations, } \\
\text { imprecise } \\
\text { (wide Cls), } \\
\text { consistency } \\
\text { unknown }\end{array}$ & Insufficient \\
\hline $\begin{array}{l}\text { History of } \\
\text { psychiatric } \\
\text { disorder or } \\
\text { TCA-exposed } \\
\text { women }\end{array}$ & $\begin{array}{l}\text { TCA } \\
\text { exposure in } \\
\text { pregnancy } \\
\text { vs. } \\
\text { unexposed } \\
\text { women with } \\
\text { psychiatric } \\
\text { disorder }\end{array}$ & $\begin{array}{l}\text { Small for } \\
\text { gestational age }\end{array}$ & $\begin{array}{l}\text { Not } \\
\text { applicable for } \\
\text { case-control }^{93}\end{array}$ & $\begin{array}{l}\text { ARR, varies by } \\
\text { trimester from } \\
0.69 \text { to } 2.12 \text {, } \\
95 \% \mathrm{Cl} \text {, spans } \\
\text { the null }\end{array}$ & $\begin{array}{l}1 \text { case- } \\
\text { control, } n \\
\text { varies by } \\
\text { trimester, } \\
>1,535^{83}\end{array}$ & $\begin{array}{l}\text { Moderate } \\
\text { study } \\
\text { limitations, } \\
\text { imprecise } \\
\text { (wide Cls), } \\
\text { consistency } \\
\text { unknown }\end{array}$ & Insufficient \\
\hline
\end{tabular}




\begin{tabular}{|c|c|c|c|c|c|c|c|}
\hline Population & $\begin{array}{l}\text { Intervention } \\
\text { and } \\
\text { Comparator }\end{array}$ & Outcome & $\begin{array}{l}\text { Incidence or } \\
\text { Mean Effect } \\
\text { by Arm }\end{array}$ & Results & $\begin{array}{l}\text { Study } \\
\text { Design and } \\
\text { Sample Size }\end{array}$ & $\begin{array}{l}\text { Factors That } \\
\text { Affect the } \\
\text { Strength of } \\
\text { Evidence }\end{array}$ & $\begin{array}{l}\text { Overall } \\
\text { Evidence } \\
\text { Strength } \\
\text { (Direction of } \\
\text { Effect) }\end{array}$ \\
\hline $\begin{array}{l}\text { History of } \\
\text { depression, } \\
\text { depression or } \\
\text { anxiety or } \\
\text { psychiatric } \\
\text { disorder or } \\
\text { TCA-exposed } \\
\text { women }\end{array}$ & $\begin{array}{l}\text { TCA } \\
\text { exposure } \\
\text { during the } \\
\text { first trimester } \\
\text { vs. } \\
\text { unexposed } \\
\text { with history of } \\
\text { depression, } \\
\text { anxiety, or } \\
\text { psychiatric } \\
\text { disorder }\end{array}$ & $\begin{array}{l}\text { Major } \\
\text { congenital } \\
\text { anomalies }\end{array}$ & $\begin{array}{l}51 / 382 \\
(13.4 \%) \text { vs. } \\
1,650 / 14,847 \\
(11.1 \%) ; 57 \\
74 / 2,428 \\
(3.0 \%) \text { vs. } \\
380 / 13,432 \\
(2.8 \%)^{72}{ }^{72} \text { Not } \\
\text { applicable for }^{9} \\
\text { case-control }^{93} \\
\end{array}$ & $\begin{array}{l}\text { Results not } \\
\text { pooled because } \\
\text { two } \\
\text { publications }{ }^{57,93} \\
\text { potentially drawn } \\
\text { from the same } \\
\text { population } \\
\text { AOR, } 1.16(95 \% \\
\text { Cl, } 0.86 \text { to } \\
1.56)^{57} \\
\text { AOR, } 1.02(95 \% \\
\text { Cl, } 0.79 \text { to } \\
1.32)^{72} \\
\text { AOR, } 0.78(95 \% \\
\text { Cl, } 0.30 \text { to } \\
2.02)^{93}\end{array}$ & $\begin{array}{l}1 \text { case- } \\
\text { control, }{ }^{93} 2 \\
\text { cohorts, } \\
\mathrm{n} \geq 31,089^{57,72} \\
\mathrm{~N} \text { from two } \\
\text { studies; }{ }^{57,72} \\
\text { (study } \\
\text { samples may } \\
\text { overlap) }\end{array}$ & $\begin{array}{l}\text { High study } \\
\text { limitations } \\
\text { (two high } \\
\text { risk-of-bias } \\
\text { studies), }{ }^{57,93} \\
\text { imprecise } \\
\text { (wide Cls), } \\
\text { consistent }\end{array}$ & Insufficient \\
\hline $\begin{array}{l}\text { History of } \\
\text { depression or } \\
\text { anxiety or } \\
\text { TCA-exposed } \\
\text { women }\end{array}$ & $\begin{array}{l}\text { TCA in first } \\
\text { trimester vs. } \\
\text { unexposed } \\
\text { women with } \\
\text { history of } \\
\text { depression }\end{array}$ & $\begin{array}{l}\text { Cardiac } \\
\text { anomalies }\end{array}$ & $\begin{array}{l}20 / 2,428 \\
(0.82 \%) \text { vs. } \\
112 / 13,432 \\
(0.83 \%) ; 72 \text { NR } \\
\text { in other } \\
\text { studies }^{7,57}\end{array}$ & $\begin{array}{l}\text { Pooled AOR, } \\
0.86(95 \% \mathrm{Cl}, \\
0.65 \text { to } 1.13), \mathrm{I}^{2} \text { : } \\
0 \%\end{array}$ & $\begin{array}{l}3 \text { cohorts, } \\
n>15,860 \\
\text { (N=NR in two } \\
\text { studies) })^{7,57,72}\end{array}$ & $\begin{array}{l}\text { High study } \\
\text { limitations (all } \\
\text { risk-of-bias } \\
\text { studies), }, 57,72 \\
\text { imprecise } \\
\text { (wide Cls), } \\
\text { consistent }\end{array}$ & Insufficient \\
\hline $\begin{array}{l}\text { Psychiatric } \\
\text { disorder or } \\
\text { clomipramine } \\
\text {-exposed } \\
\text { women }\end{array}$ & $\begin{array}{l}\text { Clomipramine } \\
\text { vs. } \\
\text { unexposed } \\
\text { women with } \\
\text { history of } \\
\text { psychiatric } \\
\text { disorder }\end{array}$ & $\begin{array}{l}\text { Autism } \\
\text { spectrum } \\
\text { disorder }\end{array}$ & $\begin{array}{l}16 / 235 \\
(6.8 \%) \text { vs. } \\
353 / 12,325 \\
(2.9 \%) ; 51 \text { NR } \\
\text { in second } \\
\text { study }^{56}\end{array}$ & $\begin{array}{l}\text { AOR, } 1.76 \\
(-95 \% \mathrm{Cl}, 1.01 \\
\text { to } 3.05) ; 51 \mathrm{ARR}, \\
3.36(95 \% \mathrm{Cl}, \\
1.39 \text { to } 8.13) \text {, } \\
\text { results not } \\
\text { statistically } \\
\text { significant when } \\
\text { corrected for } \\
\text { multiple testing } \\
\geq 1 \text { psychiatric } \\
\text { disorder: ARR, } \\
3.36(95 \% \mathrm{Cl}, \\
1.39 \text { to } 8.13)^{56} \\
\geq 2 \text { psychiatric } \\
\text { disorders: ARR, } \\
2.53(1.02 \text { to } \\
6.22)^{56} \\
\geq 3 \text { psychiatric } \\
\text { disorders: ARR, } \\
1.88(0.7 \text { to } \\
4.73)^{56}\end{array}$ & $\begin{array}{l}2 \text { cohorts, } \\
\mathrm{n}=36,936^{51,56} \\
\text { (potential } \\
\text { overlap of } \\
\text { participants in } \\
\text { the } \\
\text { publications) }\end{array}$ & $\begin{array}{l}\text { Moderate } \\
\text { study } \\
\text { limitations, } \\
\text { imprecise, } \\
\text { consistent }\end{array}$ & Insufficient \\
\hline
\end{tabular}




\begin{tabular}{|c|c|c|c|c|c|c|c|}
\hline Population & $\begin{array}{l}\text { Intervention } \\
\text { and } \\
\text { Comparator }\end{array}$ & Outcome & $\begin{array}{l}\text { Incidence or } \\
\text { Mean Effect } \\
\text { by Arm }\end{array}$ & Results & $\begin{array}{l}\text { Study } \\
\text { Design and } \\
\text { Sample Size }\end{array}$ & $\begin{array}{l}\text { Factors That } \\
\text { Affect the } \\
\text { Strength of } \\
\text { Evidence }\end{array}$ & $\begin{array}{l}\text { Overall } \\
\text { Evidence } \\
\text { Strength } \\
\text { (Direction of } \\
\text { Effect) }\end{array}$ \\
\hline $\begin{array}{l}\text { Psychiatric } \\
\text { disorder or } \\
\text { amitriptyline } \\
\text { or } \\
\text { nortriptyline- } \\
\text { exposed } \\
\text { women }\end{array}$ & $\begin{array}{l}\text { Amitriptyline } \\
\text { or } \\
\text { nortriptyline } \\
\text { vs. } \\
\text { unexposed } \\
\text { women with } \\
\text { history of } \\
\text { psychiatric } \\
\text { disorder }\end{array}$ & $\begin{array}{l}\text { Autism } \\
\text { spectrum } \\
\text { disorder }\end{array}$ & NR & $\begin{array}{l}\text { ARR, } 0.47 \text { (95\% } \\
\text { Cl, } 0.07 \text { to } 3.31) \\
\geq 1 \text { psychiatric } \\
\text { disorder: ARR, } \\
0.47(95 \% \mathrm{Cl} \text {, } \\
0.07 \text { to } 3.31)^{56} \\
\geq 2 \text { psychiatric } \\
\text { disorders: ARR, } \\
0.35(0.05 \text { to } \\
2.49)^{56} \\
\geq 3 \text { psychiatric } \\
\text { disorders: ARR, } \\
0.25(0.04 \text { to } \\
1.84)^{56}\end{array}$ & $\begin{array}{l}1 \text { cohort, } \\
n=24,418^{56}\end{array}$ & $\begin{array}{l}\text { Moderate } \\
\text { study } \\
\text { limitations, } \\
\text { imprecise, } \\
\text { consistency } \\
\text { unknown }\end{array}$ & Insufficient \\
\hline
\end{tabular}

$\mathrm{AOR}=$ adjusted odds ratio; $\mathrm{ARR}=$ adjusted risk ratio; $\mathrm{CI}=$ confidence interval; $\mathrm{n} / \mathrm{N}=$ number; $\mathrm{NR}=$ not reported; $\mathrm{TCA}=$ tricyclic antidepressant; vs. = versus.

All publications focused on exposure during pregnancy. Publications compared pregnant women exposed to TCAs with women with untreated depression, ${ }^{7,57,64,72,75,81}$ depression and anxiety, ${ }^{56,79}$ mood or anxiety disorders, ${ }^{74}$ or history of a psychiatric disorder. ${ }^{51,83,93}$ Studies specifying a history of disorders varied significantly in their definition. The most inclusive specified the control arm as having a lifetime history of at least one diagnosis of anxiety or depression but no drug exposure during pregnancy ${ }^{56}$ Among those specifying the control arm with respect to exposure during pregnancy, the allowable period of exposure also varied: no exposure during or in the year prior to pregnancy ${ }^{64}$ no exposure in the past year to 20 weeks gestation ${ }^{81}$ no exposure in the trimester of interest,${ }^{79,83}$ and a history of anxiety or depression but no supply of antidepressants in the 5 months before delivery in another. ${ }^{74}$

The evidence from two studies ${ }^{75,81}$ suggests low strength of evidence for preeclampsia with TCAs; the risk of residual confounding cannot be ruled out. The evidence for TCAs, when analyzed individually or as a class, is insufficient to judge the risk of harms for maternal (spontaneous abortion, postpartum hemorrhage) or child outcomes (perinatal mortality, small for gestational age, major congenital anomalies, cardiac anomalies, or autism spectrum disorder) (Table B-28).

Two publications from the same data source (Quebec pregnancy, children cohort) reported on major congenital anomalies, but because they drew on different samples and used different designs, their results were reported separately. Notably, the results are not consistent in direction of effect, although neither publication demonstrates an association. Pooled results for cardiac anomalies also do not indicate an association (AOR, $0.86 ; 95 \% \mathrm{CI}, 0.65$ to $1.13 ; \mathrm{I}^{2}, 0 \%$; Figure B-7). $7,57,72$

Two publications from Swedish birth cohorts, with potential overlaps in the cohort, found no association between TCA exposure during pregnancy among women with a current or past psychiatric disorder and autism spectrum disorder in offspring. ${ }^{51,56}$ When the referent group was restricted to women with more than one psychiatric diagnosis, the point estimate for relative risk for clomipramine exposure moved from 3.36 ( $\geq 1$ disorder with statistically significant results) to 
1.88 ( $\geq 3$ disorders with CIs spanning the null). ${ }^{56}$ Similarly, the point estimate for amitriptyline/nortriptyline exposure also indicated reduced risk with more disorders. The relative risk moved from 0.47 ( $\geq 1$ disorder with CIs spanning the null) to 0.25 ( $\geq 3$ disorders also with CIs spanning the null). ${ }^{56}$ This adjustment for confounding further suggests that the incidence of autism cannot be attributed to exposure to TCAs.

\section{Brexanolone}

\section{Overview}

- The prescribing information for brexanolone exposure for depression onset in the third trimester of pregnancy or within 4 weeks of birth includes a boxed warning regarding risk of excessive sedation or sudden loss of consciousness during administration of the drug. ${ }^{150}$

- Evidence was insufficient to judge the risks of any adverse events or dizziness in the brexanolone arms when compared with the. placebo arms.

\section{Detailed Results}

As noted in KQ 1, three RCTs (rated low risk of bias) reported on the harms of brexanolone versus placebo for depression symptoms with onset in the third trimester of pregnancy or within 4 weeks of birth (Table B-29). ${ }^{17,19}$ Brexanolone was administered as a 60-hour continuous infusion with a peak dose of either $60 \mu \mathrm{g} / \mathrm{kg}$ per hour or $90 \mu \mathrm{g} / \mathrm{kg}$ per hour. The study reported similar rates of any adverse events (pooled RR, 0.93; 95\% CI, 0.72 to 1.21 ; I, 1.7\%; Figure B-8) during infusion in the brexanolone and placebo groups and a higher rate of dizziness (pooled RR, 1.56; $95 \%$ CI, 0.52 to $4.66 ; \mathrm{I}^{2}, 31.4 \%$; Figure B-9) and loss of consciousness in the brexanolone versus placebo arms (pooled RR, 2.00; 95\% CI, 0.78 to 5.16; $\mathrm{I}^{2}, 0 \%$; Figure B-9). The prescribing information warns of excessive sedation or loss of consciousness in the active arm. Although results could not be pooled for excessive sedation or dose interruption based on the data in the published studies, the prescribing information notes that 5 percent of brexanolonetreated patients compared to 0 percent of placebo-treated patients had sedation or somnolence that required dose interruption. ${ }^{151}$ Rates of loss of consciousness did not appear to increase with dose intensity: 5 percent of women randomized to BRX60 versus 3\% for BRX90 experienced loss of consciousness. The pooled results for any adverse events and dizziness are imprecise and inconsistent and graded as insufficient. 
Table B-29. Strength of evidence for harms: Brexanolone versus no placebo

\begin{tabular}{|c|c|c|c|c|c|c|c|}
\hline Population & $\begin{array}{l}\text { Intervention } \\
\text { and } \\
\text { Comparator }\end{array}$ & Outcome & $\begin{array}{l}\text { Incidence or } \\
\text { Mean Effect by } \\
\text { Arm }\end{array}$ & Results & $\begin{array}{l}\text { Study } \\
\text { Design and } \\
\text { Sample } \\
\text { Size }\end{array}$ & $\begin{array}{l}\text { Factors That } \\
\text { Affect the } \\
\text { Strength of } \\
\text { Evidence }\end{array}$ & $\begin{array}{l}\text { Overall } \\
\text { Evidence } \\
\text { Strength } \\
\text { (Direction } \\
\text { of Effect) }\end{array}$ \\
\hline $\begin{array}{l}\text { Women } \\
\text { with } \\
\text { postpartum } \\
\text { depression }\end{array}$ & $\begin{array}{l}\text { Brexanolone } \\
\text { vs. placebo }\end{array}$ & $\begin{array}{l}\text { Any adverse } \\
\text { event }\end{array}$ & $\begin{array}{l}\text { BRX60: 19/38 } \\
\text { (50\%); } \\
\text { BRX90: 51/102 } \\
\text { (50\%); } \\
\text { Placebo: } \\
\text { 54/107 (50.5\%) }\end{array}$ & $\begin{array}{l}\text { Pooled } \mathrm{RR}, \\
0.93,95 \% \mathrm{Cl} \text {, } \\
0.72 \text { to } 1.21 \\
\mathrm{I}^{2}=1.7 \%\end{array}$ & $3 \mathrm{RCTs}^{17,19}$ & $\begin{array}{l}\text { Low study } \\
\text { limitations, } \\
\text { inconsistent, } \\
\text { imprecise (wide } \\
\text { Cls) }\end{array}$ & Insufficient \\
\hline $\begin{array}{l}\text { Women } \\
\text { with } \\
\text { postpartum } \\
\text { depression }\end{array}$ & $\begin{array}{l}\text { Brexanolone } \\
\text { vs. placebo }\end{array}$ & Dizziness & $\begin{array}{l}\text { BRX60: } 6 / 38 \\
(15.8 \%) ; \\
\text { BRX90: } 13 / 102 \\
(12.7 \%) \\
\text { Placebo: } 8 / 107 \\
(7.5 \%)\end{array}$ & $\begin{array}{l}\text { Pooled RR, } \\
1.56,95 \% \mathrm{Cl}, \\
0.52 \text { to } 4.66, \\
\mathrm{I}^{2}=31.4 \%\end{array}$ & $3 \mathrm{RCTs}^{17,19}$ & $\begin{array}{l}\text { Low study } \\
\text { limitations, } \\
\text { inconsistent, } \\
\text { imprecise (wide } \\
\text { Cls, few events) }\end{array}$ & Insufficient \\
\hline \multirow[t]{3}{*}{$\begin{array}{l}\text { Women } \\
\text { with } \\
\text { postpartum } \\
\text { depression }\end{array}$} & $\begin{array}{l}\text { Brexanolone } \\
\text { vs. placebo }\end{array}$ & $\begin{array}{l}\text { Dose interruption } \\
\text { or reduction in } \\
\text { some patients } \\
\text { during the } \\
\text { infusion due to } \\
\text { sedation or } \\
\text { somnolence }\end{array}$ & $\begin{array}{l}\text { Treatment arm: } \\
5 \% \\
\text { Placebo: } 0 \\
\text { (from FDA } \\
\text { prescribing } \\
\text { information) }^{151}\end{array}$ & $\begin{array}{l}\text { Data not } \\
\text { reported by } \\
\text { study pooled } \\
\text { RR not } \\
\text { calculable) }\end{array}$ & & $\begin{array}{l}\text { Low study } \\
\text { limitations, } \\
\text { precision and } \\
\text { consistency } \\
\text { unknown }\end{array}$ & Low \\
\hline & $\begin{array}{l}\text { Brexanolone } \\
\text { vs. placebo }\end{array}$ & $\begin{array}{l}\text { Loss of } \\
\text { consciousness }\end{array}$ & $\begin{array}{l}\text { BRX60: 5\%; } \\
\text { BRX90: 3\%; } \\
\text { Placebo: } 0 \% \\
\text { (from FDA } \\
\text { prescribing } \\
\text { information) }\end{array}$ & $\begin{array}{l}\text { Data not } \\
\text { reported by } \\
\text { study, cannot } \\
\text { be pooled } \\
\text { (from FDA } \\
\text { prescribing } \\
\text { information, } \\
151 \text { not } \\
\text { reported in } \\
\text { the appendix) }\end{array}$ & $3 \mathrm{RCTs}^{17,} 19$ & $\begin{array}{l}\text { Low study } \\
\text { limitations, } \\
\text { precision and } \\
\text { consistency } \\
\text { unknown }\end{array}$ & \\
\hline & $\begin{array}{l}\text { Brexanolone } \\
\text { vs. placebo }\end{array}$ & Somnolence & $\begin{array}{l}\text { BRX60: } 7 / 38 \\
(18.4 \%) \\
\text { BRX90: } 8 / 102 \\
(7.8 \%) \\
\text { Placebo: } 5 / 107 \\
(4.7 \%)\end{array}$ & $\begin{array}{l}\text { Pooled RR, } \\
2.00,95 \% \mathrm{Cl} \text {, } \\
0.78 \text { to } 5.16 \text {, } \\
\mathrm{I}^{2}=0 \%\end{array}$ & $3 \mathrm{RCTs}^{17,19}$ & $\begin{array}{l}\text { Low study } \\
\text { limitations, } \\
\text { inconsistent, } \\
\text { imprecise (wide } \\
\text { Cls, few events) }\end{array}$ & \\
\hline
\end{tabular}

$\mathrm{BRX}=$ brexanolone for postpartum depression; $\mathrm{CI}=$ confidence interval; FDA = Food and Drug Administration; $\mathrm{RCT}=$ randomized controlled trial; $\mathrm{RR}=$ relative risk; vs. = versus.

\section{Bupropion}

\section{Overview}

- Evidence from one study suggests an increased risk of postpartum hemorrhage with past exposure (supply of drug 1 to 5 months prior to delivery) to bupropion compared with women with mood or anxiety disorders and no exposure to bupropion during pregnancy (low strength of evidence).

- The evidence is insufficient to judge the risk of harms for postpartum hemorrhage for current (supply of drug on delivery date) or recent exposure (supply of drug 1 month before delivery) to bupropion. 
- The evidence is insufficient to judge the risk of harms for maternal outcomes (gestational diabetes, preeclampsia, postpartum hemorrhage) or child outcomes (any cardiac anomaly).

\section{Detailed Results}

Five publications reported on bupropion. ${ }^{7,74,75,104,106}$ These publications drew from three cohorts (the 2000-2007 U.S. nationwide Medicaid Analytic eXtract data, ${ }^{7,74,75}$ the Kaiser Permanente Washington Database, ${ }^{104}$ and the National Birth Defects Prevention Study). ${ }^{106}$ All were nonrandomized observational studies and were rated as "some concerns" regarding risk of bias. $^{7,74,75}$

All publications focused on exposure during pregnancy. One publication ${ }^{74}$ also analyzed current bupropion exposure (supply of drug on the delivery date), recent bupropion exposure (women with a supply of antidepressants on at least 1 day in the month before the delivery date but not on the delivery date), and past bupropion exposure (1 to 5 months before delivery). Publications compared pregnant women exposed to bupropion with women with nonpharmacologically treated depression ${ }^{7,75}$ or mood disorder and anxiety. ${ }^{74}$ Two publications ${ }^{75 \text {, }}$ ${ }^{104}$ compared pregnant women who continued versus discontinued bupropion during pregnancy. One publication compared women exposed to bupropion during early pregnancy to women not exposed during early pregnancy. ${ }^{106}$

The evidence for bupropion, when analyzed individually is insufficient to judge the risk of harms for most maternal outcomes (postpartum hemorrhage, preeclampsia, or gestational diabetes) or child outcomes (any cardiac anomalies) (Table B-30). One study with moderate study limitations that did not adjust for multiple comparisons generally reported no difference in several other birth defects. ${ }^{106}$

The publications consistently found no association between bupropion exposure and maternal or child harms with the exception of increased risk of postpartum hemorrhage at one time point (past bupropion exposure ${ }^{74}$ ). Notably, there was no association detected with recent or current bupropion exposure in this publication. ${ }^{74}$ In the one publication examining continuation versus discontinuation, bupropion continuation during pregnancy had no association with preeclampsia risk.

Table B-30. Strength of evidence for harms: Bupropion versus no treatment

\begin{tabular}{|c|c|c|c|c|c|c|c|}
\hline Population & $\begin{array}{l}\text { Intervention } \\
\text { and } \\
\text { Comparator }\end{array}$ & Outcome & $\begin{array}{l}\text { Incidence or } \\
\text { Mean Effect } \\
\text { by Arm }\end{array}$ & Results & $\begin{array}{l}\text { Study Design } \\
\text { and Sample } \\
\text { Size }\end{array}$ & $\begin{array}{l}\text { Factors That } \\
\text { Affect the } \\
\text { Strength of } \\
\text { Evidence }\end{array}$ & $\begin{array}{l}\text { Overall } \\
\text { Evidence } \\
\text { Strength } \\
\text { (Direction } \\
\text { of Effect) } \\
\end{array}$ \\
\hline $\begin{array}{l}\text { Women } \\
\text { taking anti- } \\
\text { depressant } \\
\mathrm{s} \text { at least } 6 \\
\text { months } \\
\text { before } \\
\text { pregnancy }\end{array}$ & $\begin{array}{l}\text { Bupropion } \\
\text { continuation in } \\
\text { pregnancy vs. } \\
\text { discontinuation }\end{array}$ & $\begin{array}{l}\text { Gestational } \\
\text { Diabetes }\end{array}$ & $\begin{array}{l}21 / 217 \\
(10 \%) \text { vs. } \\
90 / 1211 \\
(7 \%)^{104}\end{array}$ & $\begin{array}{l}\text { Weighted RR, } \\
1.07(95 \% \mathrm{Cl}, \\
0.63 \text { to } 1.81)^{104}\end{array}$ & $\begin{array}{l}1 \text { cohort, } \\
\mathrm{N}=1,438^{104}\end{array}$ & $\begin{array}{l}\text { Moderate study } \\
\text { limitations, } \\
\text { imprecise (large } \\
\text { Cls spanning } \\
\text { the null), } \\
\text { consistency } \\
\text { unknown }\end{array}$ & Insufficient \\
\hline $\begin{array}{l}\text { Depressed } \\
\text { or } \\
\text { bupropion- } \\
\text { exposed } \\
\text { women }\end{array}$ & $\begin{array}{l}\text { Bupropion } \\
\text { exposure in } \\
\text { pregnancy vs. } \\
\text { unexposed } \\
\text { women with } \\
\text { depression }\end{array}$ & $\begin{array}{l}\text { Any } \\
\text { preeclampsia }\end{array}$ & $\begin{array}{l}153 / 2,622 \\
(5.8 \%) \text { vs. } \\
3215 / 59,219 \\
(5.4 \%)^{75}\end{array}$ & $\begin{array}{l}\text { AOR, } 1.09 \\
(95 \% \mathrm{Cl}, 0.89 \\
\text { to } 1.37)^{75}\end{array}$ & $\begin{array}{l}1 \text { cohort, } \\
n=61,841^{75}\end{array}$ & $\begin{array}{l}\text { Moderate study } \\
\text { limitations, } \\
\text { imprecise (wide } \\
\text { Cls spanning } \\
\text { the null), } \\
\text { consistency } \\
\text { unknown }\end{array}$ & Insufficient \\
\hline
\end{tabular}




\begin{tabular}{|c|c|c|c|c|c|c|c|}
\hline Population & $\begin{array}{l}\text { Intervention } \\
\text { and } \\
\text { Comparator }\end{array}$ & Outcome & $\begin{array}{l}\text { Incidence or } \\
\text { Mean Effect } \\
\text { by Arm }\end{array}$ & Results & $\begin{array}{l}\text { Study Design } \\
\text { and Sample } \\
\text { Size }\end{array}$ & $\begin{array}{l}\text { Factors That } \\
\text { Affect the } \\
\text { Strength of } \\
\text { Evidence }\end{array}$ & $\begin{array}{l}\text { Overall } \\
\text { Evidence } \\
\text { Strength } \\
\text { (Direction } \\
\text { of Effect) }\end{array}$ \\
\hline $\begin{array}{l}\text { Depressed } \\
\text { or } \\
\text { bupropion- } \\
\text { exposed } \\
\text { women }\end{array}$ & $\begin{array}{l}\text { Bupropion } \\
\text { exposure in } \\
\text { pregnancy vs. } \\
\text { unexposed } \\
\text { women with } \\
\text { depression }\end{array}$ & $\begin{array}{l}\text { Severe } \\
\text { preeclampsia }\end{array}$ & $\begin{array}{l}34 / 2,622 \\
(1.3 \%) \text { vs. } \\
976 / 59,219 \\
(1.6 \%)^{75}\end{array}$ & $\begin{array}{l}\text { AOR, } 0.79 \\
(95 \% \mathrm{Cl}, 0.56 \\
\text { to } 1.12)^{75}\end{array}$ & $\begin{array}{l}1 \text { cohort, } \\
n=61,841^{75}\end{array}$ & $\begin{array}{l}\text { Moderate study } \\
\text { limitations, } \\
\text { imprecise (wide } \\
\text { Cls spanning } \\
\text { the null), } \\
\text { consistency } \\
\text { unknown }\end{array}$ & Insufficient \\
\hline $\begin{array}{l}\text { Depressed } \\
\text { or } \\
\text { bupropion- } \\
\text { exposed } \\
\text { women }\end{array}$ & $\begin{array}{l}\text { Bupropion } \\
\text { continuation in } \\
\text { pregnancy vs. } \\
\text { bupropion } \\
\text { discontinuation }\end{array}$ & Preeclampsia & $\begin{array}{l}21 / 360 \\
(5.8 \%) \text { vs. } \\
33 / 691 \\
(4.8 \%)^{75}\end{array}$ & $\begin{array}{l}\text { AOR, } 1.10 \\
(95 \% \mathrm{Cl}, 0.64 \\
\text { to } 1.91)^{75}\end{array}$ & $\begin{array}{l}1 \text { cohort, } \\
n=1,051^{75}\end{array}$ & $\begin{array}{l}\text { Moderate study } \\
\text { limitations, } \\
\text { imprecise (wide } \\
\text { Cls spanning } \\
\text { the null), } \\
\text { consistency } \\
\text { unknown }\end{array}$ & Insufficient \\
\hline $\begin{array}{l}\text { Mood } \\
\text { disorder or } \\
\text { anxiety or } \\
\text { bupropion- } \\
\text { exposed } \\
\text { women }\end{array}$ & $\begin{array}{l}\text { Current } \\
\text { bupropion } \\
\text { exposure in } \\
\text { pregnancy vs. } \\
\text { bupropion } \\
\text { unexposed } \\
\text { women with } \\
\text { mood disorder } \\
\text { or anxiety }\end{array}$ & $\begin{array}{l}\text { Postpartum } \\
\text { hemorrhage }\end{array}$ & $\begin{array}{l}42 / 1,162 \\
(3.6 \%) \text { vs. } \\
1,896 / 69,044 \\
(2.7 \%) \text { in }^{74}\end{array}$ & $\begin{array}{l}\text { ARR, } 1.32 \\
(95 \% \mathrm{Cl}, 0.98 \\
\text { to } 1.79)\end{array}$ & $\begin{array}{l}1 \text { cohort, } \\
n=70,206^{74}\end{array}$ & $\begin{array}{l}\text { Moderate study } \\
\text { limitations, } \\
\text { imprecise (wide } \\
\text { Cls spanning } \\
\text { the null), } \\
\text { consistency } \\
\text { unknown }\end{array}$ & Insufficient \\
\hline $\begin{array}{l}\text { Mood } \\
\text { disorder or } \\
\text { anxiety or } \\
\text { bupropion- } \\
\text { exposed } \\
\text { women }\end{array}$ & $\begin{array}{l}\text { Recent } \\
\text { bupropion } \\
\text { exposure in } \\
\text { pregnancy vs. } \\
\text { bupropion } \\
\text { unexposed } \\
\text { women with } \\
\text { mood disorder } \\
\text { or anxiety }\end{array}$ & $\begin{array}{l}\text { Postpartum } \\
\text { hemorrhage }\end{array}$ & $\begin{array}{l}21 / 660 \\
(3.2 \%) \text { vs. } \\
1,896 / 69,044 \\
(2.7 \%) \text { in }^{74}\end{array}$ & $\begin{array}{l}\text { ARR, } 1.17 \\
(95 \% \mathrm{Cl}, 0.8 \text { to } \\
1.8)^{74}\end{array}$ & $\begin{array}{l}1 \text { cohort, } \\
n=70,206^{74}\end{array}$ & $\begin{array}{l}\text { Moderate study } \\
\text { limitations, } \\
\text { imprecise (wide } \\
\text { Cls spanning } \\
\text { the null), } \\
\text { consistency } \\
\text { unknown }\end{array}$ & Insufficient \\
\hline $\begin{array}{l}\text { Mood } \\
\text { disorder or } \\
\text { anxiety or } \\
\text { bupropion- } \\
\text { exposed } \\
\text { women }\end{array}$ & $\begin{array}{l}\text { Past } \\
\text { bupropion } \\
\text { exposure in } \\
\text { pregnancy vs. } \\
\text { bupropion } \\
\text { unexposed } \\
\text { women with } \\
\text { mood disorder } \\
\text { or anxiety }\end{array}$ & $\begin{array}{l}\text { Postpartum } \\
\text { hemorrhage }\end{array}$ & $\begin{array}{l}61 / 1,712 \\
(3.6 \%) \text { vs. } \\
1,896 / 69,044 \\
(2.7 \%) \text { in }^{74}\end{array}$ & $\begin{array}{l}\text { ARR, } 1.32 \\
(95 \% \mathrm{Cl}, 1.02 \\
\text { to } 1.69)^{74}\end{array}$ & $\begin{array}{l}1 \text { cohort, } \\
n=70,206^{74}\end{array}$ & $\begin{array}{l}\text { Moderate study } \\
\text { limitations, } \\
\text { precise, } \\
\text { consistency } \\
\text { unknown }\end{array}$ & $\begin{array}{l}\text { Low for } \\
\text { harms of } \\
\text { bupropion }\end{array}$ \\
\hline $\begin{array}{l}\text { Depressed } \\
\text { or } \\
\text { bupropion- } \\
\text { exposed } \\
\text { women } \\
\end{array}$ & $\begin{array}{l}\text { Bupropion } \\
\text { exposure in } \\
\text { pregnancy vs. } \\
\text { unexposed } \\
\text { women with } \\
\text { depression or } \\
\text { unexposed in } \\
\text { early } \\
\text { pregnancy }\end{array}$ & $\begin{array}{l}\text { Cardiac } \\
\text { anomalies }\end{array}$ & $\begin{array}{l}\text { NR; }{ }^{7} \\
57 \text { cases/45 } \\
\text { controls } \\
\text { vs. } 149 \\
\text { cases/125 } \\
\text { controls }^{106}\end{array}$ & $\begin{array}{l}\text { AOR, } 0.92 \\
(95 \% \mathrm{Cl}, 0.69 \\
\text { to } 1.22) ;{ }^{7} \mathrm{AOR}, \\
1.06(0.66 \text { to } \\
1.71)^{106}\end{array}$ & 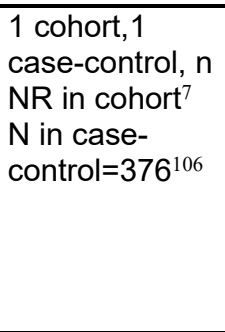 & $\begin{array}{l}\text { Serious study } \\
\text { limitations (high } \\
\text { risk of bias }{ }^{7} \text { ) } \\
\text { imprecise (wide } \\
\text { Cls spanning } \\
\text { the null), } \\
\text { consistency } \\
\text { unknown }\end{array}$ & Insufficient \\
\hline
\end{tabular}

$\overline{\mathrm{AOR}}=$ adjusted odds ratio; $\mathrm{ARR}=$ adjusted risk ratio; $\mathrm{CI}=$ confidence interval; $\mathrm{n}=$ number; $\mathrm{vs} .=$ versus. 


\section{Mirtazapine}

\section{Overview}

- The evidence is insufficient to judge the risk of harms for mirtazapine exposure during pregnancy for maternal (postpartum hemorrhage or preeclampsia) or child outcomes (preterm birth, low birth weight, NICU stay, autism spectrum disorder).

\section{Detailed Results}

Four publications reported on mirtazapine. ${ }^{56,74,75,95}$ These three publications drew from two cohorts (two publications from the U.S. Medicaid Analytic eXtract,${ }^{74,75}$ one from the Swedish Birth Cohort, ${ }^{56}$ and one from a cohort based in Turkey. ${ }^{95}$ All were nonrandomized studies and were rated as having some risk of bias.

All publications focused on exposure during pregnancy. Publications compared pregnant women exposed to mirtazapine with women with untreated depression, ${ }^{75}$ untreated mood disorder or anxiety, ${ }^{74}$ unexposed to antidepressants, ${ }^{56}$ or unmedicated psychiatric disorder. ${ }^{95}$

The evidence for mirtazapine is insufficient to judge the risk of harms for maternal (postpartum hemorrhage preeclampsia) or child outcomes (preterm birth, low birth weight, NICU stay, autism spectrum disorder) (Table B-31). When the referent group was restricted to women with more than one psychiatric diagnosis, the point estimate for relative risk for clomipramine exposure moved from 1.55 ( $\geq 1$ disorder with CIs spanning the null) to 0.99 ( $\geq 3$ disorders also with CIs spanning the null). ${ }^{56}$ This adjustment for confounding further suggests that the incidence of autism cannot be attributed to exposure to mirtazapine.

Table B-31. Strength of evidence for harms: Mirtazapine versus no treatment

\begin{tabular}{|c|c|c|c|c|c|c|c|}
\hline Population & $\begin{array}{l}\text { Intervention } \\
\text { and } \\
\text { Comparator }\end{array}$ & Outcome & $\begin{array}{l}\text { Incidence or } \\
\text { Mean Effect } \\
\text { by Arm }\end{array}$ & Results & $\begin{array}{l}\text { Study } \\
\text { Design } \\
\text { and } \\
\text { Sample } \\
\text { Size }\end{array}$ & $\begin{array}{l}\text { Factors That } \\
\text { Affect the } \\
\text { Strength of } \\
\text { Evidence }\end{array}$ & $\begin{array}{l}\text { Overall } \\
\text { Evidence } \\
\text { Strength } \\
\text { (Direction of } \\
\text { Effect) }\end{array}$ \\
\hline $\begin{array}{l}\text { Women with } \\
\text { mood } \\
\text { disorder or } \\
\text { anxiety }\end{array}$ & $\begin{array}{l}\text { Mirtazapine } \\
\text { exposure during } \\
\text { pregnancy } \\
\text { (current vs. } \\
\text { unexposed } \\
\text { women with } \\
\text { mood disorder } \\
\text { or anxiety (no } \\
\text { medication in } 5 \\
\text { months prior to } \\
\text { delivery) }\end{array}$ & $\begin{array}{l}\text { Postpartum } \\
\text { hemorrhage }\end{array}$ & $\begin{array}{l}\text { NR/129 (NR\%) } \\
\text { vs. } \\
1,896 / 69,044 \\
(2.7 \%)^{74}\end{array}$ & $\begin{array}{l}\text { ARR, } 0.87(95 \% \\
\text { Cl, } 0.29 \text { to } \\
2.66)^{74}\end{array}$ & $\begin{array}{l}1 \text { cohort, } \\
\mathrm{n}=69,173^{74}\end{array}$ & $\begin{array}{l}\text { Moderate } \\
\text { study } \\
\text { limitations, } \\
\text { imprecision } \\
\text { (wide Cls), } \\
\text { consistency } \\
\text { unknown }\end{array}$ & Insufficient \\
\hline $\begin{array}{l}\text { Women with } \\
\text { mood } \\
\text { disorder or } \\
\text { anxiety }\end{array}$ & $\begin{array}{l}\text { Mirtazapine } \\
\text { exposure during } \\
\text { pregnancy } \\
\text { (recent, 1-30 } \\
\text { days prior to } \\
\text { delivery) vs. } \\
\text { unexposed } \\
\text { women with } \\
\text { mood disorder } \\
\text { or anxiety (no } \\
\text { medication in } 5 \\
\text { months prior to } \\
\text { delivery) }\end{array}$ & $\begin{array}{l}\text { Postpartum } \\
\text { hemorrhage }\end{array}$ & $\begin{array}{l}0 / 57(0 \%) \text { vs. } \\
1,896 / 69,044 \\
(2.7 \%)^{74}\end{array}$ & ARR, NA ${ }^{74}$ & $\begin{array}{l}1 \text { cohort, } \\
\mathrm{n}=69,101^{74}\end{array}$ & $\begin{array}{l}\text { Moderate } \\
\text { study } \\
\text { limitations, } \\
\text { likely } \\
\text { imprecision } \\
\text { (few treatment } \\
\text { cases and no } \\
\text { events), } \\
\text { consistency } \\
\text { unknown }\end{array}$ & Insufficient \\
\hline
\end{tabular}




\begin{tabular}{|c|c|c|c|c|c|c|c|}
\hline Population & $\begin{array}{l}\text { Intervention } \\
\text { and } \\
\text { Comparator }\end{array}$ & Outcome & $\begin{array}{l}\text { Incidence or } \\
\text { Mean Effect } \\
\text { by Arm }\end{array}$ & Results & $\begin{array}{l}\text { Study } \\
\text { Design } \\
\text { and } \\
\text { Sample } \\
\text { Size } \\
\end{array}$ & $\begin{array}{l}\text { Factors That } \\
\text { Affect the } \\
\text { Strength of } \\
\text { Evidence }\end{array}$ & $\begin{array}{l}\text { Overall } \\
\text { Evidence } \\
\text { Strength } \\
\text { (Direction of } \\
\text { Effect) }\end{array}$ \\
\hline $\begin{array}{l}\text { Women with } \\
\text { mood } \\
\text { disorder or } \\
\text { anxiety }\end{array}$ & $\begin{array}{l}\text { Mirtazapine } \\
\text { exposure during } \\
\text { pregnancy (past } \\
\text { exposure 1-5 } \\
\text { months prior to } \\
\text { delivery) vs. } \\
\text { unexposed } \\
\text { women with } \\
\text { mood disorder } \\
\text { or anxiety (no } \\
\text { medication in } 5 \\
\text { months prior to } \\
\text { delivery) }\end{array}$ & $\begin{array}{l}\text { Postpartum } \\
\text { hemorrhage }\end{array}$ & $\begin{array}{l}\text { NR/135 (NR\%) } \\
\text { vs. } \\
1,896 / 69,044 \\
(2.7 \%)^{74}\end{array}$ & $\begin{array}{l}\text { ARR, } 1.07(95 \% \\
\mathrm{Cl}, 0.4 \text { to } 2.82)^{74}\end{array}$ & $\begin{array}{l}1 \text { cohort, } \\
\mathrm{n}=69,179^{74}\end{array}$ & $\begin{array}{l}\text { Moderate } \\
\text { study } \\
\text { limitations, } \\
\text { imprecision } \\
\text { (wide Cls), } \\
\text { consistency } \\
\text { unknown }\end{array}$ & Insufficient \\
\hline $\begin{array}{l}\text { Depressed } \\
\text { women }\end{array}$ & $\begin{array}{l}\text { Mirtazapine } \\
\text { exposure during } \\
\text { pregnancy vs. } \\
\text { unexposed } \\
\text { women with } \\
\text { depression }\end{array}$ & Preeclampsia & $\begin{array}{l}14 / 253(5.5 \%) \\
\text { vs. } \\
3,215 / 59,219 \\
(5.4 \%)^{75}\end{array}$ & $\begin{array}{l}\text { ARR, } 0.81(95 \% \\
\mathrm{Cl}, 0.5 \text { to } 1.34)^{75}\end{array}$ & $\begin{array}{l}1 \text { cohort, } \\
n=59,472^{75}\end{array}$ & $\begin{array}{l}\text { Moderate } \\
\text { study } \\
\text { limitations, } \\
\text { imprecision } \\
\text { (wide Cls), } \\
\text { consistency } \\
\text { unknown }\end{array}$ & Insufficient \\
\hline $\begin{array}{l}\text { Women with } \\
\text { psychiatric } \\
\text { diagnosis }\end{array}$ & $\begin{array}{l}\text { Mirtazapine } \\
\text { exposure during } \\
\text { pregnancy vs. } \\
\text { unmedicated } \\
\text { psychiatric } \\
\text { diagnosis }\end{array}$ & Preterm birth & $1 / 15$ vs. $3 / 19^{95}$ & $N R, p=0.767^{95}$ & $\begin{array}{l}1 \text { cohort, } \\
n=3,439^{95}\end{array}$ & $\begin{array}{l}\text { High study } \\
\text { limitations } \\
\text { (high risk of } \\
\text { bias }^{95} \text { ), } \\
\text { imprecision } \\
\text { (wide Cls), } \\
\text { consistency } \\
\text { unknown }\end{array}$ & Insufficient \\
\hline $\begin{array}{l}\text { Women with } \\
\text { psychiatric } \\
\text { diagnosis }\end{array}$ & $\begin{array}{l}\text { Mirtazapine } \\
\text { exposure during } \\
\text { pregnancy vs. } \\
\text { unmedicated } \\
\text { psychiatric } \\
\text { diagnosis }\end{array}$ & $\begin{array}{l}\text { Low birth } \\
\text { weight }\end{array}$ & $1 / 15$ vs. $3 / 19^{95}$ & $\mathrm{NR}, \mathrm{p}=0.767^{95}$ & $\begin{array}{l}1 \text { cohort, } \\
n=34^{95}\end{array}$ & $\begin{array}{l}\text { High study } \\
\text { limitations } \\
\text { (high risk of } \\
\text { bias }^{95} \text { ), } \\
\text { imprecision } \\
\text { (wide Cls), } \\
\text { consistency } \\
\text { unknown }\end{array}$ & Insufficient \\
\hline $\begin{array}{l}\text { Women with } \\
\text { psychiatric } \\
\text { diagnosis }\end{array}$ & $\begin{array}{l}\text { Mirtazapine } \\
\text { exposure during } \\
\text { pregnancy vs. } \\
\text { unmedicated } \\
\text { psychiatric } \\
\text { diagnosis }\end{array}$ & NICU stay & $2 / 15$ vs. $3 / 19^{95}$ & $N R, p=0.619^{95}$ & $\begin{array}{l}1 \text { cohort, } \\
n=3,439^{95}\end{array}$ & $\begin{array}{l}\text { High study } \\
\text { limitations } \\
\text { (high risk of } \\
\text { bias }^{95} \text { ), } \\
\text { imprecision } \\
\text { (wide Cls), } \\
\text { consistency } \\
\text { unknown }\end{array}$ & Insufficient \\
\hline
\end{tabular}




\begin{tabular}{|c|c|c|c|c|c|c|c|}
\hline Population & $\begin{array}{l}\text { Intervention } \\
\text { and } \\
\text { Comparator }\end{array}$ & Outcome & $\begin{array}{l}\text { Incidence or } \\
\text { Mean Effect } \\
\text { by Arm }\end{array}$ & Results & $\begin{array}{l}\text { Study } \\
\text { Design } \\
\text { and } \\
\text { Sample } \\
\text { Size } \\
\end{array}$ & $\begin{array}{l}\text { Factors That } \\
\text { Affect the } \\
\text { Strength of } \\
\text { Evidence }\end{array}$ & $\begin{array}{l}\text { Overall } \\
\text { Evidence } \\
\text { Strength } \\
\text { (Direction of } \\
\text { Effect) }\end{array}$ \\
\hline $\begin{array}{l}\text { Women } \\
\text { exposed to } \\
\text { antidepres- } \\
\text { sants }\end{array}$ & $\begin{array}{l}\text { Mirtazapine } \\
\text { exposure during } \\
\text { pregnancy vs. } \\
\text { unexposed } \\
\text { women with } \\
\text { prior } \\
\text { antidepressant } \\
\text { prescription }\end{array}$ & $\begin{array}{l}\text { Autism } \\
\text { spectrum } \\
\text { disorder }\end{array}$ & $\begin{array}{l}\text { NR/625 (NR) } \\
\text { vs. NR/24,285 } \\
\text { (NR) }^{56} \\
\geq 1 \text { psychiatric } \\
\text { disorder: ARR, } \\
1.55(95 \% \mathrm{Cl} \text {, } \\
0.39 \text { to } 6.29)^{56} \\
\geq 2 \text { psychiatric } \\
\text { disorders: } \\
\text { ARR, } 1.24 \\
(95 \% \mathrm{Cl}, 0.30 \\
\text { to } 5.06)^{56} \\
\geq 3 \text { psychiatric } \\
\text { disorders: } \\
\text { ARR, } 0.99 \\
(95 \% \mathrm{Cl}, 0.24 \\
\text { to } 4.09)^{56}\end{array}$ & $\begin{array}{l}\text { ARR, } 1.55(95 \% \\
\mathrm{Cl}, 0.39 \text { to } \\
6.29)^{56}\end{array}$ & $\begin{array}{l}1 \text { cohort, } \\
\mathrm{n}=24,347^{56}\end{array}$ & $\begin{array}{l}\text { Moderate } \\
\text { study } \\
\text { limitations, } \\
\text { imprecision } \\
\text { (wide Cls), } \\
\text { consistency } \\
\text { unknown }\end{array}$ & Insufficient \\
\hline
\end{tabular}

\section{Mood Stabilizers}

\section{Overview}

- The evidence for mood stabilizer exposure during pregnancy (mood stabilizers overall, lamotrigine, valproate, topiramate, carbamazepine, oxcarbazepine, lithium) is insufficient to judge the risk of harms for maternal (gestational diabetes, preeclampsia, placental abruption, preterm birth) and child outcomes (small for gestational age, IQ).

\section{Detailed Results}

Three studies reported on mood stabilizers. ${ }^{47,96,97}$ One publication with moderate risk of bias presented results for mood stabilizer monotherapy as a drug class and for individual drugs (lamotrigine, valproate, topiramate, carbamazepine, oxcarbazepine, and lithium) across a range of disorders, including bipolar disorder, migraine, epilepsy, and neuropathic pain, drawing from a cohort of deliveries in the Medicaid Analytic eXtract database. ${ }^{96}$ We requested and received subgroup results for women with a diagnosis of bipolar disorder through personal communication. A second moderate risk-of-bias study reported for lithium and antipsychotics on gestational diabetes in two Australian hospitals. ${ }^{97}$ Additionally, one high risk-of-bias publication, focusing on lithium, ${ }^{47}$ drew from a cohort in Sweden. The publication compared women exposed to mood stabilizers with women who had no exposure to mood stabilizers during pregnancy. ${ }^{47}$ These results are insufficient to judge the risk of maternal (gestational diabetes, preeclampsia, placental abruption, preterm birth) and child (small for gestational age, IQ) outcomes from mood stabilizer therapy or lithium exposure (Table B-32). 
Table B-32. Strength of evidence for harms: Mood stabilizers versus no treatment

\begin{tabular}{|c|c|c|c|c|c|c|c|}
\hline Population & $\begin{array}{l}\text { Intervention } \\
\text { and } \\
\text { Comparator }\end{array}$ & Outcome & $\begin{array}{l}\text { Incidence } \\
\text { or Mean } \\
\text { Effect by } \\
\text { Arm }\end{array}$ & Results & $\begin{array}{l}\text { Study } \\
\text { Design and } \\
\text { Sample Size }\end{array}$ & $\begin{array}{l}\text { Factors That } \\
\text { Affect the } \\
\text { Strength of } \\
\text { Evidence }\end{array}$ & $\begin{array}{l}\text { Overall } \\
\text { Evidence } \\
\text { Strength } \\
\text { (Direction of } \\
\text { Effect) } \\
\end{array}$ \\
\hline $\begin{array}{l}\text { Pregnant } \\
\text { women with } \\
\text { bipolar } \\
\text { disorder }\end{array}$ & $\begin{array}{l}\text { Mood } \\
\text { stabilizer } \\
\text { monotherapy } \\
\text { vs. no } \\
\text { exposure to } \\
\text { mood } \\
\text { stabilizers } \\
\end{array}$ & Preeclampsia & NR & $\begin{array}{l}\text { ARR, } 0.95(95 \% \\
\mathrm{Cl}, 0.79 \text { to } 1.15) \\
\text { (personal } \\
\text { communication } \\
\text { with author }{ }^{96} \text { ) }\end{array}$ & $\begin{array}{l}1 \text { cohort, N } \\
\text { NR }\end{array}$ & $\begin{array}{l}\text { Moderate study } \\
\text { limitations, likely } \\
\text { imprecise (wide } \\
\text { Cls, likely small } \\
\text { sample size), } \\
\text { consistency } \\
\text { unknown }\end{array}$ & Insufficient \\
\hline $\begin{array}{l}\text { Pregnant } \\
\text { women with } \\
\text { bipolar } \\
\text { disorder }\end{array}$ & $\begin{array}{l}\text { Mood } \\
\text { stabilizer } \\
\text { monotherapy } \\
\text { vs. no } \\
\text { exposure to } \\
\text { mood } \\
\text { stabilizers } \\
\end{array}$ & $\begin{array}{l}\text { Placental } \\
\text { abruption }\end{array}$ & NR & $\begin{array}{l}\text { ARR, } 1.32(95 \% \\
\mathrm{Cl}, 1.01 \text { to } 1.73) \\
\text { (personal } \\
\text { communication } \\
\text { with author }{ }^{96} \text { ) }\end{array}$ & $\begin{array}{l}1 \text { cohort, } \mathrm{N} \\
\text { NR }\end{array}$ & $\begin{array}{l}\text { Moderate study } \\
\text { limitations, likely } \\
\text { imprecise (wide } \\
\text { Cls, likely small } \\
\text { sample size), } \\
\text { consistency } \\
\text { unknown }\end{array}$ & Insufficient \\
\hline $\begin{array}{l}\text { Pregnant } \\
\text { women with } \\
\text { bipolar } \\
\text { disorder }\end{array}$ & $\begin{array}{l}\text { Mood } \\
\text { stabilizer } \\
\text { monotherapy } \\
\text { vs. no } \\
\text { exposure to } \\
\text { mood } \\
\text { stabilizers } \\
\end{array}$ & $\begin{array}{l}\text { Small for } \\
\text { gestational age }\end{array}$ & NR & $\begin{array}{l}\text { ARR, } 0.80(95 \% \\
\mathrm{Cl}, 0.65 \text { to } 0.97) \\
\text { (personal } \\
\text { communication } \\
\text { with author }{ }^{96} \text { ) }\end{array}$ & $\begin{array}{l}1 \text { cohort, N } \\
\text { NR }\end{array}$ & $\begin{array}{l}\text { Moderate study } \\
\text { limitations, likely } \\
\text { imprecise (wide } \\
\text { Cls, likely small } \\
\text { sample size), } \\
\text { consistency } \\
\text { unknown }\end{array}$ & Insufficient \\
\hline $\begin{array}{l}\text { Pregnant } \\
\text { women with } \\
\text { bipolar } \\
\text { disorder }\end{array}$ & $\begin{array}{l}\text { Mood } \\
\text { stabilizer } \\
\text { monotherapy } \\
\text { vs. no } \\
\text { exposure to } \\
\text { mood } \\
\text { stabilizers } \\
\end{array}$ & Preterm birth & NR & $\begin{array}{l}\text { ARR, } 0.95(95 \% \\
\mathrm{Cl}, 0.87 \text { to } 1.04) \\
\text { (personal } \\
\text { communication } \\
\text { with author }^{96} \text { ) }\end{array}$ & $\begin{array}{l}1 \text { cohort, N } \\
\text { NR }\end{array}$ & $\begin{array}{l}\text { Moderate study } \\
\text { limitations, likely } \\
\text { imprecise (wide } \\
\text { Cls, likely small } \\
\text { sample size), } \\
\text { consistency } \\
\text { unknown }\end{array}$ & Insufficient \\
\hline $\begin{array}{l}\text { Lamotrigine- } \\
\text { exposed } \\
\text { women }\end{array}$ & $\begin{array}{l}\text { Lamotrigine } \\
\text { exposure } \\
\text { during any } \\
\text { trimester of } \\
\text { pregnancy } \\
\text { vs. no } \\
\text { exposure } \\
\end{array}$ & $\begin{array}{l}\text { Gestational } \\
\text { diabetes } \\
\text { mellitus }\end{array}$ & $\begin{array}{l}10.5 \% \text { vs. } \\
8.8 \% 97\end{array}$ & $\begin{array}{l}\mathrm{RR}, 1.19(0.30 \text { to } \\
4.79)^{97}\end{array}$ & $\begin{array}{l}1 \text { cohort } \\
n<539^{97}\end{array}$ & $\begin{array}{l}\text { Moderate study } \\
\text { limitations, } \\
\text { imprecise (wide } \\
\text { Cls, small N) } \\
\text { consistency } \\
\text { unknown }\end{array}$ & \\
\hline $\begin{array}{l}\text { Pregnant } \\
\text { women with } \\
\text { bipolar } \\
\text { disorder }\end{array}$ & $\begin{array}{l}\text { Lamotrigine } \\
\text { vs. no } \\
\text { exposure to } \\
\text { mood } \\
\text { stabilizers }\end{array}$ & Preeclampsia & NR & $\begin{array}{l}\text { ARR, } 0.86(95 \% \\
\mathrm{Cl}, 0.63 \text { to } 1.16) \\
\text { (personal } \\
\text { communication } \\
\text { with author }^{96} \text { ) }\end{array}$ & $\begin{array}{l}1 \text { cohort, N } \\
\text { NR }\end{array}$ & $\begin{array}{l}\text { Moderate study } \\
\text { limitations, likely } \\
\text { imprecise (wide } \\
\text { Cls, likely small } \\
\text { sample size), } \\
\text { consistency } \\
\text { unknown }\end{array}$ & Insufficient \\
\hline $\begin{array}{l}\text { Pregnant } \\
\text { women with } \\
\text { bipolar } \\
\text { disorder }\end{array}$ & $\begin{array}{l}\text { Lamotrigine } \\
\text { vs. no } \\
\text { exposure to } \\
\text { mood } \\
\text { stabilizers }\end{array}$ & $\begin{array}{l}\text { Placental } \\
\text { abruption }\end{array}$ & NR & $\begin{array}{l}\text { ARR, } 1.28(95 \% \\
\mathrm{Cl}, 0.84 \text { to } 1.95) \\
\text { (personal } \\
\text { communication } \\
\text { with author }^{96} \text { ) }\end{array}$ & $\begin{array}{l}1 \text { cohort, N } \\
\text { NR }\end{array}$ & $\begin{array}{l}\text { Moderate study } \\
\text { limitations, likely } \\
\text { imprecise (wide } \\
\text { Cls, likely small } \\
\text { sample size), } \\
\text { consistency } \\
\text { unknown }\end{array}$ & Insufficient \\
\hline
\end{tabular}




\begin{tabular}{|c|c|c|c|c|c|c|c|}
\hline Population & $\begin{array}{l}\text { Intervention } \\
\text { and } \\
\text { Comparator }\end{array}$ & Outcome & $\begin{array}{l}\text { Incidence } \\
\text { or Mean } \\
\text { Effect by } \\
\text { Arm }\end{array}$ & Results & $\begin{array}{l}\text { Study } \\
\text { Design and } \\
\text { Sample Size }\end{array}$ & $\begin{array}{l}\text { Factors That } \\
\text { Affect the } \\
\text { Strength of } \\
\text { Evidence }\end{array}$ & $\begin{array}{l}\text { Overall } \\
\text { Evidence } \\
\text { Strength } \\
\text { (Direction of } \\
\text { Effect) }\end{array}$ \\
\hline $\begin{array}{l}\text { Pregnant } \\
\text { women with } \\
\text { bipolar } \\
\text { disorder }\end{array}$ & $\begin{array}{l}\text { Lamotrigine } \\
\text { vs. no } \\
\text { exposure to } \\
\text { mood } \\
\text { stabilizers }\end{array}$ & $\begin{array}{l}\text { Small for } \\
\text { gestational age }\end{array}$ & NR & $\begin{array}{l}\text { ARR, } 0.78(95 \% \\
\mathrm{CI}, 0.58 \text { to } 1.07) \\
\text { (personal } \\
\text { communication } \\
\text { with author }{ }^{96} \text { ) }\end{array}$ & $\begin{array}{l}1 \text { cohort, N } \\
\text { NR } 96\end{array}$ & $\begin{array}{l}\text { Moderate study } \\
\text { limitations, likely } \\
\text { imprecise (wide } \\
\text { Cls, likely small } \\
\text { sample size), } \\
\text { consistency } \\
\text { unknown }\end{array}$ & Insufficient \\
\hline $\begin{array}{l}\text { Pregnant } \\
\text { women with } \\
\text { bipolar } \\
\text { disorder }\end{array}$ & $\begin{array}{l}\text { Lamotrigine } \\
\text { vs. no } \\
\text { exposure to } \\
\text { mood } \\
\text { stabilizers }\end{array}$ & Preterm birth & NR & $\begin{array}{l}\text { ARR, } 0.97(95 \% \\
\mathrm{Cl}, 0.84 \text { to } 1.13) \\
\text { (personal } \\
\text { communication } \\
\text { with author }^{96} \text { ) }\end{array}$ & $\begin{array}{l}1 \text { cohort, N } \\
\text { NR }\end{array}$ & $\begin{array}{l}\text { Moderate study } \\
\text { limitations, likely } \\
\text { imprecise (wide } \\
\text { Cls, likely small } \\
\text { sample size), } \\
\text { consistency } \\
\text { unknown }\end{array}$ & Insufficient \\
\hline $\begin{array}{l}\text { Pregnant } \\
\text { women with } \\
\text { bipolar } \\
\text { disorder }\end{array}$ & $\begin{array}{l}\text { Valproate vs. } \\
\text { no exposure } \\
\text { to mood } \\
\text { stabilizers }\end{array}$ & Preeclampsia & NR & $\begin{array}{l}\text { ARR, } 1.01(95 \% \\
\mathrm{Cl}, 0.74 \text { to } 1.37) \\
\text { (personal } \\
\text { communication } \\
\text { with author }^{96} \text { ) }\end{array}$ & $\begin{array}{l}1 \text { cohort, N } \\
\text { NR }\end{array}$ & $\begin{array}{l}\text { Moderate study } \\
\text { limitations, likely } \\
\text { imprecise (wide } \\
\text { Cls, likely small } \\
\text { sample size), } \\
\text { consistency } \\
\text { unknown }\end{array}$ & Insufficient \\
\hline $\begin{array}{l}\text { Pregnant } \\
\text { women with } \\
\text { bipolar } \\
\text { disorder }\end{array}$ & $\begin{array}{l}\text { Valproate vs. } \\
\text { no exposure } \\
\text { to mood } \\
\text { stabilizers }\end{array}$ & $\begin{array}{l}\text { Placental } \\
\text { abruption }\end{array}$ & NR & $\begin{array}{l}\text { ARR, } 1.67(95 \% \\
\mathrm{CI}, 1.11 \text { to } 2.52) \\
\text { (personal } \\
\text { communication } \\
\text { with author }\end{array}$ & $\begin{array}{l}1 \text { cohort, N } \\
\text { NR }\end{array}$ & $\begin{array}{l}\text { Moderate study } \\
\text { limitations, likely } \\
\text { imprecise (wide } \\
\text { Cls, likely small } \\
\text { sample size), } \\
\text { consistency } \\
\text { unknown }\end{array}$ & Insufficient \\
\hline $\begin{array}{l}\text { Pregnant } \\
\text { women with } \\
\text { bipolar } \\
\text { disorder }\end{array}$ & $\begin{array}{l}\text { Valproate vs. } \\
\text { no exposure } \\
\text { to mood } \\
\text { stabilizers }\end{array}$ & $\begin{array}{l}\text { Small for } \\
\text { gestational age }\end{array}$ & NR & $\begin{array}{l}\text { ARR, } 0.70(95 \% \\
\mathrm{CI}, 0.49 \text { to } 1.00) \\
\text { (personal } \\
\text { communication } \\
\text { with author }^{96} \text { ) }\end{array}$ & $\begin{array}{l}1 \text { cohort, N } \\
\text { NR }\end{array}$ & $\begin{array}{l}\text { Moderate study } \\
\text { limitations, likely } \\
\text { imprecise (wide } \\
\text { Cls, likely small } \\
\text { sample size), } \\
\text { consistency } \\
\text { unknown }\end{array}$ & Insufficient \\
\hline $\begin{array}{l}\text { Pregnant } \\
\text { women with } \\
\text { bipolar } \\
\text { disorder }\end{array}$ & $\begin{array}{l}\text { Valproate vs. } \\
\text { no exposure } \\
\text { to mood } \\
\text { stabilizers }\end{array}$ & Preterm birth & NR & $\begin{array}{l}\text { ARR, } 1.06(95 \% \\
\mathrm{Cl}, 0.92 \text { to } 1.23) \\
\text { (personal } \\
\text { communication } \\
\text { with author }{ }^{96} \text { ) }\end{array}$ & $\begin{array}{l}1 \text { cohort, N } \\
\text { NR }\end{array}$ & $\begin{array}{l}\text { Moderate study } \\
\text { limitations, likely } \\
\text { imprecise (wide } \\
\text { Cls, likely small } \\
\text { sample size), } \\
\text { consistency } \\
\text { unknown }\end{array}$ & Insufficient \\
\hline $\begin{array}{l}\text { Pregnant } \\
\text { women with } \\
\text { bipolar } \\
\text { disorder }\end{array}$ & $\begin{array}{l}\text { Topiramate } \\
\text { vs. no } \\
\text { exposure to } \\
\text { mood } \\
\text { stabilizers }\end{array}$ & Preeclampsia & NR & $\begin{array}{l}\text { ARR, } 1.39(95 \% \\
\mathrm{Cl}, 0.94 \text { to } 2.06) \\
\text { (personal } \\
\text { communication } \\
\text { with author }{ }^{96} \text { ) }\end{array}$ & $\begin{array}{l}1 \text { cohort, N } \\
\text { NR }\end{array}$ & $\begin{array}{l}\text { Moderate study } \\
\text { limitations, likely } \\
\text { imprecise (wide } \\
\text { Cls, likely small } \\
\text { sample size), } \\
\text { consistency } \\
\text { unknown }\end{array}$ & Insufficient \\
\hline $\begin{array}{l}\text { Pregnant } \\
\text { women with } \\
\text { bipolar } \\
\text { disorder }\end{array}$ & $\begin{array}{l}\text { Topiramate } \\
\text { vs. no } \\
\text { exposure to } \\
\text { mood } \\
\text { stabilizers }\end{array}$ & $\begin{array}{l}\text { Placental } \\
\text { abruption }\end{array}$ & NR & $\begin{array}{l}\text { ARR, } 1.29(95 \% \\
\mathrm{Cl}, 0.67 \text { to } 2.50) \\
\text { (personal } \\
\text { communication } \\
\text { with author }^{96} \text { ) }\end{array}$ & $\begin{array}{l}1 \text { cohort, N } \\
\text { NR }\end{array}$ & $\begin{array}{l}\text { Moderate study } \\
\text { limitations, likely } \\
\text { imprecise (wide } \\
\text { Cls, likely small } \\
\text { sample size), } \\
\text { consistency } \\
\text { unknown }\end{array}$ & Insufficient \\
\hline
\end{tabular}




\begin{tabular}{|c|c|c|c|c|c|c|c|}
\hline Population & $\begin{array}{l}\text { Intervention } \\
\text { and } \\
\text { Comparator }\end{array}$ & Outcome & $\begin{array}{l}\text { Incidence } \\
\text { or Mean } \\
\text { Effect by } \\
\text { Arm }\end{array}$ & Results & $\begin{array}{l}\text { Study } \\
\text { Design and } \\
\text { Sample Size }\end{array}$ & $\begin{array}{l}\text { Factors That } \\
\text { Affect the } \\
\text { Strength of } \\
\text { Evidence }\end{array}$ & $\begin{array}{l}\text { Overall } \\
\text { Evidence } \\
\text { Strength } \\
\text { (Direction of } \\
\text { Effect) }\end{array}$ \\
\hline $\begin{array}{l}\text { Pregnant } \\
\text { women with } \\
\text { bipolar } \\
\text { disorder }\end{array}$ & $\begin{array}{l}\text { Topiramate } \\
\text { vs. no } \\
\text { exposure to } \\
\text { mood } \\
\text { stabilizers }\end{array}$ & $\begin{array}{l}\text { Small for } \\
\text { gestational age }\end{array}$ & NR & $\begin{array}{l}\text { ARR, } 0.58(95 \% \\
\mathrm{Cl}, 0.33 \text { to } 1.01) \\
\text { (personal } \\
\text { communication } \\
\text { with author }{ }^{96} \text { ) }\end{array}$ & $\begin{array}{l}1 \text { cohort, } \mathrm{N} \\
\text { NR }\end{array}$ & $\begin{array}{l}\text { Moderate study } \\
\text { limitations, likely } \\
\text { imprecise (wide } \\
\text { Cls, likely small } \\
\text { sample size), } \\
\text { consistency } \\
\text { unknown }\end{array}$ & Insufficient \\
\hline $\begin{array}{l}\text { Pregnant } \\
\text { women with } \\
\text { bipolar } \\
\text { disorder }\end{array}$ & $\begin{array}{l}\text { Topiramate } \\
\text { vs. no } \\
\text { exposure to } \\
\text { mood } \\
\text { stabilizers }\end{array}$ & Preterm birth & NR & $\begin{array}{l}\text { ARR, } 0.77(95 \% \\
\mathrm{Cl}, 0.59 \text { to } 1.00) \\
\text { (personal } \\
\text { communication } \\
\text { with author }^{96} \text { ) }\end{array}$ & $\begin{array}{l}1 \text { cohort, N } \\
\text { NR }\end{array}$ & $\begin{array}{l}\text { Moderate study } \\
\text { limitations, likely } \\
\text { imprecise (wide } \\
\text { Cls, likely small } \\
\text { sample size), } \\
\text { consistency } \\
\text { unknown }\end{array}$ & Insufficient \\
\hline $\begin{array}{l}\text { Pregnant } \\
\text { women with } \\
\text { bipolar } \\
\text { disorder }\end{array}$ & $\begin{array}{l}\text { Carbama- } \\
\text { zepine vs. no } \\
\text { exposure to } \\
\text { mood } \\
\text { stabilizers }\end{array}$ & Preeclampsia & NR & $\begin{array}{l}\text { ARR, } 1.16(95 \% \\
\mathrm{Cl}, 0.57 \text { to } 2.34) \\
\text { (personal } \\
\text { communication } \\
\text { with author }^{96} \text { ) }\end{array}$ & $\begin{array}{l}1 \text { cohort, N } \\
\text { NR }\end{array}$ & $\begin{array}{l}\text { Moderate study } \\
\text { limitations, likely } \\
\text { imprecise (wide } \\
\text { Cls, likely small } \\
\text { sample size), } \\
\text { consistency } \\
\text { unknown }\end{array}$ & Insufficient \\
\hline $\begin{array}{l}\text { Pregnant } \\
\text { women with } \\
\text { bipolar } \\
\text { disorder }\end{array}$ & $\begin{array}{l}\text { Carbama- } \\
\text { zepine vs. no } \\
\text { exposure to } \\
\text { mood } \\
\text { stabilizers }\end{array}$ & $\begin{array}{l}\text { Placental } \\
\text { abruption }\end{array}$ & NR & $\begin{array}{l}\text { ARR, } 1.18(95 \% \\
\mathrm{Cl}, 0.37 \text { to } 3.74) \\
\text { (personal } \\
\text { communication } \\
\text { with author }^{96} \text { ) }\end{array}$ & $\begin{array}{l}1 \text { cohort, N } \\
\text { NR }\end{array}$ & $\begin{array}{l}\text { Moderate study } \\
\text { limitations, likely } \\
\text { imprecise (wide } \\
\text { Cls, likely small } \\
\text { sample size), } \\
\text { consistency } \\
\text { unknown }\end{array}$ & Insufficient \\
\hline $\begin{array}{l}\text { Pregnant } \\
\text { women with } \\
\text { bipolar } \\
\text { disorder }\end{array}$ & $\begin{array}{l}\text { Carbama- } \\
\text { zepine vs. no } \\
\text { exposure to } \\
\text { mood } \\
\text { stabilizers }\end{array}$ & $\begin{array}{l}\text { Small for } \\
\text { gestational age }\end{array}$ & NR & $\begin{array}{l}\text { ARR, } 1.45(95 \% \\
\mathrm{Cl}, 0.76 \text { to } 2.77) \\
\text { (personal } \\
\text { communication } \\
\text { with author }^{96} \text { ) }\end{array}$ & $\begin{array}{l}1 \text { cohort, N } \\
\text { NR }\end{array}$ & $\begin{array}{l}\text { Moderate study } \\
\text { limitations, likely } \\
\text { imprecise (wide } \\
\text { Cls, likely small } \\
\text { sample size), } \\
\text { consistency } \\
\text { unknown }\end{array}$ & Insufficient \\
\hline $\begin{array}{l}\text { Pregnant } \\
\text { women with } \\
\text { bipolar } \\
\text { disorder }\end{array}$ & $\begin{array}{l}\text { Carbama- } \\
\text { zepine vs. no } \\
\text { exposure to } \\
\text { mood } \\
\text { stabilizers }\end{array}$ & Preterm birth & NR & $\begin{array}{l}\text { ARR, } 1.05(95 \% \\
\mathrm{Cl}, 0.74 \text { to } 1.48) \\
\text { (personal } \\
\text { communication } \\
\text { with author }^{96} \text { ) }\end{array}$ & $\begin{array}{l}1 \text { cohort, N } \\
\text { NR }\end{array}$ & $\begin{array}{l}\text { Moderate study } \\
\text { limitations, likely } \\
\text { imprecise (wide } \\
\text { Cls, likely small } \\
\text { sample size), } \\
\text { consistency } \\
\text { unknown }\end{array}$ & Insufficient \\
\hline $\begin{array}{l}\text { Pregnant } \\
\text { women with } \\
\text { bipolar } \\
\text { disorder }\end{array}$ & $\begin{array}{l}\text { Oxcarba- } \\
\text { zepine vs. no } \\
\text { exposure to } \\
\text { mood } \\
\text { stabilizers }\end{array}$ & Preeclampsia & NR & $\begin{array}{l}\text { ARR, } 0.95(95 \% \\
\mathrm{Cl}, 0.63 \text { to } 1.44) \\
\text { (personal } \\
\text { communication } \\
\text { with author }^{96} \text { ) }\end{array}$ & $\begin{array}{l}1 \text { cohort, N } \\
\text { NR }\end{array}$ & $\begin{array}{l}\text { Moderate study } \\
\text { limitations, likely } \\
\text { imprecise (wide } \\
\text { Cls, likely small } \\
\text { sample size), } \\
\text { consistency } \\
\text { unknown }\end{array}$ & Insufficient \\
\hline
\end{tabular}




\begin{tabular}{|c|c|c|c|c|c|c|c|}
\hline Population & $\begin{array}{l}\text { Intervention } \\
\text { and } \\
\text { Comparator }\end{array}$ & Outcome & $\begin{array}{l}\text { Incidence } \\
\text { or Mean } \\
\text { Effect by } \\
\text { Arm }\end{array}$ & Results & $\begin{array}{l}\text { Study } \\
\text { Design and } \\
\text { Sample Size }\end{array}$ & $\begin{array}{l}\text { Factors That } \\
\text { Affect the } \\
\text { Strength of } \\
\text { Evidence }\end{array}$ & $\begin{array}{l}\text { Overall } \\
\text { Evidence } \\
\text { Strength } \\
\text { (Direction of } \\
\text { Effect) }\end{array}$ \\
\hline $\begin{array}{l}\text { Pregnant } \\
\text { women with } \\
\text { bipolar } \\
\text { disorder }\end{array}$ & $\begin{array}{l}\text { Oxcarba- } \\
\text { zepine vs. no } \\
\text { exposure to } \\
\text { mood } \\
\text { stabilizers }\end{array}$ & $\begin{array}{l}\text { Placental } \\
\text { abruption }\end{array}$ & NR & $\begin{array}{l}\text { ARR, } 1.05(95 \% \\
\mathrm{CI}, 0.53 \text { to } 2.08) \\
\text { (personal } \\
\text { communication } \\
\text { with author }^{96} \text { ) }\end{array}$ & $\begin{array}{l}1 \text { cohort, N } \\
\text { NR }\end{array}$ & $\begin{array}{l}\text { Moderate study } \\
\text { limitations, likely } \\
\text { imprecise (wide } \\
\text { Cls, likely small } \\
\text { sample size), } \\
\text { consistency } \\
\text { unknown }\end{array}$ & Insufficient \\
\hline $\begin{array}{l}\text { Pregnant } \\
\text { women with } \\
\text { bipolar } \\
\text { disorder }\end{array}$ & $\begin{array}{l}\text { Oxcarba- } \\
\text { zepine vs. no } \\
\text { exposure to } \\
\text { mood } \\
\text { stabilizers }\end{array}$ & $\begin{array}{l}\text { Small for } \\
\text { gestational age }\end{array}$ & NR & $\begin{array}{l}\text { ARR, } 0.68(95 \% \\
\mathrm{CI}, 0.42 \text { to } 1.11) \\
\text { (personal } \\
\text { communication } \\
\text { with author }^{96} \text { ) }\end{array}$ & $\begin{array}{l}1 \text { cohort, N } \\
\text { NR }\end{array}$ & $\begin{array}{l}\text { Moderate study } \\
\text { limitations, likely } \\
\text { imprecise (wide } \\
\text { Cls, likely small } \\
\text { sample size), } \\
\text { consistency } \\
\text { unknown }\end{array}$ & Insufficient \\
\hline $\begin{array}{l}\text { Pregnant } \\
\text { women with } \\
\text { bipolar } \\
\text { disorder }\end{array}$ & $\begin{array}{l}\text { Oxcarba- } \\
\text { zepine vs. no } \\
\text { exposure to } \\
\text { mood } \\
\text { stabilizers }\end{array}$ & Preterm birth & NR & $\begin{array}{l}\text { ARR, } 0.97(95 \% \\
\mathrm{Cl}, 0.79 \text { to } 1.20) \\
\text { (personal } \\
\text { communication } \\
\text { with author }^{96} \text { ) }\end{array}$ & $\begin{array}{l}1 \text { cohort, N } \\
\text { NR }\end{array}$ & $\begin{array}{l}\text { Moderate study } \\
\text { limitations, likely } \\
\text { imprecise (wide } \\
\text { Cls, likely small } \\
\text { sample size), } \\
\text { consistency } \\
\text { unknown }\end{array}$ & Insufficient \\
\hline $\begin{array}{l}\text { Lithium- } \\
\text { exposed } \\
\text { women }\end{array}$ & $\begin{array}{l}\text { Lithium } \\
\text { exposure } \\
\text { during any } \\
\text { trimester of } \\
\text { pregnancy } \\
\text { vs. no } \\
\text { exposure }\end{array}$ & $\begin{array}{l}\text { Gestational } \\
\text { diabetes } \\
\text { mellitus }\end{array}$ & $\begin{array}{l}11.8 \% \text { vs. } \\
8.8 \%^{97}\end{array}$ & $\begin{array}{l}\text { RR, } 1.33 \text { (0.33 to } \\
5.31)^{97}\end{array}$ & $\begin{array}{l}1 \text { cohort } \\
\mathrm{n}<539^{97}\end{array}$ & $\begin{array}{l}\text { Moderate study } \\
\text { limitations, } \\
\text { imprecise (wide } \\
\text { Cls, small N) } \\
\text { consistency } \\
\text { unknown }\end{array}$ & Insufficient \\
\hline $\begin{array}{l}\text { Pregnant } \\
\text { women with } \\
\text { bipolar } \\
\text { disorder }\end{array}$ & $\begin{array}{l}\text { Lithium vs. } \\
\text { no exposure } \\
\text { to mood } \\
\text { stabilizers }\end{array}$ & Preeclampsia & NR & $\begin{array}{l}\text { ARR, } 0.79(95 \% \\
\mathrm{CI}, 0.51 \text { to } 1.23) \\
\text { (personal } \\
\text { communication } \\
\text { with author }^{96} \text { ) }\end{array}$ & $\begin{array}{l}1 \text { cohort, } N \\
N R, \leq 4153\end{array}$ & $\begin{array}{l}\text { Moderate study } \\
\text { limitations, likely } \\
\text { imprecise (wide } \\
\text { Cls, likely small } \\
\text { sample size), } \\
\text { consistency } \\
\text { unknown }\end{array}$ & Insufficient \\
\hline $\begin{array}{l}\text { Pregnant } \\
\text { women with } \\
\text { bipolar } \\
\text { disorder }\end{array}$ & $\begin{array}{l}\text { Lithium vs. } \\
\text { no exposure } \\
\text { to mood } \\
\text { stabilizers }\end{array}$ & $\begin{array}{l}\text { Placental } \\
\text { abruption }\end{array}$ & NR & $\begin{array}{l}\text { ARR, } 0.88(95 \% \\
\mathrm{Cl}, 0.46 \text { to } 1.66) \\
\text { (personal } \\
\text { communication } \\
\text { with author }{ }^{96} \text { ) }\end{array}$ & $\begin{array}{l}1 \text { cohort, } N \\
\text { NR }\end{array}$ & $\begin{array}{l}\text { Moderate study } \\
\text { limitations, likely } \\
\text { imprecise (wide } \\
\text { Cls, likely small } \\
\text { sample size), } \\
\text { consistency } \\
\text { unknown }\end{array}$ & Insufficient \\
\hline $\begin{array}{l}\text { Pregnant } \\
\text { women with } \\
\text { bipolar } \\
\text { disorder }\end{array}$ & $\begin{array}{l}\text { Lithium vs. } \\
\text { no exposure } \\
\text { to mood } \\
\text { stabilizers }\end{array}$ & $\begin{array}{l}\text { Small for } \\
\text { gestational age }\end{array}$ & NR & $\begin{array}{l}\text { ARR, } 0.95(95 \% \\
\mathrm{Cl}, 0.64 \text { to } 1.41) \\
\text { (personal } \\
\text { communication } \\
\text { with author }{ }^{96} \text { ) }\end{array}$ & $\begin{array}{l}1 \text { cohort, N } \\
\text { NR }\end{array}$ & $\begin{array}{l}\text { Moderate study } \\
\text { limitations, likely } \\
\text { imprecise (wide } \\
\text { Cls, likely small } \\
\text { sample size), } \\
\text { consistency } \\
\text { unknown }\end{array}$ & Insufficient \\
\hline $\begin{array}{l}\text { Pregnant } \\
\text { women with } \\
\text { bipolar } \\
\text { disorder }\end{array}$ & $\begin{array}{l}\text { Lithium vs. } \\
\text { no exposure } \\
\text { to mood } \\
\text { stabilizers }\end{array}$ & Preterm birth & NR & $\begin{array}{l}\text { ARR, } 0.83(95 \% \\
\mathrm{CI}, 0.67 \text { to } 1.02) \\
\text { (personal } \\
\text { communication } \\
\text { with author }^{96} \text { ) }\end{array}$ & $\begin{array}{l}1 \text { cohort, N } \\
\text { NR }\end{array}$ & $\begin{array}{l}\text { Moderate study } \\
\text { limitations, likely } \\
\text { imprecise (wide } \\
\text { Cls, likely small } \\
\text { sample size), } \\
\text { consistency } \\
\text { unknown }\end{array}$ & Insufficient \\
\hline
\end{tabular}




\begin{tabular}{|c|c|c|c|c|c|c|c|}
\hline Population & $\begin{array}{l}\text { Intervention } \\
\text { and } \\
\text { Comparator }\end{array}$ & Outcome & $\begin{array}{l}\text { Incidence } \\
\text { or Mean } \\
\text { Effect by } \\
\text { Arm }\end{array}$ & Results & $\begin{array}{l}\text { Study } \\
\text { Design and } \\
\text { Sample Size }\end{array}$ & $\begin{array}{l}\text { Factors That } \\
\text { Affect the } \\
\text { Strength of } \\
\text { Evidence }\end{array}$ & $\begin{array}{l}\text { Overall } \\
\text { Evidence } \\
\text { Strength } \\
\text { (Direction of } \\
\text { Effect) }\end{array}$ \\
\hline $\begin{array}{l}\text { Mood } \\
\text { disorders }\end{array}$ & $\begin{array}{l}\text { Lithium } \\
\text { exposure in } \\
\text { pregnancy } \\
\text { vs. } \\
\text { unexposed } \\
\text { women with } \\
\text { mood } \\
\text { disorders }\end{array}$ & $\begin{array}{l}\text { Child's full- } \\
\text { scale IQ at 4-5 } \\
\text { years from the } \\
\text { Wechsler } \\
\text { Preschool and } \\
\text { Primary Scale } \\
\text { of Intelligence }\end{array}$ & $\begin{array}{l}\text { Medians } \\
107.5 \text { vs. } \\
98^{47}\end{array}$ & $\begin{array}{l}\text { Regression } \\
\text { coefficient for no } \\
\text { lithium vs. lithium: } \\
-6.3, p=0.15^{47}\end{array}$ & $\begin{array}{l}1 \text { cohort, } \\
\mathrm{n}=27^{47}\end{array}$ & $\begin{array}{l}\text { High study } \\
\text { limitations (high } \\
\text { risk of bias }{ }^{47} \text { ), } \\
\text { seriously } \\
\text { imprecise (wide } \\
\text { Cls, small } \\
\text { sample size), } \\
\text { consistency } \\
\text { unknown }\end{array}$ & Insufficient \\
\hline
\end{tabular}

$\overline{\mathrm{CI}}=$ confidence interval; $\mathrm{n}=$ number; vs. $=$ versus.

\section{Antipsychotics}

\section{Overview}

- Evidence from one study with some risk-of-bias concerns suggests increased risk of gestational diabetes with continuing quetiapine or olanzapine compared with discontinuing either drug during pregnancy (low strength of evidence).

- Evidence from one study with some risk-of-bias concerns suggests increased risk of congenital anomalies with second-generation antipsychotics compared with no exposure to drugs for women with schizophrenia, bipolar disorder, or psychosis during pregnancy (low strength of evidence).

- The evidence for antipsychotics, when analyzed individually or as a class, is insufficient to judge the risk of harms for maternal (only diabetes is assessed, primarily gestational onset for second-generation antipsychotics overall, or other evaluated antipsychotics, specifically, aripiprazole, ziprasidone, and risperidone) or child outcomes (low birthweight, preterm birth, small for gestational age, large for gestational age, major congenital anomalies, or cardiac anomalies) for all evaluated antipsychotic exposure during pregnancy.

\section{Detailed Results}

Seven publications reported on antipsychotics; ${ }^{43,44,50,62,85,90,97}$ of these, four reported on antipsychotics as a class ( 1 compared second-generation antipsychotics to a control, ${ }^{50} 1$ compared antipsychotics associated with increased risks of nongestational type 2 diabetes mellitus to control, ${ }^{97}$ and 2 separately compared second-generation antipsychotics to a control and first-generation antipsychotics to a control ${ }^{62,85}$ ), and four focused on specific antipsychotics compared with control. ${ }^{43,44,62,90}$ These publications drew from four cohorts ( 3 from the National Pregnancy Registry for Atypical Antipsychotics, ${ }^{43,44,50} 2$ from the U.S. Medicaid Analytic eXtract, ${ }^{62,90} 1$ from the Taiwan National Health Insurance Research Database and birth certificate registry, ${ }^{85}$ and 1 from a cohort based in Australia. ${ }^{97}$ All were nonrandomized studies. Three were rated as high risk of bias, ${ }^{43,44,85}$ while four had some concerns. ${ }^{50,62,90,97}$

All publications focused on exposure during pregnancy; four limited their exposure to the first trimester. ${ }^{43,44,50,62}$ Publications compared pregnant women exposed to antipsychotics with those who discontinued an antipsychotic during the first half of pregnancy ${ }^{90}$ or at variably defined periods of exposure (before pregnancy or 4,6 , or 8 weeks before pregnancy) ${ }^{62}$ those with a psychiatric condition but no second-generation antipsychotic exposure (although they 
could be exposed to first-generation antipsychotics), ${ }^{79}$ or those with no psychiatric history and no receipt of an antipsychotic during pregnancy. ${ }^{85}$

The evidence for antipsychotics, when analyzed individually or as a class, is insufficient to judge the risk of harms for maternal (only diabetes is assessed, primarily gestational onset) or child outcomes (low birthweight, preterm birth, small for gestational age, or large for gestational age) (Table B-33). Most available evidence involves the newer second-generation antipsychotics.

Table B-33. Strength of evidence for harms: Antipsychotics versus no treatment

\begin{tabular}{|c|c|c|c|c|c|c|c|}
\hline Population & $\begin{array}{l}\text { Intervention } \\
\text { and } \\
\text { Comparator }\end{array}$ & Outcome & $\begin{array}{l}\text { Incidence or } \\
\text { Mean Effect by } \\
\text { Arm }\end{array}$ & Results & $\begin{array}{l}\text { Study Design } \\
\text { and Sample Size }\end{array}$ & $\begin{array}{l}\text { Factors That } \\
\text { Affect the } \\
\text { Strength of } \\
\text { Evidence }\end{array}$ & $\begin{array}{l}\text { Overall } \\
\text { Evidence } \\
\text { Strength } \\
\text { (Direction } \\
\text { of Effect) }\end{array}$ \\
\hline $\begin{array}{l}\text { Women with } \\
\text { a psychiatric } \\
\text { condition }\end{array}$ & $\begin{array}{l}\text { Second- } \\
\text { generation } \\
\text { antipsychotic } \\
\text { exposure in } \\
\text { first trimester } \\
\text { vs. unexposed } \\
\text { to second- } \\
\text { generation } \\
\text { antipsychotic }\end{array}$ & $\begin{array}{l}\text { All diabetes } \\
\text { (pre- } \\
\text { gestational } \\
\text { or } \\
\text { gestational) }\end{array}$ & $\begin{array}{l}40 / 310(12.9 \%) \\
\text { vs. } 16 / 149 \\
(10.7 \%)^{50}\end{array}$ & $\begin{array}{l}\text { AOR } \\
\text { (propensity } \\
\text { score): } 1.16 \\
(95 \% \mathrm{Cl}, 0.59 \\
\text { to } 2.28) ; \\
\text { AOR (logistic } \\
\text { regressions): } \\
0.9(95 \% \mathrm{Cl} \text {, } \\
0.44 \text { to } 1.91)^{50}\end{array}$ & 1 cohort, $n=459^{50}$ & $\begin{array}{l}\text { Moderate } \\
\text { study } \\
\text { limitations, } \\
\text { imprecise } \\
\text { (wide Cls), } \\
\text { consistency } \\
\text { unknown }\end{array}$ & Insufficient \\
\hline $\begin{array}{l}\text { Women with } \\
\text { a psychiatric } \\
\text { condition }\end{array}$ & $\begin{array}{l}\text { Second- } \\
\text { generation } \\
\text { antipsychotic } \\
\text { exposure in } \\
\text { first trimester } \\
\text { vs. unexposed } \\
\text { to second- } \\
\text { generation } \\
\text { antipsychotic } \\
\end{array}$ & $\begin{array}{l}\text { Gestational } \\
\text { diabetes }\end{array}$ & $\begin{array}{l}33 / 303(10.9 \%) \\
\text { vs. } 16 / 149 \\
(10.7 \%)^{50}\end{array}$ & $\begin{array}{l}\text { AOR } \\
\text { (propensity } \\
\text { score): } 0.79 \\
(95 \% \mathrm{Cl}, 0.40 \\
\text { to } 1.56) ; \\
\text { AOR (logistic } \\
\text { regressions): } \\
0.72(95 \% \mathrm{CI} \text {, } \\
0.34 \text { to } 1.53)^{50} \\
\end{array}$ & 1 cohort, $n=452^{50}$ & $\begin{array}{l}\text { Moderate } \\
\text { study } \\
\text { limitations, } \\
\text { imprecise } \\
\text { (wide Cls), } \\
\text { consistency } \\
\text { unknown }\end{array}$ & Insufficient \\
\hline $\begin{array}{l}\text { Women with } \\
\text { severe } \\
\text { mental illness }\end{array}$ & $\begin{array}{l}\text { Antipsychotics } \\
\text { associated with } \\
\text { increased risk } \\
\text { of } \\
\text { nongestational } \\
\text { type } 2 \text { diabetes } \\
\text { mellitus } \\
\text { (quetiapine } \\
\text { [high-dose], } \\
\text { olanzapine, } \\
\text { risperidone, or } \\
\text { clozapine) vs. } \\
\text { psychotic } \\
\text { disorders only }\end{array}$ & $\begin{array}{l}\text { Gestational } \\
\text { diabetes }\end{array}$ & NR & $\begin{array}{l}\text { ARR, } 4.39 \\
(95 \% \mathrm{Cl}, 1.8 \\
\text { to } 10.69)^{97}\end{array}$ & $\begin{array}{l}1 \text { cohort, } n=N R \text {, } \\
<539^{97}\end{array}$ & $\begin{array}{l}\text { Moderate } \\
\text { study } \\
\text { limitations, } \\
\text { imprecise } \\
\text { (wide Cls), } \\
\text { consistency } \\
\text { unknown } 97\end{array}$ & Insufficient \\
\hline $\begin{array}{l}\text { Women } \\
\text { prescribed an } \\
\text { second- } \\
\text { generation } \\
\text { antipsychotic }\end{array}$ & $\begin{array}{l}\text { Aripiprazole } \\
\text { continued vs. } \\
\text { aripiprazole } \\
\text { discontinued }\end{array}$ & $\begin{array}{l}\text { Gestational } \\
\text { diabetes }\end{array}$ & $\begin{array}{l}20 / 419(4.8 \%) \\
\text { vs. } 68 / 1,505 \\
(4.5 \%)^{90}\end{array}$ & $\begin{array}{l}\text { ARR, } 0.82 \\
(95 \% \mathrm{Cl}, 0.5 \\
\text { to } 1.33)^{90}\end{array}$ & 1 cohort, $n=924^{90}$ & $\begin{array}{l}\text { Moderate } \\
\text { study } \\
\text { limitations, } \\
\text { imprecise } \\
\text { (wide Cls), } \\
\text { consistency } \\
\text { unknown } \\
\end{array}$ & Insufficient \\
\hline $\begin{array}{l}\text { Women } \\
\text { prescribed an } \\
\text { second- } \\
\text { generation } \\
\text { antipsychotic }\end{array}$ & $\begin{array}{l}\text { Ziprasidone } \\
\text { continued vs. } \\
\text { ziprasidone } \\
\text { discontinued }\end{array}$ & $\begin{array}{l}\text { Gestational } \\
\text { diabetes }\end{array}$ & $\begin{array}{l}<11 / 167(<6.6 \%) \\
\text { vs. } 19 / 506 \\
(3.8 \%)^{90}\end{array}$ & $\begin{array}{l}\text { ARR, } 0.76 \\
(95 \% \mathrm{Cl}, 0.29 \\
\text { to } 2.0)^{90}\end{array}$ & 1 cohort, $n=673^{90}$ & $\begin{array}{l}\text { Moderate } \\
\text { study } \\
\text { limitations, } \\
\text { imprecise } \\
\text { (wide Cls), } \\
\text { consistency } \\
\text { unknown }\end{array}$ & Insufficient \\
\hline
\end{tabular}




\begin{tabular}{|c|c|c|c|c|c|c|c|}
\hline Population & $\begin{array}{l}\text { Intervention } \\
\text { and } \\
\text { Comparator }\end{array}$ & Outcome & $\begin{array}{l}\text { Incidence or } \\
\text { Mean Effect by } \\
\text { Arm }\end{array}$ & Results & $\begin{array}{l}\text { Study Design } \\
\text { and Sample Size }\end{array}$ & $\begin{array}{l}\text { Factors That } \\
\text { Affect the } \\
\text { Strength of } \\
\text { Evidence }\end{array}$ & $\begin{array}{l}\text { Overall } \\
\text { Evidence } \\
\text { Strength } \\
\text { (Direction } \\
\text { of Effect) }\end{array}$ \\
\hline $\begin{array}{l}\text { Women } \\
\text { prescribed an } \\
\text { second- } \\
\text { generation } \\
\text { antipsychotic }\end{array}$ & $\begin{array}{l}\text { Quetiapine } \\
\text { continued vs. } \\
\text { quetiapine } \\
\text { discontinued }\end{array}$ & $\begin{array}{l}\text { Gestational } \\
\text { diabetes }\end{array}$ & $\begin{array}{l}110 / 1543(7.1 \%) \\
\text { vs. } 122 / 2,990 \\
(4.1 \%)^{90}\end{array}$ & $\begin{array}{l}\text { ARR, } 1.28 \\
(95 \% \mathrm{Cl}, 1.01 \\
\text { to } 1.62)^{90}\end{array}$ & $\begin{array}{l}1 \text { cohort, } \\
n=4,533^{90}\end{array}$ & $\begin{array}{l}\text { Moderate } \\
\text { study } \\
\text { limitations, } \\
\text { precise, } \\
\text { consistency } \\
\text { unknown } \\
\end{array}$ & $\begin{array}{l}\text { Low for } \\
\text { harms of } \\
\text { continued } \\
\text { quetiapine }\end{array}$ \\
\hline $\begin{array}{l}\text { Women } \\
\text { prescribed an } \\
\text { second- } \\
\text { generation } \\
\text { antipsychotic }\end{array}$ & $\begin{array}{l}\text { Risperidone } \\
\text { continued vs. } \\
\text { risperidone } \\
\text { discontinued }\end{array}$ & $\begin{array}{l}\text { Gestational } \\
\text { diabetes }\end{array}$ & $\begin{array}{l}23 / 359(6.4 \%) \\
\text { vs. } 60 / 1,465 \\
(4.1 \%)^{90}\end{array}$ & $\begin{array}{l}\text { ARR, } 1.09 \\
(95 \% \mathrm{Cl}, 0.7 \\
\text { to } 1.7)^{90}\end{array}$ & $\begin{array}{l}1 \text { cohort, } \\
n=1,824^{90}\end{array}$ & $\begin{array}{l}\text { Moderate } \\
\text { study } \\
\text { limitations, } \\
\text { imprecise } \\
\text { (wide Cls), } \\
\text { consistency } \\
\text { unknown }\end{array}$ & Insufficient \\
\hline $\begin{array}{l}\text { Women } \\
\text { prescribed an } \\
\text { second- } \\
\text { generation } \\
\text { antipsychotic }\end{array}$ & $\begin{array}{l}\text { Olanzapine } \\
\text { continued vs. } \\
\text { olanzapine } \\
\text { discontinued }\end{array}$ & $\begin{array}{l}\text { Gestational } \\
\text { diabetes }\end{array}$ & $\begin{array}{l}46 / 384(12.0 \%) \\
\text { vs. } 49 / 1,041 \\
(4.7 \%)^{90}\end{array}$ & $\begin{array}{l}\text { ARR, } 1.61 \\
(95 \% \mathrm{Cl}, 1.13 \\
\text { to } 2.29)^{90}\end{array}$ & $\begin{array}{l}1 \text { cohort, } \\
n=1,425^{90}\end{array}$ & $\begin{array}{l}\text { Moderate } \\
\text { study } \\
\text { limitations, } \\
\text { precise, } \\
\text { consistency } \\
\text { unknown }\end{array}$ & $\begin{array}{l}\text { Low for } \\
\text { harms of } \\
\text { continued } \\
\text { quetiapine }\end{array}$ \\
\hline $\begin{array}{l}\text { Women with } \\
\text { schizophrenia }\end{array}$ & $\begin{array}{l}\text { First- } \\
\text { generation } \\
\text { antipsychotic } \\
\text { vs. no } \\
\text { antipsychotic }\end{array}$ & $\begin{array}{l}\text { Preterm } \\
\text { birth }\end{array}$ & $\begin{array}{l}35 / 194(18.0 \%) \\
\text { vs. } 37 / 454 \\
(8.1 \%)^{85}\end{array}$ & $\begin{array}{l}\text { AOR, } 2.46 \\
(95 \% \mathrm{Cl}, 1.5 \\
\text { to } 4.11)^{85}\end{array}$ & 1 cohort, $n=648^{85}$ & $\begin{array}{l}\text { High study } \\
\text { limitations } \\
(\text { high risk of } \\
\text { bias }^{85} \text { ) precise, } \\
\text { consistency } \\
\text { unknown }\end{array}$ & Insufficient \\
\hline $\begin{array}{l}\text { Women with } \\
\text { schizophrenia }\end{array}$ & $\begin{array}{l}\text { Second- } \\
\text { generation } \\
\text { antipsychotic } \\
\text { vs. no } \\
\text { antipsychotic }\end{array}$ & $\begin{array}{l}\text { Preterm } \\
\text { birth }\end{array}$ & $\begin{array}{l}6 / 48(12.5 \%) \text { vs. } \\
37 / 454(8.1 \%)^{85}\end{array}$ & $\begin{array}{l}\text { AOR, 1.61 } \\
(95 \% \mathrm{Cl}, 0.63 \\
\text { to } 4.12)^{85}\end{array}$ & 1 cohort, $\mathrm{n}=696^{85}$ & $\begin{array}{l}\text { High study } \\
\text { limitations } \\
\text { (high risk of } \text { bias }^{85} \text { ) } \\
\text { imprecise } \\
\text { (wide Cls), } \\
\text { consistency } \\
\text { unknown }\end{array}$ & Insufficient \\
\hline $\begin{array}{l}\text { Women with } \\
\text { schizophrenia }\end{array}$ & $\begin{array}{l}\text { First- } \\
\text { generation } \\
\text { antipsychotic } \\
\text { vs. no } \\
\text { antipsychotic }\end{array}$ & $\begin{array}{l}\text { Low birth } \\
\text { weight }\end{array}$ & $\begin{array}{l}16 / 194(8.2 \%) \\
\text { vs. } 41 / 454 \\
(9.0 \%)^{85}\end{array}$ & $\begin{array}{l}\text { AOR, } 0.95 \\
(95 \% \mathrm{Cl}, 0.5 \\
\text { to } 1.75)^{85}\end{array}$ & 1 cohort, $n=696^{85}$ & $\begin{array}{l}\text { High study } \\
\text { limitations } \\
\text { (high risk of } \\
\text { bias }^{85} \text { ) } \\
\text { imprecise } \\
\text { (wide Cls), } \\
\text { consistency } \\
\text { unknown }\end{array}$ & Insufficient \\
\hline $\begin{array}{l}\text { Women with } \\
\text { schizophrenia }\end{array}$ & $\begin{array}{l}\text { Second- } \\
\text { generation } \\
\text { antipsychotic } \\
\text { vs. no } \\
\text { antipsychotic }\end{array}$ & $\begin{array}{l}\text { Low birth } \\
\text { weight }\end{array}$ & $\begin{array}{l}\text { 6/48 (12.5\%) vs. } \\
41 / 454(9.0 \%)^{85}\end{array}$ & $\begin{array}{l}\text { AOR, 1.71 } \\
(95 \% \mathrm{Cl}, 0.67 \\
\text { to } 4.34)^{85}\end{array}$ & 1 cohort, $n=696^{85}$ & $\begin{array}{l}\text { High study } \\
\text { limitations } \\
\text { (high risk of } \\
\text { bias }^{85} \text { ) } \\
\text { imprecise } \text { (wide Cls), } \\
\text { consistency } \\
\text { unknown }\end{array}$ & Insufficient \\
\hline
\end{tabular}




\begin{tabular}{|c|c|c|c|c|c|c|c|}
\hline Population & $\begin{array}{l}\text { Intervention } \\
\text { and } \\
\text { Comparator }\end{array}$ & Outcome & $\begin{array}{l}\text { Incidence or } \\
\text { Mean Effect by } \\
\text { Arm }\end{array}$ & Results & $\begin{array}{l}\text { Study Design } \\
\text { and Sample Size }\end{array}$ & $\begin{array}{l}\text { Factors That } \\
\text { Affect the } \\
\text { Strength of } \\
\text { Evidence }\end{array}$ & $\begin{array}{l}\text { Overall } \\
\text { Evidence } \\
\text { Strength } \\
\text { (Direction } \\
\text { of Effect) }\end{array}$ \\
\hline $\begin{array}{l}\text { Women with } \\
\text { schizophrenia }\end{array}$ & $\begin{array}{l}\text { First- } \\
\text { generation } \\
\text { antipsychotic } \\
\text { vs. no } \\
\text { antipsychotic }\end{array}$ & $\begin{array}{l}\text { Small for } \\
\text { gestational } \\
\text { age }\end{array}$ & $\begin{array}{l}49 / 194(25.3 \%) \\
\text { vs. 92/454 } \\
(20.3 \%)^{85}\end{array}$ & $\begin{array}{l}\text { AOR, } 1.39 \\
(95 \% \mathrm{Cl}, 0.93 \\
\text { to } 2.08)^{85}\end{array}$ & 1 cohort, $n=696^{85}$ & $\begin{array}{l}\text { High study } \\
\text { limitations } \\
\text { (high risk of } \\
\text { bias }^{85} \text { ) } \\
\text { imprecise } \\
\text { (wide Cls), } \\
\text { consistency } \\
\text { unknown }\end{array}$ & Insufficient \\
\hline $\begin{array}{l}\text { Women with } \\
\text { schizophrenia }\end{array}$ & $\begin{array}{l}\text { Second- } \\
\text { generation } \\
\text { antipsychotic } \\
\text { vs. no } \\
\text { antipsychotic }\end{array}$ & $\begin{array}{l}\text { Small for } \\
\text { gestational } \\
\text { age }\end{array}$ & $\begin{array}{l}\text { 10/48(20.8\%) vs. } \\
92 / 454(20.3 \%)^{85}\end{array}$ & $\begin{array}{l}\text { AOR, } 1.15 \\
(95 \% \mathrm{Cl}, 0.55 \\
\text { to } 2.41)^{85}\end{array}$ & 1 cohort, $n=696^{85}$ & $\begin{array}{l}\text { High study } \\
\text { limitations } \\
\text { (high risk of } \\
\text { bias }^{85} \text { ) } \\
\text { imprecise } \\
\text { (wide Cls), } \\
\text { consistency } \\
\text { unknown }\end{array}$ & Insufficient \\
\hline $\begin{array}{l}\text { Women with } \\
\text { schizophrenia }\end{array}$ & $\begin{array}{l}\text { First- } \\
\text { generation } \\
\text { antipsychotic } \\
\text { vs. no } \\
\text { antipsychotic }\end{array}$ & $\begin{array}{l}\text { Large for } \\
\text { gestational } \\
\text { age }\end{array}$ & $\begin{array}{l}15 / 194(7.7 \%) \\
\text { vs. } 44 / 454 \\
(9.7 \%)^{85}\end{array}$ & $\begin{array}{l}\text { AOR, } 0.72 \\
(95 \% \mathrm{Cl}, 0.39 \\
\text { to } 1.34)^{85}\end{array}$ & 1 cohort, $n=696^{85}$ & $\begin{array}{l}\text { High study } \\
\text { limitations } \\
\text { (high risk of } \\
\text { bias }^{85} \text { ) } \\
\text { imprecise } \\
\text { (wide Cls), } \\
\text { consistency } \\
\text { unknown }\end{array}$ & Insufficient \\
\hline $\begin{array}{l}\text { Women with } \\
\text { schizophrenia }\end{array}$ & $\begin{array}{l}\text { Second- } \\
\text { generation } \\
\text { antipsychotic } \\
\text { vs. no } \\
\text { antipsychotic }\end{array}$ & $\begin{array}{l}\text { Large for } \\
\text { gestational } \\
\text { age }\end{array}$ & $\begin{array}{l}/ 48(6.3 \%) \text { vs. } \\
44 / 454(9.7 \%)^{85}\end{array}$ & $\begin{array}{l}\text { AOR, } 0.55 \\
(95 \% \mathrm{Cl}, 0.16 \\
\text { to } 1.85)^{85}\end{array}$ & 1 cohort, $n=696^{85}$ & $\begin{array}{l}\text { High study } \\
\text { limitations } \\
\text { (high risk of } \\
\text { bias }^{85} \text { ) } \\
\text { imprecise } \\
\text { (wide Cls), } \\
\text { consistency } \\
\text { unknown }\end{array}$ & Insufficient \\
\hline $\begin{array}{l}\text { Women } \\
\text { exposed to } \\
\text { risperidone } \\
\text { during or } \\
\text { before } \\
\text { pregnancy }\end{array}$ & $\begin{array}{l}\text { Risperidone } \\
\text { continued in } \\
\text { first trimester } \\
\text { vs. risperidone } \\
\text { discontinued } \\
\text { before } \\
\text { pregnancy }\end{array}$ & $\begin{array}{l}\text { Major } \\
\text { congenital } \\
\text { anomalies }\end{array}$ & $\begin{array}{l}44 / 895(4.9 \%) \\
\text { vs. } 72 / 1737 \\
\text { discontinued } \\
\text { before } \\
\text { pregnancy } \\
(4.1 \%)^{62} \\
\mathrm{~N} \text { reduces in } \\
\text { both arms as } \\
\text { more restrictive } \\
\text { definitions of } \\
\text { control group are } \\
\text { used (no } \\
\text { prescriptions } 4, \\
6, \text { and } 8 \text { weeks } \\
\text { before } \\
\text { pregnancy) }\end{array}$ & $\begin{array}{l}\text { AOR, } 1.00 \\
(95 \% \mathrm{Cl}, 0.70 \\
\text { to } 1.43)^{62} \\
\text { AOR } \\
\text { increases } \\
\text { when more } \\
\text { restrictive } \\
\text { definitions of } \\
\text { control group } \\
\text { are used (no } \\
\text { prescriptions } \\
4,6, \text { and } 8 \\
\text { weeks before } \\
\text { pregnancy) } \\
\text { from } 1.13 \text { to } \\
1.64 ; \text { Cls wide } \\
\text { in all cases } \\
\text { and span the } \\
\text { null }\end{array}$ & $\begin{array}{l}\text { cohort, } \\
\mathrm{n}=2,632^{62}\end{array}$ & $\begin{array}{l}\text { Moderate } \\
\text { study } \\
\text { limitations } \\
\text { imprecise } \\
\text { (wide Cls), } \\
\text { inconsistency } \\
\text { unknown }\end{array}$ & Insufficient \\
\hline
\end{tabular}




\begin{tabular}{|c|c|c|c|c|c|c|c|}
\hline Population & $\begin{array}{l}\text { Intervention } \\
\text { and } \\
\text { Comparator }\end{array}$ & Outcome & $\begin{array}{l}\text { Incidence or } \\
\text { Mean Effect by } \\
\text { Arm }\end{array}$ & Results & $\begin{array}{l}\text { Study } \\
\text { Design and } \\
\text { Sample Size }\end{array}$ & $\begin{array}{l}\text { Factors That } \\
\text { Affect the } \\
\text { Strength of } \\
\text { Evidence }\end{array}$ & $\begin{array}{l}\text { Overall } \\
\text { Evidence } \\
\text { Strength } \\
\text { (Direction } \\
\text { of Effect) }\end{array}$ \\
\hline $\begin{array}{l}\text { Women } \\
\text { exposed to } \\
\text { risperidone } \\
\text { during or } \\
\text { before } \\
\text { pregnancy }\end{array}$ & $\begin{array}{l}\text { Risperidone } \\
\text { continued in } \\
\text { first trimester } \\
\text { vs. risperidone } \\
\text { discontinued } \\
\text { before } \\
\text { pregnancy }\end{array}$ & $\begin{array}{l}\text { Cardiac } \\
\text { anomalies }\end{array}$ & $\begin{array}{l}18 / 895(2.0 \%) \\
\text { vs. } 26 / 1,737 \\
\text { (discontinued } \\
\text { before } \\
\text { pregnancy) } \\
(1.5 \%)^{62} \\
\mathrm{~N} \text { reduces in } \\
\text { both arms as } \\
\text { more restrictive } \\
\text { definitions of } \\
\text { control group are } \\
\text { used (no } \\
\text { prescriptions } 4, \\
6, \text { and } 8 \text { weeks } \\
\text { before } \\
\text { pregnancy) }\end{array}$ & $\begin{array}{l}\text { AOR, } 0.85 . \\
(95 \% \mathrm{Cl}, 0.49 \\
\text { to } 1.46)^{62} \\
\text { AOR } \\
\text { increases } \\
\text { with more } \\
\text { restrictive } \\
\text { definitions of } \\
\text { control group } \\
\text { are used (no } \\
\text { prescriptions } \\
4,6, \text { and } 8 \\
\text { weeks before } \\
\text { pregnancy) } \\
\text { from } 1.31 \text { to } \\
2.46 \text {; Cls } \\
\text { wide in all } \\
\text { cases and } \\
\text { span the null } \\
\end{array}$ & $\begin{array}{l}1 \text { cohort, } \\
\mathrm{n}=2,632^{62}\end{array}$ & $\begin{array}{l}\text { Moderate study } \\
\text { limitations } \\
\text { imprecise (wide } \\
\text { Cls), } \\
\text { inconsistency } \\
\text { unknown }\end{array}$ & Insufficient \\
\hline $\begin{array}{l}\text { Women with a } \\
\text { psychiatric } \\
\text { condition }\end{array}$ & $\begin{array}{l}\text { Quetiapine } \\
\text { exposure in } \\
\text { first trimester } \\
\text { vs. unexposed } \\
\text { to second- } \\
\text { generation } \\
\text { antipsychotic }\end{array}$ & $\begin{array}{l}\text { Major } \\
\text { congenital } \\
\text { anomalies }\end{array}$ & $\begin{array}{l}2 / 155(1.3 \%) \text { vs. } \\
3 / 210(1.4 \%)^{43}\end{array}$ & $\begin{array}{l}\text { AOR, } 0.9 \\
(95 \% \mathrm{Cl}, 0.15 \\
\text { to } 5.46)^{43}\end{array}$ & $\begin{array}{l}1 \text { cohort, } \\
n=357^{43}\end{array}$ & $\begin{array}{l}\text { High study } \\
\text { limitations (high } \\
\text { risk of bias }{ }^{43} \text { ) } \\
\text { imprecise (wide } \\
\text { Cls), } \\
\text { consistency } \\
\text { unknown }\end{array}$ & Insufficient \\
\hline $\begin{array}{l}\text { Women with a } \\
\text { psychiatric } \\
\text { condition or } \\
\text { women with } \\
\text { schizophrenia, } \\
\text { bipolar } \\
\text { disorder, or } \\
\text { psychosis }\end{array}$ & $\begin{array}{l}\text { Second- } \\
\text { generation } \\
\text { antipsychotic } \\
\text { exposure in } \\
\text { first trimester } \\
\text { vs. unexposed } \\
\text { to second- } \\
\text { generation } \\
\text { antipsychotic } \\
\end{array}$ & $\begin{array}{l}\text { Major } \\
\text { congenital } \\
\text { anomalies }\end{array}$ & $\begin{array}{l}209 / 3,995 \\
(5.2 \%) \text { vs. } \\
471 / 11,606 \\
(4.1 \%)^{62} \\
\\
3 / 214(1.4 \%) \text { vs. } \\
1 / 89(1.1 \%)^{44}\end{array}$ & $\begin{array}{l}\text { AOR, } 1.16 \\
(95 \% \mathrm{Cl}, 0.99 \\
\text { to } 1.35)^{62} \\
\text { AOR, } 0.69 \\
(95 \% \mathrm{Cl}, 0.06 \\
\text { to } 8.09)^{44}\end{array}$ & $\begin{array}{l}2 \text { cohorts, } \\
n=15,904^{44,}, 62\end{array}$ & $\begin{array}{l}\text { Moderate study } \\
\text { limitations (one } \\
\text { high risk of bias } \\
\text { study }{ }^{44} \text { precise, } \\
\text { inconsistent }\end{array}$ & Insufficient \\
\hline $\begin{array}{l}\text { Women with } \\
\text { schizophrenia, } \\
\text { bipolar } \\
\text { disorder, or } \\
\text { psychosis }\end{array}$ & $\begin{array}{l}\text { First- } \\
\text { generation } \\
\text { antipsychotic } \\
\text { exposure in } \\
\text { first trimester } \\
\text { vs. unexposed } \\
\text { to first- } \\
\text { generation } \\
\text { antipsychotic } \\
\end{array}$ & $\begin{array}{l}\text { Major } \\
\text { congenital } \\
\text { anomalies }\end{array}$ & $\begin{array}{l}16 / 381(4.2 \%) \\
\text { vs. } 417 / 10,418 \\
(4.0 \%)^{62}\end{array}$ & $\begin{array}{l}\text { AOR, } 0.93 \\
(95 \% \mathrm{Cl}, 0.57 \\
\text { to } 1.51)^{62}\end{array}$ & $\begin{array}{l}1 \text { cohort, } \\
n=10,799^{62}\end{array}$ & $\begin{array}{l}\text { Moderate study } \\
\text { limitations } \\
\text { imprecise (wide } \\
\text { Cls), } \\
\text { inconsistency } \\
\text { unknown }\end{array}$ & Insufficient \\
\hline $\begin{array}{l}\text { Women with } \\
\text { schizophrenia, } \\
\text { bipolar } \\
\text { disorder, or } \\
\text { psychosis }\end{array}$ & $\begin{array}{l}\text { Second- } \\
\text { generation } \\
\text { antipsychotic } \\
\text { exposure in } \\
\text { first trimester } \\
\text { vs. unexposed } \\
\text { to second- } \\
\text { generation } \\
\text { antipsychotic } \\
\end{array}$ & $\begin{array}{l}\text { Cardiac } \\
\text { anomalies }\end{array}$ & $\begin{array}{l}79 / 3,995(2.0 \%) \\
\text { vs. } 169 / 11,606 \\
(1.5 \%)^{62}\end{array}$ & $\begin{array}{l}\text { AOR, } 1.21 \\
(95 \% \mathrm{Cl}, 0.93 \\
\text { to } 1.57)^{62}\end{array}$ & $\begin{array}{l}1 \text { cohort, } \\
n=15,601^{62}\end{array}$ & $\begin{array}{l}\text { Moderate study } \\
\text { limitations } \\
\text { imprecise (wide } \\
\text { Cls), } \\
\text { inconsistency } \\
\text { unknown }\end{array}$ & Insufficient \\
\hline
\end{tabular}




\begin{tabular}{|c|c|c|c|c|c|c|c|}
\hline Population & $\begin{array}{l}\text { Intervention } \\
\text { and } \\
\text { Comparator }\end{array}$ & Outcome & $\begin{array}{l}\text { Incidence or } \\
\text { Mean Effect by } \\
\text { Arm }\end{array}$ & Results & $\begin{array}{l}\text { Study } \\
\text { Design and } \\
\text { Sample Size }\end{array}$ & $\begin{array}{l}\text { Factors That } \\
\text { Affect the } \\
\text { Strength of } \\
\text { Evidence }\end{array}$ & $\begin{array}{l}\text { Overall } \\
\text { Evidence } \\
\text { Strength } \\
\text { (Direction } \\
\text { of Effect) }\end{array}$ \\
\hline $\begin{array}{l}\text { Women with } \\
\text { schizophrenia, } \\
\text { bipolar } \\
\text { disorder, or } \\
\text { psychosis }\end{array}$ & $\begin{array}{l}\text { First- } \\
\text { generation } \\
\text { antipsychotic } \\
\text { exposure in } \\
\text { first trimester } \\
\text { vs. unexposed } \\
\text { to first- } \\
\text { generation } \\
\text { antipsychotic }\end{array}$ & $\begin{array}{l}\text { Cardiac } \\
\text { anomalies }\end{array}$ & $\begin{array}{l}\leq 10 / 381(\leq 2.6 \%) \\
\text { vs. } 152 / 10,418 \\
(2.6 \%)^{62}\end{array}$ & $\begin{array}{l}\text { AOR, } 0.91 \\
(95 \% \mathrm{Cl}, 0.43 \\
\text { to } 1.91)^{62}\end{array}$ & $\begin{array}{l}1 \text { cohort, } \\
n=10,799^{62}\end{array}$ & $\begin{array}{l}\text { Moderate study } \\
\text { limitations } \\
\text { imprecise (wide } \\
\text { Cls), } \\
\text { inconsistency } \\
\text { unknown }\end{array}$ & Insufficient \\
\hline
\end{tabular}

$\mathrm{AOR}=$ adjusted odds ratio; $\mathrm{ARR}=$ adjusted risk ratio; $\mathrm{CI}=$ confidence interval; $\mathrm{n}=$ number; vs. $=$ versus.

For maternal diabetes, data are only available for second-generation antipsychotics, and one study (which compares continuing with discontinuing second-generation antipsychotics) showed an increased risk for quetiapine $(\mathrm{N}=4,533)$ and olanzapine $(\mathrm{N}=1,425) .{ }^{90}$ The evidence is rated as low for the potential for harm: although metabolic effects of these drugs could potentially have resulted in diabetes, the potential for residual confounding limits our confidence. A smaller study ( $\mathrm{N}$ not reported but $<539$ ) reported a higher and significant risk of gestational diabetes for women with exposure to antipsychotics (first generation or second generation). The study did not control for severity of the disorder and had wide CIs, suggesting lack of precision. As a result, we graded the evidence for the risk of gestational diabetes from antipsychotics as a drug as insufficient.

For birth anomalies, the larger ${ }^{62}$ of two studies ${ }^{44,62}$ (moderate risk of bias) provided analyses in women with schizophrenia, bipolar disorder, or psychosis and adjusted for potential confounding. These results suggest a small increased risk of congenital anomalies with secondgeneration antipsychotics as a class and with risperidone in particular. Notably, however, the risk for second-generation antipsychotics is not consistently higher in all analyses; the findings could be explained by chance and residual confounding cannot be ruled out. Also, the study did not find increased risk for cardiac anomalies. Further, it found no increased risk for first-generation antipsychotics for congenital anomalies overall or cardiac anomalies. Although the risk with risperidone was higher among those who continued on medication compared with those who discontinued medication, confidence intervals included appreciable harm and appreciable benefit, suggesting imprecision.

Regarding preterm birth, one high risk-of-bias study showed a significantly increased risk of preterm birth with first-generation antipsychotics but not second-generation antipsychotics. ${ }^{85}$ Overall, however, results are not consistent in direction or magnitude of effects.

\section{Pharmacologic Combinations}

\section{Overview}

- Two publications on pharmacologic combinations reported on child outcomes (major congenital anomalies, heart anomalies, or psychomotor development) on nonspecific or undefined pharmacologic interventions compared with inactive treatment. 


\section{Detailed Results}

Two publications reported on pharmacologic combinations. ${ }^{45,72}$ These publications drew from two cohorts ( 1 from THIN ${ }^{72}$ and 1 from the Women's Mental Health Program at the Emory University School of Medicine ${ }^{45}$ ). Both were nonrandomized studies and were rated as high risk of bias.

Both publications focused on exposure during pregnancy. One compared SSRIs plus TCAs with untreated depression on child harms of major congenital and heart anomalies. ${ }^{72}$ One compared SSRIs plus non-SSRIs exposure with untreated major depressive disorder on child outcomes of infant psychomotor development. ${ }^{45}$ Because we could not determine the clinical utility of the interventions, they are not discussed in further detail.

\section{Nonspecific or Undefined Pharmacologic Intervention}

\section{Overview}

- Thirty-nine publications reported on nonspecific or undefined pharmacologic interventions compared with no treatment. Because the clinical utility is limited, we did not judge the risk of harms for maternal or child outcomes.

- These studies reported on maternal (breastfeeding at discharge, conception, ectopic pregnancy, gestational diabetes, obstetrical and/or delivery complications, induced labor, spontaneous abortion, gestational hypertension, preeclampsia, postpartum hemorrhage, severe maternal morbidity, maternal ICU, maternal extended length of hospital stay) and child outcomes (preterm birth, attention-deficit/hyperactivity disorder, asthma, Apgar scores, autism, cardiac/heart anomalies, major congenital anomalies, intellectual disabilities, low birth weight, NICU admissions and/or discharge, neonatal abstinence syndrome, prematurity/gestational age, perinatal death, psychiatric disorders, neuromotor and/or psychomotor development or other early development indicators, severe neonatal morbidity or mortality, or small for gestational age/size for gestational age).

\section{Detailed Results}

Thirty-nine publications reported on nonspecific or undefined pharmacologic interventions. ${ }^{6}$, 7, 9, 31, 33, 42, 45, 48, 49, 51-58, 60, 64, 65, 67, 68, 70, 73, 75, 77-79, 83, 84, 86, 89, 93, 94, 99, 101, 102, 104-106 These publications drew from 28 cohorts ( 1 from Antimanic Use During Pregnancy, ${ }^{89} 2$ from the Danish Medical Birth Registry, ${ }^{49,68} 2$ from the Danish National Birth Cohort, ${ }^{9,}, 60,781$ from THIN, ${ }^{79} 1$ from the Hong Kong Clinical Data Analysis and Reporting System, ${ }^{55} 1$ from Kaiser Permanente Northern California, ${ }^{67} 1$ from Kaiser Permanente Washington, ${ }^{104} 2$ from the Lombardy healthcare utilization database, ${ }^{54,58} 1$ from Massachusetts General Hospital, ${ }^{86} 1$ from Motherisk, ${ }^{77} 1$ from the National Birth Defects Study, ${ }^{106} 4$ from the QPC, ${ }^{53,57,64,93} 1$ from RAMQ,${ }^{70} 1$ from the Slone Epidemiology Center Birth Defects Study, ${ }^{84} 1$ from the Stockholm Youth Cohort, ${ }^{51} 1$ from the Swedish Medical Birth Register, ${ }^{52} 1$ from the Swedish National Registers, ${ }^{56} 1$ from Time to Conceive, ${ }^{65} 3$ from U.S. Medicaid Analytic eXtract, 6, 7, 751 from the Women's and Children's Health Network, ${ }^{48} 1$ from the OptumLabs Data Warehouse, ${ }^{99} 1$ from the Manitoba Population Research Data Repository, ${ }^{101} 1$ from the IBM MarketScan Research Databases, ${ }^{102} 1$ from the a claims databases maintained by the Japan Medical Data Center, ${ }^{105} 1$ that included multiple Danish national ${ }^{73}$ registries, ${ }^{73} 2$ that included multiple Canada-based databases, ${ }^{31,83} 2$ from populations in Australia, ${ }^{33,42}$ and 2 from populations based in the United States ${ }^{45,94}$ ). All were 
nonrandomized studies and were rated as high risk of bias or having some concerns for risk of bias.

All 39 publications focused on exposure during pregnancy. Twenty-four publications examined any antidepressants use, $7,31,33,42,48,49,51-56,58,60,64,65,67,68,73,78,83,86,99,102,104-106$ one examined new antidepressants, ${ }^{93}$ one examined antipsychotics, ${ }^{33}$ seven examined intervention groups described as other antidepressants, $7,57,58,75,79,83,106$ one examined an intervention described as antidepressant/antipsychotic ${ }^{42}$ one examined interventions described as other monotherapy ${ }^{64}$ three examined polytherapy/polypharmacy, ${ }^{64,75,79}$ four examined non-SSRIs, ${ }^{6,45}$, $51,70,84$ one examined SSRIs only, ${ }^{45}$ one examined nonsertraline SSRIs,${ }^{70}$ one examined SSRIs plus non-SSRIs,${ }^{45}$ one examined antidepressants described as SSRIs or SNRIs, ${ }_{101}^{101}$ two examined any psychotropic treatment, ${ }^{89,94}$ one examined multiple antidepressant classes, ${ }^{106}$ two examined interventions described as co-exposure, ${ }^{83,93}$ and one examined an intervention described as unclear. ${ }^{77}$ The included publications evaluated the maternal outcomes (breastfeeding at discharge, conception, obstetrical and/or delivery complications, ectopic pregnancy, gestational diabetes, induced labor, spontaneous abortion, or gestational hypertension, preeclampsia, postpartum hemorrhage, severe maternal morbidity, maternal ICU admission, maternal extended length of stay) and the child outcomes (preterm delivery, attention-deficit/hyperactivity disorder, asthma, Apgar scores, autism, behavior, cardiac anomalies, major congenital anomalies, neural tube defect, mood disorder, heart anomalies, intellectual disabilities, birth weight, neonatal abstinence syndrome, NICU admissions and/or discharge, prematurity/gestational age, perinatal death, psychiatric disorders, persistent pulmonary hypertension, intrauterine hypoxia and birth asphyxia, neonatal convulsions, neuromotor and/or psychomotor development, respiratory conditions, severe neonatal morbidity or mortality, or small for gestational age/size for gestational age). Because the interventions were not clearly defined, they are not evaluated any further given their limited clinical utility.

Key Question 4: Among reproductive-aged women with any mental health disorder, what are the comparative maternal and fetal harms of pharmacologic interventions for a mental health disorder during preconception, pregnancy, and postpartum?

\section{Anti-Anxiety Medications Versus Specific Active Comparators}

\section{Overview}

- No included publications reported on the harms of anxiolytics compared with specific active comparators.

\section{Sedative Hypnotics Versus Specific Active Comparators}

\section{Overview}

- No included publications reported on the harms of sedative hypnotics compared with specific active comparators. 


\section{SSRIs Versus SNRIs}

\section{Overview}

- The evidence is insufficient to judge the comparative harms of SSRIs versus SNRIs exposure during pregnancy for any reported maternal outcomes (preeclampsia, gestational hypertension, vaginal bleeding, diabetes during pregnancy, infection during pregnancy, or spontaneous abortion) or child outcomes (birth outcomes, childhood epilepsy, neonatal behavioral assessments, attention-deficit/hyperactivity disorder, or autism spectrum disorder).

\section{Detailed Results}

Eight publications reported on the comparative harms of SSRIs versus SNRIs during pregnancy (Table B-34). ${ }^{53,64,81,110,112,114,124,140}$ These studies drew from five cohorts (two from the Canadian QPC cohort, ${ }^{53,114}$ a second cohort using the same Canadian Quebec registry data, ${ }^{64}$ one from an Australian cohort of women with depression receiving care at a hospital's outpatient clinics, ${ }^{124}$ one from the Danish Medical Birth Registry, ${ }^{110}$ one from a Canadian British Colombian pregnant women and newborn registry, ${ }^{81}$ one from the United States and Canada MotherToBaby (MTB) USA data, ${ }^{112}$ and one from the Australian Mercy Pregnancy and Emotional Wellbeing Study. All were rated high risk of bias.

Table B-34. Strength of evidence for comparative harms: SSRIs versus SNRIs

\begin{tabular}{|c|c|c|c|c|c|c|c|}
\hline Population & $\begin{array}{l}\text { Intervention } \\
\text { and } \\
\text { Comparator }\end{array}$ & Outcome & $\begin{array}{l}\text { Incidence or } \\
\text { Mean Effect by } \\
\text { Arm }\end{array}$ & Results & $\begin{array}{l}\text { Study Design } \\
\text { and Sample } \\
\text { Size }\end{array}$ & $\begin{array}{l}\text { Factors That } \\
\text { Affect the } \\
\text { Strength of } \\
\text { Evidence }\end{array}$ & $\begin{array}{l}\text { Overall } \\
\text { Evidence } \\
\text { Strength } \\
\text { (Direction } \\
\text { of Effect) }\end{array}$ \\
\hline $\begin{array}{l}\text { Pregnant } \\
\text { women } \\
\text { who used } \\
\text { SSRIs or } \\
\text { SNRIs } \\
\text { during } \\
\text { pregnancy } \\
\text { or who had } \\
\text { depression }\end{array}$ & $\begin{array}{l}\text { SSRI vS. } \\
\text { SNRI }\end{array}$ & Preeclampsia & $\begin{array}{l}23 / 408(5.6 \%) \\
\text { vs. } 105 / 3,169 \\
(3.3 \%)^{81} \\
\\
\text { Continued AD } \\
\text { use } \geq 20 \text { weeks } \\
\text { of gestation: } \\
4 / 157(2.5 \%) \text { vs. } \\
2 / 21(9.5 \%)^{112} \\
\end{array}$ & $\begin{array}{l}\text { RR, } 0.59 \\
(95 \% \\
\mathrm{Cl}, 0.38 \text { to } \\
0.91)^{81} \\
\\
\mathrm{RR}, 0.27 \\
(95 \% \mathrm{Cl}, \\
0.052 \text { to } \\
1.37)^{112} \\
\end{array}$ & $\begin{array}{l}2 \text { cohorts; } \\
n=3,577 \text { in one } \\
\text { cohort, }^{81} \mathrm{n}=252 \\
\text { in second }^{112}\end{array}$ & $\begin{array}{l}\text { Serious study } \\
\text { limitations (high } \\
\text { risk of bias }^{81,} \\
112 \text { ), imprecise } \\
\text { (few events), } \\
\text { consistent }\end{array}$ & Insufficient \\
\hline $\begin{array}{l}\text { Pregnant } \\
\text { women } \\
\text { who used } \\
\text { SSRIs or } \\
\text { SNRIs } \\
\text { during } \\
\text { pregnancy }\end{array}$ & $\begin{array}{l}\text { SSRI vs. } \\
\text { SNRI }\end{array}$ & $\begin{array}{l}\text { Gestational } \\
\text { hypertension }\end{array}$ & $\begin{array}{l}\text { Continued AD } \\
\text { use } \geq 20 \text { weeks } \\
\text { of gestation: } \\
11 / 157(7.0 \%) \\
\text { vs } 3 / 21 \\
(14.6 \%)^{112} \\
2 / 23(8.3 \%) \text { vs. } \\
23 / 60(39.1 \%)^{140}\end{array}$ & $\begin{array}{l}\mathrm{RR}, 0.49 \\
(95 \% \mathrm{Cl}, \\
0.15 \text { to } \\
1.62)^{112} \\
\\
\mathrm{RR}, 0.235 . \\
(95 \% \mathrm{Cl}, \\
0.06 \text { to } \\
0.89)^{140} \\
\end{array}$ & $\begin{array}{l}2 \text { cohorts, } \\
n=335^{112,140}\end{array}$ & $\begin{array}{l}\text { High study } \\
\text { limitations (high } \\
\text { risk of bias }{ }^{112,} \\
{ }^{140} \text { ), imprecise } \\
\text { (wide Cls), } \\
\text { consistent }\end{array}$ & Insufficient \\
\hline $\begin{array}{l}\text { Pregnant } \\
\text { women } \\
\text { who used } \\
\text { SSRls or } \\
\text { SNRIs } \\
\text { during } \\
\text { pregnancy }\end{array}$ & $\begin{array}{l}\text { SSRI vS. } \\
\text { SNRI }\end{array}$ & $\begin{array}{l}\text { Vaginal } \\
\text { bleeding }\end{array}$ & $\begin{array}{l}2 / 27(7 \%) \text { vs. } \\
1 / 11(9 \%)^{124}\end{array}$ & $\begin{array}{l}\mathrm{RR}, 0.81 \\
(95 \% \mathrm{Cl}, \\
0.08 \text { to } \\
8.09)\end{array}$ & 1 cohort, $n=38^{124}$ & $\begin{array}{l}\text { High study } \\
\text { limitations (high } \\
\text { risk of bias }{ }^{124} \text { ), } \\
\text { seriously } \\
\text { imprecise (few } \\
\text { events, wide } \\
\text { Cls), } \\
\text { consistency } \\
\text { unknown }\end{array}$ & Insufficient \\
\hline
\end{tabular}




\begin{tabular}{|c|c|c|c|c|c|c|c|}
\hline Population & $\begin{array}{l}\text { Intervention } \\
\text { and } \\
\text { Comparator }\end{array}$ & Outcome & $\begin{array}{l}\text { Incidence or } \\
\text { Mean Effect by } \\
\text { Arm }\end{array}$ & Results & $\begin{array}{l}\text { Study Design } \\
\text { and Sample } \\
\text { Size }\end{array}$ & $\begin{array}{l}\text { Factors That } \\
\text { Affect the } \\
\text { Strength of } \\
\text { Evidence }\end{array}$ & $\begin{array}{l}\text { Overall } \\
\text { Evidence } \\
\text { Strength } \\
\text { (Direction } \\
\text { of Effect) }\end{array}$ \\
\hline $\begin{array}{l}\text { Pregnant } \\
\text { women } \\
\text { who used } \\
\text { SSRIs or } \\
\text { SNRIs } \\
\text { during } \\
\text { pregnancy }\end{array}$ & $\begin{array}{l}\text { SSRI vS. } \\
\text { SNRI }\end{array}$ & $\begin{array}{l}\text { Diabetes } \\
\text { during } \\
\text { pregnancy }\end{array}$ & $\begin{array}{l}2 / 27(7 \%) \text { vs. } \\
1 / 11(9 \%)^{124}\end{array}$ & $\begin{array}{l}\mathrm{RR}, 0.81 \\
(95 \% \mathrm{Cl} \\
0.082 \text { to } \\
8.09)\end{array}$ & 1 cohort, $n=38^{124}$ & $\begin{array}{l}\text { High study } \\
\text { limitations (high } \\
\text { risk of bias }{ }^{124} \text { ), } \\
\text { seriously } \\
\text { imprecise (few } \\
\text { events, wide } \\
\text { Cls), } \\
\text { consistency } \\
\text { unknown }\end{array}$ & Insufficient \\
\hline $\begin{array}{l}\text { Pregnant } \\
\text { women } \\
\text { who used } \\
\text { SSRIs or } \\
\text { SNRIs } \\
\text { during } \\
\text { pregnancy }\end{array}$ & $\begin{array}{l}\text { SSRI vS. } \\
\text { SNRI }\end{array}$ & $\begin{array}{l}\text { Infection during } \\
\text { pregnancy }\end{array}$ & $\begin{array}{l}1 / 27(4 \%) \text { vs. } \\
1 / 11(9 \%)^{124}\end{array}$ & $\begin{array}{l}\mathrm{RR}, 0.41 \\
(95 \% \mathrm{Cl}, \\
0.03 \text { to } \\
5.95)\end{array}$ & 1 cohort, $n=38^{124}$ & $\begin{array}{l}\text { High study } \\
\text { limitations (high } \\
\text { risk of bias }{ }^{124} \text { ), } \\
\text { seriously } \\
\text { imprecise (few } \\
\text { events, wide } \\
\text { Cls), } \\
\text { consistency } \\
\text { unknown }\end{array}$ & Insufficient \\
\hline $\begin{array}{l}\text { Pregnant } \\
\text { women } \\
\text { with } \\
\text { depression }\end{array}$ & $\begin{array}{l}\text { SSRI vS. } \\
\text { SNRI }\end{array}$ & $\begin{array}{l}\text { Spontaneous } \\
\text { abortion }\end{array}$ & $\begin{array}{l}93 / 938(10 \%) \\
\text { vs. } 20 / 137 \\
(15 \%)^{64}\end{array}$ & $\begin{array}{l}\mathrm{RR}, 0.68 \\
(95 \% \mathrm{Cl}, \\
0.434 \text { to } \\
1.064)\end{array}$ & $\begin{array}{l}1 \text { cohort, } \\
n=1075^{64}\end{array}$ & $\begin{array}{l}\text { High study } \\
\text { limitations (high } \\
\text { risk of bias }{ }^{64} \text { ), } \\
\text { imprecise (wide } \\
\text { Cls), } \\
\text { consistency } \\
\text { unknown }\end{array}$ & Insufficient \\
\hline $\begin{array}{l}\text { Pregnant } \\
\text { women } \\
\text { taking anti- } \\
\text { depressant } \\
\text { s or with } \\
\text { current or } \\
\text { past (within } \\
2 \text { years) } \\
\text { depression } \\
\text { or } \\
\text { dysthymia }\end{array}$ & $\begin{array}{l}\text { SSRI vS. } \\
\text { SNRI }\end{array}$ & $\begin{array}{l}\text { Birth outcomes } \\
\text { (small and } \\
\text { large for } \\
\text { gestational } \\
\text { age, Apgar } \\
\text { score) }\end{array}$ & NR & $\begin{array}{l}\text { NR, no } \\
\text { significant } \\
\text { associa- } \\
\text { tions }\end{array}$ & 1 cohort, $n=90^{140}$ & $\begin{array}{l}\text { High study } \\
\text { limitations (high } \\
\left.\text { risk of bias }{ }^{140}\right) \text {, } \\
\text { likely } \\
\text { imprecise, } \\
\text { consistency } \\
\text { unknown }\end{array}$ & Insufficient \\
\hline $\begin{array}{l}\text { Pregnant } \\
\text { women } \\
\text { who used } \\
\text { SSRIs or } \\
\text { SNRIs } \\
\text { during } \\
\text { pregnancy }\end{array}$ & $\begin{array}{l}\text { SSRI vS. } \\
\text { SNRI }\end{array}$ & $\begin{array}{l}\text { Childhood } \\
\text { epilepsy }\end{array}$ & $\begin{array}{l}82 / 9,728 \\
(0.008 \%) \text { vs. } \\
5 / 582 \\
(0.009 \%)^{110}\end{array}$ & $\begin{array}{l}\text { RR, } 0.98 \\
(95 \% \mathrm{Cl}, \\
0.399 \text { to } \\
2.411)\end{array}$ & $\begin{array}{l}1 \text { cohort, } \\
n=10,310^{110}\end{array}$ & $\begin{array}{l}\text { High study } \\
\text { limitations (high } \\
\text { risk of bias }{ }^{110} \text { ), } \\
\text { imprecise (wide } \\
\text { Cls), } \\
\text { consistency } \\
\text { unknown }\end{array}$ & Insufficient \\
\hline
\end{tabular}




\begin{tabular}{|c|c|c|c|c|c|c|c|}
\hline Population & $\begin{array}{l}\text { Intervention } \\
\text { and } \\
\text { Comparator }\end{array}$ & Outcome & $\begin{array}{l}\text { Incidence or } \\
\text { Mean Effect by } \\
\text { Arm }\end{array}$ & Results & $\begin{array}{l}\text { Study Design } \\
\text { and Sample } \\
\text { Size }\end{array}$ & $\begin{array}{l}\text { Factors That } \\
\text { Affect the } \\
\text { Strength of } \\
\text { Evidence }\end{array}$ & $\begin{array}{l}\text { Overall } \\
\text { Evidence } \\
\text { Strength } \\
\text { (Direction } \\
\text { of Effect) }\end{array}$ \\
\hline $\begin{array}{l}\text { Pregnant } \\
\text { women } \\
\text { who used } \\
\text { SSRIs or } \\
\text { SNRIs } \\
\text { during } \\
\text { pregnancy }\end{array}$ & $\begin{array}{l}\text { SSRI vS. } \\
\text { SNRI }\end{array}$ & $\begin{array}{l}\text { Brazelton } \\
\text { Neonatal } \\
\text { Behavioral } \\
\text { Assessment } \\
\text { Scale domain } \\
\text { means }\end{array}$ & $\begin{array}{l}\text { Means } \pm \\
\text { standard } \\
\text { deviations } \\
\text { Autonomic: } \\
5.52 \pm 0.98 \text { vs. } \\
5.47 \pm 1.59^{124} \\
\text { Habituation: } \\
6.62 \pm 1.80 \text { vs. } \\
6.46 \pm 1.65^{124} \\
\text { Motor: } \\
5.38 \pm 0.55 \text { vs. } \\
5.27 \pm 0.69^{124} \\
\text { Range: } \\
3.47 \pm 0.61 \text { vs. } \\
3.55 \pm 0.52^{124} \\
\text { Reflexes: } \\
0.74 \pm 0.08 \text { vs. } \\
0.72 \pm 0.11^{124} \\
\text { Regulation: } \\
5.91 \pm 1.13 \text { vs. } \\
6.09 \pm 1.32^{124} \\
\text { Social- } \\
\text { interactive: } \\
6.10 \pm 2.02 \text { vs. } \\
6.49 \pm 1.63^{124}\end{array}$ & $\begin{array}{l}\text { Mean diff: } \\
0.05 \text { (95\% } \\
\text { Cl, }-0.78 \text { to } \\
0.88) \\
\text { Mean diff: } \\
0.16(95 \% \\
\text { Cl, }-1.074 \\
\text { to } 1.394) \\
\text { Mean diff: } \\
0.11(95 \% \\
\text { Cl, }-0.305 \\
\text { to } 0.52) \\
\text { Mean diff: - } \\
0.08 \text { (95\% } \\
\text { Cl, }-0.49 \text { to } \\
0.331) \\
\text { Mean diff: } \\
0.02(95 \% \\
\text { Cl, }-0.043 \\
\text { to } 0.083) \\
\text { Mean } \\
\text { diff: }-0.18 \\
(95 \% \\
\text { Cl, }-1.011 \\
\text { to } 0.651) \\
\text { Mean } \\
\text { diff: }-0.39 \\
\text { (95\% } \\
\text { Cl, }-1.736 \\
\text { to } 0.956)\end{array}$ & 1 cohort, $n=38^{124}$ & $\begin{array}{l}\text { High study } \\
\text { limitations (high } \\
\text { risk of bias }{ }^{124} \text { ), } \\
\text { seriously } \\
\text { imprecise (few } \\
\text { events, wide } \\
\text { Cls), } \\
\text { consistency } \\
\text { unknown }\end{array}$ & Insufficient \\
\hline $\begin{array}{l}\text { Pregnant } \\
\text { women } \\
\text { who used } \\
\text { SSRIs or } \\
\text { SNRIs } \\
\text { during } \\
\text { pregnancy }\end{array}$ & $\begin{array}{l}\text { SSRI vS. } \\
\text { SNRI }\end{array}$ & $\begin{array}{l}\text { Attention- } \\
\text { deficit/hyper- } \\
\text { activity } \\
\text { disorder }\end{array}$ & $\begin{array}{l}86 / 1561(5.5 \%) \\
\text { vs. } 18 / 445 \\
(4 \%)^{53}\end{array}$ & $\begin{array}{l}\mathrm{RR}, 1.36 \\
(95 \% \mathrm{Cl}, \\
0.829 \text { to } \\
2.239)\end{array}$ & $\begin{array}{l}1 \text { cohort, } \\
n=2,006^{53}\end{array}$ & $\begin{array}{l}\text { High study } \\
\text { limitations (high } \\
\text { risk of bias }{ }^{53} \text { ), } \\
\text { imprecise (wide } \\
\text { Cls), } \\
\text { consistency } \\
\text { unknown }\end{array}$ & Insufficient \\
\hline $\begin{array}{l}\text { Pregnant } \\
\text { women } \\
\text { who used } \\
\text { SSRls or } \\
\text { SNRIs } \\
\text { during } \\
\text { pregnancy }\end{array}$ & $\begin{array}{l}\text { SSRI vS. } \\
\text { SNRI }\end{array}$ & $\begin{array}{l}\text { Autism } \\
\text { spectrum } \\
\text { disorder }\end{array}$ & $\begin{array}{l}22 / 1583(1.4 \%) \\
\text { vs. } 2 / 447 \\
(0.4 \%)^{114}\end{array}$ & $\begin{array}{l}\mathrm{RR}, 3.11 \\
(95 \% \mathrm{Cl}, \\
0.733 \text { to } \\
13.159)\end{array}$ & $\begin{array}{l}1 \text { cohort, } \\
n=2,030^{114}\end{array}$ & $\begin{array}{l}\text { High study } \\
\text { limitations (high } \\
\text { risk of bias }{ }^{114} \text { ), } \\
\text { imprecise (wide } \\
\text { Cls), } \\
\text { consistency } \\
\text { unknown }\end{array}$ & Insufficient \\
\hline
\end{tabular}

$\mathrm{AD}=$ antidepressants; $\mathrm{CI}=$ confidence interval; $\mathrm{n}=$ number; $\mathrm{RR}=$ relative risk; $\mathrm{SNRI}=$ serotonin-norepinephrine reuptake inhibitor; SSRI = selective serotonin reuptake inhibitor; vs. = versus.

Two publications each reported on differences in the risk of preeclampsia and gestational hypertension. For preeclampsia, one study found a significantly lower risk from SSRIs ${ }^{81}$ and a 
second found a lower but not significantly different risk in a comparison limited to women who continued using the medications for 20 or more weeks of gestation. ${ }^{112}$ For gestational hypertension, one study found a significant lower risk with SSRIs, ${ }^{140}$ and the second study did not show a reduced risk. No study controlled for any potential confounding for these two outcomes.

All other outcomes were reported in one study. One study found that the risk of miscarriage (correcting for induced abortions) was similar between the two groups. ${ }^{64}$ Other outcomes included maternal risk of vaginal bleeding, diabetes during pregnancy, and infection during pregnancy and child risks including epilepsy; Brazelton Neonatal Behavioral Assessment Scale domains; attention-deficit/hyperactivity disorder; and autism spectrum disorder. No differences were found for any of these outcomes.

\section{SSRIs Versus TCAs}

\section{Overview}

- The evidence is insufficient to judge the comparative risk of harms from SSRIs versus TCAs exposure during pregnancy for maternal outcomes (preeclampsia or spontaneous abortion) and child outcomes (preterm birth, gestational age, small for gestational age, birthweight, perinatal complications, NICU, Apgar score, anomalies, attentiondeficit/hyperactivity disorder, autism spectrum disorder, or epilepsy).

\section{Detailed Results}

Ten high risk-of-bias publications reported on the comparative harms of SSRIs versus TCAs in pregnancy. ${ }^{53,64,81,83,86,110,114,123,126,133}$ Four publications drew from Canadian cohorts from Quebec and British Columbia, ${ }^{53,64,81,83,114}$ two from Danish registries, ${ }^{110,123}$ and three from U.S. cohorts. ${ }^{86,126,133}$ All outcomes were reported in nonrandomized studies that did not control for confounding. The evidence was insufficient to grade maternal risk of preeclampsia and spontaneous abortion and child outcomes of preterm birth, small for gestational age, birthweight, perinatal complications, Apgar score, anomalies, NICU, attention-deficit/hyperactivity disorder, autism spectrum disorder, and epilepsy (Table B-35).

Table B-35. Strength of evidence for comparative harms: SSRIs versus TCAs

\begin{tabular}{|c|c|c|c|c|c|c|c|}
\hline Population & $\begin{array}{l}\text { Intervention } \\
\text { and } \\
\text { Comparator }\end{array}$ & Outcome & $\begin{array}{l}\text { Incidence or } \\
\text { Mean Effect } \\
\text { by Arm }\end{array}$ & Results & $\begin{array}{l}\text { Study Design } \\
\text { and Sample } \\
\text { Size }\end{array}$ & $\begin{array}{l}\text { Factors That } \\
\text { Affect the } \\
\text { Strength of } \\
\text { Evidence }\end{array}$ & $\begin{array}{l}\text { Overall } \\
\text { Evidence } \\
\text { Strength } \\
\text { (Direction } \\
\text { of Effect) }\end{array}$ \\
\hline $\begin{array}{l}\text { Pregnant } \\
\text { women } \\
\text { with } \\
\text { depression }\end{array}$ & $\begin{array}{l}\text { SSRIs vs. } \\
\text { TCAs }\end{array}$ & Preeclampsia & $\begin{array}{l}105 / 3,169 \\
(3.3 \%) \text { vs. } \\
14 / 146(9.6 \%)^{81}\end{array}$ & $\begin{array}{l}\mathrm{RR}, 0.34(95 \% \\
\mathrm{Cl}, 0.20 \text { to } \\
0.59)^{81}\end{array}$ & $\begin{array}{l}1 \text { cohort, } \\
n=3,315^{81}\end{array}$ & $\begin{array}{l}\text { High study } \\
\text { limitations (high } \\
\text { risk of bias }{ }^{81} \text { ), } \\
\text { imprecise (wide } \\
\text { Cls), } \\
\text { consistency } \\
\text { unknown }\end{array}$ & Insufficient \\
\hline $\begin{array}{l}\text { Pregnant } \\
\text { women } \\
\text { with } \\
\text { depression }\end{array}$ & $\begin{array}{l}\text { SSRIs vs. } \\
\text { TCAs }\end{array}$ & $\begin{array}{l}\text { Spontaneous } \\
\text { abortion }\end{array}$ & $\begin{array}{l}93 / 938(9.9 \%) \\
\text { vs. } 20 / 147 \\
(13.6 \%)^{64}\end{array}$ & $\begin{array}{l}\mathrm{RR}, 0.73(95 \% \\
\mathrm{Cl}, 0.46 \text { to } 1.14)\end{array}$ & $\begin{array}{l}1 \text { cohort, } \\
n=1,085^{64}\end{array}$ & $\begin{array}{l}\text { High study } \\
\text { limitations (high } \\
\text { risk of bias }{ }^{64} \text { ), } \\
\text { imprecise (wide } \\
\text { Cls), } \\
\text { consistency } \\
\text { unknown }\end{array}$ & Insufficient \\
\hline
\end{tabular}




\begin{tabular}{|c|c|c|c|c|c|c|c|}
\hline Population & $\begin{array}{l}\text { Intervention } \\
\text { and } \\
\text { Comparator }\end{array}$ & Outcome & $\begin{array}{l}\text { Incidence or } \\
\text { Mean Effect } \\
\text { by Arm }\end{array}$ & Results & $\begin{array}{l}\text { Study Design } \\
\text { and Sample } \\
\text { Size }\end{array}$ & $\begin{array}{l}\text { Factors That } \\
\text { Affect the } \\
\text { Strength of } \\
\text { Evidence }\end{array}$ & $\begin{array}{l}\text { Overall } \\
\text { Evidence } \\
\text { Strength } \\
\text { (Direction } \\
\text { of Effect) } \\
\end{array}$ \\
\hline $\begin{array}{l}\text { Exposed to } \\
\text { SSRIs in } \\
\text { the first } \\
\text { trimester } \\
\text { vs. } \\
\text { exposed to } \\
\text { TCAs in the } \\
\text { first } \\
\text { trimester } \\
\text { (full-term } \\
\text { infants) }\end{array}$ & $\begin{array}{l}\text { SSRIs vs. } \\
\text { TCAs }\end{array}$ & Preterm & $\begin{array}{l}166 / 1,768 \\
(9.4 \%) \text { vs. } \\
42 / 381 \\
(11.0 \%)^{126} \\
\\
3 / 42(7.1 \%) \text { vs. } \\
6 / 37(16.2 \%)^{86}\end{array}$ & $\begin{array}{l}\text { RR, } 0.85(95 \% \\
\mathrm{Cl}, 0.62 \text { to } \\
1.17)^{126} \\
\text { RR: } 2.27(95 \% \\
\mathrm{Cl}, 0.61 \text { to } \\
8.44)^{86}\end{array}$ & $\begin{array}{l}2 \text { cohort study, } \\
\mathrm{N}=2,228^{86,126}\end{array}$ & $\begin{array}{l}\text { High study } \\
\text { limitations (high } \\
\text { risk of bias }{ }^{86,126} \text { ), } \\
\text { imprecise (wide } \\
\text { Cls), } \\
\text { inconsistent }\end{array}$ & Insufficient \\
\hline $\begin{array}{l}\text { Exposed to } \\
\text { SSRIs vs. } \\
\text { exposed to } \\
\text { TCAs }\end{array}$ & $\begin{array}{l}\text { SSRIs vs. } \\
\text { TCAs }\end{array}$ & $\begin{array}{l}\text { Gestational } \\
\text { age } \leq 36 \\
\text { weeks }\end{array}$ & $\begin{array}{l}12 / 185(6.5 \%) \\
\text { vs. } 10 / 209 \\
(4.8 \%), \text { mean } \\
\text { difference: }-0.3 \\
133\end{array}$ & $\begin{array}{l}\mathrm{RR}, 1.36(95 \% \\
\mathrm{Cl}, 0.60 \text { to } \\
3.06) ; \text { Cls for } \\
\text { mean difference } \\
\text { span the null }{ }^{133}\end{array}$ & $\begin{array}{l}1 \text { cohort study, } \\
\mathrm{N}=394^{133}\end{array}$ & $\begin{array}{l}\text { High study } \\
\text { limitations (high } \\
\left.\text { risk of bias }{ }^{133}\right), \\
\text { precise, } \\
\text { consistency } \\
\text { unknown }\end{array}$ & Insufficient \\
\hline $\begin{array}{l}\text { Exposed to } \\
\text { SSRIs vs. } \\
\text { exposed to } \\
\text { TCAs }\end{array}$ & $\begin{array}{l}\text { SSRIs vs. } \\
\text { TCAs }\end{array}$ & $\begin{array}{l}\text { Birthweight } \\
\text { (birthweight } \\
\leq 2500 \mathrm{~g} \mathrm{or} \\
\text { total } \\
\text { birthweight }^{133} \\
\text { or undefined }^{86} \text { ) }\end{array}$ & $\begin{array}{l}19 / 185(10.3 \%) \\
\text { vs. } 21 / 209 \\
(10.0 \%), \text { mean } \\
\text { difference: }-80 \\
g^{133} ; 1 / 42 \\
(2.4 \%) \text { vs. } 2 / 37 \\
(5.4 \%)\end{array}$ & $\begin{array}{l}\mathrm{RR}, 1.02(95 \% \\
\mathrm{Cl}, 0.57 \text { to } \\
1.84) ; \text { Cls for } \\
\text { mean difference } \\
\text { span the null }{ }^{133} \text {; } \\
\mathrm{RR}: 2.27(95 \% \\
\mathrm{Cl}, 0.21 \text { to } \\
2.40)^{86}\end{array}$ & $\begin{array}{l}2 \text { cohort study, } \\
\mathrm{N}=473^{86,133}\end{array}$ & $\begin{array}{l}\text { High study } \\
\text { limitations (high } \\
\text { risk of bias }^{86,133} \text { ), } \\
\text { imprecise, } \\
\text { consistent }\end{array}$ & Insufficient \\
\hline $\begin{array}{l}\text { Exposed to } \\
\text { SSRIs in } \\
\text { the first } \\
\text { trimester } \\
\text { vs. } \\
\text { exposed to } \\
\text { TCAs in the } \\
\text { first } \\
\text { trimester } \\
\text { (full-term } \\
\text { infants) } \\
\end{array}$ & $\begin{array}{l}\text { SSRIs vs. } \\
\text { TCAs }\end{array}$ & $\begin{array}{l}\text { One or } \\
\text { perinatal } \\
\text { complications }\end{array}$ & $\begin{array}{l}339 / 874 \\
(38.8 \%) \text { vs. } \\
58 / 136 \\
(42.6 \%)^{126}\end{array}$ & $\begin{array}{l}\mathrm{RR}, 0.91(95 \% \\
\mathrm{Cl}, 0.74 \text { to } 1.12)\end{array}$ & $\begin{array}{l}1 \text { cohort study, } \\
\mathrm{N}=1,010^{126}\end{array}$ & $\begin{array}{l}\text { High study } \\
\text { limitations (high } \\
\text { risk of bias }{ }^{126} \text { ), } \\
\text { imprecise (wide } \\
\text { Cls), } \\
\text { consistency } \\
\text { unknown }\end{array}$ & Insufficient \\
\hline $\begin{array}{l}\text { Pregnant } \\
\text { women } \\
\text { with a } \\
\text { psychiatric } \\
\text { disorder }\end{array}$ & $\begin{array}{l}\text { SSRIs or } \\
\text { TCA by } \\
\text { trimester of } \\
\text { exposure }\end{array}$ & $\begin{array}{l}\text { Small for } \\
\text { gestational age } \\
\left(<10^{\text {th }}\right. \\
\text { percentile })\end{array}$ & $\begin{array}{l}\text { Not applicable, } \\
\text { case control }\end{array}$ & $\begin{array}{l}\text { ORs range from } \\
0.42 \text { to } 0.98 \text {, all } \\
\text { Cls cross the } \\
\text { null }\end{array}$ & $\begin{array}{l}1 \text { case-control, } \\
\mathrm{n}=172^{83}\end{array}$ & $\begin{array}{l}\text { High study } \\
\text { limitations (high } \\
\text { risk of bias, }{ }^{83} \\
\text { imprecise) wide } \\
\text { Cls, consistency } \\
\text { unknown }\end{array}$ & Insufficient \\
\hline $\begin{array}{l}\text { Exposed to } \\
\text { SSRIs vs. } \\
\text { exposed to } \\
\text { TCAs }\end{array}$ & $\begin{array}{l}\text { SSRIs vs. } \\
\text { TCAs }\end{array}$ & $\begin{array}{l}\text { Apgar scores } \\
\text { measured at } 1 \\
(\leq 5) \text { and } 5 \\
\text { minutes }(\leq 7)\end{array}$ & $\begin{array}{l}\text { Rates for low } \\
\text { Apgar scores } \\
\text { range from } \\
10.8 \% \text { to } \\
14.6 \% \text { in the } \\
\text { SSRI arm to } \\
4.8 \% \text { to } 6.7 \% \\
\text { in the TCA } \\
\text { arm; mean } \\
\text { difference } \\
\text { range } \\
\text { from }-0.28 \\
\text { to }-0.52^{133}\end{array}$ & $\begin{array}{l}\text { RR ranges from } \\
2.18 \text { to } 2.26, \text { Cls } \\
\text { do not cross the } \\
\text { null; mean } \\
\text { differences also } \\
\text { do not cross the } \\
\text { null }^{133}\end{array}$ & $\begin{array}{l}1 \text { cohort study, } \\
\mathrm{N}=394^{133}\end{array}$ & $\begin{array}{l}\text { High study } \\
\text { limitations (high } \\
\text { risk of bias }{ }^{133} \text { ), } \\
\text { precise, } \\
\text { consistency } \\
\text { unknown }\end{array}$ & Insufficient \\
\hline
\end{tabular}




\begin{tabular}{|c|c|c|c|c|c|c|c|}
\hline Population & $\begin{array}{l}\text { Intervention } \\
\text { and } \\
\text { Comparator }\end{array}$ & Outcome & $\begin{array}{l}\text { Incidence or } \\
\text { Mean Effect } \\
\text { by Arm }\end{array}$ & Results & $\begin{array}{l}\text { Study Design } \\
\text { and Sample } \\
\text { Size }\end{array}$ & $\begin{array}{l}\text { Factors That } \\
\text { Affect the } \\
\text { Strength of } \\
\text { Evidence }\end{array}$ & $\begin{array}{l}\text { Overall } \\
\text { Evidence } \\
\text { Strength } \\
\text { (Direction } \\
\text { of Effect) }\end{array}$ \\
\hline $\begin{array}{l}\text { Exposed to } \\
\text { SSRIs in } \\
\text { the first } \\
\text { trimester } \\
\text { vs. } \\
\text { exposed to } \\
\text { TCAs in the } \\
\text { first } \\
\text { trimester } \\
\text { (full-term } \\
\text { infants) }\end{array}$ & $\begin{array}{l}\text { SSRIs vs. } \\
\text { TCAs }\end{array}$ & $\begin{array}{l}\text { Anomalies } \\
\text { (one or more } \\
\text { anomalies, } \\
\text { major } \\
\text { congenital } \\
\text { anomalies, } \\
\text { minor } \\
\text { anomalies) }\end{array}$ & $\begin{array}{l}\text { Ranges from } \\
3.7 \% \text { to } 13.4 \% \\
\text { in the SSRI } \\
\text { arm vs. } 3.4 \% \text { to } \\
12.0 \% \text { in the } \\
\text { TCA arm }\end{array}$ & $\begin{array}{l}\text { RRs range from } \\
1.08 \text { to } 1.45 \text {, all } \\
\text { Cls cross the } \\
\text { null }{ }^{123,126,133}\end{array}$ & $\begin{array}{l}3 \text { cohort } \\
\text { studies, } \\
N=5,651^{123,126,} \\
133\end{array}$ & $\begin{array}{l}\text { High study } \\
\text { limitations (high } \\
\text { risk of bias }{ }^{123,126,} \\
{ }^{133} \text { ), imprecise } \\
\text { (wide Cls), } \\
\text { consistent }\end{array}$ & Insufficient \\
\hline $\begin{array}{l}\text { Exposed to } \\
\text { SSRIs vs. } \\
\text { exposed to } \\
\text { TCAs }\end{array}$ & $\begin{array}{l}\text { SSRIs vs. } \\
\text { TCAs }\end{array}$ & $\begin{array}{l}\text { Special care } \\
\text { nursery of } \\
\text { NICU }\end{array}$ & $\begin{array}{l}5 / 42(11.9 \%) \\
\text { vs. } 11 / 37 \\
(29.7 \%)\end{array}$ & $\begin{array}{l}\text { RR: } 2.50(95 \% \\
\mathrm{Cl}, 0.96 \text { to } \\
6.52)^{86}\end{array}$ & $\begin{array}{l}1 \text { cohort study, } \\
N=79^{86}\end{array}$ & $\begin{array}{l}\text { High study } \\
\text { limitations (high } \\
\text { risk of bias }{ }^{86} \text { ), } \\
\text { imprecise (wide } \\
\text { Cls), } \\
\text { consistency } \\
\text { unknown }\end{array}$ & Insufficient \\
\hline $\begin{array}{l}\text { Pregnant } \\
\text { women } \\
\text { with } \\
\text { psychiatric } \\
\text { disorders }\end{array}$ & $\begin{array}{l}\text { SSRls vs. } \\
\text { TCAs }\end{array}$ & ADHD & $\begin{array}{l}86 / 1,561 \\
(5.5 \%) \text { vs. } \\
16 / 227(7 \%)^{53}\end{array}$ & $\begin{array}{l}\mathrm{RR}, 0.78(95 \% \\
\mathrm{Cl}, 0.47 \text { to } \\
1.31)^{53}\end{array}$ & $\begin{array}{l}1 \text { cohort } \\
n=1,788^{53}\end{array}$ & $\begin{array}{l}\text { High study } \\
\text { limitations (high } \\
\text { risk of bias }{ }^{53} \text { ), } \\
\text { imprecise (wide } \\
\text { Cls), } \\
\text { consistency } \\
\text { unknown }\end{array}$ & Insufficient \\
\hline $\begin{array}{l}\text { Pregnant } \\
\text { women } \\
\text { with } \\
\text { psychiatric } \\
\text { disorders }\end{array}$ & $\begin{array}{l}\text { SSRIs vs. } \\
\text { TCAs }\end{array}$ & ASD & $\begin{array}{l}22 / 1,583 \\
(1.4 \%) \text { vs. } \\
2 / 229(0.9 \%)^{114}\end{array}$ & $\begin{array}{l}\mathrm{RR}, 1.59(95 \% \\
\mathrm{Cl}, 0.38 \text { to } 6.72)\end{array}$ & $\begin{array}{l}1 \text { cohort } \\
n=1,812^{114}\end{array}$ & $\begin{array}{l}\text { High study } \\
\text { limitations (high } \\
\text { risk of bias } 114 \text { ), } \\
\text { imprecise (wide } \\
\text { Cls), } \\
\text { consistency } \\
\text { unknown }\end{array}$ & Insufficient \\
\hline $\begin{array}{l}\text { Pregnant } \\
\text { women } \\
\text { exposed to } \\
\text { SSRIs or } \\
\text { TCAs }\end{array}$ & $\begin{array}{l}\text { SSRIs vs. } \\
\text { TCAs }\end{array}$ & Epilepsy & $\begin{array}{l}82 / 9,728 \\
(0.8 \%) \text { vs. } \\
8 / 576(1.4 \%)^{110}\end{array}$ & $\begin{array}{l}\mathrm{RR}, 0.61(95 \% \\
\mathrm{Cl}, 0.30 \text { to } \\
1.25)^{110}\end{array}$ & $\begin{array}{l}1 \text { cohort } \\
n=1,304^{110}\end{array}$ & $\begin{array}{l}\text { High study } \\
\text { limitations (high } \\
\text { risk of bias }{ }^{110} \text { ), } \\
\text { imprecise (wide } \\
\text { Cls), } \\
\text { consistency } \\
\text { unknown }\end{array}$ & Insufficient \\
\hline
\end{tabular}

$\mathrm{ADHD}=$ attention-deficit/hyperactivity disorder; $\mathrm{ASD}=$ autism spectrum disorder; $\mathrm{CI}=$ confidence interval; $\mathrm{N}=$ number; $\mathrm{RR}=$ relative risk; SNRI = selective norepinephrine reuptake inhibitor; SSRI = selective serotonin reuptake inhibitor; TCA $=$ tricyclic antidepressant; vs. = versus.

\section{SSRIs Versus SSRIs Plus Mirtazapine}

\section{Overview}

- The evidence is insufficient to judge the comparative risk of harms from SSRIs versus SSRIs plus mirtazapine exposure during pregnancy for child outcomes (preterm birth, late-term birth, low birthweight, NICU stay, macrosomia, neonatal bilirubinemia). 


\section{Detailed Results}

One high risk-of-bias publication reported on the comparative harms of SSRIs versus SSRIs plus mirtazapine in pregnancy in Turkey. ${ }^{95}$ The study did not control for confounding. The evidence was insufficient for all reported outcomes (preterm birth, late-term birth, low birthweight, birthweight, NICU stay, macrosomia, neonatal bilirubinemia) (Table B-36).

Table B-36. Strength of evidence for comparative harms: SSRIs versus SSRIs plus mirtazapine

\begin{tabular}{|c|c|c|c|c|c|c|c|}
\hline Population & $\begin{array}{l}\text { Intervention } \\
\text { and } \\
\text { Comparator }\end{array}$ & Outcome & $\begin{array}{l}\text { Incidence or } \\
\text { Mean Effect } \\
\text { by Arm }\end{array}$ & Results & $\begin{array}{l}\text { Study Design } \\
\text { and Sample } \\
\text { Size }\end{array}$ & $\begin{array}{l}\text { Factors That Affect } \\
\text { the Strength of } \\
\text { Evidence }\end{array}$ & $\begin{array}{l}\text { Overall } \\
\text { Evidence } \\
\text { Strength } \\
\text { (Direction } \\
\text { of Effect) }\end{array}$ \\
\hline $\begin{array}{l}\text { Pregnant } \\
\text { women } \\
\text { exposed to } \\
\text { SSRIs or } \\
\text { mirtazapine }\end{array}$ & $\begin{array}{l}\text { SSRIs vs. } \\
\text { SSRIs plus } \\
\text { mirtazapine }\end{array}$ & Preterm birth & $\begin{array}{l}3 / 40(7.50 \%) \\
\text { vs. } 1 / 18 \\
(5.56 \%)^{95}\end{array}$ & $\begin{array}{l}\mathrm{RR}, 1.35 \\
(95 \% \mathrm{Cl} \\
0.15,12.11)^{95}\end{array}$ & $\begin{array}{l}1 \text { cohort, } \\
n=58^{95}\end{array}$ & $\begin{array}{l}\text { High study } \\
\text { limitations (high risk } \\
\text { of bias }{ }^{95} \text { ), imprecise } \\
\text { (wide Cls), } \\
\text { consistency } \\
\text { unknown }\end{array}$ & Insufficient \\
\hline $\begin{array}{l}\text { Pregnant } \\
\text { women } \\
\text { exposed to } \\
\text { SSRIs or } \\
\text { mirtazapine }\end{array}$ & $\begin{array}{l}\text { SSRIs vS. } \\
\text { SSRIs plus } \\
\text { mirtazapine }\end{array}$ & Late-term birth & $\begin{array}{l}0 / 40(0.00 \%) \\
\text { vs. } 2 / 18 \\
(11.11 \%)^{95}\end{array}$ & $\begin{array}{l}\mathrm{RR}, 0.09 \\
(95 \% \mathrm{Cl}, \\
0.005 \text { to } \\
1.84)^{95}\end{array}$ & $\begin{array}{l}1 \text { cohort, } \\
n=58^{95}\end{array}$ & $\begin{array}{l}\text { High study } \\
\text { limitations (high risk } \\
\text { of bias }{ }^{95} \text { ), imprecise } \\
\text { (wide Cls), } \\
\text { consistency } \\
\text { unknown }\end{array}$ & Insufficient \\
\hline $\begin{array}{l}\text { Pregnant } \\
\text { women } \\
\text { exposed to } \\
\text { SSRls or } \\
\text { mirtazapine }\end{array}$ & $\begin{array}{l}\text { SSRIs vS. } \\
\text { SSRIs plus } \\
\text { mirtazapine }\end{array}$ & $\begin{array}{l}\text { Low } \\
\text { birthweight }\end{array}$ & $\begin{array}{l}4 / 40(10.00 \%) \\
\text { vs. } 1 / 18 \\
(5.56 \%)^{95}\end{array}$ & $\begin{array}{l}\mathrm{RR}, \\
1.80(95 \% \mathrm{Cl}, \\
0.22 \text { to } \\
14.99)^{95}\end{array}$ & $\begin{array}{l}1 \text { cohort, } \\
\mathrm{n}=58^{95}\end{array}$ & $\begin{array}{l}\text { High study } \\
\text { limitations (high risk } \\
\text { of bias }{ }^{95} \text { ), imprecise } \\
\text { (wide Cls), } \\
\text { consistency } \\
\text { unknown } \\
\end{array}$ & Insufficient \\
\hline $\begin{array}{l}\text { Pregnant } \\
\text { women } \\
\text { exposed to } \\
\text { mirtazapine } \\
\text { or SSRIs } \\
\text { plus } \\
\text { mirtazapine } \\
\end{array}$ & $\begin{array}{l}\text { SSRIs vs. } \\
\text { SSRIs plus } \\
\text { mirtazapine }\end{array}$ & Birthweight & $\begin{array}{l}3,153.17 \mathrm{gm} \\
\text { (SD: } 435.17) \\
\mathrm{n}=40 \text { vs. } \\
3,191.38 \mathrm{gm} \\
(\mathrm{SD}: 436.69 \\
\mathrm{n}=18)^{95}\end{array}$ & $\begin{array}{l}\text { Mean } \\
\text { difference: } \\
-38.21(95 \% \\
\mathrm{Cl},-280.55 \text { to } \\
204.13)^{95}\end{array}$ & $\begin{array}{l}1 \text { cohort, } \\
n=58^{95}\end{array}$ & $\begin{array}{l}\text { High study } \\
\text { limitations (high risk } \\
\text { of bias }{ }^{95} \text { ), imprecise } \\
\text { (wide Cls), } \\
\text { consistency } \\
\text { unknown }\end{array}$ & Insufficient \\
\hline $\begin{array}{l}\text { Pregnant } \\
\text { women } \\
\text { exposed to } \\
\text { SSRIs or } \\
\text { mirtazapine }\end{array}$ & $\begin{array}{l}\text { SSRIs vs. } \\
\text { SSRIs plus } \\
\text { mirtazapine }\end{array}$ & NICU stay & $\begin{array}{l}7 / 40(17.50 \%) \\
\text { vs. } 3 / 18 \\
(16.67 \%)^{95}\end{array}$ & $\begin{array}{l}\mathrm{RR}, 1.05 \\
(95 \% \mathrm{Cl}, \\
0.31 \text { to } \\
3.60)^{95}\end{array}$ & $\begin{array}{l}1 \text { cohort, } \\
n=58^{95}\end{array}$ & $\begin{array}{l}\text { High study } \\
\text { limitations (high risk } \\
\text { of bias }{ }^{95} \text { ), imprecise } \\
\text { (wide Cls), } \\
\text { consistency } \\
\text { unknown }\end{array}$ & Insufficient \\
\hline $\begin{array}{l}\text { Pregnant } \\
\text { women } \\
\text { exposed to } \\
\text { SSRIs or } \\
\text { mirtazapine }\end{array}$ & $\begin{array}{l}\text { SSRIs vS. } \\
\text { SSRIs plus } \\
\text { mirtazapine }\end{array}$ & Macrosomia & $\begin{array}{l}2 / 40(5.00 \%) \\
\text { vs. } 1 / 18 \\
(5.56 \%)^{95}\end{array}$ & $\begin{array}{l}\mathrm{RR}, 0.9(95 \% \\
\mathrm{Cl}, 0.09 \text { to } \\
9.30)^{95}\end{array}$ & $\begin{array}{l}1 \text { cohort, } \\
n=58^{95}\end{array}$ & $\begin{array}{l}\text { High study } \\
\text { limitations (high risk } \\
\text { of bias }{ }^{95} \text { ), imprecise } \\
\text { (wide Cls), } \\
\text { consistency } \\
\text { unknown } \\
\end{array}$ & Insufficient \\
\hline $\begin{array}{l}\text { Pregnant } \\
\text { women } \\
\text { exposed to } \\
\text { SSRls or } \\
\text { mirtazapine }\end{array}$ & $\begin{array}{l}\text { SSRIs vS. } \\
\text { SSRIs plus } \\
\text { mirtazapine }\end{array}$ & $\begin{array}{l}\text { Neonatal } \\
\text { bilirubinemia }\end{array}$ & $\begin{array}{l}19 / 40(47.50 \%) \\
\text { VS. } 4 / 18 \\
(22.22 \%)^{95}\end{array}$ & $\begin{array}{l}\mathrm{RR}, 2.14 \\
(95 \% \mathrm{Cl}, \\
0.85 \text { to } \\
5.38)^{95}\end{array}$ & $\begin{array}{l}1 \text { cohort, } \\
n=58^{95}\end{array}$ & $\begin{array}{l}\text { High study } \\
\text { limitations (high risk } \\
\text { of bias }{ }^{95} \text { ), imprecise } \\
\text { (wide Cls), } \\
\text { consistency } \\
\text { unknown }\end{array}$ & Insufficient \\
\hline
\end{tabular}

$\overline{\mathrm{CI}}=$ confidence interval; $\mathrm{n}=$ number; $\mathrm{NICU}=$ neonatal intensive care unit; $\mathrm{SD}=$ standard deviation; SSRI = selective serotonin reuptake inhibitor; $\mathrm{RR}=$ relative risk; vs. $=$ versus. 


\section{SSRIs Versus MAOIs}

\section{Overview}

- The evidence is insufficient to judge the comparative risk of harms from SSRIs versus MAOI exposure during pregnancy for child outcomes (attention-deficit/hyperactivity disorder or autism spectrum disorder).

\section{Detailed Results}

Two publications from the Canadian QPC reported on the comparative harms from treatment with SSRIs versus MAOIs among pregnant women with psychiatric disorders (Table B-37) ${ }^{53,114}$ Child attention-deficit/hyperactivity disorder outcomes were reported in one publication, ${ }^{114}$ and autism spectrum disorder outcomes were reported in a second. ${ }^{53}$ Only one woman was included in the MAOI group, and the publications were rated as high risk of bias because the analyses did not control for confounding.

Table B-37. Strength of evidence for comparative harms: SSRIs versus MAOls

\begin{tabular}{|c|c|c|c|c|c|c|c|}
\hline Population & $\begin{array}{l}\text { Intervention } \\
\text { and } \\
\text { Comparator }\end{array}$ & Outcome & $\begin{array}{l}\text { Incidence } \\
\text { or Mean } \\
\text { Effect by } \\
\text { Arm }\end{array}$ & Results & $\begin{array}{l}\text { Study } \\
\text { Design } \\
\text { and } \\
\text { Sample } \\
\text { Size } \\
\end{array}$ & $\begin{array}{l}\text { Factors That } \\
\text { Affect the } \\
\text { Strength of } \\
\text { Evidence }\end{array}$ & $\begin{array}{l}\text { Overall } \\
\text { Evidence } \\
\text { Strength } \\
\text { (Direction of } \\
\text { Effect) } \\
\end{array}$ \\
\hline $\begin{array}{l}\text { Women with } \\
\text { psychiatric } \\
\text { disorders } \\
\text { during } \\
\text { pregnancy }\end{array}$ & $\begin{array}{l}\text { SSRIs vs. } \\
\text { MAOIs }\end{array}$ & ADHD & $\begin{array}{l}86 / 1,561 \\
(5.5 \%) \text { vs. } \\
0 / 1(0 \%)^{53}\end{array}$ & $\begin{array}{l}\mathrm{RD}, 0.055 \\
(95 \% \mathrm{Cl},-0.55 \\
\text { to } 0.66)^{53}\end{array}$ & $\begin{array}{l}1 \text { cohort } \\
n=1,562\end{array}$ & $\begin{array}{l}\text { High study } \\
\text { limitations (high } \\
\text { risk of bias, } 53 \\
\text { imprecise (wide } \\
\text { Cls), consistency } \\
\text { unknown }\end{array}$ & Insufficient \\
\hline $\begin{array}{l}\text { Women with } \\
\text { psychiatric } \\
\text { disorders } \\
\text { during } \\
\text { pregnancy }\end{array}$ & $\begin{array}{l}\text { SSRIs vs. } \\
\text { MAOIs }\end{array}$ & ASD & $\begin{array}{l}22 / 1,583 \\
(0.4 \%) \text { vs. } \\
0 / 1(0 \%)^{114}\end{array}$ & $\begin{array}{l}\mathrm{RD}, 0.014 \\
(95 \% \mathrm{Cl},-0.59 \\
\text { to } 0.61)\end{array}$ & $\begin{array}{l}1 \text { cohort } \\
n=1,583\end{array}$ & $\begin{array}{l}\text { High study } \\
\text { limitations (high } \\
\text { risk of bias, }{ }^{114} \\
\text { imprecise (wide } \\
\text { Cls), consistency } \\
\text { unknown }\end{array}$ & Insufficient \\
\hline
\end{tabular}

$\mathrm{ADHD}=$ attention-deficit/hyperactivity disorder; $\mathrm{ASD}=$ autism spectrum disorder; $\mathrm{CI}=$ confidence interval; $\mathrm{MAOI}=$ monoamine oxidase inhibitors; $\mathrm{n}=$ number; $\mathrm{RD}=$ risk difference; $\mathrm{SSRI}=$ selective serotonin reuptake inhibitor; vs. $=$ versus.

\section{Citalopram Versus Bupropion}

\section{Overview}

- The evidence is insufficient to judge the comparative risk of harms from citalopram versus bupropion exposure during pregnancy for child outcomes (congenital heart disease).

\section{Detailed Results}

One publication reported on the comparative harms of citalopram versus bupropion. ${ }^{141}$ The study drew from a cohort of women who had undergone a fetal echocardiogram because of in utero exposure of the fetus to SSRIs or SNRIs. ${ }^{141}$ The study was rated as high risk of bias. The study yielded insufficient strength of evidence to judge the risk of congenital heart disease (Table B-38). 
Table B-38. Strength of evidence for comparative harms: Citalopram versus bupropion

\begin{tabular}{|c|c|c|c|c|c|c|c|}
\hline Population & $\begin{array}{l}\text { Intervention } \\
\text { and } \\
\text { Comparator }\end{array}$ & Outcome & $\begin{array}{l}\text { Incidence } \\
\text { or Mean } \\
\text { Effect by } \\
\text { Arm }\end{array}$ & Results & $\begin{array}{l}\text { Study } \\
\text { Design } \\
\text { and } \\
\text { Sample } \\
\text { Size } \\
\end{array}$ & $\begin{array}{l}\text { Factors That } \\
\text { Affect the } \\
\text { Strength of } \\
\text { Evidence }\end{array}$ & $\begin{array}{l}\text { Overall } \\
\text { Evidence } \\
\text { Strength } \\
\text { (Direction of } \\
\text { Effect) }\end{array}$ \\
\hline $\begin{array}{l}\text { Women who } \\
\text { underwent fetal } \\
\text { echocardiography } \\
\text { because of an in- } \\
\text { utero exposure to } \\
\text { either SSRIs or } \\
\text { SNRIs }\end{array}$ & $\begin{array}{l}\text { Citalopram } \\
\text { vs. bupropion }\end{array}$ & $\begin{array}{l}\text { Congenital } \\
\text { heart } \\
\text { disease }\end{array}$ & $\begin{array}{l}2 / 5 \\
(40.00 \%) \\
\text { vs. } 0 / 3 \\
(0 \%)^{141}\end{array}$ & 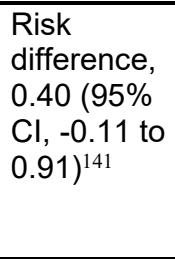 & $\begin{array}{l}1 \text { cohort, } \\
n=8^{141}\end{array}$ & $\begin{array}{l}\text { High study } \\
\text { limitations (high } \\
\text { risk of bias, } 141 \\
\text { imprecise (wide } \\
\text { Cls), } \\
\text { consistency } \\
\text { unknown }\end{array}$ & Insufficient \\
\hline
\end{tabular}

$\mathrm{CI}=$ confidence interval; $\mathrm{n}=$ number; $\mathrm{SNRI}=$ serotonin-norepinephrine reuptake inhibitor; SSRI $=$ selective serotonin reuptake inhibitor; vs. $=$ versus.

\section{Citalopram Versus Duloxetine}

\section{Overview}

- The evidence is insufficient to judge the comparative risk of harms from citalopram versus duloxetine exposure during pregnancy for child outcomes (congenital heart disease).

\section{Detailed Results}

One publication reported on the comparative harms of citalopram versus duloxetine. ${ }^{141}$ The study drew from a cohort of women who had undergone a fetal echocardiogram because of in utero exposure of the fetus to SSRIs or SNRIs. ${ }^{141}$ The study was rated as high risk of bias. The study yielded insufficient strength of evidence to judge the risk of congenital heart disease (Table B-39).

Table B-39. Strength of evidence for comparative harms: Citalopram versus duloxetine

\begin{tabular}{|c|c|c|c|c|c|c|c|}
\hline Population & $\begin{array}{l}\text { Intervention } \\
\text { and } \\
\text { Comparator }\end{array}$ & Outcome & $\begin{array}{l}\text { Incidence } \\
\text { or Mean } \\
\text { Effect by } \\
\text { Arm }\end{array}$ & Results & $\begin{array}{l}\text { Study } \\
\text { Design } \\
\text { and } \\
\text { Sample } \\
\text { Size }\end{array}$ & $\begin{array}{l}\text { Factors That } \\
\text { Affect the } \\
\text { Strength of } \\
\text { Evidence }\end{array}$ & $\begin{array}{l}\text { Overall } \\
\text { Evidence } \\
\text { Strength } \\
\text { (Direction } \\
\text { of Effect) }\end{array}$ \\
\hline $\begin{array}{l}\text { Women who } \\
\text { underwent fetal } \\
\text { echocardiography } \\
\text { because of an in-utero } \\
\text { exposure to either } \\
\text { SSRIs or SNRIs }\end{array}$ & $\begin{array}{l}\text { Citalopram } \\
\text { vs. duloxetine }\end{array}$ & $\begin{array}{l}\text { Congenital } \\
\text { heart } \\
\text { disease }\end{array}$ & $\begin{array}{l}2 / 5 \\
(40.00 \%) \\
\text { vs. 0/1 } \\
(0 \%)^{141}\end{array}$ & $\begin{array}{l}\text { Risk } \\
\text { difference, } \\
0.40(95 \% \\
\mathrm{Cl},-0.32 \text { to } \\
1.12)^{141}\end{array}$ & $\begin{array}{l}1 \text { cohort, } \\
n=4^{141}\end{array}$ & $\begin{array}{l}\text { High study } \\
\text { limitations (high } \\
\text { risk of bias, } 141 \\
\text { imprecise (wide } \\
\text { Cls), } \\
\text { consistency } \\
\text { unknown }\end{array}$ & Insufficient \\
\hline
\end{tabular}

$\mathrm{CI}=$ confidence interval; $\mathrm{n}=$ number; $\mathrm{SNRI}=$ serotonin-norepinephrine reuptake inhibitor; SSRI = selective serotonin reuptake inhibitor; vs. $=$ versus.

\section{Citalopram Versus Escitalopram}

\section{Overview}

- The evidence is insufficient to judge the comparative risk of harms from citalopram versus escitalopram exposure during pregnancy for child outcomes (congenital heart disease). 


\section{Detailed Results}

One publication reported on the comparative harms of citalopram versus escitalopram. ${ }^{141}$ The study drew from a cohort of women who had undergone a fetal echocardiogram because of in utero exposure of the fetus to SSRIs or SNRIs. ${ }^{141}$ The study was rated as high risk of bias. The study yielded insufficient strength of evidence to judge the risk of congenital heart disease (Table B-40).

Table B-40. Strength of evidence for comparative harms: Citalopram versus escitalopram

\begin{tabular}{|c|c|c|c|c|c|c|c|}
\hline Population & $\begin{array}{l}\text { Intervention } \\
\text { and } \\
\text { Comparator }\end{array}$ & Outcome & $\begin{array}{l}\text { Incidence } \\
\text { or Mean } \\
\text { Effect by } \\
\text { Arm }\end{array}$ & Results & $\begin{array}{l}\text { Study } \\
\text { Design } \\
\text { and } \\
\text { Sample } \\
\text { Size } \\
\end{array}$ & $\begin{array}{l}\text { Factors That } \\
\text { Affect the } \\
\text { Strength of } \\
\text { Evidence }\end{array}$ & $\begin{array}{l}\text { Overall } \\
\text { Evidence } \\
\text { Strength } \\
\text { (Direction of } \\
\text { Effect) }\end{array}$ \\
\hline $\begin{array}{l}\text { Women who } \\
\text { underwent fetal } \\
\text { echocardiography } \\
\text { because of } \\
\text { an in utero exposure } \\
\text { to either SSRIs or } \\
\text { SNRIs }\end{array}$ & $\begin{array}{l}\text { Citalopram } \\
\text { vs. } \\
\text { escitalopram } \\
\end{array}$ & $\begin{array}{l}\text { Congenital } \\
\text { heart } \\
\text { disease }\end{array}$ & $\begin{array}{l}2 / 5(40.00 \%) \\
\text { vs. } 1 / 9 \\
(11.11 \%)^{141}\end{array}$ & $\begin{array}{l}\text { RR, 3.60 } \\
(95 \% \mathrm{Cl}, \\
0.42 \text { to } \\
30.51)^{141}\end{array}$ & $\begin{array}{l}1 \text { cohort, } \\
n=14^{141}\end{array}$ & $\begin{array}{l}\text { High study } \\
\text { limitations (high } \\
\text { risk of bias, } 141 \\
\text { imprecise ( wide } \\
\text { Cls), consistency } \\
\text { unknown }\end{array}$ & Insufficient \\
\hline
\end{tabular}

\section{Citalopram Versus Fluvoxamine}

\section{Overview}

- The evidence is insufficient to judge the risk of child outcomes (specifically, autism spectrum disorder) when comparing exposure during pregnancy to citalopram or fluvoxamine during pregnancy.

\section{Detailed Results}

One high risk-of-bias publication reported on child harms, specifically autism spectrum disorder, of citalopram exposure compared with fluvoxamine exposure during pregnancy. ${ }^{111}$ The publication drew from the QPC in Canada. ${ }^{111}$ The study provided imprecise results on the risk of autism spectrum disorder; the evidence was rated as insufficient (Table B-41).

Table B-41. Strength of evidence for comparative harms: Citalopram versus fluvoxamine

\begin{tabular}{|c|c|c|c|c|c|c|c|}
\hline Population & $\begin{array}{l}\text { Intervention and } \\
\text { Comparator }\end{array}$ & Outcome & $\begin{array}{l}\text { Incidence or } \\
\text { Mean Effect } \\
\text { by Arm }\end{array}$ & Results & $\begin{array}{l}\text { Study } \\
\text { Design } \\
\text { and } \\
\text { Sample } \\
\text { Size }\end{array}$ & $\begin{array}{l}\text { Factors } \\
\text { That Affect } \\
\text { the } \\
\text { Strength of } \\
\text { Evidence }\end{array}$ & $\begin{array}{l}\text { Overall } \\
\text { Evidence } \\
\text { Strength } \\
\text { (Direction } \\
\text { of Effect) }\end{array}$ \\
\hline $\begin{array}{l}\text { Pregnant } \\
\text { women in } \\
\text { second or third } \\
\text { trimester } \\
\text { exposed to } \\
\text { citalopram or } \\
\text { fluvoxamine }{ }^{111}\end{array}$ & $\begin{array}{l}\text { Maternal } \\
\text { exposure to } \\
\text { citalopram vs. } \\
\text { maternal } \\
\text { exposure to } \\
\text { fluvoxamine }\end{array}$ & $\begin{array}{l}\text { Autism } \\
\text { spectrum } \\
\text { disorder } \\
\text { among } \\
\text { offspring }\end{array}$ & $\begin{array}{l}5 / 421(1.2 \%) \\
\text { vs. } 1 / 135 \\
(2.9 \%)^{111}\end{array}$ & $\begin{array}{l}\text { RR, } 0.42 \\
(95 \% \text { Cl, } \\
0.005 \text { to } \\
3.46)^{111}\end{array}$ & $\begin{array}{l}1 \text { cohort, } \\
N=556\end{array}$ & $\begin{array}{l}\text { High study } \\
\text { limitations } \\
\text { (high risk of } \\
\text { bias }^{111} \text { ), } \\
\text { imprecise, } \\
\text { consistency } \\
\text { unknown }\end{array}$ & Insufficient \\
\hline
\end{tabular}

$\overline{\mathrm{CI}}=$ confidence interval; $\mathrm{N}=$ number; $\mathrm{RR}=$ relative risk; vs. $=$ versus. 


\section{Citalopram Versus Fluoxetine}

\section{Overview}

- The evidence is insufficient to judge the risk of child outcomes (congenital heart disease, autism spectrum disorder) when comparing exposure during pregnancy to citalopram or fluoxetine during pregnancy.

\section{Detailed Results}

One publication reported on the comparative harms of citalopram versus fluoxetine. ${ }^{141}$ The study drew from a cohort of women who had undergone a fetal echocardiogram because of in utero exposure of the fetus to SSRIs or SNRIs. ${ }^{141}$ The study was rated as high risk of bias. The study yielded insufficient strength of evidence to judge the risk of congenital heart disease

Two high risk-of-bias publications reported on child harms, specifically autism spectrum disorder, of citalopram exposure versus fluoxetine exposure during pregnancy. ${ }^{111,117}$ These studies both drew from large cohorts including Danish National registries ${ }^{117}$ and the QPC in Canada. ${ }^{111}$ Two studies provided inconsistent and imprecise results on the risk of autism spectrum disorder; the evidence was rated as insufficient (Table B-42).

Table B-42. Strength of evidence for comparative harms: Citalopram versus fluoxetine

\begin{tabular}{|c|c|c|c|c|c|c|c|}
\hline Population & $\begin{array}{l}\text { Intervention and } \\
\text { Comparator }\end{array}$ & Outcome & $\begin{array}{l}\text { Incidence or } \\
\text { Mean Effect } \\
\text { by Arm }\end{array}$ & Results & $\begin{array}{l}\text { Study } \\
\text { Design } \\
\text { and } \\
\text { Sample } \\
\text { Size }\end{array}$ & $\begin{array}{l}\text { Factors That } \\
\text { Affect the } \\
\text { Strength of } \\
\text { Evidence }\end{array}$ & $\begin{array}{l}\text { Overall } \\
\text { Evidence } \\
\text { Strength } \\
\text { (Direction } \\
\text { of Effect) }\end{array}$ \\
\hline $\begin{array}{l}\text { Women who } \\
\text { underwent fetal } \\
\text { echocardio- } \\
\text { graphy } \\
\text { because of an } \\
\text { in utero } \\
\text { exposure to } \\
\text { either SSRIs or } \\
\text { SNRIs }\end{array}$ & $\begin{array}{l}\text { Citalopram vs } \\
\text { fluoxetine. }\end{array}$ & $\begin{array}{l}\text { Congenital } \\
\text { heart disease }\end{array}$ & $\begin{array}{l}2 / 5(40.00 \%) \\
\text { vs. } 2 / 9 \\
(22.22 \%)^{141}\end{array}$ & $\begin{array}{l}\text { RR, } 1.80 \\
(95 \% \mathrm{Cl}, 0.35 \\
\text { to } 9.16)^{141}\end{array}$ & $\begin{array}{l}1 \text { cohort, } \\
n=14^{141}\end{array}$ & $\begin{array}{l}\text { High study } \\
\text { limitations } \\
\text { (high risk of } \\
\text { bias, }{ }^{141} \\
\text { imprecise } \\
\text { (wide Cls), } \\
\text { consistency } \\
\text { unknown }\end{array}$ & Insufficient \\
\hline $\begin{array}{l}\text { Pregnant } \\
\text { women in } \\
\text { second or third } \\
\text { trimester }{ }^{111} \text { or } \\
\text { during } \\
\text { pregnancy }{ }^{117} \\
\text { exposed to } \\
\text { citalopram or } \\
\text { fluoxetine }\end{array}$ & $\begin{array}{l}\text { Maternal } \\
\text { exposure to } \\
\text { citalopram vs. } \\
\text { maternal } \\
\text { exposure to } \\
\text { fluoxetine }\end{array}$ & $\begin{array}{l}\text { Autism } \\
\text { spectrum } \\
\text { disorder } \\
\text { among } \\
\text { offspring }\end{array}$ & $\begin{array}{l}5 / 421(1.2 \%) \\
\text { vs. } 5 / 171 \\
(2.9 \%) ; 111 \\
14 / 1,751 \\
(0.8 \%) \text { vs. } \\
18 / 160 \\
(11.3 \%)^{117}\end{array}$ & $\begin{array}{l}\text { RR, } 0.41 \\
(95 \% \mathrm{Cl}, 0.11 \\
\text { to } 1.4) ;{ }^{111} \mathrm{RR}, \\
7.1(95 \% \mathrm{Cl}, \\
0.036 \text { to } \\
0.14)^{117}\end{array}$ & $\begin{array}{l}2 \text { cohorts, } \\
\mathrm{N}=2,503\end{array}$ & $\begin{array}{l}\text { High study } \\
\text { limitations } \\
\text { (high risk of } \\
\text { bias }^{111,117} \text { ), } \\
\text { imprecise, } \\
\text { inconsistent }\end{array}$ & Insufficient \\
\hline
\end{tabular}

\section{Citalopram Versus Paroxetine}

\section{Overview}

- The evidence is insufficient to judge the risk of child outcomes (specifically, autism spectrum disorder and congenital heart disease) when comparing exposure during pregnancy to citalopram or paroxetine during pregnancy. 


\section{Detailed Results}

Two high risk-of-bias publications reported on child harms, specifically autism spectrum disorder, of citalopram exposure compared with paroxetine exposure during pregnancy. ${ }^{111,117}$ These studies both drew from large cohorts, including Danish National registries ${ }^{117}$ and the QPC in Canada. ${ }^{111}$ Two studies provided consistent but imprecise results and had high study limitations; the evidence was rated as insufficient (Table B-43). One small high risk-of-bias study reported on congenital heart disease; the evidence was rated as insufficient.

Table B-43. Strength of evidence for comparative harms: Citalopram versus paroxetine

\begin{tabular}{|c|c|c|c|c|c|c|c|}
\hline Population & $\begin{array}{l}\text { Intervention and } \\
\text { Comparator }\end{array}$ & Outcome & $\begin{array}{l}\text { Incidence or } \\
\text { Mean Effect } \\
\text { by Arm }\end{array}$ & Results & $\begin{array}{l}\text { Study } \\
\text { Design } \\
\text { and } \\
\text { Sample } \\
\text { Size }\end{array}$ & $\begin{array}{l}\text { Factors That } \\
\text { Affect the } \\
\text { Strength of } \\
\text { Evidence }\end{array}$ & $\begin{array}{l}\text { Overall } \\
\text { Evidence } \\
\text { Strength } \\
\text { (Direction } \\
\text { of Effect) }\end{array}$ \\
\hline $\begin{array}{l}\text { Pregnant women } \\
\text { in second or third } \\
\text { trimester }{ }^{111} \text { or } \\
\text { during } \\
\text { pregnancy }{ }^{117} \\
\text { exposed to } \\
\text { citalopram or } \\
\text { paroxetine } \\
\end{array}$ & $\begin{array}{l}\text { Maternal } \\
\text { exposure to } \\
\text { citalopram vs. } \\
\text { maternal } \\
\text { exposure to } \\
\text { paroxetine }\end{array}$ & $\begin{array}{l}\text { Autism } \\
\text { spectrum } \\
\text { disorder } \\
\text { among } \\
\text { offspring }\end{array}$ & $\begin{array}{l}5 / 421(1.2 \%) \\
\text { vs. } 11 / 744 \\
(1.5 \%) ; 11 \\
14 / 1,751 \\
(0.8 \%) \text { vs. } \\
7 / 871 \\
(0.8 \%)^{117}\end{array}$ & $\begin{array}{l}\text { RR, } 0.80(95 \% \\
\mathrm{Cl}, 0.28 \text { to } \\
2.3) ;{ }^{111} \mathrm{RR}, \\
0.99(95 \% \mathrm{Cl}, \\
0.4 \text { to } 2.5)^{117}\end{array}$ & $\begin{array}{l}2 \text { cohorts, } \\
N=3,787\end{array}$ & $\begin{array}{l}\text { High study } \\
\text { limitations } \\
\text { (high risk of } \\
\text { bias }^{117} \text { ), } \\
\text { imprecise, } \\
\text { consistent }\end{array}$ & Insufficient \\
\hline $\begin{array}{l}\text { Women who } \\
\text { underwent fetal } \\
\text { echocardiography } \\
\text { because of an in } \\
\text { utero exposure to } \\
\text { either SSRIs or } \\
\text { SNRIs }\end{array}$ & $\begin{array}{l}\text { Citalopram vs. } \\
\text { paroxetine }\end{array}$ & $\begin{array}{l}\text { Congenital } \\
\text { heart disease }\end{array}$ & $\begin{array}{l}2 / 5(40.00 \%) \\
\text { vs. } 0 / 1\end{array}$ & $\begin{array}{l}\text { Risk difference } \\
0.40(95 \% \mathrm{Cl} \text {, } \\
-0.32 \text { to } 1.12^{141}\end{array}$ & $\begin{array}{l}1 \text { cohort, } \\
n=6^{141}\end{array}$ & $\begin{array}{l}\text { High study } \\
\text { limitations } \\
\text { (high risk of } \\
\text { bias, }{ }^{141} \\
\text { imprecise } \\
\text { (wide Cls), } \\
\text { consistency } \\
\text { unknown }\end{array}$ & Insufficient \\
\hline
\end{tabular}

$\mathrm{CI}=$ confidence interval; $\mathrm{n}=$ number; $\mathrm{RR}=$ relative risk; $\mathrm{SNRI}=$ serotonin-norepinephrine reuptake inhibitor; $\mathrm{SSRI}=$ selective serotonin reuptake inhibitor; vs. $=$ versus.

\section{Citalopram Versus Sertraline}

\section{Overview}

- The evidence is insufficient to judge the risk of child outcomes (specifically, autism spectrum disorder and congenital heart disease) when comparing exposure during pregnancy to citalopram or sertraline during pregnancy.

\section{Detailed Results}

Two high risk-of-bias publications reported on child harms, specifically autism spectrum disorder, of citalopram exposure compared with sertraline exposure during pregnancy. ${ }^{111,117}$ These studies both drew from large cohorts, including Danish National registries ${ }^{117}$ and the QPC in Canada. ${ }^{111}$ Two studies provided consistent but imprecise results and had high study limitations; the evidence was rated as insufficient (Table B-44). One small high risk-of-bias study reported on congenital heart disease; the evidence was rated as insufficient. 
Table B-44. Strength of evidence for comparative harms: Citalopram versus sertraline

\begin{tabular}{|c|c|c|c|c|c|c|c|}
\hline Population & $\begin{array}{l}\text { Intervention and } \\
\text { Comparator }\end{array}$ & Outcome & $\begin{array}{l}\text { Incidence or } \\
\text { Mean Effect } \\
\text { by Arm }\end{array}$ & Results & $\begin{array}{l}\text { Study } \\
\text { Design } \\
\text { and } \\
\text { Sample } \\
\text { Size }\end{array}$ & $\begin{array}{l}\text { Factors That } \\
\text { Affect the } \\
\text { Strength of } \\
\text { Evidence }\end{array}$ & $\begin{array}{l}\text { Overall } \\
\text { Evidence } \\
\text { Strength } \\
\text { (Direction } \\
\text { of Effect) }\end{array}$ \\
\hline $\begin{array}{l}\text { Pregnant women } \\
\text { in second or third } \\
\text { trimester }^{111} \text { or } \\
\text { during } \\
\text { pregnancy }{ }^{117} \\
\text { exposed to } \\
\text { citalopram or } \\
\text { sertraline }\end{array}$ & $\begin{array}{l}\text { Maternal } \\
\text { exposure to } \\
\text { citalopram vs. } \\
\text { maternal } \\
\text { exposure to } \\
\text { sertraline }\end{array}$ & $\begin{array}{l}\text { Autism } \\
\text { spectrum } \\
\text { disorder } \\
\text { among } \\
\text { offspring }\end{array}$ & $\begin{array}{l}5 / 421(1.2 \%) \\
\text { vs. } 1 / 292 \\
(0.34 \%) ; 111 \\
14 / 1,751 \\
(0.8 \%) \text { vs. } \\
9 / 1,576 \\
(0.57 \%)^{117}\end{array}$ & $\begin{array}{l}\text { RR, 3.5 (95\% } \\
\text { Cl, 0.41 to } \\
29.5)^{111} \\
\text { RR, } 1.4(95 \% \\
\text { Cl, } 0.61 \text { to } \\
3.2)^{117}\end{array}$ & $\begin{array}{l}2 \\
\text { cohorts, } \\
N=4,040\end{array}$ & $\begin{array}{l}\text { High study } \\
\text { limitations } \\
\text { (high risk of } \\
\text { bias }^{111,117} \text { ), } \\
\text { imprecise, } \\
\text { consistent }\end{array}$ & Insufficient \\
\hline $\begin{array}{l}\text { Women who } \\
\text { underwent fetal } \\
\text { echocardiography } \\
\text { because of an in } \\
\text { utero exposure to } \\
\text { either SSRIs or } \\
\text { SNRIs }\end{array}$ & $\begin{array}{l}\text { Citalopram vs. } \\
\text { sertraline }\end{array}$ & $\begin{array}{l}\text { Congenital } \\
\text { heart disease }\end{array}$ & $\begin{array}{l}2 / 5(40.00 \%) \\
\text { vs. } 0 / 7(0 \%)^{141}\end{array}$ & $\begin{array}{l}\text { Risk difference, } \\
0.40(95 \% \mathrm{Cl} \\
-0.03 \text { to } 0.83^{141}\end{array}$ & $\begin{array}{l}1 \text { cohort, } \\
\mathrm{n}=12^{141}\end{array}$ & $\begin{array}{l}\text { High study } \\
\text { limitations } \\
\text { (high risk of } \\
\text { bias, }{ }^{141} \\
\text { imprecise } \\
\text { (wide Cls), } \\
\text { consistency } \\
\text { unknown }\end{array}$ & Insufficient \\
\hline
\end{tabular}

$\mathrm{CI}=$ confidence interval; $\mathrm{n}=$ number; $\mathrm{SNRI}=$ serotonin-norepinephrine reuptake inhibitor; SSRI = selective serotonin reuptake inhibitor; $\mathrm{RR}=$ relative risk; vs. $=$ versus.

\section{Citalopram Versus Venlafaxine}

\section{Overview}

- The evidence is insufficient to judge the comparative risk of harms from exposure during pregnancy to citalopram versus venlafaxine for child outcomes (congenital heart disease).

\section{Detailed Results}

One publication reported on the comparative harms of citalopram versus venlafaxine. ${ }^{141}$ The study drew from a cohort of women who had undergone a fetal echocardiogram because of in utero exposure of the fetus to SSRIs or SNRIs. ${ }^{141}$ The study was rated as high risk of bias. The study yielded insufficient strength of evidence to judge the risk of congenital heart disease (Table B-45).

Table B-45. Strength of evidence for comparative harms: Citalopram versus venlafaxine

\begin{tabular}{|c|c|c|c|c|c|c|c|}
\hline Population & $\begin{array}{l}\text { Intervention } \\
\text { and } \\
\text { Comparator }\end{array}$ & Outcome & $\begin{array}{l}\text { Incidence } \\
\text { or Mean } \\
\text { Effect by } \\
\text { Arm }\end{array}$ & Results & $\begin{array}{l}\text { Study } \\
\text { Design } \\
\text { and } \\
\text { Sample } \\
\text { Size } \\
\end{array}$ & $\begin{array}{l}\text { Factors That } \\
\text { Affect the } \\
\text { Strength of } \\
\text { Evidence }\end{array}$ & $\begin{array}{l}\text { Overall } \\
\text { Evidence } \\
\text { Strength } \\
\text { (Direction of } \\
\text { Effect) }\end{array}$ \\
\hline $\begin{array}{l}\text { Women who underwent } \\
\text { fetal echocardiography } \\
\text { because of an in utero } \\
\text { exposure to either } \\
\text { SSRIs or SNRIs }\end{array}$ & $\begin{array}{l}\text { Citalopram } \\
\text { vs. } \\
\text { venlafaxine }\end{array}$ & $\begin{array}{l}\text { Congenital } \\
\text { heart } \\
\text { disease }\end{array}$ & $\begin{array}{l}2 / 5 \\
(40.00 \%) \\
\text { vs. } 2 / 2 \\
(100 \%)^{141}\end{array}$ & $\begin{array}{l}\text { RR, } 0.50 \\
(95 \% \mathrm{Cl}, \\
0.17 \text { to } \\
1.46)^{141}\end{array}$ & $\begin{array}{l}1 \text { cohort, } \\
N=7^{141}\end{array}$ & $\begin{array}{l}\text { High study } \\
\text { limitations (high } \\
\text { risk of bias, }{ }^{141} \\
\text { imprecise (wide } \\
\text { Cls), } \\
\text { consistency } \\
\text { unknown }\end{array}$ & Insufficient \\
\hline
\end{tabular}

$\overline{\mathrm{CI}}=$ confidence interval; $\mathrm{N}=$ number; SNRI = serotonin-norepinephrine reuptake inhibitor; SSRI = selective serotonin reuptake inhibitor; $\mathrm{RR}=$ relative risk; vs. $=$ versus. 


\section{Escitalopram Versus Bupropion}

\section{Overview}

- The evidence is insufficient to judge the comparative risk of harms from exposure to escitalopram versus bupropion during pregnancy for child outcomes (congenital heart disease).

\section{Detailed Results}

One publication reported on the comparative harms of escitalopram versus bupropion. ${ }^{141}$ The study drew from a cohort of women who had undergone a fetal echocardiogram because of in utero exposure of the fetus to SSRIs or SNRIs. ${ }^{141}$ The study was rated as high risk of bias. The study yielded insufficient strength of evidence to judge the risk of congenital heart disease (Table B-46).

Table B-46. Strength of evidence for comparative harms: Escitalopram versus bupropion

\begin{tabular}{|c|c|c|c|c|c|c|c|}
\hline Population & $\begin{array}{l}\text { Intervention } \\
\text { and } \\
\text { Comparator }\end{array}$ & Outcome & $\begin{array}{l}\text { Incidence } \\
\text { or Mean } \\
\text { Effect by } \\
\text { Arm }\end{array}$ & Results & $\begin{array}{l}\text { Study } \\
\text { Design } \\
\text { and } \\
\text { Sample } \\
\text { Size }\end{array}$ & $\begin{array}{l}\text { Factors That } \\
\text { Affect the } \\
\text { Strength of } \\
\text { Evidence }\end{array}$ & $\begin{array}{l}\text { Overall } \\
\text { Evidence } \\
\text { Strength } \\
\text { (Direction } \\
\text { of Effect) }\end{array}$ \\
\hline $\begin{array}{l}\text { Women who } \\
\text { underwent fetal } \\
\text { echocardiography } \\
\text { because of an in utero } \\
\text { exposure to either } \\
\text { SSRIs or SNRIs }\end{array}$ & $\begin{array}{l}\text { Escitalopram } \\
\text { vs. bupropion }\end{array}$ & $\begin{array}{l}\text { Congenital } \\
\text { heart } \\
\text { disease }\end{array}$ & $\begin{array}{l}1 / 9 \\
(11.11 \%) \\
\text { vs. } 0 / 3 \\
(0.00 \%)^{141}\end{array}$ & $\begin{array}{l}\text { Risk } \\
\text { difference, } \\
0.11(95 \% \\
\mathrm{Cl},-0.28 \text { to } \\
0.50)^{141}\end{array}$ & $\begin{array}{l}1 \text { cohort, } \\
n=12^{141}\end{array}$ & $\begin{array}{l}\text { High study } \\
\text { limitations (high } \\
\text { risk of bias, }{ }^{141} \\
\text { imprecise (wide } \\
\text { Cls), } \\
\text { consistency } \\
\text { unknown }\end{array}$ & Insufficient \\
\hline
\end{tabular}

$\mathrm{CI}=$ confidence interval; $\mathrm{n}=$ number; $\mathrm{SNRI}=$ serotonin-norepinephrine reuptake inhibitor; SSRI = selective serotonin reuptake inhibitor; vs. $=$ versus.

\section{Escitalopram Versus Paroxetine}

\section{Overview}

- The evidence is insufficient to judge the risk of harms for child outcomes (congenital heart disease) for exposure to escitalopram versus paroxetine during pregnancy.

\section{Detailed Results}

One publication reported on the comparative harms of paroxetine versus escitalopram (Table B-47). ${ }^{141}$ The study drew from a cohort of women who had undergone a fetal echocardiogram because of in utero exposure of the fetus to SSRIs or SNRIs. ${ }^{141}$ The study was rated as high risk of bias. The study yielded insufficient strength of evidence to judge the risk of congenital heart disease. 
Table B-47. Strength of evidence for comparative harms: Escitalopram versus paroxetine

\begin{tabular}{|c|c|c|c|c|c|c|c|}
\hline Population & $\begin{array}{l}\text { Intervention } \\
\text { and } \\
\text { Comparator }\end{array}$ & Outcome & $\begin{array}{l}\text { Incidence or } \\
\text { Mean Effect } \\
\text { by Arm }\end{array}$ & Results & $\begin{array}{l}\text { Study Design and } \\
\text { Sample Size }\end{array}$ & $\begin{array}{l}\text { Factors That } \\
\text { Affect the } \\
\text { Strength of } \\
\text { Evidence }\end{array}$ & $\begin{array}{l}\text { Overall } \\
\text { Evidence } \\
\text { Strength } \\
\text { (Direction } \\
\text { of Effect) }\end{array}$ \\
\hline $\begin{array}{l}\text { Women who } \\
\text { underwent fetal } \\
\text { echocardiography } \\
\text { because of an in } \\
\text { utero exposure to } \\
\text { either SSRIs or } \\
\text { SNRIs center }\end{array}$ & $\begin{array}{l}\text { Escitalopram } \\
\text { vs. } \\
\text { paroxetine }\end{array}$ & $\begin{array}{l}\text { Congenital } \\
\text { heart } \\
\text { disease }\end{array}$ & $\begin{array}{l}1 / 9(11 \%) \text { vs. } \\
0 / 1(0 \%)^{141}\end{array}$ & $\begin{array}{l}\text { Risk } \\
\text { difference } \\
0.11(95 \% \\
\mathrm{Cl},-0.53- \\
0.75)^{141}\end{array}$ & $\begin{array}{l}1 \text { cohort study, } \\
\mathrm{N}=10^{141}\end{array}$ & $\begin{array}{l}\text { High study } \\
\text { limitations } \\
\text { (high risk of } \\
\text { bias, }{ }^{141} \\
\text { imprecise } \\
\text { (wide Cls), } \\
\text { consistency } \\
\text { unknown }\end{array}$ & Insufficient \\
\hline
\end{tabular}

$\mathrm{CI}=$ confidence interval; $\mathrm{N}=$ number; SNRI = serotonin-norepinephrine reuptake inhibitor; SSRI = selective serotonin reuptake inhibitor; vs. $=$ versus.

\section{Escitalopram Versus Sertraline}

\section{Overview}

- The evidence is insufficient to judge the comparative risk of harms from exposure to escitalopram versus sertraline during pregnancy for child outcomes (congenital heart disease).

\section{Detailed Results}

One publication reported on the comparative harms of escitalopram versus sertraline. ${ }^{141}$ The study drew from a cohort of women who had undergone a fetal echocardiogram because of in utero exposure of the fetus to SSRIs or SNRIs. ${ }^{141}$ The study was rated as high risk of bias. The study yielded insufficient strength of evidence to judge the risk of congenital heart disease (Table B-48).

Table B-48. Strength of evidence for comparative harms: Escitalopram versus sertraline

\begin{tabular}{|c|c|c|c|c|c|c|c|}
\hline Population & $\begin{array}{l}\text { Intervention } \\
\text { and } \\
\text { Comparator }\end{array}$ & Outcome & $\begin{array}{l}\text { Incidence } \\
\text { or Mean } \\
\text { Effect by } \\
\text { Arm }\end{array}$ & Results & $\begin{array}{l}\text { Study } \\
\text { Design } \\
\text { and } \\
\text { Sample } \\
\text { Size } \\
\end{array}$ & $\begin{array}{l}\text { Factors That } \\
\text { Affect the } \\
\text { Strength of } \\
\text { Evidence }\end{array}$ & $\begin{array}{l}\text { Overall } \\
\text { Evidence } \\
\text { Strength } \\
\text { (Direction of } \\
\text { Effect) }\end{array}$ \\
\hline $\begin{array}{l}\text { Women who } \\
\text { underwent fetal } \\
\text { echocardiography } \\
\text { because of an in } \\
\text { utero exposure to } \\
\text { either SSRIs or } \\
\text { SNRIs }\end{array}$ & $\begin{array}{l}\text { Escitalopram } \\
\text { vs. sertraline }\end{array}$ & $\begin{array}{l}\text { Congenital } \\
\text { heart } \\
\text { disease }\end{array}$ & $\begin{array}{l}1 / 9 \\
(11.11 \%) \\
\text { vs. } 0 / 7 \\
(0.00 \%)^{141}\end{array}$ & 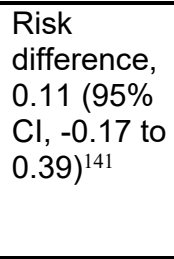 & $\begin{array}{l}1 \text { cohort, } \\
n=16^{141}\end{array}$ & $\begin{array}{l}\text { High study } \\
\text { limitations (high } \\
\text { risk of bias, } 141 \\
\text { imprecise (wide } \\
\text { Cls), } \\
\text { consistency } \\
\text { unknown }\end{array}$ & Insufficient \\
\hline
\end{tabular}

$\overline{\mathrm{CI}}=$ confidence interval; $\mathrm{n}=$ number; SNRI $=$ serotonin-norepinephrine reuptake inhibitor; SSRI $=$ selective serotonin reuptake inhibitor; vs. = versus.

\section{Escitalopram Versus Duloxetine}

\section{Overview}

- The evidence is insufficient to judge the comparative risk of harms from exposure to escitalopram versus duloxetine during pregnancy for child outcomes (congenital heart disease). 


\section{Detailed Results}

One publication reported on the comparative harms of escitalopram versus duloxetine. ${ }^{141}$ The study drew from a cohort of women who had undergone a fetal echocardiogram because of in utero exposure of the fetus to SSRIs or SNRIs. ${ }^{141}$ The study was rated as high risk of bias. The study yielded insufficient strength of evidence to judge the risk of congenital heart disease (Table B-49).

Table B-49. Strength of evidence for comparative harms: Escitalopram versus duloxetine

\begin{tabular}{|c|c|c|c|c|c|c|c|}
\hline Population & $\begin{array}{l}\text { Intervention } \\
\text { and } \\
\text { Comparator }\end{array}$ & Outcome & $\begin{array}{l}\text { Incidence } \\
\text { or Mean } \\
\text { Effect by } \\
\text { Arm }\end{array}$ & Results & $\begin{array}{l}\text { Study } \\
\text { Design } \\
\text { and } \\
\text { Sample } \\
\text { Size }\end{array}$ & $\begin{array}{l}\text { Factors That } \\
\text { Affect the } \\
\text { Strength of } \\
\text { Evidence }\end{array}$ & $\begin{array}{l}\text { Overall } \\
\text { Evidence } \\
\text { Strength } \\
\text { (Direction } \\
\text { of Effect) }\end{array}$ \\
\hline $\begin{array}{l}\text { Women who } \\
\text { underwent fetal } \\
\text { echocardiography } \\
\text { because of an in utero } \\
\text { exposure to either } \\
\text { SSRIs or SNRIs }\end{array}$ & $\begin{array}{l}\text { Escitalopram } \\
\text { vs. duloxetine }\end{array}$ & $\begin{array}{l}\text { Congenital } \\
\text { heart } \\
\text { disease }\end{array}$ & $\begin{array}{l}1 / 9 \\
(11.11 \%) \\
\text { vs. } 0 / 1 \\
(0.00 \%)^{141}\end{array}$ & $\begin{array}{l}\text { Risk } \\
\text { difference } \\
0.11(95 \% \\
\mathrm{Cl},-0.53 \\
\text { to } 0.75)^{141}\end{array}$ & $\begin{array}{l}1 \text { cohort, } \\
n=10^{141}\end{array}$ & $\begin{array}{l}\text { High study } \\
\text { limitations (high } \\
\text { risk of bias, }{ }^{141} \\
\text { imprecise (wide } \\
\text { Cls), } \\
\text { consistency } \\
\text { unknown }\end{array}$ & Insufficient \\
\hline
\end{tabular}

$\mathrm{CI}=$ confidence interval; $\mathrm{n}=$ number; $\mathrm{SNRI}=$ serotonin-norepinephrine reuptake inhibitor; SSRI = selective serotonin reuptake inhibitor; vs. $=$ versus.

\section{Fluoxetine Versus Bupropion}

\section{Overview}

- The evidence is insufficient to judge the comparative risk of harms from exposure during pregnancy to fluoxetine versus bupropion for child outcomes (congenital heart disease).

\section{Detailed Results}

One publication reported on the comparative harms of fluoxetine versus bupropion. ${ }^{141}$ The study drew from a cohort of women who had undergone a fetal echocardiogram because of in utero exposure of the fetus to SSRIs or SNRIs. ${ }^{141}$ The study was rated as high risk of bias. The study yielded insufficient strength of evidence to judge the risk of congenital heart disease (Table B-50).

Table B-50. Strength of evidence for comparative harms: Fluoxetine versus bupropion

\begin{tabular}{|c|c|c|c|c|c|c|c|}
\hline Population & $\begin{array}{l}\text { Intervention } \\
\text { and } \\
\text { Comparator }\end{array}$ & Outcome & $\begin{array}{l}\text { Incidence } \\
\text { or Mean } \\
\text { Effect by } \\
\text { Arm }\end{array}$ & Results & $\begin{array}{l}\text { Study } \\
\text { Design } \\
\text { and } \\
\text { Sample } \\
\text { Size } \\
\end{array}$ & $\begin{array}{l}\text { Factors That } \\
\text { Affect the } \\
\text { Strength of } \\
\text { Evidence }\end{array}$ & $\begin{array}{l}\text { Overall } \\
\text { Evidence } \\
\text { Strength } \\
\text { (Direction of } \\
\text { Effect) }\end{array}$ \\
\hline $\begin{array}{l}\text { Women who } \\
\text { underwent fetal } \\
\text { echocardiography } \\
\text { because of an in } \\
\text { utero exposure to } \\
\text { either SSRIs or } \\
\text { SNRIs }\end{array}$ & $\begin{array}{l}\text { Fluoxetine vs. } \\
\text { bupropion }\end{array}$ & $\begin{array}{l}\text { Congenital } \\
\text { heart } \\
\text { disease }\end{array}$ & $\begin{array}{l}2 / 9 \\
(22.22 \%) \\
\text { vs. } 0 / 3 \\
(0 \%)^{141}\end{array}$ & $\begin{array}{l}\text { Risk } \\
\text { difference, } \\
0.22(95 \% \\
\mathrm{Cl},-0.20 \text { to } \\
0.64)^{141}\end{array}$ & $\begin{array}{l}1 \text { cohort, } \\
n=12^{141}\end{array}$ & $\begin{array}{l}\text { High study } \\
\text { limitations (high } \\
\text { risk of bias, }{ }^{141} \\
\text { imprecise (wide } \\
\text { Cls), } \\
\text { consistency } \\
\text { unknown }\end{array}$ & Insufficient \\
\hline
\end{tabular}

$\mathrm{CI}=$ confidence interval; $\mathrm{n}=$ number; SNRI $=$ serotonin-norepinephrine reuptake inhibitor; SSRI $=$ selective serotonin reuptake inhibitor; vs. $=$ versus. 


\section{Fluoxetine Versus Duloxetine}

\section{Overview}

- The evidence is insufficient to judge the comparative risk of harms from exposure during pregnancy to fluoxetine versus duloxetine for child outcomes (congenital heart disease).

\section{Detailed Results}

One publication reported on the comparative harms of fluoxetine versus duloxetine. ${ }^{141}$ The study drew from a cohort of women who had undergone a fetal echocardiogram because of in utero exposure of the fetus to SSRIs or SNRIs. ${ }^{141}$ The study was rated as high risk of bias. The study yielded insufficient strength of evidence to judge the risk of congenital heart disease (Table B-51).

Table B-51. Strength of evidence for comparative harms: Fluoxetine versus duloxetine

\begin{tabular}{|c|c|c|c|c|c|c|c|}
\hline Population & $\begin{array}{l}\text { Intervention } \\
\text { and } \\
\text { Comparator }\end{array}$ & Outcome & $\begin{array}{l}\text { Incidence } \\
\text { or Mean } \\
\text { Effect by } \\
\text { Arm }\end{array}$ & Results & $\begin{array}{l}\text { Study } \\
\text { Design } \\
\text { and } \\
\text { Sample } \\
\text { Size } \\
\end{array}$ & $\begin{array}{l}\text { Factors That } \\
\text { Affect the } \\
\text { Strength of } \\
\text { Evidence }\end{array}$ & $\begin{array}{l}\text { Overall } \\
\text { Evidence } \\
\text { Strength } \\
\text { (Direction } \\
\text { of Effect) } \\
\end{array}$ \\
\hline $\begin{array}{l}\text { Women who } \\
\text { underwent fetal } \\
\text { echocardiography } \\
\text { because of an in utero } \\
\text { exposure to either } \\
\text { SSRIs or SNRIs }\end{array}$ & $\begin{array}{l}\text { Fluoxetine vs. } \\
\text { duloxetine }\end{array}$ & $\begin{array}{l}\text { Congenital } \\
\text { heart } \\
\text { disease }\end{array}$ & $\begin{array}{l}2 / 9 \\
(22.22 \%) \\
\text { vs. 0/1 } \\
(0 \%)^{141}\end{array}$ & $\begin{array}{l}\begin{array}{l}\text { Risk } \\
\text { difference, }\end{array} \\
0.22(95 \% \\
\mathrm{Cl},-0.20 \text { to } \\
0.64)^{141}\end{array}$ & $\begin{array}{l}1 \text { cohort, } \\
n=10^{141}\end{array}$ & $\begin{array}{l}\text { High study } \\
\text { limitations (high } \\
\text { risk of bias, } 141 \\
\text { imprecise (wide } \\
\text { Cls), } \\
\text { consistency } \\
\text { unknown }\end{array}$ & Insufficient \\
\hline
\end{tabular}

$\mathrm{CI}=$ confidence interval; $\mathrm{n}=$ number; SNRI = serotonin-norepinephrine reuptake inhibitor; SSRI = selective serotonin reuptake inhibitor; vs. $=$ versus.

\section{Fluoxetine Versus Escitalopram}

\section{Overview}

- The evidence is insufficient to judge the comparative risk of harms from exposure during pregnancy to fluoxetine versus escitalopram for child outcomes (congenital heart disease).

\section{Detailed Results}

One publication reported on the comparative harms of fluoxetine versus escitalopram. ${ }^{141}$ The study drew from a cohort of women who had undergone a fetal echocardiogram because of in utero exposure of the fetus to SSRIs or SNRIs. ${ }^{141}$ The study was rated as high risk of bias. The study yielded insufficient strength of evidence to judge the risk of congenital heart disease (Table B-52). 
Table B-52. Strength of evidence for comparative harms: Fluoxetine versus escitalopram

\begin{tabular}{|c|c|c|c|c|c|c|c|}
\hline Population & $\begin{array}{l}\text { Intervention } \\
\text { and } \\
\text { Comparator }\end{array}$ & Outcome & $\begin{array}{l}\text { Incidence } \\
\text { or Mean } \\
\text { Effect by } \\
\text { Arm }\end{array}$ & Results & $\begin{array}{l}\text { Study } \\
\text { Design } \\
\text { and } \\
\text { Sample } \\
\text { Size } \\
\end{array}$ & $\begin{array}{l}\text { Factors That } \\
\text { Affect the } \\
\text { Strength of } \\
\text { Evidence }\end{array}$ & $\begin{array}{l}\text { Overall } \\
\text { Evidence } \\
\text { Strength } \\
\text { (Direction of } \\
\text { Effect) }\end{array}$ \\
\hline $\begin{array}{l}\text { Women who } \\
\text { underwent fetal } \\
\text { echocardiography } \\
\text { because of an in } \\
\text { utero exposure to } \\
\text { either SSRIs or } \\
\text { SNRIs }\end{array}$ & $\begin{array}{l}\text { Fluoxetine vs. } \\
\text { escitalopram }\end{array}$ & $\begin{array}{l}\text { Congenital } \\
\text { heart } \\
\text { disease }\end{array}$ & $\begin{array}{l}2 / 9(22.22 \%) \\
\text { vs. } 1 / 9 \\
(11.11 \%)^{141}\end{array}$ & $\begin{array}{l}\text { RR, } 2.00 \\
(95 \% \mathrm{Cl}, \\
0.22 \text { to } \\
18.33)^{141}\end{array}$ & $\begin{array}{l}1 \text { cohort, } \\
n=18^{141}\end{array}$ & $\begin{array}{l}\text { High study } \\
\text { limitations (high } \\
\text { risk of bias, } 141 \\
\text { imprecise (wide } \\
\text { Cls), consistency } \\
\text { unknown }\end{array}$ & Insufficient \\
\hline
\end{tabular}

\section{Fluoxetine Versus Sertraline}

\section{Overview}

- The evidence is insufficient to judge the comparative risk of harms from exposure during pregnancy to fluoxetine versus sertraline for child outcomes (congenital heart disease).

\section{Detailed Results}

One publication reported on the comparative harms of Fluoxetine versus sertraline. ${ }^{141}$ The study drew from a cohort of women who had undergone a fetal echocardiogram because of in utero exposure of the fetus to SSRIs or SNRIs. ${ }^{141}$ The study was rated as high risk of bias. The study yielded insufficient strength of evidence to judge the risk of congenital heart disease (Table B-53).

Table B-53. Strength of evidence for comparative harms: Fluoxetine versus sertraline

\begin{tabular}{|c|c|c|c|c|c|c|c|}
\hline Population & $\begin{array}{l}\text { Intervention } \\
\text { and } \\
\text { Comparator }\end{array}$ & Outcome & $\begin{array}{l}\text { Incidence } \\
\text { or Mean } \\
\text { Effect by } \\
\text { Arm }\end{array}$ & Results & $\begin{array}{l}\text { Study } \\
\text { Design } \\
\text { and } \\
\text { Sample } \\
\text { Size } \\
\end{array}$ & $\begin{array}{l}\text { Factors That } \\
\text { Affect the } \\
\text { Strength of } \\
\text { Evidence }\end{array}$ & $\begin{array}{l}\text { Overall } \\
\text { Evidence } \\
\text { Strength } \\
\text { (Direction of } \\
\text { Effect) }\end{array}$ \\
\hline $\begin{array}{l}\text { Women who } \\
\text { underwent fetal } \\
\text { echocardiography } \\
\text { because of an in } \\
\text { utero exposure to } \\
\text { either SSRIs or } \\
\text { SNRIs }\end{array}$ & $\begin{array}{l}\text { Fluoxetine vs. } \\
\text { sertraline }\end{array}$ & $\begin{array}{l}\text { Congenital } \\
\text { heart } \\
\text { disease }\end{array}$ & $\begin{array}{l}2 / 9 \\
(22.22 \%) \\
\text { vs. } 0 / 7 \\
(0 \%)^{141}\end{array}$ & 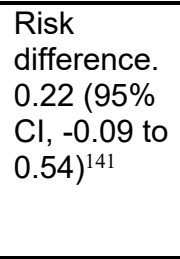 & $\begin{array}{l}1 \text { cohort, } \\
n=16^{141}\end{array}$ & $\begin{array}{l}\text { High study } \\
\text { limitations (high } \\
\text { risk of bias, } 141 \\
\text { imprecise (wide } \\
\text { Cls), } \\
\text { consistency } \\
\text { unknown }\end{array}$ & Insufficient \\
\hline
\end{tabular}

$\overline{\mathrm{CI}}=$ confidence interval; $\mathrm{n}=$ number; $\mathrm{SNRI}=$ serotonin-norepinephrine reuptake inhibitor; $\mathrm{SSRI}=$ selective serotonin reuptake inhibitor; vs. = versus.

\section{Fluoxetine Versus Venlafaxine}

\section{Overview}

- The evidence is insufficient to judge the comparative risk of harms from exposure during pregnancy to fluoxetine versus venlafaxine for child outcomes (congenital heart disease). 


\section{Detailed Results}

One publication reported on the comparative harms of fluoxetine versus venlafaxine. ${ }^{141}$ The study drew from a cohort of women who had undergone a fetal echocardiogram because of in utero exposure of the fetus to SSRIs or SNRIs. ${ }^{141}$ The study was rated as high risk of bias. The study yielded insufficient strength of evidence to judge the risk of congenital heart disease (Table B-54).

Table B-54. Strength of evidence for comparative harms: Fluoxetine versus venlafaxine

\begin{tabular}{|c|c|c|c|c|c|c|c|}
\hline Population & $\begin{array}{l}\text { Intervention } \\
\text { and } \\
\text { Comparator }\end{array}$ & Outcome & $\begin{array}{l}\text { Incidence } \\
\text { or Mean } \\
\text { Effect by } \\
\text { Arm }\end{array}$ & Results & $\begin{array}{l}\text { Study } \\
\text { Design } \\
\text { and } \\
\text { Sample } \\
\text { Size } \\
\end{array}$ & $\begin{array}{l}\text { Factors That } \\
\text { Affect the } \\
\text { Strength of } \\
\text { Evidence }\end{array}$ & $\begin{array}{l}\text { Overall } \\
\text { Evidence } \\
\text { Strength } \\
\text { (Direction of } \\
\text { Effect) }\end{array}$ \\
\hline $\begin{array}{l}\text { Women who } \\
\text { underwent fetal } \\
\text { echocardiography } \\
\text { because of an in } \\
\text { utero exposure to } \\
\text { either SSRIs or } \\
\text { SNRIs }\end{array}$ & $\begin{array}{l}\text { Fluoxetine vs. } \\
\text { venlafaxine }\end{array}$ & $\begin{array}{l}\text { Congenital } \\
\text { heart } \\
\text { disease }\end{array}$ & $\begin{array}{l}2 / 9 \\
(22.22 \%) \\
\text { vs. } 2 / 2 \\
(100 \%)^{141}\end{array}$ & $\begin{array}{l}\text { RR, } 0.03 \\
(95 \% \mathrm{Cl} \text {, } \\
0.09 \text { to } \\
0.98)^{141}\end{array}$ & $\begin{array}{l}1 \text { cohort, } \\
n=11^{141}\end{array}$ & $\begin{array}{l}\text { High study } \\
\text { limitations (high } \\
\text { risk of bias, }{ }^{141} \\
\text { imprecise (wide } \\
\text { Cls), consistency } \\
\text { unknown }\end{array}$ & Insufficient \\
\hline
\end{tabular}

\section{Fluoxetine Versus Citalopram or Escitalopram}

\section{Overview}

- The evidence is limited to one study and is insufficient to judge the comparative risk of harms from exposure during pregnancy to fluoxetine monotherapy versus citalopram or escitalopram monotherapy for maternal outcomes (convulsive seizures, nonconvulsive seizures, or spontaneous abortion or stillbirth) or child outcomes (congenital anomaly or birth weight) among women with epilepsy.

\section{Detailed Results}

One high risk-of-bias publication reported on the comparative harms of fluoxetine versus citalopram or escitalopram. ${ }^{107}$ The population consisted of pregnant women with depression, anxiety, or bipolar disorder, as well as epilepsy, drawn from the Australian Antiepileptic Drugs in Pregnancy register. No significant differences were found in the risk of harms for any maternal (convulsive seizures, nonconvulsive seizures, spontaneous abortion, or stillbirth) or child outcomes (congenital anomalies or birth weight) (Table B-55). 
Table B-55. Strength of evidence for comparative harms: Fluoxetine monotherapy versus SSRI monotherapy (citalopram or escitalopram)

\begin{tabular}{|c|c|c|c|c|c|c|c|}
\hline Population & $\begin{array}{l}\text { Intervention } \\
\text { and } \\
\text { Comparator }\end{array}$ & Outcome & $\begin{array}{l}\text { Incidence or } \\
\text { Mean Effect } \\
\text { by Arm }\end{array}$ & Results & $\begin{array}{l}\text { Study } \\
\text { Design } \\
\text { and } \\
\text { Sample } \\
\text { Size } \\
\end{array}$ & $\begin{array}{l}\text { Factors That } \\
\text { Affect the } \\
\text { Strength of } \\
\text { Evidence }\end{array}$ & $\begin{array}{l}\text { Overall } \\
\text { Evidence } \\
\text { Strength } \\
\text { (Direction } \\
\text { of Effect) } \\
\end{array}$ \\
\hline $\begin{array}{l}\text { Women with } \\
\text { epilepsy and } \\
\text { depression, } \\
\text { anxiety or } \\
\text { bipolar } \\
\text { disorder }\end{array}$ & $\begin{array}{l}\text { Fluoxetine } \\
\text { only vs. SSRI } \\
\text { monotherapy } \\
\text { (citalopram or } \\
\text { escitalopram) }\end{array}$ & $\begin{array}{l}\text { Convulsive } \\
\text { seizures: } \\
\text { From } \\
\text { enrollment } \\
\text { to } 7 \text { months } \\
\text { gestation } \\
\text { From } 7 \\
\text { months } \\
\text { gestation to } \\
\text { delivery }\end{array}$ & $\begin{array}{l}2 / 5(40 \%) \text { vs. } \\
2 / 20(10 \%)^{107} \\
1 / 5(20 \%) \text { vs. } \\
1 / 20(5 \%)^{107}\end{array}$ & $\begin{array}{l}\mathrm{RR}, 4(95 \% \mathrm{Cl} \\
0.73 \text { to } 21.84)^{107} \\
\mathrm{RR}, 4(95 \% \mathrm{Cl}, \\
0.30 \text { to } 53.47)^{107}\end{array}$ & $\begin{array}{l}1 \text { cohort, } \\
n=25\end{array}$ & $\begin{array}{l}\text { High study } \\
\text { limitations (high } \\
\text { risk of bias }{ }^{107} \text { ), } \\
\text { seriously } \\
\text { imprecise (few } \\
\text { events, wide } \\
\text { Cls), } \\
\text { consistency } \\
\text { unknown }\end{array}$ & Insufficient \\
\hline $\begin{array}{l}\text { Women with } \\
\text { epilepsy and } \\
\text { depression, } \\
\text { anxiety or } \\
\text { bipolar } \\
\text { disorder }\end{array}$ & $\begin{array}{l}\text { Fluoxetine } \\
\text { only vs. SSRI } \\
\text { monotherapy } \\
\text { (citalopram or } \\
\text { escitalopram) }\end{array}$ & $\begin{array}{l}\text { Noncon- } \\
\text { vulsive } \\
\text { seizures: } \\
\text { From } \\
\text { enrollment } \\
\text { to } 7 \text { months } \\
\text { gestation } \\
\text { From } 7 \\
\text { months } \\
\text { gestation to } \\
\text { delivery } \\
\end{array}$ & $\begin{array}{l}1 / 5(20 \%) \text { vs. } \\
4 / 20(20 \%)^{107} \\
1 / 5(20 \%) \text { vs. } \\
3 / 20(15 \%)^{107}\end{array}$ & $\begin{array}{l}\mathrm{RR}, 1(95 \% \mathrm{Cl} \\
0.14 \text { to } 7.10)^{107} \\
\mathrm{RR}, 1.33(95 \% \\
\mathrm{Cl}, 0.173 \text { to } \\
10.254)^{107}\end{array}$ & $\begin{array}{l}1 \text { cohort, } \\
n=25\end{array}$ & $\begin{array}{l}\text { High study } \\
\text { limitations (high } \\
\text { risk of bias }{ }^{107} \text { ), } \\
\text { seriously } \\
\text { imprecise (few } \\
\text { events, wide } \\
\text { Cls), } \\
\text { consistency } \\
\text { unknown }\end{array}$ & Insufficient \\
\hline $\begin{array}{l}\text { Women with } \\
\text { epilepsy and } \\
\text { depression, } \\
\text { anxiety or } \\
\text { bipolar } \\
\text { disorder }\end{array}$ & $\begin{array}{l}\text { Fluoxetine } \\
\text { only vs. SSRI } \\
\text { monotherapy } \\
\text { (citalopram or } \\
\text { escitalopram) }\end{array}$ & $\begin{array}{l}\text { Spontan- } \\
\text { eous } \\
\text { abortion }\end{array}$ & $\begin{array}{l}0 / 5(0 \%) \text { vs. } \\
0 / 20(0 \%)^{107}\end{array}$ & $\begin{array}{l}\text { RR not } \\
\text { calculable: no } \\
\text { events }\end{array}$ & $\begin{array}{l}1 \text { cohort, } \\
n=25\end{array}$ & $\begin{array}{l}\text { High study } \\
\text { limitations (high } \\
\left.\text { risk of bias }{ }^{107}\right) \text {, } \\
\text { likely imprecise } \\
\text { (small Ns), small } \\
\text { Ns), consistency } \\
\text { unknown }\end{array}$ & Insufficient \\
\hline $\begin{array}{l}\text { Women with } \\
\text { epilepsy and } \\
\text { depression, } \\
\text { anxiety or } \\
\text { bipolar } \\
\text { disorder }\end{array}$ & $\begin{array}{l}\text { Fluoxetine } \\
\text { only vs. SSRI } \\
\text { monotherapy } \\
\text { (citalopram or } \\
\text { escitalopram) }\end{array}$ & Stillbirth & $\begin{array}{l}0 / 5(0 \%) \text { vs. } \\
0 / 20(0 \%)^{107}\end{array}$ & $\begin{array}{l}\text { RR not } \\
\text { calculable: no } \\
\text { events }\end{array}$ & $\begin{array}{l}1 \text { cohort, } \\
n=25\end{array}$ & $\begin{array}{l}\text { High study } \\
\text { limitations (high } \\
\text { risk of bias }{ }^{107} \text { ), } \\
\text { likely imprecise } \\
\text { (no events, } \\
\text { small Ns), } \\
\text { consistency } \\
\text { unknown }\end{array}$ & Insufficient \\
\hline $\begin{array}{l}\text { Women with } \\
\text { epilepsy and } \\
\text { depression, } \\
\text { anxiety or } \\
\text { bipolar } \\
\text { disorder }\end{array}$ & $\begin{array}{l}\text { Fluoxetine } \\
\text { only vs. SSRI } \\
\text { monotherapy } \\
\text { (citalopram or } \\
\text { escitalopram) }\end{array}$ & $\begin{array}{l}\text { Congenital } \\
\text { anomaly }\end{array}$ & $\begin{array}{l}\text { 0/0 (0\%) vs. } \\
4 / 20(19 \%)^{107}\end{array}$ & $\begin{array}{l}\text { RD: } 0.41(95 \% \\
\mathrm{Cl}, 0.03 \text { to } \\
6.56)^{107}\end{array}$ & $\begin{array}{l}1 \text { cohort, } \\
n=25\end{array}$ & $\begin{array}{l}\text { High study } \\
\text { limitations (high } \\
\text { risk of bias }{ }^{107} \text { ), } \\
\text { seriously } \\
\text { imprecise (few } \\
\text { events, wide } \\
\text { Cls), } \\
\text { consistency } \\
\text { unknown } \\
\end{array}$ & Insufficient \\
\hline $\begin{array}{l}\text { Women with } \\
\text { epilepsy and } \\
\text { depression, } \\
\text { anxiety or } \\
\text { bipolar } \\
\text { disorder }\end{array}$ & $\begin{array}{l}\text { Fluoxetine } \\
\text { only vs. SSRI } \\
\text { monotherapy } \\
\text { (citalopram or } \\
\text { escitalopram) }\end{array}$ & Birth weight & $\begin{array}{l}\text { Median } \pm \\
\text { IQR: } \\
2,790 \pm 497 \\
\text { vs. } \\
3,087 \pm 286^{107}\end{array}$ & Not calculable & $\begin{array}{l}1 \text { cohort, } \\
n=25\end{array}$ & $\begin{array}{l}\text { High study } \\
\text { limitations (high } \\
\text { risk of bias }{ }^{107} \text { ), } \\
\text { likely imprecise, } \\
\text { consistency } \\
\text { unknown }\end{array}$ & Insufficient \\
\hline
\end{tabular}

$\mathrm{CI}=$ confidence interval; $\mathrm{IQR}=$ interquartile ratio; $\mathrm{n}=$ number; $\mathrm{RD}=$ risk difference; $\mathrm{RR}=$ relative risk; $\mathrm{SSRI}=$ selective serotonin reuptake inhibitor; vs. $=$ versus. 


\section{Fluoxetine Versus Escitalopram or Fluvoxamine}

\section{Overview}

- Based on evidence from a single study of the comparative risk of exposure during pregnancy to fluoxetine monotherapy versus fluvoxamine monotherapy among a sample of women with epilepsy, the evidence was insufficient in relation to maternal outcomes (convulsive seizures, nonconvulsive seizures, or spontaneous abortion or stillbirth) and child outcomes (congenital anomaly or birth weight). Based on the evidence from two studies, the comparative risk of autism spectrum disorder is also graded as insufficient.

\section{Detailed Results}

Three high risk-of-bias publications reported on the comparative harms of fluoxetine monotherapy versus fluvoxamine monotherapy. ${ }^{107,111,117}$ One study combined fluvoxamine monotherapy or escitalopram monotherapy as the comparison group. ${ }^{117}$ One analysis reported on a population of pregnant women with depression, anxiety, or bipolar disorder as well as epilepsy, drawn from the Australian Antiepileptic Drugs in Pregnancy register. ${ }^{107}$ The authors found no significant differences in the risk of harms for any maternal (convulsive seizures, nonconvulsive seizures, spontaneous abortion, or stillbirth) or child outcomes (congenital anomalies or birth weight). Each of these outcomes was graded as insufficient strength of evidence.

Two publications reported on the relative risk of autism spectrum disorder. Data from the Danish Birth registry ${ }^{117}$ were analyzed in one study, and data from the Canadian QPC were analyzed in the second. ${ }^{111}$ A significantly smaller risk of autism spectrum disorder was found from fluvoxamine or escitalopram compared with fluoxetine in one study ${ }^{117}$ but not in a second study where the comparison group was limited to fluvoxamine. ${ }^{111}$ Both studies did not control for confounding, resulting in the comparison being graded as insufficient strength of evidence (Table B-56).

Table B-56. Strength of evidence for comparative harms: Fluoxetine monotherapy versus fluvoxamine monotherapy

\begin{tabular}{|c|c|c|c|c|c|c|c|}
\hline Population & $\begin{array}{l}\text { Intervention } \\
\text { and } \\
\text { Comparator }\end{array}$ & Outcome & $\begin{array}{l}\text { Incidence or } \\
\text { Mean Effect by } \\
\text { Arm }\end{array}$ & Results & $\begin{array}{l}\text { Study } \\
\text { Design and } \\
\text { Sample } \\
\text { Size }\end{array}$ & $\begin{array}{l}\text { Factors That } \\
\text { Affect the } \\
\text { Strength of } \\
\text { Evidence }\end{array}$ & $\begin{array}{l}\text { Overall } \\
\text { Evidence } \\
\text { Strength } \\
\text { (Direction } \\
\text { of Effect) }\end{array}$ \\
\hline $\begin{array}{l}\text { Pregnant } \\
\text { women with } \\
\text { epilepsy and } \\
\text { depression, } \\
\text { anxiety or } \\
\text { bipolar } \\
\text { disorder }\end{array}$ & $\begin{array}{l}\text { Fluoxetine } \\
\text { monotherapy vs. } \\
\text { fluvoxamine } \\
\text { monotherapy }\end{array}$ & $\begin{array}{l}\text { Convulsive } \\
\text { seizures: } \\
\text { From } \\
\text { enrollment to } 7 \\
\text { months } \\
\text { gestation } \\
\text { From } 7 \text { months } \\
\text { gestation to } \\
\text { delivery }\end{array}$ & $\begin{array}{l}2 / 5(40 \%) \text { vs. } 0 / 1 \\
(0 \%)^{107} \\
1 / 5(20 \%) \text { vs. } 0 / 1 \\
(0 \%)^{107} \\
\end{array}$ & $\begin{array}{l}\mathrm{RD}, 0.40 \\
(95 \% \mathrm{Cl} \text {, } \\
-0.32 \text { to } \\
1.12) \\
\mathrm{RD}, 0.20 \\
(95 \% \mathrm{Cl}, \\
-0.64 \text { to } \\
0.64)\end{array}$ & $\begin{array}{l}1 \text { cohort, } \\
n=6\end{array}$ & $\begin{array}{l}\text { High study } \\
\text { limitations } \\
\text { (high risk of } \\
\text { bias }^{107} \text { ), } \\
\text { seriously } \\
\text { imprecise } \\
\text { (few events, } \\
\text { wide Cls), } \\
\text { consistency } \\
\text { unknown }\end{array}$ & Insufficient \\
\hline
\end{tabular}




\begin{tabular}{|c|c|c|c|c|c|c|c|}
\hline Population & $\begin{array}{l}\text { Intervention } \\
\text { and } \\
\text { Comparator }\end{array}$ & Outcome & $\begin{array}{l}\text { Incidence or } \\
\text { Mean Effect by } \\
\text { Arm }\end{array}$ & Results & $\begin{array}{l}\text { Study } \\
\text { Design and } \\
\text { Sample } \\
\text { Size }\end{array}$ & $\begin{array}{l}\text { Factors That } \\
\text { Affect the } \\
\text { Strength of } \\
\text { Evidence }\end{array}$ & $\begin{array}{l}\text { Overall } \\
\text { Evidence } \\
\text { Strength } \\
\text { (Direction } \\
\text { of Effect) }\end{array}$ \\
\hline $\begin{array}{l}\text { Pregnant } \\
\text { women with } \\
\text { epilepsy and } \\
\text { depression, } \\
\text { anxiety or } \\
\text { bipolar } \\
\text { disorder }\end{array}$ & $\begin{array}{l}\text { Fluoxetine } \\
\text { monotherapy vs. } \\
\text { fluvoxamine } \\
\text { monotherapy }\end{array}$ & $\begin{array}{l}\text { Nonconvulsive } \\
\text { seizures: } \\
\text { From } \\
\text { enrollment to } 7 \\
\text { months } \\
\text { gestation } \\
\text { From } 7 \text { months } \\
\text { gestation to } \\
\text { delivery }\end{array}$ & $\begin{array}{l}1 / 5(20 \%) \text { vs. } 0 / 1 \\
(0 \%)^{107} \\
1 / 5(20 \%) \text { vs. } \\
0 / 1(0 \%)^{107} \\
\end{array}$ & $\begin{array}{l}\text { RD, } 0.20 \\
(95 \% \mathrm{Cl}, \\
-0.64 \text { to } \\
0.64) \\
\\
\mathrm{RD}, 0.20 \\
(95 \% \mathrm{Cl}, \\
-0.64 \text { to } \\
0.64)\end{array}$ & $\begin{array}{l}1 \text { cohort, } \\
n=6\end{array}$ & $\begin{array}{l}\text { High study } \\
\text { limitations } \\
\text { (high risk of } \\
\text { bias }^{107} \text { ), } \\
\text { seriously } \\
\text { imprecise } \\
\text { (few events, } \\
\text { wide Cls), } \\
\text { consistency } \\
\text { unknown }\end{array}$ & Insufficient \\
\hline $\begin{array}{l}\text { Pregnant } \\
\text { women with } \\
\text { epilepsy and } \\
\text { depression, } \\
\text { anxiety or } \\
\text { bipolar } \\
\text { disorder }\end{array}$ & $\begin{array}{l}\text { Fluoxetine } \\
\text { monotherapy vs. } \\
\text { fluvoxamine } \\
\text { monotherapy }\end{array}$ & $\begin{array}{l}\text { Spontaneous } \\
\text { abortion }\end{array}$ & $\begin{array}{l}0 / 5(0 \%) \text { vs. } \\
0 / 1(0 \%)^{107}\end{array}$ & $\begin{array}{l}\text { RR not } \\
\text { calculable: } \\
\text { no events }\end{array}$ & $\begin{array}{l}1 \text { cohort, } \\
n=6\end{array}$ & $\begin{array}{l}\text { High study } \\
\text { limitations } \\
\text { (high risk of } \\
\text { bias }^{107} \text { ), likely } \\
\text { imprecise (no } \\
\text { events, small } \\
\mathrm{N} \text { ), } \\
\text { consistency } \\
\text { unknown }\end{array}$ & Insufficient \\
\hline $\begin{array}{l}\text { Pregnant } \\
\text { women with } \\
\text { epilepsy and } \\
\text { depression, } \\
\text { anxiety or } \\
\text { bipolar } \\
\text { disorder }\end{array}$ & $\begin{array}{l}\text { Fluoxetine } \\
\text { monotherapy vs. } \\
\text { fluvoxamine } \\
\text { monotherapy }\end{array}$ & Stillbirth & $\begin{array}{l}0 / 5(0 \%) \text { vs. } 0 / 1 \\
(0 \%)^{107}\end{array}$ & $\begin{array}{l}\text { RR not } \\
\text { calculable: } \\
\text { no events }\end{array}$ & $\begin{array}{l}1 \text { cohort, } \\
n=6\end{array}$ & $\begin{array}{l}\text { High study } \\
\text { limitations } \\
\text { (high risk of } \\
\text { bias }^{107} \text { ), likely } \\
\text { imprecise (no } \\
\text { events, small } \\
\mathrm{N} \text { ), } \\
\text { consistency } \\
\text { unknown }\end{array}$ & Insufficient \\
\hline $\begin{array}{l}\text { Pregnant } \\
\text { women with } \\
\text { epilepsy and } \\
\text { depression, } \\
\text { anxiety or } \\
\text { bipolar } \\
\text { disorder }\end{array}$ & $\begin{array}{l}\text { Fluoxetine } \\
\text { monotherapy vs. } \\
\text { fluvoxamine } \\
\text { monotherapy }\end{array}$ & $\begin{array}{l}\text { Congenital } \\
\text { anomaly }\end{array}$ & $\begin{array}{l}0 / 5(0 \%) \text { vs. } 0 / 1 \\
(0 \%)^{107}\end{array}$ & $\begin{array}{l}\text { RR not } \\
\text { calculable: } \\
\text { no events }\end{array}$ & $\begin{array}{l}1 \text { cohort, } \\
n=6\end{array}$ & $\begin{array}{l}\text { High study } \\
\text { limitations } \\
\text { (high risk of } \\
\text { bias }^{107} \text { ), likely } \\
\text { imprecise (no } \\
\text { events, small } \\
\mathrm{N} \text { ), } \\
\text { consistency } \\
\text { unknown }\end{array}$ & Insufficient \\
\hline $\begin{array}{l}\text { Pregnant } \\
\text { women with } \\
\text { epilepsy and } \\
\text { depression, } \\
\text { anxiety or } \\
\text { bipolar } \\
\text { disorder }\end{array}$ & $\begin{array}{l}\text { Fluoxetine } \\
\text { monotherapy vs. } \\
\text { fluvoxamine } \\
\text { monotherapy }\end{array}$ & Birthweight & $\begin{array}{l}\text { Median } \pm \text { IQR: } \\
2,790 \pm 497 \text { vs. } \\
3,310^{107}\end{array}$ & $\begin{array}{l}\text { Difference } \\
\text { not } \\
\text { calculable }\end{array}$ & $\begin{array}{l}1 \text { cohort, } \\
n=6\end{array}$ & $\begin{array}{l}\text { High study } \\
\text { limitations } \\
\text { (high risk of } \\
\text { bias }^{107} \text { ), likely } \\
\text { imprecise } \\
\text { (small Ns), } \\
\text { consistency } \\
\text { unknown }\end{array}$ & Insufficient \\
\hline $\begin{array}{l}\text { Pregnant } \\
\text { women } \\
\text { taking } \\
\text { fluoxetine, } \\
\text { fluvoxamine } \\
\text { or } \\
\text { escitalopram }\end{array}$ & $\begin{array}{l}\text { Fluoxetine } \\
\text { monotherapy vs. } \\
\text { fluvoxamine or } \\
\text { escitalopram } \\
\text { monotherapy; }{ }^{117} \\
\text { Fluoxetine } \\
\text { monotherapy vs. } \\
\text { fluvoxamine }\end{array}$ & $\begin{array}{l}\text { Autism } \\
\text { spectrum } \\
\text { disorder }\end{array}$ & $\begin{array}{l}\text { 18/160 (11\%) vs. } \\
1 / 1,047(0.09 \%)^{117} \\
5 / 171(2.9 \%) \text { vs. } \\
1 / 35(2.9 \%)^{111} \\
\end{array}$ & $\begin{array}{l}\mathrm{RR}, 117.79 \\
(95 \% \mathrm{Cl}, \\
15.83 \text { to } \\
876.27) \\
\\
\mathrm{RR}, 1.02 \\
(95 \% \mathrm{Cl}, \\
0.12 \text { to } \\
8.49)\end{array}$ & $\begin{array}{l}2 \text { cohorts; } \\
n=1,207 \text { in } \\
\text { one } \\
\text { cohort, }{ }^{117} \\
n=206 \text { in } \\
\text { one } \\
\text { cohort }^{111}\end{array}$ & $\begin{array}{l}\text { High study } \\
\text { limitations } \\
\text { (high risk of } \\
\text { bias }^{111,117} \text { ), } \\
\text { imprecise } \\
\text { (wide Cls), } \\
\text { consistent }\end{array}$ & $\begin{array}{l}\text { Insufficient } \\
\text { Low }\end{array}$ \\
\hline
\end{tabular}

$\mathrm{CI}=$ confidence interval; $\mathrm{IQR}=$ interquartile ratio; $\mathrm{n}=$ number; $\mathrm{RD}=$ risk difference; $\mathrm{RR}=$ relative risk; vs. $=$ versus. 


\section{Paroxetine Versus Duloxetine}

\section{Overview}

- The evidence is insufficient to judge the comparative risk of harms from exposure during pregnancy to paroxetine versus duloxetine for child outcomes (congenital heart disease).

\section{Detailed Results}

One publication reported on the comparative harms of paroxetine versus duloxetine. ${ }^{141}$ The study drew from a cohort of women who had undergone a fetal echocardiogram because of in utero exposure of the fetus to SSRIs or SNRIs. ${ }^{141}$ The study was rated as high risk of bias. The study yielded insufficient strength of evidence to judge the risk of congenital heart disease (Table B-57).

Table B-57. Strength of evidence for comparative harms: Paroxetine versus duloxetine

\begin{tabular}{|c|c|c|c|c|c|c|c|}
\hline Population & $\begin{array}{l}\text { Intervention } \\
\text { and } \\
\text { Comparator }\end{array}$ & Outcome & $\begin{array}{l}\text { Incidence } \\
\text { or Mean } \\
\text { Effect by } \\
\text { Arm }\end{array}$ & Results & $\begin{array}{l}\text { Study } \\
\text { Design } \\
\text { and } \\
\text { Sample } \\
\text { Size }\end{array}$ & $\begin{array}{l}\text { Factors That } \\
\text { Affect the } \\
\text { Strength of } \\
\text { Evidence }\end{array}$ & $\begin{array}{l}\text { Overall } \\
\text { Evidence } \\
\text { Strength } \\
\text { (Direction } \\
\text { of Effect) }\end{array}$ \\
\hline $\begin{array}{l}\text { Women who } \\
\text { underwent fetal } \\
\text { echocardiography } \\
\text { because of an in } \\
\text { utero exposure to } \\
\text { either SSRIs or } \\
\text { SNRIs }\end{array}$ & $\begin{array}{l}\text { Paroxetine } \\
\text { vs. duloxetine }\end{array}$ & $\begin{array}{l}\text { Congenital } \\
\text { heart } \\
\text { disease }\end{array}$ & $\begin{array}{l}0 / 1(0 \%) \\
\text { vs. 0/1 } \\
(0 \%)^{141}\end{array}$ & $\begin{array}{l}\text { RR not } \\
\text { calculable }^{141}\end{array}$ & $\begin{array}{l}1 \text { cohort, } \\
n=2^{141}\end{array}$ & $\begin{array}{l}\text { High study } \\
\text { limitations (high } \\
\text { risk of bias, }{ }^{141} \\
\text { imprecise (small } \\
\text { Ns), } \\
\text { consistency } \\
\text { unknown }\end{array}$ & Insufficient \\
\hline
\end{tabular}

$\mathrm{CI}=$ confidence interval; $\mathrm{n}=$ number; SNRI = serotonin-norepinephrine reuptake inhibitor; SSRI = selective serotonin reuptake inhibitor; $\mathrm{RR}=$ relative risk; vs. $=$ versus .

\section{Paroxetine Versus Fluoxetine}

\section{Overview}

- Based on evidence from single studies, the evidence is insufficient to judge the comparative risk of harms from exposure during pregnancy to paroxetine monotherapy versus fluoxetine monotherapy in relation to maternal outcomes (preeclampsia, convulsive seizures, nonconvulsive seizures, or spontaneous abortion or stillbirth) or child outcomes (congenital anomaly/congenital heart disease or birth weight). The evidence was graded as insufficient in relation to the comparative risk of autism spectrum disorder based on the findings from two unadjusted high risk-of-bias studies.

\section{Detailed Results}

Five publications reported on the comparative harms of paroxetine monotherapy versus fluoxetine monotherapy. ${ }^{81,107,111,117,141}$ One medium risk-of-bias analysis reported on preeclampsia outcomes among women with depression, based on data in a Canadian British Columbia province-wide pregnancy and newborn database. The risk was not found to be significantly different between the two groups after adjusting for potential confounding characteristics. ${ }^{81}$ One high risk-of-bias analysis reported on a population of pregnant women with depression, anxiety, or bipolar disorder as well as epilepsy, drawn from the Australian Antiepileptic Drugs in Pregnancy register. ${ }^{107}$ In this study, the authors found no significant 
differences in the risk of harms for any maternal (convulsive seizures, nonconvulsive seizures, spontaneous abortion, or still birth) or child outcomes (congenital anomalies or birth weight). Each of these outcomes was graded as insufficient strength of evidence study drew from a cohort of women who had undergone a fetal echocardiogram because of in utero exposure of the fetus to SSRIs or SNRIs. ${ }^{141}$ The study was rated as high risk of bias. The study yielded insufficient strength of evidence to judge the risk of congenital heart disease (Table B-58).

Table B-58. Strength of evidence for comparative harms: Paroxetine monotherapy versus fluoxetine monotherapy

\begin{tabular}{|c|c|c|c|c|c|c|c|}
\hline Population & $\begin{array}{l}\text { Intervention } \\
\text { and } \\
\text { Comparator }\end{array}$ & Outcome & $\begin{array}{l}\text { Incidence or } \\
\text { Mean Effect } \\
\text { by Arm }\end{array}$ & Results & $\begin{array}{l}\text { Study } \\
\text { Design } \\
\text { and } \\
\text { Sample } \\
\text { Size }\end{array}$ & $\begin{array}{l}\text { Factors That } \\
\text { Affect the } \\
\text { Strength of } \\
\text { Evidence }\end{array}$ & $\begin{array}{l}\text { Overall } \\
\text { Evidence } \\
\text { Strength } \\
\text { (Direction of } \\
\text { Effect) }\end{array}$ \\
\hline $\begin{array}{l}\text { Pregnant } \\
\text { women } \\
\text { with } \\
\text { depression }\end{array}$ & $\begin{array}{l}\text { Paroxetine } \\
\text { monotherapy } \\
\text { vs. fluoxetine } \\
\text { monotherapy }\end{array}$ & Preeclampsia & NR & $\begin{array}{l}\text { ARR, } 1.70 \\
(95 \% \mathrm{Cl}, 0.93 \\
\text { to } 3.11)^{81}\end{array}$ & $\begin{array}{l}1 \text { cohort, } \\
n=N^{81}\end{array}$ & $\begin{array}{l}\text { Moderate study } \\
\text { limitations, } \\
\text { imprecise (wide } \\
\text { Cls), } \\
\text { consistency } \\
\text { unknown }\end{array}$ & Insufficient \\
\hline $\begin{array}{l}\text { Pregnant } \\
\text { women } \\
\text { with } \\
\text { epilepsy } \\
\text { and } \\
\text { depression, } \\
\text { anxiety or } \\
\text { bipolar } \\
\text { disorder }\end{array}$ & $\begin{array}{l}\text { Paroxetine } \\
\text { monotherapy } \\
\text { vs. fluoxetine } \\
\text { monotherapy }\end{array}$ & $\begin{array}{l}\text { Convulsive } \\
\text { seizures: } \\
\text { From } \\
\text { enrollment to } 7 \\
\text { months } \\
\text { gestation } \\
\text { From } 7 \\
\text { months } \\
\text { gestation to } \\
\text { delivery }\end{array}$ & $\begin{array}{l}2 / 7(29 \%) \text { vs. } \\
2 / 5(40 \%)^{107} \\
2 / 7(29 \%) \text { vs. } \\
1 / 5(20 \%)^{107}\end{array}$ & $\begin{array}{l}\text { RR, } 0.20(95 \% \\
\mathrm{Cl}, 0.04 \text { to } \\
1.10)^{107} \\
\mathrm{RR}, 0.20(95 \% \\
\mathrm{Cl}, 0.02 \text { to } \\
2.69)^{107}\end{array}$ & $\begin{array}{l}1 \text { cohort, } \\
n=12^{107}\end{array}$ & $\begin{array}{l}\text { High study } \\
\text { limitations (high } \\
\text { risk of bias }{ }^{107} \text { ), } \\
\text { seriously } \\
\text { imprecise (few } \\
\text { events, wide } \\
\text { Cls), } \\
\text { consistency } \\
\text { unknown }\end{array}$ & Insufficient \\
\hline $\begin{array}{l}\text { Pregnant } \\
\text { women } \\
\text { with } \\
\text { epilepsy } \\
\text { and } \\
\text { depression, } \\
\text { anxiety or } \\
\text { bipolar } \\
\text { disorder }\end{array}$ & $\begin{array}{l}\text { Paroxetine } \\
\text { monotherapy } \\
\text { vs. fluoxetine } \\
\text { monotherapy }\end{array}$ & $\begin{array}{l}\text { Nonconvulsive } \\
\text { seizures: } \\
\text { From } \\
\text { enrollment to } 7 \\
\text { months } \\
\text { gestation } \\
\text { From } 7 \\
\text { months } \\
\text { gestation to } \\
\text { delivery } \\
\end{array}$ & $\begin{array}{l}3 / 7(43 \%) \text { vs. } \\
1 / 5(20 \%)^{107} \\
2 / 7(29 \%) \text { vs. } \\
1 / 5(20 \%)^{107}\end{array}$ & $\begin{array}{l}\text { RR, } 0.60(95 \% \\
\mathrm{Cl}, 0.08 \text { to } \\
4.66)^{107} \\
\mathrm{RR}, 0.20(95 \% \\
\mathrm{Cl}, 0.015 \text { to } \\
2.69)^{107}\end{array}$ & $\begin{array}{l}1 \text { cohort, } \\
n=12^{107}\end{array}$ & $\begin{array}{l}\text { High study } \\
\text { limitations (high } \\
\text { risk of bias }{ }^{107} \text { ), } \\
\text { seriously } \\
\text { imprecise (few } \\
\text { events, wide } \\
\text { Cls), } \\
\text { consistency } \\
\text { unknown }\end{array}$ & Insufficient \\
\hline $\begin{array}{l}\text { Pregnant } \\
\text { women } \\
\text { with } \\
\text { epilepsy } \\
\text { and } \\
\text { depression, } \\
\text { anxiety or } \\
\text { bipolar } \\
\text { disorder }\end{array}$ & $\begin{array}{l}\text { Paroxetine } \\
\text { monotherapy } \\
\text { vs. fluoxetine } \\
\text { monotherapy }\end{array}$ & $\begin{array}{l}\text { Spontaneous } \\
\text { abortion }\end{array}$ & $\begin{array}{l}\text { 0/7 (0\%) vs. } \\
0 / 5(0 \%)^{107}\end{array}$ & $\begin{array}{l}\text { RR not } \\
\text { calculable: no } \\
\text { events }\end{array}$ & $\begin{array}{l}1 \text { cohort, } \\
n=12^{107}\end{array}$ & $\begin{array}{l}\text { High study } \\
\text { limitations (high } \\
\text { risk of bias }{ }^{107} \text { ), } \\
\text { likely imprecise } \\
\text { (no events, } \\
\text { small N), } \\
\text { consistency } \\
\text { unknown }\end{array}$ & Insufficient \\
\hline $\begin{array}{l}\text { Pregnant } \\
\text { women } \\
\text { with } \\
\text { epilepsy } \\
\text { and } \\
\text { depression, } \\
\text { anxiety or } \\
\text { bipolar } \\
\text { disorder }\end{array}$ & $\begin{array}{l}\text { Paroxetine } \\
\text { monotherapy } \\
\text { vs. fluoxetine } \\
\text { monotherapy }\end{array}$ & Stillbirth & $\begin{array}{l}0 / 7(0 \%) \text { vs. } \\
0 / 5(0 \%)^{107}\end{array}$ & $\begin{array}{l}\text { RR not } \\
\text { calculable: no } \\
\text { events }\end{array}$ & $\begin{array}{l}1 \text { cohort, } \\
n=12^{107}\end{array}$ & $\begin{array}{l}\text { High study } \\
\text { limitations (high } \\
\text { risk of bias }{ }^{107} \text { ), } \\
\text { likely imprecise } \\
\text { (no events, } \\
\text { small N), } \\
\text { consistency } \\
\text { unknown }\end{array}$ & Insufficient \\
\hline
\end{tabular}




\begin{tabular}{|c|c|c|c|c|c|c|c|}
\hline Population & $\begin{array}{l}\text { Intervention } \\
\text { and } \\
\text { Comparator }\end{array}$ & Outcome & $\begin{array}{l}\text { Incidence or } \\
\text { Mean Effect } \\
\text { by Arm }\end{array}$ & Results & $\begin{array}{l}\text { Study } \\
\text { Design } \\
\text { and } \\
\text { Sample } \\
\text { Size } \\
\end{array}$ & $\begin{array}{l}\text { Factors That } \\
\text { Affect the } \\
\text { Strength of } \\
\text { Evidence }\end{array}$ & $\begin{array}{l}\text { Overall } \\
\text { Evidence } \\
\text { Strength } \\
\text { (Direction of } \\
\text { Effect) } \\
\end{array}$ \\
\hline $\begin{array}{l}\text { Pregnant } \\
\text { women } \\
\text { with } \\
\text { epilepsy } \\
\text { and } \\
\text { depression, } \\
\text { anxiety or } \\
\text { bipolar } \\
\text { disorder } \\
\end{array}$ & $\begin{array}{l}\text { Paroxetine } \\
\text { monotherapy } \\
\text { vs. fluoxetine } \\
\text { monotherapy }\end{array}$ & $\begin{array}{l}\text { Congenital } \\
\text { anomaly }\end{array}$ & $\begin{array}{l}0 / 7(0 \%) \text { vs. } \\
0 / 5(0 \%)^{107}\end{array}$ & $\begin{array}{l}\text { RR not } \\
\text { calculable: no } \\
\text { events }\end{array}$ & $\begin{array}{l}1 \text { cohort, } \\
n=12^{107}\end{array}$ & $\begin{array}{l}\text { High study } \\
\text { limitations (high } \\
\text { risk of bias }{ }^{107} \text { ), } \\
\text { likely imprecise } \\
\text { (no events, } \\
\text { small N), } \\
\text { consistency } \\
\text { unknown }\end{array}$ & Insufficient \\
\hline $\begin{array}{l}\text { Women } \\
\text { who } \\
\text { underwent } \\
\text { fetal } \\
\text { echocardio } \\
\text { graphy } \\
\text { because of } \\
\text { an in utero } \\
\text { exposure to } \\
\text { either } \\
\text { SSRIs or } \\
\text { SNRIs } \\
\end{array}$ & $\begin{array}{l}\text { Paroxetine } \\
\text { vs. fluoxetine }\end{array}$ & $\begin{array}{l}\text { Congenital } \\
\text { heart disease }\end{array}$ & $\begin{array}{l}0 / 1(0 \%) \text { vs. } \\
2 / 9 \\
(22.00 \%)^{141}\end{array}$ & $\begin{array}{l}\text { Risk difference } \\
-0.22(95 \% \mathrm{Cl} \text {, } \\
-0.88 \text { to } 0 .)^{141}\end{array}$ & $\begin{array}{l}1 \text { cohort, } \\
n=10^{141}\end{array}$ & $\begin{array}{l}\text { High study } \\
\text { limitations (high } \\
\text { risk of bias, }{ }^{141} \\
\text { imprecise (wide } \\
\text { Cls), } \\
\text { consistency } \\
\text { unknown }\end{array}$ & Insufficient \\
\hline $\begin{array}{l}\text { Pregnant } \\
\text { women } \\
\text { with } \\
\text { epilepsy } \\
\text { and } \\
\text { depression, } \\
\text { anxiety or } \\
\text { bipolar } \\
\text { disorder }\end{array}$ & $\begin{array}{l}\text { Paroxetine } \\
\text { monotherapy } \\
\text { vs. fluoxetine } \\
\text { monotherapy } \\
\end{array}$ & Birth weight & $\begin{array}{l}\text { Median } \pm \text { IQR: } \\
3,010 \pm 670 \text { vs. } \\
2,790 \pm 497^{107}\end{array}$ & $\begin{array}{l}\text { Difference not } \\
\text { calculable }\end{array}$ & $\begin{array}{l}1 \text { cohort, } \\
n=12^{107}\end{array}$ & $\begin{array}{l}\text { High study } \\
\text { limitations (high } \\
\text { risk of bias }{ }^{107} \text { ), } \\
\text { likely imprecise } \\
\text { (small Ns), } \\
\text { consistency } \\
\text { unknown }\end{array}$ & Insufficient \\
\hline $\begin{array}{l}\text { Pregnant } \\
\text { women } \\
\text { taking } \\
\text { paroxetine } \\
\text { or } \\
\text { fluoxetine }\end{array}$ & $\begin{array}{l}\text { Paroxetine } \\
\text { monotherapy } \\
\text { vs. fluoxetine } \\
\text { monotherapy }\end{array}$ & $\begin{array}{l}\text { Autism } \\
\text { spectrum } \\
\text { disorder }\end{array}$ & $\begin{array}{l}11 / 744(1.5 \%) \\
\text { vs. } 5 / 171 \\
(2.9 \%)^{111} \\
7 / 871(0.6 \%) \\
\text { vs. } 18 / 160 \\
(11 \%)^{117}\end{array}$ & $\begin{array}{l}\text { RR, } 0.51(95 \% \\
\mathrm{Cl}, 0.18 \text { to } \\
1.44)^{111} \\
\mathrm{RR}, 0.07(95 \% \\
\mathrm{Cl}, 0.03 \text { to } \\
0.17)^{117}\end{array}$ & $\begin{array}{l}2 \text { cohorts, } \\
n=915 \text { in } \\
\text { one } \\
\text { cohort, }{ }^{111} \\
n=1,031 \text { in } \\
\text { one } \\
\text { cohort }^{117}\end{array}$ & $\begin{array}{l}\text { High study } \\
\text { limitations (high } \\
\text { risk of bias }{ }^{107} \text { ), } \\
\text { imprecise (few } \\
\text { events), } \\
\text { consistent }\end{array}$ & Insufficient \\
\hline
\end{tabular}

$\mathrm{ARR}=$ adjusted risk ratio; $\mathrm{CI}=$ confidence interval; $\mathrm{n}=$ number; $\mathrm{NR}=$ not reported; $\mathrm{NS}=$ not significant; $\mathrm{RR}=$ relative risk; SNRI = serotonin-norepinephrine reuptake inhibitor; SSRI = selective serotonin reuptake inhibitor; vs. = versus.

Two publications reported on the relative risk of autism spectrum disorder. Data from the Danish Birth registry ${ }^{117}$ were analyzed in one study, and data from the Canadian QPC were analyzed in the second. ${ }^{111}$ While both studies found the same direction of effect, a smaller risk of autism spectrum disorder from paroxetine compared with fluoxetine, the difference was statistically significant in one analysis but not in a second. Both studies were rated as high risk of bias and did not control for confounding, resulting in a grade of insufficient strength of evidence. 


\section{Paroxetine Versus Venlafaxine}

\section{Overview}

- The evidence is insufficient to judge the risk of harms for maternal (spontaneous abortion) or child outcomes (congenital heart disease) for exposure during pregnancy to paroxetine versus venlafaxine.

\section{Detailed Results}

Two high risk-of-bias publication compared the maternal or infant harms of paroxetine versus other pharmacologic interventions for mental health disorders during pregnancy. ${ }^{122,141}$ One case-control study, set in Canada, quantified the association between dispensing of paroxetine or venlafaxine in early pregnancy and spontaneous abortion risk and found no difference between paroxetine versus venlafaxine. ${ }^{122}$ One publication reported on the comparative harms of venlafaxine versus paroxetine. ${ }^{141}$ The study drew from a cohort of women who had undergone a fetal echocardiogram because of in utero exposure of the fetus to SSRIs or SNRIs. ${ }^{141}$ The study was rated as high risk of bias. The study yielded insufficient strength of evidence to judge the risk of congenital heart disease. The evidence was insufficient to judge harms for the outcome (Table B-59).

Table B-59. Strength of evidence for comparative harms: Paroxetine versus venlafaxine

\begin{tabular}{|c|c|c|c|c|c|c|c|}
\hline Population & $\begin{array}{l}\text { Intervention } \\
\text { and } \\
\text { Comparator }\end{array}$ & Outcome & $\begin{array}{l}\text { Incidence or } \\
\text { Mean Effect } \\
\text { by Arm }\end{array}$ & Results & $\begin{array}{l}\text { Study Design } \\
\text { and Sample } \\
\text { Size }\end{array}$ & $\begin{array}{l}\text { Factors That } \\
\text { Affect the } \\
\text { Strength of } \\
\text { Evidence }\end{array}$ & $\begin{array}{l}\text { Overall } \\
\text { Evidence } \\
\text { Strength } \\
\text { (Direction } \\
\text { of Effect) } \\
\end{array}$ \\
\hline $\begin{array}{l}\text { Women who filled } \\
\text { a prescription for } \\
\text { an SSRI or SNRI } \\
\text { in first trimester of } \\
\text { pregnancy }\end{array}$ & $\begin{array}{l}\text { Paroxetine } \\
\text { vs. } \\
\text { venlafaxine }\end{array}$ & $\begin{array}{l}\text { Spontaneous } \\
\text { abortion }\end{array}$ & $\begin{array}{l}84 / 569 \\
(14.76 \%) \text { vs, } \\
33 / 161 \\
(20.50 \%)^{22}\end{array}$ & $\begin{array}{l}\text { OR } 0.67 \\
(95 \% \mathrm{Cl}, \\
0.43- \\
1.05)^{22}\end{array}$ & $\begin{array}{l}1 \text { case-control } \\
\text { study, } \mathrm{N}=730^{122}\end{array}$ & $\begin{array}{l}\text { High study } \\
\text { limitations } \\
\text { (high risk of }_{\text {bias }^{122} \text { ), }} \\
\text { imprecise } \\
\text { (wide Cls), } \\
\text { consistency } \\
\text { unknown }\end{array}$ & Insufficient \\
\hline $\begin{array}{l}\text { Women who } \\
\text { underwent fetal } \\
\text { echocardiography } \\
\text { because of an in } \\
\text { utero exposure to } \\
\text { either SSRIs or } \\
\text { SNRIs }\end{array}$ & $\begin{array}{l}\text { Venlafaxine } \\
\text { vs. } \\
\text { paroxetine }\end{array}$ & $\begin{array}{l}\text { Congenital } \\
\text { heart } \\
\text { disease }\end{array}$ & $\begin{array}{l}2 / 2(100 \%) \\
\text { vs. } 0 / 1(0 \%)^{141}\end{array}$ & $\begin{array}{l}\text { Risk } \\
\text { difference } \\
-1(95 \% \\
\text { Cl, }-1.73 \\
\text { to }- \\
0.27)^{141}\end{array}$ & 1 cohort, $n=3^{141}$ & $\begin{array}{l}\text { High study } \\
\text { limitations } \\
\text { (high risk of } \\
\text { bias, }{ }^{141} \\
\text { imprecise } \\
\text { (wide Cls), } \\
\text { consistency } \\
\text { unknown }\end{array}$ & Insufficient \\
\hline
\end{tabular}

$\mathrm{CI}=$ confidence interval; $\mathrm{N}=$ number; $\mathrm{OR}=$ odds ratio; $\mathrm{SNRI}=$ serotonin-norepinephrine reuptake inhibitor; $\mathrm{SSRI}=$ selective serotonin reuptake inhibitor; vs. $=$ versus.

\section{Paroxetine Versus Citalopram or Escitalopram}

\section{Overview}

- The evidence is insufficient to judge the risk of harms for child outcomes (stillbirth, birth weight, or congenital anomalies) for exposure during pregnancy to paroxetine versus citalopram or escitalopram. 


\section{Detailed Results}

One high risk-of-bias publication from the Australian Pregnancy Register of Anti-Epileptic Drugs in Pregnancy ${ }^{107}$ reported on outcomes among women with epilepsy who were treated with both anti-epileptic drugs (AEDs) and antidepressants for depression or anxiety. No differences were found in risk for stillbirth, median birthweight, or congenital anomalies for paroxetine compared with citalopram or escitalopram. The evidence was insufficient to judge harms for all outcomes (Table B-60).

Table B-60. Strength of evidence for comparative harms: Paroxetine versus citalopram or escitalopram

\begin{tabular}{|c|c|c|c|c|c|c|c|}
\hline Population & $\begin{array}{l}\text { Intervention } \\
\text { and } \\
\text { Comparator }\end{array}$ & Outcome & $\begin{array}{l}\text { Incidence or } \\
\text { Mean Effect by } \\
\text { Arm }\end{array}$ & Results & $\begin{array}{l}\text { Study Design } \\
\text { and Sample Size }\end{array}$ & $\begin{array}{l}\text { Factors That } \\
\text { Affect the } \\
\text { Strength of } \\
\text { Evidence }\end{array}$ & $\begin{array}{l}\text { Overall } \\
\text { Evidence } \\
\text { Strength } \\
\text { (Direction } \\
\text { of Effect) }\end{array}$ \\
\hline $\begin{array}{l}\text { Pregnant } \\
\text { women with } \\
\text { epilepsy } \\
\text { and anxiety } \\
\text { or } \\
\text { depression, } \\
\text { on AEDs } \\
\end{array}$ & $\begin{array}{l}\text { Paroxetine } \\
\text { vs. } \\
\text { citalopram or } \\
\text { escitalopram }\end{array}$ & Stillbirths & $\begin{array}{l}0 / 7(0 \%) \text { vs. } 0 / 21 \\
(0 \%)^{107}\end{array}$ & $\begin{array}{l}\text { No } \\
\text { events }{ }^{107}\end{array}$ & 1 cohort, $N=28^{107}$ & $\begin{array}{l}\text { High study } \\
\text { limitations (high } \\
\left.\text { risk of bias }{ }^{107}\right), \\
\text { likely imprecise, } \\
\text { consistency } \\
\text { unknown }\end{array}$ & Insufficient \\
\hline $\begin{array}{l}\text { Pregnant } \\
\text { women with } \\
\text { epilepsy } \\
\text { and anxiety } \\
\text { or } \\
\text { depression, } \\
\text { on AEDs } \\
\end{array}$ & $\begin{array}{l}\text { Paroxetine } \\
\text { vs. } \\
\text { citalopram or } \\
\text { escitalopram }\end{array}$ & Birthweight & $\begin{array}{l}\text { Median (IQR) } \\
\text { Paroxetine: } \\
3,010 \mathrm{~g}(670) \\
\text { Citalopram or } \\
\text { escitalopram: } \\
3,087 \mathrm{~g}(286)^{107}\end{array}$ & $\begin{array}{l}\text { Difference } \\
\text { not } \\
\text { calculable }\end{array}$ & 1 cohort, $\mathrm{N}=28^{107}$ & $\begin{array}{l}\text { High study } \\
\text { limitations (high } \\
\text { risk of bias }{ }^{107} \text { ), } \\
\text { likely imprecise } \\
\text { (small Ns), } \\
\text { consistency } \\
\text { unknown }\end{array}$ & Insufficient \\
\hline $\begin{array}{l}\text { Pregnant } \\
\text { women with } \\
\text { epilepsy } \\
\text { and anxiety } \\
\text { or } \\
\text { depression, } \\
\text { on AEDs }\end{array}$ & $\begin{array}{l}\text { Paroxetine } \\
\text { vs. } \\
\text { citalopram or } \\
\text { escitalopram }\end{array}$ & $\begin{array}{l}\text { Congenital } \\
\text { anomaly }\end{array}$ & $\begin{array}{l}\text { Paroxetine: } 0 / 7 \\
(0 \%) \\
\text { Citalopram or } \\
\text { escitalopram: } \\
4 / 21(19.0 \%)^{107}\end{array}$ & $\begin{array}{l}\text { RD, }-0.19 \\
(95 \% \\
\mathrm{Cl},-0.43 \\
\text { to } 0.05)^{107}\end{array}$ & 1 cohort, $\mathrm{N}=28^{107}$ & $\begin{array}{l}\text { High study } \\
\text { limitations (high } \\
\text { risk of bias }{ }^{107} \text { ), } \\
\text { imprecise (wide } \\
\text { Cls), consistency } \\
\text { unknown }\end{array}$ & Insufficient \\
\hline
\end{tabular}

$\mathrm{AED}=$ anti-epileptic drug; $\mathrm{CI}=$ confidence interval; $\mathrm{IQR}=$ interquartile ratio; $\mathrm{N}$ = number; $\mathrm{RD}=$ risk difference; vs. $=$ versus.

\section{Paroxetine Versus Sertraline}

\section{Overview}

- The evidence is insufficient to judge the risk of harms for child outcomes (birth weight, congenital anomalies, congenital heart disease, or autism spectrum disorder) for exposure during pregnancy to paroxetine versus sertraline.

\section{Detailed Results}

Four high risk-of-bias publications compared the maternal or infant harms of paroxetine versus sertraline for mental health disorders during pregnancy. ${ }^{107,111,117,141}$ A publication from the Australian Pregnancy Register of Anti-Epileptic Drugs in Pregnancy ${ }^{107}$ reported on outcomes among women with epilepsy who were treated with both AEDs and antidepressants for depression or anxiety. No differences were found in risk for stillbirth, median birthweight, or congenital anomalies for paroxetine compared with sertraline. Two publications ${ }^{111,117}$ evaluated autism spectrum disorder diagnosis among children exposed in utero to sertraline versus 
paroxetine and found no association. One study evaluated the risk of congenital heart disease among a cohort of women who had undergone a fetal echocardiogram because of in utero exposure of the fetus to SSRIs or SNRIs. ${ }^{141}$ The evidence was insufficient to judge harms for all outcomes (Table B-61).

Table B-61. Strength of evidence for comparative harms: Paroxetine versus sertraline

\begin{tabular}{|c|c|c|c|c|c|c|c|}
\hline Population & $\begin{array}{l}\text { Intervention } \\
\text { and } \\
\text { Comparator }\end{array}$ & Outcome & $\begin{array}{l}\text { Incidence or } \\
\text { Mean Effect by } \\
\text { Arm }\end{array}$ & Results & $\begin{array}{l}\text { Study Design and } \\
\text { Sample Size }\end{array}$ & $\begin{array}{l}\text { Factors That } \\
\text { Affect the } \\
\text { Strength of } \\
\text { Evidence }\end{array}$ & $\begin{array}{l}\text { Overall } \\
\text { Evidence } \\
\text { Strength } \\
\text { (Direction } \\
\text { of Effect) } \\
\end{array}$ \\
\hline $\begin{array}{l}\text { Pregnant } \\
\text { women with } \\
\text { epilepsy and } \\
\text { anxiety or } \\
\text { depression, on } \\
\text { AEDs } \\
\end{array}$ & $\begin{array}{l}\text { Paroxetine } \\
\text { vs. sertraline }\end{array}$ & Stillbirth & $\begin{array}{l}0 / 7(0 \%) \text { vs. } \\
0 / 25(0 \%)^{107}\end{array}$ & $\begin{array}{l}\text { RR not } \\
\text { calculable: } \\
\text { no events }\end{array}$ & 1 cohort, $\mathrm{N}=32^{107}$ & $\begin{array}{l}\text { High study } \\
\text { limitations (high } \\
\text { risk of bias, }{ }^{107} \text { likely } \\
\text { imprecise, } \\
\text { consistency } \\
\text { unknown }\end{array}$ & Insufficient \\
\hline $\begin{array}{l}\text { Pregnant } \\
\text { women with } \\
\text { epilepsy and } \\
\text { anxiety or } \\
\text { depression, on } \\
\text { AEDs } \\
\end{array}$ & $\begin{array}{l}\text { Paroxetine } \\
\text { vs. sertraline }\end{array}$ & Birthweight & $\begin{array}{l}\text { Median (IQR) } \\
\text { Paroxetine: } \\
3,010 \mathrm{~g}(670) \\
\text { Sertraline: } \\
3,350 \mathrm{~g}(808)^{107} \\
\end{array}$ & $\begin{array}{l}\text { RR not } \\
\text { calculable: } \\
\text { no events }\end{array}$ & 1 cohort, $\mathrm{N}=32^{107}$ & $\begin{array}{l}\text { High study } \\
\text { limitations (high } \\
\text { risk of bias, }{ }^{107} \text { likely } \\
\text { imprecise (small } \\
\text { Ns), consistency } \\
\text { unknown }\end{array}$ & Insufficient \\
\hline $\begin{array}{l}\text { Women who } \\
\text { underwent fetal } \\
\text { echocardiograp } \\
\text { hy because of } \\
\text { an in utero } \\
\text { exposure to } \\
\text { either SSRIs or } \\
\text { SNRIs }\end{array}$ & $\begin{array}{l}\text { Paroxetine } \\
\text { vs. sertraline }\end{array}$ & $\begin{array}{l}\text { Congenital } \\
\text { heart } \\
\text { disease }\end{array}$ & $\begin{array}{l}0 / 1(0 \%) \text { vs. } 0 / 7 \\
(0 \%)^{141}\end{array}$ & $\begin{array}{l}\text { RR not } \\
\text { calculable: } \\
\text { no events }\end{array}$ & 1 cohort, $n=8^{141}$ & $\begin{array}{l}\text { High study } \\
\text { limitations (high } \\
\text { risk of bias, } 141 \\
\text { imprecise (small } \\
\text { Ns, no events), } \\
\text { consistency } \\
\text { unknown }\end{array}$ & Insufficient \\
\hline $\begin{array}{l}\text { Women } \\
\text { exposed to } \\
\text { antidepressants } \\
\text { in } 2 \text { nd/3rd } \\
\text { trimester of } \\
\text { pregnancy }\end{array}$ & $\begin{array}{l}\text { Paroxetine } \\
\text { vs. sertraline }\end{array}$ & $\begin{array}{l}\text { Autism } \\
\text { spectrum } \\
\text { disorder }\end{array}$ & $\begin{array}{l}\text { Paroxetine: } \\
\text { 11/744 }(1.5 \%) \\
\text { vs. sertraline: } \\
1 / 292 \\
(0.34 \%)^{111} \\
\\
\text { Paroxetine: } \\
7 / 871(0.8 \%) \\
\text { vs. sertraline: } \\
9 / 1,576 \\
(0.57 \%)^{111}\end{array}$ & $\begin{array}{l}\text { RR, } 4.3 \\
(95 \% \mathrm{Cl}, \\
0.56 \text { to } \\
33.3)^{111} \\
\mathrm{RR}, 1.41 \\
(95 \% \mathrm{Cl}, \\
0.53 \text { to } \\
3.77)^{117}\end{array}$ & $\begin{array}{l}2 \text { cohorts, } \\
\mathrm{N}=3,483^{111,117}\end{array}$ & $\begin{array}{l}\text { High study } \\
\text { limitations (high } \\
\text { risk of bias }{ }^{111,117} \text { ), } \\
\text { imprecise (wide } \\
\text { Cls), consistent }\end{array}$ & Insufficient \\
\hline
\end{tabular}

$\mathrm{AED}=$ anti-epileptic drug; $\mathrm{CI}=$ confidence interval; $\mathrm{IQR}=$ interquartile ratio; $\mathrm{N}=$ number; $\mathrm{RR}=$ relative risk; $\mathrm{SNRI}=$ serotonin-norepinephrine reuptake inhibitor; SSRI = selective serotonin reuptake inhibitor; vs. = versus.

\section{Paroxetine Versus SNRIs}

\section{Overview}

- The evidence is insufficient to judge the risk of harms for maternal (spontaneous abortion) or child outcomes (stillbirth, birth weight, or congenital anomalies) for exposure during pregnancy to paroxetine versus SNRIs. 


\section{Detailed Results}

One high risk-of-bias publication from the Australian Pregnancy Register of Anti-Epileptic Drugs in Pregnancy ${ }^{107}$ reported on outcomes among women with epilepsy who were treated with both AEDs and antidepressants for depression or anxiety. No differences were found in risk for stillbirth, median birthweight, or congenital anomalies for paroxetine compared with SNRIs. The evidence was insufficient to judge harms for all outcomes (Table B-62).

Table B-62. Strength of evidence for comparative harms: Paroxetine versus SNRIs

\begin{tabular}{|c|c|c|c|c|c|c|c|}
\hline Population & $\begin{array}{l}\text { Intervention } \\
\text { and } \\
\text { Comparator }\end{array}$ & Outcome & $\begin{array}{l}\text { Incidence or } \\
\text { Mean Effect } \\
\text { by Arm }\end{array}$ & Results & $\begin{array}{l}\text { Study } \\
\text { Design } \\
\text { and } \\
\text { Sample } \\
\text { Size }\end{array}$ & $\begin{array}{l}\text { Factors That Affect } \\
\text { the Strength of } \\
\text { Evidence }\end{array}$ & $\begin{array}{l}\text { Overall } \\
\text { Evidence } \\
\text { Strength } \\
\text { (Direction of } \\
\text { Effect) }\end{array}$ \\
\hline $\begin{array}{l}\text { Pregnant } \\
\text { women with } \\
\text { epilepsy and } \\
\text { anxiety or } \\
\text { depression, } \\
\text { on AEDs }\end{array}$ & $\begin{array}{l}\text { Paroxetine vs. } \\
\text { SNRI } \\
\text { monotherapy }\end{array}$ & Stillbirth & $\begin{array}{l}\text { Paroxetine:0/ } \\
7(0 \%) \\
\text { SNRI } \\
\text { monotherapy: } \\
\text { 1/13 }(7.7 \%)^{107}\end{array}$ & $\begin{array}{l}\text { No } \\
\text { difference }{ }^{107}\end{array}$ & $\begin{array}{l}1 \text { cohort, } \\
\mathrm{N}=20^{107}\end{array}$ & $\begin{array}{l}\text { High study } \\
\text { limitations (high risk } \\
\text { of bias }{ }^{107} \text { ), imprecise } \\
\text { (wide Cls), } \\
\text { consistency } \\
\text { unknown }\end{array}$ & Insufficient \\
\hline $\begin{array}{l}\text { Pregnant } \\
\text { women with } \\
\text { epilepsy and } \\
\text { anxiety or } \\
\text { depression, } \\
\text { on AEDs }\end{array}$ & $\begin{array}{l}\text { Paroxetine vs. } \\
\text { SNRI } \\
\text { monotherapy }\end{array}$ & Birthweight & $\begin{array}{l}\text { Median (IQR) } \\
\text { Paroxetine: } \\
3010 \mathrm{~g}(670) \\
\text { SNRI } \\
\text { monotherapy: } \\
3530 \mathrm{~g} \\
(561)^{107}\end{array}$ & $\begin{array}{l}\text { Difference } \\
\text { not } \\
\text { calculable }\end{array}$ & $\begin{array}{l}1 \text { cohort, } \\
\mathrm{N}=20^{107}\end{array}$ & $\begin{array}{l}\text { High study } \\
\text { limitations (high risk } \\
\text { of bias }{ }^{107} \text { ), likely } \\
\text { imprecise (small } \\
\text { Ns), consistency } \\
\text { unknown }\end{array}$ & Insufficient \\
\hline $\begin{array}{l}\text { Pregnant } \\
\text { women with } \\
\text { epilepsy and } \\
\text { anxiety or } \\
\text { depression, } \\
\text { on AEDs }\end{array}$ & $\begin{array}{l}\text { Paroxetine vs. } \\
\text { SNRI } \\
\text { monotherapy }\end{array}$ & $\begin{array}{l}\text { Congenital } \\
\text { anomaly }\end{array}$ & $\begin{array}{l}\text { Paroxetine: } \\
\text { 0/7 }(0 \%) \\
\text { SNRI } \\
\text { monotherapy: } \\
0 / 13(0 \%)^{107}\end{array}$ & $\begin{array}{l}\text { RD, } 0(95 \% \\
C l,-0.19 \text { to } \\
0.19)^{107}\end{array}$ & $\begin{array}{l}1 \text { cohort, } \\
\mathrm{N}=20^{107}\end{array}$ & $\begin{array}{l}\text { High study } \\
\text { limitations (high risk } \\
\text { of bias }{ }^{107} \text { ), imprecise } \\
\text { (wide Cls), } \\
\text { consistency } \\
\text { unknown }\end{array}$ & Insufficient \\
\hline
\end{tabular}

$\mathrm{AED}=$ anti-epileptic drug; $\mathrm{CI}=$ confidence interval; $\mathrm{IQR}=$ interquartile ratio; $\mathrm{N}=$ number; $\mathrm{RD}=$ risk difference; $\mathrm{SNRI}=$ serotonin-norepinephrine reuptake inhibitor; vs. $=$ versus.

\section{Paroxetine Versus Other SSRI Comparators}

\section{Overview}

- The evidence is insufficient to judge the risk of harms for child outcomes (preterm birth, birth weight, congenital anomalies status, or respiratory distress) for exposure during pregnancy to paroxetine versus other SSRIs.

\section{Detailed Results}

Two high risk-of-bias publications compared the maternal or infant harms of paroxetine versus other SSRIs for mental health disorders during pregnancy. ${ }^{87,}{ }^{131}$ A Swedish Medical Birth Registry publication compared neonatal outcomes among women exposed to paroxetine versus other SSRIs (mainly citalopram) during pregnancy and reported no difference in preterm birth, low birthweight, small for gestational age, large for gestational age, or respiratory distress. ${ }^{131} \mathrm{~A}$ high risk-of-bias case-control study within the $\mathrm{QPC}^{87}$ evaluated major congenital and cardiac anomalies among pregnancies exposed to paroxetine versus other SSRIs in the first trimester and found no differences in outcomes (Table B-63). 
Table B-63. Strength of evidence for comparative harms: Paroxetine versus other SSRIs

\begin{tabular}{|c|c|c|c|c|c|c|c|}
\hline Population & $\begin{array}{l}\text { Intervention } \\
\text { and } \\
\text { Comparator }\end{array}$ & Outcome & $\begin{array}{l}\text { Incidence } \\
\text { or Mean } \\
\text { Effect by } \\
\text { Arm }\end{array}$ & Results & $\begin{array}{l}\text { Study } \\
\text { Design and } \\
\text { Sample } \\
\text { Size }\end{array}$ & $\begin{array}{l}\text { Factors That } \\
\text { Affect the } \\
\text { Strength of } \\
\text { Evidence }\end{array}$ & $\begin{array}{l}\text { Overall } \\
\text { Evidence } \\
\text { Strength } \\
\text { (Direction } \\
\text { of Effect) }\end{array}$ \\
\hline $\begin{array}{l}\text { Pregnant } \\
\text { women with 1st } \\
\text { trimester } \\
\text { exposure to an } \\
\text { antidepressant }\end{array}$ & $\begin{array}{l}\text { Paroxetine vs. } \\
\text { other SSRI } \\
\text { antidepressant }\end{array}$ & $\begin{array}{l}\text { Major } \\
\text { congenital } \\
\text { anomalies }\end{array}$ & $\begin{array}{l}\text { Paroxetine: } \\
43 \text { cases, } \\
499 \\
\text { controls } \\
\text { Other SSRI } \\
\text { antidepres- } \\
\text { sants: } 28 \\
\text { cases, } 415 \\
\text { controls }^{87}\end{array}$ & $\begin{array}{l}\text { Crude OR } \\
1.28(95 \% \\
\mathrm{Cl}, 0.78 \\
\text { to } 2.11)^{87}\end{array}$ & $\begin{array}{l}1 \text { case: } \\
\text { control, } \\
n=985^{87}\end{array}$ & $\begin{array}{l}\text { High study } \\
\text { limitations (high } \\
\text { risk of bias }{ }^{87} \text { ), } \\
\text { imprecise (wide } \\
\text { Cls), consistency } \\
\text { unknown }\end{array}$ & Insufficient \\
\hline $\begin{array}{l}\text { Pregnant } \\
\text { women with 1st } \\
\text { trimester } \\
\text { exposure to an } \\
\text { antidepressant }\end{array}$ & $\begin{array}{l}\text { Paroxetine vs. } \\
\text { other SSRI } \\
\text { antidepressant }\end{array}$ & $\begin{array}{l}\text { Major cardiac } \\
\text { anomalies }\end{array}$ & $\begin{array}{l}\text { Paroxetine: } \\
10 \text { cases, } \\
499 \\
\text { controls } \\
\text { Other SSRI } \\
\text { antidepres- } \\
\text { sants: } 6 \\
\text { cases, } 415 \\
\text { controls }\end{array}$ & $\begin{array}{l}\text { Crude OR } \\
1.39(95 \% \\
\mathrm{Cl}, 0.50 \\
\text { to } 4.14)^{87}\end{array}$ & $\begin{array}{l}1 \text { case- } \\
\text { control, } \\
n=930^{87}\end{array}$ & $\begin{array}{l}\text { High study } \\
\text { limitations (high } \\
\text { risk of bias }{ }^{87} \text { ), } \\
\text { imprecise (wide } \\
\text { Cls), consistency } \\
\text { unknown }\end{array}$ & Insufficient \\
\hline $\begin{array}{l}\text { Women with } \\
\text { antidepressant } \\
\text { use in } \\
\text { pregnancy }\end{array}$ & $\begin{array}{l}\text { Paroxetine vs. } \\
\text { other SSRIs, } \\
\text { mainly } \\
\text { citalopram }\end{array}$ & $\begin{array}{l}\text { Preterm } \\
\text { delivery }<37 \\
\text { weeks }\end{array}$ & $\begin{array}{l}11 / 104 \\
(10.6 \%) \\
\text { s. } 38 / 449 \\
(8.5 \%)^{131}\end{array}$ & $\begin{array}{l}\mathrm{RR}, 1.25 \\
(95 \% \mathrm{Cl}, \\
0.66 \text { to } \\
2.36)^{131}\end{array}$ & $\begin{array}{l}1 \text { cohort, } \\
N=519^{131}\end{array}$ & $\begin{array}{l}\text { High study } \\
\text { limitations (high } \\
\text { risk of bias }{ }^{131} \text { ), } \\
\text { imprecise (wide } \\
\text { Cls), consistency } \\
\text { unknown }\end{array}$ & Insufficient \\
\hline $\begin{array}{l}\text { Women with } \\
\text { antidepressant } \\
\text { use in } \\
\text { pregnancy }\end{array}$ & $\begin{array}{l}\text { Paroxetine vs. } \\
\text { other SSRIs, } \\
\text { mainly } \\
\text { citalopram }\end{array}$ & $\begin{array}{l}\text { Low birth } \\
\text { weight }\end{array}$ & $\begin{array}{l}5 / 94(5.3 \%) \\
\text { vs. } 16 / 425 \\
(3.8 \%)^{131}\end{array}$ & $\begin{array}{l}\text { RR, } 1.41 \\
(95 \% \mathrm{Cl}, \\
0.53 \text { to } \\
3.76)^{131}\end{array}$ & $\begin{array}{l}1 \text { cohort, } \\
\mathrm{N}=519^{131}\end{array}$ & $\begin{array}{l}\text { High study } \\
\text { limitations (high } \\
\text { risk of bias }{ }^{131} \text { ), } \\
\text { imprecise (wide } \\
\text { Cls), consistency } \\
\text { unknown }\end{array}$ & Insufficient \\
\hline $\begin{array}{l}\text { Women with } \\
\text { antidepressant } \\
\text { use in } \\
\text { pregnancy }\end{array}$ & $\begin{array}{l}\text { Paroxetine vs. } \\
\text { other SSRIs, } \\
\text { mainly } \\
\text { citalopram }\end{array}$ & SGA & $\begin{array}{l}2 / 94(2.3 \%) \\
\text { Vs. } 10 / 425 \\
(2.4 \%)^{131}\end{array}$ & $\begin{array}{l}\mathrm{RR}, 0.90 \\
(95 \% \mathrm{Cl}, \\
0.20 \text { to } \\
4.06)^{131}\end{array}$ & $\begin{array}{l}1 \text { cohort, } \\
N=519^{131}\end{array}$ & $\begin{array}{l}\text { High study } \\
\text { limitations (high } \\
\text { risk of bias }{ }^{131} \text { ), } \\
\text { imprecise (wide } \\
\text { Cls), consistency } \\
\text { unknown }\end{array}$ & Insufficient \\
\hline $\begin{array}{l}\text { Women with } \\
\text { antidepressant } \\
\text { use in } \\
\text { pregnancy }\end{array}$ & $\begin{array}{l}\text { Paroxetine vs. } \\
\text { other SSRIs, } \\
\text { mainly } \\
\text { citalopram }\end{array}$ & LGA & $\begin{array}{l}9 / 94(9.6 \%) \\
\text { Vs. } 21 / 425 \\
(4.9 \%)^{131}\end{array}$ & $\begin{array}{l}\mathrm{RR}, 1.94 \\
(95 \% \mathrm{Cl} \\
0.92 \text { to } \\
4.09)^{131}\end{array}$ & $\begin{array}{l}1 \text { cohort, } \\
N=519^{131}\end{array}$ & $\begin{array}{l}\text { High study } \\
\text { limitations (high } \\
\text { risk of bias }{ }^{131} \text { ), } \\
\text { imprecise (wide } \\
\text { Cls), consistency } \\
\text { unknown }\end{array}$ & Insufficient \\
\hline $\begin{array}{l}\text { Women with } \\
\text { antidepressant } \\
\text { use in } \\
\text { pregnancy }\end{array}$ & $\begin{array}{l}\text { Paroxetine vs. } \\
\text { other SSRIs, } \\
\text { mainly } \\
\text { citalopram }\end{array}$ & $\begin{array}{l}\text { Respiratory } \\
\text { distress }\end{array}$ & $\begin{array}{l}7 / 109 \\
(6.4 \%) \text { vs. } \\
24 / 455 \\
(5.3 \%)^{131}\end{array}$ & $\begin{array}{l}\mathrm{RR}, 1.22 \\
(95 \% \mathrm{Cl} \\
0.54 \text { to } \\
2.75 \%)^{131}\end{array}$ & $\begin{array}{l}1 \text { cohort, } \\
N=564^{131}\end{array}$ & $\begin{array}{l}\text { High study } \\
\text { limitations (high } \\
\text { risk of bias }{ }^{131} \text { ), } \\
\text { imprecise (wide } \\
\text { Cls), consistency } \\
\text { unknown }\end{array}$ & Insufficient \\
\hline
\end{tabular}

$\mathrm{CI}=$ confidence interval; $\mathrm{LGA}=$ large for gestational age; $\mathrm{n} / \mathrm{N}=$ number; $\mathrm{OR}=$ odds ratio; $\mathrm{RR}=$ relative risk; $\mathrm{SGA}=$ small for gestational age; SSRI $=$ selective serotonin reuptake inhibitor; vs. $=$ versus . 


\section{Paroxetine Versus TCAs}

\section{Overview}

- The evidence is insufficient to judge the risk of harms for child outcomes (one or more congenital anomalies) for exposure to paroxetine versus TCAs during pregnancy.

\section{Detailed Results}

One high risk-of-bias study provided information on the risks of one or more congenital anomalies in women exposed to paroxetine or TCAs. ${ }^{126}$ The study used data from five health maintenance organizations (HMOs) participating in the HMO Research Network's Center for Education and Research on Therapeutics. The evidence is insufficient to judge the risk of harms when comparing paroxetine with TCAs (Table B-64).

Table B-64. Strength of evidence for comparative harms: Paroxetine versus TCAs

\begin{tabular}{|c|c|c|c|c|c|c|c|}
\hline Population & $\begin{array}{l}\text { Intervention } \\
\text { and } \\
\text { Comparator }\end{array}$ & Outcome & $\begin{array}{l}\text { Incidence or } \\
\text { Mean Effect } \\
\text { by Arm }\end{array}$ & Results & $\begin{array}{l}\text { Study Design } \\
\text { and Sample } \\
\text { Size }\end{array}$ & $\begin{array}{l}\text { Factors That } \\
\text { Affect the } \\
\text { Strength of } \\
\text { Evidence }\end{array}$ & $\begin{array}{l}\text { Overall } \\
\text { Evidence } \\
\text { Strength } \\
\text { (Direction } \\
\text { of Effect) }\end{array}$ \\
\hline $\begin{array}{l}\text { Exposed to } \\
\text { paroxetine in } \\
\text { the first } \\
\text { trimester vs. } \\
\text { exposed to } \\
\text { TCAs in the } \\
\text { first trimester } \\
\text { (full-term } \\
\text { infants) }\end{array}$ & $\begin{array}{l}\text { Paroxetine } \\
\text { vs. TCAs }\end{array}$ & $\begin{array}{l}\text { One or } \\
\text { more } \\
\text { anomalies }\end{array}$ & $\begin{array}{l}26 / 182(14.2 \%) \\
\text { vs. } 20 / 167 \\
(12.0 \%)^{126}\end{array}$ & $\begin{array}{l}\text { RR, } 1.19 \\
(95 \% \mathrm{Cl} \\
0.69 \text { to } \\
2.05)^{126}\end{array}$ & $\begin{array}{l}1 \text { cohort study, } \\
\mathrm{N}=349^{126}\end{array}$ & $\begin{array}{l}\text { High study } \\
\text { limitations } \\
\text { (high risk of } \\
\text { bias }^{126} \text { ), } \\
\text { imprecise } \\
\text { (wide Cls), } \\
\text { consistency } \\
\text { unknown }\end{array}$ & Insufficient \\
\hline
\end{tabular}

$\mathrm{CI}=$ confidence interval; $\mathrm{N}=$ number; $\mathrm{RR}=$ relative risk; $\mathrm{TCA}=$ tricyclic antidepressant; $\mathrm{vs}$. = versus.

\section{Fluoxetine Versus SNRIs}

\section{Overview}

- The evidence is limited to one study and is insufficient to judge the comparative risk of harms from exposure to fluoxetine monotherapy versus SNRI monotherapy (venlafaxine or desvenlafaxine) during pregnancy for maternal outcomes (convulsive seizures, nonconvulsive seizures, spontaneous abortion, or still birth) or child outcomes (congenital anomaly or birth weight) among women with epilepsy.

\section{Detailed Results}

One high risk-of-bias publication reported on the comparative harms of fluoxetine monotherapy versus SNRI monotherapy (venlafaxine or desvenlafaxine) ${ }^{107}$ The population in the analysis was pregnant women with depression, anxiety, or bipolar disorder as well as epilepsy, drawn from the Australian Antiepileptic Drugs in Pregnancy register. No significant differences were found in the risk of harms for any maternal (convulsive seizures, nonconvulsive seizures, spontaneous abortion, or still birth) or child outcomes (congenital anomalies or birth weight) (Table B-65). 
Table B-65. Strength of evidence for comparative harms: Fluoxetine monotherapy versus SNRI monotherapy (venlafaxine or desvenlafaxine)

\begin{tabular}{|c|c|c|c|c|c|c|c|}
\hline Population & $\begin{array}{l}\text { Intervention } \\
\text { and } \\
\text { Comparator }\end{array}$ & Outcome & $\begin{array}{l}\text { Incidence or } \\
\text { Mean Effect by } \\
\text { Arm }\end{array}$ & Results & $\begin{array}{l}\text { Study } \\
\text { Design } \\
\text { and } \\
\text { Sample } \\
\text { Size }\end{array}$ & $\begin{array}{l}\text { Factors That } \\
\text { Affect the } \\
\text { Strength of } \\
\text { Evidence }\end{array}$ & $\begin{array}{l}\text { Overall } \\
\text { Evidence } \\
\text { Strength } \\
\text { (Direction } \\
\text { of Effect) }\end{array}$ \\
\hline $\begin{array}{l}\text { Women } \\
\text { with } \\
\text { epilepsy } \\
\text { and } \\
\text { depression, } \\
\text { anxiety or } \\
\text { bipolar } \\
\text { disorder }\end{array}$ & $\begin{array}{l}\text { Fluoxetine only } \\
\text { vs. SNRI } \\
\text { monotherapy } \\
\text { (venlafaxine or } \\
\text { desvenlafaxine) }\end{array}$ & $\begin{array}{l}\text { Convulsive } \\
\text { seizures: } \\
\text { From } \\
\text { enrollment to } \\
7 \text { months } \\
\text { gestation } \\
\text { From } 7 \\
\text { months } \\
\text { gestation to } \\
\text { delivery }\end{array}$ & $\begin{array}{l}2 / 5(40 \%) \text { vs. } 0 / 13 \\
(0 \%)^{107} \\
1 / 5(20 \%) \text { vs. } 0 / 13 \\
(0 \%)^{107}\end{array}$ & $\begin{array}{l}\text { RD, } 14.67 \\
(95 \% \mathrm{Cl}, 0.65 \\
\text { to } 208.21)^{107} \\
\mathrm{RD}, 7(95 \% \mathrm{Cl} \text {, } \\
0.33 \text { to } \\
148.46)^{107}\end{array}$ & $\begin{array}{l}1 \\
\text { cohort, } \\
\mathrm{n}=18^{107}\end{array}$ & $\begin{array}{l}\text { High study } \\
\text { limitations (high } \\
\text { risk of bias }{ }^{107} \text { ), } \\
\text { seriously } \\
\text { imprecise (few } \\
\text { events, wide } \\
\text { Cls), } \\
\text { consistency } \\
\text { unknown }\end{array}$ & Insufficient \\
\hline $\begin{array}{l}\text { Women } \\
\text { with } \\
\text { epilepsy } \\
\text { and } \\
\text { depression, } \\
\text { anxiety or } \\
\text { bipolar } \\
\text { disorder }\end{array}$ & $\begin{array}{l}\text { Fluoxetine only } \\
\text { vs. SNRI } \\
\text { monotherapy } \\
\text { (venlafaxine or } \\
\text { desvenlafaxine) }\end{array}$ & $\begin{array}{l}\text { Nonconvulsive } \\
\text { seizures: } \\
\text { From } \\
\text { enrollment to } \\
7 \text { months } \\
\text { gestation } \\
\text { From } 7 \\
\text { months } \\
\text { gestation to } \\
\text { delivery }\end{array}$ & $\begin{array}{l}1 / 5(20 \%) \text { vs. } 1 / 13 \\
(7.7 \%)^{107} \\
1 / 5(20 \%) \text { vs. } 0 / 13 \\
(0 \%)^{107}\end{array}$ & $\begin{array}{l}\mathrm{RR}, 2.6(95 \% \\
\mathrm{Cl}, 0.20 \text { to } \\
34.07)^{107} \\
\mathrm{RD}, 7(95 \% \mathrm{Cl} \text {, } \\
0.33 \text { to } \\
148.457)^{107}\end{array}$ & $\begin{array}{l}1 \\
\text { cohort, } \\
n=18^{107}\end{array}$ & $\begin{array}{l}\text { High study } \\
\text { limitations (high } \\
\text { risk of bias }{ }^{107} \text { ), } \\
\text { seriously } \\
\text { imprecise (few } \\
\text { events, wide } \\
\text { Cls), } \\
\text { consistency } \\
\text { unknown }\end{array}$ & Insufficient \\
\hline $\begin{array}{l}\text { Women } \\
\text { with } \\
\text { epilepsy } \\
\text { and } \\
\text { depression, } \\
\text { anxiety or } \\
\text { bipolar } \\
\text { disorder }\end{array}$ & $\begin{array}{l}\text { Fluoxetine only } \\
\text { vs. SNRI } \\
\text { monotherapy } \\
\text { (venlafaxine or } \\
\text { desvenlafaxine) }\end{array}$ & $\begin{array}{l}\text { Spontaneous } \\
\text { abortion }\end{array}$ & $\begin{array}{l}0 / 5(0 \%) \text { vs. } 0 / 13 \\
(0 \%)^{107}\end{array}$ & $\begin{array}{l}\text { RR not } \\
\text { calculable: no } \\
\text { events }\end{array}$ & $\begin{array}{l}1 \\
\text { cohort, } \\
\mathrm{n}=18^{107}\end{array}$ & $\begin{array}{l}\text { High study } \\
\text { limitations (high } \\
\text { risk of bias }{ }^{107} \text { ), } \\
\text { likely imprecise } \\
\text { (no events, } \\
\text { small Ns), } \\
\text { consistency } \\
\text { unknown }\end{array}$ & Insufficient \\
\hline $\begin{array}{l}\text { Women } \\
\text { with } \\
\text { epilepsy } \\
\text { and } \\
\text { depression, } \\
\text { anxiety or } \\
\text { bipolar } \\
\text { disorder }\end{array}$ & $\begin{array}{l}\text { Fluoxetine only } \\
\text { vs. SNRI } \\
\text { monotherapy } \\
\text { (venlafaxine or } \\
\text { desvenlafaxine) }\end{array}$ & Still birth & $\begin{array}{l}0 / 5(0 \%) \text { vs. } 1 / 13 \\
(7.7 \%)^{107}\end{array}$ & $\begin{array}{l}\mathrm{RD}, 0.78(95 \% \\
\mathrm{Cl}, 0.037 \text { to } \\
16.50)^{107}\end{array}$ & $\begin{array}{l} \\
\text { cohort, } \\
n=18^{107}\end{array}$ & $\begin{array}{l}\text { High study } \\
\text { limitations (high } \\
\text { risk of bias }{ }^{107} \text { ), } \\
\text { seriously } \\
\text { imprecise (few } \\
\text { events, wide } \\
\text { Cls), } \\
\text { consistency } \\
\text { unknown }\end{array}$ & Insufficient \\
\hline
\end{tabular}

$\mathrm{CI}=$ confidence interval; $\mathrm{n} / \mathrm{N}=$ number; $\mathrm{RD}=$ risk difference; $\mathrm{RR}=$ relative risk; $\mathrm{SNRI}=$ serotonin-norepinephrine reuptake inhibitor; vs. $=$ versus.

\section{Fluoxetine Versus TCAs}

\section{Overview}

- The evidence is insufficient to judge the comparative risk of harms from exposure to fluoxetine versus TCAs during pregnancy for any child outcomes (gestational age, birthweight, major congenital anomalies, or infant mental development).

\section{Detailed Results}

Two publications reported on the comparative harms of fluoxetine versus TCAs as a class. ${ }^{134}$, ${ }^{136}$ Both publications reported findings from the Canadian Motherisk cohort of women with 
major depression. One publication reported on women who used one of the medications during the first trimester or longer. ${ }^{39,} 136$ The third publication was limited to women who used one of the medications throughout their pregnancy. ${ }^{134}$ The studies were rated as high risk of bias.

Results for four outcomes were reported in single studies. No significant differences were found in the risk of harms for any child outcomes (gestational age, birthweight, or major congenital anomalies). Differences in infant development based on the Bayley Mental Development Index were reported in two publications. ${ }^{134,136}$ While the sample sizes differed in the two publications, it is unknown whether they overlap. Both studies reported no significant differences between the two groups. The strength of evidence was rated as insufficient for all outcomes (Table B-66).

Table B-66. Strength of evidence for comparative harms: Fluoxetine versus TCAs

\begin{tabular}{|c|c|c|c|c|c|c|c|}
\hline Population & $\begin{array}{l}\text { Intervention } \\
\text { and } \\
\text { Comparator }\end{array}$ & Outcome & $\begin{array}{l}\text { Incidence or } \\
\text { Mean Effect by } \\
\text { Arm }\end{array}$ & Results & $\begin{array}{l}\text { Study } \\
\text { Design and } \\
\text { Sample Size }\end{array}$ & $\begin{array}{l}\text { Factors That } \\
\text { Affect the } \\
\text { Strength of } \\
\text { Evidence }\end{array}$ & $\begin{array}{l}\text { Overall } \\
\text { Evidence } \\
\text { Strength } \\
\text { (Direction } \\
\text { of Effect) }\end{array}$ \\
\hline $\begin{array}{l}\text { Pregnant } \\
\text { women } \\
\text { with } \\
\text { depression }\end{array}$ & $\begin{array}{l}\text { Fluoxetine } \\
\text { vs. TCAs }\end{array}$ & $\begin{array}{l}\text { Gestational } \\
\text { age (weeks) }\end{array}$ & $\begin{array}{l}\text { Mean (SD): } 39.4 \\
(1.6) \text { vs. } 39.1 \\
(2.3)^{39}\end{array}$ & $\begin{array}{l}\text { Mean } \\
\text { difference: } \\
0.3(95 \% \\
\mathrm{Cl},-0.34 \text { to } \\
0.94)^{39}\end{array}$ & $\begin{array}{l}1 \text { cohort, } \\
n=85^{39}\end{array}$ & $\begin{array}{l}\text { High study } \\
\text { limitations (high } \\
\text { risk of bias }{ }^{39} \text { ), } \\
\text { imprecise (wide } \\
\text { Cls), } \\
\text { inconsistency } \\
\text { unknown }\end{array}$ & Insufficient \\
\hline $\begin{array}{l}\text { Pregnant } \\
\text { women } \\
\text { with } \\
\text { depression }\end{array}$ & $\begin{array}{l}\text { Fluoxetine } \\
\text { vs. TCAs }\end{array}$ & $\begin{array}{l}\text { Birthweight } \\
\text { (gram) }\end{array}$ & $\begin{array}{l}\text { Median } \pm \text { IQR: } \\
3,421.9 \pm 664.1 \\
\text { vs. } \\
3,515.9 \pm 672.3^{39}\end{array}$ & $\begin{array}{l}\text { Mean } \\
\text { difference: } \\
-94(95 \% \\
\mathrm{Cl},-309.3 \\
\text { to } 121.3)^{39}\end{array}$ & $\begin{array}{l}1 \text { cohort, } \\
n=85^{39}\end{array}$ & $\begin{array}{l}\text { High study } \\
\text { limitations (high } \\
\text { risk of bias }^{39} \text { ), } \\
\text { imprecise (wide } \\
\text { Cls), } \\
\text { inconsistency } \\
\text { unknown }\end{array}$ & Insufficient \\
\hline $\begin{array}{l}\text { Pregnant } \\
\text { women } \\
\text { with } \\
\text { depression }\end{array}$ & $\begin{array}{l}\text { Fluoxetine } \\
\text { vs. TCAs }\end{array}$ & $\begin{array}{l}\text { Major } \\
\text { congenital } \\
\text { anomalies }\end{array}$ & $\begin{array}{l}2 / 58(3 \%) \text { vs. } 0.6 \\
(\% \text { not } \\
\text { calculable })^{39}\end{array}$ & $\begin{array}{l}\text { Not } \\
\text { calculated, } \\
\text { likely } \\
\text { typographic } \\
\text { error in } \\
\text { primary } \\
\text { source } \\
\end{array}$ & $\begin{array}{l}1 \text { cohort, } \\
\mathrm{n}=85^{39}\end{array}$ & $\begin{array}{l}\text { High study } \\
\text { limitations (high } \\
\text { risk of bias }{ }^{39} \text { ), } \\
\text { likely imprecise } \\
\text { (small Ns), } \\
\text { inconsistency } \\
\text { unknown }\end{array}$ & Insufficient \\
\hline $\begin{array}{l}\text { Women } \\
\text { exposed to } \\
\text { antidepress } \\
\text { ants in } \\
\text { pregnancy }\end{array}$ & $\begin{array}{l}\text { Fluvoxamine } \\
\text { exposure vs. } \\
\text { citalopram/ } \\
\text { escitalopram } \\
\text { exposure in } \\
\text { pregnancy }\end{array}$ & $\begin{array}{l}\text { Convulsive } \\
\text { seizures in } \\
\text { women with } \\
\text { epilepsy at } \\
\text { enrollment to } \\
7 \text { months } \\
\text { gestation }\end{array}$ & $\begin{array}{l}0 / 1(0 \%) \text { vs. } 2 / 20 \\
(10 \%)^{107}\end{array}$ & $\begin{array}{l}\mathrm{RR}, 0.48 \\
(95 \% \mathrm{Cl}, \\
0.03 \text { to } \\
6.86)^{107}\end{array}$ & $\begin{array}{l}1 \text { cohort, } \\
n=22^{107}\end{array}$ & $\begin{array}{l}\text { High study } \\
\text { limitations (high } \\
\text { risk of bias }{ }^{107} \text { ), } \\
\text { seriously } \\
\text { imprecise (wide } \\
\text { Cls, few events), } \\
\text { consistency } \\
\text { unknown }\end{array}$ & Insufficient \\
\hline $\begin{array}{l}\text { Women } \\
\text { exposed to } \\
\text { antidepress } \\
\text { ants in } \\
\text { pregnancy }\end{array}$ & $\begin{array}{l}\text { Fluvoxamine } \\
\text { exposure vs. } \\
\text { citalopram/ } \\
\text { escitalopram } \\
\text { exposure in } \\
\text { pregnancy }\end{array}$ & $\begin{array}{l}\text { Convulsive } \\
\text { seizures in } \\
\text { women with } \\
\text { epilepsy at } 7 \\
\text { months to } \\
\text { delivery }\end{array}$ & $\begin{array}{l}0 / 1(0 \%) \text { vs. } 1 / 20 \\
(5 \%)^{107}\end{array}$ & $\begin{array}{l}\mathrm{RR}, 0.29 \\
(95 \% \mathrm{Cl}, \\
0.02 \text { to } \\
4.95)^{107}\end{array}$ & $\begin{array}{l}1 \text { cohort, } \\
n=21^{107}\end{array}$ & $\begin{array}{l}\text { High study } \\
\text { limitations (high } \\
\text { risk of bias }{ }^{107} \text { ), } \\
\text { seriously } \\
\text { imprecise (wide } \\
\text { Cls, few events), } \\
\text { consistency } \\
\text { unknown }\end{array}$ & Insufficient \\
\hline
\end{tabular}




\begin{tabular}{|c|c|c|c|c|c|c|c|}
\hline Population & $\begin{array}{l}\text { Intervention and } \\
\text { Comparator }\end{array}$ & Outcome & $\begin{array}{l}\text { Incidence or } \\
\text { Mean Effect } \\
\text { by Arm }\end{array}$ & Results & $\begin{array}{l}\text { Study } \\
\text { Design } \\
\text { and } \\
\text { Sample } \\
\text { Size } \\
\end{array}$ & $\begin{array}{l}\text { Factors That } \\
\text { Affect the } \\
\text { Strength of } \\
\text { Evidence }\end{array}$ & $\begin{array}{l}\text { Overall } \\
\text { Evidence } \\
\text { Strength } \\
\text { (Direction } \\
\text { of Effect) } \\
\end{array}$ \\
\hline $\begin{array}{l}\text { Women } \\
\text { exposed to } \\
\text { antidepress } \\
\text { ants in } \\
\text { pregnancy }\end{array}$ & $\begin{array}{l}\text { Fluvoxamine } \\
\text { exposure vs. } \\
\text { citalopram/ } \\
\text { escitalopram } \\
\text { exposure in } \\
\text { pregnancy }\end{array}$ & $\begin{array}{l}\text { Non- } \\
\text { convulsive } \\
\text { seizures in } \\
\text { women with } \\
\text { epilepsy at } \\
\text { enrollment to } 7 \\
\text { months } \\
\text { gestation }\end{array}$ & $\begin{array}{l}0 / 1(0 \%) \text { vs. } \\
4 / 20(20 \%)^{107}\end{array}$ & $\begin{array}{l}\mathrm{RR}, 0.86(95 \% \\
\mathrm{Cl}, 0.07 \text { to } \\
10.8)^{107}\end{array}$ & $\begin{array}{l}1 \text { cohort, } \\
n=21^{107}\end{array}$ & $\begin{array}{l}\text { High study } \\
\text { limitations (high } \\
\text { risk of bias }{ }^{107} \text { ), } \\
\text { seriously } \\
\text { imprecise (wide } \\
\text { Cls, few events), } \\
\text { consistency } \\
\text { unknown }\end{array}$ & Insufficient \\
\hline $\begin{array}{l}\text { Women } \\
\text { exposed to } \\
\text { antidepress } \\
\text { ants in } \\
\text { pregnancy }\end{array}$ & $\begin{array}{l}\text { Fluvoxamine } \\
\text { exposure vs. } \\
\text { citalopram/ } \\
\text { escitalopram } \\
\text { exposure in } \\
\text { pregnancy }\end{array}$ & $\begin{array}{l}\text { Non- } \\
\text { convulsive } \\
\text { seizures in } \\
\text { women with } \\
\text { epilepsy at } 7 \\
\text { months to } \\
\text { delivery }\end{array}$ & $\begin{array}{l}0 / 1(0 \%) \text { vs. } \\
3 / 20(15 \%)^{107}\end{array}$ & $\begin{array}{l}\mathrm{RR}, 0.67(95 \% \\
\mathrm{Cl}, 0.07 \text { to } \\
10.8)^{107}\end{array}$ & $\begin{array}{l}1 \text { cohort, } \\
n=21^{107}\end{array}$ & $\begin{array}{l}\text { High study } \\
\text { limitations (high } \\
\text { risk of bias }{ }^{107} \text { ), } \\
\text { seriously } \\
\text { imprecise (wide } \\
\text { Cls, few events), } \\
\text { consistency } \\
\text { unknown }\end{array}$ & Insufficient \\
\hline $\begin{array}{l}\text { Women } \\
\text { exposed to } \\
\text { antidepress } \\
\text { ants in } \\
\text { pregnancy }\end{array}$ & $\begin{array}{l}\text { Fluvoxamine } \\
\text { exposure vs. } \\
\text { citalopram/ } \\
\text { escitalopram } \\
\text { exposure in } \\
\text { pregnancy }\end{array}$ & Birth weight & $\mathrm{NR}^{107}$ & $\begin{array}{l}\text { NR other than } \\
\text { "specific ADDs } \\
\text { were not } \\
\text { associated with } \\
\text { birth weight"107 }\end{array}$ & $\begin{array}{l}1 \text { cohort, } \\
n=21^{107}\end{array}$ & $\begin{array}{l}\text { High study } \\
\text { limitations (high } \\
\text { risk of bias }{ }^{107} \text { ), } \\
\text { seriously } \\
\text { imprecise } \\
\text { (nonsignificant, } \\
\text { small sample } \\
\text { size), consistency } \\
\text { unknown }\end{array}$ & Insufficient \\
\hline $\begin{array}{l}\text { Women } \\
\text { exposed to } \\
\text { antidepress } \\
\text { ants in } \\
\text { pregnancy }\end{array}$ & $\begin{array}{l}\text { Fluvoxamine } \\
\text { exposure vs. } \\
\text { citalopram/ } \\
\text { escitalopram } \\
\text { exposure in } \\
\text { pregnancy }\end{array}$ & $\begin{array}{l}\text { Congenital } \\
\text { anomalies }\end{array}$ & $\begin{array}{l}0 / 1(0 \%) \text { vs. } \\
4 / 21 \\
(19.0 \%)^{107}\end{array}$ & $\begin{array}{l}\text { ARR, } 0.82 \\
(95 \% \mathrm{Cl}, 0.06 \\
\text { to } 10.35)^{107}\end{array}$ & $\begin{array}{l}1 \text { cohort, } \\
n=22^{107}\end{array}$ & $\begin{array}{l}\text { High study } \\
\text { limitations (high } \\
\text { risk of bias }{ }^{107} \text { ), } \\
\text { seriously } \\
\text { imprecise (wide } \\
\text { Cls, few events), } \\
\text { consistency } \\
\text { unknown }\end{array}$ & Insufficient \\
\hline $\begin{array}{l}\text { Women } \\
\text { exposed to } \\
\text { antidepress } \\
\text { ants in } \\
\text { pregnancy }\end{array}$ & $\begin{array}{l}\text { Fluvoxamine } \\
\text { exposure vs. } \\
\text { citalopram/ } \\
\text { escitalopram } \\
\text { exposure in } \\
\text { pregnancy }\end{array}$ & $\begin{array}{l}\text { Autism } \\
\text { spectrum } \\
\text { disorder }\end{array}$ & $\begin{array}{l}1 / 35(2.9 \%) \\
\text { vs. } 5 / 421 \\
(1.2 \%)^{111}\end{array}$ & $\begin{array}{l}\text { ARR, } 0.42 \\
(95 \% \mathrm{Cl}, 0.05 \\
\text { to } 3.5)^{111}\end{array}$ & $\begin{array}{l}1 \text { cohort, } \\
n=456^{111}\end{array}$ & $\begin{array}{l}\text { High study } \\
\text { limitations (high } \\
\text { risk of bias }{ }^{111} \text { ), } \\
\text { seriously } \\
\text { imprecise (wide } \\
\text { Cls, few events), } \\
\text { consistency } \\
\text { unknown }\end{array}$ & Insufficient \\
\hline
\end{tabular}

$\mathrm{ADD}=$ attention deficit disorder; $\mathrm{ARR}=$ adjusted risk ratio; $\mathrm{CI}=$ confidence interval; $\mathrm{n}=$ number; $\mathrm{NR}=$ not reported; $\mathrm{RD}=$ risk difference; $\mathrm{RR}=$ relative risk; vs. $=$ versus.

\section{Fluvoxamine Versus Fluoxetine}

\section{Overview}

- The evidence for exposure to fluvoxamine versus fluoxetine monotherapy during pregnancy is insufficient to judge the risk of harms for the maternal outcomes (spontaneous abortion, still birth, convulsive seizures in women with epilepsy, or nonconvulsive seizures in women with epilepsy) and child outcomes (birth weight, congenital anomalies, or autism spectrum disorder). 


\section{Detailed Results}

Two publications reported on fluvoxamine versus fluoxetine monotherapy. ${ }^{107,111}$ These publications drew from two cohorts (the Australian Pregnancy Register of Antiepileptic Drugs ${ }^{107}$ and the $\left.\mathrm{QPC}^{111}\right)$. Both were nonrandomized observational studies and were rated as high risk of bias.

Both publications focused on exposure during pregnancy. One study further examined outcomes at three time frames: enrollment to 7 months gestation, 7 months gestation to delivery, and within the first postnatal month. ${ }^{107}$ Publications compared pregnant women exposed to fluvoxamine versus fluoxetine monotherapy. ${ }^{107,111}$

The evidence for fluvoxamine versus fluoxetine monotherapy is insufficient to judge the risk of harms for the maternal outcomes (spontaneous abortion, still birth, convulsive seizures in women with epilepsy, or nonconvulsive seizures in women with epilepsy) and child outcomes (birth weight, congenital anomalies, or autism spectrum disorder) (Table B-67). These studies found no association between fluvoxamine versus fluoxetine monotherapy use in pregnancy with any outcome.

Table B-67. Strength of evidence for comparative harms: Fluvoxamine versus fluoxetine

\begin{tabular}{|c|c|c|c|c|c|c|c|}
\hline Population & $\begin{array}{l}\text { Intervention } \\
\text { and } \\
\text { Comparator }\end{array}$ & Outcome & $\begin{array}{l}\text { Incidence } \\
\text { or Mean } \\
\text { Effect by } \\
\text { Arm }\end{array}$ & Results & $\begin{array}{l}\text { Study } \\
\text { Design and } \\
\text { Sample } \\
\text { Size }\end{array}$ & $\begin{array}{l}\text { Factors That } \\
\text { Affect the } \\
\text { Strength of } \\
\text { Evidence }\end{array}$ & $\begin{array}{l}\text { Overall } \\
\text { Evidence } \\
\text { Strength } \\
\text { (Direction of } \\
\text { Effect) }\end{array}$ \\
\hline $\begin{array}{l}\text { ADD- } \\
\text { exposed } \\
\text { women }\end{array}$ & $\begin{array}{l}\text { Fluvoxamine vs. } \\
\text { fluoxetine } \\
\text { monotherapy } \\
\text { exposure in } \\
\text { pregnancy }\end{array}$ & $\begin{array}{l}\text { Spontaneo } \\
\text { us abortion }\end{array}$ & $0 / 1$ vs. $0 / 5^{107}$ & $\begin{array}{l}\text { RD, } 0(95 \% \\
C l,-0.64 \text { to } \\
0.64)^{107}\end{array}$ & $\begin{array}{l}1 \text { cohort, } \\
n=6^{107}\end{array}$ & $\begin{array}{l}\text { High study } \\
\text { limitations (high } \\
\text { risk of bias }{ }^{107} \text { ), } \\
\text { seriously } \\
\text { imprecise (wide } \\
\text { Cls, few events), } \\
\text { consistency } \\
\text { unknown }\end{array}$ & Insufficient \\
\hline $\begin{array}{l}\text { ADD- } \\
\text { exposed } \\
\text { women }\end{array}$ & $\begin{array}{l}\text { Fluvoxamine vs. } \\
\text { fluoxetine } \\
\text { monotherapy } \\
\text { exposure in } \\
\text { pregnancy }\end{array}$ & Still birth & $0 / 1$ vs. $0 / 5^{107}$ & $\begin{array}{l}\text { RD, } 0(95 \% \\
C l,-0.64 \text { to } \\
0.64)^{107}\end{array}$ & $\begin{array}{l}1 \text { cohort, } \\
n=6^{107}\end{array}$ & $\begin{array}{l}\text { High study } \\
\text { limitations (high } \\
\text { risk of bias }{ }^{107} \text { ), } \\
\text { seriously } \\
\text { imprecise (wide } \\
\text { Cls, few events), } \\
\text { consistency } \\
\text { unknown }\end{array}$ & Insufficient \\
\hline $\begin{array}{l}\text { ADD- } \\
\text { exposed } \\
\text { women }\end{array}$ & $\begin{array}{l}\text { Fluvoxamine vs. } \\
\text { fluoxetine } \\
\text { monotherapy } \\
\text { exposure in } \\
\text { pregnancy }\end{array}$ & $\begin{array}{l}\text { Convulsive } \\
\text { seizures in } \\
\text { women } \\
\text { with } \\
\text { epilepsy at } \\
\text { enrollment } \\
\text { to } 7 \text { months } \\
\text { gestation }\end{array}$ & $0 / 1$ vs. $2 / 5^{107}$ & $\begin{array}{l}\mathrm{RD}, 0.4(95 \% \\
\mathrm{Cl},-0.32 \text { to } \\
1.12)^{107}\end{array}$ & $\begin{array}{l}1 \text { cohort, } \\
n=6^{107}\end{array}$ & $\begin{array}{l}\text { High study } \\
\text { limitations (high } \\
\text { risk of bias }{ }^{107} \text { ), } \\
\text { seriously } \\
\text { imprecise (wide } \\
\text { Cls, few events), } \\
\text { consistency } \\
\text { unknown }\end{array}$ & Insufficient \\
\hline $\begin{array}{l}\text { ADD- } \\
\text { exposed } \\
\text { women }\end{array}$ & $\begin{array}{l}\text { Fluvoxamine vs. } \\
\text { fluoxetine } \\
\text { monotherapy } \\
\text { exposure in } \\
\text { pregnancy }\end{array}$ & $\begin{array}{l}\text { Convulsive } \\
\text { seizures in } \\
\text { women } \\
\text { with } \\
\text { epilepsy at } \\
7 \text { months to } \\
\text { delivery }\end{array}$ & $0 / 1$ vs. $1 / 5^{107}$ & $\begin{array}{l}\mathrm{RD}, 0.2(95 \% \\
\mathrm{Cl},-0.49 \text { to } \\
0.89)^{107}\end{array}$ & $\begin{array}{l}1 \text { cohort, } \\
n=6^{107}\end{array}$ & $\begin{array}{l}\text { High study } \\
\text { limitations (high } \\
\text { risk of bias }{ }^{107} \text { ), } \\
\text { seriously } \\
\text { imprecise (wide } \\
\text { Cls, few events), } \\
\text { consistency } \\
\text { unknown }\end{array}$ & Insufficient \\
\hline
\end{tabular}




\begin{tabular}{|c|c|c|c|c|c|c|c|}
\hline Population & $\begin{array}{l}\text { Intervention } \\
\text { and } \\
\text { Comparator }\end{array}$ & Outcome & $\begin{array}{l}\text { Incidence } \\
\text { or Mean } \\
\text { Effect by } \\
\text { Arm }\end{array}$ & Results & $\begin{array}{l}\text { Study } \\
\text { Design and } \\
\text { Sample } \\
\text { Size }\end{array}$ & $\begin{array}{l}\text { Factors That } \\
\text { Affect the } \\
\text { Strength of } \\
\text { Evidence }\end{array}$ & $\begin{array}{l}\text { Overall } \\
\text { Evidence } \\
\text { Strength } \\
\text { (Direction of } \\
\text { Effect) } \\
\end{array}$ \\
\hline $\begin{array}{l}\text { ADD- } \\
\text { exposed } \\
\text { women }\end{array}$ & $\begin{array}{l}\text { Fluvoxamine vs. } \\
\text { fluoxetine } \\
\text { monotherapy } \\
\text { exposure in } \\
\text { pregnancy }\end{array}$ & $\begin{array}{l}\text { Nonconvul- } \\
\text { sive } \\
\text { seizures in } \\
\text { women } \\
\text { with } \\
\text { epilepsy at } \\
\text { enrollment } \\
\text { to } 7 \text { months } \\
\text { gestation }\end{array}$ & $0 / 1$ vs. $1 / 5^{107}$ & $\begin{array}{l}\mathrm{RR}, 0.2(95 \% \\
\mathrm{Cl},-0.49 \text { to } \\
0.89)^{107}\end{array}$ & $\begin{array}{l}1 \text { cohort, } \\
n=6^{107}\end{array}$ & $\begin{array}{l}\text { High study } \\
\text { limitations (high } \\
\text { risk of bias }{ }^{107} \text { ), } \\
\text { seriously } \\
\text { imprecise (wide } \\
\text { Cls, few events), } \\
\text { consistency } \\
\text { unknown }\end{array}$ & Insufficient \\
\hline $\begin{array}{l}\text { ADD- } \\
\text { exposed } \\
\text { women }\end{array}$ & $\begin{array}{l}\text { Fluvoxamine vs. } \\
\text { fluoxetine } \\
\text { monotherapy } \\
\text { exposure in } \\
\text { pregnancy }\end{array}$ & $\begin{array}{l}\text { Nonconvul- } \\
\text { sive } \\
\text { seizures in } \\
\text { women } \\
\text { with } \\
\text { epilepsy at } \\
7 \text { months to } \\
\text { delivery } \\
\end{array}$ & $0 / 1$ vs. $1 / 5^{107}$ & $\begin{array}{l}\mathrm{RD}, 0.2(95 \% \\
\mathrm{Cl},-0.49 \text { to } \\
0.89)^{107}\end{array}$ & $\begin{array}{l}1 \text { cohort, } \\
n=6^{107}\end{array}$ & $\begin{array}{l}\text { High study } \\
\text { limitations (high } \\
\text { risk of bias }{ }^{107} \text { ), } \\
\text { seriously } \\
\text { imprecise (wide } \\
\text { Cls, few events), } \\
\text { consistency } \\
\text { unknown }\end{array}$ & Insufficient \\
\hline $\begin{array}{l}\text { ADD- } \\
\text { exposed } \\
\text { women }\end{array}$ & $\begin{array}{l}\text { Fluvoxamine vs. } \\
\text { fluoxetine } \\
\text { monotherapy } \\
\text { exposure in } \\
\text { pregnancy }\end{array}$ & Birth weight & $\mathrm{NR}^{107}$ & $\begin{array}{l}\text { NR other than } \\
\text { "specific } \\
\text { ADDs were } \\
\text { not } \\
\text { associated } \\
\text { with birth } \\
\text { weight" } 107\end{array}$ & $\begin{array}{l}1 \text { cohort, } \\
\mathrm{n}=6^{107}\end{array}$ & $\begin{array}{l}\text { High study } \\
\text { limitations (high } \\
\text { risk of bias }{ }^{107} \text { ), } \\
\text { seriously } \\
\text { imprecise (wide } \\
\text { Cls, few events), } \\
\text { consistency } \\
\text { unknown }\end{array}$ & Insufficient \\
\hline $\begin{array}{l}\text { ADD- } \\
\text { exposed } \\
\text { women }\end{array}$ & $\begin{array}{l}\text { Fluvoxamine vs. } \\
\text { fluoxetine } \\
\text { monotherapy } \\
\text { exposure in } \\
\text { pregnancy }\end{array}$ & $\begin{array}{l}\text { Congenital } \\
\text { anomalies }\end{array}$ & $0 / 1$ vs. $0 / 5^{107}$ & $\begin{array}{l}\text { RD, } 0(95 \% \\
\mathrm{Cl},-0.64 \text { to } \\
0.64)^{107}\end{array}$ & $\begin{array}{l}1 \text { cohort, } \\
n=6^{107}\end{array}$ & $\begin{array}{l}\text { High study } \\
\text { limitations (high } \\
\text { risk of bias }{ }^{107} \text { ), } \\
\text { seriously } \\
\text { imprecise (wide } \\
\text { Cls, few events), } \\
\text { consistency } \\
\text { unknown }\end{array}$ & Insufficient \\
\hline $\begin{array}{l}\text { ADD- } \\
\text { exposed } \\
\text { women }\end{array}$ & $\begin{array}{l}\text { Fluvoxamine vs. } \\
\text { fluoxetine } \\
\text { monotherapy } \\
\text { exposure in } \\
\text { pregnancy }\end{array}$ & $\begin{array}{l}\text { Autism } \\
\text { spectrum } \\
\text { disorder }\end{array}$ & $\begin{array}{l}\text { 1/35 vs. } \\
5 / 171^{111}\end{array}$ & $\begin{array}{l}\text { ARR, } 1.02 \\
(95 \% \mathrm{Cl}, 0.12 \\
\text { to } 8.49)^{111}\end{array}$ & $\begin{array}{l}1 \text { cohort, } \\
n=206^{111}\end{array}$ & $\begin{array}{l}\text { High study } \\
\text { limitations (high } \\
\text { risk of bias }^{111} \text { ), } \\
\text { imprecise, } \\
\text { consistency } \\
\text { unknown }\end{array}$ & Insufficient \\
\hline
\end{tabular}

$\mathrm{ADD}=$ attention deficit disorder; $\mathrm{ARR}=$ adjusted risk ratio; $\mathrm{CI}=$ confidence interval; $\mathrm{n}=$ number; $\mathrm{RD}=$ risk difference; $\mathrm{RR}=$ relative risk; vs. $=$ versus.

\section{Fluvoxamine Versus Paroxetine}

\section{Overview}

- The evidence for exposure to fluvoxamine versus paroxetine monotherapy during pregnancy is insufficient to judge the risk of harms for the maternal outcomes (spontaneous abortion, still birth, convulsive seizures in women with epilepsy, or nonconvulsive seizures in women with epilepsy) and child outcomes (birth weight, congenital anomalies, or autism spectrum disorder). 


\section{Detailed Results}

Two publications reported on fluvoxamine versus paroxetine monotherapy. ${ }^{107,111}$ These publications drew from two cohorts (the Australian Pregnancy Register of Antiepileptic Drugs ${ }^{107}$ and the $\left.\mathrm{QPC}^{111}\right)$. Both were nonrandomized observational studies and were rated as high risk of bias.

Both publications focused on exposure during pregnancy. One study further examined outcomes at three time frames: enrollment to 7 months gestation, 7 months gestation to delivery, and within the first postnatal month. ${ }^{107}$ Publications compared pregnant women exposed to fluvoxamine versus paroxetine monotherapy. ${ }^{107,111}$

The evidence for fluvoxamine versus paroxetine monotherapy is insufficient to judge the risk of harms for the maternal outcomes (spontaneous abortion, still birth, convulsive seizures in women with epilepsy, or nonconvulsive seizures in women with epilepsy) and child outcomes (birth weight, congenital anomalies, or autism spectrum disorder) (Table B-68). These studies found no association between fluvoxamine versus paroxetine monotherapy use in pregnancy with any outcome.

Table B-68. Strength of evidence for comparative harms: Fluvoxamine versus paroxetine monotherapy

\begin{tabular}{|c|c|c|c|c|c|c|c|}
\hline Population & $\begin{array}{l}\text { Intervention } \\
\text { and } \\
\text { Comparator }\end{array}$ & Outcome & $\begin{array}{l}\text { Incidence } \\
\text { or Mean } \\
\text { Effect by } \\
\text { Arm }\end{array}$ & Results & $\begin{array}{l}\text { Study } \\
\text { Design and } \\
\text { Sample } \\
\text { Size }\end{array}$ & $\begin{array}{l}\text { Factors That } \\
\text { Affect the } \\
\text { Strength of } \\
\text { Evidence }\end{array}$ & $\begin{array}{l}\text { Overall } \\
\text { Evidence } \\
\text { Strength } \\
\text { (Direction of } \\
\text { Effect) }\end{array}$ \\
\hline $\begin{array}{l}\text { ADD- } \\
\text { exposed } \\
\text { women }\end{array}$ & $\begin{array}{l}\text { Fluvoxamine vs. } \\
\text { paroxetine } \\
\text { monotherapy } \\
\text { exposure in } \\
\text { pregnancy }\end{array}$ & $\begin{array}{l}\text { Spontaneous } \\
\text { abortion }\end{array}$ & $0 / 1$ vs. $0 / 7^{107}$ & $\begin{array}{l}\text { RD, } 0(95 \% \\
C l,-0.62 \text { to } \\
0.62)^{107}\end{array}$ & $\begin{array}{l}1 \text { cohort, } \\
n=22^{107}\end{array}$ & $\begin{array}{l}\text { High study } \\
\text { limitations (high } \\
\text { risk of bias }{ }^{107} \text { ), } \\
\text { seriously } \\
\text { imprecise (wide } \\
\text { Cls, few events), } \\
\text { consistency } \\
\text { unknown }\end{array}$ & Insufficient \\
\hline $\begin{array}{l}\text { ADD- } \\
\text { exposed } \\
\text { women }\end{array}$ & $\begin{array}{l}\text { Fluvoxamine vs. } \\
\text { paroxetine } \\
\text { monotherapy } \\
\text { exposure in } \\
\text { pregnancy }\end{array}$ & Still birth & $0 / 1$ vs. $0 / 7^{107}$ & $\begin{array}{l}\mathrm{RD}, 0(95 \% \\
\mathrm{Cl}, 0.62 \text { to } \\
0.62)^{107}\end{array}$ & $\begin{array}{l}1 \text { cohort, } \\
n=22^{107}\end{array}$ & $\begin{array}{l}\text { High study } \\
\text { limitations (high } \\
\text { risk of bias }{ }^{107} \text { ), } \\
\text { seriously } \\
\text { imprecise (wide } \\
\text { Cls, few events), } \\
\text { consistency } \\
\text { unknown }\end{array}$ & Insufficient \\
\hline $\begin{array}{l}\text { ADD- } \\
\text { exposed } \\
\text { women }\end{array}$ & $\begin{array}{l}\text { Fluvoxamine vs. } \\
\text { paroxetine } \\
\text { monotherapy } \\
\text { exposure in } \\
\text { pregnancy }\end{array}$ & $\begin{array}{l}\text { Convulsive } \\
\text { seizures in } \\
\text { women with } \\
\text { epilepsy at } \\
\text { enrollment to } \\
7 \text { months } \\
\text { gestation }\end{array}$ & $0 / 1$ vs. $2 / 7^{107}$ & $\begin{array}{l}\text { RD, } 0.29 \\
(95 \% \mathrm{Cl} \text {, } \\
-0.39 \text { to } \\
0.97)^{107}\end{array}$ & $\begin{array}{l}1 \text { cohort, } \\
n=8^{107}\end{array}$ & $\begin{array}{l}\text { High study } \\
\text { limitations (high } \\
\text { risk of bias }{ }^{107} \text { ), } \\
\text { seriously } \\
\text { imprecise (wide } \\
\text { Cls, few events), } \\
\text { consistency } \\
\text { unknown }\end{array}$ & Insufficient \\
\hline $\begin{array}{l}\text { ADD- } \\
\text { exposed } \\
\text { women }\end{array}$ & $\begin{array}{l}\text { Fluvoxamine vs. } \\
\text { paroxetine } \\
\text { monotherapy } \\
\text { exposure in } \\
\text { pregnancy }\end{array}$ & $\begin{array}{l}\text { Convulsive } \\
\text { seizures in } \\
\text { women with } \\
\text { epilepsy at } 7 \\
\text { months to } \\
\text { delivery }\end{array}$ & $0 / 1$ vs. $2 / 7^{107}$ & $\begin{array}{l}\text { RD, } 0.29 \\
(95 \% \\
C l,-0.39 \text { to } \\
0.97)^{107}\end{array}$ & $\begin{array}{l}1 \text { cohort, } \\
n=8^{107}\end{array}$ & $\begin{array}{l}\text { High study } \\
\text { limitations (high } \\
\text { risk of bias }{ }^{107} \text { ), } \\
\text { seriously } \\
\text { imprecise (wide } \\
\text { Cls, few events), } \\
\text { consistency } \\
\text { unknown }\end{array}$ & Insufficient \\
\hline
\end{tabular}




\begin{tabular}{|c|c|c|c|c|c|c|c|}
\hline Population & $\begin{array}{l}\text { Intervention } \\
\text { and } \\
\text { Comparator }\end{array}$ & Outcome & $\begin{array}{l}\text { Incidence } \\
\text { or Mean } \\
\text { Effect by } \\
\text { Arm }\end{array}$ & Results & $\begin{array}{l}\text { Study } \\
\text { Design and } \\
\text { Sample } \\
\text { Size }\end{array}$ & $\begin{array}{l}\text { Factors That } \\
\text { Affect the } \\
\text { Strength of } \\
\text { Evidence }\end{array}$ & $\begin{array}{l}\text { Overall } \\
\text { Evidence } \\
\text { Strength } \\
\text { (Direction of } \\
\text { Effect) }\end{array}$ \\
\hline $\begin{array}{l}\text { ADD- } \\
\text { exposed } \\
\text { women }\end{array}$ & $\begin{array}{l}\text { Fluvoxamine vs. } \\
\text { paroxetine } \\
\text { monotherapy } \\
\text { exposure in } \\
\text { pregnancy }\end{array}$ & $\begin{array}{l}\text { Nonconvulsive } \\
\text { seizures in } \\
\text { women with } \\
\text { epilepsy at } \\
\text { enrollment to } \\
7 \text { months } \\
\text { gestation }\end{array}$ & $0 / 1$ vs. $3 / 7^{107}$ & $\begin{array}{l}\text { RR, } 0.43 \\
(95 \% \\
\mathrm{Cl},-0.26 \text { to } \\
1.12)^{107}\end{array}$ & $\begin{array}{l}1 \text { cohort, } \\
n=8^{107}\end{array}$ & $\begin{array}{l}\text { High study } \\
\text { limitations (high } \\
\text { risk of bias }{ }^{107} \text { ), } \\
\text { seriously } \\
\text { imprecise (wide } \\
\text { Cls, few events), } \\
\text { consistency } \\
\text { unknown }\end{array}$ & Insufficient \\
\hline $\begin{array}{l}\text { ADD- } \\
\text { exposed } \\
\text { women }\end{array}$ & $\begin{array}{l}\text { Fluvoxamine vs. } \\
\text { paroxetine } \\
\text { monotherapy } \\
\text { exposure in } \\
\text { pregnancy }\end{array}$ & $\begin{array}{l}\text { Nonconvulsive } \\
\text { seizures in } \\
\text { women with } \\
\text { epilepsy at } 7 \\
\text { months to } \\
\text { delivery }\end{array}$ & $0 / 1$ vs. $2 / 7^{107}$ & $\begin{array}{l}\text { RD, } 0.29 \\
(95 \% \mathrm{Cl}, \\
-0.39 \text { to } \\
0.97)^{107}\end{array}$ & $\begin{array}{l}1 \text { cohort, } \\
n=8^{107}\end{array}$ & $\begin{array}{l}\text { High study } \\
\text { limitations (high } \\
\text { risk of bias }{ }^{107} \text { ), } \\
\text { seriously } \\
\text { imprecise (wide } \\
\text { Cls, few events), } \\
\text { consistency } \\
\text { unknown }\end{array}$ & Insufficient \\
\hline $\begin{array}{l}\text { ADD- } \\
\text { exposed } \\
\text { women }\end{array}$ & $\begin{array}{l}\text { Fluvoxamine vs. } \\
\text { paroxetine } \\
\text { monotherapy } \\
\text { exposure in } \\
\text { pregnancy }\end{array}$ & Birth weight & $\mathrm{NR}^{107}$ & $\begin{array}{l}\text { NR other } \\
\text { than "specific } \\
\text { ADDs were } \\
\text { not } \\
\text { associated } \\
\text { with birth } \\
\text { weight"107 }\end{array}$ & $\begin{array}{l}1 \text { cohort, } \\
n=8^{107}\end{array}$ & $\begin{array}{l}\text { High study } \\
\text { limitations (high } \\
\text { risk of bias }{ }^{107} \text { ), } \\
\text { seriously } \\
\text { imprecise (wide } \\
\text { Cls, few events), } \\
\text { consistency } \\
\text { unknown }\end{array}$ & Insufficient \\
\hline $\begin{array}{l}\text { ADD- } \\
\text { exposed } \\
\text { women }\end{array}$ & $\begin{array}{l}\text { Fluvoxamine vs. } \\
\text { paroxetine } \\
\text { monotherapy } \\
\text { exposure in } \\
\text { pregnancy }\end{array}$ & $\begin{array}{l}\text { Congenital } \\
\text { anomalies }\end{array}$ & $0 / 1$ vs. $0 / 7^{107}$ & $\begin{array}{l}\text { RD, } 0(95 \% \\
\mathrm{Cl},-0.62 \text { to } \\
0.62)^{107}\end{array}$ & $\begin{array}{l}1 \text { cohort, } \\
n=8^{107}\end{array}$ & $\begin{array}{l}\text { High study } \\
\text { limitations (high } \\
\text { risk of bias }{ }^{107} \text { ), } \\
\text { seriously } \\
\text { imprecise (wide } \\
\text { Cls, few events), } \\
\text { consistency } \\
\text { unknown }\end{array}$ & Insufficient \\
\hline $\begin{array}{l}\text { ADD- } \\
\text { exposed } \\
\text { women }\end{array}$ & $\begin{array}{l}\text { Fluvoxamine vs. } \\
\text { paroxetine } \\
\text { monotherapy } \\
\text { exposure in } \\
\text { pregnancy }\end{array}$ & $\begin{array}{l}\text { Autism } \\
\text { spectrum } \\
\text { disorder }\end{array}$ & $\begin{array}{l}1 / 35 \text { vs. } \\
11 / 744^{111}\end{array}$ & $\begin{array}{l}\text { ARR, } 1.93 \\
(95 \% \mathrm{Cl}, 0.26 \\
\text { to } 14.55)^{111}\end{array}$ & $\begin{array}{l}1 \text { cohort, } \\
n=779^{111}\end{array}$ & $\begin{array}{l}\text { High study } \\
\text { limitations (high } \\
\text { risk of bias }{ }^{111} \text { ), } \\
\text { seriously } \\
\text { imprecise (wide } \\
\text { Cls, few events), } \\
\text { consistency } \\
\text { unknown }\end{array}$ & Insufficient \\
\hline
\end{tabular}

$\mathrm{ARR}=$ adjusted risk ratio; $\mathrm{CI}=$ confidence interval; $\mathrm{n}=$ number; $\mathrm{NR}=$ not reported; $\mathrm{RD}=$ risk difference; $\mathrm{RR}=$ relative risk; $\mathrm{vs}$. $=$ versus.

\section{Fluvoxamine Versus Sertraline}

\section{Overview}

- The evidence for exposure to fluvoxamine versus sertraline monotherapy during pregnancy is insufficient to judge the risk of harms for the maternal outcomes (spontaneous abortion, still birth, convulsive seizures in women with epilepsy, or nonconvulsive seizures in women with epilepsy) and child outcomes (birth weight, congenital anomalies, or autism spectrum disorder). 


\section{Detailed Results}

Two publications reported on fluvoxamine versus sertraline monotherapy. ${ }^{107,111}$ These publications drew from two cohorts (the Australian Pregnancy Register of Antiepileptic Drugs ${ }^{107}$ and the $\left.\mathrm{QPC}^{111}\right)$. Both were nonrandomized observational studies and were rated as high risk of bias.

Both publications focused on exposure during pregnancy. One study further examined outcomes at three time frames: enrollment to 7 months gestation, 7 months gestation to delivery, and within the first postnatal month. ${ }^{107}$ Publications compared pregnant women exposed to fluvoxamine versus sertraline monotherapy. ${ }^{107,111}$

The evidence for fluvoxamine versus sertraline monotherapy is insufficient to judge the risk of harms for the maternal outcomes (spontaneous abortion, still birth, convulsive seizures in women with epilepsy, or nonconvulsive seizures in women with epilepsy) and child outcomes (birth weight, congenital anomalies, or autism spectrum disorder) (Table B-69). These studies found no association between fluvoxamine versus sertraline monotherapy use in pregnancy with any outcome.

Table B-69. Strength of evidence for comparative harms: Fluvoxamine versus sertraline monotherapy

\begin{tabular}{|c|c|c|c|c|c|c|c|}
\hline Population & $\begin{array}{l}\text { Intervention } \\
\text { and } \\
\text { Comparator }\end{array}$ & Outcome & $\begin{array}{l}\text { Incidence } \\
\text { or Mean } \\
\text { Effect by } \\
\text { Arm }\end{array}$ & Results & $\begin{array}{l}\text { Study } \\
\text { Design and } \\
\text { Sample } \\
\text { Size }\end{array}$ & $\begin{array}{l}\text { Factors That } \\
\text { Affect the } \\
\text { Strength of } \\
\text { Evidence }\end{array}$ & $\begin{array}{l}\text { Overall } \\
\text { Evidence } \\
\text { Strength } \\
\text { (Direction of } \\
\text { Effect) }\end{array}$ \\
\hline $\begin{array}{l}\text { ADD- } \\
\text { exposed } \\
\text { women }\end{array}$ & $\begin{array}{l}\text { Fluvoxamine vs. } \\
\text { sertraline } \\
\text { monotherapy } \\
\text { exposure in } \\
\text { pregnancy }\end{array}$ & $\begin{array}{l}\text { Spontaneous } \\
\text { abortion }\end{array}$ & $\begin{array}{l}\text { 0/1 vs. } \\
0 / 25^{107}\end{array}$ & $\begin{array}{l}\text { RD, } 0(95 \% \\
C l,-0.60 \text { to } \\
0.60)^{107}\end{array}$ & $\begin{array}{l}1 \text { cohort, } \\
n=26^{107}\end{array}$ & $\begin{array}{l}\text { High study } \\
\text { limitations (high } \\
\text { risk of bias }{ }^{107} \text { ), } \\
\text { seriously } \\
\text { imprecise (wide } \\
\text { Cls, few events), } \\
\text { consistency } \\
\text { unknown }\end{array}$ & Insufficient \\
\hline $\begin{array}{l}\text { ADD- } \\
\text { exposed } \\
\text { women }\end{array}$ & $\begin{array}{l}\text { Fluvoxamine vs. } \\
\text { sertraline } \\
\text { monotherapy } \\
\text { exposure in } \\
\text { pregnancy }\end{array}$ & Still birth & $\begin{array}{l}\text { 0/1 vs. } \\
0 / 25^{107}\end{array}$ & $\begin{array}{l}\text { RD, } 0(95 \% \\
C l,-0.60 \text { to } \\
0.60)^{107}\end{array}$ & $\begin{array}{l}1 \text { cohort, } \\
n=26^{107}\end{array}$ & $\begin{array}{l}\text { High study } \\
\text { limitations (high } \\
\text { risk of bias }{ }^{107} \text { ), } \\
\text { seriously } \\
\text { imprecise (wide } \\
\text { Cls, few events), } \\
\text { consistency } \\
\text { unknown }\end{array}$ & Insufficient \\
\hline $\begin{array}{l}\text { ADD- } \\
\text { exposed } \\
\text { women }\end{array}$ & $\begin{array}{l}\text { Fluvoxamine vs. } \\
\text { sertraline } \\
\text { monotherapy } \\
\text { exposure in } \\
\text { pregnancy }\end{array}$ & $\begin{array}{l}\text { Convulsive } \\
\text { seizures in } \\
\text { women with } \\
\text { epilepsy at } \\
\text { enrollment to } \\
7 \text { months } \\
\text { gestation }\end{array}$ & $\begin{array}{l}\text { 0/1 vs. } \\
2 / 25^{107}\end{array}$ & $\begin{array}{l}\text { RD, } 0.08 \\
(95 \% \mathrm{Cl}, \\
-0.53 \text { to } \\
0.69)^{107}\end{array}$ & $\begin{array}{l}1 \text { cohort, } \\
n=26^{107}\end{array}$ & $\begin{array}{l}\text { High study } \\
\text { limitations (high } \\
\text { risk of bias }{ }^{107} \text { ), } \\
\text { seriously } \\
\text { imprecise (wide } \\
\text { Cls, few events), } \\
\text { consistency } \\
\text { unknown }\end{array}$ & Insufficient \\
\hline $\begin{array}{l}\text { ADD- } \\
\text { exposed } \\
\text { women }\end{array}$ & $\begin{array}{l}\text { Fluvoxamine vs. } \\
\text { sertraline } \\
\text { monotherapy } \\
\text { exposure in } \\
\text { pregnancy }\end{array}$ & $\begin{array}{l}\text { Convulsive } \\
\text { seizures in } \\
\text { women with } \\
\text { epilepsy at } 7 \\
\text { months to } \\
\text { delivery }\end{array}$ & $\begin{array}{l}0 / 1 \text { vs. } \\
1 / 25^{107}\end{array}$ & $\begin{array}{l}\mathrm{RD}, 0.29 \\
(95 \% \mathrm{Cl}, \\
-0.57 \text { to } \\
0.65)^{107}\end{array}$ & $\begin{array}{l}1 \text { cohort, } \\
n=26^{107}\end{array}$ & $\begin{array}{l}\text { High study } \\
\text { limitations (high } \\
\text { risk of bias }{ }^{107} \text { ), } \\
\text { seriously } \\
\text { imprecise (wide } \\
\text { Cls, few events), } \\
\text { consistency } \\
\text { unknown }\end{array}$ & Insufficient \\
\hline
\end{tabular}




\begin{tabular}{|c|c|c|c|c|c|c|c|}
\hline Population & $\begin{array}{l}\text { Intervention } \\
\text { and } \\
\text { Comparator }\end{array}$ & Outcome & $\begin{array}{l}\text { Incidence } \\
\text { or Mean } \\
\text { Effect by } \\
\text { Arm }\end{array}$ & Results & $\begin{array}{l}\text { Study } \\
\text { Design and } \\
\text { Sample } \\
\text { Size }\end{array}$ & $\begin{array}{l}\text { Factors That } \\
\text { Affect the } \\
\text { Strength of } \\
\text { Evidence }\end{array}$ & $\begin{array}{l}\text { Overall } \\
\text { Evidence } \\
\text { Strength } \\
\text { (Direction of } \\
\text { Effect) }\end{array}$ \\
\hline $\begin{array}{l}\text { ADD- } \\
\text { exposed } \\
\text { women }\end{array}$ & $\begin{array}{l}\text { Fluvoxamine vs. } \\
\text { sertraline } \\
\text { monotherapy } \\
\text { exposure in } \\
\text { pregnancy }\end{array}$ & $\begin{array}{l}\text { Nonconvulsive } \\
\text { seizures in } \\
\text { women with } \\
\text { epilepsy at } \\
\text { enrollment to } \\
7 \text { months } \\
\text { gestation }\end{array}$ & $\begin{array}{l}\text { 0/1 vs. } \\
3 / 25^{107}\end{array}$ & $\begin{array}{l}\mathrm{RR}, 0.43(95 \% \\
\mathrm{Cl} \\
-0.49 \text { to } \\
0.73)^{107}\end{array}$ & $\begin{array}{l}1 \text { cohort, } \\
n=26^{107}\end{array}$ & $\begin{array}{l}\text { High study } \\
\text { limitations (high } \\
\text { risk of bias }{ }^{107} \text { ), } \\
\text { seriously } \\
\text { imprecise (wide } \\
\text { Cls, few events), } \\
\text { consistency } \\
\text { unknown }\end{array}$ & Insufficient \\
\hline $\begin{array}{l}\text { ADD- } \\
\text { exposed } \\
\text { women }\end{array}$ & $\begin{array}{l}\text { Fluvoxamine vs. } \\
\text { sertraline } \\
\text { monotherapy } \\
\text { exposure in } \\
\text { pregnancy }\end{array}$ & $\begin{array}{l}\text { Nonconvulsive } \\
\text { seizures in } \\
\text { women with } \\
\text { epilepsy at } 7 \\
\text { months to } \\
\text { delivery }\end{array}$ & $\begin{array}{l}0 / 1 \text { vs. } \\
1 / 25^{107}\end{array}$ & $\begin{array}{l}\text { RD, } 0.29 \\
(95 \% \mathrm{Cl}, \\
-0.57 \text { to } \\
0.65)^{107}\end{array}$ & $\begin{array}{l}1 \text { cohort, } \\
n=26^{107}\end{array}$ & $\begin{array}{l}\text { High study } \\
\text { limitations (high } \\
\text { risk of bias }{ }^{107} \text { ), } \\
\text { seriously } \\
\text { imprecise (wide } \\
\text { Cls, few events), } \\
\text { consistency } \\
\text { unknown }\end{array}$ & Insufficient \\
\hline $\begin{array}{l}\text { ADD- } \\
\text { exposed } \\
\text { women }\end{array}$ & $\begin{array}{l}\text { Fluvoxamine vs. } \\
\text { sertraline } \\
\text { monotherapy } \\
\text { exposure in } \\
\text { pregnancy }\end{array}$ & Birth weight & $\mathrm{NR}^{107}$ & $\begin{array}{l}\text { NR other than } \\
\text { "specific } \\
\text { ADDs were } \\
\text { not } \\
\text { associated } \\
\text { with birth } \\
\text { weight"107 }\end{array}$ & $\begin{array}{l}1 \text { cohort, } \\
n=26^{107}\end{array}$ & $\begin{array}{l}\text { High study } \\
\text { limitations (high } \\
\text { risk of bias }{ }^{107} \text { ), } \\
\text { seriously } \\
\text { imprecise (wide } \\
\text { Cls, few events), } \\
\text { consistency } \\
\text { unknown }\end{array}$ & Insufficient \\
\hline $\begin{array}{l}\text { ADD- } \\
\text { exposed } \\
\text { women }\end{array}$ & $\begin{array}{l}\text { Fluvoxamine vs. } \\
\text { sertraline } \\
\text { monotherapy } \\
\text { exposure in } \\
\text { pregnancy }\end{array}$ & $\begin{array}{l}\text { Congenital } \\
\text { anomalies }\end{array}$ & $\begin{array}{l}0 / 1 \text { vs. } \\
3 / 25^{107}\end{array}$ & $\begin{array}{l}\text { RD, } 0.12 \\
(95 \% \mathrm{Cl}, \\
-0.49 \text { to } \\
0.73)^{107}\end{array}$ & $\begin{array}{l}1 \text { cohort, } \\
n=26^{107}\end{array}$ & $\begin{array}{l}\text { High study } \\
\text { limitations (high } \\
\text { risk of bias }{ }^{107} \text { ), } \\
\text { seriously } \\
\text { imprecise (wide } \\
\text { Cls, few events), } \\
\text { consistency } \\
\text { unknown }\end{array}$ & Insufficient \\
\hline $\begin{array}{l}\text { ADD- } \\
\text { exposed } \\
\text { women }\end{array}$ & $\begin{array}{l}\text { Fluvoxamine vs. } \\
\text { sertraline } \\
\text { monotherapy } \\
\text { exposure in } \\
\text { pregnancy }\end{array}$ & $\begin{array}{l}\text { Autism } \\
\text { spectrum } \\
\text { disorder }\end{array}$ & $\begin{array}{l}1 / 35 \text { vs. } \\
1 / 292^{111}\end{array}$ & $\begin{array}{l}\text { ARR, 8.34 } \\
(95 \% \mathrm{Cl}, 0.53 \\
\text { to } 130.45)^{111}\end{array}$ & $\begin{array}{l}1 \text { cohort, } \\
n=327^{111}\end{array}$ & $\begin{array}{l}\text { High study } \\
\text { limitations (high } \\
\text { risk of bias }{ }^{111} \text { ), } \\
\text { seriously } \\
\text { imprecise (wide } \\
\text { Cls, few events), } \\
\text { consistency } \\
\text { unknown }\end{array}$ & Insufficient \\
\hline
\end{tabular}

$\mathrm{ADD}=$ attention deficit disorder; $\mathrm{ARR}=$ adjusted risk ratio; $\mathrm{CI}=$ confidence interval; $\mathrm{NR}=$ not reported; $\mathrm{RD}=$ risk difference; $\mathrm{RR}=$ relative risk; vs. $=$ versus.

\section{Fluvoxamine Versus SNRI (Venlafaxine or Desvenlafaxine)}

\section{Overview}

- The evidence for exposure to fluvoxamine versus SNRI monotherapy during pregnancy is insufficient to judge the risk of harms for the maternal outcomes (spontaneous abortion, still birth, convulsive seizures in women with epilepsy, or nonconvulsive seizures in women with epilepsy) and child outcomes (birth weight or congenital anomalies). 


\section{Detailed Results}

One publication reported on fluvoxamine versus SNRI monotherapy (venlafaxine or desvenlafaxine). ${ }^{107}$ This publication drew from one cohort (the Australian Pregnancy Register of Antiepileptic Drugs ${ }^{107}$ ), which was a nonrandomized observational study and was rated as high risk of bias.

This publication focused on exposure during pregnancy and further examined outcomes at three time frames: enrollment to 7 months gestation, 7 months gestation to delivery, and within the first postnatal month. ${ }^{107}$ This publication compared pregnant women exposed to fluvoxamine versus SNRI monotherapy. ${ }^{107}$

The evidence for fluvoxamine versus SNRI monotherapy is insufficient to judge the risk of harms for the maternal outcomes (spontaneous abortion, still birth, convulsive seizures in women with epilepsy, or nonconvulsive seizures in women with epilepsy) and child outcomes (birth weight or congenital anomalies) (Table B-70). This study found no association between fluvoxamine versus SNRI monotherapy use in pregnancy with any outcome.

Table B-70. Strength of evidence for comparative harms: Fluvoxamine versus SNRI (venlafaxine or desvenlafaxine)

\begin{tabular}{|c|c|c|c|c|c|c|c|}
\hline Population & $\begin{array}{l}\text { Intervention } \\
\text { and } \\
\text { Comparator }\end{array}$ & Outcome & $\begin{array}{l}\text { Incidence } \\
\text { or Mean } \\
\text { Effect by } \\
\text { Arm }\end{array}$ & Results & $\begin{array}{l}\text { Study } \\
\text { Design and } \\
\text { Sample } \\
\text { Size }\end{array}$ & $\begin{array}{l}\text { Factors That } \\
\text { Affect the } \\
\text { Strength of } \\
\text { Evidence }\end{array}$ & $\begin{array}{l}\text { Overall } \\
\text { Evidence } \\
\text { Strength } \\
\text { (Direction of } \\
\text { Effect) }\end{array}$ \\
\hline $\begin{array}{l}\text { ADD- } \\
\text { exposed } \\
\text { women }\end{array}$ & $\begin{array}{l}\text { Fluvoxamine } \\
\text { exposure vs. } \\
\text { SNRI } \\
\text { monotherapy } \\
\text { (venlafaxine or } \\
\text { desvenlafaxine) } \\
\text { exposure in } \\
\text { pregnancy }\end{array}$ & $\begin{array}{l}\text { Spontaneous } \\
\text { abortion }\end{array}$ & $\begin{array}{l}\text { 0/1 vs. } \\
0 / 13^{107}\end{array}$ & $\begin{array}{l}\text { RD, } 0(95 \% \\
C l,-0.62 \text { to } \\
0.62)^{107}\end{array}$ & $\begin{array}{l}1 \text { cohort, } \\
n=14^{107}\end{array}$ & $\begin{array}{l}\text { High study } \\
\text { limitations (high } \\
\text { risk of bias }{ }^{107} \text { ), } \\
\text { seriously } \\
\text { imprecise (wide } \\
\text { Cls, few events), } \\
\text { consistency } \\
\text { unknown }\end{array}$ & Insufficient \\
\hline $\begin{array}{l}\text { ADD- } \\
\text { exposed } \\
\text { women }\end{array}$ & $\begin{array}{l}\text { Fluvoxamine } \\
\text { exposure vs. } \\
\text { SNRI } \\
\text { monotherapy } \\
\text { (venlafaxine or } \\
\text { desvenlafaxine) } \\
\text { exposure in } \\
\text { pregnancy }\end{array}$ & Still birth & $\begin{array}{l}0 / 1 \text { vs. } \\
1 / 13^{107}\end{array}$ & $\begin{array}{l}\text { RD, } 0(95 \% \\
\mathrm{Cl}, 0.62 \text { to } \\
0.62)^{107}\end{array}$ & $\begin{array}{l}1 \text { cohort, } \\
n=14^{107}\end{array}$ & $\begin{array}{l}\text { High study } \\
\text { limitations (high } \\
\text { risk of bias }{ }^{107} \text { ), } \\
\text { seriously } \\
\text { imprecise (wide } \\
\text { Cls, few events), } \\
\text { consistency } \\
\text { unknown }\end{array}$ & Insufficient \\
\hline $\begin{array}{l}\text { ADD- } \\
\text { exposed } \\
\text { women }\end{array}$ & $\begin{array}{l}\text { Fluvoxamine } \\
\text { exposure vs. } \\
\text { SNRI } \\
\text { monotherapy } \\
\text { (venlafaxine or } \\
\text { desvenlafaxine) } \\
\text { exposure in } \\
\text { pregnancy }\end{array}$ & $\begin{array}{l}\text { Convulsive } \\
\text { seizures in } \\
\text { women with } \\
\text { epilepsy at } \\
\text { enrollment to } \\
7 \text { months } \\
\text { gestation }\end{array}$ & $\begin{array}{l}\text { 0/1 vs. } \\
0 / 13^{107}\end{array}$ & $\begin{array}{l}\text { RR, 0.48 } \\
(95 \% \mathrm{Cl}, 0.03 \\
\text { to } 6.86)^{107}\end{array}$ & $\begin{array}{l}1 \text { cohort, } \\
n=14^{107}\end{array}$ & $\begin{array}{l}\text { High study } \\
\text { limitations (high } \\
\text { risk of bias }{ }^{107} \text { ), } \\
\text { seriously } \\
\text { imprecise (wide } \\
\text { Cls, few events), } \\
\text { consistency } \\
\text { unknown }\end{array}$ & Insufficient \\
\hline $\begin{array}{l}\text { ADD- } \\
\text { exposed } \\
\text { women }\end{array}$ & $\begin{array}{l}\text { Fluvoxamine } \\
\text { exposure vs. } \\
\text { SNRI } \\
\text { monotherapy } \\
\text { (venlafaxine or } \\
\text { desvenlafaxine) } \\
\text { exposure in } \\
\text { pregnancy }\end{array}$ & $\begin{array}{l}\text { Convulsive } \\
\text { seizures in } \\
\text { women with } \\
\text { epilepsy at } 7 \\
\text { months to } \\
\text { delivery }\end{array}$ & $\begin{array}{l}0 / 1 \text { vs. } \\
0 / 13^{107}\end{array}$ & $\begin{array}{l}\text { RR, 0.29 } \\
(95 \% \mathrm{Cl}, 0.02 \\
\text { to } 4.95)^{107}\end{array}$ & $\begin{array}{l}1 \text { cohort, } \\
n=14^{107}\end{array}$ & $\begin{array}{l}\text { High study } \\
\text { limitations (high } \\
\text { risk of bias }{ }^{107} \text { ), } \\
\text { seriously } \\
\text { imprecise (wide } \\
\text { Cls, few events), } \\
\text { consistency } \\
\text { unknown }\end{array}$ & Insufficient \\
\hline
\end{tabular}




\begin{tabular}{|c|c|c|c|c|c|c|c|}
\hline Population & $\begin{array}{l}\text { Intervention } \\
\text { and } \\
\text { Comparator }\end{array}$ & Outcome & $\begin{array}{l}\text { Incidence } \\
\text { or Mean } \\
\text { Effect by } \\
\text { Arm }\end{array}$ & Results & $\begin{array}{l}\text { Study } \\
\text { Design and } \\
\text { Sample } \\
\text { Size }\end{array}$ & $\begin{array}{l}\text { Factors That } \\
\text { Affect the } \\
\text { Strength of } \\
\text { Evidence }\end{array}$ & $\begin{array}{l}\text { Overall } \\
\text { Evidence } \\
\text { Strength } \\
\text { (Direction of } \\
\text { Effect) }\end{array}$ \\
\hline $\begin{array}{l}\text { ADD- } \\
\text { exposed } \\
\text { women }\end{array}$ & $\begin{array}{l}\text { Fluvoxamine } \\
\text { exposure vs. } \\
\text { SNRI } \\
\text { monotherapy } \\
\text { (venlafaxine or } \\
\text { desvenlafaxine) } \\
\text { exposure in } \\
\text { pregnancy }\end{array}$ & $\begin{array}{l}\text { Nonconvulsiv } \\
\text { e seizures in } \\
\text { women with } \\
\text { epilepsy at } \\
\text { enrollment to } \\
7 \text { months } \\
\text { gestation }\end{array}$ & $\begin{array}{l}0 / 1 \text { vs. } \\
1 / 13^{107}\end{array}$ & $\begin{array}{l}\text { RR, } 0.86 \\
(95 \% \mathrm{Cl}, 0.07 \\
\text { to } 10.8)^{107}\end{array}$ & $\begin{array}{l}1 \text { cohort, } \\
n=14^{107}\end{array}$ & $\begin{array}{l}\text { High study } \\
\text { limitations (high } \\
\text { risk of bias }{ }^{107} \text { ), } \\
\text { seriously } \\
\text { imprecise (wide } \\
\text { Cls, few events), } \\
\text { consistency } \\
\text { unknown }\end{array}$ & Insufficient \\
\hline $\begin{array}{l}\text { ADD- } \\
\text { exposed } \\
\text { women }\end{array}$ & $\begin{array}{l}\text { Fluvoxamine } \\
\text { exposure vs. } \\
\text { SNRI } \\
\text { monotherapy } \\
\text { (venlafaxine or } \\
\text { desvenlafaxine) } \\
\text { exposure in } \\
\text { pregnancy }\end{array}$ & $\begin{array}{l}\text { Nonconvulsiv } \\
\text { e seizures in } \\
\text { women with } \\
\text { epilepsy at } 7 \\
\text { months to } \\
\text { delivery }\end{array}$ & $\begin{array}{l}\text { 0/1 vs. } \\
0 / 13^{107}\end{array}$ & $\begin{array}{l}\text { RR, } 0.67 \\
(95 \% \mathrm{Cl}, 0.07 \\
\text { to } 10.8)^{107}\end{array}$ & $\begin{array}{l}1 \text { cohort, } \\
n=14^{107}\end{array}$ & $\begin{array}{l}\text { High study } \\
\text { limitations (high } \\
\text { risk of bias }{ }^{107} \text { ), } \\
\text { seriously } \\
\text { imprecise (wide } \\
\text { Cls, few events), } \\
\text { consistency } \\
\text { unknown }\end{array}$ & Insufficient \\
\hline $\begin{array}{l}\text { ADD- } \\
\text { exposed } \\
\text { women }\end{array}$ & $\begin{array}{l}\text { Fluvoxamine } \\
\text { exposure vs. } \\
\text { SNRI } \\
\text { monotherapy } \\
\text { (venlafaxine or } \\
\text { desvenlafaxine) } \\
\text { exposure in } \\
\text { pregnancy }\end{array}$ & Birth weight & $\mathrm{NR}^{107}$ & $\begin{array}{l}\text { NR other than } \\
\text { "specific } \\
\text { ADDs were } \\
\text { not } \\
\text { associated } \\
\text { with birth } \\
\text { weight"107 }\end{array}$ & $\begin{array}{l}1 \text { cohort, } \\
n=14^{107}\end{array}$ & $\begin{array}{l}\text { High study } \\
\text { limitations (high } \\
\text { risk of bias }{ }^{107} \text { ), } \\
\text { seriously } \\
\text { imprecise (wide } \\
\text { Cls, few events), } \\
\text { consistency } \\
\text { unknown }\end{array}$ & Insufficient \\
\hline $\begin{array}{l}\text { ADD- } \\
\text { exposed } \\
\text { women }\end{array}$ & $\begin{array}{l}\text { Fluvoxamine } \\
\text { exposure vs. } \\
\text { SNRI } \\
\text { monotherapy } \\
\text { (venlafaxine or } \\
\text { desvenlafaxine) } \\
\text { exposure in } \\
\text { pregnancy }\end{array}$ & $\begin{array}{l}\text { Congenital } \\
\text { anomalies }\end{array}$ & $\begin{array}{l}0 / 1 \text { vs. } \\
0 / 13^{107}\end{array}$ & $\begin{array}{l}\text { ARR, } 0.82 \\
(95 \% \mathrm{Cl}, 0.06 \\
\text { to } 10.35)^{107}\end{array}$ & $\begin{array}{l}1 \text { cohort, } \\
n=14^{107}\end{array}$ & $\begin{array}{l}\text { High study } \\
\text { limitations (high } \\
\text { risk of bias }{ }^{107} \text { ), } \\
\text { seriously } \\
\text { imprecise (wide } \\
\text { Cls, few events), } \\
\text { consistency } \\
\text { unknown }\end{array}$ & Insufficient \\
\hline
\end{tabular}

$\mathrm{ADD}=$ attention deficit disorder; $\mathrm{ARR}=$ adjusted risk ratio; $\mathrm{CI}=$ confidence interval; $\mathrm{n}=$ number; $\mathrm{NR}=$ not reported; $\mathrm{RD}=$ risk difference; $\mathrm{RR}=$ relative risk; SNRI = serotonin-norepinephrine reuptake inhibitor; vs. $=$ versus.

\section{Sertraline Only Versus Citalopram or Escitalopram Only}

\section{Overview}

- The evidence is limited to two studies and is insufficient to judge the comparative risk of harms from exposure to sertraline only versus citalopram or escitalopram only during pregnancy for maternal outcomes (convulsive seizures, nonconvulsive seizures, or spontaneous abortion or still birth) or child outcomes (congenital anomaly, birth weight, or congenital heart disease) among women with epilepsy.

\section{Detailed Results}

Two high risk-of-bias publications reported on the comparative harms of sertraline versus citalopram or escitalopram only. ${ }^{107,141}$

For one study, the population consisted of pregnant women with depression, anxiety, or bipolar disorder, as well as epilepsy, drawn from the Australian Antiepileptic Drugs in Pregnancy register. This publication focused on exposure during pregnancy and further examined outcomes at 3 time frames: enrollment to 7 months gestation, 7 months gestation to 
delivery, and within the first postnatal month. This publication compared pregnant women exposed to sertraline versus citalopram or escitalopram only. ${ }^{107}$

The second study consisted of pregnant women who underwent fetal echocardiography because of an in utero exposure at any point during pregnancy to either SSRIs or SNRIs in a retrospective review of institutional medical records at Children's Hospital of New Orleans. ${ }^{141}$

The evidence is insufficient to judge the comparative risk of harms from sertraline only versus citalopram or escitalopram only for maternal outcomes (convulsive seizures, nonconvulsive seizures, or spontaneous abortion or still birth) or child outcomes (congenital anomaly, birth weight, or congenital heart disease) among women with epilepsy (Table B-71).

Table B-71. Strength of evidence for comparative harms: Sertraline monotherapy versus SSRI monotherapy (citalopram or escitalopram)

\begin{tabular}{|c|c|c|c|c|c|c|c|}
\hline Population & $\begin{array}{l}\text { Intervention } \\
\text { and } \\
\text { Comparator }\end{array}$ & Outcome & $\begin{array}{l}\text { Incidence or } \\
\text { Mean Effect } \\
\text { by Arm }\end{array}$ & Results & $\begin{array}{l}\text { Study } \\
\text { Design } \\
\text { and } \\
\text { Sample } \\
\text { Size }\end{array}$ & $\begin{array}{l}\text { Factors That } \\
\text { Affect the } \\
\text { Strength of } \\
\text { Evidence }\end{array}$ & $\begin{array}{l}\text { Overall } \\
\text { Evidence } \\
\text { Strength } \\
\text { (Direction } \\
\text { of Effect) }\end{array}$ \\
\hline $\begin{array}{l}\text { Women } \\
\text { with } \\
\text { epilepsy } \\
\text { and } \\
\text { depression, } \\
\text { anxiety or } \\
\text { bipolar } \\
\text { disorder }\end{array}$ & $\begin{array}{l}\text { Sertraline } \\
\text { only vs. SSRI } \\
\text { monotherapy } \\
\text { (citalopram } \\
\text { or } \\
\text { escitalopram) }\end{array}$ & $\begin{array}{l}\text { Convulsive } \\
\text { seizures: } \\
\text { From } \\
\text { enrollment to } 7 \\
\text { months } \\
\text { gestation } \\
\text { From } 7 \text { months } \\
\text { gestation to } \\
\text { delivery }\end{array}$ & $\begin{array}{l}2 / 25(8 \%) \text { vs. } \\
2 / 20(10 \%)^{107} \\
1 / 25(4 \%) \text { vs. } \\
1 / 20(5 \%)^{107}\end{array}$ & $\begin{array}{l}\text { RR, } 0.8(95 \% \\
\mathrm{Cl}, 0.12 \text { to } \\
5.19)^{107} \\
\mathrm{RR}, 0.8(95 \% \\
\mathrm{Cl}, 0.05 \text { to } \\
12.01)^{107}\end{array}$ & $\begin{array}{l}1 \text { cohort, } \\
n=45^{107}\end{array}$ & $\begin{array}{l}\text { High study } \\
\text { limitations (high } \\
\text { risk of bias }{ }^{107} \text { ), } \\
\text { seriously } \\
\text { imprecise (few } \\
\text { events, wide Cls), } \\
\text { consistency } \\
\text { unknown }\end{array}$ & Insufficient \\
\hline $\begin{array}{l}\text { Women } \\
\text { with } \\
\text { epilepsy } \\
\text { and } \\
\text { depression, } \\
\text { anxiety or } \\
\text { bipolar } \\
\text { disorder }\end{array}$ & $\begin{array}{l}\text { Sertraline } \\
\text { only vs. SSRI } \\
\text { monotherapy } \\
\text { (citalopram } \\
\text { or } \\
\text { escitalopram) } \\
\end{array}$ & $\begin{array}{l}\text { Nonconvulsive } \\
\text { seizures: } \\
\text { From } \\
\text { enrollment to } 7 \\
\text { months } \\
\text { gestation } \\
\text { From } 7 \text { months } \\
\text { gestation to } \\
\text { delivery }\end{array}$ & $\begin{array}{l}3 / 25(12 \%) \\
\text { vs. } 4 / 20 \\
(20 \%)^{107} \\
1 / 25(4 \%) \text { vs. } \\
3 / 20(15 \%)^{107} \\
\end{array}$ & $\begin{array}{l}\mathrm{RR}, 0.60 \\
(95 \% \mathrm{Cl}, 0.15 \\
\text { to } 2.38)^{107} \\
\\
\mathrm{RR}, 0.27 \\
(95 \% \mathrm{Cl}, 0.03 \\
\text { to } 2.37)^{107}\end{array}$ & $\begin{array}{l}1 \text { cohort, } \\
\mathrm{n}=45^{107}\end{array}$ & $\begin{array}{l}\text { High study } \\
\text { limitations (high } \\
\text { risk of bias }{ }^{107} \text { ), } \\
\text { seriously } \\
\text { imprecise (few } \\
\text { events, wide Cls), } \\
\text { consistency } \\
\text { unknown }\end{array}$ & Insufficient \\
\hline $\begin{array}{l}\text { Women } \\
\text { with } \\
\text { epilepsy } \\
\text { and } \\
\text { depression, } \\
\text { anxiety or } \\
\text { bipolar } \\
\text { disorder } \\
\end{array}$ & $\begin{array}{l}\text { Sertraline } \\
\text { only vs. SSRI } \\
\text { monotherapy } \\
\text { (citalopram } \\
\text { or } \\
\text { escitalopram) }\end{array}$ & $\begin{array}{l}\text { Spontaneous } \\
\text { abortion }\end{array}$ & $\begin{array}{l}0 / 25(0 \%) \text { vs. } \\
0 / 21(0 \%)^{107}\end{array}$ & $\begin{array}{l}\text { RR not } \\
\text { calculable: no } \\
\text { events }\end{array}$ & $\begin{array}{l}1 \text { cohort, } \\
n=46^{107}\end{array}$ & $\begin{array}{l}\text { High study } \\
\text { limitations (high } \\
\text { risk of bias }{ }^{107} \text { ), } \\
\text { likely imprecise } \\
\text { (no events, small } \\
\text { Ns), consistency } \\
\text { unknown }\end{array}$ & Insufficient \\
\hline $\begin{array}{l}\text { Women } \\
\text { with } \\
\text { epilepsy } \\
\text { and } \\
\text { depression, } \\
\text { anxiety or } \\
\text { bipolar } \\
\text { disorder }\end{array}$ & $\begin{array}{l}\text { Sertraline } \\
\text { only vs. SSRI } \\
\text { monotherapy } \\
\text { (citalopram } \\
\text { or } \\
\text { escitalopram) }\end{array}$ & Still birth & $\begin{array}{l}0 / 25(0 \%) \text { vs. } \\
0 / 21(0 \%)^{107}\end{array}$ & $\begin{array}{l}\text { RR not } \\
\text { calculable: no } \\
\text { events }\end{array}$ & $\begin{array}{l}1 \text { cohort, } \\
\mathrm{n}=46^{107}\end{array}$ & $\begin{array}{l}\text { High study } \\
\text { limitations (high } \\
\text { risk of bias }{ }^{107} \text { ), } \\
\text { likely imprecise } \\
\text { (no events, small } \\
\text { Ns), consistency } \\
\text { unknown }\end{array}$ & Insufficient \\
\hline
\end{tabular}




\begin{tabular}{|c|c|c|c|c|c|c|c|}
\hline Population & $\begin{array}{l}\text { Intervention } \\
\text { and } \\
\text { Comparator }\end{array}$ & Outcome & $\begin{array}{l}\text { Incidence or } \\
\text { Mean Effect } \\
\text { by Arm }\end{array}$ & Results & $\begin{array}{l}\text { Study } \\
\text { Design } \\
\text { and } \\
\text { Sample } \\
\text { Size }\end{array}$ & $\begin{array}{l}\text { Factors That } \\
\text { Affect the } \\
\text { Strength of } \\
\text { Evidence }\end{array}$ & $\begin{array}{l}\text { Overall } \\
\text { Evidence } \\
\text { Strength } \\
\text { (Direction } \\
\text { of Effect) }\end{array}$ \\
\hline $\begin{array}{l}\text { Women } \\
\text { with } \\
\text { epilepsy } \\
\text { and } \\
\text { depression, } \\
\text { anxiety or } \\
\text { bipolar } \\
\text { disorder }\end{array}$ & $\begin{array}{l}\text { Sertraline } \\
\text { only vs. SSRI } \\
\text { monotherapy } \\
\text { (citalopram } \\
\text { or } \\
\text { escitalopram) }\end{array}$ & $\begin{array}{l}\text { Congenital } \\
\text { anomaly }\end{array}$ & $\begin{array}{l}3 / 25(12 \%) \\
\text { vs. } 4 / 21 \\
(19 \%)^{107}\end{array}$ & $\begin{array}{l}\text { RR, 0.63 } \\
(95 \% \mathrm{Cl}, 0.16 \\
\text { to } 2.50)^{107}\end{array}$ & $\begin{array}{l}1 \text { cohort, } \\
\mathrm{n}=46^{107}\end{array}$ & $\begin{array}{l}\text { High study } \\
\text { limitations (high } \\
\text { risk of bias }{ }^{107} \text { ), } \\
\text { seriously } \\
\text { imprecise (few } \\
\text { events, wide Cls), } \\
\text { consistency } \\
\text { unknown }\end{array}$ & Insufficient \\
\hline $\begin{array}{l}\text { Women } \\
\text { with } \\
\text { epilepsy } \\
\text { and } \\
\text { depression, } \\
\text { anxiety or } \\
\text { bipolar } \\
\text { disorder }\end{array}$ & $\begin{array}{l}\text { Sertraline } \\
\text { only vs. SSRI } \\
\text { monotherapy } \\
\text { (citalopram } \\
\text { or } \\
\text { escitalopram) }\end{array}$ & Birth weight & $\begin{array}{l}\text { Median } \pm \text { IQR: } \\
3,350 \pm 808 \\
\text { vs. } \\
3,087 \pm 286^{107}\end{array}$ & $\begin{array}{l}\text { Difference not } \\
\text { calculable }\end{array}$ & $\begin{array}{l}1 \text { cohort, } \\
\mathrm{n}=45^{107}\end{array}$ & $\begin{array}{l}\text { High study } \\
\text { limitations (high } \\
\text { risk of bias }{ }^{107} \text { ), } \\
\text { likely imprecise } \\
\text { (small Ns), } \\
\text { consistency } \\
\text { unknown }\end{array}$ & Insufficient \\
\hline $\begin{array}{l}\text { Women } \\
\text { who } \\
\text { underwent } \\
\text { fetal } \\
\text { echocardio } \\
\text { graphy } \\
\text { because of } \\
\text { an in-utero } \\
\text { exposure to } \\
\text { either } \\
\text { SSRIs or } \\
\text { SNRIs }\end{array}$ & $\begin{array}{l}\text { Sertraline vs. } \\
\text { SSRI } \\
\text { monotherapy } \\
\text { (citalopram } \\
\text { or } \\
\text { escitalopram) } \\
\text {; sertraline } \\
\text { vs. } \\
\text { citalopram; } \\
\text { sertraline vs. } \\
\text { escitalopram }\end{array}$ & $\begin{array}{l}\text { Congenital } \\
\text { heart disease }\end{array}$ & $\begin{array}{l}\text { Sertraline vs. } \\
\text { citalopram or } \\
\text { escitalopram } \\
0 / 7(0 \%) \text { vs. } \\
3 / 14 \\
(21 \%))^{141} \\
\text { Sertraline vs. } \\
\text { citalopram, } \\
0 / 7(0 \%) \text { vs. } \\
2 / 5(40 \%){ }^{141} \\
\text { Sertraline vs. } \\
\text { escitalopram } \\
0 / 7(0 \%) \text { vs. } \\
1 / 9(11 \%)^{141}\end{array}$ & $\begin{array}{l}\text { Risk } \\
\text { difference for } \\
\text { sertraline vs. } \\
\text { citalopram or } \\
\text { escitalopram, } \\
-0.21(95 \% \mathrm{Cl} \text {, } \\
-0.49 \text { to } 0.06) \text {; } \\
\text { for sertraline } \\
\text { vs. citalopram, } \\
-0.20(95 \% \mathrm{Cl} \text {, } \\
-0.59 \text { to } 0.19) \text {; } \\
\text { for sertraline } \\
\text { vs. } \\
\text { escitalopram, } \\
0.1195 \% \mathrm{Cl} \text {, - } \\
0.39 \text { to } \\
0.17)^{141}\end{array}$ & $\begin{array}{l}1 \text { cohort } \\
(\mathrm{n}=40)^{141}\end{array}$ & $\begin{array}{l}\text { High study } \\
\text { limitations (high } \\
\text { risk of bias }{ }^{141} \text { ), } \\
\text { likely imprecise } \\
\text { (small Ns), } \\
\text { consistency } \\
\text { unknown }\end{array}$ & Insufficient \\
\hline
\end{tabular}
inhibitor; SSRI = selective serotonin reuptake inhibitor; vs. $=$ versus.

\section{Sertraline Versus Duloxetine}

\section{Overview}

- The evidence is insufficient to judge the comparative risk of harms from exposure to sertraline versus duloxetine during pregnancy for child outcomes (congenital heart disease).

\section{Detailed Results}

One publication reported on the comparative harms of sertraline versus duloxetine. ${ }^{141}$ The study drew from a cohort of women who had undergone a fetal echocardiogram because of in utero exposure of the fetus to SSRIs or SNRIs. ${ }^{141}$ The study was rated as high risk of bias. The study yielded insufficient strength of evidence to judge the risk of congenital heart disease (Table B-72). 
Table B-72. Strength of evidence for comparative harms: Sertraline versus duloxetine

\begin{tabular}{|c|c|c|c|c|c|c|c|}
\hline Population & $\begin{array}{l}\text { Intervention } \\
\text { and } \\
\text { Comparator }\end{array}$ & Outcome & $\begin{array}{l}\text { Incidence } \\
\text { or Mean } \\
\text { Effect by } \\
\text { Arm }\end{array}$ & Results & $\begin{array}{l}\text { Study } \\
\text { Design } \\
\text { and } \\
\text { Sample } \\
\text { Size } \\
\end{array}$ & $\begin{array}{l}\text { Factors That } \\
\text { Affect the } \\
\text { Strength of } \\
\text { Evidence }\end{array}$ & $\begin{array}{l}\text { Overall } \\
\text { Evidence } \\
\text { Strength } \\
\text { (Direction of } \\
\text { Effect) }\end{array}$ \\
\hline $\begin{array}{l}\text { Women who } \\
\text { underwent fetal } \\
\text { echocardiography } \\
\text { because of } \\
\text { an in utero exposure } \\
\text { to either SSRIs or } \\
\text { SNRIs }\end{array}$ & $\begin{array}{l}\text { Sertraline vs. } \\
\text { duloxetine }\end{array}$ & $\begin{array}{l}\text { Congenital } \\
\text { heart } \\
\text { disease }\end{array}$ & $\begin{array}{l}0 / 7(0 \%) \text { vs. } \\
0 / 1(0 \%)^{141}\end{array}$ & $\begin{array}{l}\text { RR not } \\
\text { calculable: } \\
\text { no events }{ }^{141}\end{array}$ & $\begin{array}{l}1 \text { cohort, } \\
n=2^{141}\end{array}$ & $\begin{array}{l}\text { High study } \\
\text { limitations (high } \\
\text { risk of bias, } 141 \\
\text { imprecise (wide } \\
\text { Cls), } \\
\text { consistency } \\
\text { unknown }\end{array}$ & Insufficient \\
\hline
\end{tabular}

$\mathrm{CI}=$ confidence interval; $\mathrm{n}=$ number; $\mathrm{SNRI}=$ serotonin-norepinephrine reuptake inhibitor; SSRI = selective serotonin reuptake inhibitor; $\mathrm{RR}=$ relative risk; vs. = versus.

\section{Sertraline Versus Escitalopram or Fluvoxamine}

\section{Overview}

- The evidence is insufficient to judge the comparative risk of harms of exposure to sertraline versus escitalopram or fluvoxamine during pregnancy for child outcomes (autism spectrum disorder).

\section{Detailed Results}

One high risk-of-bias publication reported on the comparative harms of sertraline versus escitalopram or fluvoxamine. ${ }^{117}$ The population consisted of pregnant women with depression or anxiety exposed to antidepressants during pregnancy. The sample was drawn from multiple Danish National registries.

The evidence is insufficient to judge the comparative risk of harms from sertraline versus escitalopram or fluvoxamine for child outcomes (autism spectrum disorder [Table B-73]).

Table B-73. Strength of evidence for comparative harms: Sertraline versus escitalopram or fluvoxamine

\begin{tabular}{|c|c|c|c|c|c|c|c|}
\hline Population & $\begin{array}{l}\text { Intervention } \\
\text { and } \\
\text { Comparator }\end{array}$ & Outcome & $\begin{array}{l}\text { Incidence or } \\
\text { Mean Effect } \\
\text { by Arm }\end{array}$ & Results & $\begin{array}{l}\text { Study } \\
\text { Design } \\
\text { and } \\
\text { Sample } \\
\text { Size }\end{array}$ & $\begin{array}{l}\text { Factors That } \\
\text { Affect the } \\
\text { Strength of } \\
\text { Evidence }\end{array}$ & $\begin{array}{l}\text { Overall } \\
\text { Evidence } \\
\text { Strength } \\
\text { (Direction of } \\
\text { Effect) }\end{array}$ \\
\hline $\begin{array}{l}\text { Women exposed } \\
\text { to SSRIs during } \\
\text { pregnancy }{ }^{117}\end{array}$ & $\begin{array}{l}\text { Maternal } \\
\text { exposure to } \\
\text { sertraline vs. } \\
\text { escitalopram } \\
\text { or } \\
\text { fluvoxamine }\end{array}$ & $\begin{array}{l}\text { Autism } \\
\text { spectrum } \\
\text { disorder } \\
\text { among } \\
\text { offspring }\end{array}$ & $\begin{array}{l}9 / 1,576 \\
(0.6 \%) \text { vs. } \\
1 / 1047 \\
(0.1 \%)^{117}\end{array}$ & $\begin{array}{l}\mathrm{RR}, 5.98 \\
(95 \% \mathrm{Cl}, \\
0.76 \text { to } \\
47.12)^{117}\end{array}$ & $\begin{array}{l}1 \text { cohort, } \\
\mathrm{N}=2,623^{117}\end{array}$ & $\begin{array}{l}\text { High study } \\
\text { limitations } \\
\text { (high risk of } \\
\text { bias }^{117} \text { ), } \\
\text { seriously } \\
\text { imprecise } \\
\text { (few events, } \\
\text { Cls span the } \\
\text { null), } \\
\text { consistency } \\
\text { unknown }\end{array}$ & Insufficient \\
\hline
\end{tabular}

$\overline{\mathrm{CI}}=$ confidence interval; $\mathrm{N}=$ number; $\mathrm{RR}=$ relative risk; $\mathrm{SSRI}=$ selective serotonin reuptake inhibitor; vs. $=$ versus. 


\section{Sertraline Versus Fluoxetine}

\section{Overview}

- The strength of evidence is insufficient for judging a smaller risk of child autism spectrum disorder from exposure to sertraline monotherapy versus fluoxetine monotherapy during pregnancy.

- Among a sample of women with epilepsy, the evidence was insufficient in relation to maternal outcomes (convulsive seizures, nonconvulsive seizures, or spontaneous abortion or still birth) and child outcomes (congenital anomaly or birth weight).

\section{Detailed Results}

Four publications reported on the comparative harms of sertraline monotherapy versus fluoxetine monotherapy. ${ }^{81,107,111,117}$ Three studies were rated high risk of bias. ${ }^{107,111,117}$ One medium risk-of-bias analysis reported on preeclampsia outcomes among women with depression based on data in a Canadian British Columbia province-wide pregnancy and newborn database. ${ }^{81}$ The risk was not found to be significantly different between the two groups after adjusting for potential confounding characteristics. One high risk-of-bias analysis reported on a population of pregnant women with depression, anxiety, or bipolar disorder as well epilepsy, drawn from the Australian Antiepileptic Drugs in Pregnancy register. ${ }^{107}$ The authors found no significant differences in the risk of harms for any maternal (convulsive seizures, nonconvulsive seizures, spontaneous abortion, or still birth) or child outcomes (congenital anomalies or birth weight). Each of these outcomes was graded as insufficient strength of evidence.

Two publications reported on the relative risk of autism spectrum disorder. Data from the Danish Birth registry ${ }^{117}$ were analyzed in one study, and data from the Canadian QPC were analyzed in the second. ${ }^{111}$ A significantly smaller risk of autism spectrum disorder was found from sertraline compared with fluoxetine in both analyses, although we have contacted the author regarding the accuracy of the data and are awaiting a response. While both studies were rated as high risk of bias and did not control for confounding, differences in effect sizes were large. However, the uncertainty regarding accuracy resulted in the comparison being graded as insufficient (Table B-74).

Table B-74. Strength of evidence for comparative harms: Sertraline monotherapy versus fluoxetine monotherapy

\begin{tabular}{|c|c|c|c|c|c|c|c|}
\hline Population & $\begin{array}{l}\text { Intervention } \\
\text { and } \\
\text { Comparator }\end{array}$ & Outcome & $\begin{array}{l}\text { Incidence or } \\
\text { Mean Effect } \\
\text { by Arm }\end{array}$ & Results & $\begin{array}{l}\text { Study } \\
\text { Design } \\
\text { and } \\
\text { Sample } \\
\text { Size } \\
\end{array}$ & $\begin{array}{l}\text { Factors That } \\
\text { Affect the } \\
\text { Strength of } \\
\text { Evidence }\end{array}$ & $\begin{array}{l}\text { Overall } \\
\text { Evidence } \\
\text { Strength } \\
\text { (Direction } \\
\text { of Effect) } \\
\end{array}$ \\
\hline $\begin{array}{l}\text { Pregnant } \\
\text { women with } \\
\text { depression }\end{array}$ & $\begin{array}{l}\text { Sertraline } \\
\text { monotherapy } \\
\text { vs. fluoxetine } \\
\text { monotherapy }\end{array}$ & Preeclampsia & NR & $\begin{array}{l}\text { ARR, } 1.83(95 \% \\
\mathrm{Cl}, 0.92 \text { to } 3.64)^{81}\end{array}$ & $\begin{array}{l}1 \text { cohort, } \\
\mathrm{n}=\mathrm{NR}^{81}\end{array}$ & $\begin{array}{l}\text { Moderate study } \\
\text { limitations } \\
\text { imprecise (wide } \\
\text { Cls), consistency } \\
\text { unknown }\end{array}$ & Insufficient \\
\hline $\begin{array}{l}\text { Pregnant } \\
\text { women with } \\
\text { epilepsy and } \\
\text { depression, } \\
\text { anxiety or } \\
\text { bipolar } \\
\text { disorder }\end{array}$ & $\begin{array}{l}\text { Sertraline } \\
\text { monotherapy } \\
\text { vs. fluoxetine } \\
\text { monotherapy }\end{array}$ & $\begin{array}{l}\text { Convulsive } \\
\text { seizures: } \\
\text { From enrollment to } \\
7 \text { months gestation } \\
\text { From } 7 \text { months } \\
\text { gestation to } \\
\text { delivery }\end{array}$ & $\begin{array}{l}2 / 25(8 \%) \text { vs. } \\
2 / 5(40 \%)^{107} \\
1 / 25(4 \%) \text { vs. } \\
1 / 5(20 \%)^{107}\end{array}$ & $\begin{array}{l}\mathrm{RR}, 0.2(95 \% \mathrm{Cl} \\
0.04 \text { to } 1.10)^{107} \\
\mathrm{RR}, 0.2(95 \% \mathrm{Cl} \text {, } \\
0.02 \text { to } 2.69)^{107}\end{array}$ & $\begin{array}{l}1 \text { cohort, } \\
n=30^{107}\end{array}$ & $\begin{array}{l}\text { High study } \\
\text { limitations (high } \\
\text { risk of bias }{ }^{107} \text { ), } \\
\text { seriously } \\
\text { imprecise (few } \\
\text { events, wide Cls), } \\
\text { consistency } \\
\text { unknown }\end{array}$ & Insufficient \\
\hline
\end{tabular}




\begin{tabular}{|c|c|c|c|c|c|c|c|}
\hline Population & $\begin{array}{l}\text { Intervention } \\
\text { and } \\
\text { Comparator }\end{array}$ & Outcome & $\begin{array}{l}\text { Incidence or } \\
\text { Mean Effect } \\
\text { by Arm }\end{array}$ & Results & $\begin{array}{l}\text { Study } \\
\text { Design } \\
\text { and } \\
\text { Sample } \\
\text { Size } \\
\end{array}$ & $\begin{array}{l}\text { Factors That } \\
\text { Affect the } \\
\text { Strength of } \\
\text { Evidence }\end{array}$ & $\begin{array}{l}\text { Overall } \\
\text { Evidence } \\
\text { Strength } \\
\text { (Direction } \\
\text { of Effect) } \\
\end{array}$ \\
\hline $\begin{array}{l}\text { Pregnant } \\
\text { women with } \\
\text { epilepsy and } \\
\text { depression, } \\
\text { anxiety or } \\
\text { bipolar } \\
\text { disorder }\end{array}$ & $\begin{array}{l}\text { Sertraline } \\
\text { monotherapy } \\
\text { vs. fluoxetine } \\
\text { monotherapy }\end{array}$ & $\begin{array}{l}\text { Nonconvulsive } \\
\text { seizures: } \\
\text { From enrollment to } \\
7 \text { months gestation } \\
\text { From } 7 \text { months } \\
\text { gestation to } \\
\text { delivery }\end{array}$ & $\begin{array}{l}3 / 25(12 \%) \text { vs. } \\
1 / 5(20 \%)^{107} \\
1 / 25(4 \%) \text { vs. } \\
1 / 5(20 \%)^{107}\end{array}$ & $\begin{array}{l}\mathrm{RR}, 0.6(95 \% \mathrm{Cl} \\
0.077 \text { to } 4.66)^{107} \\
\mathrm{RR}, 0.2(95 \% \mathrm{Cl} \text {, } \\
0.015 \text { to } 2.69)^{107}\end{array}$ & $\begin{array}{l}1 \text { cohort, } \\
n=30^{107}\end{array}$ & $\begin{array}{l}\text { High study } \\
\text { limitations (high } \\
\text { risk of bias }{ }^{107} \text { ), } \\
\text { seriously } \\
\text { imprecise (few } \\
\text { events, wide Cls), } \\
\text { consistency } \\
\text { unknown }\end{array}$ & Insufficient \\
\hline $\begin{array}{l}\text { Pregnant } \\
\text { women with } \\
\text { epilepsy and } \\
\text { depression, } \\
\text { anxiety or } \\
\text { bipolar } \\
\text { disorder }\end{array}$ & $\begin{array}{l}\text { Sertraline } \\
\text { monotherapy } \\
\text { vs. fluoxetine } \\
\text { monotherapy }\end{array}$ & $\begin{array}{l}\text { Spontaneous } \\
\text { abortion }\end{array}$ & $\begin{array}{l}0 / 25(0 \%) \text { vs. } \\
0 / 5(0 \%)^{107}\end{array}$ & $\begin{array}{l}\text { RR not } \\
\text { calculable: no } \\
\text { events }\end{array}$ & $\begin{array}{l}1 \text { cohort, } \\
n=30^{107}\end{array}$ & $\begin{array}{l}\text { High study } \\
\text { limitations (high } \\
\text { risk of bias }{ }^{107} \text { ), } \\
\text { likely imprecise } \\
\text { (small N), } \\
\text { consistency } \\
\text { unknown }\end{array}$ & Insufficient \\
\hline $\begin{array}{l}\text { Pregnant } \\
\text { women with } \\
\text { epilepsy and } \\
\text { depression, } \\
\text { anxiety or } \\
\text { bipolar } \\
\text { disorder }\end{array}$ & $\begin{array}{l}\text { Sertraline } \\
\text { monotherapy } \\
\text { vs. fluoxetine } \\
\text { monotherapy }\end{array}$ & Stillbirth & $\begin{array}{l}0 / 25(0 \%) \text { vs. } \\
0 / 5(0 \%)^{107}\end{array}$ & $\begin{array}{l}\text { RR not } \\
\text { calculable: no } \\
\text { events }\end{array}$ & $\begin{array}{l}1 \text { cohort, } \\
n=30^{107}\end{array}$ & $\begin{array}{l}\text { High study } \\
\text { limitations (high } \\
\text { risk of bias }{ }^{107} \text { ), } \\
\text { likely imprecise } \\
\text { (small N), } \\
\text { consistency } \\
\text { unknown }\end{array}$ & Insufficient \\
\hline $\begin{array}{l}\text { Pregnant } \\
\text { women with } \\
\text { epilepsy and } \\
\text { depression, } \\
\text { anxiety or } \\
\text { bipolar } \\
\text { disorder }\end{array}$ & $\begin{array}{l}\text { Sertraline } \\
\text { monotherapy } \\
\text { vs. fluoxetine } \\
\text { monotherapy }\end{array}$ & $\begin{array}{l}\text { Congenital } \\
\text { anomaly }\end{array}$ & $\begin{array}{l}3 / 25(12 \%) \text { vs. } \\
0 / 5(0 \%)^{107}\end{array}$ & $\begin{array}{l}\mathrm{RD}, 0.12(95 \% \\
\mathrm{Cl},-0.14 \text { to } \\
0.38)^{107}\end{array}$ & $\begin{array}{l}1 \text { cohort, } \\
n=30^{107}\end{array}$ & $\begin{array}{l}\text { High study } \\
\text { limitations (high } \\
\text { risk of bias }{ }^{107} \text { ), } \\
\text { seriously } \\
\text { imprecise (few } \\
\text { events, wide Cls), } \\
\text { consistency } \\
\text { unknown }\end{array}$ & Insufficient \\
\hline $\begin{array}{l}\text { Pregnant } \\
\text { women with } \\
\text { epilepsy and } \\
\text { depression, } \\
\text { anxiety or } \\
\text { bipolar } \\
\text { disorder }\end{array}$ & $\begin{array}{l}\text { Sertraline } \\
\text { monotherapy } \\
\text { vs. fluoxetine } \\
\text { monotherapy }\end{array}$ & Birth weight & $\begin{array}{l}\text { Median } \pm \text { IQR: } \\
3,330 \pm 808 \text { vs. } \\
2,790 \pm 497^{107}\end{array}$ & $\begin{array}{l}\text { Difference not } \\
\text { calculable }\end{array}$ & $\begin{array}{l}1 \text { cohort, } \\
n=30^{107}\end{array}$ & $\begin{array}{l}\text { High study } \\
\text { limitations (high } \\
\left.\text { risk of bias }{ }^{107}\right) \text {, } \\
\text { likely imprecise } \\
\text { (small N), } \\
\text { consistency } \\
\text { unknown }\end{array}$ & Insufficient \\
\hline $\begin{array}{l}\text { Pregnant } \\
\text { women } \\
\text { taking } \\
\text { fluoxetine or } \\
\text { sertraline }\end{array}$ & $\begin{array}{l}\text { Sertraline } \\
\text { monotherapy } \\
\text { vs. fluoxetine } \\
\text { monotherapy }\end{array}$ & $\begin{array}{l}\text { Autism spectrum } \\
\text { disorder }\end{array}$ & $\begin{array}{l}9 / 1,576(0.6 \%) \\
\text { vs. } 18 / 160 \\
(11 \%)^{117} \\
\\
1 / 292(0.3 \%) \\
\text { vs. } 5 / 171 \\
(2.9 \%)^{111}\end{array}$ & $\begin{array}{l}\text { RR, } 0.05(95 \% \\
\mathrm{Cl}, 0.02 \text { to } \\
0.11)^{117} \\
\mathrm{RR}, 0.12(95 \% \\
\mathrm{Cl}, 0.01 \text { to } \\
0.99)^{111}\end{array}$ & $\begin{array}{l}2 \\
\text { cohorts; } \\
n=1,736 \\
\text { in one } \\
\text { cohort, }{ }^{117} \\
n=463 \text { in } \\
\text { one } \\
\text { cohort }^{111}\end{array}$ & $\begin{array}{l}\text { High study } \\
\text { limitations (high } \\
\text { risk of bias, } \\
\text { potential } \\
\text { inaccuracy in } \\
\text { data, } 111,117 \\
\text { precise, } \\
\text { consistent }\end{array}$ & Insufficient \\
\hline
\end{tabular}

$\mathrm{ARR}=$ adjusted risk ratio; $\mathrm{CI}=$ confidence interval; $\mathrm{IQR}=$ interquartile ratio; $\mathrm{n}=$ number; $\mathrm{NR}=$ not reported; $\mathrm{RD}=$ risk difference $\mathrm{RR}=$ relative risk; vs. = versus. 


\section{Sertraline Versus Nonsertraline SSRIs}

\section{Overview}

- The evidence is insufficient to judge the comparative risk of harms from exposure to sertraline versus nonsertraline SSRIs during pregnancy for child outcomes (major congenital abnormalities).

\section{Detailed Results}

One high risk-of-bias publication reported on the comparative harms of sertraline versus nonsertraline SSRIs. ${ }^{70}$ The population consisted of pregnant women with depression or anxiety identified in the year prior to pregnancy who were exposed to antidepressants during pregnancy. The sample was drawn from Canada's QPC.

The evidence is insufficient to judge the comparative risk of harms from sertraline only versus nonsertraline SSRIs for child outcomes (major congenital abnormalities) (Table B-75).

Table B-75. Strength of evidence for comparative harms: Sertraline versus nonsertraline SSRIs

\begin{tabular}{|c|c|c|c|c|c|c|c|}
\hline Population & $\begin{array}{l}\text { Intervention } \\
\text { and } \\
\text { Comparator }\end{array}$ & Outcome & $\begin{array}{l}\text { Incidence } \\
\text { or Mean } \\
\text { Effect by } \\
\text { Arm }\end{array}$ & Results & $\begin{array}{l}\text { Study Design and Sample } \\
\text { Size }\end{array}$ & $\begin{array}{l}\text { Factors } \\
\text { That Affect } \\
\text { the } \\
\text { Strength } \\
\text { of } \\
\text { Evidence }\end{array}$ & $\begin{array}{l}\text { Overall } \\
\text { Evidence } \\
\text { Strength } \\
\text { (Direction } \\
\text { of Effect) }\end{array}$ \\
\hline $\begin{array}{l}\text { Women } \\
\text { with } \\
\text { depression } \\
\text { or anxiety } \\
\text { in the year } \\
\text { prior to } \\
\text { pregnancy }\end{array}$ & $\begin{array}{l}\text { Exposed to } \\
\text { sertraline vs. } \\
\text { exposed to } \\
\text { non- } \\
\text { sertraline } \\
\text { SSRIs }\end{array}$ & $\begin{array}{l}\text { Major } \\
\text { congenital } \\
\text { anomalies }\end{array}$ & $\begin{array}{l}45 / 366 \\
(12.3 \%) \text { vs. } \\
236 / 1,963 \\
(12.0 \%)^{70}\end{array}$ & $\begin{array}{l}\text { ARR, } \\
1.02 \\
(95 \% \mathrm{Cl}, \\
0.76 \text { to } \\
1.38)^{70}\end{array}$ & 1 cohort, $\mathrm{N}=2,329^{70}$ & $\begin{array}{l}\text { High study } \\
\text { limitations } \\
\text { (high risk of } \\
\text { bias }^{70} \\
\text { imprecise } \\
\text { (Cls span } \\
\text { the null), } \\
\text { consistency } \\
\text { unknown }\end{array}$ & Insufficient \\
\hline
\end{tabular}

$\overline{\mathrm{ARR}}=$ adjusted risk ratio; $\mathrm{CI}=$ confidence interval; $\mathrm{N}=$ number; $\mathrm{SSRI}=$ selective serotonin reuptake inhibitor; vs. $=$ versus.

\section{Sertraline Only Versus SNRI Monotherapy (Venlafaxine or Desvenlafaxine)}

\section{Overview}

- The evidence is limited to one study and is insufficient to judge the comparative risk of harms from exposure to sertraline only versus SNRI monotherapy (venlafaxine or desvenlafaxine) during pregnancy for maternal outcomes (convulsive seizures, nonconvulsive seizures, or spontaneous abortion or still birth) or child outcomes (congenital anomaly or birth weight) among women with epilepsy.

\section{Detailed Results}

One high risk-of-bias publication reported on the comparative harms of sertraline versus SNRI monotherapy (venlafaxine or desvenlafaxine). ${ }^{107}$ The population consisted of pregnant women with depression, anxiety, or bipolar disorder, as well as epilepsy, drawn from the Australian Antiepileptic Drugs in Pregnancy register.

This publication focused on exposure during pregnancy and further examined outcomes at 3 time frames: enrollment to 7 months gestation, 7 months gestation to delivery, and within the 
first postnatal month. This publication compared pregnant women exposed to sertraline versus SNRI monotherapy.

The evidence is insufficient to judge the comparative risk of harms from sertraline only versus SNRI monotherapy (venlafaxine or desvenlafaxine) for maternal outcomes (convulsive seizures, nonconvulsive seizures, or spontaneous abortion or still birth) or child outcomes (congenital anomaly or birth weight) among women with epilepsy (Table B-76).

Table B-76. Strength of evidence for comparative harms: Sertraline monotherapy versus SNRI monotherapy (venlafaxine or desvenlafaxine)

\begin{tabular}{|c|c|c|c|c|c|c|c|}
\hline Population & $\begin{array}{l}\text { Intervention } \\
\text { and } \\
\text { Comparator }\end{array}$ & Outcome & $\begin{array}{l}\text { Incidence or } \\
\text { Mean Effect } \\
\text { by Arm }\end{array}$ & Results & $\begin{array}{l}\text { Study } \\
\text { Design } \\
\text { and } \\
\text { Sample } \\
\text { Size } \\
\end{array}$ & $\begin{array}{l}\text { Factors That Affect } \\
\text { the Strength of } \\
\text { Evidence }\end{array}$ & $\begin{array}{l}\text { Overall } \\
\text { Evidence } \\
\text { Strength } \\
\text { (Direction } \\
\text { of Effect) } \\
\end{array}$ \\
\hline $\begin{array}{l}\text { Women with } \\
\text { epilepsy and } \\
\text { depression, } \\
\text { anxiety or } \\
\text { bipolar } \\
\text { disorder }\end{array}$ & $\begin{array}{l}\text { Sertraline only } \\
\text { vs. SNRI } \\
\text { monotherapy } \\
\text { (venlafaxine or } \\
\text { desvenlafaxine) }\end{array}$ & $\begin{array}{l}\text { Convulsive } \\
\text { seizures: } \\
\text { From enrollment } \\
\text { to } 7 \text { months } \\
\text { gestation } \\
\text { From } 7 \text { months } \\
\text { gestation to } \\
\text { delivery }\end{array}$ & $\begin{array}{l}2 / 25(8 \%) \text { vs. } \\
0 / 13(0 \%)^{107} \\
1 / 25(4 \%) \text { vs. } \\
0 / 13(0 \%)^{107}\end{array}$ & $\begin{array}{l}\text { RD: } 0.08(95 \% \\
C I,-0.07 \text { to } \\
0.23)^{107} \\
\text { RD, } 0.04(95 \% \\
C l,-0.09 \text { to } \\
0.17)^{107}\end{array}$ & $\begin{array}{l}1 \\
\text { cohort, } \\
n=38^{107}\end{array}$ & $\begin{array}{l}\text { High study limitations } \\
\text { (high risk of bias }{ }^{107} \text { ), } \\
\text { seriously imprecise } \\
\text { (few events, wide } \\
\text { Cls), consistency } \\
\text { unknown }\end{array}$ & Insufficient \\
\hline $\begin{array}{l}\text { Women with } \\
\text { epilepsy and } \\
\text { depression, } \\
\text { anxiety or } \\
\text { bipolar } \\
\text { disorder }\end{array}$ & $\begin{array}{l}\text { Sertraline only } \\
\text { vs. SNRI } \\
\text { monotherapy } \\
\text { (venlafaxine or } \\
\text { desvenlafaxine) }\end{array}$ & $\begin{array}{l}\text { Nonconvulsive } \\
\text { seizures: } \\
\text { From enrollment } \\
\text { to } 7 \text { months } \\
\text { gestation } \\
\text { From } 7 \text { months } \\
\text { gestation to } \\
\text { delivery }\end{array}$ & $\begin{array}{l}3 / 25(12 \%) \\
\text { vs. } 1 / 13 \\
(8 \%)^{107} \\
1 / 25(4 \%) \text { vs. } \\
0 / 13(0 \%)^{107}\end{array}$ & $\begin{array}{l}\text { RR, } 1.56(95 \% \\
C l, 0.18 \text { to } \\
13.55)^{107} \\
\text { RD, } 0.04(95 \% \\
C l,-0.09 \text { to } \\
0.17)^{107}\end{array}$ & $\begin{array}{l}1 \\
\text { cohort, } \\
n=38^{107}\end{array}$ & $\begin{array}{l}\text { High study limitations } \\
\text { (high risk of bias }{ }^{107} \text { ), } \\
\text { seriously imprecise } \\
\text { (few events, wide } \\
\text { Cls), consistency } \\
\text { unknown }\end{array}$ & Insufficient \\
\hline $\begin{array}{l}\text { Women with } \\
\text { epilepsy and } \\
\text { depression, } \\
\text { anxiety or } \\
\text { bipolar } \\
\text { disorder } \\
\end{array}$ & $\begin{array}{l}\text { Sertraline only } \\
\text { vs. SNRI } \\
\text { monotherapy } \\
\text { (venlafaxine or } \\
\text { desvenlafaxine) }\end{array}$ & $\begin{array}{l}\text { Spontaneous } \\
\text { abortion }\end{array}$ & $\begin{array}{l}\text { 0/25 (0\%) vs. } \\
0 / 13(0 \%)^{107}\end{array}$ & $\begin{array}{l}\text { RR not } \\
\text { calculable: no } \\
\text { events }\end{array}$ & $\begin{array}{l}1 \\
\text { cohort, } \\
n=38^{107}\end{array}$ & $\begin{array}{l}\text { High study limitations } \\
\text { (high risk of bias }{ }^{107} \text { ), } \\
\text { likely imprecise (no } \\
\text { events, small Ns), } \\
\text { consistency unknown }\end{array}$ & Insufficient \\
\hline $\begin{array}{l}\text { Women with } \\
\text { epilepsy and } \\
\text { depression, } \\
\text { anxiety or } \\
\text { bipolar } \\
\text { disorder } \\
\end{array}$ & $\begin{array}{l}\text { Sertraline only } \\
\text { vs. SNRI } \\
\text { monotherapy } \\
\text { (venlafaxine or } \\
\text { desvenlafaxine) }\end{array}$ & Still birth & $\begin{array}{l}0 / 25(0 \%) \text { vs. } \\
1 / 13(8 \%)^{107}\end{array}$ & $\begin{array}{l}\text { RD, }-0.08 \\
(95 \% \mathrm{Cl},-0.24 \\
\text { to } 0.09)^{107}\end{array}$ & $\begin{array}{l}1 \\
\text { cohort, } \\
n=38^{107}\end{array}$ & $\begin{array}{l}\text { High study limitations } \\
\text { (high risk of bias }{ }^{107} \text { ), } \\
\text { likely imprecise (no } \\
\text { events, small Ns), } \\
\text { consistency unknown }\end{array}$ & Insufficient \\
\hline $\begin{array}{l}\text { Women with } \\
\text { epilepsy and } \\
\text { depression, } \\
\text { anxiety or } \\
\text { bipolar } \\
\text { disorder }\end{array}$ & $\begin{array}{l}\text { Sertraline only } \\
\text { vs. SNRI } \\
\text { monotherapy } \\
\text { (venlafaxine or } \\
\text { desvenlafaxine) }\end{array}$ & Birth weight & $\begin{array}{l}\text { Median } \pm \\
\text { IQR: } \\
3,350 \pm 808 \text { vs. } \\
3,087 \pm 286^{107}\end{array}$ & $\begin{array}{l}\text { Difference not } \\
\text { calculable }\end{array}$ & $\begin{array}{l}1 \\
\text { cohort, } \\
n=45^{107}\end{array}$ & $\begin{array}{l}\text { High study limitations } \\
\text { (high risk of bias }{ }^{107} \text { ), } \\
\text { likely imprecise (small } \\
\text { Ns), consistency } \\
\text { unknown }\end{array}$ & Insufficient \\
\hline
\end{tabular}

$\mathrm{CI}=$ confidence interval; $\mathrm{n}=$ number; $\mathrm{RD}=$ risk difference; $\mathrm{RR}=$ relative risk; $\mathrm{SNRI}=$ serotonin-norepinephrine reuptake inhibitor; vs. $=$ versus. 


\section{Sertraline Versus Bupropion}

\section{Overview}

- The evidence is insufficient to judge the comparative risk of harms of exposure to sertraline versus bupropion during pregnancy for child outcomes (congenital heart defect).

\section{Detailed Results}

One high risk-of-bias retrospective chart review of institutional medical records at Children's Hospital of New Orleans reported on the comparative effectiveness of sertraline versus bupropion for women with an in utero exposure of the fetus to SSRIs or SNRIs. ${ }^{141}$

The evidence is insufficient to judge the comparative risk of harms from sertraline only versus bupropion for child outcomes (congenital heart defects) (Table B-77).

Table B-77. Strength of evidence for comparative harms outcomes: Sertraline versus bupropion

\begin{tabular}{|c|c|c|c|c|c|c|c|}
\hline Population & $\begin{array}{l}\text { Intervention } \\
\text { and } \\
\text { Comparator }\end{array}$ & Outcome & $\begin{array}{l}\text { Incidence } \\
\text { or Mean } \\
\text { Effect by } \\
\text { Arm }\end{array}$ & Results & $\begin{array}{l}\text { Study } \\
\text { Design } \\
\text { and } \\
\text { Sample } \\
\text { Size }\end{array}$ & $\begin{array}{l}\text { Factors That } \\
\text { Affect the } \\
\text { Strength of } \\
\text { Evidence }\end{array}$ & $\begin{array}{l}\text { Overall } \\
\text { Evidence } \\
\text { Strength } \\
\text { (Direction } \\
\text { of Effect) }\end{array}$ \\
\hline $\begin{array}{l}\text { Women who } \\
\text { underwent fetal } \\
\text { echocardiography } \\
\text { because of an in } \\
\text { utero exposure to } \\
\text { either SSRIs or } \\
\text { SNRIs }\end{array}$ & $\begin{array}{l}\text { Sertraline vs. } \\
\text { duloxetine }\end{array}$ & $\begin{array}{l}\text { Congenital } \\
\text { heart } \\
\text { disease }\end{array}$ & $\begin{array}{l}0 / 7(0 \%) \\
\text { vs. } 0 / 1 \\
(0 \%)^{141}\end{array}$ & $\begin{array}{l}\text { RR not } \\
\text { calculable }\end{array}$ & $\begin{array}{l}1 \text { cohort } \\
(n=8)^{141}\end{array}$ & $\begin{array}{l}\text { High study } \\
\text { limitations } \\
\text { (high risk of } \\
\text { bias }^{141} \text { ), likely } \\
\text { imprecise (no } \\
\text { events, small } \\
\text { Ns), } \\
\text { consistency } \\
\text { unknown }\end{array}$ & Insufficient \\
\hline
\end{tabular}

$\mathrm{CI}=$ confidence interval; $\mathrm{n}=$ number; $\mathrm{RR}=$ relative risk; $\mathrm{SNRI}=$ serotonin-norepinephrine reuptake inhibitor; $\mathrm{SSRI}=$ selective serotonin reuptake inhibitor; vs. $=$ versus.

\section{Sertraline Versus Nortriptyline}

\section{Overview}

- One high risk-of-bias trial did not report any differences between sertraline and nortriptyline exposure during pregnancy for any adverse events (insufficient).

\section{Detailed Results}

One RCT (rated high risk of bias) reported on the comparative effectiveness of sertraline versus nortriptyline for women with postpartum depression. ${ }^{36}$ Some women may also have had chronic depression: the study began including women with chronic depression after the trial started. Women were treated with a fixed dose strategy of $25 \mathrm{mg} / \mathrm{d}$ of sertraline or $10 \mathrm{mg} / \mathrm{d}$ of nortriptyline initially. The dose was escalated to $50 \mathrm{mg} / \mathrm{d}$ of sertraline or $25 \mathrm{mg} / \mathrm{d}$ of nortriptyline after 2 days and then gradually increased up to a maximum of $200 \mathrm{mg} / \mathrm{d}$ of sertraline and 150 $\mathrm{mg} / \mathrm{d}$ nortriptyline if response or side effects did not prohibit further escalation. Although the study reported no differences in adverse events, it was not powered to test for equivalence. The study had higher attrition in the sertraline arm than in the nortriptyline arm (23/55 [41.8\%] vs. $13 / 54$ [24.1\%]). The authors noted that the proportion of women withdrawing by personal choice differed significantly between groups $(20 \%$ vs. $6 \%, \mathrm{p}=0.03)$, but other reasons for withdrawal, 
like side effects, did not differ between groups. We graded the evidence as insufficient as a result of imprecision and potential for bias due to attrition (Table B-78).

Table B-78. Strength of evidence for comparative harms outcomes: Sertraline versus nortriptyline

\begin{tabular}{|c|c|c|c|c|c|c|c|}
\hline Population & $\begin{array}{l}\text { Intervention } \\
\text { and } \\
\text { Comparator }\end{array}$ & Outcome & $\begin{array}{l}\text { Incidence } \\
\text { or Mean } \\
\text { Effect by } \\
\text { Arm }\end{array}$ & Results & $\begin{array}{l}\text { Study } \\
\text { Design } \\
\text { and } \\
\text { Sample } \\
\text { Size }\end{array}$ & $\begin{array}{l}\text { Factors That } \\
\text { Affect the } \\
\text { Strength of } \\
\text { Evidence }\end{array}$ & $\begin{array}{l}\text { Overall } \\
\text { Evidence } \\
\text { Strength } \\
\text { (Direction of } \\
\text { Effect) }\end{array}$ \\
\hline $\begin{array}{l}\text { Women with } \\
\text { postpartum } \\
\text { depression (with } \\
\text { or without } \\
\text { chronic } \\
\text { depression) }\end{array}$ & $\begin{array}{l}\text { Sertraline vs. } \\
\text { nortriptyline }\end{array}$ & $\begin{array}{l}\text { Asberg Side } \\
\text { Effects Rating } \\
\text { Scale (any } \\
\text { adverse } \\
\text { events) }\end{array}$ & $\begin{array}{l}0 / 55(0 \%) \\
\text { vs. } 0 / 54 \\
(0 \%)^{36}\end{array}$ & $\begin{array}{l}\text { No events } \\
\text { in either } \\
\mathrm{arm}^{36}\end{array}$ & $\begin{array}{l}1 \text { trial, } \\
\mathrm{N}=108^{36}\end{array}$ & $\begin{array}{l}\text { High study } \\
\text { limitations (high } \\
\text { risk of bias, }{ }^{36} \\
\text { imprecise, } \\
\text { consistency } \\
\text { unknown }\end{array}$ & Insufficient \\
\hline $\begin{array}{l}\text { Women with } \\
\text { postpartum } \\
\text { depression (with } \\
\text { or without } \\
\text { chronic } \\
\text { depression) }\end{array}$ & $\begin{array}{l}\text { Sertraline vs. } \\
\text { nortriptyline }\end{array}$ & $\begin{array}{l}\text { Overall study } \\
\text { withdrawal }\end{array}$ & $\begin{array}{l}23 / 55 \\
(41.8 \%) \text { vs. } \\
13 / 54 \\
(24.1 \%)^{36}\end{array}$ & $\begin{array}{l}\mathrm{RR}, 1.73 \\
(95 \% \mathrm{Cl}, \\
0.99 \text { to } \\
3.06)^{36}\end{array}$ & $\begin{array}{l}1 \text { trial, } \\
\mathrm{N}=108^{36}\end{array}$ & $\begin{array}{l}\text { High study } \\
\text { limitations (high } \\
\text { risk of bias, }{ }^{36} \\
\text { imprecise, } \\
\text { consistency } \\
\text { unknown }\end{array}$ & Insufficient \\
\hline
\end{tabular}

$\mathrm{CI}=$ confidence interval; $\mathrm{N}=$ number; $\mathrm{RR}=$ relative risk; vs. $=$ versus.

\section{Duloxetine Versus SSRIs}

\section{Overview}

- The evidence is insufficient to judge the comparative risk of harms from duloxetine versus SSRIs during pregnancy for maternal (preeclampsia and postpartum hemorrhage) and child outcomes (preterm birth, being small for gestational age, cardiovascular anomalies, and major anomalies).

\section{Detailed Results}

One publication, with some risk-of-bias concerns reported on the comparative harms of second trimester use of duloxetine versus SSRIs as a class, from a cohort study of women in the Medicaid Analytic eXtract for 2004-2013. ${ }^{98}$

Maternal outcomes include preeclampsia and postpartum hemorrhage; child outcomes include preterm birth, being small for gestational age, cardiovascular anomalies, and major anomalies. Each of these outcomes with the exception of cardiovascular anomalies was evaluated in a single study; all with the exception of one study on cardiovascular anomalies showed no association. The strength of evidence for all outcomes was rated as insufficient (Table B-79). 
Table B-79. Strength of evidence for comparative harms: Duloxetine versus SSRIs

\begin{tabular}{|c|c|c|c|c|c|c|c|}
\hline Population & $\begin{array}{l}\text { Intervention } \\
\text { and } \\
\text { Comparator }\end{array}$ & Outcome & $\begin{array}{l}\text { Incidence or } \\
\text { Mean Effect by } \\
\text { Arm }\end{array}$ & Results & $\begin{array}{l}\text { Study } \\
\text { Design and } \\
\text { Sample Size }\end{array}$ & $\begin{array}{l}\text { Factors That } \\
\text { Affect the } \\
\text { Strength of } \\
\text { Evidence }\end{array}$ & $\begin{array}{l}\text { Overall } \\
\text { Evidence } \\
\text { Strength } \\
\text { (Direction } \\
\text { of Effect) } \\
\end{array}$ \\
\hline $\begin{array}{l}\text { Women } \\
\text { exposed to } \\
\text { SSRIs vs. } \\
\text { duloxetine } \\
\text { during } \\
\text { pregnancy }\end{array}$ & $\begin{array}{l}\text { Duloxetine } \\
\text { vs. SSRIs }\end{array}$ & Preeclampsia & $\begin{array}{l}\text { Early exposure: } \\
119 / 2,104(5.66 \%) \\
\text { vs. } 2,540 / 70,926 \\
(3.58 \%)^{98} \\
\\
\text { Late exposure: } \\
42 / 662(6.34 \%) \\
\text { vs. } 1,835 / 4,8672 \\
(3.77 \%)^{98} \\
\end{array}$ & $\begin{array}{l}\text { ARR for early } \\
\text { exposure: } 1.16 \\
(95 \% \mathrm{Cl}, 0.96 \text { to } \\
1.40)^{98} \\
\text { ARR for late } \\
\text { exposure: }(1.05 \text {, } \\
95 \% \mathrm{Cl}, 0.77 \text { to } \\
1.44)^{98}\end{array}$ & $\begin{array}{l}1 \text { cohort } \\
(n=14,391)^{98}\end{array}$ & $\begin{array}{l}\text { Moderate } \\
\text { study } \\
\text { limitations, } \\
\text { imprecise } \\
\text { (wide Cls), } \\
\text { consistency } \\
\text { unknown }\end{array}$ & Insufficient \\
\hline $\begin{array}{l}\text { Women } \\
\text { exposed to } \\
\text { SSRIs vs. } \\
\text { duloxetine } \\
\text { during } \\
\text { pregnancy }\end{array}$ & $\begin{array}{l}\text { Duloxetine } \\
\text { vs. SSRIs }\end{array}$ & $\begin{array}{l}\text { Postpartum } \\
\text { hemorrhage }\end{array}$ & $\begin{array}{l}33 / 869(3.80 \%) \\
\text { vs. } 1879 / 65,303 \\
(2.88 \%)^{98}\end{array}$ & $\begin{array}{l}\text { ARR, } 1.48(95 \% \\
\mathrm{Cl}, 1.03 \text { to } 2.12)^{98}\end{array}$ & $\begin{array}{l}1 \text { cohort } \\
(n=66,172)^{98}\end{array}$ & $\begin{array}{l}\text { Moderate } \\
\text { study } \\
\text { limitations, } \\
\text { imprecise } \\
\text { (wide Cls), } \\
\text { consistency } \\
\text { unknown }\end{array}$ & Insufficient \\
\hline $\begin{array}{l}\text { Women } \\
\text { exposed to } \\
\text { SSRIs vs. } \\
\text { duloxetine } \\
\text { during } \\
\text { pregnancy }\end{array}$ & $\begin{array}{l}\text { Duloxetine } \\
\text { vs. SSRIs }\end{array}$ & Preterm birth & $\begin{array}{l}\text { Early exposure: } \\
282 / 1,989 \\
(14.18 \%) \text { vs. } \\
9013 / 67,222 \\
(13.41 \%)^{98} \\
\\
\text { Late exposure: } \\
128 / 621(20.61 \%) \\
\text { vs. } 7,685 / 46,098 \\
(16.67 \%)^{98} \\
\end{array}$ & $\begin{array}{l}\text { ARR for early } \\
\text { exposure: } 0.95 \\
(95 \% \mathrm{Cl}, 0.84 \text { to } \\
1.06)^{98} \\
\text { ARR for late } \\
\text { exposure: }(1.02 \text {, } \\
95 \% \mathrm{Cl}, 0.87 \text { to } \\
1.21)^{98}\end{array}$ & $\begin{array}{l}1 \text { cohort } \\
\left(n=115,930^{98}\right.\end{array}$ & $\begin{array}{l}\text { Moderate } \\
\text { study } \\
\text { limitations, } \\
\text { imprecise } \\
\text { (wide Cls), } \\
\text { consistency } \\
\text { unknown }\end{array}$ & Insufficient \\
\hline $\begin{array}{l}\text { Women } \\
\text { exposed to } \\
\text { SSRIs vs. } \\
\text { duloxetine } \\
\text { during } \\
\text { pregnancy }\end{array}$ & $\begin{array}{l}\text { Duloxetine } \\
\text { vs. SSRIs }\end{array}$ & $\begin{array}{l}\text { Small for } \\
\text { gestational } \\
\text { age }\end{array}$ & $\begin{array}{l}\text { Early exposure: } \\
60 / 1,989(3.02 \%) \\
\text { vs. } 1697 / 67,222 \\
(2.52 \%)^{98} \\
\text { Late exposure: } \\
18 / 621(2.90 \%) \\
\text { vs. } 1189 / 46,098 \\
(2.58 \%)^{98} \\
\end{array}$ & $\begin{array}{l}\text { ARR for early } \\
\text { exposure: } 1.26 \\
(95 \% \mathrm{Cl}, 0.97 \text { to } \\
1.64)^{98} \\
\text { ARR for late } \\
\text { exposure: }(1.17 \text {, } \\
95 \% \mathrm{Cl}, 0.73 \text { to } \\
1.88)^{98}\end{array}$ & $\begin{array}{l}1 \text { cohort } \\
(n=115,930)^{98}\end{array}$ & $\begin{array}{l}\text { Moderate } \\
\text { study } \\
\text { limitations, } \\
\text { imprecise } \\
\text { (wide Cls), } \\
\text { consistency } \\
\text { unknown }\end{array}$ & $\overline{\text { Insufficient }}$ \\
\hline $\begin{array}{l}\text { Women } \\
\text { exposed to } \\
\text { SSRIs vs. } \\
\text { duloxetine } \\
\text { during } \\
\text { pregnancy }\end{array}$ & $\begin{array}{l}\text { Duloxetine } \\
\text { vs. SSRIs }\end{array}$ & $\begin{array}{l}\text { Any congenital } \\
\text { malformations }\end{array}$ & $\begin{array}{l}99 / 1966(5.04 \%) \\
\text { vs. } 2,192 / 52,018 \\
(4.21 \%)^{98}\end{array}$ & $\begin{array}{l}\text { ARR, } 1.09(95 \% \\
\mathrm{Cl}, 0.89 \text { to } 1.35^{98}\end{array}$ & $\begin{array}{l}1 \text { cohort } \\
(n=53,984)^{98}\end{array}$ & $\begin{array}{l}\text { Moderate } \\
\text { study } \\
\text { limitations, } \\
\text { imprecise } \\
\text { (wide Cls), } \\
\text { consistency } \\
\text { unknown }\end{array}$ & Insufficient \\
\hline $\begin{array}{l}\text { Women } \\
\text { exposed to } \\
\text { SSRIs vs. } \\
\text { duloxetine } \\
\text { during } \\
\text { pregnancy }\end{array}$ & $\begin{array}{l}\text { Duloxetine } \\
\text { vs. SSRIs }\end{array}$ & $\begin{array}{l}\text { Cardiovascular } \\
\text { anomalies }\end{array}$ & $\begin{array}{l}46 / 1,966(2.34 \%) \\
\text { vs. } \\
810 / 52,018 \\
(1.56 \%)^{98}\end{array}$ & $\begin{array}{l}\text { ARR, } 1.27(95 \% \\
\mathrm{Cl}, 0.93 \text { to } 1.74^{98}\end{array}$ & $\begin{array}{l}1 \text { cohort } \\
(n=53,984)^{98}\end{array}$ & $\begin{array}{l}\text { Moderate } \\
\text { study } \\
\text { limitations, } \\
\text { imprecise } \\
\text { (wide Cls), } \\
\text { consistency } \\
\text { unknown }\end{array}$ & Insufficient \\
\hline
\end{tabular}

$\overline{\mathrm{ARR}}=$ adjusted relative risk; $\mathrm{CI}=$ confidence interval; $\mathrm{n}=$ number; $\mathrm{SNRI}=$ serotonin-norepinephrine reuptake inhibitor; $\mathrm{SSRI}=$ selective serotonin reuptake inhibitor; vs. = versus. 


\section{Duloxetine Versus Venlafaxine}

\section{Overview}

- The evidence is insufficient to judge the comparative risk of harms from duloxetine versus venlafaxine during pregnancy for maternal (preeclampsia and postpartum hemorrhage) and child outcomes (preterm birth, being small for gestational age, cardiovascular anomalies, and major anomalies).

\section{Detailed Results}

Two publications reported on the comparative harms of duloxetine versus venlafaxine. ${ }^{98,141}$ One is a cohort study of women in the Medicaid Analytic eXtract for 2004-2013. ${ }^{98}$ The second is a cohort of women who had undergone a fetal echocardiogram because of in utero exposure of the fetus to SSRIs or SNRIs. ${ }^{141}$ One study was rated as high risk of bias. ${ }^{141}$

Maternal outcomes include preeclampsia and postpartum hemorrhage; child outcomes include preterm birth, being small for gestational age, cardiovascular anomalies, and major anomalies. Each of these outcomes with the exception of cardiovascular anomalies was evaluated in a single study; all with the exception of one study on cardiovascular anomalies showed no association. The studies on cardiovascular anomalies were inconsistent in direction of the association. The strength of evidence for all outcomes was rated as insufficient (Table B-80).

Table B-80. Strength of evidence for comparative harms: Duloxetine versus venlafaxine

\begin{tabular}{|c|c|c|c|c|c|c|c|}
\hline Population & $\begin{array}{l}\text { Intervention } \\
\text { and } \\
\text { Comparator }\end{array}$ & Outcome & $\begin{array}{l}\text { Incidence or } \\
\text { Mean Effect by } \\
\text { Arm }\end{array}$ & Results & $\begin{array}{l}\text { Study } \\
\text { Design and } \\
\text { Sample Size }\end{array}$ & $\begin{array}{l}\text { Factors That } \\
\text { Affect the } \\
\text { Strength of } \\
\text { Evidence }\end{array}$ & $\begin{array}{l}\text { Overall } \\
\text { Evidence } \\
\text { Strength } \\
\text { (Direction } \\
\text { of Effect) }\end{array}$ \\
\hline $\begin{array}{l}\text { Women } \\
\text { exposed to } \\
\text { venlafaxine } \\
\text { vs. } \\
\text { duloxetine } \\
\text { during } \\
\text { pregnancy }\end{array}$ & $\begin{array}{l}\text { Duloxetine } \\
\text { vs. } \\
\text { venlafaxine }\end{array}$ & Preeclampsia & $\begin{array}{l}\text { Early exposure: } \\
161 / 2,927 \\
(5.50 \%) \text { vs. } \\
357 / 7,642 \\
(4.67 \%)^{98} \\
\\
\text { Late exposure: } \\
55 / 879(6.26 \%) \\
\text { vs. } 185 / 2,943 \\
(6.29 \%)^{98}\end{array}$ & $\begin{array}{l}\text { ARR for early } \\
\text { exposure: } 0.96 \\
(95 \% \mathrm{Cl}, 0.8 \text { to } \\
1.16)^{98} \\
\text { ARR for late } \\
\text { exposure: } 0.8 \\
(95 \% \mathrm{Cl}, 0.59 \text { to } \\
1.08)^{98}\end{array}$ & $\begin{array}{l}1 \text { cohort } \\
(n=14,391)^{98}\end{array}$ & $\begin{array}{l}\text { Moderate study } \\
\text { limitations, } \\
\text { imprecise (wide } \\
\text { Cls), } \\
\text { consistency } \\
\text { unknown }\end{array}$ & Insufficient \\
\hline $\begin{array}{l}\text { Women } \\
\text { exposed to } \\
\text { venlafaxine } \\
\text { vs. } \\
\text { duloxetine } \\
\text { during } \\
\text { pregnancy }\end{array}$ & $\begin{array}{l}\text { Duloxetine } \\
\text { vs. } \\
\text { venlafaxine }\end{array}$ & $\begin{array}{l}\text { Postpartum } \\
\text { hemorrhage }\end{array}$ & $\begin{array}{l}34 / 948(3.59 \%) \\
\text { vs. } 135 / 3,573 \\
(3.78 \%)^{98}\end{array}$ & $\begin{array}{l}\text { ARR: } 1.04(95 \% \\
\mathrm{Cl}, 0.69 \text { to } 1.56)^{98}\end{array}$ & $\begin{array}{l}1 \text { cohort } \\
(n=4,521)\end{array}$ & $\begin{array}{l}\text { Moderate study } \\
\text { limitations, } \\
\text { imprecise (wide } \\
\text { Cls), } \\
\text { consistency } \\
\text { unknown }\end{array}$ & Insufficient \\
\hline $\begin{array}{l}\text { Women } \\
\text { exposed to } \\
\text { venlafaxine } \\
\text { vs. } \\
\text { duloxetine } \\
\text { during } \\
\text { pregnancy }\end{array}$ & $\begin{array}{l}\text { Duloxetine } \\
\text { vs. } \\
\text { venlafaxine }\end{array}$ & Preterm birth & $\begin{array}{l}\text { Early exposure: } \\
402 / 2,779 \\
(14.47 \%) \text { vs. } \\
1,008 / 7,258 \\
(13.89 \%)^{98} \\
\\
\text { Late exposure: } \\
167 / 828 \\
(20.17 \%) \text { vs. } \\
559 / 2,770 \\
(20.18 \%)^{98}\end{array}$ & $\begin{array}{l}\text { ARR for early } \\
\text { exposure: } 0.94 \\
(95 \% \mathrm{Cl}, 0.84 \text { to } \\
1.06)^{98} \\
\text { ARR for late } \\
\text { exposure: } 0.88 \\
(95 \% \mathrm{Cl}, 0.75 \text { to } \\
1.04)^{98}\end{array}$ & $\begin{array}{l}1 \text { cohort } \\
(n=13,635)\end{array}$ & $\begin{array}{l}\text { Moderate study } \\
\text { limitations, } \\
\text { imprecise (wide } \\
\text { Cls), } \\
\text { consistency } \\
\text { unknown }\end{array}$ & Insufficient \\
\hline
\end{tabular}




\begin{tabular}{|c|c|c|c|c|c|c|c|}
\hline Population & $\begin{array}{l}\text { Intervention } \\
\text { and } \\
\text { Comparator }\end{array}$ & Outcome & $\begin{array}{l}\text { Incidence or } \\
\text { Mean Effect by } \\
\text { Arm }\end{array}$ & Results & $\begin{array}{l}\text { Study } \\
\text { Design and } \\
\text { Sample Size }\end{array}$ & $\begin{array}{l}\text { Factors That } \\
\text { Affect the } \\
\text { Strength of } \\
\text { Evidence }\end{array}$ & $\begin{array}{l}\text { Overall } \\
\text { Evidence } \\
\text { Strength } \\
\text { (Direction } \\
\text { of Effect) }\end{array}$ \\
\hline $\begin{array}{l}\text { Women } \\
\text { exposed to } \\
\text { venlafaxine } \\
\text { vs. } \\
\text { duloxetine } \\
\text { during } \\
\text { pregnancy }\end{array}$ & $\begin{array}{l}\text { Duloxetine } \\
\text { vs. } \\
\text { venlafaxine }\end{array}$ & $\begin{array}{l}\text { Small for } \\
\text { gestational age }\end{array}$ & $\begin{array}{l}\text { Early exposure: } \\
79 / 2,779 \\
(2.84 \%) \text { vs. } \\
166 / 7,258 \\
(2.29 \%)^{98} \\
\\
\text { Late exposure: } \\
26 / 828(3.14 \%) \\
\text { vs. } 61 / 2,770 \\
(2.20 \%)^{98}\end{array}$ & $\begin{array}{l}\text { ARR for early } \\
\text { exposure: } 1.18 \\
(95 \% \mathrm{Cl}, 0.90 \text { to } \\
1.56)^{98} \\
\text { ARR for late } \\
\text { exposure: } 1.32 \\
(95 \% \mathrm{Cl}, 0.83 \text { to } \\
2.09)^{98}\end{array}$ & $\begin{array}{l}1 \text { cohort } \\
(n=13,635)^{98}\end{array}$ & $\begin{array}{l}\text { Moderate study } \\
\text { limitations, } \\
\text { imprecise (wide } \\
\text { Cls), } \\
\text { consistency } \\
\text { unknown }\end{array}$ & Insufficient \\
\hline $\begin{array}{l}\text { Women } \\
\text { exposed to } \\
\text { venlafaxine } \\
\text { vs. } \\
\text { duloxetine } \\
\text { during } \\
\text { pregnancy }\end{array}$ & $\begin{array}{l}\text { Duloxetine } \\
\text { vs. } \\
\text { venlafaxine }\end{array}$ & $\begin{array}{l}\text { Any congenital } \\
\text { malformations }\end{array}$ & $\begin{array}{l}127 / 2,467 \\
(5.15 \%) \text { vs. } \\
270 / 6,369 \\
(4.24 \%)^{98}\end{array}$ & $\begin{array}{l}\text { ARR: } 1.09(95 \% \\
\mathrm{Cl}, 0.88 \text { to } 1.36)^{98}\end{array}$ & $\begin{array}{l}1 \text { cohort } \\
(n=8,836)^{98}\end{array}$ & $\begin{array}{l}\text { Moderate study } \\
\text { limitations, } \\
\text { imprecise (wide } \\
\text { Cls), } \\
\text { consistency } \\
\text { unknown }\end{array}$ & Insufficient \\
\hline $\begin{array}{l}\text { Women } \\
\text { exposed to } \\
\text { venlafaxine } \\
\text { vs. } \\
\text { duloxetine } \\
\text { during } \\
\text { pregnancy }\end{array}$ & $\begin{array}{l}\text { Duloxetine } \\
\text { vs. } \\
\text { venlafaxine }\end{array}$ & $\begin{array}{l}\text { Congenital heart } \\
\text { disease/cardiova } \\
\text { scular anomalies }\end{array}$ & $\begin{array}{l}0 / 1(0 \%) \text { vs. } 2 / 2 \\
(100 \%)^{141}{ }^{141} \\
58 / 2,467(2.35 \%) \\
\text { vs. } 96 / 6,369 \\
(1.51 \%)^{152}\end{array}$ & $\begin{array}{l}\text { RR: } 0.30(95 \% \mathrm{Cl} \text {, } \\
0.30 \text { to } 3.49): 141 \\
\text { ARR: } 1.17(95 \% \\
\mathrm{Cl}, 0.84 \text { to } 1.63)^{98}\end{array}$ & $\begin{array}{l}2 \text { cohorts } \\
(n=8,839)\end{array}$ & $\begin{array}{l}\text { Moderate study } \\
\text { limitations (high } \\
\text { risk of bias }{ }^{141} \text { ), } \\
\text { imprecise, } \\
\text { inconsistent }\end{array}$ & Insufficient \\
\hline
\end{tabular}

$\mathrm{ARR}=$ adjusted relative risk; $\mathrm{CI}=$ confidence interval; $\mathrm{n}=$ number; $\mathrm{RR}=$ relative risk; $\mathrm{vs} .=$ versus.

\section{Venlafaxine Versus SSRIs}

\section{Overview}

- The evidence is insufficient to judge the comparative risk of harms from venlafaxine versus SSRIs during pregnancy for maternal spontaneous abortion and child outcomes (preterm delivery, gestational age, birthweight, small for gestational age, any congenital malformations, and any major congenital malformations).

\section{Detailed Results}

Three publications reported on the comparative harms of second trimester use of venlafaxine versus SSRIs as a class. One was a cohort study of women participating in the Motherisk Program, providing pregnancy counseling in centers in selected sites in North America, Europe, and Brazil. ${ }^{135}$ The second was a case-control study using the Canadian Quebec Pregnancy Registry. ${ }^{83}$ Both studies were rated as high risk of bias. A third publication, rated as having some concerns for risk of bias, reported on the comparative harms of venlafaxine versus SSRIs during pregnancy. The study cohort was drawn from surveillance data collected by the U.K. Teratology Information Service. ${ }^{144}$

Outcomes include differences in the risk of maternal spontaneous abortion, preterm delivery, child gestational age, birth weight, being small for gestational age, any congenital malformations, and major anomalies. Each of these outcomes, with the exception of spontaneous abortion, was evaluated in a single study and none showed an association. Two studies reported 
on spontaneous abortion and were consistent in not reporting an association between exposure to venlafaxine and spontaneous abortion when compared with SSRIs. However, wide CIs for both studies effects spanned appreciable benefit and appreciable harm, so we graded the evidence as imprecise and the overall evidence as insufficient. The strength of evidence for all outcomes was rated as insufficient (Table B-81).

Table B-81. Strength of evidence for comparative harms: Venlafaxine versus SSRIs

\begin{tabular}{|c|c|c|c|c|c|c|c|}
\hline Population & $\begin{array}{l}\text { Intervention } \\
\text { and } \\
\text { Comparator }\end{array}$ & Outcome & $\begin{array}{l}\text { Incidence or } \\
\text { Mean Effect } \\
\text { by Arm }\end{array}$ & Results & $\begin{array}{l}\text { Study } \\
\text { Design and } \\
\text { Sample } \\
\text { Size }\end{array}$ & $\begin{array}{l}\text { Factors That } \\
\text { Affect the } \\
\text { Strength of } \\
\text { Evidence }\end{array}$ & $\begin{array}{l}\text { Overall } \\
\text { Evidence } \\
\text { Strength } \\
\text { (Direction } \\
\text { of Effect) }\end{array}$ \\
\hline $\begin{array}{l}\text { Pregnant } \\
\text { women with } \\
\text { depression, } \\
\text { pregnant } \\
\text { women who } \\
\text { used SSRIs or } \\
\text { venlafaxine } \\
\text { during } \\
\text { pregnancy }\end{array}$ & $\begin{array}{l}\text { Venlafaxine or } \\
\text { SSRI, first } \\
\text { trimester } \\
\text { exposure }^{135}\end{array}$ & $\begin{array}{l}\text { Spontaneous } \\
\text { abortion }\end{array}$ & $\begin{array}{l}18 / 150(12 \%) \\
\text { vs. } 16 / 150 \\
(10.7 \%)^{135} \\
\\
46 / 281 \\
(16.37 \%) \text { vs. } \\
144 / 843 \\
(17.1 \%)^{144}\end{array}$ & $\begin{array}{l}\mathrm{RR}, 1.1(95 \% \\
\mathrm{Cl}, 0.60 \text { to } \\
2.12)^{135} \\
\mathrm{AOR}, 1.0(95 \% \\
\mathrm{Cl}, 0.655 \text { to } \\
1.53)^{144}\end{array}$ & $\begin{array}{l}2 \text { cohorts, } \\
\mathrm{n}=1,424^{135} \\
144\end{array}$ & $\begin{array}{l}\text { Moderate } \\
\text { study } \\
\text { limitations } \\
\text { (high risk of } \\
\text { bias }^{135} \text { ) } \\
\text { imprecise } \\
\text { (wide Cls), } \\
\text { consistent }\end{array}$ & Insufficient \\
\hline $\begin{array}{l}\text { Pregnant } \\
\text { women who } \\
\text { used SSRIs or } \\
\text { venlafaxine } \\
\text { during } \\
\text { pregnancy }\end{array}$ & $\begin{array}{l}\text { Venlafaxine } \\
\text { vs. SSRI } \\
\text { during first or } \\
\text { second } \\
\text { trimester }\end{array}$ & $\begin{array}{l}\text { Preterm } \\
\text { delivery }\end{array}$ & $\begin{array}{l}33 / 198(16.7 \%) \\
\text { vs. } 81 / 614 \\
(13.2 \%)^{144}\end{array}$ & $\begin{array}{l}\text { AOR, } 1.32 \\
(95 \% \mathrm{Cl}, 0.844 \\
\text { to } 2.06)^{144}\end{array}$ & $\begin{array}{l}1 \text { cohort, } \\
\mathrm{n}=812^{144}\end{array}$ & $\begin{array}{l}\text { Moderate } \\
\text { study } \\
\text { limitations } \\
\text { imprecise } \\
\text { (wide Cls), } \\
\text { inconsistency } \\
\text { unknown }\end{array}$ & Insufficient \\
\hline $\begin{array}{l}\text { Pregnant } \\
\text { women with } \\
\text { depression }\end{array}$ & $\begin{array}{l}\text { Venlafaxine or } \\
\text { SSRI, second } \\
\text { trimester } \\
\text { exposure }\end{array}$ & $\begin{array}{l}\text { Gestational } \\
\text { age at birth } \\
\text { (weeks) }\end{array}$ & $\begin{array}{l}\text { Mean (SD): } 39 \\
(2) \text { vs. } 38(2)^{135}\end{array}$ & NR & $\begin{array}{l}1 \text { cohort, } \\
n=300\end{array}$ & $\begin{array}{l}\text { High study } \\
\text { limitations } \\
\text { (high risk of } \\
\text { bias, }{ }^{135} \\
\text { imprecise) } \\
\text { wide Cls, } \\
\text { consistency } \\
\text { unknown }\end{array}$ & Insufficient \\
\hline $\begin{array}{l}\text { Pregnant } \\
\text { women with a } \\
\text { psychiatric } \\
\text { disorder }\end{array}$ & $\begin{array}{l}\text { Venlafaxine or } \\
\text { SSRI, second } \\
\text { trimester } \\
\text { exposure }\end{array}$ & $\begin{array}{l}\text { Birthweight } \\
\text { (g) }\end{array}$ & $\begin{array}{l}\text { Mean (SD): } \\
3,332(609) \text { vs. } \\
3,429(482)^{135}\end{array}$ & NR & $\begin{array}{l}1 \text { cohort, } \\
\mathrm{N}=300^{135}\end{array}$ & $\begin{array}{l}\text { High study } \\
\text { limitations } \\
\text { (high risk of } \\
\text { bias, } \\
\text { imprecise) } \\
\text { wide Cls, } \\
\text { consistency } \\
\text { unknown }\end{array}$ & Insufficient \\
\hline $\begin{array}{l}\text { Pregnant } \\
\text { women who } \\
\text { used SSRIs or } \\
\text { venlafaxine } \\
\text { during } \\
\text { pregnancy }\end{array}$ & $\begin{array}{l}\text { Venlafaxine } \\
\text { vs. SSRI }\end{array}$ & $\begin{array}{l}\text { Term infants } \\
\text { with low birth } \\
\text { weight }\end{array}$ & $\begin{array}{l}4 / 133(3.01 \%) \\
\text { vs. } 22 / 438 \\
(5.02 \%)^{144}\end{array}$ & $\begin{array}{l}\text { AOR, } 0.610 \\
(95 \% \mathrm{Cl}, 0.175 \\
\text { to } 1.64)^{144}\end{array}$ & $\begin{array}{l}1 \text { cohort, } \\
\mathrm{n}=600^{144}\end{array}$ & $\begin{array}{l}\text { Moderate } \\
\text { study } \\
\text { limitations } \\
\text { imprecise } \\
\text { (wide Cls), } \\
\text { inconsistency } \\
\text { unknown }\end{array}$ & Insufficient \\
\hline
\end{tabular}




\begin{tabular}{|c|c|c|c|c|c|c|c|}
\hline Population & $\begin{array}{l}\text { Intervention } \\
\text { and } \\
\text { Comparator }\end{array}$ & Outcome & $\begin{array}{l}\text { Incidence or } \\
\text { Mean Effect } \\
\text { by Arm }\end{array}$ & Results & $\begin{array}{l}\text { Study } \\
\text { Design and } \\
\text { Sample } \\
\text { Size }\end{array}$ & $\begin{array}{l}\text { Factors That } \\
\text { Affect the } \\
\text { Strength of } \\
\text { Evidence }\end{array}$ & $\begin{array}{l}\text { Overall } \\
\text { Evidence } \\
\text { Strength } \\
\text { (Direction } \\
\text { of Effect) }\end{array}$ \\
\hline $\begin{array}{l}\text { Pregnant } \\
\text { women with a } \\
\text { psychiatric } \\
\text { disorder } \\
\text { (second } \\
\text { trimester) or } \\
\text { exposed to } \\
\text { venlafaxine or } \\
\text { SSRIs (during } \\
\text { pregnancy) }\end{array}$ & $\begin{array}{l}\text { Venlafaxine } \\
\text { vs. SSRI } \\
\text { during second } \\
\text { trimester }\end{array}$ & $\begin{array}{l}\text { SGA }\left(<10^{\text {th }}\right. \\
\text { percentile) }\end{array}$ & $\begin{array}{l}5 \text { cases } / 12 \\
\text { controls vs. } 24 \\
\text { cases } / 131 \\
\text { controls } \\
12 / 133(9.02 \%) \\
\text { vs. } 55 / 438 \\
(12.6 \%)^{144}\end{array}$ & $\begin{array}{l}\text { OR, } 3.19,95 \% \\
\mathrm{Cl}, 0.93 \text { to } \\
10.90^{83} \mathrm{AOR}, \\
0.714(95 \% \mathrm{Cl}, \\
0.353 \text { to } \\
1.35)^{144}\end{array}$ & $\begin{array}{l}1 \text { cohort, } 1 \\
\text { case- } \\
\text { control, } \\
n=772^{83,144}\end{array}$ & $\begin{array}{l}\text { Moderate } \\
\text { study } \\
\text { limitations } \\
\text { (high risk of } \\
\text { bias, }{ }^{83} \\
\text { imprecise) } \\
\text { wide Cls, } \\
\text { inconsistent }\end{array}$ & Insufficient \\
\hline $\begin{array}{l}\text { Pregnant } \\
\text { women with a } \\
\text { psychiatric } \\
\text { disorder } \\
\text { (second } \\
\text { trimester) or } \\
\text { exposed to } \\
\text { venlafaxine or } \\
\text { SSRls (during } \\
\text { pregnancy) }\end{array}$ & $\begin{array}{l}\text { Venlafaxine } \\
\text { vs. SSRI } \\
\text { during second } \\
\text { trimester }\end{array}$ & $\begin{array}{l}\text { Major } \\
\text { anomaly }\end{array}$ & $\begin{array}{l}2 / 150(1.6 \%) \\
\text { vs. } 3 / 150 \\
(2.4 \%)^{135} \\
4 / 281(1.42 \%) \\
\text { vs. } 14 / 838 \\
(1.67 \%)^{144}\end{array}$ & $\begin{array}{l}\text { RR, } 0.67(95 \% \\
\mathrm{Cl}, 0.11 \text { to } \\
3.93)^{135} \\
\text { AOR, } 0.864 \\
(95 \% \mathrm{Cl}, 0.243 \\
\text { to } 2.44^{144}\end{array}$ & $\begin{array}{l}1 \text { cohort, } \\
n=1,419^{135,} \\
144\end{array}$ & $\begin{array}{l}\text { Moderate } \\
\text { study } \\
\text { limitations } \\
\text { (high risk of } \\
\text { bias, }{ }^{135} \\
\text { imprecise) } \\
\text { wide Cls, } \\
\text { consistent }\end{array}$ & Insufficient \\
\hline $\begin{array}{l}\text { Pregnant } \\
\text { women who } \\
\text { used SSRIs or } \\
\text { venlafaxine } \\
\text { during } \\
\text { pregnancy }\end{array}$ & $\begin{array}{l}\text { Venlafaxine } \\
\text { vs. SSRI } \\
\text { during first or } \\
\text { second } \\
\text { trimester }\end{array}$ & $\begin{array}{l}\text { Any } \\
\text { congenital } \\
\text { malformation }\end{array}$ & $\begin{array}{l}7 / 281(2.49 \%) \\
\text { vs. } 33 / 838 \\
(3.94 \%)^{144}\end{array}$ & $\begin{array}{l}\text { AOR, } 0.641 \\
(95 \% \mathrm{Cl}, 0.257 \\
\text { to } 1.39)^{144}\end{array}$ & $\begin{array}{l}1 \text { cohort, } \\
n=1,119^{144}\end{array}$ & $\begin{array}{l}\text { Moderate } \\
\text { study } \\
\text { limitations } \\
\text { imprecise } \\
\text { (wide Cls), } \\
\text { inconsistency } \\
\text { unknown }\end{array}$ & Insufficient \\
\hline $\begin{array}{l}\text { Pregnant } \\
\text { women who } \\
\text { used SSRIs or } \\
\text { venlafaxine } \\
\text { during first } \\
\text { pregnancy } \\
\text { trimester }\end{array}$ & $\begin{array}{l}\text { Venlafaxine } \\
\text { vs. SSRI } \\
\text { during first or } \\
\text { second } \\
\text { trimester }\end{array}$ & $\begin{array}{l}\text { Any } \\
\text { congenital } \\
\text { malformation }\end{array}$ & $\begin{array}{l}7 / 270(2.59 \%) \\
\text { vs. } 28 / 716 \\
(3.9 \%)^{144}\end{array}$ & $\begin{array}{l}\text { AOR, } 0.567 \\
(95 \% \mathrm{Cl}, 0.209 \\
\text { to } 1.30)^{144}\end{array}$ & $\begin{array}{l}1 \text { cohort, } \\
n=986^{144}\end{array}$ & $\begin{array}{l}\text { Moderate } \\
\text { study } \\
\text { limitations } \\
\text { imprecise } \\
\text { (wide Cls), } \\
\text { inconsistency } \\
\text { unknown }\end{array}$ & Insufficient \\
\hline
\end{tabular}

$\mathrm{AOR}=$ adjusted odds ratio; $\mathrm{CI}=$ confidence interval; $\mathrm{n}=$ number; $\mathrm{NR}=$ not reported; $\mathrm{RR}=$ relative risk; $\mathrm{SD}=$ standard deviation; SSRI = selective serotonin reuptake inhibitor; vs. = versus.

\section{Venlafaxine Versus Bupropion}

\section{Overview}

- The evidence is insufficient to judge the comparative risk of harms from venlafaxine versus bupropion during pregnancy for child outcomes (congenital heart disease).

\section{Detailed Results}

One publication reported on the comparative harms of venlafaxine versus bupropion. ${ }^{141}$ The study drew from a cohort of women who had undergone a fetal echocardiogram because of in utero exposure of the fetus to SSRIs or SNRIs. ${ }^{141}$ The study was rated as high risk of bias. The study yielded insufficient strength of evidence to judge the risk of congenital heart disease (Table B-82). 
Table B-82. Strength of evidence for comparative harms: Venlafaxine versus bupropion

\begin{tabular}{|c|c|c|c|c|c|c|c|}
\hline Population & $\begin{array}{l}\text { Intervention } \\
\text { and } \\
\text { Comparator }\end{array}$ & Outcome & $\begin{array}{l}\text { Incidence } \\
\text { or Mean } \\
\text { Effect by } \\
\text { Arm }\end{array}$ & Results & $\begin{array}{l}\text { Study } \\
\text { Design } \\
\text { and } \\
\text { Sample } \\
\text { Size } \\
\end{array}$ & $\begin{array}{l}\text { Factors That } \\
\text { Affect the } \\
\text { Strength of } \\
\text { Evidence }\end{array}$ & $\begin{array}{l}\text { Overall } \\
\text { Evidence } \\
\text { Strength } \\
\text { (Direction of } \\
\text { Effect) }\end{array}$ \\
\hline $\begin{array}{l}\text { Women who } \\
\text { underwent fetal } \\
\text { echocardiography } \\
\text { because of } \\
\text { an in utero exposure } \\
\text { to either SSRIs or } \\
\text { SNRIs }\end{array}$ & $\begin{array}{l}\text { Venlafaxine } \\
\text { vs. bupropion } \\
\end{array}$ & $\begin{array}{l}\text { Congenital } \\
\text { heart } \\
\text { disease }\end{array}$ & $\begin{array}{l}2 / 2(100 \%) \\
\text { vs. } 0 / 3 \\
(0 \%)^{141}\end{array}$ & $\begin{array}{l}\text { Risk } \\
\text { difference, } \\
1.00(95 \% \\
C l, 0.47 \text { to } \\
1.53)^{141}\end{array}$ & $\begin{array}{l}1 \text { cohort, } \\
n=5^{141}\end{array}$ & $\begin{array}{l}\text { High study } \\
\text { limitations (high } \\
\text { risk of bias, } 141 \\
\text { imprecise (wide } \\
\text { Cls), } \\
\text { consistency } \\
\text { unknown }\end{array}$ & Insufficient \\
\hline
\end{tabular}

$\mathrm{CI}=$ confidence interval; $\mathrm{n}=$ number; $\mathrm{SNRI}=$ serotonin-norepinephrine reuptake inhibitor; SSRI = selective serotonin reuptake inhibitor; vs. $=$ versus.

\section{Venlafaxine Versus Escitalopram}

\section{Overview}

- The evidence is insufficient to judge the comparative risk of harms from venlafaxine versus escitalopram during pregnancy for child outcomes (congenital heart disease)

\section{Detailed Results}

One publication reported on the comparative harms of venlafaxine versus escitalopram. ${ }^{141}$ The study drew from a cohort of women who had undergone a fetal echocardiogram because of in utero exposure of the fetus to SSRIs or SNRIs. ${ }^{141}$ The study was rated as high risk of bias. The study yielded insufficient strength of evidence to judge the risk of congenital heart disease (Table B-83).

Table B-83. Strength of evidence for comparative harms: Venlafaxine versus escitalopram

\begin{tabular}{|c|c|c|c|c|c|c|c|}
\hline Population & $\begin{array}{l}\text { Intervention } \\
\text { and } \\
\text { Comparator }\end{array}$ & Outcome & $\begin{array}{l}\text { Incidence } \\
\text { or Mean } \\
\text { Effect by } \\
\text { Arm }\end{array}$ & Results & $\begin{array}{l}\text { Study } \\
\text { Design } \\
\text { and } \\
\text { Sample } \\
\text { Size } \\
\end{array}$ & $\begin{array}{l}\text { Factors That } \\
\text { Affect the } \\
\text { Strength of } \\
\text { Evidence }\end{array}$ & $\begin{array}{l}\text { Overall } \\
\text { Evidence } \\
\text { Strength } \\
\text { (Direction of } \\
\text { Effect) }\end{array}$ \\
\hline $\begin{array}{l}\text { Women who } \\
\text { underwent fetal } \\
\text { echocardiography } \\
\text { because of an in } \\
\text { utero exposure to } \\
\text { either SSRIs or } \\
\text { SNRIs }\end{array}$ & $\begin{array}{l}\text { Venlafaxine } \\
\text { vs. } \\
\text { escitalopram }\end{array}$ & $\begin{array}{l}\text { Congenital } \\
\text { heart } \\
\text { disease }\end{array}$ & $\begin{array}{l}2 / 2(100 \%) \\
\text { vs. } 1 / 9 \\
(11.11 \%)^{141}\end{array}$ & $\begin{array}{l}\text { RR, 5.56 } \\
\text { (95\% Cl, } \\
1.17 \text { to } \\
26.43)^{141}\end{array}$ & $\begin{array}{l}1 \text { cohort, } \\
n=11^{141}\end{array}$ & $\begin{array}{l}\text { High study } \\
\text { limitations (high } \\
\text { risk of bias, }{ }^{141} \\
\text { imprecise (wide } \\
\text { Cls), consistency } \\
\text { unknown }\end{array}$ & Insufficient \\
\hline
\end{tabular}

\section{Venlafaxine Versus Sertraline}

\section{Overview}

- The evidence is insufficient to judge the comparative risk of harms from venlafaxine versus sertraline during pregnancy for child outcomes (congenital heart disease). 


\section{Detailed Results}

One publication reported on the comparative harms of venlafaxine versus sertraline. ${ }^{141}$ The study drew from a cohort of women who had undergone a fetal echocardiogram because of in utero exposure of the fetus to SSRIs or SNRIs. ${ }^{141}$ The study was rated as high risk of bias. The study yielded insufficient strength of evidence to judge the risk of congenital heart disease (Table B-84).

Table B-84. Strength of evidence for comparative harms: Venlafaxine versus sertraline

\begin{tabular}{|c|c|c|c|c|c|c|c|}
\hline Population & $\begin{array}{l}\text { Intervention } \\
\text { and } \\
\text { Comparator }\end{array}$ & Outcome & $\begin{array}{l}\text { Incidence } \\
\text { or Mean } \\
\text { Effect by } \\
\text { Arm }\end{array}$ & Results & $\begin{array}{l}\text { Study } \\
\text { Design } \\
\text { and } \\
\text { Sample } \\
\text { Size }\end{array}$ & $\begin{array}{l}\text { Factors That } \\
\text { Affect the } \\
\text { Strength of } \\
\text { Evidence }\end{array}$ & $\begin{array}{l}\text { Overall } \\
\text { Evidence } \\
\text { Strength } \\
\text { (Direction } \\
\text { of Effect) }\end{array}$ \\
\hline $\begin{array}{l}\text { Women who } \\
\text { underwent fetal } \\
\text { echocardiography } \\
\text { because of an in } \\
\text { utero exposure to } \\
\text { either SSRIs or } \\
\text { SNRIs }\end{array}$ & $\begin{array}{l}\text { Venlafaxine } \\
\text { vs. sertraline }\end{array}$ & $\begin{array}{l}\text { Congenital } \\
\text { heart } \\
\text { disease }\end{array}$ & $\begin{array}{l}2 / 2(100 \%) \\
\text { vs. 0/7 } \\
(0 \%)^{141}\end{array}$ & $\begin{array}{l}\text { Risk difference } \\
1.00(95 \% \mathrm{Cl} \\
-0.55 \text { to } 1.45)^{141}\end{array}$ & $\begin{array}{l}1 \text { cohort, } \\
n=9^{141}\end{array}$ & $\begin{array}{l}\text { High study } \\
\text { limitations } \\
\text { (high risk of } \\
\text { bias, } \\
\text { imprecise, } \\
\text { wide Cls, } \\
\text { consistency } \\
\text { unknown }\end{array}$ & Insufficient \\
\hline
\end{tabular}

$\mathrm{CI}=$ confidence interval; $\mathrm{n}=$ number; $\mathrm{SNRI}=$ serotonin-norepinephrine reuptake inhibitor; SSRI = selective serotonin reuptake inhibitor; vs. $=$ versus.

\section{SNRIs Versus MAOls}

\section{Overview}

- The evidence is insufficient to judge the comparative risk of harms from SNRIs versus MAOIs during pregnancy for child outcomes (attention-deficit/hyperactivity disorder or autism spectrum disorder).

\section{Detailed Results}

Two publications from the Canadian QPC reported on the comparative harms from treatment with SNRIs versus MAOIs among pregnant women with psychiatric disorders. ${ }^{53,114}$ Child attention-deficit/hyperactivity disorder outcomes were reported in one publication, ${ }^{114}$ and autism spectrum disorder outcomes were reported in a second. ${ }^{53}$ Only one woman was included in the MAOI group and the publications were rated as high risk of bias because the analyses did not control for confounding, leading to insufficient ratings (Table B-85).

Table B-85. Strength of evidence for comparative harms: SNRIs versus MAOls

\begin{tabular}{|c|c|c|c|c|c|c|c|}
\hline Population & $\begin{array}{l}\text { Intervention } \\
\text { and } \\
\text { Comparator }\end{array}$ & Outcome & $\begin{array}{l}\text { Incidence or } \\
\text { Mean Effect } \\
\text { by Arm }\end{array}$ & Results & $\begin{array}{l}\text { Study } \\
\text { Design } \\
\text { and } \\
\text { Sample } \\
\text { Size } \\
\end{array}$ & $\begin{array}{l}\text { Factors That Affect } \\
\text { the Strength of } \\
\text { Evidence }\end{array}$ & $\begin{array}{l}\text { Overall } \\
\text { Evidence } \\
\text { Strength } \\
\text { (Direction of } \\
\text { Effect) } \\
\end{array}$ \\
\hline $\begin{array}{l}\text { Psychiatric } \\
\text { disorder }\end{array}$ & $\begin{array}{l}\text { SNRIs vs. } \\
\text { MAOIs }\end{array}$ & ADHD & $\begin{array}{l}18 / 445(4 \%) \\
\text { vs. } 0 / 1(0 \%)^{53}\end{array}$ & $\begin{array}{l}\mathrm{RD}, 0.04 \\
(95 \% \\
\mathrm{Cl},-0.56 \text { to } \\
0.64)^{53}\end{array}$ & $\begin{array}{l}1 \text { cohort } \\
n=446^{53}\end{array}$ & $\begin{array}{l}\text { High study } \\
\text { limitations (high risk } \\
\text { of bias, }{ }^{53} \text { imprecise } \\
\text { (wide Cls), } \\
\text { consistency } \\
\text { unknown }\end{array}$ & Insufficient \\
\hline
\end{tabular}




\begin{tabular}{|c|c|c|c|c|c|c|c|}
\hline Population & $\begin{array}{l}\text { Intervention } \\
\text { and } \\
\text { Comparator }\end{array}$ & Outcome & $\begin{array}{l}\text { Incidence or } \\
\text { Mean Effect } \\
\text { by Arm }\end{array}$ & Results & $\begin{array}{l}\text { Study } \\
\text { Design } \\
\text { and } \\
\text { Sample } \\
\text { Size } \\
\end{array}$ & $\begin{array}{l}\text { Factors That Affect } \\
\text { the Strength of } \\
\text { Evidence }\end{array}$ & $\begin{array}{l}\text { Overall } \\
\text { Evidence } \\
\text { Strength } \\
\text { (Direction of } \\
\text { Effect) } \\
\end{array}$ \\
\hline $\begin{array}{l}\text { Psychiatric } \\
\text { disorder }\end{array}$ & $\begin{array}{l}\text { SNRIs vs. } \\
\text { MAOIs }\end{array}$ & ASD & $\begin{array}{l}2 / 447(0.4 \%) \\
\text { vs. 0/1 } \\
(0 \%)^{114}\end{array}$ & $\begin{array}{l}\text { RD, } 0.004 \\
(95 \% \\
C I,-0.60 \text { to } \\
0.60)^{114}\end{array}$ & $\begin{array}{l}1 \text { cohort } \\
n=448^{114}\end{array}$ & $\begin{array}{l}\text { High study } \\
\text { limitations (high risk } \\
\text { of bias, }{ }^{114} \text { imprecise } \\
\text { (wide Cls), } \\
\text { consistency } \\
\text { unknown }\end{array}$ & Insufficient \\
\hline
\end{tabular}

$\mathrm{ADHD}=$ attention-deficit/hyperactivity disorder; $\mathrm{ASD}=$ autism spectrum disorder; $\mathrm{CI}=$ confidence interval; $\mathrm{MAOI}=$ monoamine oxidase inhibitors; $\mathrm{n}=$ number; $\mathrm{RD}=$ risk difference; $\mathrm{SNRI}=$ serotonin-norepinephrine reuptake inhibitor; vs. $=$ versus.

\section{SNRIs Versus TCAs}

\section{Overview}

- The evidence is insufficient to judge the comparative risk of harms from SNRIs versus TCAs during pregnancy for maternal outcomes (preeclampsia or spontaneous abortion) and child outcomes (attention-deficit/hyperactivity disorder or autism spectrum disorder).

\section{Detailed Results}

Four high risk-of-bias publications reported on the comparative harms of SNRIs versus TCAs in pregnancy. ${ }^{53,64,81,114}$ These studies drew from three cohorts (2 from the Canadian QPC, ${ }^{53,114}$ a second cohort using the same Canadian Quebec registry data ${ }^{64}$ and 1 from a Canadian British Colombian pregnant women and newborn registry).$^{81}$ All outcomes were reported in a single nonrandomized study that did not control for confounding. These include maternal risk of preeclampsia and spontaneous abortion and child risk of attentiondeficit/hyperactivity disorder and autism spectrum disorder. No differences were found for any of these outcomes (Table B-86).

Table B-86. Strength of evidence for comparative harms: SNRIs versus TCAs

\begin{tabular}{|c|c|c|c|c|c|c|c|}
\hline Population & $\begin{array}{l}\text { Intervention } \\
\text { and } \\
\text { Comparator }\end{array}$ & Outcome & $\begin{array}{l}\text { Incidence or } \\
\text { Mean Effect by } \\
\text { Arm }\end{array}$ & Results & $\begin{array}{l}\text { Study } \\
\text { Design } \\
\text { and } \\
\text { Sample } \\
\text { Size }\end{array}$ & $\begin{array}{l}\text { Factors That } \\
\text { Affect the } \\
\text { Strength of } \\
\text { Evidence }\end{array}$ & $\begin{array}{l}\text { Overall } \\
\text { Evidence } \\
\text { Strength } \\
\text { (Direction } \\
\text { of Effect) }\end{array}$ \\
\hline $\begin{array}{l}\text { Pregnant } \\
\text { women } \\
\text { with } \\
\text { depression }\end{array}$ & $\begin{array}{l}\text { SNRIs vs. } \\
\text { TCAs }\end{array}$ & Preeclampsia & $\begin{array}{l}23 / 408(5.6 \%) \\
\text { vs. } 14 / 146 \\
(9.6 \%)^{81}\end{array}$ & $\begin{array}{l}\mathrm{RR}, 0.59 \\
(95 \% \mathrm{Cl} \text {, } \\
0.31 \text { to } \\
1.11)^{81}\end{array}$ & $\begin{array}{l}1 \text { cohort, } \\
n=554^{81}\end{array}$ & $\begin{array}{l}\text { High study } \\
\text { limitations (high } \\
\text { risk of bias }{ }^{81} \text { ), } \\
\text { imprecise (wide } \\
\text { Cls), consistency } \\
\text { unknown }\end{array}$ & Insufficient \\
\hline $\begin{array}{l}\text { Pregnant } \\
\text { women } \\
\text { with } \\
\text { depression }\end{array}$ & $\begin{array}{l}\text { SNRIs vs. } \\
\text { TCAs }\end{array}$ & $\begin{array}{l}\text { Spontaneous } \\
\text { abortion }\end{array}$ & $\begin{array}{l}20 / 137(15 \%) \\
\text { vs. } 20 / 147 \\
(14 \%)^{64}\end{array}$ & $\begin{array}{l}\mathrm{RR}, 1.07 \\
(95 \% \mathrm{Cl} \text {, } \\
0.60 \text { to } \\
1.91)^{64}\end{array}$ & $\begin{array}{l}1 \text { cohort, } \\
\mathrm{n}=284^{64}\end{array}$ & $\begin{array}{l}\text { High study } \\
\text { limitations (high } \\
\text { risk of bias }{ }^{64} \text { ), } \\
\text { imprecise (wide } \\
\text { Cls), consistency } \\
\text { unknown }\end{array}$ & Insufficient \\
\hline
\end{tabular}




\begin{tabular}{|c|c|c|c|c|c|c|c|}
\hline Population & $\begin{array}{l}\text { Intervention } \\
\text { and } \\
\text { Comparator }\end{array}$ & Outcome & $\begin{array}{l}\text { Incidence or } \\
\text { Mean Effect by } \\
\text { Arm }\end{array}$ & Results & $\begin{array}{l}\text { Study } \\
\text { Design } \\
\text { and } \\
\text { Sample } \\
\text { Size }\end{array}$ & $\begin{array}{l}\text { Factors That } \\
\text { Affect the } \\
\text { Strength of } \\
\text { Evidence }\end{array}$ & $\begin{array}{l}\text { Overall } \\
\text { Evidence } \\
\text { Strength } \\
\text { (Direction } \\
\text { of Effect) }\end{array}$ \\
\hline $\begin{array}{l}\text { Pregnant } \\
\text { women } \\
\text { with } \\
\text { psychiatric } \\
\text { disorders }\end{array}$ & $\begin{array}{l}\text { SNRIs vs. } \\
\text { TCAs }\end{array}$ & ADHD & $\begin{array}{l}18 / 445(4 \%) \text { vs. } \\
16 / 227(7 \%)^{53}\end{array}$ & $\begin{array}{l}\text { RR, } 0.57 \\
(95 \% \mathrm{Cl}, \\
0.30 \text { to } \\
1.10)^{53}\end{array}$ & $\begin{array}{l}1 \text { cohort } \\
n=672^{53}\end{array}$ & $\begin{array}{l}\text { High study } \\
\text { limitations (high } \\
\text { risk of bias }{ }^{53} \text { ), } \\
\text { imprecise (wide } \\
\text { Cls), consistency } \\
\text { unknown }\end{array}$ & Insufficient \\
\hline $\begin{array}{l}\text { Pregnant } \\
\text { women } \\
\text { with } \\
\text { psychiatric } \\
\text { disorders }\end{array}$ & $\begin{array}{l}\text { SNRIs vs. } \\
\text { TCAs }\end{array}$ & ASD & $\begin{array}{l}2 / 447(0.4 \%) \\
\text { vs. } 2 / 229 \\
(0.9 \%)^{114}\end{array}$ & $\begin{array}{l}\mathrm{RR}, 0.51 \\
(95 \% \mathrm{Cl} \text {, } \\
0.07 \text { to } \\
3.61)^{114}\end{array}$ & $\begin{array}{l}1 \text { cohort } \\
n=676^{114}\end{array}$ & $\begin{array}{l}\text { High study } \\
\text { limitations (high } \\
\text { risk of bias } 114 \text { ), } \\
\text { imprecise (wide } \\
\text { Cls), consistency } \\
\text { unknown }\end{array}$ & Insufficient \\
\hline
\end{tabular}

$\mathrm{ADHD}=$ attention-deficit/hyperactivity disorder; $\mathrm{ASD}=$ autism spectrum disorder; $\mathrm{CI}=$ confidence interval; $\mathrm{n}=$ number; $\mathrm{RR}=$ relative risk; SNRI $=$ serotonin-norepinephrine reuptake inhibitor; TCA $=$ tricyclic antidepressant; vs. $=$ versus.

\section{MAOls Versus TCAs}

\section{Overview}

- The evidence is insufficient to judge the comparative risk of harms from MAOIs versus TCAs during pregnancy for child outcomes (attention-deficit/hyperactivity disorder or autism spectrum disorder).

\section{Detailed Results}

Two publications from the Canadian QPC reported on the comparative harms from treatment with MAOIs versus TCAs among pregnant women with psychiatric disorders. ${ }^{53,114}$ Child attention-deficit/hyperactivity disorder outcomes were reported in one publication, ${ }^{114}$ and autism spectrum disorder outcomes were reported in a second. ${ }^{53}$ Only one woman was included in the MAOI group, and the publications were rated as high risk of bias because the analyses did not control for confounding (Table B-87).

Table B-87. Strength of evidence for comparative harms: MAOls versus TCAs

\begin{tabular}{|c|c|c|c|c|c|c|c|}
\hline Population & $\begin{array}{l}\text { Intervention } \\
\text { and } \\
\text { Comparator }\end{array}$ & Outcome & $\begin{array}{l}\text { Incidence or } \\
\text { Mean Effect } \\
\text { by Arm }\end{array}$ & Results & $\begin{array}{l}\text { Study } \\
\text { Design } \\
\text { and } \\
\text { Sample } \\
\text { Size } \\
\end{array}$ & $\begin{array}{l}\text { Factors That Affect } \\
\text { the Strength of } \\
\text { Evidence }\end{array}$ & $\begin{array}{l}\text { Overall } \\
\text { Evidence } \\
\text { Strength } \\
\text { (Direction of } \\
\text { Effect) } \\
\end{array}$ \\
\hline $\begin{array}{l}\text { Psychiatric } \\
\text { disorder }\end{array}$ & $\begin{array}{l}\text { MAOls vs. } \\
\text { TCAs }\end{array}$ & ADHD & $\begin{array}{l}0 / 1(0 \%) \text { vs. } \\
16 / 227 \\
(7.0 \%)^{53}\end{array}$ & $\begin{array}{l}\mathrm{RD},-0.07 \\
(95 \% \\
\mathrm{Cl},-0.67 \text { to } \\
0.53)^{53}\end{array}$ & $\begin{array}{l}1 \text { cohort } \\
n=227^{53}\end{array}$ & $\begin{array}{l}\text { High study } \\
\text { limitations (high risk } \\
\text { of bias, }{ }^{53} \text { imprecise } \\
\text { (wide Cls), } \\
\text { consistency } \\
\text { unknown }\end{array}$ & Insufficient \\
\hline $\begin{array}{l}\text { Psychiatric } \\
\text { disorder }\end{array}$ & $\begin{array}{l}\text { MAOIs vs. } \\
\text { TCAs }\end{array}$ & ASD & $\begin{array}{l}0 / 1(0 \%) \text { vs. } \\
2 / 229 \\
(0.9 \%)^{114}\end{array}$ & $\begin{array}{l}\text { RD, }-0.009 \\
(95 \% \\
\mathrm{Cl},-0.61 \text { to } \\
0.59)^{114}\end{array}$ & $\begin{array}{l}1 \text { cohort } \\
n=230^{114}\end{array}$ & $\begin{array}{l}\text { High study } \\
\text { limitations (high risk } \\
\text { of bias, }{ }^{114} \text { imprecise } \\
\text { (wide Cls), } \\
\text { consistency } \\
\text { unknown }\end{array}$ & Insufficient \\
\hline
\end{tabular}

$\overline{\mathrm{ADHD}}=$ attention-deficit/hyperactivity disorder; $\mathrm{ASD}=$ autism spectrum disorder; $\mathrm{CI}=$ confidence interval; $\mathrm{MAOI}=$ monoamine oxidase inhibitors; $\mathrm{n}=$ number; $\mathrm{RD}=$ risk difference; $\mathrm{RR}=$ relative risk; $\mathrm{TCA}=$ tricyclic antidepressant; $\mathrm{vs} .=$ versus. 


\section{Venlafaxine Versus TCAs}

\section{Overview}

- The evidence is insufficient to judge the comparative risk of harms from venlafaxine versus TCAs during pregnancy for the child outcome of small for gestational age.

\section{Detailed Results}

One publication reported on the comparative harms of second trimester use of venlafaxine versus TCAs as a class; a case-control study using the Canadian QPC. ${ }^{83}$ The study was rated as high risk of bias.

The one outcome reported in this study is small for gestational age. ${ }^{83}$ The strength of evidence was rated as insufficient (Table B-88).

Table B-88. Strength of evidence for comparative harms: Venlafaxine versus TCAs

\begin{tabular}{|c|c|c|c|c|c|c|c|}
\hline Population & $\begin{array}{l}\text { Intervention } \\
\text { and } \\
\text { Comparator }\end{array}$ & Outcome & $\begin{array}{l}\text { Incidence or Mean } \\
\text { Effect by Arm }\end{array}$ & Results & $\begin{array}{l}\text { Study Design } \\
\text { and Sample } \\
\text { Size }\end{array}$ & $\begin{array}{l}\text { Factors } \\
\text { That Affect } \\
\text { the } \\
\text { Strength of } \\
\text { Evidence }\end{array}$ & $\begin{array}{l}\text { Overall } \\
\text { Evidence } \\
\text { Strength } \\
\text { (Direction of } \\
\text { Effect) }\end{array}$ \\
\hline $\begin{array}{l}\text { Pregnant } \\
\text { women with } \\
\text { a psychiatric } \\
\text { disorder }\end{array}$ & $\begin{array}{l}\text { Venlafaxine } \\
\text { or TCAs, 2nd } \\
\text { trimester } \\
\text { exposure }\end{array}$ & $\begin{array}{l}\text { Small for } \\
\text { gestational } \\
\text { age }\left(<10^{\text {th }}\right. \\
\text { percentile })\end{array}$ & $\begin{array}{l}5 \text { case/17controls vs. } \\
1 \text { cases } / 10 \text { controls }^{83}\end{array}$ & $\begin{array}{l}\text { OR, } 5.71 \\
(95 \% \mathrm{Cl}, \\
0.53 \text { to } \\
61.41)^{83}\end{array}$ & $\begin{array}{l}1 \text { case-control, } \\
\mathrm{n}=27^{83}\end{array}$ & $\begin{array}{l}\text { High study } \\
\text { limitations } \\
\text { (high risk of } \\
\text { bias }^{83} \text { ), } \\
\text { seriously } \\
\text { imprecise } \\
\text { (few events, } \\
\text { wide Cls), } \\
\text { consistency } \\
\text { unknown }\end{array}$ & Insufficient \\
\hline
\end{tabular}

$\mathrm{CI}=$ confidence interval; $\mathrm{n}=$ number; $\mathrm{OR}=$ odds ratio; $\mathrm{TCA}=$ tricyclic antidepressant; $\mathrm{vs.}=$ versus.

\section{Bupropion Versus Duloxetine}

\section{Overview}

- The evidence is insufficient to judge the comparative risk of harms from bupropion versus duloxetine during pregnancy for child outcomes (congenital heart disease).

\section{Detailed Results}

One publication reported on the comparative harms of bupropion versus duloxetine. ${ }^{141}$ The study drew from a cohort of women who had undergone a fetal echocardiogram because of in utero exposure of the fetus to SSRIs or SNRIs. ${ }^{141}$ The study was rated as high risk of bias. The study yielded insufficient strength of evidence to judge the risk of congenital heart disease (Table B-89). 
Table B-89. Strength of evidence for comparative harms: Bupropion versus duloxetine

\begin{tabular}{|c|c|c|c|c|c|c|c|}
\hline Population & $\begin{array}{l}\text { Intervention } \\
\text { and } \\
\text { Comparator }\end{array}$ & Outcome & $\begin{array}{l}\text { Incidence or } \\
\text { Mean Effect } \\
\text { by Arm }\end{array}$ & Results & $\begin{array}{l}\text { Study } \\
\text { Design and } \\
\text { Sample } \\
\text { Size }\end{array}$ & $\begin{array}{l}\text { Factors That } \\
\text { Affect the } \\
\text { Strength of } \\
\text { Evidence }\end{array}$ & $\begin{array}{l}\text { Overall } \\
\text { Evidence } \\
\text { Strength } \\
\text { (Direction } \\
\text { of Effect) }\end{array}$ \\
\hline $\begin{array}{l}\text { Women who } \\
\text { underwent fetal } \\
\text { echocardiography } \\
\text { because of an } \\
\text { in utero exposure } \\
\text { to either SSRIs or } \\
\text { SNRIs }\end{array}$ & $\begin{array}{l}\text { Bupropion } \\
\text { vs. } \\
\text { duloxetine }\end{array}$ & $\begin{array}{l}\text { Congenital } \\
\text { heart disease }\end{array}$ & $\begin{array}{l}0 / 3(0 \%) \text { vs. } \\
0 / 1(0 \%)^{141}\end{array}$ & $\begin{array}{l}\text { RR not } \\
\text { calculable: } \\
\text { no events }{ }^{141}\end{array}$ & $\begin{array}{l}1 \text { cohort, } \\
n=4^{141}\end{array}$ & $\begin{array}{l}\text { High study } \\
\text { limitations (high } \\
\text { risk of bias, }{ }^{141} \\
\text { likely imprecise } \\
\text { (no events, } \\
\text { small Ns), } \\
\text { consistency } \\
\text { unknown) }\end{array}$ & Insufficient \\
\hline
\end{tabular}

$\overline{\mathrm{CI}}=$ confidence interval; $\mathrm{RR}=$ relative risk; SNRI = serotonin and norepinephrine reuptake inhibitor; SSRI $=$ selective serotonin reuptake inhibitor; vs $=$ versus.

\section{Bupropion Versus Paroxetine}

\section{Overview}

- The evidence is insufficient to judge the comparative risk of harms from bupropion versus paroxetine during pregnancy for child outcomes (congenital heart disease).

\section{Detailed Results}

One publication reported on the comparative harms of bupropion versus paroxetine. ${ }^{141}$ The study drew from a cohort of women who had undergone a fetal echocardiogram because of in utero exposure of the fetus to SSRIs or SNRIs. ${ }^{141}$ The study was rated as high risk of bias. The study yielded insufficient strength of evidence to judge the risk of congenital heart disease (Table B-90).

Table B-90. Strength of evidence for comparative harms: Bupropion versus paroxetine

\begin{tabular}{|c|c|c|c|c|c|c|c|}
\hline Population & $\begin{array}{l}\text { Intervention } \\
\text { and } \\
\text { Comparator }\end{array}$ & Outcome & $\begin{array}{l}\text { Incidence or } \\
\text { Mean Effect } \\
\text { by Arm }\end{array}$ & Results & $\begin{array}{l}\text { Study } \\
\text { Design and } \\
\text { Sample } \\
\text { Size }\end{array}$ & $\begin{array}{l}\text { Factors That } \\
\text { Affect the } \\
\text { Strength of } \\
\text { Evidence }\end{array}$ & $\begin{array}{l}\text { Overall } \\
\text { Evidence } \\
\text { Strength } \\
\text { (Direction } \\
\text { of Effect) }\end{array}$ \\
\hline $\begin{array}{l}\text { Women who } \\
\text { underwent fetal } \\
\text { echocardiography } \\
\text { because of an in } \\
\text { utero exposure to } \\
\text { either SSRIs or } \\
\text { SNRIs }\end{array}$ & $\begin{array}{l}\text { Bupropion } \\
\text { vs. } \\
\text { paroxetine }\end{array}$ & $\begin{array}{l}\text { Congenital } \\
\text { heart disease }\end{array}$ & $\begin{array}{l}0 / 3(0 \%) \text { vs. } \\
0 / 1(0 \%)^{141}\end{array}$ & $\begin{array}{l}\text { RR not } \\
\text { calculable: } \\
\text { no events }{ }^{141}\end{array}$ & $\begin{array}{l}1 \text { cohort, } \\
n=4^{141}\end{array}$ & $\begin{array}{l}\text { High study } \\
\text { limitations (high } \\
\text { risk of bias, }{ }^{141} \\
\text { likely imprecise } \\
\text { (no events, } \\
\text { small Ns), } \\
\text { consistency } \\
\text { unknown }\end{array}$ & Insufficient \\
\hline
\end{tabular}

$\mathrm{CI}=$ confidence interval; $\mathrm{n}=$ number; $\mathrm{RR}=$ relative risk; $\mathrm{SNRI}=$ serotonin and norepinephrine reuptake inhibitor; $\mathrm{SSRI}=$ selective serotonin reuptake inhibitor; vs. $=$ versus. 


\section{Bupropion Versus Sertraline}

\section{Overview}

- The evidence is insufficient to judge the comparative risk of harms from bupropion versus sertraline during pregnancy for child outcomes (congenital heart disease).

\section{Detailed Results}

One publication reported on the comparative harms of bupropion versus sertraline. ${ }^{141}$ The study drew from a cohort of women who had undergone a fetal echocardiogram because of in utero exposure of the fetus to SSRIs or SNRIs. ${ }^{141}$ The study was rated as high risk of bias. The study yielded insufficient strength of evidence to judge the risk of congenital heart disease (Table B-91).

Table B-91. Strength of evidence for comparative harms: Bupropion versus sertraline

\begin{tabular}{|c|c|c|c|c|c|c|c|}
\hline Population & $\begin{array}{l}\text { Intervention } \\
\text { and } \\
\text { Comparator }\end{array}$ & Outcome & $\begin{array}{l}\text { Incidence } \\
\text { or Mean } \\
\text { Effect by } \\
\text { Arm }\end{array}$ & Results & $\begin{array}{l}\text { Study } \\
\text { Design } \\
\text { and } \\
\text { Sample } \\
\text { Size }\end{array}$ & $\begin{array}{l}\text { Factors That } \\
\text { Affect the } \\
\text { Strength of } \\
\text { Evidence }\end{array}$ & $\begin{array}{l}\text { Overall } \\
\text { Evidence } \\
\text { Strength } \\
\text { (Direction } \\
\text { of Effect) }\end{array}$ \\
\hline $\begin{array}{l}\text { Women who } \\
\text { underwent fetal } \\
\text { echocardiography } \\
\text { because of an in } \\
\text { utero exposure to } \\
\text { either SSRIs or } \\
\text { SNRIs }\end{array}$ & $\begin{array}{l}\text { Bupropion } \\
\text { vs. sertraline }\end{array}$ & $\begin{array}{l}\text { Congenital } \\
\text { heart disease }\end{array}$ & $\begin{array}{l}0 / 3(0 \%) \text { vs. } \\
0 / 7(0 \%)^{141}\end{array}$ & $\begin{array}{l}\text { RR not } \\
\text { calculable: } \\
\text { no events }\end{array}$ & $\begin{array}{l}1 \text { cohort, } \\
n=10^{141}\end{array}$ & $\begin{array}{l}\text { High study } \\
\text { limitations (high } \\
\text { risk of bias, }{ }^{141} \\
\text { likely imprecise } \\
\text { (no events, small } \\
\text { Ns), consistency } \\
\text { unknown }\end{array}$ & Insufficient \\
\hline
\end{tabular}

$\mathrm{CI}=$ confidence interval; $\mathrm{n}=$ number; $\mathrm{RR}=$ relative risk; $\mathrm{SNRI}=$ serotonin and norepinephrine reuptake inhibitor; $\mathrm{SSRI}=$ selective serotonin reuptake inhibitor; vs. = versus.

\section{Brexanolone Versus Specific Active Comparators}

\section{Overview}

- No included publications reported on the harms of brexanolone compared with specific active comparators.

\section{Mirtazapine Versus SSRIs}

\section{Overview}

- The evidence is insufficient to judge the risk of harms for maternal (spontaneous abortion or stillbirth) or child outcomes (preterm birth late-term birth, low birthweight, major birth defects, NICU stay, macrosomia, neonatal bilirubinemia) for mirtazapine versus SSRIs during pregnancy.

\section{Detailed Results}

One publication reported on mirtazapine compared with SSRIs. ${ }^{116}$ The study drew from a cohort from the European Network of Teratology Information Services. The study was nonrandomized and was rated as high risk of bias. The publication focused on exposure to mirtazapine compared with exposure to SSRIs during pregnancy. ${ }^{116}$ A second high risk-of-bias publication reported on the comparative harms of SSRIs versus mirtazapine in pregnancy in 
Turkey. ${ }^{95}$ The study did not control for confounding. The evidence is insufficient to judge the risk of harms for maternal (spontaneous abortion or stillbirth) or child outcomes (preterm birth late-term birth, low birthweight, birthweight, major birth defects, NICU stay, macrosomia, neonatal bilirubinemia) for mirtazapine versus SSRIs (Table B-92).

Table B-92. Strength of evidence for comparative harms: Mirtazapine versus SSRIs

\begin{tabular}{|c|c|c|c|c|c|c|c|}
\hline Population & $\begin{array}{l}\text { Intervention and } \\
\text { Comparator }\end{array}$ & Outcome & $\begin{array}{l}\text { Incidence or } \\
\text { Mean Effect } \\
\text { by Arm }\end{array}$ & Results & $\begin{array}{l}\text { Study } \\
\text { Design } \\
\text { and } \\
\text { Sample } \\
\text { Size } \\
\end{array}$ & $\begin{array}{l}\text { Factors That } \\
\text { Affect the } \\
\text { Strength of } \\
\text { Evidence }\end{array}$ & $\begin{array}{l}\text { Overall } \\
\text { Evidence } \\
\text { Strength } \\
\text { (Direction of } \\
\text { Effect) }\end{array}$ \\
\hline $\begin{array}{l}\text { Women } \\
\text { exposed to } \\
\text { therapeutic } \\
\text { agents during } \\
\text { pregnancy }\end{array}$ & $\begin{array}{l}\text { Mirtazapine } \\
\text { exposure during } \\
\text { pregnancy vs. } \\
\text { SSRI exposure } \\
\text { during pregnancy }\end{array}$ & $\begin{array}{l}\text { Spontaneous } \\
\text { abortion or } \\
\text { stillbirth }\end{array}$ & $\begin{array}{l}39 / 323 \\
(12.1 \%) \text { vs. } \\
41 / 342 \\
(12 \%)^{116}\end{array}$ & $\begin{array}{l}\text { OR, } 1.01(95 \% \\
\mathrm{Cl},(0.63 \text { to } \\
1.61)^{116}\end{array}$ & $\begin{array}{l}1 \text { cohort, } \\
n=665^{116}\end{array}$ & $\begin{array}{l}\text { High study } \\
\text { limitations } \\
\text { (high risk of } \\
\text { bias }^{116} \text { ), } \\
\text { imprecise, } \\
\text { consistent }\end{array}$ & Insufficient \\
\hline $\begin{array}{l}\text { Women } \\
\text { exposed to } \\
\text { therapeutic } \\
\text { agents during } \\
\text { pregnancy }\end{array}$ & $\begin{array}{l}\text { Mirtazapine } \\
\text { exposure during } \\
\text { pregnancy vs. } \\
\text { SSRI exposure } \\
\text { during pregnancy }\end{array}$ & $\begin{array}{l}\text { Preterm } \\
\text { delivery }\end{array}$ & $\begin{array}{l}31 / 279 \\
(11.1 \%) \text { vs. } \\
32 / 302 \\
(10.6 \%)^{116} \\
1 / 16(6.25 \%) \\
\text { vs. } 3 / 40 \\
(7.50 \%)^{95}\end{array}$ & $\begin{array}{l}\text { OR, } 1.06(95 \% \\
\mathrm{Cl},(0.63 \text { to } \\
1.78) ; 116 \text { the OR } \\
\text { did not change } \\
\text { after adjusting } \\
\text { for concomitant } \\
\text { drug treatment } \\
\mathrm{RR}, 0.83(95 \% \\
\mathrm{Cl}, 0.09 \text { to } \\
7.43)^{95}\end{array}$ & $\begin{array}{l}2 \text { cohorts, } \\
n=637^{95,116}\end{array}$ & $\begin{array}{l}\text { High study } \\
\text { limitations } \\
\text { (high risk of } \text { bias }^{95,116} \text { ), } \\
\text { imprecise, } \\
\text { consistent }\end{array}$ & Insufficient \\
\hline $\begin{array}{l}\text { Pregnant } \\
\text { women } \\
\text { exposed to } \\
\text { mirtazapine } \\
\text { or SSRIs }\end{array}$ & $\begin{array}{l}\text { Mirtazapine } \\
\text { exposure during } \\
\text { pregnancy vs. } \\
\text { SSRI exposure } \\
\text { during pregnancy }\end{array}$ & $\begin{array}{l}\text { Late-term } \\
\text { birth }\end{array}$ & $\begin{array}{l}2 / 16(12.50 \%) \\
\text { vs. } 0 / 40 \\
(0.00 \%)^{95}\end{array}$ & $\begin{array}{l}\text { Risk difference, } \\
0.13(95 \% \mathrm{Cl} \\
-0.05 \text { to } 0.30)^{95}\end{array}$ & $\begin{array}{l}1 \text { cohort, } \\
n=56^{95}\end{array}$ & $\begin{array}{l}\text { High study } \\
\text { limitations } \\
\text { (high risk of } \\
\text { bias }^{95} \text { ), } \\
\text { imprecise } \\
\text { (wide Cls), } \\
\text { consistency } \\
\text { unknown }\end{array}$ & Insufficient \\
\hline $\begin{array}{l}\text { Women } \\
\text { exposed to } \\
\text { therapeutic } \\
\text { agents during } \\
\text { pregnancy }\end{array}$ & $\begin{array}{l}\text { Mirtazapine } \\
\text { exposure during } \\
\text { pregnancy vs. } \\
\text { SSRI exposure } \\
\text { during pregnancy }\end{array}$ & $\begin{array}{l}\text { Major birth } \\
\text { defects }\end{array}$ & $\begin{array}{l}13 / 292(4.4 \%) \\
\text { vs. } 13 / 307 \\
(4.2 \%)^{116}\end{array}$ & $\begin{array}{l}\text { OR, } 1.05(95 \% \\
\mathrm{Cl}, 0.48 \text { to } \\
2.31)^{116} \\
\text { The OR did not } \\
\text { change after } \\
\text { adjusting for } \\
\text { concomitant drug } \\
\text { treatment }\end{array}$ & $\begin{array}{l}1 \text { cohort, } \\
\mathrm{n}=599^{116}\end{array}$ & $\begin{array}{l}\text { High study } \\
\text { limitations } \\
\text { (high risk of } \\
\text { bias }^{116} \text { ), } \\
\text { imprecise } \\
\text { (wide Cls),, } \\
\text { consistent }\end{array}$ & Insufficient \\
\hline
\end{tabular}




\begin{tabular}{|c|c|c|c|c|c|c|c|}
\hline Population & $\begin{array}{l}\text { Intervention and } \\
\text { Comparator }\end{array}$ & Outcome & $\begin{array}{l}\text { Incidence or } \\
\text { Mean Effect } \\
\text { by Arm }\end{array}$ & Results & $\begin{array}{l}\text { Study } \\
\text { Design } \\
\text { and } \\
\text { Sample } \\
\text { Size }\end{array}$ & $\begin{array}{l}\text { Factors } \\
\text { That Affect } \\
\text { the } \\
\text { Strength } \\
\text { of } \\
\text { Evidence }\end{array}$ & $\begin{array}{l}\text { Overall Evidence } \\
\text { Strength } \\
\text { (Direction of } \\
\text { Effect) }\end{array}$ \\
\hline $\begin{array}{l}\text { Women } \\
\text { exposed to } \\
\text { therapeutic } \\
\text { agents during } \\
\text { pregnancy }\end{array}$ & $\begin{array}{l}\text { Mirtazapine } \\
\text { exposure during } \\
\text { pregnancy vs. } \\
\text { SSRI exposure } \\
\text { during pregnancy }\end{array}$ & $\begin{array}{l}\text { Birth weight } \\
\text { (grams) }\end{array}$ & $\begin{array}{l}39 \text { (IQR 38-40) } \\
\text { vs. } 39 \text { (IQR } \\
38-40)^{116} \\
3,251.25 \mathrm{gm} \\
\text { (SD: } 502.27 \\
\text { n=16 vs. } \\
3,153.17 \mathrm{gm} \\
\text { (SD: } 435.17) \\
\mathrm{n}=40^{95}\end{array}$ & $\begin{array}{l}\text { No difference } \\
\text { between } \\
\text { groups; }{ }^{116} \text { mean } \\
\text { difference, } 98.08 \\
\text { (955 } \mathrm{Cl},-165.60 \\
\text { to } 361.76)^{95} \\
\\
\text { The OR did not } \\
\text { change after } \\
\text { adjusting for } \\
\text { concomitant drug } \\
\text { treatment }^{116}\end{array}$ & $\begin{array}{l}2 \text { cohorts, } \\
\mathrm{n}=652^{95,116}\end{array}$ & $\begin{array}{l}\text { High study } \\
\text { limitations } \\
\text { (high risk of } \\
\text { bias }^{95,116} \text { ), } \\
\text { imprecise } \\
\text { (wide Cls), } \\
\text { consistent }\end{array}$ & Insufficient \\
\hline $\begin{array}{l}\text { Pregnant } \\
\text { women } \\
\text { exposed to } \\
\text { mirtazapine } \\
\text { or SSRIs }\end{array}$ & $\begin{array}{l}\text { Mirtazapine } \\
\text { exposure during } \\
\text { pregnancy vs. } \\
\text { SSRI exposure } \\
\text { during pregnancy }\end{array}$ & NICU stay & $\begin{array}{l}2 / 16(12.50 \%) \\
\text { vs. } 7 / 40 \\
(17.50 \%)^{95}\end{array}$ & $\begin{array}{l}\mathrm{RR}, 0.71(95 \% \\
\mathrm{Cl}, 0.17 \text { to } \\
3.08)^{95}\end{array}$ & $\begin{array}{l}1 \text { cohort, } \\
n=56^{95}\end{array}$ & $\begin{array}{l}\text { High study } \\
\text { limitations } \\
\text { (high risk of } \\
\text { bias }^{95} \text { ), } \\
\text { imprecise } \\
\text { (wide Cls), } \\
\text { consistency } \\
\text { unknown }\end{array}$ & Insufficient \\
\hline $\begin{array}{l}\text { Pregnant } \\
\text { women } \\
\text { exposed to } \\
\text { mirtazapine } \\
\text { or SSRIs }\end{array}$ & $\begin{array}{l}\text { Mirtazapine } \\
\text { exposure during } \\
\text { pregnancy vs. } \\
\text { SSRI exposure } \\
\text { during pregnancy }\end{array}$ & $\begin{array}{l}\text { Neonatal } \\
\text { bilirubinemia }\end{array}$ & $\begin{array}{l}3 / 16(18.75 \%) \\
\text { vs. } 19 / 40 \\
(47.50 \%)^{95}\end{array}$ & $\begin{array}{l}\mathrm{RR}, 0.39(95 \% \\
\mathrm{Cl}, 0.14 \text { to } \\
1.15)^{95}\end{array}$ & $\begin{array}{l}1 \text { cohort, } \\
n=56^{95}\end{array}$ & $\begin{array}{l}\text { High study } \\
\text { limitations } \\
(\text { high risk of } \\
\text { bias }^{95} \text { ), } \\
\text { imprecise } \\
\text { (wide Cls), } \\
\text { consistency } \\
\text { unknown }\end{array}$ & 更 \\
\hline
\end{tabular}

$\overline{\mathrm{CI}}=$ confidence interval; $\mathrm{IQR}=$ interquartile ratio; $\mathrm{n}=$ number; $\mathrm{OR}=$ odds ratio; $\mathrm{RR}=$ relative risk; SSRI $=$ selective serotonin reuptake inhibitor; vs. = versus. 


\section{Mirtazapine Versus SSRIs Plus Mirtazapine}

\section{Overview}

- The evidence is insufficient to judge the comparative risk of harms from mirtazapine plus SSRIs vs. mirtazapine during pregnancy for child outcomes (preterm birth, late-term birth, low birthweight, NICU stay, macrosomia, neonatal bilirubinemia).

\section{Detailed Results}

One high risk-of-bias publication reported on the comparative harms of mirtazapine versus SSRIs plus mirtazapine in pregnancy in Turkey. ${ }^{95}$ The study did not control for confounding. The evidence was insufficient for all reported outcomes (preterm birth, late-term birth, low birthweight, NICU stay, macrosomia, neonatal bilirubinemia) (Table B-93).

Table B-93. Strength of evidence for comparative harms: Mirtazapine versus SSRls plus mirtazapine

\begin{tabular}{|c|c|c|c|c|c|c|c|}
\hline Population & $\begin{array}{l}\text { Intervention } \\
\text { and } \\
\text { Comparator }\end{array}$ & Outcome & $\begin{array}{l}\text { Incidence or } \\
\text { Mean Effect } \\
\text { by Arm }\end{array}$ & Results & $\begin{array}{l}\text { Study Design } \\
\text { and Sample } \\
\text { Size }\end{array}$ & $\begin{array}{l}\text { Factors That } \\
\text { Affect the } \\
\text { Strength of } \\
\text { Evidence }\end{array}$ & $\begin{array}{l}\text { Overall } \\
\text { Evidence } \\
\text { Strength } \\
\text { (Direction } \\
\text { of Effect) } \\
\end{array}$ \\
\hline $\begin{array}{l}\text { Pregnant } \\
\text { women } \\
\text { exposed to } \\
\text { mirtazapine } \\
\text { or SSRIs } \\
\text { plus } \\
\text { mirtazapine } \\
\end{array}$ & $\begin{array}{l}\text { Mirtazapine } \\
\text { vs. SSRIs } \\
\text { plus } \\
\text { mirtazapine } \\
\end{array}$ & Preterm birth & $\begin{array}{l}1 / 16(6.25 \%) \\
\text { vs. } 1 / 18 \\
(5.56 \%)^{95}\end{array}$ & $\begin{array}{l}\mathrm{RR}, 1.13(95 \% \\
\mathrm{CI}, 0.08 \text { to } \\
16.55)^{95}\end{array}$ & $\begin{array}{l}1 \text { cohort, } \\
n=34^{95}\end{array}$ & $\begin{array}{l}\text { High study } \\
\text { limitations (high } \\
\text { risk of bias }{ }^{95} \text { ), } \\
\text { imprecise (wide } \\
\text { Cls), } \\
\text { consistency } \\
\text { unknown }\end{array}$ & Insufficient \\
\hline $\begin{array}{l}\text { Pregnant } \\
\text { women } \\
\text { exposed to } \\
\text { mirtazapine } \\
\text { or SSRIs } \\
\text { plus } \\
\text { mirtazapine } \\
\end{array}$ & $\begin{array}{l}\text { Mirtazapine } \\
\text { vs. SSRIs } \\
\text { plus } \\
\text { mirtazapine } \\
\end{array}$ & Late-term birth & $\begin{array}{l}2 / 16(12.50 \%) \\
\text { vs. } 2 / 18 \\
(11.11 \%)^{95}\end{array}$ & $\begin{array}{l}\text { RR, } 1.13(95 \% \\
\mathrm{Cl}, 0.18 \text { to } \\
7.09)^{95}\end{array}$ & $\begin{array}{l}1 \text { cohort, } \\
n=34^{95}\end{array}$ & $\begin{array}{l}\text { High study } \\
\text { limitations (high } \\
\text { risk of bias }{ }^{95} \text { ), } \\
\text { imprecise (wide } \\
\text { Cls), } \\
\text { consistency } \\
\text { unknown }\end{array}$ & Insufficient \\
\hline $\begin{array}{l}\text { Pregnant } \\
\text { women } \\
\text { exposed to } \\
\text { mirtazapine } \\
\text { or SSRIs } \\
\text { plus } \\
\text { mirtazapine } \\
\end{array}$ & $\begin{array}{l}\text { Mirtazapine } \\
\text { vs. SSRIs } \\
\text { plus } \\
\text { mirtazapine } \\
\end{array}$ & $\begin{array}{l}\text { Low } \\
\text { birthweight }\end{array}$ & $\begin{array}{l}1 / 16(6.25 \%) \\
\text { vs. } 1 / 18 \\
(5.56 \%)^{95}\end{array}$ & $\begin{array}{l}\mathrm{RR}, 1.13(95 \% \\
\mathrm{Cl}, 0.08 \text { to } \\
16.55)^{95}\end{array}$ & $\begin{array}{l}1 \text { cohort, } \\
n=34^{95}\end{array}$ & $\begin{array}{l}\text { High study } \\
\text { limitations (high } \\
\text { risk of bias }{ }^{95} \text { ), } \\
\text { imprecise (wide } \\
\text { Cls), } \\
\text { consistency } \\
\text { unknown }\end{array}$ & Insufficient \\
\hline $\begin{array}{l}\text { Pregnant } \\
\text { women } \\
\text { exposed to } \\
\text { mirtazapine } \\
\text { or SSRIs } \\
\text { plus } \\
\text { mirtazapine }\end{array}$ & $\begin{array}{l}\text { Mirtazapine } \\
\text { vs. SSRIs } \\
\text { plus } \\
\text { mirtazapine } \\
\end{array}$ & Birthweight & $\begin{array}{l}3251.25 \mathrm{gm} \\
\text { (SD: } 502.27) \\
n=16 \text { vs. } \\
3191.38 \mathrm{gm} \\
(\mathrm{SD}: 436.69 \\
\mathrm{n}=18)^{95}\end{array}$ & $\begin{array}{l}\text { Mean } \\
\text { difference, } \\
59.87(95 \% \mathrm{Cl} \text {, } \\
-255.68 \text { to } \\
375.42)^{95}\end{array}$ & $\begin{array}{l}1 \text { cohort, } \\
n=34^{95}\end{array}$ & $\begin{array}{l}\text { High study } \\
\text { limitations (high } \\
\text { risk of bias }{ }^{95} \text { ), } \\
\text { imprecise (wide } \\
\text { Cls), } \\
\text { consistency } \\
\text { unknown }\end{array}$ & Insufficient \\
\hline $\begin{array}{l}\text { Pregnant } \\
\text { women } \\
\text { exposed to } \\
\text { mirtazapine } \\
\text { or SSRIs } \\
\text { plus } \\
\text { mirtazapine }\end{array}$ & $\begin{array}{l}\text { Mirtazapine } \\
\text { vs. SSRIs } \\
\text { plus } \\
\text { mirtazapine } \\
\end{array}$ & NICU stay & $\begin{array}{l}2 / 16(12.50 \%) \\
\text { Vs. } 3 / 18 \\
(16.67 \%)^{95}\end{array}$ & $\begin{array}{l}\text { RR, } 0.75(95 \% \\
\mathrm{Cl}, 0.14 \text { to } \\
3.94)^{95}\end{array}$ & $\begin{array}{l}1 \text { cohort, } \\
n=34^{95}\end{array}$ & $\begin{array}{l}\text { High study } \\
\text { limitations (high } \\
\text { risk of bias }{ }^{95} \text { ), } \\
\text { imprecise (wide } \\
\text { Cls), } \\
\text { consistency } \\
\text { unknown }\end{array}$ & Insufficient \\
\hline
\end{tabular}




\begin{tabular}{|c|c|c|c|c|c|c|c|}
\hline Population & $\begin{array}{l}\text { Intervention } \\
\text { and } \\
\text { Comparator }\end{array}$ & Outcome & $\begin{array}{l}\text { Incidence or } \\
\text { Mean Effect } \\
\text { by Arm }\end{array}$ & Results & $\begin{array}{l}\text { Study Design } \\
\text { and Sample } \\
\text { Size }\end{array}$ & $\begin{array}{l}\text { Factors That } \\
\text { Affect the } \\
\text { Strength of } \\
\text { Evidence }\end{array}$ & $\begin{array}{l}\text { Overall } \\
\text { Evidence } \\
\text { Strength } \\
\text { (Direction } \\
\text { of Effect) }\end{array}$ \\
\hline $\begin{array}{l}\text { Pregnant } \\
\text { women } \\
\text { exposed to } \\
\text { mirtazapine } \\
\text { or SSRIs } \\
\text { plus } \\
\text { mirtazapine } \\
\end{array}$ & $\begin{array}{l}\text { Mirtazapine } \\
\text { vs. SSRIs } \\
\text { plus } \\
\text { mirtazapine } \\
\end{array}$ & Macrosomia & $\begin{array}{l}0 / 16(0 \%) \text { vs. } \\
1 / 18(5.56 \%)^{95}\end{array}$ & $\begin{array}{l}\text { Risk difference:, } \\
-0.06(95 \% \mathrm{Cl} \\
-0.20 \text { to } 0.09)^{95}\end{array}$ & $\begin{array}{l}1 \text { cohort, } \\
n=34^{95}\end{array}$ & $\begin{array}{l}\text { High study } \\
\text { limitations (high } \\
\text { risk of bias }{ }^{95} \text { ), } \\
\text { imprecise (wide } \\
\text { Cls), } \\
\text { consistency } \\
\text { unknown }\end{array}$ & Insufficient \\
\hline $\begin{array}{l}\text { Pregnant } \\
\text { women } \\
\text { exposed to } \\
\text { mirtazapine } \\
\text { or SSRIs } \\
\text { plus } \\
\text { mirtazapine }\end{array}$ & $\begin{array}{l}\text { Mirtazapine } \\
\text { vs. SSRIs } \\
\text { plus } \\
\text { mirtazapine } \\
\end{array}$ & $\begin{array}{l}\text { Neonatal } \\
\text { bilirubinemia }\end{array}$ & $\begin{array}{l}3 / 16(18.75 \%) \\
\text { vs. } 4 / 18 \\
(22.22 \%)^{95}\end{array}$ & $\begin{array}{l}\text { RR, } 0.84(95 \% \\
C l, 0.22 \text { to } \\
3.21)^{95}\end{array}$ & $\begin{array}{l}1 \text { cohort, } \\
n=34^{95}\end{array}$ & $\begin{array}{l}\text { High study } \\
\text { limitations (high } \\
\text { risk of bias } 95 \text { ), } \\
\text { imprecise (wide } \\
\text { Cls), } \\
\text { consistency } \\
\text { unknown }\end{array}$ & Insufficient \\
\hline
\end{tabular}

$\overline{\mathrm{CI}}=$ confidence interval; $\mathrm{n}=$ number; $\mathrm{RR}=$ relative risk; SSRI $=$ selective serotonin reuptake inhibitor; vs. $=$ versus.

\section{Lithium Versus Lamotrigine}

\section{Overview}

- The evidence from one high risk-of-bias study indicates a higher risk of overall and cardiac fetal anomalies with lithium during pregnancy (low strength of evidence), which can inform the decision to switch a medication in a successfully treated individual.

- The evidence from one high risk-of-bias was insufficient to judge the comparative risk of harms from lithium versus lamotrigine during pregnancy for gestational diabetes.

\section{Detailed Results}

One high risk-of-bias publication comparing lithium with lamotrigine ${ }^{108}$ drew from a cohort in the United States. The publication, which focused on first-trimester exposure, ${ }^{108}$ found a substantially higher risk for fetal cardiac anomalies and overall congenital anomalies with lithium. ${ }^{108}$ The strength of evidence is low because of the limitations of the study (Table B-94).

Another high risk-of-bias publication comparing lithium or lamotrigine exposure during any trimester of pregnancy to no exposure did not find a significantly increased risk of gestational diabetes with exposure to either lithium or lamotrigine. ${ }^{97}$ The strength of the evidence is low due to the small sample size, wide CIs, and lack of adjustment for possible confounders. 
Table B-94. Strength of evidence for comparative harms: Lithium versus lamotrigine

\begin{tabular}{|c|c|c|c|c|c|c|c|}
\hline Population & $\begin{array}{l}\text { Intervention } \\
\text { and } \\
\text { Comparator }\end{array}$ & Outcome & $\begin{array}{l}\text { Incidence } \\
\text { or Mean } \\
\text { Effect by } \\
\text { Arm }\end{array}$ & Results & $\begin{array}{l}\text { Study } \\
\text { Design and } \\
\text { Sample } \\
\text { Size }\end{array}$ & $\begin{array}{l}\text { Factors That } \\
\text { Affect the } \\
\text { Strength of } \\
\text { Evidence }\end{array}$ & $\begin{array}{l}\text { Overall } \\
\text { Evidence } \\
\text { Strength } \\
\text { (Direction of } \\
\text { Effect) }\end{array}$ \\
\hline $\begin{array}{l}\text { Women exposed } \\
\text { to psychotropic } \\
\text { exposure } \\
\text { medication during } \\
\text { any trimester }\end{array}$ & $\begin{array}{l}\text { Lithium vs. } \\
\text { lamotrigine }\end{array}$ & $\begin{array}{l}\text { Gestational } \\
\text { diabetes }\end{array}$ & $\begin{array}{l}2 / 17 \\
(11.7 \%) \text { vs. } \\
2 / 19 \\
(10.5 \%)^{97}\end{array}$ & $\begin{array}{l}\text { RR, 1.12 } \\
(0.18 \text { to } \\
7.09)^{97}\end{array}$ & $\begin{array}{l}1 \text { cohort, } \\
n=36^{97}\end{array}$ & $\begin{array}{l}\text { High study } \\
\text { limitations (high } \\
\text { risk of bias, }{ }^{97} \\
\text { imprecise (wide } \\
\text { Cls, small N) } \\
\text { consistency } \\
\text { unknown }\end{array}$ & Insufficient \\
\hline $\begin{array}{l}\text { Lithium or } \\
\text { lamotrigine- } \\
\text { exposed women }\end{array}$ & $\begin{array}{l}\text { Lithium vs. } \\
\text { lamotrigine } \\
\text { exposure in } \\
\text { first-trimester } \\
\text { pregnancy }\end{array}$ & $\begin{array}{l}\text { Fetal } \\
\text { cardiac } \\
\text { anomalies }\end{array}$ & $\begin{array}{l}16 / 663 \\
(2.4 \%) \text { vs. } \\
27 / 1,945 \\
(1.4 \%)^{108}\end{array}$ & $\begin{array}{l}\text { ARR, } 2.25 \\
(95 \% \mathrm{Cl} \\
1.17 \text { to } \\
4.34)^{108}\end{array}$ & $\begin{array}{l}1 \text { cohort, } \\
n=2,608^{108}\end{array}$ & $\begin{array}{l}\text { High study } \\
\text { limitations (high } \\
\text { risk of bias, }{ }^{108} \\
\text { precise, large } \\
\text { effect size, } \\
\text { consistency } \\
\text { unknown }\end{array}$ & $\begin{array}{l}\text { Low for } \\
\text { harms with } \\
\text { lithium }\end{array}$ \\
\hline $\begin{array}{l}\text { Lithium or } \\
\text { lamotrigine- } \\
\text { exposed women }\end{array}$ & $\begin{array}{l}\text { Lithium vs. } \\
\text { lamotrigine } \\
\text { exposure in } \\
\text { first-trimester } \\
\text { pregnancy }\end{array}$ & $\begin{array}{l}\text { Overall } \\
\text { congenital } \\
\text { anomalies }\end{array}$ & $\begin{array}{l}38 / 663 \\
(5.7 \%) \text { vs. } \\
76 / 1,945 \\
(3.9 \%)^{108}\end{array}$ & $\begin{array}{l}\text { ARR, } 1.85 \\
\text { (95\% Cl, } \\
1.23 \text { to } \\
2.78)^{108}\end{array}$ & $\begin{array}{l}1 \text { cohort, } \\
n=2,608^{108}\end{array}$ & $\begin{array}{l}\text { High study } \\
\text { limitations (high } \\
\text { risk of bias, }{ }^{108} \\
\text { precise, large } \\
\text { effect size, } \\
\text { consistency } \\
\text { unknown }\end{array}$ & $\begin{array}{l}\text { Low for } \\
\text { harms with } \\
\text { lithium }\end{array}$ \\
\hline $\begin{array}{l}\text { Lithium or } \\
\text { lamotrigine- } \\
\text { exposed women }\end{array}$ & $\begin{array}{l}\text { Lithium vs. } \\
\text { lamotrigine } \\
\text { exposure in } \\
\text { first-trimester } \\
\text { pregnancy }\end{array}$ & $\begin{array}{l}\text { Noncardiac } \\
\text { fetal } \\
\text { anomalies }\end{array}$ & $\begin{array}{l}22 / 663 \\
(3.3 \%) \text { vs. } \\
49 / 1,945 \\
(2.5 \%)^{108}\end{array}$ & $\begin{array}{l}\text { ARR, } 1.63 \\
(95 \% \mathrm{Cl}, \\
0.96 \text { to } \\
2.78)^{108}\end{array}$ & $\begin{array}{l}1 \text { cohort, } \\
n=2,608^{108}\end{array}$ & $\begin{array}{l}\text { High study } \\
\text { limitations (high } \\
\text { risk of bias, } \\
\text { imprecise, } \\
\text { consistency } \\
\text { unknown }\end{array}$ & Insufficient \\
\hline
\end{tabular}

$\overline{\mathrm{ARR}}=$ adjusted risk ratio; $\mathrm{CI}=$ confidence interval; $\mathrm{n}=$ number; $\mathrm{RR}=$ relative risk; $\mathrm{vs} .=$ versus.

\section{Haloperidol Versus Olanzapine}

\section{Overview}

- The evidence for haloperidol versus olanzapine during pregnancy is insufficient to judge the risk of harms for the child outcomes reported (high birth weight, low birth weight, neonatal complications [respiratory], NICU admission, preterm birth, mean Apgar score at 1 minute, or mean Apgar score at 5 minutes).

\section{Detailed Results}

One publication reported on the comparative harms of haloperidol versus olanzapine in a sample collected at the Emory Women's Mental Health Program. ${ }^{127}$ The study was a nonrandomized cohort study and was rated high risk of bias. The study did not require a diagnosis of schizophrenia but required antipsychotic exposure during pregnancy and a stable dose at delivery per laboratory confirmation for $>5$ elimination half-lives at delivery as determined by high-performance liquid chromatography. Only fetal outcomes were reported.

The evidence for haloperidol versus olanzapine is insufficient to judge the risk of harms for the child outcomes reported (high birth weight, low birth weight, neonatal complications [respiratory], NICU admission, preterm birth, mean Apgar score at 1 minute, or mean Apgar 
score at 5 minutes) (Table B-95). These studies found no association between haloperidol versus olanzapine use in pregnancy with any outcome.

Table B-95. Strength of evidence for comparative harms: Haloperidol versus olanzapine

\begin{tabular}{|c|c|c|c|c|c|c|c|}
\hline Population & $\begin{array}{l}\text { Intervention } \\
\text { and } \\
\text { Comparator }\end{array}$ & Outcome & $\begin{array}{l}\text { Incidence or } \\
\text { Mean Effect } \\
\text { by Arm }\end{array}$ & Results & $\begin{array}{l}\text { Study Design } \\
\text { and Sample } \\
\text { Size }\end{array}$ & $\begin{array}{l}\text { Factors That } \\
\text { Affect the } \\
\text { Strength of } \\
\text { Evidence }\end{array}$ & $\begin{array}{l}\text { Overall } \\
\text { Evidence } \\
\text { Strength } \\
\text { (Direction } \\
\text { of Effect) } \\
\end{array}$ \\
\hline \multirow[t]{7}{*}{$\begin{array}{l}\text { Women } \\
\text { exposed to an } \\
\text { antipsychotic } \\
\text { during } \\
\text { pregnancy } \\
\text { (with a stable } \\
\text { dose at } \\
\text { delivery per } \\
\text { laboratory } \\
\text { confirmation) } \\
\end{array}$} & $\begin{array}{l}\text { Haloperidol } \\
\text { exposure vs. } \\
\text { olanzapine } \\
\text { exposure }\end{array}$ & $\begin{array}{l}\text { High birth } \\
\text { weight } \\
\text { (>4,000 } \\
\text { grams) }\end{array}$ & $\begin{array}{l}1 / 13(7.7 \%) \\
\text { vs. } 1 / 14 \\
(7.1 \%)^{127}\end{array}$ & $\begin{array}{l}\text { Calculated RR, } \\
1.07(95 \% \mathrm{Cl}, \\
0.07 \text { to } 15.5)^{127}\end{array}$ & $\begin{array}{l}1 \text { cohort, } \\
n=27^{127}\end{array}$ & $\begin{array}{l}\text { High study } \\
\text { limitations (high risk } \\
\text { of bias }{ }^{116} \text { ), seriously } \\
\text { imprecise (few } \\
\text { events, wide Cls), } \\
\text { consistency } \\
\text { unknown }\end{array}$ & Insufficient \\
\hline & & $\begin{array}{l}\text { Low birth } \\
\text { weight } \\
(<2,500 \\
\text { grams })\end{array}$ & $\begin{array}{l}0 / 13(0 \%) \text { vs. } \\
4 / 14 \\
(28.6 \%)^{127}\end{array}$ & $\begin{array}{l}\text { Calculated } \mathrm{RR} \text {, } \\
0.22(95 \% \mathrm{Cl} \\
0.007 \text { to } \\
2.02)^{127}\end{array}$ & $\begin{array}{l}1 \text { cohort, } \\
\mathrm{n}=27^{127}\end{array}$ & $\begin{array}{l}\text { High study } \\
\text { limitations (high risk } \\
\text { of bias }{ }^{116} \text { ), seriously } \\
\text { imprecise (few } \\
\text { events, wide Cls), } \\
\text { consistency } \\
\text { unknown }\end{array}$ & Insufficient \\
\hline & & $\begin{array}{l}\text { Neonatal } \\
\text { complications } \\
\text { (respiratory) }\end{array}$ & $\begin{array}{l}1 / 13(7.7 \%) \\
\text { vs. } 4 / 14 \\
(28.6 \%)^{127}\end{array}$ & $\begin{array}{l}\text { Calculated RR, } \\
0.27(95 \% \mathrm{Cl}, \\
0.03 \text { to } 2.11)^{127}\end{array}$ & $\begin{array}{l}1 \text { cohort, } \\
n=27^{127}\end{array}$ & $\begin{array}{l}\text { High study } \\
\text { limitations (high risk } \\
\text { of bias }{ }^{116} \text { ), seriously } \\
\text { imprecise (few } \\
\text { events, wide Cls), } \\
\text { consistency } \\
\text { unknown }\end{array}$ & Insufficient \\
\hline & & $\begin{array}{l}\text { NICU } \\
\text { admission }\end{array}$ & $\begin{array}{l}0 / 13(0 \%) \text { vs. } \\
4 / 14 \\
(28.6 \%)^{127}\end{array}$ & $\begin{array}{l}\text { Calculated RR, } \\
0.12(95 \% \mathrm{Cl}, \\
0.07 \text { to } 2.02)^{127}\end{array}$ & $\begin{array}{l}1 \text { cohort, } \\
n=27^{127}\end{array}$ & $\begin{array}{l}\text { High study } \\
\text { limitations (high risk } \\
\text { of bias }^{116} \text { ), seriously } \\
\text { imprecise (few } \\
\text { events, wide Cls), } \\
\text { consistency } \\
\text { unknown }\end{array}$ & Insufficient \\
\hline & & $\begin{array}{l}\text { Preterm birth } \\
\text { (<37 weeks) }\end{array}$ & $\begin{array}{l}0 / 13(0 \%) \text { vs. } \\
3 / 14 \\
(21.4 \%)^{127}\end{array}$ & $\begin{array}{l}\text { Calculated } \mathrm{RR} \text {, } \\
0.15(95 \% \mathrm{Cl} \text {, } \\
0.008 \text { to } \\
2.71)^{127}\end{array}$ & $\begin{array}{l}1 \text { cohort, } \\
\mathrm{n}=27^{127}\end{array}$ & $\begin{array}{l}\text { High study } \\
\text { limitations (high risk } \\
\text { of bias }{ }^{116} \text { ), seriously } \\
\text { imprecise (few } \\
\text { events, wide Cls), } \\
\text { consistency } \\
\text { unknown }\end{array}$ & Insufficient \\
\hline & & $\begin{array}{l}\text { Mean Apgar } \\
\text { score at } 1 \\
\text { minute }\end{array}$ & $\begin{array}{l}7.4(n=13) \\
\text { vs. } 7.6 \\
(n=14)^{127}\end{array}$ & $\begin{array}{l}\text { Calculated } \\
\text { mean } \\
\text { difference: } \\
-0.2(95 \% \mathrm{Cl},- \\
1.52 \text { to } 1.12)^{127} \\
\end{array}$ & $\begin{array}{l}1 \text { cohort, } \\
n=27^{127}\end{array}$ & $\begin{array}{l}\text { High study } \\
\text { limitations (high risk } \\
\text { of bias }{ }^{116} \text { ), } \\
\text { imprecise (wide } \\
\text { Cls), consistent }\end{array}$ & Insufficient \\
\hline & & $\begin{array}{l}\text { Mean Apgar } \\
\text { score at } 5 \\
\text { minutes }\end{array}$ & $\begin{array}{l}8.9(n=13) \\
\text { vs. } 8.8 \\
(n=14)^{127}\end{array}$ & $\begin{array}{l}\text { Calculated } \\
\text { mean } \\
\text { difference: } 0.10 \\
(95 \% \mathrm{Cl},-0.17 \\
\text { to } 0.37)^{127}\end{array}$ & $\begin{array}{l}1 \text { cohort, } \\
\mathrm{n}=27^{127}\end{array}$ & $\begin{array}{l}\text { High study } \\
\text { limitations (high risk } \\
\text { of bias }{ }^{116} \text { ), } \\
\text { imprecise (wide } \\
\text { Cls), consistent }\end{array}$ & Insufficient \\
\hline
\end{tabular}

$\overline{\mathrm{CI}}=$ confidence interval; $\mathrm{n}=$ number; $\mathrm{NICU}=$ neonatal intensive care unit; $\mathrm{RR}=$ relative risk; vs. $=$ versus. 


\section{Haloperidol Versus Quetiapine}

\section{Overview}

- The evidence for haloperidol versus quetiapine during pregnancy is insufficient to judge the risk of harms for the child outcomes reported (high birth weight, low birth weight, neonatal complications [respiratory], NICU admission, preterm birth, mean Apgar score at 1 minute, or mean Apgar score at 5 minutes).

\section{Detailed Results}

One publication reported on the comparative harms of haloperidol versus quetiapine in a sample collected at the Emory Women's Mental Health Program. ${ }^{127}$ The study was a nonrandomized cohort study and was rated high risk of bias. The study did not require a diagnosis of schizophrenia but required antipsychotic exposure during pregnancy and a stable dose at delivery per laboratory confirmation for $>5$ elimination half-lives at delivery as determined by high-performance liquid chromatography. Only fetal outcomes were reported.

The evidence for haloperidol versus quetiapine is insufficient to judge the risk of harms for the child outcomes reported (high birth weight, low birth weight, neonatal complications [respiratory], NICU admission, preterm birth, mean Apgar score at 1 minute, or mean Apgar score at 5 minutes) (Table B-96). These studies found no association between haloperidol versus quetiapine use in pregnancy with any outcome.

Table B-96. Strength of evidence for comparative harms: Haloperidol versus quetiapine

\begin{tabular}{|c|c|c|c|c|c|c|c|}
\hline Population & $\begin{array}{l}\text { Intervention } \\
\text { and } \\
\text { Comparator }\end{array}$ & Outcome & $\begin{array}{l}\text { Incidence or } \\
\text { Mean Effect } \\
\text { by Arm }\end{array}$ & Results & $\begin{array}{l}\text { Study } \\
\text { Design and } \\
\text { Sample Size }\end{array}$ & $\begin{array}{l}\text { Factors That } \\
\text { Affect the } \\
\text { Strength of } \\
\text { Evidence }\end{array}$ & $\begin{array}{l}\text { Overall } \\
\text { Evidence } \\
\text { Strength } \\
\text { (Direction } \\
\text { of Effect) }\end{array}$ \\
\hline \multirow[t]{3}{*}{$\begin{array}{l}\text { Women } \\
\text { exposed to } \\
\text { an } \\
\text { antipsychotic } \\
\text { during } \\
\text { pregnancy } \\
\text { (with a stable } \\
\text { dose at } \\
\text { delivery per } \\
\text { laboratory } \\
\text { confirmation) }\end{array}$} & $\begin{array}{l}\text { Haloperidol } \\
\text { exposure vs. } \\
\text { quetiapine } \\
\text { exposure }\end{array}$ & $\begin{array}{l}\text { High birth } \\
\text { weight } \\
\text { (>4,000 } \\
\text { grams) }\end{array}$ & $\begin{array}{l}1 / 13(7.7 \%) \text { vs. } \\
0 / 21(0 \%)^{127}\end{array}$ & $\begin{array}{l}\text { Calculated } \\
\text { RR, } 4.71 \\
(95 \% \mathrm{Cl} \\
0.21 \text { to } \\
107.8)^{127}\end{array}$ & $\begin{array}{l}1 \text { cohort, } \\
n=34^{127}\end{array}$ & $\begin{array}{l}\text { High study } \\
\text { limitations (high } \\
\text { risk of bias }{ }^{116} \text { ), } \\
\text { seriously } \\
\text { imprecise (few } \\
\text { events, wide } \\
\text { Cls), consistency } \\
\text { unknown }\end{array}$ & Insufficient \\
\hline & & $\begin{array}{l}\text { Low birth } \\
\text { weight } \\
(<2,500 \\
\text { grams })\end{array}$ & $\begin{array}{l}0 / 13(0 \%) \text { vs. } \\
1 / 21(4.8 \%)^{127}\end{array}$ & $\begin{array}{l}\text { Calculated } \\
\text { RR, } 0.52 \\
(95 \% \mathrm{Cl} \\
0.02 \text { to } \\
11.98)^{127}\end{array}$ & $\begin{array}{l}1 \text { cohort, } \\
n=34^{127}\end{array}$ & $\begin{array}{l}\text { High study } \\
\text { limitations (high } \\
\text { risk of bias }{ }^{116} \text { ), } \\
\text { seriously } \\
\text { imprecise (few } \\
\text { events, wide } \\
\text { Cls), consistency } \\
\text { unknown }\end{array}$ & Insufficient \\
\hline & & $\begin{array}{l}\text { Neonatal } \\
\text { complications } \\
\text { (respiratory) }\end{array}$ & $\begin{array}{l}1 / 13(7.7 \%) \text { vs. } \\
7 / 21(33.3 \%)^{127}\end{array}$ & $\begin{array}{l}\text { Calculated } \\
\text { RR, } 0.23 \\
(95 \% \mathrm{Cl} \\
0.03 \text { to } \\
1.67)^{127}\end{array}$ & $\begin{array}{l}1 \text { cohort, } \\
n=34^{127}\end{array}$ & $\begin{array}{l}\text { High study } \\
\text { limitations (high } \\
\text { risk of bias }{ }^{116} \text { ), } \\
\text { seriously } \\
\text { imprecise (few } \\
\text { events, wide } \\
\text { Cls), consistency } \\
\text { unknown }\end{array}$ & Insufficient \\
\hline
\end{tabular}




\begin{tabular}{|c|c|c|c|c|c|c|c|}
\hline Population & $\begin{array}{l}\text { Intervention } \\
\text { and } \\
\text { Comparator }\end{array}$ & Outcome & $\begin{array}{l}\text { Incidence or } \\
\text { Mean Effect } \\
\text { by Arm }\end{array}$ & Results & $\begin{array}{l}\text { Study } \\
\text { Design and } \\
\text { Sample Size }\end{array}$ & $\begin{array}{l}\text { Factors That } \\
\text { Affect the } \\
\text { Strength of } \\
\text { Evidence }\end{array}$ & $\begin{array}{l}\text { Overall } \\
\text { Evidence } \\
\text { Strength } \\
\text { (Direction } \\
\text { of Effect) }\end{array}$ \\
\hline \multirow[t]{4}{*}{$\begin{array}{l}\text { Women } \\
\text { exposed to } \\
\text { an } \\
\text { antipsychotic } \\
\text { during } \\
\text { pregnancy } \\
\text { (with a stable } \\
\text { dose at } \\
\text { delivery per } \\
\text { laboratory } \\
\text { confirmation) } \\
\text { (continued) } \\
\end{array}$} & $\begin{array}{l}\text { Haloperidol } \\
\text { exposure vs. } \\
\text { quetiapine } \\
\text { exposure } \\
\text { (continued) } \\
\end{array}$ & $\begin{array}{l}\mathrm{NICU} \\
\text { admission }\end{array}$ & $\begin{array}{l}0 / 13(0 \%) \text { vs. } \\
2 / 21(9.5 \%)^{127}\end{array}$ & $\begin{array}{l}\text { Calculated } \\
\text { RR, } \\
0.31(95 \% \mathrm{Cl}, \\
0.02 \text { to } \\
6.07)^{127}\end{array}$ & $\begin{array}{l}1 \text { cohort, } \\
\mathrm{n}=34^{127}\end{array}$ & $\begin{array}{l}\text { High study } \\
\text { limitations (high } \\
\text { risk of bias }{ }^{116} \text { ), } \\
\text { seriously } \\
\text { imprecise (few } \\
\text { events, wide } \\
\text { Cls), consistency } \\
\text { unknown }\end{array}$ & Insufficient \\
\hline & & $\begin{array}{l}\text { Preterm birth } \\
\text { (<37 weeks) }\end{array}$ & $\begin{array}{l}0 / 13(0 \%) \text { vs. } \\
1 / 21(4.8 \%)^{127}\end{array}$ & $\begin{array}{l}\text { Calculated } \\
\text { RR, } 0.52 \\
(95 \% \mathrm{Cl} \\
0.02 \text { to } \\
11.98)^{127}\end{array}$ & $\begin{array}{l}1 \text { cohort, } \\
n=34^{127}\end{array}$ & $\begin{array}{l}\text { High study } \\
\text { limitations (high } \\
\text { risk of bias }{ }^{116} \text { ), } \\
\text { seriously } \\
\text { imprecise (few } \\
\text { events, wide } \\
\text { Cls), consistency } \\
\text { unknown }\end{array}$ & Insufficient \\
\hline & & $\begin{array}{l}\text { Mean Apgar } \\
\text { score at } 1 \\
\text { minute }\end{array}$ & $\begin{array}{l}7.4(n=13) \text { vs. } \\
7.6(n=21)^{127}\end{array}$ & $\begin{array}{l}\text { Calculated } \\
\text { mean } \\
\text { difference: } \\
-0.2(95 \% \mathrm{Cl} \text {, } \\
-1.17 \text { to } \\
0.77)^{127}\end{array}$ & $\begin{array}{l}1 \text { cohort, } \\
\mathrm{n}=34^{127}\end{array}$ & $\begin{array}{l}\text { High study } \\
\text { limitations (high } \\
\text { risk of bias }{ }^{116} \text { ), } \\
\text { imprecise (wide } \\
\text { Cls), consistent }\end{array}$ & Insufficient \\
\hline & & $\begin{array}{l}\text { Mean Apgar } \\
\text { score at } 5 \\
\text { minutes }\end{array}$ & $\begin{array}{l}8.9(n=13) v s . \\
8.9(n=21)^{127}\end{array}$ & $\begin{array}{l}\text { Calculated } \\
\text { mean } \\
\text { difference: } 0 \\
(95 \% \mathrm{Cl} \text {, } \\
-0.25 \text { to } \\
0.25)^{127}\end{array}$ & $\begin{array}{l}1 \text { cohort, } \\
n=34^{127}\end{array}$ & $\begin{array}{l}\text { High study } \\
\text { limitations (high } \\
\text { risk of bias }{ }^{116} \text { ), } \\
\text { imprecise (wide } \\
\text { Cls), consistent }\end{array}$ & Insufficient \\
\hline
\end{tabular}

$\overline{\mathrm{CI}}=$ confidence interval; $\mathrm{n}=$ number; $\mathrm{NICU}=$ neonatal intensive care unit; $\mathrm{RR}=$ relative risk; vs. $=$ versus.

\section{Haloperidol Versus Risperidone}

\section{Overview}

- The evidence for haloperidol versus risperidone during pregnancy is insufficient to judge the risk of harms for the child outcomes reported (high birth weight, low birth weight, neonatal complications [respiratory], NICU admission, preterm birth, mean Apgar score at 1 minute, or mean Apgar score at 5 minutes).

\section{Detailed Results}

One publication reported on the comparative harms of haloperidol versus risperidone in a sample collected at the Emory Women's Mental Health Program. ${ }^{127}$ The study was a nonrandomized cohort study and was rated high risk of bias. The study did not require a diagnosis of schizophrenia but required antipsychotic exposure during pregnancy and a stable dose at delivery per laboratory confirmation for $>5$ elimination half-lives at delivery as determined by high-performance liquid chromatography. Only fetal outcomes were reported. 
The evidence for haloperidol versus risperidone is insufficient to judge the risk of harms for the child outcomes reported (high birth weight, low birth weight, neonatal complications [respiratory], NICU admission, preterm birth, mean Apgar score at 1 minute, or mean Apgar score at 5 minutes) (Table B-97). Mean Apgar scores have uncertain clinical value when compared with ordinal measures of $<7$ versus $\geq 7$. These studies found no association between haloperidol versus risperidone use in pregnancy with any outcome.

Table B-97. Strength of evidence for comparative harms: Haloperidol versus risperidone

\begin{tabular}{|c|c|c|c|c|c|c|c|}
\hline Population & $\begin{array}{l}\text { Intervention } \\
\text { and } \\
\text { Comparator }\end{array}$ & Outcome & $\begin{array}{l}\text { Incidence or } \\
\text { Mean Effect } \\
\text { by Arm }\end{array}$ & Results & $\begin{array}{l}\text { Study } \\
\text { Design and } \\
\text { Sample } \\
\text { Size }\end{array}$ & $\begin{array}{l}\text { Factors That Affect } \\
\text { the Strength of } \\
\text { Evidence }\end{array}$ & $\begin{array}{l}\text { Overall } \\
\text { Evidence } \\
\text { Strength } \\
\text { (Direction } \\
\text { of Effect) }\end{array}$ \\
\hline \multirow{7}{*}{$\begin{array}{l}\text { Women } \\
\text { exposed to } \\
\text { an } \\
\text { antipsychotic } \\
\text { during } \\
\text { pregnancy } \\
\text { (with a stable } \\
\text { dose at } \\
\text { delivery per } \\
\text { laboratory } \\
\text { confirmation) }\end{array}$} & $\begin{array}{l}\text { Haloperidol } \\
\text { exposure vs. } \\
\text { risperidone } \\
\text { exposure }\end{array}$ & $\begin{array}{l}\text { High birth } \\
\text { weight } \\
(>4,000 \\
\text { grams) }\end{array}$ & $\begin{array}{l}1 / 13(7.7 \%) \\
\text { vs. } 0 / 6(0 \%)^{127}\end{array}$ & $\begin{array}{l}\text { Calculated } \\
\text { RR, } 1.5 \text { (95\% } \\
\text { Cl, } 0.07 \text { to } \\
32.3)^{127}\end{array}$ & $\begin{array}{l}1 \text { cohort, } \\
\mathrm{n}=19^{127}\end{array}$ & $\begin{array}{l}\text { High study limitations } \\
\text { (high risk of bias }^{116} \text { ), } \\
\text { seriously imprecise } \\
\text { (few events, wide } \\
\text { Cls), consistency } \\
\text { unknown }\end{array}$ & Insufficient \\
\hline & & $\begin{array}{l}\text { Low birth } \\
\text { weight } \\
(<2,500 \\
\text { grams })\end{array}$ & $\begin{array}{l}0 / 13(0 \%) \text { vs. } \\
1 / 6(16.7 \%)^{127}\end{array}$ & $\begin{array}{l}\text { Calculated } \\
\text { RR, } 0.17 \\
(95 \% \mathrm{Cl}, \\
0.007 \text { to } \\
3.59)^{127}\end{array}$ & $\begin{array}{l}1 \text { cohort, } \\
n=19^{127}\end{array}$ & $\begin{array}{l}\text { High study limitations } \\
\text { (high risk of bias }^{116} \text { ), } \\
\text { seriously imprecise } \\
\text { (few events, wide } \\
\text { Cls), consistency } \\
\text { unknown }\end{array}$ & Insufficient \\
\hline & & $\begin{array}{l}\text { Neonatal } \\
\text { complications } \\
\text { (respiratory) }\end{array}$ & $\begin{array}{l}1 / 13(7.7 \%) \\
\text { vs. } 0 / 6(0 \%)^{127}\end{array}$ & $\begin{array}{l}\text { Calculated } \\
\text { RR, } 1.5 \text { (95\% } \\
\text { Cl, } 0.07 \text { to } \\
32.3)^{127}\end{array}$ & $\begin{array}{l}1 \text { cohort, } \\
\mathrm{n}=19^{127}\end{array}$ & $\begin{array}{l}\text { High study limitations } \\
\text { (high risk of bias }^{116} \text { ), } \\
\text { seriously imprecise } \\
\text { (few events, wide } \\
\text { Cls), consistency } \\
\text { unknown }\end{array}$ & Insufficient \\
\hline & & $\begin{array}{l}\text { NICU } \\
\text { admission }\end{array}$ & $\begin{array}{l}0 / 13(0 \%) \text { vs. } \\
0 / 6(0 \%)^{127}\end{array}$ & No events & $\begin{array}{l}1 \text { cohort, } \\
n=19^{127}\end{array}$ & $\begin{array}{l}\text { High study limitations } \\
\left.\text { (high risk of bias }^{116}\right) \text {, } \\
\text { imprecise (no events), } \\
\text { consistent }\end{array}$ & Insufficient \\
\hline & & $\begin{array}{l}\text { Preterm birth } \\
\text { (<37 weeks) }\end{array}$ & $\begin{array}{l}0 / 13(0 \%) \text { vs. } \\
0 / 6(0 \%)^{127}\end{array}$ & No events & $\begin{array}{l}1 \text { cohort, } \\
n=19^{127}\end{array}$ & $\begin{array}{l}\text { High study limitations } \\
\left.\text { (high risk of bias }{ }^{116}\right) \text {, } \\
\text { imprecise (no events), } \\
\text { consistent }\end{array}$ & Insufficient \\
\hline & & $\begin{array}{l}\text { Mean Apgar } \\
\text { score at } 1 \\
\text { minute }\end{array}$ & $\begin{array}{l}7.4(n=13) \text { vs. } \\
8.7(n=6)^{127}\end{array}$ & $\begin{array}{l}\text { Calculated } \\
\text { mean } \\
\text { difference: } \\
-1.3(95 \% \mathrm{Cl} \text {, } \\
-2.87 \text { to } \\
2.67)^{127} \\
\end{array}$ & $\begin{array}{l}1 \text { cohort, } \\
\mathrm{n}=19^{127}\end{array}$ & $\begin{array}{l}\text { High study limitations } \\
\left.\text { (high risk of bias }{ }^{116}\right), \\
\text { imprecise (wide Cls), } \\
\text { consistent }\end{array}$ & Insufficient \\
\hline & & $\begin{array}{l}\text { Mean Apgar } \\
\text { score at } 5 \\
\text { minutes }\end{array}$ & $\begin{array}{l}8.9(n=13) \text { vs. } \\
9.2(n=6)^{127}\end{array}$ & $\begin{array}{l}\text { Calculated } \\
\text { mean } \\
\text { difference: } \\
-0.3(95 \% \mathrm{Cl} \text {, } \\
-0.62 \text { to } \\
0.02)^{127}\end{array}$ & $\begin{array}{l}1 \text { cohort, } \\
n=19^{127}\end{array}$ & $\begin{array}{l}\text { High study limitations } \\
\text { (high risk of bias } 116), \\
\text { imprecise (wide Cls), } \\
\text { consistent }\end{array}$ & Insufficient \\
\hline
\end{tabular}

$\overline{\mathrm{CI}}=$ confidence interval; $\mathrm{n}=$ number; NICU $=$ neonatal intensive care unit; $\mathrm{RR}=$ relative risk; vs. $=$ versus. 


\section{Aripiprazole Versus Risperidone}

\section{Overview}

- The evidence for aripiprazole versus risperidone during pregnancy is insufficient to judge the risk of harms for developing gestational diabetes.

\section{Detailed Results}

One publication reported on the comparative harms of aripiprazole versus risperidone in a sample collected from two Australian tertiary obstetric hospitals (Mercy Hospital for Women in Melbourne, Victoria, and King Edward Memorial Hospital in Perth, Western Australia). ${ }^{97}$ The study was a retrospective cohort study and was rated high risk of bias. The study required a charted mental health disorder diagnosis. Only the single maternal outcome was reported.

The evidence for aripiprazole versus risperidone is insufficient to judge the risk of harms for developing gestational diabetes (Table B-98). This study found no association between aripiprazole versus risperidone use in pregnancy with any outcome.

Table B-98. Strength of evidence for comparative harms: Aripiprazole versus risperidone

\begin{tabular}{|c|c|c|c|c|c|c|c|}
\hline Population & $\begin{array}{l}\text { Intervention } \\
\text { and } \\
\text { Comparator }\end{array}$ & Outcome & $\begin{array}{l}\text { Incidence or } \\
\text { Mean Effect } \\
\text { by Arm }\end{array}$ & Results & $\begin{array}{l}\text { Study } \\
\text { Design and } \\
\text { Sample Size }\end{array}$ & $\begin{array}{l}\text { Factors That } \\
\text { Affect the } \\
\text { Strength of } \\
\text { Evidence }\end{array}$ & $\begin{array}{l}\text { Overall } \\
\text { Evidence } \\
\text { Strength } \\
\text { (Direction } \\
\text { of Effect) }\end{array}$ \\
\hline $\begin{array}{l}\text { Women } \\
\text { exposed to } \\
\text { psychotropic } \\
\text { exposure } \\
\text { medication } \\
\text { during any } \\
\text { trimester }\end{array}$ & $\begin{array}{l}\text { Aripiprazole } \\
\text { exposure vs. } \\
\text { risperidone } \\
\text { exposure }\end{array}$ & $\begin{array}{l}\text { Gestational } \\
\text { diabetes } \\
\text { diagnosed at } \\
28 \text { weeks } \\
\text { gestation }\end{array}$ & $\begin{array}{l}1 / 14(7.1 \%) \\
\text { vs. } 4 / 14 \\
(28.6 \%)^{97}\end{array}$ & $\begin{array}{l}\text { Calculated } \\
\text { RR, } 0.25 \\
(95 \% \mathrm{Cl}, 0.03 \\
\text { to } 1.97)^{97}\end{array}$ & $\begin{array}{l}1 \text { cohort, } \\
\mathrm{n}=28^{97}\end{array}$ & $\begin{array}{l}\text { High study } \\
\text { limitations (high } \\
\text { risk of bias }{ }^{97} \text { ), } \\
\text { seriously } \\
\text { imprecise (few } \\
\text { events, wide } \\
\text { Cls), consistency } \\
\text { unknown }\end{array}$ & Insufficient \\
\hline
\end{tabular}

$\overline{\mathrm{CI}}=$ confidence interval; $\mathrm{n}=$ number; $\mathrm{RR}=$ relative risk; vs. $=$ versus.

\section{Clozapine Versus Aripiprazole}

\section{Overview}

- The evidence for clozapine versus aripiprazole during pregnancy is insufficient to judge the risk of harms for developing gestational diabetes.

\section{Detailed Results}

One publication reported on the comparative harms of clozapine versus aripiprazole in a sample collected from two Australian tertiary obstetric hospitals (Mercy Hospital for Women in Melbourne, Victoria, and King Edward Memorial Hospital in Perth, Western Australia). ${ }^{97}$ The study was retrospective cohort study and was rated high risk of bias. The study required a charted mental health disorder diagnosis. Only the single maternal outcome was reported.

The evidence for clozapine versus aripiprazole is insufficient to judge the risk of harms for developing gestational diabetes (Table B-99). This study found an increased association between clozapine and gestational diabetes when compared with aripiprazole use in pregnancy. High study limitations, imprecision due to small study sizes, and no information on consistency limit our confidence in these results. 
Table B-99. Strength of evidence for comparative harms: Clozapine versus aripiprazole

\begin{tabular}{|c|c|c|c|c|c|c|c|}
\hline Population & $\begin{array}{l}\text { Intervention } \\
\text { and } \\
\text { Comparator }\end{array}$ & Outcome & $\begin{array}{l}\text { Incidence or } \\
\text { Mean Effect } \\
\text { by Arm }\end{array}$ & Results & $\begin{array}{l}\text { Study } \\
\text { Design and } \\
\text { Sample Size }\end{array}$ & $\begin{array}{l}\text { Factors That } \\
\text { Affect the } \\
\text { Strength of } \\
\text { Evidence }\end{array}$ & $\begin{array}{l}\text { Overall } \\
\text { Evidence } \\
\text { Strength } \\
\text { (Direction } \\
\text { of Effect) }\end{array}$ \\
\hline $\begin{array}{l}\text { Women } \\
\text { exposed to } \\
\text { psychotropic } \\
\text { exposure } \\
\text { medication } \\
\text { during any } \\
\text { trimester }\end{array}$ & $\begin{array}{l}\text { Clozapine } \\
\text { exposure vs. } \\
\text { aripiprazole } \\
\text { exposure }\end{array}$ & $\begin{array}{l}\text { Gestational } \\
\text { diabetes } \\
\text { diagnosed at } \\
28 \text { weeks } \\
\text { gestation }\end{array}$ & $\begin{array}{l}8 / 11(72.7 \%) \\
\text { vs. } 1 / 14 \\
(7.1 \%)^{97}\end{array}$ & $\begin{array}{l}\text { Calculated } \\
\text { RR, } 10.18 \\
(95 \% \mathrm{Cl} \\
1.49 \text { to } \\
69.67)^{97}\end{array}$ & $\begin{array}{l}1 \text { cohort, } \\
n=25^{97}\end{array}$ & $\begin{array}{l}\text { High study } \\
\text { limitations (high } \\
\text { risk of bias }^{97} \text { ), } \\
\text { seriously } \\
\text { imprecise (few } \\
\text { events, wide } \\
\text { Cls), consistency } \\
\text { unknown }\end{array}$ & Insufficient \\
\hline
\end{tabular}

$\overline{\mathrm{CI}}=$ confidence interval; $\mathrm{n}=$ number; $\mathrm{RR}=$ relative risk; vs. $=$ versus.

\section{Clozapine Versus Risperidone}

\section{Overview}

- The evidence for clozapine versus risperidone during pregnancy is insufficient to judge the risk of harms for developing gestational diabetes.

\section{Detailed Results}

One publication reported on the comparative harms of clozapine versus risperidone in a sample collected from two Australian tertiary obstetric hospitals (Mercy Hospital for Women in Melbourne, Victoria, and King Edward Memorial Hospital in Perth, Western Australia). ${ }^{97}$ The study was a retrospective cohort study and was rated high risk of bias. The study required a charted mental health disorder diagnosis. Only the single maternal outcome was reported.

The evidence for clozapine versus risperidone is insufficient to judge the risk of harms for developing gestational diabetes (Table B-100). This study found an increased association between clozapine and gestational diabetes when compared with risperidone use in pregnancy. High study limitations, imprecision due to small study sizes, and no information on consistency limit our confidence in these results.

Table B-100. Strength of evidence for comparative harms: Clozapine versus risperidone

\begin{tabular}{|c|c|c|c|c|c|c|c|}
\hline Population & $\begin{array}{l}\text { Intervention } \\
\text { and } \\
\text { Comparator }\end{array}$ & Outcome & $\begin{array}{l}\text { Incidence or } \\
\text { Mean Effect } \\
\text { by Arm }\end{array}$ & Results & $\begin{array}{l}\text { Study } \\
\text { Design and } \\
\text { Sample Size }\end{array}$ & $\begin{array}{l}\text { Factors That } \\
\text { Affect the } \\
\text { Strength of } \\
\text { Evidence }\end{array}$ & $\begin{array}{l}\text { Overall } \\
\text { Evidence } \\
\text { Strength } \\
\text { (Direction } \\
\text { of Effect) }\end{array}$ \\
\hline $\begin{array}{l}\text { Women } \\
\text { exposed to } \\
\text { psychotropic } \\
\text { exposure } \\
\text { medication } \\
\text { during any } \\
\text { trimester }\end{array}$ & $\begin{array}{l}\text { Clozapine } \\
\text { exposure vs. } \\
\text { risperidone } \\
\text { exposure }\end{array}$ & $\begin{array}{l}\text { Gestational } \\
\text { diabetes } \\
\text { diagnosed at } \\
28 \text { weeks } \\
\text { gestation }\end{array}$ & $\begin{array}{l}8 / 11(72.7 \%) \\
\text { vs. } 4 / 14 \\
(28.6 \%)^{97}\end{array}$ & $\begin{array}{l}\text { Calculated } \\
\text { RR, } 2.55 \\
(95 \% \mathrm{Cl} \\
1.03 \text { to } \\
6.28)^{97}\end{array}$ & $\begin{array}{l}1 \text { cohort, } \\
n=25^{97}\end{array}$ & $\begin{array}{l}\text { High study } \\
\text { limitations (high } \\
\text { risk of bias }{ }^{97} \text { ), } \\
\text { seriously } \\
\text { imprecise (few } \\
\text { events, wide } \\
\text { Cls), consistency } \\
\text { unknown }\end{array}$ & Insufficient \\
\hline
\end{tabular}

$\overline{\mathrm{CI}}=$ confidence interval; $\mathrm{n}=$ number; $\mathrm{RR}=$ relative risk; vs. $=$ versus. 


\section{Olanzapine Versus Aripiprazole}

\section{Overview}

- The evidence for olanzapine versus aripiprazole during pregnancy is insufficient to judge the risk of harms for developing gestational diabetes.

\section{Detailed Results}

One publication reported on the comparative harms of olanzapine versus aripiprazole in a sample collected from two Australian tertiary obstetric hospitals (Mercy Hospital for Women in Melbourne, Victoria, and King Edward Memorial Hospital in Perth, Western Australia). ${ }^{97}$ The study was a retrospective cohort study and was rated high risk of bias. The study required a charted mental health disorder diagnosis. Only the single maternal outcome was reported.

The evidence for olanzapine versus aripiprazole is insufficient to judge the risk of harms for developing gestational diabetes (Table B-101). This study found no association between olanzapine versus aripiprazole use in pregnancy with any outcome.

Table B-101. Strength of evidence for comparative harms: Olanzapine versus aripiprazole

\begin{tabular}{|c|c|c|c|c|c|c|c|}
\hline Population & $\begin{array}{l}\text { Intervention } \\
\text { and } \\
\text { Comparator }\end{array}$ & Outcome & $\begin{array}{l}\text { Incidence or } \\
\text { Mean Effect } \\
\text { by Arm }\end{array}$ & Results & $\begin{array}{l}\text { Study } \\
\text { Design and } \\
\text { Sample Size }\end{array}$ & $\begin{array}{l}\text { Factors That } \\
\text { Affect the } \\
\text { Strength of } \\
\text { Evidence }\end{array}$ & $\begin{array}{l}\text { Overall } \\
\text { Evidence } \\
\text { Strength } \\
\text { (Direction } \\
\text { of Effect) }\end{array}$ \\
\hline $\begin{array}{l}\text { Women } \\
\text { exposed to } \\
\text { psychotropic } \\
\text { exposure } \\
\text { medication } \\
\text { during any } \\
\text { trimester }\end{array}$ & $\begin{array}{l}\text { Olanzapine } \\
\text { exposure vs. } \\
\text { aripiprazole } \\
\text { exposure }\end{array}$ & $\begin{array}{l}\text { Gestational } \\
\text { diabetes } \\
\text { diagnosed at } \\
28 \text { weeks } \\
\text { gestation }\end{array}$ & $\begin{array}{l}\text { 9/49 (18.4\%) } \\
\text { vs. } 1 / 14 \\
(7.1 \%)^{97}\end{array}$ & $\begin{array}{l}\text { Calculated } \\
\text { RR, } 2.57 \\
(95 \% \mathrm{Cl}, \\
0.36 \text { to } \\
18.60)^{97}\end{array}$ & $\begin{array}{l}1 \text { cohort, } \\
n=63^{97}\end{array}$ & $\begin{array}{l}\text { High study } \\
\text { limitations (high } \\
\text { risk of bias }^{97} \text { ), } \\
\text { seriously } \\
\text { imprecise (few } \\
\text { events, wide } \\
\text { Cls), consistency } \\
\text { unknown }\end{array}$ & Insufficient \\
\hline
\end{tabular}

$\overline{\mathrm{CI}}=$ confidence interval; $\mathrm{n}=$ number; $\mathrm{RR}=$ relative risk; vs. $=$ versus.

\section{Olanzapine Versus Clozapine}

\section{Overview}

- The evidence for olanzapine versus clozapine during pregnancy is insufficient to judge the risk of harms for developing gestational diabetes.

\section{Detailed Results}

One publication reported on the comparative harms of olanzapine versus clozapine in a sample collected from two Australian tertiary obstetric hospitals (Mercy Hospital for Women in Melbourne, Victoria, and King Edward Memorial Hospital in Perth, Western Australia). ${ }^{97}$ The study was a retrospective cohort study and was rated high risk of bias. The study required a charted mental health disorder diagnosis. Only the single maternal outcome was reported.

The evidence for olanzapine versus clozapine is insufficient to judge the risk of harms for developing gestational diabetes (Table B-102). This study found a reduced association between exposure to olanzapine and gestational diabetes when compared with clozapine use in pregnancy. High study limitations, imprecision due to small study sizes, and no information on consistency limit our confidence in these results. 
Table B-102. Strength of evidence for comparative harms: Olanzapine versus clozapine

\begin{tabular}{|c|c|c|c|c|c|c|c|}
\hline Population & $\begin{array}{l}\text { Intervention } \\
\text { and } \\
\text { Comparator }\end{array}$ & Outcome & $\begin{array}{l}\text { Incidence or } \\
\text { Mean Effect } \\
\text { by Arm }\end{array}$ & Results & $\begin{array}{l}\text { Study } \\
\text { Design and } \\
\text { Sample Size }\end{array}$ & $\begin{array}{l}\text { Factors That } \\
\text { Affect the } \\
\text { Strength of } \\
\text { Evidence }\end{array}$ & $\begin{array}{l}\text { Overall } \\
\text { Evidence } \\
\text { Strength } \\
\text { (Direction } \\
\text { of Effect) }\end{array}$ \\
\hline $\begin{array}{l}\text { Women } \\
\text { exposed to } \\
\text { psychotropic } \\
\text { exposure } \\
\text { medication } \\
\text { during any } \\
\text { trimester }\end{array}$ & $\begin{array}{l}\text { Olanzapine } \\
\text { exposure vs. } \\
\text { clozapine } \\
\text { exposure }\end{array}$ & $\begin{array}{l}\text { Gestational } \\
\text { diabetes } \\
\text { diagnosed at } \\
28 \text { weeks } \\
\text { gestation }\end{array}$ & $\begin{array}{l}9 / 49(18.4 \%) \\
\text { Vs. } 8 / 11 \\
(72.7 \%)^{97}\end{array}$ & $\begin{array}{l}\text { Calculated } \\
\text { RR,: } 0.25 \\
(0.13 \text { to } 0.51) \\
\text { vs. } 8.23 \\
(4.55 \text { to } \\
14.86)^{97}\end{array}$ & $\begin{array}{l}1 \text { cohort, } \\
n=60^{97}\end{array}$ & $\begin{array}{l}\text { High study } \\
\text { limitations (high } \\
\text { risk of bias }{ }^{97} \text { ), } \\
\text { seriously } \\
\text { imprecise (few } \\
\text { events, wide Cls, } \\
\text { small Ns), } \\
\text { consistency } \\
\text { unknown }\end{array}$ & Insufficient \\
\hline
\end{tabular}

$\mathrm{CI}=$ confidence interval; $\mathrm{n}=$ number; $\mathrm{RR}=$ relative risk; $\mathrm{vs.}=$ versus.

\section{Olanzapine Versus Quetiapine}

\section{Overview}

- The evidence for olanzapine versus quetiapine during pregnancy is insufficient to judge the risk of harms for the child outcomes reported (high birth weight, low birth weight, neonatal complications [respiratory], NICU admission, preterm birth, or gestational diabetes).

\section{Detailed Results}

Two publications reported on the comparative harms of olanzapine versus quetiapine, one in a sample collected at the Emory Women's Mental Health Program in a nonrandomized prospective cohort study ${ }^{127}$ and the other from two Australian tertiary obstetric hospitals (Mercy Hospital for Women in Melbourne, Victoria, and King Edward Memorial Hospital in Perth, Western Australia) in a retrospective cohort study. ${ }^{97}$ Both studies were rated high risk of bias.

The Emory study did not require a diagnosis of schizophrenia but required antipsychotic exposure during pregnancy and a stable dose at delivery per laboratory confirmation, and it only reported fetal outcomes for $>5$ elimination half-lives at delivery as determined by highperformance liquid chromatography. ${ }^{127}$ The Australian study required a charted mental health disorder diagnosis the single maternal outcomes.

The evidence for olanzapine versus quetiapine is insufficient to judge the risk of harms for the child outcomes reported (high birth weight, low birth weight, neonatal complications [respiratory], NICU admission, preterm birth, or gestational diabetes) (Table B-103). These studies found no association between olanzapine versus quetiapine use in pregnancy with any outcome. 
Table B-103. Strength of evidence for comparative harms: Olanzapine versus quetiapine

\begin{tabular}{|c|c|c|c|c|c|c|c|}
\hline Population & $\begin{array}{l}\text { Intervention } \\
\text { and } \\
\text { Comparator }\end{array}$ & Outcome & $\begin{array}{l}\text { Incidence or } \\
\text { Mean Effect } \\
\text { by Arm }\end{array}$ & Results & $\begin{array}{l}\text { Study } \\
\text { Design and } \\
\text { Sample Size }\end{array}$ & $\begin{array}{l}\text { Factors That } \\
\text { Affect the } \\
\text { Strength of } \\
\text { Evidence }\end{array}$ & $\begin{array}{l}\text { Overall } \\
\text { Evidence } \\
\text { Strength } \\
\text { (Direction } \\
\text { of Effect) }\end{array}$ \\
\hline $\begin{array}{l}\text { Women } \\
\text { exposed to } \\
\text { psychotropic } \\
\text { exposure } \\
\text { medication } \\
\text { during any } \\
\text { trimester }\end{array}$ & $\begin{array}{l}\text { Olanzapine } \\
\text { exposure vs. } \\
\text { quetiapine } \\
\text { exposure }\end{array}$ & $\begin{array}{l}\text { Gestational } \\
\text { diabetes } \\
\text { diagnosed at } \\
28 \text { weeks } \\
\text { gestation }\end{array}$ & $\begin{array}{l}9 / 49(18.4 \%) \\
\text { Vs. } 10 / 96 \\
(10.4 \%)^{97}\end{array}$ & $\begin{array}{l}\text { Calculated } \\
\text { RR, } 1.76(95 \% \\
\mathrm{Cl}, 0.77 \text { to } \\
4.05)^{97}\end{array}$ & $\begin{array}{l}1 \text { cohort, } \\
\mathrm{n}=145^{97}\end{array}$ & $\begin{array}{l}\text { High study } \\
\text { limitations (high } \\
\text { risk of bias }{ }^{97} \text { ), } \\
\text { seriously } \\
\text { imprecise (few } \\
\text { events, wide } \\
\text { Cls), } \\
\text { consistency } \\
\text { unknown }\end{array}$ & Insufficient \\
\hline \multirow{5}{*}{$\begin{array}{l}\text { Women } \\
\text { exposed to } \\
\text { an } \\
\text { antipsychotic } \\
\text { during } \\
\text { pregnancy } \\
\text { (with a stable } \\
\text { dose at } \\
\text { delivery per } \\
\text { laboratory } \\
\text { confirmation) }\end{array}$} & $\begin{array}{l}\text { Olanzapine } \\
\text { exposure vs. } \\
\text { quetiapine } \\
\text { exposure }\end{array}$ & $\begin{array}{l}\text { High birth } \\
\text { weight } \\
\text { (>4,000 } \\
\text { grams) }\end{array}$ & $\begin{array}{l}1 / 14(7.1 \%) \\
\text { vs. } 0 / 21 \\
(0 \%)^{127}\end{array}$ & $\begin{array}{l}\text { Calculated } \\
\text { RR, } 4.4(95 \% \\
\mathrm{Cl}, 0.19 \text { to } \\
100.9)^{127}\end{array}$ & $\begin{array}{l}1 \text { cohort, } \\
n=34^{127}\end{array}$ & $\begin{array}{l}\text { High study } \\
\text { limitations (high } \\
\text { risk of bias }{ }^{116} \text { ), } \\
\text { imprecise (no } \\
\text { events), } \\
\text { consistent }\end{array}$ & Insufficient \\
\hline & & $\begin{array}{l}\text { Low birth } \\
\text { weight } \\
(<2,500 \\
\text { grams) }\end{array}$ & $\begin{array}{l}4 / 14(28.6 \%) \\
\text { vs. } 1 / 21 \\
(4.8 \%)^{127}\end{array}$ & $\begin{array}{l}\text { Calculated } \\
\text { RR, } 6(95 \% \\
\mathrm{Cl}, 0.75 \text { to } \\
48.2)^{127}\end{array}$ & $\begin{array}{l}1 \text { cohort, } \\
n=34^{127}\end{array}$ & $\begin{array}{l}\text { High study } \\
\text { limitations (high } \\
\text { risk of bias }{ }^{116} \text { ), } \\
\text { imprecise (no } \\
\text { events), } \\
\text { consistent }\end{array}$ & Insufficient \\
\hline & & $\begin{array}{l}\text { Neonatal } \\
\text { complications } \\
\text { (respiratory) }\end{array}$ & $\begin{array}{l}4 / 14(28.6 \%) \\
\text { vs. } 7 / 21 \\
(33.3 \%)^{127}\end{array}$ & $\begin{array}{l}\text { Calculated } \\
\text { RR, } 0.86(95 \% \\
\mathrm{Cl}, 0.31 \text { to } \\
2.39)^{127}\end{array}$ & $\begin{array}{l}1 \text { cohort, } \\
n=34^{127}\end{array}$ & $\begin{array}{l}\text { High study } \\
\text { limitations (high } \\
\text { risk of bias }{ }^{116} \text { ), } \\
\text { imprecise (no } \\
\text { events), } \\
\text { consistent }\end{array}$ & Insufficient \\
\hline & & $\begin{array}{l}\text { NICU } \\
\text { admission }\end{array}$ & $\begin{array}{l}4 / 14(28.6 \%) \\
\text { vs. } 2 / 21 \\
(9.5 \%)^{127}\end{array}$ & $\begin{array}{l}\text { Calculated } \\
\text { RR), } 3 \text { (95\% } \\
\mathrm{Cl}, 0.63 \text { to } \\
14.2)^{127}\end{array}$ & $\begin{array}{l}1 \text { cohort, } \\
n=34^{127}\end{array}$ & $\begin{array}{l}\text { High study } \\
\text { limitations (high } \\
\left.\text { risk of bias }{ }^{116}\right), \\
\text { imprecise (no } \\
\text { events), } \\
\text { consistent }\end{array}$ & Insufficient \\
\hline & & $\begin{array}{l}\text { Preterm birth } \\
\text { (<37 weeks) }\end{array}$ & $\begin{array}{l}3 / 14(21.4 \%) \\
\text { vs. } 1 / 21 \\
(4.8 \%)^{127}\end{array}$ & $\begin{array}{l}\text { Calculated } \\
\mathrm{RR}, 4.5(95 \% \\
\mathrm{Cl}, 0.52 \text { to } \\
39.0)^{127}\end{array}$ & $\begin{array}{l}1 \text { cohort, } \\
n=34^{127}\end{array}$ & $\begin{array}{l}\text { High study } \\
\text { limitations (high } \\
\text { risk of bias }{ }^{116} \text { ), } \\
\text { imprecise (no } \\
\text { events), } \\
\text { consistent }\end{array}$ & Insufficient \\
\hline
\end{tabular}

$\overline{\mathrm{CI}}=$ confidence interval; $\mathrm{n}=$ number; $\mathrm{NICU}=$ neonatal intensive care unit; $\mathrm{RR}=$ relative risk; vs. $=$ versus.

\section{Olanzapine Versus Risperidone}

\section{Overview}

- The evidence for olanzapine versus risperidone during pregnancy is insufficient to judge the risk of harms for the child outcomes reported (high birth weight, low birth weight, 
neonatal complications [respiratory], NICU admission, preterm birth, or development of gestational diabetes).

\section{Detailed Results}

Two publications reported on the comparative harms of olanzapine versus risperidone, one in a sample collected at the Emory Women's Mental Health Program in a nonrandomized prospective cohort study ${ }^{127}$ and the other from two Australian tertiary obstetric hospitals (Mercy Hospital for Women in Melbourne, Victoria, and King Edward Memorial Hospital in Perth, Western Australia) in a retrospective cohort study. ${ }^{97}$ Both studies were rated high risk of bias.

The Emory study did not require a diagnosis of schizophrenia but required antipsychotic exposure during pregnancy and a stable dose at delivery per laboratory confirmation, and it only reported fetal outcomes for $>5$ elimination half-lives at delivery as determined by highperformance liquid chromatography. ${ }^{127}$ The Australian study required a charted mental health disorder diagnosis the single maternal outcomes.

The evidence for olanzapine versus risperidone is insufficient to judge the risk of harms for the child outcomes reported (high birth weight, low birth weight, neonatal complications [respiratory], NICU admission, preterm birth, or gestational diabetes) (Table B-104). These studies found no association between olanzapine versus risperidone use in pregnancy with any outcome.

Table B-104. Strength of evidence for comparative harms: Olanzapine versus risperidone

\begin{tabular}{|c|c|c|c|c|c|c|c|}
\hline Population & $\begin{array}{l}\text { Intervention } \\
\text { and } \\
\text { Comparator }\end{array}$ & Outcome & $\begin{array}{l}\text { Incidence or } \\
\text { Mean Effect } \\
\text { by Arm }\end{array}$ & Results & $\begin{array}{l}\text { Study } \\
\text { Design and } \\
\text { Sample Size }\end{array}$ & $\begin{array}{l}\text { Factors That } \\
\text { Affect the } \\
\text { Strength of } \\
\text { Evidence }\end{array}$ & $\begin{array}{l}\text { Overall } \\
\text { Evidence } \\
\text { Strength } \\
\text { (Direction } \\
\text { of Effect) }\end{array}$ \\
\hline \multirow{3}{*}{$\begin{array}{l}\text { Women } \\
\text { exposed to } \\
\text { an } \\
\text { antipsychotic } \\
\text { during } \\
\text { pregnancy } \\
\text { (with a stable } \\
\text { dose at } \\
\text { delivery per } \\
\text { laboratory } \\
\text { confirmation) }\end{array}$} & $\begin{array}{l}\text { Olanzapine } \\
\text { exposure vs. } \\
\text { risperidone } \\
\text { exposure }\end{array}$ & $\begin{array}{l}\text { High birth } \\
\text { weight } \\
\text { (>4,000 } \\
\text { grams) }\end{array}$ & $\begin{array}{l}1 / 14(7.7 \%) \text { vs. } \\
0 / 6(0 \%)^{127}\end{array}$ & $\begin{array}{l}\text { Calculated } \\
\text { RR, } 1.4 \\
(95 \% \mathrm{Cl} \\
0.06 \text { to } \\
30.2)^{127}\end{array}$ & $\begin{array}{l}1 \text { cohort, } \\
n=20^{127}\end{array}$ & $\begin{array}{l}\text { High study } \\
\text { limitations (high risk } \\
\text { of bias }^{116} \text { ), seriously } \\
\text { imprecise (few } \\
\text { events, wide Cls), } \\
\text { consistency } \\
\text { unknown }\end{array}$ & Insufficient \\
\hline & & $\begin{array}{l}\text { Low birth } \\
\text { weight } \\
(<2,500 \\
\text { grams) }\end{array}$ & $\begin{array}{l}4 / 14(28.6 \%) \\
\text { vs. } 1 / 6 \\
(16.7 \%)^{127}\end{array}$ & $\begin{array}{l}\text { Calculated } \\
\text { RR, } 1.7 \\
(95 \% \mathrm{Cl} \\
0.24 \text { to } \\
12.3)^{127}\end{array}$ & $\begin{array}{l}1 \text { cohort, } \\
n=20^{127}\end{array}$ & $\begin{array}{l}\text { High study } \\
\text { limitations (high risk } \\
\text { of bias }{ }^{116} \text { ), seriously } \\
\text { imprecise (few } \\
\text { events, wide Cls), } \\
\text { consistency } \\
\text { unknown }\end{array}$ & Insufficient \\
\hline & & $\begin{array}{l}\text { Neonatal } \\
\text { complications } \\
\text { (respiratory) }\end{array}$ & $\begin{array}{l}4 / 14(28.6 \%) \\
\text { vs. } 0 / 6(0 \%)^{127}\end{array}$ & $\begin{array}{l}\text { Calculated } \\
\text { RR, } 4.2 \\
(95 \% \mathrm{Cl} \\
0.26 \text { to } \\
67.7)^{127}\end{array}$ & $\begin{array}{l}1 \text { cohort, } \\
n=20^{127}\end{array}$ & $\begin{array}{l}\text { High study } \\
\text { limitations (high risk } \\
\text { of bias }{ }^{116} \text { ), seriously } \\
\text { imprecise (few } \\
\text { events, wide Cls), } \\
\text { consistency } \\
\text { unknown }\end{array}$ & Insufficient \\
\hline
\end{tabular}




\begin{tabular}{|c|c|c|c|c|c|c|c|}
\hline Population & $\begin{array}{l}\text { Intervention } \\
\text { and } \\
\text { Comparator }\end{array}$ & Outcome & $\begin{array}{l}\text { Incidence or } \\
\text { Mean Effect } \\
\text { by Arm }\end{array}$ & Results & $\begin{array}{l}\text { Study } \\
\text { Design and } \\
\text { Sample Size }\end{array}$ & $\begin{array}{l}\text { Factors That } \\
\text { Affect the } \\
\text { Strength of } \\
\text { Evidence }\end{array}$ & $\begin{array}{l}\text { Overall } \\
\text { Evidence } \\
\text { Strength } \\
\text { (Direction } \\
\text { of Effect) }\end{array}$ \\
\hline \multirow{2}{*}{$\begin{array}{l}\text { Women } \\
\text { exposed to } \\
\text { an } \\
\text { antipsychotic } \\
\text { during } \\
\text { pregnancy } \\
\text { (with a stable } \\
\text { dose at } \\
\text { delivery per } \\
\text { laboratory } \\
\text { confirmation) } \\
\text { (continued) } \\
\end{array}$} & & $\begin{array}{l}\text { NICU } \\
\text { admission }\end{array}$ & $\begin{array}{l}4 / 14(28.6 \%) \\
\text { vs. } 0 / 6(0 \%)^{127}\end{array}$ & $\begin{array}{l}\text { Calculated } \\
\text { RR, } 4.2 \\
(95 \% \mathrm{Cl} \\
0.26 \text { to } \\
67.7)^{127}\end{array}$ & $\begin{array}{l}1 \text { cohort, } \\
n=20^{127}\end{array}$ & $\begin{array}{l}\text { High study } \\
\text { limitations (high risk } \\
\text { of bias }{ }^{116} \text { ), seriously } \\
\text { imprecise (few } \\
\text { events, wide Cls), } \\
\text { consistency } \\
\text { unknown }\end{array}$ & Insufficient \\
\hline & & $\begin{array}{l}\text { Preterm birth } \\
\text { (<37 weeks) }\end{array}$ & $\begin{array}{l}3 / 14(21.4 \%) \\
\text { vs. 0/6 }(0 \%)^{127}\end{array}$ & $\begin{array}{l}\text { Calculated } \\
\text { RR, } 3.3 \\
(95 \% \mathrm{Cl} \\
0.19 \text { to } \\
55.0)^{127}\end{array}$ & $\begin{array}{l}1 \text { cohort, } \\
n=20^{127}\end{array}$ & $\begin{array}{l}\text { High study } \\
\text { limitations (high risk } \\
\text { of bias }{ }^{116} \text { ), seriously } \\
\text { imprecise (few } \\
\text { events, wide Cls), } \\
\text { consistency } \\
\text { unknown }\end{array}$ & Insufficient \\
\hline $\begin{array}{l}\text { Women } \\
\text { exposed to } \\
\text { psychotropic } \\
\text { exposure } \\
\text { medication } \\
\text { during any } \\
\text { trimester }\end{array}$ & $\begin{array}{l}\text { Olanzapine } \\
\text { exposure vs. } \\
\text { risperidone } \\
\text { exposure }\end{array}$ & $\begin{array}{l}\text { Gestational } \\
\text { diabetes } \\
\text { diagnosed at } \\
28 \text { weeks } \\
\text { gestation }\end{array}$ & $\begin{array}{l}9 / 49(18.4 \%) \\
\text { Vs. } 4 / 14 \\
(28.6 \%)^{97}\end{array}$ & $\begin{array}{l}\text { Calculated } \\
\text { RR, } 0.64 \\
(95 \% \mathrm{Cl}, \\
0.23 \text { to } \\
1.78)^{97}\end{array}$ & $\begin{array}{l}1 \text { cohort, } \\
n=63^{97}\end{array}$ & $\begin{array}{l}\text { High study } \\
\text { limitations (high risk } \\
\text { of bias }{ }^{97} \text { ), seriously } \\
\text { imprecise (few } \\
\text { events, wide Cls), } \\
\text { consistency } \\
\text { unknown }\end{array}$ & Insufficient \\
\hline
\end{tabular}

$\overline{\mathrm{CI}}=$ confidence interval; $\mathrm{n}=$ number; $\mathrm{NICU}=$ neonatal intensive care unit; $\mathrm{RR}=$ relative risk; vs. $=$ versus.

\section{Quetiapine Versus Clozapine}

\section{Overview}

- The evidence for quetiapine versus clozapine during pregnancy is insufficient to judge the risk of harms for developing gestational diabetes.

\section{Detailed Results}

One publication reported on the comparative harms of quetiapine versus clozapine in a sample collected from two Australian tertiary obstetric hospitals (Mercy Hospital for Women in Melbourne, Victoria, and King Edward Memorial Hospital in Perth, Western Australia). ${ }^{97}$ The study was a retrospective cohort study and was rated high risk of bias. The study required a charted mental health disorder diagnosis. Only the single maternal outcome was reported.

The evidence for quetiapine versus clozapine is insufficient to judge the risk of harms for developing gestational diabetes (Table B-105). This study found a reduced association between exposure to quetiapine and gestational diabetes when compared with clozapine use in pregnancy. High study limitations, imprecision due to small study sizes, and no information on consistency limit our confidence in these results. 
Table B-105. Strength of evidence for comparative harms: Quetiapine versus clozapine

\begin{tabular}{|c|c|c|c|c|c|c|c|}
\hline Population & $\begin{array}{l}\text { Intervention } \\
\text { and } \\
\text { Comparator }\end{array}$ & Outcome & $\begin{array}{l}\text { Incidence or } \\
\text { Mean Effect } \\
\text { by Arm }\end{array}$ & Results & $\begin{array}{l}\text { Study } \\
\text { Design and } \\
\text { Sample Size }\end{array}$ & $\begin{array}{l}\text { Factors That } \\
\text { Affect the } \\
\text { Strength of } \\
\text { Evidence }\end{array}$ & $\begin{array}{l}\text { Overall } \\
\text { Evidence } \\
\text { Strength } \\
\text { (Direction } \\
\text { of Effect) }\end{array}$ \\
\hline $\begin{array}{l}\text { Women } \\
\text { exposed to } \\
\text { psychotropic } \\
\text { exposure } \\
\text { medication } \\
\text { during any } \\
\text { trimester }\end{array}$ & $\begin{array}{l}\text { Quetiapine } \\
\text { exposure vs. } \\
\text { clozapine } \\
\text { exposure }\end{array}$ & $\begin{array}{l}\text { Gestational } \\
\text { diabetes } \\
\text { diagnosed at } \\
28 \text { weeks } \\
\text { gestation }\end{array}$ & $\begin{array}{l}\text { 10/96 (10.4\%) } \\
\text { vs. } 8 / 11 \\
(72.7 \%)^{97}\end{array}$ & $\begin{array}{l}\text { Calculated } \\
\text { RR, } 0.14 \\
(95 \% \mathrm{Cl} \\
0.07 \text { to } \\
0.29)^{97}\end{array}$ & $\begin{array}{l}1 \text { cohort, } \\
n=107^{97}\end{array}$ & $\begin{array}{l}\text { High study } \\
\text { limitations (high } \\
\text { risk of bias }{ }^{97} \text { ), } \\
\text { seriously } \\
\text { imprecise (few } \\
\text { events, small } \\
\text { Ns), consistency } \\
\text { unknown }\end{array}$ & Insufficient \\
\hline
\end{tabular}

$\mathrm{CI}=$ confidence interval; $\mathrm{n}=$ number; $\mathrm{RR}=$ relative risk; vs. $=$ versus.

\section{Quetiapine Versus Aripiprazole}

\section{Overview}

- The evidence for quetiapine versus aripiprazole during pregnancy is insufficient to judge the risk of harms for developing gestational diabetes.

\section{Detailed Results}

One publication reported on the comparative harms of quetiapine versus aripiprazole in a sample collected from two Australian tertiary obstetric hospitals (Mercy Hospital for Women in Melbourne, Victoria, and King Edward Memorial Hospital in Perth, Western Australia). ${ }^{97}$ The study was a retrospective cohort study and was rated high risk of bias. The study required a charted mental health disorder diagnosis. Only the single maternal outcome was reported.

The evidence for quetiapine versus aripiprazole is insufficient to judge the risk of harms for developing gestational diabetes (Table B-106). This study found no association between olanzapine versus aripiprazole use in pregnancy with any outcome.

Table B-106. Strength of evidence for comparative harms: Quetiapine versus aripiprazole

\begin{tabular}{|c|c|c|c|c|c|c|c|}
\hline Population & $\begin{array}{l}\text { Intervention } \\
\text { and } \\
\text { Comparator }\end{array}$ & Outcome & $\begin{array}{l}\text { Incidence or } \\
\text { Mean Effect } \\
\text { by Arm }\end{array}$ & Results & $\begin{array}{l}\text { Study } \\
\text { Design and } \\
\text { Sample Size }\end{array}$ & $\begin{array}{l}\text { Factors That } \\
\text { Affect the } \\
\text { Strength of } \\
\text { Evidence }\end{array}$ & $\begin{array}{l}\text { Overall } \\
\text { Evidence } \\
\text { Strength } \\
\text { (Direction } \\
\text { of Effect) }\end{array}$ \\
\hline $\begin{array}{l}\text { Women } \\
\text { exposed to } \\
\text { psychotropic } \\
\text { exposure } \\
\text { medication } \\
\text { during any } \\
\text { trimester }\end{array}$ & $\begin{array}{l}\text { Quetiapine } \\
\text { exposure vs. } \\
\text { aripiprazole } \\
\text { exposure }\end{array}$ & $\begin{array}{l}\text { Gestational } \\
\text { diabetes } \\
\text { diagnosed at } \\
28 \text { weeks } \\
\text { gestation }\end{array}$ & $\begin{array}{l}10 / 96(10.4 \%) \\
\text { vs. } 1 / 14 \\
(7.1 \%)^{97}\end{array}$ & $\begin{array}{l}\text { Calculated } \\
\text { RR, } 1.46 \\
(95 \% \mathrm{Cl} \\
0.20 \text { to } \\
10.54)^{97}\end{array}$ & $\begin{array}{l}1 \text { cohort, } \\
\mathrm{n}=110^{97}\end{array}$ & $\begin{array}{l}\text { High study } \\
\text { limitations (high } \\
\text { risk of bias }{ }^{97} \text { ), } \\
\text { seriously } \\
\text { imprecise (few } \\
\text { events, wide } \\
\text { Cls), consistency } \\
\text { unknown }\end{array}$ & Insufficient \\
\hline
\end{tabular}

$\overline{\mathrm{CI}}=$ confidence interval; $\mathrm{n}=$ number; $\mathrm{RR}=$ relative risk; vs. $=$ versus.

\section{Quetiapine Versus Risperidone}

\section{Overview}

- The evidence for quetiapine versus risperidone during pregnancy is insufficient to judge the risk of harms for the child outcomes reported (high birth weight, low birth weight, 
neonatal complications [respiratory], NICU admission, preterm birth, or gestational diabetes).

\section{Detailed Results}

Two publications reported on the comparative harms of quetiapine versus risperidone, one in a sample collected at the Emory Women's Mental Health Program in a nonrandomized prospective cohort study ${ }^{127}$ and the other from two Australian tertiary obstetric hospitals (Mercy Hospital for Women in Melbourne, Victoria, and King Edward Memorial Hospital in Perth, Western Australia) in a retrospective cohort study. ${ }^{97}$ Both studies were rated high risk of bias.

The Emory study did not require a diagnosis of schizophrenia but required antipsychotic exposure during pregnancy and a stable dose at delivery per laboratory confirmation, and it only reported fetal outcomes for $>5$ elimination half-lives at delivery as determined by highperformance liquid chromatography. ${ }^{127}$ The Australian study required a charted mental health disorder diagnosis the single maternal outcomes.

The evidence for quetiapine versus risperidone is insufficient to judge the risk of harms for the child outcomes reported (high birth weight, low birth weight, neonatal complications [respiratory], NICU admission, preterm birth, or gestational diabetes) (Table B-107). These studies found no association between olanzapine versus risperidone use in pregnancy with any outcome.

Table B-107. Strength of evidence for comparative harms: Quetiapine versus risperidone

\begin{tabular}{|c|c|c|c|c|c|c|c|}
\hline Population & $\begin{array}{l}\text { Intervention } \\
\text { and } \\
\text { Comparator }\end{array}$ & Outcome & $\begin{array}{l}\text { Incidence or } \\
\text { Mean Effect } \\
\text { by Arm }\end{array}$ & Results & $\begin{array}{l}\text { Study } \\
\text { Design and } \\
\text { Sample Size }\end{array}$ & $\begin{array}{l}\text { Factors That Affect } \\
\text { the Strength of } \\
\text { Evidence }\end{array}$ & $\begin{array}{l}\text { Overall } \\
\text { Evidence } \\
\text { Strength } \\
\text { (Direction of } \\
\text { Effect) }\end{array}$ \\
\hline $\begin{array}{l}\text { Women } \\
\text { exposed to } \\
\text { psychotropic } \\
\text { exposure } \\
\text { medication } \\
\text { during any } \\
\text { trimester }\end{array}$ & $\begin{array}{l}\text { Quetiapine } \\
\text { exposure vs. } \\
\text { risperidone } \\
\text { exposure }\end{array}$ & $\begin{array}{l}\text { Gestational } \\
\text { diabetes } \\
\text { diagnosed at } \\
28 \text { weeks } \\
\text { gestation }\end{array}$ & $\begin{array}{l}10 / 96(10.4 \%) \\
\text { vs. } 4 / 14 \\
(28.6 \%)^{97}\end{array}$ & $\begin{array}{l}\text { Calculated } \\
\text { RR, } 0.36 \\
(95 \% \mathrm{Cl} \\
0.13 \text { to } \\
1.01)^{97}\end{array}$ & $\begin{array}{l}1 \text { cohort, } \\
n=110^{97}\end{array}$ & $\begin{array}{l}\text { High study limitations } \\
\text { (high risk of bias }{ }^{97} \text { ), } \\
\text { seriously imprecise } \\
\text { (few events, wide } \\
\text { Cls), consistency } \\
\text { unknown }\end{array}$ & Insufficient \\
\hline \multirow{3}{*}{$\begin{array}{l}\text { Women } \\
\text { exposed to an } \\
\text { antipsychotic } \\
\text { during } \\
\text { pregnancy } \\
\text { (with a stable } \\
\text { dose at } \\
\text { delivery per } \\
\text { laboratory } \\
\text { confirmation) } \\
\end{array}$} & $\begin{array}{l}\text { Quetiapine } \\
\text { exposure vs. } \\
\text { risperidone } \\
\text { exposure }\end{array}$ & $\begin{array}{l}\text { High birth } \\
\text { weight } \\
\text { (>4,000 } \\
\text { grams) }\end{array}$ & $\begin{array}{l}0 / 21(0 \%) \text { vs. } \\
0 / 6(0 \%)^{127}\end{array}$ & No events & $\begin{array}{l}1 \text { cohort, } \\
n=27^{127}\end{array}$ & $\begin{array}{l}\text { High study limitations } \\
\text { (high risk of bias }{ }^{116} \text { ), } \\
\text { imprecise (no } \\
\text { events), consistent }\end{array}$ & Insufficient \\
\hline & & $\begin{array}{l}\text { Low birth } \\
\text { weight } \\
(<2,500 \\
\text { grams })\end{array}$ & $\begin{array}{l}1 / 21(4.8 \%) \\
\text { vs. } 1 / 6 \\
(16.7 \%)^{127}\end{array}$ & $\begin{array}{l}\text { Calculated } \\
\text { RR, } 0.29 \\
(95 \% \mathrm{Cl} \\
0.02 \text { to } \\
3.92)^{127}\end{array}$ & $\begin{array}{l}1 \text { cohort, } \\
n=27^{127}\end{array}$ & $\begin{array}{l}\text { High study limitations } \\
\text { (high risk of bias }^{116} \text { ), } \\
\text { seriously imprecise } \\
\text { (few events, wide } \\
\text { Cls), consistency } \\
\text { unknown }\end{array}$ & $x^{2}$ \\
\hline & & $\begin{array}{l}\text { Neonatal } \\
\text { complications } \\
\text { (respiratory) }\end{array}$ & $\begin{array}{l}7 / 21(33.3 \%) \\
\text { vs. } 0 / 6(0 \%)^{127}\end{array}$ & $\begin{array}{l}\text { Calculated } \\
\text { RR, } 4.8 \\
(95 \% \mathrm{Cl} \\
0.31 \text { to } \\
73.4)^{127}\end{array}$ & $\begin{array}{l}1 \text { cohort, } \\
n=27^{127}\end{array}$ & $\begin{array}{l}\text { High study limitations } \\
\text { (high risk of bias }^{116} \text { ), } \\
\text { seriously imprecise } \\
\text { (few events, wide } \\
\text { Cls), consistency } \\
\text { unknown }\end{array}$ & Insufficient \\
\hline
\end{tabular}




\begin{tabular}{|c|c|c|c|c|c|c|c|}
\hline Population & $\begin{array}{l}\text { Intervention } \\
\text { and } \\
\text { Comparator }\end{array}$ & Outcome & $\begin{array}{l}\text { Incidence or } \\
\text { Mean Effect } \\
\text { by Arm }\end{array}$ & Results & $\begin{array}{l}\text { Study } \\
\text { Design and } \\
\text { Sample Size }\end{array}$ & $\begin{array}{l}\text { Factors That Affect } \\
\text { the Strength of } \\
\text { Evidence }\end{array}$ & $\begin{array}{l}\text { Overall } \\
\text { Evidence } \\
\text { Strength } \\
\text { (Direction of } \\
\text { Effect) }\end{array}$ \\
\hline \multirow{2}{*}{$\begin{array}{l}\text { Women } \\
\text { exposed to } \\
\text { an } \\
\text { antipsychotic } \\
\text { during } \\
\text { pregnancy } \\
\text { (with a stable } \\
\text { dose at } \\
\text { delivery per } \\
\text { laboratory } \\
\text { confirmation) } \\
\text { (continued) } \\
\end{array}$} & & $\begin{array}{l}\mathrm{NICU} \\
\text { admission }\end{array}$ & $\begin{array}{l}2 / 21(9.5 \%) \\
\text { vs. } 0 / 6(0 \%)^{127}\end{array}$ & $\begin{array}{l}\text { Calculated } \\
\text { RR, } 1.6 \\
(95 \% \mathrm{Cl} \\
0.09 \text { to } \\
29.3)^{127}\end{array}$ & $\begin{array}{l}1 \text { cohort, } \\
n=27^{127}\end{array}$ & $\begin{array}{l}\text { High study limitations } \\
\text { (high risk of bias }{ }^{116} \text { ), } \\
\text { seriously imprecise } \\
\text { (few events, wide } \\
\text { Cls), consistency } \\
\text { unknown }\end{array}$ & Insufficient \\
\hline & & $\begin{array}{l}\text { Preterm birth } \\
\text { (<37 weeks) }\end{array}$ & $\begin{array}{l}1 / 21(4.8 \%) \\
\text { vs. } 0 / 6(0 \%)^{127}\end{array}$ & $\begin{array}{l}\text { Calculated } \\
\text { RR, } 0.95 \\
(95 \% \mathrm{Cl} \\
0.04 \text { to } \\
20.9)^{127}\end{array}$ & $\begin{array}{l}1 \text { cohort, } \\
n=27^{127}\end{array}$ & $\begin{array}{l}\text { High study limitations } \\
\text { (high risk of bias }{ }^{116} \text { ), } \\
\text { seriously imprecise } \\
\text { (few events, wide } \\
\text { Cls), consistency } \\
\text { unknown }\end{array}$ & Insufficient \\
\hline
\end{tabular}

$\overline{\mathrm{CI}}=$ confidence interval; $\mathrm{n}=$ number; $\mathrm{NICU}=$ neonatal intensive care unit; $\mathrm{RR}=$ relative risk; vs. $=$ versus.

\section{First-Generation Antipsychotics Versus Second-Generation Antipsychotics}

\section{Overview}

- The evidence for first-generation antipsychotics (as a class) versus second-generation antipsychotics (as a class) during pregnancy is insufficient to judge the risk of harms for the child outcomes reported (large for gestational age, low birth weight, small for gestational age preterm birth, or abnormal neuromotor development).

\section{Detailed Results}

Three publications reported on the comparative harms of first-generation antipsychotics versus second-generation antipsychotics, ${ }^{85,119,125}$ but only two provided usable data. ${ }^{85,119}$ These samples were drawn from the Emory Women's Mental Health Program ${ }^{119}$ and the Taiwan National Health Insurance Research Dataset..$^{85}$ The studies were each nonrandomized cohort studies. One was rated some concern, ${ }^{119}$ and one was rated high risk of bias. ${ }^{85}$

Both publications focused on exposure during pregnancy. One study providing data required a maternal diagnosis of schizophrenia, ${ }^{85}$ while the other only required exposure to antipsychotics during pregnancy without a confirmed schizophrenia diagnosis. ${ }^{119}$ Only fetal outcomes alone were reported.

The evidence for first-generation antipsychotics versus second-generation antipsychotics is insufficient to judge the risk of harms for the child outcomes reported (large for gestational age, low birth weight, small for gestational age preterm birth, or abnormal neuromotor development) (Table B-108). These studies found no association between first-generation antipsychotics versus second-generation antipsychotics use in pregnancy with any outcome. 
Table B-108. Strength of evidence for comparative harms: First-generation antipsychotics versus second-generation antipsychotics

\begin{tabular}{|c|c|c|c|c|c|c|c|}
\hline Population & $\begin{array}{l}\text { Intervention } \\
\text { and } \\
\text { Comparator }\end{array}$ & Outcome & $\begin{array}{l}\text { Incidence or } \\
\text { Mean Effect } \\
\text { by Arm }\end{array}$ & Results & $\begin{array}{l}\text { Study } \\
\text { Design and } \\
\text { Sample Size }\end{array}$ & $\begin{array}{l}\text { Factors That } \\
\text { Affect the } \\
\text { Strength of } \\
\text { Evidence }\end{array}$ & $\begin{array}{l}\text { Overall } \\
\text { Evidence } \\
\text { Strength } \\
\text { (Direction } \\
\text { of Effect) }\end{array}$ \\
\hline $\begin{array}{l}\text { Women } \\
\text { exposed to } \\
\text { an } \\
\text { antipsychotic } \\
\text { during } \\
\text { pregnancy }\end{array}$ & $\begin{array}{l}\text { First- } \\
\text { generation } \\
\text { antipsychotic } \\
\text { vs. second- } \\
\text { generation } \\
\text { antipsychotic }\end{array}$ & $\begin{array}{l}\text { Abnormal } \\
\text { neuromotor } \\
\text { development } \\
\text { (measured } \\
\text { by INFANIB, } \\
\text { higher score } \\
\text { is better) }\end{array}$ & NR & $\begin{array}{l}\text { After controlling for } \\
\text { maternal psychiatric } \\
\text { history using the } \\
\text { severity/ } \\
\text { chronicity index, } \\
\text { results revealed a } \\
\text { modest but } \\
\text { nonsignificant } \\
\text { difference } \\
\text { (F2,21=2.96; } p=0.10) \text {, } \\
\text { with adjusted means } \\
\text { suggesting higher } \\
\text { scores for first- } \\
\text { generation } \\
\text { antipsychotic-exposed } \\
\text { infants (mean } \\
\text { [SE]=67.1 [1.84]) than } \\
\text { with second- } \\
\text { generation } \\
\text { antipsychotic-exposed } \\
\text { infants (mean } \\
\text { [SE]=62.9 [1.60]) } \text { (119 }^{2}\end{array}$ & $\begin{array}{l}1 \text { cohort, } \\
\mathrm{n}=21^{119}\end{array}$ & $\begin{array}{l}\text { Moderate } \\
\text { study } \\
\text { limitations, } \\
\text { likely imprecise } \\
\text { (small Ns), } \\
\text { consistency } \\
\text { unknown }\end{array}$ & Insufficient \\
\hline \multirow[t]{3}{*}{$\begin{array}{l}\text { Women with } \\
\text { schizophrenia } \\
\text { and exposed } \\
\text { to an } \\
\text { antipsychotic } \\
\text { during } \\
\text { pregnancy }\end{array}$} & $\begin{array}{l}\text { First- } \\
\text { generation } \\
\text { antipsychotic } \\
\text { vs. second- } \\
\text { generation } \\
\text { antipsychotic }\end{array}$ & $\begin{array}{l}\text { Large for } \\
\text { gestational } \\
\text { age } \\
\text { (birthweight } \\
>10^{\text {th }} \\
\text { percentile for } \\
\text { gestational } \\
\text { age) }\end{array}$ & $\begin{array}{l}15 / 194 \\
(7.7 \%) \text { vs. } \\
3 / 48(6.3 \%)^{85} \\
\end{array}$ & $\begin{array}{l}\text { Calculated RR, } 1.23 \\
(95 \% \mathrm{Cl}, 0.37 \text { to } \\
4.10)^{127}\end{array}$ & $\begin{array}{l}1 \text { cohort, } \\
\mathrm{n}=242^{127}\end{array}$ & $\begin{array}{l}\text { High study } \\
\text { limitations } \\
\text { (high risk of } \text { bias }^{127} \text { ), } \\
\text { seriously } \\
\text { imprecise (few } \\
\text { events, wide } \\
\text { Cls), } \\
\text { consistency } \\
\text { unknown }\end{array}$ & Insufficient \\
\hline & & $\begin{array}{l}\text { Low birth } \\
\text { weight } \\
(<2,500 \\
\text { grams })\end{array}$ & $\begin{array}{l}16 / 194 \\
(8.2 \%) \text { vs. } \\
6 / 48 \\
(12.5 \%)^{85}\end{array}$ & $\begin{array}{l}\text { Calculated RR, } 0.66 \\
(95 \% \mathrm{Cl}, 0.27 \text { to } \\
1.60)^{127}\end{array}$ & $\begin{array}{l}1 \text { cohort, } \\
n=242^{127}\end{array}$ & $\begin{array}{l}\text { High study } \\
\text { limitations } \\
\text { (high risk of } \text { bias }^{127} \text { ), } \\
\text { seriously } \\
\text { imprecise (few } \\
\text { events, wide } \\
\text { Cls), } \\
\text { consistency } \\
\text { unknown }\end{array}$ & Insufficient \\
\hline & & $\begin{array}{l}\text { Small for } \\
\text { gestational } \\
\text { age } \\
\text { (birthweight } \\
<10 \text { th } \\
\text { percentile for } \\
\text { gestational } \\
\text { age) }\end{array}$ & $\begin{array}{l}49 / 194 \\
(25.3 \%) \text { vs. } \\
10 / 48 \\
(20.8 \%)^{85}\end{array}$ & $\begin{array}{l}\text { Calculated RR, } 1.21 \\
(95 \% \mathrm{Cl}, 0.66 \text { to } \\
2.21)^{127}\end{array}$ & $\begin{array}{l}1 \text { cohort, } \\
\mathrm{n}=242^{127}\end{array}$ & $\begin{array}{l}\text { High study } \\
\text { limitations } \\
\text { (high risk of } \text { bias }^{127} \text { ), } \\
\text { seriously } \\
\text { imprecise (few } \\
\text { events, wide } \\
\text { Cls), } \\
\text { consistency } \\
\text { unknown }\end{array}$ & Insufficient \\
\hline
\end{tabular}




\begin{tabular}{|c|c|c|c|c|c|c|c|}
\hline Population & $\begin{array}{l}\text { Intervention } \\
\text { and } \\
\text { Comparator }\end{array}$ & Outcome & $\begin{array}{l}\text { Incidence or } \\
\text { Mean Effect } \\
\text { by Arm }\end{array}$ & Results & $\begin{array}{l}\text { Study } \\
\text { Design and } \\
\text { Sample Size }\end{array}$ & $\begin{array}{l}\text { Factors That } \\
\text { Affect the } \\
\text { Strength of } \\
\text { Evidence }\end{array}$ & $\begin{array}{l}\text { Overall } \\
\text { Evidence } \\
\text { Strength } \\
\text { (Direction } \\
\text { of Effect) }\end{array}$ \\
\hline $\begin{array}{l}\text { Women with } \\
\text { schizophrenia } \\
\text { and exposed } \\
\text { to an } \\
\text { antipsychotic } \\
\text { during } \\
\text { pregnancy } \\
\text { (continued) }\end{array}$ & & Preterm birth & $\begin{array}{l}35 / 194 \\
(18.0 \%) \text { vs. } \\
6 / 48 \\
(12.5 \%)^{85}\end{array}$ & $\begin{array}{l}\text { Calculated RR, } 1.44 \\
(95 \% \mathrm{Cl}, 0.64 \text { to } \\
3.23)^{127}\end{array}$ & $\begin{array}{l}1 \text { cohort, } \\
n=242^{127}\end{array}$ & $\begin{array}{l}\text { High study } \\
\text { limitations } \\
\text { (high risk of } \text { bias }^{127} \text { ), } \\
\text { seriously } \\
\text { imprecise (few } \\
\text { events, wide } \\
\text { Cls), } \\
\text { consistency } \\
\text { unknown }\end{array}$ & Insufficient \\
\hline
\end{tabular}

$\mathrm{CI}=$ confidence interval; INFANIB $=$ Infant Neurological International Battery; $\mathrm{n}=$ number; $\mathrm{RR}=$ relative risk; $\mathrm{SE}=$ standard error; vs. $=$ versus.

\section{Second-Generation Antipsychotics Versus Mood Stabilizers}

\section{Overview}

- The evidence for specific second-generation antipsychotics versus specific mood stabilizers during pregnancy is insufficient to judge the risk of harms for developing gestational diabetes.

\section{Detailed Results}

One publication reported on the comparative harms of a number of second-generation antipsychotics (quetiapine, olanzapine, risperidone, clozapine, or aripiprazole) versus mood stabilizers (lithium or lamotrigine), respectively, in a sample collected from two Australian tertiary obstetric hospitals (Mercy Hospital for Women in Melbourne, Victoria, and King Edward Memorial Hospital in Perth, Western Australia). ${ }^{97}$ The study was a retrospective cohort study and rated high risk of bias. The study required a charted mental health disorder diagnosis. Only the single maternal outcome was reported. There were 10 specific comparisons available of a single agent versus a single agent (Table B-109).

The evidence for specific second-generation antipsychotics versus specific mood stabilizers is insufficient to judge the risk of harms for developing gestational diabetes (Table B-109). This study found no association between olanzapine versus aripiprazole use in pregnancy. One study found an increased association between gestational diabetes and exposure to clozapine when compared with exposure to lamotrigine or lithium. High study limitations, imprecision due to small study sizes, and no information on consistency limit our confidence in these results. 
Table B-109. Strength of evidence for comparative harms: Second-generation antipsychotics versus mood stabilizers

\begin{tabular}{|c|c|c|c|c|c|c|c|}
\hline Population & $\begin{array}{l}\text { Intervention } \\
\text { and } \\
\text { Comparator }\end{array}$ & Outcome & $\begin{array}{l}\text { Incidence or } \\
\text { Mean Effect } \\
\text { by Arm }\end{array}$ & Results & $\begin{array}{l}\text { Study } \\
\text { Design and } \\
\text { Sample Size }\end{array}$ & $\begin{array}{l}\text { Factors That } \\
\text { Affect the } \\
\text { Strength of } \\
\text { Evidence }\end{array}$ & $\begin{array}{l}\text { Overall } \\
\text { Evidence } \\
\text { Strength } \\
\text { (Direction } \\
\text { of Effect) }\end{array}$ \\
\hline $\begin{array}{l}\text { Women } \\
\text { exposed to } \\
\text { psychotropic } \\
\text { exposure } \\
\text { medication } \\
\text { during any } \\
\text { trimester }\end{array}$ & $\begin{array}{l}\text { Olanzapine } \\
\text { exposure vs. } \\
\text { lithium } \\
\text { exposure }\end{array}$ & $\begin{array}{l}\text { Gestational } \\
\text { diabetes } \\
\text { diagnosed at } \\
28 \text { weeks } \\
\text { gestation }\end{array}$ & $\begin{array}{l}9 / 49(18.4 \%) \\
\text { vs. } 2 / 17 \\
(11.7 \%)^{97}\end{array}$ & $\begin{array}{l}\text { Calculated } \\
\text { RR, } 1.56 \\
(95 \% \mathrm{Cl} \\
0.37 \text { to } \\
6.52)^{97}\end{array}$ & $\begin{array}{l}1 \text { cohort, } \\
n=66^{97}\end{array}$ & $\begin{array}{l}\text { High study } \\
\text { limitations (high } \\
\text { risk of bias }{ }^{97} \text { ), } \\
\text { seriously } \\
\text { imprecise (few } \\
\text { events, wide } \\
\text { Cls), consistency } \\
\text { unknown }\end{array}$ & Insufficient \\
\hline $\begin{array}{l}\text { Women } \\
\text { exposed to } \\
\text { psychotropic } \\
\text { exposure } \\
\text { medication } \\
\text { during any } \\
\text { trimester }\end{array}$ & $\begin{array}{l}\text { Olanzapine } \\
\text { exposure vs. } \\
\text { lamotrigine } \\
\text { exposure }\end{array}$ & $\begin{array}{l}\text { Gestational } \\
\text { diabetes } \\
\text { diagnosed at } \\
28 \text { weeks } \\
\text { gestation }\end{array}$ & $\begin{array}{l}9 / 49(18.4 \%) \\
\text { vs. } 2 / 19 \\
(10.5 \%)^{97}\end{array}$ & $\begin{array}{l}\text { Calculated } \\
\text { RR, } 1.74 \\
(95 \% \mathrm{Cl} \\
0.41 \text { to } \\
7.35)^{97}\end{array}$ & $\begin{array}{l}1 \text { cohort, } \\
\mathrm{n}=68^{97}\end{array}$ & $\begin{array}{l}\text { High study } \\
\text { limitations (high } \\
\text { risk of bias }{ }^{97} \text { ), } \\
\text { seriously } \\
\text { imprecise (few } \\
\text { events, wide } \\
\text { Cls), consistency } \\
\text { unknown }\end{array}$ & Insufficient \\
\hline $\begin{array}{l}\text { Women } \\
\text { exposed to } \\
\text { psychotropic } \\
\text { exposure } \\
\text { medication } \\
\text { during any } \\
\text { trimester }\end{array}$ & $\begin{array}{l}\text { Quetiapine } \\
\text { exposure vs. } \\
\text { lithium } \\
\text { exposure }\end{array}$ & $\begin{array}{l}\text { Gestational } \\
\text { diabetes } \\
\text { diagnosed at } \\
28 \text { weeks } \\
\text { gestation }\end{array}$ & $\begin{array}{l}10 / 96(10.4 \%) \\
\text { vs. } 2 / 17 \\
(11.7 \%)^{97}\end{array}$ & $\begin{array}{l}\text { Calculated } \\
\text { RR, } 0.99 \\
(95 \% \mathrm{Cl} \\
0.24 \text { to } \\
4.16)^{97}\end{array}$ & $\begin{array}{l}1 \text { cohort, } \\
n=113^{97}\end{array}$ & $\begin{array}{l}\text { High study } \\
\text { limitations (high } \\
\text { risk of bias }{ }^{97} \text { ), } \\
\text { seriously } \\
\text { imprecise (few } \\
\text { events, wide } \\
\text { Cls), consistency } \\
\text { unknown }\end{array}$ & Insufficient \\
\hline $\begin{array}{l}\text { Women } \\
\text { exposed to } \\
\text { psychotropic } \\
\text { exposure } \\
\text { medication } \\
\text { during any } \\
\text { trimester }\end{array}$ & $\begin{array}{l}\text { Quetiapine } \\
\text { exposure vs. } \\
\text { lamotrigine } \\
\text { exposure }\end{array}$ & $\begin{array}{l}\text { Gestational } \\
\text { diabetes } \\
\text { diagnosed at } \\
28 \text { weeks } \\
\text { gestation }\end{array}$ & $\begin{array}{l}10 / 96(10.4 \%) \\
\text { vs. } 2 / 19 \\
(10.5 \%)^{97}\end{array}$ & $\begin{array}{l}\text { Calculated } \\
\text { RR, } 0.89 \\
(95 \% \mathrm{Cl}, \\
0.21 \text { to } \\
3.69)^{97}\end{array}$ & $\begin{array}{l}1 \text { cohort, } \\
n=115^{97}\end{array}$ & $\begin{array}{l}\text { High study } \\
\text { limitations (high } \\
\text { risk of bias }{ }^{97} \text { ), } \\
\text { seriously } \\
\text { imprecise (few } \\
\text { events, wide } \\
\text { Cls), consistency } \\
\text { unknown }\end{array}$ & Insufficient \\
\hline $\begin{array}{l}\text { Women } \\
\text { exposed to } \\
\text { psychotropic } \\
\text { exposure } \\
\text { medication } \\
\text { during any } \\
\text { trimester }\end{array}$ & $\begin{array}{l}\text { Aripiprazole } \\
\text { exposure vs. } \\
\text { lithium } \\
\text { exposure }\end{array}$ & $\begin{array}{l}\text { Gestational } \\
\text { diabetes } \\
\text { diagnosed at } \\
28 \text { weeks } \\
\text { gestation }\end{array}$ & $\begin{array}{l}1 / 14(7.1 \%) \\
\text { vs. } 2 / 17 \\
(11.7 \%)^{97}\end{array}$ & $\begin{array}{l}\text { Calculated } \\
\mathrm{RR}, 0.61 \\
(95 \% \mathrm{Cl} \\
0.06 \text { to } \\
6.02)^{97}\end{array}$ & $\begin{array}{l}1 \text { cohort, } \\
n=31^{97}\end{array}$ & $\begin{array}{l}\text { High study } \\
\text { limitations (high } \\
\text { risk of bias }{ }^{97} \text { ), } \\
\text { seriously } \\
\text { imprecise (few } \\
\text { events, wide } \\
\text { Cls), consistency } \\
\text { unknown }\end{array}$ & Insufficient \\
\hline $\begin{array}{l}\text { Women } \\
\text { exposed to } \\
\text { psychotropic } \\
\text { exposure } \\
\text { medication } \\
\text { during any } \\
\text { trimester }\end{array}$ & $\begin{array}{l}\text { Aripiprazole } \\
\text { exposure vs. } \\
\text { lamotrigine } \\
\text { exposure }\end{array}$ & $\begin{array}{l}\text { Gestational } \\
\text { diabetes } \\
\text { diagnosed at } \\
28 \text { weeks } \\
\text { gestation }\end{array}$ & $\begin{array}{l}1 / 14(7.1 \%) \\
\text { vs. } 2 / 19 \\
(10.5 \%)^{97}\end{array}$ & $\begin{array}{l}\text { Calculated } \\
\text { RR, 0.68 } \\
(95 \% \mathrm{Cl}, \\
0.07 \text { to } \\
6.76)^{153}\end{array}$ & $\begin{array}{l}1 \text { cohort, } \\
n=33^{97}\end{array}$ & $\begin{array}{l}\text { High study } \\
\text { limitations (high } \\
\text { risk of bias }{ }^{97} \text { ), } \\
\text { seriously } \\
\text { imprecise (few } \\
\text { events, wide } \\
\text { Cls), consistency } \\
\text { unknown }\end{array}$ & Insufficient \\
\hline
\end{tabular}




\begin{tabular}{|c|c|c|c|c|c|c|c|}
\hline Population & $\begin{array}{l}\text { Intervention } \\
\text { and } \\
\text { Comparator }\end{array}$ & Outcome & $\begin{array}{l}\text { Incidence or } \\
\text { Mean Effect } \\
\text { by Arm }\end{array}$ & Results & $\begin{array}{l}\text { Study } \\
\text { Design and } \\
\text { Sample Size }\end{array}$ & $\begin{array}{l}\text { Factors That } \\
\text { Affect the } \\
\text { Strength of } \\
\text { Evidence }\end{array}$ & $\begin{array}{l}\text { Overall } \\
\text { Evidence } \\
\text { Strength } \\
\text { (Direction } \\
\text { of Effect) }\end{array}$ \\
\hline $\begin{array}{l}\text { Women } \\
\text { exposed to } \\
\text { psychotropic } \\
\text { exposure } \\
\text { medication } \\
\text { during any } \\
\text { trimester }\end{array}$ & $\begin{array}{l}\text { Clozapine } \\
\text { exposure vs. } \\
\text { lithium } \\
\text { exposure }\end{array}$ & $\begin{array}{l}\text { Gestational } \\
\text { diabetes } \\
\text { diagnosed at } \\
28 \text { weeks } \\
\text { gestation }\end{array}$ & $\begin{array}{l}8 / 11(72.7 \%) \\
\text { vs. } 2 / 17 \\
(11.7 \%)^{97}\end{array}$ & $\begin{array}{l}\text { Calculated } \\
\text { RR, } 6.18 \\
(95 \% \mathrm{Cl}, \\
1.60 \text { to } \\
23.87)^{97}\end{array}$ & $\begin{array}{l}1 \text { cohort, } \\
n=28^{97}\end{array}$ & $\begin{array}{l}\text { High study } \\
\text { limitations (high } \\
\text { risk of bias }{ }^{97} \text { ), } \\
\text { seriously } \\
\text { imprecise (few } \\
\text { events, wide } \\
\text { Cls), consistency } \\
\text { unknown }\end{array}$ & Insufficient \\
\hline $\begin{array}{l}\text { Women } \\
\text { exposed to } \\
\text { psychotropic } \\
\text { exposure } \\
\text { medication } \\
\text { during any } \\
\text { trimester }\end{array}$ & $\begin{array}{l}\text { Clozapine } \\
\text { exposure vs. } \\
\text { lamotrigine } \\
\text { exposure }\end{array}$ & $\begin{array}{l}\text { Gestational } \\
\text { diabetes } \\
\text { diagnosed at } \\
28 \text { weeks } \\
\text { gestation }\end{array}$ & $\begin{array}{l}8 / 11(72.7 \%) \\
\text { vs. } 2 / 19 \\
(10.5 \%)^{97}\end{array}$ & $\begin{array}{l}\text { Calculated } \\
\text { RR, 6.91 } \\
(95 \% \mathrm{Cl}, \\
1.77 \text { to } \\
26.92)^{97}\end{array}$ & $\begin{array}{l}1 \text { cohort, } \\
\mathrm{n}=30^{97}\end{array}$ & $\begin{array}{l}\text { High study } \\
\text { limitations (high } \\
\text { risk of bias }{ }^{97} \text { ), } \\
\text { seriously } \\
\text { imprecise (few } \\
\text { events, wide } \\
\text { Cls), consistency } \\
\text { unknown }\end{array}$ & Insufficient \\
\hline $\begin{array}{l}\text { Women } \\
\text { exposed to } \\
\text { psychotropic } \\
\text { exposure } \\
\text { medication } \\
\text { during any } \\
\text { trimester }\end{array}$ & $\begin{array}{l}\text { Risperidone } \\
\text { exposure vs. } \\
\text { lithium } \\
\text { exposure }\end{array}$ & $\begin{array}{l}\text { Gestational } \\
\text { diabetes } \\
\text { diagnosed at } \\
28 \text { weeks } \\
\text { gestation }\end{array}$ & $\begin{array}{l}4 / 14(28.6 \%) \\
\text { vs. } 2 / 17 \\
(11.7 \%)^{97}\end{array}$ & $\begin{array}{l}\text { Calculated } \\
\text { RR, } 2.43 \\
(95 \% \mathrm{Cl} \\
0.52 \text { to } \\
11.36)^{97}\end{array}$ & $\begin{array}{l}1 \text { cohort, } \\
n=31^{97}\end{array}$ & $\begin{array}{l}\text { High study } \\
\text { limitations (high } \\
\text { risk of bias }{ }^{97} \text { ), } \\
\text { seriously } \\
\text { imprecise (few } \\
\text { events, wide } \\
\text { Cls), consistency } \\
\text { unknown }\end{array}$ & Insufficient \\
\hline $\begin{array}{l}\text { Women } \\
\text { exposed to } \\
\text { psychotropic } \\
\text { exposure } \\
\text { medication } \\
\text { during any } \\
\text { trimester }\end{array}$ & $\begin{array}{l}\text { Risperidone } \\
\text { exposure vs. } \\
\text { lamotrigine } \\
\text { exposure }\end{array}$ & $\begin{array}{l}\text { Gestational } \\
\text { diabetes } \\
\text { diagnosed at } \\
28 \text { weeks } \\
\text { gestation }\end{array}$ & $\begin{array}{l}4 / 14(28.6 \%) \\
\text { vs. } 2 / 19 \\
(10.5 \%)^{97}\end{array}$ & $\begin{array}{l}\text { Calculated } \\
\text { RR, } 2.71 \\
(95 \% \mathrm{Cl} \\
0.58 \text { to } \\
12.80)^{97}\end{array}$ & $\begin{array}{l}1 \text { cohort, } \\
n=33^{97}\end{array}$ & $\begin{array}{l}\text { High study } \\
\text { limitations (high } \\
\text { risk of bias }{ }^{97} \text { ), } \\
\text { seriously } \\
\text { imprecise (few } \\
\text { events, wide } \\
\text { Cls), consistency } \\
\text { unknown }\end{array}$ & Insufficient \\
\hline
\end{tabular}

$\mathrm{CI}=$ confidence interval; $\mathrm{n}=$ number; $\mathrm{RR}=$ relative risk; vs. $=$ versus.

\section{Pharmacologic Combinations}

\section{Overview}

- Six publications reported on child outcomes (breastfeeding difficulty, transient neonatal symptoms, 5-minute Apgar $<9$, NICU admission, respiratory distress, birth weight, gestational age, internalizing problems, or congenital heart disease) for women receiving pharmacologic combinations.

\section{Detailed Results}

Six publications reported on pharmacologic combinations. ${ }^{45,115,120,129,132,141}$ These publications drew from six cohorts ( 3 from the British Columbia Children's and Women's Health Centre, ${ }^{120}, 129,1321$ from The Women's Mental Health Program at the Emory University School of Medicine, ${ }^{45}$ and 2 cohorts based in the United States). ${ }^{115}$ All were nonrandomized studies and were rated as high risk of bias.

All six publications focused on exposure during pregnancy. Three compared SSRIs plus clonazepam with SSRIs, ${ }^{120,129,132}$ one compared SSRIs plus benzodiazepine with SSRIs, ${ }^{115}$ one 
compared SSRIs plus non-SSRIs with SRIs only, and one compared combinations of antidepressants (e.g., fluoxetine and bupropion, sertraline and paroxetine, and venlafaxine and bupropion with individual SSRIs [e.g., citalopram, escitalopram, fluoxetine, sertraline, or paroxetine] or SNRIs [e.g., venlafaxine, bupropion, or duloxetine]). ${ }^{141}$ These studies evaluated the outcomes of breastfeeding difficulty, transient neonatal symptoms, 5-minute Apgar $<9$, NICU admission, respiratory distress, birth weight, gestational age, internalizing problems, infant psychomotor development, and congenital heart disease. These studies are not evaluated any further due to limited clinical utility.

\section{Nonspecific or Undefined Pharmacologic Interventions}

\section{Overview}

- Thirty-one publications reported on nonspecific or undefined pharmacologic interventions compared with another active treatment. Because the clinical utility is limited, we do not judge the risk of harms for maternal or child outcomes.

\section{Detailed Results}

Thirty-one publications reported on nonspecific/undefined pharmacologic interventions

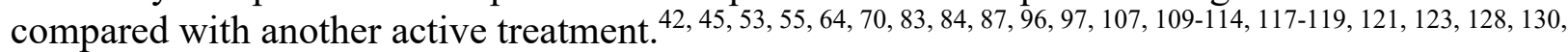
137, 138, 142, 143, 145, 146 These publications drew from 24 cohorts (1 from the Australian Pregnancy Register of Antiepileptic Drugs in Pregnancy, ${ }^{107} 1$ from the Danish Medical Birth Registry, ${ }^{110}$ one from the Emory Women's Mental Health Program, ${ }^{119} 1$ from the U.S. Medicaid Analytic eXtract, ${ }^{96} 2$ from the U.S. Food and Drug Administration Adverse Event Reporting System, ${ }^{143} 1$ from the Hong Kong Clinical Data Analysis and Reporting System, ${ }^{55} 1$ from Ingenix Research Data Mart [claims data from UnitedHealthcare], ${ }^{146} 5$ from Motherisk, ${ }^{77,}$ 121, 128, 130, 1381 from Blue Cross Blue Shield of Michigan claims data, ${ }^{145} 1$ from the Norwegian Mother and Child Cohort Study, ${ }^{142} 5$ from the QPC, ${ }^{53,64,87,111,114} 1$ from RAMQ,${ }^{70} 1$ from the Slone Epidemiology Center Birth Defects Study, ${ }^{84} 1$ from the national Quality Register of Assisted Reproductions, ${ }^{113} 1$ from MoBa, ${ }^{118} 1$ from MTB,${ }^{112} 2$ that included multiple Danish national registries, ${ }^{117,}{ }^{123} 1$ from birth registries in Sweden, ${ }^{137} 1$ that included multiple Canada-based databases, ${ }^{83} 1$ from a population in the United States, ${ }^{45}$ and 3 from populations in Australia). ${ }^{42,97,109}$ All were nonrandomized studies and were rated as high risk of bias $42,45,53,70,84,87,107,109-114,117,118,121,123,128,130,137,138$ or having some concerns for risk of bias. ${ }^{55,64,83,119}$

All 31 publications focused on exposure during pregnancy. Six publications examined any antidepressants use, ${ }^{42,55,109,111,119,143,145}$ eight examined interventions groups described as other antidepressants, ${ }^{53,83,87,114,121,128,130,146}$ one examined an intervention described as other antidepressants/TCAs, ${ }^{118}$ three publications examined an intervention described as antidepressant plus antipsychotics, ${ }^{42,55,109}$ one examined an intervention described as antidepressants coexposed with benzodiazepines, ${ }^{146}$ two examined interventions described as other monotherapy, ${ }^{64}$,

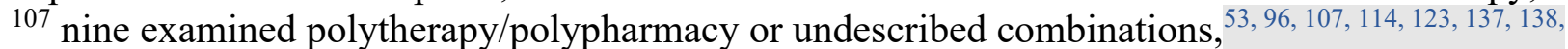
154, 155 three examined non-SSRIs, ${ }^{55,70,113}$ one examined non-SSRIs and non-SSRIs plus SSRIs, ${ }^{45}$ one examined nonsertraline and nonsertraline SSRIs, ${ }^{70}$ one examined any psychotropic treatment, ${ }^{137}$ one examined interventions described as other psychotropic medication, ${ }^{97}$ one examined an intervention described as co-exposure,${ }^{83}$ two examined interventions described as other, ${ }^{110,123}$ two examined interventions described as other monotherapy, ${ }^{64,107}$ and one examined an intervention described as only SNRI/TCA/MAOI/others. ${ }^{111}$ The included publications 
evaluated the maternal outcomes of anemia, gestational diabetes, spontaneous abortion, gestational hypertension, preeclampsia, vaginal bleeding, placental abruption, postpartum hemorrhage, and the child outcomes of preterm delivery, attention-deficit/hyperactivity disorder, Apgar scores, autism, cardiac anomalies, convulsive seizures, epilepsy, major congenital anomalies, heart anomalies, birth weight, NICU admissions, gestational age/prematurity, perinatal death, neuromotor and psychomotor development, and small for gestational age. Because the interventions were not clearly defined, they are not evaluated any further due to limited clinical utility.

\section{Meta-Analysis}

Figure B-2. Pooled odds ratios for cardiac anomalies for SSRIs versus no treatment

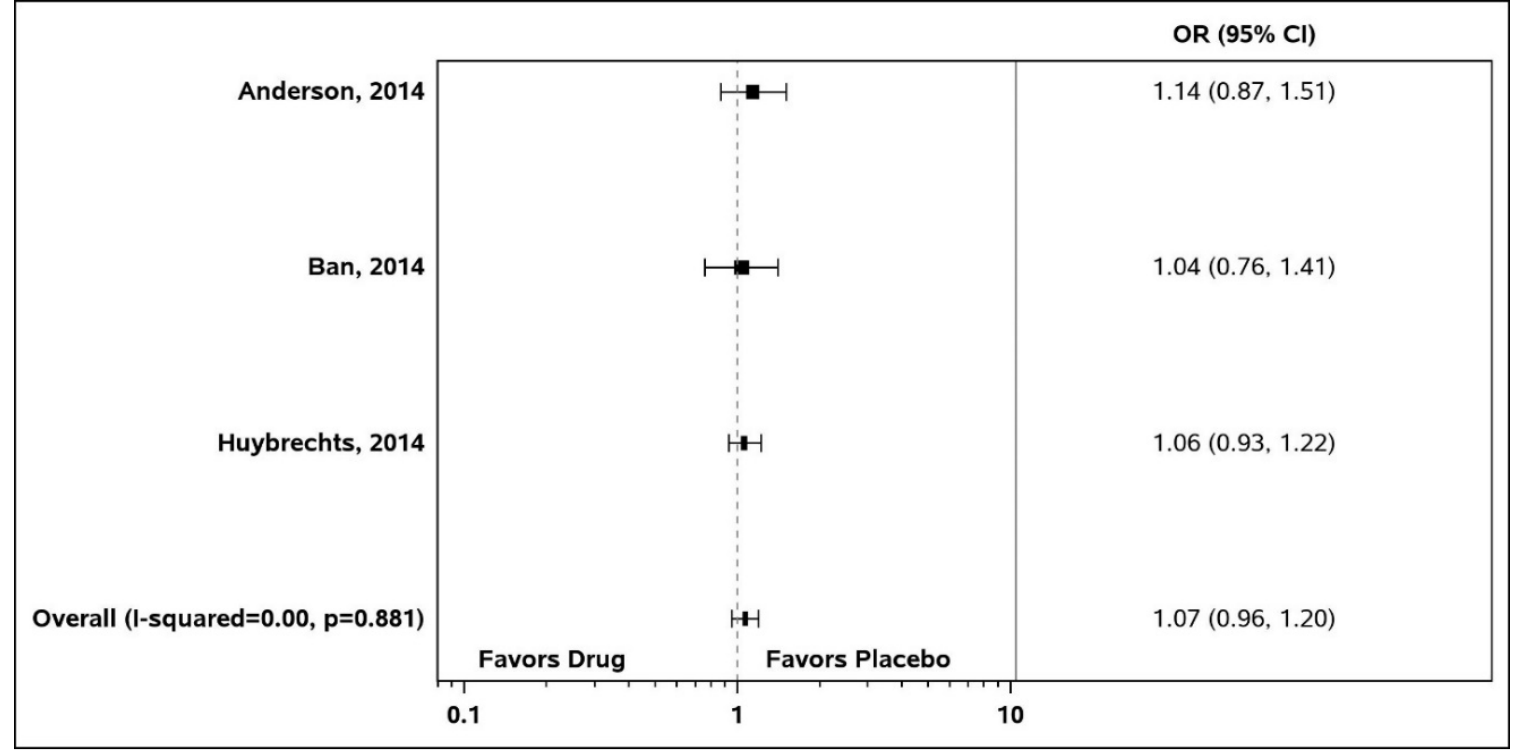

Figure B-3. Pooled odds ratios for cardiac anomalies for citalopram versus no treatment

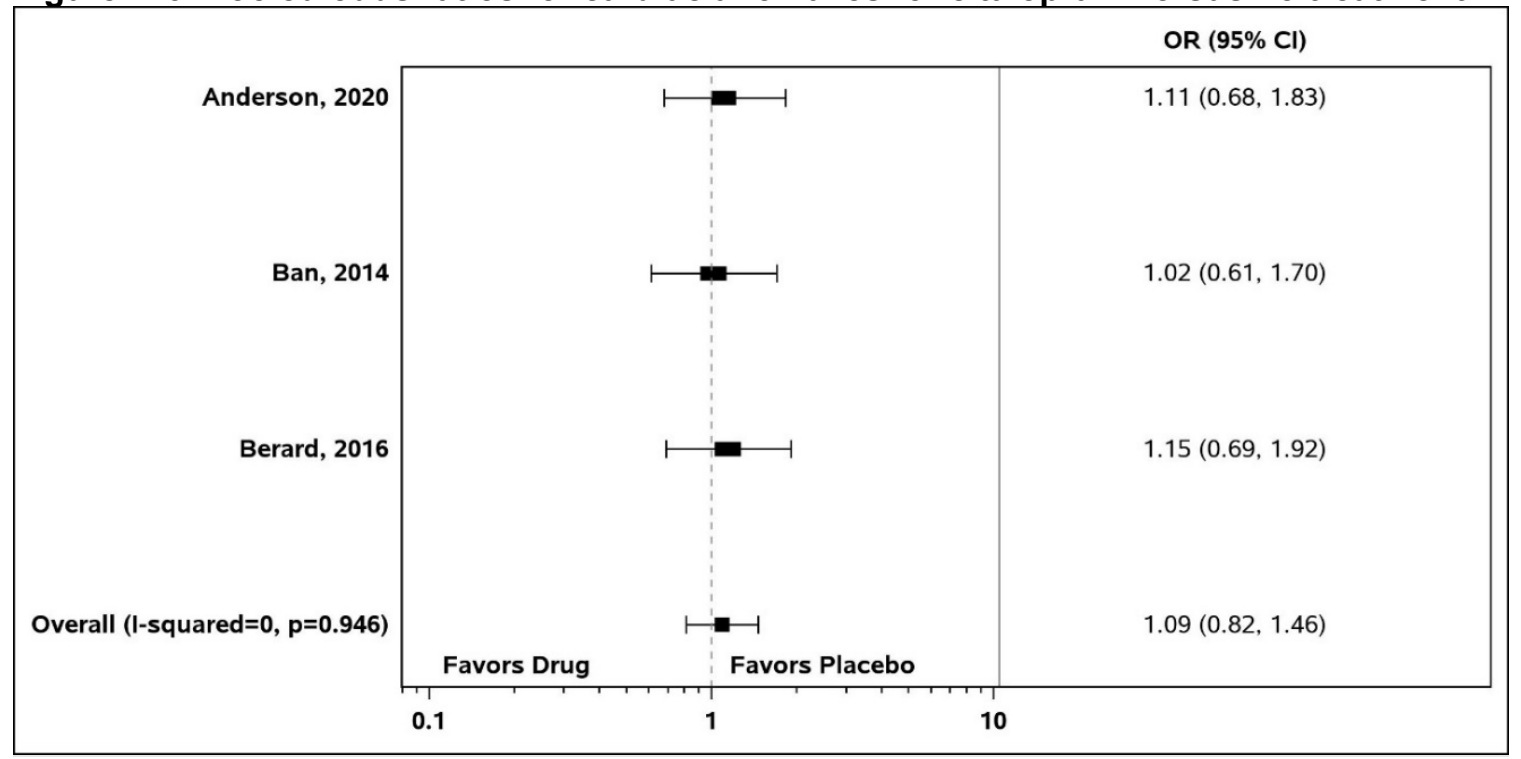


Figure B-4. Pooled odds ratios for cardiac anomalies for fluoxetine versus no treatment

\begin{tabular}{|c|c|c|}
\hline & & OR $(95 \% \mathrm{Cl})$ \\
\hline Anderson, 2020 & -1 & $1.33(0.91,1.95)$ \\
\hline Ban, 2014 & $\longmapsto-1$ & $0.79(0.49,1.27)$ \\
\hline Berard, 2017 & -1 & $0.42(0.10,1.75)$ \\
\hline Huybrechts, 2014 & $1-1$ & $1.14(0.90,1.44)$ \\
\hline Overall (I-squared $=35.94, p=0.179$ ) & $\mapsto-1$ & $1.06(0.81,1.39)$ \\
\hline & Favors Placebo & \\
\hline & 1 & \\
\hline
\end{tabular}

Figure B-5. Pooled odds ratios for cardiac anomalies for paroxetine versus placebo

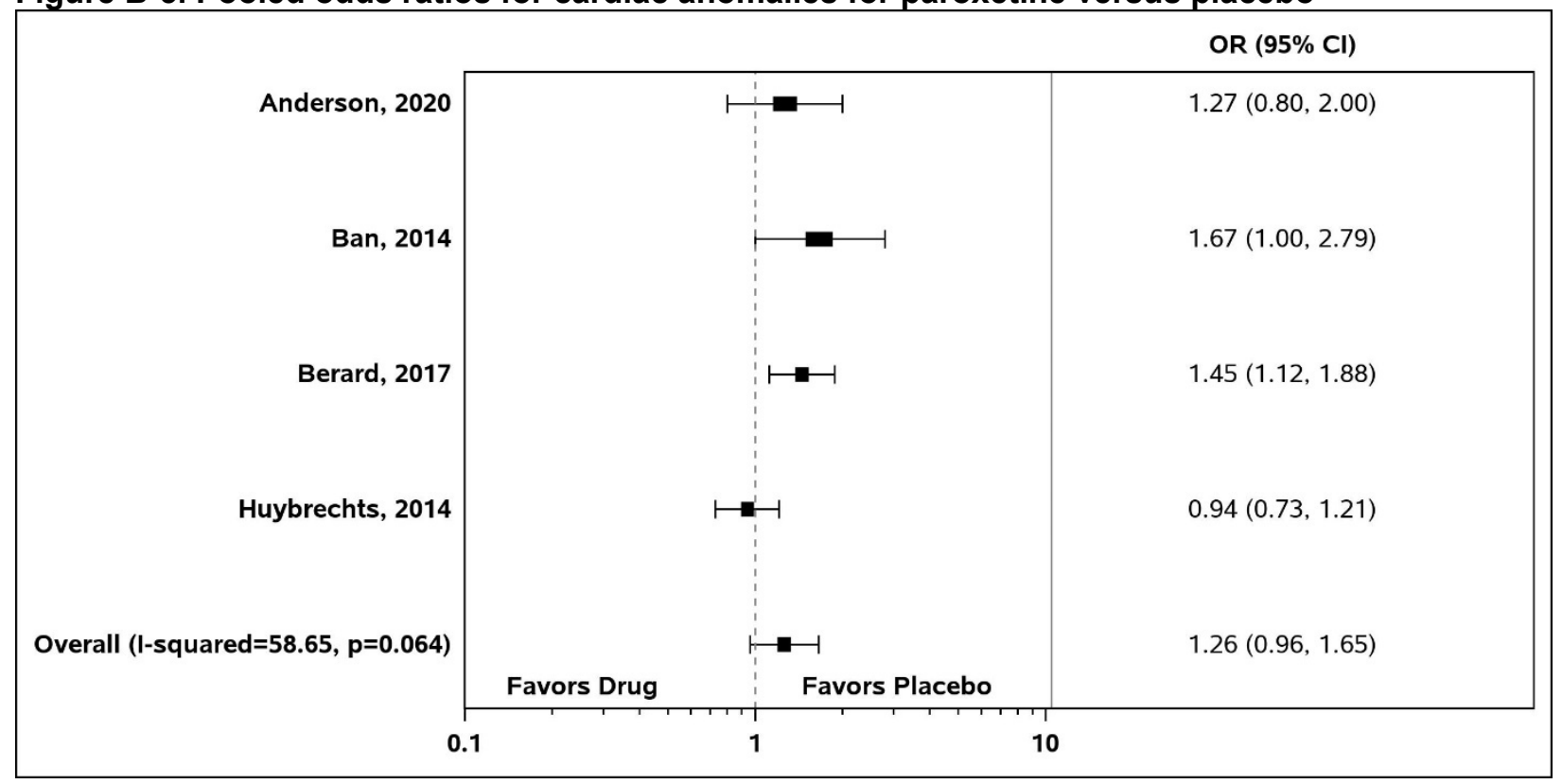


Figure B-6. Pooled odds ratios for cardiac anomalies for sertraline versus placebo

\begin{tabular}{|c|c|c|}
\hline & & OR $(95 \% \mathrm{Cl})$ \\
\hline Anderson, 2020 & $\longmapsto-1$ & $0.97(0.69,1.37)$ \\
\hline Ban, 2014 & $\longmapsto=-$ & $1.39(0.70,2.75)$ \\
\hline Berard, 2017 & $\longmapsto-1$ & $1.14(0.60,2.16)$ \\
\hline Huybrechts, 2014 & $H$ & $1.09(0.88,1.35)$ \\
\hline Overall (I-squared $=0.00, p=0.816$ ) & $H$ & $1.08(0.91,1.28)$ \\
\hline & Favors Placebo & \\
\hline & 1 & \\
\hline
\end{tabular}

Figure B-7. Pooled odds ratios for cardiac anomalies for tricyclic antidepressants versus placebo

\begin{tabular}{|c|c|c|}
\hline & & OR $(95 \% \mathrm{Cl})$ \\
\hline Ban, 2014 & $\longmapsto \square$ & $0.90(0.54,1.50)$ \\
\hline Berard, 2017 & $\longmapsto-1$ & $1.05(0.55,2.00)$ \\
\hline Huybrechts, 2014 & $\longmapsto$ & $0.77(0.52,1.14)$ \\
\hline Overall (I-squared $=0.00, p=0.703$ ) & $\mapsto-1$ & $0.86(0.65,1.13)$ \\
\hline & Favors Placebo & \\
\hline & 1 & \\
\hline
\end{tabular}


Figure B-8. Pooled relative risks for adverse events for brexanolone versus placebo

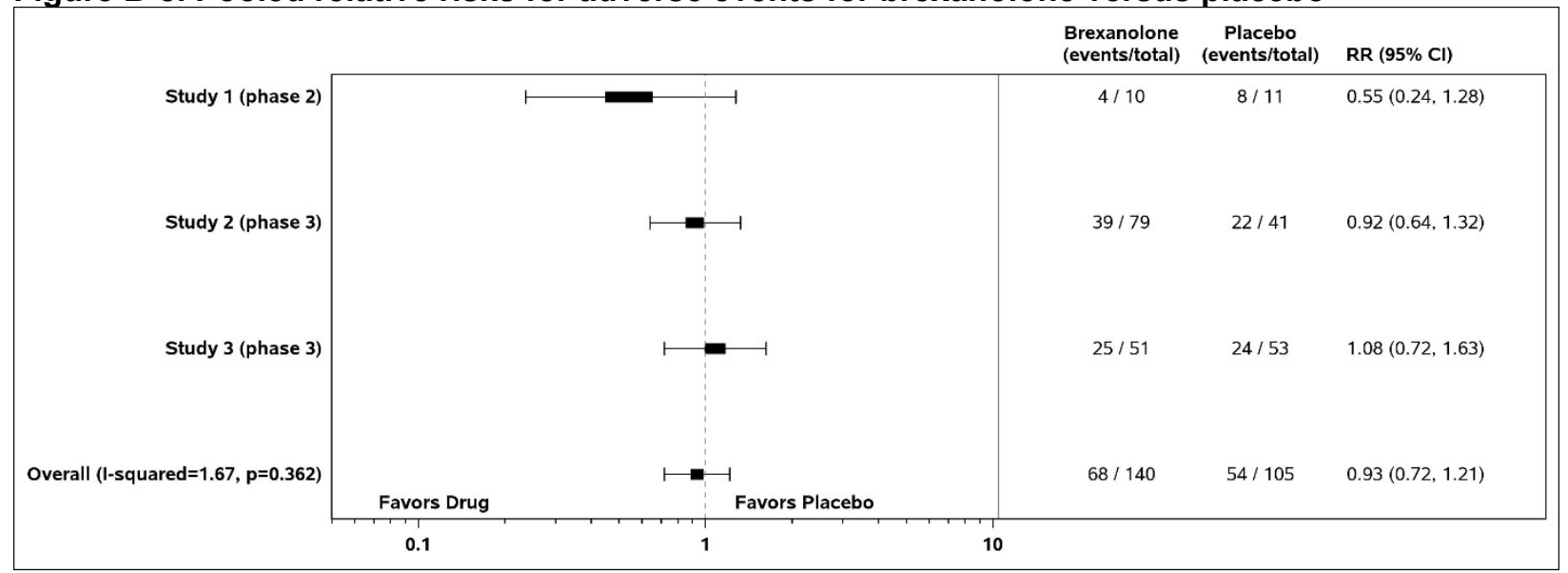

Figure B-9. Pooled relative risks for dizziness for brexanolone versus placebo

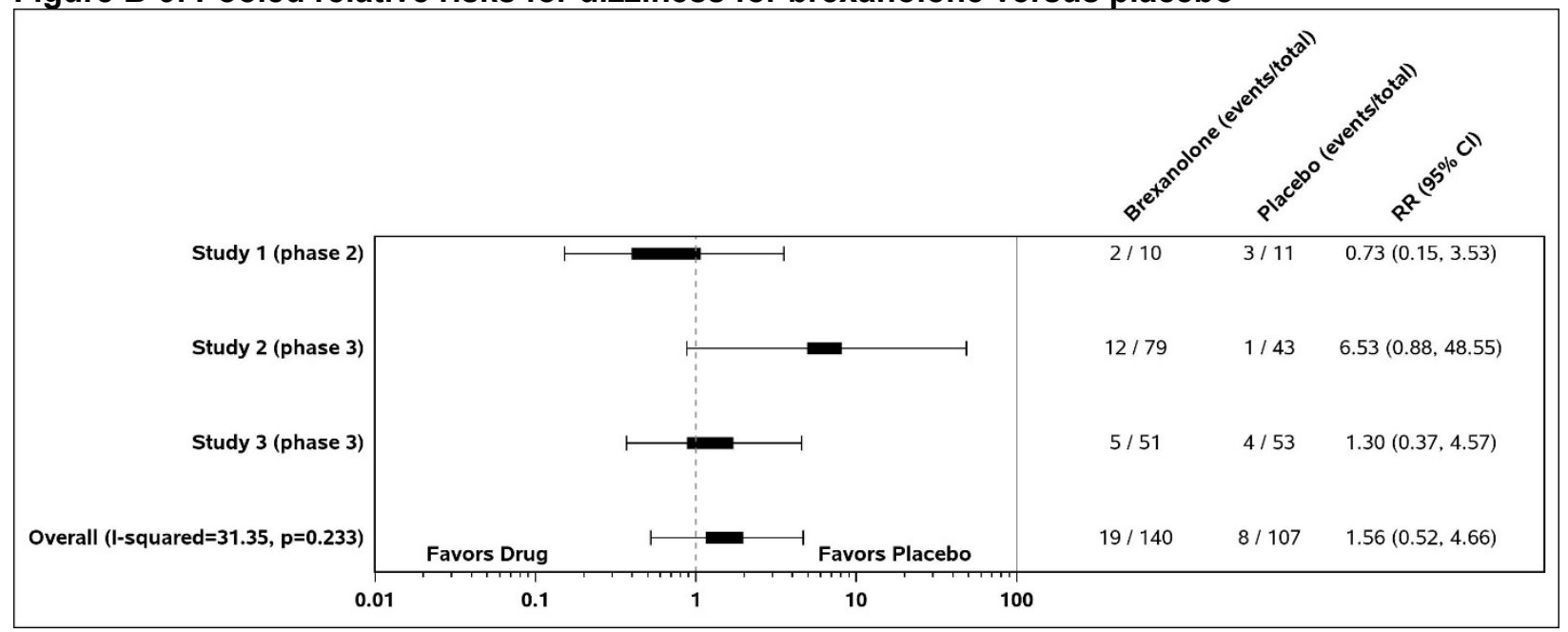


Figure B-10. Pooled relative risks for somnolence for brexanolone versus placebo

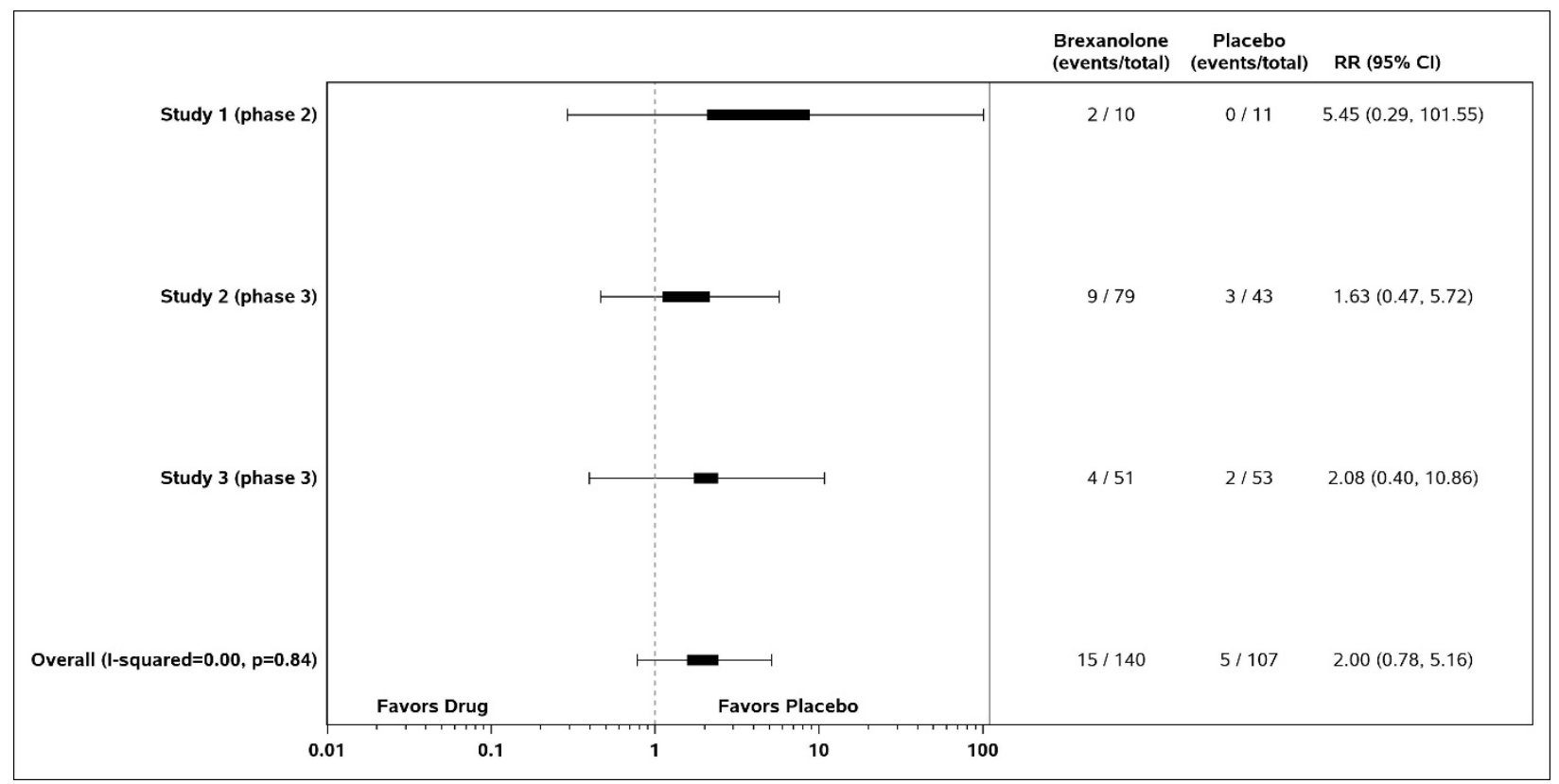




\section{Excluded Studies}

X1: Not Original Research

$\mathrm{X} 2$ : Ineligible Population

$\mathrm{X} 3$ : Ineligible Intervention

X4: Ineligible Comparator

X5: Ineligible Outcome

X6: Ineligible Time Frame

X7: Ineligible Study Design

X8: Not English

X9: Abstract Only

X10: Overlapping Arms

X11: Irretrievable

X12: Indirect Comparison

X13: Dose-Comparison Only

1. Lithium: useful drug or hazardous teratogen? Food Cosmet Toxicol. 1973

Feb;11(1):135-9. PMID: 4577298.

Exclusion Code: X1.

2. [Schizophrenia and depression. Special aspects in the therapy of women]. Nervenarzt. 1998 Jul;69(7 Suppl

Besondere):1-8. PMID: 9713002. Exclusion Code: X8.

3. Depression during pregnancy and after. Harv Ment Health Lett. 2002 Sep;19(3):6-8. PMID: 12356558. Exclusion Code: X1.

4. SSRIs linked with birth defects. Pharm J. 2005;275(7366):303. Exclusion Code: X1.

5. Erratum: SSRI treatment during pregnancy: Deceleration of weight gain due to depression or drug? (American Journal of Psychiatry (2006) 163 (986-991)). Am J Psychiatry. 2006;163(10):1843. Exclusion Code: X1.

6. Prescribing during pregnancy. Prenatal drug exposure and an untreated psychiatric disorder both present risks. Harv Ment Health Lett. 2008 Dec;25(6):1-3. PMID: 19160573. Exclusion Code: X1.

7. Safety of SSRIs in Pregnancy. Med Lett Drugs Ther. 2008 Nov 17;50(1299):89-91. PMID: 19008787. Exclusion Code: X1.
8. $\quad$ SSRIs and risk of postpartum hemorrhage. Harv Ment Health Lett. 2008 Jul;25(1):7. PMID: 18780416. Exclusion Code: X1.

9. The costs of discontinuing antidepressants during pregnancy. Drug Benefit Trends. 2009;21(7):218+20. Exclusion Code: X1.

10. Depression, SSRIs, and premature birth. The American Journal of Psychiatry. 2009;166(5):A22-A. PMID: 2009-09562001. Exclusion Code: X1.

11. The Cochrane Database of Systematic Reviews - Issue 11 of 2010. J Evid Based Med. 2010;3(4):226-7. doi: 10.1111/j.17565391.2010.01103.x. Exclusion Code: X7.

12. In utero SSRI associated with adverse perinatal events. J Natl Med Assoc. 2010;102(2):152-3. Exclusion Code: X1.

13. In utero antipsychotic exposure and neonatal extrapyramidal and withdrawal adverse effects. Australian Prescriber. 2011;34(4):110. Exclusion Code: X1.

14. Valproate in pregnancy linked to autism in children. BMJ. 2013 Apr 24;346:f2602. doi: 10.1136/bmj.f2602. PMID: 23616359. Exclusion Code: X7.

15. SSRI antidepressants: altered psychomotor development following exposure in utero? Prescrire Int. 2013 Feb;22(135):43-4. PMID: 23444502. Exclusion Code: X1. 
16. Erratum: Does Fetal Exposure to SSRIs or Maternal Depression Impact Infant Growth? (American Journal of Psychiatry (2013) 117 (485-493)). Am J Psychiatry. 2013;170(10):1218. Exclusion Code: X1.

17. The Cochrane Database of Systematic Reviews - Issue 10 2013. J Evid Based Med. 2013;6(4):305-6. doi: 10.1111/jebm.12079. Exclusion Code: X7.

18. Antidepressant use in pregnancy linked to anxiety in 3-year-olds. 2015;40. Exclusion Code: X1.

19. Antidepressants in pregnancy and autism. Arch Dis Child. 2016 Mar;101(3):240. doi: 10.1136/archdischild-2016-310462. PMID: 26787608. Exclusion Code: X1.

20. SSRI antidepressants and pregnancy: a link with autism? Prescrire Int. 2017;26(186):239-40. Exclusion Code: X1.

21. Risk of oral cleft from topiramate exposure increases with dosage. Clinical Pharmacist. 2018;10(4). doi:

10.1211/CP.2018.20204441. Exclusion Code: X1.

22. Erratum: brexanolone injection in postpartum depression: two multicentre, doubleblind, randomised, placebo-controlled, phase 3 trials (The Lancet (2018) 392(10152) (1058-1070), (S0140673618315514) (10.1016/S0140-6736(18)31551-4)). Lancet. 2018;392(10153):1116. doi: 10.1016/S01406736\%2818\%2932288-8. PMID: CN01922333. Exclusion Code: X1.

23. Phase 3 study evaluating brexanolone, a gabaa receptor modulator, in severe postpartum depression. Obstet Gynecol. 2018;Conference: 66th Annual Clinical and Scientific Meeting of the American College of Obstetricians and Gynecologists. United States. 131(Supplement 1):27S. PMID: CN01616736. Exclusion Code: X9.

24. Antiepileptics and pregnancy: Potential long-term effects in children. Prescrire Int. 2020;29(211):13-20. Exclusion Code: X7.

25. Abadie D, Hurault Delarue C, Damase Michel C, et al. Drug use and spontaneous abortion: A case-control study. Drug Saf. 2011;34(10):1001. Exclusion Code: X4.
26. Abdulaziz M, Alsaad S, Chaudhry S, et al. First trimester exposure to topiramate and the risk of oral clefts in the offspring: A systematic review and meta-analysis. Birth Defects Research Part A - Clinical and Molecular Teratology. 2015;103(5):464. doi: 10.1002/bdra.23387. Exclusion Code: $\mathrm{X} 7$.

27. Abel KM. Pregnancy prescribing of psychotropic drugs: Keeping pace in a contemporary landscape. In: Pariante CM, Conroy S, Dazzan P, Howard L, Pawlby S, Seneviratne T, eds. Perinatal psychiatry: The legacy of Channi Kumar. New York, NY: Oxford University Press; 2014:53-66. Exclusion Code: X1.

28. Abou-Ali A, Callaham M, Kuyateh F, et al. SSRIs use during pregnancy and the risk of cardiac congenital malformations using the general practice research database. Pharmacoepidemiol Drug Saf. 2011;20:S43. doi: 10.1002/pds.2206. Exclusion Code: X4.

29. Abou-Saleh MT. The mentally ill mother: The use of drug therapy during pregnancy and lactation. In: Göpfert M, Webster J, Seeman MV, eds. Parental psychiatric disorder: Distressed parents and their families. New York, NY: Cambridge University Press; 1996:81-6. Exclusion Code: X11.

30. Abou-Saleh MT, Coppen A. Puerperal affective disorders and response to lithium. Br J Psychiatry. 1983 May;142:539. PMID: 6871572. Exclusion Code: X7.

31. Abramowitz JS, Schwartz SA, Moore KM, et al. Obsessive-complusive symptoms in pregnancy and the puerperium: A review of the literature. J Anxiety Disord. 2003;17(4):461-78. doi: 10.1016/S08876185(02)00206-2. PMID: 2003-06850-008. Exclusion Code: X1.

32. Achtyes E, Keaton SA, Smart L, et al. Inflammation and kynurenine pathway dysregulation in post-partum women with severe and suicidal depression. Brain Behav Immun. 2020 Jan;83:239-47. doi: 10.1016/j.bbi.2019.10.017. PMID: 31698012. Exclusion Code: X3. 
33. Ackerman S, Schoenbrun S, Hudac C, et al. Interactive effects of prenatal antidepressant exposure and likely gene disrupting mutations on the severity of autism spectrum disorder. J Autism Dev Disord. 2017 Nov;47(11):3489-96. doi: 10.1007/s10803017-3246-6. PMID: 28770524. Exclusion Code: X5.

34. Acs N, Banhidy F, Horvath-Puho E, et al. Maternal panic disorder and congenital abnormalities: a population-based casecontrol study. Birth Defects Res A Clin Mol Teratol. 2006 Apr;76(4):253-61. doi: 10.1002/bdra.20250. PMID: 16583439. Exclusion Code: X12.

35. Adab N. Does prenatal valproate exposure cause cognitive impairment? Epilepsia. 2009;50:31. doi: 10.1111/j.15281167.2009.02063.x. Exclusion Code: X2.

36. Adab N, Tudur SC, Vinten J, et al. Common antiepileptic drugs in pregnancy in women with epilepsy. Cochrane Database Syst Rev. 2004(3):Cd004848. doi: 10.1002/14651858.cd004848. PMID: 15266543. Exclusion Code: X2.

37. Adams J. Prenatal exposure to teratogenic agents and neurodevelopmental outcome. Birth Defects Orig Artic Ser. 1989;25(6):6372. PMID: 2481517. Exclusion Code: X1.

38. Adams J. Anxiety and depression. Diagnosis and treatment during pregnancy. J Womens Health. 1998 Jun;7(5):601-2. PMID: 9650162. Exclusion Code: X1.

39. Addis A, Koren G. Safety of fluoxetine during the first trimester of pregnancy: a meta-analytical review of epidemiological studies. Psychol Med. 2000 Jan;30(1):89-94. PMID: 10722179. Exclusion Code: X7.

40. Agell I. Use of antidepressants by nursing mothers. Br J Psychiatry. 2002 Jan;180:856. PMID: 11772860. Exclusion Code: X1.

41. Aguilera C, Agustí A. Characteristics of enquiries requested to a teratogen information service and outcome of pregnancies. Basic and Clinical Pharmacology and Toxicology. 2016;119:37. Exclusion Code: X2.
42. Ahmadpanah M, Nazaribadie M, Aghaei E, et al. Influence of adjuvant detached mindfulness and stress management training compared to pharmacologic treatment in primiparae with postpartum depression. Arch Womens Ment Health. 2017:1-9. doi: 10.1007/s00737-017-0753-6. PMID: CN01393997. Exclusion Code: X4.

43. Ait-Daoud N, Hamby AS, Sharma S, et al. A review of alprazolam use, misuse, and withdrawal. J Addict Med. 2018 Jan/Feb;12(1):4-10. doi: 10.1097/adm.0000000000000350. PMID: 28777203. Exclusion Code: X1.

44. Akioyamen LE, Minhas H, Holloway AC, et al. Effects of depression pharmacotherapy in fertility treatment on conception, birth, and neonatal health: a systematic review. J Psychosom Res. 2016 May;84:69-80. doi: 10.1016/j.jpsychores.2016.03.015. PMID: 27095162. Exclusion Code: X7.

45. Aktepe E, Ozkorumak E, Tanriover-Kandil S. Pregnancy and delivery complications and treatment approach in attention deficit hyperactivity disorder. Turk J Pediatr. 2009 Sep-Oct;51(5):478-84. PMID: 20112604. Exclusion Code: X2.

46. Aleksy LM, Smith MA. Sedatives and hypnotics in lactation. J Hum Lact. 1998 Mar;14(1):61-4. doi: 10.1177/089033449801400118. PMID: 9543962. Exclusion Code: X1.

47. Aliaga A, Cunillera O, Amador MDC, et al. Association between affective disorders presenting before and during pregnancy and pre-term birth, considering sociodemographic factors, obstetric factors, health conditions, and use of medication. Aten Primaria. 2018 Nov 16. doi: 10.1016/j.aprim.2018.06.009. PMID: 30454957. Exclusion Code: X2.

48. Alisky JM. Pregnancy outcomes for women with epilepsy and bipolar disorder could be improved with intraventricular or intrathecal medication administration. Med Hypotheses. 2009 Dec;73(6):1073-4. doi: 10.1016/j.mehy.2009.05.024. PMID: 19556068. Exclusion Code: X1. 
49. Allan LD, Desai G, Tynan MJ. Prenatal echocardiographic screening for Ebstein's anomaly for mothers on lithium therapy. Lancet. 1982 Oct 16;2(8303):875-6. PMID: 6126733. Exclusion Code: X7.

50. Almond P. Is postnatal depression a public health problem? A review of the literature. Arch Womens Ment Health. 2011;14:S59. doi: 10.1007/s00737-010-0203-1. Exclusion Code: X1.

51. Altamura AC, De Gaspari IF, Rovera C, et al. Safety of SSRIs during pregnancy: a controlled study. Hum Psychopharmacol. 2013 Jan;28(1):25-8. doi: 10.1002/hup. 2276. PMID: 23166037. Exclusion Code: X2.

52. Altemus M. Obsessive-compulsive disorder during pregnancy and postpartum. In: Yonkers K, Little B, eds. Management of psychiatric disorders in pregnancy. New York, NY: Oxford University Press; 2001:149-63. Exclusion Code: X1.

53. Altshuler LL. The use of SSRIs in depressive disorders specific to women. J Clin Psychiatry. 2002;63 Suppl 7:3-8. PMID: 11995776. Exclusion Code: X1.

54. Altshuler LL, Cohen L, Szuba MP, et al. Pharmacologic management of psychiatric illness during pregnancy: dilemmas and guidelines. Am J Psychiatry. 1996 May;153(5):592-606. doi: 10.1176/ajp.153.5.592. PMID: 8615404. Exclusion Code: X7.

55. Alvarez SL, Meltzer-Brody S, Mandel M, et al. Maternal depression and early intervention: A call for an integration of services. Infants \& Young Children. 2015;28(1):72-87. doi: 10.1097/IYC.0000000000000024. PMID: 2014-54074-006. Exclusion Code: X1.

56. Alwan S, Friedman JM. Safety of selective serotonin reuptake inhibitors in pregnancy. CNS Drugs. 2009;23(6):493-509. doi: 10.2165/00023210-200923060-00004. PMID: 19480468. Exclusion Code: X7.

57. Alwan S, Reefhuis J, Botto LD, et al. Maternal use of bupropion and risk for congenital heart defects. Am J Obstet Gynecol. 2010 Jul;203(1):52 e1-6. doi: 10.1016/j.ajog.2010.02.015. PMID: 20417496. Exclusion Code: X4.
58. Alwan S, Reefhuis J, Rasmussen SA, et al. Use of selective serotonin-reuptake inhibitors in pregnancy and the risk of birth defects. N Engl J Med. 2007 Jun 28;356(26):2684-92. doi: 10.1056/NEJMoa066584. PMID: 17596602. Exclusion Code: X2.

59. Alwan S, Reefhuis J, Rasmussen SA, et al. Use of selective serotonin-reuptake inhibitors in pregnancy and the risk of birth defects. Obstet Gynecol Surv.

2007;62(11):702-3. doi: 10.1097/01.ogx.0000286792.22798.b7. Exclusion Code: X4.

60. Ananth CV, Friedman AM. Late pregnancy use of selective serotonin reuptake inhibitors and serotonin and norepinephrine reuptake inhibitors is associated with increased risk of postpartum haemorrhage. Evid Based Nurs. 2014 Jul;17(3):76. doi: 10.1136/eb-2013101595. PMID: 24288247. Exclusion Code: $\mathrm{X} 1$.

61. Ananth J. Congenital malformations with psychopharmacologic agents. Compr Psychiatry. 1975;16(5):437-45. doi: 10.1016/0010-440X(75)90033-4. PMID: 1976-27491-001. Exclusion Code: X1.

62. Ananth J. Congenital malformations with psychopharmacologic agents. Compr Psychiatry. 1975 Sep-Oct;16(5):437-45. PMID: 240643. Exclusion Code: X1.

63. Andalib S, Emamhadi MR, YousefzadehChabok S, et al. Maternal SSRI exposure increases the risk of autistic offspring: A meta-analysis and systematic review. Eur Psychiatry. 2017 Sep;45:161-6. doi: 10.1016/j.eurpsy.2017.06.001. PMID: 28917161. Exclusion Code: X7.

64. Andersen HR, Debes F, Wohlfahrt-Veje C, et al. Occupational pesticide exposure in early pregnancy associated with sex-specific neurobehavioral deficits in the children at school age. Neurotoxicol Teratol. 2015;47:1-9. doi: 10.1016/j.ntt.2014.10.006. Exclusion Code: X2.

65. Andersen JT, Andersen NL, Horwitz H, et al. Exposure to selective serotonin reuptake inhibitors in early pregnancy and the risk of miscarriage. Obstet Gynecol. 2014 Oct;124(4):655-61. doi: 10.1097/aog.0000000000000447. PMID: 25198261. Exclusion Code: X4. 
66. Andersen JT, Jimenez-Solem E, Andersen NL, et al. SSRI and the risk of miscarriage-a register based nation wide cohort study. Pharmacoepidemiol Drug Saf. 2013;22:1967. doi: 10.1002/pds.3512. Exclusion Code: X9.

67. Anderson KN, Ailes EC, Lind JN, et al. Atypical antipsychotic use during pregnancy and birth defect risk: National Birth Defects Prevention Study, 1997-2011. Schizophr Res. 2020 Jan;215:81-8. doi: 10.1016/j.schres.2019.11.019. PMID: 31761471. Exclusion Code: X4.

68. Anderson KN, Lind JN, Simeone R, et al. Maternal early pregnancy use of specific antidepressant medications and risk for birth defects. Pharmacoepidemiol Drug Saf. 2019;28:367-8. doi: 10.1002/pds.4864. Exclusion Code: X4.

69. Anderson KN, Lind JN, Simeone R, et al. Maternal early pregnancy antidepressant use by medication class and birth defect risk. Pharmacoepidemiol Drug Saf. 2019;28:382. doi: 10.1002/pds.4864. Exclusion Code: X4.

70. Andolsek KM. Effects of fluoxetine use during pregnancy. Am Fam Physician. 1997;55(2):641-2. Exclusion Code: X1.

71. Andrade C. Selective serotonin reuptake inhibitors and persistent pulmonary hypertension of the newborn. J Clin Psychiatry. 2012 May;73(5):e601-5. doi: 10.4088/JCP.12f07731. PMID: 22697207. Exclusion Code: X1.

72. Andrade C. Antidepressant use in pregnancy and risk of autism spectrum disorders: a critical examination of the evidence. J Clin Psychiatry. 2013 Sep;74(9):940-1. doi: 10.4088/JCP.13ac08607. PMID: 24107768. Exclusion Code: X1.

73. Andrade C. Antenatal exposure to selective serotonin reuptake inhibitors and duration of gestation. J Clin Psychiatry. 2013 Jul;74(7):e633-5. doi: 10.4088/JCP.13f08617. PMID: 23945457. Exclusion Code: X1.

74. Andrade C. Adverse outcomes following serotonin reuptake inhibitor exposure during pregnancy. J Clin Psychiatry. 2016

Feb;77(2):e199-200. doi: 10.4088/JCP.15com10041. PMID: 26930536. Exclusion Code: X1.
75. Andrade C. Antidepressant exposure during pregnancy and risk of autism in the offspring, 1: meta-review of meta-analyses. J Clin Psychiatry. 2017

Sep/Oct;78(8):e1047-e51. doi: 10.4088/JCP.17f1 1903. PMID: 28994903. Exclusion Code: X7.

76. Annegers JF, Elveback LR, Hauser WA, et al. Do anticonvulsants have a teratogenic effect? Arch Neurol. 1974 Dec;31(6):36473. PMID: 4441320. Exclusion Code: X1.

77. Ansah DA, Reinking BE, Colaizy TT, et al. A Prospective Study Evaluating the Effects of SSRI Exposure on Cardiac Size and Function in Newborns. Neonatology. 2019;115(4):320-7. doi: 10.1159/000496451. PMID: 30836356. Exclusion Code: X4.

78. Anzai T, Takahashi K, Watanabe M, et al. Adverse event reports in patients taking psychiatric medication during pregnancy from spontaneous reports in Japan and the United States: An approach using latent class analysis. BMC Psychiatry. 2020;20. doi: 10.1186/s12888-020-02525-z. PMID: 2020-19203-001. Exclusion Code: X7.

79. Appleby L. A controlled study of fluoxetine and cognitive -behavioural counselling in the treatment of postnatal depression. 9th congress of the association of european psychiatrists. Copenhagen, denmark. 20 24th september 1998. 1998. PMID: CN00642401. Exclusion Code: X9.

80. Applehy L, Warner R, Whitton A, et al. Fluoxetine vs counselling for postnatal depression. N Z Med J. 1997;110(1046):221. Exclusion Code: X1.

81. Araujo JSA, Delgado IF, Paumgartten FJR. Antenatal exposure to antidepressant drugs and the risk of neurodevelopmental and psychiatric disorders: a systematic review. Cad Saude Publica. 2020;36(2):e00026619. doi: 10.1590/0102-311x00026619. PMID: 32022173. Exclusion Code: X7.

82. Arbat A, Danes I. [Postpartum depression]. Med Clin (Barc). 2003 Nov 15;121(17):6735. PMID: 14642231. Exclusion Code: X8. 
83. Ardau R, Bocchetta A, Carta P, et al. A retrospective case series of bipolar patients during pregnancy: Lithium prophylaxis, risk of recurrences and maternal/neonatal complications. Int J Psychiatry Clin Pract. 2012;16:21-2. doi: 10.3109/13651501.2012.736163. Exclusion Code: X7.

84. Aref A, Wali M, Watson H. The myth behind antipsychotic use during pregnancy. 'a review of literature'. BJOG. 2019;126:190. doi: 10.1111/14710528.16_15703. Exclusion Code: X7.

85. Arkilo D, Hanna JE, Dickens D, et al. Pregnancy outcomes with newer antiepileptic agents. Neurology. 2013;80(1). Exclusion Code: X2.

86. Arkilo D, Vaou EO. Antiepileptic drug use during pregnancy: sodium valproate is associated with lower offspring IQ. Evid Based Nurs. 2015 Oct;18(4):109. doi: 10.1136/eb-2014-102045. PMID: 25736074. Exclusion Code: X1.

87. Arnon J, Shechtman S, Ornoy A. The use of psychiatric drugs in pregnancy and lactation. Isr J Psychiatry Relat Sci. 2000;37(3):20522. PMID: 2001-14363-007. Exclusion Code: X1.

88. Arsova S, Hadzhihamza K, Bajraktarov S, et al. Treatment of Depressive Conditions in Pregnancy. Open Access Maced J Med Sci. 2018 Nov 25;6(11):2079-83. doi: 10.3889/oamjms.2018.433. PMID: 30559864. Exclusion Code: X4.

89. Auerbach JG, Hans SL, Marcus J, et al. Maternal psychotropic medication and neonatal behavior. Neurotoxicol Teratol. 1992 Nov-Dec;14(6):399-406. PMID: 1488034. Exclusion Code: X4.

90. Austin MP, Karatas JC, Mishra P, et al. Infant neurodevelopment following in utero exposure to antidepressant medication. Acta Paediatr. 2013 Nov;102(11):1054-9. doi: 10.1111/apa.12379. PMID: 23927695. Exclusion Code: X4.

91. Austin MP, Mitchell PB. Use of psychotropic medications in breast-feeding women: acute and prophylactic treatment. Aust N Z J Psychiatry. 1998 Dec;32(6):77884. doi: 10.3109/00048679809073866. PMID: 10084341. Exclusion Code: X7.
92. Austin MP, Mitchell PB. Psychotropic medications in pregnant women: treatment dilemmas. Med J Aust. 1998 Oct 19;169(8):428-31. PMID: 9830392. Exclusion Code: X7.

93. Avitan T, Lim K, Brain U, et al. Fetal SSRIexposure and increased cord red cell counts suggesting chronic fetal hypoxia. Am J Obstet Gynecol. 2017;216(1):S330. Exclusion Code: X4.

94. Avram MJ, Stika CS, Rasmussen-Torvik LJ, et al. Rationale and design for an investigation to optimize selective serotonin reuptake inhibitor treatment for pregnant women with depression. Clin Pharmacol Ther. 2016 Jul;100(1):31-3. doi: 10.1002/cpt.375. PMID: 27037844. Exclusion Code: X7.

95. Axelsdottir TO, Sigurdsson EL, Gudmundsdottir AM, et al. Drug use during early pregnancy: Cross-sectional analysis from the Childbirth and Health Study in Primary Care in Iceland. Scand J Prim Health Care. 2014;32(3):139-45. doi: 10.3109/02813432.2014.965884. PMID: 2014-43840-007. Exclusion Code: X2.

96. Ayoub A, Fraser WD, Low N, et al. Risk of central nervous system defects in offspring of women with and without mental illness. Arch Womens Ment Health. 2018. doi: 10.1007/s00737-018-0819-0. PMID: 2018 08141-001. Exclusion Code: X2.

97. Azoulay C. [Which antidepressants to use during pregnancy?]. Soins Pediatr Pueric. 2014 Sep-Oct(280):12. PMID: 25518242. Exclusion Code: X8.

98. Babu GN, Thippeswamy H, Chandra PS. Use of electroconvulsive therapy (ECT) in postpartum psychosis--a naturalistic prospective study. Arch Womens Ment Health. 2013 Jun;16(3):247-51. doi: 10.1007/s00737-013-0342-2. PMID: 23568390. Exclusion Code: X3.

99. Baer R, Chambers CD, Jelliffe-Pawlowski LL. Risk of preterm birth among pregnant Medi-Cal participants with mental illness. Am J Obstet Gynecol. 2016 Jan;214(1):S221-S. doi: DOI 10.1016/j.ajog.2015.10.443. PMID: WOS:000367092800396. Exclusion Code: $\mathrm{X} 3$. 
100. Baer RJ, Chambers CD, Ryckman KK, et al. Risk of preterm and early term birth by maternal drug use. J Perinatol. 2019

Feb;39(2):286-94. doi: 10.1038/s41372-0180299-0. PMID: 30573752. Exclusion Code: $\mathrm{X} 2$.

101. Bågedahl-Strindlund M. Parapartum mental illness: An interview follow-up study. Acta Psychiatr Scand. 1997;95(5):389-95. doi: 10.1111/j.1600-0447.1997.tb09651.x. PMID: 1998-00159-006. Exclusion Code: X7.

102. Bailey BA, Janisse J, McCook JG, et al. The potential "double whammy" of cigarette smoking and SSRI use: Reduced infant weight and length. Reprod Sci. 2015;22:262A. Exclusion Code: X9.

103. Bakaysa SL, Kelly J, Urato A. Does SSRI antidepressant use influence APGAR scores? Obstet Gynecol. 2016;127:122S. doi:

10.1097/01.AOG.0000483498.81885.6d. Exclusion Code: X4.

104. Baker R, Kendrick D, Tata LJ, et al. Association between maternal depression and anxiety episodes and rates of childhood injuries: a cohort study from England. Inj Prev. 2017 Dec;23(6):396-402. doi: 10.1136/injuryprev-2016-042294. PMID: 28232401. Exclusion Code: X6.

105. Ball DE, Morrison P. Oestrogen transdermal patches for post partum depression in lactating mothers--a case report. Cent Afr J Med. 1999 Mar;45(3):68-70. PMID: 10565065. Exclusion Code: X7.

106. Ban L, Gibson JE, West J, et al. Risks of major congenital anomaly in the children of women with clinically diagnosed depression: A United Kingdom populationbased cohort study. Eur J Epidemiol. 2013;28(1):S18-S9. doi: 10.1007/s10654013-9820-0. Exclusion Code: X9.

107. Ban L, Gibson JE, West J, et al. Risks of major congenital anomaly in the children of women with clinically diagnosed depression: A population-based cohort study from the United Kingdom. BJOG. 2013;120:2-3. doi: 10.1111/14710528.12332. Exclusion Code: X9.
108. Ban L, West J, Gibson JE, et al. Antenatal exposure to anti-anxiety and hypnotic drugs and the risks of major congenital anomalies: A United Kingdom population-based cohort study. Eur J Epidemiol. 2013;28(1):S19. doi: 10.1007/s10654-013-9820-0. Exclusion Code: X4.

109. Banach R, Boskovic R, Einarson T, et al. Long-term developmental outcome of children of women with epilepsy, unexposed or exposed prenatally to antiepileptic drugs: a meta-analysis of cohort studies. Drug Saf. 2010 Jan 1;33(1):73-9. doi: 10.2165/11317640-000000000-00000. PMID: 20000869. Exclusion Code: X7.

110. Bandoli G, Chambers C, Palmsten K. Prenatal antidepressant exposure and perinatal outcomes: An analysis utilizing longitudinal clustering methods. Pharmacoepidemiol Drug Saf. 2019;28:32. doi: 10.1002/pds.4864. Exclusion Code: X9.

111. Bandoli G, Kuo G, Sugathan R, et al. Longitudinal trajectories of antidepressant use in pregnancy and the postnatal period. Am J Obstet Gynecol. 2018;218(1):S442S3. Exclusion Code: X5.

112. Banga A, Handratta V, Ibrahim L, et al. SSRIs and placental dysfunction. Med Hypotheses. 2011 Aug;77(2):311-2. doi: 10.1016/j.mehy.2011.05.023. PMID: 21664765. Exclusion Code: X1.

113. Bank AM, Stowe ZN, Newport DJ, et al. Placental passage of antiepileptic drugs at delivery and neonatal outcomes. Epilepsia. 2017 May;58(5):e82-e6. doi: 10.1111/epi.13733. PMID: 28387929. Exclusion Code: X4.

114. Banu N, Subair O, Sadler S. Depression relapse rates in pregnant women who discontinue their antidepressants. Arch Dis Child Fetal Neonatal Ed. 2011;96:Fa105. doi: 10.1136/adc.2011.300163.27. Exclusion Code: X9.

115. Barbui C, Conti V, Purgato M, et al. Use of antipsychotic drugs and mood stabilizers in women of childbearing age with schizophrenia and bipolar disorder: epidemiological survey. Epidemiol Psychiatr Sci. 2013 Dec;22(4):355-61. doi: 10.1017/s2045796013000012. PMID: 23369654. Exclusion Code: X5. 
116. Barbui C, Conti V, Purgato M, et al. Use of antipsychotic drugs and mood stabilizers in women of childbearing age with schizophrenia and bipolar disorder: Epidemiological survey. Epidemiology and Psychiatric Sciences. 2014;22(4):355-61. doi: $10.1017 / \mathrm{S} 2045796013000012$. Exclusion Code: X5.

117. Barbui C, Ostuzzi G. Beneficial and harmful consequences of prepartum and postpartum antidepressant exposure. Evid Based Ment Health. 2014 Aug;17(3):69-71. doi: 10.1136/eb-2014-101884. PMID: 24938274. Exclusion Code: X1.

118. Bar-Oz B, Einarson T, Einarson A, et al. Paroxetine and congenital malformations: meta-Analysis and consideration of potential confounding factors. Clin Ther. 2007 May;29(5):918-26. doi: 10.1016/j.clinthera.2007.05.003. PMID: 17697910. Exclusion Code: X7.

119. Bartens MA, Wennink HJMB, Honig A. Risk of autism after antidepressant use during pregnancy. Ned Tijdschr Geneeskd. 2016;160(17). Exclusion Code: X8.

120. Barton S, Nadebaum C, Anderson VA, et al. Memory dysfunction in school-aged children exposed prenatally to antiepileptic drugs. Neuropsychology. 2018;32(7):78496. doi: $10.1037 /$ neu0000465

10.1037/neu0000465.supp (Supplemental). PMID: 2018-32565-001. Exclusion Code: X2.

121. Basar HM, Yalcin SU, Aydin N. Is it possible doing both medical treatment and breastfeeding for acute psychiatric inpatients? Klinik Psikofarmakoloji Bulteni. 2016;26:S15. Exclusion Code: X4.

122. Bascoul C, Franchitto L, Parant O, et al. [Psychotropic drugs during pregnancy and lactation: development practice]. Presse Med. 2015 Mar;44(3):271-83. doi: 10.1016/j.lpm.2014.07.026. PMID: 25595818. Exclusion Code: X8.

123. Bateman BT, Huybrechts KF, Patorno E, et al. Attention deficit hyperactivity medications during pregnancy and the risk of congenital cardiac malformations: A cohort study. Pharmacoepidemiol Drug Saf. 2015;24:206-7. doi: 10.1002/pds.3838.

Exclusion Code: X3.
124. Battino D. Cognitive outcome after exposure to antiepileptic drugs in utero. Lancet Neurol. 2009 Jul;8(7):600-1. doi: 10.1016/s1474-4422(09)70154-1. PMID: 19539227. Exclusion Code: X1.

125. Battle CL, Salisbury AL. Treatment of antenatal depression. J Midwifery Womens Health. 2010 Sep-Oct;55(5):479; author reply -80. doi: 10.1016/j.jmwh.2010.06.004. PMID: 20732671. Exclusion Code: X1.

126. Battle CL, Uebelacker LA, Magee SR. Patient-centered care for antenatal depression. Am J Obstet Gynecol. 2012 Nov;207(5):e10-1; author reply e1. doi: 10.1016/j.ajog.2012.06.062. PMID: 22917485. Exclusion Code: X1.

127. Battle CL, Zlotnick C, Miller IW, et al. Clinical characteristics of perinatal psychiatric patients: a chart review study. J Nerv Ment Dis. 2006 May;194(5):369-77. doi: 10.1097/01.nmd.0000217833.49686.c0. PMID: 16699387. Exclusion Code: X1.

128. Batton B, Batton E, Weigler K, et al. In utero antidepressant exposure and neurodevelopment in preterm infants. Am J Perinatol. 2013 Apr;30(4):297-301. doi: 10.1055/s-0032-1324697. PMID: 22893558. Exclusion Code: X2.

129. Baum AL, Misri S. Selective serotoninreuptake inhibitors in pregnancy and lactation. Harv Rev Psychiatry. 1996;4(3):117-25. doi: 10.3109/10673229609030534. PMID: 199606340-001. Exclusion Code: X1.

130. Bavoux F, Lanfranchi C, Olive G, et al. [Adverse effects on newborns from intra uterine exposure to benzodiazepines and other psychotropic agents (author's transl)]. Therapie. 1981 May-Jun;36(3):305-12. PMID: 6117137. Exclusion Code: X8.

131. Baylor SD, Patterson BY. Psychotropic medication during pregnancy. 2009;34((Baylor S.D.) Clinical Pharmacy Practice Resident in Public Health, Division of Pharmacy Practice, University of Missouri-Kansas City, Kansas City, MO, United States):58-68. Exclusion Code: X1. 
132. Beaulac-Baillargeon L, Boucher N. Neonatal adverse reactions after in utero exposure to selective serotonin reuptake inhibitors: still controversial. Arch Pediatr Adolesc Med. 2006 Aug;160(8):855-6; author reply 6 . doi:

10.1001/archpedi.160.8.855-b. PMID: 16894088. Exclusion Code: X1.

133. Bech LF, Polcwiartek C, Kragholm K, et al. In utero exposure to antiepileptic drugs is associated with learning disabilities among offspring. J Neurol Neurosurg Psychiatry. 2018 Dec;89(12):1324-31. doi: 10.1136/jnnp-2018-318386. PMID: 30076271. Exclusion Code: X2.

134. Bedard P. Prenatal antidepressant use and risk of autism spectrum disorders in children. JAMA Pediatr. 2016 Jul 1;170(7):712-3. doi: 10.1001/jamapediatrics.2016.0730. PMID: 27244697. Exclusion Code: X1.

135. Beeley L. Adverse effects of drugs in later pregnancy. Clin Obstet Gynaecol. 1986 Jun;13(2):197-214. PMID: 2426027. Exclusion Code: X1.

136. Bellantuono C, Bozzi F, Orsolini L, et al. The safety of escitalopram during pregnancy and breastfeeding: a comprehensive review. Hum Psychopharmacol. 2012 Nov;27(6):534-9. doi: 10.1002/hup.2265. PMID: 23044635. Exclusion Code: X7.

137. Bellantuono C, Migliarese G, Gentile S. Serotonin reuptake inhibitors in pregnancy and the risk of major malformations: A systematic review. Human Psychopharmacology: Clinical and Experimental. 2007;22(3):121-8. doi: 10.1002/hup.836. PMID: 2007-06820-001. Exclusion Code: X7.

138. Bellantuono C, Migliarese G, Gentile S. 'Serotonin reuptake inhibitors in pregnancy and the risk of major malformations: A systematic review': Erratum. Human Psychopharmacology: Clinical and Experimental. 2007;22(6):413-. doi: 10.1002/hup.867. PMID: 2007-13298-010. Exclusion Code: X7.

139. Bellantuono C, Orsolini L, Bozzi F. [The safety profile of escitalopram in pregnancy and breastfeeding]. Riv Psichiatr. 2013 NovDec;48(6):407-14. doi: 10.1708/1379.15335. PMID: 24441517. Exclusion Code: X8.
140. Bellantuono C, Santone G. [Antidepressants-SSRIs in pregnancy and risk of major malformations: treat or not to treat]. Recenti Prog Med. 2014

Jun;105(6):254-61. doi: 10.1701/1543.16855. PMID: 25002286. Exclusion Code: X8.

141. Bellantuono C, Vargas M, Mandarelli G, et al. The safety of serotonin-noradrenaline reuptake inhibitors (SNRIs) in pregnancy and breastfeeding: A comprehensive review. Human Psychopharmacology: Clinical and Experimental. 2015;30(3):143-51. doi: 10.1002/hup.2473. PMID: 2015-18066-003. Exclusion Code: X1.

142. Bellet F, Beyens MN, Bernard N, et al. Pregnancy outcome in women exposed to aripiprazole during the embryonic period: A prospective multicentric cohort. Drug Saf. 2013;36(9):807. doi: 10.1007/s40264-0130087-x. Exclusion Code: X4.

143. Bellissima V, Visser GH, Ververs TF, et al. Antenatal maternal antidepressants drugs affect $\mathrm{S} 100 \mathrm{~B}$ concentrations in fetalmaternal biological fluids. CNS Neurol Disord Drug Targets. 2015;14(1):49-54. PMID: 25613501. Exclusion Code: X4.

144. Ben-Amitai D, Merlob P. Neonatal fever and cyanotic spells from maternal chlorpromazine. DICP. 1991 Sep;25(9):1009-10. PMID: 1949962. Exclusion Code: X7.

145. Bénard-Laribière A, Pambrun E, SutterDallay AL, et al. Impact of pregnancy on antidepressant treatment course: A populationbased comparative cohort study in France. Fundam Clin Pharmacol. 2019;33:50. doi: 10.1111/fcp.12470. Exclusion Code: X5.

146. Bender S, Mukadam N. Evaluation of psychotropic medications and the incidence of post-partum haemorrhage (PPH). Aust N Z J Obstet Gynaecol. 2018;58:68. doi: 10.1111/ajo.12874. Exclusion Code: X4.

147. Bennedsen BE, Mortensen PB, Olesen AV, et al. Congenital malformations, stillbirths, and infant deaths among children of women with schizophrenia. Arch Gen Psychiatry. 2001 Jul;58(7):674-9. PMID: 11448375. Exclusion Code: X4. 
148. Berard A. Antidepressants and spontaneous abortion. CMAJ. 2011 Aug 9;183(11):1283. doi: 10.1503/cmaj.111-2057. PMID: 21825056. Exclusion Code: X1.

149. Bérard A. Re: Association between antidepressant use during pregnancy and infants born small for gestational age. Canadian Journal of Psychiatry. 2011;56(8):509-10. Exclusion Code: X1.

150. Berard A, Abbas-Chorfa F, Kassai B, et al. The french pregnancy cohort: Prevalence and trend of medication use during pregnancy in the French population. Birth Defects Research. 2019;111(9):507. doi: 10.1002/bdr2.v111.9. Exclusion Code: X5.

151. Berard A, Abbas-Chorfa F, Kassai B, et al. The French pregnancy cohort: Prevalence and trend of medication use during pregnancy in the French population. Pharmacoepidemiol Drug Saf. 2019;28:381. doi: 10.1002/pds.4864. Exclusion Code: X5.

152. Bérard A, Abbas-Chorfa F, Kassai B, et al. The PregMed-France cohort: Prevalence and trend of medication use during pregnancy in the French population. Fundam Clin Pharmacol. 2019;33:61. doi: 10.1111/fcp.12470. Exclusion Code: X5.

153. Bérard A, Abbas-Chorfa F, Kassai B, et al. The French Pregnancy Cohort: Medication use during pregnancy in the French population. PLoS One. 2019;14(7):e0219095. doi: 10.1371/journal.pone.0219095. PMID: 31314794. Exclusion Code: X3.

154. Berard A, Chaabane S, Boukhris T. Antidepressant use in pregnancy and the risk of cardiac defects. N Engl J Med. 2014 Sep 18;371(12):1167-8. doi: 10.1056/NEJMc1409203. PMID: 25229934. Exclusion Code: X1.

155. Bérard A, Iessa N, Chaabane S, et al. The risk of major cardiac malformations associated with paroxetine use during the first trimester of pregnancy: A systematic review and meta-analysis. Br J Clin Pharmacol. 2016;81(4):589-604. doi: 10.1111/bcp.12849. Exclusion Code: X7.
156. Bérard A, Karam F, Gaegick A, et al. CYP2D6 genotypes, depression, and antidepressant discontinuation during pregnancy: Results from the antidepressants in pregnancy discontinuation study. Pharmacoepidemiology and Drug Safety (PDS). 2009;18(S1):S161. doi: 10.1002/pds.1806. Exclusion Code: X5.

157. Berard A, Karam F, Sheehy O, et al. Antidepressant use during pregnancy and the risk of delay in overall cognitive development at one year old: Results from the otis antidepressants study. Birth Defects Research Part A - Clinical and Molecular Teratology. 2012;94(5):368. doi: 10.1002/bdra.23023. Exclusion Code: X4.

158. Berard A, Karam F, Sheehy O, et al. Antidepressant use during pregnancy and the risk of delay in ooverall cognitive development at 1 year old: Results from the otis antidepressants study.

Pharmacoepidemiol Drug Saf. 2012;21:323. doi: 10.1002/pds.3324. Exclusion Code: $\mathrm{X} 4$.

159. Berard A, Karam F, Sheehy O, et al. Relapse of depression in pregnant users and discontinued users of antidepressants: Results from the OTIS Antidepressants Study. J Popul Ther Clin Pharmacol. 2011;18(2):e186-e7. Exclusion Code: X9.

160. Bérard A, Karam F, Sheehy O, et al. Relapse of depressive and anxiety symptoms, and quality-of-life in pregnant users and discontinued users of antidepressants: Results from the OTIS Antidepressants Study. J Popul Ther Clin Pharmacol. 2011;18(2):e285-e6. Exclusion Code: X9.

161. Berard A, Nakhai-Pour HR, Broy P. Antidepressant use (during pregnancy) and miscarriage. CMAJ. 2010 Jul 13;182(10):1079. doi: 10.1503/cmaj.1102084. PMID: 20624881. Exclusion Code: $\mathrm{X} 1$.

162. Berard A, Sheehy O. [Quebec Pregnancy Cohort: prevalence of medication use during gestation and pregnancy outcomes]. Therapie. 2014 Jan-Feb;69(1):71-81. doi: 10.2515/therapie/2014011. PMID: 24698191. Exclusion Code: X2. 
163. Bérard A, Sheehy O, Bernatsky S, et al. SNRI, bupropion and mirtazapine use during pregnancy and the risk of PPHN.

Pharmacoepidemiol Drug Saf. 2015;24:2634. doi: 10.1002/pds.3838. Exclusion Code:

X9.

164. Berard A, Sheehy O, Leung V. First trimester exposure to sertraline and the risk of major congenital malformations in a cohort of depressed women.

Pharmacoepidemiol Drug Saf. 2013;22:1434. doi: 10.1002/pds.3512. Exclusion Code: X9.

165. Berard A, Sheehy O, Zhao JP, et al. SSRI and SNRI use during pregnancy and the risk of persistent pulmonary hypertension of the newborn. Br J Clin Pharmacol. 2017

May;83(5):1126-33. doi:

10.1111/bcp.13194. PMID: 27874994. Exclusion Code: X4.

166. Bérard AB, Sheehy OS, Bernatsky SB, et al. Snri, bupropion, and mirtazapine use during pregnancy and the risk of pphn. Birth Defects Research Part A - Clinical and Molecular Teratology. 2015;103(5):411. doi: 10.1002/bdra.23387. Exclusion Code: X9.

167. Bergink V. Prevention of postpartum psychosis and mania in women at high risk. Eur Psychiatry. 2013;28. Exclusion Code: $\mathrm{X} 4$.

168. Bergink V, Bouvy P, Vervoort J, et al. Prevention of postpartum psychosis in women at high risk. Eur Psychiatry. 2012;27. doi: 10.1016/S09249338(12)75622-5. Exclusion Code: X4.

169. Bergink V, Bouvy P, Vervoort J, et al. Prevention of postpartum psychosis in women at high risk. Arch Womens Ment Health. 2013;16:S60. doi: 10.1007/s00737013-0355-x. Exclusion Code: X4.

170. Bergink V, Bouvy PF, Vervoort JS, et al. Prevention of postpartum psychosis and mania in women at high risk. Am J Psychiatry. 2012 Jun;169(6):609-15. doi: 10.1176/appi.ajp.2012.11071047. PMID: 22407083. Exclusion Code: X4.
171. Bergink V, Burgerhout KM, Koorengevel $\mathrm{KM}$, et al. Treatment of psychosis and mania in the postpartum period. Arch Womens Ment Health. 2015;18(2):390. doi: 10.1007/s00737-014-0488-6. Exclusion Code: X4.

172. Bergink V, Burgerhout KM, Koorengevel $\mathrm{KM}$, et al. Treatment of psychosis and mania in the postpartum period. Am J Psychiatry. 2015 Feb 1;172(2):115-23. doi: 10.1176/appi.ajp.2014.13121652. PMID: 25640930. Exclusion Code: X4.

173. Bergink V, Koorengevel KM. Treatment of postpartum depression with psychotic features. Arch Womens Ment Health. 2011;14:S60. doi: 10.1007/s00737-0100203-1. Exclusion Code: X7.

174. Bergink V, Rasgon N, Wisner KL. Postpartum psychosis: madness, mania, and melancholia in motherhood. Am J Psychiatry. 2016 Dec 1;173(12):1179-88. doi: 10.1176/appi.ajp.2016.16040454. PMID: 27609245. Exclusion Code: X1.

175. Bergman K, Sarkar P, O'Connor TG, et al. Maternal stress during pregnancy predicts cognitive ability and fearfulness in infancy. J Am Acad Child Adolesc Psychiatry. 2007 Nov;46(11):1454-63. doi: 10.1097/chi.0b013e31814a62f6. PMID: 18049295. Exclusion Code: X2.

176. Bergman U, Rosa FW, Baum C, et al. Effects of exposure to benzodiazepine during fetal life. Lancet. 1992 Sep 19;340(8821):694-6. PMID: 1355799. Exclusion Code: X7.

177. Berihu BA, Welderufael AL, Berhe Y, et al. Maternal risk factors associated with neural tube defects in Tigray regional state of Ethiopia. Brain Dev. 2019 Jan;41(1):11-8. doi: 10.1016/j.braindev.2018.07.013. PMID: 30075882. Exclusion Code: X2.

178. Berle JO, Steen VM, Aamo TO, et al. Breastfeeding during maternal antidepressant treatment with serotonin reuptake inhibitors: infant exposure, clinical symptoms, and cytochrome p450 genotypes. J Clin Psychiatry. 2004 Sep;65(9):1228-34. PMID: 15367050. Exclusion Code: X4. 
179. Bernard N, Forest JC, Tarabulsy GM, et al. Use of antidepressants and anxiolytics in early pregnancy and the risk of preeclampsia and gestational hypertension: a prospective study. BMC Pregnancy Childbirth. 2019 Apr 30;19(1):146. doi: 10.1186/s12884-0192285-8. PMID: 31039756. Exclusion Code: X5.

180. Bertino V, Caldiroli A, Dobrea C, et al. Are obstetrical complications really involved in the aetiology and outcome of psychotic and mood disorders? Eur

Neuropsychopharmacol. 2015;25:S367. Exclusion Code: X2.

181. Bhat A, Grote NK, Russo J, et al. Collaborative care for perinatal depression among socioeconomically disadvantaged women: adverse neonatal birth events and treatment response. Psychiatr Serv. 2017 Jan 1;68(1):17-24. doi: 10.1176/appi.ps.201600002. PMID: 27691376. Exclusion Code: X4.

182. Bhimanadham NN, Mainali P, Robert CA, et al. Hospital Outcomes in Antepartum Mental Disorders: A Study on 897,397 Pregnant Inpatients. Behav Sci (Basel). 2019 Sep 29;9(10). doi: 10.3390/bs9100105. PMID: 31569495 . Exclusion Code: X3.

183. Biffi A, Cantarutti A, Rea F, et al. Use of antidepressants during pregnancy and neonatal outcomes: An umbrella review of meta-analyses of observational studies. J Psychiatr Res. 2020 May;124:99-108. doi: 10.1016/j.jpsychires.2020.02.023. PMID: 32135392. Exclusion Code: X7.

184. Bíró A, Elek P. Caesarean delivery and the use of antidepressants. Eur J Public Health. 2020 Mar 27. doi: 10.1093/eurpub/ckaa047. PMID: 32219394. Exclusion Code: X4.

185. Bliddal S, Boas M, Hilsted L, et al. Increase in thyroid dysfunction and autoimmunity in danish pregnant women upon the iodine fortification is not associated with negative obstetric outcomes. Thyroid. 2015;25:A81. doi: 10.1089/thy.2015.29004.abstracts. Exclusion Code: X3.
186. Bloch M, Meiboom H, Lorberblat M, et al. Treatment of postpartum depression with psychotherapy and add-on sertraline: a double-blind, randomised, placebocontrolled study. European neuropsychopharmacology [abstracts from the 24th congress of the european college of neuropsychopharmacology, ECNP 2011 paris france, 3-7 sept]. 2011;21(Suppl 3):S359-60. PMID: CN-00850529. Exclusion Code: X9.

187. Bloem BR, Lammers GJ, Roofthooft DW, et al. Clomipramine withdrawal in newborns. Arch Dis Child Fetal Neonatal Ed. 1999 Jul;81(1):F77. PMID: 10744432. Exclusion Code: $\mathrm{X} 7$.

188. Bloomhardt H, Young ME, Salisbury AL. Sleep state development in early infancy after prenatal exposure to maternal depression and antidepressant medication use. Sleep. 2012;35:A372. Exclusion Code: $\mathrm{X} 5$.

189. Blotiere PO, Miranda S, Weill A, et al. Risk of early neurodevelopmental disorders associated with prenatal exposure to valproate: A nationwide cohort study based on the French health care databases. Pharmacoepidemiol Drug Saf. 2018;27:380. doi: 10.1002/pds.4629. Exclusion Code: X9.

190. Blotière PO, Miranda S, Weill A, et al. Risk of early neurodevelopmental outcomes associated with prenatal exposure to antiepileptic drugs: A Nationwide cohort study based on the French national healthcare databases. Pharmacoepidemiol Drug Saf. 2019;28:369-70. doi: 10.1002/pds.4864. Exclusion Code: X9.

191. Blotière PO, Raguideau F, Ehrhardt C, et al. Anticonvulsants and mood stabilizers prescribing during pregnancy in France between 2007 and 2014: A study based on the French healthcare databases. Pharmacoepidemiol Drug Saf. 2017;26:5056. doi: 10.1002/pds.4275. Exclusion Code: $\mathrm{X} 4$.

192. Blum LD. Postpartum depression. N Engl J Med. 2003 Mar 27;348(13):1294. doi: 10.1056/nejm200303273481319. PMID: 12660400. Exclusion Code: X1. 
193. Boath E, Bradley E, Henshaw C. The prevention of postnatal depression: a narrative systematic review. J Psychosom Obstet Gynaecol. 2005 Sep;26(3):185-92. PMID: 16295516. Exclusion Code: X7.

194. Boath E, Henshaw C. The treatment of postnatal depression: A comprehensive literature review. J Reprod Infant Psychol. 2001;19(3):215-48. Exclusion Code: X7.

195. Bobes J, Montes JM, Mostaza J, et al. Gynaecological and obstetric comorbidities in patients with bipolar disorder: A systematic review. Eur Psychiatry. 2009;24:S592. Exclusion Code: X7.

196. Boden R, Lundgren M, Brandt L, et al. Risks of adverse pregnancy and birth outcomes in women treated or not treated with mood stabilisers for bipolar disorder: population based cohort study. BMJ. 2012 Nov 8;345:e7085. doi: 10.1136/bmj.e7085. PMID: 23137820. Exclusion Code: X10.

197. Bodén R, Lundgren M, Brandt L, et al. Bipolar disorder, mood stabilizers and adverse pregnancy outcome-a population based cohort study. Pharmacoepidemiol Drug Saf. 2012;21:31. doi: 10.1002/pds.3324. Exclusion Code: X9.

198. Bogen DL, Hanusa BH, Moses-Kolko E, et al. Are maternal depression or symptom severity associated with breastfeeding intention or outcomes? J Clin Psychiatry. 2010 Aug;71(8):1069-78. doi: 10.4088/JCP.09m05383blu. PMID: 20584521. Exclusion Code: X2.

199. Borkowska A, Rybakowski J. [Factors connected with the effect of risperidone on psychopathological symptoms and working memory in schizophrenia]. Psychiatr Pol. 2002 Nov-Dec;36(6 Suppl):225-33. PMID: 12647443. Exclusion Code: X8.

200. Bosco Guerreiro da Silva J. Acupuncture for mild to moderate emotional complaints in pregnancy--a prospective, quasi-randomised, controlled study. Acupunct Med. 2007 Sep;25(3):65-71. PMID: 17906599. Exclusion Code: X3.
201. Bot P, Semmekrot BA, van der Stappen J. Neonatal effects of exposure to selective serotonin reuptake inhibitors during pregnancy. Arch Dis Child Fetal Neonatal Ed. 2006 Mar;91(2):F153. doi: 10.1136/adc.2004.066431. PMID: 16492956. Exclusion Code: X1.

202. Boucher N, Bairam A, Beaulac-Baillargeon L. A new look at the neonate's clinical presentation after in utero exposure to antidepressants in late pregnancy. J Clin Psychopharmacol. 2008 Jun;28(3):334-9. doi: 10.1097/JCP.0b013e318173aa2e. PMID: 18480693. Exclusion Code: X4.

203. Boukhris T. Antidepressant use during pregnancy and ADHD risk in children: current knowledge. BJOG. 2018;125(9):1085. doi: 10.1111/14710528.15118. Exclusion Code: X1.

204. Boukhris T, Muanda-Tsobo F, Bérard A. Antidepressant, especially SSRI use, during pregnancy and the risk of autism spectrum disorder: A meta-analysis.

Pharmacoepidemiol Drug Saf. 2015;24:246. doi: 10.1002/pds.3838. Exclusion Code: X7.

205. Boukhris T, Sheehy O, Berard A.

Antidepressant use during pregnancy and risk of autism spectrum disorders in children above or beyond of genetic background. Birth Defects Research Part A - Clinical and Molecular Teratology. 2014;100(5):384. Exclusion Code: X9.

206. Boukhris T, Sheehy O, Berard A. Antidepressant use in pregnancy and the risk of attention deficit with or without hyperactivity disorder in children. Pharmacoepidemiol Drug Saf. 2016;25:299. doi: 10.1002/pds.4070. Exclusion Code: X4.

207. Boukhris T, Sheehy O, Bérard A. Antidepressant use during pregnancy and risk of autism spectrum disorders in children: A population-based cohort study. Value Health. 2014;17(3):A211. doi: 10.1016/j.jval.2014.03.1236. Exclusion Code: X9.

208. Bowen A, Bowen R, Butt P, et al. Patterns of depression and treatment in pregnant and postpartum in women. The Canadian Journal of Psychiatry / La Revue canadienne de psychiatrie. 2012;57(3):161-7. PMID: 201210627-005. Exclusion Code: X4. 
209. Boyle B, Garne E, Loane M, et al. The changing epidemiology of Ebstein's anomaly and its relationship with maternal mental health conditions: a European registry-based study. Cardiol Young. 2017 May;27(4):677-85. doi: 10.1017/s1047951116001025. PMID: 27572669. Exclusion Code: X4.

210. Boyle RJ. Effects of certain prenatal drugs on the fetus and newborn. Pediatr Rev. 2002 Jan;23(1):17-24. PMID: 11773589.

Exclusion Code: X1.

211. Bozzo P, Koren G, Nava-Ocampo AA, et al. The incidence of nausea and vomiting of pregnancy (NVP): a comparison between depressed women treated with antidepressants and non-depressed women. Clin Invest Med. 2006 Dec;29(6):347-50. PMID: 17330450. Exclusion Code: X4.

212. Bracken MB. Case-control studies require appropriate population controls: an example of error in the SSRI birth defect literature. Eur J Epidemiol. 2015 Nov;30(11):1217-8. doi: 10.1007/s10654-015-0097-3. PMID: 26518772. Exclusion Code: X1.

213. Braillon A, Bewley S. Paucity of data on the safety of drugs for treating depression in pregnancy. BMJ. 2016 May 11;353:i2582. doi: 10.1136/bmj.i2582. PMID: 27170305. Exclusion Code: X1.

214. Brandlistuen RE, Ystrom E, Eberhard-Gran $\mathrm{M}$, et al. Behavioural effects of fetal antidepressant exposure in a Norwegian cohort of discordant siblings. Int J Epidemiol. 2015 Aug;44(4):1397-407. doi: 10.1093/ije/dyv030. PMID: 25873178. Exclusion Code: X4.

215. Brandlistuen RE, Ystrom E, HernandezDiaz S, et al. Association of prenatal exposure to benzodiazepines and child internalizing problems: A sibling-controlled cohort study. PLoS One.

2017;12(7):e0181042. doi:

10.1371/journal.pone.0181042. PMID: 28746341. Exclusion Code: X2.

216. Brennan PA, Pargas R, Walker EF, et al. Maternal depression and infant cortisol: influences of timing, comorbidity and treatment. J Child Psychol Psychiatry. 2008 Oct;49(10):1099-107. doi: 10.1111/j.14697610.2008.01914.x. PMID: 18492036. Exclusion Code: X5.
217. Bro SP, Kjaersgaard MIS, Parner ET, et al. Adverse pregnancy outcomes after exposure to methylphenidate or atomoxetine during pregnancy. Clin Epidemiol. 2015;7:139-47. doi: 10.2147/CLEP.S72906. Exclusion Code: X3.

218. Broeks SC, Thisted Horsdal H, Glejsted Ingstrup K, et al. Psychopharmacological drug utilization patterns in pregnant women with bipolar disorder-A nationwide register-based study. J Affect Disord. 2017;210:158-65. doi: 10.1016/j.jad.2016.12.001. PMID: 201706493-023. Exclusion Code: X4.

219. Bromley RL, Baker GA. Fetal antiepileptic drug exposure and cognitive outcomes. Seizure. 2017 Jan;44:225-31. doi: 10.1016/j.seizure.2016.10.006. PMID: 27784632. Exclusion Code: X1.

220. Bromley RL, Mawer G, Clayton-Smith J, et al. Autism spectrum disorders following in utero exposure to antiepileptic drugs. Neurology. 2008 Dec 2;71(23):1923-4. doi: 10.1212/01.wnl.0000339399.64213.1a. PMID: 19047565. Exclusion Code: X2.

221. Bromley RL, Wieck A, Makarova D, et al. Fetal effects of selective serotonin reuptake inhibitor treatment during pregnancy: Immediate and longer term child outcomes. Fetal Matern Med Rev. 2012;23(3-4):23075. doi: $10.1017 / \mathrm{S} 0965539512000095$. Exclusion Code: X7.

222. Bröms G, Hernández-Díaz S, Bateman B, et al. ADHD drugs during pregnancy and the risk of congenital malformations: a study from the international pregnancy safety study (inpress) consortium. Pharmacoepidemiol Drug Saf. 2017;26:4689. doi: 10.1002/pds.4275. Exclusion Code: $\mathrm{X} 7$.

223. Brouwer ME, Williams AD, van Grinsven SE, et al. Offspring outcomes after prenatal interventions for common mental disorders: a meta-analysis. BMC Med. 2018 Nov 15;16(1):208. doi: 10.1186/s12916-0181192-6. PMID: 30428883. Exclusion Code: $\mathrm{X} 7$.

224. Brown AS. Maternal influenza and other respiratory infections: Specificity to schizophrenia. Schizophr Bull. 2011;37:48. doi: $10.1093 /$ schbul/sbq173. Exclusion Code: X2. 
225. Brown HK, Hussain-Shamsy N, Lunsky Y, et al. The association between antenatal exposure to selective serotonin reuptake inhibitors and autism: a systematic review and meta-analysis. J Clin Psychiatry. 2017 Jan;78(1):e48-e58. doi: 10.4088/JCP.15r10194. PMID: 28129495. Exclusion Code: X7.

226. Brown HK, Ray JG, Wilton AS, et al. Association between serotonergic antidepressant use during pregnancy and autism spectrum disorder in children. JAMA. 2017 Apr 18;317(15):1544-52. doi: 10.1001/jama.2017.3415. PMID: 28418480. Exclusion Code: X10.

227. Brownell M, Singal D, Dahl M, et al. Inutero SSRI and SNRI exposure and the risk of long-term adverse mental and educational outcomes in children: A population-based retrospective cohort study utilizing linked administrative data. Pharmacoepidemiol Drug Saf. 2018;27:379-80. doi: 10.1002/pds.4629. Exclusion Code: X9.

228. Brummelte S, Galea LA, Devlin AM, et al. Antidepressant use during pregnancy and serotonin transporter genotype (SLC6A4) affect newborn serum reelin levels. Dev Psychobiol. 2013 Jul;55(5):518-29. doi: 10.1002/dev.21056. PMID: 22692766. Exclusion Code: X2.

229. Bschor T. [Tricyclic antidepressants for initial treatment of depressive episodes? Pro]. Nervenarzt. 2016 May;87(5):558-60. doi: 10.1007/s00115-015-0035-3. PMID: 26628045. Exclusion Code: X8.

230. Buckingham-Howes S, Mazza D, Wang Y, et al. Prenatal drug exposure and adolescent cortisol reactivity: association with behavioral concerns. J Dev Behav Pediatr. 2016 Sep;37(7):565-72. doi: 10.1097/dbp.0000000000000338. PMID: 27541579. Exclusion Code: X2.

231. Budenholzer B. ACP Journal Club. Use of SSRIs during pregnancy was not associated with increased risk for stillbirth or neonatal mortality. Ann Intern Med. 2013 Jun 18;158(12):Jc12. doi: 10.7326/0003-4819158-12-201306180-02012. PMID: 23778924. Exclusion Code: X1.
232. Buist A, Janson H. Effect of exposure to dothiepin and northiaden in breast milk on child development. Br J Psychiatry. 1995 Sep;167(3):370-3. PMID: 7496646. Exclusion Code: X4.

233. Bulik CM. Pregnancy and eating disorders: Reproductive and psychiatric outcomes. Arch Womens Ment Health. 2011;14:S12. doi: 10.1007/s00737-010-0203-1. Exclusion Code: X2.

234. Burbeck R, Pilling S, Tomson D, et al. Antidepressants in the postpartum period: additional considerations. Am J Psychiatry. 2008 Feb;165(2):262-3; author reply 3. doi: 10.1176/appi.ajp.2007.07091462. PMID: 18245193. Exclusion Code: X1.

235. Burian I, Lelli L, Giardinelli L, et al. The effects of treatment with selective serotonin reuptake inhibitors in pregnant depressed women on fetal and neonatal outcomes. Eur Neuropsychopharmacol. 2016;26:S475. Exclusion Code: X4.

236. Burt V, Miller L, Einarson A. SSRIs and birth defects. Epidemiology. 2007 May;18(3):410-1; author reply 2-3. doi: 10.1097/01.ede.0000259343.32599.a2. PMID: 17435452. Exclusion Code: X1.

237. Burt VK, Suri R, Altshuler L, et al. The use of psychotropic medications during breastfeeding. Am J Psychiatry. 2001

Jul;158(7):1001-9. doi: 10.1176/appi.ajp.158.7.1001. PMID: 11431219. Exclusion Code: X7.

238. Butz AM, Pulsifer MB, Belcher HME, et al. Infant head growth and cognitive status at 36 months in children with in-utero drug exposure. J Child Adolesc Subst Abuse. 2005;14(4):15-39. doi: 10.1300/J029v14n04_02. PMID: 200510091-002. Exclusion Code: X3.

239. Byatt N, Deligiannidis KM, Freeman MP. Antidepressant use in pregnancy: a critical review focused on risks and controversies. Acta Psychiatr Scand. 2013 Feb;127(2):94114. doi: 10.1111/acps.12042. PMID: 23240634. Exclusion Code: X7. 
240. Byatt N, Freeman MP. Exposure to selective serotonin reuptake inhibitors in late pregnancy increases the risk of persistent pulmonary hypertension of the newborn, but the absolute risk is low. Evid Based Nurs. 2015 Jan;18(1):15-6. doi: 10.1136/eb-2014101786. PMID: 24858440. Exclusion Code: $\mathrm{X} 1$.

241. Cantarutti A, Patorno E, Corrao G, et al. Antidepressant use in pregnancy and the risk of low Apgar score. Pharmacoepidemiol Drug Saf. 2017;26:406. doi: 10.1002/pds.4275. Exclusion Code: X4.

242. Capdevila E, Lasheras G, Porta R, et al. Postpartum depression, motherinfant bonding and postpartum coping strategies in mothers of newborns admitted to the neonatal unit. Journal of Maternal-Fetal and Neonatal Medicine. 2016;29:176-7. doi: 10.1080/14767058.2016.1191212. Exclusion Code: X3.

243. Cardenas A, Faleschini S, Cortes Hidalgo A, et al. Prenatal maternal antidepressants, anxiety, and depression and offspring DNA methylation: epigenome-wide associations at birth and persistence into early childhood. Clin Epigenetics. 2019 Mar 29;11(1):56. doi: 10.1186/s13148-019-0653-x. PMID: 30925934. Exclusion Code: X5.

244. Casilla-Lennon MM, Chantala K, MeltzerBrody S, et al. The effect of antidepressants on fertility. Fertil Steril. 2015;103(2):e16e7. doi: 10.1016/j.fertnstert.2014.12.023. Exclusion Code: X4.

245. Casparie AF, Miedema K, Peters PJ, et al. [Lithium intoxication during pregnancy].

Ned Tijdschr Geneeskd. 1974 Sep 14;118(37):1406-9. PMID: 4410030. Exclusion Code: X8.

246. Casper R. Selective serotonin reuptake inhibitors (SSRI) in pregnancy: The effects on the offspring. Biol Psychiatry.

2012;71(8):101S. doi: 10.1016/j.biopsych.2012.02.012. Exclusion Code: X7.
247. Casper RC, Gilles AA, Fleisher BE, et al. Length of prenatal exposure to selective serotonin reuptake inhibitor (SSRI) antidepressants: effects on neonatal adaptation and psychomotor development. Psychopharmacology (Berl). 2011 Sep;217(2):211-9. doi: 10.1007/s00213-0112270-z. PMID: 21499702. Exclusion Code: $\mathrm{X} 4$.

248. Castro VM, Kong SW, Clements CC, et al. Absence of evidence for increase in risk for autism or attention-deficit hyperactivity disorder following antidepressant exposure during pregnancy: a replication study. Transl Psychiatry. 2016 Jan 5;6:e708. doi: 10.1038/tp.2015.190. PMID: 26731445. Exclusion Code: X4.

249. Cesta CE, Olsson H, Cnattingius S, et al. Psychological distress and involuntary childlessness in women: A register-based Swedish cohort study. Hum Reprod. 2013;28:i70. doi: 10.1093/humrep/det177. Exclusion Code: X3.

250. Chambers CD, Hernandez-Diaz S, Van Marter LJ, et al. Selective serotoninreuptake inhibitors and risk of persistent pulmonary hypertension of the newborn. $\mathrm{N}$ Engl J Med. 2006 Feb 9;354(6):579-87. doi: 10.1056/NEJMoa052744. PMID: 16467545. Exclusion Code: X5.

251. Chambers CD, Johnson KA, Dick LM, et al. Birth outcomes in pregnant women taking fluoxetine. N Engl J Med. 1996 Oct 3;335(14):1010-5. doi: 10.1056/nejm199610033351402. PMID: 8793924. Exclusion Code: X4.

252. Chan RL, Jonsson Funk ML, Savitz DA, et al. Self-reported antidepressants use and spontaneous abortion. Am J Epidemiol. 2011;173:S140. doi: 10.1093/aje/kwr181. Exclusion Code: X4.

253. Charlton BG. Self-management and pregnancy--safe interventions for panic, phobia and other anxiety-disorders might include over-the-counter (OTC) 'SSRI' antihistamines such as diphenhydramine and chlorpheniramine. Acta Psychiatr Scand. 2005 Oct;112(4):323; author reply doi: 10.1111/j.1600-0447.2005.00610.x. PMID: 16156843. Exclusion Code: X1. 
254. Charlton BG. Self-management and pregnancy-safe interventions for panic, phobia and other anxiety-disorders might include over-the counter (OTC) 'SSRF antihistamines such as diphenhydramine and chlorpheniramine. Acta Psychiatr Scand. 2005;112(4):323-. doi: 10.1111/j.16000447.2005.00610.x. PMID: 2005-11155013. Exclusion Code: X1.

255. Chatillon O, Even C. [Antepartum depression: prevalence, diagnosis and treatment]. Encephale. 2010 Dec;36(6):44351. doi: 10.1016/j.encep.2010.02.004. PMID: 21130227. Exclusion Code: X8.

256. Chaudron LH. Treating pregnant women with antidepressants: the gray zone. J Womens Health (Larchmt). 2007

May;16(4):551-3. doi: 10.1089/jwh.2006.E073. PMID: 17521259. Exclusion Code: X1.

257. Chaudron LH, Jefferson JW. Mood stabilizers during breastfeeding: a review. J Clin Psychiatry. 2000 Feb;61(2):79-90. PMID: 10732654. Exclusion Code: X1.

258. Christensen J. Prenatal valproate exposure and risk of Autism-a population based study. Epilepsy Curr. 2012;12(1). Exclusion Code: $\mathrm{X} 2$.

259. Christensen J, Gronborg TK, Sorensen MJ, et al. Prenatal valproate exposure and risk of autism spectrum disorders and childhood autism. JAMA. 2013 Apr 24;309(16):1696703. doi: 10.1001/jama.2013.2270. PMID: 23613074. Exclusion Code: X2.

260. Christensen J, Grønborg TK, Sørensen MJ, et al. Prenatal valproate exposure and risk of autism spectrum disorders and childhood autism. Obstet Gynecol Surv.

2013;68(9):613-4. doi:

10.1097/OGX.0b013e3182a4a03f. Exclusion Code: X9.

261. Christensen J, Pedersen L, Sun Y, et al. Association of Prenatal Exposure to Valproate and Other Antiepileptic Drugs With Risk for AttentionDeficit/Hyperactivity Disorder in Offspring. JAMA Netw Open. 2019 Jan 4;2(1):e186606. doi: 10.1001/jamanetworkopen.2018.6606. PMID: 30646190 . Exclusion Code: X2.
262. Chua TE, Allen JC, Ang L, et al. Patterns and predictors of treatment outcome for antenatal major depression. Singapore Med J. 2017 Nov;58(11):642-8. doi: 10.11622/smedj.2016100. PMID: 27245863. Exclusion Code: X4.

263. Chung Y, Lee E, Noh J. A study on the effects of psychotropic drug use during pregnancy on fetal development in female patients with psychiatric disorders. Eur Neuropsychopharmacol. 2013;23:S213-S4. doi: 10.1016/S0924-977X(13)70329-8. Exclusion Code: X9.

264. Ciesielski TH, Marsit CJ, Williams SM. Maternal psychiatric disease and epigenetic evidence suggest a common biology for poor fetal growth. BMC Pregnancy Childbirth. 2015 Aug 25;15:192. doi: 10.1186/s12884-015-0627-8. PMID: 26303856. Exclusion Code: X4.

265. Clapp MA, Little SE, Mittal LP, et al. A multi-state analysis of the trends in psychiatric disease and substance use in pregnancy. Am J Obstet Gynecol. 2016;214(1):S201-S2. Exclusion Code: X5.

266. Clark CT, Klein AM, Wisner KL. Lamotrigine dosing for pregnant patients with bipolar disorder. Arch Womens Ment Health. 2013;16:S89-S90. doi: 10.1007/s00737-013-0355-x. Exclusion Code: X7.

267. Clements CC, Castro VM, Blumenthal SR, et al. Prenatal antidepressant exposure is associated with risk for attention-deficit hyperactivity disorder but not autism spectrum disorder in a large health system. Mol Psychiatry. 2014. doi: 10.1038/mp.2014.90. Exclusion Code: X4.

268. Clements CC, Castro VM, Blumenthal SR, et al. Prenatal antidepressant exposure is associated with risk for attention-deficit hyperactivity disorder but not autism spectrum disorder in a large health system. Mol Psychiatry. 2015 Jun;20(6):727-34. doi: 10.1038/mp.2014.90. PMID: 25155880. Exclusion Code: X4. 
269. Clemson C. S.08.01 Development of brexanolone iv, a GABA-A receptor positive allosteric modulator, for postpartum depression. Eur Neuropsychopharmacol. 2019;29:S10-S1. doi:

10.1016/j.euroneuro.2018.11.928. Exclusion Code: X9.

270. Clemson C, Meltzer-Brody S, Colquhoun H, et al. 86: Brexanolone iv efficacy in postpartum depression in three pivotal trials: montgomery-åsberg depression rating scale assessment. Am J Obstet Gynecol.

2019;220(1):S69-S70. doi:

10.1016/j.ajog.2018.11.095. Exclusion Code: X9.

271. Clinebell K, Gannon J, Debrunner S, et al. Long-acting risperidone injections in a pregnant patient with bipolar disorder. Bipolar Disord. 2017 Nov;19(7):606-7. doi: 10.1111/bdi.12572. PMID: 29120507. Exclusion Code: X1.

272. Cohen JM, Hernandez-Diaz S, Bateman BT, et al. ADHD medication use in pregnancy and risk of preeclampsia and small for gestational age birth. Pharmacoepidemiol Drug Saf. 2017;26:502-3. doi: 10.1002/pds.4275. Exclusion Code: X9.

273. Cohen JM, Patorno E, Huybrechts KF, et al. Mood stabilizer use and risk of ischemic placental disease. Pharmacoepidemiol Drug Saf. 2016;25:297. doi: 10.1002/pds.4070. Exclusion Code: X4.

274. Cohen L, Viguera A, Freeman M, et al. The national pregnancy registry for atypical antipsychotics: Effects of fetal exposure on risk for major malformations.

Neuropsychopharmacology. 2015;40:S321S2. doi: 10.1038/npp.2015.326. Exclusion Code: X4.

275. Cohen L, Viguera A, Freeman M, et al. The national pregnancy registry for atypical antipsychotics: effects of first trimester exposure to aripiprazole and quetiapine on risk for major malformations. Neuropsychopharmacology. 2016;41:S496. doi: 10.1038/npp.2016.242. Exclusion Code: $\mathrm{X} 4$.
276. Cohen L, Viguera AC, McInerney KA, et al. The national pregnancy registry for atypical antipsychotics: Effects of fetal exposure on risk for major malformations and extrapyramidal symptoms.

Neuropsychopharmacology. 2014;39:S459S60. doi: 10.1038/npp.2014.281. Exclusion Code: X4.

277. Cohen LS. Pharmacologic treatment of depression in women: PMS, pregnancy, and the postpartum period. Depress Anxiety. 1998;8 Suppl 1:18-26. PMID: 9809210. Exclusion Code: X1.

278. Cohen LS, Friedman JM, Jefferson JW, et al. A reevaluation of risk of in utero exposure to lithium. JAMA. 1994 Jan 12;271(2):146-50. PMID: 8031346. Exclusion Code: X7.

279. Cohen LS, Heller VL. On the use of anticonvulsants for manic depression during pregnancy. Psychosomatics. 1990 Fall;31(4):462-4. doi: 10.1016/s00333182(90)72150-0. PMID: 2078236. Exclusion Code: X1.

280. Cohen LS, Heller VL, Bailey JW, et al. Birth outcomes following prenatal exposure to fluoxetine. Biol Psychiatry. $2000 \mathrm{Nov}$ 15;48(10):996-1000. PMID: 11082474. Exclusion Code: X4.

281. Cohen LS, Nonacs R. Neurodevelopmental Implications of Fetal Exposure to Selective Serotonin Reuptake Inhibitors and Untreated Maternal Depression: Weighing Relative Risks. JAMA Psychiatry. 2016 Nov 1;73(11):1170-2. doi: 10.1001/jamapsychiatry.2016.2705. PMID: 27732715. Exclusion Code: X7.

282. Cohen LS, Rosenbaum JF. Birth outcomes in pregnant women taking fluoxetine. N Engl J Med. 1997 Mar 20;336(12):872; author reply 3. doi: 10.1056/nejm199703203361211. PMID: 9072682. Exclusion Code: X1.

283. Cohen LS, Rosenbaum JF, Heller VL. Prescribing lithium for pregnant women. Am J Psychiatry. 1988 Jun;145(6):772-3. PMID: 3369582. Exclusion Code: X1.

284. Cohen LS, Sichel DA, Dimmock JA, et al. Postpartum course in women with preexisting panic disorder. J Clin Psychiatry. 1994 Jul;55(7):289-92. PMID: 7915273. Exclusion Code: X2. 
285. Cohen LS, Sichel DA, Robertson LM, et al. Postpartum prophylaxis for women with bipolar disorder. Am J Psychiatry. 1995 Nov;152(11):1641-5. doi: 10.1176/ajp.152.11.1641. PMID: 7485628. Exclusion Code: X7.

286. Cohen LS, Viguera AC, Bouffard SM, et al. Venlafaxine in the treatment of postpartum depression. J Clin Psychiatry. 2001 Aug;62(8):592-6. PMID: 11561929. Exclusion Code: X7.

287. Cohen LS, Viguera AC, Masnik S, et al. The national pregnancy registry for atypical antipsychotics: Effects of fetal exposure on risk for congenital malformations and maternal/newborn outcomes. Birth Defects Research Part A - Clinical and Molecular Teratology. 2013;97(5):296. doi: 10.1002/bdra.23146. Exclusion Code: X7.

288. Cohen LS, Viguera AC, McLnerney KA, et al. Establishment of the national pregnancy registry for atypical antipsychotics. The Journal of Clinical Psychiatry.

2015;76(7):986-9. doi: 10.4088/JCP.14br09418. PMID: 201538869-020. Exclusion Code: X7.

289. Cohen MJ, Meador KJ, Browning N, et al. Fetal antiepileptic drug exposure: motor, adaptive, and emotional/behavioral functioning at age 3 years. Epilepsy Behav. 2011 Oct;22(2):240-6. doi: 10.1016/j.yebeh.2011.06.014. PMID: 21783425. Exclusion Code: X2.

290. Colvin L, Slack-Smith L, Stanley FJ, et al. Dispensing patterns and pregnancy outcomes for women dispensed selective serotonin reuptake inhibitors in pregnancy. Birth Defects Res A Clin Mol Teratol. 2011 Mar;91(3):142-52. doi: 10.1002/bdra.20773. PMID: 21381184. Exclusion Code: X10.

291. Colvin L, Slack-Smith L, Stanley FJ, et al. Early morbidity and mortality following in utero exposure to selective serotonin reuptake inhibitors: a population-based study in Western Australia. CNS Drugs. 2012 Jul 1;26(7):e1-14. doi: 10.2165/11634190-000000000-00000. PMID: 22712699. Exclusion Code: X2.
292. Condon J. Serotonergic symptoms in neonates exposed to SSRIs during pregnancy. Aust N Z J Psychiatry. 2003 Dec;37(6):777-8. doi: 10.1080/j.14401614.2003.01282.x. PMID: 14636401. Exclusion Code: X5.

293. Cooper M, Kilvert H, Eldar-Lissai A, et al. Evaluating pharmacotherapies for postpartum depression: A match-adjusted, indirect comparison of ssris to brexanolone injection, a novel investigational compound. J Manag Care Spec Pharm. 2019;25:S66-S7. Exclusion Code: X1.

294. Cooper MC, Kilvert HS, Hodgkins P, et al. Using Matching-Adjusted Indirect Comparisons and Network Meta-analyses to Compare Efficacy of Brexanolone Injection with Selective Serotonin Reuptake Inhibitors for Treating Postpartum Depression. CNS Drugs. 2019 Oct;33(10):1039-52. doi: 10.1007/s40263-019-00672-w. PMID: 31642037. Exclusion Code: X7.

295. Cooper SJ. Poisoned people: psychotropic drugs in pregnancy: morphological and psychological adverse effects on offspring. J Biosoc Sci. 1978 Jul;10(3):321-34. PMID: 690165. Exclusion Code: X1.

296. Coppola D, Russo LJ, Kwarta RF, Jr., et al. Evaluating the postmarketing experience of risperidone use during pregnancy:

pregnancy and neonatal outcomes. Drug Saf. 2007;30(3):247-64. doi: 10.2165/00002018200730030-00006. PMID: 17343431. Exclusion Code: X4.

297. Cornelius MD, De Genna NM, Goldschmidt L, et al. Adverse environmental exposures during gestation and childhood: Direct associations to adolescent drinking. Alcoholism: Clinical and Experimental Research. 2015;39:72A. doi: 10.1111/acer.12741. Exclusion Code: X2.

298. Corti S, Pileri P, Mandò C, et al. Neonatal outcomes of newborns exposed to SSRI during pregnancy: a pharmacokinetic and pharmacogenetic analysis. Reprod Sci. 2017;24(1):253A. doi: 10.1177/1933719117699773. Exclusion Code: X4. 
299. Corti S, Pileri P, Mazzocco M, et al. Exposure to SSRI during pregnancy and postpartum hemorrhage risk. Reprod Sci. 2017;24(1):189A. doi:

10.1177/1933719117699773. Exclusion Code: X4.

300. Corti S, Pileri P, Mazzocco MI, et al. Neonatal Outcomes in Maternal Depression in Relation to Intrauterine Drug Exposure. Front Pediatr. 2019;7:309. doi: 10.3389/fped.2019.00309. PMID: 31403037. Exclusion Code: X4.

301. Cossa N, Garlipp H, Kretz R, et al. Development and behavior of children aged 2 to 5 years after exposure to antiepileptic monotherapy in utero. Epilepsia. 2012;53:162. doi: 10.1111/j.15281167.2012.03677.x. Exclusion Code: X9.

302. Costei AM, Kozer E, Ho T, et al. Perinatal outcome following third trimester exposure to paroxetine. Arch Pediatr Adolesc Med. 2002 Nov;156(11):1129-32. PMID: 12413342. Exclusion Code: X4.

303. Costoloni G, Pierantozzi E, Goracci A, et al. [Mood stabilisers and pregnancy outcomes a review]. Psychiatr Pol. 2014 SepOct;48(5):865-87. PMID: 25639010. Exclusion Code: X1.

304. Courtney K. Use of SSRIs in pregnancy: neonatal implications. Nurs Womens Health. 2009 Jun;13(3):234-8. doi: 10.1111/j.1751486X.2009.01424.x. PMID: 19523137. Exclusion Code: X7.

305. Courtney MA, Bader AM, Hartwell B, et al. Perioperative analgesia with subarachnoid sufentanil administration. Reg Anesth. 1992 Sep-Oct;17(5):274-8. PMID: 1419940. Exclusion Code: X3.

306. Coverdale JH, McCullough LB, Chervenak FA. The ethics of randomized placebocontrolled trials of antidepressants with pregnant women: a systematic review. Obstet Gynecol. 2008 Dec;112(6):1361-8. doi: 10.1097/AOG.0b013e31818c2a27. PMID: 19037048. Exclusion Code: X7.

307. Craig M, Howard L. Postnatal depression. BMJ Clin Evid. 2009 Jan 26;2009. PMID: 19445768. Exclusion Code: X7.
308. Cramoy CC, Liu Z, Quirk MN, et al. Preconception treatment with antiepileptic drugs and the occurrence of fetal chromosomal abnormalities: Insights from the North American AED pregnancy registry. Birth Defects Research Part A Clinical and Molecular Teratology. 2015;103(5):388. doi: 10.1002/bdra.23387. Exclusion Code: X2.

309. Crane T, Neece C, Tagge E.

Neurobehavioral performace of infants in the neonatal intensive care unit. J Investig Med. 2014;62(1):167-8. doi: 10.231/JIM.0000000000000033. Exclusion Code: X2.

310. Crawford-Faucher A. Safety of psychotropic medications in breastfeeding. Am Fam Physician. 2010;81(11):1369-70. Exclusion Code: X7.

311. Creeley CE, Denton LK. Use of Prescribed Psychotropics during Pregnancy: A Systematic Review of Pregnancy, Neonatal, and Childhood Outcomes. Brain Sci. 2019 Sep 14;9(9). doi: 10.3390/brainsci9090235. PMID: 31540060 . Exclusion Code: X7.

312. Croen LA, Grether JK, Yoshida CK, et al. Antidepressant use during pregnancy and childhood autism spectrum disorders. Arch Gen Psychiatry. 2011 Nov;68(11):1104-12. doi: 10.1001/archgenpsychiatry.2011.73. PMID: 21727247. Exclusion Code: X4.

313. Csaky-Szunyogh M, Vereczkey A, Kosa Z, et al. Risk factors in the origin of congenital left-ventricular outflow-tract obstruction defects of the heart: a population-based case-control study. Pediatr Cardiol. 2014 Jan;35(1):108-20. doi: 10.1007/s00246-0130749-6. PMID: 23843102. Exclusion Code: $\mathrm{X} 4$.

314. Cummings C, Stewart M, Stevenson M, et al. Neurodevelopment of children exposed in utero to lamotrigine, sodium valproate and carbamazepine. Arch Dis Child. 2011 Jul;96(7):643-7. doi: 10.1136/adc.2009.176990. PMID: 21415043. Exclusion Code: X2. 
315. Cuomo A, Goracci A, Fagiolini A. Aripiprazole use during pregnancy, peripartum and lactation. A systematic literature search and review to inform clinical practice. J Affect Disord. 2018 Mar 1;228:229-37. doi: 10.1016/j.jad.2017.12.021. PMID: 29275156. Exclusion Code: X7.

316. Curry SJ, Krist AH, Owens DK, et al. Interventions to Prevent Perinatal Depression: US Preventive Services Task Force Recommendation Statement. JAMA. 2019 Feb 12;321(6):580-7. doi: 10.1001/jama.2019.0007. PMID: 30747971. Exclusion Code: X1.

317. Czeizel AE. Attempted suicide and pregnancy. J Inj Violence Res. 2011 Jan;3(1):45-54. doi: 10.5249/jivr.v3i1.77. PMID: 21483214. Exclusion Code: X2.

318. Czeizel AE, Erös E, Rockenbauer M, et al. Short-term oral diazepam treatment during pregnancy: A population-based teratological case-control study. Clin Drug Investig. 2003;23(7):451-62. Exclusion Code: X4.

319. Czeizel AE, Rockenbauer M, Sorensen HT, et al. A population-based case-control study of oral chlordiazepoxide use during pregnancy and risk of congenital abnormalities. Neurotoxicol Teratol. 2004 Jul-Aug;26(4):593-8. doi: 10.1016/j.ntt.2004.03.009. PMID: 15203182. Exclusion Code: X2.

320. Czeizel AE, Szegal BA, Joffe JM, et al. The effect of diazepam and promethazine treatment during pregnancy on the somatic development of human offspring. Neurotoxicol Teratol. 1999 MarApr;21(2):157-67. PMID: 10192276. Exclusion Code: X2.

321. Czeizel AE, Szegal BA, Joffe JM, et al. The effect of diazepam and promethazine treatment during pregnancy on the somatic development of human offspring. Neurotoxicol Teratol. 1999;21(2):157-67. doi: 10.1016/S0892-0362(98)00043-9. PMID: 1999-10883-005. Exclusion Code: $\mathrm{X} 2$.
322. Dahal KA, Patten SB, Lee S, et al. Adherence and persistence to antidepressant medication during pregnancy: Does it differ by the class of antidepressant medication prescribed? Arch Womens Ment Health. 2019;22(5):692. doi: 10.1007/s00737-01900996-y. Exclusion Code: X5.

323. Dahl ML, Olhager E, Ahlner J. Paroxetine withdrawal syndrome in a neonate. Br J Psychiatry. 1997 Oct;171:391-2. PMID: 9373435. Exclusion Code: X7.

324. Dalen K, Bruaroy S, Wentzel-Larsen T, et al. Cognitive functioning in children prenatally exposed to alcohol and psychotropic drugs. Neuropediatrics. 2009 Aug;40(4):162-7. doi: 10.1055/s-00291243176. PMID: 20135573. Exclusion Code: X2.

325. Dally P. Anorexia tardive-late onset marital anorexia nervosa. J Psychosom Res. 1984;28(5):423-8. doi: 10.1016/00223999(84)90074-6. PMID: 1985-28419-001. Exclusion Code: X2.

326. Dalton K, Herxheimer A. Treatment of postnatal depression. Additional information would enhance value of study. BMJ. 1997 Jul 12;315(7100):122. PMID: 9240067. Exclusion Code: X1.

327. Damkier P, Videbech P. The safety of second-generation antipsychotics during pregnancy: a clinically focused review. CNS Drugs. 2018;32(4):351-66. doi: 10.1007/s40263-018-0517-5. Exclusion Code: X7.

328. Dandjinou M, Sheehy O, Berard A. Should we treat or not mildly to moderately depressed pregnant women during pregnancy? Pharmacoepidemiol Drug Saf. 2016;25:328. doi: 10.1002/pds.4070. Exclusion Code: X9.

329. Dandjinou M, Sheehy O, Berard A. Antidepressants use during pregnancy and the risk of gestational diabetes mellitus. Birth defects research. Conference: 58th annual teratology society meeting. United states. 2018;110(9):775. doi: 10.1002/bdr2.1355. PMID: CN-01613448. Exclusion Code: X9. 
330. Dandjinou M, Sheehy O, Bérard A. Antidepressants use during pregnancy and the risk of gestational diabetes mellitus. Pharmacoepidemiol Drug Saf. 2018;27:3789. doi: 10.1002/pds.4629. Exclusion Code:

$\mathrm{X} 4$.

331. Dandjinou M, Sheehy O, Vigod S, et al. Prevalence and trends in depression/anxiety, antidepressant use and key maternal conditions during pregnancy, 1998-2015. Birth Defects Research. 2019;111(9):486. doi: 10.1002/bdr2.v111.9. Exclusion Code: X5.

332. Dandjinou M, Sheehy O, Vigod S, et al. Prevalence and trends in depression and anxiety, antidepressant use and key maternal conditions during pregnancy, 1998-2015. Pharmacoepidemiol Drug Saf. 2019;28:3823. doi: 10.1002/pds.4864. Exclusion Code: $\mathrm{X} 5$.

333. Das A, Gordon-Ocejo G, Kumar M, et al. Association of the previous history of maternal depression with post-partum depression, anxiety, and stress in the neonatal intensive care unit. J Matern Fetal Neonatal Med. 2019 Aug 4:1-6. doi: 10.1080/14767058.2019.1647162. PMID: 31327298. Exclusion Code: X2.

334. Dathe K, Schaefer C. The Use of Medication in Pregnancy. Dtsch Arztebl Int. 2019 Nov 15;116(46):783-90. doi: 10.3238/arztebl.2019.0783. PMID: 31920194. Exclusion Code: X2.

335. Davanzo R, Copertino M, De Cunto A, et al. Antidepressant drugs and breastfeeding: a review of the literature. Breastfeed Med. 2011 Apr;6(2):89-98. doi: 10.1089/bfm.2010.0019. PMID: 20958101. Exclusion Code: X1.

336. Davies E, Al-Muzaffar I. Neonatal outcome following maternal antidepressants use. Arch Dis Child. 2018;103:A80-A1. doi: 10.1136/archdischild-2018-rcpch.191. Exclusion Code: X4.

337. Dawes SD. Can SSRIs reduce the risk of preeclampsia in pregnant, depressed patients? Med Hypotheses. 2005;64(1):33-6. doi: 10.1016/j.mehy.2003.10.038. PMID: 15533606. Exclusion Code: X1.
338. Daya S. Aspirin in assisted reproduction. Evidence-based Obstetrics and Gynecology. 2004;6(4):218-9. doi: 10.1016/j.ebobgyn.2004.09.018. Exclusion Code: X3.

339. Dayan J, Graignic-Philippe R, Seligmann C, et al. Use of antipsychotics and breastfeeding. Current Women's Health Reviews. 2011;7(1):37-45. doi: 10.2174/157340411794474694. Exclusion Code: X7.

340. De Crescenzo F, Perelli F, Armando M, et al. Selective serotonin reuptake inhibitors (SSRIs) for post-partum depression (PPD): a systematic review of randomized clinical trials. J Affect Disord. 2014 Jan;152-154:3944. doi: 10.1016/j.jad.2013.09.019. PMID: 24139299. Exclusion Code: X7.

341. De Jonghe F, Swinkels J. Selective serotonin reuptake inhibitors: Relevance of differences in their pharmacological and clinical profiles. CNS Drugs. 1997;7(6):452-67. Exclusion Code: X7.

342. De las Cuevas C, J. Sanz E. Lithium use during early, late pregnancy, and breastfeeding. Current Women's Health Reviews. 2011;7(1):46-9. doi: 10.2174/157340411794474810. Exclusion Code: X1.

343. De Magistris A, Coni E, Puddu M, et al. Screening of postpartum depression: Comparison between mothers in the neonatal intensive care unit and in the neonatal section. Journal of Maternal-Fetal and Neonatal Medicine. 2010;23(SUPPL. 3):101-3. doi: 10.3109/14767058.2010.506759. Exclusion Code: X5.

344. De Matteis T, D'Andrea G, Lal J, et al. The impact of peri-natal stress on psychosis risk: results from the Bo-FEP incidence study. BMC Res Notes. 2020 Mar 16;13(1):153. doi: 10.1186/s13104-020-04992-9. PMID: 32178713. Exclusion Code: X3.

345. De Vera M, Bérard A. Antidepressant use during pregnancy and the risk of pregnancyinduced hypertension. J Popul Ther Clin Pharmacol. 2011;18(2):e280. Exclusion Code: X4. 
346. De Vera MA, Berard A. Antidepressant use during pregnancy and the risk of pregnancyinduced hypertension. Br J Clin Pharmacol. 2012 Aug;74(2):362-9. doi: 10.1111/j.13652125.2012.04196.x. PMID: 22435711.

Exclusion Code: X4.

347. De Vera MA, Hanley G, Oberlander T, et al. Antidepressant use during pregnancy and the risk of gestational hypertension. J Popul Ther Clin Pharmacol. 2011;18(2):e178. Exclusion Code: X4.

348. De Vera MA, Hanley G, Oberlander T, et al. Antidepressant use during pregnancy and the risk of gestational hypertension. Am J Epidemiol. 2011;173:S237. doi:

10.1093/aje/kwr181. Exclusion Code: X4.

349. De Vera MA, Rey E, St-Andre M, et al. Antidepressant use during pregnancy and the risk of gestational hypertension. Pharmacoepidemiol Drug Saf. 2011;20:S125-S6. doi: 10.1002/pds.2206. Exclusion Code: X4.

350. de Vries NK, van der Veere CN, Reijneveld $\mathrm{SA}$, et al. Early neurological outcome of young infants exposed to selective serotonin reuptake inhibitors during pregnancy: results from the observational SMOK study. PLoS One. 2013;8(5):e64654. doi: 10.1371/journal.pone.0064654. PMID: 23785389. Exclusion Code: X5.

351. Dean JC, Hailey H, Moore SJ, et al. Long term health and neurodevelopment in children exposed to antiepileptic drugs before birth. J Med Genet. 2002 Apr;39(4):251-9. PMID: 11950853. Exclusion Code: X2.

352. Defoort P, Thiery M, Maes R. Uterine and perinatal effects of lorazepam. IRCS MED.SCI.CLIN.PHARMACOL.THER. 1975;3(6):290. Exclusion Code: X2.

353. Degremont A, Le Nautout B, Rault C, et al. Impact of French Medicines Agency's communication on sodium valproate prescription in women of childbearing potential age. Fundam Clin Pharmacol. 2018;32:6. doi: 10.1111/fcp.12370. Exclusion Code: X5.

354. Dehn C, Dalhoff KP. [Do antipsychotic drugs during pregnancy affect the unborn baby?]. Ugeskr Laeger. 2007 Apr 30;169(18):1659-63. PMID: 17532870. Exclusion Code: X8.
355. Deiana V, Chillotti C, Manchia M, et al. Continuation versus discontinuation of lithium during pregnancy: a retrospective case series. J Clin Psychopharmacol. 2014 Jun;34(3):407-10. doi: 10.1097/jcp.0000000000000059. PMID: 24525653. Exclusion Code: X7.

356. Dekel S, Ein-Dor T, Ruohomäki A, et al. The dynamic course of peripartum depression across pregnancy and childbirth. J Psychiatr Res. 2019 Jun;113:72-8. doi: 10.1016/j.jpsychires.2019.03.016. PMID: 30921631. Exclusion Code: X3.

357. Deligiannidis K, Huang MY, Suthoff E, et al. Health-related quality of life in a phase 3, randomized, placebo-controlled trial of the neuroactive steroid GABAA receptor positive allosteric modulator SAGE-217 in postpartum depression.

Neuropsychopharmacology. 2019;44:299300. doi: 10.1038/s41386-019-0546-x. Exclusion Code: X3.

358. Deligiannidis K, Lasser R, Gunduz-Bruce H, et al. Evaluation of depression and anxiety in a phase 3 , double-blind, placebocontrolled trial of the neuroactive steroid GABAA receptor positive allosteric modulator SAGE-217 in postpartum depression. Neuropsychopharmacology. 2019;44:426-7. doi: 10.1038/s41386-0190547-9. Exclusion Code: X3.

359. Deligiannidis KM. Therapeutic drug monitoring in pregnant and postpartum women: recommendations for SSRIs, lamotrigine, and lithium. J Clin Psychiatry. 2010 May;71(5):649-50. doi: 10.4088/JCP.10ac06132gre. PMID: 20492855. Exclusion Code: X1.

360. Dellicour SOMC, Campbell J, Cotton S, et al. Utilising the CPRD pregnancy register to examine the pattern of antiepileptic drug use during pregnancy in the United Kingdom. Pharmacoepidemiol Drug Saf. 2018;27:3812. doi: 10.1002/pds.4629. Exclusion Code: $\mathrm{X} 5$.

361. Dennis CL, Allen K. Interventions (other than pharmacological, psychosocial or psychological) for treating antenatal depression. Cochrane Database Syst Rev. 2008 Oct 8(4):Cd006795. doi: 10.1002/14651858.CD006795.pub2. PMID: 18843730. Exclusion Code: X3. 
362. Dennis CL, Birken C, Hoch J, et al. Evaluating collaborative care for postpartum depression in early childhood primary care settings: a randomized controlled trial protocol. Arch Womens Ment Health. 2015;18(2):301. doi: 10.1007/s00737-0140488-6. PMID: CN-01084776. Exclusion Code: X3.

363. Dennis CL, Brown HK. Psychosocial, psychological, and pharmacological interventions for treating antenatal anxiety. Cochrane Database Syst Rev. 2017(11). doi: 10.1002/14651858.CD012870. PMID: CD012870. Exclusion Code: X7.

364. Dennis CL, Brown HK, Morrell J. Interventions (other than psychosocial, psychological and pharmacological) for preventing postpartum depression. Cochrane Database Syst Rev. 2016(5). doi: 10.1002/14651858.CD012201. PMID: CD012201. Exclusion Code: X3.

365. Dennis CL, Dowswell T. Interventions (other than pharmacological, psychosocial or psychological) for treating antenatal depression. Cochrane Database Syst Rev. 2013 Jul 31(7):Cd006795. doi: 10.1002/14651858.CD006795.pub3. PMID: 23904069. Exclusion Code: X3.

366. Dennis CL, Ross LE, Herxheimer A. Oestrogens and progestins for preventing and treating postpartum depression. Cochrane Database Syst Rev. 2008 Oct 8(4):Cd001690. doi: 10.1002/14651858.CD001690.pub2. PMID: 18843619. Exclusion Code: X7.

367. Dennis CL, Stewart DE. Treatment of postpartum depression, part 1: a critical review of biological interventions. J Clin Psychiatry. 2004 Sep;65(9):1242-51. PMID: 15367053. Exclusion Code: X7.

368. Deshmukh U, Adams J, Macklin EA, et al. Behavioral outcomes in children exposed prenatally to lamotrigine, valproate, or carbamazepine. Neurotoxicol Teratol. 2016 Mar-Apr;54:5-14. doi: 10.1016/j.ntt.2016.01.001. PMID: 26791321. Exclusion Code: X2.
369. Devarajan S, Devarajan K, Dursun S. Re: Treatment of perinatal mood and anxiety disorders: a review. Can J Psychiatry. 2008 Mar;53(3):209-10; author reply 10. doi: 10.1177/070674370805300315. PMID: 18444281. Exclusion Code: X1.

370. Dhaliwal G, Weikum W, JolicoeurMartineau A, et al. Household confusion, maternal depression and prenatal SSRI exposure and self-regulation from infancy to 6 years. Arch Womens Ment Health. 2019;22(5):653. doi: 10.1007/s00737-01900996-y. Exclusion Code: X5.

371. Di Florio A, Gordon-Smith K, Forty L, et al. Stratification of the risk of bipolar disorder recurrences in pregnancy and postpartum. $\mathrm{Br}$ J Psychiatry. 2018 Sep;213(3):542-7. doi: 10.1192/bjp.2018.92. PMID: 30113284. Exclusion Code: X3.

372. Di Giacomo E, Anniverno R, Bonfiglio M, et al. Escitalopram in pregnancy and postpartum: Maternal efficacy and neonatal outcomes. Arch Womens Ment Health. 2013;16:S115-S6. doi: 10.1007/s00737-0130355-x. Exclusion Code: X4.

373. di Scalea TL, Wisner KL. Pharmacotherapy of postpartum depression. Expert Opin Pharmacother. 2009 Nov;10(16):2593-607. doi: 10.1517/14656560903277202. PMID: 19874247. Exclusion Code: X7.

374. Dias MC, Jones I. Perinatal psychiatry. Medicine (United Kingdom). 2016;44(12):720-3. doi: 10.1016/j.mpmed.2016.09.006. Exclusion Code: X1.

375. Diav-Citrin O, Shechtman S, Bar-Oz B, et al. Pregnancy outcome after in utero exposure to valproate : evidence of dose relationship in teratogenic effect. CNS Drugs. 2008;22(4):325-34. doi: 10.2165/00023210-200822040-00004. PMID: 18336060. Exclusion Code: X2.

376. Diav-Citrin O, Shechtman S, Tahover E, et al. Is Lithium only a cardiovascular teratogen? A prospective comparative multicenter observational study. Birth Defects Research Part A - Clinical and Molecular Teratology. 2012;94(5):405. doi: 10.1002/bdra.23023. Exclusion Code: X4. 
377. Diav-Citrin O, Shechtman S, Zvi N, et al. Is it safe to use lamotrigine during pregnancy? A prospective comparative observational study. Birth Defects Res. 2017 Sep 1;109(15):1196-203. doi: 10.1002/bdr2.1058. PMID: 28657171. Exclusion Code: X2.

378. Dickson RA, Edwards A. Clozapine and fertility. Am J Psychiatry. 1997 Apr;154(4):582-3. PMID: 9090358. Exclusion Code: X1.

379. Dilaghi A, Mannaioni G, Ieri A, et al. Outcome of antiepileptic drug treated pregnant women. Clin Toxicol. 2010;48(3):297-8. doi: 10.3109/15563651003740240. Exclusion Code: X2.

380. Dilaghi A, Occupati B, Ieri A, et al. Use and safety of mood stabilizers and antipsychotic drugs during pregnancy: The experience of the Florence Teratology Information Service. Clin Toxicol. 2018;56(6):488. doi: 10.1080/15563650.2018.1457818.

Exclusion Code: X4.

381. Dolev Z, Muzik M. Perinatal psychiatry. Arch Womens Ment Health. 2011;14:S107. doi: 10.1007/s00737-011-0238-y. Exclusion Code: X7.

382. Dolk H, McCullough N, Callaghan S, et al. Risk factors for congenital heart disease: The Baby Hearts Study, a population-based case-control study. PLoS One. 2020;15(2):e0227908. doi: 10.1371/journal.pone.0227908. PMID: 32092068. Exclusion Code: X4.

383. Dolk H, McElhatton P. Assessing epidemiological evidence for the teratogenic effects of anticonvulsant medications. J Med Genet. 2002 Apr;39(4):243-4. PMID: 11950850. Exclusion Code: X1.

384. Dolk H, Wemakor A, Casson K, et al. Selective serotonin reuptake inhibitor antidepressant use in first trimester pregnancy and risk of congenital anomalies: A European register-based study in 12 European countries. Arch Womens Ment Health. 2015;18(2):366-7. doi: 10.1007/s00737-014-0488-6. Exclusion Code: X4.
385. Domar AD, Moragianni VA, Ryley DA, et al. The risks of selective serotonin reuptake inhibitor use in infertile women: a review of the impact on fertility, pregnancy, neonatal health and beyond. Hum Reprod. 2013 Jan;28(1):160-71. doi: 10.1093/humrep/des383. PMID: 23117129. Exclusion Code: X1.

386. Doucet S, Jones I, Letourneau N, et al. Interventions for the prevention and treatment of postpartum psychosis: a systematic review. Arch Womens Ment Health. 2011 Apr;14(2):89-98. doi: 10.1007/s00737-010-0199-6. PMID: 21128087. Exclusion Code: X7.

387. Driebe AM, Herrero T, Fratto V, et al. Elevated antenatal Edinburgh Depression Scale (EDS), maternal and neonatal outcomes and the association with behavioral versus pharmacologic therapy. Am J Obstet Gynecol. 2017;216(1):S464S5. Exclusion Code: X2.

388. Driscoll K. Lost in translation: Treating perinatal bipolar disorder. Biol Psychiatry. 2014;75(9):276S-7S. doi: 10.1016/j.biopsych.2014.03.016. Exclusion Code: X9.

389. Dubnov-Raz G, Hemila H, Vurembrand Y, et al. Maternal use of selective serotonin reuptake inhibitors during pregnancy and neonatal bone density. Early Hum Dev. 2012 Mar;88(3):191-4. doi: 10.1016/j.earlhumdev.2011.08.005. PMID: 21890289. Exclusion Code: X2.

390. Dubnov-Raz G, Juurlink DN, Fogelman R, et al. Antenatal use of selective serotoninreuptake inhibitors and QT interval prolongation in newborns. Pediatrics. 2008 Sep;122(3):e710-5. doi: 10.1542/peds.20080658. PMID: 18762507. Exclusion Code: $\mathrm{X} 4$.

391. Duncan D, Taylor D. Which antidepressants are safe to use in breast-feeding mothers? Psychiatric Bulletin. 1995;19(9):551-2. Exclusion Code: X1.

392. Duwe K, Rasmussen S, Louik C, et al. Maternal exposure to venlafaxine and risk for birth defects, National birth defects prevention study, 1997-2007. Pharmacoepidemiol Drug Saf. 2011;20:S264-S5. doi: 10.1002/pds.2206. Exclusion Code: X4. 
393. Dzhafarova N, Bindra T, Andriola M, et al. Neurodevelopmental outcome in children exposed to antiepileptic drugs in utero. Epilepsia. 2009;50:247. doi: 10.1111/j.15281167.2009.02377.x. Exclusion Code: X2.

394. Eberhard-Gran M, Eskild A, Opjordsmoen $\mathrm{S}$. Use of psychotropic medications in treating mood disorders during lactation : practical recommendations. CNS Drugs. 2006;20(3):187-98. doi: 10.2165/00023210200620030-00002. PMID: 16529525.

Exclusion Code: X7.

395. Edelson PK, Prabhu M, Berry M, et al. 745: SSRIs are not associated with increased severity of neonatal abstinence among women on opioid agonists. Am J Obstet Gynecol. 2020;222(1):S471. doi: 10.1016/j.ajog.2019.11.759. Exclusion Code: X2.

396. Edvinsson $\AA$, Hoyer A, Hansson M, et al. Placental glucocorticoid receptors are not affected by maternal depression or SSRI treatment. Ups J Med Sci. 2020

Feb;125(1):30-6. doi: 10.1080/03009734.2019.1702126. PMID: 31960733. Exclusion Code: X5.

397. Eggermont E. Withdrawal symptoms in neonates associated with maternal imipramine therapy. Lancet. $1973 \mathrm{Sep}$ 22;2(7830):680. PMID: 4125653. Exclusion Code: X7.

398. Eggermont E, Raveschot J, Deneve V, et al. The adverse influence of imipramine on the adaptation of the newborn infant to extrauterine life. Acta Paediatr Belg. 1972;26(4):197-204. PMID: 4656174.

Exclusion Code: X11.

399. Einarson A. Risks/safety of psychotropic medication use during pregnancy-Motherisk Update 2008. Can J Clin Pharmacol. 2009 Winter;16(1):e58-65. PMID: 19164847. Exclusion Code: X1.

400. Einarson A. Association between antidepressant use during pregnancy and infants born small for gestational age. Can J Psychiatry. 2011 Aug;56(8):509; author reply -10 . doi:

10.1177/070674371105600811. PMID: 21878163. Exclusion Code: X1.
401. Einarson A. Publishing statistically significant results with questionable clinical importance: focus on antidepressant use in pregnancy. J Clin Psychiatry. 2012 Nov;73(11):1443-6. doi: 10.4088/JCP.12com08192. PMID: 23218161. Exclusion Code: X1.

402. Einarson A, Bozzo P, Koren G, et al. Rates of spontaneous abortion and preterm birth in women who took an antidepressant during pregnancy: A comparison between continuers and discontinuers. Arch Womens Ment Health. 2011;14:S18. doi: 10.1007/s00737-010-0203-1. Exclusion Code: X9.

403. Einarson A, Choi J, Bozzo P, et al. Increased risk for spontaneous abortion and preterm birth associated with antidepressant use in pregnancy: A comparison between continuers and discontinuers.

Pharmacoepidemiol Drug Saf. 2010;19:S67. doi: 10.1002/pds.2019. Exclusion Code: X9.

404. Einarson A, Choi J, Einarson TR, et al. Rates of spontaneous and therapeutic abortions following use of antidepressants in pregnancy: results from a large prospective database. J Obstet Gynaecol Can. 2009 May;31(5):452-6. PMID: 19604427. Exclusion Code: X2.

405. Einarson A, Choi J, Einarson TR, et al. Incidence of major malformations in infants following antidepressant exposure in pregnancy: results of a large prospective cohort study. Can J Psychiatry. 2009 Apr;54(4):242-6. doi: 10.1177/070674370905400405. PMID: 19321030. Exclusion Code: X2.

406. Einarson A, Choi J, Einarson TR, et al. Adverse effects of antidepressant use in pregnancy: an evaluation of fetal growth and preterm birth. Depress Anxiety. 2010;27(1):35-8. doi: 10.1002/da.20598. PMID: 19691030. Exclusion Code: X2.

407. Einarson A, Davis W. Barriers to the pharmacological treatment of women with psychiatric disorders during pregnancy and breastfeeding: results of a survey. J Obstet Gynaecol Can. 2013 Jun;35(6):504-5. doi: 10.1016/s1701-2163(15)30905-1. PMID: 23870771. Exclusion Code: X2. 
408. Einarson A, Ito S. Use of contemporary antidepressants during breastfeeding [1]. Drug Saf. 2007;30(7):643. Exclusion Code: $\mathrm{X} 1$.

409. Einarson A, McKenna K, Levinson A. Review: women with schizophrenia have poorer pregnancy outcomes than other women, but it is unclear whether antipsychotic medications affect their infants. Evid Based Ment Health. 2003 Aug;6(3):89. PMID: 12893800. Exclusion Code: X7.

410. Einarson A, Pistelli A, DeSantis M, et al. Evaluation of the risk of congenital cardiovascular defects associated with use of paroxetine during pregnancy. Am J Psychiatry. 2008 Jun;165(6):749-52. doi: 10.1176/appi.ajp.2007.07060879. PMID: 18381907. Exclusion Code: X4.

411. Einarson A, Smart K, Vial T, et al. Rates of major malformations in infants following exposure to duloxetine during pregnancy: a preliminary report. J Clin Psychiatry. 2012 Nov;73(11):1471. doi: 10.4088/JCP.12108013. PMID: 23218163. Exclusion Code: X1.

412. Einarson A, Snyder C, Robinson G. Prenatal antidepressant use and risk of autism spectrum disorders in children. JAMA Pediatr. 2016 Jul 1;170(7):710-1. doi: 10.1001/jamapediatrics.2016.0742. PMID: 27243820. Exclusion Code: X1.

413. Einarson A, Weitzner B, Klieger-Grossmann C. Evaluation of pregnancy outcomes following use of escitalopram in pregnancy: A prospective comparative cohort study. Arch Womens Ment Health. 2011;14:S65. doi: 10.1007/s00737-010-0203-1. Exclusion Code: X9.

414. Einarson TR, Einarson A. Newer antidepressants in pregnancy and rates of major malformations: a meta-analysis of prospective comparative studies. Pharmacoepidemiol Drug Saf. 2005 Dec;14(12):823-7. doi: 10.1002/pds.1084. PMID: 15742359. Exclusion Code: X7.
415. Einarson TR, Koren G, Einarson A. Problems with maternal antidepressant treatment and neonatal outcomes study. Arch Gen Psychiatry. 2007 Jul;64(7):866; author reply 7-8. doi: 10.1001/archpsyc.64.7.866-a. PMID: 17606822. Exclusion Code: X1.

416. Eke AC, Saccone G, Berghella V. Selective serotonin reuptake inhibitor (SSRI) use during pregnancy and risk of preterm birth: a systematic review and meta-analysis. BJOG. 2016 Nov;123(12):1900-7. doi: 10.1111/1471-0528.14144. PMID: 27239775. Exclusion Code: X7.

417. El Marroun H. Use of SSRI during pregnancy and symptoms of autism in children. Tijdschrift voor Psychiatrie. 2015;57(4):288. Exclusion Code: X8.

418. El Marroun H, Jaddoe VW, Hudziak JJ, et al. Maternal use of selective serotonin reuptake inhibitors, fetal growth, and risk of adverse birth outcomes. Arch Gen Psychiatry. 2012 Jul;69(7):706-14. doi: 10.1001/archgenpsychiatry.2011.2333. PMID: 22393202. Exclusion Code: X12.

419. El Marroun H, White T, Verhulst FC, et al. Maternal use of antidepressant or anxiolytic medication during pregnancy and childhood neurodevelopmental outcomes: a systematic review. Eur Child Adolesc Psychiatry. 2014 Oct;23(10):973-92. doi: 10.1007/s00787014-0558-3. PMID: 24863148. Exclusion Code: X7.

420. El Marroun H, White TJ, van der Knaap NJ, et al. Prenatal exposure to selective serotonin reuptake inhibitors and social responsiveness symptoms of autism: population-based study of young children. Br J Psychiatry. 2014 Aug;205(2):95-102. doi: 10.1192/bjp.bp.113.127746. PMID: 25252317. Exclusion Code: X12.

421. Elbeshir LE, Pretlove SP, Coccia FC, et al. To evaluate the effects of antipsychotic medications taken in pregnancy, compared with antidepressants and a control group who had mental illness but who were not on medication, in the setting of a specialist antenatal liaison mental health clinic. BJOG. 2013;120:15-6. doi: 10.1111/14710528.12333. Exclusion Code: X7. 
422. Elbeshir LE, Pretlove SP, Coccia FC, et al. To evaluate the effects of anti-psychotic medications taken in pregnancy, compared to anti-depressants and a control group who had mental illness, but were not on medication. the setting was a specialist antenatal liaison mental health clinic. Arch Dis Child Fetal Neonatal Ed. 2013;98. doi: 10.1136/archdischild-2013-303966.147. Exclusion Code: X4.

423. Eldar-Lissai A, Meltzer-Brody S, Gerbasi $\mathrm{M}$, et al. PMH51 BREXANOLONE INJECTION FOR THE TREATMENT OF POSTPARTUM DEPRESSION: MINIMAL IMPORTANT DIFFERENCE AND MEANINGFUL CHANGE. Value Health. 2019;22:S690. doi:

10.1016/j.jval.2019.09.1525. Exclusion Code: X9.

424. Eldar-Lissai A, Meltzer-Brody S, Gerbasi $\mathrm{M}$, et al. PMH2 BREXANOLONE INJECTION IN POSTPARTUM DEPRESSION: SUSTAINED REMISSION, SUSTAINED RESPONSE, AND NUMBER NEEDED TO TREAT. Value Health. 2019;22:S681. doi: 10.1016/j.jval.2019.09.1476. Exclusion Code: X9.

425. Emory EK, Dieter JN. Maternal depression and psychotropic medication effects on the human fetus. Ann N Y Acad Sci. 2006 Dec;1094:287-91. doi: 10.1196/annals.1376.036. PMID: 17347363. Exclusion Code: X2.

426. Englerová K, Takács L. The effects of prenatal, perinatal and neonatal factors on academic performance in primary school age children. Ceska Gynekol. 2020 Winter;85(1):71-9. PMID: 32414288. Exclusion Code: X8.

427. Ennis ZN, Damkier P. Pregnancy exposure to olanzapine, quetiapine, risperidone, aripiprazole and risk of congenital malformations. A systematic review. Basic Clin Pharmacol Toxicol. 2015

Apr;116(4):315-20. doi:

10.1111/bcpt.12372. PMID: 25536446. Exclusion Code: X7.

428. Epperson CN, Anderson GM, McDougle CJ. Sertraline and breast-feeding. N Engl J Med. 1997 Apr 17;336(16):1189-90. doi: 10.1056/nejm199704173361615. PMID: 9102576. Exclusion Code: X1.
429. Epperson CN, McDougle CJ, Ward-O'Brien $\mathrm{D}$, et al. A controlled study of antidepressant treatment of postpartum depression. 149th annual meeting of the american psychiatric association; 1996 may 4-9; new york, NY. 1996:0028. PMID: CN-00212263. Exclusion Code: X9.

430. Epperson CN, Wisner KL, Yamamoto B. Gonadal steroids in the treatment of mood disorders. Psychosom Med. 1999 SepOct;61(5):676-97. PMID: 10511016. Exclusion Code: X1.

431. Epperson N, Czarkowski KA, Ward-O'Brien $\mathrm{D}$, et al. Maternal sertraline treatment and serotonin transport in breast-feeding motherinfant pairs. Am J Psychiatry. 2001

Oct;158(10):1631-7. doi:

10.1176/appi.ajp.158.10.1631. PMID: 11578995. Exclusion Code: X7.

432. Erickson NL, Hancock GR, Oberlander TF, et al. Prenatal SSRI antidepressant use and maternal internalizing symptoms during pregnancy and postpartum: Exploring effects on infant temperament trajectories for boys and girls. J Affect Disord. 2019 Nov 1;258:179-94. doi: 10.1016/j.jad.2019.08.003. PMID: 31437600. Exclusion Code: X4.

433. Eriksson K, Viinikainen K, Monkkonen A, et al. Children exposed to valproate in utero-population based evaluation of risks and confounding factors for long-term neurocognitive development. Epilepsy Res. 2005 Jul;65(3):189-200. doi: 10.1016/j.eplepsyres.2005.06.001. PMID: 16029946. Exclusion Code: X2.

434. Eros E, Czeizel AE, Rockenbauer M, et al. A population-based case-control teratologic study of nitrazepam, medazepam, tofisopam, alprazolum and clonazepam treatment during pregnancy. Eur J Obstet Gynecol Reprod Biol. 2002 Mar 10;101(2):147-54. PMID: 11858890. Exclusion Code: X2.

435. Escande M, Gayral L, Goldberger E. [Depression and antidepressive agents during treatments by prolonged-action neuroleptics. Side effects and "emergent metatherapeutic syndrome"]. Ann Med Psychol (Paris). 1974 Dec;2(5):669-94. PMID: 4458592. Exclusion Code: X8. 
436. Espinosa JP, Roige ES, Sagué M, et al. P.333 Obstetric outcomes in women with bipolar disorder treated with lithium in the perinatal period: a case-control study. Eur Neuropsychopharmacol. 2020;31:S71-S2. doi: 10.1016/j.euroneuro.2019.12.096.

Exclusion Code: X9.

437. Evans-Hoeker EA, Eisenberg E, Legro RS, et al. Depressive symptoms, antidepressant use and fertility treatment outcomes. Fertil Steril. 2017 Sep;108(3):E298-E9. doi: DOI 10.1016/j.fertnstert.2017.07.882. PMID: WOS:000409446002095. Exclusion Code: $\mathrm{X} 2$.

438. Evatt ML, DeLong MR, Grant WB, et al. Autism spectrum disorders following in utero exposure to antiepileptic drugs. Neurology. 2009 Sep 22;73(12):997. doi: 10.1212/WNL.0b013e3181af0b95. PMID: 19770480. Exclusion Code: X1.

439. Farriaux JP, Ghisolfi J, Lenoir G, et al. [Psychotropic drugs and breast feeding]. Arch Fr Pediatr. 1986 Mar;43(3):211-4. PMID: 2875699. Exclusion Code: X8.

440. Favreliere S, Nourrisson A, Jaafari N, et al. [Treatment of depressed pregnant women by selective serotonin reuptake inhibitors: risk for the foetus and the newborn]. Encephale. 2010 Jun;36(Suppl 2):D133-8. doi: 10.1016/j.encep.2009.06.005. PMID: 20513456. Exclusion Code: X8.

441. Feldman M. Lithium and pregnancy. Isr J Psychiatry Relat Sci. 2008;45(4):300-1; author reply 1. PMID: 19452657 . Exclusion Code: X1.

442. Fenger-Gron J, Thomsen M, Andersen KS, et al. Paediatric outcomes following intrauterine exposure to serotonin reuptake inhibitors: a systematic review. Dan Med Bull. 2011 Sep;58(9):A4303. PMID: 21893008. Exclusion Code: X7.

443. Fergusson DM, Horwood LJ, Lynskey MT. Maternal depressive symptoms and depressive symptoms in adolescents. Child Psychology \& Psychiatry \& Allied Disciplines. 1995;36(7):1161-78. doi: 10.1111/j.1469-7610.1995.tb01363.x. PMID: 1996-14518-001. Exclusion Code: X3.
444. Ferreira E, Carceller AM, Agogue C, et al. Effects of selective serotonin reuptake inhibitors and venlafaxine during pregnancy in term and preterm neonates. Pediatrics. 2007 Jan;119(1):52-9. doi: 10.1542/peds.2006-2133. PMID: 17200271. Exclusion Code: X4.

445. Figueroa R. Use of antidepressants during pregnancy and risk of attentiondeficit/hyperactivity disorder in the offspring. J Dev Behav Pediatr. 2010 Oct;31(8):641-8. doi: 10.1097/DBP.0b013e3181e5ac93. PMID: 20613624. Exclusion Code: X4.

446. Fitton CA, Steiner MFC, Aucott L, et al. In utero exposure to antidepressant medication and neonatal and child outcomes: a systematic review. Acta Psychiatr Scand. 2020 Jan;141(1):21-33. doi: 10.1111/acps.13120. PMID: 31648376. Exclusion Code: X7.

447. Flores-Ramos M, Galindo-Sevilla N, Córdova Barrios A, et al. Depression and anxiety during pregnancy: Clinical aspects. Current Psychiatry Reviews. 2013;9(4):32530. doi: $10.2174 / 15734005113096660010$. PMID: 2014-02850-006. Exclusion Code: $\mathrm{X} 7$.

448. Fluegge K. Prenatal antidepressant use and risk of autism spectrum disorders in children. JAMA Pediatr. 2016 Jul 1;170(7):710. doi: 10.1001/jamapediatrics.2016.0739. PMID: 27243632. Exclusion Code: X1.

449. Fombonne E. Prenatal antidepressant use and risk of autism spectrum disorders in the children. JAMA Pediatr. 2016 Jul 1;170(7):711-2. doi: 10.1001/jamapediatrics.2016.0745. PMID: 27244054. Exclusion Code: X1.

450. Fong A, Simon-Freeman R, Westermann M, et al. Maternal depression in pregnancy and its association with adverse perinatal outcomes. Am J Obstet Gynecol. 2017;216(1):S439. Exclusion Code: X3.

451. Ford JB. Further research supports association between antidepressant use and elevated risk of postpartum haemorrhage. Evid Based Nurs. 2017 Jul;20(3):76. doi: 10.1136/eb-2016-102600. PMID: 28424199. Exclusion Code: X1. 
452. Ford JB, Morris JM. Peripartum antidepressant use is associated with an increased risk of postpartum haemorrhage. Evid Based Med. 2014 Apr;19(2):79. doi: 10.1136/eb-2013-101570. PMID: 24282177. Exclusion Code: X1.

453. Fornaro M, Maritan E, Ferranti R, et al. Lithium Exposure During Pregnancy and the Postpartum Period: A Systematic Review and Meta-Analysis of Safety and Efficacy Outcomes. Am J Psychiatry. 2020 Jan 1;177(1):76-92. doi: 10.1176/appi.ajp.2019.19030228. PMID: 31623458. Exclusion Code: X7.

454. Forsberg L, Berglund G, Navér L, et al. Bipolar disorder and pregnancyneurodevelopmental outcome of children exposed to maternal illness with or without lithium during pregnancy. Arch Dis Child. 2012;97:A184-A5. doi:

10.1136/archdischild-2012-302724.0636. Exclusion Code: X9.

455. Forsberg L, Navér L, Gustafsson LL, et al. Neonatal adaptation in infants prenatally exposed to antidepressants; clinical monitoring using neonatal abstinence score. Arch Dis Child. 2014;99:A476. doi: 10.1136/archdischild-2014-307384.1319. Exclusion Code: X4.

456. Fortinguerra F, Clavenna A, Bonati M. Psychotropic drug use during breastfeeding: a review of the evidence. Pediatrics. 2009 Oct;124(4):e547-56. doi: 10.1542/peds.2009-0326. PMID: 19736267. Exclusion Code: X7.

457. Frayne J, Nguyen T, Mok T, et al. Lithium exposure during pregnancy: outcomes for women who attended a specialist antenatal clinic. J Psychosom Obstet Gynaecol. 2018 Sep;39(3):211-9. doi: 10.1080/0167482x.2017.1337743. PMID: 28617151. Exclusion Code: X7.

458. Freeman MP. Pregnancy and psychiatric disorders: inherent risks and treatment decisions. J Clin Psychiatry. 2013

Apr;74(4):373-4. doi: 10.4088/JCP.13f08356. PMID: 23656843. Exclusion Code: X1.
459. Freeman MP, Goez-Mogollon L, Sosinsky AZ, et al. The impact of obesity on pregnancy outcomes among women with psychiatric disorders: Results from a prospective pregnancy registry. J Psychosom Res. 2019 Aug;123:109735. doi: 10.1016/j.jpsychores.2019.109735. PMID: 31376871. Exclusion Code: X3.

460. Freitas C, Macedo S, Queirós S. Psychotropic drugs and pregnancy. Eur Neuropsychopharmacol. 2014;24:S749. Exclusion Code: X7.

461. Friedman EH. Neurobiology of the use of selective serotonin reuptake inhibitors during pregnancy. Can J Psychiatry. 2001 May;46(4):371. doi: 10.1177/070674370104600419. PMID: 11387796. Exclusion Code: X1.

462. Friedman SH, Nagle-Yang S, Amin J, et al. Perinatal psychiatry in the neonatal intensive care unit. Arch Womens Ment Health. 2011;14:S67. doi: 10.1007/s00737-0100203-1. Exclusion Code: X5.

463. Fritel X, Tsegan YE, Pierre F, et al. Association of postpartum depressive symptoms and urinary incontinence. A cohort study. Eur J Obstet Gynecol Reprod Biol. 2016 Mar;198:62-7. doi: 10.1016/j.ejogrb.2015.12.028. PMID: 26799771. Exclusion Code: X2.

464. Furu K, Kieler H, Haglund B, et al. Selective serotonin reuptake inhibitors and venlafaxine in early pregnancy and risk of birth defects: population based cohort study and sibling design. BMJ. $2015 \mathrm{Apr}$ 17;350:h1798. doi: 10.1136/bmj.h1798. PMID: 25888213. Exclusion Code: X4.

465. Furu K, Kieler H, Haglund B, et al. Selective serotonin reuptake inhibitors and venlafaxine in early pregnancy and risk of birth defects: Population based cohort study and sibling design. Obstet Gynecol Surv. 2015;70(9):553-4. Exclusion Code: X9.

466. Furukawa TA. Adverse effects of antidepressants during pregnancy. Evid Based Ment Health. 2014 Nov;17(4):103-4. doi: 10.1136/eb-2014-101964. PMID: 25326493. Exclusion Code: X1. 
467. Galbally M, Ijzendoorn M, Permezel M, et al. Mercy Pregnancy and Emotional Wellbeing Study (MPEWS): Understanding maternal mental health, fetal programming and child development Study design and cohort profile. Int J Methods Psychiatr Res. 2017;26(4):1-10. doi: 10.1002/mpr.1558. PMID: 2017-54618-002. Exclusion Code: $\mathrm{X} 7$.

468. Galbally M, Lewis A, Gentile S, et al. Maternal antidepressant treatment in pregnancy and child developmental outcomes. Arch Womens Ment Health. 2013;16:S53. doi: 10.1007/s00737-0130355-x. Exclusion Code: X7.

469. Galbally M, Lewis AJ, Buist A. Developmental outcomes of children exposed to antidepressants in pregnancy. Aust N Z J Psychiatry. 2011 May;45(5):3939. doi: $10.3109 / 00048674.2010 .549995$. PMID: 21314237. Exclusion Code: X2.

470. Galbally M, Lewis AJ, Buist A. Child developmental outcomes in preschool children following antidepressant exposure in pregnancy. Aust N Z J Psychiatry. 2015 Jul;49(7):642-50. doi: 10.1177/0004867415569800. PMID: 25698806. Exclusion Code: X4.

471. Galbally M, Lewis AJ, Lum J, et al. Serotonin discontinuation syndrome following in utero exposure to antidepressant medication: prospective controlled study. Aust N Z J Psychiatry. 2009 Sep;43(9):846-54. doi: 10.1080/00048670903107583. PMID: 19670058. Exclusion Code: X2.

472. Galbally M, Roberts M, Buist A. Mood stabilizers in pregnancy: a systematic review. Aust N Z J Psychiatry. 2010 Nov;44(11):967-77. doi: 10.3109/00048674.2010.506637. PMID: 21034180. Exclusion Code: X7.

473. Galbally M, Ryan J, van IM, et al. Maternal depression, antidepressant use and placental oxytocin receptor DNA methylation: Findings from the MPEWS study. Psychoneuroendocrinology. 2018 Apr;90:18. doi: 10.1016/j.psyneuen.2018.01.004. PMID: 29407512. Exclusion Code: X5.
474. Galbally M, Snellen M, Walker S, et al. Management of antipsychotic and mood stabilizer medication in pregnancy: recommendations for antenatal care. Aust N Z J Psychiatry. 2010 Feb;44(2):99-108. doi: 10.3109/00048670903487217. PMID: 20113298. Exclusion Code: X7.

475. Galbally M, Spigset O, Johnson AR, et al. Neonatal adaptation following intrauterine antidepressant exposure: assessment, drug assay levels, and infant development outcomes. Pediatr Res. 2017 Nov;82(5):80613. doi: 10.1038/pr.2017.156. PMID: 28665925. Exclusion Code: X4.

476. Galbally M, Watson SJ, Ball H, et al. Breastfeeding, antidepressants, and depression in the mercy pregnancy and emotional well-being study. J Hum Lact. 2018 Mar 1:890334418758658. doi: 10.1177/0890334418758658. PMID: 29596759. Exclusion Code: X12.

477. Galbally M, Watson SJ, Teti D, et al. Perinatal maternal depression, antidepressant use and infant sleep outcomes: Exploring cross-lagged associations in a pregnancy cohort study. J Affect Disord. 2018 Oct 1;238:218-25. doi: 10.1016/j.jad.2018.05.025. PMID: 29886202. Exclusion Code: X5.

478. Galbally M, Watson SJ, van IM, et al. The role of glucocorticoid and mineralocorticoid receptor DNA methylation in antenatal depression and infant stress regulation. Psychoneuroendocrinology. 2020

May;115:104611. doi: 10.1016/j.psyneuen.2020.104611. PMID: 32087522. Exclusion Code: X5.

479. Gao SY, Wu QJ, Zhang TN, et al. Fluoxetine and congenital malformations: a systematic review and meta-analysis of cohort studies. Br J Clin Pharmacol. 2017 Oct;83(10):2134-47. doi:

10.1111/bcp.13321. PMID: 28513059. Exclusion Code: X7.

480. García-Esteve L, Luisa Imaz M, Roca A, et al. Selective serotonin reuptake inhibitors (SSRI) discontinuation during pregnancy: Risk or benefit? Arch Womens Ment Health. 2013;16:S69. doi: 10.1007/s00737-0130355-x. Exclusion Code: X9. 
481. Garcia-Esteve L, Torres A, Imaz ML, et al. Long-term developmental effects on children exposed to selective serotonin reuptake inhibitors in Utero: Preliminary findings. Eur Psychiatry. 2012;27. doi: 10.1016/S0924-9338(12)75633-X. Exclusion Code: X4.

482. Gardener H, Spiegelman D, Buka SL. Prenatal risk factors for autism: comprehensive meta-analysis. Br J Psychiatry. $2009 \mathrm{Jul} ; 195(1): 7-14$. doi: 10.1192/bjp.bp.108.051672. PMID: 19567888. Exclusion Code: X7.

483. Garne E, Hansen AV, Birkelund AS, et al. Major congenital anomalies in a Danish region. Dan Med J. 2014 Jun;61(6):A4825. PMID: 24947618. Exclusion Code: X4.

484. Garriga M, Fernandez-Egea E, Mallorqui A, et al. Antipsychotic-induced weight gain and birth weight in psychosis: A fetal programming model. J Psychiatr Res. 2019 Aug;115:29-35. doi:

10.1016/j.jpsychires.2019.05.004. PMID: 31085376. Exclusion Code: X2.

485. Garrison-Desany HM, Nawa N, Kim Y, et al. Polydrug Use During Pregnancy and Preterm Birth in a Low-Income, Multiethnic Birth Cohort, Boston, 1998-2018. Public Health Rep. 2020 May/Jun;135(3):383-92. doi: 10.1177/0033354920915437. PMID: 32311304. Exclusion Code: X3.

486. Gartstein MA, Hookenson KV, Brain U, et al. Sculpting infant soothability: the role of prenatal SSRI antidepressant exposure and neonatal SLC6A4 methylation status. Dev Psychobiol. 2016 Sep;58(6):745-58. doi: 10.1002/dev.21414. PMID: 27254389. Exclusion Code: X4.

487. Gavin AR, Holzman C, Siefert K, et al. Maternal depressive symptoms, depression, and psychiatric medication use in relation to risk of preterm delivery. Womens Health Issues. 2009 Sep-Oct;19(5):325-34. doi: 10.1016/j.whi.2009.05.004. PMID: 19733802. Exclusion Code: X4.

488. Gawade PL, Oeffinger KC, Sklar CA, et al. Lifestyle, distress, and pregnancy outcomes in the Childhood Cancer Survivor Study cohort. Am J Obstet Gynecol. 2014. doi: 10.1016/j.ajog.2014.07.036. Exclusion Code: X2.
489. Geisler JG. 2,4 dinitrophenol as medicine. Cells. 2019;8(3). doi: 10.3390/cells8030280. Exclusion Code: X1.

490. Gelisse P, Genton P, Crespel A. Is there a difference between various presentations of valproate for cognitive outcome after in utero exposure? Epilepsia. 2016 Mar;57(3):523-4. doi: 10.1111/epi.13301. PMID: 26948846. Exclusion Code: X1.

491. Genbacev O, Ratkovic M, Kraincanic M, et al. Effect of prostaglandin PGE2alpha on the synthesis of placental proteins and human placental lactogen (HPL). Prostaglandins. 1977 Apr;13(4):723-33. PMID: 854581. Exclusion Code: X3.

492. Gentile S. Clinical utilization of atypical antipsychotics in pregnancy and lactation. Ann Pharmacother. 2004 Jul-Aug;38(78):1265-71. doi: 10.1345/aph.1D485. PMID: 15150376. Exclusion Code: X7.

493. Gentile S. Prophylactic treatment of bipolar disorder in pregnancy and breastfeeding: focus on emerging mood stabilizers. Bipolar Disord. 2006 Jun;8(3):207-20. doi: 10.1111/j.1399-5618.2006.00295.x. PMID: 16696822. Exclusion Code: X7.

494. Gentile S. Serotonin reuptake inhibitorinduced perinatal complications. Paediatr Drugs. 2007;9(2):97-106. doi: 10.2165/00148581-200709020-00003. PMID: 17407365. Exclusion Code: X7.

495. Gentile S. Pregnancy exposure to serotonin reuptake inhibitors and the risk of spontaneous abortions. CNS Spectr. 2008 Nov;13(11):960-6. PMID: 19037175. Exclusion Code: X7.

496. Gentile S. Infant safety with antipsychotic therapy in breast-feeding: a systematic review. J Clin Psychiatry. 2008 Apr;69(4):666-73. PMID: 18370569. Exclusion Code: X7.

497. Gentile S. Neurodevelopmental effects of prenatal exposure to psychotropic medications. Depress Anxiety. 2010 Jul;27(7):675-86. doi: 10.1002/da.20706. PMID: 20583298. Exclusion Code: X7. 
498. Gentile S. On categorizing gestational, birth, and neonatal complications following late pregnancy exposure to antidepressants: the prenatal antidepressant exposure syndrome. CNS Spectr. 2010 Mar;15(3):167-85. PMID: 20414166. Exclusion Code: X7.

499. Gentile S. Antipsychotic therapy during early and late pregnancy. A systematic review. Schizophr Bull. 2010

May;36(3):518-44. doi:

10.1093/schbul/sbn107. PMID: 18787227.

Exclusion Code: X7.

500. Gentile S. Selective serotonin reuptake inhibitor exposure during early pregnancy and the risk of birth defects. Acta Psychiatr Scand. 2011 Apr;123(4):266-75. doi: 10.1111/j.1600-0447.2011.01673.x. PMID: 21261600. Exclusion Code: X7.

501. Gentile S. A safety evaluation of aripiprazole for treating schizophrenia during pregnancy and puerperium. Expert Opin Drug Saf. 2014 Dec;13(12):1733-42. doi: $10.1517 / 14740338.2014 .951325$. PMID: 25139223. Exclusion Code: X1.

502. Gentile S. Early pregnancy exposure to selective serotonin reuptake inhibitors, risks of major structural malformations, and hypothesized teratogenic mechanisms. Expert Opin Drug Metab Toxicol. 2015;11(10):1585-97. doi: 10.1517/17425255.2015.1063614. PMID: 26135630. Exclusion Code: X7.

503. Gentile S. Prenatal antidepressant exposure and the risk of autism spectrum disorders in children. Are we looking at the fall of Gods? J Affect Disord. 2015 Aug 15;182:132-7. doi: 10.1016/j.jad.2015.04.048. PMID: 25985383. Exclusion Code: X1.

504. Gentile S, Bellantuono C. Selective serotonin reuptake inhibitor exposure during early pregnancy and the risk of fetal major malformations: focus on paroxetine. J Clin Psychiatry. 2009 Mar;70(3):414-22. PMID: 19254517. Exclusion Code: X7.

505. Gentile S, Fusco ML. In children 7 years of age, prenatal antidepressant exposure is not associated with increased risk of poor behavioural outcomes after adjusting for maternal factors including antenatal mood. Evid Based Med. 2017 Jun;22(3):113-4. doi: 10.1136/ebmed-2016-110650. PMID: 28348049. Exclusion Code: X1.
506. Gentile S, Fusco ML. Neurodevelopmental outcomes in infants exposed in utero to antipsychotics: a systematic review of published data. CNS Spectr. 2017 Jun;22(3):273-81. doi: 10.1017/s1092852916000699. PMID: 27866497. Exclusion Code: X7.

507. Gentile S, Fusco ML. Placental and fetal effects of antenatal exposure to antidepressants or untreated maternal depression. J Matern Fetal Neonatal Med. 2017 May;30(10):1189-99. doi: 10.1080/14767058.2016.1209184. PMID: 27379818. Exclusion Code: X1.

508. Gentile S, Fusco ML. Schizophrenia and motherhood. Psychiatry Clin Neurosci. 2019 Jul;73(7):376-85. doi: 10.1111/pcn.12856. PMID: 31026107 . Exclusion Code: X7.

509. Gentile S, Galbally M. Prenatal exposure to antidepressant medications and neurodevelopmental outcomes: A systematic review. J Affect Disord. 2010. doi: 10.1016/j.jad.2010.02.125. Exclusion Code: $\mathrm{X} 7$.

510. Gentile S, Galbally M. Prenatal exposure to antidepressant medications and neurodevelopmental outcomes: a systematic review. J Affect Disord. 2011 Jan;128(12):1-9. doi: 10.1016/j.jad.2010.02.125. PMID: 20303599. Exclusion Code: X7.

511. Georgiopoulos AM, Bryan TL, Wollan P, et al. Routine screening for postpartum depression. J Fam Pract. 2001 Feb;50(2):117-22. PMID: 11219558. Exclusion Code: X3.

512. Gidai J, Acs N, Banhidy F, et al. No association found between use of very large doses of diazepam by 112 pregnant women for a suicide attempt and congenital abnormalities in their offspring. Toxicol Ind Health. 2008 Feb-Mar;24(1-2):29-39. doi: 10.1177/0748233708089019. PMID: 18818179. Exclusion Code: X2.

513. Gidaya NB, Lee BK, Burstyn I, et al. In utero exposure to selective serotonin reuptake inhibitors (SSRIS) and risk for AUTISM SPECTRUM DISORDERS (ASD). Am J Epidemiol. 2012;175:S127. doi: 10.1093/aje/kws258. Exclusion Code: $\mathrm{X} 4$. 
514. Gidaya NB, Lee BK, Burstyn I, et al. In utero exposure to selective serotonin reuptake inhibitors and risk for autism spectrum disorder. J Autism Dev Disord. 2014 Oct;44(10):2558-67. doi: 10.1007/s10803-014-2128-4. PMID: 24803368. Exclusion Code: X7.

515. Gilbert H. The national register of antipsychotic medication in pregnancy (NRAMP), the first 100 babies. Arch Womens Ment Health. 2011;14:S117. doi: 10.1007/s00737-011-0238-y. Exclusion Code: X5.

516. Gillis S. Pharmacologic treatment of depression during pregnancy. J Midwifery Womens Health. 2000 Jul-Aug;45(4):357-9. PMID: 10983439. Exclusion Code: X1.

517. Giménez A, Pacchiarotti I, Gil J, et al. Adverse outcomes during pregnancy and major congenital malformations in infants of patients with bipolar and schizoaffective disorders treated with antiepileptic drugs: A systematic review. Psychiatr Pol. 2019 Apr 30;53(2):223-44. doi: 10.12740/pp/105906. PMID: 31317955 . Exclusion Code: X7.

518. Ginsberg DL. Paroxetine use during pregnancy associated with neonatal intracerebral bleeding. Primary Psychiatry. 2004;11(7):21-2. Exclusion Code: X1.

519. Gissler M, Artama M, Ritvanen A, et al. Use of psychotropic drugs before pregnancy and the risk for induced abortion: populationbased register-data from Finland 1996-2006. BMC Public Health. 2010 Jun 30;10:383. doi: 10.1186/1471-2458-10-383. PMID: 20591182. Exclusion Code: X5.

520. Given JE, Loane M, Garne E, et al. Gastroschisis in Europe - a case-malformedcontrol study of medication and maternal illness during pregnancy as risk factors. Paediatr Perinat Epidemiol. 2017 Nov;31(6):549-59. doi: 10.1111/ppe.12401. PMID: 28841756. Exclusion Code: X4.

521. Gjerdingen D. The effectiveness of various postpartum depression treatments and the impact of antidepressant drugs on nursing infants. J Am Board Fam Pract. 2003 SepOct;16(5):372-82. PMID: 14645327. Exclusion Code: X7.
522. Gjerdingen D, Crow S, McGovern P, et al. Stepped care treatment of postpartum depression: impact on treatment, health, and work outcomes. J Am Board Fam Med. 2009;22(5):473-82. doi: 10.3122/jabfm.2009.05.080192. PMID: CN00731182. Exclusion Code: X3.

523. Goedhart G, Snijders AC, Hesselink AE, et al. Maternal depressive symptoms in relation to perinatal mortality and morbidity: results from a large multiethnic cohort study. Psychosom Med. 2010 Oct;72(8):769-76. doi: 10.1097/PSY.0b013e3181 ee4a62. PMID: 20668282. Exclusion Code: X3.

524. Goldfield M, Weinstein MR. Lithium in pregnancy: a review with recommendations. Am J Psychiatry. 1971 Jan;127(7):888-93. doi: 10.1176/ajp.127.7.888. PMID: 4924779. Exclusion Code: X1.

525. Goldstein DJ. Effects of third trimester fluoxetine exposure on the newborn. J Clin Psychopharmacol. 1995 Dec;15(6):417-20. PMID: 8748430. Exclusion Code: X4.

526. Goldstein DJ, Corbin LA, Fung MC. Olanzapine-exposed pregnancies and lactation: early experience. J Clin Psychopharmacol. 2000 Aug;20(4):399-403. PMID: 10917399. Exclusion Code: X4.

527. Goldstein DJ, Corbin LA, Sundell KL. Effects of first-trimester fluoxetine exposure on the newborn. Obstet Gynecol. 1997 May;89(5 Pt 1):713-8. PMID: 9166307. Exclusion Code: X4.

528. Goldstein DJ, Sundell KL, Corbin LA. Birth outcomes in pregnant women taking fluoxetine. N Engl J Med. 1997 Mar 20;336(12):872-3; author reply 3. PMID: 9072683. Exclusion Code: X7.

529. González-Rodríguez A, Imaz ML, Romans $\mathrm{C}$, et al. Exposure to lithium during late pregnancy and neonatal outcomes. Eur Psychiatry. 2013;28. Exclusion Code: X9.

530. Goodman SH, Cullum KA, Dimidjian S, et al. Opening windows of opportunities: Evidence for interventions to prevent or treat depression in pregnant women being associated with changes in offspring's developmental trajectories of psychopathology risk. Dev Psychopathol. 2018 Aug;30(3):1179-96. doi: 10.1017/s0954579418000536. PMID: 30068424. Exclusion Code: X7. 
531. Goodman SH, Newport DJ, Rouse MH, et al. Perinatal depression and antidepressant usage: Associations with 12-month-old infant bayley scores. Arch Womens Ment Health. 2011;14:S25. doi: 10.1007/s00737010-0203-1. Exclusion Code: X5.

532. Goodwin G, Young AH. Using guidelines in real clinical situations: clozapine and breast feeding in bipolar disorder. J

Psychopharmacol. 2005 May;19(3):317-8. doi: $10.1177 / 0269881105054667$. PMID: 15888520. Exclusion Code: X1.

533. Goracci A, Valdagno M, Maltinti E, et al. [Antidepressant use in pregnancy: a critical review of the risk and benefits]. Riv Psichiatr. 2015 May-Jun;50(3):118-26. doi: 10.1708/1910.20792. PMID: 26156817. Exclusion Code: X8.

534. Gordon A, Mikocka-Walus A, Grzeskowiak LE, et al. Antidepressants for depression during pregnancy. Cochrane Database Syst Rev. 2013(8). doi: 10.1002/14651858.CD010710. PMID: CD010710. Exclusion Code: X7.

535. Gracious BL, Hanusa BH, Wisner KL, et al. Weight changes in postpartum women with remitted depression. J Clin Psychiatry. 2005 Mar;66(3):291-3. PMID: 15766293.

Exclusion Code: X3.

536. Gramaglia C, Ressico F, Coppola I, et al. Perinatal and neonatal outcomes of lithiumtreated and untreated bipolar women during pregnancy: A review of present literature. Current Psychopharmacology.

2013;2(2):132-42. doi:

10.2174/2211556011302020004. Exclusion Code: X1.

537. Granroth G. Defects of the central nervous system in Finland: III. Disease and drugs in pregnancy. Early Hum Dev. 1978 Jul;2(2):147-62. PMID: 569047. Exclusion Code: X4.

538. Granström AL, Skoglund C, Wester T. Selective serotonin reuptake inhibitors during pregnancy Do not increase the risk of Hirschsprung disease. J Pediatr Surg. 2019 Nov;54(11):2398-401. doi: 10.1016/j.jpedsurg.2019.02.015. PMID: 30867098. Exclusion Code: X4.
539. Gréger J, Czeizel E, Rácz J. Evaluation of the effect of drug intake during pregnancy based on the Monitoring Surveillance of the Pathogenesis of Congenital Anomalies. Orv Hetil. 1992;133(7):407-8, 13-5. PMID: CN00082216. Exclusion Code: X8.

540. Gregoire A, Pearson S. Risk of pregnancy when changing to atypical antipsychotics. $\mathrm{Br}$ J Psychiatry. 2002 Jan;180:83-4. PMID: 11772858. Exclusion Code: X7.

541. Grieve PG, Fifer WP, Cousy NP, et al. Neonatal infant EEG bursts are altered by prenatal maternal depression and serotonin selective reuptake inhibitor use. Clin Neurophysiol. 2019 Nov;130(11):2019-25. doi: 10.1016/j.clinph.2019.08.021. PMID: 31539768. Exclusion Code: X5.

542. Grigoriadis S, Graves L, Peer M, et al. The impact of perinatal anxiety on mothers and babies: A systematic review and metaanalysis. J Womens Health. 2017;26(4):A21-A2. doi: 10.1089/jwh.2017.29011.abstracts. Exclusion Code: X7.

543. Grigoriadis S, Graves L, Peer M, et al. Benzodiazepine Use During Pregnancy Alone or in Combination With an Antidepressant and Congenital Malformations: Systematic Review and Meta-Analysis. J Clin Psychiatry. $2019 \mathrm{Jul}$ 9;80(4). doi: 10.4088/JCP.18r12412. PMID: 31294935. Exclusion Code: X7.

544. Grigoriadis S, Mamisashvili L, Ross LE. Achieving the balance: treating depressed pregnant women with antidepressants. J Clin Psychiatry. 2013 Apr;74(4):375-6. doi: 10.4088/JCP.13com08407. PMID: 23656844. Exclusion Code: X1.

545. Grigoriadis S, VonderPorten EH, Mamisashvili L, et al. The effect of prenatal antidepressant exposure on neonatal adaptation: a systematic review and metaanalysis. J Clin Psychiatry. 2013 Apr;74(4):e309-20. doi: 10.4088/JCP.12r07967. PMID: 23656856. Exclusion Code: X7. 
546. Grigoriadis S, VonderPorten EH, Mamisashvili L, et al. Antidepressant exposure during pregnancy and congenital malformations: is there an association? A systematic review and meta-analysis of the best evidence. J Clin Psychiatry. 2013

Apr;74(4):e293-308. doi: 10.4088/JCP.12r07966. PMID: 23656855. Exclusion Code: X7.

547. Grigoriadis S, Vonderporten EH, Mamisashvili L, et al. Prenatal exposure to antidepressants and persistent pulmonary hypertension of the newborn: systematic review and meta-analysis. BMJ. 2014 Jan 14;348:f6932. doi: 10.1136/bmj.f6932. PMID: 24429387. Exclusion Code: X7.

548. Grimaldi R. The clinical use of fluphenazine in pregnancy. N Y Med Coll News Notes. 1960 Jun-Sep;2:42-9. PMID: 13708693. Exclusion Code: X11.

549. Grossmann CK, Weitzner B, Pistelli A, et al. Escitalopram use in pregnancy: An observational cohort study. Am J Obstet Gynecol. 2012;206(1):S58. doi: 10.1016/j.ajog.2011.10.116. Exclusion Code: X9.

550. Grote N, Katon W, Lohr MJ. Momcare: culturally relevant treatment services for perinatal depression. Archives of women's mental health. 2013;16:S4. doi: 10.1007/s00737-013-0355-x. PMID: CN01058120. Exclusion Code: X3.

551. Grote NK, Katon WJ, Russo JE, et al. Collaborative care for perinatal depression in socioeconomically disadvantaged women: a randomized trial. Depress Anxiety. 2015 Nov;32(11):821-34. doi: 10.1002/da.22405. PMID: 26345179. Exclusion Code: X3.

552. Grush LR, Cohen LS. Treatment of depression during pregnancy: balancing the risks. Harv Rev Psychiatry. 1998 JulAug;6(2):105-9. PMID: 10370454. Exclusion Code: X1.

553. Grzeskowiak L. Antidepressant use in late pregnancy. Midwives. 2015 Winter;18:2930. PMID: 26867234. Exclusion Code: X1.
554. Grzeskowiak LE, Gilbert AL, Morrison JL. Conception and beyond: Using populationBased record linkage to monitor long-term effects of medications used during pregnancy. Journal of Pharmacy Practice and Research. 2010;40(1):46-9. Exclusion Code: X1.

555. Grzeskowiak LE, Gilbert AL, Morrison JL. Neonatal outcomes following late gestation SSRI exposure. Pharmacoepidemiol Drug Saf. 2012;21:373. doi: 10.1002/pds.3324. Exclusion Code: X9.

556. Grzeskowiak LE, Gilbert AL, Sorensen TIA, et al. Childhood overweight following fetal exposure to selective serotonin reuptake inhibitors and maternal psychiatric illness. Pharmacoepidemiol Drug Saf. 2012;21:32. doi: 10.1002/pds.3324. Exclusion Code: X9.

557. Grzeskowiak LE, Leggett C, Costi L, et al. Antidepressant use in late gestation and breastfeeding rates at discharge from hospital. Pharmacoepidemiol Drug Saf. 2013;22:367. doi: 10.1002/pds.3512. Exclusion Code: X9.

558. Grzeskowiak LE, Leggett C, Costi L, et al. Use of serotonin reuptake inhibitors in late gestation and lactation difficulties. Pharmacoepidemiol Drug Saf. 2013;22:1501. doi: 10.1002/pds.3512. Exclusion Code: X9.

559. Grzeskowiak LE, Leggett C, Costi L, et al. Late gestation use of serotonin reuptake inhibitor antidepressants is not associated with an increased risk for low milk supply in mothers of preterm infants.

Pharmacoepidemiol Drug Saf. 2018;27:2334. doi: 10.1002/pds.4629. Exclusion Code: X9.

560. Grzeskowiak LE, Leggett C, Costi L, et al. Impact of serotonin reuptake inhibitor use on breast milk supply in mothers of preterm infants: a retrospective cohort study. Br J Clin Pharmacol. 2018;84(6):1373-9. doi: 10.1111/bcp.13575. Exclusion Code: X4.

561. Grzeskowiak LE, Morrison JL. Long-term effects of prenatal SSRI exposure on child growth: weighing the evidence. Am J Psychiatry. 2013 Nov;170(11):1364. doi: 10.1176/appi.ajp.2013.13040472. PMID: 24185243. Exclusion Code: X1. 
562. Grzeskowiak LE, Morrison JL, Henriksen TB, et al. Prenatal antidepressant exposure and child behavioural outcomes at 7-years of age. Pharmacoepidemiol Drug Saf. 2013;22:151-2. doi: 10.1002/pds.3512. Exclusion Code: X9.

563. Grzeskowiak LE, Pedersen LH, Costi L, et al. Continuation vs. Cessation of antidepressant use in the pre-and post-natal period and impact on duration of breastfeeding. J Paediatr Child Health. 2014;50:12-3. Exclusion Code: X9.

564. Grzeskowiak LE, Pedersen LH, Costi L, et al. Prenatal antidepressant exposure and impact on duration of breastfeeding. $\mathrm{J}$ Paediatr Child Health. 2014;50:86-7. Exclusion Code: X9.

565. Guan HB, Wei Y, Wang LL, et al. Prenatal selective serotonin reuptake inhibitor use and associated risk for gestational hypertension and preeclampsia: a metaanalysis of cohort studies. J Womens Health (Larchmt). 2018 Jun;27(6):791-800. doi: 10.1089/jwh.2017.6642. PMID: 29489446. Exclusion Code: X7.

566. Guille C, Epperson CN. Relapse of major depression in women who continue or discontinue antidepressant medication during pregnancy. Am J Psychiatry. 2013 May;170(5):558. doi: 10.1176/appi.ajp.2013.13010092. PMID: 23632837. Exclusion Code: X1.

567. Guinchat V, Thorsen P, Laurent C, et al. Pre-, peri- and neonatal risk factors for autism. Acta Obstet Gynecol Scand. 2012 Mar;91(3):287-300. doi: 10.1111/j.16000412.2011.01325.x. PMID: 22085436. Exclusion Code: X7.

568. Guitart M, Imaz ML, Soy D, et al. Pharmacokinetics of lithium during delivery and in the neonatal period. A preliminary data. Eur Psychiatry. 2013;28. Exclusion Code: X9.

569. Gunduz-Bruce H, Riesenberg R, Sankoh A, et al. SAGE-217 in subjects with major depressive disorder: efficacy and safety results from open-label Part A of a phase 2A study. Eur Neuropsychopharmacol. 2017 Oct;27(Supplement 4):S856-S7. PMID: WOS:000413847703023. Exclusion Code: $\mathrm{X} 2$.
570. Gupta M, Mulvihill AO, Lascaratos G, et al. Nystagmus and reduced visual acuity secondary to drug exposure in utero: longterm follow-up. J Pediatr Ophthalmol Strabismus. 2012 Jan-Feb;49(1):58-63. doi: 10.3928/01913913-20110308-01. PMID: 21417186. Exclusion Code: X7.

571. Gupta S, Rangwani S. SSRIs in pregnancy. J Fam Pract. 1998 Jul;47(1):72. PMID: 9673612. Exclusion Code: X1.

572. Gurnot C, Martin-Subero I, Mah SM, et al. Prenatal antidepressant exposure associated with CYP2E1 DNA methylation change in neonates. Epigenetics. 2015;10(5):361-72. doi: 10.1080/15592294.2015.1026031. PMID: 25891251. Exclusion Code: X5.

573. Gutierrez-Esteinou R, Maximos B, Riesenberg R, et al. Safety and Efficacy of Intravenous Ganaxolone in Severe Postpartum Depression: Results From a Double-Blind, Placebo-Controlled Phase 2 Study. Biol Psychiatry. 2019;85(10):S181S2. doi: 10.1016/j.biopsych.2019.03.459. Exclusion Code: X3.

574. Habermann F, Fritzsche J, Fuhlbruck F, et al. Atypical antipsychotic drugs and pregnancy outcome: a prospective, cohort study. J Clin Psychopharmacol. 2013 Aug;33(4):453-62. doi: 10.1097/JCP.0b013e318295fe12. PMID: 23764684. Exclusion Code: X4.

575. Haeri S, Baker A, Johnson N, et al. Do pregnant women with depression have a proinflammatory profile? Am J Obstet Gynecol. 2011;204(1):S322. doi: 10.1016/j.ajog.2010.10.847. Exclusion Code: X3.

576. Hærvig KB, Mortensen LH, Hansen AV, et al. Use of ADHD drugs during pregnancy from 1995 to 2010: A Danish register-based study. Eur J Epidemiol. 2013;28(1):S122. doi: 10.1007/s10654-013-9820-0. Exclusion Code: X3.

577. Hagberg KW, Robijn A, Jick SS. Maternal depression and antidepressant use during pregnancy and risk of autism spectrum disorders in offspring: Population-based cohort and bidirectional case-crossover sibling study. Pharmacoepidemiol Drug Saf. 2017;26:470. doi: 10.1002/pds.4275.

Exclusion Code: X9. 
578. Hagberg KW, Robijn AL, Jick S. Maternal depression and antidepressant use during pregnancy and the risk of autism spectrum disorder in offspring. Clin Epidemiol. 2018;10:1599-612. doi: 10.2147/clep.s180618. PMID: 30464639. Exclusion Code: X12.

579. Hale TW, Kendall-Tackett K, Cong Z, et al. Discontinuation syndrome in newborns whose mothers took antidepressants while pregnant or breastfeeding. Breastfeed Med. 2010 Dec;5(6):283-8. doi: 10.1089/bfm.2010.0011. PMID: 20807106. Exclusion Code: X4.

580. Hall NR, Rutledge EC, Ramin SM, et al. Utilization of inpatient psychiatry consults at an urban obstetric hospital. Reprod Sci. 2017;24(1):123A. doi: 10.1177/1933719117699773. Exclusion Code: X3.

581. Hallberg P, Odlind V, Sjoblom V. Selective serotonin-reuptake inhibitors and persistent pulmonary hypertension of the newborn. $\mathrm{N}$ Engl J Med. 2006 May 18;354(20):2188-90; author reply -90. PMID: 16710910. Exclusion Code: X1.

582. Hallberg P, Odlind V, Sjöblom V. Selective serotonin-reuptake inhibitors and persistent pulmonary hypertension of the newborn [13]. N Engl J Med. 2006;354(20):2189. Exclusion Code: X1.

583. Halvorsen A, Hesel B, Østergaard SD, et al. In utero exposure to selective serotonin reuptake inhibitors and development of mental disorders: a systematic review and meta-analysis. Acta Psychiatr Scand. 2019 Jun;139(6):493-507. doi:

10.1111/acps.13030. PMID: 30937904. Exclusion Code: X7.

584. Hampton T. Antidepressants and pregnancy: weighing risks and benefits no easy task. JAMA. 2006 Apr 12;295(14):1631-3. doi: 10.1001/jama.295.14.1631. PMID: 16609077. Exclusion Code: X1.

585. Hancock RL. An economic evaluation of teratology information services: ProQuest Information \& Learning; 2012. Exclusion Code: X2.
586. Handal M, Skurtveit S, Furu K, et al. Motor development in children prenatally exposed to selective serotonin reuptake inhibitors: a large population-based pregnancy cohort study. BJOG. 2016 Nov;123(12):1908-17. doi: 10.1111/1471-0528.13582. PMID: 26374234. Exclusion Code: X4.

587. Handal M, Skurtveit S, Furu K, et al. Prenatal exposure to antidepressants and motor development in the 3 years old child. Results from MoBa, a large population based pregnancy cohort in Norway. Pharmacoepidemiol Drug Saf. 2013;22:152. doi: 10.1002/pds.3512. Exclusion Code: X9.

588. Handal M, Skurtveit S, Roth C, et al. Use of folic acid and antidepressants during pregnancy and child language development. Pharmacoepidemiol Drug Saf. 2015;24:258. doi: 10.1002/pds.3838. Exclusion Code: X4.

589. Hanley GE, Brain U, Oberlander TF. Infant developmental outcomes following prenatal exposure to antidepressants, and maternal depressed mood and positive affect. Early Hum Dev. 2013 Aug;89(8):519-24. doi: 10.1016/j.earlhumdev.2012.12.012. PMID: 23384962. Exclusion Code: X4.

590. Hanley GE, Brain U, Oberlander TF. Prenatal exposure to serotonin reuptake inhibitor antidepressants and childhood behavior. Pediatr Res. 2015 Aug;78(2):17480. doi: 10.1038/pr.2015.77. PMID: 25897539. Exclusion Code: X4.

591. Hannerfors AK, Hellgren C, Schijven D, et al. Treatment with serotonin reuptake inhibitors during pregnancy is associated with elevated corticotropin-releasing hormone levels. Psychoneuroendocrinology. 2015 Aug;58:104-13. doi: 10.1016/j.psyneuen.2015.04.009. PMID: 25978816. Exclusion Code: X10.

592. Hanson JW, Smith DW. Fetal hydantoin syndrome. Lancet. 1976 Mar 27;1(7961):692. PMID: 73661. Exclusion Code: X1. 
593. Harden CL, Meador KJ, Pennell PB, et al. Practice parameter update: management issues for women with epilepsy--focus on pregnancy (an evidence-based review): teratogenesis and perinatal outcomes: report of the Quality Standards Subcommittee and Therapeutics and Technology Assessment Subcommittee of the American Academy of Neurology and American Epilepsy Society. Neurology. 2009 Jul 14;73(2):133-41. doi: 10.1212/WNL.0b013e3181a6b312. PMID: 19398681. Exclusion Code: X2.

594. Harrington RA, Lee LC, Crum RM, et al. Serotonin hypothesis of autism: implications for selective serotonin reuptake inhibitor use during pregnancy. Autism Res. 2013 Jun;6(3):149-68. doi: 10.1002/aur.1288. PMID: 23495208. Exclusion Code: X7.

595. Harrington RA, Lee LC, Crum RM, et al. Prenatal SSRI use and offspring with autism spectrum disorder or developmental delay. Pediatrics. 2014 May;133(5):e1241-8. doi: 10.1542/peds.2013-3406. PMID: 24733881. Exclusion Code: X4.

596. Hartlage LC. Effects of chlorpromazine on learning. Psychol Bull. 1965;64(4):235-45. doi: 10.1037/h0022419. PMID: 196515585-001. Exclusion Code: X2.

597. Hartmann-Boyce J, Hong B, LivingstoneBanks J, et al. Additional behavioural support as an adjunct to pharmacotherapy for smoking cessation. Cochrane Database Syst Rev. 2019(6). doi: 10.1002/14651858.CD009670.pub4. PMID: CD009670. Exclusion Code: X2.

598. Hashimoto K. Neonatal signs following exposure to SSRIs. Hum Psychopharmacol. 2005 Oct;20(7):522. doi: 10.1002/hup.721. PMID: 16184524. Exclusion Code: X1.

599. Haskey C, Galbally M. Mood stabilizers in pregnancy and child developmental outcomes: a systematic review. Aust N Z J Psychiatry. 2017 Nov;51(11):1087-97. doi: 10.1177/0004867417726175. PMID: 28825316. Exclusion Code: X7.

600. Hatters Friedman S, Moller-Olsen C, Prakash C, et al. Atypical antipsychotic use and outcomes in an urban maternal mental health service. Int J Psychiatry Med. 2016 Aug;51(6):521-33. doi: 10.1177/0091217417696739. PMID: 28629296. Exclusion Code: X4.
601. Haug NA. Motivational enhancement therapy for cigarette smoking in methadonemaintained pregnant women: ProQuest Information \& Learning; 2003. Exclusion Code: X2.

602. Hauser LA. Pregnancy and psychiatric drugs. Hosp Community Psychiatry. 1985 Aug;36(8):817-8. PMID: 2863210. Exclusion Code: X1.

603. Healy D, Le Noury J, Mangin D. Links between serotonin reuptake inhibition during pregnancy and neurodevelopmental delay/spectrum disorders: A systematic review of epidemiological and physiological evidence. Int J Risk Saf Med. 2016 Sep 17;28(3):125-41. doi: 10.3233/jrs-160726. PMID: 27662278. Exclusion Code: X7.

604. Heath AC, Yonkers KA. Somatic treatments in depression: Concerns during pregnancy and breastfeeding. In: Yonkers K, Little B, eds. Management of psychiatric disorders in pregnancy. New York, NY: Oxford University Press; 2001:82-104. Exclusion Code: X1.

605. Heath AC, Yonkers KA, Rush AJ. Paroxetine in the treatment of postpartum depression. 153rd annual meeting of the american psychiatric association; 2000 may 13-18; chicago, IL. 2000:No. 68B. PMID: CN-00306347. Exclusion Code: X9.

606. Heath AC, Yonkers Kimberly A, Rush AJ. Paroxetine in the treatment of postpartum depression. 155th annual meeting of the american psychiatric association; 2002 may 18-23rd; philadelphia, PA, USA. 2002. PMID: CN-00794948. Exclusion Code: X1.

607. Heikkinen T, Ekblad U, Kero P, et al. Citalopram in pregnancy and lactation. Clin Pharmacol Ther. 2002 Aug;72(2):184-91. doi: 10.1067/mcp.2002.126181. PMID: 12189365. Exclusion Code: X4.

608. Heinonen E, Szymanska-von Schultz B, Kaldo V, et al. MAGDALENA: study protocol of a randomised, placebocontrolled trial on cognitive development at 2 years of age in children exposed to SSRI in utero. BMJ Open. 2018 Aug 5;8(8):e023281. doi: 10.1136/bmjopen2018-023281. PMID: 30082365. Exclusion Code: X7. 
609. Heller HM, Ravelli ACJ, Bruning AHL, et al. Increased postpartum haemorrhage, the possible relation with serotonergic and other psychopharmacological drugs: a matched cohort study. BMC Pregnancy Childbirth. 2017 Jun 2;17(1):166. doi: 10.1186/s12884017-1334-4. PMID: 28577352. Exclusion Code: X4.

610. Hemels ME, Einarson A, Koren G, et al. Antidepressant use during pregnancy and the rates of spontaneous abortions: a metaanalysis. Ann Pharmacother. 2005 May;39(5):803-9. doi: 10.1345/aph.1E547. PMID: 15784808. Exclusion Code: X7.

611. Hendrick V, Altshuler L, Wertheimer A, et al. Venlafaxine and breast-feeding. Am J Psychiatry. 2001 Dec;158(12):2089-90. PMID: 11729040. Exclusion Code: X7.

612. Hendrick V, Fukuchi A, Altshuler L, et al. Use of sertraline, paroxetine and fluvoxamine by nursing women. Br J Psychiatry. 2001 Aug;179:163-6. PMID: 11483479. Exclusion Code: X5.

613. Hendrick V, Smith LM, Hwang S, et al. Weight gain in breastfed infants of mothers taking antidepressant medications. J Clin Psychiatry. 2003 Apr;64(4):410-2. PMID: 12716242. Exclusion Code: X4.

614. Hendrick V, Suri R, Gitlin MJ, et al. Bupropion use during pregnancy: a systematic review. Prim Care Companion CNS Disord. 2017 Sep 21;19(5). doi: 10.4088/PCC.17r02160. PMID: 28973846. Exclusion Code: X7.

615. Henriksen DP, Pottegard A, Jimenez-Solem E, et al. In utero SSRI exposure and risk of clubfoot. Epidemiology. 2015 May;26(3):e34-5. doi: 10.1097/ede.0000000000000276. PMID: 25835138. Exclusion Code: X1.

616. Hermansen TK, Yrttiaho S, Roysamb E, et al. Perceptual interference processing in preschool children, with and without prenatal exposure to selective serotonin reuptake inhibitors. Psychopharmacology (Berl). 2017 Feb;234(3):339-51. doi: 10.1007/s00213-016-4467-7. PMID: 27826628. Exclusion Code: X5.
617. Hernandez-Diaz S, Huybrechts KF, Desai $\mathrm{RJ}$, et al. Topiramate use early in pregnancy and the risk of oral clefts: A pregnancy cohort study. Neurology. 2018 Jan 23;90(4):e342-e51. doi: 10.1212/wnl.0000000000004857. PMID: 29282333. Exclusion Code: X2.

618. Hernández-Martínez C, Arija V, Balaguer A, et al. Do the emotional states of pregnant women affect neonatal behaviour? Early Hum Dev. 2008;84(11):745-50. doi: 10.1016/j.earlhumdev.2008.05.002. PMID: 2008-16357-008. Exclusion Code: X3.

619. Hernandez-Nieto CA, Nazem TG, Lee JA, et al. Selective serotonin reuptake inhibitors, IVF and PGT: how does exposure affect outcome? Fertil Steril. 2017;108(3):e324. Exclusion Code: X4.

620. Herrero T, Driebe A, Fratto V, et al. Positive antenatal Edinburgh Depression Scale: examining behavioral and pharmacological therapy on maternal and neonatal outcomes. J Matern Fetal Neonatal Med. 2018 Jul 22:15. doi: 10.1080/14767058.2018.1488162. PMID: 30033785. Exclusion Code: X12.

621. Hertzberg T. [Postpartum depression]. Duodecim. 2000;116(14):1491-7; quiz 7, 71. PMID: 12001467. Exclusion Code: X8.

622. Hill R, Law D, Yelland C, et al. What is optimal care for mother-infant pairs affected by postpartum psychosis? Treatment and outcomes in the Australian context. Aust N Z J Psychiatry. 2017;51(1):128. doi: 10.1177/0004867417702054. Exclusion Code: X4.

623. Hill RM. Will this drug harm the unborn infant? The doctor's dilemma. South Med J. 1974 Dec;67(12):1476-80. PMID: 4473825. Exclusion Code: X1.

624. Hill RM, Verniaud WM, Horning MG, et al. Infants exposed in utero to antiepileptic drugs. A prospective study. Am J Dis Child. 1974 May;127(5):645-53. PMID: 4825584. Exclusion Code: X4.

625. Hisle-Gorman E, Susi A, Stokes T, et al. Prenatal, perinatal, and neonatal risk factors of autism spectrum disorder. Pediatr Res. 2018 Aug;84(2):190-8. doi: 10.1038/pr.2018.23. PMID: 29538366. Exclusion Code: X4. 
626. Hjorth S, Bromley R, Ystrom E, et al. Use and validity of child neurodevelopment outcome measures in studies on prenatal exposure to psychotropic and analgesic medications - A systematic review. PLoS One. 2019;14(7):e0219778. doi: 10.1371/journal.pone.0219778. PMID: 31295318. Exclusion Code: X3.

627. Hodnett RM, Wijesiriwardana A. District General Hospital experience of managing perinatal mental illness. Arch Dis Child Fetal Neonatal Ed. 2010;95:Fa56. doi: 10.1136/adc.2010.189753.76. Exclusion Code: X5.

628. Hoedjes M, Berks D, Vogel I, et al. Symptoms of post-traumatic stress after preeclampsia. Journal of Psychosomatic Obstetrics \& Gynecology. 2011;32(3):12634. doi: 10.3109/0167482X.2011.599460. PMID: 2011-17785-003. Exclusion Code: $\mathrm{X} 2$.

629. Hoffbrand S, Howard L, Crawley H. Antidepressant drug treatment for postnatal depression. Cochrane Database Syst Rev. 2001(2):Cd002018. doi: 10.1002/14651858.cd002018. PMID: 11406023. Exclusion Code: X7.

630. Hoffbrand S, Howard L, Crawley H. Antidepressant treatment for post-natal depression. Nurs Times. 2001 Nov 814;97(45):35. PMID: 11966146. Exclusion Code: X1.

631. Hoffman MC, Mazzoni S, Valdez C, et al. Health service utilization and charges of mothers with bipolar disorder and their children. Am J Obstet Gynecol. 2016;214(1):S323-S4. Exclusion Code: X5.

632. Holland D. An observation of the effect of sertraline on breast milk supply. Aust N Z J Psychiatry. 2000 Dec;34(6):1032. doi: 10.1080/000486700283. PMID: 11127615. Exclusion Code: X7.

633. Hoog SL, Cheng Y, Elpers J, et al. Duloxetine and pregnancy outcomes: safety surveillance findings. Int J Med Sci. 2013;10(4):413-9. doi: 10.7150/ijms.5213. PMID: 23471302. Exclusion Code: X4.

634. Howard L. Postnatal depression. Clin Evid. 2004 Jun(11):1872-85. PMID: 15652086. Exclusion Code: X1.
635. Howard L. Erratum: Safety of antipsychotic drugs for pregnant and breastfeeding women with non-affective psychosis (British Medical Journal (October 23) (933-934)). Br Med J. 2004;329(7476):1236. Exclusion Code: X1.

636. Howard L, Chew-Graham CA, Tylee A, et al. The RESPOND trial: a randomized evaluation of antidepressants and support for women with postnatal depression. Arch Womens Ment Health. 2011;14:S30. doi: 10.1007/s00737-010-0203-1. PMID: CN01021074. Exclusion Code: X4.

637. Howard LM, Boath E, Henshaw C. Antidepressant prevention of postnatal depression. PLoS Med. 2006

Oct;3(10):e389. doi: 10.1371/journal.pmed.0030389. PMID: 17020407. Exclusion Code: X1.

638. Howard LM, Hoffbrand S, Henshaw C, et al. Antidepressant prevention of postnatal depression. Cochrane Database Syst Rev. 2005 Apr 18(2):Cd004363. doi: 10.1002/14651858.CD004363.pub2. PMID: 15846711. Exclusion Code: X7.

639. Howell EA, Balbierz A, Jason W, et al. Mothers avoiding depression through empowerment intervention trial (made it). J Gen Intern Med. 2011;26:S222-S3. doi: 10.1007/s11606-011-1730-9. Exclusion Code: X3.

640. Howland RH. Prescribing psychotrophic medications during pregnancy and lactation: Principles and guidelines. J Psychosoc Nurs Ment Health Serv. 2009;47(5):19-23. doi: 10.3928/02793695-20090331-05. PMID: 2009-07495-002. Exclusion Code: X1.

641. Howley MM, Keppler-Noreuil KM, Cunniff $\mathrm{CM}$, et al. Descriptive epidemiology of cerebellar hypoplasia in the National Birth Defects Prevention Study. Birth Defects Res. 2018 Nov 15;110(19):1419-32. doi: 10.1002/bdr2.1388. PMID: 30230717. Exclusion Code: X2.

642. Hoyer J, Wieder G, Höfler M, et al. Do lifetime anxiety disorders (anxiety liability) and pregnancy-related anxiety predict complications during pregnancy and delivery? Early Hum Dev. 2020 Mar 25;144:105022. doi: 10.1016/j.earlhumdev.2020.105022. PMID: 32220767. Exclusion Code: X3. 
643. Huang H, Coleman S, Bridge JA, et al. A meta-analysis of the relationship between antidepressant use in pregnancy and the risk of preterm birth and low birth weight. Gen Hosp Psychiatry. 2014 Jan-Feb;36(1):13-8. doi: 10.1016/j.genhosppsych.2013.08.002. PMID: 24094568. Exclusion Code: X7.

644. Huang M, Cook K, Birk E, et al. Healthcare resource utilization and costs associated with postpartum depression: Commercially insured households. J Manag Care Spec Pharm. 2019;25:S51. Exclusion Code: X5.

645. Huang MY, Deligiannidis K, Suthoff E, et al. SAGE-217 in Postpartum Depression (PPD): Number Needed to Treat (NNT) From a Phase 3, Randomized, PlaceboControlled Trial. Biol Psychiatry. 2020;87(9):S334-S5. doi: 10.1016/j.biopsych.2020.02.859. Exclusion Code: X3.

646. Huang MY, Suthoff E, Deligiannidis K, et al. 934: Phase 3, randomized, placebocontrolled trial of SAGE-217 in postpartum depression: Association between HAM-D and PHQ-9. Am J Obstet Gynecol. 2020;222(1):S578. doi: 10.1016/j.ajog.2019.11.945. Exclusion Code: X3.

647. Huang MY, Xiao RS, Eldar-Lissai A, et al. PMH60 SURVEY OF PATIENT EXPERIENCE AND BURDEN OF ILLNESS IN WOMEN WITH POSTPARTUM DEPRESSION. Value Health. 2020;23:S211. doi: 10.1016/j.jval.2020.04.676. Exclusion Code: X7.

648. Huber-Mollema Y, Oort FJ, Lindhout D, et al. Behavioral problems in children of mothers with epilepsy prenatally exposed to valproate, carbamazepine, lamotrigine, or levetiracetam monotherapy. Epilepsia. 2019 Jun;60(6):1069-82. doi: 10.1111/epi.15968. PMID: 31166022. Exclusion Code: X2.

649. Huisjes HJ, Hadders-Algra M, Touwen BC. Is clonidine a behavioural teratogen in the human? Early Hum Dev. 1986 Jul;14(1):438. PMID: 3732118 . Exclusion Code: X4.
650. Hunter SK, Mendoza JH, D'Anna K, et al. Antidepressants may mitigate the effects of prenatal maternal anxiety on infant auditory sensory gating. Am J Psychiatry. 2012 Jun;169(6):616-24. doi: 10.1176/appi.ajp.2012.11091365. PMID: 22581104. Exclusion Code: X5.

651. Huntington J, Zantop V. Antidepressant medications in pregnancy. Am Fam Physician. 2004 Dec 1;70(11):2195-6. PMID: 15606068. Exclusion Code: X1.

652. Husain N, Munshi T, Jafri F, et al. Antenatal depression is not associated with low birth weight: A study from urban Pakistan. Frontiers in Psychiatry. 2014;5(NOV). doi: 10.3389/fpsyt.2014.00175. Exclusion Code: $\mathrm{X} 3$.

653. Husebye ESN, Daltveit AK, Spigset O, et al. Language delay in children of mothers with epilepsy is associated with the use of antiepileptic drugs - The Norwegian Mother and Child Cohort Study. Eur J Neurol. 2019;26:102. doi: 10.1111/ene.14017. Exclusion Code: X2.

654. Hutchison SM, Masse LC, Brain U, et al. A 6-year longitudinal study: Are maternal depressive symptoms and Selective Serotonin Reuptake Inhibitor (SSRI) antidepressant treatment during pregnancy associated with everyday measures of executive function in young children? Early Hum Dev. 2018 Nov 14;128:21-6. doi: 10.1016/j.earlhumdev.2018.10.009. PMID: 30447406. Exclusion Code: X4.

655. Hutchison SM, Mâsse LC, Glier MB, et al. Impact of Prenatal Selective Serotonin Reuptake Inhibitor Antidepressant Exposure and Maternal Mood on Physical Activity, Dietary Intake, and Markers of Adiposity at Age 6 Years. J Dev Behav Pediatr. 2019 May;40(4):266-74. doi: 10.1097/dbp.0000000000000658. PMID: 30950906. Exclusion Code: X4.

656. Hüttemann D. Drug dependence: Pregnant women substituting safely. Pharm Ztg. 2015;160(22). Exclusion Code: X8.

657. Huybrechts K, Bateman B, Levin R, et al. Comparative safety of depression treatments in late pregnancy for the neonate. Pharmacoepidemiol Drug Saf. 2014;23:166. doi: 10.1002/pds.3701. Exclusion Code: X4. 
658. Huybrechts KF, Bateman BT, Desai RJ, et al. Risk of neonatal drug withdrawal after intrauterine co-exposure to opioids and psychotropic medications: cohort study. BMJ. 2017 Aug 2;358:j3326. doi:

10.1136/bmj.j3326. PMID: 28768628. Exclusion Code: X4.

659. Huybrechts KF, Bateman BT, HernandezDiaz S, et al. Psychotropic medications and the risk of neonatal withdrawal.

Pharmacoepidemiol Drug Saf. 2016;25:260. doi: 10.1002/pds.4070. Exclusion Code: X4.

660. Huybrechts KF, Bröms G, Christensen LB, et al. Association between methylphenidate and amphetamine use in pregnancy and risk of congenital malformations: A cohort study from the international pregnancy safety study consortium. JAMA Psychiatry. 2018;75(2):167-75. doi:

10.1001/jamapsychiatry.2017.3644. Exclusion Code: X3.

661. Huybrechts KF, Hernandez-Diaz S, Avorn J. Antidepressant use in pregnancy and the risk of cardiac defects. N Engl J Med. 2014 Sep 18;371(12):1168-9. doi:

10.1056/NEJMc1409203. PMID: 25229932. Exclusion Code: X1.

662. Huybrechts KF, Palmsten K, Avorn J, et al. Antidepressant use in pregnancy and the risk for cardiac defects. Obstet Gynecol Surv. 2015;69(10):579-81. Exclusion Code: X4.

663. Huybrechts KF, Palmsten K, Mogun H, et al. Specific selective serotonin reuptake inhibitors (SSRIS) during pregnancy and major cardiac defects: A national us cohort study in publicly-insured women. Pharmacoepidemiol Drug Saf. 2013;22:368. doi: 10.1002/pds.3512. Exclusion Code: X9.

664. Huybrechts KF, Sanghani RS, Avorn J, et al. Preterm birth and antidepressant medication use during pregnancy: a systematic review and meta-analysis. PLoS One.

2014;9(3):e92778. doi:

10.1371/journal.pone.0092778. PMID: 24671232. Exclusion Code: X7.

665. Huybrechts KF, Shah RC, Avorn J, et al. Antidepressant medication use during pregnancy and the risk of preterm birth. Pharmacoepidemiol Drug Saf. 2013;22:154. doi: 10.1002/pds.3512. Exclusion Code: X7.
666. Hviid A, Melbye M, Pasternak B. Use of selective serotonin reuptake inhibitors during pregnancy and risk for autism. Obstet Gynecol Surv. 2014;69(4):187-9. doi: 10.1097/OGX.0000000000000050. Exclusion Code: X9.

667. Illamola SM, Young V, Job K, et al. Clinical management of antidepressants during pregnancy. Clinical Pharmacology in Drug Development. 2017;6:11-2. doi: 10.1002/cpdd.385. Exclusion Code: X5.

668. Imaz ML, Gonzalez A, Garcia M, et al. In utero exposure to lithium or politherapy and third trimester fetal biometry. Arch Womens Ment Health. 2013;16:S93-S4. doi: 10.1007/s00737-013-0355-x. Exclusion Code: X5.

669. Imaz ML, Gonzalez A, Hidalgo D, et al. Anthropometric parameters in neonates exposed prenatally to lithium or polytherapy. Bipolar Disorders. 2012;14:84. doi: 10.1111/j.1399-5618.2012.00981.x. Exclusion Code: X9.

670. Imaz ML, Hernández C, Torra M, et al. Obstetrical and neonatal outcomes following prenatal exposure to lithium. Bipolar Disorders. 2011;13:56. doi: 10.1111/j.13995618.2011.00912.x. Exclusion Code: X4.

671. Imaz ML, Torra M, Soy D, et al. Clinical Lactation Studies of Lithium: A Systematic Review. Front Pharmacol. 2019;10:1005. doi: 10.3389/fphar.2019.01005. PMID: 31551795. Exclusion Code: X7.

672. Iniesta-Sepúlveda M, Storch EA. Cognitivebehavioral therapy as an effective, safe, and acceptable intervention for OCD during pregnancy. Revista Brasileira de Psiquiatria. 2017;39(1):84-. doi: 10.1590/1516-44462016-1952. PMID: 2017-36140-011. Exclusion Code: X1.

673. Innocenti D, Lanzi C, Missanelli A, et al. \#42 Maternal exposure to venlafaxine during the first trimester: 10-years experience of the Florence Teratology Information Service. Reprod Toxicol. 2019;88:147. doi: 10.1016/j.reprotox.2019.05.047. Exclusion Code: X4. 
674. Iqbal MM, Kunwar A, Lee K, et al. Effects of commonly used antipsychotics (typical and atypical) in pregnancy and lactation. $\mathrm{J}$ La State Med Soc. 2005 Mar-Apr;157(2):947. PMID: 16022275. Exclusion Code: X7.

675. Isakovich N, Smith E. Delving further into discontinuation risk: addressing the use of mood stabilizers during pregnancy. Am J Psychiatry. 2008 May;165(5):646; author reply 7-8. doi:

10.1176/appi.ajp.2008.08010072. PMID: 18450942. Exclusion Code: X1.

676. Ishii S, Hashimoto-Torii K. Impact of prenatal environmental stress on cortical development. Front Cell Neurosci.

2015;9(MAY). doi:

10.3389/fncel.2015.00207. Exclusion Code: $\mathrm{X} 7$.

677. Izquierdo de la Torre JA. [Maintenance therapy using lithium]. Actas Luso Esp Neurol Psiquiatr Cienc Afines. 1977 NovDec;5(6):311-26. PMID: 564134. Exclusion Code: X8.

678. Jacobson SJ, Jones K, Johnson K, et al. Prospective multicentre study of pregnancy outcome after lithium exposure during first trimester. Lancet. $1992 \mathrm{Feb}$ 29;339(8792):530-3. PMID: 1346886. Exclusion Code: X4.

679. Jaeger DA, Suchan B, Scholmerich A, et al. Attention functioning in children with prenatal drug exposure. Infant Ment Health J. 2015 Sep-Oct;36(5):522-30. doi: 10.1002/imhj.21530. PMID: 26331969. Exclusion Code: X2.

680. Jaeger KM, Roberts TA, Mu TS. Effect of Selective Serotonin Reuptake Inhibitor Use During Pregnancy On Maternal And Neonatal Outcomes Among Women With Depression In The United States Military Healthcare System: A Retrospective Cohort Study. J Adolesc Health. 2019;64(2):S66. doi: $10.1016 /$ j.jadohealth.2018.10.143. Exclusion Code: X9.

681. Jaiswal S, Coombs RC, Isbister GK. Paroxetine withdrawal in a neonate with historical and laboratory confirmation. Eur J Pediatr. 2003 Oct;162(10):723-4. doi: 10.1007/s00431-003-1293-9. PMID: 12905013. Exclusion Code: X7.
682. Janecka M, Kodesh A, Levine SZ, et al. Association of Autism Spectrum Disorder With Prenatal Exposure to Medication Affecting Neurotransmitter Systems. JAMA Psychiatry. 2018 Dec 1;75(12):1217-24. doi: 10.1001/jamapsychiatry.2018.2728. PMID: 30383108. Exclusion Code: X4.

683. Jarde A, Morais M, Kingston D, et al. Neonatal outcomes in women with untreated antenatal depression compared with women without depression: a systematic review and meta-analysis. JAMA Psychiatry. 2016 Aug 1;73(8):826-37. doi: 10.1001/jamapsychiatry.2016.0934. PMID: 27276520. Exclusion Code: X7.

684. Jayaratne K, Karunasena C, Rajapakse T, et al. Counting and reviewing maternal suicides in resource-limited settings: Lessons from Sri Lanka. Arch Womens Ment Health. 2019;22(5):688. doi: 10.1007/s00737-019-00996-y. Exclusion Code: X3.

685. Jazayeri D, Graham J, Hitchcock A, et al. Outcomes of pregnancies in women taking antiepileptic drugs for non-epilepsy indications. Seizure. 2018 Mar;56:111-4. doi: 10.1016/j.seizure.2018.02.009. PMID: 29471258. Exclusion Code: X4.

686. Jensen HM, Gron R, Lidegaard O, et al. The effects of maternal depression and use of antidepressants during pregnancy on risk of a child small for gestational age. Psychopharmacology (Berl). 2013 Jul;228(2):199-205. doi: 10.1007/s00213013-3029-5. PMID: 23455598. Exclusion Code: X12.

687. Jensen HM, Gron R, Lidegaard O, et al. Maternal depression, antidepressant use in pregnancy and Apgar scores in infants. $\mathrm{Br} \mathrm{J}$ Psychiatry. 2013 May;202(5):347-51. doi: 10.1192/bjp.bp.112.115931. PMID: 23429204. Exclusion Code: X4.

688. Jentink J, Loane MA, Dolk H, et al. Valproic acid monotherapy in pregnancy and major congenital malformations. Obstet Gynecol Surv. 2010;65(10):619-20. doi: 10.1097/OGX.0b013e3182021f65. Exclusion Code: X9. 
689. Jeret JS. Neurodevelopment after in utero exposure to phenytoin and carbamazepine. JAMA. 1994 Sep 21;272(11):850; author reply 1. PMID: 7521401. Exclusion Code: $\mathrm{X} 1$.

690. Jermain DM. Treatment of postpartum depression. Am Pharm. 1995

Jan;Ns35(1):33-8, 45. PMID: 7887372. Exclusion Code: X1.

691. Jerome L. The use of selective serotonin reuptake inhibitors during pregnancy and lactation: current knowledge. Can J Psychiatry. 2000 Dec;45(10):940. PMID: 11190371. Exclusion Code: X1.

692. Jessel N, Stiskal JA. Neonatal encephalopathy after antidepressant exposure during pregnancy. Herbst F, Gortner L. Paroxetine withdrawal syndrome as differential diagnosis of acute neonatal encephalophathy? Z Geburtsh Neonatol 2003; 207: 232-234. Z Geburtshilfe Neonatol. 2004 Apr;208(2):75-6; author reply 6. doi: 10.1055/s-2004-818962. PMID: 15112141. Exclusion Code: X1.

693. Jha SC, Meltzer-Brody S, Steiner RJ, et al. Antenatal depression, treatment with selective serotonin reuptake inhibitors, and neonatal brain structure: A propensitymatched cohort study. Psychiatry Res Neuroimaging. 2016 Jul 30;253:43-53. doi: 10.1016/j.pscychresns.2016.05.004. PMID: 27254086. Exclusion Code: X4.

694. Jiang HY, Peng CT, Zhang X, et al. Antidepressant use during pregnancy and the risk of attention-deficit/hyperactivity disorder in the children: a meta-analysis of cohort studies. BJOG. 2018;125(9):1077-84. doi: 10.1111/1471-0528.15059. Exclusion Code: X7.

695. Jiang HY, Xu LL, Li YC, et al. Antidepressant use during pregnancy and risk of postpartum hemorrhage: A systematic review and meta-analysis. J Psychiatr Res. 2016 Dec;83:160-7. doi: 10.1016/j.jpsychires.2016.09.001. PMID: 27637098. Exclusion Code: X7.

696. Jimenez-Solem E. Exposure to antidepressants during pregnancy-prevalences and outcomes. Dan Med J. 2014 Sep;61(9):B4916. PMID: 25186551. Exclusion Code: X1.
697. Jimenez-Solem E, Andersen JT, Andersen $\mathrm{NL}$, et al. In utero exposure to antipsychotics and congenital malformations-a nation wide cohort study. Pharmacoepidemiol Drug Saf. 2013;22:154. doi: 10.1002/pds.3512. Exclusion Code: X9.

698. Jimenez-Solem E, Andersen JT, Petersen M, et al. SSRI use during pregnancy and risk of stillbirth and neonatal mortality. Am J Psychiatry. 2013 Mar;170(3):299-304. doi: 10.1176/appi.ajp.2012.11081251. PMID: 23361562. Exclusion Code: X10.

699. Johnson EE, Bardsley T, Schulte K, et al. Long acting opioids and antiepileptic medications prolong inpatient length of stay and pharmacologic treatment of infants with neonatal opioid withdrawal syndrome (NOWS). J Investig Med. 2019;67(1):88. doi: 10.1136/jim-2018-000939.57. Exclusion Code: X2.

700. Johnson GL. Birth outcomes in pregnant women taking fluoxetine. J Fam Pract. 1997 Jan;44(1):32. PMID: 9010368. Exclusion Code: X1.

701. Johnson JA, Canavan TP. Analysis of maternal characteristics of patients with fetal hypoplastic left heart syndrome. Congenit Heart Dis. 2018;13(5):848-9. doi: 10.1111/chd.12693. Exclusion Code: X2.

702. Jones GL. Abnormal lactation associated with fluphenazine enanthate. J Med Soc N J. 1968 May;65(5):192-3. PMID: 5239952. Exclusion Code: X7.

703. Jones I, Shakespeare J. Postnatal depression. BMJ. 2014 Aug 14;349:g4500. doi: 10.1136/bmj.g4500. PMID: 25125284. Exclusion Code: X1.

704. Jonquiere-Wichmann M. [Post-partum psychoses]. Rev Med Suisse Romande. 1974 Jul;94(7):511-23. PMID: 4842888. Exclusion Code: X8.

705. Jordan S, Charlton RA, Tingay K, et al. EURO medi CAT: Safety of medication use in pregnancy. Arch Womens Ment Health. 2015;18(2):337. doi: 10.1007/s00737-0140488-6. Exclusion Code: X2. 
706. Jordan S, Davies GI, Thayer DS, et al. Antidepressant prescriptions, discontinuation, depression and perinatal outcomes, including breastfeeding: A population cohort analysis. PLoS One. 2019;14(11):e0225133. doi: 10.1371/journal.pone.0225133. PMID: 31738813. Exclusion Code: X4.

707. Jordan S, Morris JK, Davies GI, et al. Selective serotonin reuptake inhibitor (SSRI) antidepressants in pregnancy and congenital anomalies: analysis of linked databases in Wales, Norway and Funen, Denmark. PLoS One. 2016;11(12):e0165122. doi: 10.1371/journal.pone.0165122. PMID: 27906972. Exclusion Code: X7.

708. Joseph KS, Sheehy O, Mehrabadi A, et al. Can drug effects explain the recent temporal increase in atonic postpartum haemorrhage? Paediatr Perinat Epidemiol. 2015

May;29(3):220-31. doi: 10.1111/ppe.12190. PMID: 25847112. Exclusion Code: X4.

709. Julyan TE, Wood C, Cameron M, et al. Characteristics and consequences of antenatal exposure to psychotropic medication 3: neonatal outcomes. Arch Womens Ment Health. 2015;18(2):339. doi: 10.1007/s00737-014-0488-6. Exclusion Code: X4.

710. Junge-Hoffmeister J, Bittner A, Richter J, et al. Preventing peripartal psychosomatic symptoms in pregnant women - Is there an impact on pregnancy and birth complications or neonatal outcomes. Arch Womens Ment Health. 2011;14:S32. doi: 10.1007/s00737-010-0203-1. Exclusion Code: X3.

711. Junker H. A Phase 3, double-blind, placebocontrolled trial of SAGE-217 in postpartum depression: Assessment of depressive symptoms across multiple measures. Pharmacopsychiatry. 2020;53(2):94. doi: 10.1055/s-0039-3403036. Exclusion Code: X9.
712. Junker H, Kanes S, Meltzer-Brody S, et al. The gamma-aminobutyric acid type A receptor positive allosteric modulators brexanolone injection and SAGE-217 in the treatment of mood disorders: Results from recent placebo-controlled studies. Pharmacopsychiatry. 2019;52(2):103. doi: 10.1055/s-0039-1679163. Exclusion Code: $\mathrm{X} 9$.

713. Jutla A. Prenatal antidepressant exposure as a risk factor for autism spectrum disorder: An examination of the evidence. J Am Acad Child Adolesc Psychiatry. 2016;55(10):S104. doi: 10.1016/j.jaac.2016.09.016. Exclusion Code: X7.

714. Kaabi W, El Aidli S, Kastalli S, et al. Pregnancy outcomes in women using antiepileptic drugs. Drug Saf. 2013;36(9):844. doi: 10.1007/s40264-0130087-x. Exclusion Code: X2.

715. Kadrmas A, Winokur G, Crowe RR. Postpartum mania. Br J Psychiatry. 1979;135:551-4. doi: 10.1192/bjp.135.6.551. PMID: 1981-25968-001. Exclusion Code: $\mathrm{X} 1$.

716. Kaihola H, Olivier J, Poromaa IS, et al. The effect of antenatal depression and selective serotonin reuptake inhibitor treatment on nerve growth factor signaling in human placenta. PLoS One. 2015;10(1):e0116459. doi: 10.1371/journal.pone.0116459. PMID: 25611484. Exclusion Code: X5.

717. Kalin M, Cochran A, Newport DJ, et al. Fetal Antidepressant Exposure and Outcomes. Biol Psychiatry. 2019;85(10):S319. doi: 10.1016/j.biopsych.2019.03.809. Exclusion Code: X5.

718. Kallen B. Maternal use of antidepressant drugs and twin deliveries. Eur J Obstet Gynecol Reprod Biol. 2012 Oct;164(2):235. doi: 10.1016/j.ejogrb.2012.06.005. PMID: 22770631. Exclusion Code: X1.

719. Kallen B, Olausson PO. Maternal use of selective serotonin re-uptake inhibitors and persistent pulmonary hypertension of the newborn. Pharmacoepidemiol Drug Saf. 2008 Aug;17(8):801-6. doi: 10.1002/pds.1570. PMID: 18314924. Exclusion Code: X4. 
720. Kallen B, Robert-Gnansia E. Maternal drug use, fertility problems, and infant craniostenosis. Cleft Palate Craniofac J. 2005 Nov;42(6):589-93. doi: 10.1597/04031.1. PMID: 16241169. Exclusion Code: $\mathrm{X} 2$.

721. Kallen BA, Otterblad Olausson P. Maternal use of selective serotonin re-uptake inhibitors in early pregnancy and infant congenital malformations. Birth Defects Res A Clin Mol Teratol. 2007 Apr;79(4):301-8. doi: 10.1002/bdra.20327. PMID: 17216624. Exclusion Code: X4.

722. Kamenou I, Wilson A, Miller J. Managing bipolar disorder in pregnancy-use of lithium. BJOG. 2016;123:27-8. doi:

10.1111/14710528.13987. Exclusion Code: $\mathrm{X} 7$.

723. Kandall SR, Lee YJ, Siljestrom K. Polydrug vs. methadone use in pregnancy: studies on offspring. Pediatr Res. 1978;12(4 II):No.983. Exclusion Code: X2.

724. Kanes S, Colquhoun H, Gunduz-Bruce H, et al. SAGE-547 for the treatment of severe postpartum depression.

Neuropsychopharmacology. Conference: 55 th annual meeting of the american college of neuropsychopharmacology, ACNP 2016. United states. Conference start: 20161204. Conference end: 20161208. 2016;41:S165S6. doi: 10.1038/npp.2016.240. PMID: CN01296353. Exclusion Code: X9.

725. Kanes S, Colquhoun H, Riesenberg R, et al. Phase 3 study evaluating brexanolone, a GABAA receptor modulator, in moderate postpartum depression. Obstet Gynecol. 2018 May;131(Supplement 1):94s-s. doi: 10.1097/01.AOG.0000533413.36000.6e. PMID: WOS:000442482900321. Exclusion Code: X9.

726. Kanes S, Colquhoun H, Riesenberg R, et al. Phase 3 study evaluating brexanolone, a gabaa receptor modulator, in moderate postpartum depression. Obstet Gynecol. 2018;131:93S. Exclusion Code: X7.

727. Kanes S, Nomikos G, Quirk M, et al. A single-ascending dose study of the neuroactive steroid SAGE-217. Biol Psychiatry. 2017 May 15;81(10):S31-S. doi: DOI 10.1016/j.biopsych.2017.02.086. PMID: WOS:000400348700076. Exclusion Code: X2.
728. Kanes SJ, Colquhoun H, Doherty J, et al. Open-label, proof-of-concept study of brexanolone in the treatment of severe postpartum depression. Hum Psychopharmacol. 2017 Mar;32(2). doi: 10.1002/hup.2576. PMID: 28370307. Exclusion Code: X4.

729. Kanes SJ, Colquhoun H, Doherty J, et al. The open-label, proof-of-concept study of sage-547 (allopregnanolone) in the treatment of postpartum depression. Biol Psychiatry. 2016;79(9):133S. doi: 10.1016/j.biopsych.2016.03.1748. Exclusion Code: X4.

730. Kaplan YC, Keskin-Arslan E, Acar S. Prenatal antidepressant use and risk of autism spectrum disorders in children. JAMA Pediatr. 2016 Jul 1;170(7):712. doi: 10.1001/jamapediatrics.2016.0727. PMID: 27244647. Exclusion Code: X1.

731. Kaplan YC, Keskin-Arslan E, Acar S, et al. Prenatal selective serotonin reuptake inhibitor use and the risk of autism spectrum disorder in children: A systematic review and meta-analysis. Reprod Toxicol. 2016 Dec;66:31-43. doi: 10.1016/j.reprotox.2016.09.013. PMID: 27667009. Exclusion Code: X7.

732. Kaplan YC, Keskin-Arslan E, Acar S, et al. Maternal SSRI discontinuation, use, psychiatric disorder and the risk of autism in children: a meta-analysis of cohort studies.

Br J Clin Pharmacol. 2017

Dec;83(12):2798-806. doi:

10.1111/bcp.13382. PMID: 28734011. Exclusion Code: X7.

733. Kara S, Tan S, Aldemir S, et al. Investigation of perceived social support in mothers of infants hospitalized in neonatal Intensive Care Unit. Hippokratia. 2013;17(2):130-5. Exclusion Code: X5.

734. Karam F, Berard A, Sheehy O, et al. Impact of maternal attachment on infant development at one year of age: Results from the otis antidepressants in pregnancy study. Birth Defects Research Part A Clinical and Molecular Teratology. 2012;94(5):347. doi: 10.1002/bdra.23023. Exclusion Code: X3. 
735. Karam F, Sheehy O, Berard A. Maternal prenatal and parental postnatal stress in a cohort of depressed women and their partners. Pharmacoepidemiol Drug Saf. 2011;20:S52-S3. doi: 10.1002/pds.2206. Exclusion Code: X5.

736. Karam F, Sheehy O, Bérard A. Maternal prenatal and parental postnatal stress in a cohort of depressed women and their partners. Am J Epidemiol. 2011;173:S45. doi: 10.1093/aje/kwr181. Exclusion Code: X5.

737. Karam F, Sheehy O, Huneau MC, et al. Maternal prenatal and parental postnatal stress in a cohort of depressed women and their partners. J Popul Ther Clin Pharmacol. 2011;18(2):e296-e7. Exclusion Code: X5.

738. Karlsson L, Ekholm E, Korja R, et al. Prenatal psychological distress and the risk of pre-eclampsia-the finnbrain birth cohort study. Psychother Psychosom. 2019;88:63. doi: 10.1159/000502467. Exclusion Code: X3.

739. Kashani L, Eslatmanesh S, Saedi N, et al. Comparison of saffron versus fluoxetine in treatment of mild to moderate postpartum depression: a double-blind, randomized clinical trial. Pharmacopsychiatry. 2017 Mar;50(2):64-8. doi: 10.1055/s-0042115306. PMID: 27595298. Exclusion Code: $\mathrm{X} 3$.

740. Kaunitz AM. Making the patient-pill match: Considerations in OC selection. Sexuality, Reproduction and Menopause. 2008;6(2):13-6. Exclusion Code: X1.

741. Kawada T. Selective serotonin reuptake inhibitors exposure during pregnancy and neonatal outcomes. J Clin Psychopharmacol. 2014 Dec;34(6):751. doi: 10.1097/jcp.0000000000000110. PMID: 24781437. Exclusion Code: X1.

742. Kellner CH, Beale MD, Pritchett JT. The risk of in utero exposure to lithium. JAMA. 1994 Jun 15;271(23):1828-9. PMID: 8196137. Exclusion Code: X1.

743. Kellner CH, Pasculli RM, Briggs MC. Treatment of depression during pregnancy. J ECT. 2012 Sep;28(3):195-6. doi: 10.1097/YCT.0b013e318250e585. PMID: 22914631. Exclusion Code: X1.
744. Kellogg CK, Chisholm J, Simmons RD, et al. Neural and behavioral consequences of prenatal exposure to diazepam. Monogr Neural Sci. 1983;9:119-29. PMID: 6684207. Exclusion Code: X2.

745. Kelly BD, Lane A, Agartz I, et al. Craniofacial dysmorphology in Swedish schizophrenia patients. Acta Psychiatr Scand. 2005 Mar;111(3):202-7. doi: 10.1111/j.1600-0447.2004.00473.x. PMID: 15701104. Exclusion Code: X2.

746. Kelly LE, Poon S, Madadi P, et al. Neonatal benzodiazepines exposure during breastfeeding. J Pediatr. 2012 Sep;161(3):448-51. doi: 10.1016/j.jpeds.2012.03.003. PMID: 22504099. Exclusion Code: X4.

747. Kelly MB, Wisner KL, Cornelius MD. SSRIs and birth defects. Epidemiology. 2007 May;18(3):411-2; author reply 2-3. doi: 10.1097/01.ede.0000259346.39498.eb. PMID: 17435454. Exclusion Code: X1.

748. Kelsey FO. Drugs in pregnancy and their effects on pre- and postnatal development. Res Publ Assoc Res Nerv Ment Dis. 1973;51:233-43. PMID: 4590403. Exclusion Code: X1.

749. Kempny A, Swan L, Dimopoulos K. Antidepressant use in pregnancy and the risk of cardiac defects. N Engl J Med. 2014 Sep 18;371(12):1167. doi: 10.1056/NEJMc1409203. PMID: 25229933. Exclusion Code: X1.

750. Kenar ANI, Cimen E. P.227 Psychiatric drug use in pregnancy: retrospective analysis of data from a psychiatry hospital. Eur Neuropsychopharmacol. 2019;29:S175. doi: 10.1016/j.euroneuro.2019.09.270. Exclusion Code: X7.

751. Kernizan N, Forinash A, Yancey AM. Evaluation of prescribing patterns for the treatment of bipolar disorder in pregnancy. JACCP Journal of the American College of Clinical Pharmacy. 2018;1(2):284. doi: 10.1002/jac5.1059. Exclusion Code: X7. 
752. Kheir YN, Yoder R. 3.3 EFFECTS OF PARENTAL DEPRESSION TREATMENT ON THEIR CHILDREN'S MENTAL HEALTH OUTCOMES: SYSTEMATIC REVIEW OF CLINICAL TRIALS AND OBSERVATIONAL STUDIES. J Am Acad Child Adolesc Psychiatry. 2019;58(10):S195. doi: 10.1016/j.jaac.2019.08.166. Exclusion Code: X2.

753. Kieler H, Artama M, Engeland A, et al. Selective serotonin reuptake inhibitors during pregnancy and risk of persistent pulmonary hypertension in the newborn: population based cohort study from the five Nordic countries. BMJ. 2012 Jan 12;344:d8012. doi: 10.1136/bmj.d8012. PMID: 22240235. Exclusion Code: X10.

754. Kieler H, Furu K, Gissler M, et al. Selective serotonin-reuptake inhibitors and risks of persistent fetal circulation and pulmonary hypertension in the newborn.

Pharmacoepidemiol Drug Saf. 2011;20:S10. doi: 10.1002/pds.2206. Exclusion Code: X4.

755. Kieler H, Malm H, Artama M, et al. Use of antidepressants and association with elective termination of pregnancy: population based case-control study. BJOG. 2015

Nov;122(12):1618-24. doi: 10.1111/14710528.13164. PMID: 25395328. Exclusion Code: X5.

756. Kieviet N, de Jong F, Scheele F, et al. Use of antidepressants during pregnancy in the Netherlands: observational study into postpartum interventions. BMC Pregnancy Childbirth. 2017 Jan 11;17(1):23. doi: 10.1186/s12884-016-1184-5. PMID: 28077067. Exclusion Code: X4.

757. Kieviet N, Duijn M, Dolman KM, et al. Poor neonatal adaptation after in utero exposure to fluvoxamine. J Obstet Gynaecol. 2015 Feb;35(2):201-2. doi: 10.3109/01443615.2014.940303. PMID: 25162141. Exclusion Code: X7.

758. Kieviet N, Hoppenbrouwers C, Dolman $\mathrm{KM}$, et al. Risk factors for poor neonatal adaptation after exposure to antidepressants in utero. Acta Paediatr. 2015

Apr;104(4):384-91. doi: 10.1111/apa.12921. PMID: 25559357. Exclusion Code: X5.
759. Kieviet N, van Keulen V, van de Ven PM, et al. Serotonin and poor neonatal adaptation after antidepressant exposure in utero. Acta Neuropsychiatr. 2017 Feb;29(1):43-53. doi: 10.1017/neu.2016.30. PMID: 27387606. Exclusion Code: X4.

760. Kim DR, Epperson CN, Weiss AR, et al. Pharmacotherapy of postpartum depression: an update. Expert Opin Pharmacother. 2014 Jun;15(9):1223-34. doi: 10.1517/14656566.2014.911842. PMID: 24773410. Exclusion Code: X7.

761. Kim DR, Pinheiro E, Luther JF, et al. Is third trimester serotonin reuptake inhibitor use associated with postpartum hemorrhage? J Psychiatr Res. 2016 Feb;73:79-85. doi: 10.1016/j.jpsychires.2015.11.005. PMID: 26692255. Exclusion Code: X4.

762. Kim JY, Son MJ, Son CY, et al. Environmental risk factors and biomarkers for autism spectrum disorder: an umbrella review of the evidence. Lancet Psychiatry. 2019 Jul;6(7):590-600. doi: 10.1016/s22150366(19)30181-6. PMID: 31230684.

Exclusion Code: X7.

763. Kimbui E, Kuria M, Yator O, et al. A crosssectional study of depression with comorbid substance use dependency in pregnant adolescents from an informal settlement of Nairobi: Drawing implications for treatment and prevention work. Annals of General Psychiatry. 2018;17(1). doi: 10.1186/s12991-018-0222-2. Exclusion Code: X3.

764. King BH. Association between maternal use of SSRI medications and autism in their children. JAMA. 2017 Apr

18;317(15):1568-9. doi: 10.1001/jama.2016.20614. PMID: 28418468. Exclusion Code: X1.

765. King-Hele SA, Abel KM, Webb RT, et al. Risk of sudden infant death syndrome with parental mental illness. Arch Gen Psychiatry. 2007 Nov;64(11):1323-30. doi: 10.1001/archpsyc.64.11.1323. PMID: 17984401. Exclusion Code: X3.

766. Kiss H. Prevention of CMV infection in newborns and foetuses -study design and epidemiological results of a phase III trial. J Inherit Metab Dis. 2011;34:S7. doi: 10.1007/s10545-011-9312-x. Exclusion Code: X2. 
767. Kittel-Schneider S, Preis K, Menke A, et al. Therapeutic drug level measurement in breast milk. Eur Neuropsychopharmacol. 2019;29:S92-S3. doi:

10.1016/j.euroneuro.2018.11.1077.

Exclusion Code: X5.

768. Kittel-Schneider S, Preis K, Van Braam L, et al. Therapeutic drug monitoring in pregnancy and post-partum periodantidepressant substances.

Pharmacopsychiatry. 2020;53(3):142. doi: 10.1055/s-0040-1710121. Exclusion Code: $\mathrm{X} 5$.

769. Kivisto J, Lehto SM, Halonen K, et al. Maternal use of selective serotonin reuptake inhibitors and lengthening of the umbilical cord: indirect evidence of increased foetal activity-a retrospective cohort study. PLoS One. 2016;11(4):e0154628. doi:

10.1371/journal.pone.0154628. PMID: 27128030. Exclusion Code: X4.

770. Kjaersgaard MI, Parner ET, Vestergaard M, et al. Prenatal antidepressant exposure and risk of spontaneous abortion - a populationbased study. PLoS One. 2013;8(8):e72095. doi: 10.1371/journal.pone.0072095. PMID: 24015208. Exclusion Code: X12.

771. Klein DF. Pregnancy and panic disorder. J Clin Psychiatry. 1994 Jul;55(7):293-4. PMID: 7915274. Exclusion Code: X1.

772. Klieger-Grossmann C, Weitzner B, Einarson $\mathrm{T}$, et al. The safety of escitalopram in pregnancy: A prospective cohort study. J Popul Ther Clin Pharmacol. 2010;17(1):e230. Exclusion Code: X5.

773. Klinger G, Frankenthal D, Merlob P, et al. Long-term outcome following selective serotonin reuptake inhibitor induced neonatal abstinence syndrome. J Perinatol. 2011 Sep;31(9):615-20. doi: 10.1038/jp.2010.211. PMID: 21311497. Exclusion Code: X2.

774. Klinger G, Stahl B, Fusar-Poli P, et al. Antipsychotic drugs and breastfeeding. Pediatr Endocrinol Rev. 2013 MarApr;10(3):308-17. PMID: 23724438. Exclusion Code: X7.
775. Knickmeyer R, Meltzer-Brody S, Woolson $\mathrm{S}$, et al. Increased rate of chiari $\mathrm{i}$ malformation in children of depressed mothers treated with selective serotonin reuptake inhibitors during pregnancy. Neuropsychopharmacology. 2013;38:S112. doi: 10.1038/npp.2013.279. Exclusion Code: X9.

776. Knickmeyer RC, Meltzer-Brody S, Woolson $\mathrm{S}$, et al. Rate of Chiari I malformation in children of mothers with depression with and without prenatal SSRI exposure. Neuropsychopharmacology. 2014 Oct;39(11):2611-21. doi: 10.1038/npp.2014.114. PMID: 24837031. Exclusion Code: X5.

777. Knight M. MBRRACE-UK Update: Key messages from the UK and Ireland Confidential Enquiries into Maternal Death and Morbidity 2018. Obstetrician and Gynaecologist. 2019;21(1):69-71. doi: 10.1111/tog.12548. Exclusion Code: X1.

778. Ko JY, Farr SL, Dietz PM, et al. Depression and treatment among U.S. pregnant and nonpregnant women of reproductive age, 2005-2009. J Womens Health (Larchmt). 2012 Aug;21(8):830-6. doi: 10.1089/jwh.2011.3466. PMID: 22691031. Exclusion Code: X2.

779. Ko JY, Farr SL, Dietz PM, et al. Depression and treatment among US pregnant and nonpregnant women of reproductive age, 2005-2009. J Womens Health.

2012;21(8):830-6. doi: 10.1089/jwh.2011.3466. PMID: 201221336-003. Exclusion Code: X5.

780. Kobayashi T, Matsuyama T, Takeuchi M, et al. Autism spectrum disorder and prenatal exposure to selective serotonin reuptake inhibitors: A systematic review and metaanalysis. Reprod Toxicol. 2016 Oct;65:1708. doi: 10.1016/j.reprotox.2016.07.016. PMID: 27474253. Exclusion Code: X7.

781. Koga E, Takahashi Y, Seki K, et al. Pregnancy outcomes vary with disease progression in pregnant women with schizophrenia. Clin Exp Obstet Gynecol. 2019;46(2):201-5. doi: 10.12891/ceog4396.2019. Exclusion Code: $\mathrm{X} 13$. 
782. Kojima S, Ogura H, Yokoyama M, et al. [The effect of diazepam on neonatal neurobehavioral response]. Nihon Sanka Fujinka Gakkai Zasshi. 1985 Dec;37(12):2784-90. PMID: 4086907. Exclusion Code: X8.

783. Kola L, Oladeji BD, Bello T, et al. A comparison of the prevalence, correlates and outcomes of perinatal depression in adolescent and adult mothers-Results from the EXPONATE randomized controlled trial in Nigeria. Arch Womens Ment Health. 2019;22(5):670. doi: 10.1007/s00737-01900996-y. Exclusion Code: X3.

784. Kong L, Zhou T, Wang B, et al. The risks associated with the use of lamotrigine during pregnancy. Int J Psychiatry Clin Pract. 2018 Mar;22(1):2-5. doi: 10.1080/13651501.2017.1341986. PMID: 28657488. Exclusion Code: X7.

785. Koren G. First-trimester exposure to fluoxetine (prozac). Does it affect pregnancy outcome? Can Fam Physician. 1996 Jan;42:43-4. PMID: 8924812. Exclusion Code: X1.

786. Koren G. SSRIs in pregnancy--are they safe? Pediatr Res. 2002 Apr;51(4):424-5. doi: 10.1203/00006450-200204000-00005. PMID: 11919325. Exclusion Code: X1.

787. Koren G. SSRIs in late pregnancy: the risk of neonatal respiratory distress and seizures. Acta Psychiatr Scand. 2011 Apr;123(4):318; author reply -9. doi: 10.1111/j.16000447.2010.01642.x. PMID: 21198456. Exclusion Code: X1.

788. Koren G. What adversely affects the unborn--psychotropic drugs or maternal morbidity? J Clin Psychiatry. 2015 Jul;76(7):e904-5. doi: 10.4088/JCP.14com09439. PMID: 26231024. Exclusion Code: X1.

789. Koren G, Boucher N. Adverse effects in neonates exposed to SSRIs and SNRI in late gestation--Motherisk Update 2008. Can J Clin Pharmacol. 2009 Winter;16(1):e66-7. PMID: 19164848. Exclusion Code: X1.

790. Koren G, Cohn T, Chitayat D, et al. Use of atypical antipsychotics during pregnancy and the risk of neural tube defects in infants. Am J Psychiatry. 2002 Jan;159(1):136-7. doi: 10.1176/appi.ajp.159.1.136. PMID: 11772703. Exclusion Code: X4.
791. Koren G, Kalra S, Einarson A. Antidepressant use in late pregnancy. Canadian Pharmacists Journal. 2005;138(8):67-8. Exclusion Code: X1.

792. Koren G, Matsui D, Einarson A, et al. Is maternal use of selective serotonin reuptake inhibitors in the third trimester of pregnancy harmful to neonates? CMAJ. 2005 May 24;172(11):1457-9. doi: 10.1503/cmaj.1041100. PMID: 15911861. Exclusion Code: X1.

793. Korhonen LS, Karlsson L, Scheinin NM, et al. Prenatal Maternal Psychological Distress and Offspring Risk for Recurrent Respiratory Infections. J Pediatr. 2019 May;208:229-35.e1. doi: 10.1016/j.jpeds.2018.12.050. PMID: 30723014. Exclusion Code: X3.

794. Kornetsky C. Psychoactive drugs in the immature organism. Psychopharmacologia. 1970;17(2):105-36. PMID: 4910308. Exclusion Code: X1.

795. Kowalik E, Ward K, Ye Y. SSRI use in pregnancy and congenital heart defects: A metaanalysis of population-based cohort studies. Pharmacotherapy. 2016;36(12):e302. doi: 10.1002/phar.1877. Exclusion Code: X4.

796. Kragholm K, Andersen MP, Mortensen RN, et al. Exposure to selective serotonin reuptake inhibitors in utero and early elementary school outcomes. Acta Psychiatr Scand. 2018;137(6):481-90. doi: 10.1111/acps.12867. Exclusion Code: X4.

797. Krause L, Einsle F, Petzoldt J, et al. The role of maternal anxiety and depressive disorders prior to and during pregnancy and perinatal psychopathological symptoms for early infant diseases and drug administration. Early Hum Dev. 2017 Jun;109:7-14. doi: 10.1016/j.earlhumdev.2017.03.009. PMID: 28399458. Exclusion Code: X2.

798. Kris EB, Carmichael DM. Chlorpromazine maintenance therapy during pregnancy and confinement. Psychiatr Q. 1957;31(4):690-5. PMID: 13518422. Exclusion Code: X7.

799. Kronenfeld N, Ziv Baran T, Berlin M, et al. Chronic use of psychotropic medications in breastfeeding women: Is it safe? PLoS One. 2018;13(5):e0197196. doi: 10.1371/journal.pone.0197196. PMID: 29782546. Exclusion Code: X4. 
800. Kronenfeld N, Ziv Baran T, Berlin M, et al. Chronic use of psychotropic medications in breastfeeding women: Is it safe? PLoS One. 2018;13(5):e0197196. doi: 10.1371/journal.pone.0197196. PMID: 29782546. Exclusion Code: X4.

801. Kuijten RR, Bunin GR, Nass CC, et al. Gestational and familial risk factors for childhood astrocytoma: results of a casecontrol study. Cancer Res. 1990 May 1;50(9):2608-12. PMID: 2328486.

Exclusion Code: X2.

802. Kulkarni J, Gilbert H, Gurvich C, et al. The national register of antipsychotic medication in pregnancy (NRAMP): The first one hundred babies. Aust N Z J Psychiatry. 2010;44:A45. doi:

10.3109/00048674.2010.507066. Exclusion Code: X5.

803. Kulkarni J, Gilbert H, Gurvich C, et al. The first 100 women in the national register of antipsychotic medication in pregnancy (NRAMP). Schizophr Bull. 2011;37:27. doi: 10.1093/schbul/sbq173. Exclusion Code: $\mathrm{X} 5$.

804. Kulkarni J, McCauley-Elsom K, Marston N, et al. Preliminary findings from the National Register of Antipsychotic Medication in Pregnancy. Aust N Z J Psychiatry. 2008;42(1):38-44. doi: 10.1080/00048670701732723. PMID: 200812695-006. Exclusion Code: X7.

805. Kulkarni J, Worsley R, Gilbert H, et al. A prospective cohort study of antipsychotic medications in pregnancy: the first 147 pregnancies and 100 one year old babies. PLoS One. 2014;9(5):e94788. doi: 10.1371/journal.pone.0094788. PMID: 24787688. Exclusion Code: X4.

806. Kullander S, Kallen B. A prospective study of drugs and pregnancy. I. Psychopharmaca. Acta Obstet Gynecol Scand. 1976;55(1):2533. PMID: 1251690 . Exclusion Code: X4.

807. Kunugi H, Nanko S, Takei N, et al. Perinatal complications and schizophrenia: Data from the Maternal and Child Health Handbook in Japan. J Nerv Ment Dis. 1996;184(9):542-6. doi: 10.1097/00005053-199609000-00005. PMID: 1996-00486-005. Exclusion Code: $\mathrm{X} 3$.
808. Kurasaki A, Hasegawa J, Harada S, et al. Analysis of characteristics in puerperium women predictive of perinatal psychiatric disorders within a year. J Obstet Gynaecol Res. 2019;45(8):1749. doi: 10.1111//jog.14030. Exclusion Code: X3.

809. Lacroix I, Hurault-Delarue C, Guitard C, et al. Psychomotor effects of in utero exposure to psychotropic medications: A comparative study in EFEMERIS database. Fundam Clin Pharmacol. 2012;26:9-10. doi: 10.1111/j.1472-8206.2012.01032.x. Exclusion Code: X4.

810. Lacroix I, Hurault-Delarue C, Montastruc JL, et al. Psychomotor effects of in utero exposure to psychotropic medications: A comparative study in efemeris database. Pharmacoepidemiol Drug Saf. 2012;21:141. doi: 10.1002/pds.3324. Exclusion Code: X4.

811. Laegreid L. Clinical observations in children after prenatal benzodiazepine exposure. Dev Pharmacol Ther. 1990;15(3-4):186-8. PMID: 1983095. Exclusion Code: X7.

812. Laegreid L. Potential hazards of benzodiazepines for the fetus. Acta Obstet Gynecol Scand. 1992;71(8):655-6. Exclusion Code: X1.

813. Laegreid L, Conradi N, Hagberg G, et al. Psychotropic drug use in pregnancy and perinatal death. Acta Obstet Gynecol Scand. 1992 Aug;71(6):451-7. PMID: 1356291. Exclusion Code: X2.

814. Laegreid L, Hagberg G, Lundberg A. The effect of benzodiazepines on the fetus and the newborn. Neuropediatrics. 1992 Feb;23(1):18-23. doi: 10.1055/s-20081071305. PMID: 1348849 . Exclusion Code: $\mathrm{X} 2$.

815. Laegreid L, Hagberg G, Lundberg A. Neurodevelopment in late infancy after prenatal exposure to benzodiazepines--a prospective study. Neuropediatrics. 1992 Apr;23(2):60-7. doi: 10.1055/s-20081071314. PMID: 1351263. Exclusion Code: $\mathrm{X} 4$.

816. Laegreid L, Olegard R, Conradi N, et al. Congenital malformations and maternal consumption of benzodiazepines: a casecontrol study. Dev Med Child Neurol. 1990 May;32(5):432-41. PMID: 1972364. Exclusion Code: X4. 
817. Laegreid L, Olegard R, Wahlstrom J, et al. Abnormalities in children exposed to benzodiazepines in utero. Lancet. 1987 Jan 10;1(8524):108-9. PMID: 2879165.

Exclusion Code: X7.

818. Laegreid L, Olegard R, Walstrom J, et al. Teratogenic effects of benzodiazepine use during pregnancy. J Pediatr. 1989 Jan;114(1):126-31. PMID: 2562851. Exclusion Code: X7.

819. Laine K, Heikkinen T, Ekblad U, et al. Effects of exposure to selective serotonin reuptake inhibitors during pregnancy on serotonergic symptoms in newborns and cord blood monoamine and prolactin concentrations. Arch Gen Psychiatry. 2003 Jul;60(7):720-6. doi:

10.1001/archpsyc.60.7.720. PMID: 12860776. Exclusion Code: X5.

820. Lajeunie E, Barcik U, Thorne JA, et al. Craniosynostosis and fetal exposure to sodium valproate. J Neurosurg. 2001 Nov;95(5):778-82. doi: 10.3171/jns.2001.95.5.0778. PMID: 11702867. Exclusion Code: X7.

821. Lamberg L. Safety of antidepressant use in pregnant and nursing women. JAMA. 1999 Jul 21;282(3):222-3. PMID: 10422977. Exclusion Code: X1.

822. Lamberg L. Risks and benefits key to psychotropic use during pregnancy and postpartum period. JAMA. 2005 Oct 5;294(13):1604-8. doi: 10.1001/jama.294.13.1604. PMID: 16204654. Exclusion Code: X1.

823. Lambregtse-Van Den Berg M, Burger H, Bockting CL, et al. Stop or go? Tapering antidepressants in pregnancy: a pragmatic multicenter RCT to investigate risk and benefits for mother and child. Archives of women's mental health. 2015;18(2):403-4. doi: 10.1007/s00737-014-0488-6. PMID: CN-01100607. Exclusion Code: X5.

824. Lan TH, Wu BJ, Chiu HJ, et al. Depression status as a confounder in the effects of antidepressants on the gestation age. Am J Psychiatry. 2008 Mar;165(3):399-400; author reply doi:

10.1176/appi.ajp.2007.07081361. PMID: 18316436. Exclusion Code: X1.
825. Lane S. Maternal ADHD and preterm birth: interpret with care. BJOG. 2020 May 8. doi: 10.1111/1471-0528.16311. PMID: 32384217. Exclusion Code: X1.

826. Lanza di Scalea T, Wisner KL. Antidepressant medication use during breastfeeding. Clin Obstet Gynecol. 2009 Sep;52(3):483-97. doi: 10.1097/GRF.0b013e3181b52bd6. PMID: 19661763. Exclusion Code: X7.

827. Larsen ER, Saric K. Pregnancy and bipolar disorder: the risk of recurrence when discontinuing treatment with mood stabilisers: a systematic review. Acta Neuropsychiatr. 2017 Oct;29(5):259-66. doi: 10.1017/neu.2016.60. PMID: 27852343. Exclusion Code: X7.

828. Lassen D, Ennis ZN, Damkier P. Firsttrimester pregnancy exposure to venlafaxine or duloxetine and risk of major congenital malformations: a systematic review. Basic Clin Pharmacol Toxicol. 2016 Jan;118(1):32-6. doi: 10.1111/bcpt.12497. PMID: 26435496. Exclusion Code: X7.

829. Lasser R, Deligiannidis K, Gunduz-Bruce H, et al. P.308 A double-blind, randomized, placebo-controlled phase 3 study of sage217 in postpartum depression: improvements in unidimensional measures of depression and anxiety. Eur Neuropsychopharmacol. 2019;29:S219-S20. doi: 10.1016/j.euroneuro.2019.09.328. Exclusion Code: X9.

830. Latendresse G, Ruiz RJ. Maternal corticotropin-releasing hormone and the use of selective serotonin reuptake inhibitors independently predict the occurrence of preterm birth. J Midwifery Womens Health. 2011 Mar-Apr;56(2):118-26. doi: 10.1111/j.1542-2011.2010.00023.x. PMID: 21429075. Exclusion Code: X2.

831. Latendresse G, Ruiz RJ. Maternal corticotropin-releasing hormone and the use of selective serotonin reuptake inhibitors independently predict the occurrence of preterm birth. J Midwifery Womens Health. 2011;56(2):118-26. doi: 10.1111/j.15422011.2010.00023.x. PMID: 2012-21856006. Exclusion Code: X2. 
832. Lattimore KA, Donn SM, Kaciroti N, et al. Selective serotonin reuptake inhibitor (SSRI) use during pregnancy and effects on the fetus and newborn: a meta-analysis. J Perinatol. 2005 Sep;25(9):595-604. doi: 10.1038/sj.jp.7211352. PMID: 16015372. Exclusion Code: X7.

833. Laugesen K, Olsen M, Andersen ABT, et al. In utero exposure to antidepressant drugs and risk of attention deficit hyperactivity disorder (ADHD): A nationwide danish cohort study. Pharmacoepidemiol Drug Saf. 2013;22:366-7. doi: 10.1002/pds.3512. Exclusion Code: X9.

834. Laugesen K, Olsen MS, Andersen ABT, et al. In utero exposure to antidepressant drugs and risk of attention deficit hyperactivity disorder: A nationwide Danish cohort study. BMJ Open. 2013;3(9). doi:

10.1136/bmjopen-2013-003507. Exclusion Code: X4.

835. Lavenstein B. Neonatal signs after in utero exposure to selective serotonin reuptake inhibitors. JAMA. 2005 Nov 9;294(18):2300; author reply -1. doi: 10.1001/jama.294.18.2300-a. PMID: 16278356. Exclusion Code: X1.

836. Lawrie TA, Herxheimer A, Dalton K. Oestrogens and progestogens for preventing and treating postnatal depression. Cochrane Database Syst Rev. 2000(2):CD001690. doi: 10.1002/14651858.CD001690. PMID: 10796270. Exclusion Code: X7.

837. Leibson T, Yini V, Bozzo P, et al. Adverse effects in infants exposed to second generation antipsychotics during lactation. Birth Defects Research Part A - Clinical and Molecular Teratology. 2015;103(5):463. doi: 10.1002/bdra.23387. Exclusion Code: X9.

838. Lemelin M, Sheehy O, Bérard A. Maternal ADHD medication use during pregnancy and the risk of ADHD in children. Pharmacoepidemiol Drug Saf. 2018;27:165. doi: 10.1002/pds.4629. Exclusion Code: X4.

839. Leppée M, Culig J, Eric M, et al. The effects of benzodiazepines in pregnancy. Acta Neurol Belg. 2010;110(2):163-7. PMID: 2010-19863-003. Exclusion Code: X4.
840. Lesage A, Diallo FB. Prenatal antidepressant use and risk of autism spectrum disorders in children. JAMA Pediatr. 2016 Jul 1;170(7):714. doi: 10.1001/jamapediatrics.2016.0733. PMID: 27243219. Exclusion Code: X1.

841. Letourneau NL, Dennis CL, Cosic N, et al. The effect of perinatal depression treatment for mothers on parenting and child development: A systematic review. Depress Anxiety. 2017;34(10):928-66. doi: 10.1002/da.22687. PMID: 2017-44421-001. Exclusion Code: X7.

842. Leung EY. A report from \#BlueJC: is prozac going to affect my baby's development? BJOG. 2015 May;122(6):886. doi: 10.1111/1471-0528.13414. PMID: 25892473. Exclusion Code: X1.

843. Levinson-Castiel R, Merlob P, Linder N, et al. Neonatal abstinence syndrome after in utero exposure to selective serotonin reuptake inhibitors in term infants. Arch Pediatr Adolesc Med. 2006 Feb;160(2):1736. doi: 10.1001/archpedi.160.2.173. PMID: 16461873. Exclusion Code: X5.

844. Lewis AJ, Bailey C, Galbally M. Antidepressant use during pregnancy in Australia: findings from the Longitudinal Study of Australian Children. Aust N Z J Public Health. 2012 Oct;36(5):487-8. doi: 10.1111/j.1753-6405.2012.00917.x. PMID: 23025373. Exclusion Code: X5.

845. Lewis AJ, Galbally M, Opie G, et al. Neonatal growth outcomes at birth and one month postpartum following in utero exposure to antidepressant medication. Aust N Z J Psychiatry. 2010 May;44(5):482-7. doi: 10.3109/00048670903559593. PMID: 20397792. Exclusion Code: X2.

846. Lewis BA, Schuver K, Gjerdingen D, et al. The relationship between prenatal antidepressant use and the decision to breastfeed among women enrolled in a randomized exercise intervention trial. J Hum Lact. 2016 Aug;32(3):Np67-72. doi: 10.1177/0890334415592153. PMID: 26112472. Exclusion Code: X3. 
847. Lewis C, Robinson-Wolrath S, Kohan R, et al. SSRI/SNRI dose during pregnancy is associated with increased need for resuscitation at birth and special nursery admission. J Paediatr Child Health. 2015;51:25. doi: 10.1111/jpc.12884-2. Exclusion Code: X9.

848. Lewkowitz AK, Rosenbloom JI, Keller M, et al. 123: Is severe intrapartum maternal morbidity associated with acute psychiatric illness within a year of delivery? Am J Obstet Gynecol. 2019;220(1):S97-S8. doi: 10.1016/j.ajog.2018.11.144. Exclusion Code: X3.

849. Li DK, Ferber JR. Treatment of depression during pregnancy and its effect on infant nicu admission. Pharmacoepidemiol Drug Saf. 2012;21:31. doi: 10.1002/pds.3324. Exclusion Code: X9.

850. Li X, Guo JJ, Wigle PR, et al. Drug utilization of pregnancy category $\mathrm{D}$ or $\mathrm{X}$ drugs among child-bearing age women with depression or bipolar disorder in Medicaid. Pharmacoepidemiology and Drug Safety (PDS). 2009;18(S1):S73-S4. doi: 10.1002/pds.1806. Exclusion Code: X5.

851. Li YJ, Li YM. Gestational exposure to selective serotonin reuptake inhibitors and offspring psychiatric disorders: need for further investigation. J Am Acad Child Adolesc Psychiatry. 2016 Aug;55(8):726. doi: 10.1016/j.jaac.2016.04.010. PMID: 27453087. Exclusion Code: X1.

852. Liepman MR, Kothari CL, Tareen RS, et al. Community-wide intervention reduces post partum depression through promotion of perinatal screening and treatment. Arch Womens Ment Health. 2011;14:S34. doi: 10.1007/s00737-010-0203-1. Exclusion Code: X3.

853. Lilliecreutz C, Theodorsson E, Sydsjö G, et al. Salivary cortisol in pregnant women suffering from blood and injection phobia. Arch Womens Ment Health. 2011;14(5):405-11. doi: 10.1007/s00737011-0234-2. PMID: 2011-22324-005. Exclusion Code: X2.
854. Lim K, Sanders A, Brain U, et al. Third trimester fetal pulmonary artery Doppler blood flow velocity characteristics following prenatal selective serotonin reuptake inhibitor (SSRI) exposure. Early Hum Dev. 2012 Aug;88(8):609-15. doi: 10.1016/j.earlhumdev.2012.01.004. PMID: 22305713. Exclusion Code: X2.

855. Lin AE, Peller AJ, Westgate MN, et al. Clonazepam use in pregnancy and the risk of malformations. Birth Defects Res A Clin Mol Teratol. 2004 Aug;70(8):534-6. doi: 10.1002/bdra.20051. PMID: 15329832. Exclusion Code: X4.

856. Lind JN, Reefhuis J, Friedman JM, et al. Maternal use of specific selective serotoninreuptake inhibitors in early pregnancy and the risk of birth defects. Pharmacoepidemiol Drug Saf. 2015;24:243-4. doi: 10.1002/pds.3838. Exclusion Code: X4.

857. Lind JN, Reefhuis J, Friedman JM, et al. First trimester antidepressant use in the national birth defects prevention study. Pharmacoepidemiol Drug Saf. 2016;25:3278. doi: 10.1002/pds.4070. Exclusion Code: X5.

858. Lindensmith R. Interventions to Improve Maternal-Infant Relationships in Mothers With Postpartum Mood Disorders. MCN Am J Matern Child Nurs. 2018 Nov/Dec;43(6):334-40. doi: 10.1097/nmc.0000000000000471. PMID: 30339579. Exclusion Code: X3.

859. Lindqvist PG, Nasiell J, Gustafsson LL, et al. Selective serotonin reuptake inhibitor use during pregnancy increases the risk of postpartum hemorrhage and anemia: a hospital-based cohort study. J Thromb Haemost. 2014 Dec;12(12):1986-92. doi: 10.1111/jth.12757. PMID: 25322909. Exclusion Code: X4.

860. Link NA, Temple-Cooper ME. Prenatal antidepressant use and risk of autism spectrum disorders in children. JAMA Pediatr. 2016 Jul 1;170(7):713-4. doi: 10.1001/jamapediatrics.2016.0736. PMID: 27243468. Exclusion Code: X1.

861. Lint D. Antidepressants and spontaneous abortion. CMAJ. 2011 Aug 9;183(11):1283. doi: 10.1503/cmaj.111-2056. PMID: 21825057. Exclusion Code: X1. 
862. Liu C, Cnattingius S, Bergstrom M, et al. Prenatal parental depression and preterm birth: a national cohort study. BJOG. 2016 Nov;123(12):1973-82. doi: 10.1111/14710528.13891. PMID: 26786413. Exclusion Code: X2.

863. Liu X, Musliner KL, Agerbo E, et al. Treatment indications for antidepressants prescribed to pregnant women: A population-based descriptive study from Denmark. Pharmacoepidemiol Drug Saf. 2020;29(3):347-51. doi: 10.1002/pds.4953. Exclusion Code: X5.

864. Liu X, Plana-Ripoll O, Ingstrup KG, et al. Postpartum psychiatric disorders and subsequent live birth: a population-based cohort study in Denmark. Hum Reprod. 2020 Apr 28;35(4):958-67. doi:

10.1093/humrep/deaa016. PMID: 32227097. Exclusion Code: X4.

865. Liu YH, Wang L, Huang YG. Cognitive function after fetal exposure to antiepileptic drugs. N Engl J Med. 2009 Aug 6;361(6):629; author reply -30. doi: 10.1056/NEJMc091029. PMID: 19657130. Exclusion Code: X1.

866. Loebstein R, Koren G. Pregnancy outcome and neurodevelopment of children exposed in utero to psychoactive drugs: the Motherisk experience. J Psychiatry Neurosci. 1997 May;22(3):192-6. PMID: 9183118. Exclusion Code: X1.

867. Logsdon MC, Wisner K, Hanusa BH. Does maternal role functioning improve with antidepressant treatment in women with postpartum depression? J Womens Health (Larchmt). 2009 Jan-Feb;18(1):85-90. doi: 10.1089/jwh.2007.0635. PMID: 19132881. Exclusion Code: X4.

868. Logsdon MC, Wisner K, Hanusa BH, et al. Role functioning and symptom remission in women with postpartum depression after antidepressant treatment. Arch Psychiatr Nurs. 2003 Dec;17(6):276-83. PMID: 14685952. Exclusion Code: X7.

869. Logsdon MC, Wisner K, Sit D, et al. Depression treatment and maternal functioning. Depress Anxiety. 2011 Nov;28(11):1020-6. doi: 10.1002/da.20892. PMID: 21898714. Exclusion Code: X4.
870. Longhurst JG, Weiss E. Use of psychotropic medications during lactation. Am J Psychiatry. 1998 Nov;155(11):1643-4. PMID: 9812155. Exclusion Code: X1.

871. Loomans EM, van der Stelt O, van Eijsden $\mathrm{M}$, et al. Antenatal maternal anxiety is associated with problem behaviour at age five. Early Hum Dev. 2011;87(8):565-70. doi: 10.1016/j.earlhumdev.2011.04.014. PMID: 2011-16156-011. Exclusion Code: $\mathrm{X} 3$.

872. Lopez-Yarto M, Ruiz-Mirazo E, Holloway AC, et al. Do psychiatric medications, especially antidepressants, adversely impact maternal metabolic outcomes? J Affect Disord. 2012 Dec 10;141(2-3):120-9. doi: 10.1016/j.jad.2012.01.043. PMID:

22370064. Exclusion Code: X7.

873. Lorenz K, Gannon M, Ramage M, et al. 321: Psychotropic medications do not appear to increase NAS risk in women on MAT. Am J Obstet Gynecol. 2019;220(1):S224-S5. doi: 10.1016/j.ajog.2018.11.342. Exclusion Code: X2.

874. Lorenzo L, Byers B, Einarson A. Antidepressant use in pregnancy. Expert Opin Drug Saf. 2011 Nov;10(6):883-9. doi: 10.1517/14740338.2011.583917. PMID: 21545242. Exclusion Code: X1.

875. Lorenzo L, Einarson A. Antidepressant use in pregnancy: an evaluation of adverse outcomes excluding malformation. Isr J Psychiatry Relat Sci. 2014;51(2):94-104. PMID: 25372558. Exclusion Code: X7.

876. Lorenzo LS, Einarson A, Einarson TR. Contribution of Latin America to mental health in pregnancy. Value Health. 2013;16(7):A695-A6. doi: 10.1016/j.jval.2013.08.2092. Exclusion Code: X1.

877. Loring DW, Meador KJ, Thompson WO. Neurodevelopment after in utero exposure to phenytoin and carbamazepine. JAMA. 1994 Sep 21;272(11):850-1. PMID: 7521402. Exclusion Code: X1.

878. Loudin S, Murray S, Prunty L, et al. An atypical withdrawal syndrome in neonates prenatally exposed to gabapentin and opioids. J Pediatr. 2017 Feb;181:286-8. doi: 10.1016/j.jpeds.2016.11.004. PMID: 27889067. Exclusion Code: X2. 
879. Louik C, Kerr S, Mitchell AA. First trimester exposure to bupropion and cardiac defects. Pharmacoepidemiol Drug Saf. 2013;22:157-8. doi: 10.1002/pds.3512. Exclusion Code: X9.

880. Louik C, Kerr S, Mitchell AA. Firsttrimester exposure to bupropion and risk of cardiac malformations. Pharmacoepidemiol Drug Saf. 2014 Oct;23(10):1066-75. doi: 10.1002/pds.3661. PMID: 24920293. Exclusion Code: X4.

881. Louik C, Lin AE, Werler MM, et al. Firsttrimester use of selective serotonin-reuptake inhibitors and the risk of birth defects. $\mathrm{N}$ Engl J Med. 2007 Jun 28;356(26):2675-83. doi: 10.1056/NEJMoa067407. PMID: 17596601. Exclusion Code: X2.

882. Lugoboni F, Zadra N, Urli N, et al. Use of benzodiazepines during pregnancy. A survey in a cohort of pregnant women in northern Italy. Acta Neurol Belg. 2011 Jun;111(2):172-3. PMID: 21748948. Exclusion Code: X1.

883. Lugo-Candelas C, Cha J, Hong S, et al. Altered brain structure and connectivity in infants exposed to serotonin reuptake inhibitors during pregnancy.

Neuropsychopharmacology. 2017;43:S508. doi: 10.1038/npp.2017.266. Exclusion Code: X5.

884. Lugo-Candelas C, Cha J, Hong S, et al. Associations between brain structure and connectivity in infants and exposure to selective serotonin reuptake inhibitors during pregnancy. JAMA Pediatr. 2018 Jun 1;172(6):525-33. doi: 10.1001/jamapediatrics.2017.5227. PMID: 29630692. Exclusion Code: X5.

885. Lund N, Pedersen LH, Henriksen TB. Selective serotonin reuptake inhibitor exposure in utero and pregnancy outcomes. Arch Pediatr Adolesc Med. 2009 Oct;163(10):949-54. doi: 10.1001/archpediatrics.2009.164. PMID: 19805715. Exclusion Code: X12.

886. Lupattelli A, Chambers CD, Bandoli G, et al. Maternal use of benzodiazepines and zhypnotics during pregnancy and developmental outcomes in offspring at 5 years of age. Pharmacoepidemiol Drug Saf. 2018;27:173. doi: 10.1002/pds.4629.

Exclusion Code: X4.
887. Lupattelli A, Mahic M, Handal M, et al. Risk of attention deficit hyperactivity disorder in childhood after exposure to serotonergic antidepressants in pregnancy. Pharmacoepidemiol Drug Saf. 2019;28:3701. doi: 10.1002/pds.4864. Exclusion Code: X9.

888. Lupattelli A, Mahic M, Handal M, et al. Maternal use of serotonergic antidepressants during pregnancy and risk of ADHD diagnosis and symptoms in offspring. Birth Defects Research. 2019;111(9):555. doi: 10.1002/bdr2.v111.9. Exclusion Code: X9.

889. Lupattelli A, Spigset O, Björnsdóttir I, et al. Patterns and factors associated with low adherence to psychotropic medications during pregnancy — a cross-sectional, multinational web-based study. Depress Anxiety. 2015;32(6):426-36. doi: 10.1002/da.22352. PMID: 2015-08249-001. Exclusion Code: X5.

890. Lupattelli A, Twigg MJ, Zagorodnikova K, et al. Self-reported postnatal depressive symptom severity after treatment with antidepressants in pregnancy:A crosssectional study in 12 European countries using the Edinburgh Postnatal Depression Scale. Pharmacoepidemiol Drug Saf. 2018;27:173-4. doi: 10.1002/pds.4629. Exclusion Code: X4.

891. Lupattelli A, Wood M, Spigset O, et al. Gestational exposure to SSRIs and risk of preeclampsia in pregnancy: A study from the norwegian mother and child cohort study accounting for time-varying severity of depressive and anxiety symptoms. Pharmacoepidemiol Drug Saf. 2016;25:235. doi: 10.1002/pds.4070. Exclusion Code: X9.

892. Lupattelli A, Ystrom E, Wood M, et al. Effect of time-dependent SSRI exposure during pregnancy on child's neurobehavioral development by age 5 . Pharmacoepidemiol Drug Saf. 2017;26:470-1. doi: 10.1002/pds.4275. Exclusion Code: X4.

893. Lynch MM, Amoozegar JB, McClure EM, et al. Improving safe use of medications during pregnancy: The roles of patients, physicians, and pharmacists. Qual Health Res. 2017;27(13):2071-80. doi: 10.1177/1049732317732027. PMID: 201749815-015. Exclusion Code: X7. 
894. Maayan-Metzger A, Kuint J, Lubetsky A, et al. Maternal selective serotonin reuptake inhibitor intake does not seem to affect neonatal platelet function tests. Acta Haematol. 2006;115(3-4):157-61. doi: 10.1159/000090929. PMID: 16549890. Exclusion Code: X4.

895. MacBeth A, McSkimming P, Bhattacharya $\mathrm{S}$, et al. General fertility rate and perinatal outcomes in women with schizophrenia. Eur Arch Psychiatry Clin Neurosci. 2017;267(1):S41-S2. doi: 10.1007/s00406017-0824-8. Exclusion Code: X3.

896. Magalhaes PV, Pinheiro RT. Pharmacological treatment of postpartum depression. Acta Psychiatr Scand. 2006 Jan;113(1):75-6; author reply 6-7. doi: 10.1111/j.1600-0447.2005.00690.x. PMID: 16390378. Exclusion Code: X1.

897. Maimburg RD, Vaeth M. Perinatal risk factors and infantile autism. Acta Psychiatr Scand. 2006 Oct;114(4):257-64. doi: 10.1111/j.1600-0447.2006.00805.x. PMID: 16968363. Exclusion Code: X2.

898. Maina G, Rosso G. Perinatal obsessivecompulsive disorder. Psychother Psychosom. 2019;88:79. doi: 10.1159/000502467. Exclusion Code: X3.

899. Makatsariya N, Bitsadze V, Panfilova O, et al. Maternal and paternal hypergomocysteinemia and prophylaxis of recurrent antenatal fetal death. J Perinat Med. 2013;41. doi: 10.1515/jpm-2013-2003. Exclusion Code: X2.

900. Makikyro T, Leinonen E, Koponen H, et al. Early developmental differences between DSM-III-R schizophrenics treated with clozapine and typical neuroleptics. J Psychiatr Res. 1998 Mar-Apr;32(2):105-10. doi: 10.1016/s0022-3956(98)00056-9. PMID: 9694006. Exclusion Code: X2.

901. Malek-Ahmadi P. The use of serotonin reuptake inhibitors in pregnancy. Can J Psychiatry. 2000 Dec;45(10):939-40. PMID: 11190370. Exclusion Code: X1.

902. Malm H. Prenatal exposure to selective serotonin reuptake inhibitors and infant outcome. Ther Drug Monit. 2012

Dec;34(6):607-14. doi: 10.1097/FTD.0b013e31826d07ea. PMID: 23042258. Exclusion Code: X1.
903. Malm H, Artama M, Brown AS, et al. Infant and childhood neurodevelopmental outcomes following prenatal exposure to selective serotonin reuptake inhibitors (SSRIS). Pharmacoepidemiol Drug Saf. 2012;21:141-2. doi: 10.1002/pds.3324. Exclusion Code: X7.

904. Malm H, Artama M, Brown AS, et al. Infant and childhood neurodevelopmental outcomes following prenatal exposure to selective serotonin reuptake inhibitors: overview and design of a Finnish RegisterBased Study (FinESSI). BMC Psychiatry. 2012 Dec 4;12:217. doi: 10.1186/1471244x-12-217. PMID: 23206294. Exclusion Code: X5.

905. Malm H, Klaukka T, Neuvonen PJ. Risks associated with selective serotonin reuptake inhibitors in pregnancy. Obstet Gynecol. 2005 Dec;106(6):1289-96. doi: 10.1097/01.aog.0000187302.61812.53. PMID: 16319254. Exclusion Code: X5.

906. Malone K, Papagni K, Ramini S, et al. Antidepressants, antipsychotics, benzodiazepines, and the breastfeeding dyad. Perspect Psychiatr Care. 2004 AprJun;40(2):73-85. PMID: 15323416. Exclusion Code: X1.

907. Malt U. Lithium treatment during pregnancy and the postpartum period. J Psychosom Res. 2011;70(6):605. doi: 10.1016/j.jpsychores.2011.03.006. Exclusion Code: X7.

908. Man KK, Tong HH, Wong LY, et al. Exposure to selective serotonin reuptake inhibitors during pregnancy and risk of autism spectrum disorder in children: a systematic review and meta-analysis of observational studies. Neurosci Biobehav Rev. 2015 Feb;49:82-9. doi: 10.1016/j.neubiorev.2014.11.020. PMID: 25498856. Exclusion Code: X7.

909. Man KKC, Chan EW, Coghill DR, et al. Prenatal antidepressant exposure and the risk of autism spectrum disorder and attention-deficit hyperactivity disorder. Pharmacoepidemiol Drug Saf. 2015;24:234. doi: 10.1002/pds.3838. Exclusion Code: X9. 
910. Man KKC, Chan EW, Ip P, et al. Prenatal antidepressant exposure and the risk of attention-deficit hyperactivity disorder in children: A systematic review and metaanalysis. Neurosci Biobehav Rev.

2018;86:1-11. doi:

10.1016/j.neubiorev.2017.12.007. Exclusion Code: X7.

911. Manakova E, Hubickova L. Antidepressant drug exposure during pregnancy. CZTIS small prospective study. Neuro Endocrinol Lett. 2011;32 Suppl 1:53-6. PMID: 22167208. Exclusion Code: X4.

912. Marc I, Toureche N, Ernst E, et al. Mindbody interventions during pregnancy for preventing or treating women's anxiety. Cochrane Database Syst Rev. 2011 Jul 6(7):Cd007559. doi: 10.1002/14651858.CD007559.pub2. PMID: 21735413. Exclusion Code: X7.

913. Marchesi C, Ossola P, Amerio A, et al. Clinical management of perinatal anxiety disorders: a systematic review. J Affect Disord. 2016 Jan 15;190:543-50. doi: 10.1016/j.jad.2015.11.004. PMID: 26571104. Exclusion Code: X7.

914. Marcon C, Géniaux H, Coubret A, et al. Child psychomotor development after in utero exposure to an antiepileptic drug monotherapy. Fundam Clin Pharmacol. 2015;29:4. Exclusion Code: X2.

915. Marcus SM, Flynn HA. Depression, antidepressant medication, and functioning outcomes among pregnant women. Int J Gynaecol Obstet. 2008 Mar;100(3):248-51. doi: 10.1016/j.ijgo.2007.09.016. PMID: 18005968. Exclusion Code: X5.

916. Margulies AI, Berris B. Jaundice associated with the administration of trifluoperazine. Can Med Assoc J. 1968 Jun 1;98(22):10634. PMID: 5652451. Exclusion Code: X2.

917. Markovitz PJ, Calabrese JR. Use of anticonvulsants for manic depression during pregnancy. Psychosomatics. 1990 Winter;31(1):118. doi: 10.1016/s00333182(90)72237-2. PMID: 2405447. Exclusion Code: X1.

918. Martins V, Garrido P, Pimenta S, et al. Obstetrical and neonatal outcomes after prenatal exposure to psychiatric medicationexperience of the early intervention unit. Eur Psychiatry. 2013;28. Exclusion Code: X4.
919. Martins V, Murta I, Moinho R, et al. Experience of the early intervention unit of Bissaya Barreto maternity-review of six years retrospective follow up. Psychother Psychosom. 2013;82:62. doi: 10.1159/000354142. Exclusion Code: X4.

920. Martins V, Pimenta S, Murta I, et al. Neonatal outcomes after prenatal exposure to psychotropic medication-a case control study. Psychother Psychosom. 2013;82:62. doi: 10.1159/000354142. Exclusion Code: $\mathrm{X} 4$.

921. Martins V, Silva R, Mendes E, et al. Neonatal outcomes after prenatal exposure to psychotropic drugs for sleep disorders-a case-control study. Eur

Neuropsychopharmacol. 2014;24:S748. Exclusion Code: X4.

922. Masarwa R, Bar-Oz B, Gorelik E, et al. Prenatal exposure to SSRIs and SNRIs and risk for persistent pulmonary hypertension of the newborn: a systematic review, metaanalysis and network meta-analysis. Am J Obstet Gynecol. 2018 Aug 28. doi: 10.1016/j.ajog.2018.08.030. PMID: 30170040. Exclusion Code: X7.

923. Masarwa R, Bar-Oz B, Gorelik E, et al. Prenatal exposure to selective serotonin reuptake inhibitors and serotonin norepinephrine reuptake inhibitors and risk for persistent pulmonary hypertension of the newborn: a systematic review, metaanalysis, and network meta-analysis. Am J Obstet Gynecol. 2018. doi: 10.1016/j.ajog.2018.08.030. Exclusion Code: X7.

924. Maschi S, Clavenna A, Campi R, et al. Neonatal outcome following pregnancy exposure to antidepressants: a prospective controlled cohort study. BJOG. 2008 Jan;115(2):283-9. doi: 10.1111/j.14710528.2007.01518.x. PMID: 17903222. Exclusion Code: X5.

925. Masud Iqbal M. The effects of lithium on fetuses, neonates, and nursing infants. Psychiatric Annals. 2000;30(3):159-64. doi: 10.3928/0048-5713-20000301-03. PMID: 2000-07408-001. Exclusion Code: X1. 
926. Matteo B, Miriam I, Giulia B, et al. 'Determinants of ante-partum depression: A multicenter study': Erratum. Social Psychiatry and Psychiatric Epidemiology: The International Journal for Research in Social and Genetic Epidemiology and Mental Health Services. 2013;48(3):513-. doi: 10.1007/s00127-012-0557-y. PMID: 2013-04950-017. Exclusion Code: X1.

927. Mattera J, Miller-Loncar C, Anderson GM, et al. Circadian neurohormone excretion and objective sleep measures in toddlers prenatally exposed to maternal depression and antidepressant medication. Sleep. Conference: 31 st anniversary meeting of the associated professional sleep societies, LLC, SLEEP 2017. United states. 2017;40:A20. PMID: CN-01375422. Exclusion Code: X5.

928. Mattera JA, Stone KC, Salisbury AL. Longitudinal and multimodal sleep assessment in children of mothers with depression during pregnancy: Prenatal antidepressant exposure associated with decreased sleep duration at 18-months and earlier sleep schedules at 18- and 36months. Sleep. 2019;42:A313. doi: 10.1093/sleep/zsz067.778. Exclusion Code: X5.

929. Mattson SN, Calarco KE, Chambers CD, et al. Interaction of maternal smoking and other in-pregnancy exposures: analytic considerations. Neurotoxicol Teratol. 2002 May-Jun;24(3):359-67. doi: 10.1016/s08920362(02)00198-8. PMID: 12009491. Exclusion Code: X2.

930. Mazaira S. [Effects of psychiatric drugs on the fetus and newborn children. Consequences of the treatment of psychiatric disorders during pregnancy and lactation]. Vertex. 2005 Jan-Feb;16(59):3542. PMID: 15785787 . Exclusion Code: X8.

931. Mazer-Poline C, Rifkin A, Geisler S, et al. Mood stabilizer discontinuation in pregnant women with bipolar disorder. Am J Psychiatry. 2008 May;165(5):646-7; author reply 7-8. doi:

10.1176/appi.ajp.2008.08010036. PMID: 18450941. Exclusion Code: X1.
932. McCauley C-O, Casson K. A qualitative study into how guidelines facilitate general practitioners to empower women to make decisions regarding antidepressant use in pregnancy. International Journal of Mental Health Promotion. 2013;15(1):3-28. doi: 10.1080/14623730.2013.781872. PMID: 2013-33694-002. Exclusion Code: X7.

933. McCauley-Elsom K, Gurvich C, Elsom SJ, et al. Antipsychotics in pregnancy. $\mathrm{J}$ Psychiatr Ment Health Nurs. 2010 Mar;17(2):97-104. doi: 10.1111/j.13652850.2009.01481.x. PMID: 20465753. Exclusion Code: X7.

934. McClendon J. Reexamining paroxetine and cognitive-behavioral therapy in postpartum depression and anxiety. J Clin Psychiatry. 2005 Nov;66(11):1494-5; author reply 5. PMID: 16420094. Exclusion Code: X1.

935. McClure CK, Katz KD, Patrick TE, et al. The epidemiology of acute poisonings in women of reproductive age and during pregnancy, California, 2000-2004. Matern Child Health J. 2011;15(7):964-73. doi: 10.1007/s10995-010-0571-1. PMID: 201120756-017. Exclusion Code: X2.

936. McConnell PJ, Linn K, Filkins K. Depression and pregnancy: Use of selective serotonin reuptake inhibitors in pregnancy. Prim Care Update Ob Gyns. 1998;5(1):11-5. doi: 10.1016/S1068-607X(97)00116-9. Exclusion Code: X4.

937. McDonagh M, Matthews A, Phillipi C, et al. Antidepressant treatment of depression during pregnancy and the postpartum period. Evid Rep Technol Assess (Full Rep). 2014 Jul(216):1-308. doi: 10.23970/ahrqepcerta216. PMID: 30313002. Exclusion Code: X7.

938. McDonagh MS, Matthews A, Phillipi C, et al. Depression drug treatment outcomes in pregnancy and the postpartum period: a systematic review and meta-analysis. Obstet Gynecol. 2014 Sep;124(3):526-34. doi: 10.1097/aog.0000000000000410. PMID: 25004304. Exclusion Code: X7. 
939. McDonald L, Jones I. Erratum: Depression and SSRI use in pregnancy associated with traits of autism in children (BMJ (Online) (2014) 349 (g4835) DOI:

10.1136/bmj.g4835). BMJ (Online).

2014;349. doi: 10.1136/bmj.g4989.

Exclusion Code: X1.

940. McDowell ML, Tonismae TR, James SE, et al. 346: The impact of breastfeeding on neonatal abstinence syndrome in buprenorhpine exposed neonates. Am J Obstet Gynecol. 2019;220(1):S241. doi: 10.1016/j.ajog.2018.11.367. Exclusion Code: X4.

941. McElhatton PR. The use of phenothiazines during pregnancy and lactation. Reprod Toxicol. 1992;6(6):475-90. PMID: 1288758. Exclusion Code: X1.

942. McEvoy KM, Rayapati D, Washington Cole KO, et al. Poor Postpartum Sleep Quality Predicts Subsequent Postpartum Depressive Symptoms in a High-Risk Sample. J Clin Sleep Med. 2019 Sep 15;15(9):1303-10. doi: 10.5664/jcsm.7924. PMID: 31538601. Exclusion Code: X3.

943. McGowen KR, Miller MN. Depression treatment during pregnancy. Am J Psychiatry. 2004 Nov;161(11):2137-8. doi: 10.1176/appi.ajp.161.11.2137-a. PMID: 15514429. Exclusion Code: X1.

944. McGregor M, Coghlan M, Dennis CL. The effect of physician-based cognitive behavioural therapy among pregnant women with depressive symptomatology: a pilot quasi-experimental trial. Early Interv Psychiatry. 2014 Nov;8(4):348-57. doi: 10.1111/eip.12074. PMID: 23855406. Exclusion Code: X3.

945. McKean M. Psychiatric Care During Pregnancy and Postpartum. Psychopharm Review. 2013;48(3):17-23. doi: 10.1097/01.PSYPHR.0000428766.86387.f4. Exclusion Code: X1.

946. McKenna K, Koren G, Tetelbaum M, et al. Pregnancy outcome of women using atypical antipsychotic drugs: a prospective comparative study. J Clin Psychiatry. 2005 Apr;66(4):444-9; quiz 546. PMID: 15816786. Exclusion Code: X5.
947. McKnight RF, Adida M, Budge K, et al. Lithium toxicity profile: a systematic review and meta-analysis. Lancet. 2012 Feb 25;379(9817):721-8. doi: 10.1016/s01406736(11)61516-x. PMID: 22265699. Exclusion Code: X7.

948. McMahon CA, Boivin J, Gibson FL, et al. Pregnancy-specific anxiety, ART conception and infant temperament at 4 months postpartum. Hum Reprod. 2013 Apr;28(4):9971005. doi: 10.1093/humrep/det029. PMID: 23427229. Exclusion Code: X2.

949. McNeil TF, Cantor-Graae E. Minor physical anomalies and obstetric complications in schizophrenia. Aust N Z J Psychiatry. 2000;34(Suppl):S65-S73. doi: 10.1046/j.1440-1614.2000.00784.x. PMID: 2000-12630-010. Exclusion Code: X1.

950. McNicholas F. Eating psychopathology and its effect on pregnancy, infant growth and development. Ir J Psychol Med.

1996;13(2):67-9. doi: 10.1017/S0790966700002469. PMID: 199604907-007. Exclusion Code: X2.

951. Meador KJ. Breastfeeding and antiepileptic drugs. JAMA. 2014 May 7;311(17):1797-8. doi: 10.1001/jama.2014.967. PMID: 24794373. Exclusion Code: X1.

952. Meador KJ. Fetal Valproate Exposure and Attention-Deficit/Hyperactivity Disorder.

JAMA Netw Open. 2019 Jan 4;2(1):e186603. doi: 10.1001/jamanetworkopen.2018.6603. PMID: 30646187. Exclusion Code: X1.

953. Meador KJ, Baker GA, Browning N, et al. Cognitive function at 3 years of age after fetal exposure to antiepileptic drugs. N Engl J Med. 2009 Apr 16;360(16):1597-605. doi: 10.1056/NEJMoa0803531. PMID: 19369666. Exclusion Code: X2.

954. Meador KJ, Baker GA, Browning N, et al. Cognitive function at three years of age after fetal exposure to antiepileptic drugs. Obstet Gynecol Surv. 2009;64(8):517-8. doi: 10.1097/01.ogx.0000356745.41545.57. Exclusion Code: X2. 
955. Meador KJ, Baker GA, Browning N, et al. Breastfeeding in children of women taking antiepileptic drugs: cognitive outcomes at age 6 years. JAMA Pediatr. 2014

Aug;168(8):729-36. doi:

10.1001/jamapediatrics.2014.118. PMID: 24934501. Exclusion Code: X2.

956. Meador KJ, Loring DW. Prenatal valproate exposure is associated with autism spectrum disorder and childhood autism. J Pediatr. 2013 Sep;163(3):924. doi: 10.1016/j.jpeds.2013.06.050. PMID: 23973243. Exclusion Code: X1.

957. Medveczky E, Puho E, Czeizel EA. The use of drugs in mothers of offspring with neuraltube defects. Pharmacoepidemiol Drug Saf. 2004 Jul;13(7):443-55. doi:

10.1002/pds.900. PMID: 15269928. Exclusion Code: X4.

958. Mehta R, Bhandari R, Kuhad A, et al. GABAA receptor positive allosteric modulator Treatment of major depressive disorder Treatment of postpartum depression. Drugs of the Future. 2019;44(6):443-51. doi: 10.1358/dof.2019.44.6.2958473. Exclusion Code: X11.

959. Mehta TM, Van Lieshout RJ. A review of the safety of clozapine during pregnancy and lactation. Arch Womens Ment Health. 2017 Feb;20(1):1-9. doi: 10.1007/s00737-0160670-0. PMID: 27704220. Exclusion Code: $\mathrm{X} 7$.

960. Meltzer HY. Long-term effects of neuroleptic drugs on the neuroendocrine system. Adv Biochem Psychopharmacol. 1985;40:59-68. PMID: 2862768. Exclusion Code: X1.

961. Meltzer-Brody S, Bankole K, Riesenberg R, et al. Time to antidepressant response in double-blind, randomized, placebocontrolled trials of brexanolone injection in postpartum depression.

Neuropsychopharmacology. 2019;44:305-6. doi: 10.1038/s41386-019-0546-x. Exclusion Code: X9.

962. Meltzer-Brody S, Brandon A, Bullard E, et al. The new UNC perinatal psychiatry inpatient unit (USA): Report on the first year. Arch Womens Ment Health. 2013;16:S97. doi: 10.1007/s00737-0130355-x. Exclusion Code: X5.
963. Meltzer-Brody S, Colquhoun H, Doherty J, et al. Brexanolone injection for post-partum depression treatment - Authors' reply. Lancet. 2019 Aug 3;394(10196):380. doi: 10.1016/s0140-6736(19)30703-2. PMID: 31255299. Exclusion Code: X1.

964. Meltzer-Brody S, Colquhoun H, Riesenberg $\mathrm{R}$, et al. Brexanolone iv, a GABA-A receptor modulator, in postpartum depression: Pooled analysis of HAM-D subitems. Eur Neuropsychopharmacol. 2019;29:S63. doi: 10.1016/j.euroneuro.2018.11.1040. Exclusion Code: X9.

965. Meltzer-Brody S, Colquhoun H, Riesenberg R, et al. P.309 Double-blind, randomized, placebo-controlled trials of brexanolone injection in postpartum depression: analysis of ham-d depression subscales and individual symptom items. Eur Neuropsychopharmacol. 2019;29:S220-S1. doi: 10.1016/j.euroneuro.2019.09.329. Exclusion Code: X9.

966. Meltzer-Brody S, Colquhoun H, Riesenberg $\mathrm{R}$, et al. Efficacy and safety of Brexanolone injection, a GABA-A receptor modulator, in women with postpartum depression: Integrated analysesof clinical data. Arch Womens Ment Health. 2019;22(5):701-2. doi: 10.1007/s00737-019-00996-y. Exclusion Code: X9.

967. Meltzer-Brody S, Colquhoun H, Riesenberg $\mathrm{R}$, et al. Brexanolone Injection in Postpartum Depression: Two Multicenter, Double-Blind, Randomized, PlaceboControlled, Phase 3 Trials. Obstet Gynecol Surv. 2019;74(4):219-20. doi: 10.1097/01.ogx.0000554376.54568.64. Exclusion Code: X9.

968. Meltzer-Brody S, Colquhoun H, Riesenberg $\mathrm{R}$, et al. Phase 2 and 3 evaluation of brexanolone, a GABA a receptor positive allosteric modulator, in postpartum depression. Neuropsychopharmacology. Conference: 56th annual meeting of the american college of neuropsychopharmacology, ACNP 2017. United states. 2017;43(Supplement 1):S549. doi: 10.1038/npp.2017.266. PMID: CN01439224. Exclusion Code: X9. 
969. Meltzer-Brody S, Colquhoun H, Riesenberg $R$, et al. Efficacy of Brexanolone Injection in Subjects With Postpartum Depression With and Without Baseline Antidepressant Therapy: Insights From an Integrated Analysis of Three Pivotal Trials. Biol Psychiatry. 2019;85(10):S241. doi: 10.1016/j.biopsych.2019.03.610. Exclusion Code: X9.

970. Meltzer-Brody S, Deligiannidis KM, Colquhoun $\mathrm{H}$, et al. Brexanolone injection for postpartum depression. Current Psychiatry. 2019;18(9):43-8. Exclusion Code: X7.

971. Meltzer-Brody S, Kanes S, Riesenberg R, et al. Efficacy and safety of brexanolone IV across phase $2 / 3$ studies: a first-in-class GABAA receptor positive allosteric modulator for postpartum depression. Biol Psychiatry. 2018 May 1;83(9):S385-S6. doi: DOI 10.1016/j.biopsych.2018.02.991. PMID: WOS:000433001900386. Exclusion Code: X9.

972. Meltzer-Brody S, Kanes S, Riesenberg R, et al. Phase 3 study evaluating brexanolone, a gabaa receptor modulator, in severe postpartum depression. Obstet Gynecol. 2018 May;Conference: 66th Annual Clinical and Scientific Meeting of the American College of Obstetricians and Gynecologists. United States. 131(Supplement 1):27S. doi: 10.1097/01.AOG.0000532931.73344.b7. PMID: CN-01616736. Exclusion Code: X9.

973. Meltzer-Brody S, Kanes S, Riesenberg R, et al. Phase 3 study evaluating brexanolone, a gabaa receptor modulator, in severe postpartum depression. Obstet Gynecol. 2018;131:27S. Exclusion Code: X9.

974. Meltzer-Brody S, Riesenberg R, Epperson $\mathrm{CN}$, et al. Reduction in Depressive Symptoms Over the First 24 Hours in Patients With PPD Treated With the Neuroactive Steroid Brexanolone Injection. Biol Psychiatry. 2020;87(9):S329. doi: 10.1016/j.biopsych.2020.02.845. Exclusion Code: X9.
975. Merikukka M, Räsänen S, Hakko H, et al. Parental hospital-treated somatic illnesses during offspring's childhood associated with later offspring use of psychotropic medication during childhood to young adult — The 1987 Finnish Birth Cohort study. Prev Med. 2018;111:254-64. doi: 10.1016/j.ypmed.2018.02.020. Exclusion Code: X3.

976. Merlo GB, Elliot L, Campbell S, et al. Critical appraisal of systematic reviews assessing safety outcomes of SSRIs in the perinatal period. Value Health. 2010;13(7):A539. Exclusion Code: X7.

977. Merlob P, Stahl B, Sulkes J. Paroxetine during breast-feeding: infant weight gain and maternal adherence to counsel. Eur J Pediatr. 2004 Mar;163(3):135-9. doi: 10.1007/s00431-003-1391-8. PMID: 14745552. Exclusion Code: X4.

978. Meyer MR, Yeaton-Massey A, Cheng YW, et al. History of depression and pregnancy: Are there differences in perinatal outcomes. Reprod Sci. 2011;18(3):181A. doi: 10.1177/193371912011183s067. Exclusion Code: X3.

979. Mezzacappa A, Lasica PA, Gianfagna F, et al. Risk for autism spectrum disorders according to period of prenatal antidepressant exposure: a systematic review and meta-analysis. JAMA Pediatr. 2017 Jun 1;171(6):555-63. doi: 10.1001/jamapediatrics.2017.0124. PMID: 28418571. Exclusion Code: X7.

980. Michels R. Remission of maternal depression and children's psychopathology. JAMA. 2006 Sep 13;296(10):1232; author reply 3-4. doi: 10.1001/jama.296.10.1232-a. PMID: 16968845. Exclusion Code: X1.

981. Milgrom J, Holt CJ, Gemmill AW, et al. Treating postnatal depressive symptoms in primary care: a randomised controlled trial of GP management, with and without adjunctive counselling. BMC Psychiatry. 2011;11:95. doi: 10.1186/1471-244X-11-95. PMID: CN-00796876. Exclusion Code: X3. 
982. Millard SJ, Weston-Green K, Newell KA. The effects of maternal antidepressant use on offspring behaviour and brain development: Implications for risk of neurodevelopmental disorders. Neurosci Biobehav Rev. 2017 Sep;80:743-65. doi: 10.1016/j.neubiorev.2017.06.008. PMID: 28629713. Exclusion Code: X7.

983. Miller ES, Grobman WA, Culhane J, et al. Antenatal depression, psychotropic medication use, and inflammation among pregnant women. Arch Womens Ment Health. 2018 Dec;21(6):785-90. doi: 10.1007/s00737-018-0855-9. PMID: 29862416. Exclusion Code: X5.

984. Miller ES, Saade GR, Simhan HN, et al. 87: Trajectories of antenatal depression and adverse pregnancy outcomes. Am J Obstet Gynecol. 2019;220(1):S70. doi: 10.1016/j.ajog.2018.11.096. Exclusion Code: X3.

985. Miller LJ. Pharmacotherapy during the perinatal period. The Hatherleigh guide to psychopharmacology. New York, NY: Hatherleigh Press; 1999:251-78. Exclusion Code: X1.

986. Minick G, Atlas M. What's the best strategy for bipolar disorder during pregnancy? The Journal of Family Practice. 2007;56(8):6658. PMID: 2007-12879-001. Exclusion Code: $\mathrm{X} 1$.

987. Mintzes B, Abi-Jaoude E, Ford AR. Antidepressants and pregnancy. CMAJ. 2011 Mar 22;183(5):585; author reply doi: 10.1503/cmaj.111-2027. PMID: 21422144. Exclusion Code: X1.

988. Mintzes B, Jureidini J. Should paroxetine be used to treat depression during pregnancy? Am J Psychiatry. 2008 Nov;165(11):1487; author reply -8 . doi: 10.1176/appi.ajp.2008.08040573. PMID: 18981077. Exclusion Code: X1.

989. Misri S. From conception to cradle: Combatting anxiety disorders. Arch Womens Ment Health. 2011;14:S114-S5. doi: 10.1007/s00737-011-0238-y. Exclusion Code: X7.

990. Misri S, Abizadeh J. Bipolar disorder across the female life cycle. Bipolar Disorders. 2010;12:38. doi: 10.1111/j.13995618.2010.00782.x. Exclusion Code: X7.
991. Misri S, Abizadeh J, Albert G, et al. Restoration of functionality in postpartum depressed mothers: an open-label study with escitalopram. J Clin Psychopharmacol. 2012 Oct;32(5):729-32. doi: 10.1097/JCP.0b013e31826867c9. PMID: 22926619. Exclusion Code: X1.

992. Misri S, Abizadeh J, Nirwan S. Depression during pregnancy and the postpartum period. In: Wenzel A, ed The Oxford handbook of perinatal psychology. New York, NY: Oxford University Press; 2016:111-31. Exclusion Code: X1.

993. Misri S, Burgmann A, Kostaras D. Are SSRIs safe for pregnant and breastfeeding women? Can Fam Physician. 2000 Mar;46:626-8, 31-3. PMID: 10752001. Exclusion Code: X7.

994. Misri S, Kendrick K. Treatment of perinatal mood and anxiety disorders: a review. Can J Psychiatry. 2007 Aug;52(8):489-98. doi: 10.1177/070674370705200803. PMID: 17955910. Exclusion Code: X7.

995. Misri S, Kendrick K, Oberlander TF, et al. Antenatal depression and anxiety affect postpartum parenting stress: a longitudinal, prospective study. Can J Psychiatry. 2010 Apr;55(4):222-8. doi: 10.1177/070674371005500405. PMID: 20416145. Exclusion Code: X2.

996. Misri S, Kostaras D, Kostaras X. The use of selective serotonin reuptake inhibitors during pregnancy and lactation: current knowledge. Can J Psychiatry. 2000 Apr;45(3):285-7. doi: 10.1177/070674370004500309. PMID: 10779887. Exclusion Code: X1.

997. Misri S, Milis L. Obsessive-compulsive disorder in the postpartum: open-label trial of quetiapine augmentation. J Clin Psychopharmacol. 2004 Dec;24(6):624-7. PMID: 15538124. Exclusion Code: X4.

998. Misri S, Reebye P, Corral M, et al. The use of paroxetine and cognitive-behavioral therapy in postpartum depression and anxiety: a randomized controlled trial. J Clin Psychiatry. 2004 Sep;65(9):1236-41. PMID: 15367052. Exclusion Code: X4. 
999. Misri S, Sivertz K. Tricyclic drugs in pregnancy and lactation: a preliminary report. Int J Psychiatry Med. 1991;21(2):157-71. doi: 10.2190/jdtx-byc3k3vp-lwah. PMID: 1894455. Exclusion Code: X5.

1000. Misri S, Swift E, Abizadeh J, et al. Overcoming functional impairment in postpartum depressed or anxious women: a pilot trial of desvenlafaxine with flexible dosing. Ther Adv Psychopharmacol. 2016 Aug;6(4):269-76. doi: 10.1177/2045125316656297. PMID: 27536346. Exclusion Code: X4.

1001. Mitchell EA, Thompson JM, Stewart AW, et al. Postnatal depression and SIDS: a prospective study. J Paediatr Child Health. 1992;28 Suppl 1:S13-6. PMID: 1524875. Exclusion Code: X4.

1002. Mitchell J, Goodman J. Comparative effects of antidepressant medications and untreated major depression on pregnancy outcomes: a systematic review. Arch Womens Ment Health. 2018 Oct;21(5):505-16. doi: 10.1007/s00737-018-0844-z. PMID: 29644439. Exclusion Code: X7.

1003. Mittal A, Deligiannidis K, Huang MY, et al. Effect of SAGE-217 on Anxiety Outcomes in Postpartum Depression in a Randomized, Placebo-Controlled Trial. Biol Psychiatry. 2020;87(9):S278-S9. doi: 10.1016/j.biopsych.2020.02.719. Exclusion Code: X3.

1004. Mohammad Masud I, Sobhan T, Ryals T. Effects of commonly used benzodiazepines on the fetus, the neonate, and the nursing infant. Psychiatr Serv. 2002;53(1):39-49. doi: 10.1176/appi.ps.53.1.39. PMID: 200210112-001. Exclusion Code: X1.

1005. Mohan MR, Guirguis J. Pregnant women should be carefully supervised [9]. Pharm J. 2005;274(7345):452. Exclusion Code: X1.

1006. Mohr P, Hnidek D, Hanka J, et al. Pharmacotherapy in pregnancy and breastfeeding: Clinical database. Int J Neuropsychopharmacol. 2012;15:240. doi: 10.1017/S1461145712000508. Exclusion Code: X4.
1007. Molenaar NM, Brouwer ME, Bockting CL, et al. Stop or go? Preventive cognitive therapy with guided tapering of antidepressants during pregnancy: study protocol of a pragmatic multicentre noninferiority randomized controlled trial. BMC Psychiatry. 2016 Mar 18;16:72. doi: 10.1186/s12888-016-0752-6. PMID: 26993629. Exclusion Code: X7.

1008. Molenaar NM, Brouwer ME, Kamperman AM, et al. Recurrence of depression in the perinatal period: Clinical features and associated vulnerability markers in an observational cohort. PLoS One. 2019;14(2):e0212964. doi: 10.1371/journal.pone.0212964. PMID: 30794709. Exclusion Code: X2.

1009. Molenaar NM, Houtman D, Bijma HH, et al. Dose-effect of maternal serotonin reuptake inhibitor use during pregnancy on birth outcomes: A prospective cohort study. J Affect Disord. 2020 Apr 15;267:57-62. doi: 10.1016/j.jad.2020.02.003. PMID: 32063573. Exclusion Code: X13.

1010. Moller-Olsen C, Friedman SH, Prakash C, et al. Clinical characteristics of maternal mental health service users treated with mood stabilizing or antipsychotic medication. Asia Pac Psychiatry. 2018 Jun;10(2):e12304. doi: 10.1111/appy.12304. PMID: 29226616. Exclusion Code: X5.

1011. Møller-Olsen C, Hatters-Friedman S, Prakash C, et al. Mood stabilizer and antipsychotic utilization among maternal mental health service users in pregnancy. Aust N Z J Psychiatry. 2016;50:161-2. doi: 10.1177/0004867416640967. Exclusion Code: X4.

1012. Molyneaux E, Howard LM, McGeown HR, et al. Antidepressant treatment for postnatal depression. Cochrane Database Syst Rev. 2014 Sep 11(9):Cd002018. doi: 10.1002/14651858.CD002018.pub2. PMID: 25211400. Exclusion Code: X1.

1013. Molyneaux E, Howard LM, McGeown HR, et al. Antidepressant treatment for postnatal depression. Issues Ment Health Nurs. 2017 Feb;38(2):188-90. doi: 10.1080/01612840.2016.1182409. PMID: 27362508. Exclusion Code: X1. 
1014. Molyneaux E, Telesia LA, Henshaw C, et al. Antidepressants for preventing postnatal depression. Cochrane Database Syst Rev. 2018 Apr 18;4:Cd004363. doi:

10.1002/14651858.CD004363.pub3. PMID: 29669175. Exclusion Code: X7.

1015. Molyneaux E, Trevillion K, Howard LM. Antidepressant treatment for postnatal depression. JAMA. 2015 May

19;313(19):1965-6. doi:

10.1001/jama.2015.2276. PMID: 25988466.

Exclusion Code: X7.

1016. Momen NC, Munk-Olsen T, Li J, et al. Antidepressant use during pregnancy and childhood cancer in the offspring. Pharmacoepidemiol Drug Saf. 2018 Jan;27(1):114-8. doi: 10.1002/pds.4352. PMID: 29141273. Exclusion Code: X5.

1017. Monk C, Fifer W, Myers M, et al. Perinatal endophenotype related to women's antenatal depression and SSRI use. Biol Psychiatry. 2009;65(8):20S-1S. Exclusion Code: X5.

1018. Monk C, Newport DJ, Korotkin JH, et al. Uterine blood flow in a psychiatric population: impact of maternal depression, anxiety, and psychotropic medication. Biol Psychiatry. 2012 Sep 15;72(6):483-90. doi: 10.1016/j.biopsych.2012.05.006. PMID: 22695184. Exclusion Code: X5.

1019. Montastruc F. Antipsychotic use in pregnancy: Is reproductive safety different according to the indication? Pharmacoepidemiol Drug Saf. 2017;26:408. doi: 10.1002/pds.4275. Exclusion Code: X4.

1020. Montastruc F, Hurault-Delarue C, Pariente A, et al. Antipsychotic use in pregnancy in France: is reproductive safety different according to the indication? Fundam Clin Pharmacol. 2017;31:53-4. Exclusion Code: $\mathrm{X} 4$.

1021. Morales DR, Slattery J, Evans S, et al. Ssris during pregnancy and risk of autism spectrum disorder and attention deficit hyperactivity disorder: Systematic review of observational studies and methodological considerations. Pharmacoepidemiol Drug Saf. 2017;26:418-9. doi: 10.1002/pds.4275. Exclusion Code: X7.
1022. Morales DR, Slattery J, Evans S, et al. Antidepressant use during pregnancy and risk of autism spectrum disorder and attention deficit hyperactivity disorder: systematic review of observational studies and methodological considerations. BMC Med. 2018 Jan 15;16(1):6. doi: 10.1186/s12916-017-0993-3. PMID: 29332605. Exclusion Code: X7.

1023. Moreira Soares Macedo S, Daniela Do Vale Faria EMC, Freitas C. Psychotropic drugs and breastfeeding. Eur

Neuropsychopharmacol. 2014;24:S743. Exclusion Code: X1.

1024. Moriyama Y, Tachi A, Nomoto M, et al. Safe and autonomic breastfeeding of mothers with major depression disorder. J Obstet Gynaecol Res. 2017;43:74-5. doi: 10.1111/jog.13388. Exclusion Code: X4.

1025. Morrow J. Bipolar affective disorder and pregnancy registers. Prog Neurol Psychiatry. 2009;13(1):11. Exclusion Code: X1.

1026. Mostacci B, D'Alessandro R, Zenesini C, et al. Antiepileptic drug exposure in pregnancy and mode of delivery: Data from the emilia romagna study on pregnancy and exposure to antiepileptic drugs (Espea). Epilepsia. 2018;59:S186. doi: 10.1111/epi.14612. Exclusion Code: X4.

1027. Mrad A, Mechri A, Slama H, et al. Correlations between obstetric complications and neurological soft signs in Tunisian patients with schizophrenia. Psychiatry Clin Neurosci. 2010;64(6):645-8. doi: 10.1111/j.1440-1819.2010.02149.x. PMID: 2011-00870-009. Exclusion Code: $\mathrm{X} 7$.

1028. Mueller PS. Carbamazepine in pregnancy. Psychosomatics. 1986 Nov;27(11):797-8. doi: 10.1016/s0033-3182(86)72609-1. PMID: 3797613. Exclusion Code: X1.

1029. Mulder EJ, Ververs FF, de Heus R, et al. Selective serotonin reuptake inhibitors affect neurobehavioral development in the human fetus. Neuropsychopharmacology. 2011 Sep;36(10):1961-71. doi: 10.1038/npp.2011.67. PMID: 21525859. Exclusion Code: X4. 
1030. Munk-Olsen T, Laursen TM, Meltzer-Brody $\mathrm{S}$, et al. Psychiatric disorders with postpartum onset: Possible early manifestations of bipolar affective disorders. Arch Gen Psychiatry. 2012;69(4):428-34. doi: 10.1001/archgenpsychiatry.2011.157. PMID: 2012-09587-011. Exclusion Code: $\mathrm{X} 3$.

1031. Munk-Olsen T, Liu X, Bergink V. Antidepressant treatment postpartum and the risk of treatment-emergent mania. J Affect Disord. 2017 Dec 1;223:26-7. doi: 10.1016/j.jad.2017.07.025. PMID: 28715725. Exclusion Code: X1.

1032. Munk-Olsen T, Liu X, Viktorin A, et al. Maternal and infant outcomes associated with lithium use in pregnancy: an international collaborative meta-analysis of six cohort studies. Lancet Psychiatry. 2018 Aug;5(8):644-52. doi: 10.1016/s22150366(18)30180-9. PMID: 29929874. Exclusion Code: X7.

1033. Murala K, Karunyam B, Rao LV, et al. Prinatal mental health assessment and care in obstetrics. BJOG. 2014;121:49. doi: 10.1111/1471-0528.13165. Exclusion Code: X3.

1034. Muzik M, Marcus SM, Flynn HA. Psychotherapeutic treatment options for perinatal depression: Emphasis on maternalinfant dyadic outcomes. The Journal of Clinical Psychiatry. 2009;70(9):1318-9. doi: 10.4088/JCP.09com05451. PMID: 201202479-022. Exclusion Code: X1.

1035. Nadebaum C, Anderson V, Vajda F, et al. Academic achievement of Australian children prenatally exposed to antiepileptic drugs. Epilepsia. 2011;52:249. doi: 10.1111/j.1528-1167.2011.03207.x. Exclusion Code: X2.

1036. Naguy A. Brexanolone and postpartum depression: what does it have to do with GABA? Arch Womens Ment Health. 2019 Dec;22(6):833-4. doi: 10.1007/s00737-01900986-0. PMID: 31302763. Exclusion Code: $\mathrm{X} 1$.

1037. Naguy A. Mirtazapine for Major Depression Developed After Hyperemesis Gravidarum. Am J Ther. 2019 Sep/Oct;26(5):e661-e2. doi: $10.1097 / \mathrm{mjt} .000000000000860$. PMID: 30277906. Exclusion Code: X1.
1038. Nahas Z, Bohning DE, Molloy MA, et al. Safety and feasibility of repetitive transcranial magnetic stimulation in the treatment of anxious depression in pregnancy: a case report. J Clin Psychiatry. 1999;60(1):50-2. PMID: CN-00160595. Exclusion Code: X3.

1039. Nair MT, Black MA, Whitmore J, et al. Psychotropic drugs during pregnancy: An audit of what information women are given at first presentation to the psychiatric antenatal clinic. Arch Womens Ment Health. 2015;18(2):331. doi: 10.1007/s00737-0140488-6. Exclusion Code: X5.

1040. Nakhai-Pour HR, Broy P, Bérarp A. Antidepressant medication use during pregnancy and the risk of miscarriage. Birth Defects Research Part A - Clinical and Molecular Teratology. 2009;85(5):437. doi: 10.1002/bdra.20605. Exclusion Code: X4.

1041. Nassr DG. Use of lithium in pregnancy. Br J Psychiatry. 1969 Sep;115(526):1102. PMID: 5387000. Exclusion Code: X7.

1042. Nayar B, Patel M, Grant W. Joint Perinatal Mental Health Clinic (PMH) in North West London hospital-a success story of collaborative working. BJOG. 2016;123:141-2. doi: 10.1111/14710528.14104. Exclusion Code: X3.

1043. Nct. Clinical trial of estrogen for postpartum depression.

Https:/clinicaltrials.gov/show/nct00059228. 2003. PMID: CN-01590858. Exclusion Code: X5.

1044. Nct. Pilot study of bupropion for smoking cessation in postpartum non-breastfeeding women.

Https://clinicaltrials.gov/show/nct00119210. 2005. PMID: CN-01510475. Exclusion Code: X5.

1045. Nct. Sertraline for the prevention of recurrent postpartum depression. Https://clinicaltrials.gov/show/nct00276900. 2006. PMID: CN-01513112. Exclusion Code: X5.

1046. Nct. Evaluation of effectiveness and safety of flexible-dose paliperidone extended release in patients with schizoaffective disorder.

Https://clinicaltrials.gov/show/nct00412373. 2006. PMID: CN-01513745. Exclusion Code: X5. 
1047. Nct. Effectiveness of sertraline alone and interpersonal psychotherapy alone in treating women with postpartum depression. Https://clinicaltrials.gov/show/nct00602355. 2008. PMID: CN-01516689. Exclusion Code: X5.

1048. Nct. Postpartum depression: transdermal estradiol versus sertraline. Https://clinicaltrials.gov/show/nct00744328. 2008. PMID: CN-01484242. Exclusion Code: X5.

1049. Nct. Effectiveness of group interpersonal psychotherapy in treating women with prenatal depression.

Https://clinicaltrials.gov/show/nct00633178. 2008. PMID: CN-01517473. Exclusion Code: X5.

1050. Nct. Interpersonal treatment program to prevent depression and post-traumatic stress disorder in low-income pregnant women with partner abuse.

Https://clinicaltrials.gov/show/nct00602732. 2008. PMID: CN-01516704. Exclusion Code: X5.

1051. Nct. The effect of selective serotonin reuptake inhibitor (SSRI) treatment on affective symptoms and fertility treatment outcome in women undergoing in vitro fertilization (IVF).

Https://clinicaltrials.gov/show/nct00989053. 2009. PMID: CN-01526130. Exclusion Code: X5.

1052. Nct. Bupropion for smoking cessation during pregnancy. Https://clinicaltrials.gov/show/nct01390246. 2011. PMID: CN-01487453. Exclusion Code: X5.

1053. Nct. Buprenorphine for treatment of neonatal abstinence syndrome in infants with in utero exposure to benzodiazepines. Https://clinicaltrials.gov/show/nct01671410. 2012. PMID: CN-01585246. Exclusion Code: X5.

1054. Nct. Partner-assisted interpersonal psychotherapy or antidepressant medication for antenatal depression.

Https://clinicaltrials.gov/show/nct01732055. 2012. PMID: CN-01538831. Exclusion Code: X5.
1055. Nct. Effects and consequences for mother and child from treatment for depression. Https://clinicaltrials.gov/show/nct02185547. 2014. PMID: CN-01547092. Exclusion Code: X5.

1056. Nct. Prophylactic use of postpartum sertraline to prevent postpartum depression. Https://clinicaltrials.gov/show/nct02235064. 2014. PMID: CN-01548551. Exclusion Code: X5.

1057. Nct. A randomised trial of sertraline, cognitive behaviour therapy and combined therapy for postnatal depression.

Https://clinicaltrials.gov/show/nct02122393. 2014. PMID: CN-01545279. Exclusion Code: X5.

1058. Nct. Placebo-controlled trial of bupropion for smoking cessation in pregnant women. Https://clinicaltrials.gov/show/nct02188459. 2014. PMID: CN-01576758. Exclusion Code: X5.

1059. Nct. Efficacy of nepeta menthoides extract on anxiety of patients with depression: a double-blind randomized controlled clinical trial.

Https://clinicaltrials.gov/show/nct02565316. 2015. PMID: CN-01492662. Exclusion Code: X5.

1060. Nct. Adjunctive psychotherapy for perinatal bipolar disorder.

Https://clinicaltrials.gov/show/nct02402738. 2015. PMID: CN-01505365. Exclusion Code: X5.

1061. Nct. Adjunctive use of celecoxib in the treatment of bipolar postpartum depression. Https://clinicaltrials.gov/show/nct02726659. 2016. PMID: CN-01557038. Exclusion Code: X5.

1062. Nct. Low-dose ketamine and postpartum depression in parturients with prenatal depression.

Https://clinicaltrials.gov/show/nct03336541. 2017. PMID: CN-01565614. Exclusion Code: X5.

1063. Nct. Behavioural Interventions for postnatal depression: a RCT study. Https://clinicaltrials.gov/show/nct03196726. 2017. PMID: CN-01495181. Exclusion Code: X5. 
1064. Nct. PDA for antidepressant use in pregnancy.

Https:/clinicaltrials.gov/show/nct03632863. 2018. PMID: CN-01626278. Exclusion Code: X5.

1065. Nebhinani N, Soni S. Low Apgar scores in neonates with prenatal antidepressant exposure. Br J Psychiatry. 2013 Jun;202:464. doi: 10.1192/bjp.202.6.464. PMID: 23732937. Exclusion Code: X1.

1066. Nevriana A, Pierce M, Dalman C, et al. Association between maternal and paternal mental illness and risk of injuries in children and adolescents: nationwide register based cohort study in Sweden. BMJ. $2020 \mathrm{Apr}$ 8;369:m853. doi: 10.1136/bmj.m853. PMID: 32269017. Exclusion Code: X3.

1067. Newmark RL, Bogen DL, Wisner KL, et al. Risk-Benefit assessment of infant exposure to lithium through breast milk: a systematic review of the literature. Int Rev Psychiatry. 2019 May;31(3):295-304. doi: 10.1080/09540261.2019.1586657. PMID: 31180257. Exclusion Code: X7.

1068. Newport DJ, Fernandez SV, Juric S, et al. Psychopharmacology during pregnancy and lactation. In: Schatzberg AF, Nemeroff CB, eds. The American Psychiatric Publishing textbook of psychopharmacology. Arlington, VA: American Psychiatric Publishing, Inc.; 2009:1323-412. Exclusion Code: X7.

1069. Newport DJ, Hostetter AL, Juul SH, et al. Prenatal psychostimulant and antidepressant exposure and risk of hypertensive disorders of pregnancy. J Clin Psychiatry. 2016 Nov;77(11):1538-45. doi: 10.4088/JCP.15m10506. PMID: 28076672. Exclusion Code: X10.

1070. Newport DJ, Stowe ZN. Clinical management of perinatal depression: focus on paroxetine. Psychopharmacol Bull. 2003 Spring;37 Suppl 1:148-66. PMID: 14566197. Exclusion Code: X7.

1071. Newport DJ, Viguera AC, Beach AJ, et al. Lithium placental passage and obstetrical outcome: implications for clinical management during late pregnancy. Am J Psychiatry. 2005 Nov;162(11):2162-70. doi: 10.1176/appi.ajp.162.11.2162. PMID: 16263858. Exclusion Code: X7.
1072. Nezvalova-Henriksen K, Spigset O, Brandlistuen RE, et al. Effect of selective serotonin reuptake inhibitor (SSRI) exposure during pregnancy on birth weight and gestational age: A sibling- controlled cohort study. Pharmacoepidemiol Drug Saf. 2015;24:208. doi: 10.1002/pds.3838. Exclusion Code: X9.

1073. Nezvalova-Henriksen K, Spigset O, Brandlistuen RE, et al. Effect of prenatal selective serotonin reuptake inhibitor (SSRI) exposure on birthweight and gestational age: a sibling-controlled cohort study. Int $\mathbf{J}$ Epidemiol. 2016 Dec 1;45(6):2018-29. doi: 10.1093/ije/dyw049. PMID: 27188860. Exclusion Code: X4.

1074. Nezvalova-Henriksen K, Spigset O, Brandlistuen RE, et al. Effect of prenatal selective serotonin reuptake inhibitor (SSRI) exposure on birthweight and gestational age: a sibling-controlled cohort study. Int J Clin Pharm. 2017;39(1):311. doi: 10.1007/s11096-016-0404-4. Exclusion Code: X4.

1075. Ng QX, Venkatanarayanan N, Ho CYX, et al. Selective serotonin reuptake inhibitors and persistent pulmonary hypertension of the newborn: an update meta-analysis. J Womens Health (Larchmt). 2018 Nov 8. doi: 10.1089/jwh.2018.7319. PMID: 30407100. Exclusion Code: X7.

1076. Ng RC, Hirata CK, Yeung W, et al. Pharmacologic treatment for postpartum depression: a systematic review. Pharmacotherapy. 2010 Sep;30(9):928-41. doi: 10.1592/phco.30.9.928. PMID: 20795848. Exclusion Code: X7.

1077. Ngai FW, Wong PW, Leung KY, et al. The effect of telephone-based cognitivebehavioral therapy on postnatal depression: a randomized controlled trial. Psychother Psychosom. 2015;84(5):294-303. doi: 10.1159/000430449. PMID: CN-01096388. Exclusion Code: X3.

1078. Nguyen HT, Sharma V, McIntyre RS. Teratogenesis associated with antibipolar agents. Adv Ther. 2009 Mar;26(3):281-94. doi: 10.1007/s12325-009-0011-z. PMID: 19330496. Exclusion Code: X7. 
1079. Nguyen NT, Gorman M, Caughey AB. Pregnancy outcomes in women with schizophrenia: A retrospective cohort study. Am J Obstet Gynecol. 2016;214(1):S296. Exclusion Code: X3.

1080. Nguyen TN, Faulkner D, Frayne JS, et al. Obstetric and neonatal outcomes of pregnant women with severe mental illness at a specialist antenatal clinic. Med J Aust. 2013 Aug 5;199(3 Suppl):S26-9. PMID: 25369845. Exclusion Code: X3.

1081. Nicolai J, Vles JS, Aldenkamp AP. Neurodevelopmental delay in children exposed to antiepileptic drugs in utero: a critical review directed at structural studybias. J Neurol Sci. 2008 Aug 15;271(1-2):114. doi: 10.1016/j.jns.2008.03.004. PMID: 18479711. Exclusion Code: X7.

1082. Nielsen RE, Damkier P. Pharmacological treatment of unipolar depression during pregnancy and breast-feeding - A clinical overview. Nordic Journal of Psychiatry. 2012;66(3):159-66. doi: 10.3109/08039488.2011.650198. PMID: 2012-13469-003. Exclusion Code: X1.

1083. Nielsen SW, Ljungdalh PM, Nielsen J, et al. Maternal use of selective serotonin reuptake inhibitors during pregnancy is associated with Hirschsprung's disease in newborns - a nationwide cohort study. Orphanet J Rare Dis. 2017 Jun 20;12(1):116. doi: 10.1186/s13023-017-0667-4. PMID: 28633635. Exclusion Code: X5.

1084. Nijenhuis CM, ter Horst PG, van Rein N, et al. Disturbed development of the enteric nervous system after in utero exposure of selective serotonin re-uptake inhibitors and tricyclic antidepressants. Part 2: Testing the hypotheses. Br J Clin Pharmacol. 2012 Jan;73(1):126-34. doi: 10.1111/j.13652125.2011.04081.x. PMID: 21848990. Exclusion Code: X2.

1085. Nijhuis IJ, Kok-Van Rooij GW, Bosschaart AN. Withdrawal reactions of a premature neonate after maternal use of paroxetine. Arch Dis Child Fetal Neonatal Ed. 2001 Jan;84(1):F77. PMID: 11199858. Exclusion Code: X7.
1086. Nikfar S, Abdollahi M, Hendoiee N, et al. Pregnancy outcomes folowing exposure to serotonin reuptake inhibitors: A metaanalysis. Value Health. 2012;15(7):A638. doi: 10.1016/j.jval.2012.08.212. Exclusion Code: X7.

1087. Nikfar S, Rahimi R, Hendoiee N, et al. Increasing the risk of spontaneous abortion and major malformations in newborns following use of serotonin reuptake inhibitors during pregnancy: A systematic review and updated meta-analysis. DARU, Journal of Pharmaceutical Sciences. 2012;20(1). doi: 10.1186/2008-2231-20-75. Exclusion Code: X7.

1088. Nillni YI, Mehralizade A, Mayer L, et al. Treatment of depression, anxiety, and trauma-related disorders during the perinatal period: A systematic review. Clin Psychol Rev. 2018 Dec;66:136-48. doi: 10.1016/j.cpr.2018.06.004. PMID: 29935979. Exclusion Code: X7.

1089. Nilsson E, Lichtenstein P, Cnattingius S, et al. Women with schizophrenia: Pregnancy outcome and infant death among their offspring. Schizophr Res. 2002;58(2-3):2219. doi: 10.1016/S0920-9964(01)00370-X. PMID: 2002-06655-014. Exclusion Code: $\mathrm{X} 2$.

1090. Noe K. Are Antidepressant Medications Safe for Pregnant Women With Epilepsy? The Signs Point to Yes. Epilepsy Curr. 2019 May-Jun;19(3):161-2. doi: 10.1177/1535759719841382. PMID: 31035821. Exclusion Code: X1.

1091. Noera K, Fokke DJ, Fedde S, et al. Psychotropic drug use during pregnancy: Is observation of mother and infant needed postpartum? J Perinat Med. 2015;43. doi: 10.1515/jpm-2015-2002. Exclusion Code: $\mathrm{X} 4$.

1092. Non AL, Binder AM, Kubzansky LD, et al. Genome-wide DNA methylation in neonates exposed to maternal depression, anxiety, or SSRI medication during pregnancy. Epigenetics. 2014 Jul;9(7):964-72. doi: 10.4161/epi.28853. PMID: 24751725. Exclusion Code: X5. 
1093. Nonacs RM, Soares CN, Viguera AC, et al. Bupropion SR for the treatment of postpartum depression: a pilot study. Int $\mathrm{J}$ Neuropsychopharmacol. 2005 Sep;8(3):4459. doi: $10.1017 / \mathrm{s} 1461145705005079$.

PMID: 15817137. Exclusion Code: X4.

1094. Noorbala AA, Afzali HM, Abedinia N, et al. Investigation of the effectiveness of psychiatric interventions on the mental health of pregnant women in Kashan City Iran: A clinical trial study. Asian J Psychiatr. 2019 Dec;46:79-86. doi: 10.1016/j.ajp.2019.09.036. PMID: 31639554. Exclusion Code: X2.

1095. Nordeng H, Einarson A, Eberhard-Gran M, et al. Safety of antidepressants during pregnancy. Arch Womens Ment Health. 2011;14:S40. doi: 10.1007/s00737-0100203-1. Exclusion Code: X9.

1096. Nordeng H, Hansen C, Garthus-Niegel S, et al. Fear of childbirth, mental health, and medication use during pregnancy. Arch Womens Ment Health. 2012 Jun;15(3):2039. doi: 10.1007/s00737-012-0278-y. PMID: 22526406. Exclusion Code: X4.

1097. Nordeng H, van Gelder MM, Spigset O, et al. Pregnancy outcome after exposure to antidepressants and the role of maternal depression: results from the Norwegian Mother and Child Cohort Study. J Clin Psychopharmacol. 2012 Apr;32(2):186-94. doi: 10.1097/JCP.0b013e3182490eaf. PMID: 22367660. Exclusion Code: X10.

1098. Norwitz ER, Bellanger K, Funai EF, et al. Post-Traumatic stress disorder (PTSD) and panic disorder are independent risk factors for preterm birth, but not low birth weight or small-for-gestational age: The Yale 'Pink \&Blue' Prospective cohort study. Reprod Sci. 2010;17(3):352A. doi: 10.1177/193371912010173s067. Exclusion Code: X4.

1099. Norwitz ER, Lockwood CJ, Bellanger K, et al. Effect of SSRI medications on preterm birth is independent of birth weight: The yale 'pink \& blue' prospective cohort study. Reprod Sci. 2010;17(3):284A. doi: 10.1177/193371912010173s067. Exclusion Code: X4.
1100. Nulman I. Neurodevelopment of children exposed in utero to venlafaxine: Preliminary results. Clin Pharmacol Ther. 2010;87:S8. doi: 10.1038/clpt.2009.269. Exclusion Code: X9.

1101. Nulman I, Barrera M, Pulver A, et al. Neurodevelopment of children exposed in utero to venlafaxine: Preliminary results. Birth Defects Research Part A - Clinical and Molecular Teratology. 2010;88(5):363. doi: 10.1002/bdra.20677. Exclusion Code: X9.

1102. Nulman I, Barrera M, Streimer D, et al. Neurodevelopment of children exposed in utero to venlafaxine: Children exposed to SSRIs and non-teratogens as comparison groups. Birth Defects Research Part A Clinical and Molecular Teratology. 2009;85(5):455. doi: 10.1002/bdra.20605. Exclusion Code: X9.

1103. Nulman I, Koren G, Rovet J, et al. Neurodevelopment of children prenatally exposed to selective reuptake inhibitor antidepressants: Toronto sibling study. J Clin Psychiatry. 2015 Jul;76(7):e842-7. doi: 10.4088/JCP.14m09240. PMID: 26231010. Exclusion Code: X4.

1104. Nulman I, Sadowski A, Todorow M, et al. Pregnancy outcomes following maternal exposure to second generation antipsychotics: Considering polytherapy. FASEB J. 2013;27. Exclusion Code: X9.

1105. Nulman I, Terrana N, Koren G, et al. Psychotropic medications in pregnancy with a focus on antipsychotic drugs. Basic and Clinical Pharmacology and Toxicology. 2014;115:84. Exclusion Code: X7.

1106. O’Connor E, Senger CA, Henninger M, et al. U.S. Preventive Services Task Force Evidence Syntheses, formerly Systematic Evidence Reviews. Interventions to Prevent Perinatal Depression: A Systematic Evidence Review for the U.S. Preventive Services Task Force. Rockville (MD): Agency for Healthcare Research and Quality (US); 2019. Exclusion Code: X2.

1107. Oakley GP, Jr. Drug influences on malformations. Clin Perinatol. 1979 Sep;6(2):403-14. PMID: 42506. Exclusion Code: X1. 
1108. Oates M, Cantwell R. Chapter 11: Deaths from psychiatric causes. BJOG. 2011;118(SUPPL. 1):132-42. Exclusion Code: X1.

1109. Oates MR. The confidential enquiry into maternal deaths 1997-2005: implications for practice. Psychiatry. 2009;8(1):13-6. doi: 10.1016/j.mppsy.2008.10.013. Exclusion Code: X1.

1110. Oberlander TF. Prenata serotonin reuptake inhibitor (SRI) antidepressant exposure and serotonin transporter promoter genotype (SLC6A4) influence executive functions at 6 years of age. Biol Psychiatry.

2014;75(9):276S. doi:

10.1016/j.biopsych.2014.03.016. Exclusion Code: X4.

1111. Oberlander TF, Bonaguro RJ, Misri S, et al. Infant serotonin transporter (SLC6A4) promoter genotype is associated with adverse neonatal outcomes after prenatal exposure to serotonin reuptake inhibitor medications. Mol Psychiatry. 2008

Jan;13(1):65-73. doi:

10.1038/sj.mp.4002007. PMID: 17519929.

Exclusion Code: X4.

1112. Oberlander TF, Eckstein Grunau R, Fitzgerald C, et al. Prolonged prenatal psychotropic medication exposure alters neonatal acute pain response. Pediatr Res. 2002 Apr;51(4):443-53. doi: 10.1203/00006450-200204000-00008. PMID: 11919328. Exclusion Code: X4.

1113. Oberlander TF, Grunau R, Mayes L, et al. Hypothalamic-pituitary-adrenal (HPA) axis function in 3-month old infants with prenatal selective serotonin reuptake inhibitor (SSRI) antidepressant exposure. Early Hum Dev. 2008 Oct;84(10):689-97. doi: 10.1016/j.earlhumdev.2008.06.008. PMID: 18639992. Exclusion Code: X4.

1114. Oberlander TF, Grunau RE, Brain U, et al. Prenatal serotonin reuptake inhibitor (SRI) antidepressant exposure and serotonin transporter promoter genotype (SLC6A4) influence executive functions at 6 years of age. Arch Womens Ment Health. 2013;16:S22. doi: 10.1007/s00737-0130355-x. Exclusion Code: X4.
1115. Oberlander TF, Grunau RE, Fitzgerald C, et al. Pain reactivity in 2-month-old infants after prenatal and postnatal serotonin reuptake inhibitor medication exposure. Pediatrics. 2005 Feb;115(2):411-25. doi: 10.1542/peds.2004-0420. PMID: 15687451. Exclusion Code: X5.

1116. Oberlander TF, Papsdorf M, Brain UM, et al. Prenatal effects of selective serotonin reuptake inhibitor antidepressants, serotonin transporter promoter genotype (SLC6A4), and maternal mood on child behavior at 3 years of age. Arch Pediatr Adolesc Med. 2010 May;164(5):444-51. doi: 10.1001/archpediatrics.2010.51. PMID: 20439795. Exclusion Code: X2.

1117. Oberlander TF, Reebye P, Misri S, et al. Externalizing and attentional behaviors in children of depressed mothers treated with a selective serotonin reuptake inhibitor antidepressant during pregnancy. Arch Pediatr Adolesc Med. 2007 Jan;161(1):22-9. doi: 10.1001/archpedi.161.1.22. PMID: 17199063. Exclusion Code: X4.

1118. Oberlander TF, Warburton W, Misri S, et al. Effects of timing and duration of gestational exposure to serotonin reuptake inhibitor antidepressants: population-based study. $\mathrm{Br}$ J Psychiatry. 2008 May;192(5):338-43. doi: 10.1192/bjp.bp.107.037101. PMID: 18450656. Exclusion Code: X4.

1119. O'Brien L, Schachtschneider AM, Koren G, et al. Longitudinal study of depression, anxiety, irritability, and stress in pregnancy following evidence-based counseling on the use of antidepressants. J Psychiatr Pract. 2007 Jan;13(1):33-9. PMID: 17242590. Exclusion Code: X4.

1120. O'Brien LA-M. Critical determinants of the risk-benefit assessment of antidepressants in pregnancy: Pharmacokinetic, safety and economic considerations: ProQuest Information \& Learning; 2010. Exclusion Code: X7.

1121. O'Connor AB, O'Brien L, Alto WA, et al. Does concurrent in utero exposure to buprenorphine and antidepressant medications influence the course of neonatal abstinence syndrome? J Matern Fetal Neonatal Med. 2016;29(1):112-4. doi: 10.3109/14767058.2014.987750. PMID: 25394611. Exclusion Code: X4. 
1122. O'Connor E, Rossom RC, Henninger M, et al. Primary care screening for and treatment of depression in pregnant and postpartum women: evidence report and systematic review for the US Preventive Services Task Force. JAMA. 2016 Jan 26;315(4):388-406. doi: 10.1001/jama.2015.18948. PMID: 26813212. Exclusion Code: X7.

1123. O'Connor E, Rossom RC, Henninger M, et al. U.S. Preventive Services Task Force evidence syntheses, formerly systematic evidence reviews. Screening for Depression in Adults: An Updated Systematic Evidence Review for the U.S. Preventive Services Task Force. Rockville (MD): Agency for Healthcare Research and Quality (US); 2016. Exclusion Code: X7.

1124. O'Connor E, Senger CA, Henninger ML, et al. Interventions to Prevent Perinatal Depression: Evidence Report and Systematic Review for the US Preventive Services Task Force. JAMA. 2019 Feb 12;321(6):588-601. doi: 10.1001/jama.2018.20865. PMID: 30747970. Exclusion Code: X2.

1125. O'Donnell M, Maclean MJ, Sims S, et al. Maternal mental health and risk of child protection involvement: mental health diagnoses associated with increased risk. J Epidemiol Community Health. 2015;69(12):1175-83. doi: 10.1136/jech2014-205240. PMID: 2015-56012-002. Exclusion Code: X2.

1126. Odsbu I, Skurtveit S, Selmer R, et al. Prenatal exposure to anxiolytics and hypnotics and language competence at 3 years of age. Eur J Clin Pharmacol. 2015 Mar;71(3):283-91. doi: 10.1007/s00228014-1797-4. PMID: 25547568. Exclusion Code: X4.

1127. Øgendahl BK, Agerbo E, Byrne M, et al. Indicators of fetal growth and bipolar disorder: A Danish national register-based study. Psychol Med. 2006;36(9):1219-24. doi: 10.1017/S0033291706008269. PMID: 2006-11586-003. Exclusion Code: X2.

1128. Ogunyemi D, Jovanovski A, Liu J, et al. The contribution of untreated and treated anxiety and depression to prenatal, intrapartum, and neonatal outcomes. AJP Rep. 2018 Jul;8(3):e146-e57. doi: 10.1055/s-00381661379. PMID: 29998037. Exclusion Code: X12.
1129. O'Keane V, Lightman S, Marsh M, et al. Increased pituitary-adrenal activation and shortened gestation in depressed pregnant women. J Affect Disord. 2010;122:S41. doi: 10.1016/j.jad.2010.02.025. Exclusion Code: $\mathrm{X} 4$.

1130. Okun ML, Kiewra K, Luther JF, et al. Sleep disturbances in depressed and nondepressed pregnant women. Depress Anxiety. 2011 Aug;28(8):676-85. doi: 10.1002/da.20828. PMID: 21608086. Exclusion Code: X5.

1131. Olfson M, Marcus S. Neonatal risks of maternal treatment with mood stabilizers. Arch Gen Psychiatry. 2007 Jul;64(7):866-7; author reply 7-8. doi:

10.1001/archpsyc.64.7.866-b. PMID: 17606821. Exclusion Code: X1.

1132. Olie JP, Lloret-Loy C. [Lithium treatment and adverse effects]. Ann Med Psychol (Paris). 1995 May;153(3):207-11; discussion 11-2. PMID: 7598357. Exclusion Code: X8.

1133. Oliff HS. Saffron Comparable to Fluoxetine in the Treatment of Postpartum Depression. Herbalgram. 2017(115):30-. PMID: CN02112944. Exclusion Code: X4.

1134. Oliveira CIF, Fett-Conte AC. Birth defects: Risk factors and consequences. Journal of Pediatric Genetics. 2013;2(2):85-90. doi: 10.3233/PGE-13052. Exclusion Code: X1.

1135. Olleman R, Wennink H, Honig A. Use of SSRI during pregnancy and the risk of autism spectrum disorder in the child. Tijdschrift voor Psychiatrie. 2015;57(1):58. Exclusion Code: X8.

1136. Olson JG. Retrospective analysis of puerperal depression, assessment, and treatment: ProQuest Information \& Learning; 2010. Exclusion Code: X4.

1137. O'Mahen H, Himle JA, Fedock G, et al. A pilot randomized controlled trial of cognitive behavioral therapy for perinatal depression adapted for women with low incomes. Depress Anxiety. 2013;30(7):67987. doi: 10.1002/da.22050. PMID: 201323976-011. Exclusion Code: X3.

1138. Öndemark M, Lindqvist PG. Is the risk of bleeding complications related to SSRI dose-dependent? A cohort study. Thromb Res. 2017;151:S107. Exclusion Code: X4. 
1139. Ong LL, Chua TE, Wang J, et al. Clinical audit of antenatal depression management in Singapore. Arch Womens Ment Health. 2011;14:S77-S8. doi: 10.1007/s00737-0100203-1. Exclusion Code: X4.

1140. Onken M, Mick I, Schaefer C. Paliperidone and pregnancy-an evaluation of the German Embryotox database. Arch Womens Ment Health. 2018 Dec;21(6):657-62. doi: 10.1007/s00737-018-0828-z. PMID: 29569043. Exclusion Code: X4.

1141. Onken M, Mick I, Schaefer C. Paliperidone and pregnancy - An evaluation of the German Embryotox database. Arch Womens Ment Health. 2018;21(6):657-62. doi: 10.1007/s00737-018-0828-z. PMID: 201812727-001. Exclusion Code: X4.

1142. Ornoy A, Arnon J, Shechtman S, et al. Is benzodiazepine use during pregnancy really teratogenic? Reprod Toxicol. 1998 SepOct;12(5):511-5. PMID: 9763242. Exclusion Code: X4.

1143. Orsolini L, Bellantuono C. Serotonin reuptake inhibitors and breastfeeding: a systematic review. Hum Psychopharmacol. 2015 Jan;30(1):4-20. doi: 10.1002/hup.2451. PMID: 25572308. Exclusion Code: X7.

1144. Orsolini L, Bozzi F, Bellantuono C. Psychopharmacological treatment in pregnancy and in breastfeeding: Focus on escitalopram. Eur Neuropsychopharmacol. 2013;23:S399-S400. doi: 10.1016/S0924977X(13)70631-X. Exclusion Code: X7.

1145. Orsolini L, Valchera A, Vecchiotti R, et al. Suicide during perinatal period: Epidemiology, risk factors, and clinical correlates. Frontiers in Psychiatry. 2016;7(AUG). doi: 10.3389/fpsyt.2016.00138. Exclusion Code: $\mathrm{X} 7$.

1146. Osadchy A, Weitzner B, Lam J, et al. Therapeutic use of opioids: A prospective, comparative study of pregnancy outcomes. J Popul Ther Clin Pharmacol. 2010;17(1):e235. Exclusion Code: X2.

1147. O'Shea M, Kenton K, Mueller M, et al. Postpartum contraception after obstetric anal sphincter injury. Female Pelvic Medicine and Reconstructive Surgery.

2018;24(5):S149. doi:

10.1097/SPV.0000000000000616.

Exclusion Code: X2.
1148. Ottomani H, Gouraud A, Jonville-Béra AP, et al. First-trimester exposure to valproic acid treatment in bipolar disorders: A prospective comparative study using the Terappel database. Fundam Clin Pharmacol. 2014;28:68. doi: 10.1111/fcp.12065. Exclusion Code: X9.

1149. Ou JJ, Li YM. Autism spectrum disorder and prenatal exposure to selective serotonin reuptake inhibitors: need for further analysis. Reprod Toxicol. 2017;67:146-7. doi: 10.1016/j.reprotox.2016.11.017. Exclusion Code: X1.

1150. Özerdem A, Akdeniz F. Pregnancy and postpartum in bipolar disorder. Neuropsychiatry. 2014;4(1):95-107. doi: 10.2217/npy.13.87. Exclusion Code: X7.

1151. Ozkan M. Psychotropic drug use in pregnancy and lactation. Klinik Psikofarmakoloji Bulteni. 2013;23:S55. Exclusion Code: X1.

1152. Oztürk Z, Olmez E, Gurpinar T, et al. Prenatal psychotropic drug exposure, birth outcome and tendency to elective termination of pregnancy. Eur Neuropsychopharmacol. 2015;25:S355. Exclusion Code: X4.

1153. Paarlberg Marieke K, Upmeijer JG, Pot DJ. Development and structure of psychiatricobstetric-pediatric consultation in a general teaching hospital. Journal of Psychosomatic Obstetrics and Gynecology. 2010;31:29. doi: 10.3109/0167482x.2010.536387. Exclusion Code: X3.

1154. Pacchiarotti I, Leon-Caballero J, Murru A, et al. Mood stabilizers and antipsychotics during breastfeeding: Focus on bipolar disorder. Eur Neuropsychopharmacol. 2016 Oct;26(10):1562-78. doi: 10.1016/j.euroneuro.2016.08.008. PMID: 27568278. Exclusion Code: X7.

1155. Packer CH, Chatroux LR, Valent AM, et al. 840: Influence of depression on neonatal outcomes. Am J Obstet Gynecol. 2019;220(1):S548-S9. doi: 10.1016/j.ajog.2018.11.863. Exclusion Code: X3.

1156. Pae CU. Filicide: Needing psychiatrists' attention. Psychiatry Investig. 2014;11(2):214-5. doi: 10.4306/pi.2014.11.2.214. Exclusion Code: $\mathrm{X} 1$. 
1157. Page L. Prescribing for pregnancy: managing prescribing for women with mental health diagnoses. Drug Ther Bull. 2020 Jan;58(1):8-11. doi: 10.1136/dtb.2019.000006. PMID: 31740448. Exclusion Code: X1.

1158. Pahuja S. Impact of prenatal exposure to antidepressants on adverse birth outcomes. Value Health. 2017;20(5):A291-A2. Exclusion Code: X9.

1159. Palladino CL, Flynn HA, Richardson C, et al. Lengthened predelivery stay and antepartum complications in women with depressive symptoms during pregnancy. J Womens Health. 2011;20(6):953-62. doi: 10.1089/jwh.2010.2380. PMID: 201123511-012. Exclusion Code: X3.

1160. Palmsten K, Hernandez-Diaz S. Can nonrandomized studies on the safety of antidepressants during pregnancy convincingly beat confounding, chance, and prior beliefs? Epidemiology. 2012

Sep;23(5):686-8. doi: 10.1097/EDE.0b013e318258fbb2. PMID: 22872113. Exclusion Code: X1.

1161. Palmsten K, Huybrechts K, Setoguchi S, et al. Antidepressant use during pregnancy and risk for preeclampsia in the us medicaid population. Pharmacoepidemiol Drug Saf. 2012;21:374. doi: 10.1002/pds.3324.

Exclusion Code: X9.

1162. Palmsten K, Setoguchi S, Margulis AV, et al. Elevated risk of preeclampsia in pregnantwomen with depression: disease vs. antidepressants. Pharmacoepidemiol Drug Saf. 2010;19:S210-S1. doi: 10.1002/pds.2019. Exclusion Code: X9.

1163. Palmsten K, Setoguchi S, Patrick AR, et al. Elevated risk of preeclampsia in pregnant women with depression: disease vs. anti depressants. Am J Epidemiol. 2010;171:S101. doi: 10.1093/aje/kwq151. Exclusion Code: X9.

1164. Palmsten KK, Hernández-Díaz S, Huybrechts KF, et al. Antidepressant use near delivery increases the risk of postpartum hemorrhage.

Pharmacoepidemiol Drug Saf. 2013;22:366. doi: 10.1002/pds.3512. Exclusion Code: X9.
1165. Palmsten KK, Huybrechts KF, Kowal MK, et al. Antidepressant use late in pregnancy and risk for persistent pulmonary hypertension of the newborn (PPHN) among low-income women. Pharmacoepidemiol Drug Saf. 2013;22:159. doi: 10.1002/pds.3512. Exclusion Code: X9.

1166. Panelli DM, Chan C, Shaw JG, et al. 504: Post-traumatic stress disorder in pregnancy: Does treatment impact the risk of preterm birth? Am J Obstet Gynecol. 2020;222(1):S328. doi: 10.1016/j.ajog.2019.11.520. Exclusion Code: X9.

1167. Panesar K. Pharmacologic agents used in obstetrics. 2012;37((Panesar K.)):HS2-HS6. Exclusion Code: X1.

1168. Pantell MS, Baer RJ, Torres JM, et al. Associations between unstable housing, obstetric outcomes, and perinatal health care utilization. American Journal of Obstetrics and Gynecology MFM. 2019;1(4). doi: 10.1016/j.ajogmf.2019.100053. Exclusion Code: X2.

1169. Pariante CM. Depression and antidepressants in pregnancy: molecular and psychosocial mechanisms affecting offspring's physical and mental health. Neuropsychopharmacology. 2015 Jan;40(1):246-7. doi: 10.1038/npp.2014.219. PMID: 25482175. Exclusion Code: X7.

1170. Pariente G, Leibson T, Shulman T, et al. Pregnancy outcomes following in utero exposure to lamotrigine: a systematic review and meta-analysis. CNS Drugs. 2017 Jun;31(6):439-50. doi: 10.1007/s40263-0170433-0. PMID: 28434134. Exclusion Code: $\mathrm{X} 7$.

1171. Parikh T, Goyal D, Chaudhari G, et al. 3.21 mood stabilizers, breastfeeding, and infant safety: a literature review. J Am Acad Child Adolesc Psychiatry. 2018;57(10):S188. doi: 10.1016/j.jaac.2018.09.179. Exclusion Code: X2.

1172. Parikh T, Goyal D, Chaudhari G, et al. Mood Stabilizers, Breastfeeding, and Infant Safety: A Literature Review. J Am Acad Child Adolesc Psychiatry. 2018;57(10):S188. doi: 10.1016/j.jaac.2018.09.179. Exclusion Code: X7. 
1173. Parikh T, Goyal D, Makani R, et al. Serotonin norepinephrine reuptake inhibitors (SNRI), selective serotonin reuptake inhibitors (SSRI), breastfeeding, and concerns for infant safety. J Am Acad Child Adolesc Psychiatry. 2017;56(10):S274. doi: 10.1016/j.jaac.2017.09.341. Exclusion Code: X7.

1174. Park M, Brain U, Grunau RE, et al. Maternal depression trajectories from pregnancy to 3 years postpartum are associated with children's behavior and executive functions at 3 and 6 years. Arch Womens Ment Health. 2018;21(3):353-63. doi: 10.1007/s00737-017-0803-0. PMID: 201802379-001. Exclusion Code: X4.

1175. Park M, Oberlander TF. In-utero Selective Serotonin Reuptake Inhibitor Antidepressant Exposure: Fetal Programing and Developmental Interactions With Context. Clin Pharmacol Ther. 2018 Oct;104(4):6168. doi: 10.1002/cpt.1134. PMID: 30054914. Exclusion Code: X1.

1176. Park Y, Hernández-Díaz S, Bateman BT, et al. Utilization of antipsychotics and stimulants during pregnancy among publicly insured women in the United States. Pharmacoepidemiol Drug Saf. 2015;24:2056. doi: 10.1002/pds.3838. Exclusion Code: $\mathrm{X} 5$.

1177. Partridge MC, Salisbury AL, LaGasse LL. Fine Motor Differences and Prenatal Serotonin Reuptake Inhibitors Exposure. J Pediatr. 2016 Aug;175:144-9.e1. doi: 10.1016/j.jpeds.2016.04.065. PMID: 27215778. Exclusion Code: X5.

1178. Passoforte P, Frappampina R, De Venuto C, et al. An important and unusual cause of hypoalbuminaemia. Dig Liver Dis. 2013;45:S174-S5. Exclusion Code: X7.

1179. Pastuszak A, Milich V, Chan S, et al. Prospective assessment of pregnancy outcome following first trimester exposure to benzodiazepines. Canadian Journal of Clinical Pharmacology. 1996;3(4):167-71. Exclusion Code: X3.

1180. Patrick MJ, Tilstone WJ, Reavey P. Diazepam and breast-feeding. Lancet. 1972 Mar 4;1(7749):542-3. PMID: 4110044. Exclusion Code: X7.
1181. Patton SW, Misri S, Corral MR, et al. Antipsychotic medication during pregnancy and lactation in women with schizophrenia: evaluating the risk. Can J Psychiatry. 2002 Dec;47(10):959-65. doi: 10.1177/070674370204701008. PMID: 12553132. Exclusion Code: X7.

1182. Paulus W, Schloemp S, Stoz F. Paroxetine exposure during pregnancy: Increased risk of congenital malformations? Arch Gynecol Obstet. 2010;282:S79. doi: 10.1007/s00404010-1634-7. Exclusion Code: X4.

1183. Paulzen M, Schoretsanitis G, Gründer G, et al. Pregnancy exposure to venlafaxineTherapeutic drug monitoring in maternal blood, amniotic fluid and umbilical cord blood and obstetrical outcomes. J Affect Disord. 2020;266:578-84. doi: 10.1016/j.jad.2020.02.010. PMID: 202022174-076. Exclusion Code: X5.

1184. Paulzen M, Schoretsanitis G, Gründer G, et al. Pregnancy exposure to venlafaxineTherapeutic drug monitoring in maternal blood, amniotic fluid and umbilical cord blood and obstetrical outcomes. J Affect Disord. 2020 Apr 1;266:578-84. doi: 10.1016/j.jad.2020.02.010. PMID: 32056930. Exclusion Code: X5.

1185. Pawlby SJ, Pariante CM. Mental disorders in the perinatal period: Effects on mothers and babies. Arch Womens Ment Health. 2013;16:S71-S2. doi: 10.1007/s00737-0130355-x. Exclusion Code: X7.

1186. Pawluski JL, Brain UM, Underhill CM, et al. Prenatal SSRI exposure alters neonatal corticosteroid binding globulin, infant cortisol levels, and emerging HPA function. Psychoneuroendocrinology. 2012 Jul;37(7):1019-28. doi: 10.1016/j.psyneuen.2011.11.011. PMID: 22177580. Exclusion Code: X2.

1187. Pawluski JL, Galea LA, Brain U, et al. Neonatal S100B protein levels after prenatal exposure to selective serotonin reuptake inhibitors. Pediatrics. 2009 Oct;124(4):e66270. doi: 10.1542/peds.2009-0442. PMID: 19786426. Exclusion Code: X2. 
1188. Payne JL. Use of antidepressants in the second trimester is associated with reduced pregnancy duration, and third trimester antidepressant use with infant convulsions. Evid Based Nurs. 2013 Jul;16(3):74-5. doi: 10.1136/eb-2012-101002. PMID: 23171571. Exclusion Code: X1.

1189. Payne JL. Antidepressant exposure during the first trimester does not increase the risk of cardiac malformations in infants. Evid Based Ment Health. 2015 May;18(2):48. doi: 10.1136/eb-2014-101933. PMID: 25398873. Exclusion Code: X1.

1190. Payne JL, Kimmel M. Family history, not lack of medication use, is associated with the development of postpartum depression in a high risk sample. Biol Psychiatry. 2014;75(9):125S-6S. doi: 10.1016/j.biopsych.2014.03.014. Exclusion Code: X4.

1191. Pedersen L, Henriksen T, Bech B, et al. Antidepressant exposure during pregnancy and child behavior. Acta Obstet Gynecol Scand. 2012;91:28-9. doi: 10.1111/j.16000412.2012.01435.x. Exclusion Code: X9.

1192. Pedersen LH, Henriksen TB, Olsen J. Fetal exposure to antidepressants and normal milestone development at 6 and 19 months of age. Pediatrics. 2010 Mar;125(3):e600-8. doi: 10.1542/peds.2008-3655. PMID: 20176667. Exclusion Code: X5.

1193. Peindl KS. The use of nortriptyline for prevention of postpartum depression in a high-risk group of women. 152nd annual meeting of the american psychiatric association. 1999 may 15-20, washington DC. 1999. PMID: CN-00283536. Exclusion Code: X9.

1194. Peng M, Gao K, Ding Y, et al. Effects of prenatal exposure to atypical antipsychotics on postnatal development and growth of infants: a case-controlled, prospective study. Psychopharmacology (Berl). 2013 Aug;228(4):577-84. doi: 10.1007/s00213013-3060-6. PMID: 23559219. Exclusion Code: X4.
1195. Pereira CM, Pacagnella RC, Parpinelli MA, et al. Drug Use during Pregnancy and its Consequences: A Nested Case Control Study on Severe Maternal Morbidity. Rev Bras Ginecol Obstet. 2018 Sep;40(9):51826. doi: 10.1055/s-0038-1667291. PMID: 30064145. Exclusion Code: X2.

1196. Perera C, Patterson S, Bruxner G. 'Conceivably Neglected' - Are prescribers sufficiently aware of the risks of prescribing sodium valproate to women with mental illness? Australas Psychiatry. 2019 Apr;27(2):125-8. doi: 10.1177/1039856219828175. PMID: 30763123. Exclusion Code: X1.

1197. Perkins C, Jr. Treatment discontinuation increases risk of symptom recurrence for pregnant women with bipolar. Primary Psychiatry. 2008;15(2):20-1. PMID: 200818360-005. Exclusion Code: X1.

1198. Perlis RH. Autism and antidepressant use in pregnancy. J Pediatr. 2016 Jul;174:278. doi: 10.1016/j.jpeds.2016.04.072. PMID: 27346512. Exclusion Code: X1.

1199. Perucca E, Tomson T. Prenatal exposure to antiepileptic drugs. Lancet. 2006 May 6;367(9521):1467-9. doi: 10.1016/s01406736(06)68627-3. PMID: 16679145. Exclusion Code: X1.

1200. Petersen GL, Hougaard CO, Pinborg A, et al. Mortality among 42,915 women in Denmark treated with assisted reproductive technology ñ a register-based nationwide cohort study. Hum Reprod. 2013;28:i81. doi: 10.1093/humrep/det183. Exclusion Code: X2.

1201. Petersen I, Evans S, Marston L, et al. Selective serotonin reuptake inhibitors and congenital heart anomalies: Comparative cohort studies. Arch Womens Ment Health. 2015;18(2):367. doi: 10.1007/s00737-0140488-6. Exclusion Code: X9.

1202. Petersen I, Gilbert R, Evans S, et al. SSRI and risk of congenital cardiac abnormalities. Pharmacoepidemiol Drug Saf. 2010;19:S211. doi: 10.1002/pds.2019. Exclusion Code: X9. 
1203. Petersen I, McCrea RL, Sammon CJ, et al. Antipsychotics in pregnancy: Comparative cohort studies of women treated before and during pregnancy. Pharmacoepidemiol Drug Saf. 2015;24:260. doi: 10.1002/pds.3838. Exclusion Code: X9.

1204. Petersen I, McCrea RL, Sammon CJ, et al. Risks and benefits of psychotropic medication in pregnancy: cohort studies based on UK electronic primary care health records. Health Technol Assess. 2016 Mar;20(23):1-176. doi: 10.3310/hta20230. PMID: 27029490. Exclusion Code: X7.

1205. Petersen I, Peltola T, Kaski S, et al. Postnatal depression and pharmacological and non-pharmacological treatment. Pharmacoepidemiol Drug Saf. 2017;26:4101. doi: 10.1002/pds.4275. Exclusion Code: $\mathrm{X} 4$.

1206. Petersen I, Sammon C, Walters K, et al. Associations between treatment with Selective Serotonin Reuptakeinhibitors (SSRIs) before and after pregnancy. Pharmacoepidemiology and drug safety. Conference: 32 nd international conference on pharmacoepidemiology and therapeutic risk management. Ireland. Conference start: 20160825. Conference end: 20160828. 2016;25:236-7. doi: 10.1002/pds.4070. PMID: CN-01293587. Exclusion Code: X5.

1207. Pinheiro E, Bogen DL, Hoxha D, et al. Sertraline and breastfeeding: review and meta-analysis. Arch Womens Ment Health. 2015 Apr;18(2):139-46. doi: 10.1007/s00737-015-0499-y. PMID: 25589155. Exclusion Code: X7.

1208. Pinheiro EA, Wisner KL, Clark CT. Quetiapine dose adjustments in pregnant and postpartum women with bipolar disorder. J Clin Psychopharmacol. 2018 Feb;38(1):8991. doi: 10.1097/jcp.0000000000000820. PMID: 29194089. Exclusion Code: X1.

1209. Pinkofsky HB, Fitz-Gerald MJ, Reeves RR. Psychotropic treatment during pregnancy. Am J Psychiatry. 1997 May;154(5):718-9. PMID: 9137147. Exclusion Code: X7.

1210. Pinto N, Koren G. Research on maternal and fetal safety after exposure to antidepressants in utero. Am J Obstet Gynecol. 2003 Dec;189(6):1810-1; author reply 1. PMID: 14710127. Exclusion Code: X1.
1211. Pinzon R. Fetal exposure to valproate was associated with lower IQ scores at 3 years of age than exposure to other antiepileptic drugs. Evid Based Med. 2009;14(5):153. doi: 10.1136/ebm.14.5.153. Exclusion Code: $\mathrm{X} 2$.

1212. Platt JE, Friedhoff AJ, Broman SH, et al. Effects of prenatal exposure to neuroleptic drugs on children's growth.

Neuropsychopharmacology. 1988

Sep;1(3):205-12. PMID: 2908019.

Exclusion Code: X4.

1213. Podrebarac SK, Duerden EG, Chau V, et al. Antenatal exposure to antidepressants is associated with altered brain development in very preterm-born neonates. Neuroscience. 2017 Feb 7;342:252-62. doi: 10.1016/j.neuroscience.2016.11.025. PMID: 27890829. Exclusion Code: X2.

1214. Poels EMP, Schrijver L, Kamperman AM, et al. Long-term neurodevelopmental consequences of intrauterine exposure to lithium and antipsychotics: a systematic review and meta-analysis. Eur Child Adolesc Psychiatry. 2018 Sep;27(9):120930. doi: 10.1007/s00787-018-1177-1. PMID: 29948232. Exclusion Code: X7.

1215. Poinsat T, Lachuer C, Poinsignon V, et al. Prescription analysis in women of childbearing age hospitalised in psychiatry:

Teratogenic risk. Eur Neuropsychopharmacol. 2016;26:S587. Exclusion Code: X5.

1216. Pokorny A, Melkova J, Broscheova M, et al. [Diazepam, premature labor and the status of the neonate]. Cesk Gynekol. 1986 Sep;51(8):601-6. PMID: 3779855. Exclusion Code: X8.

1217. Pokorny A, Melkova J, Broscheova M, et al. [The side-effects of diazepam administered to the mother at the end of pregnancy on the condition of the neonate]. Cesk Pediatr. 1986 Aug;41(8):455-8. PMID: 3757128. Exclusion Code: X8.

1218. Polen KN, Rasmussen SA, Riehle-Colarusso $\mathrm{T}$, et al. Association between reported venlafaxine use in early pregnancy and birth defects, national birth defects prevention study, 1997-2007. Birth Defects Res A Clin Mol Teratol. 2013 Jan;97(1):28-35. doi: 10.1002/bdra.23096. PMID: 23281074. Exclusion Code: X2. 
1219. Polytherapy Nulman I, Sadowski A, Todorow M, et al. In utero exposure to second generation antipsychotics: Pregnancy outcomes considering. Birth Defects Research Part A - Clinical and Molecular Teratology. 2013;97(5):364. doi: 10.1002/bdra.23146. Exclusion Code: X9.

1220. Ponder KL, Salisbury A, McGonnigal B, et al. Maternal depression and anxiety are associated with altered gene expression in the human placenta without modification by antidepressant use: implications for fetal programming. Dev Psychobiol. 2011 Nov;53(7):711-23. doi: 10.1002/dev.20549. PMID: 21547899. Exclusion Code: X5.

1221. Potvin W, Evans MF. Outcome of pregnancy following mothers' use of new SSRIs. Can Fam Physician. 1999 Jun;45:1477-9. PMID: 10386211. Exclusion Code: X1.

1222. Poulton A, Nanan R. Reproductive outcomes of women treated with stimulant medication for AttentionDeficit/Hyperactivity Disorder (ADHD). ADHD Attention Deficit and Hyperactivity Disorders. 2015;7:S72-S3. doi: 10.1007/s12402-015-0169-y. Exclusion Code: X2.

1223. Powell J, Manoharan M, Reed J. How should we manage bipolar II disorder in the perinatal period? BJPsych Advances. 2020;26(1):61-3. doi: 10.1192/bja.2019.27. Exclusion Code: X1.

1224. Powell JG, Garland S, Preston K, et al. Brexanolone (Zulresso): Finally, an FDAApproved Treatment for Postpartum Depression. Ann Pharmacother. 2020 Feb;54(2):157-63. doi: 10.1177/1060028019873320. PMID: 31476884. Exclusion Code: X7.

1225. Pradeep B, Sruthi K, Soumya Lakshmi K, et al. Drug utilization assessment in pregnancy women: A cross sectional study. International Research Journal of Pharmacy. 2019;10(2):109-13. doi: 10.7897/22308407.100252. Exclusion Code: X2.
1226. Prady SL, Hanlon I, Fraser LK, et al. A systematic review of maternal antidepressant use in pregnancy and short- and long-term offspring's outcomes. Arch Womens Ment Health. 2018 Apr;21(2):127-40. doi: 10.1007/s00737-017-0780-3. PMID: 29027013. Exclusion Code: X7.

1227. Prakash C, Hatters-Friedman S, MollerOlsen C, et al. Maternal and fetal outcomes after lamotrigine use in pregnancy: a retrospective analysis from an urban maternal mental health centre in New Zealand. Psychopharmacol Bull. 2016 Aug 15;46(2):63-9. PMID: 27738382. Exclusion Code: X4.

1228. Pray WS. Treating common problems in the pregnant patient. 2009;34((Pray W.S.) Nonprescription Products and Devices, College of Pharmacy, Southwestern Oklahoma State University, Weatherford, OK, United States):12-5. Exclusion Code: $\mathrm{X} 1$.

1229. Preisig R, Landman ME. Use of perphenazine in anxiety states and hyperemesis; report of 135 cases. Am Pract Dig Treat. 1958 May;9(5):740-4. PMID: 13521249. Exclusion Code: X7.

1230. Previti G, Marino M, Pawlby S, et al. Neuro developmental outcome in off spring of women treated for antenatal depression: A systematic review. Arch Womens Ment Health. 2015;18(2):347. doi: 10.1007/s00737-014-0488-6. Exclusion Code: X7.

1231. Previti G, Pawlby S, Chowdhury S, et al. Neurodevelopmental outcome for offspring of women treated for antenatal depression: a systematic review. Arch Womens Ment Health. 2014 Dec;17(6):471-83. doi: 10.1007/s00737-014-0457-0. PMID: 25212663. Exclusion Code: X7.

1232. Punamäki R-L, Repokari L, Vilska S, et al. Maternal mental health and medical predictors of infant developmental and health problems from pregnancy to one year: Does former infertility matter? Infant Behav Dev. 2006;29(2):230-42. doi: 10.1016/j.infbeh.2005.12.001. PMID: 200602877-009. Exclusion Code: X2. 
1233. Qiu CL, Xiao B, Xie WJ. A controlled clinical trial of citalopram and citalopram combined with psychotherapy in the treatment of postpartum depression. Chinese mental health journal. 2005;19(12):847-8.

PMID: CN-00712781. Exclusion Code: X8.

1234. Rabinowitz I, Baruch Y, Barak Y. Highdose escitalopram for the treatment of obsessive-compulsive disorder. Int Clin Psychopharmacol. 2008 Jan;23(1):49-53. doi: 10.1097/YIC.0b013e3282f0f0c5. PMID: 18090508. Exclusion Code: X2.

1235. Rado JT, Hernandez EI, Janicak PG. Novel approaches for managing schizophrenia. Psychopharm Review. 2013;48(11):81-7. doi: 10.1097/01.Psyphr.0000437783.35936.18. Exclusion Code: X1.

1236. Radojcic MR, El Marroun H, Miljkovic B, et al. Prenatal exposure to anxiolytic and hypnotic medication in relation to behavioral problems in childhood: A population-based cohort study. Neurotoxicol Teratol. 2017 May;61:58-65. doi: 10.1016/j.ntt.2017.02.005. PMID: 28259732. Exclusion Code: X4.

1237. Rahimi R, Nikfar S, Abdollahi M. Pregnancy outcomes following exposure to serotonin reuptake inhibitors: a metaanalysis of clinical trials. Reprod Toxicol. 2006 Nov;22(4):571-5. doi: 10.1016/j.reprotox.2006.03.019. PMID: 16720091. Exclusion Code: X7.

1238. Rahman A, Fisher J, Bower P, et al. Interventions for common perinatal mental disorders in women in low- and middleincome countries: a systematic review and meta-analysis. Bull World Health Organ. 2013 Aug 1;91(8):593-601i. doi: 10.2471/blt.12.109819. PMID: 23940407. Exclusion Code: X7.

1239. Rai D, Lee BK, Dalman C, et al. Parental depression, maternal antidepressant use during pregnancy, and risk of autism spectrum disorders: population based casecontrol study. BMJ. 2013 Apr 19;346:f2059. doi: 10.1136/bmj.f2059. PMID: 23604083. Exclusion Code: X10.
1240. Rais TB, Rais A. Association between antidepressants use during pregnancy and autistic spectrum disorders: A meta-analysis. Innov Clin Neurosci. 2014;11(5-6):18-22. Exclusion Code: X7.

1241. Rajkumar AP, Jacob KS. Are antidepressants safe during pregnancy? $\mathrm{Br} \mathrm{J}$ Psychiatry. 2008 Dec;193(6):508; author reply -9. doi: 10.1192/bjp.193.6.508. PMID: 19043161. Exclusion Code: X1.

1242. Ram D, Gandotra S. Antidepressants, anxiolytics, and hypnotics in pregnancy and lactation. Indian J Psychiatry. 2015 Jul;57(Suppl 2):S354-71. doi: 10.4103/0019-5545.161504. PMID: 26330654. Exclusion Code: X7.

1243. Rasalam AD, Hailey H, Williams JH, et al. Characteristics of fetal anticonvulsant syndrome associated autistic disorder. Dev Med Child Neurol. 2005 Aug;47(8):551-5. PMID: 16108456. Exclusion Code: X2.

1244. Rasgon N. Tying it all together: Creating an optimal management paradigm for women with mood disorders during perinatal period. Arch Womens Ment Health. 2013;16:S76. doi: 10.1007/s00737-013-0355-x. Exclusion Code: X7.

1245. Raudzus J, Misri S. Managing unipolar depression in pregnancy. Curr. Opin. Psychiatry. 2009 Jan;22(1):13-8. doi: 10.1097/YCO.0b013e3283169375. PMID: 19122529. Exclusion Code: X1.

1246. Redhead K, Walsh J, Galbally M, et al. Obstructive sleep apnea is associated with depressive symptoms in pregnancy. Sleep. 2020 May 12;43(5). doi: 10.1093/sleep/zsz270. PMID: 31782959. Exclusion Code: X2.

1247. Redpath TH, Gayford JJ. The side effects of carbamazepine therapy. Oral Surg Oral Med Oral Pathol. 1968 Sep;26(3):299-303. PMID: 5243906. Exclusion Code: X7.

1248. Reebye P, Morison SJ, Panikkar H, et al. Affect expression in prenatally psychotropic exposed and nonexposed mother-infant dyads. Infant Ment Health J. 2002;23(4):403-16. doi: 10.1002/imhj.10025. PMID: 2002-17492004. Exclusion Code: X4. 
1249. Reefhuis J, Devine O, Friedman JM, et al. Specific SSRIs and birth defects: Bayesian analysis to interpret new data in the context of previous reports. BMJ. $2015 \mathrm{Jul}$ 8;351:h3190. doi: 10.1136/bmj.h3190. PMID: 26156519. Exclusion Code: X4.

1250. Reeves I, Kall M, Creighton S, et al. Using protease inhibitors in first-line HAART: Is clinician anxiety about poor adherence justified? HIV Med. 2010;11:29. doi: 10.1111/j.1468-1293.2010.00841-2.x. Exclusion Code: X2.

1251. Reinhart M, Patton C, Chawla A, et al. Treatment of postpartum depression: a systematic literature review. Value Health. 2017;20(9):A717. doi: 10.1016/j.jval.2017.08.3056. Exclusion Code: X7.

1252. Reinisch JM, Simon NG, Gandelman R. Prenatal exposure to prednisone permanently alters fighting behavior of female mice. Pharmacol Biochem Behav. 1980 Feb;12(2):213-6. PMID: 7189593. Exclusion Code: X2.

1253. Reis M, Kallen B. Maternal use of antipsychotics in early pregnancy and delivery outcome. J Clin Psychopharmacol. 2008 Jun;28(3):279-88. doi: 10.1097/JCP.0b013e318172b8d5. PMID: 18480684. Exclusion Code: X10.

1254. Rejnö G, Lundholm C, Öbeg S, et al. Maternal anxiety, depression and asthma and adverse pregnancy outcomes-a population based study. International Journal of Gynecology and Obstetrics. 2018;143:188. doi: 10.1002/ijgo.12582. Exclusion Code: X3.

1255. Rejnö G, Lundholm C, Öberg S, et al. Maternal anxiety, depression and asthma and adverse pregnancy outcomes - a population based study. Sci Rep. 2019 Sep 11;9(1):13101. doi: 10.1038/s41598-01949508-z. PMID: 31511586 . Exclusion Code: $\mathrm{X} 2$.

1256. Richards N, Reith D, Stitely M, et al. Antiepileptic drug exposure in pregnancy and pregnancy outcome from national drug usage data. BMC Pregnancy Childbirth. 2018;18(1). doi: 10.1186/s12884-018-1728y. Exclusion Code: X4.
1257. Rifkin A. Lithium discontinuation during pregnancy. Am J Psychiatry. 2001 Oct;158(10):1741-2. doi: 10.1176/appi.ajp.158.10.1741. PMID: 11579024. Exclusion Code: X1.

1258. Rifkin A, Rifkin W. Antidepressant treatment and relapse of depression during pregnancy. JAMA. 2006 Jul 12;296(2):1656; author reply 6-7. doi: 10.1001/jama.296.2.165-b. PMID: 16835415. Exclusion Code: X1.

1259. Riggin L, Frankel Z, Moretti M, et al. The fetal safety of fluoxetine: a systematic review and meta-analysis. J Obstet Gynaecol Can. 2013 Apr;35(4):362-9. doi: 10.1016/s1701-2163(15)30965-8. PMID: 23660045. Exclusion Code: X7.

1260. Robakis TK, Williams KE. Biologically based treatment approaches to the patient with resistant perinatal depression. Arch Womens Ment Health. 2013 Oct;16(5):34351. doi: 10.1007/s00737-013-0366-7. PMID: 23828097. Exclusion Code: X1.

1261. Robbins NM. A longitudinal investigation of change in maternal depression, parenting practices and child attachment: ProQuest Information \& Learning; 2008. Exclusion Code: X7.

1262. Roberson EK. Using pregnancy risk assessment monitoring system data to investigate prescription drug use during pregnancy in Hawai' $i$ : ProQuest Information \& Learning; 2015. Exclusion Code: X7.

1263. Robertson Blackmore E, Craddock N, Rubinow D, et al. Correlates of risk for recurrent illness inwomen with postpartum psychosis. Arch Womens Ment Health. 2011;14:S44-S5. doi: 10.1007/s00737-0100203-1. Exclusion Code: X3.

1264. Robinson DS. Psychotropic drugs and pregnancy: Guidance for antidepressants. Primary Psychiatry. 2005;12(6):22-3. PMID: 2005-07400-004. Exclusion Code: $\mathrm{X} 1$.

1265. Robinson GE. Treatment of schizophrenia in pregnancy and postpartum. J Popul Ther Clin Pharmacol. 2012;19(3):e380-6. PMID: 23075483. Exclusion Code: X1. 
1266. Robinson GE, Einarson A. Risks of untreated depression outweigh any risks of SSRIs. Hum Reprod. 2013 Apr;28(4):11456. doi: 10.1093/humrep/det006. PMID: 23406973. Exclusion Code: X1.

1267. Robinson R, Lahti-Pulkkinen M, Schnitzlein $\mathrm{D}$, et al. Mental health outcomes of adults born very preterm or with very low birth weight: A systematic review. Semin Fetal Neonatal Med. 2020 Apr 28:101113. doi: 10.1016/j.siny.2020.101113. PMID: 32402835. Exclusion Code: X2.

1268. Robinson-Wolrath S, Lewis C, Kohan R, et al. SSRI/SNRI use during pregnancy is associated with increased antenatal and labour complications. J Paediatr Child Health. 2015;51:24. doi: 10.1111/jpc.128842. Exclusion Code: X9.

1269. Robinson-Wolrath S, Lewis C, Kohan R, et al. SSRI and SNRI use during pregnancy is associated with both obstetric and neonatal complications. BJOG. 2016;123:168-9. doi: 10.1111/1471-0528.14106. Exclusion Code: $\mathrm{X} 4$.

1270. Roca A, Garcia-Esteve L, Imaz ML, et al. Obstetrical and neonatal outcomes after prenatal exposure to selective serotonin reuptake inhibitors: the relevance of dose. $\mathrm{J}$ Affect Disord. 2011 Dec;135(1-3):208-15. doi: 10.1016/j.jad.2011.07.022. PMID: 21890210. Exclusion Code: X2.

1271. Rogers SC, Wheatley D, Galbraith AW, et al. Psychotropic drugs and fertility. J Psychosom Res. 1970;14(4):383-6. doi: 10.1016/0022-3999(70)90005-X. PMID: 1972-01118-001. Exclusion Code: X5.

1272. Romaine E, McAllister-Williams RH. Guidelines on prescribing psychotropic medication during the perinatal period. $\mathrm{Br} \mathrm{J}$ Hosp Med (Lond). 2019 Jan 2;80(1):27-32. doi: 10.12968/hmed.2019.80.1.27. PMID: 30592677. Exclusion Code: X1.

1273. Rönnqvist I, Brus O, Hammar Å, et al. Rehospitalization of Postpartum Depression and Psychosis After Electroconvulsive Therapy: A Population-Based Study With a Matched Control Group. J ECT. 2019 Feb 20. doi: 10.1097/yct.0000000000000578. PMID: 30807499. Exclusion Code: X3.
1274. Rose S, Borowski KS, White WM, et al. Adderall and pregnancy: Neonatal outcomes following prenatal prescription mixed amphetamine exposure. Obstet Gynecol. 2018;131:39S-40S. Exclusion Code: X3.

1275. Ross LE, Grigoriadis S, Mamisashvili L, et al. Selected pregnancy and delivery outcomes after exposure to antidepressant medication: a systematic review and metaanalysis. JAMA Psychiatry. 2013 Apr;70(4):436-43. doi: 10.1001/jamapsychiatry.2013.684. PMID: 23446732. Exclusion Code: X7.

1276. Rosso G, Albert U, Di Salvo G, et al. Lithium prophylaxis during pregnancy and the postpartum period in women with lithium-responsive bipolar I disorder. Arch Womens Ment Health. 2016 Apr;19(2):42932. doi: 10.1007/s00737-016-0601-0. PMID: 26790685. Exclusion Code: X4.

1277. Rotem-Kohavi N, Williams LJ, Muller AM, et al. Hub distribution of the brain functional networks of newborns prenatally exposed to maternal depression and SSRI antidepressants. Depress Anxiety. 2019 Aug;36(8):753-65. doi: 10.1002/da.22906. PMID: 31066992 . Exclusion Code: X5.

1278. Rubertsson C, Pallant JF, Sydsjö G, et al. Maternal depressive symptoms have a negative impact on prenatal attachmentFindings from a Swedish community sample. J Reprod Infant Psychol. 2015;33(2):153-64. doi: 10.1080/02646838.2014.992009. PMID: 2015-12022-005. Exclusion Code: X2.

1279. Rubinchik SM, Kablinger AS, Gardner JS. Medications for panic disorder and generalized anxiety disorder during pregnancy. Prim Care Companion J Clin Psychiatry. 2005;7(3):100-5. PMID: 16027764. Exclusion Code: X7.

1280. Ruchkin V, Martin A. SSRIs and the developing brain. Lancet. 2005 Feb 511;365(9458):451-3. doi: 10.1016/s01406736(05)17877-5. PMID: 15705440. Exclusion Code: X1. 
1281. Rurak D, Lim K, Sanders A, et al. Third trimester fetal heart rate and Doppler middle cerebral artery blood flow velocity characteristics during prenatal selective serotonin reuptake inhibitor exposure. Pediatr Res. 2011 Jul;70(1):96-101. doi: 10.1203/PDR.0b013e31821ba11a. PMID: 21436759. Exclusion Code: X2.

1282. Ryan D, Milis L, Misri N. Depression during pregnancy. Can Fam Physician. 2005 Aug;51:1087-93. PMID: 16121830. Exclusion Code: X7.

1283. Rybakowski J, Cubała WJ, Gałecki P, et al. Recommendations of the Polish Psychiatric Association regarding the treatment of affective disorders in women of childbearing age. Part II: Bipolar disorder. Psychiatr Pol. 2019 Apr 30;53(2):263-76. doi: 10.12740/pp/103555. PMID: 31317957. Exclusion Code: X1.

1284. Saatçioğlu Ö, Tomruk NB. Antidepressant treatment strategies in the perinatal period with a focus on SSRI use. Nöropsikiyatri Arşivi. 2013;50(1):93-4. doi: 10.4274/Npa.y7029. PMID: 2013-11432017. Exclusion Code: X1.

1285. Saccone G, Eke AC, Berghella V. Selective serotonin reuptake inhibitor (SSRI) use during pregnancy and risk of preterm birth: A systematic review and metaanalysis. Am J Obstet Gynecol. 2016;214(1):S443. Exclusion Code: X7.

1286. Saeedi S. Effect of exercise program on symptoms of postpartum depression. Iranian journal of obstetrics, gynecology and infertility. 2013;15(34):26-31. PMID: CN00906776. Exclusion Code: X8.

1287. Sagiv SK, Epstein JN, Bellinger DC, et al. Pre- and postnatal risk factors for ADHD in a nonclinical pediatric population. J Atten Disord. 2013 Jan; 17(1):47-57. doi: 10.1177/1087054711427563. PMID: 22298092. Exclusion Code: X4.

1288. Saiz Garcia H, Álvarez De Mon MA, Pereira V, et al. Antipsychotics drugs during pregnancy. Eur Psychiatry. 2018;48:S325. doi: 10.1016/j.eurpsy.2017.12.016. Exclusion Code: X7.

1289. Sajdak S, Paluch A, Kampioni M, et al. Antiphospholipid syndrome in the course of pregnancy. Ginekologia i Poloznictwo. 2011;20(2):52-67. Exclusion Code: X2.
1290. Sakai T, Ohtsu F, Mori C, et al. Signal of miscarriage with aripiprazole: a disproportionality analysis of the Japanese adverse drug event report database. Drug Saf. 2017 Nov;40(11):1141-6. doi: 10.1007/s40264-017-0560-z. PMID: 28664356. Exclusion Code: X7.

1291. Salameh TN, Hall LA. Depression, Anxiety, and Substance Use Disorders and Treatment Receipt Among Pregnant Women in the United States: A Systematic Review of Trend and Population-Based Studies. Issues Ment Health Nurs. 2020 Jan;41(1):7-23. doi: 10.1080/01612840.2019.1667460. PMID: 31855501. Exclusion Code: X3.

1292. Salisbury AL. Examination of fetal neurobehavior at the time of exposure to maternal depression and antidepressant use. Arch Womens Ment Health. 2011;14:S47S8. doi: 10.1007/s00737-010-0203-1. Exclusion Code: X5.

1293. Salisbury AL. Influences of maternal mood, SRI treatment, anxiety, and sleep on fetal and infant neurobehavior. Arch Womens Ment Health. 2011;14:S48. doi: 10.1007/s00737-010-0203-1. Exclusion Code: X5.

1294. Salisbury AL, Mattera J, Miller-Loncar C, et al. The effects of prenatal exposure to maternal depression and antidepressant treatment on sleep state development in toddlers. Sleep. Conference: 31st anniversary meeting of the associated professional sleep societies, LLC, SLEEP 2017. United states. 2017;40:A20. PMID: $\mathrm{CN}-01375417$. Exclusion Code: X5.

1295. Salisbury AL, Papandonatos GD, Stroud LR, et al. Prenatal antidepressant exposures and gastrointestinal complaints in childhood: A gut-brain axis connection? Dev Psychobiol. 2020 Mar 13. doi: 10.1002/dev.21966. PMID: 32167584. Exclusion Code: X4.

1296. Salisbury AL, Young ME, Singer-Clark T. Fetal and newborn behavioral state development during and after antidepressant exposure. Sleep. 2013;36:A352-A3. Exclusion Code: X5. 
1297. Salkeld E, Ferris LE, Juurlink DN. The risk of postpartum hemorrhage with selective serotonin reuptake inhibitors and other antidepressants. J Clin Psychopharmacol. 2008 Apr;28(2):230-4. doi: 10.1097/JCP.0b013e318166c52e. PMID: 18344737. Exclusion Code: X5.

1298. Samalin L, Abbar M, Courtet P, et al. [French Society for Biological Psychiatry and Neuropsychopharmacology task force: Formal Consensus for the prescription of depot antipsychotics]. Encephale. 2013 Dec;39 Suppl 4:189-203. doi: 10.1016/s0013-7006(13)70121-0. PMID: 24373464. Exclusion Code: X8.

1299. Samyraju M, Natarajan D, Waller K, et al. Managing mental health for women with previous history of schizophrenia during pregnancy - How can we improve our care? BJOG. 2012;119:106. doi: 10.1111/j.14710528.2012.03376.x. Exclusion Code: X3.

1300. Sanlorenzo LA, Cooper WO, Dudley JA, et al. Increased Severity of Neonatal Abstinence Syndrome Associated With Concomitant Antenatal Opioid and Benzodiazepine Exposure. Hosp Pediatr. 2019 Aug;9(8):569-75. doi: 10.1542/hpeds.2018-0227. PMID: 31262946. Exclusion Code: X2.

1301. Santone G, Ricchi G, Rocchetti D, et al. Is the exposure to antidepressant drugs in early pregnancy a risk factor for spontaneous abortion? A review of available evidences. Epidemiol Psichiatr Soc. 2009 JulSep;18(3):240-7. PMID: 20034202. Exclusion Code: X7.

1302. Santos F, Sola I, Rigau D, et al. Quality assessment of clinical practice guidelines for the prescription of antidepressant drugs during pregnancy. Pharmacoepidemiol Drug Saf. 2011;20:S59-S60. doi: 10.1002/pds.2206. Exclusion Code: X7.

1303. Santucci AK, Singer LT, Wisniewski SR, et al. Does prenatal exposure to serotonin reuptake inhibitors or maternal major depression impact infant developmental outcomes? Birth Defects Research Part A Clinical and Molecular Teratology. 2015;103(5):457. doi: 10.1002/bdra.23387. Exclusion Code: X9.
1304. Sanz EJ, De-las-Cuevas C, Kiuru A, et al. Selective serotonin reuptake inhibitors in pregnant women and neonatal withdrawal syndrome: a database analysis. Lancet. 2005 Feb 5-11;365(9458):482-7. doi: 10.1016/s0140-6736(05)17865-9. PMID: 15705457. Exclusion Code: X4.

1305. Savage DD, Reyes E. Prenatal exposure to ethanol retards the development of kindling in adult rats. Exp Neurol. 1985 Sep;89(3):583-91. PMID: 4029337. Exclusion Code: X2.

1306. Scheffers JWT, Dorning H, Petersen I. A systematic review on prescribing patterns of Antidepresssants before, during, and after pregnancy. Pharmacoepidemiol Drug Saf. 2019;28:382. doi: 10.1002/pds.4864. Exclusion Code: X5.

1307. Scheidell J, Khan M. Stress, post-traumatic stress disorder, and sexual and reproductive health in a nationally-representative sample of us women. Sex Transm Infect. 2019;95:A174. doi: 10.1136/sextrans-2019sti.439. Exclusion Code: X3.

1308. Schifrin BS, Harwell R, Rubinstein T, et al. Maternal heart rate pattern: a confounding factor in intrapartum fetal surveillance. Prenat Neonatal Med. 2001 Feb;6(1):75-82. PMID: WOS:000167935800010. Exclusion Code: X7.

1309. Schmiedel K. Pregnant and depressed: more psychological diseases in children when the pregnant woman has taken antidepressants. Dtsch Apoth Ztg. 2017;157(43). Exclusion Code: X8.

1310. Schnabel A, Hahn N, Muellenbach R, et al. [Obstetric analgesia in German clinics. Remifentanil as alternative to regional analgesia]. Anaesthesist. 2011 Nov;60(11):995-1001. doi: 10.1007/s00101011-1933-9. PMID: 21918824. Exclusion Code: X8.

1311. Schoretsanitis G, Spigset O, Stingl JC, et al. The impact of pregnancy on the pharmacokinetics of antidepressants: a systematic critical review and meta-analysis. Expert Opin Drug Metab Toxicol. 2020 May;16(5):431-40. doi: 10.1080/17425255.2020.1750598. PMID: 32238008. Exclusion Code: X5. 
1312. Schou M. What happened later to the lithium babies? A follow-up study of children born without malformations. Acta Psychiatr Scand. 1976 Sep;54(3):193-7. PMID: 970196. Exclusion Code: X4.

1313. Schou M, Weinstein MR. Problems of lithium maintenance treatment during pregnancy, delivery and lactation. Agressologie. 1980;21(A):7-9. PMID: 7246885. Exclusion Code: X11.

1314. Scolnik D, Nulman I, Rovet J, et al. Neurodevelopment of children exposed in utero to phenytoin and carbamazepine monotherapy. JAMA. 1994 Mar 9;271(10):767-70. PMID: 7509419. Exclusion Code: X4.

1315. Scrandis DA. Bipolar disorder in pregnancy: a review of pregnancy outcomes. J Midwifery Womens Health. 2017 Nov;62(6):673-83. doi: 10.1111/jmwh.12645. PMID: 29083536. Exclusion Code: X7.

1316. Scrandis DA. Antidepressant use in breastfeeding women. Nurse Pract. 2018 Oct;43(10):18-21. doi: 10.1097/01.npr.0000545001.45758.48. PMID: 30234822. Exclusion Code: X1.

1317. Seip M. Effects of antiepileptic drugs in pregnancy on the fetus and newborn infant. Ann Clin Res. 1973;5:205-7. Exclusion Code: X2.

1318. Serafini P, Lobo DS, Grosman A, et al. Fluoxetine treatment for anxiety in women undergoing in vitro fertilization. Int $\mathbf{J}$ Gynaecol Obstet. 2009 May;105(2):136-9. doi: 10.1016/j.ijgo.2008.12.013. PMID: 19201400. Exclusion Code: X5.

1319. Serati M, Buoli M, Redaelli M, et al. Which pregnant bipolar women have an increased risk of delayed psychiatric treatment? Eur Neuropsychopharmacol. 2015;25:S392-S3. Exclusion Code: X3.

1320. Serrano Drozdowskyj E. Antenatal depression and newborn outcome: Case study. Arch Womens Ment Health. 2011;14:S80. doi: 10.1007/s00737-0100203-1. Exclusion Code: X7.
1321. Seth S, Lewis AJ, Saffery R, et al. Maternal prenatal mental health and placental 11 betaHSD2 gene expression: initial findings from the mercy pregnancy and emotional wellbeing study. Int J Mol Sci. 2015 Nov 17;16(11):27482-96. doi: 10.3390/ijms 161126034. PMID: 26593902. Exclusion Code: X5.

1322. Shabbir O, Khan KS, Jawaid H. Brexanolone Therapy for Postpartum Depression. J Pak Med Assoc. 2020 Mar;70(3):565-6. doi: 10.5455/jpma.48397. PMID: 32207453. Exclusion Code: X1.

1323. Shah N. Mood disorder in the perinatal period. BMJ. 2012 Mar 1;344:e1209. doi: 10.1136/bmj.e1209. PMID: 22381523. Exclusion Code: X1.

1324. Shaikh K, Premji SS, Lalani S, et al. Ethnic disparity and exposure to supplements rather than adverse childhood experiences linked to preterm birth in Pakistani women. J Affect Disord. 2020 Apr 15;267:49-56. doi: 10.1016/j.jad.2020.01.180. PMID: 32063572. Exclusion Code: X2.

1325. Shallcross R, Bromley R, Irwin B, et al. The cognitive and language abilities of children exposed in utero to levetiracetam and sodium valproate: 3 -4 years of age. Epilepsia. 2011;52:249. doi: 10.1111/j.15281167.2011.03207.x. Exclusion Code: X2.

1326. Shallcross R, Bromley RL, Irwin B, et al. A first look at the language and developmental abilities of children aged three to four years exposed in utero to levetiracetam. on behalf of the liverpool and manchester neurodevelopment group and the UK epilepsy and pregnancy register. Epilepsy Curr. 2011;11(1). Exclusion Code: X2.

1327. Shallcross R, Bromley RL, Irwin B, et al. Developmental abilities of children exposed in utero to antiepileptic drugs: A comparison between sodium valproate and levetiracetam. Epilepsia. 2010;51:9. doi: 10.1111/j.1528-1167.2010.02658.x. Exclusion Code: X9.

1328. Sharma V, Burt VK, Ritchie HL. Assessment and treatment of bipolar II postpartum depression: a review. J Affect Disord. 2010 Sep;125(1-3):18-26. doi: 10.1016/j.jad.2009.09.014. PMID: 19837461. Exclusion Code: X7. 
1329. Sharma V, Doobay M, Baczynski C. Bipolar postpartum depression: an update and recommendations. J Affect Disord. 2017

Sep;219:105-11. doi:

10.1016/j.jad.2017.05.014. PMID:

28535448. Exclusion Code: X1.

1330. Sharma V, Khan M. Quetiapine in the acute treatment of bipolar postpartum depression. Bipolar Disorders. 2015;17:79. doi: 10.1111/bdi.12309. Exclusion Code: X4.

1331. Sharma V, Khan M, Sommerdyk C. Quetiapine in the acute treatment of bipolar postpartum depression: a chart review. J Clin Psychopharmacol. 2015 Dec;35(6):7335. doi: 10.1097/jcp.0000000000000415. PMID: 26444950. Exclusion Code: X1.

1332. Sharma V, Pope C1J. Pregnancy and bipolar disorders: A systematic review. The Journal of Clinical Psychiatry. 2012;73(11):144755. doi: 10.4088/JCP.11r07499. PMID: 2013-12487-012. Exclusion Code: X7.

1333. Sharma V, Sommerdyk C. Are antidepressants effective in the treatment of postpartum depression? A systematic review. Prim Care Companion CNS Disord. 2013;15(6). doi: 10.4088/PCC.13r01529. PMID: 24800125. Exclusion Code: X7.

1334. Sharma V, Sommerdyk C, Xie B. Aripiprazole augmentation of antidepressants for postpartum depression: a preliminary report. Arch Womens Ment Health. 2015 Feb;18(1):131-4. doi: 10.1007/s00737-014-0462-3. PMID: 25223952. Exclusion Code: X4.

1335. Sharma V, Sommerdyk C, Xie B, et al. Pharmacotherapy of bipolar II disorder during and after pregnancy. Curr Drug Saf. 2013 Sep;8(4):246-52. PMID: 23859430. Exclusion Code: X4.

1336. Sharp D. Antidepressant drug therapy vs a community-based psychosocial intervention for the treatment of moderate postnatal depression: a pragmatic randomised controlled trial. National research register. 2004. PMID: CN-00497269. Exclusion Code: X5.
1337. Sharp DJ, Chew-Graham C, Tylee A, et al. A pragmatic randomised controlled trial to compare antidepressants with a communitybased psychosocial intervention for the treatment of women with postnatal depression: the RESPOND trial. Health Technol Assess. 2010 Sep;14(43):iii-iv, ixxi, 1-153. doi: 10.3310/hta14430. PMID: 20860888. Exclusion Code: X4.

1338. Sharts-Hopko NC. Lithium and pregnancy. MCN Am J Matern Child Nurs. 1993 NovDec;18(6):344. PMID: 8295523. Exclusion Code: X1.

1339. Shaw AK, Infante-Rivard C, Morrison HI. Use of medication during pregnancy and risk of childhood leukemia (Canada). Cancer Causes Control. 2004 Nov;15(9):931-7. doi: 10.1007/s10552-004-2230-6. PMID: 15577295. Exclusion Code: X4.

1340. Shear MK, Mammen O. Anxiety disorders in pregnant and postpartum women. Psychopharmacol Bull. 1995;31(4):693-703. PMID: 1996-03326-008. Exclusion Code: $\mathrm{X} 1$.

1341. Shearer WT, Schreiner RL, Marshall RE. Urinary retention in a neonate secondary to maternal ingestion of nortriptyline. J Pediatr. 1972 Sep;81(3):570-2. PMID: 5049831. Exclusion Code: X7.

1342. Sheehan J, Crotty FM. Depressed mood during pregnancy and after childbirth. Treatment for depression is important confounding variable. BMJ. $2001 \mathrm{Dec}$ 8;323(7325):1367; author reply 8. PMID: 11791255. Exclusion Code: X1.

1343. Sheffield LJ. A multicentre prospective study of the effects on the foetus of anticonvulsant drugs taken during pregnancy. Aust Paediatr J. 1979;15(3):201. Exclusion Code: X2.

1344. Shen X, Raisch DW. Evaluating outcomes from prenatal antidepressant exposure: A meta-analytic review. Value Health. 2012;15(4):A81. doi: 10.1016/j.jval.2012.03.447. Exclusion Code: X7.

1345. Shoar Z, Fuld KB. Case study of a patient with pseudohypoparathyroidism and learning disability. Endocr Rev. 2012;33(3). Exclusion Code: X7. 
1346. Silbermann RM, Beenen F, de Jong H. Clinical treatment of post partum delirium with perfenazine and lithium carbonate. Psychiatr Clin (Basel). 1975;8(6):314-26. PMID: 1233538. Exclusion Code: X11.

1347. Silver R, Adams M, Straub H, et al. Antenatal depressive symptoms increase the likelihood of preterm birth. Am J Obstet Gynecol. 2012;206(1):S235. doi: 10.1016/j.ajog.2011.10.538. Exclusion Code: X3.

1348. Silverman BC, Gross AF. Weighing risks and benefits of prescribing antidepressants during pregnancy. Virtual Mentor. 2013 Sep 1;15(9):746-52. doi:

10.1001/virtualmentor.2013.15.9.ecas11309. PMID: 24021101. Exclusion Code: $\mathrm{X} 7$.

1349. Sim M. Imipramine and pregnancy. Br Med J. 1972 Apr 1;2(5804):45. PMID: 4259413. Exclusion Code: X7.

1350. Simoncelli M, Martin BZ, Berard A. Antidepressant use during pregnancy: a critical systematic review of the literature. Curr Drug Saf. 2010 Apr;5(2):153-70. PMID: 19534639. Exclusion Code: X7.

1351. Simonetti A, Koukopoulos AE, Kotzalidis GD, et al. Stabilization Beyond Mood: Stabilizing Patients With Bipolar Disorder in the Various Phases of Life. Front Psychiatry. 2020;11:247. doi: 10.3389/fpsyt.2020.00247. PMID: 32395107. Exclusion Code: X7.

1352. Singal D, Brownell M, Chateau D, et al. Neonatal and childhood neurodevelopmental, health and educational outcomes of children exposed to antidepressants and maternal depression during pregnancy: protocol for a retrospective population-based cohort study using linked administrative data. BMJ Open. 2016 Nov 29;6(11):e013293. doi: 10.1136/bmjopen-2016-013293. PMID: 27899401. Exclusion Code: X7.

1353. Singal D, Chateau D, Brownell M. Prenatal antidepressant use and autism spectrum disorder. JAMA. 2017 Aug 15;318(7):6645. doi: 10.1001/jama.2017.8640. PMID: 28810014. Exclusion Code: X1.
1354. Singal D, Chateau D, Dahl M, et al. In utero SSRI and SNRI exposure and the risk of neurodevelopmental outcomes in children: A population-based retrospective cohort study utilizing linked administrative data. Pharmacoepidemiol Drug Saf. 2018;27:2401. doi: 10.1002/pds.4629. Exclusion Code: X9.

1355. Singh I, Srinivasan A. Higher risk of low birth weight and preterm delivery associated with antidepressant exposure: are we in the right direction? Gen Hosp Psychiatry. 2014 May-Jun;36(3):358. doi: 10.1016/j.genhosppsych.2014.01.004. PMID: 24556259. Exclusion Code: X1.

1356. Singh MK, DelBello MP, Soutullo C, et al. Obstetrical complications in children at high risk for bipolar disorder. J Psychiatr Res. 2007;41(8):680-5. doi: 10.1016/j.jpsychires.2006.02.009. PMID: 2007-05381-009. Exclusion Code: X3.

1357. Singh S. Valproate use during pregnancy was linked to autism spectrum disorder and childhood autism in offspring. Ann Intern Med. 2013 Aug 20;159(4):Jc13. doi: 10.7326/0003-4819-159-4-20130820002013. PMID: 24026277. Exclusion Code: $\mathrm{X} 1$.

1358. Singh S, Gulati S, Narang A, et al. Nonnarcotic withdrawal syndrome in a neonate due to maternal clomipramine therapy. J Paediatr Child Health. 1990 Apr;26(2):110. PMID: 2361068. Exclusion Code: X1.

1359. Sit D, Luther J, Dills JL, et al. Abnormal screening for gestational diabetes, maternal mood disorder, and preterm birth. Bipolar Disord. 2014 May;16(3):308-17. doi: 10.1111/bdi.12129. PMID: 24164892. Exclusion Code: X4.

1360. Sit D, Perel JM, Wisniewski SR, et al. Mother-infant antidepressant concentrations, maternal depression, and perinatal events. J Clin Psychiatry. 2011 Jul;72(7):994-1001. doi: 10.4088/JCP.10m06461. PMID: 21824458. Exclusion Code: X5.

1361. Sit D, Wisner K. Glucose metabolism and mothers with major depressive disorder, bipolar disorder and controls: Pregnancy outcomes. Arch Womens Ment Health. 2013;16:S101-S2. doi: 10.1007/s00737-0130355-x. Exclusion Code: X4. 
1362. Sit D, Wisner KL, O'Shea K, et al.

Pharmacological Treatments for Bipolar

Disorders: Pregnancy and Newborn

Outcomes. Biol Psychiatry.

2019;85(10):S345. doi:

10.1016/j.biopsych.2019.03.877. Exclusion

Code: X9.

1363. Sit DKY, Luther J, Wisniewksi S, et al. Diabetes and obesity in pregnant women with unipolar and bipolar disorders: Adverse birth outcomes. Bipolar Disorders.

2011;13:90-1. doi: 10.1111/j.1399-

5618.2011.00912.x. Exclusion Code: X4.

1364. Sit DKY, Perel J, Luther J, et al. Disposition of antidepressants across childbearing: Impact on dosing. Arch Womens Ment Health. 2011;14:S49. doi: 10.1007/s00737010-0203-1. Exclusion Code: X7.

1365. Siu AL, Bibbins-Domingo K, Grossman DC, et al. Screening for depression in adults: US Preventive Services Task Force Recommendation Statement. JAMA. 2016 Jan 26;315(4):380-7. doi: 10.1001/jama.2015.18392. PMID: 26813211. Exclusion Code: X1.

1366. Sjaarda LA, Radoc JG, Flannagan KS, et al. Antidepressant medication exposure: time to pregnancy and risk of pregnancy loss. Fertil Steril. 2019;112(3):e2. doi:

10.1016/j.fertnstert.2019.07.139. Exclusion Code: X2.

1367. Skarsgard ED, Meaney C, Bassil K, et al. Maternal risk factors for gastroschisis in Canada. Birth Defects Res A Clin Mol Teratol. 2015 Feb;103(2):111-8. doi: 10.1002/bdra.23349. PMID: 25684659. Exclusion Code: X4.

1368. Skurtveit S, Selmer R, Roth C, et al. Prenatal exposure to antidepressants and language competence at 3 years of age. Results from a large population based pregnancy cohort in Norway.

Pharmacoepidemiol Drug Saf. 2013;22:1612. doi: $10.1002 / p d s .3512$. Exclusion Code: X9.
1369. Skurtveit S, Selmer R, Roth C, et al. Prenatal exposure to antidepressants and language competence at age three: results from a large population-based pregnancy cohort in Norway. BJOG. 2014 Dec;121(13):1621-31. doi: 10.1111/14710528.12821. PMID: 24726047. Exclusion Code: X2.

1370. Smit M, Dolman KM, Honig A. Mirtazapine in pregnancy and lactation - a systematic review. Eur Neuropsychopharmacol. 2016 Jan;26(1):126-35. doi: 10.1016/j.euroneuro.2015.06.014. PMID: 26631373. Exclusion Code: X7.

1371. Smit M, Wennink H, Heres M, et al. Mirtazapine in pregnancy and lactation: data from a case series. J Clin Psychopharmacol. 2015 Apr;35(2):163-7. doi: 10.1097/jcp.0000000000000279. PMID: 25689290. Exclusion Code: X7.

1372. Smith A, Twynstra J, Seabrook JA. Antenatal depression and offspring health outcomes. Obstetric Medicine. 2019. doi: 10.1177/1753495X19843015. Exclusion Code: X3.

1373. Smith AK, Conneely KN, Newport DJ, et al. Prenatal antiepileptic exposure associates with neonatal DNA methylation differences. Epigenetics. 2012 May;7(5):458-63. doi: 10.4161/epi.19617. PMID: 22419127. Exclusion Code: X5.

1374. Smith LM, Lagasse LL, Derauf C, et al. Growth and neurodevelopmental outcomes in children prenatally exposed to methamphetamine. Birth Defects Research Part A - Clinical and Molecular Teratology. 2012;94(5):303. doi: 10.1002/bdra.23023. Exclusion Code: X3.

1375. Smith LM, Paz MS, Lagasse LL, et al. Maternal depression and prenatal exposure to methamphetamine: Neurodevelopmental findings from the infant development, environment, and lifestyle (ideal) study. Depress Anxiety. 2012. doi: 10.1002/da.21956. Exclusion Code: X3.

1376. Smith MV, Sung A, Shah B, et al. Neurobehavioral assessment of infants born at term and in utero exposure to serotonin reuptake inhibitors. Early Hum Dev. 2013 Feb;89(2):81-6. doi: 10.1016/j.earlhumdev.2012.08.001. PMID: 22999988. Exclusion Code: X2. 
1377. Smith V, Brown N. Prenatal valproate exposure and risk of autism spectrum disorders and childhood autism. Arch Dis Child Educ Pract Ed. 2014 Oct;99(5):198. doi: 10.1136/archdischild-2013-305636. PMID: 24692263. Exclusion Code: X1.

1378. Sockol LE, Epperson CN, Barber JP. A meta-analysis of treatments for perinatal depression. Clin Psychol Rev. 2011 Jul;31(5):839-49. doi: 10.1016/j.cpr.2011.03.009. PMID: 21545782. Exclusion Code: X7.

1379. Solmi M. P.3.09 Systematic review and meta-analysis of lithium safety and efficacy in pregnancy and peri-partum. Eur Neuropsychopharmacol. 2019;29:S685. doi: 10.1016/j.euroneuro.2019.01.071. Exclusion Code: X7.

1380. Sommerlad S, Schermelleh-Engel K, Raddatz LM, et al. The impact of perceived control during delivery in the context of traumatic birth-experience. European Journal of Obstetrics Gynecology and Reproductive Biology. 2019;234:e167. doi: 10.1016/j.ejogrb.2018.08.526. Exclusion Code: X3.

1381. Son M, Silver RM, Haas DM, et al. 347: Patient psychosocial traits and perinatal outcomes. Am J Obstet Gynecol. 2019;220(1):S241-S2. doi: 10.1016/j.ajog.2018.11.368. Exclusion Code: X3.

1382. Sontheimer DL, Ables AZ. Safety of antidepressant medications during pregnancy. JAMA. 2000 Mar 1;283(9):1139. PMID: 10703770. Exclusion Code: X1.

1383. Soubry A, Murphy S, Huang Z, et al. The effects of depression and use of antidepressive medicines during pregnancy on the methylation status of the IGF2 imprinted control regions in the offspring. Clin Epigenetics. 2011 Oct 26;3:2. doi: 10.1186/1868-7083-3-2. PMID: 22414206. Exclusion Code: X5.

1384. Speisman BB, Storch EA, Abramowitz JS. Postpartum obsessive-compulsive disorder. J Obstet Gynecol Neonatal Nurs. 2011 NovDec;40(6):680-90. doi: 10.1111/j.15526909.2011.01294.x. PMID: 22092284. Exclusion Code: X1.
1385. Spielvogel A, Wile J. Treatment of the psychotic pregnant patient. Psychosomatics. 1986 Jul;27(7):487-92. doi: 10.1016/s00333182(86)72654-6. PMID: 3526381. Exclusion Code: X1.

1386. Spinelli M. Antidepressant treatment during pregnancy. Am J Psychiatry. 2012

Feb;169(2):121-4. doi: 10.1176/appi.ajp.2011.11111622. PMID: 22318792. Exclusion Code: X1.

1387. Stadelmaier R, Nasri H, Deutsch CK, et al. Exposure to sodium valproate during pregnancy: facial features and signs of autism. Birth Defects Res. 2017 Aug 15;109(14):1134-43. doi: 10.1002/bdr2.1052. PMID: 28635121. Exclusion Code: X2.

1388. Staszewski C, Herrera K, Persad MD, et al. 313: Medication-Assisted Treatment (MAT) in pregnancy: methadone and buprenorphine. Am J Obstet Gynecol. 2019;220(1):S219-S20. doi: 10.1016/j.ajog.2018.11.334. Exclusion Code: X2.

1389. Staunstrup N, Viuff AC, Sharp G, et al. EPIGENETIC DIFFERENCES IN CORD BLOOD OF NEWBORNS EXPOSED TO ANTIDEPRESSANT MEDICATION DURING PREGNANCY - A STUDY IN THE AARHUS BIRTH COHORT. Eur Neuropsychopharmacol. 2019;29:S983-S4. doi: 10.1016/j.euroneuro.2017.08.360. Exclusion Code: X5.

1390. Stein A, Murphy S, Arteche A, et al. Effects of reboxetine and citalopram on appraisal of infant facial expressions and attentional biases. J Psychopharmacol. 2012 May;26(5):670-6. doi: 10.1177/0269881111421970. PMID: 21948858. Exclusion Code: X2.

1391. Steinberg JR, Laursen TM, Adler NE, et al. Examining the association of antidepressant prescriptions with first abortion and first childbirth. JAMA Psychiatry. 2018;75(8):828-34. doi: 10.1001/jamapsychiatry.2018.0849. Exclusion Code: X4. 
1392. Steinberg JR, Laursen TM, Adler NE, et al. Examining the association of antidepressant prescriptions with first abortion and first childbirth. JAMA Psychiatry. 2018 Aug 1;75(8):828-34. doi: 10.1001/jamapsychiatry.2018.0849. PMID: 29847626. Exclusion Code: X5.

1393. Steinberg SI, Bellavance F. Characteristics and treatment of women with antenatal and postpartum depression. Int J Psychiatry Med. 1999;29(2):209-33. doi: 10.2190/j96yyu6c-5pxq-c4j9. PMID: 10587816.

Exclusion Code: X4.

1394. Steiner M, Latz A, Blum I, et al. Propranolol versus chlorpromazine in the treatment of psychoses associated with childbearing. Psychiatr Neurol Neurochir. 1973 NovDec;76(6):421-6. PMID: 4781521. Exclusion Code: X2.

1395. Steinig J, Nagl M, Linde K, et al. Antenatal and postnatal depression in women with obesity: A systematic review. Arch Womens Ment Health. 2017;20(4):569-85. doi: 10.1007/s00737-017-0739-4. PMID: 201726419-001. Exclusion Code: X7.

1396. Stephansson O, Kieler H, Haglund B, et al. Selective serotonin reuptake inhibitors during pregnancy and risk of stillbirth and infant mortality. JAMA. 2013 Jan 2;309(1):48-54. doi: 10.1001/jama.2012.153812. PMID: 23280224. Exclusion Code: X2.

1397. Stephens S, Adams S, Richardson JL, et al. Outcome of pregnancy after first trimester exposure to venlafaxine. Birth Defects Research Part A - Clinical and Molecular Teratology. 2011;91(5):414. doi: 10.1002/bdra.20834. Exclusion Code: X4.

1398. Stevens A, Goossens PJJ, Knoppert-van der Klein EAM, et al. Risk of recurrence of mood disorders during pregnancy and the impact of medication: A systematic review. J Affect Disord. 2019 Apr 15;249:96-103. doi: 10.1016/j.jad.2019.02.018. PMID: 30769297. Exclusion Code: X7.

1399. Stewart DE, Vigod S. Postpartum depression. N Engl J Med. 2016 Dec 1;375(22):2177-86. doi: 10.1056/NEJMcp1607649. PMID: 27959754. Exclusion Code: X7.
1400. Stewart M, Pretlove S, Berrisford G, et al. Antipsychotic use in pregnancy: Maternal and fetal outcomes. Arch Womens Ment Health. 2015;18(2):303-4. doi: 10.1007/s00737-014-0488-6. Exclusion Code: X7.

1401. Stone KC, Salisbury AL, Miller-Loncar CL, et al. Combined hypnotic and antidepressant treatment: Potential moderator of change in perinatal depression severity over time. Sleep. 2016;39:A294-A5. Exclusion Code: $\mathrm{X} 4$.

1402. Stone KC, Salisbury AL, Miller-Loncar CL, et al. Pregnancy and postpartum antidepressant use moderates the effects of sleep quality on depression severity. Sleep. 2017;40:A411. Exclusion Code: X9.

1403. Stringer E, Johnson J, Jackson C, et al. Antenatal depression in an urban population of pregnant adolescents and young adults may contribute to preterm birth. Am J Obstet Gynecol. 2016;214(1):S344. Exclusion Code: X3.

1404. Stuebe AM, Meltzer-Brody S, Propper C, et al. The Mood, Mother, and Infant Study: Associations Between Maternal Mood in Pregnancy and Breastfeeding Outcome. Breastfeed Med. 2019 Oct;14(8):551-9. doi: 10.1089/bfm.2019.0079. PMID: 31424266. Exclusion Code: X3.

1405. Suarez A, Lahti JMT, Girchenko P, et al. Maternal depression during pregnancy and epigenetic age in their newborns: The PREDO study. Psychoneuroendocrinology. 2016;71:63. doi: 10.1016/j.psyneuen.2016.07.163. Exclusion Code: X3.

1406. Sujan A, Rickert M, Sara Oberg A, et al. Maternal antidepressant use during the first trimester of pregnancy and offspring neurodevelopmental problems. Behav Genet. 2017;47(6):691. doi: 10.1007/s10519-017-9879-6. Exclusion Code: X4. 
1407. Sujan AC, Rickert ME, Oberg AS, et al. Associations of maternal antidepressant use during the first trimester of pregnancy with preterm birth, small for gestational age, autism spectrum disorder, and attentiondeficit/hyperactivity disorder in offspring. JAMA. 2017 Apr 18;317(15):1553-62. doi: 10.1001/jama.2017.3413. PMID: 28418479. Exclusion Code: X4.

1408. Sujan AC, Rickert ME, Öberg AS, et al. Associations of maternal antidepressant use during the first trimester of pregnancy with preterm birth, small for gestational age, autism spectrum disorder, and attentiondeficit/hyperactivity disorder in offspring. Obstet Gynecol Surv. 2017;72(9):523-4. doi: 10.1097/01.ogx.0000524510.66825.09. Exclusion Code: X4.

1409. Sumption LA, Garay SM, John RM. Low serum placental lactogen at term is associated with postnatal symptoms of depression and anxiety in women delivering female infants. Psychoneuroendocrinology. 2020 Mar 20;116:104655. doi: 10.1016/j.psyneuen.2020.104655. PMID: 32247203. Exclusion Code: X2.

1410. Sun Y, Pedersen L, Christensen J, et al. Prenatal valproate exposure and risk of attention deficit hyperactivity disorder. Epilepsia. 2018;59:S90. doi: 10.1111/epi.14612. Exclusion Code: X2.

1411. Sundaresh S, Conroy S, Pawlby S, et al. Depression in pregnancy: Adverse obstetric and fetal outcomes. Arch Womens Ment Health. 2019;22(5):706. doi: 10.1007/s00737-019-00996-y. Exclusion Code: X2.

1412. Sundaresh S, Conroy S, Pawlby S, et al. Depression in pregnancy: Adverse obstetric and fetal outcomes. Arch Womens Ment Health. 2015;18(2):284-5. doi:

10.1007/s00737-014-0488-6. Exclusion Code: X9.

1413. Sundbakk LM, Wood M, Gran JM, et al. Impact of prenatal exposure to benzodiazepines and z-hypnotics on behavioral problems at 5 years of age: A study from the Norwegian Mother and Child Cohort Study. PLoS One. 2019;14(6):e0217830. doi: 10.1371/journal.pone.0217830. PMID: 31170221. Exclusion Code: X4.
1414. Sunder K, Wisner KL. Antidepressants and premature labor. Am J Psychiatry. 2004 May;161(5):925-6; author reply 6. doi: 10.1176/appi.ajp.161.5.925. PMID: 15121667. Exclusion Code: X1.

1415. Sunder KR, Wisner KL, Hanusa BH, et al. Postpartum depression recurrence versus discontinuation syndrome: observations from a randomized controlled trial. J Clin Psychiatry. 2004 Sep;65(9):1266-8. PMID: 15367055. Exclusion Code: X3.

1416. Sunnqvist C, Sjöström K, Finnbogadóttir H. Depressive symptoms during pregnancy and postpartum in women and use of antidepressant treatment - a longitudinal cohort study. Int J Womens Health. 2019;11:109-17. doi: 10.2147/ijwh.s185930. PMID: 30799960. Exclusion Code: X2.

1417. Suri R, Burt VK, Altshuler LL. Nefazodone for the treatment of postpartum depression. Arch Womens Ment Health. 2005 May;8(1):55-6. doi: 10.1007/s00737-0050071-2. PMID: 15868388. Exclusion Code: $\mathrm{X} 1$.

1418. Suri R, Burt VK, Altshuler LL, et al. Fluvoxamine for postpartum depression. Am J Psychiatry. 2001 Oct;158(10):1739-40. doi: 10.1176/appi.ajp.158.10.1739. PMID: 11579021. Exclusion Code: X7.

1419. Suri R, Hellemann G, Cohen L, et al. Saliva estriol levels in women with and without prenatal antidepressant treatment. Biol Psychiatry. 2008 Sep 15;64(6):533-7. doi: 10.1016/j.biopsych.2008.04.015. PMID: 18495086. Exclusion Code: X5.

1420. Suri R, Lin AS, Cohen LS, et al. Acute and long-term behavioral outcome of infants and children exposed in utero to either maternal depression or antidepressants: a review of the literature. J Clin Psychiatry. 2014 Oct;75(10):e1142-52. doi: 10.4088/JCP.13r08926. PMID: 25373125. Exclusion Code: X1.

1421. Sutter-Dallay AL, Bales M, Pambrun E, et al. Impact of prenatal exposure to psychotropic drugs on neonatal outcome in infants of mothers with serious psychiatric illnesses. J Clin Psychiatry. 2015 Jul;76(7):967-73. doi: 10.4088/JCP.14m09070. PMID: 25844580. Exclusion Code: X4. 
1422. Sutter-Dallay AL, Bales M, Pambrun E, et al. Infants hospitalized in french MBUS: Impact of prenatal exposure to psychotropic drugs on neonatal outcome. Arch Womens Ment Health. 2015;18(2):363. doi: 10.1007/s00737-014-0488-6. Exclusion Code: X9.

1423. Sutter-Dallay AL, Lacaze I, Chazaud C, et al. Influence of psychotropic drugs intake during pregnancy on early newborn adaptation. Arch Womens Ment Health. 2011;14:S51. doi: 10.1007/s00737-0100203-1. Exclusion Code: X9.

1424. Suvisaari J, Häkkinen L, Haukka J, et al. Mortality in offspring of mothers with psychotic disorder. Psychol Med. 2008;38(8):1203-10. doi: 10.1017/S0033291707002383. PMID: 200816143-016. Exclusion Code: X2.

1425. Suzuki S. Relapse of depression during pregnancy and postpartum periods in Japanese women associated with selfinterruption of their medications: Selective serotonin reuptake inhibitors. Asian J Psychiatr. 2015 Dec;18:99. doi: 10.1016/j.ajp.2015.10.013. PMID: 26603370. Exclusion Code: X7.

1426. Swain J, Ho S. Resting State Functional Connectivity \& Parental Bonding in Postpartum Women on Buprenorphine for Opioid Use Disorder. Biol Psychiatry. 2020;87(9):S438. doi:

10.1016/j.biopsych.2020.02.1116. Exclusion Code: X2.

1427. Szegda K, Markenson G, Bertone-Johnson ER, et al. Depression during pregnancy: a risk factor for adverse neonatal outcomes? A critical review of the literature. J Matern Fetal Neonatal Med. 2014 Jun;27(9):960-7. doi: 10.3109/14767058.2013.845157.

PMID: 24044422. Exclusion Code: X7.

1428. Tahmasian M, Khazaie H, Ghadami M, et al. Insomnia treatment in the third trimester of pregnancy prevents postpartum depression: a randomized clinical trial. Sleep medicine. 2013;14:e39. doi: 10.1016/j.sleep.2013.11.056. PMID: CN01063516. Exclusion Code: X2.
1429. Tak CR, Job KM, Schoen-Gentry K, et al. The impact of exposure to antidepressant medications during pregnancy on neonatal outcomes: a review of retrospective database cohort studies. Eur J Clin Pharmacol. 2017 Sep;73(9):1055-69. doi: 10.1007/s00228017-2269-4. PMID: 28600701. Exclusion Code: X7.

1430. Talati A, Weissman MM. Commentary: Studies of prenatal antidepressant exposures: what can you recommend? A reflection on Sujan et al. (2019). J Child Psychol Psychiatry. 2019 Apr;60(4):377-9. doi: 10.1111/jcpp.13036. PMID: 30919484. Exclusion Code: X1.

1431. Tam WH, Chung T. Psychosomatic disorders in pregnancy. Curr Opin Obstet Gynecol. 2007 Apr;19(2):126-32. doi: 10.1097/GCO.0b013e3280825614. PMID: 17353680. Exclusion Code: X1.

1432. Tanaka T, Ito S. Novel influenza A/H1N1 during pregnancy and lactation: Safety of vaccines and drug treatment. Expert Review of Obstetrics and Gynecology. 2010;5(2):169-75. doi: 10.1586/eog.10.11. Exclusion Code: X2.

1433. Tang CH, Chang WC, Lee HC. Safety of Zolpidem And Zopiclone during pregnancy: a nationwide retrospective cohort study of risks of preterm delivery and low birth weight at birth and intellectual disability during 7-year follow-up. Sleep Med. 2019;64:S216. doi: 10.1016/j.sleep.2019.11.603. Exclusion Code: X2.

1434. Tang GY, Parekh J. Brexanolone injection for post-partum depression treatment. Lancet. 2019 Aug 3;394(10196):379. doi: 10.1016/s0140-6736(19)30714-7. PMID: 31255300. Exclusion Code: X1.

1435. Tanne JH. Psychiatric drugs can be used with caution in pregnancy, say experts. BMJ. 2013 Jun 13;346:f3852. doi: 10.1136/bmj.f3852. PMID: 23766493. Exclusion Code: X1.

1436. Targum SD. Dealing with psychosis during pregnancy. Am Pharm. 1979 Aug;19(9):1821. PMID: 506913. Exclusion Code: X1. 
1437. Taş Hí, Pek İ E. An investigation of probable relationships between depressive symptoms, mixed symptoms and affective temperaments in a sample of pregnant women. Yeni Symposium. 2018;56(3):9-14. doi: 10.5455/NYS.20181204073521. Exclusion Code: X3.

1438. Taylor CL, Khalifeh H, Pasupathy D, et al. Use of UK hospital data and mental health records to investigate maternal physical health and obstetric outcomes for pregnant women with serious mental illness. Arch Womens Ment Health. 2019;22(5):666. doi: 10.1007/s00737-019-00996-y. Exclusion Code: X9.

1439. Taylor CL, Stewart RJ, Howard LM. Relapse in the first three months postpartum in women with history of serious mental illness. Schizophr Res. 2019 Feb;204:46-54. doi: 10.1016/j.schres.2018.07.037. PMID: 30089534. Exclusion Code: X3.

1440. Te Winkel B, Beghin D, Pistelli A, et al. Venlafaxine exposure in pregnancy, a multicenter ENTIS study.

Pharmacoepidemiology and drug safety. Conference: 32 nd international conference on pharmacoepidemiology and therapeutic risk management. Ireland. Conference start: 20160825. Conference end: 20160828. 2016;25:325-6. doi: 10.1002/pds.4070. PMID: CN-01293592. Exclusion Code: X4.

1441. Ter Horst PG, Smit JP. [Antidepressants during pregnancy and lactation]. Tijdschr Psychiatr. 2009;51(5):307-14. PMID: 19434568. Exclusion Code: X8.

1442. ter Horst PG, van der Linde S, Smit JP, et al. Clomipramine concentration and withdrawal symptoms in 10 neonates. Br J Clin Pharmacol. 2012 Feb;73(2):295-302. doi: 10.1111/j.1365-2125.2011.04072.x. PMID: 21801198. Exclusion Code: X4.

1443. Terao T. Antidepressant treatment and relapse of depression during pregnancy. JAMA. 2006 Jul 12;296(2):165; author reply 6-7. doi: 10.1001/jama.296.2.165-a. PMID: 16835416. Exclusion Code: X1.
1444. Terrana N, Koren G, Pivovarov J, et al. Pregnancy outcomes following in utero exposure to second-generation antipsychotics: a systematic review and meta-analysis. J Clin Psychopharmacol. 2015 Oct;35(5):559-65. doi: 10.1097/jcp.0000000000000391. PMID: 26274044. Exclusion Code: X7.

1445. Thomas P, Severus WE. Managing bipolar disorder during pregnancy and lactation: is there a safe and effective option? Eur Psychiatry. 2003 Dec;18 Suppl 1:3s-8s. PMID: 23573634. Exclusion Code: X1.

1446. Tinker SC, Reefhuis J, Bitsko RH, et al. Use of benzodiazepine medications during pregnancy and potential risk for birth defects, National Birth Defects Prevention Study, 1997-2011. Birth Defects Res. 2019 Jun 1;111(10):613-20. doi: 10.1002/bdr2.1497. PMID: 30891943. Exclusion Code: X2.

1447. Toh S, Li Q, Cheetham TC, et al. Prevalence and trends in the use of antipsychotic medications during pregnancy in the US, 2001-2007: A population-based study of 585,615 deliveries. Arch Womens Ment Health. 2013;16(2):149-57. doi: 10.1007/s00737-013-0330-6. PMID: 201304553-001. Exclusion Code: X5.

1448. Toh S, Mitchell AA, Louik C, et al. Selective serotonin reuptake inhibitor use and risk of gestational hypertension. Am J Psychiatry. 2009 Mar;166(3):320-8. doi: 10.1176/appi.ajp.2008.08060817. PMID: 19122006. Exclusion Code: X4.

1449. Toh S, Mitchell AA, Louik C, et al. Use of antidepressants during pregnancy and the risk of preterm delivery and fetal growth restriction. Pharmacoepidemiology and Drug Safety (PDS). 2009;18(S1):S13-S4. doi: 10.1002/pds.1806. Exclusion Code: X4.

1450. Tong HHY, Man KKC, Chan EW, et al. Selective serotonin reuptake inhibitor exposure during pregnancy and risk of autism spectrum disorders in children: A meta-analysis of observational studies. Drug Saf. 2014;37(10):836. doi: 10.1007/s40264014-0208-1. Exclusion Code: X7. 
1451. Toppozada M, Parmar C, Fotherby K. Effect of injecTable Bontraceptives Depo-Provera and norethisterone oenanthate on pituitary gonadotropin response to luteinizing hormone-releasing hormone. Fertil Steril. 1978 Nov;30(5):545-8. PMID: 363460. Exclusion Code: X3.

1452. Torjesen I. Depression and SSRI use in pregnancy associated with traits of autism in children. BMJ. 2014 Jul 31;349:g4835. doi: 10.1136/bmj.g4835. PMID: 25084785. Exclusion Code: X1.

1453. Torjesen I. Antidepressants in pregnancy are not associated with autism risk, new study finds. BMJ (Online). 2018;357. doi: 10.1136/bmj.j1923. Exclusion Code: X1.

1454. Torres A, Ascaso C, Roca A, et al. Longterm psychopathological effects on children exposed to selective serotonin reuptake inhibitors in the uterus. Arch Womens Ment Health. 2015;18(2):340-1. doi: 10.1007/s00737-014-0488-6. Exclusion Code: X4.

1455. Tosato S, Albert U, Tomassi S, et al. A systematized review of atypical antipsychotics in pregnant women: balancing between risks of untreated illness and risks of drug-related adverse effects. J Clin Psychiatry. 2017 May;78(5):e477-e89. doi: 10.4088/JCP.15r10483. PMID: 28297592. Exclusion Code: X7.

1456. Tran DT, Havard A, Preen DB, et al. Birth outcomes associated with the use of smoking cessation pharmacotherapies in pregnancy: Findings from the smoking mums (maternal use of medications and safety) study. Pharmacoepidemiol Drug Saf. 2017;26:435-6. doi: 10.1002/pds.4275. Exclusion Code: X2.

1457. Trixler M, Gati A, Tenyi T. Risks associated with childbearing in schizophrenia. Acta Psychiatr Belg. 1995 May-Jun;95(3):159-62. PMID: 8525858. Exclusion Code: X3.

1458. Trønnes JN, Lupattelli A, Nordeng H. Safety profile of medication used in pregnancy: Results of a multinational European study. Pharmacoepidemiol Drug Saf. 2016;25:336. doi: 10.1002/pds.4070. Exclusion Code: X2.
1459. Tuccori M, Montagnani S, Testi A, et al. Use of selective serotonin reuptake inhibitors during pregnancy and risk of major and cardiovascular malformations: an update. Postgrad Med. 2010 Jul;122(4):4965. doi: 10.3810/pgm.2010.07.2175. PMID: 20675971. Exclusion Code: X7.

1460. Tuccori M, Testi A, Antonioli L, et al. Safety concerns associated with the use of serotonin reuptake inhibitors and other serotonergic/noradrenergic antidepressants during pregnancy: a review. Clin Ther. 2009 Jun;31 Pt 1:1426-53. doi: 10.1016/j.clinthera.2009.07.009. PMID: 19698902. Exclusion Code: X7.

1461. Tufts JA. Brexanolone injection for postpartum depression treatment. Lancet. 2019 Aug 3;394(10196):379. doi: 10.1016/s01406736(19)30727-5. PMID: 31255297. Exclusion Code: X1.

1462. Turner E, Jones M, Vaz LR, et al. Systematic review and meta-analysis to assess the safety of buproprion and varenicline in pregnancy. Nicotine Tob Res. 2018 Mar 22. doi: 10.1093/ntr/nty055. PMID: 29579233. Exclusion Code: X7.

1463. Turner E, Jones M, Vaz LR, et al. Systematic Review and Meta-Analysis to Assess the Safety of Bupropion and Varenicline in Pregnancy. Nicotine Tob Res. 2019 Jul 17;21(8):1001-10. doi: 10.1093/ntr/nty055. PMID: 29579233. Exclusion Code: X7.

1464. Turner K, Zambrelli E, Bassanese G, et al. Effects of postpartum depression on the behaviour of children born to mothers with epilepsy. Seizure. 2019 Dec;73:31-8. doi: 10.1016/j.seizure.2019.10.018. PMID: 31715519. Exclusion Code: X2.

1465. Tylden E. Psychiatric disorders including drug therapy and addiction. Clin Obstet Gynaecol. 1977 Aug;4(2):435-49. PMID: 340108. Exclusion Code: X4.

1466. Tze-Ern C. Understanding impact of peripartum mood and anxiety disorders and how to intervene early. Annals of the Academy of Medicine Singapore. 2011;40(7):S5. Exclusion Code: X7. 
1467. Udechuku A, Nguyen T, Hill R, et al. Antidepressants in pregnancy: a systematic review. Aust N Z J Psychiatry. 2010 Nov;44(11):978-96. doi: 10.3109/00048674.2010.507543. PMID: 21034181. Exclusion Code: X7.

1468. Uguz F. Is there any association between use of antidepressants and preeclampsia or gestational hypertension?: A systematic review of current studies. J Clin Psychopharmacol. 2017 Feb;37(1):72-7. doi: 10.1097/jcp.0000000000000618. PMID: 27941417. Exclusion Code: X7.

1469. Uguz F. Prophylactic use of olanzapine and quetiapine from pregnancy to the postpartum period in women with bipolar disorder: a case series. J Matern Fetal Neonatal Med. 2017 Nov;30(21):2569-71. doi: 10.1080/14767058.2016.1256991. PMID: 27809629. Exclusion Code: X7.

1470. Uguz F. Short-term safety of paroxetine plus low-dose mirtazapine during lactation. Breastfeed Med. 2018 Nov 29. doi: 10.1089/bfm.2018.0197. PMID: 30489153. Exclusion Code: X4.

1471. Uguz F. Maternal antidepressant use during pregnancy and the risk of attentiondeficit/hyperactivity disorder in children: a systematic review of the current literature. J Clin Psychopharmacol. 2018 Jun;38(3):2549. doi: 10.1097/jcp.0000000000000868. PMID: 29596147. Exclusion Code: X7.

1472. Uguz F. Pharmacotherapy of ObsessiveCompulsive Disorder During Breastfeeding. Am J Ther. 2018 Sep/Oct;25(5):e541-e7. doi: 10.1097/mjt.0000000000000500. PMID: 27661749. Exclusion Code: X1.

1473. Uguz F. A New Safety Scoring System for the Use of Psychotropic Drugs During Lactation. Am J Ther. 2019 Apr 1. doi: 10.1097/mjt.0000000000000909. PMID: 30601177. Exclusion Code: X7.

1474. Uguz F, Arpaci N. Short-term safety of paroxetine and sertraline in breastfed infants: a retrospective cohort study from a university hospital. Breastfeed Med. 2016 Nov;11:487-9. doi: 10.1089/bfm.2016.0095. PMID: 27575664. Exclusion Code: X5.
1475. Uguz F, Sharma V. Mood stabilizers during breastfeeding: a systematic review of the recent literature. Bipolar Disord. 2016 Jun;18(4):325-33. doi: 10.1111/bdi.12398. PMID: 27297617. Exclusion Code: X7.

1476. Uguz F, Subasi E, Dalboy F, et al. Pharmacological prophylaxis of postpartum exacerbation in depressive and anxiety symptoms: A retrospective chart review. Eur Neuropsychopharmacol. 2017;27:S784-S5. Exclusion Code: X2.

1477. Uguz F, Subasi E, Dalboy F, et al. Pharmacological prophylaxis of postpartum exacerbation in depressive and anxiety symptoms: a retrospective study. J Matern Fetal Neonatal Med. 2018 Mar 12:1-3. doi: 10.1080/14767058.2018.1447559. PMID: 29495902. Exclusion Code: X4.

1478. Ulbrich KA, Shah M, Miller E, et al. Identifying need for acute resuscitation in delivery room for SSRI exposed neonates. Birth Defects Research. 2019;111(9):555. doi: 10.1002/bdr2.v111.9. Exclusion Code: $\mathrm{X} 1$.

1479. Upmeijer JG. Prevalence and manifestation of psychiatric disorders in pregnancy. Journal of Psychosomatic Obstetrics and Gynecology. 2010;31:29. doi:

10.3109/0167482x.2010.536387. Exclusion Code: X5.

1480. Urato AC. Concerns regarding antidepressant drug use during pregnancy. J Psychiatry Neurosci. 2006 Nov;31(6):411; author reply -2 . PMID: 17136220 . Exclusion Code: X1.

1481. Urato AC. Antidepressant treatment and relapse of depression during pregnancy. JAMA. 2006 Jul 12;296(2):166; author reply -7. doi: 10.1001/jama.296.2.166-a. PMID: 16835417. Exclusion Code: X1.

1482. Urato AC. 'Concerns regarding antidepressant drug use during pregnancy:' Comment. J Psychiatry Neurosci. 2006;31(6):411-. PMID: 2007-03389-007. Exclusion Code: X1.

1483. Urato AC. Antidepressant use and preterm birth. Am J Psychiatry. 2009 Oct;166(10):1189; author reply -90. doi: 10.1176/appi.ajp.2009.09050712. PMID: 19797450. Exclusion Code: X1. 
1484. Urato AC, Mintzes B, Mangin D, et al.

Diagnosis, pathophysiology, and management of mood disorders in pregnant and postpartum women. Obstet Gynecol. 2011;118(3):708. doi:

10.1097/AOG.0b013e31822bd7e3.

Exclusion Code: X1.

1485. Uygur OF, Annagür BBA, Annagür A. Prevalence of and factors associated with postpartum depression among mothers of preterm infants. Eur

Neuropsychopharmacol. 2016;26:S352.

Exclusion Code: X3.

1486. Vajda F. Epilepsy: Effects of exposure to antiepileptic drugs during development. Nat Rev Neurol. 2014 Jan;10(1):11-2. doi: 10.1038/nrneurol.2013.254. PMID: 24323050. Exclusion Code: X2.

1487. Vajda FJ, Solinas C. Potential risks associated with high-dose valproate in pregnancy in psychiatric patients. Aust N Z J Psychiatry. 2005 Jun;39(6):526-7. doi: 10.1080/j.1440-1614.2005.01613 6.x. PMID: 15943661. Exclusion Code: X1.

1488. Valenzuela SK. The power of natural progesterone: treating hormone-related postpartum depression. Midwifery Today Int Midwife. 2012 Autumn(103):22-5. PMID: 23061144. Exclusion Code: X1.

1489. Van Den Bergh BRH, Otte RA, Braeken M, et al. Prenatal exposure to maternal anxiety is associated with sensory-cognitive development in 2-month-olds. Dev Med Child Neurol. 2012;54:22-3. doi: 10.1111/j.1469-8749.2012.04279.x. Exclusion Code: X3.

1490. van der Lugt NM, van de Maat JS, van Kamp IL, et al. Fetal, neonatal and developmental outcomes of lithium-exposed pregnancies. Early Hum Dev. 2012 Jun;88(6):375-8. doi: 10.1016/j.earlhumdev.2011.09.013. PMID: 22000820. Exclusion Code: X2.

1491. Van Der Veere CN, De Vries NKS, Bos AF. Poorer cognitive and gross motor outcome at age 2.5 years after intrauterine exposure to SSRI. Proceedings from the dutch smok trial. Arch Dis Child. 2014;99:A103-A4. doi: 10.1136/archdischild-2014-307384.276. Exclusion Code: X4.
1492. van Ravesteyn LM, Lambregtse-van den Berg MP, Hoogendijk WJ, et al. Interventions to treat mental disorders during pregnancy: a systematic review and multiple treatment meta-analysis. PLoS One. 2017;12(3):e0173397. doi: 10.1371/journal.pone.0173397. PMID: 28358808. Exclusion Code: X7.

1493. Vasilakis-Scaramozza C, Aschengrau A, Cabral H, et al. Antidepressant use during early pregnancy and the risk of congenital anomalies. Pharmacotherapy. 2013 Jul;33(7):693-700. doi: 10.1002/phar.1211. PMID: 23744675. Exclusion Code: X10.

1494. Vasile D, Vasiliu O, Mangalagiu AG, et al. Mirtazapine versus sertraline in the treatment of post-psychotic depression. Eur Neuropsychopharmacol. 2013;23:S320. doi: 10.1016/S0924-977X(13)70502-9.

Exclusion Code: X2.

1495. Vasudev K, Mead A. Use of valproate in women of childbearing potential: Pre- and post-NICE bipolar guidelines. Arch Womens Ment Health. 2010;13(4):371-2. doi: 10.1007/s00737-010-0166-2. PMID: 2010-14061-010. Exclusion Code: X1.

1496. Vatturi M, Dovari A, Inuganti B, et al. PND11 EFFICACY AND SAFETY OF BREXANOLONE IN THE TREATMENT OF POSTPARTUM DEPRESSION: A SYSTEMATIC REVIEW AND METAANALYSIS. Value Health. 2020;23:S260. doi: 10.1016/j.jval.2020.04.904. Exclusion Code: X7.

1497. Vavasour E. Nutritional implications of observed neurobehavioral disturbances and reduced gestation length in infants of mothers using selective serotonin reuptake inhibitors during pregnancy. Pediatrics. 2004 Jul;114(1):325. PMID: 15231956. Exclusion Code: X1.

1498. Vega ML, Newport GC, Bozhdaraj D, et al. Implementation of Advanced Methods for Reproductive Pharmacovigilance in Autism: A Meta-Analysis of the Effects of Prenatal Antidepressant Exposure. Am J Psychiatry. 2020 Jun 1;177(6):506-17. doi: 10.1176/appi.ajp.2020.18070766. PMID: 32375539. Exclusion Code: X7. 
1499. Veiby G, Bjork M, Engelsen BA, et al. Epilepsy and recommendations for breastfeeding. Seizure. 2015 May;28:57-65. doi: 10.1016/j.seizure.2015.02.013. PMID: 25837494. Exclusion Code: X1.

1500. Veiby G, Daltveit AK, Schjolberg S, et al. Exposure to antiepileptic drugs in utero and child development: a prospective population-based study. Epilepsia. 2013 Aug;54(8):1462-72. doi: 10.1111/epi.12226. PMID: 23865818. Exclusion Code: X2.

1501. Vemuri M, Williams K. Treating bipolar disorder during pregnancy. Current Psychiatry. 2011;10(9):58-66. Exclusion Code: X1.

1502. Venkatesh KK, Castro VM, Perlis RH, et al. Impact of antidepressant treatment during pregnancy on obstetric outcomes among women previously treated for depression: an observational cohort study. J Perinatol. 2017 Sep;37(9):1003-9. doi: 10.1038/jp.2017.92. PMID: 28682318. Exclusion Code: X4.

1503. Venkatesh KK, Castro VM, Perlis RH, et al. Impact of antidepressant treatment during pregnancy on obstetric outcomes among women previously treated for depression. Am J Obstet Gynecol. 2017;216(1):S466S7. Exclusion Code: X4.

1504. Venkatesh KK, Ferguson K, Smith NA, et al. Association of maternal depression in pregnancy with clinical pathways of preterm birth. Am J Obstet Gynecol. 2018 Jan;218(1):S425-S. PMID: WOS:000423616600206. Exclusion Code: $\mathrm{X} 4$.

1505. Venkatesh KK, Ferguson KK, Smith NA, et al. Association of Antenatal Depression with Clinical Subtypes of Preterm Birth. Am J Perinatol. 2019 May;36(6):567-73. doi: 10.1055/s-0038-1675646. PMID: 30551235. Exclusion Code: X3.

1506. Venkatesh KK, Pate V, Boggess KA, et al. 566: Co-prescription of opioids and psychotropics among commercially insured US pregnant women and neonatal abstinence syndrome. Am J Obstet Gynecol. 2019;220(1):S377. doi:

10.1016/j.ajog.2018.11.588. Exclusion Code: X2.
1507. Venkatesh KK, Riley L, Castro VM, et al. Association of antenatal depression symptoms and antidepressant treatment with preterm birth. Obstet Gynecol. 2016 May;127(5):926-33. doi: 10.1097/aog.0000000000001397. PMID: 27054941. Exclusion Code: X4.

1508. Veroniki AA, Rios P, Cogo E, et al. Comparative safety of antiepileptic drugs for neurological development in children exposed during pregnancy and breast feeding: a systematic review and network meta-analysis. BMJ Open. 2017 Jul 20;7(7):e017248. doi: 10.1136/bmjopen2017-017248. PMID: 28729328. Exclusion Code: X7.

1509. Ververs TF, van Wensen K, Freund MW, et al. Association between antidepressant drug use during pregnancy and child healthcare utilisation. BJOG. 2009 Nov;116(12):156877. doi: 10.1111/j.1471-0528.2009.02292.x. PMID: 19681852. Exclusion Code: X2.

1510. Videman M, Stjerna S, Roivainen R, et al. Evidence for spared attention to faces in 7 month-old infants after prenatal exposure to antiepileptic drugs. Epilepsy Behav. 2016 Nov;64(Pt A):62-8. doi: 10.1016/j.yebeh.2016.09.023. PMID: 27732918. Exclusion Code: X2.

1511. Videman M, Tokariev A, Saikkonen H, et al. Newborn brain function is affected by fetal exposure to maternal serotonin reuptake inhibitors. Cereb Cortex. 2017 Jun 1;27(6):3208-16. doi: 10.1093/cercor/bhw153. PMID: 27269962. Exclusion Code: X4.

1512. Viggedal G, Hagberg BS, Laegreid L, et al. Mental development in late infancy after prenatal exposure to benzodiazepines--a prospective study. J Child Psychol Psychiatry. 1993 Mar;34(3):295-305. PMID: 8463369. Exclusion Code: X4.

1513. Vigod SN, Fung K, Amartey A, et al. Maternal schizophrenia and adverse birth outcomes: what mediates the risk? Soc Psychiatry Psychiatr Epidemiol. 2020 May;55(5):561-70. doi: 10.1007/s00127019-01814-7. PMID: 31811316 . Exclusion Code: X3. 
1514. Vigod SN, Wilson CA, Howard LM. Depression in pregnancy. BMJ. 2016 Mar 24;352:i1547. doi: 10.1136/bmj.i1547. PMID: 27013603. Exclusion Code: X1.

1515. Viguera AC. 'Risk of recurrence in women with bipolar disorder during pregnancy: Prospective study of mood stabilizer discontinuation': Dr Viguera replies. The American Journal of Psychiatry. 2008;165(5):647-8. doi: 10.1176/appi.ajp.2008.08010072r. PMID: 2008-06006-023. Exclusion Code: X1.

1516. Viguera AC, Cohen LS, Nonacs RM, et al. Management of bipolar disorder during pregnancy and the postpartum period: weighing the risks and benefits. In: Cohen LS, Nonacs RM, eds. Mood and anxiety disorders during pregnancy and postpartum. Arlington, VA: American Psychiatric Publishing, Inc.; 2005:53-76. Exclusion Code: X1.

1517. Viinikainen K, Eriksson K, Monkkonen A, et al. The effects of valproate exposure in utero on behavior and the need for educational support in school-aged children. Epilepsy Behav. 2006 Dec;9(4):636-40. doi: 10.1016/j.yebeh.2006.08.007. PMID: 17049311. Exclusion Code: X2.

1518. Viktorin A, Lichtenstein P, Lundholm C, et al. Selective serotonin re-uptake inhibitor use during pregnancy: association with offspring birth size and gestational age. Int $\mathrm{J}$ Epidemiol. 2016 Feb;45(1):170-7. doi: 10.1093/ije/dyv351. PMID: 26748846. Exclusion Code: X12.

1519. Vitale SG, Lagana AS, Muscatello MR, et al. Psychopharmacotherapy in pregnancy and breastfeeding. Obstet Gynecol Surv. 2016 Dec;71(12):721-33. doi: 10.1097/ogx.0000000000000369. PMID: 28005135. Exclusion Code: X7.

1520. Viuff AC, Pedersen LH, Kyng K, et al. Antidepressant medication during pregnancy and epigenetic changes in umbilical cord blood: a systematic review. Clin Epigenetics. 2016;8(1):94. doi: 10.1186/s13148-016-0262-x. PMID: 27610205. Exclusion Code: X7.
1521. Vlenterie R, Van Gelder M, Pop-Purceleanu $\mathrm{M}$, et al. Maternal depression and use of antidepressants during pregnancy increase the risk of adverse pregnancy outcomes: An IPD meta-analysis. Pharmacoepidemiol Drug Saf. 2016;25:328-9. doi: 10.1002/pds.4070. Exclusion Code: X7.

1522. Vlenterie R, Van Gelder MMHJ, PopPurceleanu M, et al. Maternal depression and use of antidepressant during pregnancy and the risk of adverse pregnancy outcomes: An IPD metaanalysis. Eur J Epidemiol. 2015;30(8):928. doi: 10.1007/s10654-0150072-z. Exclusion Code: X7.

1523. Vorster D. Psychiatric drugs and treatment in pregnancy. Br J Psychiatry. 1965 May;111:431-8. PMID: 14327541. Exclusion Code: X1.

1524. Vu H, Shaya FT. Predicting factors of depression, antidepressant use and positive response to antidepressants in perinatal and postpartum women. Clin Pract Epidemiol Ment Health. 2017;13:49-60. doi: 10.2174/1745017901713010049. PMID: 28761500. Exclusion Code: X3.

1525. Wachman EM, Warden AH, Thomas Z, et al. Impact of psychiatric medication coexposure on Neonatal Abstinence Syndrome severity. Drug Alcohol Depend. 2018 Nov 1;192:45-50. doi: 10.1016/j.drugalcdep.2018.07.024. PMID: 30205307. Exclusion Code: X2.

1526. Walch TJ, Tinkelman A. Selective serotonin reuptake inhibitors and autism. Am J Ther. 2016 Mar-Apr;23(2):e325. doi: 10.1097/mjt.0000000000000441. PMID: 26950585. Exclusion Code: X1.

1527. Waldman MD, Safferman AZ. Pregnancy and clozapine. The American Journal of Psychiatry. 1993;150(1):168-9. doi: 10.1176/ajp.150.1.168a. PMID: 199318788-001. Exclusion Code: X1.

1528. Waldmann KD, Greger J, Kluge H. [Indications, adverse effects and complications of lithium therapy]. Z Gesamte Inn Med. 1976 Jul 15;31(14):5368. PMID: 960878. Exclusion Code: X8.

1529. Walling AD. Does valproate prevent relapse in patients with bipolar I disorder? Am Fam Physician. 2010;82(7):827-8. PMID: CN01016674. Exclusion Code: X2. 
1530. Wall-Wieler E, Carmichael SL, Urquia ML, et al. Severe maternal morbidity and postpartum mental health-related outcomes in Sweden: a population-based matchedcohort study. Arch Womens Ment Health. 2018 Oct 17. doi: 10.1007/s00737-0180917-z. PMID: 30334101. Exclusion Code: $\mathrm{X} 4$.

1531. Wan MW, Sharp DJ, Howard LM, et al. Attitudes and adjustment to the parental role in mothers following treatment for postnatal depression. J Affect Disord. 2011 Jun;131(1-3):284-92. doi: 10.1016/j.jad.2011.01.009. PMID: 21349585. Exclusion Code: X5.

1532. Wang C, Cheng Y, Liang J. Effect of diazepam on fetus after intravenous administration during the active phase in labour. Hunan yi ke da xue xue bao [Bulletin of Hunan Medical University]. 1999;24(1):53-5. PMID: CN-00408529. Exclusion Code: X8.

1533. Wang Z, Alfageh B, Mongkhon P, et al. The association between prenatal antipsychotics exposure and adverse obstetric and offspring outcomes: A systematic review and metaanalysis. Br J Clin Pharmacol. 2019;85(7):1645. doi: 10.1111/bcp.13937. Exclusion Code: X7.

1534. Warburton W, Hertzman C, Oberlander TF. A register study of the impact of stopping third trimester selective serotonin reuptake inhibitor exposure on neonatal health. Acta Psychiatr Scand. 2010 Jun;121(6):471-9. doi: 10.1111/j.1600-0447.2009.01490.x. PMID: 19878137. Exclusion Code: X4.

1535. Warner R, Appleby L, Whitton A, et al. A controlled study of fluoxetine and cognitive behavioural counselling in the treatment of post-natal depression conference abstract. Royal college of psychiatrists winter meeting, cardiff, wales. 21-24 january 1997. 1997. PMID: CN-00790279. Exclusion Code: X4.

1536. Warnock FF, Bakeman R, Shearer K, et al. Caregiving behavior and interactions of prenatally depressed mothers (antidepressant-treated and nonantidepressant-treated) during newborn acute pain. Infant Ment Health J. 2009 Jul;30(4):384-406. doi: 10.1002/imhj.20220. PMID: 28636285. Exclusion Code: X5.
1537. Wartko PD, Weiss NS, Enquobahrie DA, et al. Association of antidepressant continuation in pregnancy with maternal gestational weight gain and gestational diabetes. Pharmacoepidemiol Drug Saf. 2018;27:174. doi: 10.1002/pds.4629. Exclusion Code: X4.

1538. Warton FL, Meintjes EM, Warton CMR, et al. Prenatal methamphetamine exposure is associated with reduced subcortical volumes in neonates. Neurotoxicol Teratol.

2018;65:51-9. doi: 10.1016/j.ntt.2017.10.005. Exclusion Code: $\mathrm{X} 2$.

1539. Watanabe O. [Current evaluation of teratogenic and fetotoxic effects of psychotropic drugs]. Seishin Shinkeigaku Zasshi. 2014;116(12):996-1004. PMID: 25823351. Exclusion Code: X8.

1540. Webb RT, Howard L, Abel KM. Antipsychotic drugs for non-affective psychosis during pregnancy and postpartum. Cochrane Database Syst Rev.

2004(2):Cd004411. doi: 10.1002/14651858.CD004411.pub2. PMID: 15106251. Exclusion Code: X7.

1541. Webb RT, Pickles AR, King-Hele SA, et al. Parental mental illness and fatal birth defects in a national birth cohort. Psychol Med. 2008 Oct;38(10):1495-503. doi: 10.1017/s0033291707002280. PMID: 18076770. Exclusion Code: X2.

1542. Webster PA. Withdrawal symptoms in neonates associated with maternal antidepressant therapy. Lancet. 1973 Aug 11;2(7824):318-9. PMID: 4124794. Exclusion Code: X7.

1543. Weedin N, Meltzer-Brody S, Deligiannidis $\mathrm{K}$, et al. 1068: Insights on GABAergic mechanism of postpartum depression from pivotal studies of brexanolone injection and SAGE-217. Am J Obstet Gynecol. 2020;222(1):S659-S60. doi: 10.1016/j.ajog.2019.11.1081. Exclusion Code: X9. 
1544. Weikum WM, Brain U, Chau CM, et al. Prenatal serotonin reuptake inhibitor (SRI) antidepressant exposure and serotonin transporter promoter genotype (SLC6A4) influence executive functions at 6 years of age. Front Cell Neurosci. 2013; 7:180. doi: 10.3389/fncel.2013.00180. PMID: 24130516. Exclusion Code: X4.

1545. Weikum WM, Mayes LC, Grunau RE, et al. The impact of prenatal serotonin reuptake inhibitor (SRI) antidepressant exposure and maternal mood on mother-infant interactions at 3 months of age. Infant Behav Dev. 2013 Dec;36(4):485-93. doi: 10.1016/j.infbeh.2013.04.001. PMID: 23728194. Exclusion Code: X4.

1546. Weikum WM, Mayes LC, Grunau RE, et al. The impact of prenatal serotonin reuptake inhibitor (SRI) antidepressant exposure and maternal mood on mother-infant interactions at 3 months of age. Infant Behav Dev. 2013;36(4):485-93. doi: 10.1016/j.infbeh.2013.04.001. PMID: 201343995-002. Exclusion Code: X4.

1547. Weinstein MR, Goldfield M. Cardiovascular malformations with lithium use during pregnancy. Am J Psychiatry. 1975

May;132(5):529-31. doi:

10.1176/ajp.132.5.529. PMID: 1119612. Exclusion Code: X7.

1548. Weinstein MR, Goldfield MD. Wanted: reports of lithium babies. Pediatrics. 1971 Jul;48(1):161-2. PMID: 5561872. Exclusion Code: X1.

1549. Weiser M, Levi L, Levine SZ, et al. A randomized, double-blind, placebo- and risperidone-controlled study on valnoctamide for acute mania. Bipolar Disord. 2017 Jun;19(4):285-94. doi: 10.1111/bdi.12506. PMID: 28605109. Exclusion Code: X2.

1550. Weisskopf E, Guidi M, Fischer CJ, et al. A population pharmacokinetic model for escitalopram and its major metabolite in depressive patients during the perinatal period: Prediction of infant drug exposure through breast milk. Br J Clin Pharmacol. 2020 Mar 12. doi: 10.1111/bcp.14278. PMID: 32162723. Exclusion Code: X4.
1551. Wemakor A, Casson K, Garne E, et al. Selective serotonin reuptake inhibitor antidepressant use in first trimester pregnancy and risk of specific congenital anomalies: a European register-based study. Eur J Epidemiol. 2015 Nov;30(11):1187-98. doi: 10.1007/s10654-015-0065-y. PMID: 26148560. Exclusion Code: X4.

1552. Wen SW, Walker M. The use of selective serotonin reuptake inhibitors in pregnancy. $\mathrm{J}$ Obstet Gynaecol Can. 2004 Sep;26(9):81922. PMID: 15361279. Exclusion Code: X11.

1553. Wen T, Overton E, Huang Y, et al. 684: Continuation of psychiatric medications during pregnancy. Am J Obstet Gynecol. 2020;222(1):S433. doi: 10.1016/j.ajog.2019.11.698. Exclusion Code: X4.

1554. Wendland BE, Atkinson L, Steiner M, et al. Low maternal sensitivity at 6 months of age predicts higher BMI in 48 month old girls but not boys. Appetite. 2014;82:97-102. doi: 10.1016/j.appet.2014.07.012. Exclusion Code: X2.

1555. Weobong B, ten Asbroek AH, Soremekun S, et al. Association of antenatal depression with adverse consequences for the mother and newborn in rural Ghana: findings from the DON population-based cohort study. PLoS One. 2014;9(12):e116333. doi: 10.1371/journal.pone.0116333. PMID: 25549334. Exclusion Code: X3.

1556. Wesseloo R, Burgerhout KM, Koorengevel $\mathrm{KM}$, et al. [Postpartum psychosis in clinical practice: diagnostic considerations, treatment and prevention]. Tijdschr Psychiatr. 2015;57(1):25-33. PMID: 25601625. Exclusion Code: X8.

1557. Wesseloo R, Wierdsma Al, van Kamp IL, et al. Lithium dosing strategies during pregnancy and the postpartum period. Br J Psychiatry. 2017;211(1):31-6. doi: 10.1192/bjp.bp.116.192799. PMID: 201747009-004. Exclusion Code: X4.

1558. Whalen OM, Campbell LE, Murphy VE, et al. Observational study of mental health in asthmatic women during the prenatal and postnatal periods. J Asthma. 2019 May 31:113. doi: 10.1080/02770903.2019.1621888. PMID: 31148493. Exclusion Code: X2. 
1559. Whitehall J, Smith J. Valproate and babies. Aust N Z J Psychiatry. 2008 Sep;42(9):837. doi: 10.1080/00048670802277313. PMID: 18696289. Exclusion Code: X1.

1560. Whitmore JJ, Berrisford G, Coccia F, et al. A register of women referred to the perinatal mental health service during pregnancy with regard to their physical and mental health and that of their baby. Arch Womens Ment Health. 2013;16:S144-S5. doi:

10.1007/s00737-013-0355-x. Exclusion Code: X5.

1561. Whitworth M, Dowswell T. Routine prepregnancy health promotion for improving pregnancy outcomes. Cochrane Database Syst Rev. 2009 Oct 7(4):CD007536. doi: 10.1002/14651858.CD007536.pub2. PMID: 19821424. Exclusion Code: X7.

1562. Wibroe MA, Mathiasen R, Pagsberg AK, et al. Risk of impaired cognition after prenatal exposure to psychotropic drugs. Acta Psychiatr Scand. 2017 Aug;136(2):177-87. doi: 10.1111/acps.12754. PMID: 28561934. Exclusion Code: X4.

1563. Wichman CL. Atypical antipsychotic use in pregnancy: A retrospective review. Arch Womens Ment Health. 2009;12(1):53-7. doi: 10.1007/s00737-008-0044-3. PMID: 200902370-007. Exclusion Code: X1.

1564. Wichman CL, Moore KM, Lang TR, et al. Congenital heart disease associated with selective serotonin reuptake inhibitor use during pregnancy. Mayo Clin Proc. 2009;84(1):23-7. PMID: 19121250. Exclusion Code: X4.

1565. Wickramaratne P, Gameroff MJ, Pilowsky DJ, et al. Children of depressed mothers 1 year after remission of maternal depression: Findings from the STARD-Child study. The American Journal of Psychiatry. 2011;168(6):593-602. doi: 10.1176/appi.ajp.2010.10010032. PMID: 2011-15374-007. Exclusion Code: X2.

1566. Wide K, Heinonen E, Forsberg L, et al. \#13 MAGDALENA - A randomized study on neurodevelopment after fetal exposure to SSRIs. Reprod Toxicol. 2019;88:137. doi: 10.1016/j.reprotox.2019.05.023. Exclusion Code: X5.
1567. Wieck A. A randomised controlled trial of sertraline in postnatal depression. National research register. 1997. PMID: CN00712808. Exclusion Code: X5.

1568. Wieck A, Reis M. Pharmacological treatment of mental health problems in pregnancy and lactation. In: Castle DJ, Abel KM, eds. Comprehensive women's mental health. New York, NY: Cambridge University Press; 2016:122-36. Exclusion Code: X1.

1569. Wilbanks GD, Bressler B, Peete CH, Jr., et al. Toxic effects of lithium carbonate in a mother and newborn infant. JAMA. 1970 Aug 3;213(5):865-7. PMID: 5468637. Exclusion Code: X7.

1570. Williams JH, Ross L. Consequences of prenatal toxin exposure for mental health in children and adolescents: a systematic review. Eur Child Adolesc Psychiatry. 2007 Jun;16(4):243-53. doi: 10.1007/s00787-0060596-6. PMID: 17200791. Exclusion Code: X7.

1571. Williams K, Oke S. Lithium and pregnancy. Psychiatric Bulletin. 2000;24(6):229-31. Exclusion Code: X1.

1572. Wilmshurst J. ADHD and epilepsy - Myths and facts. J Neurol Sci. 2019;405:58. doi: 10.1016/j.jns.2019.10.163. Exclusion Code: $\mathrm{X} 1$.

1573. Wintz CJB. Difficult decisions: Women of childbearing age, mental illness, and psychopharmacologic therapy. J Am Psychiatr Nurses Assoc. 1999;5(1):5-14. doi: 10.1016/S1078-3903(99)90060-5. PMID: 1999-13324-001. Exclusion Code: $\mathrm{X} 1$.

1574. Wise J. SSRI exposure in pregnancy linked to speech disorders in offspring. BMJ (Online). 2016;355. doi: 10.1136/bmj.i5536. Exclusion Code: X1.

1575. Wise J. Study finds lamotrigine to be safe during pregnancy. BMJ (Online). 2017;359. doi: 10.1136/bmj.j4827. Exclusion Code: $\mathrm{X} 1$.

1576. Wisner K. Estradiol for postpartum depression: translational challenges. Neuropsychopharmacology. 2014;39:S32. doi: 10.1038/npp.2014.279. PMID: CN-

01066391. Exclusion Code: X4. 
1577. Wisner KL. Postpartum depression: transdermal estradiol versus sertraline. Clinicaltrials.gov [www.clinicaltrials.gov]. 2008. PMID: CN-00852184. Exclusion Code: X5.

1578. Wisner KL. SSRI treatment during pregnancy: are we asking the right questions? Depress Anxiety. 2010 Aug;27(8):695-8. doi: 10.1002/da.20729. PMID: 20694964. Exclusion Code: X1.

1579. Wisner KL. Impact of SSRI antidepressants and major depression on pregnancy and infant outcomes. Arch Womens Ment Health. 2011;14:S113. doi: 10.1007/s00737011-0238-y. Exclusion Code: X9.

1580. Wisner KL. Should estradiol be prescribed for postpartum depression? Biological psychiatry. 2014;75(9 SUPPL. 1):299S. doi: 10.1016/j.biopsych.2014.03.016. PMID: CN-01060460. Exclusion Code: X3.

1581. Wisner KL. Does prenatal exposure to sris or maternal major depression impact infant developmental outcomes? Biol Psychiatry. 2014;75(9):276S. doi:

10.1016/j.biopsych.2014.03.016. Exclusion Code: X9.

1582. Wisner KL. Impact of prenatal exposure to SSRIs or maternal depression disorder on infant developmental outcomes. Eur Neuropsychopharmacol. 2015;25:S156. Exclusion Code: X9.

1583. Wisner KL, Costantino M, Sit DK, et al. Transdermal estradiol for postpartum major depression. Arch Womens Ment Health. 2011;14:S86. doi: 10.1007/s00737-0100203-1. Exclusion Code: X5.

1584. Wisner KL, Gelenberg AJ, Leonard H, et al. Pharmacologic treatment of depression during pregnancy. JAMA. 1999 Oct 6;282(13):1264-9. PMID: 10517430. Exclusion Code: X7.

1585. Wisner KL, Peindl KS, Perel JM, et al. Sertraline prevents postpartum depression. 156th annual meeting of the american psychiatric association, may 17-22, san francisco CA. 2003:No. 101. PMID: CN00542603. Exclusion Code: X2.

1586. Wisner KL, Perel JM. Nortriptyline treatment of breast-feeding women. Am J Psychiatry. 1996 Feb;153(2):295. PMID: 8561220. Exclusion Code: X7.
1587. Wisner KL, Perel JM, Findling RL. Antidepressant treatment during breastfeeding. Am J Psychiatry. 1996 Sep;153(9):1132-7. doi: 10.1176/ajp.153.9.1132. PMID: 8780414. Exclusion Code: X7.

1588. Wisner KL, Perel JM, Peindl KS, et al. Prevention of recurrent postpartum depression: a randomized clinical trial. J Clin Psychiatry. 2001 Feb;62(2):82-6. PMID: 11247106. Exclusion Code: X2.

1589. Wisner KL, Perel JM, Peindl KS, et al. Prevention of postpartum depression: a pilot randomized clinical trial. Am J Psychiatry. 2004 Jul;161(7):1290-2. doi: 10.1176/appi.ajp.161.7.1290. PMID: 15229064. Exclusion Code: X3.

1590. Wisner KL, Stika CS, Ciolino JD. The First Food and Drug Administration-Indicated Drug for Postpartum DepressionBrexanolone. JAMA Psychiatry. 2019 Jun 26. doi: 10.1001/jamapsychiatry.2019.1546. PMID: 31241747. Exclusion Code: X1.

1591. Wisner KL, Wheeler SB. Prevention of recurrent postpartum major depression. Hosp Community Psychiatry. 1994 Dec;45(12):1191-6. PMID: 7868100. Exclusion Code: X4.

1592. Witlin AG. Risk of fetal anomalies with exposure to selective serotonin reuptake inhibitors. JAMA. 1998 Jun 17;279(23):1873. PMID: 9634253. Exclusion Code: X1.

1593. Witt WP, DeLeire T, Hagen EW, et al. The prevalence and determinants of antepartum mental health problems among women in the USA: A nationally representative population-based study. Arch Womens Ment Health. 2010;13(5):425-37. doi: 10.1007/s00737-010-0176-0. PMID: 201019591-007. Exclusion Code: X7.

1594. Womersley K, Ripullone K, Agius M. What are the risks associated with different Selective Serotonin Re-uptake Inhibitors (SSRIs) to treat depression and anxiety in pregnancy? An evaluation of current evidence. Psychiatr Danub. 2017 Sep;29(Suppl 3):629-44. PMID: 28953843. Exclusion Code: X7. 
1595. Wood A. Prenatal exposure to sodium valproate is associated with increased risk of childhood autism and autistic spectrum disorder. Evid Based Nurs. 2014 Jul;17(3):84. doi: 10.1136/eb-2013-101422. PMID: 23999195. Exclusion Code: X1.

1596. Wood AG, Nadebaum C, Anderson V, et al. Prospective assessment of autism traits in children exposed to antiepileptic drugs during pregnancy. Epilepsia. 2015 Jul;56(7):1047-55. doi: 10.1111/epi.13007. PMID: 25963613. Exclusion Code: X2.

1597. Wood ME, Hernandez-Diaz S, Nordeng HME. Are associations between prenatal antidepressant exposure and toddler neurodevelopment mediated by gestational age at birth? Pharmacoepidemiol Drug Saf. 2018;27:174-5. doi: 10.1002/pds.4629.

Exclusion Code: X4.

1598. Wooltorton E. Persistent pulmonary hypertension of the newborn and maternal use of SSRIs. CMAJ. 2006 May 23;174(11):1555-6. doi: 10.1503/cmaj.060509. PMID: 16670395. Exclusion Code: X1.

1599. Worly BL, Gur TL. The effect of mental illness and psychotropic medication on gametes and fertility: a systematic review. J Clin Psychiatry. 2015 Jul;76(7):974-85. doi: 10.4088/JCP.14r09080. PMID: 25700201. Exclusion Code: X7.

1600. Worsley R, Gilbert H, Gavrilidis E, et al. Breastfeeding and psychotropic medications. Lancet. 2013 Mar 16;381(9870):905. doi: 10.1016/s0140-6736(13)60671-6. PMID: 23499040. Exclusion Code: X1.

1601. Wurtz AM, Rytter D, Vestergaard CH, et al. Prenatal exposure to antiepileptic drugs and use of primary healthcare during childhood: a population-based cohort study in Denmark. BMJ Open. 2017 Jan 5;7(1):e012836. doi: 10.1136/bmjopen2016-012836. PMID: 28069620. Exclusion Code: X2.

1602. Wurtz AML, Hostrup Vestergaard C, Rytter D, et al. Prenatal exposure to antipsychotic medication and use of primary health care system in childhood: a population-based cohort study in Denmark. Clin Epidemiol. 2017;9:657-66. doi: 10.2147/clep.s145524. PMID: 29238228. Exclusion Code: X5.
1603. Yacobi S, Ornoy A. Is lithium a real teratogen? What can we conclude from the prospective versus retrospective studies? A review. Isr J Psychiatry Relat Sci. 2008;45(2):95-106. PMID: 18982835. Exclusion Code: X7.

1604. Yadav DS. SSRIs and congenital defects. Case registers in pregnancy? BMJ. 2009 Oct 21;339:b4286. doi: 10.1136/bmj.b4286. PMID: 19846493. Exclusion Code: X1.

1605. Yakuwa N, Watanabe O, Nakajima K, et al. Second-generation antipsychotic drugs use in first-trimester and the risk for major malformations: A prospective cohort study from Japan drug information institute in pregnancy. Birth Defects Research. 2019;111(9):560. doi: 10.1002/bdr2.v111.9. Exclusion Code: X4.

1606. Yamashita H, Yoshida K, Kanba S. Maternal attachment style, mother-infant interaction and their toddlers' socioemotional outcome. Arch Womens Ment Health. 2015;18(2):326-7. doi: 10.1007/s00737-014-0488-6. Exclusion Code: X2.

1607. Yan Y, Cheng Y, Crowe B, et al. First trimester fluoxetine use and major malformations: A meta-analysis of epidemiological studies. Pharmacoepidemiol Drug Saf. 2013;22:168-9. doi: 10.1002/pds.3512. Exclusion Code: X7.

1608. Yang F, Chen J, Miao MH, et al. Risk of autism spectrum disorder in offspring following paternal use of selective serotonin reuptake inhibitors before conception: a population-based cohort study. BMJ Open. 2017 Dec 22;7(12):e016368. doi: 10.1136/bmjopen-2017-016368. PMID: 29275337. Exclusion Code: X2.

1609. Yaris F, Ulku C, Kesim M, et al. Psychotropic drugs in pregnancy: a casecontrol study. Prog Neuropsychopharmacol Biol Psychiatry. 2005 Feb;29(2):333-8. doi: 10.1016/j.pnpbp.2004.11.020. PMID: 15694243. Exclusion Code: X4.

1610. Yawn BP, Dietrich AJ, Wollan P, et al. TRIPPD: A practice-based network effectiveness study of postpartum depression screening and management. Ann Fam Med. 2012;10(4):320-9. doi: 10.1370/afm.1418. PMID: 2012-20921-005. Exclusion Code: $\mathrm{X} 4$. 
1611. Yazdy MM, Mitchell AA, Louik C, et al. Use of selective serotonin-reuptake inhibitors during pregnancy and the risk of clubfoot. Epidemiology. 2014

Nov;25(6):859-65. doi: 10.1097/ede.0000000000000157. PMID: 25171134. Exclusion Code: X10.

1612. Yazdy MM, Mitchell AM, Louik C, et al. Use of selective serotonin-reuptake inhibitors during pregnancy and the risk of clubfoot. Pharmacoepidemiol Drug Saf. 2012;21:376. doi: 10.1002/pds.3324. Exclusion Code: X4.

1613. Yazici E, Kirkan TS, Aslan PA, et al. Untreated depression in the first trimester of pregnancy leads to postpartum depression: High rates from a natural follow-up study. Neuropsychiatr Dis Treat. 2015;11:405-11. doi: 10.2147/NDT.S77194. Exclusion Code: $\mathrm{X} 4$.

1614. Yogev Y, Ben-Haroush A, Kaplan B. Maternal clozapine treatment and decreased fetal heart rate variability. Int J Gynaecol Obstet. 2002 Dec;79(3):259-60. PMID: 12445996. Exclusion Code: X7.

1615. Yonkers KA, Gilstad-Hayden K, Forray A, et al. Association of panic disorder, generalized anxiety disorder, and benzodiazepine treatment during pregnancy with risk of adverse birth outcomes. JAMA Psychiatry. 2017 Nov 1;74(11):1145-52. doi: 10.1001/jamapsychiatry.2017.2733. PMID: 28903165. Exclusion Code: X4.

1616. Yonkers KA, Gotman N, Smith MV, et al. Does antidepressant use attenuate the risk of a major depressive episode in pregnancy? Epidemiology. 2011 Nov;22(6):848-54. doi: 10.1097/EDE.0b013e3182306847. PMID: 21900825. Exclusion Code: X2.

1617. Yonkers KA, Norwitz ER, Smith MV, et al. Depression and serotonin reuptake inhibitor treatment as risk factors for preterm birth. Epidemiology. 2012 Sep;23(5):677-85. doi: 10.1097/EDE.0b013e31825838e9. PMID: 22627901. Exclusion Code: X12.

1618. Yonkers KA, Smith MV, Forray A, et al. Pregnant women with posttraumatic stress disorder and risk of preterm birth. JAMA Psychiatry. 2014 Aug;71(8):897-904. doi: 10.1001/jamapsychiatry.2014.558. PMID: 24920287. Exclusion Code: X4.
1619. Yonkers KA, Wisner KL, Stewart DE, et al. The management of depression during pregnancy: a report from the American Psychiatric Association and the American College of Obstetricians and Gynecologists. Gen Hosp Psychiatry. 2009 SepOct;31(5):403-13. doi: 10.1016/j.genhosppsych.2009.04.003. PMID: 19703633. Exclusion Code: X7.

1620. Yonkers KA, Wisner KL, Stewart DE, et al. The management of depression during pregnancy: a report from the American Psychiatric Association and the American College of Obstetricians and Gynecologists. Obstet Gynecol. 2009 Sep;114(3):703-13. doi: 10.1097/AOG.0b013e3181ba0632. PMID: 19701065. Exclusion Code: X7.

1621. Yoshida K, Fujinaga Y, Yamashita H, et al. Clinical survey of the mother-infant mental health clinic: Psychiatric characteristics of consecutive 109 patients.

Neuropsychopharmacology. 2011;36:S162S3. doi: 10.1038/npp.2011.291. Exclusion Code: X5.

1622. Yoshida K, Kumar RC, Smith B, et al. Psychotropic drugs in breast milk: no evidence for adverse effects on prepulse modulation of startle reflex or on cognitive level in infants. Dev Psychobiol. 1998 Apr;32(3):249-56. PMID: 9553734. Exclusion Code: X4.

1623. Yoshida K, Smith B, Craggs M, et al. Neuroleptic drugs in breast-milk: a study of pharmacokinetics and of possible adverse effects in breast-fed infants. Psychol Med. 1998 Jan;28(1):81-91. PMID: 9483685. Exclusion Code: X4.

1624. Yoshida K, Smith B, Craggs M, et al. Investigation of pharmacokinetics and of possible adverse effects in infants exposed to tricyclic antidepressants in breast-milk. J Affect Disord. 1997 May;43(3):225-37. PMID: 9186793. Exclusion Code: X5.

1625. Yoshida K, Yamashita H. Clinical survey of themother-infant mental health clinic in kyushu university hospital: Psychiatric characteristics of consecutive 109 women and their infants. Arch Womens Ment Health. 2013;16:S104-S5. doi: 10.1007/s00737-013-0355-x. Exclusion Code: X5. 
1626. Youash S, Sharma V. Depression, Antidepressants and Hypertensive Disorders of Pregnancy: A Systematic Review. Curr Drug Saf. 2019;14(2):102-8. doi: 10.2174/1574886314666190121144711. PMID: 30666916. Exclusion Code: X7.

1627. Yu H, Liu C, Wang X. The therapeutic effects of paroxetine addition of psychological intervention on post-partum depression. Progress in obstetrics and gynecology. 2006;15(4):289-91. PMID: CN00710808. Exclusion Code: X8.

1628. Yuen S, Rezai S, Henderson CE. Exposure to selective serotonin reuptake inhibitors in early pregnancy and the risk of miscarriage. Obstet Gynecol. 2015 Feb;125(2):493. doi: 10.1097/aog.0000000000000656. PMID: 25611632. Exclusion Code: X1.

1629. Yuksel G, Alptekin FB, Atar AO, et al. Unintended pregnancies as a burden for women with psychiatric diagnoses. Klinik Psikofarmakoloji Bulteni. 2015;25:S204. Exclusion Code: X5.

1630. Yuksel G, Ozer E, Eskiler S, et al. Why ECT in pregnancy? Klinik Psikofarmakoloji Bulteni. 2014;24:S340. Exclusion Code: X5.

1631. Yuksel G, Sahingoz M, Uguz F. Birth weight and preterm birth in babies of pregnant women with major depression in relation to treatment with antidepressants. Arch Womens Ment Health. 2015;18(2):395. doi: 10.1007/s00737-0140488-6. Exclusion Code: X9.

1632. Zainul Rashid MR, Das S. Antidepressant medication use patterns during pregnancy and pregnancy outcomes: an insight. Am J Obstet Gynecol. 2013 Feb;208(2):159-60. doi: 10.1016/j.ajog.2012.11.002. PMID: 23159746. Exclusion Code: X1.

1633. Zalzstein E, Koren G, Einarson T, et al. A case-control study on the association between first trimester exposure to lithium and Ebstein's anomaly. Am J Cardiol. 1990 Mar 15;65(11):817-8. PMID: 2107734. Exclusion Code: X1.

1634. Zelson C, Ja Lee S. Neonatal narcotic addiction exposure to heroin and methadone. Pediatr Res. 1973;7(4):289. Exclusion Code: $\mathrm{X} 2$.
1635. Zhang TN, Gao SY, Shen ZQ, et al. Use of selective serotonin-reuptake inhibitors in the first trimester and risk of cardiovascularrelated malformations: a meta-analysis of cohort studies. Sci Rep. 2017 Feb 21;7:43085. doi: 10.1038/srep43085. PMID: 28220881. Exclusion Code: X7.

1636. Zhao JP, Sheehy O, Iessa N, et al. First trimester exposure to citalopram and the risk of major congenital malformations in a cohort of depressed women.

Pharmacoepidemiol Drug Saf. 2015;24:2089. doi: 10.1002/pds.3838. Exclusion Code: X9.

1637. Zhao JPZ, Sheehy OS, Iessa NI, et al. First trimester exposure to citalopram and the risk of major congenital malformations in a cohort of depressed women. Birth Defects Research Part A - Clinical and Molecular Teratology. 2015;103(5):386. doi: 10.1002/bdra.23387. Exclusion Code: X9.

1638. Zhao X, Liu Q, Cao S, et al. A meta-analysis of selective serotonin reuptake inhibitors (SSRIs) use during prenatal depression and risk of low birth weight and small for gestational age. J Affect Disord. 2018 Dec 1;241:563-70. doi: 10.1016/j.jad.2018.08.061. PMID: 30153640. Exclusion Code: X7.

1639. Zheng W, Cai DB, Zheng W, et al. Brexanolone for postpartum depression: A meta-analysis of randomized controlled studies. Psychiatry Res. 2019 Sep;279:83-9. doi: 10.1016/j.psychres.2019.07.006. PMID: 31323375. Exclusion Code: X7.

1640. Zhou XH, Li YJ, Ou JJ, et al. Association between maternal antidepressant use during pregnancy and autism spectrum disorder: an updated meta-analysis. Mol Autism. 2018;9:21. doi: 10.1186/s13229-018-02077. PMID: 29599960. Exclusion Code: X7.

1641. Zitner D, Bischoff A. Using antidepressants during pregnancy. CMAJ. 2005 Nov 8;173(10):1205; author reply -6 . doi: 10.1503/cmaj.1050149. PMID: 16275973. Exclusion Code: X1. 
1642. Zlotnick C, Stuart S. Placebo controlled trial of sertraline and interpersonal psychotherapy for postpartum depression.

Http://clinicaltrials.gov/ct2/show/nct006023 55.

2008(http://www.clinicaltrials.gov/ct2/show/ NCT00602355 (accessed 12 March 2013)). PMID: CN-00850586. Exclusion Code: X5.
1643. Zuccotti GV, Fabiano V, Manfredini V. Neonates born to mothers using antidepressant drugs. Early Hum Dev. 2012 May;88 Suppl 2:S84-5. doi: 10.1016/s03783782(12)70023-3. PMID: 22633523. Exclusion Code: X1.

1644. Zuckerman B, Bauchner H, Parker S, et al. Maternal depressive symptoms during pregnancy, and newborn irritability. J Dev Behav Pediatr. 1990 Aug;11(4):190-4.

PMID: 2212032. Exclusion Code: X3. 


\section{Appendix C. References for Appendixes}

1. Sterne JA, Hernan MA, Reeves BC, et al. ROBINS-I: a tool for assessing risk of bias in non-randomised studies of interventions. BMJ. 2016 Oct 12;355:i4919. doi: 10.1136/bmj.i4919. PMID: 27733354.

2. Higgins JPT, Green S. Cochrane handbook for systematic reviews of interventions: John Wiley \& Sons, Ltd; 2011.

3. Agency for Healthcare Research and Quality. Methods guide for effectiveness and comparative effectiveness reviews. Rockville, MD: Agency for Healthcare Research and Quality; 2014. https://effectivehealthcare.ahrq.gov/p roducts/cer-methods-guide/overview.

4. West S, Gartlehner G, Mansfield A, et al. Comparative effectiveness review methods: clinical heterogeneity. (Prepared by RTI International-University of North Carolina Evidence-based Practice Center under Contract No. 2902007-10056-I). Rockville, MD: Agency for Healthcare Research and Quality; 2010.

5. Eke AC, Saccone G, Berghella V. Selective serotonin reuptake inhibitor (SSRI) use during pregnancy and risk of preterm birth: a systematic review and metaanalysis. BJOG. 2016

Nov;123(12):1900-7. doi: 10.1111/1471-0528.14144. PMID: 27239775.

6. Huybrechts KF, Bateman BT, Palmsten K, et al. Antidepressant use late in pregnancy and risk of persistent pulmonary hypertension of the newborn. JAMA. 2015 Jun
2;313(21):2142-51. doi: 10.1001/jama.2015.5605. PMID: 26034955.

7. Huybrechts KF, Palmsten K, Avorn $\mathrm{J}$, et al. Antidepressant use in pregnancy and the risk of cardiac defects. N Engl J Med. 2014 Jun 19;370(25):2397-407. doi: 10.1056/NEJMoa1312828. PMID: 24941178.

8. Lupattelli A, Wood M, Ystrom E, et al. Effect of time-dependent selective serotonin reuptake inhibitor antidepressants during pregnancy on behavioral, emotional, and social development in preschool-aged children. J Am Acad Child Adolesc Psychiatry. 2018 Mar;57(3):200-8. doi: 10.1016/j.jaac.2017.12.010. PMID: 29496129.

9. Grzeskowiak LE, Gilbert AL, Sorensen TI, et al. Prenatal exposure to selective serotonin reuptake inhibitors and childhood overweight at 7 years of age. Ann Epidemiol. 2013 Nov;23(11):681-7. doi: 10.1016/j.annepidem.2013.08.005. PMID: 24113367.

10. Palmsten K, Huybrechts KF, Mogun $\mathrm{H}$, et al. Harnessing the Medicaid Analytic eXtract (MAX) to Evaluate Medications in Pregnancy: Design Considerations. PLoS One. 2013;8(6):e67405. doi: 10.1371/journal.pone.0067405. PMID: 23840692.

11. Petersen I, McCrea RL, Sammon CJ, et al. Risks and benefits of psychotropic medication in pregnancy: cohort studies based on UK electronic primary care health records. Health Technol Assess. 2016 Mar;20(23):1-176. doi: 
10.3310/hta20230. PMID:

27029490.

12. Airways Group. The transitivity assumption. London, United Kingdom: Cochrane.

https://airways.cochrane.org/sites/air ways.cochrane.org/files/public/uploa ds/The $\% 20$ transitivity $\% 20$ assumptio n\%20CJC.pdf. Accessed on November 132019.

13. Akl E, Mustafa R, Wiercioch N, et al. 1. Overview of the GRADE approach. In: Schunemann H, Brozek J, Guyatt G, Oxman A, eds. GRADE handbook. Geneva, Switzerland: World Health Organization; 2013.

14. Berkman ND, Lohr KN, Ansari MT, et al. Grading the strength of a body of evidence when assessing health care interventions: an EPC update. J Clin Epidemiol. 2015 Nov;68(11):1312-24. doi: 10.1016/j.jclinepi.2014.11.023. PMID: 25721570.

15. Atkins D, Chang SM, Gartlehner G, et al. Assessing applicability when comparing medical interventions: AHRQ and the Effective Health Care Program. J Clin Epidemiol. 2011 Nov;64(11):1198-207. doi: 10.1016/j.jclinepi.2010.11.021. PMID: 21463926.

16. O'Hara MW, Pearlstein T, Stuart S, et al. A placebo controlled treatment trial of sertraline and interpersonal psychotherapy for postpartum depression. J Affect Disord. 2018 Oct 31;245:524-32. doi: 10.1016/j.jad.2018.10.361. PMID: 30447565.

17. Meltzer-Brody S, Colquhoun H, Riesenberg R, et al. Brexanolone injection in post-partum depression: two multicentre, double-blind, randomised, placebo-controlled, phase 3 trials. Lancet. 2018 Sep 22;392(10152):1058-70. doi: 10.1016/s0140-6736(18)31551-4. PMID: 30177236.

18. Lupattelli A, Twigg MJ, Zagorodnikova K, et al. Selfreported perinatal depressive symptoms and postnatal symptom severity after treatment with antidepressants in pregnancy: a cross-sectional study across 12 European countries using the Edinburgh Postnatal Depression Scale. Clin Epidemiol. 2018;10:65569. doi: 10.2147/clep.s156210. PMID: 29922092.

19. Kanes S, Colquhoun H, GunduzBruce H, et al. Brexanolone (SAGE547 injection) in post-partum depression: a randomised controlled trial. Lancet. 2017 Jul 29;390(10093):480-9. doi: 10.1016/s0140-6736(17)31264-3. PMID: 28619476.

20. Swanson SA, Hernandez-Diaz S, Palmsten K, et al. Methodological considerations in assessing the effectiveness of antidepressant medication continuation during pregnancy using administrative data. Pharmacoepidemiol Drug Saf. 2015 Sep;24(9):934-42. doi: 10.1002/pds.3798. PMID: 26045370.

21. Milgrom J, Gemmill AW, Ericksen $\mathrm{J}$, et al. Treatment of postnatal depression with cognitive behavioural therapy, sertraline and combination therapy: a randomised controlled trial. Aust N Z J Psychiatry. 2015 Mar;49(3):236-45. doi: $10.1177 / 0004867414565474$. PMID: 25586754.

22. Hantsoo L, Ward-O'Brien D, Czarkowski KA, et al. A randomized, placebo-controlled, double-blind trial of sertraline for 
postpartum depression.

Psychopharmacology (Berl). 2014

Mar;231(5):939-48. doi:

10.1007/s00213-013-3316-1. PMID:

24173623.

23. Bloch M, Meiboom H, Lorberblatt $\mathrm{M}$, et al. The effect of sertraline addon to brief dynamic psychotherapy for the treatment of postpartum depression: a randomized, doubleblind, placebo-controlled study. J Clin Psychiatry. 2012 Feb;73(2):23541. doi: $10.4088 / \mathrm{JCP} .11 \mathrm{~m} 07117$. PMID: 22401479.

24. Newport DJ, Stowe ZN, Viguera $\mathrm{AC}$, et al. Lamotrigine in bipolar disorder: efficacy during pregnancy. Bipolar Disord. 2008 May;10(3):432-6. doi: 10.1111/j.1399-5618.2007.00565.x. PMID: 18402631

25. Yonkers KA, Lin H, Howell HB, et al. Pharmacologic treatment of postpartum women with new-onset major depressive disorder: a randomized controlled trial with paroxetine. J Clin Psychiatry. 2008 Apr;69(4):659-65. PMID: 18363420.

26. Viguera AC, Whitfield T, Baldessarini RJ, et al. Risk of recurrence in women with bipolar disorder during pregnancy: prospective study of mood stabilizer discontinuation. Am J Psychiatry. 2007 Dec;164(12):1817-24; quiz 923. doi:

10.1176/appi.ajp.2007.06101639. PMID: 18056236.

27. Oberlander TF, Warburton W, Misri $S$, et al. Neonatal outcomes after prenatal exposure to selective serotonin reuptake inhibitor antidepressants and maternal depression using population-based linked health data. Arch Gen Psychiatry. 2006 Aug;63(8):898-906. doi: 10.1001/archpsyc.63.8.898. PMID: 16894066.

28. Cohen LS, Altshuler LL, Harlow BL, et al. Relapse of major depression during pregnancy in women who maintain or discontinue antidepressant treatment. JAMA. 2006 Feb 1;295(5):499-507. doi: 10.1001/jama.295.5.499. PMID: 16449615.

29. Appleby L, Warner R, Whitton A, et al. A controlled study of fluoxetine and cognitive-behavioural counselling in the treatment of postnatal depression. BMJ. 1997 Mar 29;314(7085):932-6. PMID: 9099116.

30. Reisner JG. Hydroxyzine for controlling postpartum anxiety: a double-blind study. Nebr State Med J. 1967 Nov;52(11):498-9. PMID: 4230037.

31. Adhikari K, Patten SB, Lee S, et al. Risk of adverse perinatal outcomes among women with pharmacologically treated and untreated depression during pregnancy: A retrospective cohort study. Paediatr Perinat Epidemiol. 2019 Sep;33(5):323-31. doi: 10.1111/ppe.12576. PMID: 31556142.

32. Bérard A, Sheehy O, Zhao JP, et al. Impact of antidepressant use, discontinuation, and dosage modification on maternal depression during pregnancy. Eur Neuropsychopharmacol. 2019 Jul;29(7):803-12. doi: 10.1016/j.euroneuro.2019.06.007. PMID: 31248651.

33. Schaffer AL, Zoega H, Tran DT, et al. Trajectories of antipsychotic use before and during pregnancy and associated maternal and birth characteristics. Aust N Z J 
Psychiatry. 2019 Dec;53(12):1208-

21. doi:

10.1177/0004867419847764. PMID: 31088287.

34. Solé E, Roca A, Torres A, et al. Obstetric complications in bipolar disorder: psychiatric factors and the risk of caesarean section. Eur Neuropsychopharmacol. 2020 Mar;32:47-55. doi: 10.1016/j.euroneuro.2019.12.115. PMID: 31911063.

35. Lanza di Scalea T, Hanusa BH, Wisner KL. Sexual function in postpartum women treated for depression: results from a randomized trial of nortriptyline versus sertraline. J Clin Psychiatry. 2009 Mar;70(3):423-8. PMID: 19284932.

36. Wisner KL, Hanusa BH, Perel JM, et al. Postpartum depression: a randomized trial of sertraline versus nortriptyline. J Clin Psychopharmacol. 2006 Aug;26(4):353-60. doi: 10.1097/01.jcp.0000227706.56870.d d. PMID: 16855451.

37. Wesseloo R, Liu X, Clark CT, et al. Risk of postpartum episodes in women with bipolar disorder after lamotrigine or lithium use during pregnancy: a population-based cohort study. J Affect Disord. 2017 Aug 15;218:394-7. doi: 10.1016/j.jad.2017.04.070. PMID: 28501739 .

38. Sharma V, Smith A, Mazmanian D. Olanzapine in the prevention of postpartum psychosis and mood episodes in bipolar disorder. Bipolar Disord. 2006 Aug;8(4):400-4. doi: 10.1111/j.1399-5618.2006.00335.x. PMID: 16879140.

39. Koren G, Nulman I, Addis A. Outcome of children exposed in utero to fluoxetine: a critical review. Depress Anxiety. 1998;8 Suppl 1:2731. PMID: 9809211.

40. Pastuszak A, Schick-Boschetto B, Zuber C, et al. Pregnancy outcome following first-trimester exposure to fluoxetine (Prozac). JAMA. 1993 May 5;269(17):2246-8. PMID: 8474204.

41. Lebedevs T, Gan M, Teoh SWK, et al. Analysis of perinatal women attending a mother and baby unit taking sodium valproate or lithium with a diagnosis of bipolar affective disorder. Psychiatr Q. 2020 Mar 10. doi: 10.1007/s11126-020-09729-2. PMID: 32157548.

42. Frayne J, Nguyen T, Hauck Y, et al. The relationship between pregnancy exposure to antidepressant and atypical antipsychotic medications and placental weight and birth weight ratio: a retrospective cohort study. J Clin Psychopharmacol. 2018 Dec;38(6):563-9. doi: 10.1097/jcp.0000000000000964. PMID: 30346334.

43. Cohen LS, Goez-Mogollon L, Sosinsky AZ, et al. Risk of major malformations in infants following first-trimester exposure to quetiapine. Am J Psychiatry. 2018 Aug 16:appiajp201818010098. doi: 10.1176/appi.ajp.2018.18010098. PMID: 30111186.

44. Cohen LS, Viguera AC, McInerney $\mathrm{KA}$, et al. Reproductive safety of second-generation antipsychotics: Current data from the Massachusetts General Hospital National Pregnancy Registry for atypical antipsychotics. Am J Psychiatry. 2016 Mar 1;173(3):263-70. doi: 10.1176/appi.ajp.2015.15040506. PMID: 26441156. 
45. Gustafsson HC, Goodman SH, Feng

$\mathrm{T}$, et al. Major depressive disorder during pregnancy: psychiatric medications have minimal effects on the fetus and infant yet development is compromised. Dev Psychopathol. 2018 Aug;30(3):773-85. doi: $10.1017 / \mathrm{s} 0954579418000639$. PMID: 30068426.

46. Freeman MP, Goez-Mogollon L, McInerney KA, et al. Obstetrical and neonatal outcomes after benzodiazepine exposure during pregnancy: results from a prospective registry of women with psychiatric disorders. Gen Hosp Psychiatry. 2018 Jul - Aug;53:73-9. doi:

10.1016/j.genhosppsych.2018.05.010 . PMID: 29958100.

47. Forsberg L, Adler M, Romer Ek I, et al. Maternal mood disorders and lithium exposure in utero were not associated with poor cognitive development during childhood. Acta Paediatr. 2018 Aug;107(8):1379-88. doi: 10.1111/apa.14152. PMID: 29150869.

48. Leggett C, Costi L, Morrison JL, et al. Antidepressant use in late gestation and breastfeeding rates at discharge from hospital. J Hum Lact. 2017 Nov;33(4):701-9. doi: $10.1177 / 0890334416678209$. PMID: 28984528.

49. Liu X, Agerbo E, Ingstrup KG, et al. Antidepressant use during pregnancy and psychiatric disorders in offspring: Danish nationwide register based cohort study. BMJ. 2017 Sep 6;358:j3668. doi: 10.1136/bmj.j3668. PMID: 28877907.

50. Panchaud A, Hernandez-Diaz S, Freeman MP, et al. Use of atypical antipsychotics in pregnancy and maternal gestational diabetes. $\mathrm{J}$ Psychiatr Res. 2017 Dec;95:84-90. doi:

10.1016/j.jpsychires.2017.07.025. PMID: 28810177.

51. Rai D, Lee BK, Dalman C, et al. Antidepressants during pregnancy and autism in offspring: population based cohort study. BMJ. 2017 Jul 19;358:j2811. doi: 10.1136/bmj.j2811. PMID: 28724519.

52. Viktorin A, Uher R, Kolevzon A, et al. Association of antidepressant medication use during pregnancy with intellectual disability in offspring. JAMA Psychiatry. 2017 Oct 1;74(10):1031-8. doi: 10.1001/jamapsychiatry.2017.1727. PMID: 28700807.

53. Boukhris T, Sheehy O, Berard A. Antidepressant use in pregnancy and the risk of attention deficit with or without hyperactivity disorder in children. Paediatr Perinat Epidemiol. 2017 Jul;31(4):363-73. doi:

10.1111/ppe.12378. PMID: 28640459.

54. Cantarutti A, Merlino L, Giaquinto $\mathrm{C}$, et al. Use of antidepressant medication in pregnancy and adverse neonatal outcomes: a populationbased investigation.

Pharmacoepidemiol Drug Saf. 2017 Sep;26(9):1100-8. doi: 10.1002/pds.4242. PMID: 28594145.

55. Man KKC, Chan EW, Ip P, et al. Prenatal antidepressant use and risk of attention-deficit/hyperactivity disorder in offspring: population based cohort study. BMJ. 2017 May 31;357:j2350. doi: 10.1136/bmj.j2350. PMID: 28566274.

56. Viktorin A, Uher R, Reichenberg A, et al. Autism risk following 
antidepressant medication during pregnancy. Psychol Med. 2017

Dec;47(16):2787-96. doi:

$10.1017 / \mathrm{s} 0033291717001301$.

PMID: 28528584.

57. Berard A, Zhao JP, Sheehy O.

Antidepressant use during pregnancy and the risk of major congenital malformations in a cohort of depressed pregnant women: an updated analysis of the Quebec Pregnancy Cohort. BMJ Open. 2017 Jan 12;7(1):e013372. doi:

10.1136/bmjopen-2016-013372. PMID: 28082367.

58. Cantarutti A, Merlino L, Monzani E, et al. Is the risk of preterm birth and low birth weight affected by the use of antidepressant agents during pregnancy? A population-based investigation. PLoS One. 2016;11(12):e0168115. doi:

10.1371/journal.pone.0168115.

PMID: 27977749.

59. Brown AS, Gyllenberg D, Malm H, et al. Association of selective serotonin reuptake inhibitor exposure during pregnancy with speech, scholastic, and motor disorders in offspring. JAMA Psychiatry. 2016 Nov 1;73(11):1163-70. doi: 10.1001/jamapsychiatry.2016.2594. PMID: 27732704.

60. Grzeskowiak LE, Morrison JL, Henriksen TB, et al. Prenatal antidepressant exposure and child behavioural outcomes at 7 years of age: a study within the Danish National Birth Cohort. BJOG. 2016 Nov;123(12):1919-28. doi: 10.1111/1471-0528.13611. PMID: 26374344.

61. El Marroun H, White TJ, Fernandez $\mathrm{G}$, et al. Prenatal exposure to selective serotonin reuptake inhibitors and non-verbal cognitive functioning in childhood. $\mathrm{J}$

Psychopharmacol. 2017

Mar;31(3):346-55. doi:

10.1177/0269881116665335. PMID: 27624153.

62. Huybrechts KF, Hernandez-Diaz S, Patorno E, et al. Antipsychotic use in pregnancy and the risk for congenital malformations. JAMA Psychiatry. 2016 Sep 1;73(9):938-46. doi: 10.1001/jamapsychiatry.2016.1520. PMID: 27540849.

63. Malm H, Brown AS, Gissler M, et al. Gestational exposure to selective serotonin reuptake inhibitors and offspring psychiatric disorders: a national register-based study. J Am Acad Child Adolesc Psychiatry. 2016 May;55(5):359-66. doi: 10.1016/j.jaac.2016.02.013. PMID: 27126849.

64. Almeida ND, Basso O, Abrahamowicz M, et al. Risk of miscarriage in women receiving antidepressants in early pregnancy, correcting for induced abortions. Epidemiology. 2016 Jul;27(4):53846. doi: 10.1097/ede.0000000000000484. PMID: 27031036.

65. Casilla-Lennon MM, Meltzer-Brody $\mathrm{S}$, Steiner AZ. The effect of antidepressants on fertility. Am J Obstet Gynecol. 2016 Sep;215(3):314.e1-5. doi: 10.1016/j.ajog.2016.01.170. PMID: 26827878.

66. Malm H, Sourander A, Gissler M, et al. Pregnancy complications following prenatal exposure to SSRIs or maternal psychiatric disorders: results from population-based national register data. Am J Psychiatry. 2015 Dec;172(12):122432. doi: 
10.1176/appi.ajp.2015.14121575.

PMID: 26238606.

67. Avalos LA, Chen H, Li DK.

Antidepressant medication use, depression, and the risk of preeclampsia. CNS Spectr. 2015

Feb;20(1):39-47. doi:

10.1017/s1092852915000024.

PMID: 25778691.

68. Liu X, Olsen J, Pedersen LH, et al.

Antidepressant use during pregnancy and asthma in the offspring.

Pediatrics. 2015 Apr;135(4):e911-7.

doi: 10.1542/peds.2014-4073.

PMID: 25755245.

69. Eriksen HL, Kesmodel US, Pedersen

LH, et al. No association between

prenatal exposure to psychotropics

and intelligence at age five. Acta

Obstet Gynecol Scand. 2015

May;94(5):501-7. doi:

10.1111/aogs.12611. PMID:

25711407.

70. Berard A, Zhao JP, Sheehy O.

Sertraline use during pregnancy and

the risk of major malformations. Am

J Obstet Gynecol. 2015

Jun;212(6):795.e1-.e12. doi:

10.1016/j.ajog.2015.01.034. PMID:

25637841.

71. Ban L, West J, Gibson JE, et al. First trimester exposure to anxiolytic and hypnotic drugs and the risks of major congenital anomalies: a United

Kingdom population-based cohort study. PLoS One.

2014;9(6):e100996. doi:

10.1371/journal.pone.0100996.

PMID: 24963627.

72. Ban L, Gibson JE, West J, et al. Maternal depression, antidepressant prescriptions, and congenital anomaly risk in offspring: a population-based cohort study. BJOG. 2014 Nov;121(12):1471-81. doi: 10.1111/1471-0528.12682.

PMID: 24612301.

73. Sorensen MJ, Gronborg TK, Christensen J, et al. Antidepressant exposure in pregnancy and risk of autism spectrum disorders. Clin Epidemiol. 2013;5:449-59. doi: 10.2147/clep.s53009. PMID: 24255601.

74. Palmsten K, Hernandez-Diaz S, Huybrechts KF, et al. Use of antidepressants near delivery and risk of postpartum hemorrhage: cohort study of low income women in the United States. BMJ. 2013 Aug 21;347:f4877. doi:

10.1136/bmj.f4877. PMID: 23965506.

75. Palmsten K, Huybrechts KF, Michels $\mathrm{KB}$, et al. Antidepressant use and risk for preeclampsia. Epidemiology. 2013 Sep;24(5):682-91. doi: 10.1097/EDE.0b013e31829e0aaa. PMID: 23873072.

76. Wisner KL, Bogen DL, Sit D, et al. Does fetal exposure to SSRIs or maternal depression impact infant growth? Am J Psychiatry. 2013 May;170(5):485-93. doi: 10.1176/appi.ajp.2012.11121873. PMID: 23511234.

77. Nulman I, Koren G, Rovet J, et al. Neurodevelopment of children following prenatal exposure to venlafaxine, selective serotonin reuptake inhibitors, or untreated maternal depression. Am J Psychiatry. 2012 Nov;169(11):116574. doi: 10.1176/appi.ajp.2012.11111721. PMID: 23128923.

78. Pedersen LH, Henriksen TB, Bech $\mathrm{BH}$, et al. Prenatal antidepressant exposure and behavioral problems in early childhood--a cohort study. Acta Psychiatr Scand. 2013 
Feb;127(2):126-35. doi:

10.1111/acps.12032. PMID:

23126521.

79. Ban L, Tata LJ, West J, et al. Live and non-live pregnancy outcomes among women with depression and anxiety: a population-based study. PLoS One. 2012;7(8):e43462. doi: 10.1371/journal.pone.0043462. PMID: 22937052.

80. Grzeskowiak LE, Gilbert AL, Morrison JL. Neonatal outcomes after late-gestation exposure to selective serotonin reuptake inhibitors. J Clin Psychopharmacol. 2012 Oct;32(5):615-21. doi: 10.1097/JCP.0b013e31826686bc. PMID: 22926594.

81. Palmsten K, Setoguchi S, Margulis $\mathrm{AV}$, et al. Elevated risk of preeclampsia in pregnant women with depression: depression or antidepressants? Am J Epidemiol. 2012 May 15;175(10):988-97. doi: 10.1093/aje/kwr394. PMID: 22442287.

82. Salisbury AL, Wisner KL, Pearlstein $\mathrm{T}$, et al. Newborn neurobehavioral patterns are differentially related to prenatal maternal major depressive disorder and serotonin reuptake inhibitor treatment. Depress Anxiety. 2011 Nov;28(11):1008-19. doi: 10.1002/da.20883. PMID: 21898709.

83. Ramos E, St-Andre M, Berard A. Association between antidepressant use during pregnancy and infants born small for gestational age. Can J Psychiatry. 2010 Oct;55(10):643-52. PMID: 20964943.

84. Toh S, Mitchell AA, Louik C, et al. Antidepressant use during pregnancy and the risk of preterm delivery and fetal growth restriction. J Clin

Psychopharmacol. 2009

Dec;29(6):555-60. doi:
10.1097/JCP.0b013e3181bf344c.

PMID: 19910720.

85. Lin HC, Chen IJ, Chen YH, et al. Maternal schizophrenia and pregnancy outcome: does the use of antipsychotics make a difference? Schizophr Res. 2010 Jan;116(1):5560. doi:

10.1016/j.schres.2009.10.011. PMID: 19896335.

86. Pearson $\mathrm{KH}$, Nonacs RM, Viguera AC, et al. Birth outcomes following prenatal exposure to antidepressants. J Clin Psychiatry. 2007 Aug;68(8):1284-9. PMID: 17854255.

87. Berard A, Ramos E, Rey E, et al. First trimester exposure to paroxetine and risk of cardiac malformations in infants: the importance of dosage. Birth Defects Res B Dev Reprod Toxicol. 2007 Feb;80(1):18-27. doi: 10.1002/bdrb.20099. PMID: 17187388.

88. Casper RC, Fleisher BE, LeeAncajas JC, et al. Follow-up of children of depressed mothers exposed or not exposed to antidepressant drugs during pregnancy. J Pediatr. 2003 Apr;142(4):402-8. doi: 10.1067/mpd.2003.139. PMID: 12712058.

89. Santucci AK, Singer LT, Wisniewski $\mathrm{SR}$, et al. One-year developmental outcomes for infants of mothers with bipolar disorder. J Clin Psychiatry. 2017 Sep/Oct;78(8):1083-90. doi: 10.4088/JCP.15m10535. PMID: 28068465.

90. Park Y, Hernandez-Diaz S, Bateman BT, et al. Continuation of atypical antipsychotic medication during early pregnancy and the risk of gestational diabetes. Am J 
Psychiatry. 2018;175(6):564-74. doi: 10.1176/appi.ajp.2018.17040393.

91. Jimenez-Solem E, Andersen JT, Petersen M, et al. Exposure to selective serotonin reuptake inhibitors and the risk of congenital malformations: a nationwide cohort study. BMJ Open. 2012;2(3). doi: 10.1136/bmjopen-2012-001148.

92. Juric S, Newport DJ, Ritchie JC, et al. Zolpidem (Ambien $\left.{ }^{\circledR}\right)$ in pregnancy: placental passage and outcome. Arch Womens Ment Health. 2009;12(6):441-6. doi: 10.1007/s00737-009-0100-7. PMID: 2009-21413-008.

93. Ramos É, St-André M, Rey É, et al. Duration of antidepressant use during pregnancy and risk of major congenital malformations. Br J Psychiatry. 2008;192(5):344-50. doi: 10.1192/bjp.bp.107.042523. PMID: 2008-07004-007.

94. Wisner KL, Sit D, O'Shea K, et al. Bipolar disorder and psychotropic medication: impact on pregnancy and neonatal outcomes. J Affect Disord. 2019 Jan 15;243:220-5. doi: 10.1016/j.jad.2018.09.045. PMID: 30248632.

95. Güngör BB, Öztürk N, Atar AÖ, et al. Comparison of the groups treated with mirtazapine and selective serotonine reuptake inhibitors with respect to birth outcomes and severity of psychiatric disorder.

Psychiatry and Clinical

Psychopharmacology. 2019;29(4):822-31. doi: 10.1080/24750573.2019.1673936.

96. Cohen JM, Huybrechts KF, Patorno E, et al. Anticonvulsant mood stabilizer and lithium use and risk of adverse pregnancy outcomes. J Clin Psychiatry. 2019 Jun 18;80(4). doi:
10.4088/JCP.18m12572. PMID: 31237992.

97. Galbally M, Frayne J, Watson SJ, et al. The association between gestational diabetes mellitus, antipsychotics and severe mental illness in pregnancy: A multicentre study. Aust N Z J Obstet Gynaecol. 2020 Feb;60(1):63-9. doi: 10.1111/ajo.12986. PMID: 31141172.

98. Huybrechts KF, Bateman BT, Pawar A, et al. Maternal and fetal outcomes following exposure to duloxetine in pregnancy: cohort study. BMJ. 2020 Feb 19;368:m237. doi: 10.1136/bmj.m237. PMID: 32075794.

99. Palmsten K, Chambers CD, Wells A, et al. Patterns of prenatal antidepressant exposure and risk of preeclampsia and postpartum haemorrhage. Paediatr Perinat Epidemiol. 2020 Mar 24. doi: 10.1111/ppe.12660. PMID: 32207549.

100. Sheehy O, Zhao JP, Bérard A. Association between incident exposure to benzodiazepines in early pregnancy and risk of spontaneous abortion. JAMA Psychiatry. 2019 May 15;76(9):948-57. doi: 10.1001/jamapsychiatry.2019.0963. PMID: 31090881.

101. Singal D, Chateau D, Struck S, et al. In utero antidepressants and neurodevelopmental outcomes in kindergarteners. Pediatrics. 2020 May;145(5). doi: 10.1542/peds.2019-1157. PMID: 32341177.

102. Wall-Wieler E, Robakis TK, Cesta $\mathrm{CE}$, et al. Antidepressant use around conception, prepregnancy depression, and risk of ectopic pregnancy. Can J Psychiatry. 2020 
May 21:706743720927829. doi:

10.1177/0706743720927829. PMID:

32436752 .

103. Wall-Wieler E, Robakis TK, Lyell

DJ, et al. Benzodiazepine use before conception and risk of ectopic pregnancy. Hum Reprod. 2020 Jun 3. doi: 10.1093/humrep/deaa082.

PMID: 32485732.

104. Wartko PD, Weiss NS, Enquobahrie DA, et al. Antidepressant continuation in pregnancy and risk of gestational diabetes.

Pharmacoepidemiol Drug Saf. 2019

Sep;28(9):1194-203. doi:

10.1002/pds.4799. PMID: 31298445.

105. Yamamoto-Sasaki M, Yoshida S,

Takeuchi M, et al. Association between antidepressant use during pregnancy and autism spectrum disorder in children: a retrospective cohort study based on Japanese claims data. Matern Health Neonatol Perinatol. 2019;5:1. doi: 10.1186/s40748-018-0096-y. PMID: 30652008 .

106. Anderson KN, Lind JN, Simeone $\mathrm{RM}$, et al. Maternal use of specific antidepressant medications during early pregnancy and the risk of selected birth defects. JAMA Psychiatry. 2020 Aug 5. doi: 10.1001/jamapsychiatry.2020.2453. PMID: 32777011.

107. Sivathamboo N, Hitchcock A, Graham J, et al. The use of antidepressant drugs in pregnant women with epilepsy: a study from the Australian Pregnancy Register. Epilepsia. 2018 Sep;59(9):1696-704. doi: 10.1111/epi.14539. PMID: 30142256.

108. Patorno E, Huybrechts KF, Bateman BT, et al. Lithium use in pregnancy and the risk of cardiac malformations. N Engl J Med. 2017
Jun 8;376(23):2245-54. doi: 10.1056/NEJMoa1612222. PMID: 28591541.

109. Frayne J, Nguyen T, Bennett K, et al. The effects of gestational use of antidepressants and antipsychotics on neonatal outcomes for women with severe mental illness. Aust N Z J Obstet Gynaecol. 2017 Oct;57(5):526-32. doi: 10.1111/ajo.12621. PMID: 28386942.

110. Mao Y, Pedersen LH, Christensen J, et al. Prenatal exposure to antidepressants and risk of epilepsy in childhood. Pharmacoepidemiol Drug Saf. 2016 Nov;25(11):1320-30. doi: 10.1002/pds.4072. PMID: 27477111.

111. Berard A, Boukhris T, Sheehy O. Selective serotonin reuptake inhibitors and autism: additional data on the Quebec Pregnancy/Birth Cohort. Am J Obstet Gynecol. 2016 Dec;215(6):803-5. doi: 10.1016/j.ajog.2016.08.021. PMID: 27589900.

112. De Ocampo MPG, Araneta MRG, Macera CA, et al. Risk of gestational hypertension and preeclampsia in women who discontinued or continued antidepressant medication use during pregnancy. Arch Womens Ment Health. 2016 Dec;19(6):105161. doi: 10.1007/s00737-016-0655-z. PMID: 27558246.

113. Cesta CE, Viktorin A, Olsson H, et al. Depression, anxiety, and antidepressant treatment in women: association with in vitro fertilization outcome. Fertil Steril. 2016 Jun;105(6):1594-602.e3. doi: 10.1016/j.fertnstert.2016.01.036. PMID: 26920258.

114. Boukhris T, Sheehy O, Mottron L, et al. Antidepressant use during 
pregnancy and the risk of autism spectrum disorder in children. JAMA Pediatr. 2016 Feb;170(2):117-24.

doi:

10.1001/jamapediatrics.2015.3356. PMID: 26660917.

115. Salisbury AL, O'Grady KE, Battle $\mathrm{CL}$, et al. The roles of maternal depression, serotonin reuptake inhibitor treatment, and concomitant benzodiazepine use on infant neurobehavioral functioning over the first postnatal month. Am J

Psychiatry. 2016 Feb 1;173(2):14757. doi:

10.1176/appi.ajp.2015.14080989.

PMID: 26514656.

116. Winterfeld U, Klinger G, Panchaud A, et al. Pregnancy outcome following maternal exposure to mirtazapine: a multicenter, prospective study. J Clin

Psychopharmacol. 2015

Jun;35(3):250-9. doi: 10.1097/jcp.0000000000000309.

PMID: 25830592.

117. Hviid A, Melbye M, Pasternak B. Use of selective serotonin reuptake inhibitors during pregnancy and risk of autism. N Engl J Med. 2013 Dec 19;369(25):2406-15. doi:

10.1056/NEJMoa1301449. PMID: 24350950

118. Lupattelli A, Spigset O, Koren G, et al. Risk of vaginal bleeding and postpartum hemorrhage after use of antidepressants in pregnancy: a study from the Norwegian Mother and Child Cohort Study. J Clin

Psychopharmacol. 2014

Feb;34(1):143-8. doi:

10.1097/jcp.0000000000000036.

PMID: 24135843.

119. Johnson KC, LaPrairie JL, Brennan

PA, et al. Prenatal antipsychotic exposure and neuromotor performance during infancy. Arch Gen Psychiatry. 2012

Aug;69(8):787-94. doi:

10.1001/archgenpsychiatry.2012.160

. PMID: 22474072.

120. Reebye PN, Ng TW, Misri S, et al. Affect expression and self-regulation capacities of infants exposed in utero to psychotropics. Front Psychiatry. 2012;3:11. doi: 10.3389/fpsyt.2012.00011. PMID: 22403552.

121. Klieger-Grossmann C, Weitzner B, Panchaud A, et al. Pregnancy outcomes following use of escitalopram: a prospective comparative cohort study. J Clin Pharmacol. 2012 May;52(5):766-70. doi: $10.1177 / 0091270011405524$. PMID: 22075232.

122. Nakhai-Pour HR, Broy P, Berard A. Use of antidepressants during pregnancy and the risk of spontaneous abortion. CMAJ. 2010 Jul 13;182(10):1031-7. doi: 10.1503/cmaj.091208. PMID: 20513781.

123. Pedersen LH, Henriksen TB, Vestergaard M, et al. Selective serotonin reuptake inhibitors in pregnancy and congenital malformations: population based cohort study. BMJ. 2009 Sep 23;339:b3569. doi: 10.1136/bmj.b3569. PMID: 19776103.

124. Rampono J, Simmer K, Ilett KF, et al. Placental transfer of SSRI and SNRI antidepressants and effects on the neonate. Pharmacopsychiatry. 2009 May;42(3):95-100. doi: 10.1055/s-0028-1103296. PMID: 19452377.

125. Newham JJ, Thomas SH, MacRitchie K, et al. Birth weight of infants after maternal exposure to 
typical and atypical antipsychotics: prospective comparison study. Br J Psychiatry. 2008 May;192(5):333-7. doi: 10.1192/bjp.bp.107.041541. PMID: 18450655.

126. Davis RL, Rubanowice D, McPhillips H, et al. Risks of congenital malformations and perinatal events among infants exposed to antidepressant medications during pregnancy. Pharmacoepidemiol Drug Saf. 2007 Oct;16(10):1086-94. doi: 10.1002/pds.1462. PMID: 17729378.

127. Newport DJ, Calamaras MR, DeVane CL, et al. Atypical antipsychotic administration during late pregnancy: placental passage and obstetrical outcomes. Am J Psychiatry. 2007 Aug;164(8):121420. doi:

10.1176/appi.ajp.2007.06111886.

PMID: 17671284.

128. Djulus J, Koren G, Einarson TR, et al. Exposure to mirtazapine during pregnancy: a prospective, comparative study of birth outcomes. J Clin Psychiatry. 2006 Aug;67(8):1280-4. PMID: 16965209.

129. Misri S, Reebye P, Kendrick K, et al. Internalizing behaviors in 4-year-old children exposed in utero to psychotropic medications. Am J Psychiatry. 2006 Jun;163(6):102632. doi: 10.1176/ajp.2006.163.6.1026. PMID: 16741203.

130. Chun-Fai-Chan B, Koren G, Fayez I, et al. Pregnancy outcome of women exposed to bupropion during pregnancy: a prospective comparative study. Am J Obstet Gynecol. 2005 Mar;192(3):932-6. doi: 10.1016/j.ajog.2004.09.027. PMID: 15746694.
131. Kallen B. Neonate characteristics after maternal use of antidepressants in late pregnancy. Arch Pediatr Adolesc Med. 2004 Apr;158(4):3126. doi: 10.1001/archpedi.158.4.312. PMID: 15066868.

132. Oberlander TF, Misri S, Fitzgerald $\mathrm{CE}$, et al. Pharmacologic factors associated with transient neonatal symptoms following prenatal psychotropic medication exposure. J Clin Psychiatry. 2004 Feb;65(2):2307. PMID: 15003078.

133. Simon GE, Cunningham ML, Davis RL. Outcomes of prenatal antidepressant exposure. Am J Psychiatry. 2002 Dec;159(12):205561. doi: 10.1176/appi.ajp.159.12.2055. PMID: 12450956.

134. Nulman I, Rovet J, Stewart DE, et al. Child development following exposure to tricyclic antidepressants or fluoxetine throughout fetal life: a prospective, controlled study. Am J Psychiatry. 2002 Nov;159(11):188995. doi:

10.1176/appi.ajp.159.11.1889. PMID: 12411224.

135. Einarson A, Fatoye B, Sarkar M, et al. Pregnancy outcome following gestational exposure to venlafaxine: a multicenter prospective controlled study. Am J Psychiatry. 2001 Oct;158(10):1728-30. doi: 10.1176/appi.ajp.158.10.1728. PMID: 11579012.

136. Nulman I, Rovet J, Stewart DE, et al. Neurodevelopment of children exposed in utero to antidepressant drugs. N Engl J Med. 1997 Jan 23;336(4):258-62. doi: 10.1056/nejm199701233360404. PMID: 8995088.

137. Kallen B, Tandberg A. Lithium and pregnancy. A cohort study on manic- 
depressive women. Acta Psychiatr

Scand. 1983 Aug;68(2):134-9.

PMID: 6624510.

138. Sadowski A, Todorow M, Brojeni

PY, et al. Pregnancy outcomes

following maternal exposure to

second-generation antipsychotics

given with other psychotropic drugs:

a cohort study. BMJ Open.

2013;3(7). doi: 10.1136/bmjopen-

2013-003062.

139. Babu GN, Desai G, Tippeswamy H, et al. Birth weight and use of olanzapine in pregnancy: a prospective comparative study. J Clin Psychopharmacol.

2010;30(3):331-2. doi:

10.1097/JCP.0b013e3181db8734.

PMID: 2010-10616-020.

140. Galbally M, Watson SJ, Boyce P, et al. The mother, the infant and the mother-infant relationship: What is the impact of antidepressant medication in pregnancy. J Affect Disord. 2020;272:363-70. doi: 10.1016/j.jad.2020.03.116.

141. Desai PH, Yagnik PJ, Ross Ascuitto $\mathrm{N}$, et al. Risk of congenital heart disease in newborns with prenatal exposure to anti-depressant medications. Cureus. 2019 May 15;11(5):e4673. doi:

10.7759/cureus.4673. PMID: 31328065.

142. Lupattelli A, Chambers CD, Bandoli $\mathrm{G}$, et al. Association of maternal use of benzodiazepines and z-hypnotics during pregnancy with motor and communication skills and attentiondeficit/hyperactivity disorder symptoms in preschoolers. JAMA

Netw Open. 2019 Apr

5;2(4):e191435. doi:

10.1001/jamanetworkopen.2019.143

5. PMID: 30951155.
143. Perrotta C, Giordano F, Colombo A, et al. Postpartum bleeding in pregnant women receiving SSRIs/SNRIs: new insights from a descriptive observational study and an analysis of data from the FAERS database. Clin Ther. 2019

Sep;41(9):1755-66. doi: 10.1016/j.clinthera.2019.06.008. PMID: 31371035.

144. Richardson JL, Martin F, Dunstan H, et al. Pregnancy outcomes following maternal venlafaxine use: A prospective observational comparative cohort study. Reprod Toxicol. 2019 Mar;84:108-13. doi: 10.1016/j.reprotox.2019.01.003. PMID: 30639403.

145. Thombre Kulkarni M, Holzman C, Wasilevich E, et al. Pregnancy hypertension and its associations with pre-pregnancy depression, anxiety, antidepressants, and anxiolytics. Pregnancy Hypertens. 2019 Apr;16:67-74. doi: 10.1016/j.preghy.2019.03.003. PMID: 31056161.

146. Cole JA, Modell JG, Haight BR, et al. Bupropion in pregnancy and the prevalence of congenital malformations. Pharmacoepidemiol Drug Saf. 2007 May;16(5):474-84. doi: 10.1002/pds.1296. PMID: 16897811.

147. Milliren CE, Gupta M, Graham DA, et al. Hospital variation in neonatal abstinence syndrome incidence, treatment modalities, resource use, and costs across pediatric hospitals in the United States, 2013 to 2016. Hosp Pediatr. 2018 Jan;8(1):15-20. doi: 10.1542/hpeds.2017-0077. PMID: 29263122.

148. Steurer MA, Baer RJ, Oltman S, et al. Morbidity of persistent pulmonary hypertension of the 
newborn in the first year of life. J Pediatr. 2019 Oct;213:58-65 e4. doi: 10.1016/j.jpeds.2019.06.053. PMID: 31399244

149. Botto LD, Lin AE, Riehle-Colarusso $\mathrm{T}$, et al. Seeking causes: classifying and evaluating congenital heart defects in etiologic studies. Birth Defects Res A Clin Mol Teratol. 2007 Oct; 79(10):714-27. doi: 10.1002/bdra.20403. PMID: 17729292.

150. Sage Therapeutics I. ZULRESSO. Highlights of prescribing information. Medication guide. Cambridge, MA: Sage Therapeutics, Inc; 2019.

https://assets.sagerx.com/zulresso/pr escribing-information.pdf. Accessed on February 6, 2020.

151. U.S. Food and Drug Administration. Highlights of prescribing information. Reference ID: 4405779. Silver Spring, MD: U.S. Food and Drug Administration; 2019. https://www.accessdata.fda.gov/drug satfda docs/label/2019/211371lbl.pd f. Accessed on February 17, 2020.

152. Koechlin H, Donado C, Berde CB, et al. Effects of childhood life events on adjustment problems in adolescence: A longitudinal study. J Dev Behav Pediatr. 2018;39(8):62941. PMID: 2019-06562-004.

153. Chaki S, Funakoshi T, Hirota-Okuno $\mathrm{S}$, et al. Anxiolytic- and antidepressant-like profile of ATC0065 and ATC0175: nonpeptidic and orally active melanin-concentrating hormone receptor 1 antagonists. J Pharmacol Exp Ther. 2005 May;313(2):831-9. doi: 10.1124/jpet.104.081711. PMID: 15677346.

154. Abouleish E, Taylor FH. Effect of morphine-diazepam on signs of anesthesia, awareness, and dreams of patients under $\mathrm{N} 2 \mathrm{O}$ for cesarean section. Anesth Analg. 1976 SepOct;55(5):702-5. PMID: 987727.

155. Wilson CA, Terry AV, Jr. Variable maternal stress in rats alters locomotor activity, social behavior, and recognition memory in the adult offspring. Pharmacol Biochem Behav. 2013 Mar;104:47-61. doi: 10.1016/j.pbb.2012.12.015. PMID: 23287801 . 